\title{
Water Resources of Monroe County, New York, Water Years 1989-93, with Emphasis on Water Quality in the Irondequoit Creek Basin
}

Part 1. Water-Resources Data

\section{By DONALD A. SHERWOOD}

Prepared in cooperation with the

Monroe County Department of Health 


\section{BRUCE BABBITT, Secretary}

\section{U.S. GEOLOGICAL SURVEY}

Mark Schaefer, Acting Director

Any use of trade, product, or firm names in this publication is for descriptive purposes only and does not imply endorsement by the U.S. Geological Survey

For additional information write to:

Subdistrict Chief

U.S. Geological Survey 903 Hanshaw Road Ithaca, NY 14850
Copies of this report can be purchased from:

U.S. Geological Survey Branch of Information Services Box 25286

Denver, CO 80225-0286 


\section{CONTENTS}

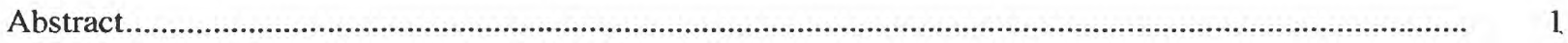

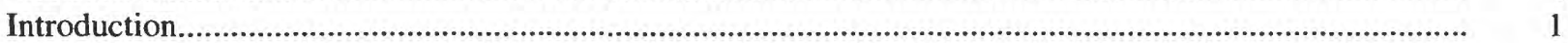

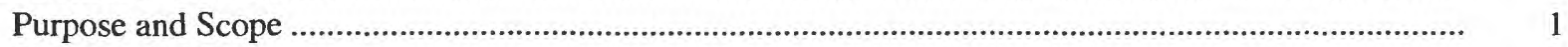

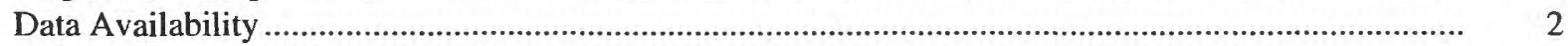

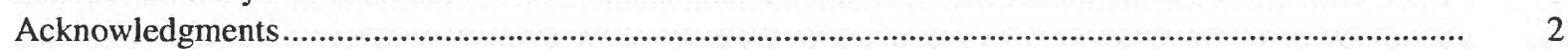

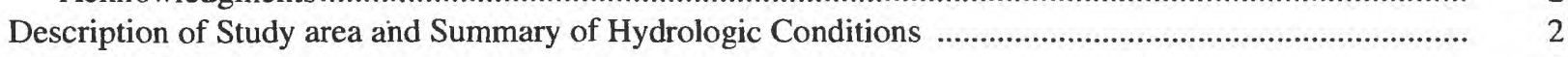

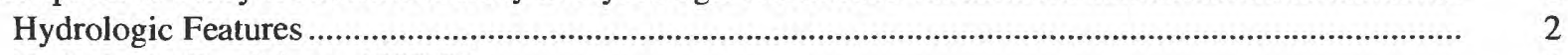

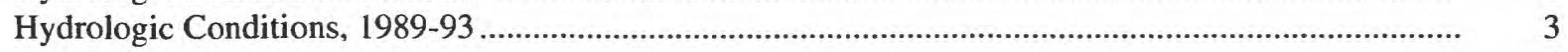

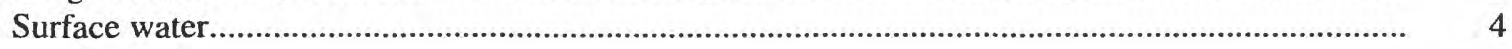

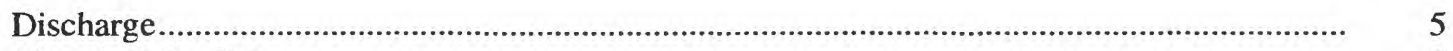

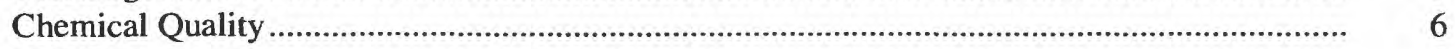

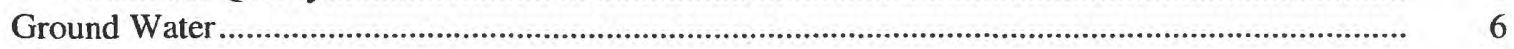

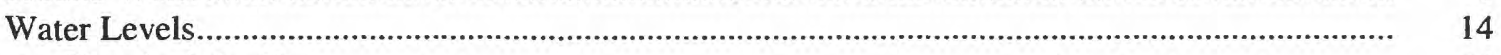

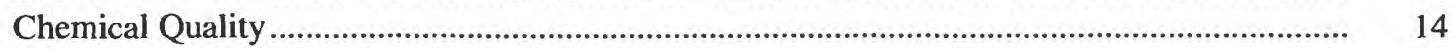

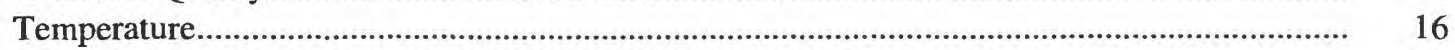

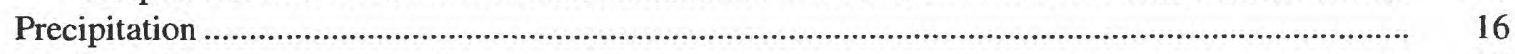

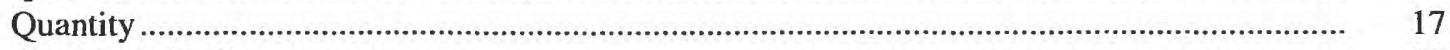

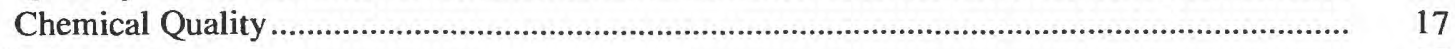

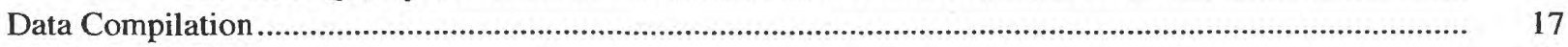

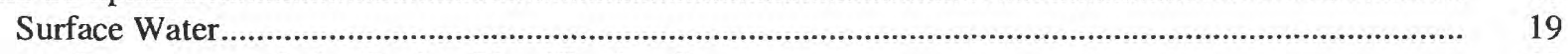

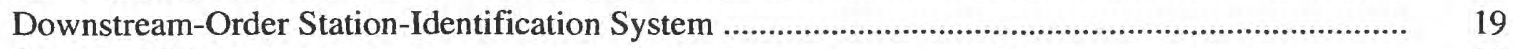

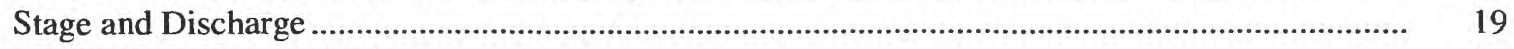

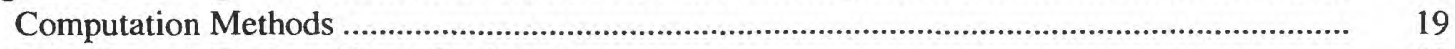

Data Format (Surface-Water Stations) ................................................................................... 20

Accuracy and Precision of Records ......................................................................................... 22

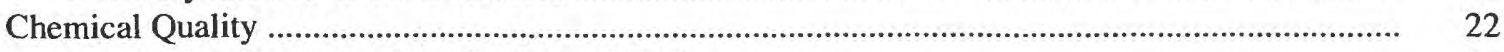

Continuing-Record and Partial-Record Stations ....................................................................... 22

Field and Laboratory Methods.............................................................................................. 22

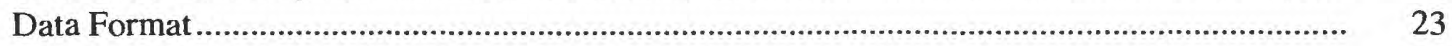

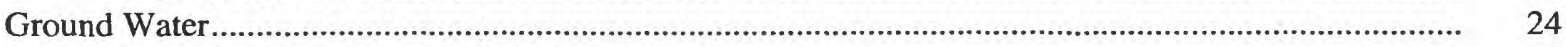

Latitude and Longitude Identification System .......................................................................... 24

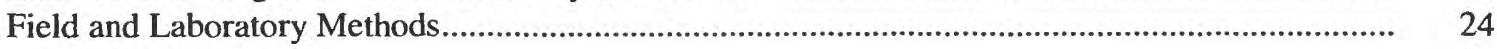

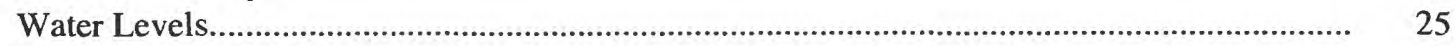

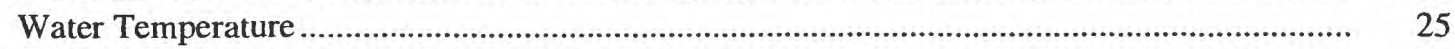

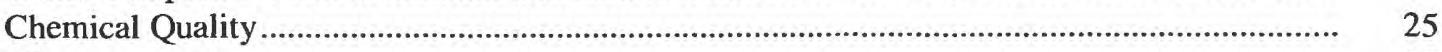

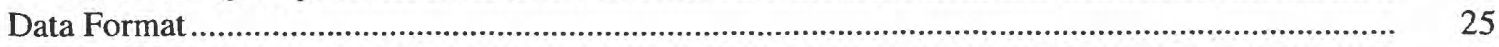

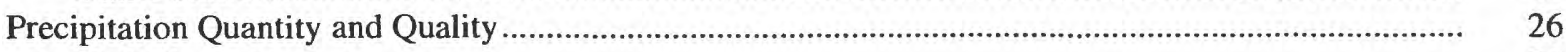

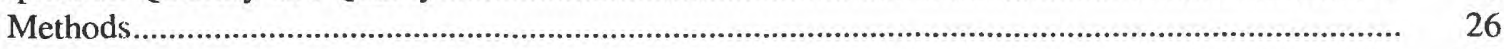

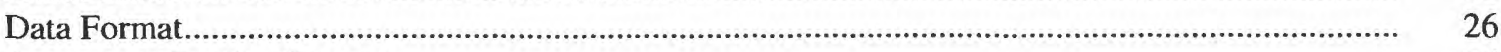

Quality Assurance/Quality Control .................................................................................................... 26

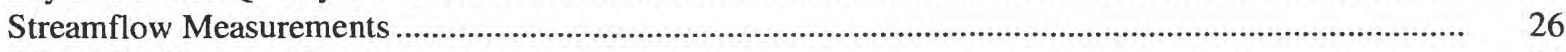

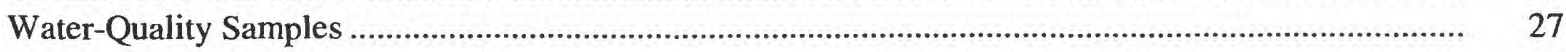

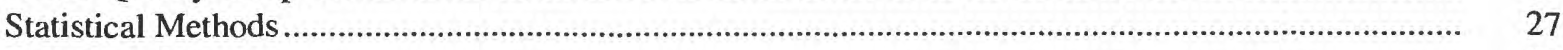

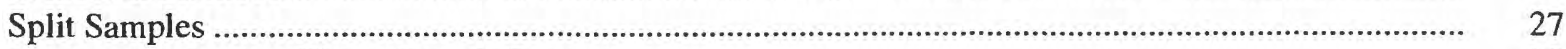

Standard Reference Water-Sample Program ....................................................................................... 29

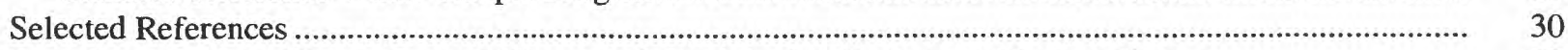

Data From Surface-Water, Ground-Water, and Precipitation Stations in Monroe County .......................... 32

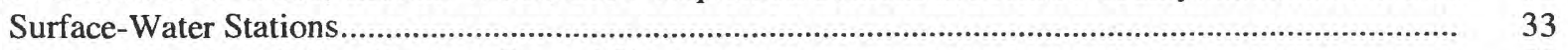

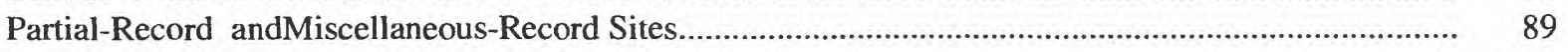

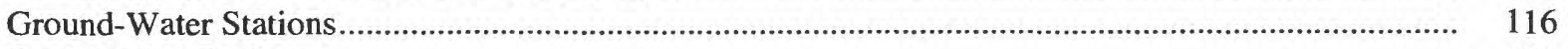

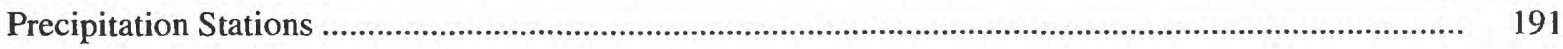




\section{FIGURES}

1. Map of Monroe County showing location of streamflow-gaging stations and principal geographic features

2. Map showing location of gages within Irondequoit Creek watershed.

3. Hydrographs for Allen Creek near Rochester showing monthly mean discharge throughout given water years with median of monthly mean discharges for period of record, and daily discharges throughout given water years with median discharge for $1970-88$ period of record:
A. Water year 1989
B. Water year 1990
C. Water year 1991
D. Water year 1992
E. Water year 1993

4. Box plots showing concentrations of eight constituents in samples from Northrup Creek at North Greece and six sites in the Irondequoit Creek basin.

5. Box plots showing concentrations of eight constituents in water samples from Allen Creek near Rochester and Irondequoit Creek at Blossom road for water years 1984-88 and 1989-93

6. Hydrograph showing water levels for water years 1989-93 at well Ot 900 in northern Ontario County with monthly mean, maximum, and minimum water level throughout period of record, 1955-88

7. Graph showing water-temperature profiles from a well screened in (A) a confined aquifer, and (B) a shallow water-table in Powder Mill Park, 1989-93

8. Graphs showing monthly precipitation at Rochester Airport, October 1989 through September 1993 and normal monthly precipitation based on average for 1951-80.....

9. Diagram showing system for numbering wells

\section{TABLES}

1. Annual mean discharges, water year 1989-93, with average discharge for the period of record for selected stations in Monroe County

2. Annual mean, maximum, and minimum water levels for water years 1989-93 and those for period of record 1955-83 at well Ot 900

3. Total annual precipitation at Rochester Airport, by water year, and departures from the normal of 31.27 inches per year

4. Statistical analysis of split samples collected to determine representativeness of samples collected by automatic sampler

5. Statistical analysis of split samples collected for laboratory comparison.

6. Rating of cooperating laboratory's analysis of U.S. Geological Survey standard reference water samples. 


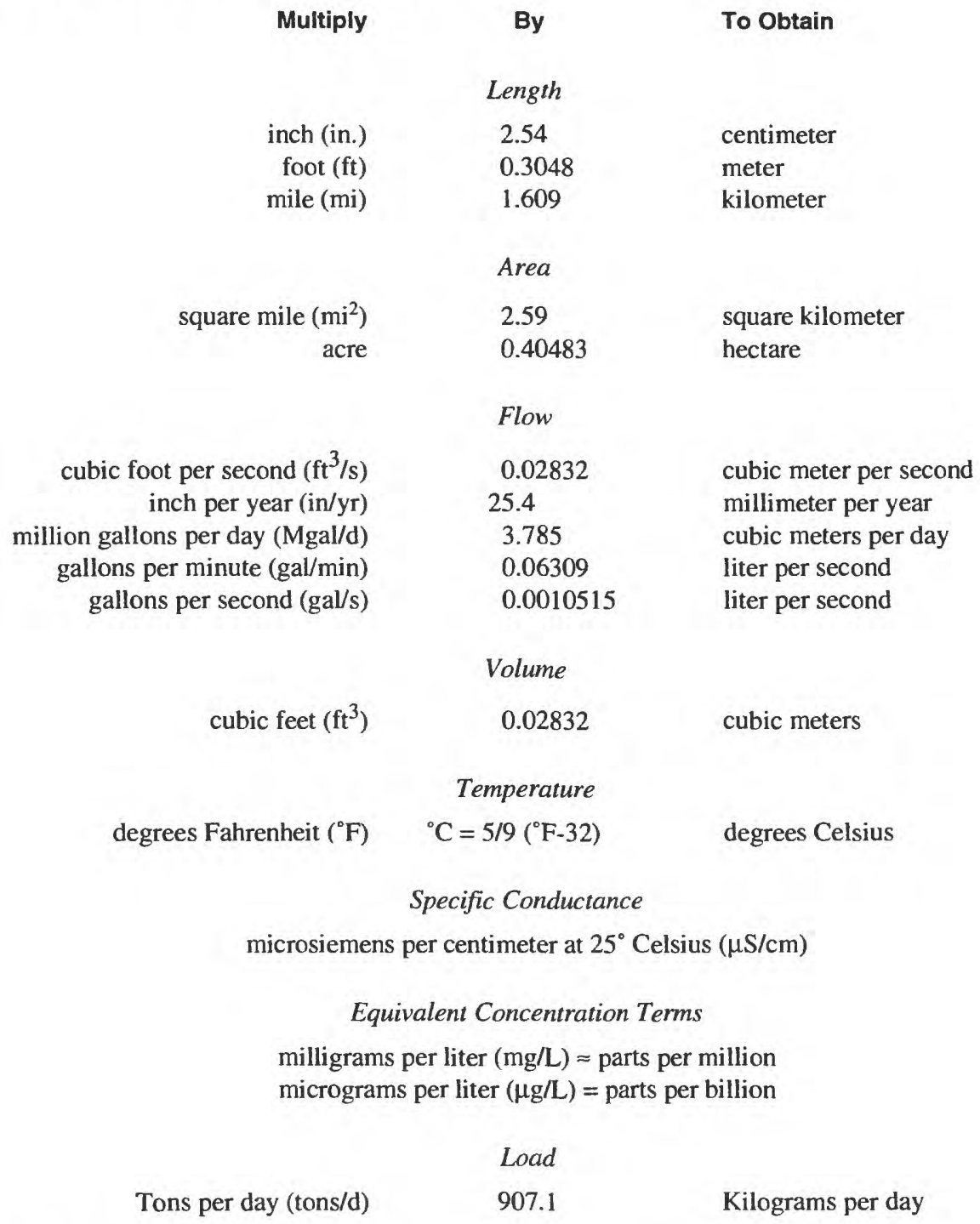

National Geodetic Vertical Datum of 1929 (NGVD) is a geodetic datum derived from a general adjustment of the first-order level nets of both the United States and Canada, formerly called Sea Level Datum of 1929. 


\title{
Water Resources of Monroe County, New York, Water Years 1989-93, with Emphasis on Water Quality in the Irondequoit Creek Basin Part 1. Water-Resources Data
}

\author{
By Donald A. Sherwood
}

\section{Abstract}

Water quality in the 169-square-mile Irondequoit Creek basin, in eastern Monroe County, N.Y., has been documented for more than 100 years. In the past, sediment and contamination carried by Irondequioit Creek have contributed to the accelerated eutrophication of Irondequoit bay on Lake Ontario. During 198081, the U.S. Geological Survey and the Monroe County Environmental Health Laboratory collected streamflow and water-quality data in the Irondequoit Creek basin near Rochester, as part of the National Urban Runoff Program (NURP) study, and have continued to collect data at several sites in the basin and elsewhere in Monroe County to document changes in the concentrations of chemical constituents since the beginning of the NURP study. Data collected during 198488 are given in two companion reports; this third report presents data collected during 1989-93.

This report includes records of: (1) streamflow, and chemical quality of streams; (2) groundwater levels and quality of ground water; and (3) quantity and quality of precipitation in Monroe County, during water years 1989-93, by water year. Streamflow and (or) water-quality data were collected at 14 sites, four of which were discontinued during the 1989-93 period covered by this report. Ground-water levels were measured and water-samples collected at 15 wells. Precipitation-volume data were collected at four sites, and atmospheric-quality (wetfall, dustfall, and bulk deposition) data at three.

\section{INTRODUCTION}

Water quality in the $169-\mathrm{mi}^{2}$ Irondequoit Creek basin, in eastern Monroe County, N.Y., has been documented for more than 100 years. In the past, sediment and contamination carried by Irondequoit Creek have contributed to the accelerated eutrophication of Irondequoit bay on Lake Ontario. In 1979, the U. S. Geological Survey (USGS), in cooperation with the Monroe County Department of Health, monitored the quantity and quality of storm runoff in the Irondequoit Creek basin (Zarriello and others, 1984; Kappel and others, 1986) as part of the National Urban Runoff Program (NURP). Since completion of that study in 1981, the USGS, the Monroe County Health Department, and the Monroe County Environmental Health Laboratory (MCEHL) have continued to collect data on precipitation, streamflow, and ground water at selected sites in the basin to document temporal changes in concentration and loads of chemical constituents and to evaluate the success of county programs designed to improve water quality in the basin.

Data collected during 1984-88 are presented in Johnston and Sherwood (1994) and interpreted in Johnston and Sherwood (1996). This report, the third in the series, includes stream-discharge and (or) waterquality data from fourteen stream-gaging stations, precipitation data from four sites, and ground-water data from 15 wells during 1989-93.

\section{Purpose and Scope}

This report summarizes the hydrologic conditions in Monroe County from October 1988 through September 1993 in hydrographs and box plots and presents (1) records of chemical quality of streams; (2) 
ground-water levels and quality at wells; and (3) chemical quality and daily precipitation at rain gages; statistical summaries of records of streamflow are included. The records represent (1) streamflow and water quality at eight continuous-record gaging stations; (2) water levels, chemical quality and temperature at 15 observation wells; and (3) precipitation quality at three rain-gage sites, and total daily precipitation at four sites. This report does not contain data that have been previously published in the USGS annual water-data reports. Data collected during 1984-88 are given in two companion reports; This report presents data collected during 1989-93. All hydrologic data collected in Monroe County since 1993 have been published annually in the USGS New York water data report.

\section{Data Availability}

Long-term streamflow data from the St. Lawrence River Basin collected in Monroe County by the USGS through cooperative programs with State and other federal agencies, are available from USGS files, as are water-quality data from Genesee River at Charlotte Docks at Rochester (station discontinued in 1994) near the mouth of the Genesee River. These long-term data can be useful in defining historical trends in streamflow and water quality.

All information used in preparation of records in this report, such as discharge-measurement notes, water-temperature measurements, gage-height records, and rating tables, is on file in the USGS office in Ithaca, N.Y. Most gaging-station records are also available in computer-readable form, and many statistical analyses are available. Information on the availability of unpublished data or statistical analyses can be obtained from the USGS office in Ithaca or Troy, N.Y.

Records of streamflow, chemical quality of streams, ground-water levels, chemical quality of ground water, precipitation amounts, and chemical quality of atmospheric deposition for water years 1984-88 are given in Johnston and Sherwood (1994).

\section{Acknowledgments}

Special thanks are extended to MCEHL for assistance in the collection, analysis, and verification of the data presented in this report. Richard Burton, lab- oratory administrator, provided guidance and suggestions throughout the data-collection period. Charles Knauf of MCEHL organized and prepared the chemical-quality data for entry into the USGS data base.

Staff of the Powder Mill Park fish hatchery assisted in the measurement of ground-water levels at the Powder Mill Park wells.

\section{DESCRIPTION OF STUDY AREA AND SUMMARY OF HYDROLOGIC CONDITIONS}

Monroe County, in the Lake Ontario Plain region of western New York (fig. 1), has a total area of about $673 \mathrm{mi}^{2}$ (Heffner and Goodman, 1973). Rochester, the county seat and largest city, is in the northern part of the county.

\section{Hydrologic Features}

The Genesee River, the largest in Monroe County, has a drainage area of $2,480 \mathrm{mi}^{2}$ at its mouth (Wagner and Dixson, 1985) and flows northward through the city of Rochester into Lake Ontario. Streams in the several smaller drainage basins (ranging from less than $5 \mathrm{mi}^{2}$ to about $88 \mathrm{mi}^{2}$ ) west of the Genesee River flow northeastward into Lake Ontario or one of the several bays of the western part of the Rochester Embayment, and those in several small drainage basins (ranging from less than $0.2 \mathrm{mi}^{2}$ in size to nearly $24 \mathrm{mi}^{2}$ ) east of the Genesee River flow north or northwestward into Lake Ontario and the Irondequoit Creek basin (169 $\mathrm{mi}^{2}$ ).

Irondequoit Creek drains into Lake Ontario through Irondequoit Bay (fig. 2). The drainage basin is mostly in eastern Monroe County and includes drainage from the east side of the city of Rochester and from neighboring Ontario and Wayne Counties. A more complete basin description, that includes stormwater and sanitary-sewer systems, drinking-water supplies, surface geology and climate, is given in Kappel and others (1986). The glacial history and geohydrology of the Irondequoit Creek valley are discussed in Kappel and Young (1989).

The Erie Canal flows southeastward through the middle of the county and receives flow from the headwater areas of many of these streams. Diversion structures at several points along the canal allow water to 


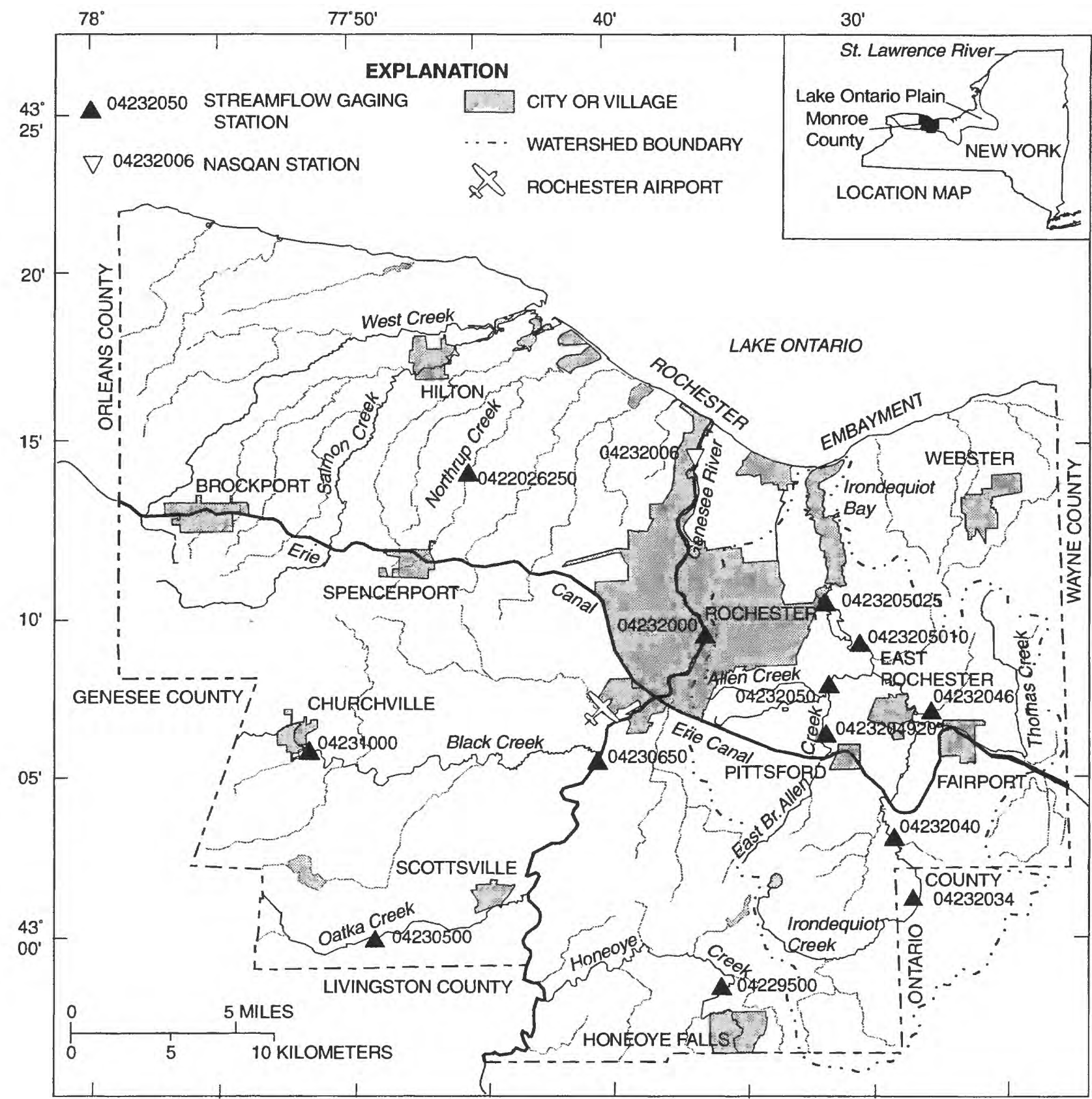

Base from U.S. Geological Survey

Digital line graph, 1:100,000

Figure 1. Location of streamflow-gaging stations and principal geographic features of Monroe County, N.Y. (From Johnston and Sherwood, 1996, fig. 1).

be discharged from the canal to augment the flow of several small streams during low-flow conditions. The canal crosses the Genesee River 11.8 mi upstream from its mouth. Water diverted by the canal from Lake Erie is discharged into the Genesee River from the west; a smaller amount of water is then diverted from the Genesee River eastward into the canal.

\section{Hydrologic Conditions during 1989-93, by Water Year}

Hydrologic conditions in Monroe County fluctuated considerably during water years 1989-93. Surface-water, ground-water, and precipitation patterns are discussed separately further on. 


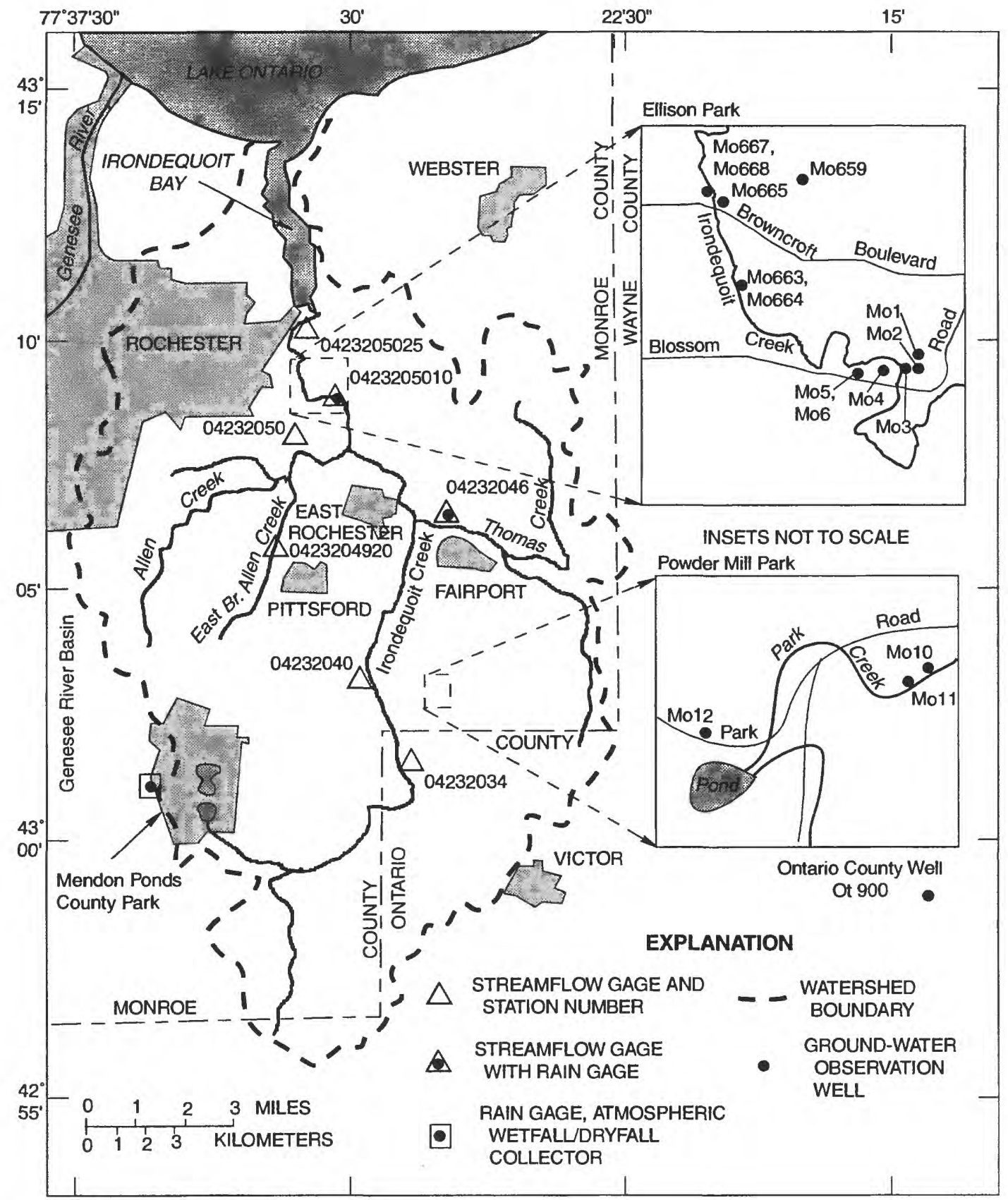

Base from U.S. Geological Survey State base map 1:500,000, 1974

Figure 2. Locations of gages and wells within the Irondequoit Creek watershed, Monroe County, N.Y. (From Johnston and Sherwood, 1996, fig. 1)

Annual mean stream discharges in the county (table 1) ranged from 77 percent to 145 percent of long-term averages, and annual mean ground-water levels in a long-term observation well (Ot 900) southeast of Monroe County (fig. 1), were below normal. Total precipitation for the 5-year period, recorded at the Rochester Airport, was slightly more than 8 in. above normal, however.

\section{Surface Water}

Discharge-frequency statistics were computed for stations on unregulated streams with more than 9 years of record and were compared with 1989-93 data to indicate the representativeness of streamflow during 1989-93. Records from Oatka Creek at Garbutt and Black Creek at Churchville, each with 48 years of 
record, were judged suitable for hydrologic comparison (table 1). In the Irondequoit Creek basin, only Allen Creek near Rochester, with 33 years of record, and Irondequoit Creek at Blossom Road, with 13 years of record, have been in operation long enough to provide a record adequate for hydrologic comparison.

Although streamflow at these sites is affected by minor diversions from the Erie Canal (as at several other sites in the basin), comparison of mean annual flows (table 1) indicates similar departures, for most years from the period-of-record average discharge.

\section{Discharge}

Annual mean discharges in north-central New York State during water years 1989-93 ranged from above average to below average (table 1). Monthly means for Allen Creek near Rochester during the same period were commonly well above or below the median value for the particular month. The median of monthly means was determined from a statistical analysis of daily streamflow data that computed monthly means for all months of water years 1970-88. The means for each month were then ranked, and the median selected such that, for any particular month, 50 percent of the values would fall above the median, and 50 percent below.

The normal range for daily values was determined from a statistical analysis of daily streamflow data for water years 1970-88. Means for each day of the year were ranked, and 20-percent, 50-percent, and 80-percent points were selected such that 20 percent of the means for any particular day would exceed the 20th percentile, and 20 percent would be less than the 80 th percentile; the remaining 60 percent of the values would fall between the 20 th and 80 th percentiles (in the normal range). The 50th percentile value represents the median discharge for any particular day.

1989 water year---Mean monthly streamflow was less than the median from October through April as result of below-normal precipitation for those months (fig. 3A). Mean monthly streamflow was greater than the median only during May and June, when precipitation was much greater than normal; from July through September it again fell to less than the median as a result of below-normal or near-normal precipitation for those months. Daily streamflows reflected the monthly means-largely below normal from October through March, normal or slightly above normal during April, May, and June, and normal or below normal from July through September (fig. 3A).
Table 1. Annual mean discharge for selected stations in Monroe County, water years 1989-93, with average discharge for period of record.

[ft ${ }^{3} / \mathrm{s}$, cubic feet per second. Locations are shown in fig. 1]

\begin{tabular}{|c|c|c|c|c|}
\hline $\begin{array}{l}\text { Water } \\
\text { year }\end{array}$ & $\begin{array}{c}\text { Annual } \\
\text { Mean } \\
\text { discharge } \\
\left(\mathrm{ft}^{3} / \mathrm{s}\right)\end{array}$ & $\begin{array}{c}\text { Percent } \\
\text { of } \\
\text { average } \\
\text { discharge }\end{array}$ & $\begin{array}{l}\text { Period of } \\
\text { record }\end{array}$ & $\begin{array}{c}\text { Average } \\
\text { discharge } \\
\left(\mathrm{ft}^{3} / \mathrm{s}\right)\end{array}$ \\
\hline \multicolumn{5}{|c|}{04230500 Oatka Creek at Garbutt } \\
\hline 1989 & 227 & 105 & $1946-93$ & 217 \\
\hline 1990 & 238 & 110 & & \\
\hline 1991 & 256 & 118 & & \\
\hline 1992 & 192 & 88 & & \\
\hline 1993 & 281 & 129 & & \\
\hline
\end{tabular}

\begin{tabular}{llrrr}
\hline 04231000 Black & Creek at Churchville & & \\
\hline 1989 & 100 & 86 & $1946-93$ & 116 \\
1990 & 130 & 112 & & \\
1991 & 135 & 116 & & \\
1992 & 113 & 97 & & \\
1993 & 168 & 145 & & \\
\hline
\end{tabular}

04232050 Allen Creek near Rochester

$\begin{array}{lrrrr}1989 & 24.6 & 77 & 1961-93 & 32.1 \\ 1990 & 35.9 & 112 & & \\ 1991 & 32.0 & 100 & & \\ 1992 & 28.1 & 88 & & \\ 1993 & 30.6 & 95 & & \end{array}$

\begin{tabular}{lllll}
\hline 0423205010 Irondequoit & Creek at Blossom road \\
\hline 1989 & 108 & 81 & $1982-93$ & 134 \\
1990 & 144 & 107 & & \\
1991 & 155 & 116 & & \\
1992 & 136 & 101 & & \\
1993 & 182 & 136 & \\
\hline
\end{tabular}

1990 water year.--Daily and monthly mean streamflow were in the below-normal range from October through December, above or near normal from January through May, and close to or in the normal range from June through September (fig. 3B). The above normal streamflow from January through May resulted from above-normal precipitation and runoff from snowmelt for those months, and the near-normal streamflow from June through September resulted from near-normal precipitation for those months.

1991 water year.--With the exception of December and March, monthly mean and daily mean streamflows for October through May were near normal. Above-normal precipitation contributed to the higherthan-normal flows in December and March. Monthly mean flows from May and through the end of the water year were below median values, and daily streamflows were in the low-normal range (fig. $3 \mathrm{C}$ ). 

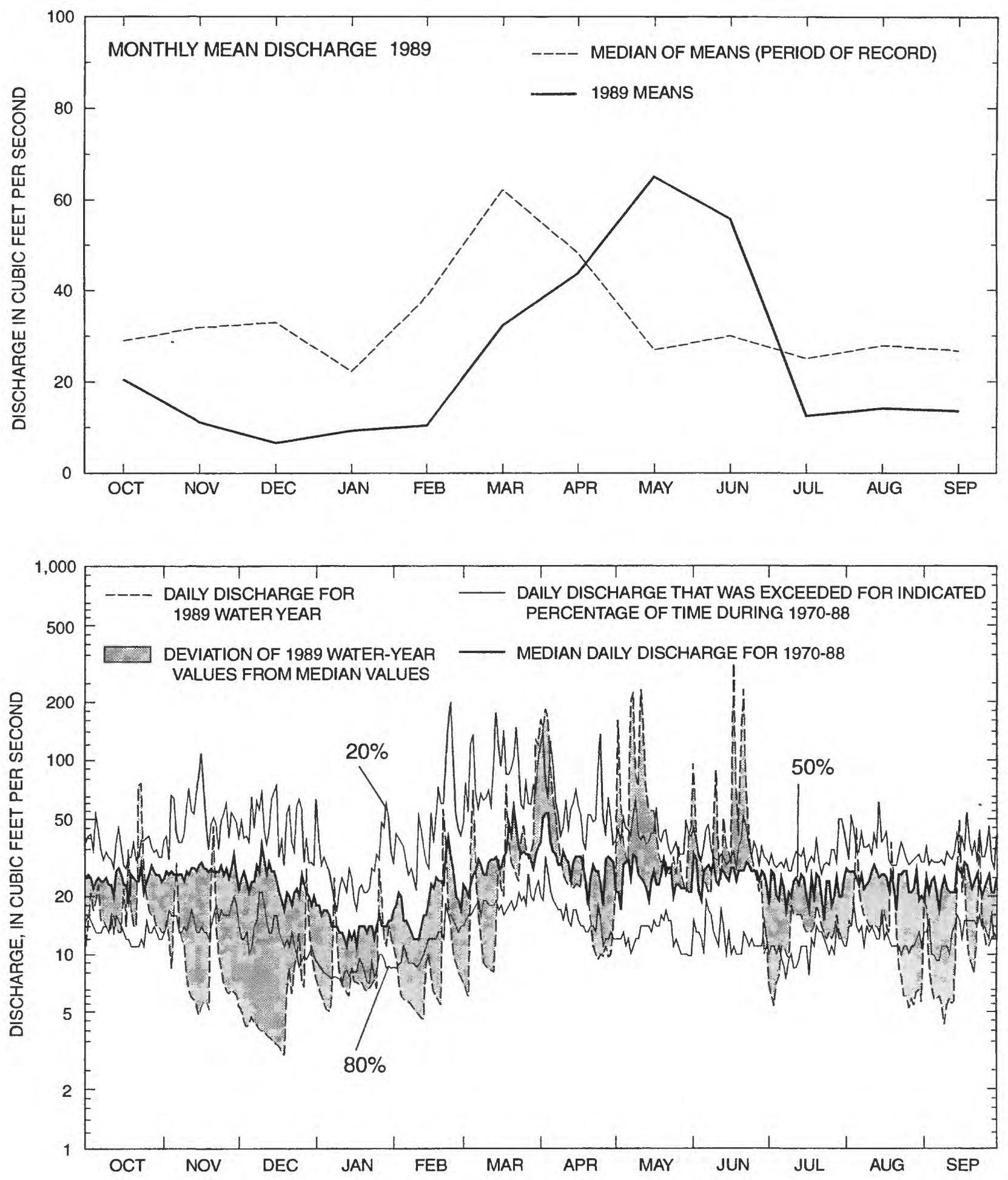

Figure 3A. Hydrographs for Allen Creek near Rochester, water year 1989: above monthly mean discharge during water year 1989 with median of monthly mean discharges for period of record, Below, median, 20-percent, and 80-percent discharges for 1970-88 period of record and daily discharges during water year 1989 with deviation from median values. 

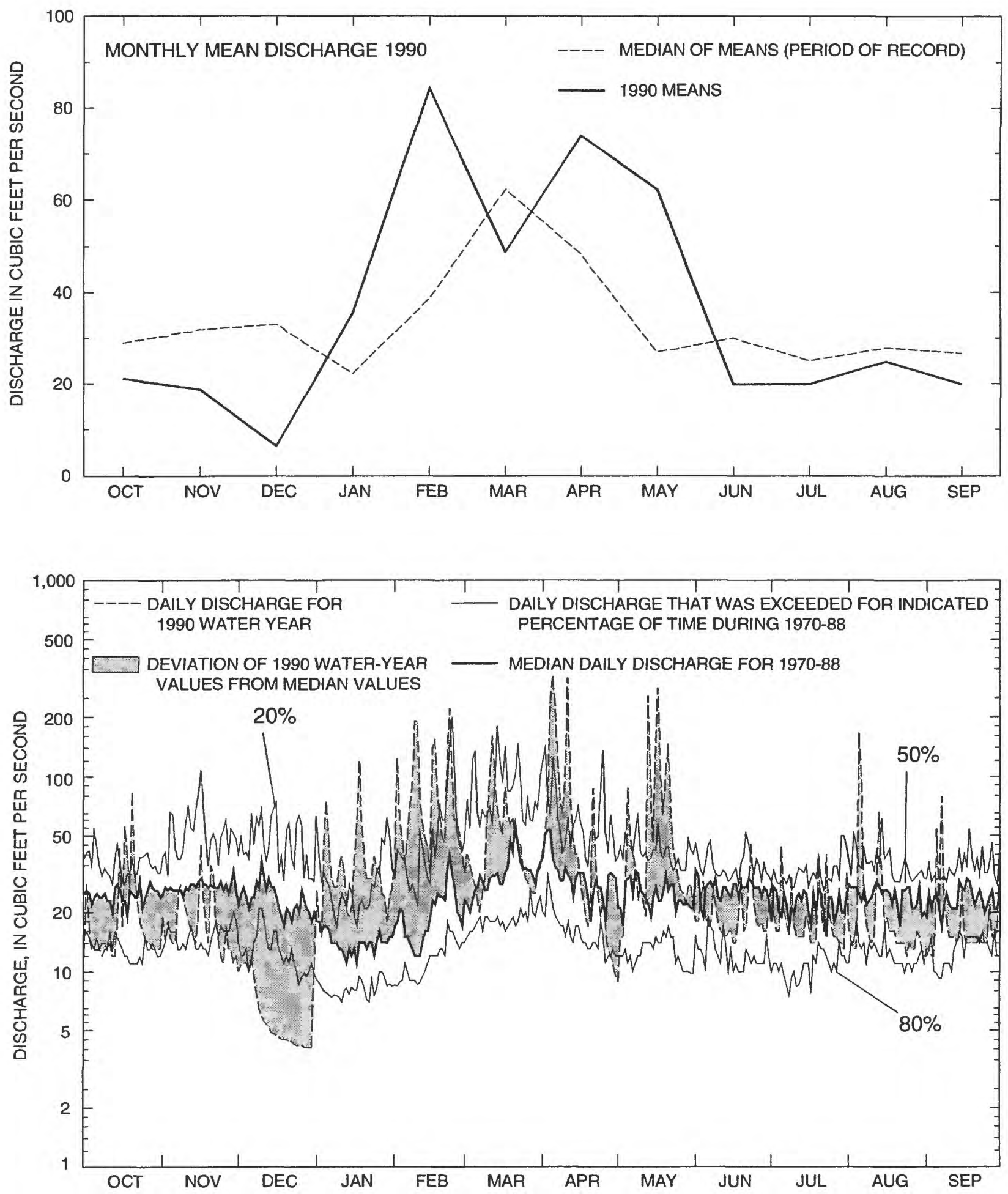

Figure 3B. Hydrographs for Allen Creek near Rochester, water year 1990: above monthly mean discharge during water year 1989 with median of monthly mean discharges for period of record, Below, median, 20-percent, and 80-percent discharges for 1970-88 period of record and daily discharges during water year 1990 with deviation from median values. 

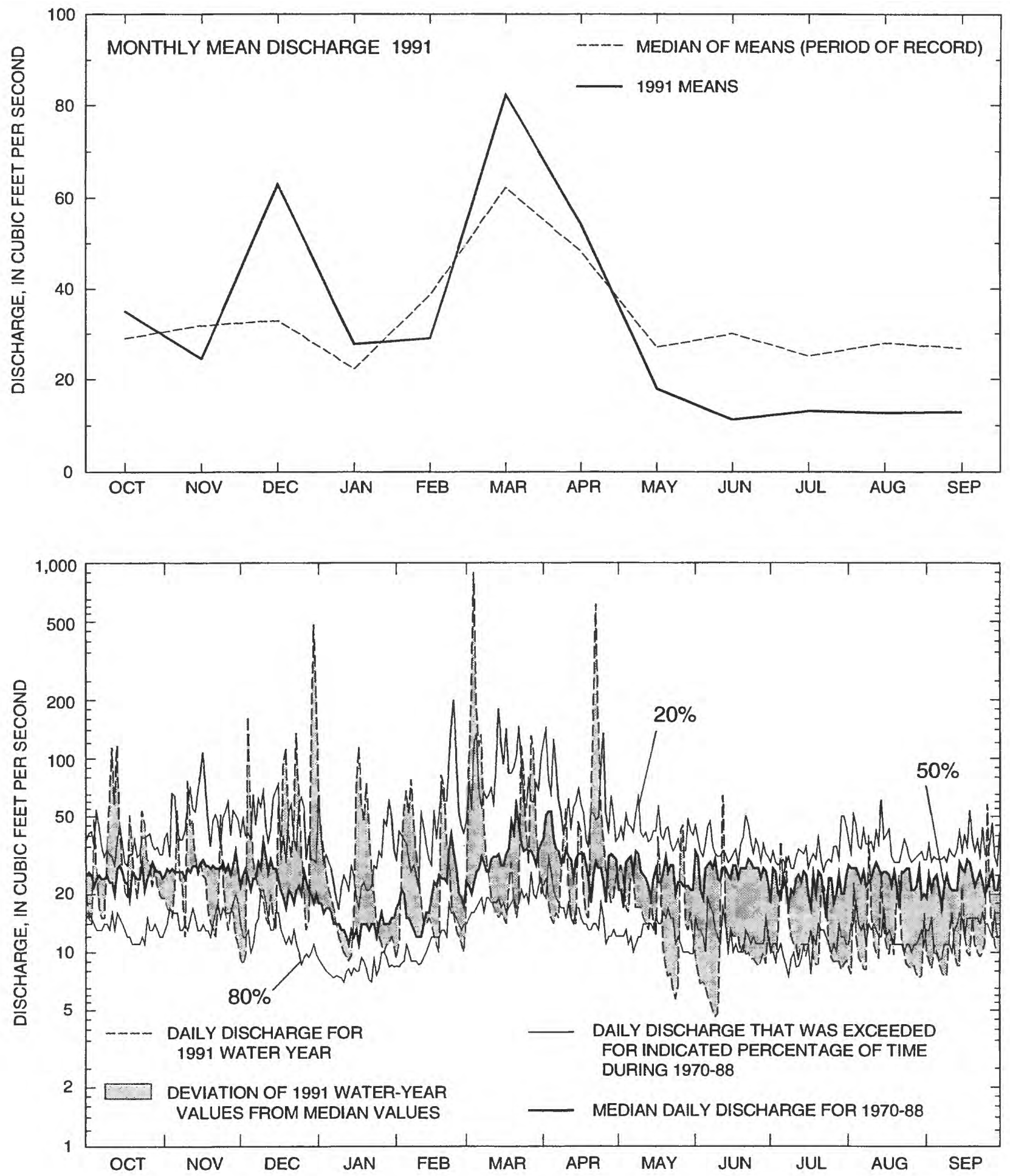

Figure 3C. Hydrographs for Allen Creek near Rochester, water year 1991: above monthly mean discharge during water year 1991 with median of monthly mean discharges for period of record, Below, median, 20-percent, and 80-percent discharges for 1970-88 period of record and daily discharges during water year 1991 with deviation from median values. 

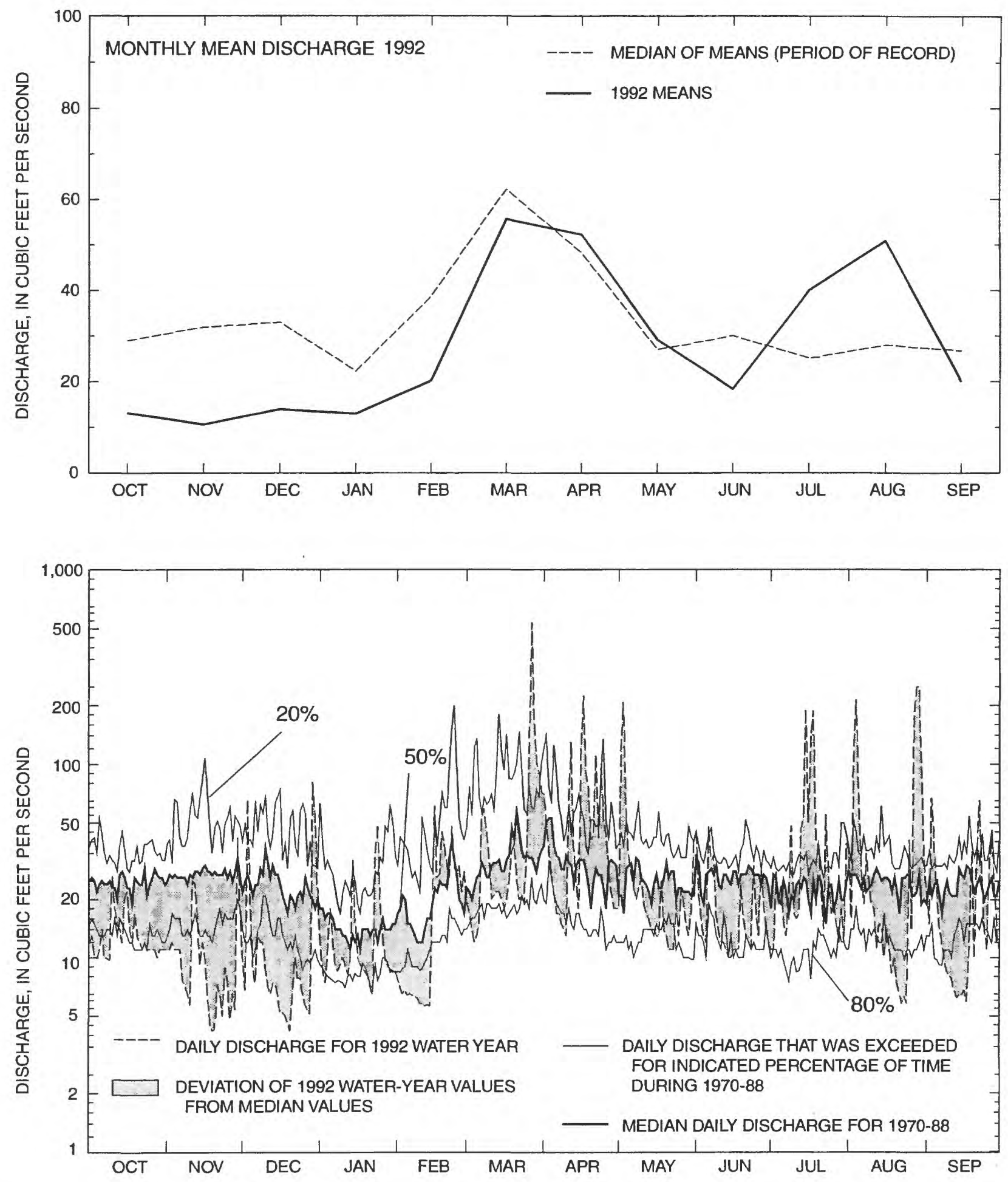

Figure 3D. Hydrographs for Allen Creek near Rochester, water year 1992: above monthly mean discharge during water year 1992 with median of monthly mean discharges for period of record, Below, median, 20-percent, and 80-percent discharges for 1970-88 period of record and daily discharges during water year 1992 with deviation from median values. 

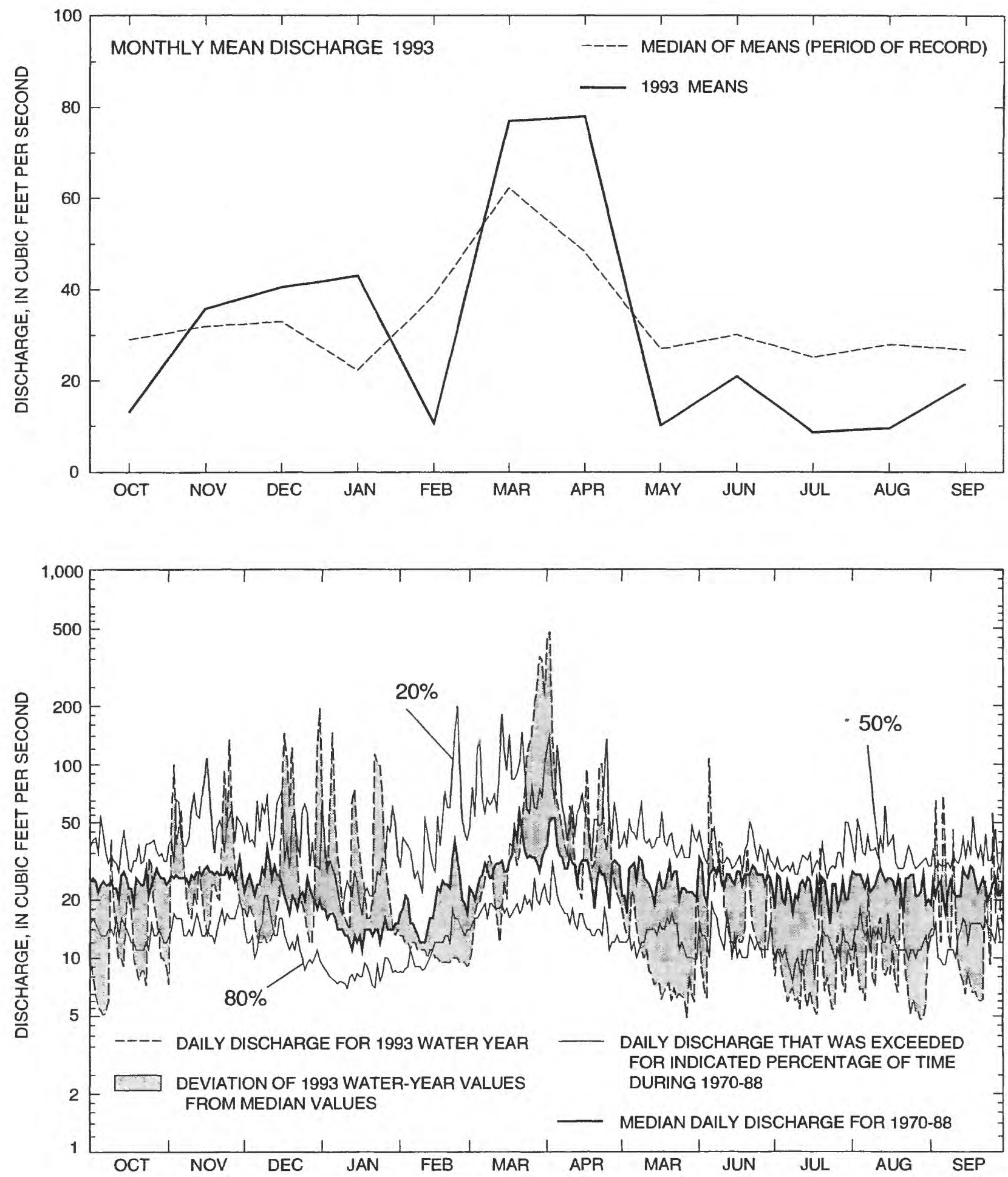

Figure 3E. Hydrographs for Allen Creek near Rochester, water year 1993: above monthly mean discharge during water year 1993 with median of monthly mean discharges for period of record, Below, median, 20-percent, and 80 percent discharges for 1970-88 period of record and daily discharges during water year 1993 with deviation from median values. 
1992 water year---Monthly mean streamflows were below the median until March, when above- normal precipitation for March and April caused streamflows to nearly equal median values. The monthly mean for June was again below the median value, but much greater-than-normal precipitation from July through August caused monthly mean streamflows to exceed median values for those months (fig. 3D). Daily mean streamflows were below normal for much of the time from October through February. Snowmelt and greater-than-normal precipitation from March through the remainder of the water year resulted in daily mean streamflows in the low-normal range (fig. 3D).

1993 water year.--Monthly mean streamflow for November, December, and January was higher than the median values for those months in response to slightly greater-than-normal precipitation, and melting of the snowpack in March and above-normal precipitation in April caused streamflow values for those months to be above the median (fig. 3E). Deficient rainfall in the spring and summer caused mean monthly streamflow to be below median. Daily mean streamflows for the water year were generally in the normal range until March, when they fell below normal for the remainder of the year (fig. 3E).

\section{Chemical Quality}

Chemical data from the Irondequoit Creek Basin and at Northrup Creek at North Greece in western Monroe County indicated that yearly mean concentrations of most constituents were relatively constant during 1989-93. Boxplots for each station (fig. 4) show the distribution of constituent concentrations over the 5-year period. Median concentrations of dissolved sulfate and dissolved chloride showed the most variability among sites, and median concentrations of total phosphorus and orthophosphorus at the Northrup Creek site were much higher than at the six Irondequoit basin sites during the study period.

Comparison of median concentrations of chemical constituents at Allen Creek and Irondequoit Creek at Blossom road in 1984-88 with those for 1989-93 indicates virtually no change (fig. 5).

\section{Ground Water}

Community water suppliers deliver about 4.47 $\mathrm{Mgal} / \mathrm{d}$ of ground water to more than 45,000 residents of Monroe County (D.S. Lumia, U.S. Geological Sur- vey, oral commun., 1992). In addition about 25,400 homes in rural areas of the county obtain a total of 1.9 $\mathrm{Mgal} / \mathrm{d}$ from wells. Ground water also is the scurce of base flow and maintains streamflow during periods of limited rainfall. Ground-water recharge generclly begins at the end of the growing season in the fall and receives much of its recharge during snowmelt periods, which are often accompanied by rain. Rerharge during the growing season occurs only when rinfall exceeds evapotranspiration demands.

Ground-water flow in the unconsolidated aquifers in the Irondequoit valley, as described in detail by Kappel and Young (1989), is continuous but restricted by deposits of low-permeability in the buried P:nnacle Hills Moraine (Kappel and Young, 1989, fig. 3 and pls. $1 \mathrm{~A}$ and $1 \mathrm{~B})$, which transects the valley north of the Ellison Park wells and south of well Mo 659 (B.86-2) (fig. 2). The aquifers north and south of the moraine have only limited subsurface connections through that part of the moraine, which is continuously incised by Irondequoit Creek (Kappel and Young, 1989). Ground water discharges from the Powder Mill Park area as seepage directly into Irondequoit Creek, as springs along the base of the east valley wall, and as underflow northward through the unconsolidated deposits of the valley. Similarly, ground water in the Ellison Park area discharges northward into Irondequoit Creak and as northward underflow (Kappel and Young, 1989).

Data on water from 15 wells in Monroe County, all in the Irondequoit Creek basin, are presented in the ground-water section of this report, which also includes water levels and seasonal temperature profiles at each of these wells. Three of the wells are ir Powder Mill Park (see inset, fig. 2), and 12 are in Ellison Park. Six of the Ellison Park wells are near Blossom Road and together form a line that transects the valley, another five are in the wetlands of Ellison Park near Browncroft Boulevard, and one (Mo 659) on th? eastern boundary of Ellison Park and north of Brow'ncroft Boulevard. Two of the Powder Mill Park wells -Mo 10 (PM 83-1) and Mo 11( PM 83-2) - are comoleted in the water-table aquifer, and the third-Mo 12 (PM 83-4) - is completed in the confined aquifer. All Ellison Park wells except Mo 659 are screened in the water-table aquifer. The four sets of paired wells-Mo 1 (El 84-1) and Mo 2 (El 84-2), Mo 5 (El 84-5) and Mo 6 (E1 84-6), Mo 663 (B88-3s), ant Mo 664 (B88-3d) and Mo 667 (B88-2s) and Mo 668 (B88$2 \mathrm{~d}$ )-indicate the variability of potentiometric $\mathrm{l} \mathrm{sad}$ at differing depths in the water-table aquifer. Well Mo 

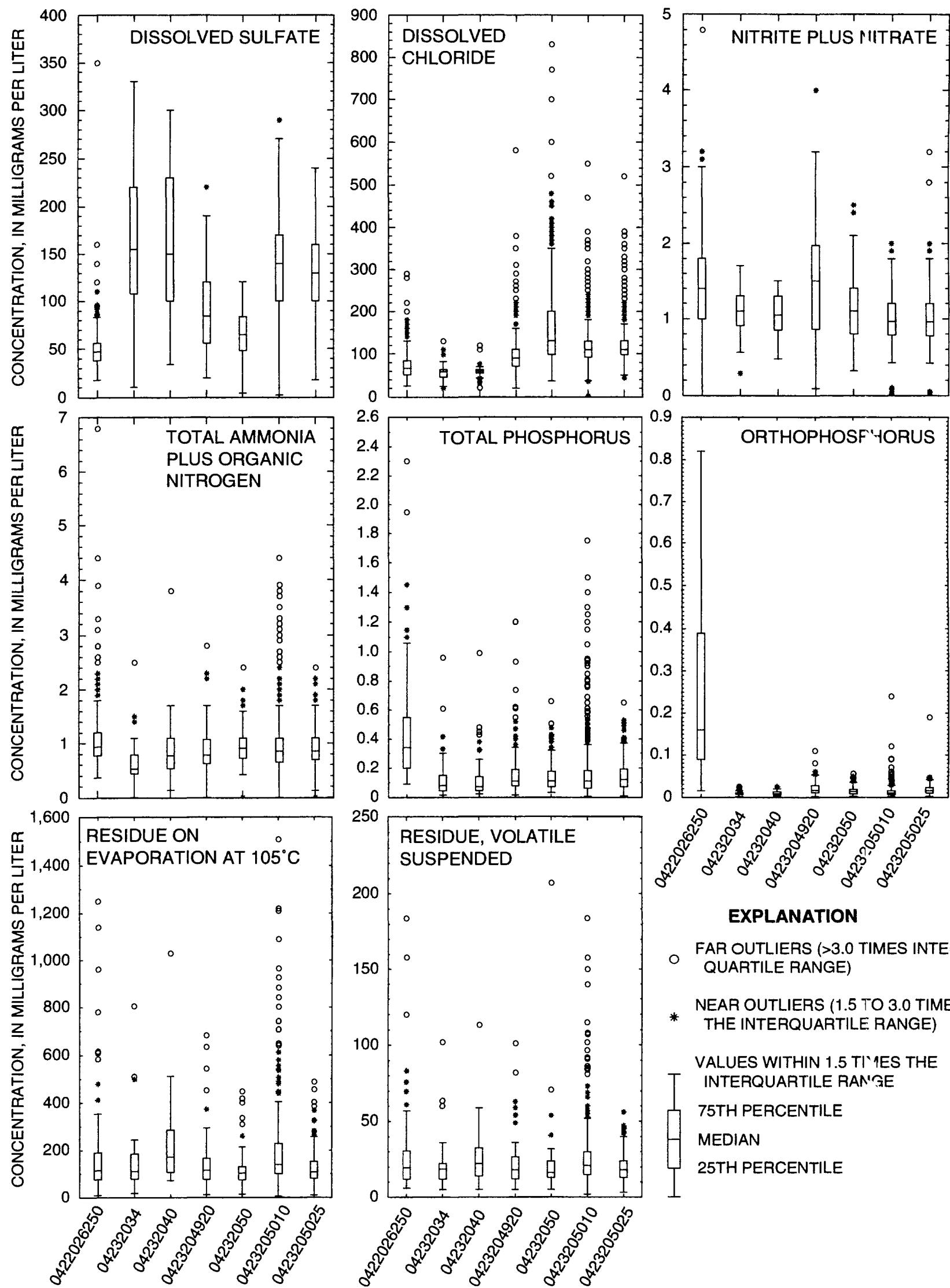

FAR OUTLIERS (>3.0 TIMES INTERQUARTILE RANGE)

NEAR OUTLIERS (1.5 TO 3.0 TIMES THE INTERQUARTILE RANGE)

VALUES WITHIN 1.5 TI 1 IES THE

T INTERQUARTILE RAN'GE

75TH PERCENTILE MEDIAN

25TH PERCENTILE

Figure 4. Concentrations of eight constituents in samples from Northrup Creek at North Greece, and at six sites in the Irondequoit Creek basin, 1989-93. (Locations are shown in fig. 1.) 

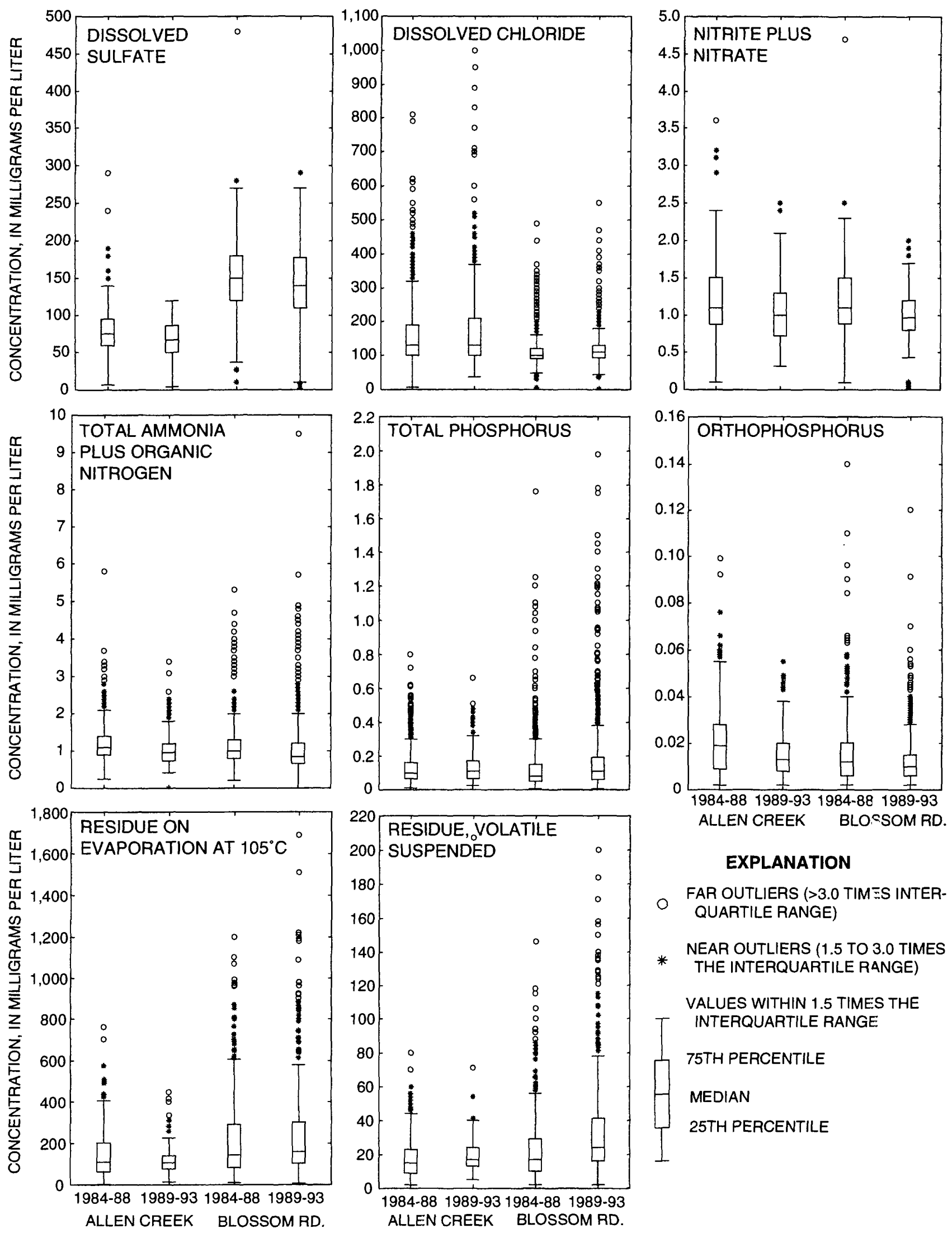

\section{EXPLANATION}

FAR OUTLIERS (>3.0 TIM $=$ S INTERQUARTILE RANGE)

NEAR OUTLIERS (1.5 TO 3.0 TIMES THE INTERQUARTILE RANGE)

VALUES WITHIN 1.5 TIMES THE

T INTERQUARTILE RANGE:

75TH PERCENTILE

MEDIAN

25TH PERCENTILE

Figure 5. Concentrations of eight constituents in samples from Allen Creek near Rochester (04232050) $\varepsilon$ nd Irondequoit Creek at Blossom road (0423205010), water years 1984-88 and 1989-93. 
659 (B86-2) is screened in the confined aquifer. All wells are considered to represent the same aquifer system (Kappel and Young, 1989).

\section{Water Levels}

Monroe County has no observation wells from which USGS has collected long-term records; therefore, well Ot 900, in the northern part of Ontario County, to the southwest (fig. 2), 8.5 mi east of the village of Victor, was selected as an indicator of annual water-level trends because it is the well nearest to Monroe County with a long term-record. This well penetrates a confined aquifer and, thus, might not fully reflect trends of water-table aquifers in Monroe County. Water-table aquifers in other parts of New York State indicate annual trends similar to those at well Ot 900, however, although the fluctuations at $\mathrm{Ot}$ 900 are more subdued (U.S. Geological Survey, 1988). The monthly mean water levels at this long-term observation well during water years 1989-93 (table 2) are generally in the below-average range (fig. 6). The annual maximum water levels for this period range from $2.15 \mathrm{ft}$ to $2.90 \mathrm{ft}$ below the maximum for the period of record. A new period-of-record minimum (4.44 ft) was observed during the 1992 water year. Monthly precipitation at the Rochester Airport for water years 1989-93 and normal monthly precipitation are shown in figure 8 (p. 13) and discussed in the following section of this report.

1989 water year.--Water levels in well Ot 900 were substantially below the long-term monthly mean at the beginning of the 1989 water year, in response to below-normal precipitation. But much higher-thannormal precipitation in May and June (+3.41 in. and +2.87 in., respectively) caused water levels to approach near long-term means, after which belownormal precipitation in July and August and near-normal precipitation in September caused water levels to decline to slightly below the long-term means for the remainder of the year.

1990 water year.--Ground-water levels at well Ot 900 were slightly below the long-term monthly means but closely followed them. Above-normal precipitation in February, April, and May brought water levels up to long term monthly means for May and part of June. Normal precipitation for the remainder of the water year was insufficient to reverse the seasonal decline in water levels during the summer months.
Table 2. Annual mean, maximum, and minimum water levels for period of record (1955-88) and water years 1989-93 at well Ot 900, Ontario County, $\Lambda^{\prime}$. $Y$.

[Water levels are in feet above land surface. Locations shown in fig. 2.]

\begin{tabular}{ccccccc} 
& $\begin{array}{c}\text { Period } \\
\text { of } \\
\text { record }\end{array}$ & \multicolumn{6}{c}{ Water year } \\
\cline { 3 - 7 } & 1989 & 1990 & 1991 & 1992 & 1993 \\
\hline Mean & 8.21 & 6.98 & 7.52 & 7.05 & 6.87 & 7.76 \\
Max. & 11.14 & 8.52 & 8.98 & 8.24 & 8.58 & 8.99 \\
Min. & 4.59 & 5.17 & 6.04 & 4.65 & 4.44 & 5.40 \\
\hline
\end{tabular}

1991 water year---Heavy precipitaton in October caused water levels in well Ot 900 to recover brieffy to slightly above long term means. Despite above-normal precipitation in December, below- normal precipitation in January and February, and well-above-normal precipitation in March and April, water levels remained relatively constant until May, when they began a decline to record minimums.

1992 water year.--Water levels began the 1992 water year with a period-of-record minimum $(4.44 \mathrm{ft})$ on October 28 but increased over the n?xt several months to slightly above period-of-record lows for each month. After the slightly above-normal precipitation of March and April, water levels decreased slightly but were closer to the long-term monthly means. Recharge from heavy precipitation in July (6.03 in.) and August (4.45 in.) caused water levels at well Ot 900 to climb substantially above long-term monthly means for the remainder of the water year.

1993 water year--Despite slightly below-normal precipitation for October and near-normal precipitation from November through January, water levels remained above the long-term monthly means until February, when low temperatures and deficient precipitation caused them to decline in late February and March. Snowmelt and near-normal precipitation during April and May caused water levels to recover to near long-term means, but seasonal declines in precipitation kept water levels below monthly means for the remainder of the year.

\section{Chemical Quality}

Ground-water samples were collected from 15 wells in Monroe County ( 3 from Powdermill Park, 12 from Ellison Park), and water temperatures were measured at successive depths in the wells to provide water-temperature profiles. Results are given in the section on ground-water data. 

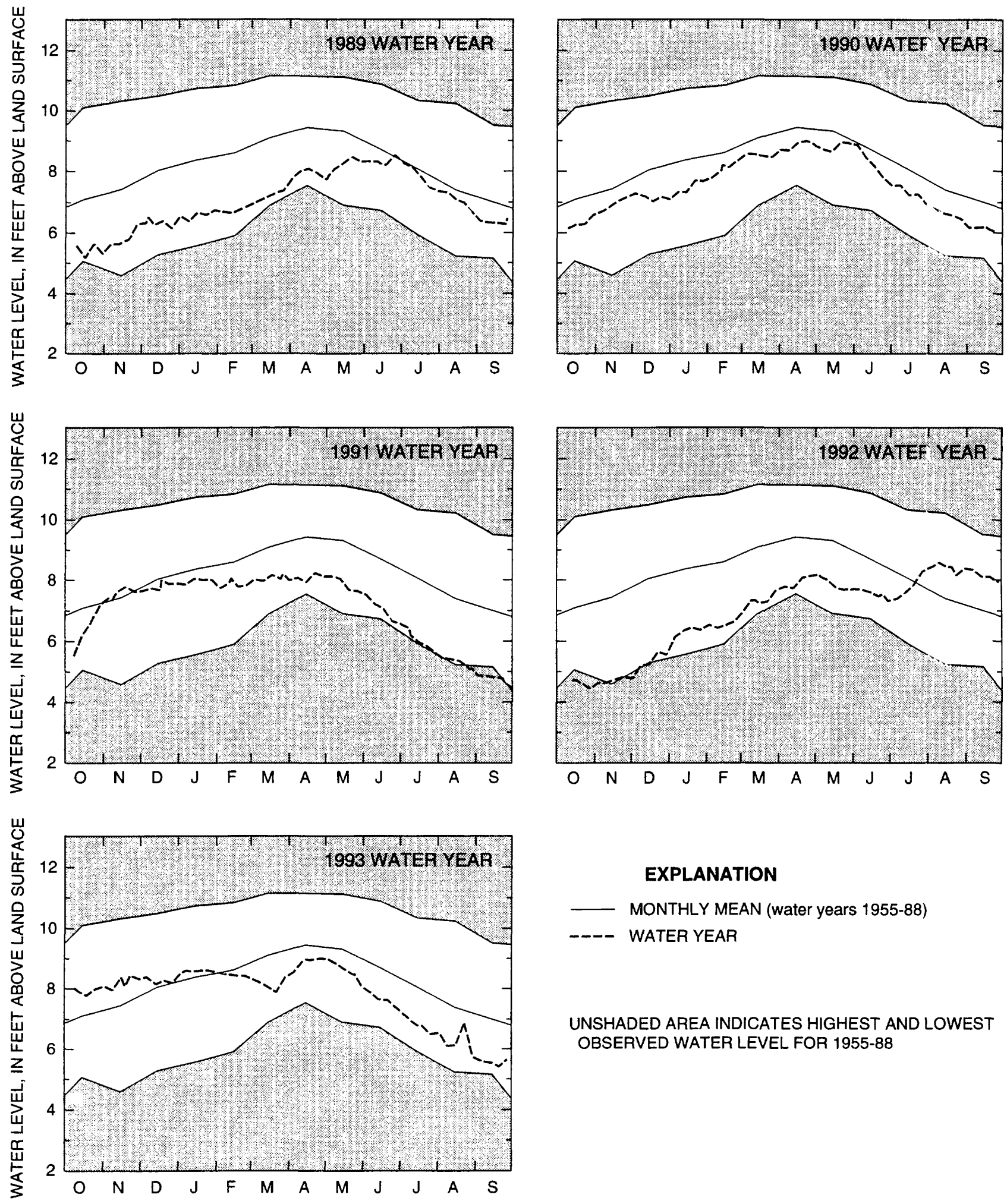

\section{EXPLANATION}

- MONTHLY MEAN (water years 1955-88)

WATER YEAR

UNSHADED AREA INDICATES HIGHEST AND LOWEST OBSERVED WATER LEVEL FOR 1955-88

Figure 6. Observed water levels for water years 1989-93 at well Ot 900 in northern Ontario County with month'v mean, maximum, and minimum water level throughout period of record, 1955-88. (Location is shown on fig. 2.) 
All wells (fig. 2) were sampled quarterly. Two wells (Mo 5 and Mo 6) were discontinued in June 1989, and two more (Mo 1 and Mo 4) were discontinued at the end of September 1990. Analyses of ground-water samples from both parks indicate that water in the aquifer system has high specific conductance. Specific conductance values at all but two of the wells (Mo 659 and Mo 668), averaged from 830 to $2,800 \mu \mathrm{S} / \mathrm{cm}$. Specific conductance at Mo 659 averaged $670 \mu \mathrm{S} / \mathrm{cm}$ and Mo $66420,000 \mu \mathrm{S} / \mathrm{cm}$. Likewise, hardness values at all but wells Mo 659 and Mo 664 averaged from 290 to $845 \mathrm{mg} / \mathrm{L}$ as $\mathrm{CaCO}_{3}$; hardness values at Mo 659 and Mo 664 had averaged of 126 and $5900 \mathrm{mg} / \mathrm{L}$ as $\mathrm{CaCO}_{3}$, respectivly. Average alkalinity values ranged from 200 to $345 \mathrm{mg} / \mathrm{L}$ as $\mathrm{CaCO}_{3}$.

\section{Temperature}

Water temperatures were measured seasonally during 1989-93 in 11 water-table wells and one confined aquifer well (Mo 659) in Ellison Park, and in two water-table wells and one confined-aquifer well in Powder Mill Park. Water temperatures were not measured in wells Mo 659, Mo 665, Mo 667, Mo 668, Mo 663 , or Mo 664 until the 1991 water year. Seasonal changes in water temperature profiles are useful in estimating the vertical component of hydraulic conductivity and, together with concentration data for selected chemicals, can be used to predict the downward movement of chemical contaminants (fig. 7).

\section{Precipitation}

Precipitation data have been collected in the Rochester area since 1827. Normal, monthly, and annual precipitation data used in this report (fig. 8 and table 3 ) are from records published by the National Oceanic and Atmospheric Administration for the Rochester Airport. Normal precipitation values are based on the average precipitation during 1951-80. Precipitation-quantity data in this report represents three sites in the Irondequoit Creek basin and one site in the Genesee River basin near the drainage divide between these two basins. Data on chemical quality of precipitation are also collected at the Genesee River basin site in Mendon Ponds County Park, Empire Boulevard at the Irondequoit Bay wetlands, and at the State University of New York (SUNY) Brockport in western Monroe County (fig. 2).

Much of the precipitation-quantity data collected at the four sites contain large gaps (missing and ques-
A. Confined aquifer Well Mo 12

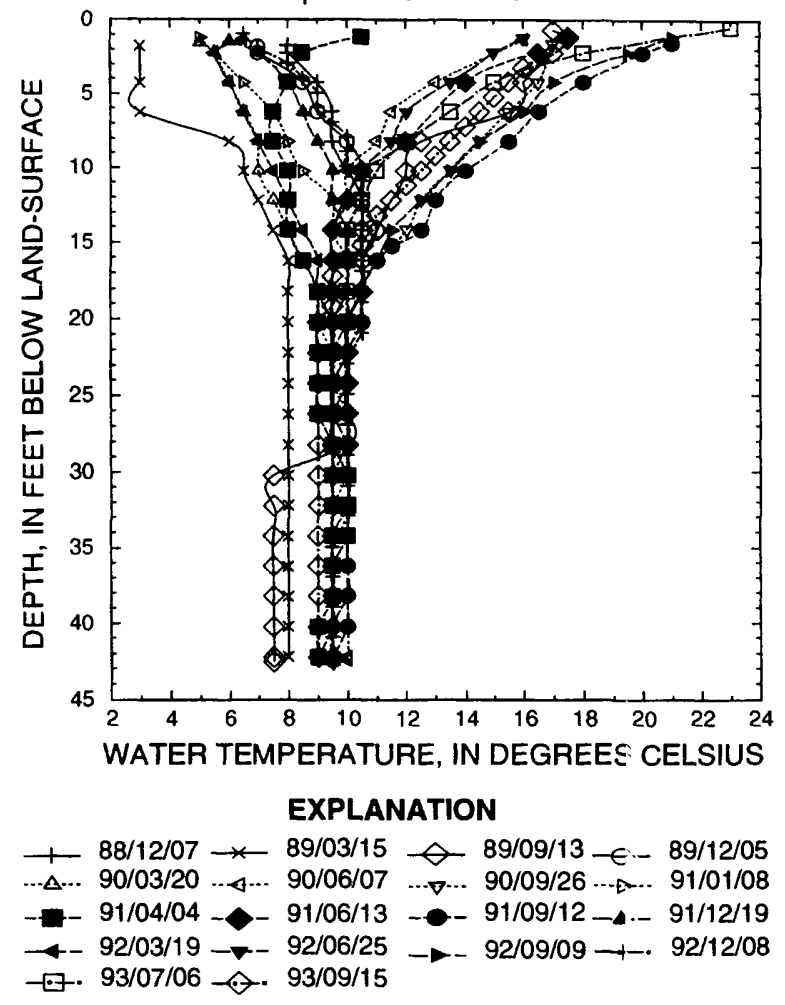

B. Water-table aquifer Mo 10

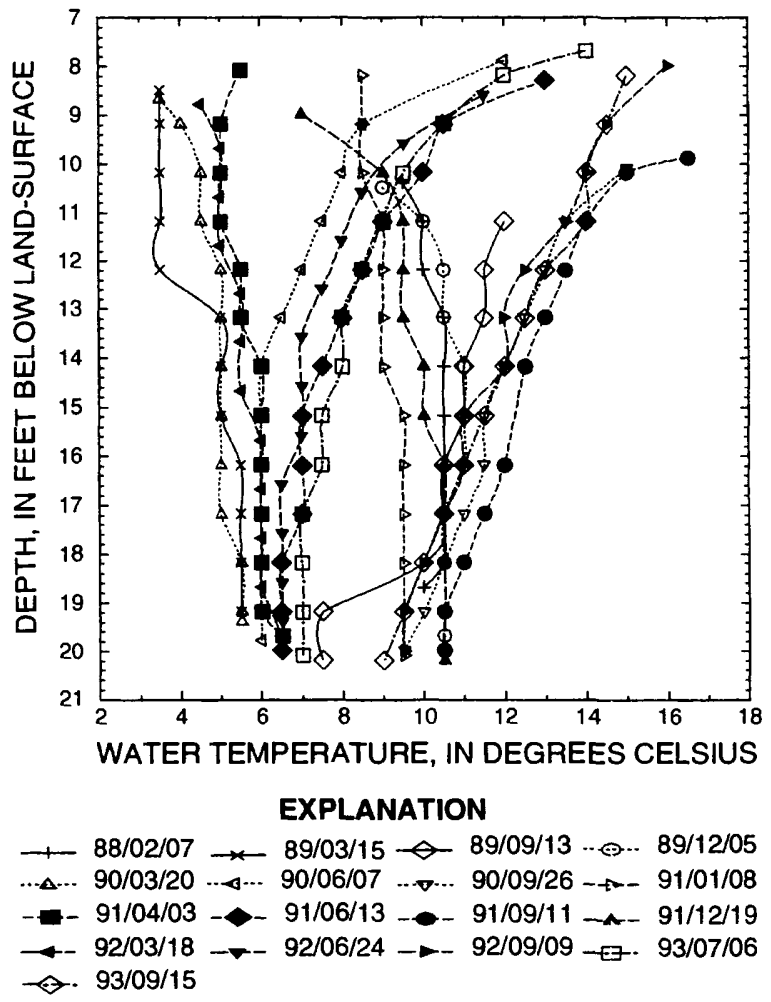

Figure 7. Water-temperature profiles from a well screened in (A) a confined aquifer, and (B) a shallow water-table aquifer, in Powder Mill Park, 1989-93. Note differing scales.

(Locations are shown in fig. 2, tables and graphs are given in the ground-water tables (p. 116-190). 
tionable data); no attempt was made to estimate missing data, and questionable data were deleted.

Precipitation-quantity data collected at Thomas Creek at Fairport and Irondequoit Creek near Pittsford contained smaller gaps, and these values were estimated.

Table 3. Total annual precipitation at Rochester airport, by water year, and departures from the normal ${ }^{1}$ of 31.27 inches per year.

[values are in inches]

\begin{tabular}{lrrrrr}
\hline & 1989 & 1990 & 1991 & 1992 & 1993 \\
\hline \hline Precipitation & 31.07 & 36.04 & 33.09 & 35.56 & 28.72 \\
Departure & -0.20 & 4.77 & 1.82 & 4.29 & -2.55 \\
\hline
\end{tabular}

${ }^{1}$ The value for "normal" monthly or annual precipitation as used by NOAA is computed as the average of the appropriate values for 1951-80. This is not the same as the statistical normal used by the USGS, when referring to normal runoff or normal water level, where half of the values for the specified period are above the normal and half below.

\section{Quantity}

Annual total precipitation at the Rochester Airport for water years 1989-93, from records published by the National Oceanic and Atmospheric Administration (NOAA) (1988-93), ranges from 4.77 in. above normal (31.27 in. for 1951-80) in 1990 to 2.55 in. below normal in 1993 (table 3 ). The cumulative total for the 5 -year period was 8.13 in. above normal. Monthly precipitation recorded at the Rochester airport during each of the 5 water years is shown in figure 8 .

1989 water year.--Precipitation was below normal from October through February, was well above normal during March, and below normal for April. The May and June totals were well above normal, and the July total was well below normal. The August total was slightly below normal, and the September total slightly above normal. Total precipitation for the year was slightly ( 0.20 in.) below normal.

1990 water year.--Precipitation was above normal for October and below normal for November, December, and January. Precipitation for February was substantially above normal, and that for March was well below normal. Precipitation for April and May also was much higher than normal, and that for June, July, August, and September was slightly above normal. Total precipitation for the 1990 water year was well above (4.77 in.) normal.

1991 water year.--Total precipitation for the 1991 water year was 1.82 in. above normal. Totals for October, December, March, and April were well above normal (avg. 1.78 in.). Precipitation for all remaining months was below normal except September, in which precipitation was near normal.

1992 water year.--Precipitation recorded at the Rochester Airport during the 1992 water year was 4.29 in. above normal. Except for July, in which precipitation was $3.55 \mathrm{in}$. above normal, and August, in which it was 1.25 in. above normal, the monthly totals for the rest of the months were within 1.00 in. of normal.

1993 water year.--Most months during the 1993 water year had near-normal precipitation. Maj', June, July, and August values were moderately below normal, while the September value was well above normal. Total precipitation for the year was $2.55 \mathrm{in}$. below normal.

\section{Chemical Quality}

Data on chemical quality of precipitation are collected by MCEHL at Mendon Ponds Park, Iron Jequoit Creek at Empire Boulevard, and at the SUNY Brockport campus (fig. 2). Three forms of precipitatin at Mendon Ponds Park were analyzed for chemic $`$ quality: (1) wetfall (liquid deposition), (2) dustfall (dry deposition, which is that fraction of precipitation that settles out of the atmosphere as dust), and (3) l'lk (composite) deposition, which consists of the r'et and dry forms combined. Only wetfall and dustfall are collected at the Empire Boulevard and SUNY Brockport sites. These analyses provide information on the atmospheric contribution of various chemical constituents to streams and land surface.

The three forms of deposition were analyzed for common ions, nutrients, lead, and physical characteristics such as $\mathrm{pH}$ and specific conductance. $\mathrm{pH}$ values indicated moderate acidity (4.0 to 5.0 ), which is typical for precipitation in this area. Specific conductance was generally less than $100 \mu \mathrm{S} / \mathrm{cm}$. Concentrations of lead in late fall and winter and during the summer were slightly above those during the rest of the year.

\section{DATA COMPILATION}

The surface-water, ground-water, and precipitation data in the following compilation represent the water years that began October 1, 1988 and ented September 30, 1993. The data include (1) streamflow summaries and surface-water-quality data, (2) ground-water levels and quality, and (3) precipitation quantity and quality. Locations of the stations and wells at which data were collected are shown in fig- 

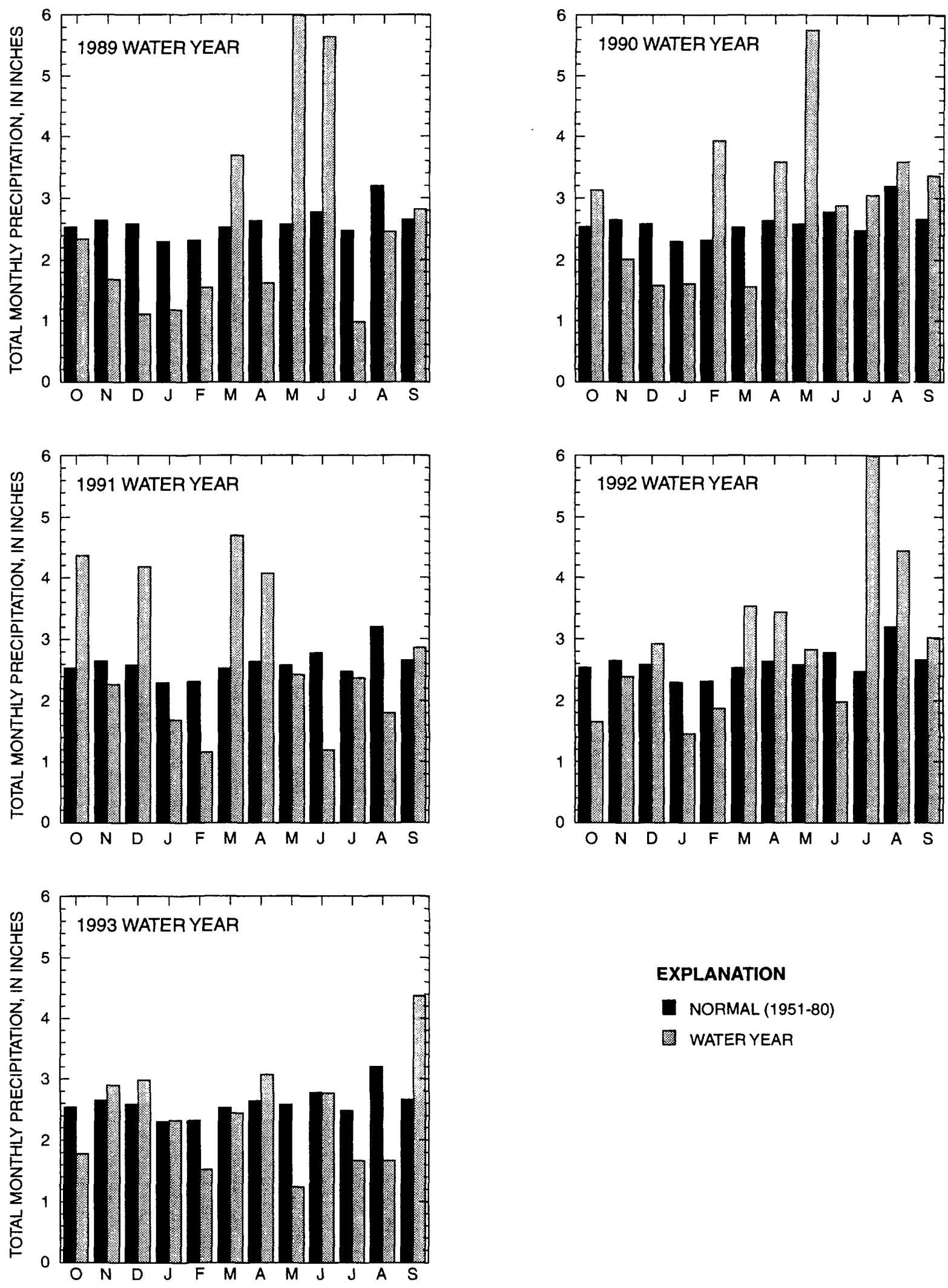

Figure 8. Monthly precipitation at Rochester airport, October 1989 through September 1993 and normal monthly precipitation based on average for 1951-80. (Data from National Oceanic and Atmospheric Administration, Climatological Data Annual Summary, New York series.) 
ures 1 and 2. The following paragraphs explain how the data were collected, analyzed, computed, and arranged for presentation.

\section{Surface Water}

The surface-water part of this compilation is arranged by station (by downstream-order station identification number), and the data for each station are grouped into two sections-summaries of daily streamflow data and water-quality data.

\section{Downstream-Order Station-Identification System}

Since October 1, 1950, surface-water station records in USGS reports are listed in a downstream order along the main stream. All stations on a tributary entering upstream from a main-stream station are listed before that station, and a station on a tributary that enters between two main-stream stations is listed between them. The rank of a tributary with respect to the stream to which it is immediately tributary is indicated by an indention in the list of stations on page 41 . The downstream order and system of indention show which stations are on tributaries between any two stations in a basin.

Gaps are left in the station-number sequence to allow for new stations; hence, the numbers are not consecutive. The complete 8-digit number for each station, such as 04232050 , consists of a 2-digit part "04" that represents the major river basin (St. Lawrence) plus the 6-digit downstream-order number "232050." Wherever no gap is available for a new station, two digits are added to make a 10-digit number.

\section{Stage and Discharge}

The data collected at streamflow-gaging stations consist of records of stage, measurements of discharge throughout a range of stages, and notations regarding factors that can affect the relation between stage and discharge. Records of stage were obtained from a water-stage recorder that gives either a continuous graph or a tape punched at selected time intervals. Measurements of discharge are made with a current meter through methods adopted by the USGS and described in Rantz and others, (1982, v. 1).

\section{Computation Methods}

Results of individual discharge measurements at streamflow-gaging stations are plotted against corresponding stages to develop stage-to-discharge relation curves. These curves are used to prepare rating tables that indicate the approximate discharge for any stage within the range of measurements. If the discrarge to be expressed exceeds the measured value, the rating curves are extended from indirect measurements of peak discharge, step-backwater techniques (Bailey and Ray, 1966; Shearman, 1976), slope-conveyance, studies (Rantz and others, 1982, v. 1), and logarithmic plotting (Kennedy, 1984). Indirect measurement techniques include (1) slope-area measurements (Dalrymple and Benson, 1967), (2) contracted-opening measurements (Matthai, 1967), (3) compu'ation of flow over dams or weirs (Hulsing, 1967) and (4) computation of flow through culverts (Bodhaire, 1968). Most of these topics are also covered in Rantz and others, (1982, v. 1).

Daily mean discharges are computed through a process whereby the instantaneous stages (gage heights) are applied to the stage-to-discharge curves or rating tables, and the resulting discharges are averaged for each day. Monthly and yearly mean discharges are computed from the daily values. If the stage-to-discharge relation is subject to change as a result of frequent or continual change in the physical featu'es that form the control, the daily mean discharge is computed by the shifting-control method (Kennedy, 1983; Rantz and others, 1982, v. 2). Correction factors based on individual discharge measurements and notes by the person making the measurement are applied to the gage heights before the discharges are read from the curves or tables. This shifting-control method also is used if the stage-to-discharge relation is tempo-arily altered by aquatic growth or debris on the control.

Ice formation in the winter can so obscure the stage-to-discharge relation at some stations that daily mean discharges must be estimated from gage-height record, occasional discharge measurements, an 7 other information such as temperature and precipitation records, notes by hydrographers, and records of discharge at other stations in the same or nearby basins for comparable periods.

Some gaging stations have periods when the gage-height record either is unavailable or is sc faulty that it cannot be used to compute daily discharge. This happens when, for example, the recorder stops or fails to operate properly, stilling well intakes are plugged, 
or the float is frozen in the well. The daily discharges for such periods are estimated from the recorded range in stage, previous and following records, discharge measurements, weather records, and comparison with other station records in the same or nearby basins. Designation of estimated values in the tables of station records is explained below.

\section{Data Format (Surface-water stations)}

The tables of surface-water data in this report are presented in two parts-(A) Discharge and WaterQuality Stations (p. 33-88), and (B) Partial-record and Miscellaneous-record Sites (p. 89-115). Part A represents continuous-record stations and includes information on the station, as well as discharge statistics and water-quality data. Part B includes water-quality data for each site and only brief site information.

Part A provides a description of each continuousrecord station (location and drainage area), followed by (1) water-discharge records, and (2) water-quality records. The water-discharge records include the following information: period of record; type of gage; remarks on record accuracy and other factors pertinent to station operation and regulation; cooperating agencies; and historical extremes (for 1989-93 and for the station's period of record). This information is followed by a table of statistics on monthly mean discharge for water years 1989-93 and a table of summary statistics for the 1992 calendar year, the 1993 water year and water years 1989-93. A graph of monthly mean discharge for 1989-93 is included for each continuous-record station.

Part B (water-quality records) provides information on the period of record for each station, the years for which chemical data are given, and in parentheses, a letter designating the sampling frequency for those years (defined on p. 25); it also includes cooperating agencies and may include remarks. The tables of water-quality are given by water year.

The headings and the types of information provided for each continuous-record station are as follows:

Location.--Information on location is obtained from topographic maps (usually 1:24,000 scale). The location of the gage is given with respect to the cultural and physical features in the vicinity and the reference place mentioned in the station name. River mileage, given for some stations, is that determined and used by the U.S. Army Corps of Engineers or other agencies.
Drainage Area.--Drainage areas are measured from topographic maps (usually 1:24,000 scale). Because the types of maps available differ from one drainage basin to another, the accurac' of the drainage areas likewise varies. Drainage-area values are updated as revised maps become available.

Period of Record.--Identifies the period for which published records for the station (or an equivalent station) are available.

Revised Records.--Published reccrds are occasionally revised in light of new information. Listed under this heading are all reports in which revisions for the station have been published, ard the water years for which revisions apply. If a revision did not include daily, monthly, or annual discharge figures, that fact is noted after the year dates as follows: (M) means that only the instantaneous maximum discharge was revised; $(\mathrm{m})$ that only the instantaneous minimum was revised; and (P) that only peak discharges were revised. If the drainage area has been revised, the report in which the most recently revised value was first published is cited.

Gage.--Under this heading are listed the type of gage in use, the datum of the current g'ge above mean sea level, and a condensed history of the types, locations, and datum of previous gages.

Remarks.--This paragraph gives information on the accuracy of the records, special methods of computation, conditions that affect natural flow at the station, and other pertinent items. The accuracy of the records for some stations varies from year to year; where this occurs a general statement explains the accuracy for the 5 years represented in this report, and a statement at the top of the table for each water year describes the accuracy of that year's data.

Cooperation.--Records provided ty a cooperating organization or obtained for USGS by a cooperating organization are identified here.

Extremes For Period.--This paragraph includes information on extremes that occurred from the beginning of the period of record until the record was either discontinued, or until September 1993 (the end of the period covered in this report). Extremes include maximum and minimum stages and maxim um and minimum discharges. Unless otherwise qualified, the maximum discharge is the instantaneo's maximum corresponding to the highest stage recorded on a stage recorder (graphic or digital), a crest-stage gage, or a nonrecording gage read at the time of the crest. If the maximum gage height did not occur on the same day 
as the maximum discharge, it is given separately. Similarly, the minimum is the instantaneous minimum unless otherwise qualified.

\section{Extremes Outside Period Of Record.--Included} here is information concerning major floods or unusually low flows that occurred outside the stated period of record. The information may or may not have been obtained by the USGS or by other agencies.

Statistics of Monthly Mean Discharge Data.- The preceding information is followed by a table titled "Statistics of monthly mean discharge for water years 19 ___, by water year," which lists mean, maximum, and minimum values for each month, for the period designated. The two lines headed (WY) immediately below the MAX and MIN lines indicate the water year of the first occurrence of the maximum and minimum monthly flows. The period for which data are given (designated in the table heading) includes all partial water years, if any. The water years for which the statistics are computed are consecutive unless a break in the station record is indicated in the heading.

Summary Statistics.- A second table, "Summary Statistics," gives values for several statistics, such as annual, daily, and instantaneous discharges, for the designated period. This table contains three columns for each statistic: the first lists the values for the calendar year preceding the last water year of the designated period; the second lists values for the last water year of the designated period, and the third lists values for the entire designated period. The third column also indicates all of the station record within the specified water years, including complete months of record for partial water years, if any; this period may coincide with the period of record for the station. The water years for which the statistics are computed are consecutive unless a break in the station record is indicated in the general information for that station. All calculations for the statistical characteristics in rows designated ANNUAL (See line headings below), except the "ANNUAL 7-DAY MINIMUM" statistic, are done for the designated period and are based on complete water years. Calculations of the other characteristics may be based on partial water years.

The date or water year (as appropriate) of each statistic reporting extreme values of discharge is provided adjacent to the statistic. In some instances, these extremes may occur on more than one date or year. These repeated occurrences are identified with a letter symbol and printed in the footnotes. Because the designated period may not be the same as the station period of record published in the heading, the dates of occurrence listed for the daily and instantaneous extremes in the designated-period column may not always be within the selected water years lister in the heading. When this occurs, it will be noted in the REMARKS paragraph or in footnotes. Selected streamflow duration curve statistics and runoff data are also given. Runoff data may be omitted if there is extensive regulation or diversion of flow in the drainage basin.

The following types of data listed in the summary statistics column, are provided with each continuous record of discharge. The row headings of the summary statistics table are defined as follows;

Annual Total.--The sum of the daily mear values of discharge for the year. At some stations the annual total discharge is adjusted for reservoir storage or diversion. The adjusted figures are identified by a symbol and corresponding footnotes.

Annual Mean.--The arithmetic mean of the individual daily mean discharges for the year notet or for the designated period. At some stations, the yearly mean discharge is adjusted for reservoir storage or diversion. The adjusted figures are identified ly a symbol and corresponding footnotes.

Average Discharge.--This is the discharge value given to the arithmetic mean of the water-year mean discharges. It is computed for stations having at least 5 water years of complete record.

Highest Annual Mean.--The maximum annual mean discharge occurring for the designated prriod.

Lowest Annual Mean.--The minimum anrual mean discharge occurring for the designated pariod.

Highest Daily Mean.--The maximum dail:' mean discharge for the year or for the designated period.

Lowest Daily Mean.--The minimum daily mean discharge for the year or for the designated period.

Annual 7-Day Minimum.--The lowest mean discharge for 7 consecutive days for a calendar year or a water year. Note that most low-flow frequency analyses of annual 7-day minimum flows use a climatic year (April 1-March 31). The data shown in the summary statistics table is the initial date of the 7-day period. (This value should not be confused with the 7-day 10year low-flow statistic.)

Instantaneous Peak Flow.--The maximum instantaneous discharge occurring for the water year or for the designated period.

Instantaneous Peak Stage.--The maximum instantaneous stage occurring for the water year or 
for the designated period. If the dates of occurrence for the instantaneous peak flow and instantaneous peak stage differ, the REMARKS paragraph in the manuscript or a footnote may be used to provide further information.

Instantaneous Low Flow.--The minimum instantaneous discharge occurring for the water year or for the designated period.

Annual Runoff.--Indicates the total quantity of water in runoff for a drainage area for the year. Data reports may use any of the following units of measurement in presenting annual runoff data:

Cubic feet per second per square mile (CFSM) is the average number of cubic feet of water flowing per second from each square mile of area drained, assuming the runoff is distributed uniformly in time and area.

Inches (INCHES) indicates the depth to which the drainage area would be covered if all of the runoff for a given time period were uniformly distributed on it.

10 Percent Exceeds.--The discharge that has been exceeded 10 percent of the time for the designated period.

50 Percent Exceeds.--The discharge that has been exceeded 50 percent of the time for the designated period.

90 Percent Exceeds.--The discharge that has been exceeded 90 percent of the time for the designated period.

\section{Accuracy and Precision of Records}

The accuracy of the streamflow records depends primarily on (1) the stability of the stage-to-discharge relation or, if the control is unstable, the frequency of discharge measurements, and (2) the accuracy of stage observations, discharge measurements, and records interpretations. The accuracy attributed to the records for each station is indicated in the "REMARKS" paragraph of each station description. "Excellent" means that about 95 percent of the daily discharges are within 5 percent of the true discharge; "good," means that about 95 percent are within 10 percent; and "fair," means that about 95 percent are within 15 percent, and "poor" means that the daily discharges have less than fair accuracy.

\section{Chemical Quality}

This report presents chemical-quality data from eight continuous-record streamflow-gaging stations and eight partial-record and miscellanecus-record sites. The water-quality records for the eight continuous-record streamflow-gaging stations follow the summary streamflow data for that site. Stat in number and name are the same for both records unless the water-quality-site location differs significantly from that of the streamflow station, in which case the waterquality site is given a separate number and name in the downstream-order sequence.

Water samples are generally collected as close to streamflow-gaging stations as possible tecause streamflow data are essential to the interpretation of water-quality data. Each streamflow-gaging station in the Irondequoit Creek basin was visited two or three times each week. Samples were collecter hourly at all sites by automatic sampler and combine- 1 into flowrelated composite samples during storms. Samples from Blossom Road and Empire Boulevard also were combined into 2-to-4 day baseline composite samples two or three times per week; samples from the other six sites were combined into 2-to-4 day baseline composite samples at least once monthly. The records of surface-water quality presented herein ganerally include physical properties, such as turbidity and dissolved solids, and chemical constituents. such as nitrogen and phosphorus species and common ions such as chloride and sulfate.

\section{Continuing-Record ${ }^{\boldsymbol{t}}$ and Partial-Reco-d Stations}

Each surface-water-quality site is classified as either (1) a continuing-record station-a site at which data are collected on a regular schedule, such as once or more daily, weekly, monthly, or quart srly, or (2) a partial-record station-a site at which limited waterquality data are collected systematically over a period of years, usually less than quarterly. All stations represented in this report are in the continuing-record category; their locations are shown in figure 2.

\section{Field and Laboratory Methoc's}

Carefully prescribed procedures were followed in the collection and processing of the samfles and in preservation of the samples to minimize chemical or physical changes between time of collection and analysis, to ensure that analytical results obtair ed in the laboratory accurately reflected the in-stream chemistry of

\footnotetext{
l "Continuing record" differs from "continuors record," which refers to a continuous graph or a series of discrete values recorded at predetermined intervals.
} 
the water. Procedures for collecting, treating, and transporting samples are given in Britton and Greeson (1989), Goerlitz and Brown (1972), Guy and Norman (1970), Skougstad and others (1979), and Wood (1976).

Most of the samples reported herein were collected by automatic samplers. Automatic samplers are capable of collecting either discrete or composite samples. Discrete samples are collected at a particular instant and assumed to represent only the water quality at that time, whereas composite samples consist of two or more discrete samples collected and combined over a period of time, such as several hours or days, to reflect average water-quality conditions for that period. The limitation of automatic water samplers is that they collect the sample from only one point in the stream cross section. Although a sample from a single point in the stream can adequately define the water quality for that time if the water is homogeneous, variations in turbulence can cause uneven mixing and result in local differences in the concentration of solutes throughout the cross section, depending on rate of flow and the source of the solutes. For this reason, placement of the automatic sampler intake in the stream cross section is occasionally checked for representativeness. (See Quality Assurance/Quality Control section, further on, for detailed information.)

Chemical-quality data published herein are considered to be the most representative values available for the stations listed, and they describe, as closely as possible within the limits of available sampling techniques and methods of analysis, the water-quality conditions at the time of sampling.

MCEHL analyzed all samples using analytical methods described in American Public Health Association (1985). Some samples were split into two parts, one of which was sent to the USGS National WaterQuality Laboratory (NWQL) at Denver, Colo., for analysis as part of the QA/QC program.

\section{Data Format}

The water-quality table for each station are given by water year. Each table of chemical data is preceded by a station description, which includes information pertinent to the history of station operation, including location, drainage area, period of record, type of data available, instrumentation, general remarks, and cooperation. If the location is identical to that of the discharge-gaging station, neither the Location nor the Drainage Area statements are repeated. The headings and types of information provided under each are explained below.

Location.--Information on locations is obtained from the most accurate maps available. The lccation of the gage is given with respect to the cultural and physical features in the vicinity and to the reference place mentioned in the station name. River mileage, given for some stations, is that determined and used by the U.S. Army Corps of Engineers or other agencies.

Drainage Area.--Drainage areas are measured in square miles from USGS topographic maps. Eecause the types of maps available differ from one drainage area to another, the accuracy of drainage areas likewise varies. Drainage areas are updated as revised maps become available.

Period Of Record.--This statement indicates (1) the periods for which published water-quality records for the station are available, (2) the categories of data to which these records pertain (chemical, mincr elements, organic compounds, nutrients, and biological constituents), and (3) the amount of data available, as specified by the following letter codes:

(a) 1 or 2 samples per year

(b) 3 to 5 samples per year

(c) 6 to 9 samples per year

(d) 10 to 20 samples per year

(e) more than 20 samples per year

For example, "CHEMICAL DATA: 1972-74(c), 1977-82(a)." indicates from 6 to 9 analyses for each year for the first 3 years of record, no data for this category in 1975 and 1976, and one or two samples for each of the 6 additional years.

Instrumentation.--Information on instrumentation is given only if a water-quality monitor or other automatic sampling device is in operation at the station.

Remarks.--Remarks provide added information pertinent to the collection, analysis, or computation of the records. The following remark codes appear in the water-quality tables:

$\mathrm{K}$ results based on colony count outside the ideal range (nonideal colony c sunt);

E estimated value;

$>\quad$ actual value known to be greater than value shown;

$<\quad$ actual value known to be less than value shown;

$\mathrm{N}$ presumptive evidence of presence of material. 
Cooperation.--Records provided by a cooperating organization or obtained for USGS by a cooperating organization are identified here.

Tables of chemical, physical, biological data, and so forth that were obtained at a frequency less than daily at each station follow the information on station history.

Chemical Data.--Generally include most of the major ions and some of the following physical properties: specific conductance, $\mathrm{pH}$, temperature, color, turbidity, dissolved oxygen.

Nutrient Data.--Constituents containing nitrogen or phosphorus. Analytical results usually include several of the following species: nitrite plus nitrate, phosphorus, ammonia nitrogen, organic nitrogen, and ammonia plus organic nitrogen.

\section{Ground Water}

Ground-water records consist of water-level measurements made in observation wells, analyses of water samples collected quarterly from these wells, and seasonal water-temperature profiles based on measurements made at successive depths. Ground-water records are presented by locality in order of latitude and longitude. (See fig. 9.) Locations of observation wells are shown in figure 2 .

\section{Latitude-Longitude Identification Sy-tem}

The well-identification and precipitation-station numbers are based on the grid system of latitude and longitude. The number consists of 15 digits; the first six denote the degrees, minutes, and seronds of latitude, and the next seven denote degrees. minutes, and seconds of longitude. The last two digits (assigned sequentially) identify the wells or precipitation gages within a 1-second grid (fig. 9).

\section{Field and Laboratory Methods}

Water levels were measured in 15 wells in the Irondequoit Creek Basin, 3 wells in Powder Mill Park, 11 wells in Ellison Park, and 1 well (Mo 659) on the eastern boundary of Ellison Park and on the north side of the Pinnacle Hills Moraine (fig. 2). Water temperatures were measured at successive dept's in the Powder Mill and Ellison Park wells to obtain watertemperature profiles that can be used as an indicator of (1) similar (or dissimilar) stratigraphy ly their shape and spread, and (2) anomalous features, by any sudden change in temperature with depth. The seasonal temperature profiles can also provide an estimate of aquifer permeability (Lapham, 1989). Water samples were collected from the Powder Mill and Ellison Park wells for comparison of ground-water crality in differing parts of the aquifer system. The procedures used are discussed in the following paragraphs.

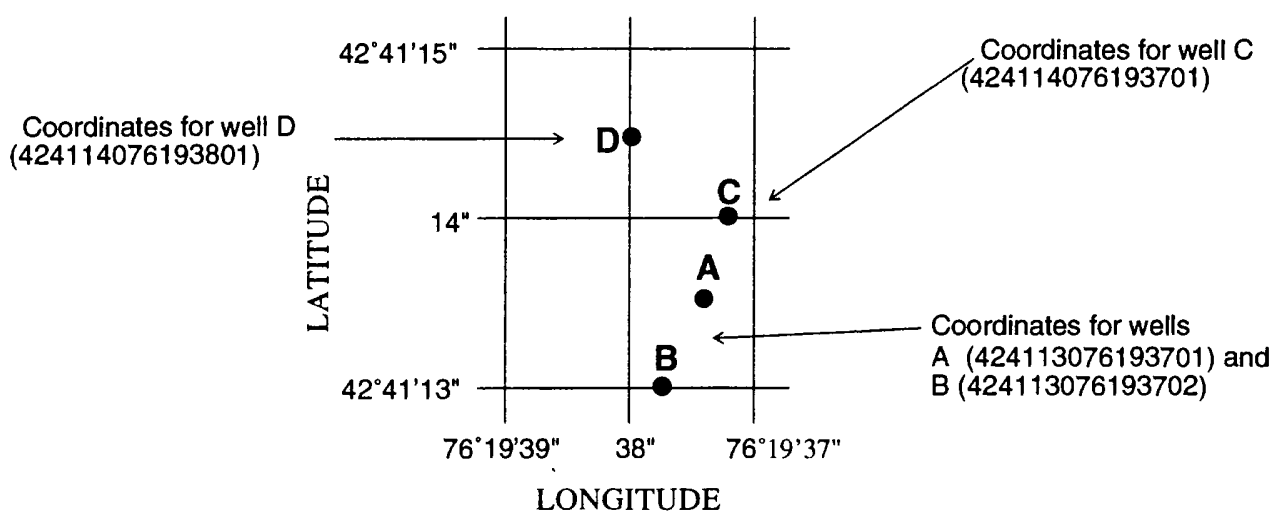

Figure 9. Latitude and longitude system for well numbering. 


\section{Water Levels}

Water-level records are taken from direct measurements made with a weighted steel tape and recorded in feet below land-surface datum, a datum plane that approximates land surface at each well. Water levels in wells are measured periodically (usually monthly) and are recorded to the nearest hundredth of a foot. Each well description herein includes the land-surface datum above mean sea level and the height of the measuring point above or below land-surface datum.

\section{Water Temperature}

Water temperature is measured seasonally in most wells at various depths with a temperature probe. The depth intervals between measurements range from $1 \mathrm{ft}$ to about $3 \mathrm{ft}$, and temperatures are recorded to the nearest hundredth of a degree Celsius.

\section{Chemical Quality}

Water samples were collected quarterly from 15 wells in the Irondequoit Creek basin during 1989-93. All samples were collected with a peristaltic pump. At least three casing volumes of water were removed to purge the well before sampling, and the water level was then allowed to recover before sample collection to ensure that samples would be representative of fresh aquifer water, not water that had been standing in the well.

Results of the chemical analyses document the water quality of the aquifer system and indicate temporal and areal differences in the quality of water within the aquifer, as well as areas that may be affected by contamination. Ground-water samples were analyzed for specific conductance, $\mathrm{pH}$, and concentrations of common ions, nutrients, metals, dissolved solids, alkalinity, and hardness. These constituents generally provide an indication of the general water quality of an aquifer.

\section{Data Format}

Ground-water data from 3 wells in Powder Mill Park, and 12 wells in Ellison Park, are presented; these data include water levels, temperature, and chemical quality.

Each well record consists of four parts-the well description, a table and graph of water levels measured during 1989-93, chemical analyses for each water year, and a table and depth profile of water tempera- ture. The well description includes such information as location, aquifer, well characteristics and in strumentation, datum, period of record, historical extremes, and remarks giving other pertinent information. The headings used in the well descriptions are explained below.

Location.--Gives the latitude and longitude (in degrees, minutes, and seconds); the hydrologic unit number; the distance and direction from a georraphic point of reference; and the owner's name.

Aquifer.--Identifies by name (if a name exists) and geologic age of the aquifer(s) open to the well.

Well Characteristics.--Describes the deptl , diameter, casing depth and(or) screened interval, method of construction, and use of the well, and additional information such as casing breaks, collapsed screer. and other changes since construction.

Instrumentation.--Describes frequency of measurements and the method used.

Datum.--Describes both the measuring point and the land-surface elevation at the well. The measuring point is described physically (such as top of cc'lar, notch in top of casing, plug in pump base and so on), and in relation to land surface (such as $1.3 \mathrm{ft}$ above land-surface datum). The elevation of land-surface datum is described in feet above mean sea level; precision depends on the method of determinatior.

Remarks.--Describes factors that could affect the water level in a well or the measurement of the water level and identifies wells that also are water-quality observation wells; it also acknowledges the assistance of local (non-USGS) observers.

Period of Record.--Identifies the period(s) for which published records are available.

Extremes For Period.--Indicates the highert and lowest water levels of the period of published record, with respect to land-surface datum, and the dates of their occurrence.

The second part of the well record is a table that lists water levels for each of the water years, in feet above or below land-surface datum and the measurement dates. A hydrograph of water levels for the period of record follows the water-level tables. The annual water-level tables and hydrograph for ea-h well are followed by the water-quality tables. The fourth part of the well record is table of water-temperatures and a water-temperature profile to allow a visual comparison of seasonal patterns. 


\section{Precipitation Quantity and Quality}

Precipitation-quantity data were collected at four sites, three of which are in the Irondequoit Creek basin, and one (Mendon Ponds) just southwest of the Irondequoit Creek basin, in the Genesee River basin near the drainage divide. Precipitationquality data were collected at Mendon Ponds and at two other sites- one in the western Monroe County at SUNY Brockport, and one at Empire Boulevard near Rochester.

\section{Methods}

Total precipitation at the three Irondequoit sites was measured with a precipitation-collection tube with float and counterweight whose values were coded at 15-minute intervals by punched-tape recorders. The positive difference between two successive readings was computed and recorded as the total precipitation for that 15 -minute interval. The 96 values of 15-minute data were summed to give the total daily precipitation value, which was subsequently entered into the WATSTORE data-storage system and is presented here.

Precipitation at the Mendon Ponds site was recorded continuously on a strip chart from a weighing-bucket rain gage. A bulk collector and a wetfall/ dryfall sampler were used to collect composite samples. The dryfall (or dustfall) container was removed monthly for analysis of the contents, and the wetfall container was removed and the contents analyzed after selected storms. MCEHL collected and analyzed these samples in accordance with procedures outlined by USGS.

\section{Data Format}

The precipitation records herein consist of (1) a site description, (2) a table of total daily precipitation values by water year, (3) precipitation-quality data, which include chemical analyses of dustfall, wetfall, and bulk deposition for each water year.

The site descriptions include information on location, period of record, annual maximum, and equipment and remarks giving other pertinent information. The used in the site descriptions are as follows:

Location.--Information on locations is obtained from USGS topographic maps. The location of the gage is given with respect to the cultural and physical features in the vicinity and to the reference place mentioned in the station name.
Period of Record.--This indicates the period for which published precipitation or atmospheric-quality records for the station are available.

Equipment.--Describes the type $c^{f}$ equipment used at the site, the type of data collested by each, and the location of the equipment with respect to ground level.

Remarks.--Provides added information pertinent to the collection, analysis, or computat in of the records.

The site description for each precipitation gage is followed by a table of total daily precipitation values by water year, with monthly and yearly' summaries. The "TOTAL" line for each water year gives the sum of the daily figures for each month.

The chemical data are presented in order of monthly dustfall, monthly wetfall, and, if available, monthly composite.

\section{QUALITY ASSURANCE/QUALITY CONTROL}

Much of the data presented in this repcrt were collected and analyzed by MCEHL, whos: responsibilities included completion of most of the discharge measurements at stations with establisl ad ratings, monthly inspections at all sites, removal of recorded data, and collection and laboratory analysis of water samples. A Quality-Assurance/Quality-Control (QA/ QC) program is an integral part of this cooperative data-collection effort to ensure that the data meet standards for publication set by the USGS. The basic QA/ QC protocol devised for the NURP stuty (Zarriello and others, 1984; Kappel and others, 1986) has continued and been extended under the USGS-MCEHL cooperative program. The program contains two parts: (1) streamflow measurements, and (2) water-quality samples. The procedures and guidelines used in the QA/QC program are summarized below.

\section{Streamflow Measurements}

General guidelines and procedures accepted by the USGS for gaging streams (Carter and Davidian, 1968) were followed throughout the period represented by this report. More detailed procedures were followed in regard to specific phases of data collection, which include stage measurement at gaging stations (Buchanan and Somers, 1968) and discharge measurement by current meter (Buchanan and Somers, 
1969). Interpretation of the data by USGS staff followed recommended procedures and include stage-todischarge rating development (Kennedy, 1984) and computation of records for publication (Kennedy 1983). These topics are also covered in Rantz and others (1982, v. 1 and 2).

The USGS provides further quality control of the streamflow data-collection efforts of MCEHL by (1) monthly review of stream-discharge measurements and equipment-inspection notes, and (2) semiannual onsite inspections of gaging facilities and completion of discharge measurements. These semiannual discharge measurements, which check the validity of the rating developed for that particular year, have consistently indicated that discharge measurements made by MCEHL fully meet USGS standards. In addition, USGS personnel make additional discharge measurements for the first year after the establishment of a new gaging station.

\section{Water-Quality Samples}

The QA/QC program for water-quality samples includes sample collection and laboratory analysis. Continuing-record water samples are collected from a single point in the stream at surface-water sites by automatic sampler. Part of this program is designed to determine whether these samples are representative of water quality throughout the stream cross section; the program also is designed to ensure that laboratory analysis of water samples by MCEHL meet standards for publication set by USGS. This is done by two procedures: (1) split-sample collection and analysis, and (2) participation in the USGS Standard Reference Water-Sample (SRWS) program. All aspects of the QA/QC procedures for water-quality samples are evaluated by statistical methods and are discussed more fully in the following sections.

\section{Statistical Methods}

A paired $t$ test was used to compare mean differences between (1) constituent concentrations in the split samples analyzed by the USGS Central Laboratory and those analyzed by MCEHL, and (2) samples collected by hand and those collected by automatic sampler. A paired $t$ test uses the difference method to test the null hypothesis that the mean difference between the two sample groups is zero. If the calculated $t$ statistic is greater than the $t$ statistic from the $t$ distribution table for a particular confidence level and number of degrees of freedom, then the null hypothesis is rejected, and a difference is indicated between the two groups of data, with a less than 5-percent (95percent confidence level) chance that the difference is due to random causes. If the $t$ statistic is less than that found in the table, the null hypothesis is not rejocted and indicates a chance of less than 5 percent that the means are different.

The data were tested for bias to determine whether constituent concentrations in samples collected by the automatic sampler were consistently high or low. The mean bias (in percent) was calcula ${ }^{+}$ed from the equation:

$$
\text { Bias }=\frac{C_{a}-C_{b}}{C_{b}} \times 100
$$

where:

$$
\begin{aligned}
C_{a}= & \text { concentrations either in samples } \\
& \text { taken from the automatic sampler } \\
& \text { or determined by } \\
& \text { MCEHL, and } \\
C_{b}= & \text { concentrations in samples col- } \\
& \text { lected by hand from the stream } \\
& \text { cross section or determined by the } \\
& \text { USGS laboratory. }
\end{aligned}
$$

A $t$ test was then done on the mean bias to determine significance at the 95-percent confidence level. Statistical methods used are outlined in Friedman and Erdmann (1982).

Statistical analysis of the sample results, discussed in some detail below, indicate some significant differences, as well as instances of bias, but the results are considered inconclusive because only a small number of split samples were involved in the analysis.

\section{Split Samples}

The split samples collected by Monroe County were used to (1) compare concentrations of constituents in samples collected by the automatic sampler with those collected by hand from the stream cross section, and (2) assess any differences in analytical results between MCEHL and the USGS Central Laboratory. Split samples are samples divided into equal parts to obtain a statistical comparison of analytical results.

Part of the QA/QC protocol is designed to determine whether samples collected by the automatic sam- 
plers are representative of water quality throughout the stream cross section. Periodically at each site, depthintegrated cross-sectional samples were collected from the stream, while the automatic sampler was induced to take samples. The results of the analysis of the two sets of samples were then compared to detect any systematic bias in samples collected by the automatic sampler.

Six cases showed a statistically significant difference between mean concentrations in samples collected by the automatic sampler and those collected by hand (table 4). In all but one of those cases, mean concentrations in samples collected by the automatic sampler were higher than those collected by hand. Mean concentrations of total phosphorus were significantly higher in samples collected by the automatic sampler at the new Irondequoit Creek site above Blossom Road (On October 1, 1991 the Blossom Roal site was moved several hundred feet upstream because of bridge construction) on July 20, 1993 and at Irondequoit Creek at Empire Boulevard on July 29, 1992. Mean concentrations of dissolved ammonia plus organic nitrogen were higher in autom tic samples collected at Irondequoit Creek near Pittsford on June 20,1989 , and at the upstream (new) Blossom road site on September 18, 1991. Nitrite plus nitrate was significantly higher in samples collected by the automatic sampler at Blossom road (old site) on June 20, 1989. The only case wherein the automatic s`mpler produced a lower concentration was in a total phosphorus result from a sample collected at the (row) Blossom road site on July 29,1992 . Wherever a statistically

Table 4. Statistical analysis of split samples collected to assess representativeness of samples collected by the automatic sampler.

[DKN, dissolved ammonia plus organic nitrogen; TKN, total ammonia plus organic nitrogen; NOx, nitrite plus nitrate; TP, total phosphorus; Hand, samples collected from stream using depth integrated equal width increment method; Auto, automatic sampler; locations are shown in fig. 2]

\begin{tabular}{|c|c|c|c|c|c|c|c|c|c|c|c|}
\hline \multirow[b]{3}{*}{ Site } & \multirow[b]{3}{*}{ Date } & \multirow{3}{*}{$\begin{array}{c}\text { Consti- } \\
\text { tuent }\end{array}$} & \multicolumn{5}{|c|}{ Paired t-test on differences } & \multicolumn{4}{|c|}{ t-test on bias } \\
\hline & & & \multirow{2}{*}{$\begin{array}{l}\text { Number } \\
\text { of pairs } \\
(n)\end{array}$} & \multicolumn{2}{|c|}{ Mean values } & \multirow{2}{*}{$\begin{array}{l}\text { Mean } \\
\text { diff. }\end{array}$} & \multirow{2}{*}{$\begin{array}{c}\text { Test } \\
\text { statistic } \\
(t)\end{array}$} & \multirow{2}{*}{$\begin{array}{c}\text { Mean } \\
\text { bias }\end{array}$} & \multirow{2}{*}{$\begin{array}{l}\text { Standardd } \\
\text { eviation }\end{array}$} & \multirow{2}{*}{$\begin{array}{c}\text { Number } \\
\text { of pairs } \\
\text { (n) }\end{array}$} & \multirow{2}{*}{$\begin{array}{c}\text { Test } \\
\text { statistic }(t\end{array}$} \\
\hline & & & & Hand & Auto & & & & & & \\
\hline \multirow{4}{*}{$\begin{array}{l}\text { Irondequoit } \\
\text { Creek } \\
\text { near } \\
\text { Pittsford, } \\
\text { NY }\end{array}$} & $6-20-89$ & DKN & 8 & 0.561 & 0.738 & -0.177 & $-2.493^{*}$ & 30.61 & 32.78 & $\overline{8}$ & $2.642^{*}$ \\
\hline & & NOx & 8 & 0.874 & 0.863 & 0.011 & 2.183 & -1.270 & 1.658 & 8 & -2.166 \\
\hline & $8-21-90$ & DKN & 4 & 0.425 & 0.400 & 0.025 & 1.000 & -5.000 & 10.00 & 4 & -1.414 \\
\hline & & NOx & 4 & 1.300 & 1.300 & 0 & 0 & 0 & 0 & 4 & 0 \\
\hline \multirow{6}{*}{$\begin{array}{c}\text { Irondequoit } \\
\text { Creek } \\
\text { at } \\
\text { Blossom } \\
\text { Rd. } \\
\text { (old site) }\end{array}$} & $6-20-89$ & DKN & 8 & 0.783 & 0.719 & 0.064 & 0.635 & -4.168 & 33.53 & 8 & -0.352 \\
\hline & & NOx & 8 & 1.225 & 1.300 & -0.075 & $-4.583^{*}$ & 6.250 & 3.858 & 8 & $4.582^{*}$ \\
\hline & $8-21-90$ & DKN & 4 & 0.325 & 0.325 & 0 & 0 & 4.167 & 47.87 & 4 & 0.174 \\
\hline & & NOx & 4 & 0.900 & 0.900 & 0 & 0 & 0 & 0 & 4 & 0 \\
\hline & $9-18-91$ & DKN & 8 & 0.550 & 0.433 & 0.117 & 1.817 & -17.66 & 24.34 & 8 & -2.047 \\
\hline & & NOx & 8 & 0.686 & 0.700 & -0.014 & -1.429 & 2.098 & 4.057 & 8 & 1.463 \\
\hline \multirow{7}{*}{$\begin{array}{c}\text { Irondequoit } \\
\text { Creek } \\
\text { at } \\
\text { Empire } \\
\text { Blvd. }\end{array}$} & $7-29-92$ & TKN & 4 & 0.475 & 0.425 & 0.050 & 1.732 & -10.00 & 11.55 & 4 & -1.732 \\
\hline & & NOx & 4 & 1.000 & 1.000 & 0 & 0 & 0 & 0 & 4 & 0 \\
\hline & & $\mathrm{TP}$ & 4 & 0.043 & 0.053 & -0.010 & $-2.449 *$ & 27.92 & 28.00 & 4 & $2.418^{*}$ \\
\hline & $7-20-93$ & TKN & 3 & 0.837 & 0.830 & 0.007 & 0.068 & 0.460 & 19.43 & 3 & 0.041 \\
\hline & & TP & 3 & 0.120 & 0.123 & -0.003 & -1.000 & 2.778 & 4.811 & 3 & 1.000 \\
\hline & $10-20-93$ & TKN & 8 & 0.479 & 0.541 & -0.062 & -0.959 & 25.32 & 60.15 & 8 & 1.191 \\
\hline & & TP & 8 & 0.035 & 0.032 & 0.003 & 0.886 & 10.98 & 58.62 & 8 & 0.530 \\
\hline \multirow{7}{*}{$\begin{array}{l}\text { Irondequoit } \\
\text { Creek } \\
\text { above } \\
\text { Blossom } \\
\text { Rd. } \\
\text { (new gage } \\
\text { site) }\end{array}$} & $9-18-91$ & $\overline{\mathrm{DKN}}$ & 8 & 0.434 & 0.697 & -0.263 & $-3.074^{*}$ & 78.04 & 68.24 & 8 & $3.235^{*}$ \\
\hline & & NOx & 8 & 0.714 & 0.714 & 0 & 0 & -0.044 & 1.673 & 8 & -0.074 \\
\hline & $7-29-92$ & TKN & 4 & 0.425 & 0.400 & 0.025 & 1.000 & -5.000 & 10.00 & 4 & -1.000 \\
\hline & & NOx & 4 & 1.100 & 1.100 & 0 & 0 & 0 & 0 & 4 & 0 \\
\hline & & TP & 4 & 0.043 & 0.022 & 0.021 & $4.899 *$ & -45.83 & 8.333 & 4 & $-11.00^{*}$ \\
\hline & $7-20-93$ & TKN & 3 & 0.650 & 0.793 & -0.143 & -0.959 & 25.35 & 43.34 & 3 & 1.013 \\
\hline & & TP & 3 & 0.072 & 0.100 & -0.028 & $-17.00 *$ & 39.68 & 5.499 & 3 & $12.50^{*}$ \\
\hline
\end{tabular}

* differences are statistically significant at the 95 percent confidence level. 
significant difference in constituent concentration between samples collected by the automatic sampler and those collected by hand was detected, a statistically significant bias also was noted.

A paired $t$ test was also used to statistically compare constituent concentrations determined by MCEHL with those determined by USGS. Of the split samples collected on September 18, 1991, only nitrite plus nitrate showed a statistically significant difference in concentration between the two laboratories (table 5). In samples collected on October 20,1993, total ammonia plus organic nitrogen and total phosphorus showed a significant difference in concentration between the two laboratories. Samples collected for laboratory comparison were not tested for bias.

\section{Standard Reference Water-Sample Program}

As part of USGS quality-assurance program for cooperating laboratories, MCEHL was requiret to participate in a standard reference water-sample (SRWS) program. Under this program, the USGS Central Laboratory submits reference samples (major constituents, trace constituents, and nutrients) twice yearly to laboratories that analyze water samples as part of a cooperative program. The analytical results from all participating laboratories are sent to the USGS Central Laboratory and analyzed statistically to determine the "most probable value" (MPV) for each constit ıent. Each laboratory's results are then compared against the MPV and rated (table 6) by increments of standard deviation from the MPV.

Table 5. Statistical analysis of split samples collected for laboratory comparison.

[USGS = U. S. Geological Survey; MCEHL = Monroe County Environmental Health Laboratory; DKN = Dissolved ammonia plus organic nitrogen; $\mathrm{NOx}=$ nitrite plus nitrate; $\mathrm{TKN}=$ total ammonia plus organic nitrogen; $\mathrm{TP}=$ total phosphorus]

\begin{tabular}{|c|c|c|c|c|c|c|c|}
\hline \multirow[b]{3}{*}{ Date } & \multirow{3}{*}{$\begin{array}{l}\text { Constit } \\
\text {-uent }\end{array}$} & \multicolumn{6}{|c|}{ Paired t-test on differences } \\
\hline & & \multirow{2}{*}{$\begin{array}{c}\text { Number of } \\
\text { pairs }(n)\end{array}$} & \multicolumn{2}{|c|}{ Mean Values } & \multirow{2}{*}{$\begin{array}{c}\text { Mean } \\
\text { difference }\end{array}$} & \multirow{2}{*}{$\begin{array}{c}\text { Test } \\
\text { statistic } \\
(t)\end{array}$} & \multirow{2}{*}{$\begin{array}{c}\text { Significant } \\
\text { at } \\
95 \% \\
\end{array}$} \\
\hline & & & USGS & MCEHL & & & \\
\hline \multirow[t]{2}{*}{$6-20-89$} & $\overline{\mathrm{DKN}}$ & 16 & 0.744 & 0.656 & 0.088 & 1.577 & no \\
\hline & NOx & 16 & 1.062 & 1.068 & -0.006 & -0.570 & no \\
\hline \multirow[t]{2}{*}{$9-18-91$} & DKN & 16 & 0.612 & 0.519 & 0.093 & 0.941 & no \\
\hline & NOx & 16 & 0.686 & 0.721 & -0.035 & -3.075 & yes \\
\hline \multirow[t]{2}{*}{$10-20-93$} & TKN & 20 & 0.315 & 0.611 & -0.296 & -9.267 & yes \\
\hline & TP & 20 & 0.018 & 0.042 & -0.024 & -7.765 & yes \\
\hline
\end{tabular}

Table 6. Rating of cooperating laboratory's analysis of U.S. Geological Survey standard reference water samples. [First number is rating, explained in footnote. Numbers in parentheses are the number of constituents analyzed in each group.]

\begin{tabular}{|c|c|c|c|c|c|c|}
\hline \multirow[b]{2}{*}{ Date } & \multicolumn{6}{|c|}{ Constituents } \\
\hline & $\begin{array}{c}\text { Trace } \\
\text { elements }\end{array}$ & $\begin{array}{l}\text { Major } \\
\text { ions }\end{array}$ & Nutrients & Precipitation & Mercury & $\begin{array}{c}\text { All } \\
\text { constituents }\end{array}$ \\
\hline Aug. 89 & $2.47(17)$ & $3.46(13)$ & $3.50(6)$ & $2.29(7)$ & $4.00(1)$ & $3.18(44)$ \\
\hline Jan. 90 & $2.33(18)$ & $2.92(13)$ & $3.83(6)$ & $2.80(10)$ & -- & $2.85(52)$ \\
\hline Jul. 90 & $3.1(15)$ & $3.6(13)$ & $3.3(11)$ & $3.1(10)$ & $4.0(1)$ & $3.3(51)$ \\
\hline Feb. 91 & $2.5(15)$ & $3.5(12)$ & $3.2(22)$ & $3.1(10)$ & -- & $3.1(59)$ \\
\hline Sept. 91 & $2.3(18)$ & $3.0(13)$ & $3.6(14)$ & $3.3(9)$ & $4.0(1)$ & $3.0(56)$ \\
\hline Oct. 92 & $2.0(15)$ & $3.7(13)$ & $3.7(18)$ & $3.1(8)$ & $3.0(1)$ & $3.1(55)$ \\
\hline Apr. 93 & $2.6(28)$ & $2.7(13)$ & $3.8(18)$ & -- & -- & $3.0(59)$ \\
\hline
\end{tabular}

Rating system:

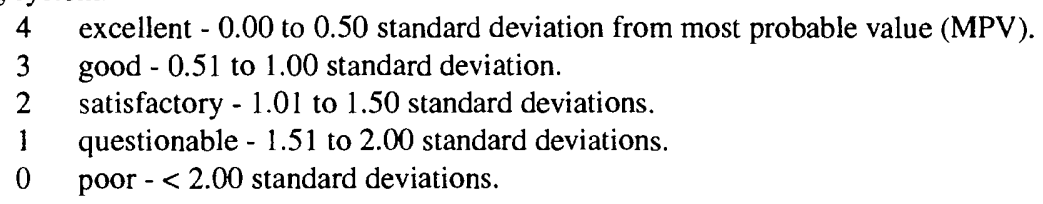




\section{SELECTED REFERENCES}

American Public Health Association, American Water Works Association, and Water Pollution Control Federation, 1985, Standard methods for the examination of water and waste water: Washington, D.C., American Public Health Association, 16th ed., 1,134 p.

Bailey, J.F., and Ray, H.A., 1966, Definition of stage-discharge relation in natural channels by step-back-water analysis: U.S. Geological Survey Water-Supply Paper 1869-A, $24 \mathrm{p}$

Britton, L.J., and Greeson, P.E., 1989, Methods for collection and analysis of aquatic biological and microbiological samples: U.S. Geological Survey Techniques of Water-Resources Investigations, book 5, chap. A4, $363 \mathrm{p}$.

Bodhaine, G. L., 1968, Measurement of peak discharge at culverts by indirect methods: U.S. Geological Survey Techniques of Water-Resources Investigations, book 3, chap. A3, 60 p.

Buchanan, T.J., and Somers, W.P., 1968, Stage measurements at gaging stations: U.S. Geological Survey Techniques of Water-Resources Investigations, book 3, chap. A7, 28 p.

1969, Discharge measurements at gaging stations:

U.S. Geological Survey Techniques of Water-

Resources Investigations, book 3, chap. A8, 65 p.

Campbell, J.B., Coon, W.F., Sherwood, D.A., and Deloff,

D.D., 1989, Water-resources data, New York, Water

Year 1989, volume 3-Western New York: U.S. Geological Survey Water-Data Report NY-89-3, 196 p.

Campbell, J.B., Szabo, C.O., Sherwood, D.A., and Deloff, D.D., 1990, Water-Resources Data, New York, Water Year 1990, volume 3-Western New York: U.S. Geological Survey Water Data Report NY-90-3, 202 p. 1991, Water-Resources Data, New York, Water Year 1991, volume 3-Western New York: U.S. Geological Survey Water Data Report NY-91-3, 200 p.

Carter, R.W., and Davidian, Jacob, 1968, General procedure for gaging streams: U.S. Geological Survey Techniques of Water-Resources Investigations, book 3, chap. A6, 13 p.

Dalrymple, Tate, and Benson, M.A., 1967, Measurement of peak discharge by the slope-area method: U.S. Geological Survey Techniques of Water-Resources Investigations, book 3, chap. A2, 12 p.

Friedman, L.C., and Erdmann, D.E., 1982, Quality assurance practices for the chemical and biological analyses of water and fluvial/sediments: U.S. Geological Survey Techniques of Water-Resources Investigations, book 5, chap. A6, $181 \mathrm{p}$.

Goerlitz, D.F., and Brown, Eugene, 1972, Methods for analysis of organic substances in water: U.S. Geological
Survey Techniques of Water-Resources Investigations, book 5, chap. A3, $40 \mathrm{p}$.

Guy, H.P., and Norman, V.W., 1970, Field methods for measurement of fluvial sediment: U.S. Geological Survey Techniques of Water-Resources Investigations, book 3, chap. C2, $59 \mathrm{p}$.

Heffner, R.L., and Goodman, S.D., 1973, Soil survey of Monroe County, New York: U.S. Dejartment of Agriculture, $172 \mathrm{p}$.

Hornlein, J.F., Szabo, C.O., Zajd, H.J., Jr., and Deloff, D.D., 1992, Water-resources data, New York, Water Year 1992, volume 3--Western New York: U.S. Geological Survey Water-Data Report NY-92-3, 289 p.

1993, Water-resources data, New Ycrk, Water Year 1993, volume 3-Western New York: U.S. Geological Survey Water-Data Report NY-93-3, 275 p.

Hulsing, Harry, 1967, Measurement of peak discharge at dams by indirect methods: U.S. Geo'ngical Survey Techniques of Water-Resources Investigations, book 3, chap. A5, 29 p.

Johnston, W.H., and Sherwood, D.A., 1994, Water Resources of Monroe County, New York, Water Years 1984-88 with emphasis on Water Quelity in the Irondequoit Creek Basin. U.S. Geological Survey Open File Report 93-370, 311p.

Kappel, W.M., Yager, R.M., and Zarriello, P.J., 1986, Quantity and quality of urban storm runoff in the Irondequoit Creek basin near Rochester, Ne'v York, Part 2-Quality of storm runoff and atmospheric deposition, rainfall-runoff quality modeling, and potential of wetlands for sediment and nutrient retention: U.S. Geological Survey Water-Resources Investigations Report 85-4113, 93 p.

Kappel, W.M., and Young, R.A., 1989, Glacial history and geohydrology of the Irondequoit Creek valley, Monroe County, New York: U.S. Geological Survey WaterResources Investigations Report 88-4145, 34 p.

Kennedy, E.J., 1983, Computation of continuous records of streamflow: U.S. Geological Survey Techniques of Water-Resources Investigations, book 3, chap. A13, 53 p.

1984, Discharge ratings at gaging stations: U.S. Geological Survey Techniques of Water-Resources Investigations, book 3, chap. A10, $59 \mathrm{p}$.

Lapham, W.W., 1989, Use of temperature profiles beneath streams to determine rates of vertical ground-water flow and vertical hydraulic conductivity: U.S. Geological Survey Water-Supply Paper 2337, 35 p.

Matthai, H.J., 1967, Measurement of peak discharge at width contractions by indirect methods: U.S. Geological Survey Techniques of Water-Reso'ırces Investigations, book 3, chap. A4, 44 p.

National Oceanic and Atmospheric Administration, 1989, Climatological data, annual summary, New York: 
Asheville, N.C., National Climatic Data Center, v. 101, no. $13,39 \mathrm{p}$.

1990, Climatological data, annual summary, New

York: Asheville, N.C., National Climatic Data Center, v. 102 , no. 13,30 p. 1991, Climatological data, annual summary, New

York: Asheville, N.C., National Climatic Data Center, v. 103 , no. 13,30 p.

1992, Climatological data, annual summary, New

York: Asheville, N.C., National Climatic Data Center, v. 104 , no. 13,29 p.

1993, Climatological data, annual summary, New

York: Asheville, N.C., National Climatic Data Center, v. 105 , no. 13,29 p.

Rantz, S.E., and others, 1982, Measurement and computation of streamflow--volume 1 - measurement of stage and discharge: U.S. Geological Survey Water-Supply Paper 2175, $284 \mathrm{p}$.

1982, Measurement and computation of streamflow-volume 2 - computation of discharge: U.S. Geological Survey Water-Supply Paper 2175, 347 p.
Shearman, J.O., 1976, Computer applications for step-backwater and floodway analysis: U.S. Geological survey Open-File Report 76-499, 103 p.

Skougstad, M.W., and others, 1979, Methods for determination of inorganic substances in water and fluvial sediments: U.S. Geological Survey Techniques of W/aterResources Investigations, book 5, chap. A1, $626 \mathrm{p}$.

Wagner, L.A., and Dixson, H.L., 1985, Drainage areas of New York streams by river basins--a stream gaz tteer: U.S. Geological Survey Open-File Report 81-1055, $359 \mathrm{p}$.

Wood, W.W., 1976, Guidelines for collection and fie $e^{1 / d}$ analysis of ground-water samples for selected unstahle constituents: U.S. Geological Survey Techniques of Water-Resources Investigations, book 1, chap. D2, 24 p.

Zarriello, P.J., Harding, W.E., Yager, R.M., and Kapfel, W.M., 1984, Quality and quantity of storm runoff in the Irondequoit Creek basin near Rochester, New York, part 1--Data-collection network and methods, qualityassurance program and description of available data: U.S. Geological Survey Open-File Report 84-610, 29 p. 


\section{DATA FROM SURFACE-WATER, GROUND-WATER, AND PRECIPITATION STAT'ONS IN MONROE COUNTY}

\section{Surface-Water Stations}

[Letter after station name designates type of data: (d) discharge, (e) gage height,

(c) chemical, (b) biological, (t) water temperature, (s) sediment, (p) precipitation.]

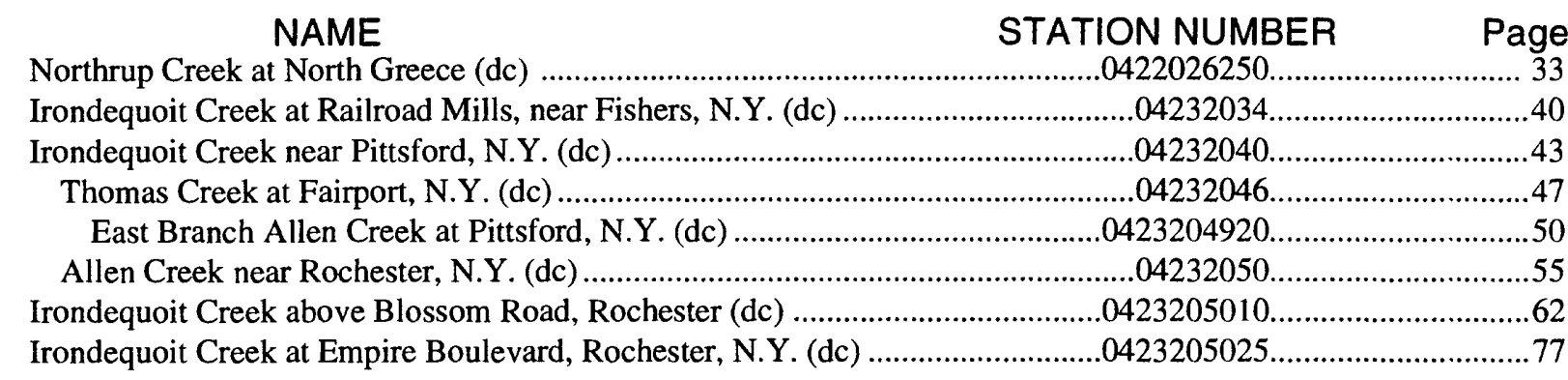

\section{Miscellaneous and Partial-Record Stations}

Genesee River at Charlotte Pump station, at Rochester

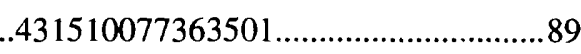

Cartersville Waste Channel at Pittsford, N.Y. .430449077294201

E. Br. Allen Creek above Erie Canal Siphon nr Pittsford, N.Y 430526077315201

E. Br. Allen Creek below Erie Canal Siphon nr Pittsford, N.Y .430526077315202

E. Br. Allen Creek Erie Canal Siphon nr Pittsford, N.Y .430526077315203

Allen Creek below Erie Canal Siphon nr Rochester, N.Y 430557077344402

\section{Ground-Water Stations}

[Letter after station identification designates type of data:

(e) water level, (c) chemical, (t) water temperature]

Powder Mill Park Mo 10 (ect)

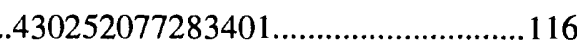

Powder Mill Park Mo 11 (ect)

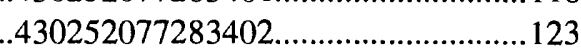

Powder Mill Park Mo 12 (ect) $430249077284501 \ldots \ldots \ldots \ldots \ldots \ldots \ldots \ldots \ldots \ldots . . . . .130$

Ellison Park Mo 1 (ect)

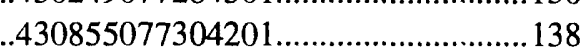

Ellison Park Mo 2 (ect)

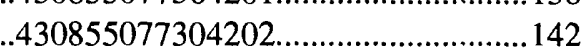

Ellison Park Mo 3 (ect) .430854077304601

Ellison Park Mo 4 (ect) .430854077304901

Ellison Park Mo 5 (ect) .430855077305201

Ellison Park Mo 6 (ect) .430855077305202

Ellison Park Mo 659 (ect) .430932077311501

Ellison Park Mo 663 (ect) . .430912077313301

Ellison Park Mo 664 (ect) .430912077313302

Ellison Park Mo 665 (ect) 430928077313802

Ellison Park Mo 667 (ect) 430928077314001

Ellison Park Mo 668 (ect)

430928077314002

\section{Precipitation Stations}

[Letter after station identification designates type of data: (p) precipitation, (c) chemical]

At Mendon Ponds (pc)

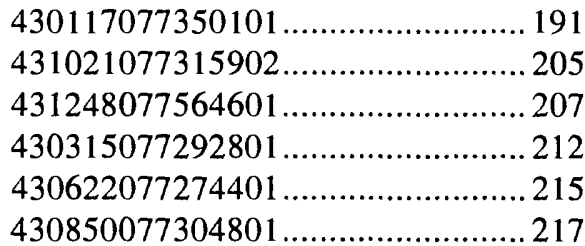

At Empire Boulevard (c)

At SUNY Brockport (c) 


\section{Surface-Water Stations}

\section{A. Discharge and water quality}

\section{Northrup Creek At North Greece, N.Y.}

.OCATION.--Lat $43^{\circ} 15^{\prime} 13^{\prime \prime}$, long $77^{\circ} 43^{\prime} 33^{\prime \prime}$, Monroe County, Hydrologic Unit 04130001 , on right bank 75 ft downstream from bridge on State Highway 18 (Latta Road), $0.5 \mathrm{mi}$ west of North Greece and $5.1 \mathrm{mi}$ upstream from mouth.

DRAINAGE AREA. $\cdots 11.7 \mathrm{mi}^{2}$.

'ERIOD OF RECORD.--August 1989 to current year

\section{WATER-DISCHARGE RECORDS}

TAGE--Water-stage recorder. Elevation of gage is $306 \mathrm{ft}$ above sea level, from topographic map.

REMARKS.--Records fair. Unpublished water-quality records for prior years are available in files of Monroe County Department of Health.

SOOPERATION.--Gage-height record and 9 discharge measurements were provided by the Monroe County Environmental Health Laboratory at Rochester, N.Y.

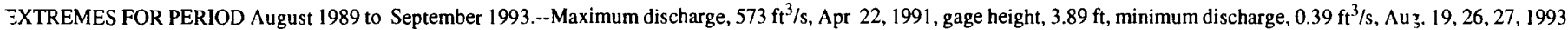

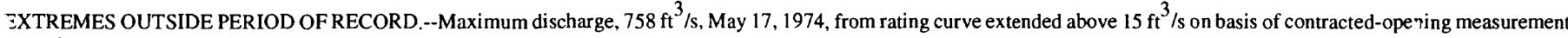
of peak flow.

STATISTICS OF MONTHLY MEAN DISCHARGE (in cubic feet per second) FOR WATER YEARS 1989-93, BY WATER YEAR

\begin{tabular}{|c|c|c|c|c|c|c|c|c|c|c|c|c|}
\hline & OCT & NOV & DEC & JAN & FEB & MAR & APR & MAY & JUN & JUL & AUG & SEP \\
\hline MEAN & 7.88 & 10.4 & 13.5 & 17.8 & 19.5 & 30.0 & 28.2 & 13.6 & 4.28 & 3.79 & 3.82 & 3.08 \\
\hline MAX & 14.7 & 18.8 & 23.1 & 25.0 & 38.9 & 40.7 & 31.7 & 27.1 & 5.12 & 6.31 & 7.43 & 5.33 \\
\hline (WY) & 1990 & 1993 & 1991 & 1993 & 1990 & 1993 & 1991 & 1990 & 1990 & 1992 & 1992 & 1992 \\
\hline MIN & 2.54 & 2.49 & 3.65 & 10.2 & 7.82 & 17.1 & 26.7 & 4.77 & 3.06 & 1.96 & 1.60 & 1.10 \\
\hline (WY) & 1992 & 1992 & 1992 & 1992 & 1993 & 1990 & 1993 & 1993 & [99] & 1993 & 1993 & 1989 \\
\hline
\end{tabular}

STATISTIC

ANNUAL TOTAL

ANNUAL MEAN

AVERAGE DISCHARGE

HIGHEST ANNUAL MEAN

LOWEST ANNUAL MEAN

HIGHEST DAILY MEAN

LOWEST DAILY MEAN

ANNUAL SEVEN-DAY MINIMUM

INSTANTANEOUS PEAK FLOW

INSTANTANEOUS PEAK STAGE

INSTANTANEOUS LOW FLOW

ANNUAL RUNOFF (CFSM)

ANNUAL RUNOFF (INCHES)

10 PERCENT EXCEEDS

50 PERCENT EXCEEDS

90 PERCENT EXCEEDS

a Aug 19, 26, 27, 1993.

\section{SUMMARY STATISTICS}

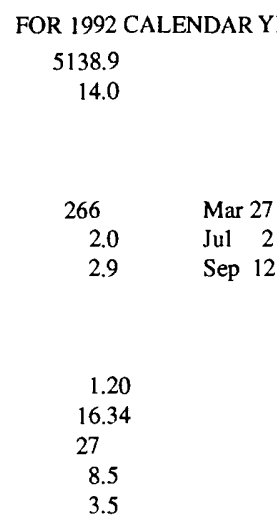

\section{FOR 1993 WATER YEAR 4954.8}

13.6

$\begin{array}{cll}229 & & \\ 1.1 & & \text { Apr 1 } \\ 1.4 & \text { Aug 19 } \\ 327 & \text { Aug 22 } \\ 3.11 & \text { Apr } 1 \\ .39 & \text { Apr } & \text { a } \\ 1.16 & & \\ 15.75 & & \\ 26 & & \\ 7.0 & & \\ 1.7 & & \end{array}$

WATER YEARS $1987-1993$

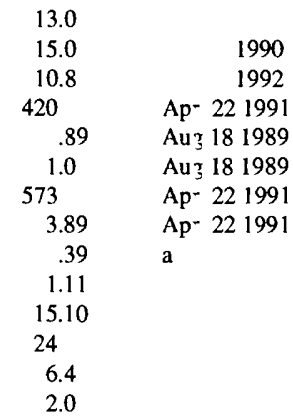

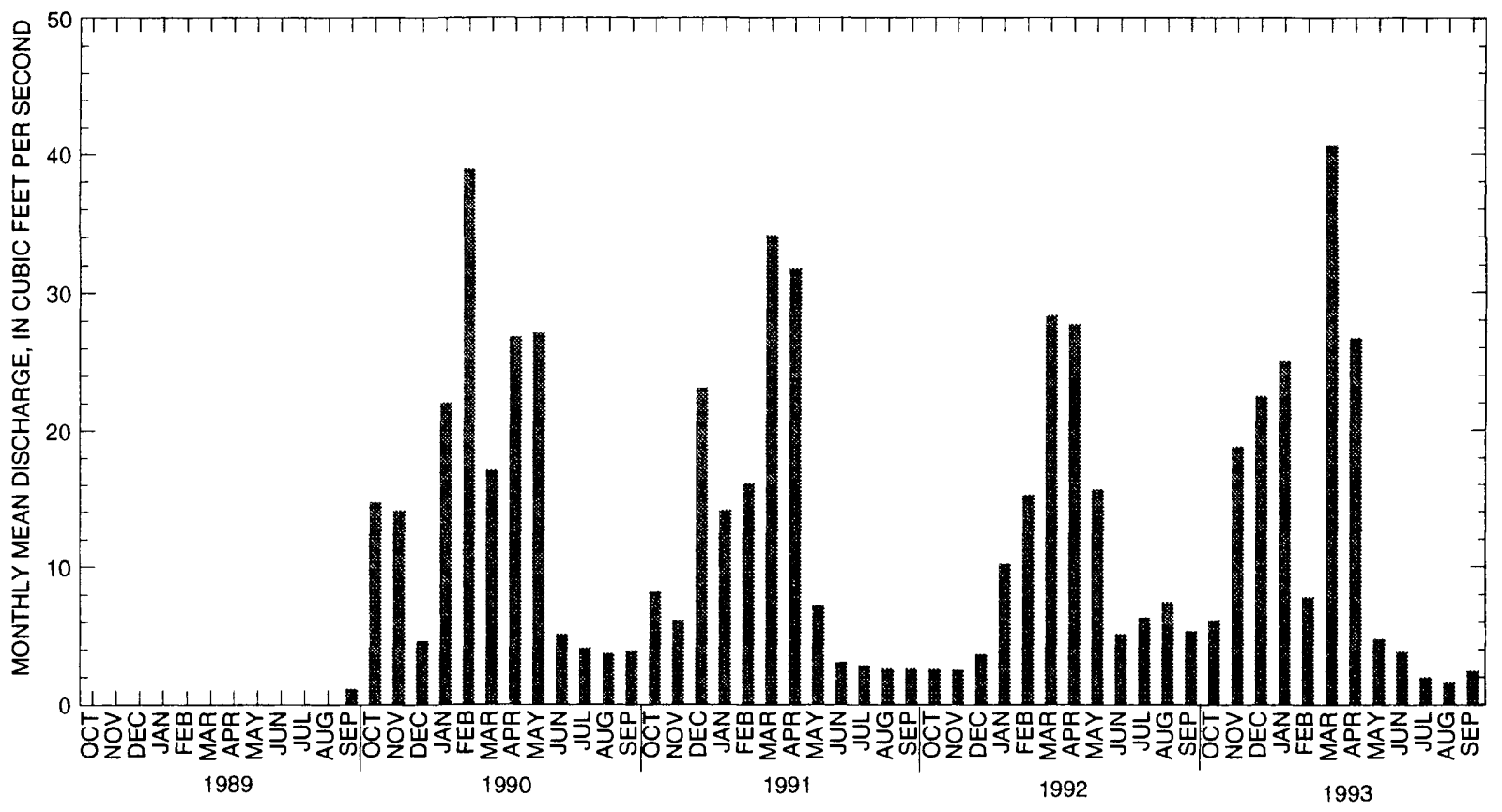




\section{Surface-Water Stations}

\section{A. Discharge and water quality}

\section{Northrup Creek At North Greece, N.Y.}

\section{WATER-QUALITY RECORDS}

PERIOD OF RECORD.--October 1989 to current year.

CHEMICAL DATA: 1989-95 (e).

NUTRIENT DATA: 1989-95 (e)

COOPERATION.--Water-quality samples were collected and analyzed by the Monroe County Environmental Health Laboratory at Rochester, N.Y.

\begin{tabular}{|c|c|c|c|c|c|c|c|c|c|c|c|c|c|c|}
\hline & & & $\begin{array}{c}\text { DIS- } \\
\text { CHARGE, } \\
\text { IN } \\
\text { CUBIC }\end{array}$ & & $\begin{array}{l}\text { SPE- } \\
\text { CIFIC } \\
\text { CON- }\end{array}$ & $\begin{array}{l}\text { ESIDUE } \\
\text { OTAL } \\
\text { T } 105 \\
\text { EG. C, }\end{array}$ & $\begin{array}{l}\text { RESIDUE } \\
\text { VOLA- } \\
\text { TILE, }\end{array}$ & $\begin{array}{l}\text { NITRO- } \\
\text { GEN, } \\
\text { AMMONIA } \\
\text { DIS- }\end{array}$ & $\begin{array}{l}\text { NITRO- } \\
\text { GEN, AM- } \\
\text { MONIA + } \\
\text { ORGANIC }\end{array}$ & $\begin{array}{c}\text { NITRO- } \\
\text { GEN, } \\
\mathrm{NO}_{2}+\mathrm{NO}_{3}\end{array}$ & & $\begin{array}{l}\text { PHOS- } \\
\text { PHORUS } \\
\text { ORTHO, } \\
\text { DIS- }\end{array}$ & $\begin{array}{l}\text { CHLO- } \\
\text { RIDE, } \\
\text { DIS- }\end{array}$ & $\begin{array}{l}\text { SULFATE } \\
\text { DIS- }\end{array}$ \\
\hline & $\mathrm{ME}$ & NG & $\begin{array}{l}\text { FEET } \\
\text { PER } \\
\text { SECOND }\end{array}$ & $\begin{array}{l}\text { BID- } \\
\text { ITY } \\
\text { (NTU) }\end{array}$ & $\begin{array}{l}\text { DUCT- } \\
\text { ANCE } \\
(\mu \mathrm{s} / \mathrm{Cm})\end{array}$ & $\begin{array}{l}\text { SUS- } \\
\text { PENDED } \\
(\mathrm{mg} / \mathrm{L})\end{array}$ & $\begin{array}{l}\text { SUS- } \\
\text { PENDED } \\
(\mathrm{mg} / \mathrm{L})\end{array}$ & $\begin{array}{l}\text { SOLVED } \\
\text { (mg/L } \\
\text { as N) }\end{array}$ & $\begin{array}{l}\text { TOTAL } \\
(\mathrm{mg} / \mathrm{L} \\
\mathrm{as} \mathrm{N})\end{array}$ & $\begin{array}{l}\text { TOTAL } \\
\text { (mg/L } \\
\text { as N) }\end{array}$ & $\begin{array}{l}\text { TOTAL } \\
\text { (mg/L } \\
\text { as P) }\end{array}$ & $\begin{array}{l}\text { SOLVED } \\
(\mathrm{mg} / \mathrm{L} \\
\text { as } \mathrm{P})\end{array}$ & $\begin{array}{l}\text { SOLVED } \\
\text { (mg/L } \\
\text { as Cl) }\end{array}$ & $\begin{array}{l}\text { SOLVED } \\
\text { (mg/L } \\
\text { as SO })\end{array}$ \\
\hline
\end{tabular}

OCT

\begin{tabular}{|c|c|c|c|c|c|c|c|c|c|c|c|c|c|c|}
\hline $01-03$ & 0800 & 0700 & 15 & 11 & 452 & -- & -- & 0.02 & 0.54 & 0.60 & 0.220 & 0.125 & 39 & 61 \\
\hline $13-14$ & 0935 & 2035 & 14 & 1.8 & 407 & -- & -- & 0.01 & 0.36 & 0.74 & 0.160 & 0.120 & 26 & 45 \\
\hline $14-15$ & 2135 & 2035 & 16 & 6.0 & 412 & -- & -- & 0.01 & 0.79 & 0.86 & 0.260 & 0.130 & 29 & 47 \\
\hline $15-16$ & 2135 & 2035 & 14 & 3.1 & 426 & -- & -- & 0.01 & 0.38 & 0.62 & 0.180 & 0.135 & 28 & 47 \\
\hline $16-17$ & 2135 & 0835 & 14 & 2.7 & 411 & -- & -- & 0.01 & 0.41 & 0.55 & 0.200 & 0.150 & 27 & 4 \\
\hline $17-18$ & 0745 & 0645 & 16 & 4.4 & 409 & -- & -- & 0.02 & 0.56 & 0.59 & 0.200 & 0.150 & 29 & 4 \\
\hline $18-19$ & 0745 & 0645 & 14 & 5.3 & 433 & -- & -- & 0.02 & 0.48 & 0.69 & 0.200 & 0.155 & 32 & 48 \\
\hline $19-20$ & 0745 & 0645 & 15 & 5.3 & 455 & -- & -- & 0.02 & 0.65 & 0.75 & 0.190 & 0.155 & 32 & 54 \\
\hline $20-22$ & 1000 & 0900 & 17 & 8.0 & 456 & -- & -- & 0.02 & 0.82 & 0.90 & 0.240 & 0.145 & 37 & 57 \\
\hline $22-24$ & 1000 & 0900 & 14 & 3.4 & 453 & -- & -- & 0.06 & 0.40 & 0.81 & 0.180 & 0.145 & 36 & 55 \\
\hline \multicolumn{14}{|l|}{$\mathrm{V}$} & \\
\hline $06-07$ & 0755 & 1555 & 13 & 1.9 & 452 & -- & -- & 0.32 & 0.73 & 0.70 & 0.220 & 0.170 & 36 & \\
\hline $07-08$ & 1655 & 1555 & 17 & 6.0 & 434 & -- & -- & 0.29 & 1.0 & 0.79 & 0.230 & 0.160 & 34 & \\
\hline $08-09$ & 1655 & 0655 & 15 & 3.4 & 509 & -- & -- & 0.27 & 1.1 & 0.84 & 0.190 & 0.145 & 42 & \\
\hline $09-10$ & 0800 & 1500 & 15 & 1.8 & 480 & -- & -- & 0.22 & 0.77 & 0.80 & 0.180 & 0.145 & 41 & \\
\hline $10-11$ & 1600 & 2300 & 15 & 2.2 & 514 & -- & -- & 0.17 & 0.67 & 0.85 & 0.170 & 0.135 & 43 & 6 \\
\hline $11-13$ & 2400 & 0700 & -- & 2.5 & 516 & -- & -- & 0.16 & 0.62 & 0.87 & 0.180 & 0.145 & 43 & \\
\hline $13-15$ & 0800 & 2200 & 13 & 1.8 & 480 & -- & -- & 0.05 & 0.40 & 0.79 & 0.220 & 0.120 & 39 & \\
\hline $15-16$ & 2300 & 0700 & 13 & 2.8 & 524 & -- & -- & 0.06 & 0.41 & 0.94 & 0.180 & 0.135 & 42 & \\
\hline $16-18$ & 0945 & 0045 & 16 & 3.7 & -- & -- & -- & 0.14 & 0.72 & 0.83 & 0.210 & 0.145 & 48 & \\
\hline $18-19$ & 0145 & 2045 & 14 & 3.0 & -- & -- & 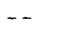 & 0.09 & 0.59 & 1.10 & 0.170 & 0.130 & 63 & 6 \\
\hline $19-20$ & 2145 & 0745 & 14 & 3.7 & -- & -- & -- & 0.07 & 0.59 & 1.20 & 0.180 & 0.140 & 55 & \\
\hline $20-22$ & 0800 & 0700 & 18 & 6.2 & 639 & -- & -- & 0.02 & 0.88 & 1.10 & 0.200 & 0.115 & 100 & 75 \\
\hline \multicolumn{15}{|l|}{$N$} \\
\hline $02-03$ & 0900 & 0800 & 71 & 4.9 & 1370 & -. & $\ldots$ & 0.41 & 1.8 & 2.10 & 0.270 & 0.135 & 280 & \\
\hline $03-04$ & 0900 & 0800 & 65 & 5.3 & 1400 & -- & -- & 0.40 & 1.3 & 2.10 & 0.230 & 0.125 & 290 & \\
\hline $04-05$ & 0900 & 0700 & 92 & 26 & 1140 & -- & -- & 0.37 & 2.2 & 1.80 & 0.310 & 0.110 & 220 & 77 \\
\hline $05-06$ & 0755 & 0055 & 93 & 15 & 954 & -- & -- & 0.27 & 1.6 & 2.20 & 0.250 & 0.015 & 170 & \\
\hline $06-06$ & 0155 & 2155 & 65 & 4.7 & 1110 & -- & -- & 0.31 & 1.2 & 2.50 & 0.210 & 0.110 & 200 & 8 \\
\hline $06-07$ & 2255 & 1655 & 53 & 4.9 & 1140 & -- & -- & 0.33 & 1.2 & 2.50 & 0.200 & 0.120 & 200 & \\
\hline \multicolumn{15}{|l|}{ B } \\
\hline $22-22$ & 0955 & 2025 & 80 & 80 & 686 & 209 & 26 & 0.10 & 1.6 & 1.30 & 0.410 & 0.064 & 87 & 4 \\
\hline $22-23$ & 2155 & 0755 & 174 & 70 & 431 & 166 & 20 & 0.10 & 1.6 & 1.10 & 0.350 & 0.057 & 54 & \\
\hline $23-24$ & 0805 & 0105 & 63 & 35 & 499 & -- & -- & 0.08 & 1.1 & 1.30 & 0.190 & 0.052 & 64 & \\
\hline \multicolumn{15}{|l|}{$\mathrm{R}$} \\
\hline $09-10$ & 0805 & 0705 & 8.4 & 18 & 667 & -- & -- & 0.20 & 1.2 & 1.80 & 0.220 & 0.110 & 92 & 44 \\
\hline $10-11$ & 0805 & 0705 & 21 & 50 & 465 & 148 & 17 & 0.11 & 1.3 & 1.20 & 0.320 & 0.063 & 54 & \\
\hline $11-12$ & 0805 & 0705 & 64 & 6.0 & 828 & -- & -- & 0.24 & 0.95 & 3.10 & 0.260 & 0.165 & 110 & \\
\hline $12-13$ & 0810 & 1510 & 59 & -- & -- & 76 & 11 & 0.06 & 1.0 & 1.30 & 0.220 & 0.062 & 51 & 33 \\
\hline $13-15$ & 1610 & 0710 & 22 & 8.1 & -- & -- & -- & 0.06 & 0.83 & 1.30 & 0.150 & 0.064 & 63 & 36 \\
\hline $15-17$ & 0735 & 0635 & 12 & -- & 633 & -- & -- & 0.06 & 0.93 & 1.40 & 0.180 & 0.070 & 77 & 43 \\
\hline $17-19$ & 0735 & 0635 & 24 & -- & 585 & -- & -- & 0.09 & 1.2 & 1.20 & 0.240 & 0.077 & 70 & \\
\hline \multicolumn{15}{|l|}{$\mathrm{R}$} \\
\hline $02-03$ & 0815 & 1915 & 12 & 2.8 & -- & -- & -- & 0.02 & 0.76 & 1.30 & 0.280 & 0.076 & 82 & 46 \\
\hline $02 \ldots$ & 0825 & - & 14 & 3.7 & -- & -- & - & 0.07 & 0.98 & 1.20 & 0.130 & 0.082 & 76 & \\
\hline $03-05$ & 2015 & 0715 & 74 & 25 & -- & -- & -- & 0.03 & 1.3 & 0.84 & 0.290 & 0.061 & 57 & \\
\hline $05-09$ & 0925 & 0025 & 47 & 26 & -- & 90 & 11 & 0.02 & 1.0 & 1.20 & 0.200 & 0.035 & 64 & \\
\hline $09-09$ & 0125 & 0725 & 13 & 6.5 & -- & $\cdots$ & -- & 0.02 & 0.75 & 1.60 & 0.100 & 0.032 & 82 & 46 \\
\hline $09-10$ & 0815 & 2215 & 17 & 14 & -- & $\rightarrow$ & -- & 0.01 & 1.1 & 1.50 & 0.210 & 0.033 & 84 & 4 \\
\hline $11-12$ & 2015 & 0715 & 43 & 60 & -- & 124 & 17 & 0.02 & 1.6 & 1.10 & 0.300 & 0.035 & 50 & \\
\hline $12-13$ & 0815 & 1515 & 24 & 36 & -- & -- & -- & 0.03 & 0.94 & 1.30 & 0.130 & 0.041 & 66 & 35 \\
\hline $13-16$ & 1615 & 0715 & 16 & 5.1 & -- & -- & -- & 0.02 & 0.91 & 1.50 & 0.100 & 0.041 & 72 & 38 \\
\hline $20-21$ & 0800 & 0100 & 11 & 3.9 & 710 & -- & -- & 0.03 & 1.5 & 1.70 & 0.200 & 0.061 & 90 & 4 \\
\hline $21-23$ & 1100 & 0700 & 21 & 18 & 561 & -- & -- & 0.03 & 1.3 & 0.87 & 0.210 & 0.048 & 61 & 3 \\
\hline
\end{tabular}


0422026250 Northrup Creek At North Greece, N.Y.

\section{WATER-QUALITY RECORDS}

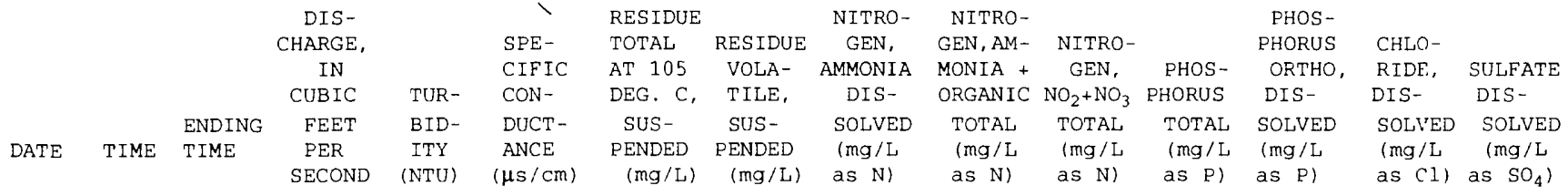

MAY

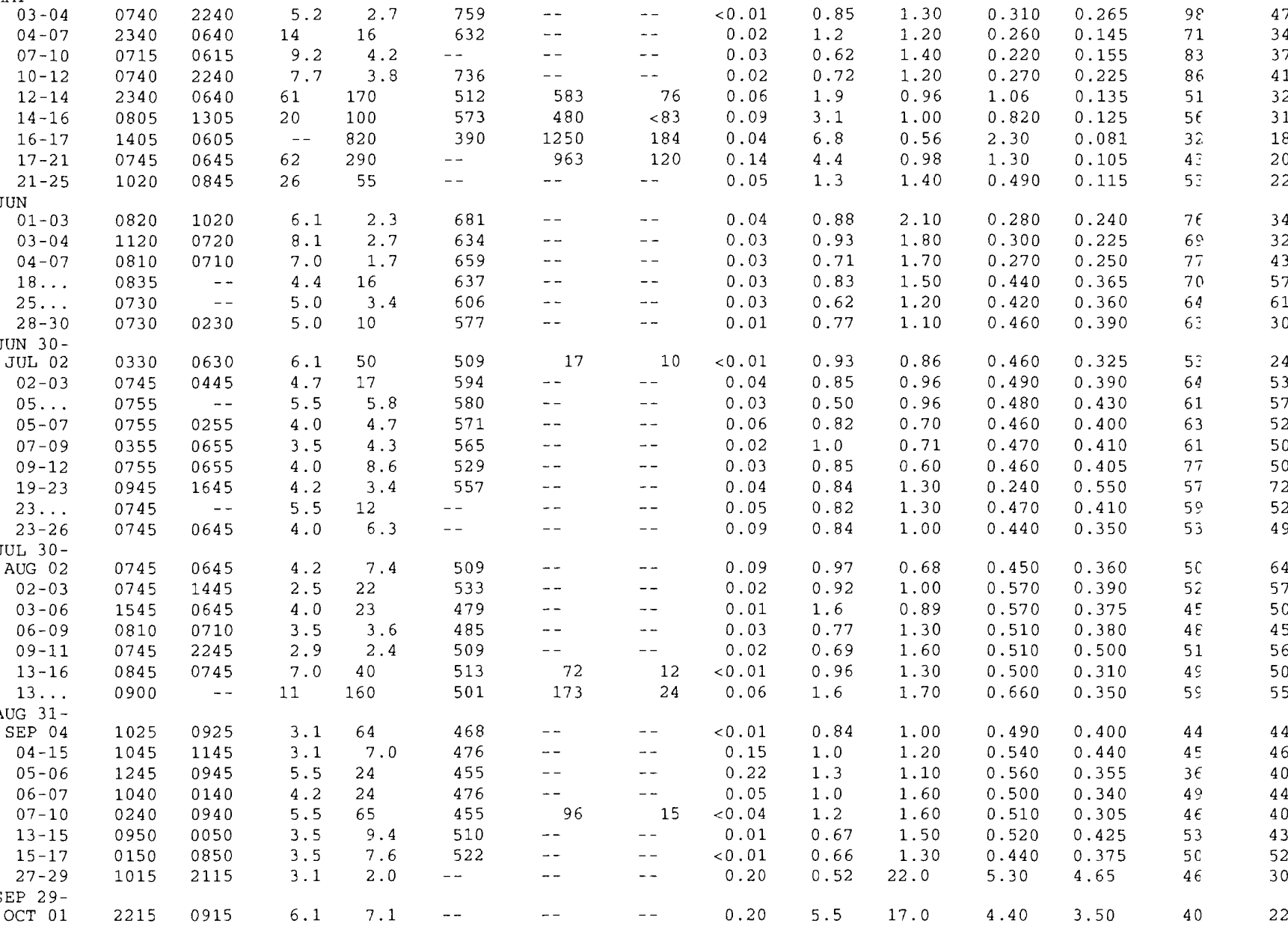

OCT

\section{WATER YEAR OCTOBER 1990 TO SEPTEMBER 1991}

\begin{tabular}{|c|c|c|c|c|c|c|c|c|c|c|c|c|c|c|}
\hline $01-04$ & 1000 & 0900 & 3.5 & 2.6 & -- & -- & -- & $<0.01$ & 0.57 & 2.10 & 0.480 & 0.405 & 50 & 35 \\
\hline $11-13$ & 0950 & 0450 & 25 & 40 & 493 & 92 & 15 & 0.04 & 1.1 & 1.40 & 0.420 & 0.205 & 48 & 33 \\
\hline $13-15$ & 0550 & 0850 & 18 & 9.5 & 605 & -. & - - & 0.05 & 0.83 & 1.50 & 0.260 & 0.190 & 63 & 42 \\
\hline $23-25$ & 1530 & 0830 & 8.8 & 5.0 & 701 & -. & -- & 0.03 & 0.97 & 1.70 & 0.360 & 0.285 & 77 & 50 \\
\hline \multicolumn{15}{|c|}{$\mathrm{OV}$} \\
\hline $06-09$ & 0135 & 0835 & 6.7 & 7.3 & 709 & -- & -- & 0.05 & 0.70 & 1.80 & 0.320 & 0.240 & 76 & 51 \\
\hline $09-10$ & 1015 & 0115 & 5.0 & 1.6 & $\cdots$ & -- & -- & 0.03 & 0.88 & 2.10 & 0.340 & 0.262 & 81 & 34 \\
\hline $10-13$ & 0215 & 0915 & 11 & 7.5 & 701 & -- & -- & 0.03 & 0.92 & 2.00 & 0.300 & 0.173 & 79 & 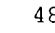 \\
\hline \multicolumn{15}{|l|}{$\mathrm{C}$} \\
\hline $04-04$ & 0045 & 1045 & 37 & 65 & -- & 240 & 37 & 0.06 & 2.6 & 1.40 & 0.825 & 0.225 & 110 & 7 \\
\hline $04-06$ & 1245 & 0845 & 19 & 21 & 676 & - & -- & 0.03 & 1.4 & 1.50 & 0.325 & 0.230 & 89 & 40 \\
\hline $17-18$ & 0935 & 2335 & 16 & 9.8 & -- & -- & -- & 0.04 & 1.2 & 1.80 & 0.110 & 0.160 & 82 & 1 \\
\hline $19-20$ & 0035 & 0835 & 24 & 21 & 495 & -- & -- & 0.03 & 1.0 & 1.40 & 0.100 & 0.110 & 66 & 36 \\
\hline $20-21$ & 1340 & 2040 & 12 & 4.9 & 692 & -- & -. & 0.10 & 0.90 & 1.90 & 0.240 & 0.140 & 81 & \\
\hline $21-24$ & 2140 & 0740 & 31 & 80 & 530 & 270 & 35 & 0.07 & 2.3 & 1.40 & 0.580 & 0.074 & 58 & 3 \\
\hline
\end{tabular}




\section{Surface-Water Stations}

\section{A. Discharge and water quality}

0422026250 Northrup Creek At North Greece, N.Y.

\section{WATER-QUALITY RECORDS}

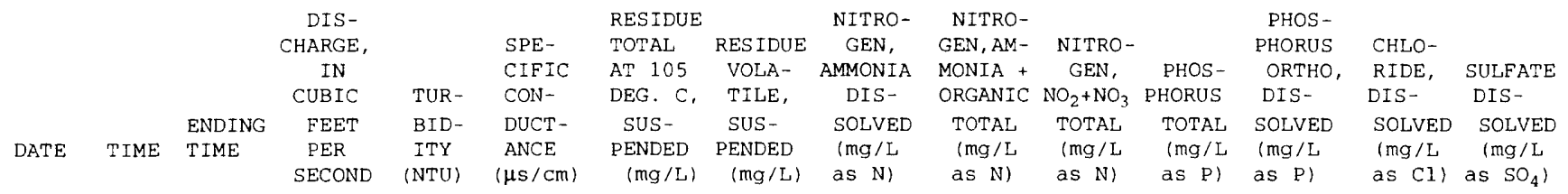

JAN

\begin{tabular}{|c|c|c|c|c|c|c|c|c|c|c|c|c|c|c|}
\hline & 0945 & - & 18 & 7.9 & 612 & -- & -- & 0.17 & 0.67 & 1.90 & 0.130 & 0.090 & 71 & 43 \\
\hline $04 \ldots$ & 0955 & -- & 17 & 6.0 & 788 & -- & -- & 0.15 & 1.0 & 2.30 & 0.170 & 0.121 & 110 & 50 \\
\hline $14-15$ & 1015 & 1215 & 5.2 & 5.3 & -- & -- & -- & 0.17 & 1.0 & 2.90 & 0.210 & 0.160 & 110 & 53 \\
\hline $15-17$ & 1315 & 0915 & 26 & 18 & 700 & -. & - & 0.17 & 1.0 & 1.90 & 0.220 & 0.120 & 100 & 43 \\
\hline $17-22$ & 0955 & 0855 & 28 & 7.2 & 590 & -- & -- & 0.11 & 0.76 & 1.70 & 0.130 & 0.085 & 73 & 36 \\
\hline \multicolumn{15}{|l|}{ EEB } \\
\hline $04-07$ & 0940 & 0840 & 37 & 13 & 536 & -. & -- & 0.05 & 0.76 & 1.30 & 0.150 & 0.075 & 66 & 34 \\
\hline $07-11$ & 0925 & 0825 & 20 & 3.7 & 644 & -- & -- & 0.03 & 0.74 & 1.80 & 0.150 & 0.095 & 79 & 41 \\
\hline $19-19$ & 0940 & 1640 & 55 & 9.6 & 722 & -. & -- & 0.04 & 0.82 & 1.70 & 0.190 & 0.110 & 120 & 39 \\
\hline $19-21$ & 1740 & 0840 & 41 & 36 & 545 & 141 & 31 & 0.02 & 1.7 & 1.30 & 0.460 & 0.071 & 75 & 33 \\
\hline $24-25$ & 1350 & 0850 & -- & 6.3 & 570 & -- & -- & 0.03 & 0.80 & 1.10 & 0.120 & 0.068 & 64 & 37 \\
\hline \multicolumn{15}{|l|}{ MAR } \\
\hline $01-02$ & 0815 & 1915 & 13 & 30 & -- & -- & -- & 0.01 & 0.85 & 1.90 & 0.220 & 0.124 & 110 & 44 \\
\hline $02-03$ & 2015 & 0815 & 42 & 70 & 547 & 272 & 37 & 0.02 & 2.1 & 0.97 & 0.700 & 0.054 & 72 & 34 \\
\hline $06 \ldots$ & 0955 & -. & 62 & 12 & 414 & -- & -- & 0.10 & 0.86 & 1.00 & 0.110 & 0.052 & 44 & 24 \\
\hline $07 \ldots$ & 1000 & $\cdots$ & 99 & 21 & 422 & -- & $\cdots$ & 0.14 & 0.97 & 0.89 & 0.140 & 0.048 & 46 & 24 \\
\hline $07-11$ & 1010 & 0910 & 21 & 5.1 & 573 & -. & -- & 0.07 & 0.76 & 1.40 & 0.095 & 0.047 & 70 & 36 \\
\hline $21 \ldots$ & 0940 & - & -- & 1.7 & 696 & -- & -- & 0.01 & 1.1 & 1.60 & 0.170 & 0.083 & 81 & 47 \\
\hline $27-27$ & 0645 & 1445 & 43 & 21 & 551 & -- & -- & 0.03 & 1.0 & 1.40 & 0.260 & 0.089 & 61 & 38 \\
\hline $27-28$ & 1545 & 0845 & 110 & 62 & 377 & 136 & 21 & 0.05 & 1.5 & 0.77 & 0.360 & 0.063 & 37 & 26 \\
\hline \multicolumn{15}{|l|}{ MAR 28- } \\
\hline APR 01 & 0940 & 0840 & 26 & 5.0 & 589 & -- & -- & 0.02 & 0.81 & 1.40 & 0.140 & 0.078 & 63 & 38 \\
\hline $01-02$ & 0950 & 0250 & 11 & 1.6 & 664 & - & -- & 0.02 & 0.70 & 1.70 & 0.160 & 0.093 & 77 & 41 \\
\hline $02-04$ & 0350 & 0850 & 11 & 1.6 & 654 & -- & -- & 0.02 & 0.60 & 1.70 & 0.160 & 0.100 & 74 & 41 \\
\hline $08-10$ & 0930 & 0230 & 9.5 & 12 & 679 & -- & -. & 0.03 & 0.70 & 1.30 & 0.320 & 0.225 & 80 & 42 \\
\hline $10-11$ & 0330 & 0830 & 27 & 20 & 489 & -- & -- & 0.05 & 0.89 & 0.88 & 0.260 & 0.120 & 46 & 29 \\
\hline $11-14$ & 0945 & 1645 & 8.8 & 2.2 & 653 & -- & - & 0.03 & 0.80 & 1.50 & 0.170 & 0.160 & 72 & 38 \\
\hline $15-15$ & 0935 & 2035 & 14 & 9.3 & 572 & -- & -- & 0.02 & 1.1 & 1.10 & 0.220 & 0.130 & 61 & 34 \\
\hline $15-18$ & 2135 & 0835 & 15 & 5.1 & 617 & -- & -. & 0.02 & 0.94 & 1.00 & 0.180 & 0.115 & 62 & 32 \\
\hline $18-20$ & 0940 & 0400 & -- & 2.2 & 676 & -- & -- & 0.01 & 0.99 & 1.50 & 0.230 & 0.032 & 75 & 35 \\
\hline $18-20$ & 0940 & 0840 & -. & 2.1 & 678 & -- & -- & 0.01 & 0.89 & 1.50 & 0.220 & 0.032 & 75 & 36 \\
\hline $20-22$ & 0140 & 0740 & 102 & 40 & 437 & 115 & 22 & 0.02 & 1.7 & 0.82 & 0.420 & 0.018 & 39 & 22 \\
\hline \multicolumn{15}{|l|}{ MAY } \\
\hline $02 \ldots$ & 0945 & -- & -- & 1.5 & 649 & - & -- & 0.01 & 0.86 & 1.70 & 0.260 & 0.084 & 70 & 35 \\
\hline $20-23$ & 0835 & 0735 & 5.8 & 50 & 683 & 229 & 57 & 0.13 & 2.8 & 1.00 & 1.30 & 0.444 & 75 & 50 \\
\hline $26-26$ & 0810 & 2210 & 4.2 & 4.5 & 676 & -- & -- & 0.06 & 1.0 & 1.30 & 0.660 & 0.558 & 71 & 55 \\
\hline $26-28$ & 2310 & 0910 & 4.4 & 4.7 & 654 & -- & -- & 0.04 & 1.2 & 1.20 & 0.640 & 0.510 & 70 & 50 \\
\hline \multicolumn{15}{|l|}{ JUL } \\
\hline $05-07$ & 0755 & 0355 & 3.1 & 7.1 & 413 & -- & -- & 0.04 & 0.39 & 1.00 & 0.720 & 0.625 & 44 & 48 \\
\hline $07-07$ & 0455 & 1255 & 6.1 & 13 & 447 & -- & -- & 0.04 & 0.60 & 1.00 & 0.850 & 0.615 & 44 & 47 \\
\hline $07-08$ & 1335 & 0655 &.- & 13 & 400 & -- & -- & 0.03 & 0.67 & 1.10 & 0.700 & 0.530 & 36 & 51 \\
\hline $11-13$ & 0810 & 1510 & 2.5 & 4.2 & 463 & -. & -- & 0.05 & 0.63 & 1.40 & 0.700 & 0.610 & 46 & 56 \\
\hline $13-14$ & 1610 & 0310 & 4.7 & 8.1 & 440 & -- & -- & 0.02 & 0.70 & 1.20 & 0.600 & 0.530 & 39 & 71 \\
\hline $14-15$ & 0410 & 0710 & 3.1 & 4.6 & 476 & -- & -- & 0.02 & 0.70 & 1.90 & 0.600 & 0.530 & 44 & 75 \\
\hline \multicolumn{15}{|l|}{ AUG } \\
\hline $02-03$ & 0835 & 0735 & 2.0 & 3.9 & 411 & -- & -- & 0.03 & 0.72 & 0.90 & 0.670 & 0.026 & 37 & 42 \\
\hline $03-03$ & 0835 & 1935 & - & 11 & 397 & -- & -- & 0.03 & 0.66 & 1.00 & 0.690 & 0.026 & 36 & 44 \\
\hline $03-05$ & 2035 & 0335 & 4.9 & 6.2 & 384 & -- & $\cdots$ & 0.02 & 0.60 & 1.10 & 0.590 & 0.021 & 35 & 38 \\
\hline $19-22$ & 0800 & 0700 & -. & 16 & 300 & -- & -- & 0.02 & 0.78 & 0.84 & 0.440 & 0.375 & 25 & 35 \\
\hline \multicolumn{15}{|l|}{ SEP } \\
\hline $05-09$ & 0925 & 0825 & 2.3 & -- & 423 & -- & -- & 0.02 & 0.69 & -- & 0.680 & 0.505 & 40 & 43 \\
\hline $09-11$ & 1015 & 0015 & 2.1 & 4.5 & 425 & 77 & 14 & 0.04 & 0.50 & 1.60 & 0.620 & 0.590 & 37 & 38 \\
\hline $11-12$ & 0115 & 0915 & 2.9 & 6.0 & 400 & -. & -- & 0.03 & 0.78 & 1.60 & 0.660 & 0.610 & 36 & 31 \\
\hline $23-24$ & 0940 & 0540 & 2.9 & 3.4 & -- & -- & -- & 0.02 & 0.55 & 1.40 & 0.630 & 0.580 & 39 & 29 \\
\hline $24-26$ & 0640 & 0840 & 6.4 & 19 & 343 & -- & - & 0.03 & 0.79 & 1.10 & 0.540 & 0.430 & 32 & 27 \\
\hline \multicolumn{15}{|c|}{ WATER YEAR OCTOBER 1991 TO SEPTEMBER 1992} \\
\hline OCT & & & & & & & & & & & & & & \\
\hline $03-05$ & 0910 & 2010 & 2.7 & 4.7 & -- & -- & -- & 0.01 & 0.84 & 1.30 & 0.560 & 0.510 & 46 & 68 \\
\hline $05-07$ & 2110 & 0810 & 3.1 & 5.1 & -. & -- & -- & 0.01 & 0.73 & 1.30 & 0.530 & 0.495 & 44 & 45 \\
\hline $10 \ldots$ & 0925 & -- & 2.9 & 2.0 & 459 & -- & -- & 0.03 & 0.60 & 1.50 & 0.550 & 0.525 & 44 & 45 \\
\hline $10-15$ & 0925 & 0825 & 2.5 & 2.4 & 478 & -- & -- & 0.02 & 0.54 & 1.30 & 0.540 & 0.485 & 46 & 51 \\
\hline
\end{tabular}

\section{WATER YEAR OCTOBER 1990 TO SEPTEMBER 1991-continued}




\section{Northrup Creek At North Greece, N.Y.}

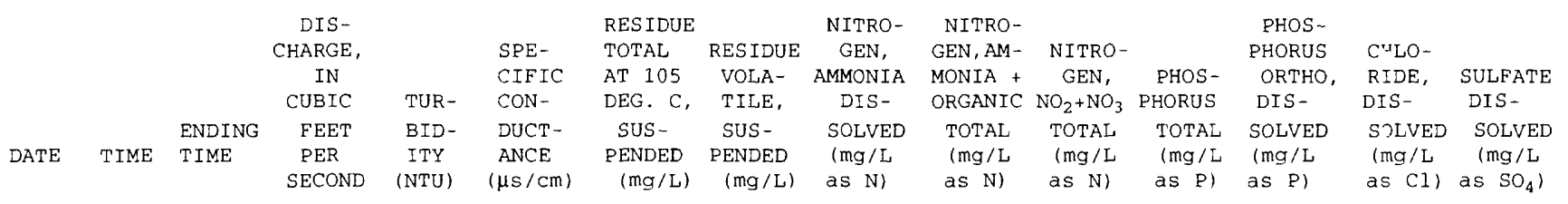

DEC

09-12

15-16

$29-30$

29-30

$30 \ldots$

$31 \ldots$

JAN 03

$03-06$

$14-17$

$14 \ldots$

$21-23$
$23-24$

$24-28$

FEB

$14-15$

15-18

$18-20$

$20-21$

$21-24$

$24 \ldots$

$27-28$
$28-29$

$29-29$

FEB $29-$

MAR 02

06-07

$07-07$

08-09

$09-10$

$10-13$
$24-25$

$25-27$

27-31

MAR 31 -

10-11

$11-12$

12-14

$16-17$

17-20

20-21

$21-22$
$22-23$

$23-24$

24-28

MAY

01-02

$02-03$
$03-05$

05-08

JUN

07-08

08-09

$09-11$

$19-23$

$24-24$

JUL

03-03 $0445 \quad 1945$

$03-06$

06-08

08-09

$09-10$

$10-12$

$12-13$ $\begin{array}{rr}0925 & 0825 \\ 2140 & 084 \\ 0625 & 2125 \\ 2225 & 0925 \\ 1655 & -\end{array}$

0800

$1600 \quad 0900$

0930
$0920 \quad 0820$

0925
0945
06545

$0645 \quad 0845$

$0935 \quad 0835$

$0930 \quad 1230$

$1330 \quad 0830$

$0310 \quad 0810$

$0930 \quad 0830$

0930
0935
1335

$1335 \quad 0435$

05352035

$\begin{array}{ll}2135 & 0835\end{array}$

$0925 \quad 2325$

$0025 \quad 0825$

$\begin{array}{ll}0935 & 2035 \\ 2135 & 0835\end{array}$

$0930 \quad 1130$

$1230 \quad 0830$

$0935 \quad 0835$

09250425

$0525 \quad 0025$

01250825

$0955 \quad 1655$

$1755 \quad 0725$

$1025 \quad 0525$

06250825

0915

1315

0515
0925

1725

0125

$0930 \quad 1130$

$0945 \quad 0845$

$0630 \quad 2330$

$\begin{array}{ll}2045 & 0745 \\ 0935 & 1635\end{array}$

$1735 \quad 0435$

05350835

$0920 \quad 1620$

$1720 \quad 1220$ $\begin{array}{ll}1330 & 0030 \\ 0130 & 0930\end{array}$

\section{WATER YEAR OCTOBER 1991 TO SEPTEMBER 1992-continued}

$\begin{array}{cc}2.7 & 1.9 \\ 4.2 & 2.6 \\ 17 & 44 \\ 28 & 35 \\ 12 & 10 \\ 24 & 6.5 \\ 7.0 & 5.0 \\ 4.4 & 4.9 \\ -- & 4.2 \\ 9.9 & 8.6 \\ 5.2 & 2.0 \\ 51 & 43\end{array}$

$\begin{array}{rlllll}1060 & -- & -- & 0.30 & 1.2 & 2.10 \\ -- & -- & -- & 1.40 & 2.0 & 2.30 \\ 752 & 124 & 25 & 1.80 & 3.9 & 1.40 \\ 667 & 97 & 23 & 1.20 & 3.3 & 1.20 \\ 932 & -- & -- & 0.72 & 1.7 & 1.60 \\ 1090 & -- & -- & 1.30 & 2.1 & 1.90 \\ & & & & & \\ -- & -- & -- & 1.20 & 1.9 & 2.50 \\ 924 & -- & -- & 0.83 & 1.8 & 2.30 \\ 969 & -- & -- & 0.79 & 1.7 & 3.20 \\ 943 & -- & -- & 0.79 & 1.7 & 2.20 \\ 1010 & -- & -- & 1.00 & 0.78 & 3.00 \\ 894 & 110 & 18 & 0.51 & 2.0 & 2.20 \\ 955 & -- & -- & 0.21 & 1.2 & 0.43\end{array}$

0.660

$180 \quad 92$

0.820
0.760

0.540

0.270

0.820

0.420

0.310

0.175

0.370

0.450

0.390

0.490

0.500

0.570
0.250

0.240

120

100

160

0.300

0.380

0.335

0.420

0.450

0.300

200

$150 \quad 350$

$150 \quad 85$

$\begin{array}{ll}26 & 8.4\end{array}$

955

$\begin{array}{ll}9.9 & 1.5 \\ 39 & 6.2 \\ 45 & 5.7\end{array}$

5.7

$\begin{array}{ll}22 & 2.3 \\ 13 & 6.5\end{array}$

9.9

6.5

$\begin{array}{rr}8.0 & 2.5 \\ 15 & 7.8\end{array}$

$15 \quad 21$

$\begin{array}{rr}9.5 & 7.9 \\ 14 & 6.1\end{array}$

$\begin{array}{ll}14 & 6.1 \\ 23 & 14 \\ 23 & 12\end{array}$

17

$15 \quad 4.0$

$\begin{array}{cc}15 & 9.0 \\ 74 & 75\end{array}$

7

--
--
75
69
75
781
780
736
69

0.24

1.24 .80

0.630

$\begin{array}{lllll}\ldots & - & 0.24 & 1.2 & 4.80 \\ \ldots & -- & 0.15 & 1.2 & 3.10 \\ \ldots & \ldots & 0.10 & 1.5 & 2.10\end{array}$

0.630
0.330

0.320

$\begin{array}{lll}0.31 & 1.5 & 1.50\end{array}$

$\begin{array}{lll}0.32 & 1.2 & 2.20\end{array}$

$\begin{array}{lll}0.30 & 1.1 & 2.50\end{array}$

$\begin{array}{lll}0.29 & 1.1 & 2.60 \\ 0.22 & 1.2 & 2.40\end{array}$

0.13

1.6

2.00

0.260

0.210

0.200

0.210

0.260

$-$

0.340

0.245

0.155

0.150

0.155

0.150

0.165

0.150

0.230

0.150

$\begin{array}{lll}0.30 & 1.3 & 2.00\end{array}$

0.13

0.71

2.20

0.200

0.120

0.190

0.105

0.16

1.1

1.70

0.09

$0.97 \quad 2.00$

0.18

0.07

0.91

2.00

0.170

0.105

0.180

0.150

0.300

0.105

0.115

$2.0 \quad 1.30$

0.390

0.074

0.120

0.047

0.07

0.80

1. 20

0.03

0.09
0.06

0.86
1.1

0.90

1.10

0.160

0.115

0. 200

0.150

0.090

0.067

$\begin{array}{lll}0.13 & 1.5 & 0.73\end{array}$

0.03

0.02

0.87

0.73
1.20

0.83

1.00

0.074

0.140

0.052

$0.110 \quad 0.069$

0.02

0.02

$0.79 \quad 1.00$

$0.84 \quad 0.80$

0.130

0.180

0.066

0.891 .00

0.160

0.081

0.180

$0.75 \quad 1.50$

$2.3 \quad 0.78$

$\begin{array}{ll}1.1 & 0.89 \\ 0.85 & 1.10\end{array}$

0.670

0.130

0.240

0.089

0.160

0.075

0.900

$1.5 \quad 2.20$

0.02

0.02

2.0

2.20
1.70

0.03

0.76

1.80

0.02

0.71
0.65

2.40

0.900

0.500

0.480

0.600

0.365

0.420

0.530

0.535

160

170

160

170

160

120
100

120

110

110

100 


\section{Surface-Water Stations}

\section{A. Discharge and water quality}

0422026250 Northrup Creek At North Greece, N.Y.

\section{WATER-QUALITY RECORDS}

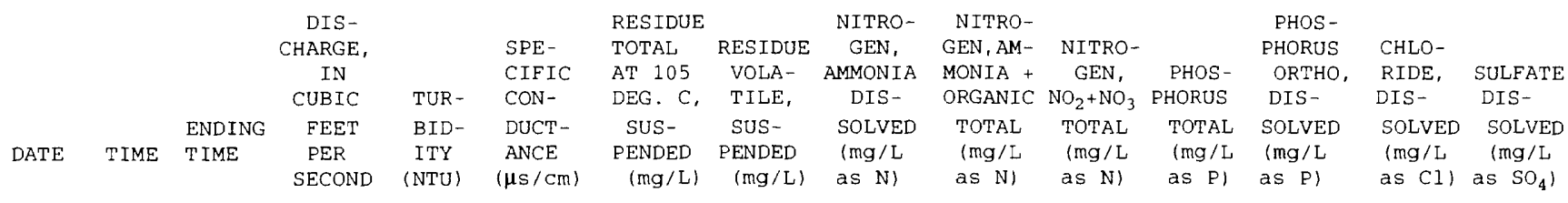

$\begin{array}{ccccc}\text { JUL-cont. } & & & & \\ 14 \ldots & 0935 & -- & 7.4 & 20 \\ 14-15 & 0940 & 1140 & 16 & 85 \\ 15-17 & 1240 & 0540 & 13 & 50 \\ 26-26 & 0925 & 2025 & 5.8 & 21 \\ 26-27 & 0925 & 2025 & -- & 21 \\ 31-31 & 0830 & 1930 & 25 & 60 \\ \text { JUL } 31- & & & & \\ \text { AUG 01 } & 2030 & 2330 & 25 & 60 \\ 03 \ldots & 0945 & -- & 30 & 40 \\ 04-04 & 0940 & 1740 & 30 & 70 \\ 04-07 & 1840 & 1440 & 11 & 26 \\ 21-24 & 0915 & 1615 & 3.5 & 5 . \\ 24-25 & 1715 & 0815 & 6.4 & 25 \\ 25-27 & 0915 & 1415 & 4.2 & 12 \\ 27-27 & 1515 & 2315 & 5.2 & 12 \\ 28-28 & 0015 & 0815 & 8.8 & 28 \\ 28-29 & 1320 & 0420 & 9.5 & 44 \\ \text { AUG 29- } & & & & \\ \text { SEP 01 } & 0520 & 0820 & 5.5 & 28 \\ 03-03 & 1245 & 2045 & 8.0 & 16 \\ 03-04 & 2145 & 0845 & 7.7 & 23 \\ 04-08 & 0940 & 0840 & 5.0 & 12 \\ 18-19 & 2115 & 0815 & 9.5 & 38 \\ 19-21 & 0915 & 1215 & 4.2 & 23 \\ 21-22 & 1315 & 0815 & 11 & 40 \\ 22-25 & 0945 & 0845 & 12 & 70\end{array}$

OCT

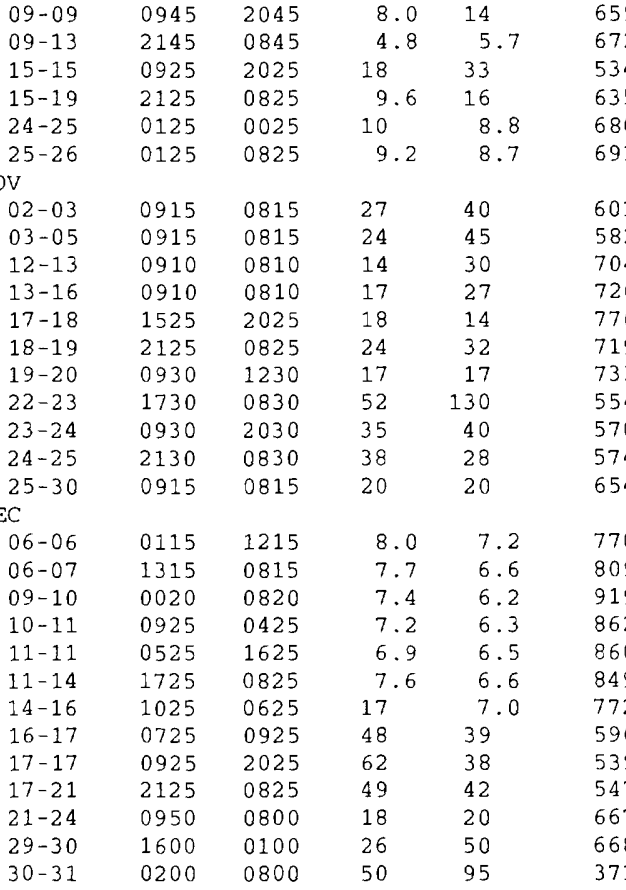

WATER YEAR OCTOBER 1991 TO SEPTEMBER 1992-continued

\begin{tabular}{|c|c|c|c|c|c|c|c|c|}
\hline 570 & -- & -- & 0.02 & 0.93 & 1.80 & 0.630 & 0.495 & 61 \\
\hline 475 & 194 & 30 & 0.02 & 2.2 & -- & -- & 0.315 & 48 \\
\hline 587 & 141 & 61 & 0.02 & 1.4 & -- & -- & 0.245 & 59 \\
\hline 659 & 26 & $<8$ & 0.01 & 0.87 & 2.40 & 0.560 & 0.410 & 79 \\
\hline 618 & 34 & $<7$ & 0.02 & 0.94 & 1.80 & 0.470 & 0.360 & 68 \\
\hline 453 & 184 & 31 & 0.03 & 1.8 & 1.50 & 0.720 & 0.300 & 47 \\
\hline 453 & 134 & 22 & 0.04 & 1.4 & 0.93 & 0.520 & 0.180 & 43 \\
\hline 466 & 59 & 10 & 0.03 & 1.1 & 0.75 & 0.350 & 0.180 & 44 \\
\hline-- & 117 & 20 & 0.02 & 1.4 & 0.84 & 0.440 & 0.155 & 45 \\
\hline-- & -- & -- & 0.02 & 1.0 & 1.10 & 0.350 & 0.215 & 59 \\
\hline 671 & $-\cdot$ & -- & 0.03 & 0.76 & 1.50 & 0.490 & 0.400 & 75 \\
\hline 601 & -- & -- & 0.01 & 0.96 & 1.30 & 0.550 & 0.405 & 65 \\
\hline 564 & -- & -- & 0.03 & 0.64 & 0.92 & 0.460 & 0.350 & 62 \\
\hline 621 & -- & -- & 0.02 & 0.56 & 1.10 & 0.500 & 0.410 & 65 \\
\hline 513 & -- & -- & 0.03 & 0.81 & 1.10 & 0.530 & 0.360 & 53 \\
\hline 542 & 92 & 15 & 0.03 & 1.2 & 0.91 & 0.550 & 0.315 & 57 \\
\hline 615 & -- & -- & 0.02 & 0.80 & 1.10 & 0.470 & 0.315 & 65 \\
\hline 646 & -- & -- & $<0.01$ & 0.70 & 1.30 & 0.540 & 0.370 & 71 \\
\hline 528 & -- & -- & 0.01 & 0.77 & 1.10 & 0.520 & 0.310 & 56 \\
\hline 638 & -- & + & 0.01 & 0.73 & 1.00 & 0.410 & 0.360 & 79 \\
\hline 560 & 95 & 19 & 0.02 & 1.4 & 1.40 & 0.740 & 0.475 & 60 \\
\hline 574 & 32 & $<6$ & 0.01 & 0.81 & 1.30 & 0.470 & 0.390 & 63 \\
\hline 534 & 86 & 15 & 0.01 & 1.1 & 0.33 & 0.570 & 0.365 & 58 \\
\hline 558 & 153 & 21 & 0.02 & 1.3 & 0.30 & 0.490 & 0.220 & 58 \\
\hline
\end{tabular}

WATER YEAR OCTOBER 1992 TO SEPTEMBER 1993

\begin{tabular}{|c|c|c|c|c|c|c|c|c|}
\hline 659 & -- & -- & $<0.01$ & 0.79 & 0.20 & 0.510 & 0.415 & 70 \\
\hline 672 & -- & -- & 0.01 & 0.48 & 1.60 & 0.420 & 0.375 & 32 \\
\hline 534 & 64 & 11 & 0.01 & 1.1 & 1.50 & 0.560 & 0.345 & 56 \\
\hline 635 & -- & -- & 0.01 & 0.76 & 1.30 & 0.340 & 0.250 & 65 \\
\hline 680 & -- & -- & $<0.01$ & 0.66 & 2.00 & 0.340 & 0.285 & 74 \\
\hline 691 & -- & -- & 0.04 & 0.80 & 1.20 & 0.290 & 0.230 & 71 \\
\hline 601 & 106 & 19 & $<0.01$ & 1.4 & 1.10 & 0.550 & 0.250 & 64 \\
\hline 582 & 70 & 12 & 0.01 & 1.3 & 0.74 & 0.340 & 0.135 & 54 \\
\hline 704 & 42 & 9 & 0.02 & 0.99 & 1.40 & 0.340 & 0.175 & 73 \\
\hline 720 & -- & -- & 0.01 & 0.86 & 1.30 & 0.220 & 0.145 & 83 \\
\hline 776 & -- & -- & 0.03 & 0.89 & 1.40 & 0.210 & 0.135 & 110 \\
\hline 719 & 76 & 17 & 0.02 & 1.6 & 1.10 & 0.420 & 0.125 & 93 \\
\hline 733 & -- & -- & 0.03 & 0.90 & 1.30 & 0.230 & 0.125 & 91 \\
\hline 554 & 286 & 51 & 0.02 & 3.1 & 1.10 & 0.870 & 0.110 & 56 \\
\hline 570 & 76 & 14 & 0.04 & 1.2 & 0.89 & 0.290 & 0.095 & 59 \\
\hline 574 & -- & -- & 0.03 & 0.92 & 1.00 & 0.240 & 0.125 & 58 \\
\hline 654 & -- & -- & 0.02 & 0.99 & 1.70 & 0.130 & 0.024 & 67 \\
\hline 770 & -- & -- & 0.10 & 0.77 & 2.10 & 0.260 & 0.195 & 86 \\
\hline 809 & -- & -- & 0.16 & 0.82 & 2.30 & 0.260 & 0.195 & 96 \\
\hline 919 & -- & -- & 0.15 & 0.88 & 2.10 & 0.250 & 0.180 & 130 \\
\hline 862 & 10 & $<6$ & 0.19 & 1.0 & 2.60 & 0.280 & 0.190 & 120 \\
\hline 860 & -- & -- & 0.27 & 1.1 & 2.10 & 0.270 & 0.175 & 120 \\
\hline 849 & -- & -- & 0.23 & 0.96 & 1.60 & 0.230 & 0.155 & 130 \\
\hline 772 & -- & -- & 0.13 & 0.85 & 1.20 & 0.180 & 0.115 & 110 \\
\hline 596 & 125 & 23 & 0.13 & 1.3 & 0.85 & 0.310 & 0.091 & 110 \\
\hline 539 & -- & -- & 0.10 & 1.6 & 0.90 & 0.400 & 0.076 & 67 \\
\hline 547 & 119 & 24 & 0.12 & 1.8 & 1.10 & 0.430 & 0.072 & 62 \\
\hline 667 & -- & -- & 0.07 & 1.0 & 0.40 & 0.250 & 0.079 & 73 \\
\hline 668 & 171 & 21 & 0.24 & 1.7 & 1.20 & 0.390 & 0.096 & 93 \\
\hline 371 & 281 & 30 & 0.14 & 1.8 & 1.00 & 0.550 & 0.095 & 41 \\
\hline
\end{tabular}


Surface-Water Stations

\section{A. Discharge and water quality}

0422026250 Northrup Creek At North Greece, N.Y.

\begin{tabular}{|c|c|c|c|c|c|c|c|c|c|c|c|c|c|}
\hline & & DIS- & & & RESIDUE & & NITRO- & NITRO- & & & PHOS- & & \\
\hline & & $\begin{array}{c}\text { CHARGE, } \\
\text { IN } \\
\text { CUBIC }\end{array}$ & TUR- & $\begin{array}{l}\text { SPE- } \\
\text { CIFIC } \\
\text { CON- }\end{array}$ & $\begin{array}{l}\text { TOTAL } \\
\text { AT } 105 \\
\text { DEG. C. }\end{array}$ & $\begin{array}{c}\text { RESIDUE } \\
\text { VOLA- } \\
\text { TILE, }\end{array}$ & $\begin{array}{l}\text { GEN, } \\
\text { AMMONIA } \\
\text { DIS- }\end{array}$ & $\begin{array}{l}\text { GEN, AM- } \\
\text { MONIA + } \\
\text { ORGANIC }\end{array}$ & $\begin{array}{c}\text { NITRO- } \\
\text { GEN, } \\
\mathrm{NO}_{2}+\mathrm{NO}_{3}\end{array}$ & $\begin{array}{l}\text { PHOS- } \\
\text { PHORUS }\end{array}$ & $\begin{array}{c}\text { PHORUS } \\
\text { ORTHO, } \\
\text { DIS- }\end{array}$ & $\begin{array}{l}\text { CHLO- } \\
\text { RIDE, } \\
\text { DIS- }\end{array}$ & $\begin{array}{l}\text { SULFATE } \\
\text { DIS- }\end{array}$ \\
\hline IME & $\begin{array}{l}\text { ENDING } \\
\text { TIME }\end{array}$ & $\begin{array}{c}\text { FEET } \\
\text { PER } \\
\text { SECOND }\end{array}$ & $\begin{array}{l}\text { BID- } \\
\text { ITY } \\
\text { (NTU) }\end{array}$ & $\begin{array}{l}\text { DUCT- } \\
\text { ANCE } \\
(\mu \mathrm{s} / \mathrm{cm})\end{array}$ & $\begin{array}{l}\text { SUS- } \\
\text { PENDED } \\
\text { (mg/L) }\end{array}$ & $\begin{array}{l}\text { SUS- } \\
\text { PENDED } \\
(\mathrm{mg} / \mathrm{L})\end{array}$ & $\begin{array}{l}\text { SOLVED } \\
\text { (mg/L } \\
\text { as N) }\end{array}$ & $\begin{array}{l}\text { TOTAL } \\
\text { (mg/L } \\
\text { as N) }\end{array}$ & $\begin{array}{l}\text { TOTAL } \\
\text { (mg/L } \\
\text { as N })\end{array}$ & $\begin{array}{c}\text { TOTAL } \\
\langle\mathrm{mg} / \mathrm{L} \\
\text { as P) }\end{array}$ & $\begin{array}{l}\text { SOLVED } \\
\text { (mg/L } \\
\text { as P) }\end{array}$ & $\begin{array}{l}\text { SOTVED } \\
\text { (my/L } \\
\text { as Cl) }\end{array}$ & $\begin{array}{l}\text { SOLVED } \\
\text { (mg/L } \\
\text { as SO })\end{array}$ \\
\hline & & & $(\mathrm{NTU})$ & $(\mu \mathrm{s} / \mathrm{cm})$ & & $(\mathrm{mg} / \mathrm{L})$ & & & & & & & \\
\hline
\end{tabular}

\section{WATER-QUALITY RECORDS}

WATER YEAR OCTOBER 1992 TO SEPTEMBER 1993-continued

\begin{tabular}{|c|c|c|c|c|c|c|c|c|c|c|c|c|c|c|}
\hline \multicolumn{15}{|c|}{ WATER YEAR OCTOBER 1992 TO SEPTEMBER 1993-continued } \\
\hline JAN 02 & 0835 & 0735 & 66 & 45 & 478 & 105 & 13 & 0.15 & 1.0 & 0.98 & 0.260 & 0.065 & 54 & 28 \\
\hline $04-05$ & 0930 & 0830 & 55 & 50 & 556 & 139 & 17 & 0.22 & 1.4 & 1.10 & 0.300 & 0.069 & $\begin{array}{l}54 \\
70\end{array}$ & $\begin{array}{l}28 \\
35\end{array}$ \\
\hline $05-07$ & 0930 & 0830 & 45 & 45 & 520 & 85 & 12 & 0.25 & 1.4 & 1.10 & 0.240 & 0.076 & 59 & 34 \\
\hline $21-22$ & 0920 & 2020 & 57 & 32 & - & 86 & 12 & 0.46 & 1.4 & 1.50 & 0.290 & 0.098 & 91 & 34 \\
\hline $22-25$ & 2120 & 0820 & 59 & 28 & -- & -- & -- & 0.18 & 0.87 & 0.95 & 0.180 & 0.054 & 66 & 28 \\
\hline $25-28$ & 0935 & 0835 & 24 & 12 & 728 & -- & -- & 0.30 & 1.1 & 1.50 & 0.140 & 0.068 & 99 & 36 \\
\hline \multicolumn{15}{|l|}{$\mathrm{FEB}$} \\
\hline $04-06$ & 0955 & 0855 & 8.8 & 4.5 & 951 & -- & -- & 0.57 & 1.4 & -- & 0.150 & 0.096 & 150 & 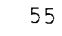 \\
\hline \multicolumn{15}{|l|}{ MAR } \\
\hline $22-25$ & 0845 & 0745 & 47 & 24 & 806 & -- & -- & 0.20 & 1.3 & 1.40 & 0.200 & 0.056 & 130 & 38 \\
\hline $25-29$ & 0905 & 0805 & 103 & 140 & 518 & 616 & 55 & 0.12 & 2.0 & 1.20 & 0.730 & 0.056 & 69 & 25 \\
\hline \multicolumn{15}{|l|}{ MAR $29-$} \\
\hline APR 01 & 0835 & 0835 & 156 & 85 & 335 & -- & -- & 0.07 & 1.2 & 0.89 & 0.360 & 0.048 & 34 & 20 \\
\hline $01-02$ & 0910 & 2010 & 195 & 110 & 292 & -- & -- & 0.06 & 1.5 & 0.84 & 0.520 & 0.055 & 31 & 19 \\
\hline $02-05$ & 2110 & 0710 & 51 & 39 & 419 & -- & -- & 0.08 & 0.94 & 1.10 & 0.230 & 0.055 & 46 & 25 \\
\hline $05-08$ & 0915 & 0815 & 24 & 14 & 533 & -- & -- & 0.07 & 0.72 & 1.40 & 0.130 & 0.046 & 58 & 26 \\
\hline $08-10$ & 0835 & 0735 & 15 & 5.7 & 609 & -- & -- & 0.05 & 0.82 & 1.60 & 0.130 & 0.037 & 70 & 33 \\
\hline $10-10$ & 0835 & 2335 & 24 & 13 & 606 & -- & -- & 0.10 & 1.2 & 1.40 & 0.220 & 0.037 & 71 & 34 \\
\hline $11-12$ & 0035 & 0735 & 19 & 18 & 602 & -- & -- & 0.08 & 0.98 & 1.30 & 0.170 & 0.053 & 70 & 32 \\
\hline $12-15$ & 0800 & 0700 & 10 & 3.7 & -- & -- & -- & 0.04 & 0.84 & $<0.02$ & 0.130 & 0.055 & 84 & 35 \\
\hline $15-17$ & 0815 & 1515 & 11 & 3.8 & 699 & -- & -- & 0.05 & 0.75 & 1.60 & 0.130 & 0.068 & 69 & 40 \\
\hline $17-19$ & 1615 & 0715 & 14 & 7.9 & 658 & -- & -- & 0.03 & 0.82 & 1.20 & 0.140 & 0.062 & 76 & 36 \\
\hline $22-24$ & 0825 & 2325 & 8.4 & 4.4 & 722 & -- & -- & 0.01 & 0.94 & 1.70 & 0.150 & 0.087 & 88 & 40 \\
\hline $24-26$ & 0025 & 0725 & 7.8 & 3.7 & 751 & -- & -- & $<0.01$ & 0.90 & 1.80 & 0.170 & 0.094 & 95 & 44 \\
\hline $26-29$ & 1010 & 0450 & 9.6 & 6.4 & 664 & -- & -- & $<0.01$ & 1.0 & 1.70 & 0.150 & 0.086 & 78 & 38 \\
\hline \multicolumn{15}{|l|}{ APR $29-$} \\
\hline MAY 03 & 0855 & 0755 & 8.6 & 4.8 & 575 & -- & -- & $<0.01$ & 0.59 & 1.50 & 0.140 & 0.082 & 64 & 37 \\
\hline \multicolumn{15}{|l|}{ JUN } \\
\hline $01-03$ & 0930 & 0830 & 3.6 & 11 & 717 & -- & -- & $<0.01$ & 1.1 & 2.10 & 0.500 & 0.410 & 88 & 64 \\
\hline $05-05$ & 0155 & 2055 & 9.0 & N150 & 655 & 413 & 70 & -- & 3.1 & 2.30 & 1.45 & 0.455 & 80 & 54 \\
\hline $05-07$ & 2155 & 0855 & 7.4 & N540 & 631 & 1140 & 158 & -- & 4.4 & 1.80 & 1.95 & 0.285 & 74 & 61 \\
\hline $08-09$ & 1445 & 0145 & 7.2 & 40 & 698 & 77 & $<23$ & -- & 1.2 & 2.10 & 0.610 & 0.430 & 87 & 59 \\
\hline $09-09$ & 0245 & 1045 & 6.7 & 70 & 611 & 113 & 19 & -- & 1.3 & 1.90 & 0.670 & 0.360 & 73 & 48 \\
\hline $09-09$ & 1145 & 1945 & 14 & 120 & 594 & 254 & 38 & -- & 2.5 & 1.60 & 0.910 & 0.310 & 66 & 50 \\
\hline $09-10$ & 2045 & 0745 & 10 & 420 & 559 & 609 & 61 & -- & 2.0 & 1.40 & 1.00 & 0.240 & 65 & 55 \\
\hline $10-14$ & 0845 & 0745 & 4.3 & 60 & 740 & 103 & 16 & 0.02 & 1.1 & 2.20 & 0.630 & 0.405 & 94 & 55 \\
\hline $19-20$ & 0100 & 0800 & 3.3 & 24 & 745 & -- & -- & 0.02 & 1.1 & 2.60 & 0.740 & 0.590 & 95 & 65 \\
\hline $20-21$ & 0900 & 0800 & 4.2 & 40 & 657 & 71 & 14 & 0.02 & 1.2 & 2.20 & 0.750 & 0.510 & 81 & 52 \\
\hline $21-24$ & 0840 & 0740 & 3.0 & 26 & 728 & -- & -- & 0.02 & 1.1 & 2.30 & 0.730 & 0.540 & 91 & 57 \\
\hline \multicolumn{15}{|l|}{ JUL } \\
\hline $11-12$ & 2120 & 0820 & 1.7 & 7.0 & 996 & - & -- & 0.01 & 0.79 & 0.94 & 0.090 & 0.025 & 150 & 110 \\
\hline $12-12$ & 0905 & 1705 & 5.0 & 41 & 596 & 114 & 21 & 0.02 & 1.6 & 2.30 & 1.15 & 0.775 & 75 & 53 \\
\hline $12 \ldots$ & 0910 & -- & 8.8 & 40 & 660 & -- & -- & $<0.01$ & 1.4 & 2.00 & 1.10 & 0.795 & 88 & 56 \\
\hline $12-15$ & 1805 & 0805 & 1.8 & 18 & 548 & -- & -- & 0.02 & 1.0 & 1.80 & 0.810 & 0.680 & 67 & 48 \\
\hline $19-19$ & 0840 & 1640 & 6.6 & 65 & -- & 172 & 33 & 0.04 & 1.7 & 2.20 & 1.10 & 0.545 & 65 & 47 \\
\hline $19-22$ & 1740 & 0740 & 2.7 & 29 & -- & -- & -- & 0.02 & 1.1 & 1.80 & 0.770 & 0.475 & 60 & 52 \\
\hline \multicolumn{15}{|l|}{ AUG } \\
\hline $05-07$ & 0850 & 2350 & 1.6 & 12 & 692 & -- & -- & $<0.01$ & 0.84 & 1.70 & 0.850 & 0.678 & 91 & 81 \\
\hline $07-09$ & 0050 & 0750 & 1.7 & 5.0 & 617 & -- & -- & $<0.01$ & 0.61 & 1.90 & 0.840 & 0.761 & 74 & 70 \\
\hline $20-21$ & 0510 & 0010 & 1.7 & 22 & 513 & -- & -- & 0.02 & 1.1 & 1.50 & 0.900 & 0.710 & 67 & 58 \\
\hline $21-23$ & 0110 & 0810 & 1.7 & 10 & 478 & -- & -- & $<0.01$ & 0.82 & 1.40 & 0.720 & 0.610 & 50 & 48 \\
\hline \multicolumn{15}{|l|}{ AUG $31-$} \\
\hline SEP 01 & 1745 & 0445 & 4.4 & 32 & 481 & 90 & 24 & $<0.01$ & 1.1 & -- & 0.960 & 0.740 & 47 & 62 \\
\hline $01-03$ & 0545 & 0845 & 2.0 & 17 & 447 & -- & -- & $<0.01$ & 0.90 & -- & 0.790 & 0.685 & 46 & 50 \\
\hline $03-06$ & 0920 & 1220 & 2.4 & 12 & 462 & -- & -- & $<0.01$ & 1.1 & 1.60 & 0.730 & 0.625 & 49 & 46 \\
\hline $06-07$ & 1320 & 0820 & 3.8 & 18 & 474 & -- & -- & $<0.01$ & 0.92 & 2.10 & 0.760 & 0.634 & 48 & 46 \\
\hline $07-09$ & 1030 & 0930 & 2.0 & 8.8 & -- & -- & -- & 0.01 & 0.70 & 2.00 & 0.710 & 0.630 & 51 & . \\
\hline $10-10$ & 0540 & 1640 & 3.2 & 8.7 & 521 & -- & -- & 0.02 & 0.69 & 2.10 & 0.770 & 0.720 & 55 & 53 \\
\hline $10-13$ & 1740 & 0840 & 2.2 & 6.7 & 499 & -- & -- & $<0.01$ & 0.57 & 2.00 & 0.730 & 0.698 & 53 & 51 \\
\hline $23-23$ & 0925 & 2025 & 2.7 & 5.8 & 499 & 11 & $<6$ & 0.01 & 0.69 & 2.20 & 0.690 & 0.600 & 51 & 59 \\
\hline $25-26$ & 2125 & 1225 & 4.9 & 30 & 470 & 71 & 12 & 0.01 & 0.98 & 2.10 & 0.840 & 0.600 & 48 & 48 \\
\hline $26-27$ & 2125 & 0825 & 3.1 & 22 & 342 & -- & -- & 0.02 & 0.71 & 1.30 & 0.550 & 0.425 & 34 & 31 \\
\hline
\end{tabular}




\section{Surface-Water Stations}

\section{A. Discharge and water quality}

\section{Irondequoit Creek At Railroad Mills Near Fishers, N.Y.}

LOCATION.--Lat $43^{\circ} 01^{\prime} 40^{\prime \prime}$, long $77^{\circ} 28^{\prime} 42^{\prime \prime}$, Ontario County, Hydrologic Unit 04140101 , on right bank $90 \mathrm{ft}$ upstream from bridge on Railroad Mills Road, 1.5 mi northwest of

Fishers, and $4.0 \mathrm{mi}$ southwest of Fairport.

DRAINAGE AREA.--39.2 $\mathrm{mi}^{2}$.

PERIOD OF RECORD.--July 1991 to current year.

\section{WATER-DISCHARGE RECORDS}

GAGE.--Water-stage recorder. Elevation of gage is $450 \mathrm{ft}$ above sea level, from topographic map.

REMARKS.--Records good except those for estimated daily discharges, which are fair. Unpublished water-quality records for prior years are available in files of Monroe County

Department of Health. Several measurements of water temperature were made during the year.

COOPERATION.--Gage-height record and 9 discharge measurements were provided by the Monroe County Environmental Health Laboratory at Rochester, N.Y.

EXTREMES FOR PERIOD July 1991 to September 1993.--Maimum discharge, $588 \mathrm{ft}^{3} / \mathrm{s}$, Apr. 2, 1993, gage height, $9.36 \mathrm{ft}$, minimum daily discharge, $9.5 \mathrm{ft}{ }^{3} / \mathrm{s}$, Aug. $2,1991$.

STATISTICS OF MONTHLY MEAN DISCHARGE (in cubic feet per second) FOR WATER YEARS 1991-93, BY WATER YEAR

\begin{tabular}{|c|c|c|c|c|c|c|c|c|c|c|c|c|}
\hline & $\mathrm{OCT}$ & NOV & DEC & JAN & FEB & MAR & APR & MAY & JUN & JUL & AUG & SEP \\
\hline MEAN & 26.8 & 42.5 & 45.2 & 50.5 & 31.8 & 80.9 & 101 & 38.8 & 25.6 & 27.7 & 28.7 & 22.4 \\
\hline MAX & 39.5 & 67.5 & 66.1 & 74.6 & 34.7 & 98.0 & 143 & 39.1 & 26.9 & 52.5 & 58.0 & 35 \\
\hline (WY) & 1993 & 1993 & 1993 & 1993 & 1993 & 1993 & 1993 & 1993 & 1993 & 1992 & 1992 & 199 \\
\hline MIN & 14.1 & 17.6 & 24.4 & 26.5 & 29.0 & 63.9 & 58.2 & 38.6 & 24.4 & 13.4 & 12.5 & 12.3 \\
\hline (WY) & 1992 & 1992 & 1992 & 1992 & 1992 & 1992 & 1992 & 1992 & 1992 & 1991 & 1991 & 91 \\
\hline
\end{tabular}

SUMMARY STATISTICS

STATISTIC

ANNUAL TOTAL

ANNUAL MEAN

AVERAGE DISCHARGE

HIGHEST ANNUAL MEAN

LOWEST ANNUAL MEAN

HIGHEST DAILY MEAN

LOWEST DAILY MEAN

ANNUAL SEVEN-DAY MINIMUM

INSTANTANEOUS PEAK FLOW

INSTANTANEOUS LOW FLOW

ANNUAL RUNOFF (CFSM)

ANNUAL RUNOFF (INCHES)

10 PERCENT EXCEEDS

50 PERCENT EXCEEDS

90 PERCENT EXCEEDS
INSTANTANEOUS PEAK STAGE

FOR 1992 CALENDAR YEAR

17116

46.8

\begin{tabular}{|c|c|}
\hline 381 & Mar 27 \\
\hline 13 & Jul \\
\hline 17 & $\mathrm{Feb}$ \\
\hline
\end{tabular}

a Aug 27, 28, 29 .
FOR 1993 WATER YEAR

19517

$$
53.5
$$

WATER YEARS 1991 - 1993

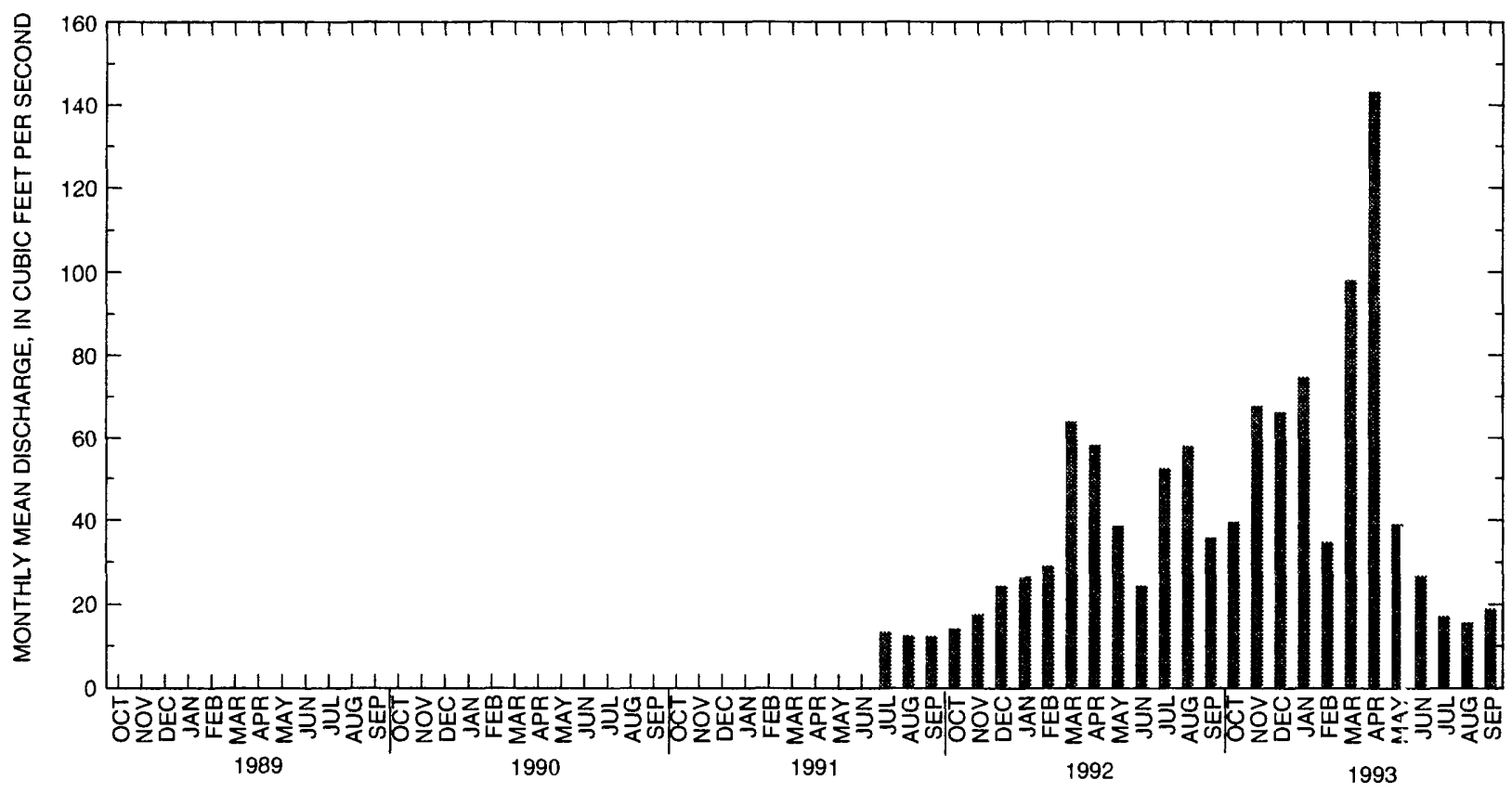

40 Water Resources of Monroe County, N.Y., 1989-93, with emphasis on Water Quality in the Irondequoit Creek Basin 


\section{Irondequoit Creek At Railroad Mills Near Fishers, N.Y.}

\section{WATER-QUALITY RECORDS}

PERIOD OF RECORD.--July 1991 to current year.

CHEMICAL DATA: 1991-93 (e).

NUTRIENT DATA: 1991-93 (e).

COOPERATION, - Water-quality samples were collected and analyzed by the Monroe County Environmental Health Laboratory at Rochester, NY.

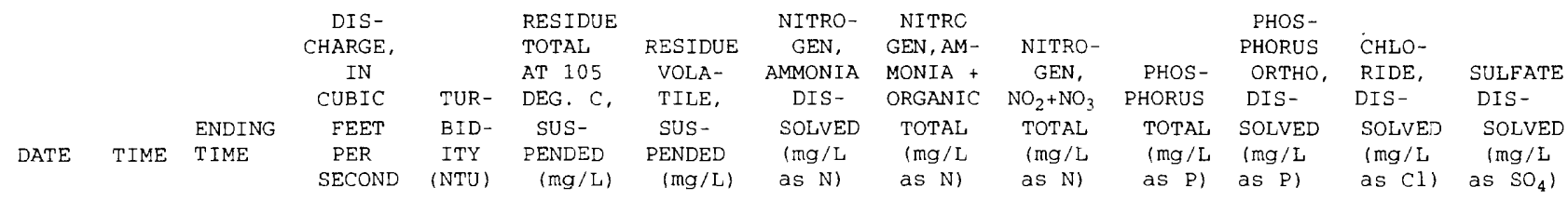

NOV

\begin{tabular}{|c|c|c|}
\hline $27-28$ & 1220 & 2220 \\
\hline $28-29$ & 2320 & 1320 \\
\hline \multicolumn{3}{|l|}{ NOV $29-$} \\
\hline DEC 02 & 1420 & 1120 \\
\hline $02-03$ & 1240 & 0540 \\
\hline $03-04$ & 0640 & 1140 \\
\hline $04-05$ & 1240 & 1140 \\
\hline $26-29$ & 1405 & 0505 \\
\hline $29-30$ & 0605 & 1305 \\
\hline \multicolumn{3}{|l|}{ DEC $30-$} \\
\hline JAN 02 & 1350 & 1250 \\
\hline $21-23$ & 1420 & 1220 \\
\hline $23-24$ & 1230 & 2330 \\
\hline \multicolumn{3}{|l|}{ FEB } \\
\hline $14-15$ & 1035 & 2155 \\
\hline $15-16$ & 2255 & 1755 \\
\hline $16-18$ & 1855 & 0955 \\
\hline $18-19$ & 1255 & 1555 \\
\hline $19-20$ & 1655 & 1055 \\
\hline $20-22$ & 1155 & 2255 \\
\hline $22-24$ & 2355 & 1055 \\
\hline $24-27$ & 1150 & 1050 \\
\hline $27-28$ & 1125 & 1825 \\
\hline $28-29$ & 1925 & 0625 \\
\hline \multicolumn{3}{|l|}{ FEB 29- } \\
\hline MAR 02 & 0725 & 1025 \\
\hline $05-07$ & 1130 & 0230 \\
\hline $07-08$ & 0330 & 0230 \\
\hline $08-09$ & 0330 & 1030 \\
\hline $09-12$ & 1235 & 1135 \\
\hline $26-28$ & 1120 & 0220 \\
\hline $28-30$ & 0320 & 1020 \\
\hline \multicolumn{3}{|l|}{ MAR $30-$} \\
\hline APR 02 & 1155 & 1055 \\
\hline $09-11$ & 1110 & 1410 \\
\hline $11-12$ & 1510 & 1410 \\
\hline $12-13$ & 1510 & 1010 \\
\hline $13-16$ & 1210 & 1110 \\
\hline $16-17$ & 1125 & 1025 \\
\hline $17-19$ & 1125 & 0425 \\
\hline $19-20$ & 0525 & 1025 \\
\hline \multicolumn{3}{|l|}{ MAY } \\
\hline $04-07$ & 1140 & 1040 \\
\hline \multicolumn{3}{|l|}{ JUN } \\
\hline $01-04$ & 1155 & 1055 \\
\hline $01 \ldots$ & 1200 & - \\
\hline $06-07$ & 0335 & 1035 \\
\hline $07-08$ & 1135 & 1035 \\
\hline $08-11$ & 1200 & 1100 \\
\hline $19-19$ & 0725 & 2225 \\
\hline $19-22$ & 2325 & 1025 \\
\hline \multicolumn{3}{|l|}{ JUL } \\
\hline $03-03$ & 1105 & 2205 \\
\hline $03-06$ & 2305 & 1005 \\
\hline $06-08$ & 1135 & 1935 \\
\hline $08-09$ & 2035 & 1035 \\
\hline $09-12$ & 1135 & 1435 \\
\hline $29-31$ & 1510 & 1010 \\
\hline
\end{tabular}

PERIOD NOVEMBER 1991 TO SEPTEMBER 1992

\begin{tabular}{|c|c|c|c|c|c|c|c|c|}
\hline-- & -- & -- & 0.42 & 1.20 & 0.025 & $<0.002$ & 61 & 330 \\
\hline-- & -- & -- & 0.49 & 0.29 & 0.045 & 0.003 & 65 & 310 \\
\hline-- & -- & -- & 0.56 & 0.98 & 0.035 & 0.003 & 64 & 280 \\
\hline-- & -- & 0.01 & 0.44 & 1.00 & 0.040 & 0.006 & 60 & 300 \\
\hline-- & -- & 0.02 & 0.76 & 0.87 & 0.075 & 0.006 & 82 & 240 \\
\hline-- & -- & 0.02 & 0.52 & 0.93 & 0.030 & 0.006 & 65 & 240 \\
\hline-- & -- & 0.01 & 0.40 & 1.20 & 0.035 & 0.006 & 63 & 310 \\
\hline 74 & 18 & 0.03 & 1.1 & 1.00 & 0.120 & 0.008 & 98 & 220 \\
\hline-- & -- & 0.02 & 0.50 & 1.70 & 0.060 & 0.009 & 69 & 210 \\
\hline-- & -- & 0.02 & 0.26 & 1.50 & 0.020 & 0.005 & 63 & 270 \\
\hline-- & -- & -- & -- & -- & 0.060 & 0.008 & 110 & $<10$ \\
\hline-- & -- & 0.01 & 0.24 & 1.30 & 0.015 & 0.005 & 73 & 270 \\
\hline-- & -- & 0.05 & 0.63 & 1.30 & 0.050 & 0.006 & 130 & 220 \\
\hline-- & -- & 0.04 & 0.56 & 1.20 & 0.055 & 0.007 & 75 & 160 \\
\hline-- & -- & 0.02 & 0.51 & 1.40 & 0.040 & 0.008 & 79 & 190 \\
\hline-- & -- & 0.02 & 0.71 & 1.50 & 0.075 & 0.009 & 71 & 150 \\
\hline-- & -- & 0.03 & 0.50 & 1.50 & 0.040 & 0.011 & 61 & 160 \\
\hline-- & -- & 0.03 & 0.73 & 1.60 & 0.095 & 0.012 & 68 & 150 \\
\hline-- & -- & 0.03 & 0.49 & 1.70 & 0.050 & 0.009 & 60 & 170 \\
\hline-- & -- & 0.03 & 0.42 & 1.70 & 0.025 & 0.008 & 57 & 190 \\
\hline-- & -- & 0.03 & 0.50 & 1.50 & 0.040 & 0.010 & 71 & 190 \\
\hline-- & -- & 0.02 & 0.43 & 1.50 & 0.025 & 0.007 & 71 & 200 \\
\hline-- & -- & 0.02 & 0.29 & -- & 0.015 & 0.007 & 59 & 130 \\
\hline-- & -- & 0.01 & 0.52 & $\cdots$ & 0.055 & 0.007 & 74 & 220 \\
\hline-- & -- & 0.01 & 0.72 & -- & 0.065 & 0.006 & 62 & 180 \\
\hline-- & -- & 0.03 & 0.43 & 1.30 & 0.030 & 0.009 & 60 & 180 \\
\hline 510 & 60 & 0.03 & 2.5 & 1.20 & 0.610 & 0.008 & 46 & 60 \\
\hline 111 & 14 & 0.03 & 0.85 & 1.30 & 0.160 & 0.009 & 44 & 69 \\
\hline-- & -- & 0.01 & 0.46 & 1.00 & 0.070 & 0.007 & 52 & 110 \\
\hline-- & -- & 0.02 & 0.28 & 1.30 & 0.015 & 0.002 & 59 & 210 \\
\hline-- & -- & 0.01 & 0.50 & 1.10 & 0.070 & 0.004 & 68 & 170 \\
\hline-- & -- & 0.01 & 0.55 & 0.80 & 0.050 & 0.004 & 54 & 130 \\
\hline-- & -- & 0.01 & 0.40 & 1.10 & 0.020 & 0.004 & 56 & - \\
\hline 133 & 20 & -- & 0.76 & 1.00 & 0.130 & 0.006 & 59 & 140 \\
\hline 69 & 13 & -- & 0.55 & 1.00 & 0.095 & 0.006 & 45 & 100 \\
\hline-- & -- & -- & 0.48 & 0.97 & 0.060 & 0.006 & 45 & 110 \\
\hline-- & -- & - & - & - & 0.040 & 0.004 & 49 & 130 \\
\hline-- & -- & -- & -- & $\sim$ & 0.060 & 0.008 & 60 & 140 \\
\hline-- & -- & -- & -- & -- & 0.065 & 0.007 & 62 & 160 \\
\hline-- & -- & -- & -- & -- & 0.090 & 0.010 & 60 & 220 \\
\hline$\ldots$ & -- & -- & -- & -- & 0.075 & 0.010 & 57 & 190 \\
\hline-- & -- & $\cdots$ & -- & -- & 0.060 & 0.008 & 56 & - \\
\hline 105 & 10 & -- & -- & - & 0.110 & 0.008 & 61 & 240 \\
\hline$\cdots$ & -- & -- & -- & -- & 0.070 & 0.009 & 64 & 250 \\
\hline 177 & 25 & -- & -- & -- & 0.160 & 0.007 & 58 & 270 \\
\hline-- & -- & -- & -- & -- & 0.080 & 0.008 & 59 & 250 \\
\hline- & -- & -- & -- & -- & 0.035 & 0.008 & 62 & 290 \\
\hline 221 & 22 & -- & -- & -- & 0.300 & 0.010 & 59 & 250 \\
\hline 92 & 10 & -- & -- & -- & 0.110 & 0.012 & 60 & 220 \\
\hline 245 & 36 & -- & -- & -- & 0.230 & 0.018 & 46 & - \\
\hline
\end{tabular}




\begin{tabular}{|c|c|c|c|c|c|c|c|c|c|c|c|c|c|}
\hline & & & $\begin{array}{c}\text { DIS- } \\
\text { CHARGE, } \\
\text { IN } \\
\text { CUBIC }\end{array}$ & TUR- & $\begin{array}{l}\text { RESIDUE } \\
\text { TOTAL } \\
\text { AT } 105 \\
\text { DEG. C, }\end{array}$ & $\begin{array}{l}\text { RES IDUE } \\
\text { VOLA- } \\
\text { TILE, }\end{array}$ & $\begin{array}{l}\text { NITRO- } \\
\text { GEN, } \\
\text { AMMONIA } \\
\text { DIS- }\end{array}$ & $\begin{array}{l}\text { NITRO- } \\
\text { GEN, AM- } \\
\text { MONIA + } \\
\text { ORGANIC }\end{array}$ & $\begin{array}{c}\text { NITRO- } \\
\text { GEN, } \\
\mathrm{NO}_{2}+\mathrm{NO}_{3}\end{array}$ & $\begin{array}{l}\text { PHOS- } \\
\text { PHORUS }\end{array}$ & $\begin{array}{c}\text { PHOS- } \\
\text { PHORUS } \\
\text { ORTHO, } \\
\text { DIS- }\end{array}$ & $\begin{array}{l}\text { CHLO- } \\
\text { RIIE, } \\
\text { DIS- }\end{array}$ & $\begin{array}{l}\text { SULFATE } \\
\text { DIS- }\end{array}$ \\
\hline DATE & TIME & $\begin{array}{l}\text { ENDING } \\
\text { TIME }\end{array}$ & $\begin{array}{c}\text { FEET } \\
\text { PER } \\
\text { SECOND }\end{array}$ & $\begin{array}{l}\text { BID- } \\
\text { ITY } \\
\text { (NTU) }\end{array}$ & $\begin{array}{l}\text { SUS- } \\
\text { PENDED } \\
(\mathrm{mg} / \mathrm{L})\end{array}$ & $\begin{array}{l}\text { SUS- } \\
\text { PENDED } \\
\text { (mg/L) }\end{array}$ & $\begin{array}{l}\text { SOLVED } \\
\text { (mg/L } \\
\text { as N) }\end{array}$ & $\begin{array}{l}\text { TOTAL } \\
(\mathrm{mg} / \mathrm{L} \\
\text { as N) }\end{array}$ & $\begin{array}{l}\text { TOTAL } \\
\text { (mg/L } \\
\text { as N) }\end{array}$ & $\begin{array}{l}\text { TOTAL } \\
\text { (mg/L } \\
\text { as P) }\end{array}$ & $\begin{array}{l}\text { SOLVED } \\
(\mathrm{mg} / \mathrm{L} \\
\text { as P) }\end{array}$ & $\begin{array}{l}\text { SOLVED } \\
\text { (mg/L } \\
\text { as } \mathrm{Cl} \text { ) }\end{array}$ & $\begin{array}{c}\text { SOLVED } \\
(\mathrm{mg} / \mathrm{L} \\
\left.\text { as } \mathrm{SO}_{4}\right)\end{array}$ \\
\hline
\end{tabular}

AUG

$\begin{array}{lllr}03-04 & 1210 & 1410 & 105 \\ 04-06 & 1510 & 1010 & 107 \\ 24-24 & 1120 & 2220 & 18 \\ 24-25 & 2320 & 0720 & 23 \\ 25-27 & 0820 & 1020 & 20 \\ 27-28 & 1520 & 1420 & 118 \\ 28-31 & 1520 & 1020 & 152 \\ \text { P } & & & \\ 03-04 & 0400 & 0300 & 50 \\ 04-04 & 0400 & 1100 & 75 \\ 04-08 & 1105 & 1005 & 36 \\ 18-19 & 1930 & 1430 & 43 \\ 19-21 & 1530 & 1030 & 28 \\ & & & \end{array}$

PERIOD NOVEMBER 1991 TO SEPTEMBER 1992-continued

OCT

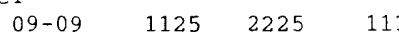

440

1125

2225

111

60

23551055

$25-26 \quad 1155 \quad 1055$

26-29 $1235 \quad 1135$

69

80
45

NOV

$\begin{array}{llll}02-03 & 1200 & 0800 & 117\end{array}$

12

$03-05$

$0900 \quad 1100$

$22-23$

$1200 \quad 1100$

$23-25$

$1210 \quad 2310$

130

90

50
130

1110

131

60

10-11

$1220 \quad 1920$

35

$16-17$

$1030 \quad 1230$

$17-18 \quad 1250 \quad 1150$

105

$18-21$

$29-31$

$1755 \quad 1055$

6.7
34
33
24
50

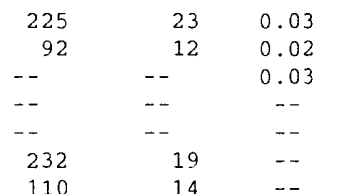

$\begin{array}{lc}0.95 & 0.86 \\ 0.89 & 0.56 \\ 0.32 & 1.30 \\ -- & -- \\ -- & -- \\ -- & -- \\ -- & -- \\ -- & 1.00 \\ -- & 0.73 \\ -- & -- \\ -- & -- \\ -- & --\end{array}$

0.160

0.160

0.022

0.025

0.045

0.035

0.290

0.005

0.006

3.6
62

21

30
12
75

$\begin{array}{rrc}47 & 9 & 0.01 \\ 62 & 12 & 0.02 \\ -- & -- & -- \\ 142 & -21 & -- \\ -- & -- & --\end{array}$

0.140

0.165

0.050
0.190

0.005

0.017

0.025

0.013

0.020

0.016

0.009

0.070

0.006

$\begin{array}{rr}41 & 120 \\ 36 & 88 \\ 58 & 180 \\ 59 & 180 \\ 60 & 180 \\ 37 & - \\ 30 & - \\ & \\ 54 & - \\ 42 & - \\ 58 & 170 \\ 58 & 150 \\ 53 & 190\end{array}$

WATER YEAR OCTOBER 1992 TO SEPTEMBER 1993

$\begin{array}{rrrrr}\text { JAN 03 } & 1110 & 0210 & 132 & 36 \\ 03-04 & 0310 & 1010 & 65 & 8.5 \\ 04-05 & 1235 & 1735 & 138 & 60 \\ 05-07 & 1835 & 1135 & 107 & 32 \\ 21-23 & 1205 & 0305 & 85 & 21 \\ 23-25 & 0405 & 1105 & 134 & 34 \\ 25-28 & 1235 & 1135 & 68 & 16 \\ \text { MAR } & & & & \\ 18-19 & 1140 & 1445 & 43 & 5.2 \\ 19-22 & 1545 & 0245 & 43 & 4.4 \\ 22-25 & 1115 & 1015 & 88 & 17 \\ 25-29 & 1100 & 1000 & 210 & 60 \\ 29-31 & 1125 & 0725 & 427 & 85 \\ \text { MAR 31- } & & & & \\ \text { APR 01 } & 0825 & 0725 & 418 & 85 \\ 01-03 & 1150 & 0250 & 540 & 80 \\ 03-05 & 0350 & 0950 & 222 & 32 \\ 05-08 & 1135 & 1035 & 173 & 22 \\ 08-10 & 1115 & 1015 & 95 & 2.8 \\ 16-18 & 1035 & 0035 & 151 & 50 \\ \text { JUL } & & & & \\ 19-21 & 1325 & 1225 & 19 & 23 \\ \text { SEP } & & & & \\ 06-07 & 0515 & 0015 & 22 & 25 \\ 07-07 & 0115 & 1015 & 23 & 29 \\ 14-15 & 2030 & 1630 & 15 & 16 \\ 23-26 & 1050 & 0150 & 18 & 50 \\ 26-27 & 0250 & 0950 & 37 & 240\end{array}$

\begin{tabular}{|c|c|c|c|c|c|}
\hline 805 & 102 & -- & -- & -- & 0.960 \\
\hline 175 & 29 & -- & -- & -- & 0.150 \\
\hline 135 & 20 & - & -- & -- & 0.160 \\
\hline 63 & 13 & -- & -- & -- & 0.120 \\
\hline-- & -. & -- & -. & 0.84 & 0.045 \\
\hline 180 & 28 & -- & -- & -- & 0.180 \\
\hline 78 & 12 & -- & - & -- & 0.130 \\
\hline 196 & 24 & -- & -- & -- & 0.270 \\
\hline 78 & 12 & -. & -- & -- & 0.150 \\
\hline 166 & 22 & -- & -- & -. & 0.330 \\
\hline 18 & $<5$ & $<0.01$ & 0.39 & 1.50 & 0.045 \\
\hline 89 & 19 & -- & -- & -- & 0.100 \\
\hline-- & -- & -- & -- & -- & 0.120 \\
\hline - & -- & -. & -- & -- & 0.090 \\
\hline-- & - & 0.01 & 1.0 & 0.95 & 0.140 \\
\hline 78 & 12 & $<0.013$ & 0.78 & 0.88 & 0.130 \\
\hline-- & -. & -- & -- & -. & 0.045 \\
\hline 96 & 14 & $<0.01$ & -- & 0.82 & 0.140 \\
\hline 49 & 9 & $<0.01$ & -- & 0.86 & 0.095 \\
\hline - & -- & -- & -- & -- & 0.090 \\
\hline 67 & 10 & -- & -- & -- & 0.120 \\
\hline-- & -. & - & -. & -- & 0.045 \\
\hline -- & -- & $<0.01$ & -- & 1.20 & 0.040 \\
\hline -- & -- & $<0.01$ & -- & 1.20 & 0.030 \\
\hline-- & -- & $<0.01$ & 0.52 & 1.10 & 0.060 \\
\hline 149 & 19 & 0.01 & 1.5 & 0.80 & 0.150 \\
\hline 201 & 20 & 0.01 & 0.94 & 0.69 & 0.210 \\
\hline 152 & 15 & 0.01 & 0.83 & 0.64 & 0.210 \\
\hline - - & -- & $\ldots$ & -- & -- & 0.170 \\
\hline-- & - & - & -- & -- & 0.120 \\
\hline -- & -. & $<0.01$ & 0.71 & 0.91 & 0.085 \\
\hline-- & -- & 0.01 & 1.4 & 1.00 & 0.080 \\
\hline 91 & 17 & -- & $\cdots$ & -- & 0.140 \\
\hline -- & -- & 0.02 & 0.0 & $\mathrm{~N} 1.60$ & 0.110 \\
\hline -- & -. & $<0.01$ & 0.78 & 1.30 & 0.160 \\
\hline-- & -. & $<0.01$ & 0.96 & 1.10 & 0.120 \\
\hline-- & -- & 0.01 & 0.59 & 1.30 & 0.060 \\
\hline 109 & 20 & -- & -- & -- & 0.160 \\
\hline 4 & 64 & -- & -- & -- & 0.420 \\
\hline
\end{tabular}

0.022

0.018

0.008

0.008

0.006

0.012

0.011

0.007

0.011

0.010

0.005

0.006

0.008

0.006

0.008

0.006

0.003

0.009

0.006

0.007

0.005

0.005

0.006

0.005

0.004

0.010

0.010

0.008

0.010

0.008

0.005

0.009

0.007

$46 \quad 150$ 


\section{urface-Water Stations}

\section{$\therefore$ Discharge and water quality}

\section{Irondequoit Creek Near Pittsford, N.Y.}

LOCATION.--Lat $43^{\circ} 03^{\circ} 15^{\prime \prime}$, long $77^{\circ} 29^{\prime} 28^{\prime \prime}$, Monroe County, Hydrologic Unit 04140101 , on right bank $140 \mathrm{ft}$ upstream from bridge on Thornell Road, $0.9 \mathrm{mi}$ south of creek passage under Erie (Barge) Canal, and $2.7 \mathrm{mi}$ southeast of Pittsford.

DRAINAGE AREA.-- $-44.4 \mathrm{mi}^{2}$.

\section{WATER-DISCHARGE RECORDS}

PERIOD OF RECORD.--Occasional low-flow measurements, water years 1955, 1961-62, 1964-66, 1968, and annual maximum, water years 1962-63, 1965-66, 196? -70, 1972. March 1980 to May 1991 (discontinued).

REVISED RECORDS.--WDR NY-81-3: Drainage area.

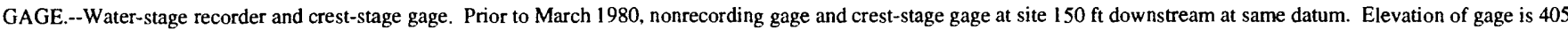
$\mathrm{ft}$ above National Geodetic Vertical Datum of 1929, from Corps of Engineers river-profile map.

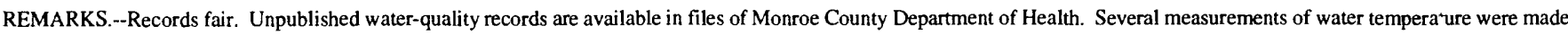
during each year.

COOPERATION.--Streamflow measurements were obtained and recorder equipment maintained by Monroe County Environmental Health Laboratory, Rochester, N.Y

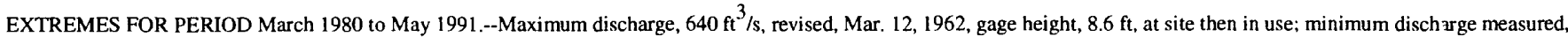
$8.10 \mathrm{ft}^{3} / \mathrm{s}$, Sept. 17, 1964; minimum gage height at present site, $2.98 \mathrm{ft}$, Sept. 12, 1983.

STATISTICS OF MONTHLY MEAN DISCHARGE (in cubic feet per second) FOR WATER YEARS 1980-90, BY WATER YEAR

\begin{tabular}{|c|c|c|c|c|c|c|c|c|c|c|c|c|}
\hline & OCT & NOV & DEC & JAN & FEB & MAR & APR & MAY & JUN & JUL & AUG & SEP \\
\hline MEAN & 26.7 & 41.9 & 45.4 & 37.2 & 56.1 & 61.5 & 65.8 & 49.0 & 30.6 & 19.7 & 23.0 & 22.1 \\
\hline MAX & 58.0 & 79.1 & 80.1 & 58.5 & 83.8 & 91.7 & 101 & 101 & 46.5 & 27.6 & 41.9 & 38.6 \\
\hline$(W Y)$ & 1987 & 1986 & 1987 & 1986 & 1990 & 1982 & 1990 & 1984 & 1989 & 1986 & 1986 & 1984 \\
\hline MIN & 15.9 & 27.1 & 18.8 & 22.1 & 24.3 & 36.4 & 34.0 & 27.4 & 14.9 & 13.1 & 13.2 & 15.6 \\
\hline (WY) & 1983 & 1989 & 1990 & 1989 & 1989 & 1988 & 1981 & 1981 & 1988 & 1983 & 1985 & 1988 \\
\hline
\end{tabular}

STATISTIC

ANNUAL TOTAL

ANNUAL MEAN

AVERAGE DISCHARGE

HIGHEST ANNUAL MEAN

LOWEST ANNUAL MEAN

HIGHEST DAILY MEAN

LOWEST DAILY MEAN

ANNUAL SEVEN-DAY MINIMUM

ANNUAL RUNOFF (CFSM)

ANNUAL RUNOFF (INCHES)

10 PERCENT EXCEEDS

50 PERCENT EXCEEDS

90 PERCENT EXCEEDS

\section{SUMMARY STATISTICS}

$\begin{array}{cl}\text { FOR } 1989 \text { CALENDAR YEAR } & \text { FOR 1990 WATER YEAR } \\ 11917 & 15608 \\ 32.6 & 42.8\end{array}$

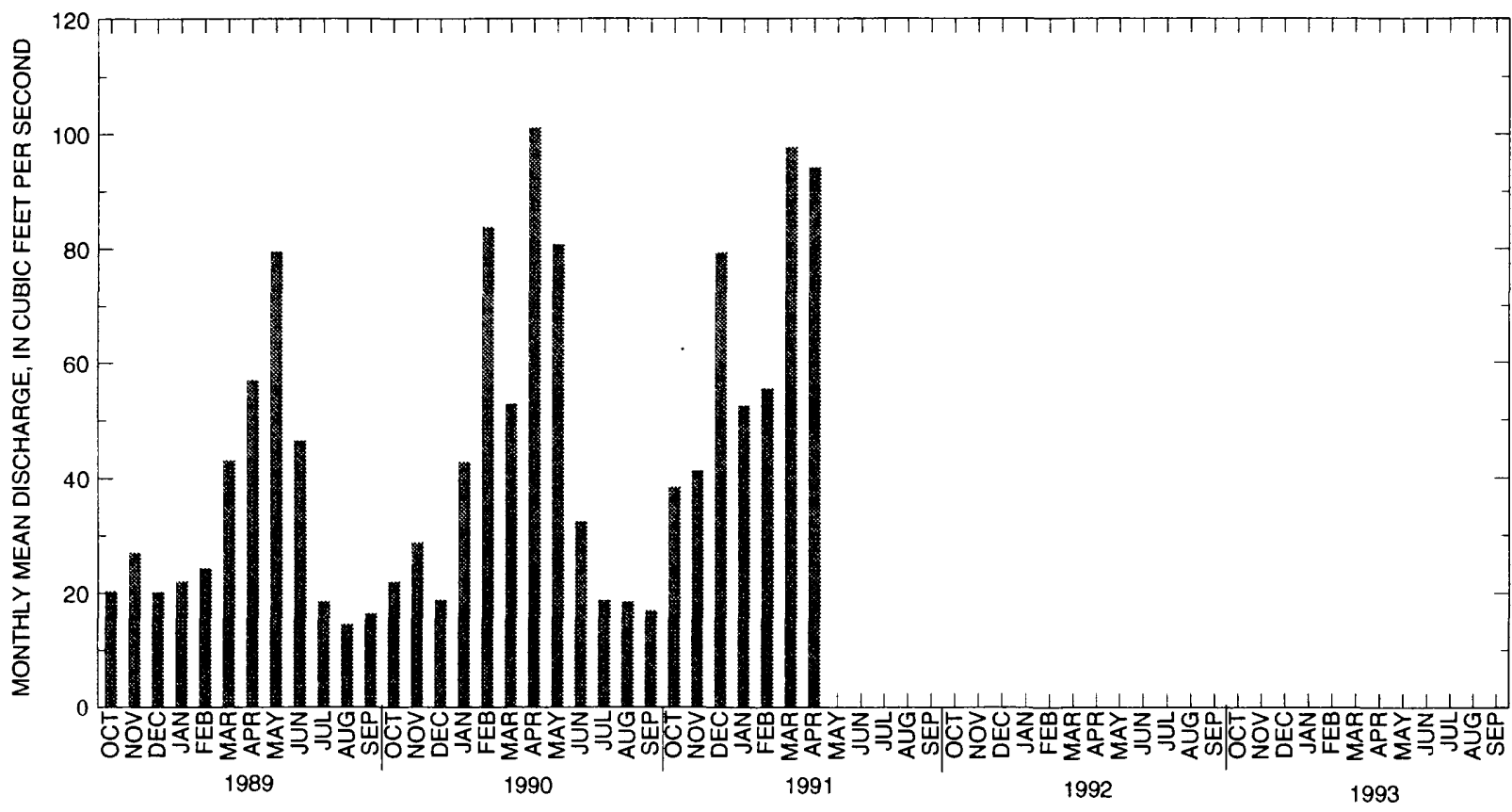




\section{Surface-Water Stations}

\section{A. Discharge and water quality}

\section{Irondequoit Creek Near Pittsford, N.Y.}

\section{WATER-QUALITY RECORDS}

PERIOD OF RECORD.--October 1983 to April, 1991, discontinued.

CHEMICAL DATA: $1983-91$ (e).

NUTRIENT DATA: $1983-91$ (e)

COOPERATION. - Water-quality samples were collected and analyzed by the Monroe County Environmental Health

Laboratory at Rochester, N.Y

REMARKS.--Prior to 1988 water year, data published in WATER RESOURCES OF MONROE COUNTY NEW YORK, WATER YEARS

1984-88. U.S. Geological Survey Open-File Report 93-370.

\begin{tabular}{|c|c|c|c|c|c|c|c|c|c|c|c|c|c|}
\hline & & & $\begin{array}{c}\text { DIS- } \\
\text { CHARGE, } \\
\text { IN }\end{array}$ & & $\begin{array}{l}\text { RESIDUE } \\
\text { TOTAL } \\
\text { AT } 105\end{array}$ & $\begin{array}{l}\text { RESIDUE } \\
\text { VOLA- } \\
\text { TILE, }\end{array}$ & $\begin{array}{l}\text { NITRO- } \\
\text { GEN, } \\
\text { AMMONIA } \\
\text { DIS- }\end{array}$ & $\begin{array}{l}\text { NITRO- } \\
\text { GEN, AM- } \\
\text { MONIA + } \\
\text { ORGANIC }\end{array}$ & $\begin{array}{c}\mathrm{NITRO}- \\
\text { GEN, } \\
\mathrm{NO}_{2}+\mathrm{NO}_{3}\end{array}$ & $\begin{array}{l}\text { PHOS- } \\
\text { PHORUS }\end{array}$ & $\begin{array}{l}\text { PHOS- } \\
\text { PHORUS } \\
\text { ORTHO, } \\
\text { DIS- }\end{array}$ & $\begin{array}{l}\text { CHLO- } \\
\text { RIDE, } \\
\text { DIS- }\end{array}$ & $\begin{array}{l}\text { SULFATE } \\
\text { DIS- }\end{array}$ \\
\hline & $\Xi$ & & $\begin{array}{l}\text { FEET } \\
\text { PER } \\
\text { SECOND }\end{array}$ & $\begin{array}{l}\text { BID- } \\
\text { ITY } \\
\text { (NTU) }\end{array}$ & $\begin{array}{l}\text { SUS- } \\
\text { PENDED } \\
\text { (mg/L) }\end{array}$ & $\begin{array}{l}\text { SUS- } \\
\text { PENDED } \\
(\mathrm{mg} / \mathrm{L})\end{array}$ & $\begin{array}{l}\text { SOLVED } \\
\text { (mg/L } \\
\text { as } \mathrm{N} \text { ) }\end{array}$ & $\begin{array}{l}\text { TOTAL } \\
(\mathrm{mg} / \mathrm{L} \\
\text { as N) }\end{array}$ & $\begin{array}{l}\text { TOTAL } \\
\text { (mg/L } \\
\text { as N) }\end{array}$ & $\begin{array}{l}\text { TOTAL } \\
\text { (mg/L } \\
\text { as P) }\end{array}$ & $\begin{array}{l}\text { SOLVED } \\
(\mathrm{mg} / \mathrm{L} \\
\text { as } \mathrm{P} \text { ) }\end{array}$ & $\begin{array}{l}\text { SOLVED } \\
\text { (mg/L } \\
\text { as } \mathrm{Cl} \text { ) }\end{array}$ & $\begin{array}{c}\text { SOLVED } \\
(\mathrm{mg} / \mathrm{L} \\
\left.\text { as } \mathrm{SO}_{4}\right)\end{array}$ \\
\hline
\end{tabular}

OCT

\begin{tabular}{|c|c|c|c|c|c|c|c|c|c|c|c|c|c|}
\hline $21-22$ & 1315 & 0615 & 18 & -- & -- & -- & $<0.01$ & 0.43 & 0.87 & 0.030 & 0.006 & 60 & 260 \\
\hline $22-24$ & 0715 & 1215 & 44 & 20 & - & -- & 0.01 & 0.70 & 0.66 & 0.040 & 0.008 & 57 & 220 \\
\hline $24-27$ & 1320 & 1220 & 24 & -- & -- & -- & $<0.01$ & 0.82 & 0.79 & 0.035 & 0.006 & 62 & 250 \\
\hline $27 \ldots$ & 1300 & -- & 24 & 2.8 & -- & -- & $<0.01$ & 0.56 & 0.99 & 0.030 & 0.005 & 62 & 270 \\
\hline \multicolumn{14}{|l|}{ NOV } \\
\hline $07 \ldots$ & 1325 & -- & 30 & 2.1 & -- & -- & $<0.01$ & 0.71 & 0.59 & 0.010 & 0.005 & 60 & 230 \\
\hline $10 \ldots$ & 1220 & -- & 26 & 1.4 & -- & -- & $<0.01$ & 0.30 & 0.87 & 0.010 & 0.007 & 62 & 270 \\
\hline $17-18$ & 1310 & 2010 & 22 & -- & - & -- & 0.03 & 0.31 & 1.02 & 0.010 & 0.007 & 58 & 280 \\
\hline $18-21$ & 2110 & 1210 & 29 & -- & -- & -- & 0.03 & 0.27 & 1.04 & 0.010 & 0.005 & 58 & 290 \\
\hline \multicolumn{14}{|l|}{$\mathrm{DEC}$} \\
\hline $19 \ldots$ & 1235 & -- & 13 & 1.2 & -- & -- & 0.03 & 0.42 & $1: 37$ & 0.010 & 0.004 & 64 & 300 \\
\hline $27-28$ & 1315 & 0315 & 21 & 1.1 & -- & -- & 0.04 & 0.55 & 1.21 & 0.020 & $<0.002$ & 72 & 240 \\
\hline $28-30$ & 0415 & 0815 & 27 & 3.4 & -- & -- & 0.03 & 0.55 & 1.17 & 0.025 & 0.004 & 71 & 240 \\
\hline \multicolumn{14}{|l|}{ JAN } \\
\hline $08-09$ & 0400 & 1100 & 28 & 4.2 & -- & -. & 0.02 & 0.91 & 1.14 & 0.035 & 0.005 & 110 & 250 \\
\hline $09-12$ & 1235 & 1535 & 25 & 1.9 & -- & -- & 0.02 & 0.29 & 1.22 & 0.025 & $<0.002$ & 76 & 230 \\
\hline $23 \ldots$ & 1220 & -- & 19 & 2.1 & -- & -. & 0.02 & 0.43 & 1.30 & 0.025 & 0.004 & 61 & 260 \\
\hline $26-30$ & 1145 & 1045 & 29 & 2.8 & -- & -- & 0.02 & 0.87 & 1.10 & 0.025 & 0.003 & 71 & 230 \\
\hline \multicolumn{14}{|l|}{ FEB } \\
\hline $06 \ldots$ & 1120 & -- & 20 & 1.5 & -- & -- & 0.01 & 0.68 & 1.20 & 0.015 & 0.006 & 65 & 250 \\
\hline $13-17$ & 1120 & 1020 & 22 & 1.1 & -- & -- & 0.01 & 0.36 & 1.20 & 0.015 & 0.002 & 74 & 250 \\
\hline $17-20$ & 1210 & 2310 & 18 & 8.5 & -- & -- & 0.02 & 0.33 & 1.20 & 0.010 & 0.003 & 72 & 260 \\
\hline $21-21$ & 0010 & 1110 & 24 & 4.5 & -- & -- & 0.02 & 0.40 & 1.20 & 0.030 & 0.007 & 75 & 250 \\
\hline $21-24$ & 1240 & 1140 & 47 & -- & -- & -. & 0.01 & 1.3 & 1.00 & 0.135 & 0.006 & 69 & 150 \\
\hline \multicolumn{14}{|l|}{ MAR } \\
\hline $02-04$ & 1315 & 0815 & 23 & 2.0 & -- & -- & 0.02 & 0.54 & 1.30 & 0.020 & 0.004 & 65 & 240 \\
\hline $04-06$ & 0915 & 1215 & 76 & 45 & 110 & 22 & 0.03 & 1.7 & 1.10 & 0.175 & 0.013 & 99 & 130 \\
\hline $13-16$ & 1240 & 1140 & 33 & 3.5 & -- & -- & $<0.01$ & 0.61 & 0.92 & 0.040 & 0.003 & 60 & 190 \\
\hline $16-18$ & 1215 & 0315 & 33 & 2.6 & -- & -. & 0.01 & 0.44 & 0.87 & 0.030 & 0.003 & 55 & 160 \\
\hline $18-20$ & 0415 & 1115 & 56 & 35 & 100 & 12 & 0.01 & 1.2 & 1.00 & 0.100 & 0.005 & 68 & 130 \\
\hline $23-24$ & 1230 & 1530 & 34 & 2.4 & -- & -- & 0.02 & 0.60 & 1.00 & 0.035 & 0.004 & 70 & 190 \\
\hline $24-27$ & 1630 & 1130 & 52 & 6.5 & -- & -- & $<0.01$ & 0.88 & 1.20 & 0.070 & 0.004 & 61 & 150 \\
\hline $27-30$ & 1200 & 0500 & 46 & 2.1 & - & -- & 0.01 & 0.57 & 0.70 & 0.030 & 0.002 & 58 & 160 \\
\hline $30-30$ & 0600 & 1100 & 51 & 45 & -- & - & 0.03 & 1.5 & 0.66 & 0.185 & 0.006 & 57 & 140 \\
\hline \multicolumn{14}{|l|}{ MAR 30- } \\
\hline APR 02 & 1245 & 1545 & 131 & 40 & 122 & 25 & 0.03 & 1.7 & 0.93 & 0.170 & 0.007 & 63 & 110 \\
\hline $02-03$ & 1645 & 1145 & 177 & 75 & 240 & 24 & 0.02 & 1.8 & 1.10 & 0.330 & 0.007 & 57 & 87 \\
\hline $03-06$ & 1215 & 1115 & 157 & 50 & 115 & 14 & 0.02 & 1.1 & 1.20 & 0.180 & 0.007 & 49 & 84 \\
\hline $06-10$ & 1210 & 1110 & 52 & 2.8 & -- & -- & 0.02 & 0.34 & 0.90 & 0.025 & 0.005 & 57 & 140 \\
\hline $10-13$ & 1200 & 1100 & 38 & 2.2 & -- & $\cdots$ & 0.03 & 0.42 & 0.51 & 0.025 & 0.004 & 60 & 170 \\
\hline $17 \ldots$ & 1145 & -. & 35 & 1.8 & -- & -- & 0.01 & 0.38 & 0.64 & 0.020 & 0.004 & 62 & 210 \\
\hline $27-27$ & 0015 & 1115 & 29 & 3.7 & -- & - & 0.03 & 0.34 & 0.99 & 0.040 & 0.003 & 59 & 220 \\
\hline $27-28$ & 1620 & 0720 & 29 & 4.6 & -- & -- & 0.01 & 0.40 & 1.00 & 0.065 & 0.003 & 60 & 230 \\
\hline \multicolumn{14}{|l|}{ APR $28-$} \\
\hline MAY 01 & 0820 & 112 & 29 & 2.9 & -- & -- & 0.01 & 0.11 & 1.10 & 0.025 & 0.003 & 61 & 250 \\
\hline $01-01$ & 1310 & 2110 & 34 & 2.5 & -- & -- & 0.02 & 0.32 & 0.93 & 0.040 & 0.005 & 59 & 170 \\
\hline $01-04$ & 2210 & 1210 & 83 & 24 & -- & -- & 0.02 & 1.2 & 0.68 & 0.115 & 0.006 & 54 & 130 \\
\hline $04-07$ & 1238 & 0338 & 38 & 3.9 & -- & -- & 0.05 & 0.48 & 0.73 & 0.030 & 0.006 & 56 & 170 \\
\hline $07-08$ & 0438 & 1138 & 173 & 60 & 224 & 26 & 0.03 & 1.1 & 0.69 & 0.225 & 0.009 & 53 & 110 \\
\hline $08-10$ & 1200 & 1700 & 186 & 28 & -- & -- & $<0.01$ & -- & 0.97 & 0.145 & 0.009 & 35 & 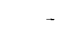 \\
\hline $08-11$ & 1200 & 1100 & 173 & -- & -. & $\ldots$ & - & 1.1 & $\ldots$ & $\ldots$ & -. & - - & - \\
\hline $10-11$ & 1800 & 1100 & 119 & 22 & - & -- & 0.02 & -- & 0.75 & 0.100 & 0.021 & 49 & 20 \\
\hline $11-14$ & 1225 & 1125 & 185 & 26 & -- & -- & 0.04 & -- & 0.86 & 0.155 & 0.010 & 38 & 57 \\
\hline $11-15$ & 1225 & 1125 & 170 & -. & - - & - & -- & 1.0 & - & -- & -- & -. & - \\
\hline
\end{tabular}


Surface-Water Stations

A. Discharge and water quality

04232040 Irondequoit Creek Near Pittsford, N.Y.

2. WATER-QUALITY RECORDS

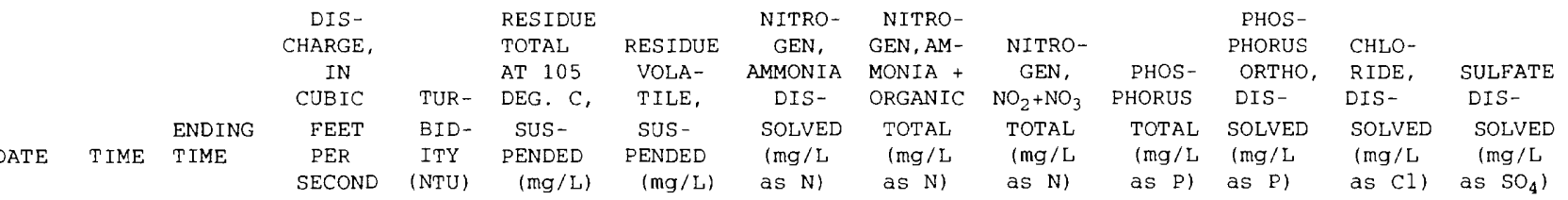

$\begin{array}{cccr}\text { MAY - cont inued } & & \\ 14-15 & 1225 & 1125 & 111 \\ 15-16 & 1225 & 0225 & 93 \\ 16-18 & 0325 & 1125 & 88 \\ 25 \ldots & 1130 & -- & 39 \\ 26 \ldots & 1000 & -- & 38 \\ 30 \ldots & 1200 & -- & 32 \\ 30-31 & 1230 & 2330 & 38 \\ \text { JUN } & & & \\ 01-02 & 0030 & 1130 & 50 \\ 02-05 & 1220 & 1120 & 34 \\ 08-09 & 1215 & 1115 & 21 \\ 09-12 & 2015 & 1115 & 52 \\ 12-13 & 1245 & 0545 & 29 \\ 13-15 & 0645 & 1145 & 38 \\ 19 . . & 1200 & -- & 35 \\ 19-20 & 1215 & 0815 & 35 \\ 20-22 & 0915 & 1115 & 154 \\ 22-26 & 1155 & 1055 & 37 \\ 26-28 & 1215 & 0215 & 27 \\ 28-29 & 0315 & 1115 & 52 \\ \text { JUL } & & & \\ 10-13 & 1230 & 113 & 17 \\ 17-20 & 1210 & 1110 & 17 \\ 20-22 & 1210 & 1910 & 18 \\ 22-24 & 2010 & 1110 & 35 \\ \text { AUG } & & & \\ 03-04 & 0155 & 0455 & 14 \\ 04-07 & 0555 & 0055 & 22 \\ 14-17 & 1230 & 1130 & 13 \\ 17-19 & 1210 & 1510 & 13 \\ 19-21 & 1610 & 1110 & 20 \\ \text { SEP } & & & \\ 14-17 & 1200 & 0700 & 24 \\ 14-18 & 1200 & 1100 & 27 \\ 17-18 & 0800 & 1100 & 36 \\ 21-22 & 1215 & 2315 & 15 \\ 22-25 & 0015 & 0915 & 17\end{array}$

WATER YEAR OCTOBER 1988 TO SEPTEMBER 1989-continued

OCT

$\begin{array}{rrrr}13-14 & 1300 & 1500 & 1 \\ 14-16 & 1600 & 1200 & 2 \\ 16-17 & 1240 & 2340 & 2 \\ 18-19 & 0040 & 1140 & 3 \\ \text { NOV } & & & \\ 06-07 & 1130 & 1630 & 2 \\ 07-09 & 1730 & 1030 & \\ 16-16 & 0245 & 1045 & 2 \\ \text { DEC } & & & \\ 11-14 & 1210 & 1110 & 1 \\ 29-31 & 1210 & 0710 & 20 \\ \text { DEC } 31- & & & \\ \text { JAN } 02 & 0810 & 1110 & 4 \\ 05 \ldots & 1200 & -\cdots & 10 \\ 16-17 & 1110 & 1310 & 42 \\ 19 \ldots & 1130 & -- & 6 \\ \text { FEB } & & & \\ 08-09 & 1105 & 0605 & 90 \\ 09-12 & 0705 & 1005 & 16 \\ 15-18 & 1230 & 0930 & 138 \\ 18-20 & 1030 & 1130 & 7 \\ 20-22 & 1245 & 1745 & 5 \\ 22-23 & 1845 & 1145 & 207 \\ & & & \end{array}$

\begin{tabular}{|c|c|c|c|c|c|}
\hline 50 & 136 & 18 & 0.03 & -- & 0.7 \\
\hline-- & -- & -- & 0.02 & 0.92 & 0 . \\
\hline 8.0 & -- & - & 0.03 & 0.95 & 0.6 \\
\hline 3.2 & - & -- & 0.03 & 0.64 & 1. \\
\hline 4.2 & -- & -- & 0.05 & 0.57 & 1. \\
\hline 4.4 & -- & -- & 0.04 & 0.52 & 1. \\
\hline 2.6 & -- & -- & 0.03 & 0.57 & 1. \\
\hline 5.8 & - & - & 0.04 & 1.1 & 0 \\
\hline 4.3 & -- & -- & 0.06 & 0.87 & \\
\hline 1.5 & -- & -- & 0.04 & 0.38 & 1. \\
\hline 30 & - & -- & 0.05 & 1.2 & \\
\hline 2.5 & - & -- & 0.04 & 0.68 & \\
\hline 5.2 & -- & -- & 0.04 & 0.86 & \\
\hline 5.2 & -- & $\cdots$ & 0.05 & 0.95 & \\
\hline 15 & -- & -- & 0.03 & 0.84 & 1. \\
\hline 100 & 256 & 62 & 0.03 & 1.9 & \\
\hline 12 & -- & -- & 0.03 & 0.66 & \\
\hline 8.4 & -- & -- & 0.02 & 0.49 & \\
\hline 32 & 96 & 20 & 0.05 & 1.3 & 1. \\
\hline 19 & -- & -- & 0.02 & 0.65 & 1. \\
\hline 2.5 & -- & -- & 0.02 & 0.38 & \\
\hline 2.5 & - & -- & 0.03 & 0.31 & 1. \\
\hline 3.9 & -- & -- & 0.03 & 0.41 & 1. \\
\hline 3.3 & -- & - & -- & 0.41 & 1. \\
\hline 19 & -- & -- & -- & 0.70 & 1. \\
\hline 2.4 & -- & -- & 0.02 & 0.39 & \\
\hline 1.8 & -- & -- & $<0.01$ & 0.61 & 1. \\
\hline 3.6 & -- & -- & 0.01 & 0.40 & \\
\hline 5.6 & -- & -- & -- & -- & - \\
\hline-- & -- & -- & 0.03 & 0.49 & 0. \\
\hline 39 & 90 & 18 & -- & -- & \\
\hline 2.9 & $\ldots$ & -. & -- & 0.23 & - \\
\hline 3.1 & -- & -- & -- & 0.29 & \\
\hline
\end{tabular}

\begin{tabular}{|c|c|c|c|}
\hline 0.185 & 0.009 & 46 & 100 \\
\hline 0.105 & 0.008 & 45 & 78 \\
\hline 0.125 & 0.007 & 44 & 87 \\
\hline 0.045 & 0.008 & 54 & 160 \\
\hline 0.055 & 0.009 & 55 & 180 \\
\hline 0.065 & 0.012 & 60 & 210 \\
\hline 0.070 & 0.009 & 59 & 390 \\
\hline 0.130 & 0.011 & 55 & 150 \\
\hline 0.080 & 0.013 & 58 & 170 \\
\hline 0.040 & 0.003 & 61 & 220 \\
\hline 0.240 & 0.015 & 50 & 130 \\
\hline 0.065 & 0.016 & 54 & 160 \\
\hline 0.100 & 0.013 & 56 & 170 \\
\hline 0.100 & 0.024 & 52 & 140 \\
\hline 0.120 & 0.023 & 53 & 150 \\
\hline 0.420 & 0.025 & 40 & 79 \\
\hline 0.095 & 0.021 & 53 & 160 \\
\hline 0.065 & 0.016 & 60 & 200 \\
\hline 0.170 & 0.020 & 49 & 140 \\
\hline 0.100 & 0.013 & 64 & 260 \\
\hline 0.060 & 0.005 & 65 & 300 \\
\hline 0.035 & 0.008 & 64 & 270 \\
\hline 0.065 & 0.008 & 60 & 270 \\
\hline 0.045 & 0.016 & 64 & 220 \\
\hline 0.075 & 0.012 & 63 & 210 \\
\hline 0.055 & 0.005 & 65 & 250 \\
\hline 0.030 & 0.005 & 65 & 29 \\
\hline 0.050 & 0.007 & 59 & 25 \\
\hline 0.075 & 0.014 & 55 & 24 \\
\hline-- & -- & -- & \\
\hline 0.175 & 0.018 & 59 & 21 \\
\hline 0.035 & 0.009 & 63 & 26 \\
\hline 0.045 & 0.010 & 63 & \\
\hline
\end{tabular}

WATER YEAR OCTOBER 1989 TO SEPTEMBER 1990

\begin{tabular}{|c|c|c|c|c|c|c|c|c|c|}
\hline 0.95 & -- & -- & -- & 0.26 & -- & 0.055 & 0.003 & 65 & 300 \\
\hline 3.2 & -- & -- & -- & 0.40 & -- & 0.065 & 0.004 & 60 & 300 \\
\hline 4.2 & -- & -- & -- & -- & -- & 0.050 & 0.007 & 61 & 260 \\
\hline 3.6 & -- & -- & -- & -- & -- & 0.050 & 0.007 & 61 & 250 \\
\hline 2.4 & -- & -- & -- & -- & -- & 0.030 & 0.005 & 63 & 260 \\
\hline 2.4 & - & -- & -- & -- & -- & 0.025 & 0.004 & 61 & 260 \\
\hline 2.4 & -- & -- & -- & -- & -- & 0.035 & 0.006 & 59 & 220 \\
\hline 0.80 & - & -- & -- & -- & 1.20 & 0.025 & 0.003 & 63 & 250 \\
\hline 0.70 & -- & -- & 0.03 & 0.23 & 1.40 & 0.020 & 0.004 & 76 & 260 \\
\hline 2.1 & -- & -- & 0.05 & 0.13 & 1.30 & 0.035 & 0.004 & 120 & 250 \\
\hline 36 & 192 & 35 & -- & -- & -- & 0.250 & 0.015 & 76 & 120 \\
\hline 34 & -- & $\ldots$ & 0.03 & 0.85 & 1.30 & 0.100 & 0.008 & 110 & 200 \\
\hline 35 & 72 & 11 & 0.04 & 1.1 & 1.50 & 0.080 & 0.015 & 53 & 120 \\
\hline 40 & 129 & 22 & 0.01 & 1.2 & 1.20 & 0.135 & 0.009 & 76 & 100 \\
\hline 50 & 176 & 26 & 0.02 & 1.2 & 1.20 & 0.185 & 0.012 & 50 & 92 \\
\hline 32 & 89 & $<5$ & 0.04 & 0.86 & 1.00 & 0.110 & 0.008 & 65 & 96 \\
\hline 17 & -- & -- & 0.04 & 0.60 & 1.10 & 0.065 & 0.007 & 58 & 100 \\
\hline 20 & -- & -- & 0.02 & 0.65 & 1.00 & 0.070 & 0.007 & 63 & 120 \\
\hline 150 & 352 & 48 & 0.04 & 1.6 & 0.87 & 0.325 & 0.015 & 51 & 72 \\
\hline
\end{tabular}


Surface-Water Stations

A. Discharge and water quality

04232040 Irondequoit Creek Near Pittsford, N.Y.

2. WATER-QUALITY RECORDS

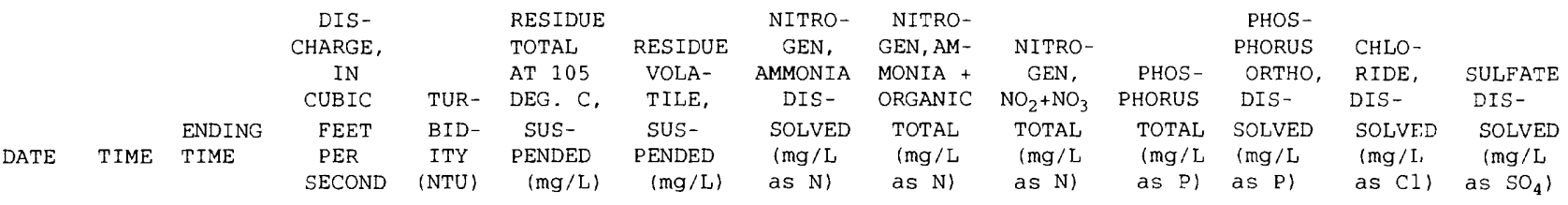

MAR

\begin{tabular}{|c|c|c|c|c|c|c|c|c|c|c|c|c|c|}
\hline $08-10$ & 1220 & 1120 & 36 & 4.1 & -- & -- & $<0.01$ & -- & 1.50 & 0.035 & 0.006 & 70 & 200 \\
\hline $10-12$ & 1220 & 1120 & 81 & 24 & -- & -- & $<0.01$ & -- & 1.10 & 0.070 & 0.006 & 60 & 150 \\
\hline $19-22$ & 1200 & 1100 & 65 & 13 & -- & -- & 0.02 & -- & 0.90 & 0.055 & 0.003 & 60 & 130 \\
\hline \multicolumn{14}{|l|}{ APR } \\
\hline $02-05$ & 1600 & 100 & 132 & 65 & 161 & 23 & 0.01 & 0.96 & 0.70 & 0.120 & 0.006 & 59 & 99 \\
\hline $05-08$ & 1055 & 1825 & 226 & 80 & 176 & 22 & 0.01 & 0.80 & 0.71 & 0.260 & 0.009 & 50 & 71 \\
\hline 09-09 & 1135 & 1335 & 90 & 400 & 1030 & 113 & $<0.01$ & 3.8 & 0.60 & 0.990 & 0.012 & 41 & 55 \\
\hline $19-20$ & 1430 & 2130 & 48 & 5.8 & -- & -- & 0.04 & 0.57 & 1.20 & 0.035 & 0.004 & 61 & 150 \\
\hline $20-23$ & 2230 & 1030 & 81 & 16 & -- & -- & 0.03 & 0.69 & 1.00 & 0.060 & 0.004 & 59 & 130 \\
\hline $26-30$ & 1230 & 1130 & 43 & 4.9 & -- & -- & 0.05 & 0.52 & 0.89 & 0.045 & 0.005 & 59 & 150 \\
\hline \multicolumn{14}{|l|}{ MAY } \\
\hline $03-04$ & 1050 & 1750 & 29 & 4.5 & - & - & 0.05 & 0.39 & 1.30 & 0.030 & 0.003 & 67 & 200 \\
\hline $04-07$ & 1850 & 0950 & 75 & 24 & -- & - & 0.03 & 1.1 & 0.48 & 0.090 & 0.004 & 59 & 140 \\
\hline $10-13$ & 1140 & 0640 & 47 & 5.5 & -- & -- & 0.04 & 0.39 & 1.00 & 0.040 & 0.004 & 61 & 160 \\
\hline $13-14$ & 0740 & 1040 & 235 & 200 & 464 & 59 & 0.05 & 1.6 & 0.91 & 0.480 & 0.012 & 42 & 82 \\
\hline \multicolumn{14}{|l|}{ JUN } \\
\hline $04-07$ & 1210 & 1110 & 40 & 1.3 & -- & -- & 0.01 & 0.82 & 1.20 & 0.025 & 0.006 & 60 & 180 \\
\hline $25 \ldots$ & 1230 & -- & 25 & 5.7 & -- & -- & 0.05 & 0.42 & 1.40 & 0.055 & 0.014 & 64 & 240 \\
\hline \multicolumn{14}{|l|}{ JUL } \\
\hline $02-04$ & 1145 & 1945 & 20 & 6.8 & -- & -- & 0.03 & 0.50 & 1.30 & 0.055 & 0.013 & 61 & 220 \\
\hline $04-05$ & 2045 & 1045 & 22 & 15 & -- & -- & 0.05 & 0.60 & 1.40 & 0.070 & 0.014 & 60 & 220 \\
\hline $05-07$ & 1230 & 1530 & 22 & 18 & -- & -- & 0.02 & -- & 1.30 & 0.080 & 0.012 & 64 & 240 \\
\hline $09 \ldots$ & 1130 & -- & 26 & 10 & -- & -- & 0.02 & -- & 1.30 & 0.055 & 0.011 & 64 & 230 \\
\hline \multicolumn{14}{|l|}{ AUG } \\
\hline $02-05$ & 1045 & 0545 & 11 & 21 & -- & -- & 0.03 & 0.42 & 1.20 & 0.065 & 0.013 & 59 & 240 \\
\hline $06 \ldots$ & 1300 & -- & 42 & 210 & 388 & 30 & 0.03 & 1.2 & 0.73 & 0.380 & 0.016 & 56 & 240 \\
\hline $06-09$ & 1300 & 1200 & 21 & 40 & 91 & 11 & 0.04 & 0.86 & 1.00 & 0.130 & 0.015 & 61 & 210 \\
\hline \multicolumn{14}{|l|}{ SEP } \\
\hline $07-10$ & 1325 & 1225 & 19 & 26 & -- & -- & 0.04 & 0.77 & 1.30 & 0.110 & 0.011 & 63 & 240 \\
\hline \multicolumn{14}{|c|}{ PERIOD OCTOBER 1990 TO APAIL 1991} \\
\hline \multicolumn{14}{|c|}{ 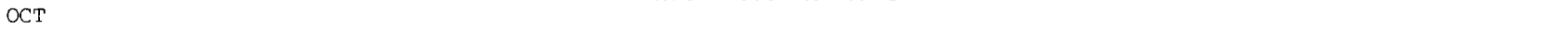 } \\
\hline $01-04$ & 1320 & 1220 & 18 & 13 & -- & -- & $<0.01$ & 0.57 & 0.95 & 0.045 & 0.010 & 56 & 260 \\
\hline 09-11 & 1400 & 1300 & 50 & 31 & 82 & 14 & 0.03 & 0.64 & 0.73 & 0.120 & 0.025 & 59 & 190 \\
\hline $11-13$ & 1315 & 0815 & 69 & 50 & 127 & 5 & 0.03 & 1.2 & 0.61 & 0.160 & 0.015 & 53 & 130 \\
\hline $13-15$ & 0915 & 1215 & 65 & 32 & 93 & 16 & 0.03 & 1.1 & 0.89 & 0.140 & 0.016 & 53 & 120 \\
\hline $18-22$ & 1210 & 1110 & 40 & 21 & -- & - & $<0.01$ & 0.62 & 0.80 & 0.070 & 0.013 & 59 & 140 \\
\hline $22-23$ & 1250 & 1150 & 41 & 7.3 & -- & -- & $<0.01$ & 0.79 & 0.85 & 0.045 & 0.012 & 63 & 190 \\
\hline $23-25$ & 1250 & 1150 & 63 & 27 & -- & - & $<0.01$ & 0.76 & 0.88 & 0.120 & 0.014 & 57 & 170 \\
\hline \multicolumn{14}{|l|}{ NOV } \\
\hline $09-09$ & 1045 & 2145 & 31 & 2.6 & -- & -- & 0.06 & 0.66 & 1.00 & 0.045 & 0.004 & 55 & 160 \\
\hline $09-13$ & 2245 & 0945 & 67 & 13 & -- & -- & 0.01 & 0.86 & 0.89 & 0.095 & 0.009 & 59 & 120 \\
\hline \multicolumn{14}{|l|}{$\mathrm{DEC}$} \\
\hline $29-31$ & 1400 & 1900 & 320 & 140 & -- & -- & 0.03 & 1.7 & 0.88 & 0.430 & 0.016 & 68 & 88 \\
\hline \multicolumn{14}{|l|}{ DEC $30-$} \\
\hline JAN 02 & 2000 & 1200 & 148 & 80 & 168 & 18 & 0.03 & 0.99 & 0.84 & 0.200 & 0.019 & 32 & 60 \\
\hline 02-04 & 1305 & 1205 & 63 & 14 & -- & - & 0.04 & 0.46 & 1.10 & 0.060 & 0.009 & 54 & 130 \\
\hline $24-27$ & 1600 & 0900 & 32 & 7.7 & -- & -- & 0.01 & 0.36 & 1.40 & 0.035 & 0.008 & 69 & 140 \\
\hline \multicolumn{14}{|l|}{ FEB } \\
\hline $19-20$ & 1335 & 0835 & 114 & 70 & 262 & 32 & -- & -- & -- & 0.250 & 0.006 & 69 & 120 \\
\hline $20-21$ & 0935 & 1235 & 122 & 45 & 137 & 20 & -- & -- & -- & 0.160 & 0.007 & 46 & 90 \\
\hline \multicolumn{14}{|l|}{ MAR } \\
\hline $02-04$ & 2055 & 1155 & 229 & 220 & 512 & 50 & 0.01 & 1.7 & 0.80 & 0.450 & 0.010 & 56 & 80 \\
\hline $06 \ldots$ & 1340 & -- & 241 & 33 & -- & - & 0.03 & 0.64 & 0.74 & 0.100 & 0.014 & 35 & 55 \\
\hline $06-07$ & 1730 & 1330 & 186 & 50 & 112 & 14 & 0.02 & 0.77 & 0.73 & 0.130 & 0.006 & 43 & 70 \\
\hline $07-10$ & 1330 & 2230 & 75 & 9.9 & -- & -- & -- & -- & -- & 0.030 & 0.005 & 56 & 110 \\
\hline APR & & & & & & & & & & & & & \\
\hline $20-22$ & 0515 & 1115 & 222 & 85 & 196 & 22 & 0.02 & 1.3 & 0.71 & 0.190 & 0.009 & 41 & 88 \\
\hline $22-22$ & 1210 & 2210 & 558 & 120 & 182 & 21 & 0.02 & 1.0 & 0.57 & 0.320 & 0.020 & 21 & 34 \\
\hline
\end{tabular}




\section{Surface-Water Stations}

\section{A. Discharge and water quality}

\section{Thomas Creek At Fairport, N.Y.}

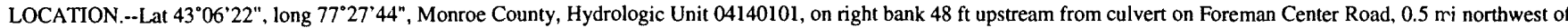
Fairport, and 0.8 mi upstream from mouth.

DRAINAGE AREA.--28.5 $\mathrm{mi}^{2}$, flow from $0.86 \mathrm{mi}^{2}$ noncontributing.

1. WATER-DISCHARGE RECORDS

PERIOD OF RECORD.-March 1980 to February 1990 (discontinued).

REVISED RECORDS.--WDR NY-81-3: Drainage area.

GAGE.--Water-stage recorder. Elevation of gage is $403 \mathrm{ft}$ above National Geodetic Vertical Datum of 1929, from topographic map.

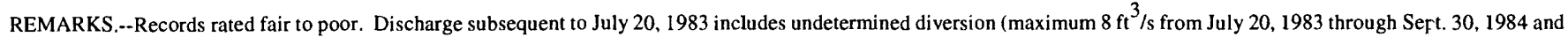

$25 \mathrm{ft}^{3} / \mathrm{s}$ thereafter) from Erie (Barge) Canal upstream from station. Several measurements of water temperature were made during each year.

COOPERATION.--Streamflow measurements were obtained and recorder equipment maintained by Monroe County Environmental Health Laboratory, Rochester, N Y.

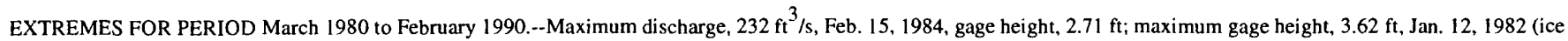
jam); minimum discharge, $1.2 \mathrm{ft}^{3} / \mathrm{s}$, Sept. 12, 1989; minimum gage height, $1.22 \mathrm{ft}$, June 7, 8, 13, 1981.

STATISTICS OF MONTHLY MEAN DISCHARGE (in cubic feet per second) FOR WATER YEARS 1980-89, BY WATER YEAR

\begin{tabular}{|c|c|c|c|c|c|c|c|c|c|c|c|c|}
\hline & OCT & NOV & DEC & JAN & FEB & MAR & APR & MAY & JUN & JUL & AUG & SEP \\
\hline MEAN & 10.6 & 18.1 & 20.4 & 12.5 & 23.4 & 28.3 & 26.6 & 21.6 & 13.3 & 6.23 & 7.18 & 6.49 \\
\hline MAX & 25.3 & 31.8 & 44.8 & 25.2 & 54.1 & 43.0 & 41.8 & 51.6 & 30.1 & 12.5 & 12.5 & 9.44 \\
\hline (WY) & 1987 & 1982 & 1984 & 1986 & 1981 & 1982 & 1987 & 1984 & 1989 & 1986 & 1986 & 1984 \\
\hline MIN & 3.59 & 7.19 & 3.86 & 6.32 & 6.97 & 12.8 & 8.04 & 9.85 & 5.07 & 3.86 & 3.74 & 4.11 \\
\hline (WY) & 1983 & 1989 & 1989 & 1989 & 1989 & 1988 & 1981 & 1986 & 1988 & 1989 & 1985 & 1989 \\
\hline
\end{tabular}

STATISTIC

ANNUAL TOTAL

ANNUAL MEAN

AVERAGE DISCHARGE

HIGHEST ANNUAL MEAN

LOWEST ANNUAL MEAN

HIGHEST DAILY MEAN

LOWEST DAILY MEAN

ANNUAL SEVEN-DAY MINIMUM

INSTANTANEOUS PEAK FLOW

INSTANTANEOUS LOW FLOW

ANNUAL RUNOFF (CFSM)

ANNUAL RUNOFF (INCHES)

10 PERCENT EXCEEDS

50 PERCENT EXCEEDS

90 PERCENT EXCEEDS

\section{SUMMARY STATISTICS}

FOR 1988 CALENDAR YEAR
3476.9

9.50
FOR 1989 WATER YEAR

5435.7
WATER YEARS $1980-1990$

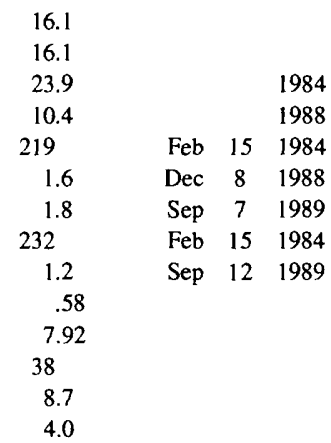

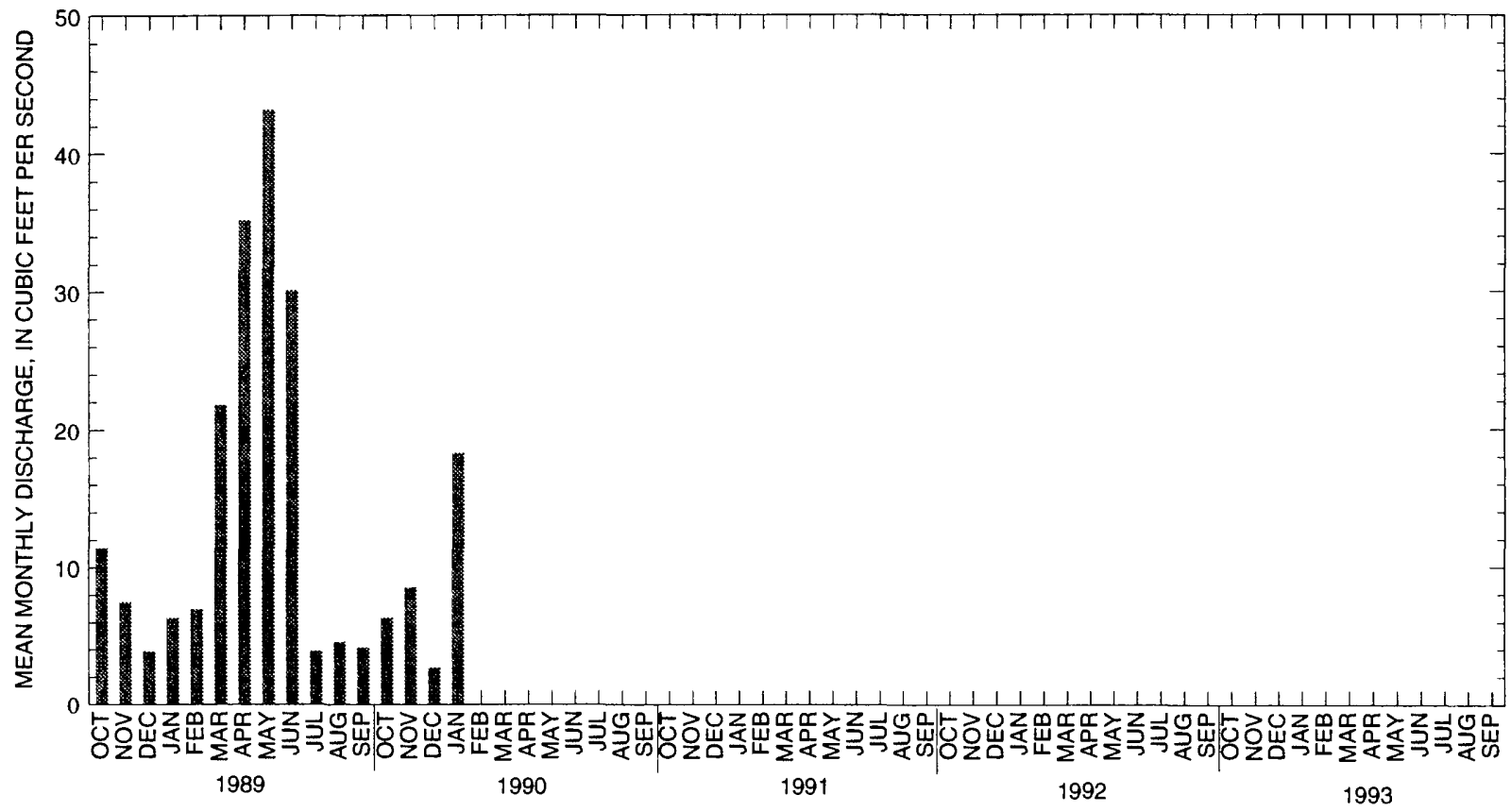




\section{Surface-Water Stations}

\section{A. Discharge and water quality}

04232046 Thomas Creek At Fairport, N.Y.

\section{WATER-QUALITY RECORDS}

PERIOD OF RECORD.--October 1983 to September 1989, discont inued.

CHEMICAL DATA: 1983-89 (e)

NUTRIENT DATA: $1983-89$ (e)

COOPERATION. - Water-quality samples were collected and analyzed by the Monroe County Environmental Health Laboratory at Rochester, N.Y.

REMARKS.--Prior to 1994 water year, data published in WATER RESOURCES OF MONROE COUNTY NEW YORK, WATER YEARS 1984-88, U. S. Geological Survey open-file report 93-370.

\begin{tabular}{|c|c|c|c|c|c|c|c|c|c|c|c|}
\hline & & & $\begin{array}{c}\text { DIS- } \\
\text { CHARGE, } \\
\text { IN } \\
\text { CUBIC }\end{array}$ & TUR- & $\begin{array}{l}\text { NITRO- } \\
\text { GEN, } \\
\text { AMMONIA } \\
\text { DIS- }\end{array}$ & $\begin{array}{l}\text { NITRO- } \\
\text { GEN, AM- } \\
\text { MONIA + } \\
\text { ORGANIC }\end{array}$ & $\begin{array}{c}\mathrm{NITRO}- \\
\mathrm{GEN} \\
\mathrm{NO}_{2}+\mathrm{NO}_{3}\end{array}$ & $\begin{array}{l}\text { PHOS- } \\
\text { PHORUS }\end{array}$ & $\begin{array}{l}\text { PHOS- } \\
\text { PHORUS } \\
\text { ORTHO, } \\
\text { DIS- }\end{array}$ & $\begin{array}{l}\text { CHLO- } \\
\text { RIDE, } \\
\text { DIS- }\end{array}$ & $\begin{array}{l}\text { SULFATE } \\
\text { DIS- }\end{array}$ \\
\hline DATE & TIME & $\begin{array}{l}\text { ENDING } \\
\text { TIME }\end{array}$ & $\begin{array}{c}\text { FEET } \\
\text { PER } \\
\text { SECOND }\end{array}$ & $\begin{array}{l}\text { BID- } \\
\text { ITY } \\
\text { (NTU) }\end{array}$ & $\begin{array}{l}\text { SOLVED } \\
\text { (mg/L } \\
\text { as } N \text { ) }\end{array}$ & $\begin{array}{l}\text { TOTAL } \\
\text { (mg/L } \\
\text { as N) }\end{array}$ & $\begin{array}{c}\text { TOTAL } \\
(\mathrm{mg} / \mathrm{L} \\
\text { as N) }\end{array}$ & $\begin{array}{c}\text { TOTAL } \\
\text { (mg/L } \\
\text { as P) }\end{array}$ & $\begin{array}{l}\text { SOLVED } \\
\text { (mg/L } \\
\text { as P) }\end{array}$ & $\begin{array}{l}\text { SOLVED } \\
\text { (mg/L } \\
\text { as Cl) }\end{array}$ & $\begin{array}{c}\text { SOLVED } \\
(\mathrm{mg} / \mathrm{L} \\
\left.\text { as } \mathrm{SO}_{4}\right)\end{array}$ \\
\hline
\end{tabular}

OCT

\section{$03-04 \quad 1230$}

$04-06 \quad 2030$

06-07 0830

$07-11 \quad 1230$

$14 \ldots \quad 1300$

$\begin{array}{ll}14-18 & 1305 \\ 18-18 & 0505\end{array}$

18-21 1045

$21-21 \quad 1235$

$\begin{array}{ll}22-24 & 0035 \\ 24-27 & 1235\end{array}$

27-28 1205

28-31 $\quad 1205$

OCT $31-$

NOV 02

$02-03$

$03-05$

$05-07$

07-10

$17-18$

DEC

15-19

23-25

$25-27$

$27-28$

28-30

JAN

05-07 1125

07-09 1925

$09-12$

$12-13$

$20-23$
$26-30$

FEB

$13-17$

$17-20$

20-21

21-22

24 .

MAR

$03 \quad 04$

04-06

$09 .$.

$13 \ldots$

$16-18$

$18-20$

$23-24$

$24-27$

$27-30$

$30-30$

MAR 30-

APR 02

$02-03$
$03-06$

06-10

2035

1115

1030

1130

1200

1130

1630

1230

1115

1115

0315

1145
1545

1120

0520

1200

1600

1115

$1245 \quad 0545$

$1320 \quad 1620$

$1720 \quad 1220$

$1310 \quad 1210$

$1235 \quad 1935$

$1210 \quad 1110$

$1130 \quad 1830$

$1930 \quad 1030$

$1230 \quad 0230$

1130

1825

1025
1040

$\begin{array}{ll}1140 & 1040 \\ 1100 & 1040\end{array}$

$1140 \quad 1040$

1015

0930

2230

1030

1200

1130

1130

0215

1015

1445
1045

0420

1020

1500

1100

1015
1030

\section{WATER YEAR OCTOBER 1988 TO SEPTEMBER 1989}

$\begin{array}{rrll}2.7 & <0.01 & 0.53 & 0.10 \\ 4.5 & 0.01 & 0.65 & 0.10 \\ 6.6 & <0.01 & 0.67 & 0.11 \\ 3.5 & <0.01 & 1.1 & 0.15 \\ 2.4 & <0.01 & 0.61 & 0.28 \\ 3.0 & <0.01 & 0.53 & 0.17 \\ 7.7 & <0.01 & 0.76 & 0.17 \\ 3.5 & <0.01 & 3.3 & 0.13 \\ -- & 0.01 & 0.72 & 0.10 \\ 13 & <0.01 & 0.90 & 0.18 \\ -- & 0.01 & 0.93 & 0.14 \\ 2.2 & 0.02 & 0.80 & 0.21 \\ 2.8 & 0.01 & 0.76 & 0.21\end{array}$

0.080

$0.023 \quad 100$

0.080

0.075

0.060

0.050

0.060

0.080
0.070

0.060

0.085

0.060

0.030

0.023

0.02183

0.01892

0.020100

$0.016 \quad 110$

0.019100

0.01599

0.01590

0.02470

$0.020 \quad 85$

0.017100

0.016100

$0.016 \quad 120$

$0.84 \quad 0.27$

$0.82 \quad 0.35$

$0.71 \quad 0.29$

$0.80 \quad 0.18$

$0.74 \quad 0.19$

$0.70 \quad 0.25$

0.080

0.065

0.045

$0.015 \quad 110$

0.012110

0.01395

$0.045 \quad 0.012 \quad 110$

$0.040 \quad 0.012 \quad 120$

$0.012 \quad 110$

$0.007 \quad 320$

$0.71 \quad 1.22$

0.030

0.045

0.050

$0.008 \quad 190$

0.621 .08

$\begin{array}{ll}1.5 & 1.06\end{array}$

0.055

$0.009 \quad 180$

$0.014 \quad 180$

$0.007 \quad 230$

0.035

1. $3 \quad 1.44$

$\begin{array}{ll}1.0 & 1.37 \\ 0.90 & 1.59\end{array}$

$0.74 \quad 1.72$

0.055

0.045

0.035

0.045

$0.007 \quad 220$

$0.007 \quad 400$

$0.008 \quad 240$

0.002180

$0.005 \quad 180$

0.009170

$0.003 \quad 310$

0.025

$1.1 \quad 1.50$

$0.94 \quad 1.50$

0.020

0.055

0.100

$\begin{array}{ll}0.004 & 290 \\ 0.007 & 260\end{array}$

$0.007 \quad 260$

$0.027 \quad 180$

0.011290

0.045

0.004200

0.014300

0.009150

$0.007 \quad 110$

0.006120

$0.003 \quad 140$

$0.004 \quad 150$

0.020130

$0.003 \quad 120$

0.005100

230

240

180

160
200

200

190

180

180

110

110
130

170

180

210

200

190

150

170
190

150

180

140

140

160

130

180

140

130

140

150
130

150

150

140

100
75

140

87
120

90

90

110

110

110
99

$\begin{array}{rrr}75 & 7.0 & 0.05 \\ 98 & 6.6 & 0.03 \\ 108 & 8.0 & 0.03 \\ 45 & 1.7 & 0.03\end{array}$

$1.4 \quad 1.50$

0.060

$0.012 \quad 110$

$1.7 \quad 1.60$

0.110

0.090

$0.015 \quad 110$

$\begin{array}{ll}0.021 & 80 \\ 0.014 & 95\end{array}$

59
61
40 
Surface-Water Stations

A. Discharge and water quality

04232046 Thomas Creek At Fairport, N.Y.

2. WATER-QUALITY RECORDS

\begin{tabular}{|c|c|c|c|c|c|c|c|c|c|c|c|}
\hline & & & $\begin{array}{c}\text { DIS- } \\
\text { CHARGE, } \\
\text { IN } \\
\text { CUBIC }\end{array}$ & TUR- & $\begin{array}{l}\text { NITRO- } \\
\text { GEN, } \\
\text { AMMONIA } \\
\text { DIS- }\end{array}$ & $\begin{array}{l}\text { NITRO- } \\
\text { GEN, AM- } \\
\text { MONIA + } \\
\text { ORGANIC }\end{array}$ & $\begin{array}{c}\text { NITRO- } \\
\text { GEN, } \\
\mathrm{NO}_{2}+\mathrm{NO}_{3}\end{array}$ & $\begin{array}{l}\text { PHOS- } \\
\text { PHORUS }\end{array}$ & $\begin{array}{l}\text { PHOS- } \\
\text { PHORUS } \\
\text { ORTHO, } \\
\text { DIS- }\end{array}$ & $\begin{array}{l}\text { CHLO- } \\
\text { RIDE, } \\
\text { DIS- }\end{array}$ & $\begin{array}{l}\text { SULFATE } \\
\text { DIS- }\end{array}$ \\
\hline DATE & TIME & $\begin{array}{l}\text { ENDING } \\
\text { TIME }\end{array}$ & $\begin{array}{l}\text { FEET } \\
\text { PER } \\
\text { SECOND }\end{array}$ & $\begin{array}{l}\text { BID- } \\
\text { ITY } \\
\text { (NTU) }\end{array}$ & $\begin{array}{l}\text { SOLVED } \\
\text { (mg/L } \\
\text { as N) }\end{array}$ & $\begin{array}{l}\text { TOTAL } \\
\text { (mg/L } \\
\text { as N) }\end{array}$ & $\begin{array}{l}\text { TOTAL } \\
(\mathrm{mg} / \mathrm{L} \\
\text { as } \mathrm{N})\end{array}$ & $\begin{array}{l}\text { TOTAL } \\
(\mathrm{mg} / \mathrm{L} \\
\text { as P) }\end{array}$ & $\begin{array}{l}\text { SOLVED } \\
\text { (mg/L } \\
\text { as P) }\end{array}$ & $\begin{array}{l}\text { SOLVED } \\
\text { (mg/L } \\
\text { as (1) }\end{array}$ & $\begin{array}{c}\text { SOLVED } \\
\left(\mathrm{mg}^{2} \mathrm{~L}\right. \\
\left.\text { as } \mathrm{SO}_{4}\right)\end{array}$ \\
\hline
\end{tabular}

\begin{tabular}{|c|c|c|c|c|c|c|c|c|c|c|c|}
\hline \\
\hline \multicolumn{12}{|c|}{ WATER YEAR OCTOBER 1988 TO SEPTEMBER 1989-continued } \\
\hline $10-13$ & 1115 & 1015 & 26 & 1.5 & 0.02 & 0.80 & 0.99 & 0.035 & 0.009 & 110 & 90 \\
\hline $13-17$ & 1115 & 1015 & 22 & 1.3 & 0.01 & 1.0 & 1.80 & 0.030 & 0.005 & 110 & 96 \\
\hline $27 \ldots$ & 1115 & -- & 10 & 280 & 0.23 & 1.6 & 0.70 & 0.490 & 0.004 & 200 & 98 \\
\hline \multicolumn{12}{|l|}{ APR $27-$} \\
\hline MAY 01 & 1530 & 1130 & 13 & 3.0 & 0.02 & 0.86 & 0.77 & 0.050 & 0.004 & 110 & 110 \\
\hline 01-04 & 1220 & 1120 & 42 & 8.2 & 0.02 & 1.1 & 0.76 & 0.085 & 0.010 & 93 & 67 \\
\hline $04-07$ & 1145 & 0245 & 32 & 1.4 & 0.03 & 1.0 & 0.81 & 0.035 & 0.008 & 99 & 98 \\
\hline $07-08$ & 0345 & 1045 & 80 & 8.6 & 0.02 & 0.89 & 0.63 & 0.085 & 0.012 & 80 & 70 \\
\hline $08-09$ & 1.100 & 2200 & 107 & 3.3 & $<0.01$ & -- & 1.00 & 0.090 & 0.015 & 65 & $<10$ \\
\hline $09-11$ & 2300 & 1000 & 85 & 3.0 & $<0.01$ & -- & 1.20 & 0.065 & 0.012 & 5.4 & $<10$ \\
\hline $11-15$ & 1135 & 1035 & 76 & 4.1 & 0.04 & 1.1 & 1.20 & 0.060 & 0.014 & 72 & 77 \\
\hline $15-18$ & 1145 & 1045 & 46 & 2.1 & 0.05 & 1.5 & 0.88 & 0.070 & 0.009 & 91 & 66 \\
\hline $22-23$ & 1140 & 1940 & 19 & 4.0 & 0.06 & 1.3 & 0.52 & 0.095 & 0.017 & 100 & 110 \\
\hline $23-25$ & 2040 & 0740 & 19 & 5.5 & 0.05 & 1.2 & 0.58 & 0.105 & 0.015 & 100 & 100 \\
\hline $25-30$ & 1115 & 1015 & 18 & 4.7 & 0.06 & 1.5 & 0.56 & 0.095 & 0.020 & 97 & 100 \\
\hline $30-31$ & 1130 & 2230 & 21 & 7.0 & 0.07 & 1.2 & 0.56 & 0.140 & 0.025 & 98 & 89 \\
\hline \multicolumn{12}{|l|}{ MAY 31- } \\
\hline JUN 02 & 2330 & 1030 & 34 & 5.1 & 0.08 & 1.2 & 0.47 & 0.130 & 0.031 & 88 & 68 \\
\hline $12-13$ & 1200 & 0500 & 17 & 2.2 & 0.28 & 1.7 & 0.26 & 0.170 & 0.045 & 95 & 81 \\
\hline $13-15$ & 0600 & 1100 & 19 & 3.4 & 0.08 & 1.3 & 0.31 & 0.190 & 0.039 & 89 & 72 \\
\hline $15-16$ & 1145 & 1045 & 21 & 1.7 & 0.03 & 0.97 & 0.44 & 0.085 & 0.033 & 98 & 76 \\
\hline $16-19$ & 1145 & 1045 & 59 & 5.7 & 0.02 & 1.1 & 0.48 & 0.140 & 0.035 & 80 & 70 \\
\hline $19-20$ & 1130 & 0730 & 53 & 10 & 0.04 & 1.3 & 0.55 & 0.130 & 0.044 & 74 & 54 \\
\hline $20-22$ & 0830 & 1030 & 70 & 4.7 & 0.04 & 1.3 & 0.57 & 0.120 & 0.045 & 62 & 46 \\
\hline $22-26$ & 1025 & 0925 & 37 & 5.5 & 0.03 & 1.4 & 0.58 & 0.120 & 0.042 & 76 & 63 \\
\hline $26-28$ & 1150 & 0150 & 12 & 5.2 & 0.07 & 1.4 & 0.46 & 0.140 & 0.048 & 99 & 90 \\
\hline $28-29$ & 0250 & 1050 & 12 & 6.3 & 0.05 & 1.3 & 0.53 & 0.145 & 0.045 & 100 & 93 \\
\hline \multicolumn{12}{|l|}{ JUL } \\
\hline $10-13$ & 1100 & 1000 & 6.1 & 2.6 & $<0.01$ & 0.79 & 0.49 & 0.105 & 0.041 & 110 & 180 \\
\hline $17-20$ & 1130 & 1030 & 3.1 & 3.0 & 0.07 & 0.95 & 0.26 & $0.12=$ & 0.021 & 130 & 270 \\
\hline $17 \ldots$ & 1135 & -- & 3.2 & 1.3 & 0.03 & 0.53 & 0.43 & 0.085 & 0.044 & 130 & 280 \\
\hline \multicolumn{12}{|l|}{ AUG } \\
\hline $03-04$ & 0110 & 0410 & 3.6 & 0.60 & 0.02 & 0.39 & 0.21 & 0.045 & 0.023 & 120 & 280 \\
\hline $04-05$ & 0510 & 0410 & 11 & 21 & 0.02 & 1.1 & 0.18 & 0.175 & 0.041 & 87 & 230 \\
\hline $05-07$ & 0510 & 0010 & 21 & 14 & 0.02 & 0.94 & 0.15 & 0.165 & 0.053 & 65 & 110 \\
\hline $14 \ldots$ & 1120 & -- & 2.4 & 1.1 & 0.02 & 0.24 & 0.34 & 0.090 & 0.058 & 120 & 250 \\
\hline $14-15$ & 1120 & 1920 & 2.3 & 0.95 & 0.04 & 0.71 & 0.21 & 0.090 & 0.041 & 120 & 260 \\
\hline $15-17$ & 2020 & 1020 & 3.7 & 1.5 & 0.02 & 0.56 & 0.22 & 0.090 & 0.037 & 110 & 250 \\
\hline $17-19$ & 1120 & 1420 & 2.7 & 0.95 & 0.01 & 0.51 & 0.18 & 0.065 & 0.030 & 100 & 320 \\
\hline $19-21$ & 1520 & .1020 & 4.6 & 2.3 & 0.01 & 0.61 & 0.13 & 0.080 & 0.034 & 100 & 290 \\
\hline \multicolumn{12}{|l|}{ AUG $31-$} \\
\hline SEP 05 & 1115 & 1015 & 2.9 & 60 & 0.06 & 1.8 & 0.07 & 0.270 & 0.013 & 120 & 320 \\
\hline $14-16$ & 1120 & 1420 & 12 & 11 & -- & $\ldots$ & -- & 0.105 & 0.025 & 74 & 230 \\
\hline $16-18$ & 1520 & 1020 & 13 & 13 & -- & -- & -- & 0.125 & 0.028 & 59 & 120 \\
\hline $25 \ldots$ & 1000 & -- & 3.0 & 1.6 & - & 0.44 & - & 0.090 & 0.054 & 110 & 230 \\
\hline
\end{tabular}




\section{Surface-Water Stations}

\section{A. Discharge and water quality}

\section{East Branch Allen Creek At Pittsford, N.Y.}

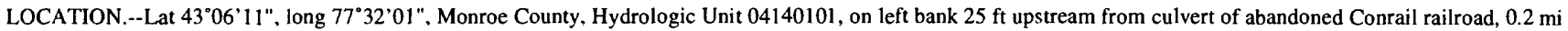
downstream from State Highway $31,0.7$ mi northwest of Pittsford and 1.8 mi upstream from mouth.

DRAINAGE AREA. $-6.96 \mathrm{mi}^{2}$, revised.

\section{WATER-DISCHARGE RECORDS}

PERIOD OF RECORD.--April 1990 to current year.

GAGE.--Water-stage recorder. Datum of gage is $400.00 \mathrm{ft}$ above sea level.

REMARKS.--Records good except those for estimated daily discharges, which are fair. Unpublished water-quality records for prior years are availat le in files of Monroe County Department of Health. Discharge includes undetermined diversion from Erie (Barge) Canal upstream from station. Several measurements of water temperature were made during the year.

COOPERATION.--Gage-height record and discharge measurements are provided by the Monroe County Health Laboratory at Rochester, N.Y

EXTREMES FOR PERIOD April 1990 to September 1993.--Maximum discharge during period April 82 to September $1993,319 \mathrm{ft}^{3} / \mathrm{s}$, Aug. 27,1992 , gage height $7.18 \mathrm{ft}$; minimum daily discharge $0.85 \mathrm{ft}^{3} / \mathrm{s}$, May 30,1993 .

STATISTICS OF MONTHLY MEAN DISCHARGE (in cubic feet per second) FOR WATER YEARS 1990 -93, BY VIATER YEAR

\begin{tabular}{|c|c|c|c|c|c|c|c|c|c|c|c|c|}
\hline & OCT & NOV & $\mathrm{DEC}$ & JAN & FEB & MAR & APR & MAY & JUN & JUL & AUG & SEP \\
\hline MEAN & 5.81 & 7.54 & 11.7 & 9.13 & 6.46 & 23.8 & 19.5 & 8.49 & 5.06 & 6.03 & 8.43 & 4.84 \\
\hline MAX & 10.3 & 13.3 & 18.1 & 13.4 & 9.67 & 26.7 & 23.4 & 16.4 & 7.52 & 12.3 & 21.7 & 6.76 \\
\hline (WY) & 1991 & 1993 & 1991 & 1993 & 1991 & 1991 & 1993 & 1990 & 1993 & 1992 & 1992 & 1992 \\
\hline MIN & 3.38 & 2.23 & 2.97 & 3.57 & 3.60 & 18.1 & 16.4 & 2.39 & 3.96 & 3.67 & 2.97 & 3.25 \\
\hline$(W Y)$ & 1993 & 1992 & 1992 & 1992 & 1993 & 1992 & 1992 & 1993 & 1991 & 1991 & 1991 & 1991 \\
\hline
\end{tabular}

SUMMARY STATISTICS

STATISTIC

ANNUAL TOTAL

ANNUAL MEAN

AVERAGE DISCHARGE

HIGHEST ANNUAL MEAN

LOWEST ANNUAL MEAN

HIGHEST DAILY MEAN

LOWEST DAILY MEAN

ANNUAL SEVEN-DAY MINIMUM

INSTANTANEOUS PEAK FLOW

INSTANTANEOUS PEAK STAGE

INSTANTANEOUS LOW FLOW

ANNUAL RUNOFF (CFSM)

ANNUAL RUNOFF (INCHES)

10 PERCENT EXCEEDS

50 PERCENT EXCEEDS

90 PERCENT EXCEEDS

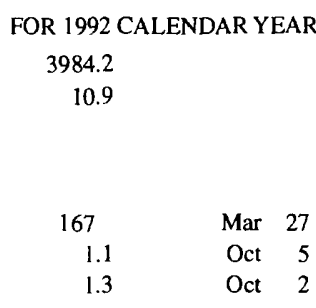

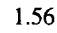

21.29

23

5.1

1.9
FOR 1993 WATER YEAR 3687.52

10.1

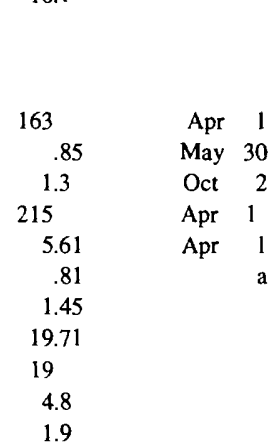

WATER YFARS 1990 - 1993

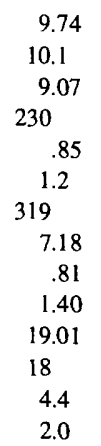

a May 30,31, 1993.

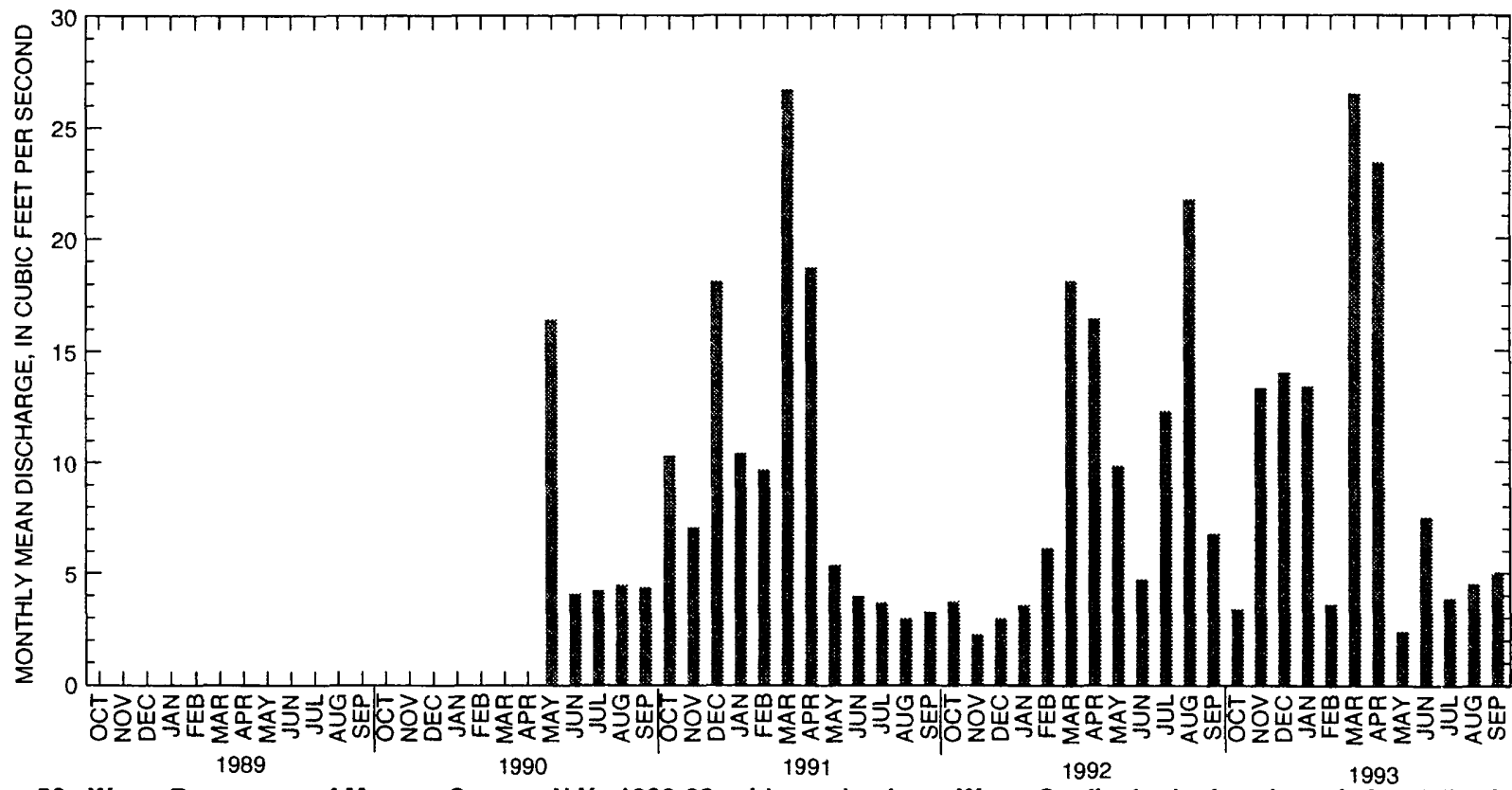

50 Water Resources of Monroe County, N.Y., 1989-93, with emphasis on Water Quality in the Irondequoit Creek Basin 
Surface-Water Stations

\section{A. Discharge and water quality}

\section{East Branch Allen Creek At Pittsford, N.Y.}

\section{WATER-QUALITY RECORDS}

PERIOD OF RECORD. --1990 to current year.

CHEMICAL DATA: $1990-93$ (e).

NUTRIENT DATA: $1990-93$ (e).

COOPERATION. - Water-quality samples were collected and analyzed by the Monroe County Environmental Health Laboratory at Rochester, N.Y.

REMARKS . - -

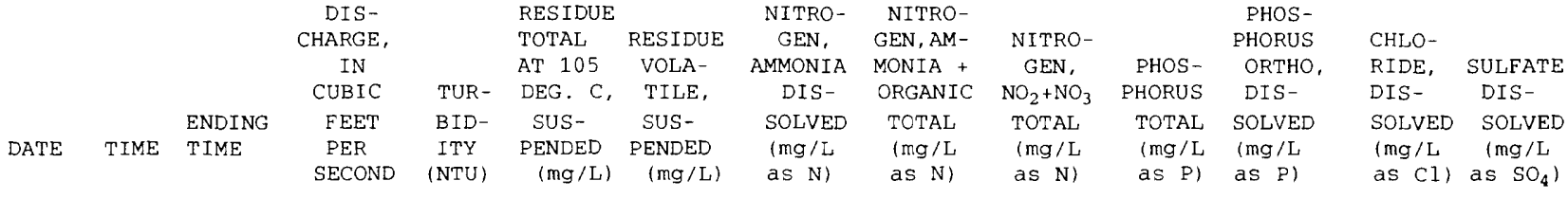

OCT

\begin{tabular}{|c|c|c|}
\hline $09-11$ & 1540 & 1240 \\
\hline $18-18$ & 1250 & 2350 \\
\hline $19-22$ & 0050 & 1150 \\
\hline $22-23$ & 1330 & 0630 \\
\hline $23-25$ & 0730 & 1230 \\
\hline \multicolumn{3}{|l|}{ NOV } \\
\hline $05-09$ & 1130 & 1030 \\
\hline $09-13$ & 1140 & 1040 \\
\hline $13-15$ & 1340 & 1240 \\
\hline $15-19$ & 1450 & 1250 \\
\hline $22-26$ & 1710 & 1010 \\
\hline \multicolumn{3}{|l|}{ DEC } \\
\hline $03-04$ & 1300 & 0300 \\
\hline $04-06$ & 0400 & 1200 \\
\hline $17-18$ & 1335 & 1535 \\
\hline $19-20$ & 1635 & 1235 \\
\hline $29-30$ & 0930 & 1430 \\
\hline \multicolumn{3}{|l|}{ DEC $30-$} \\
\hline JAN 01 & 1530 & 0830 \\
\hline $02-04$ & 1350 & 1250 \\
\hline $14-16$ & 1530 & 0330 \\
\hline $16-17$ & 0430 & 0630 \\
\hline $17 \ldots$ & 1430 & -- \\
\hline \multicolumn{3}{|l|}{ FEB } \\
\hline $07 \ldots$ & 1345 & $\ldots$ \\
\hline $14-15$ & 1400 & 1100 \\
\hline $19-20$ & 1400 & 0700 \\
\hline $20-21$ & 0800 & 1300 \\
\hline \multicolumn{3}{|l|}{ FEB 28- } \\
\hline MAR 02 & 1315 & 2015 \\
\hline $02-04$ & 2115 & 1215 \\
\hline $06 \ldots$ & 1420 & -- \\
\hline $06-08$ & 1430 & 1230 \\
\hline $08-11$ & 1210 & 1110 \\
\hline $11-14$ & 1400 & 1300 \\
\hline $22-23$ & 1340 & 0040 \\
\hline $23-25$ & 0140 & 1240 \\
\hline $27-27$ & 0455 & 1555 \\
\hline $27-28$ & 1655 & 1255 \\
\hline \multicolumn{3}{|l|}{ MAR 28- } \\
\hline APR 01 & 1335 & 1235 \\
\hline $08-10$ & 1310 & 0110 \\
\hline $10-10$ & 1010 & 1510 \\
\hline $19-20$ & 1730 & 1230 \\
\hline $22-25$ & 1250 & 1150 \\
\hline $22 \ldots$ & 1255 & -- \\
\hline \multicolumn{3}{|l|}{ MAY } \\
\hline $16-17$ & 1130 & 1030 \\
\hline $17-17$ & 1130 & 2230 \\
\hline $17-20$ & 2330 & 1030 \\
\hline $26-27$ & 1350 & 1350 \\
\hline \multicolumn{3}{|l|}{ JUN } \\
\hline $11-12$ & 1200 & 1100 \\
\hline $12-13$ & 1200 & 1100 \\
\hline \multicolumn{3}{|l|}{ JUL } \\
\hline $01-04$ & 1215 & 1515 \\
\hline $04-05$ & 1615 & 1115 \\
\hline $05-06$ & 1130 & 0730 \\
\hline $07-08$ & 0830 & 0430 \\
\hline
\end{tabular}

\begin{tabular}{|c|c|}
\hline 12 & 31 \\
\hline 27 & 50 \\
\hline 6.9 & 6.9 \\
\hline 4.8 & 13 \\
\hline 14 & 17 \\
\hline 6.2 & 12 \\
\hline 16 & 14 \\
\hline 8.5 & 6.5 \\
\hline 7.3 & 7.6 \\
\hline 7.4 & 8.0 \\
\hline 13 & 17 \\
\hline 23 & 31 \\
\hline 23 & 15 \\
\hline 19 & 20 \\
\hline 96 & 95 \\
\hline 74 & 13 \\
\hline 12 & 10 \\
\hline 7.3 & 4.6 \\
\hline 30 & 16 \\
\hline 41 & 15 \\
\hline 35 & 16 \\
\hline 4.5 & 19 \\
\hline 27 & 30 \\
\hline 20 & 11 \\
\hline 15 & 15 \\
\hline 113 & 230 \\
\hline 52 & 10 \\
\hline 37 & 45 \\
\hline 9.8 & 5.2 \\
\hline 5.9 & 3.1 \\
\hline 9.6 & 3.6 \\
\hline 24 & 36 \\
\hline 51 & 120 \\
\hline 40 & 100 \\
\hline 19 & 6.6 \\
\hline 12 & 39 \\
\hline 17 & 50 \\
\hline 15 & 12 \\
\hline 54 & 16 \\
\hline 167 & 70 \\
\hline
\end{tabular}

$\begin{array}{rrrll}58 & 10 & 0.03 & 0.84 & 0.57 \\ 100 & 16 & 0.06 & 1.5 & 0.97 \\ -- & -- & 0.02 & 0.83 & 1.50 \\ -- & -- & <0.01 & 0.13 & 1.20 \\ -- & -- & 0.02 & 0.08 & 1.80 \\ -- & -- & 0.03 & 0.85 & 1.50 \\ -- & -- & 0.01 & 1.1 & 1.90 \\ -- & -- & 0.03 & 0.79 & 2.30 \\ -- & -- & 0.02 & 1.0 & 2.00\end{array}$

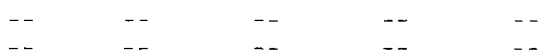

--

$265 \quad--$

$\begin{array}{lll}- & \ldots & 0.02\end{array}$

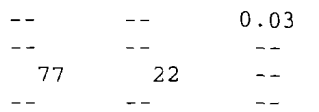

$\begin{array}{rrr}-- & -- & 0.02 \\ 543 & 63 & 0.02\end{array}$

$\begin{array}{rrr}543 & -- & 0.02\end{array}$

$\begin{array}{ccc}92 & 14 & -- \\ 149 & 19 & -- \\ -- & -- & <0.01 \\ -- & -- & 0.04 \\ 94 & 11 & 0.11\end{array}$

$\begin{array}{lc}3.4 & 7.7 \\ 6.3 & 14\end{array}$

$6.7 \quad 80$

6.033

$\begin{array}{rrr}-- & -- & <0.01 \\ -- & -- & <0.01 \\ 113 & 16 & 0.07\end{array}$

0.032

\subsection{0}

0.290

0.080

0.085

0.020

0.110

0.100

0.021

0.024

0.100

0.100

0.065

0.065

0.015

0.016

0.742 .00

0.065

$0.016 \quad 120$

0.012120

0.012100

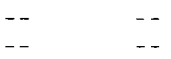

$1.7 \quad 1.80$

0.180

0.008

0.080
0.100

0.028

0.010

0.550

0.032

110

100

$0.83 \quad 2.40$

0.100

0.019

0.060

0.035

0.014

0.008

0.016

0.110

0.039

$0.70 \quad 2.40$

0.095

0.025

0.075

0.160

0.019

0.009

0.100

$0.78 \quad 1.60$

$\begin{array}{lll}2.3 & 1.40 & 0.300\end{array}$

0.003

$0.62 \quad 1.80$

0.085

0.230

0.040

0.030

0.030

0.170

0.420

0.030

0.022

0.011

0.013

0.004

$<0.002$

0.007

0.400

0.014

0.050

0.140

0.230

0.006

$\begin{array}{ll}-- & -- \\ -- & -- \\ -- & -- \\ 0.96 & 0.98\end{array}$

$\begin{array}{ll}0.96 & 0.98 \\ 0.87 & 1.60\end{array}$

0.080

0.090

0.010

0.016

1.41 .60

0.014

0.048

0.045

0.009

0.80

0.360

0.022

0.070

0.016

0.240

0.031

0.170

0.024

0.610

0.039

0.070

0.029

$0.46 \quad 0.49$

0.130

0.260

0.010

66

68

120

81

120

65

84

86

55

42
44

23

46

69

$\begin{array}{ll}250 & 92 \\ 190 & 56\end{array}$

$110 \quad 46$

$98 \quad 37$

$270 \quad 61$

$110 \quad 59$

$200 \quad 75$

$53 \quad 25$

$\begin{array}{ll}73 & 47 \\ 81 & 51\end{array}$

$98 \quad 69$

$110 \quad 82$

$96 \quad 82$

$82 \quad 55$

$\begin{array}{ll}64 & 44 \\ 59 & 34\end{array}$

$\begin{array}{ll}87 & 47\end{array}$

$81 \quad 110$

78

57
46

$0.55 \quad 0.51$

0.120 
Surface-Water Stations

A. Discharge and water quality

0423204920 East Branch Allen Creek At Pittsford, N.Y.

2. WATER-QUALITY RECORDS

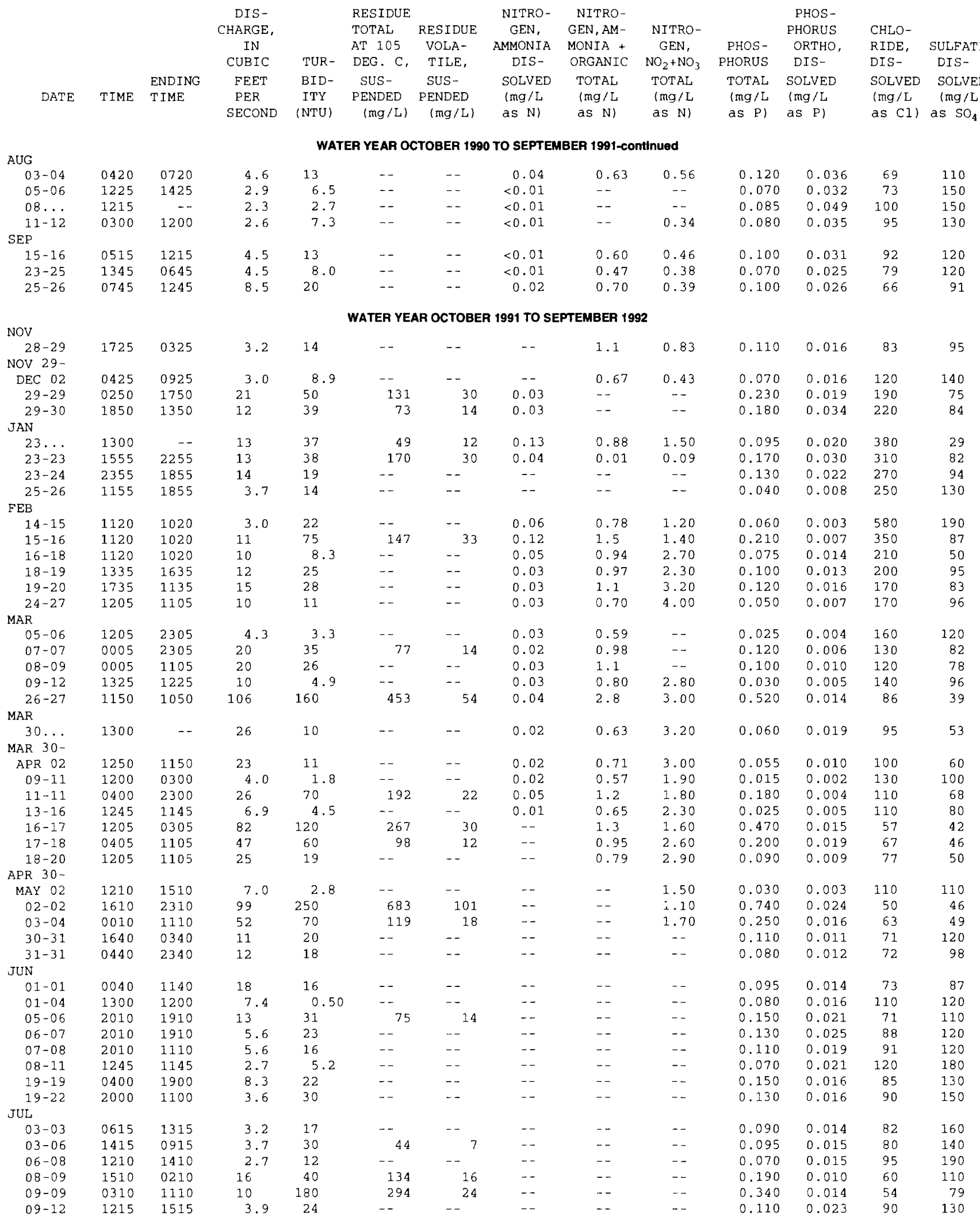




\section{3urface-Water Stations}

\section{Discharge and water quality}

0423204920 East Branch Allen Creek At Pittsford, N.Y.

\section{WATER-QUALITY RECORDS}

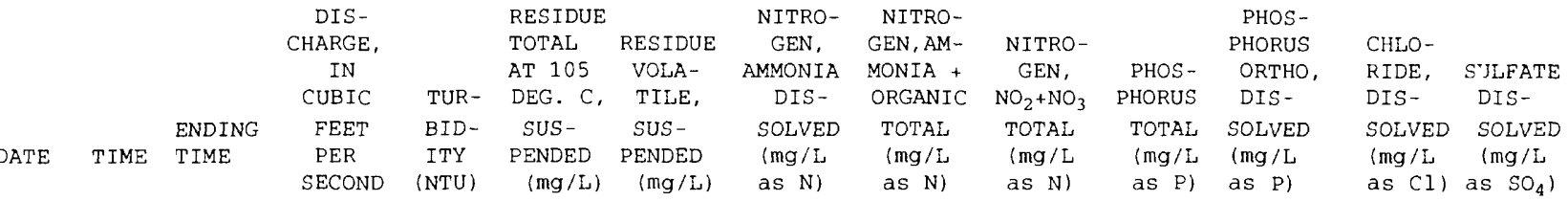

WATER YEAR OCTOBER 1991 TO SEPTEMBER 1992-continued

$\begin{array}{lcccc}\text { AUG } & & & & \\ 03-04 & 1250 & 1150 & 84 & 200 \\ 04-06 & 1250 & 1150 & 36 & 45 \\ 24-24 & 1220 & 1920 & 2.9 & 6.3 \\ 24-25 & 2020 & 0520 & 44 & 460 \\ 25-27 & 0620 & 1120 & 7.5 & 45 \\ 27-28 & 1605 & 0305 & 256 & 220 \\ 28-31 & 0405 & 1105 & 80 & 70 \\ \text { SEP } & & & & \\ 03-03 & 0440 & 1440 & 43 & 31 \\ 03-04 & 2040 & 0940 & 49 & 45 \\ 04-08 & 1025 & 0925 & 4.2 & 10 \\ 18-19 & 1610 & 0310 & 22 & 34 \\ 19-21 & 0410 & 1110 & 2.6 & 10\end{array}$
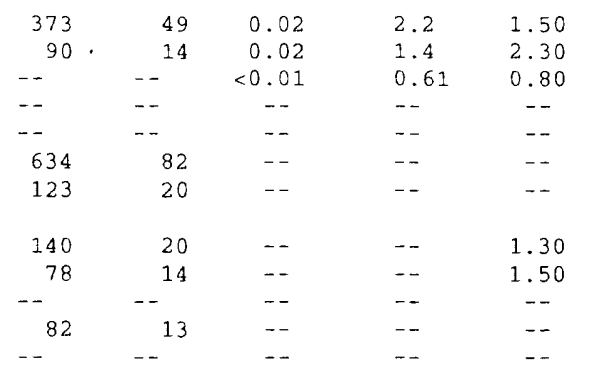

$\begin{array}{lllr}0.620 & 0.053 & 47 & 62 \\ 0.250 & 0.051 & 60 & 54 \\ 0.080 & 0.023 & 92 & 160 \\ 0.930 & 0.047 & 40 & 60 \\ 0.260 & 0.051 & 72 & 110 \\ 1.20 & 0.081 & 19 & - \\ 0.300 & 0.061 & 43 & - \\ 0.270 & 0.040 & 45 & - \\ 0.215 & 0.054 & 49 & - \\ 0.100 & 0.041 & 88 & 100 \\ 0.190 & 0.029 & 61 & 100 \\ 0.100 & 0.027 & 81 & 120\end{array}$

OCT

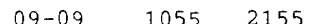

$09-13$

$23-24 \quad 2030 \quad 1930$

$24-26 \quad 2030 \quad 1130$

NOV

$02-03 \quad 1250 \quad 0850$

$03-05 \quad 0950 \quad 1150$

$\begin{array}{lll}12-13 & 1630 & 0330\end{array}$

DEC

10-11 $1255 \quad 1955$

16-17 $0815 \quad 1315$

$\begin{array}{lll}17-21 & 1330 & 1230\end{array}$

29-31 $1615 \quad 1115$

DEC $31-$

JAN $03 \quad 1140 \quad 0640$

03-04 $0740 \quad 1040$

04-05 $1310 \quad 0910$

05-07 $1010 \quad 2210$

21-22 $1235 \quad 1935$

$22-25$

$2035 \quad 1135$

FEB

04-08 $1410 \quad 1310$

MAR

13-15 $1245 \quad 1145$

$15-18 \quad 1100 \quad 1000$

22-25 $1210 \quad 1210$

APR

$\begin{array}{cc}21 & 95 \\ 3.0 & 12 \\ 11 & 42\end{array}$

\begin{tabular}{|c|c|c|c|c|c|c|c|c|}
\hline 105 & 59 & -- & -- & -- & 0.130 & 0.033 & 46 & 56 \\
\hline-- & -- & -- & -- & -- & 0.100 & 0.028 & 88 & 120 \\
\hline 56 & 10 & -- & -- & -- & 0.140 & 0.015 & 76 & 110 \\
\hline+- & -- & -- & -- & -- & 0.120 & 0.024 & 61 & 76 \\
\hline 162 & -- & -- & -- & $\ldots$ & 0.300 & 0.050 & 48 & 44 \\
\hline-- & -- & -- & -- & - & 0.130 & 0.035 & 66 & 61 \\
\hline-- & -- & -- & -- & -- & 0.110 & 0.014 & 84 & 95 \\
\hline-- & -- & -- & $-\infty$ & -- & 0.090 & 0.014 & 84 & 94 \\
\hline 11 & $<5$ & 0.03 & 0.64 & 2.20 & 0.040 & 0.009 & 200 & 96 \\
\hline-- & -- & -- & -- & -- & 0.150 & 0.021 & 150 & 40 \\
\hline-- & -- & -- & -- & -- & 0.110 & 0.020 & 79 & 39 \\
\hline 136 & 21 & 0.03 & 1.4 & 1.30 & 0.260 & 0.026 & 110 & 48 \\
\hline-- & -- & 0.02 & 0.47 & 2.00 & 0.110 & 0.014 & 93 & 66 \\
\hline-- & -- & -- & 0.70 & -- & 0.080 & 0.010 & 190 & 74 \\
\hline 129 & 20 & 0.01 & -- & 1.60 & 0.230 & 0.019 & 84 & 48 \\
\hline-- & -- & 0.01 & -- & 1.80 & 0.095 & 0.015 & 78 & 62 \\
\hline 64 & 11 & -- & -- & -- & 0.120 & 0.009 & 200 & 60 \\
\hline 59 & 9 & -- & -- & -- & 0.120 & 0.016 & 110 & 42 \\
\hline-- & -- & $\cdots$ & -- & -- & 0.045 & 0.009 & 130 & 75 \\
\hline-- & -- & 0.02 & 0.62 & 1.90 & 0.025 & 0.004 & 230 & 120 \\
\hline- & -- & -- & -- & -- & 0.320 & 0.003 & 260 & 86 \\
\hline-- & -- & -- & -- & -- & 0.400 & 0.003 & 290 & 80 \\
\hline 86 & 12 & 0.05 & 1.1 & 1.50 & 0.150 & 0.011 & 190 & 53 \\
\hline 171 & 22 & 0.05 & 1.5 & 1.70 & 0.290 & 0.018 & 90 & 33 \\
\hline 164 & 23 & 0.06 & 1.1 & 1.20 & 0.320 & 0.043 & 37 & 22 \\
\hline-- & -- & 0.02 & 0.57 & 1.80 & 0.060 & 0.009 & 73 & 54 \\
\hline-- & -- & 0.02 & 0.56 & 1.50 & 0.040 & 0.006 & 85 & 69 \\
\hline-- & -- & $<0.01$ & $<0.01$ & 1.40 & 0.045 & 0.016 & 130 & - \\
\hline 123 & 18 & -- & -- & -- & 0.230 & 0.017 & 100 & 150 \\
\hline 63 & $<11$ & -- & -- & -- & 0.160 & 0.027 & 97 & 98 \\
\hline-- & -- & 0.03 & 0.84 & 0.92 & 0.140 & 0.020 & 100 & 150 \\
\hline 80 & 13 & 0.03 & 0.96 & 0.91 & 0.085 & 0.025 & 88 & 120 \\
\hline$\cdots$ & -- & 0.03 & 0.78 & 0.96 & 0.085 & 0.031 & 93 & 150 \\
\hline 98 & 18 & -- & -- & -- & 0.200 & 0.023 & 66 & 100 \\
\hline-- & -- & 0.01 & $<0.01$ & NO. 91 & 0.120 & 0.034 & 84 & 1220 \\
\hline-- & -- & $<0.01$ & 0.62 & No. 60 & 0.085 & 0.022 & 85 & 120 \\
\hline-- & -- & $<0.01$ & -- & No. 64 & 0.065 & 0.025 & 71 & 150 \\
\hline
\end{tabular}




\section{Surface-Water Stations}

\section{A. Discharge and water quality}

\section{East Branch Allen Creek At Pittsford, N.Y.}

2. WATER-QUALITY RECORDS

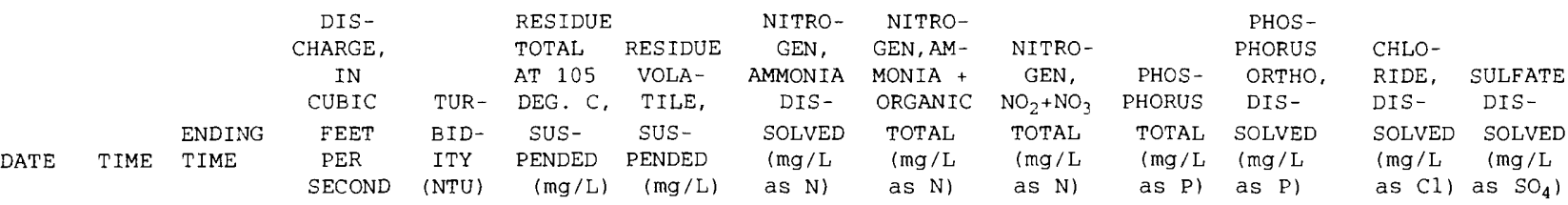

WATER YEAR OCTOBER 1992 TO SEPTEMBER 1993-continued

$\begin{array}{lll}\text { AUG } & & \\ 11-12 & 1905 & 0305 \\ 12-12 & 0405 & 1205 \\ \text { SEP } & & \\ 02-03 & 1715 & 0215 \\ 03-03 & 0315 & 1215 \\ 15-16 & 0310 & 1110 \\ 26-27 & 0320 & 1020\end{array}$

$\begin{array}{rr}6.4 & 15 \\ 4.9 & 11 \\ 18 & \\ 7.0 & 75 \\ 2.2 & 12 \\ 11 & 50\end{array}$

$\begin{array}{ccc}-- & - & <0.01 \\ -- & - & <0.01 \\ & & \\ -- & -- & 0.01 \\ -- & -- & <0.01 \\ - & -- & 0.02 \\ 75 & 12 & --\end{array}$

$\begin{array}{lc}0.62 & \text { N0.60 } \\ -- & \text { N0.64 } \\ 0.74 & 0.73 \\ 0.76 & 0.72 \\ 0.73 & 0.68 \\ -- & --\end{array}$

0.022

0.065

0.025

85

0.150

0.028

0.160

0.030

0.070

0.029

0.037

56

$100 \quad 150$ 


\section{Surface-Water Stations}

\section{A. Discharge and water quality}

\section{Allen Creek near Rochester, N.Y.}

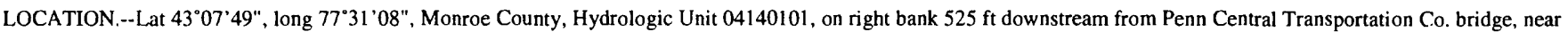
Rochester, and about $1.3 \mathrm{mi}$ upstream from Irondequoit Creek.

DRAINAGE AREA.--30.1 $\mathrm{mi}^{2}$, flow from $3.5 \mathrm{mi}^{2}$ noncontributing.

PERIOD OF RECORD.--November 1959 to current year.

\section{WATER-DISCHARGE RECORDS}

REVISED RECORDS.--WRD NY 1974: 1972(M), 1973(M, P). WDR NY-76-1: 1960-75 (M, P), 1960-63, 1972-74.

GAGE.--Water-stage recorder and concrete control. Datum of gage is $323.54 \mathrm{ft}$ above sea level.

REMARKS.--Records good except those for estimated daily discharges, which are fair. Unpublished water-quality records for prior years are available in files of Monroe County Department of Health. Discharge prior to January 1980 included undetermined diversion (maximum $20 \mathrm{ft}^{3} / \mathrm{s}$ ) from Erie (Barge) Canal upstream from station. January 1980 to present, diversion reduced to a maximum of $3 \mathrm{ft}^{3} / \mathrm{s}$ for use by several golf courses adjacent to stream. Several measurements of water temperature were made during the year. COOPERATION.--Gage-height record and 9 discharge measurements were provided by the Monroe County Health Laboratory at Rochester, N.Y.

EXTREMES FOR PERIOD November 1959 to September 1993.--Maximum discharge, 3,280 ft ${ }^{3} / \mathrm{s}$. May 17, 1974, gage height, $7.42 \mathrm{ft}$, from rating curve extended above 1,000 $\mathrm{ft}^{3} / \mathrm{s}$ on basis of contracted-opening measurement of peak discharge and step-backwater analysis; minimum daily, $1.7 \mathrm{ft} / 3$, Jan. 24. 1963; minimum gage height, $1.16 \mathrm{ft}$, Feb. 19,1962

\section{STATISTICS OF MONTHLY MEAN DISCHARGE (in cubic feet per second) FOR WATER YEARS 1960-93, BY WATER YEAR}

\begin{tabular}{|c|c|c|c|c|c|c|c|c|c|c|c|c|}
\hline & OCT & NOV & DEC & JAN & FEB & MAR & APR & MAY & JUN & JUL & AUG & SEP \\
\hline MEAN & 26.2 & 32.3 & 31.9 & 22.7 & 35.0 & 58.3 & 47.7 & 34.1 & 28.5 & 22.6 & 25.3 & 23.9 \\
\hline (WY) & 1978 & 1973 & 1978 & 1969 & 1981 & 1960 & 1969 & 1974 & 1972 & 1976 & 1992 & 1977 \\
\hline (WY) & 1962 & 1961 & 1961 & 1963 & 1989 & 1981 & 1981 & 1993 & 1991 & 1960 & 1961 & 1961 \\
\hline
\end{tabular}

SUMMARY STATISTICS

STATISTIC

ANNUAL TOTAL

ANNUAL MEAN

AVERAGE DISCHARGE

HIGHEST ANNUAL MEAN

HIGHEST DAILY MEAN

LOWEST DAILY MEAN

ANNUAL SEVEN-DAY MINIMUM

INSTANTANEOUS PEAK FLOW

INSTANTANEOUS PEAK STAGE

INSTANTANEOUS LOW FLOW

10 PERCENT EXCEEDS

50 PERCENT EXCEEDS

90 PERCENT EXCEEDS
LOWEST ANNUAL MEAN

\section{FOR 1993 WATER YEAR} 11161.7 11860.7

$$
32.4
$$

$\begin{array}{ccc}537 & \text { Mar } & 27 \\ 5.1 & \text { Oct } & 6 \\ 5.8 & \text { Feb } & 8\end{array}$

a From rating table extended above $1.000 \mathrm{ft}^{3} / \mathrm{s}$ on bsis of contracted-opening measurement of peak discharge and step-backwater analysis.

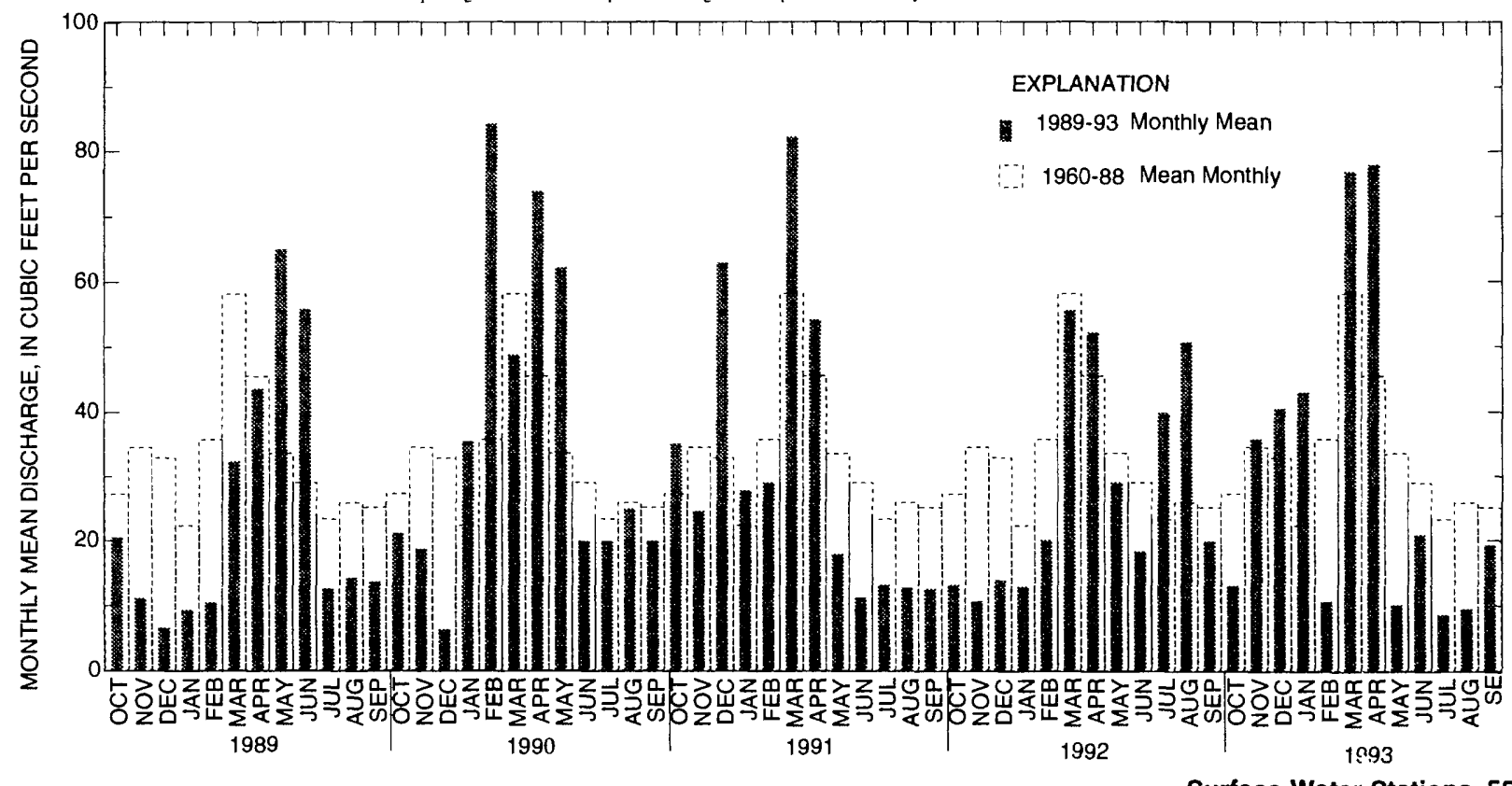




\section{Surface-Water Stations}

\section{A. Discharge and water quality}

\section{Allen Creek near Rochester, N.Y.}

\section{WATER-QUALITY RECORDS}

PERIOD OF RECORD.--October 1983 to current year.

CHEMICAL DATA: 1983-93 (e).

NUTRIENT DATA: 1983-93 (e).

COOPERATION. - Water-quality samples were collected and analyzed by the Monroe County Environmental Health Laboratory at Rochester, N.Y.

REMARKS.--Prior to 1994 water year, data published in WATER RESOURCES OF MONROE COUNTY NEW YORK, WATER YEARS 1984-88, U. S. Geological Survey open-file report 93-370.

\begin{tabular}{|c|c|c|c|c|c|c|c|c|c|c|c|c|c|}
\hline & & & $\begin{array}{c}\text { DIS- } \\
\text { CHARGE, } \\
\text { IN } \\
\text { CUBIC }\end{array}$ & TUR & $\begin{array}{l}\text { RESIDUE } \\
\text { TOTAL } \\
\text { AT } 105 \\
\text { DEG. C, }\end{array}$ & $\begin{array}{l}\text { RES IDUE } \\
\text { VOLA- } \\
\text { TILE, }\end{array}$ & $\begin{array}{l}\text { NITRO- } \\
\text { GEN, } \\
\text { AMMONIA } \\
\text { DIS- }\end{array}$ & $\begin{array}{l}\text { NITRO - } \\
\text { GEN, AM- } \\
\text { MONIA + } \\
\text { ORGANIC }\end{array}$ & $\begin{array}{c}\mathrm{NITRO}- \\
\text { GEN, } \\
\mathrm{NO}_{2}+\mathrm{NO}_{3}\end{array}$ & $\begin{array}{l}\text { PHOS- } \\
\text { PHORUS }\end{array}$ & $\begin{array}{c}\text { PHOS- } \\
\text { PHORUS } \\
\text { ORTHO, } \\
\text { DIS- }\end{array}$ & $\begin{array}{l}\text { CHLO- } \\
\text { RIDE, } \\
\text { DIS- }\end{array}$ & $\begin{array}{l}\text { SULFATE } \\
\text { DIS- }\end{array}$ \\
\hline $\mathrm{TE}$ & TIME & $\begin{array}{l}\text { ENDING } \\
\text { TIME }\end{array}$ & $\begin{array}{c}\text { FEET } \\
\text { PER } \\
\text { SECOND }\end{array}$ & $\begin{array}{l}\text { BID- } \\
\text { ITY } \\
\text { (NTU) }\end{array}$ & $\begin{array}{l}\text { SUS- } \\
\text { PENDED } \\
(\mathrm{mg} / \mathrm{L})\end{array}$ & $\begin{array}{l}\text { SUS- } \\
\text { PENDED } \\
(\mathrm{mg} / \mathrm{L})\end{array}$ & $\begin{array}{l}\text { SOLVED } \\
(\mathrm{mg} / \mathrm{L} \\
\text { as } \mathrm{N})\end{array}$ & $\begin{array}{l}\text { TOTAL } \\
\text { (mg/L } \\
\text { as N) }\end{array}$ & $\begin{array}{l}\text { TOTAL } \\
\text { (mg/L } \\
\text { as N) }\end{array}$ & $\begin{array}{l}\text { TOTAL } \\
\text { (mg/L } \\
\text { as P) }\end{array}$ & $\begin{array}{l}\text { SOLVED } \\
(\mathrm{mg} / \mathrm{L} \\
\text { as P) }\end{array}$ & $\begin{array}{l}\text { SOLVED } \\
\text { (mg/L } \\
\text { as Cl) }\end{array}$ & $\begin{array}{l}\text { SOLVED } \\
\text { ( } \mathrm{mg} / \mathrm{L} \\
\text { as } \mathrm{SO}_{4} \text { ) }\end{array}$ \\
\hline
\end{tabular}

OCT

\begin{tabular}{|c|c|c|}
\hline $03-04$ & 1125 & 18 \\
\hline $04-05$ & 1925 & \\
\hline $14-16$ & 1210 & \\
\hline $18 \ldots$ & 1010 & \\
\hline $18-21$ & 1010 & \\
\hline $21-21$ & 1150 & \\
\hline $21-24$ & 2250 & \\
\hline $24-27$ & 1150 & \\
\hline \multicolumn{3}{|l|}{ OCT $31-$} \\
\hline NOV 02 & 1215 & 08 \\
\hline $02-03$ & 0915 & \\
\hline $03-04$ & 1200 & \\
\hline $04-07$ & 1600 & \\
\hline $10 \ldots$ & 1035 & \\
\hline $17-18$ & 1130 & \\
\hline $21 \ldots$ & 1100 & \\
\hline \multicolumn{3}{|l|}{$\mathrm{DEC}$} \\
\hline $19 \ldots$ & 1110 & \\
\hline $30 \ldots$ & 1100 & \\
\hline \multicolumn{3}{|l|}{ JAN } \\
\hline $09 \ldots$ & 1050 & \\
\hline $13 \ldots$ & 1030 & \\
\hline $23 \ldots$ & 1115 & \\
\hline $30 \ldots$ & 1000 & \\
\hline \multicolumn{3}{|l|}{ FEB } \\
\hline $14 \ldots$ & 0945 & \\
\hline $14 \ldots$ & 1420 & \\
\hline $15 \ldots$ & 1130 & \\
\hline $21 \ldots$ & 1045 & \\
\hline $21 \ldots$ & 1400 & \\
\hline $21 \ldots$ & 1500 & \\
\hline $22 \ldots$ & 1230 & \\
\hline $22 \ldots$ & 1500 & \\
\hline \multicolumn{3}{|l|}{ MAR } \\
\hline $03 \ldots$ & 1230 & \\
\hline $05-06$ & 0930 & \\
\hline $14-16$ & 1115 & \\
\hline $16-18$ & 1100 & \\
\hline $18-20$ & 0300 & \\
\hline $23-24$ & 1115 & 1 \\
\hline $24-27$ & 1515 & 10 \\
\hline $27-30$ & 1045 & 03 \\
\hline $30-30$ & 0445 & \\
\hline \multicolumn{3}{|l|}{ MAR 30- } \\
\hline APR 02 & 1120 & 1 \\
\hline $02-03$ & 1520 & \\
\hline $03-06$ & 1035 & 05 \\
\hline $06-10$ & 1040 & 09 \\
\hline $10-13$ & 1015 & 0 \\
\hline $13-17$ & 1035 & \\
\hline \multicolumn{3}{|l|}{ MAY } \\
\hline $01-04$ & 1120 & 10 \\
\hline $04-07$ & 1115 & \\
\hline $07-08$ & 0315 & 1 \\
\hline $08-10$ & 1030 & 15 \\
\hline
\end{tabular}

WATER YEAR OCTOBER 1988 TO SEPTEMBER 1989

18
21
15
71
19
13

$\begin{array}{lllr}16 & -- & - & 0.02 \\ 14 & -- & -- & 0.01 \\ 15 & -- & -- & 0.01 \\ 80 & 228 & 37 & <0.01 \\ 30 & -- & -- & <0.01 \\ -- & -- & -- & <0.01 \\ 45 & -- & -- & 0.01\end{array}$

19

13

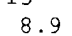

18

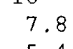

37

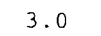

9.3

12

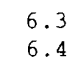

14

9.0

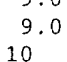

67

79

77

31

29

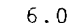

6.
55
25

19

48

35

40

34

44

142
169
128

128
41

24

22

73

29

223
97

99
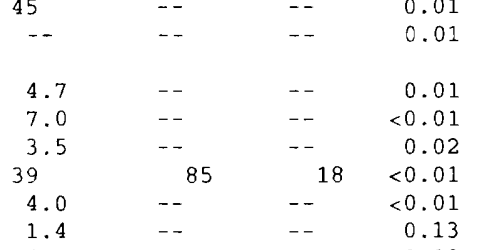

$$
\begin{array}{rr}
-- & <0.01 \\
-- & 0.02
\end{array}
$$$$
\begin{array}{rr}
18 & <0.01 \\
--\quad<0.01
\end{array}
$$$$
\text { - } \quad 0.13
$$$$
\begin{aligned}
& 0.13 \\
& 0.02
\end{aligned}
$$$$
\begin{array}{ll}
- & 0.03 \\
-- & 0.01
\end{array}
$$$$
2.1
$$$$
--
$$

0.05

$\begin{array}{rrrr}13 & -- & -- & 0.05 \\ 2.1 & -- & -- & 0.04 \\ 1.9 & & -- & <0.01\end{array}$

1. 9

$$
5.0
$$$$
-
$$$$
0.01
$$$$
\begin{array}{rrr}
- & - & <0.01 \\
-- & -- & 0.03
\end{array}
$$$$
--
$$$$
\begin{aligned}
& 169 \\
& 154
\end{aligned}
$$$$
--
$$$$
\begin{array}{rr}
-- & 0.03 \\
- & 0.12 \\
41 & 0.18 \\
32 & 0.19
\end{array}
$$$$
0.07
$$$$
\begin{array}{cc}
3.4 & - \\
75 & 130 \\
18 & - \\
3.2 & --
\end{array}
$$

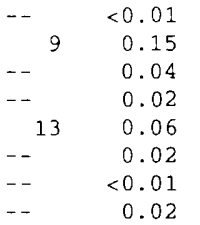

0.03
0.80

0.76

0.80

1.5

1.2

0.63

1.6
1.0

0.80

0.80

0.57

1.0
0.43

0.43

0.74
0.75

0.44

0.45

0.67

0.50

0.49

0.53

0.57

0.84

0.64

0.82

0.68

0.31

0.66

0.48

0.62

0.53

0.99

$0.65 \quad 0.91$$$
1.1
$$$$
0.53 \quad 1.12
$$$$
0.69 \quad 1.06
$$$$
0.90 \quad 0.90
$$$$
1.2 \quad 1.20
$$$$
\text { 1. } 2
$$$$
1.5
$$$$
3.1
$$$$
\begin{aligned}
& 1.20 \\
& 1.60
\end{aligned}
$$$$
1.10
$$$$
1.10
$$$$
\text { 1. } 10
$$$$
1.5 \quad 1.30
$$$$
\begin{array}{ll}
1.5 & 1.30
\end{array}
$$

$0.78 \quad 1.10$

$3.4 \quad 1.60$

$1.8 \quad 1.20$

$0.92 \quad 1.40$

1.61 .50

$1.1 \quad 1.60$

$1.0 \quad 1.50$

$\begin{array}{ll}1.6 & 0.98 \\ 1.6 & 1.10\end{array}$

$2.1 \quad 1.30$

$\begin{array}{ll}2.1 & 1.30 \\ 2.0 & 1.50\end{array}$

1.91 .80

$\begin{array}{ll}1.2 & 1.80 \\ 0.66 & 0.72\end{array}$

$0.98 \quad 1.20$

\begin{tabular}{|c|}
\hline 075 \\
\hline 0.070 \\
\hline 0.070 \\
\hline .120 \\
\hline .050 \\
\hline .040 \\
\hline .195 \\
\hline .045 \\
\hline .070 \\
\hline .105 \\
\hline 0.025 \\
\hline 0.210 \\
\hline 0.025 \\
\hline 0.060 \\
\hline 0.110 \\
\hline 0.025 \\
\hline 0.030 \\
\hline .075 \\
\hline 0.025 \\
\hline 0.030 \\
\hline 0.040 \\
\hline 0.025 \\
\hline 0.085 \\
\hline 0.040 \\
\hline 0.460 \\
\hline 0.320 \\
\hline 0.280 \\
\hline 0.095 \\
\hline 0.095 \\
\hline 0.030 \\
\hline 0.290 \\
\hline 0.135 \\
\hline 0.035 \\
\hline 0.130 \\
\hline 0.080 \\
\hline 0.085 \\
\hline 0.070 \\
\hline 0.130 \\
\hline 0.210 \\
\hline 0.220 \\
\hline 0.170 \\
\hline 0.055 \\
\hline 0.030 \\
\hline 0.025 \\
\hline 0.155 \\
\hline 0.040 \\
\hline 0.185 \\
\hline \\
\hline
\end{tabular}

$\begin{array}{ll}1.5 & 0.72 \\ 0.98 & 0.70 \\ 1.3 & 0.64 \\ -- & 1.30 \\ 2.3 & \end{array}$

0.011

$0.009 \quad 95$

0.012120

$0.035 \quad 72$

$0.010 \quad 86$

0.022100

0.03188

$0.015 \quad 130$

$0.010 \quad 130$

$0.014 \quad 130$

$0.007 \quad 140$

0.01297

0.015150

0.015180

0.02983

$<0.002 \quad 330$

$0.006 \quad 400$

$0.013 \quad 450$

$0.006 \quad 260$

$0.005 \quad 460$

$0.006 \quad 340$

$0.002 \quad 560$

0.004510

0.004950

0.014510

0.0191000

0.024890

0.024690

$0.020 \quad 710$

$0.005 \quad 410$

$0.037 \quad 350$

$0.003 \quad 380$

0.004390

$0.007 \quad 390$

0.004330

$0.004 \quad 290$

$0.002 \quad 270$

0.005230

$0.009 \quad 250$

$0.013 \quad 150$

$0.010 \quad 160$

$0.005 \quad 210$

$0.002 \quad 230$

$0.002 \quad 230$

$0.006 \quad 150$

$0.008 \quad 170$

$0.014 \quad 120$

0.015130

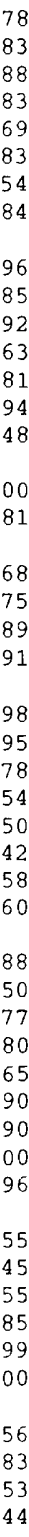

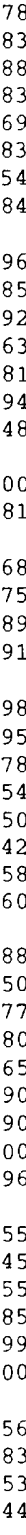




\section{Surface-Water Stations}

A. Discharge and water quality

04232050 Allen Creek near Rochester, N.Y.

\section{WATER-QUALITY RECORDS}

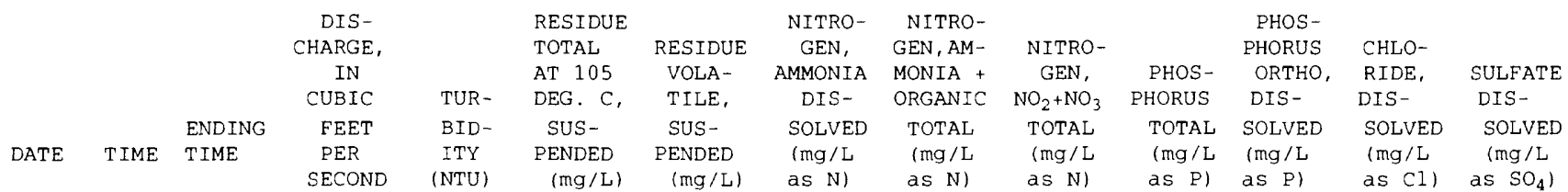

MAY-cont inued

\begin{tabular}{|c|c|c|}
\hline $10-11$ & 1630 & 0930 \\
\hline $11-14$ & 1045 & 1345 \\
\hline $11-15$ & 1045 & 0945 \\
\hline $14-15$ & 1445 & 0945 \\
\hline $15-18$ & 1045 & 0945 \\
\hline $22-23$ & 1115 & 1915 \\
\hline $23-25$ & 2015 & 1015 \\
\hline $25-30$ & 1035 & 0935 \\
\hline $30-31$ & 1030 & 2130 \\
\hline \multicolumn{3}{|l|}{ MAY 31- } \\
\hline JUN 02 & 2230 & 0930 \\
\hline $02-05$ & 1115 & 1015 \\
\hline $05-08$ & 1045 & 0945 \\
\hline $08-09$ & 1050 & 1750 \\
\hline $09-12$ & 1850 & 0950 \\
\hline $12-13$ & 1115 & 0415 \\
\hline $13-15$ & 0515 & 1015 \\
\hline $15-16$ & 1100 & 1400 \\
\hline $16-19$ & 1500 & 1000 \\
\hline $19-20$ & 1100 & 0700 \\
\hline $20-22$ & 0800 & 0900 \\
\hline $22-26$ & 0945 & 0845 \\
\hline $26-28$ & 1100 & 0100 \\
\hline $28-29$ & 0200 & 1000 \\
\hline \multicolumn{3}{|l|}{ JUL } \\
\hline $17-20$ & 1045 & 0945 \\
\hline $20-24$ & 1040 & 0940 \\
\hline \multicolumn{3}{|l|}{ AUG } \\
\hline $03-05$ & 1230 & 1530 \\
\hline $05-07$ & 1630 & 1130 \\
\hline $14-15$ & 1050 & 1850 \\
\hline $15-17$ & 1950 & 0950 \\
\hline $24 \ldots$ & 1000 & - \\
\hline \multicolumn{3}{|l|}{ AUG 31- } \\
\hline SEP 01 & 1055 & 1555 \\
\hline $01-05$ & 1655 & 0955 \\
\hline $14-16$ & 1040 & 1340 \\
\hline $14-18$ & 1040 & 0940 \\
\hline $16-18$ & 1440 & 0940 \\
\hline $21-22$ & 1030 & 2130 \\
\hline $22-25$ & 2230 & 0930 \\
\hline 28 . & 105 & - \\
\hline
\end{tabular}

$\mathrm{OCT}$

$\begin{array}{crrr}13-14 & 1155 & 0755 & 12 \\ 16 \ldots & 1130 & -- & 17 \\ 17-18 & 1300 & 1200 & 66 \\ \text { NOV } & & & \\ 06-07 & 1000 & 1500 & 14 \\ 07-09 & 1600 & 0900 & 29 \\ 14-16 & 2000 & 1000 & 23 \\ \text { DEC } & & & \\ 11-14 & 1130 & 1030 & 5.8 \\ \text { JAN } & & & \\ 19 \ldots & 1030 & -- & 58 \\ 22-25 & 1100 & 1000 & 34 \\ 25-29 & 1035 & 0935 & 27 \\ \text { FEB } & & & \\ 20-22 & 1155 & 1655 & 54 \\ 22-23 & 1755 & 1055 & 277 \\ \text { MAR } & & & \\ 01-05 & 1035 & 0930 & 47 \\ 01 \ldots & 1425 & -- & 44 \\ 08-10 & 1150 & 1050 & 42\end{array}$

\section{WATER YEAR OCTOBER 1988 TO SEPTEMBER 1989-continued}

\begin{tabular}{|c|c|c|c|c|c|c|c|c|c|}
\hline 12 & -- & -- & 0.06 & -- & 1.10 & 0.085 & 0.012 & 120 & 44 \\
\hline 8.2 & -- & -- & 0.06 & -- & 1.40 & 0.120 & 0.014 & 110 & 42 \\
\hline-- & -- & -- & -- & 1.4 & -- & -- & $\cdots$ & -- & \\
\hline 12 & -- & -- & 0.04 & -- & 1.20 & 0.105 & 0.010 & 120 & 49 \\
\hline 3.3 & -- & -- & 0.04 & 1.5 & 1.10 & 0.055 & 0.006 & 130 & 46 \\
\hline 5.2 & -- & -- & 0.06 & 0.72 & 1.00 & 0.065 & 0.008 & 110 & 59 \\
\hline 8.5 & -- & -- & 0.05 & 0.83 & 1.00 & 0.075 & 0.007 & 110 & 51 \\
\hline 13 & -- & -- & 0.03 & 0.95 & 1.10 & 0.070 & 0.011 & 110 & 62 \\
\hline 20 & -- & -- & 0.11 & 1.1 & 0.85 & 0.170 & 0.018 & 88 & 45 \\
\hline 50 & 154 & 24 & 0.09 & 1.8 & 0.78 & 0.215 & 0.018 & 91 & 45 \\
\hline 6.8 & -- & -- & 0.10 & 1.2 & 1.00 & 0.125 & 0.022 & 120 & 110 \\
\hline 16 & -- & -- & 0.04 & 1.1 & 1.10 & 0.130 & 0.018 & 120 & 67 \\
\hline 6.2 & -- & -- & 0.11 & 1.6 & 1.00 & 0.115 & 0.030 & 120 & 67 \\
\hline 34 & 109 & 15 & 0.09 & 1.5 & 0.81 & 0.275 & 0.030 & 98 & 54 \\
\hline 150 & 57 & 8 & 0.04 & 1.0 & 1.10 & 0.165 & 0.022 & 120 & 65 \\
\hline 30 & 58 & 9 & 0.05 & 1.2 & 0.89 & 0.145 & 0.014 & 100 & 48 \\
\hline 13 & -- & -- & 0.07 & 1.0 & 1.00 & 0.160 & 0.024 & 120 & 59 \\
\hline 55 & 143 & 21 & 0.05 & 1.8 & 1.10 & 0.240 & 0.025 & 93 & 46 \\
\hline 17 & -- & -- & 0.07 & 1.2 & 1.40 & 0.140 & 0.028 & 110 & 46 \\
\hline 34 & 102 & 15 & 0.06 & 1.6 & 1.00 & 0.130 & 0.033 & 74 & 25 \\
\hline 19 & -- & -- & 0.06 & 1.3 & 1.30 & 0.155 & 0.030 & 110 & 54 \\
\hline 21 & -- & -- & 0.05 & 1.2 & 1.30 & 0.190 & 0.034 & 110 & 53 \\
\hline 22 & -- & -- & 0.04 & 1.5 & 1.40 & 0.145 & 0.024 & 110 & 54 \\
\hline 18 & -- & -- & 0.04 & 0.99 & 0.64 & 0.150 & 0.013 & 120 & 100 \\
\hline 6.5 & -- & -- & 0.08 & 0.72 & 0.66 & 0.110 & 0.017 & 120 & 100 \\
\hline 25 & -- & -- & 0.11 & 1.3 & 0.38 & 0.170 & 0.011 & 98 & 91 \\
\hline 30 & 58 & 10 & 0.04 & 1.1 & 0.44 & 0.130 & 0.013 & 94 & 86 \\
\hline 6.4 & -- & -- & 0.03 & 0.89 & 0.56 & 0.155 & 0.020 & 110 & 92 \\
\hline 5.0 & -- & -- & 0.04 & 0.96 & 0.58 & 0.150 & 0.013 & 110 & 87 \\
\hline 4.0 & -- & -- & 0.04 & 0.48 & 0.71 & 0.060 & 0.022 & 150 & 110 \\
\hline 21 & -- & -- & 0.04 & 1.2 & 0.63 & 0.165 & 0.022 & 110 & 110 \\
\hline 24 & -- & -- & 0.03 & 1.3 & 0.63 & 0.165 & 0.017 & 120 & 83 \\
\hline 30 & 75 & 15 & -- & -- & -- & 0.170 & 0.025 & 71 & 64 \\
\hline-- & -- & -- & 0.04 & -- & 0.70 & -- & -- & -- & \\
\hline 17 & -- & -- & -- & -- & - & 0.120 & 0.018 & 86 & 67 \\
\hline 8.0 & -- & -- & -- & 0.58 & -- & 0.090 & 0.018 & 170 & 110 \\
\hline 24 & - & - & -- & 0.82 & -- & 0.140 & 0.015 & 92 & 68 \\
\hline & 28 & 40 & 0.03 & 0.83 & 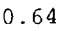 & 0.380 & 0.010 & 98 & 110 \\
\hline
\end{tabular}

\section{WATER YEAR OCTOBER 1989 TO SEPTEMBER 1990}

\begin{tabular}{|c|c|c|c|c|c|c|c|c|c|}
\hline 3.7 & -- & - & -- & 0.54 & -- & 0.095 & 0.012 & 94 & 88 \\
\hline 4.1 & - & - & -- & 0.42 & $\ldots$ & 0.065 & 0.014 & 95 & 75 \\
\hline 19 & -- & -- & -- & -- & - & 0.160 & 0.017 & 110 & 76 \\
\hline 20 & -- & -- & -- & -- & -- & 0.140 & 0.008 & 140 & 100 \\
\hline 39 & 129 & 15 & -- & -- & -- & 0.200 & 0.011 & 110 & 75 \\
\hline 8.6 & -- & -- & -- & -- & -. & 0.090 & 0.007 & 140 & 83 \\
\hline 2.1 & - & -- & - & -- & 1.10 & 0.065 & 0.005 & 360 & 110 \\
\hline 26 & -- & -- & 0.22 & 1.2 & 2.10 & 0.095 & 0.022 & 270 & 98 \\
\hline 6.8 & -- & -- & 0.13 & 0.94 & 1.90 & 0.065 & 0.007 & 460 & 100 \\
\hline 6.5 & -- & -- & 0.08 & 0.75 & 1.90 & 0.050 & 0.007 & 330 & 100 \\
\hline 29 & -- & -- & 0.05 & 1.5 & 1.60 & 0.140 & 0.006 & 270 & 81 \\
\hline 70 & 192 & 28 & 0.04 & 1.4 & 1.10 & 0.300 & 0.015 & 150 & 28 \\
\hline 13 & - & - & -- & 0.61 & $\cdots$ & 0.060 & 0.007 & 270 & 84 \\
\hline 6.5 & -- & -- & 0.09 & 0.65 & 1.90 & 0.055 & 0.009 & 340 & 90 \\
\hline 6.0 & -- & -- & 0.03 & -- & 1.70 & 0.055 & 0.007 & 240 & 99 \\
\hline
\end{tabular}


Surface-Water Stations

\section{A. Discharge and water quality}

04232050 Allen Creek near Rochester, N.Y.

\begin{tabular}{|c|c|c|c|c|c|c|c|c|c|c|c|c|c|}
\hline & & & $\begin{array}{c}\text { DIS- } \\
\text { CHARGE, } \\
\text { IN } \\
\text { CUBIC }\end{array}$ & TUR- & $\begin{array}{l}\text { RESIDUE } \\
\text { TOTAL } \\
\text { AT } 105 \\
\text { DEG. C, }\end{array}$ & $\begin{array}{l}\text { RESIDUE } \\
\text { VOLA- } \\
\text { TILE, }\end{array}$ & $\begin{array}{l}\text { NITRO- } \\
\text { GEN, } \\
\text { AMMONIA } \\
\text { DIS- }\end{array}$ & $\begin{array}{l}\text { NITRO- } \\
\text { GEN, AM- } \\
\text { MONIA + } \\
\text { ORGANIC }\end{array}$ & $\begin{array}{c}\mathrm{NITRO}- \\
\text { GEN, } \\
\mathrm{NO}_{2}+\mathrm{NO}_{3}\end{array}$ & $\begin{array}{l}\text { PHOS- } \\
\text { PHORUS }\end{array}$ & $\begin{array}{l}\text { PHOS- } \\
\text { PHORUS } \\
\text { ORTHO, } \\
\text { DIS- }\end{array}$ & $\begin{array}{l}\text { CHLO- } \\
\text { RIDE, } \\
\text { DIS- }\end{array}$ & $\begin{array}{l}\text { SULFATE } \\
\text { DIS- }\end{array}$ \\
\hline ATE & TIME & $\begin{array}{l}\text { ENDING } \\
\text { TIME }\end{array}$ & $\begin{array}{c}\text { FEET } \\
\text { PER } \\
\text { SECOND }\end{array}$ & $\begin{array}{l}\text { BID- } \\
\text { ITY } \\
\text { (NTU) }\end{array}$ & $\begin{array}{c}\text { SUS- } \\
\text { PENDED } \\
(\mathrm{mg} / \mathrm{L})\end{array}$ & $\begin{array}{l}\text { SUS - } \\
\text { PENDED } \\
(\mathrm{mg} / \mathrm{L})\end{array}$ & $\begin{array}{l}\text { SOLVED } \\
\text { (mg/L } \\
\text { as } \mathrm{N})\end{array}$ & $\begin{array}{l}\text { TOTAL } \\
\text { (mg/L } \\
\text { as N) }\end{array}$ & $\begin{array}{l}\text { TOTAL } \\
\text { (mg/L } \\
\text { as N) }\end{array}$ & $\begin{array}{l}\text { TOTAL } \\
\text { (mg/L } \\
\text { as P) }\end{array}$ & $\begin{array}{l}\text { SOLVED } \\
(\mathrm{mg} / \mathrm{L} \\
\text { as P) }\end{array}$ & $\begin{array}{l}\text { SOLVED } \\
\text { (mg/L } \\
\text { as Cl) }\end{array}$ & $\begin{array}{c}\text { SOLVED } \\
(\mathrm{mg} / \mathrm{L} \\
\left.\text { as } \mathrm{SO}_{4}\right)\end{array}$ \\
\hline
\end{tabular}

\begin{tabular}{|c|c|c|c|}
\hline \multicolumn{4}{|c|}{ MAR-cont inued } \\
\hline $10-12$ & 1150 & 1050 & 118 \\
\hline $19-22$ & 1120 & 1020 & 56 \\
\hline \multicolumn{4}{|l|}{ APR } \\
\hline $02-05$ & 1100 & 1000 & 151 \\
\hline $05-09$ & 1025 & 0925 & 10 \\
\hline $09-12$ & 1105 & 1005 & 148 \\
\hline $19-20$ & 1110 & 1110 & 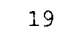 \\
\hline \multicolumn{4}{|l|}{ MAY } \\
\hline $03-04$ & 1010 & 1710 & 1 \\
\hline $04-07$ & 1810 & 0910 & 5 \\
\hline $10-13$ & 1055 & 0555 & 2 \\
\hline $13-14$ & 0655 & 0955 & 10 \\
\hline $17 \ldots$ & 1110 & -- & 2 \\
\hline \multicolumn{4}{|l|}{ JUN } \\
\hline $21 \ldots$ & 1030 & -- & \\
\hline $25 \ldots$ & 1145 & $-\rightarrow$ & \\
\hline \multicolumn{4}{|l|}{ JUL } \\
\hline $02-04$ & 1115 & 1615 & 1 \\
\hline $04-05$ & 1715 & 1015 & \\
\hline $05-08$ & 1130 & 2230 & \\
\hline $08-09$ & 2330 & 1030 & \\
\hline \multicolumn{4}{|l|}{ AUG } \\
\hline $02-05$ & 1015 & 0515 & \\
\hline $05-06$ & 0615 & 0915 & 1 \\
\hline $06-09$ & 1210 & 1110 & \\
\hline $09-12$ & 1130 & 1430 & \\
\hline $12-13$ & 1530 & 1030 & \\
\hline \multicolumn{4}{|l|}{ AUG 31- } \\
\hline SEP 04 & 1230 & 1130 & \\
\hline $04-05$ & 1230 & 0730 & \\
\hline $05-06$ & 0830 & 1130 & \\
\hline $06-07$ & 1255 & 0755 & \\
\hline $07-10$ & 0855 & 1155 & \\
\hline
\end{tabular}

WATER YEAR OCTOBER 1989 TO SEPTEMBER 1990-continued

\begin{tabular}{|c|c|c|c|c|c|c|c|c|c|}
\hline 28 & -. & - & $<0.01$ & -- & 1.30 & 0.215 & 0.010 & 160 & 57 \\
\hline 19 & -- & $\cdots$ & 0.05 & -- & 1.30 & 0.125 & 0.005 & 210 & 84 \\
\hline 33 & -- & -- & 0.06 & 1.1 & 1.00 & 0.165 & 0.006 & 160 & 65 \\
\hline 21 & -. & - & 0.03 & 0.90 & 1.50 & 0.100 & 0.007 & 160 & 51 \\
\hline 39 & 90 & 13 & 0.02 & 0.98 & 1.40 & 0.150 & 0.008 & 140 & 50 \\
\hline 6.1 & -- & -- & 0.04 & 0.80 & 1.40 & 0.055 & 0.003 & 190 & 62 \\
\hline 7.6 & - & - & 0.02 & 1.0 & 0.93 & 0.080 & 0.004 & 150 & 55 \\
\hline 23 & -- & -- & 0.02 & 1.1 & 0.92 & 0.110 & 0.004 & 110 & 43 \\
\hline 6.8 & -- & -. & 0.06 & 0.79 & 1.00 & 0.075 & 0.005 & 140 & 62 \\
\hline 65 & 164 & 24 & 0.02 & 1.5 & 0.84 & 0.320 & 0.008 & 75 & 34 \\
\hline 65 & 211 & 30 & 0.01 & 1.7 & 0.86 & 0.380 & 0.030 & 63 & 42 \\
\hline 8.1 & - & - & 0.03 & 0.90 & 1.10 & 0.085 & 0.028 & 120 & 110 \\
\hline 19 & - & $\cdots$ & 0.03 & 0.65 & 1.00 & 0.085 & 0.029 & 130 & 93 \\
\hline 4.7 & $\cdots$ & - & 0.04 & 0.63 & 0.76 & 0.050 & 0.007 & 120 & 93 \\
\hline 40 & 112 & 21 & 0.05 & 1.1 & 0.88 & 0.200 & 0.011 & 65 & 60 \\
\hline 5.0 & -- & -- & 0.02 & -- & 0.72 & 0.300 & 0.009 & 120 & 76 \\
\hline 6.5 & -- & -- & 0.02 & $\cdots$ & 0.62 & 0.075 & 0.006 & 120 & 80 \\
\hline 4.5 & -- & $\cdots$ & 0.03 & 0.48 & 0.52 & 0.050 & 0.015 & 120 & 86 \\
\hline 45 & 70 & 12 & 0.06 & 1.2 & 0.32 & 0.100 & 0.016 & 52 & 36 \\
\hline 12 & -- & -. & 0.03 & 0.84 & 0.58 & 0.075 & 0.014 & 110 & 80 \\
\hline 5.5 & -- & - & 0.03 & 0.66 & 0.70 & 0.070 & 0.019 & 120 & 100 \\
\hline 24 & 55 & 11 & 0.02 & 1.0 & 0.69 & 0.160 & 0.009 & 78 & 43 \\
\hline 8.6 & -- & -- & -- & 0.90 & 0.62 & 0.090 & 0.016 & 110 & 100 \\
\hline 5.9 & -- & -- & 0.07 & 0.73 & 0.47 & 0.075 & 0.016 & 100 & 86 \\
\hline 31 & 62 & 14 & 0.05 & 1.3 & 0.71 & 0.190 & 0.014 & 57 & 60 \\
\hline 22 & -- & -- & 0.09 & 0.81 & 0.59 & 0.140 & 0.021 & 97 & N75 \\
\hline 19 & -- & - & 0.05 & 0.74 & 0.59 & 0.100 & 0.019 & 94 & 58 \\
\hline
\end{tabular}

OCT

$\begin{array}{rrrr}09-11 & 1300 & 1200 & 54 \\ 11-13 & 1225 & 0325 & 88 \\ 13-15 & 0425 & 1125 & 61 \\ 22-23 & 1210 & 0810 & 27 \\ 23-25 & 0910 & 1110 & 50 \\ \text { NOV } & & & \\ 05-09 & 1200 & 1000 & 30 \\ 09-10 & 0950 & 0050 & 12 \\ 10-13 & 0150 & 0850 & 53 \\ \text { DEC } & & & \\ 03-04 & 1000 & 0600 & 42 \\ 04-05 & 0700 & 0500 & 137 \\ 12-16 & 1105 & 1005 & 22 \\ 16-17 & 1105 & 1005 & 44 \\ 18-18 & 0920 & 1720 & 55 \\ 18-20 & 1820 & 1020 & 106 \\ 29-30 & 0805 & 1805 & 274 \\ \text { DEC } 30- & & & \\ \text { JAN } 02 & 1905 & 1105 & 156 \\ 02-04 & 1200 & 1100 & 33 \\ 14-15 & 1350 & 1550 & 13 \\ 15-17 & 1650 & 1250 & 77 \\ 17-22 & 1315 & 1215 & 64 \\ \text { FEB } & & & \\ 04-07 & 1225 & 1125 & 64 \\ 07-11 & 1225 & 1125 & 38 \\ 19-20 & 1245 & 1145 & 107 \\ 20-21 & 1245 & 1145 & 48\end{array}$

WATER YEAR OCTOBER 1990 TO SEPTEMBER 1991

\begin{tabular}{|c|c|c|c|c|c|c|c|c|}
\hline 22 & -- & -- & 0.02 & 0.66 & 0.45 & 0.110 & 0.055 & 80 \\
\hline 19 & -- & -- & 0.05 & 0.70 & 0.54 & 0.095 & 0.012 & 74 \\
\hline 15 & -- & - & 0.03 & 0.66 & 1.10 & 0.075 & 0.012 & 75 \\
\hline 9.9 & -- & -- & 0.05 & 0.80 & 0.82 & 0.065 & 0.011 & 94 \\
\hline 16 & - & - & 0.03 & 0.72 & 0.98 & 0.075 & 0.013 & 87 \\
\hline 10 & - & -- & 0.03 & 0.82 & 1.10 & 0.065 & 0.013 & 120 \\
\hline 10 & -- & -- & 0.02 & 0.78 & 1.30 & 0.070 & 0.012 & 140 \\
\hline 14 & -- & -- & 0.01 & 0.82 & 1.20 & 0.075 & 0.016 & 180 \\
\hline 10 & -- & -- & -- & -- & -- & 0.065 & 0.008 & 300 \\
\hline 25 & -. & -- & -- & -- & -- & 0.140 & 0.023 & 130 \\
\hline 5.9 & -- & -- & -- & -. & -- & 0.035 & 0.006 & 210 \\
\hline 17 & - & -- & -- & -- & -- & 0.065 & 0.010 & 130 \\
\hline 10 & -- & -- & -- & -- & -- & 0.045 & 0.008 & 140 \\
\hline 26 & -- & -- & -- & $\cdots$ & -- & 0.110 & 0.014 & 100 \\
\hline 33 & 69 & 13 & 0.02 & 0.98 & 1.00 & 0.150 & 0.018 & 170 \\
\hline 19 & -- & -- & 0.02 & 0.73 & 1.60 & 0.090 & 0.018 & 130 \\
\hline 7.2 & -- & -- & 0.03 & 0.66 & 1.90 & 0.050 & 0.013 & 230 \\
\hline 4.5 & -- & -- & -- & -- & - & 0.035 & 0.010 & 330 \\
\hline 18 & -- & -- & -- & -. & -- & 0.095 & 0.014 & 330 \\
\hline 12 & - & -- & -- & $\cdots$ & -- & 0.075 & 0.016 & 200 \\
\hline 8.7 & -- & -. & 0.02 & 0.82 & 1.30 & 0.070 & 0.009 & 200 \\
\hline 6.2 & -- & -- & -- & - & $\ldots$ & 0.050 & 0.007 & 200 \\
\hline 30 & 85 & 14 & -- & -- & $\ldots$ & 0.150 & 0.013 & 210 \\
\hline 6.6 & -- & -- & -- & -- & -- & 0.060 & 0.006 & 210 \\
\hline
\end{tabular}


04232050 Allen Creek near Rochester, N.Y.

2. WATER-QUALITY RECORDS

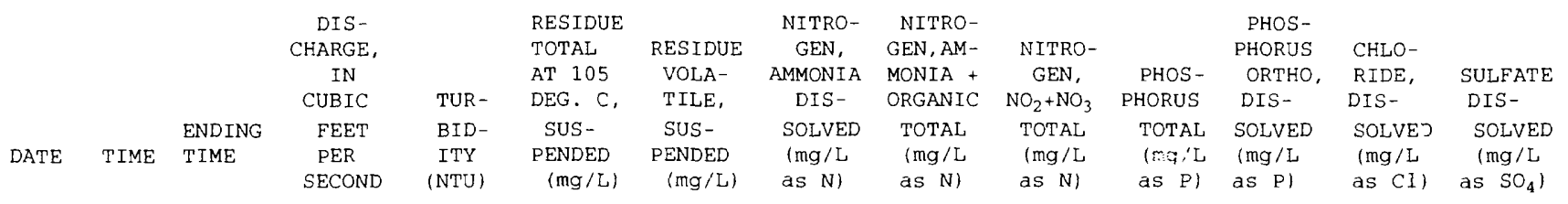

MAR

$\begin{array}{rrrr}02-04 & 2005 & 1105 & 429 \\ 06 & 1245 & -- & 91 \\ 06-08 & 1300 & 1100 & 104 \\ 08-11 & 1130 & 1030 & 29 \\ 22-23 & 1620 & 1520 & 51 \\ 23-25 & 1620 & 1120 & 89 \\ 27-27 & 0630 & 1730 & 124\end{array}$

MAR 28 -

$\begin{array}{llll}A P R & 01 & 1215 & 1115\end{array}$

08-09 $1150 \quad 0150 \quad 38$

09-10 $0250 \quad 0150 \quad 64$

$\begin{array}{llll}10-11 & 0250 & 1050 & 42 \\ 11-14 & 1215 & 1915 & 14\end{array}$

19-22 $2350 \quad 0250 \quad 128$

$\begin{array}{llll}22-22 & 0350 & 1050 & 831\end{array}$

$\begin{array}{llll}22-25 & 1110 \quad 1010 \quad 145\end{array}$

MAY

$\begin{array}{llll}16-17 & 1105 & 1005 & 10\end{array}$

17-17 $1105 \quad 2205 \quad 31$

$\begin{array}{lll}17-20 & 2305 & 1005\end{array}$

26-26 $1320 \quad 2220$

$26-28 \quad 2320 \quad 0920$

JUN

$11-12 \quad 1415 \quad 0715$

$12-12 \quad 0815 \quad 1315$

$12-13 \quad 1415 \quad 1015$ JUL

01-04 $1115 \quad 1415$

$04-05 \quad 1515 \quad 1015$

$05-05 \quad 1050 \quad 2150$

05-07 $2250 \quad 0350$

$07-07 \quad 0450 \quad 1250$

07-08 $1350 \quad 0950$

08-09 $1105 \quad 1905$

$09-11$

$2005 \quad 1005$

03-03 $0345 \quad 1445$

03-05 $1545 \quad 1045$

09-12 $0730 \quad 1030$

SE

15-16 $0820 \quad 1120$

$23-24 \quad 1240 \quad 2340$

25-26 $0040 \quad 1140$

\section{WATER YEAR OCTOBER 1990 TO SEPTEMBER 1991-continued}

\begin{tabular}{|c|c|c|c|c|c|c|c|c|c|}
\hline 120 & 260 & 10 & 0.03 & 1.7 & 0.90 & 0.420 & 0.017 & 150 & 25 \\
\hline 11 & -- & -- & 0.01 & 0.77 & 1.50 & 0.070 & 0.015 & 140 & 42 \\
\hline 18 & -- & -- & -- & $\cdots$ & -- & 0.100 & 0.011 & 140 & 45 \\
\hline 4.1 & -- & -- & -- & -- & -- & 0.040 & 0.006 & 170 & 60 \\
\hline 18 & -- & -- & -- & -- & -- & 0.100 & 0.005 & 150 & 61 \\
\hline 28 & -- & -- & -- & + & -- & 0.120 & 0.007 & 130 & 41 \\
\hline 40 & 91 & 16 & - & -- & -- & 0.170 & 0.009 & 100 & 41 \\
\hline 50 & 73 & 12 & -- & -- & -- & 0.190 & 0.015 & 89 & 33 \\
\hline 7.7 & -- & -- & -- & -- & -- & 0.055 & 0.006 & 150 & 42 \\
\hline 17 & -- & -- & -- & -- & -- & 0.100 & 0.006 & 120 & 98 \\
\hline 20 & -- & -- & -- & -- & -- & 0.095 & 0.008 & 100 & 90 \\
\hline 16 & -- & -- & -- & -- & -- & 0.095 & 0.008 & 120 & 92 \\
\hline 3.5 & $\cdots$ & -- & -- & -- & -- & 0.030 & 0.004 & 150 & 64 \\
\hline 23 & -- & -- & 0.02 & 1.0 & 0.88 & 0.100 & 0.006 & 98 & 4 \\
\hline 75 & 105 & 15 & 0.03 & 1.2 & 0.70 & 0.230 & 0.021 & 37 & 14 \\
\hline 15 & -- & -- & 0.02 & 0.92 & 1.20 & 0.100 & 0.011 & 94 & 39 \\
\hline 2.9 & -- & -- & -- & 0.91 & 1.00 & 0.045 & 0.004 & 130 & 91 \\
\hline 17 & -- & -- & -- & -- & -- & 0.140 & 0.005 & 100 & 70 \\
\hline 8.5 & -- & -- & -- & -- & -- & 0.060 & 0.005 & 120 & 61 \\
\hline 26 & -- & -- & -- & -- & -- & 0.200 & 0.014 & 120 & 80 \\
\hline 26 & -- & -- & -- & -- & -- & 0.160 & 0.014 & 77 & 52 \\
\hline 21 & $\cdots$ & -- & 0.06 & -- & 1.60 & 0.150 & 0.018 & 110 & 72 \\
\hline 31 & 130 & 24 & 0.07 & -- & 1.30 & 0.280 & 0.022 & 76 & 60 \\
\hline 16 & -- & -- & 0.07 & -- & 1.60 & 0.340 & 0.028 & 64 & 56 \\
\hline 7.5 & -- & -- & $<0.01$ & 1.0 & 0.76 & 0.130 & 0.016 & 120 & 110 \\
\hline 19 & -- & -- & $<0.01$ & 1.0 & 0.84 & 0.150 & 0.011 & 90 & 90 \\
\hline 32 & 62 & 13 & 0.05 & 0.71 & 0.91 & 0.190 & 0.022 & 69 & 80 \\
\hline 20 & 46 & 9 & 0.06 & 0.46 & 0.64 & 0.180 & 0.020 & 79 & 64 \\
\hline 20 & -- & -- & -- & -- & -- & 0.110 & 0.016 & 81 & 80 \\
\hline 9.0 & -- & -- & -- & -- & -- & 0.090 & 0.013 & 76 & 84 \\
\hline 6.0 & -- & -- & 0.03 & 0.53 & 0.57 & 0.050 & 0.010 & 110 & 92 \\
\hline 3.0 & -- & -- & 0.03 & 0.57 & 0.64 & 0.035 & 0.010 & 120 & 92 \\
\hline 21 & -- & -- & 0.04 & 1.2 & 0.82 & 0.160 & 0.023 & 95 & 80 \\
\hline 11 & -- & -- & 0.02 & 0.74 & 0.80 & 0.100 & 0.016 & 85 & 72 \\
\hline 18 & -- & -- & 0.03 & -- & 0.60 & 0.130 & 0.030 & 96 & 76 \\
\hline 18 & -- & -- & 0.03 & 0.74 & 0.85 & 0.130 & 0.018 & 80 & 46 \\
\hline 6.1 & -- & -- & 0.02 & 0.78 & 0.65 & 0.060 & 0.018 & 110 & 90 \\
\hline 27 & -- & -- & $<0.01$ & 0.93 & 0.59 & 0.140 & 0.018 & 59 & 56 \\
\hline
\end{tabular}

WATER YEAR OCTOBER 1991 TO SEPTEMBER 1992

OCT

NOV

$\begin{array}{llll}17-19 & 1220 & 0320 & 13\end{array}$

$28-29$

NOV $29-$

DEC 02

03-03

$03-05$

$29-29$

$29-30$

$30 \ldots$

6.1

0.01

$\begin{array}{lll}0.74 & 0.91 \quad 0.065\end{array}$

$0.013 \quad 120$

$2020 \quad 1020 \quad 37$

24

0.96

0.60

0.170

$0.015 \quad 220$

57

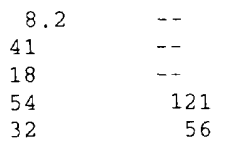

$\begin{array}{cc}-- & -- \\ - & 0.02 \\ - & 0.02 \\ 30 & 0.03 \\ 11 & 0.02\end{array}$

0.63

0.80

0.065

$0255 \quad 1355$

17
85

$1455 \quad 1055$

$0525 \quad 2025$

$2125 \quad 1225$

56

0.78

0.78

0.230

0.100

0.230
0.140

$0.014 \quad 150$

$0.016 \quad 480$

$0.018 \quad 260$

0.017350

0.025290

DEC $30-$

$1305 \quad 1205$

19

$23 .$.

$\begin{array}{rr}1140 & - \\ 1145 & 1345\end{array}$

9.3
15
25
11

--
--
--
--

$\begin{array}{cc}-- & 0.03 \\ -- & 0.11 \\ -- & --\end{array}$

$0.96 \quad 1.50$

0.075

$0.013 \quad 390$

$0.010 \quad 830$

0.011600

$0.011 \quad 520$

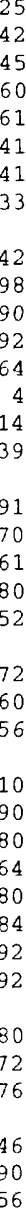

$24-25$

$\begin{array}{lll}1530 & 0130 & 29\end{array}$ 
04232050 Allen Creek near Rochester, N.Y.

\section{WATER-QUALITY RECORDS}

\begin{tabular}{|c|c|c|c|c|c|c|c|c|c|c|c|c|c|}
\hline & & & $\begin{array}{c}\text { DIS- } \\
\text { CHARGE, } \\
\text { IN } \\
\text { CUBIC }\end{array}$ & TUR- & $\begin{array}{l}\text { RESIDUE } \\
\text { TOTAL } \\
\text { AT } 105 \\
\text { DEG. C, }\end{array}$ & $\begin{array}{l}\text { RESIDUE } \\
\text { VOLA- } \\
\text { TILE, }\end{array}$ & $\begin{array}{l}\text { NITRO- } \\
\text { GEN, } \\
\text { AMMONIA } \\
\text { DIS- }\end{array}$ & $\begin{array}{l}\text { NITRO- } \\
\text { GEN, AM- } \\
\text { MONIA + } \\
\text { ORGANIC }\end{array}$ & $\begin{array}{c}\text { NITRO- } \\
\text { GEN } \\
\mathrm{NO}_{2}+\mathrm{NO}_{3}\end{array}$ & $\begin{array}{l}\text { PHOS- } \\
\text { PHORUS }\end{array}$ & $\begin{array}{l}\text { PHOS- } \\
\text { PHORUS } \\
\text { ORTHO, } \\
\text { DIS- }\end{array}$ & $\begin{array}{l}\text { CHLO- } \\
\text { RIDE, } \\
\text { DIS- }\end{array}$ & $\begin{array}{l}\text { SULFATE } \\
\text { DIS- }\end{array}$ \\
\hline DATE & TIME & $\begin{array}{l}\text { ENDING } \\
\text { TIME }\end{array}$ & $\begin{array}{c}\text { FEET } \\
\text { PER } \\
\text { SECOND }\end{array}$ & $\begin{array}{l}\text { BID- } \\
\text { ITY } \\
\text { (NTU) }\end{array}$ & $\begin{array}{c}\text { SUS- } \\
\text { PENDED } \\
(\mathrm{mg} / \mathrm{L})\end{array}$ & $\begin{array}{l}\text { SUS - } \\
\text { PENDED } \\
\text { (mg/L) }\end{array}$ & $\begin{array}{l}\text { SOLVED } \\
\text { (mg/L } \\
\text { as N) }\end{array}$ & $\begin{array}{l}\text { TOTAL } \\
(\mathrm{mg} / \mathrm{L} \\
\text { as N) }\end{array}$ & $\begin{array}{l}\text { TOTAL } \\
(\mathrm{mg} / \mathrm{L} \\
\text { as N) }\end{array}$ & $\begin{array}{l}\text { TOTAL } \\
\text { (mg/L } \\
\text { as P) }\end{array}$ & $\begin{array}{l}\text { SOLVED } \\
\text { (mg/L } \\
\text { as P) }\end{array}$ & $\begin{array}{l}\text { SOLVED } \\
\text { (mg/L } \\
\text { as C1) }\end{array}$ & $\begin{array}{l}\text { SOLVED } \\
(\mathrm{mg} / \mathrm{L} \\
\left.\text { as } \mathrm{SO}_{4}\right)\end{array}$ \\
\hline
\end{tabular}

FEB

$\begin{array}{lll}14-15 & 1035 & 0935\end{array}$

15-16 $1035 \quad 0935$

16-18 $1035 \quad 0935$

$18-19 \quad 1210 \quad 1510$

$19-20 \quad 1610 \quad 1010$

$\begin{array}{llr}20 \ldots & 1040 & -- \\ 20-22 & 1050 & 0950\end{array}$

$22-24 \quad 1050 \quad 0950$

$24-27 \quad 1120 \quad 1020$

$27-28 \quad 1040 \quad 1340$

$28-29$

MAR 29

$\begin{array}{lll}\text { MAR } 02 & 2240 \quad 0940\end{array}$

$\begin{array}{lll}05-07 & 1045 & 0145 \\ 07-08 & 0245 & 0145\end{array}$

$08-09 \quad 0245 \quad 0945$

$09-12 \quad 1155 \quad 1055$

$26-27$

$27-30$

MAR 30-

APR 02

$09-11$

$11-12$

$12-13$

$13-16$

$16-17$

$17-18$

$18-20$

APR $30-$

MAY 02

$02-03$

$03-04$

$04-07$

$30-31$

MAY 31 -

JUN 01

$01-01$
$01-04$

$05-06$

$06-08$

08-11

$19-19$

$19-22$

JUL

03-04 $0950 \quad 0050$

04-06 $0150 \quad 0850$

06-08 $1035 \quad 1535$

$\begin{array}{lll}08-09 & 1635 & 0935 \\ 09-12 & 1050 & 1350\end{array}$

AUG

03-04 $1130 \quad 1330$

$04-06 \quad 1430 \quad 1030$

24-24 $1045 \quad 2145$

$\begin{array}{lll}24-25 & 2245 & 0945\end{array}$

25-27 $1045 \quad 0945$

$27-28 \quad 1445 \quad 0945$

$28-31$

SEP

$03-03 \quad 0325 \quad 1425$

$03-04$

$04-08$

$18-19$

1525

0950

1440

$\begin{array}{ll}6.5 & \\ 48 & 5 \\ 30 & 16 \\ 36 & 34 \\ 48 & 25 \\ 39 & \\ 29 & \\ 38 & 19 \\ 27 & \\ 20 & \\ 47 & 31 \\ 21 & \\ 16 & \\ 71 & 4 \\ 53 & 33 \\ 37 & 11 \\ 330 & 140 \\ 179 & 40\end{array}$

WATER YEAR OCTOBER 1991 TO SEPTEMBER 1992-continued

$\begin{array}{cccc}-- & -- & - & 0.02 \\ 50 & 140 & 29 & 0.17 \\ 16 & -- & -- & 0.07 \\ 34 & 48 & 12 & 0.03 \\ 25 & -- & -- & 0.03 \\ -- & -- & -- & -- \\ 7.0 & -- & -- & -- \\ 19 & -- & -- & -- \\ 7.0 & -- & -- & 0.02 \\ 3.8 & -- & -- & 0.02 \\ 31 & 101 & 22 & 0.05 \\ & & & \\ 4.1 & -- & -- & 0.02 \\ 3.3 & -- & - & 0.01 \\ 45 & 113 & 22 & <0.01 \\ 33 & 70 & 14 & 0.02 \\ 11 & -- & -- & 0.02 \\ 140 & 449 & 207 & 0.05\end{array}$

$\begin{array}{lc}0.50 & 1.20 \\ 1.8 & 1.20 \\ 0.90 & 1.60 \\ 0.96 & 1.50 \\ 1.1 & 1.70 \\ -- & -- \\ 0.63 & 2.10 \\ 0.83 & 1.90 \\ 0.65 & 2.50 \\ 0.83 & 2.40 \\ 1.6 & 1.60 \\ & \\ 0.85 & 2.00 \\ 0.63 & -- \\ 1.2 & -- \\ 1.2 & -- \\ 1.0 & 1.80 \\ 2.4 & 1.40 \\ 1.3 & 1.80\end{array}$

0.035

0.260

0.085

0.120

0.140

0.045

0.075

0.050

0.045

0.170

0.060

0.035

0.160

0.120

0.065
0.660

0.200

$0.83 \quad 1.40$

0.170

$\begin{array}{ll}-- & 0.01 \\ -- & 0.02 \\ 28 & 0.04\end{array}$

$0.73 \quad 1.50$

$\begin{array}{ll}1.6 & 1.30 \\ 1.5 & 1.40\end{array}$

$\begin{array}{ll}1.5 & 1.40\end{array}$

$\begin{array}{ll}0.71 & 1.50 \\ 1.5 & 1.10 \\ 0.97 & 1.40\end{array}$

$0.97 \quad 1.40$

0.045

0.210

0.380

0.050

0.310

0.150

$\begin{array}{lll}0.94 & 1.40 & 0.120\end{array}$

$0.006 \quad 770$

$0.006 \quad 700$

$0.011 \quad 420$

$0.008 \quad 410$

$0.012 \quad 370$

$0.010 \quad 370$

$0.010 \quad 330$

$0.010 \quad 340$

$0.008 \quad 290$

$0.008 \quad 310$

$0.006 \quad 380$

$0.003 \quad 290$

0.003280

$0.008 \quad 230$

$0.006 \quad 250$

$0.012 \quad 180$

$0.014 \quad 230$

$0.008 \quad 130$

$0.003 \quad 250$

0.003200

$0.007 \quad 150$

$0.004 \quad 220$

$0.011 \quad 110$

$0.008 \quad 130$

$0.006 \quad 140$

-- 1.20

0.055

$0.002 \quad 170$

$0.014 \quad 86$

$0.011 \quad 110$

$0.004 \quad 140$

$0.009 \quad 120$

0.065

$0.011 \quad 96$

0.110

0.140

0.065

0.160

0.190

0.110

0.235

0.145

0.011

$0.013 \quad 140$

$0.015 \quad 130$

$0.015 \quad 110$

$0.017 \quad 160$

$0.014 \quad 120$

$0.020 \quad 120$

0.190

$0.012 \quad 100$

$\begin{array}{ll}0.012 & 100 \\ 0.015 & 100\end{array}$

$0.018 \quad 130$

$0.017 \quad 80$

$0.020 \quad 110$

0.230

0.120

0.04466

0.210

\section{$2.0 \quad 0.94$}

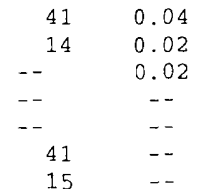

$\begin{array}{ll}1.3 & 1.20 \\ 0.52 & 0.91\end{array}$

0.210

0.055

0.400

0.180

0.240

$0.043 \quad 85$

$0.010 \quad 160$

$0.010 \quad 96$

$0.031 \quad 95$

$0.048 \quad 48$

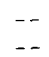

$--$

0.230

$\begin{array}{ccc}- & 1.40 & 0.155 \\ -- & 0.75 & 0.250 \\ -- & -- & 0.100 \\ -- & -- & 0.180 \\ -- & -- & 0.085\end{array}$

$0.030 \quad 100$

$0.038 \quad 55$

$0.035 \quad 130$

$0.020 \quad 100$

$0.024 \quad 99$
110

110

77

75

90

80

90

80

92

97

76

73

39

110

85

57

50

37

47$$
78
$$$$
\begin{aligned}
& 78 \\
& 37 \\
& 38
\end{aligned}
$$$$
38
$$$$
84
$$$$
100
$$$$
61
$$$$
73
$$$$
81
$$$$
77
$$$$
86
$$$$
81
$$ 


\section{Surface-Water Stations}

\section{A. Discharge and water quality}

\section{Allen Creek near Rochester, N.Y.}

\section{WATER-QUALITY RECORDS}

\begin{tabular}{|c|c|c|c|c|c|c|c|c|c|c|c|c|c|}
\hline & & & $\begin{array}{c}\text { DIS- } \\
\text { CHARGE, } \\
\text { IN } \\
\text { CUBIC }\end{array}$ & TUR - & $\begin{array}{l}\text { RESIDUE } \\
\text { TOTAL } \\
\text { AT } 105 \\
\text { DEG. C. }\end{array}$ & $\begin{array}{l}\text { RESIDUE } \\
\text { VOLA- } \\
\text { TILE, }\end{array}$ & $\begin{array}{l}\text { NITRO- } \\
\text { GEN, } \\
\text { AMMONIA } \\
\text { DIS- }\end{array}$ & $\begin{array}{l}\text { NITRO- } \\
\text { GEN, AM- } \\
\text { MONIA + } \\
\text { ORGANIC }\end{array}$ & $\begin{array}{c}\text { NITRO- } \\
\text { GEN, } \\
\mathrm{NO}_{2}+\mathrm{NO}_{3}\end{array}$ & $\begin{array}{l}\text { PHOS- } \\
\text { PHORUS }\end{array}$ & $\begin{array}{l}\text { PHOS- } \\
\text { PHORUS } \\
\text { ORTHO, } \\
\text { DIS- }\end{array}$ & $\begin{array}{l}\text { CHLO- } \\
\text { RIDI, } \\
\text { DIS- }\end{array}$ & $\begin{array}{l}\text { SULFATE } \\
\text { DIS- }\end{array}$ \\
\hline ATE & TIME & $\begin{array}{l}\text { END ING } \\
\text { T IME }\end{array}$ & $\begin{array}{c}\text { FEET } \\
\text { PER } \\
\text { SECOND }\end{array}$ & $\begin{array}{l}\text { BID- } \\
\text { ITY } \\
\text { (NTU) }\end{array}$ & $\begin{array}{l}\text { SUS- } \\
\text { PENDED } \\
\{\mathrm{mg} / \mathrm{L}\}\end{array}$ & $\begin{array}{l}\text { SUS- } \\
\text { PENDED } \\
(\mathrm{mg} / \mathrm{L})\end{array}$ & $\begin{array}{l}\text { SOLVED } \\
\text { (mg/L } \\
\text { as N) }\end{array}$ & $\begin{array}{l}\text { TOTAL } \\
\text { (mg/L } \\
\text { as N) }\end{array}$ & $\begin{array}{l}\text { TOTAL } \\
\text { (mg/L } \\
\text { as N) }\end{array}$ & $\begin{array}{c}\text { TOTAL } \\
\text { (mg/L } \\
\text { as P) }\end{array}$ & $\begin{array}{l}\text { SOLVED } \\
\text { (mg/L } \\
\text { as P) }\end{array}$ & $\begin{array}{l}\text { SOLVED } \\
\text { (mg/L } \\
\text { as Cl) }\end{array}$ & $\begin{array}{c}\text { SOLVED } \\
\left(\mathrm{mg} / L^{2}\right. \\
\left.\text { as } \mathrm{SO}_{4}\right)\end{array}$ \\
\hline
\end{tabular}

OCT

$\begin{array}{ccc}09-13 & 1015 & 0915 \\ 23-24 & 2320 & 2220 \\ 24-26 & 2320 & 1020 \\ \text { NOV } & & \\ 02-03 & 1430 & 0730 \\ 03-05 & 0830 & 1130 \\ 12-13 & 1120 & 0620 \\ 13-16 & 0720 & 1020 \\ \text { DEC } & & \\ 10-11 & 1140 & 1840 \\ 16-17 & 0645 & 1145 \\ 17-17 & 1210 & 2310 \\ 18-21 & 0010 & 1110 \\ 29-31 & 1700 & 1000 \\ \text { DC } 31- & & \\ \text { JAN } 03 & 1030 & 0530 \\ 03-04 & 0630 & 0930 \\ 04-05 & 1155 & 0755 \\ 05-07 & 0855 & 1055 \\ 21-22 & 1135 & 1835 \\ 22-25 & 1935 & 1035 \\ 25-28 & 1155 & 0900 \\ \text { EB } & & \\ 04-06 & 1245 & 0145 \\ \text { MAR } & & \\ 22-25 & 1025 & 0925 \\ 25-29 & 1020 & 0920 \\ 29-30 & 1040 & 0040 \\ \text { MAR 30- } & & \\ \text { APR 01 } & 0140 & 0940 \\ 01-02 & 1110 & 1510 \\ 02-05 & 1910 & 0910 \\ 05-08 & 1100 & 1035 \\ 08-10 & 1040 & 0940 \\ 20-24 & 1010 & 0910 \\ \text { MAY 31- } & & \\ \text { JUN 01 } & 2145 & 0845 \\ 05-05 & 0630 & 1730 \\ 05-07 & 1830 & 0930 \\ 19-20 & 0225 & 0925 \\ 20-21 & 1025 & 0925 \\ 21-24 & 1025 & 0925 \\ \text { UL } & & \\ 19-19 & 0240 & 0940 \\ 19-22 & 1235 & 1135 \\ 11-11 & 1730 & 2230 \\ 11-12 & 2330 & 1030 \\ 26-30 & 1025 & 0125 \\ \text { EP } & & \\ 02-03 & 1555 & 2055 \\ 03-06 & 2155 & 1355 \\ 26-27 & 0215 & 0915 \\ 09 & & \\ 175 & \end{array}$




\section{Surface-Water Stations}

\section{A. Discharge and water quality}

\section{Irondequoit Creek Above Blossom Road, Rochester, N.Y.}

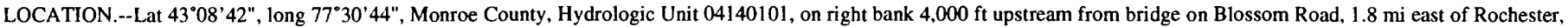

$1.7 \mathrm{mi}$ downstream from Allen Creek, and 4.4 mi upstream from mouth.

DRAINAGE AREA. - $142 \mathrm{mi}^{2}$, flow from $8.45 \mathrm{mi}^{2}$ noncontributing.

\section{WATER-DISCHARGE RECORDS}

PERIOD OF RECORD.--Occasional discharge measurements water years 1977-80. December 1980 to current year.

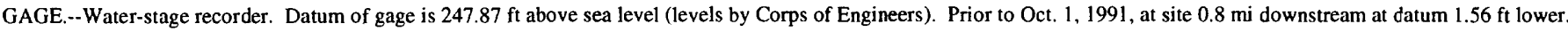

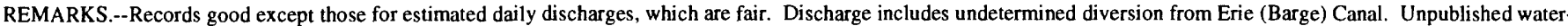
quality records for prior years are available in files of Monroe County Department of Health. Several measurements of water temperature were made durirg the year. COOPERATION.--Gage-height record and 8 discharge measurements were provided by the Monroe County Environmental Health Laboratory at Rochester, N.Y.

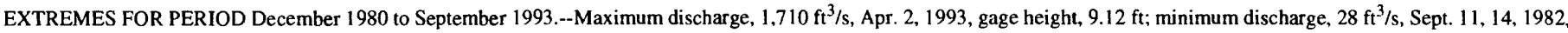
gage height, $1.69 \mathrm{ft}$.

STATISTICS OF MONTHLY MEAN DISCHARGE (in cubic feet per second) FOR WATER YEARS 1981-93, BY WATE? YEAR

\begin{tabular}{|c|c|c|c|c|c|c|c|c|c|c|c|c|}
\hline & OCT & NOV & DEC & JAN & FEB & MAR & APR & MAY & JUN & JUL & AUG & SEP \\
\hline MEAN & 87.9 & 123 & 148 & 131 & 173 & 211 & 239 & 148 & 93.7 & 72.3 & 85.9 & 73.9 \\
\hline MAX & 188 & 224 & 242 & 279 & 347 & 348 & 468 & 292 & 186 & 181 & 253 & 132 \\
\hline (WY) & 1987 & 1986 & 1987 & 1993 & 1981 & 1993 & 1993 & 1984 & 1989 & 1992 & 1992 & 1992 \\
\hline $\mathrm{MIN}$ & 39.5 & 54.5 & 49.5 & 60.8 & 67.1 & 122 & 90.8 & 67.8 & 46.9 & 42.2 & 40.8 & 44.6 \\
\hline (WY) & 1983 & 1992 & 1990 & 1989 & 1989 & 1988 & 1981 & 1982 & 1988 & 1983 & 1985 & 1991 \\
\hline
\end{tabular}

SUMMARY STATISTICS

STATISTIC

ANNUAL TOTAL

ANNUAL MEAN

AVERAGE DISCHARGE

HIGHEST ANNUAL MEAN

LOWEST ANNUAL MEAN

HIGHEST DAILY MEAN

LOWEST DAILY MEAN

ANNUAL SEVEN-DAY MINIMUM

INSTANTANEOUS PEAK FLOW

INSTANTANEOUS PEAK STAGE

INSTANTANEOUS LOW FLOW

10 PERCENT EXCEEDS

50 PERCENT EXCEEDS

90 PERCENT EXCEEDS

\section{FOR 1992 CALENDAR YEAR}

60905

166

FOR 1993 WATER YEAR

WATER YEAFS 1981 - 1993 66550

$\begin{aligned} 970 & \text { Mar } 28 \\ 39 & \text { Feb } 9 \\ 44 & \text { Feb } \quad 6\end{aligned}$

a Sept. 11, 14, 1982

182

182

\begin{tabular}{|c|c|c|}
\hline \multicolumn{3}{|l|}{134} \\
\hline 182 & & 1993 \\
\hline 98.2 & & 1988 \\
\hline 1630 & Apr & 21993 \\
\hline 29 & Aug 2 & 201985 \\
\hline 30 & Aug 1 & 171985 \\
\hline 1710 & Apr & 21993 \\
\hline 9.12 & Apr & 21993 \\
\hline 28 & $\mathbf{a}$ & \\
\hline 259 & & \\
\hline 90 & & \\
\hline 46 & & \\
\hline
\end{tabular}

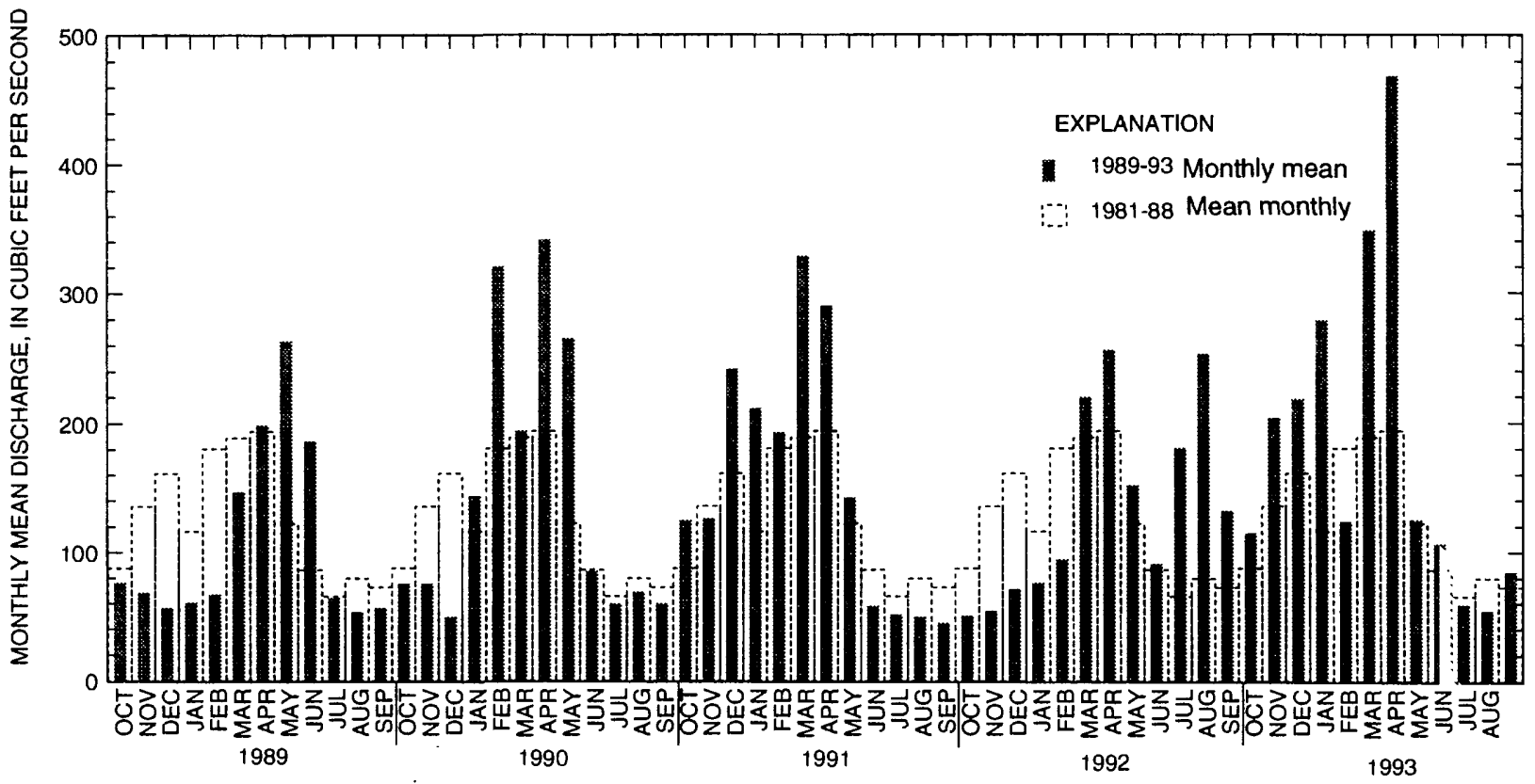

62 Water Resources of Monroe County, N.Y., 1989-93, with emphasis on Water Quality in the Irondequoit Creek Basin 


\section{Irondequoit Creek Above Blossom Road, Rochester, N.Y.}

\section{WATER-QUALITY RECORDS}

PERIOD OF RECORD.--OCtober 1983 to current year.

CHEMICAL DATA: 1983-93 (e) .

NUTRIENT DATA: 1983-93 (e) .

COOPERATION. - Water-quality samples were collected and analyzed by the Monroe County Environmental Health

Laboratory at Rochester, N.Y.

REMARKS.--Prior to 1994 water year, data published in WATER RESOURCES OF MONROE COUNTY NEW YORK, WATER YEARS 1984-88.

U.S. Geological Survey Open-File Report 93-370.

\begin{tabular}{|c|c|c|c|c|c|c|c|c|c|c|c|c|c|}
\hline & & & $\begin{array}{c}\text { DIS- } \\
\text { CHARGE, } \\
\text { IN } \\
\text { CUBIC }\end{array}$ & TUR- & $\begin{array}{l}\text { RESIDUE } \\
\text { TOTAL } \\
\text { AT } 105 \\
\text { DEG. C. }\end{array}$ & $\begin{array}{l}\text { RESIDUE } \\
\text { VOLA- } \\
\text { TILE, }\end{array}$ & $\begin{array}{l}\text { NITRO- } \\
\text { GEN, } \\
\text { AMMONIA } \\
\text { DIS- }\end{array}$ & $\begin{array}{l}\text { NITRO- } \\
\text { GEN, AM- } \\
\text { MONIA + } \\
\text { ORGANIC }\end{array}$ & $\begin{array}{c}\text { NITRO- } \\
\text { GEN, } \\
\mathrm{NO}_{2}+\mathrm{NO}_{3}\end{array}$ & $\begin{array}{l}\text { PHOS- } \\
\text { PHORUS }\end{array}$ & $\begin{array}{c}\text { PHOS- } \\
\text { PHORUS } \\
\text { ORTHO, } \\
\text { DIS- }\end{array}$ & $\begin{array}{l}\text { CHLO- } \\
\text { RIDE, } \\
\text { DIS - }\end{array}$ & $\begin{array}{l}\text { SULFATE } \\
\text { DIS- }\end{array}$ \\
\hline & IME & $\begin{array}{l}\text { NDING } \\
\text { IME }\end{array}$ & $\begin{array}{l}\text { FEET } \\
\text { PER } \\
\text { SECOND }\end{array}$ & $\begin{array}{l}\text { BID- } \\
\text { ITY } \\
\text { (NTU) }\end{array}$ & $\begin{array}{l}\text { SUS- } \\
\text { PENDED } \\
(\mathrm{mg} / \mathrm{L})\end{array}$ & $\begin{array}{l}\text { SUS- } \\
\text { PENDED } \\
(\mathrm{mg} / \mathrm{L})\end{array}$ & $\begin{array}{l}\text { SOLVED } \\
\text { (mg/L } \\
\text { as } \mathrm{N})\end{array}$ & $\begin{array}{l}\text { TOTAL } \\
\text { (mg/L } \\
\text { as N) }\end{array}$ & $\begin{array}{l}\text { TOTAL } \\
\text { (mg/L } \\
\text { as N) }\end{array}$ & $\begin{array}{l}\text { TOTAL } \\
\text { (mg/L } \\
\text { as P) }\end{array}$ & $\begin{array}{l}\text { SOLVED } \\
\text { (mg/L } \\
\text { as P) }\end{array}$ & $\begin{array}{l}\text { SOLVED } \\
\text { (mg/L } \\
\text { as Cl) }\end{array}$ & $\begin{array}{l}\text { SOLVED } \\
(\mathrm{mg} / \mathrm{L} \\
\left.\text { as } \mathrm{SO}_{4}\right)\end{array}$ \\
\hline
\end{tabular}

OCT

\begin{tabular}{|c|c|c|}
\hline & & \\
\hline $02-03$ & 1405 & 0905 \\
\hline $03-04$ & 1050 & 1750 \\
\hline $04-05$ & 1850 & 2150 \\
\hline $05-07$ & 2250 & 0950 \\
\hline 07-11 & 1100 & 1000 \\
\hline $11-14$ & 1055 & 0955 \\
\hline $14-17$ & 1110 & 0210 \\
\hline $17-18$ & 0310 & 0210 \\
\hline $18-18$ & 0310 & 0910 \\
\hline $18-21$ & 0945 & 0845 \\
\hline $21-21$ & 1105 & 1905 \\
\hline $21-24$ & 2005 & 1005 \\
\hline $24-27$ & 1100 & 1000 \\
\hline $27-31$ & 1010 & 0910 \\
\hline OCT 31- & & \\
\hline NOV 02 & 1115 & 0715 \\
\hline $02-03$ & 0815 & 1015 \\
\hline $03-05$ & 1115 & 1415 \\
\hline $05-07$ & 1515 & 1015 \\
\hline $07-10$ & 1110 & 1010 \\
\hline $10-14$ & 1020 & 0920 \\
\hline $14-17$ & 1020 & 0920 \\
\hline $17-18$ & 1030 & 1730 \\
\hline $18-21$ & 1830 & 0930 \\
\hline $21-23$ & 1045 & 0945 \\
\hline $23-25$ & 1045 & 0945 \\
\hline $25-28$ & 1055 & 0955 \\
\hline Nov 28 - & & \\
\hline DEC 01 & 1030 & 0930 \\
\hline $02-05$ & 1400 & 1300 \\
\hline $05-08$ & 1110 & 1010 \\
\hline $07 \ldots$ & 0200 & -- \\
\hline $0 B-11$ & 1125 & 0225 \\
\hline $11-12$ & 0325 & 1025 \\
\hline $15 \ldots$ & 1030 & -- \\
\hline $19-19$ & 1010 & 1010 \\
\hline $19-20$ & 1045 & 0945 \\
\hline $20-22$ & 0915 & 0815 \\
\hline $22-23$ & 1005 & 0905 \\
\hline $23 \ldots$ & 1010 & -- \\
\hline $23 \ldots$ & 1015 & -- \\
\hline $23-24$ & 1100 & 0600 \\
\hline $24-27$ & 0700 & 1000 \\
\hline $27-28$ & 1125 & 0125 \\
\hline $28-30$ & 0225 & 1025 \\
\hline JAN & & \\
\hline $03-05$ & 1125 & 0925 \\
\hline $05-07$ & 1000 & 2100 \\
\hline $07-09$ & 2200 & 0900 \\
\hline $09-13$ & 1030 & 0930 \\
\hline $13-17$ & 1000 & 0900 \\
\hline $17-20$ & 1045 & 0945 \\
\hline $20-23$ & 1030 & 0930 \\
\hline $23-25$ & 1050 & 2150 \\
\hline $25-26$ & 2250 & 0950 \\
\hline $26-30$ & 1000 & 0900 \\
\hline
\end{tabular}

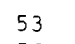

56

66
74

74
56

56
53

55

55

92
64

51

24
97

67

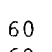

69
66

105

66
51
47

47

40
58

58
108
106

106

\section{3}

58
52

52
54

54
51

50

47
40

\begin{tabular}{|c|c|c|c|}
\hline-- & -- & -- & 0.02 \\
\hline 8.4 & -- & -- & 0.01 \\
\hline 9.4 & -- & -- & 0.02 \\
\hline 18 & -- & - & $<0.01$ \\
\hline 6.6 & -- & -- & $<0.01$ \\
\hline 14 & -- & -- & $<0.01$ \\
\hline 17 & -- & -- & $<0.01$ \\
\hline 20 & -- & -- & $<0.01$ \\
\hline 50 & 220 & 37 & $<0.01$ \\
\hline 100 & -- & -- & 0.02 \\
\hline 45 & -- & - & 0.02 \\
\hline 340 & -- & -- & 0.02 \\
\hline-- & -- & -- & 0.02 \\
\hline 4.8 & -- & -- & 0.01 \\
\hline 8.0 & -- & -. & 0.02 \\
\hline 7.1 & -- & -- & $<0.01$ \\
\hline 1.5 & -- & -- & 0.02 \\
\hline 14 & -- & -- & 0.02 \\
\hline 4.3 & $\cdots$ & -- & 0.06 \\
\hline 2.3 & -- & -- & $<0.01$ \\
\hline 2.6 & -- & -- & 0.02 \\
\hline 12 & $\cdots$ & -- & 0.03 \\
\hline 90 & -- & -- & 0.03 \\
\hline 6.9 & -- & -- & 0.02 \\
\hline 5.2 & -- & -- & 0.01 \\
\hline 3.7 & -- & -- & 0.02 \\
\hline 3.2 & -- & -- & 0.01 \\
\hline 8.0 & $\cdots$ & -- & 0.01 \\
\hline 3.0 & -- & -- & $<0.01$ \\
\hline 2.2 & -- & $\cdots$ & 0.02 \\
\hline 1.7 & -- & -- & 0.01 \\
\hline 2.9 & -- & -- & 0.01 \\
\hline 5.6 & -- & -- & 0.12 \\
\hline 2.2 & -- & - & 0.05 \\
\hline 4.1 & -- & -- & 0.04 \\
\hline 5.3 & -- & -- & 0.01 \\
\hline 3.1 & -. & $\cdots$ & 0.02 \\
\hline 4.4 & -- & -- & 0.05 \\
\hline 3.7 & -. & -- & 0.03 \\
\hline 2.7 & -- & -- & $<0.01$ \\
\hline 3.8 & -- & - & $<0.01$ \\
\hline 2.1 & -- & -- & 0.02 \\
\hline 5.3 & -- & $\cdots$ & $<0.01$ \\
\hline 3.4 & -. & -- & 0.03 \\
\hline 2.6 & -- & -- & 0.01 \\
\hline 8.7 & -- & -- & $<0.01$ \\
\hline 3.7 & -- & -- & 0.03 \\
\hline 2.0 & -- & -- & 0.01 \\
\hline 1.5 & -. & -- & 0.02 \\
\hline 2.1 & -- & -. & $<0.01$ \\
\hline 1.9 & -- & -- & 0.01 \\
\hline 3.6 & -- & -- & $<0.01$ \\
\hline 16 & -- & -- & 0.02 \\
\hline
\end{tabular}

\section{WATER YEAR OCTOBER 1988 TO SEPTEMBER 1989}




\section{A. Discharge and water quality}

0423205010 Irondequoit Creek Above Blossom Road, Rochester, N.Y.

2. WATER-QUALITY RECORDS

\begin{tabular}{|c|c|c|c|c|c|c|c|c|c|c|c|c|c|}
\hline & & & $\begin{array}{c}\text { DIS- } \\
\text { CHARGE, } \\
\text { IN }\end{array}$ & & $\begin{array}{l}\text { RESIDUE } \\
\text { TOTAL } \\
\text { AT } 105\end{array}$ & $\begin{array}{l}\text { RES IDUE } \\
\text { VOLA- }\end{array}$ & $\begin{array}{l}\text { NITRO- } \\
\text { GEN, } \\
\text { AMMONIA }\end{array}$ & $\begin{array}{l}\text { NITRO- } \\
\text { GEN, AM- } \\
\text { MONIA + }\end{array}$ & $\begin{array}{l}\text { NITRO- } \\
\text { GEN }\end{array}$ & PHOS- & $\begin{array}{l}\text { PHOS- } \\
\text { PHORUS } \\
\text { ORTHO, }\end{array}$ & $\begin{array}{l}\text { CH'O- } \\
\text { RIDE, }\end{array}$ & $\begin{array}{l}\text { SULFATE } \\
\text { DIS- }\end{array}$ \\
\hline TTE & TIME & $\begin{array}{l}\text { ENDING } \\
\text { TIME }\end{array}$ & $\begin{array}{l}\text { CUBIC } \\
\text { FEET } \\
\text { PER } \\
\text { SECOND }\end{array}$ & $\begin{array}{l}\text { TUR- } \\
\text { BID- } \\
\text { ITY } \\
\text { (NTU) }\end{array}$ & $\begin{array}{l}\text { DEG. C, } \\
\text { SUS- } \\
\text { PENDED } \\
(\mathrm{mg} / \mathrm{L})\end{array}$ & $\begin{array}{l}\text { TILE, } \\
\text { SUS- } \\
\text { PENDED } \\
(\mathrm{mg} / \mathrm{L})\end{array}$ & $\begin{array}{l}\text { DIS- } \\
\text { SOLVED } \\
\text { (mg/L } \\
\text { as N) }\end{array}$ & $\begin{array}{c}\text { ORGAN IC } \\
\text { TOTAL } \\
\text { (mg/L } \\
\text { as N) }\end{array}$ & $\begin{array}{c}\mathrm{NO}_{2}+\mathrm{NO}_{3} \\
\mathrm{TOTAL} \\
(\mathrm{mg} / \mathrm{L} \\
\text { as } \mathrm{N})\end{array}$ & $\begin{array}{c}\text { PHORUS } \\
\text { TOTAL } \\
\text { (mg/L } \\
\text { as P) }\end{array}$ & $\begin{array}{l}\text { DIS- } \\
\text { SOLVED } \\
\text { (mg/L } \\
\text { as P) }\end{array}$ & $\begin{array}{l}\text { DIS- } \\
\text { SO_VED } \\
\text { (mg/L } \\
\text { as Cl) }\end{array}$ & $\begin{array}{l}\text { DIS- } \\
\text { SOLVED } \\
(\mathrm{mg} / \mathrm{L} \\
\left.\text { as } \mathrm{SO}_{4}\right)\end{array}$ \\
\hline
\end{tabular}

\begin{tabular}{|c|c|c|c|c|c|c|c|c|c|c|c|c|c|}
\hline \multirow{3}{*}{$\begin{array}{r}\text { JAN } 30- \\
\text { FEB } 02\end{array}$} & \multicolumn{13}{|c|}{ WATER YEAR OCTOBER 1988 TO SEPTEMBER 1989-continued } \\
\hline & & & & & & & & & & & & & \\
\hline & 0940 & 0840 & 80 & 3.8 & -- & -- & $<0.01$ & 0.78 & 0.89 & 0.050 & 0.004 & 150 & 170 \\
\hline $02-06$ & 1140 & 1040 & 61 & 2.0 & -- & -- & $<0.01$ & 0.95 & 1.00 & 0.020 & 0.003 & 190 & 190 \\
\hline 06-09 & 1000 & 0900 & 51 & 2.4 & -- & -. & 0.01 & 0.80 & 1.00 & 0.030 & 0.021 & 230 & 200 \\
\hline $09-13$ & 1115 & 0915 & 44 & 2.8 & -. & -- & $<0.01$ & 0.72 & 1.10 & 0.030 & 0.002 & 200 & 210 \\
\hline $13-17$ & 0920 & 0820 & 46 & 2.2 & -- & -- & $<0.01$ & 0.73 & 1.10 & 0.020 & 0.002 & 280 & 200 \\
\hline $17-20$ & 1020 & 2120 & 45 & 1.8 & -- & -- & $<0.01$ & 0.80 & 1.10 & 0.025 & 0.006 & 260 & 210 \\
\hline $20-21$ & 2220 & 0920 & 125 & 2.8 & -- & -- & $<0.01$ & 0.78 & 1.00 & 0.025 & 0.007 & 240 & 210 \\
\hline $21-24$ & 1030 & 0930 & 150 & $\cdots$ & -- & -- & 0.01 & 2.5 & 1.10 & 0.340 & 0.003 & 220 & 120 \\
\hline $24-27$ & 0945 & 0845 & 79 & 3.2 & - & -. & 0.01 & 1.1 & 1.30 & 0.060 & 0.004 & 200 & 170 \\
\hline \multicolumn{14}{|l|}{ FEB $27-$} \\
\hline MAR 02 & 1130 & 1030 & 62 & 1.9 & -- & - & 0.02 & 0.94 & 1.20 & 0.040 & 0.004 & 270 & 180 \\
\hline $02-04$ & 1145 & 1045 & 69 & 3.6 & -- & -- & 0.02 & 1.8 & 1.10 & 0.045 & 0.004 & 200 & 190 \\
\hline $04-05$ & 1145 & 1045 & 192 & 90 & 200 & 39 & 0.02 & 2.6 & 1.20 & 0.320 & 0.003 & 440 & 120 \\
\hline $05-06$ & 1145 & 0945 & 244 & 170 & 480 & 64 & 0.03 & 3.7 & 1.30 & 0.590 & 0.007 & 200 & 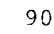 \\
\hline $06-07$ & 1015 & 0315 & 195 & 50 & 157 & 28 & 0.03 & 2.1 & 1.30 & 0.200 & 0.004 & 170 & 94 \\
\hline $07-09$ & 0415 & 0915 & 135 & 13 & -- & -- & 0.02 & 1.3 & 1.40 & 0.115 & 0.003 & 180 & 130 \\
\hline $09-13$ & 1015 & 0915 & 69 & 2.1 & -- & -- & 0.02 & 0.84 & 1.30 & 0.035 & 0.004 & 200 & 180 \\
\hline $13-16$ & 1045 & 0945 & 102 & 25 & 81 & 11 & 0.01 & 1.2 & 1.10 & 0.155 & 0.002 & 210 & 150 \\
\hline $15 \ldots$ & 0200 & -. & 135 & 20 & -- & -- & 0.02 & 0.72 & 1.10 & 0.035 & 0.002 & 200 & 140 \\
\hline $16-18$ & 1000 & 0100 & 128 & 3.7 & -- & -- & 0.05 & 1.0 & 0.95 & 0.090 & 0.004 & 160 & 120 \\
\hline $18-20$ & 0200 & 0900 & 211 & 100 & 370 & 41 & 0.05 & 2.4 & 1.30 & 0.345 & 0.005 & 200 & 100 \\
\hline $20-23$ & 1030 & 0930 & 149 & 28 & -- & -- & 0.02 & 1.3 & 1.40 & 0.115 & 0.005 & 230 & 120 \\
\hline $23-24$ & 1015 & 1315 & 122 & 2.1 & -- & - & 0.09 & 0.55 & 1.20 & 0.090 & 0.006 & 190 & 120 \\
\hline $24-27$ & 1415 & 0915 & 160 & 45 & 200 & 19 & 0.03 & 0.50 & 1.40 & 0.265 & 0.005 & 170 & 120 \\
\hline $27-28$ & 1000 & 1200 & 136 & 25 & 91 & 28 & 0.08 & 1.7 & 1.10 & 0.280 & 0.008 & 140 & 130 \\
\hline $28-30$ & 1300 & 0300 & 158 & 50 & 250 & 25 & 0.03 & 2.2 & 0.94 & 0.395 & 0.005 & 150 & 130 \\
\hline $30-30$ & 0400 & 0900 & 312 & 55 & -. & -. & 0.03 & 2.1 & 0.88 & 0.250 & 0.005 & 140 & 130 \\
\hline \multicolumn{14}{|l|}{ MAR 30- } \\
\hline APR 02 & 1045 & 1345 & 459 & 270 & 849 & 128 & 0.06 & 4.8 & 1.30 & 1.06 & 0.011 & 160 & 86 \\
\hline $02-03$ & 1445 & 0945 & 590 & 300 & 853 & 136 & 0.04 & 4.6 & 1.50 & 1.10 & 0.010 & 130 & 69 \\
\hline $03-06$ & 1045 & 0945 & 558 & 210 & 818 & 83 & 0.03 & 4.5 & 1.70 & 1.10 & 0.013 & 100 & 70 \\
\hline $06-06$ & 1000 & 1300 & 371 & 90 & -- & -- & 0.04 & 2.9 & 1.70 & 0.575 & 0.019 & 110 & 96 \\
\hline $06-10$ & 1400 & 0900 & 241 & 55 & 174 & 26 & 0.02 & 1.6 & 1.40 & 0.245 & 0.014 & 120 & 110 \\
\hline $10-13$ & 0950 & 0850 & 137 & 13 & $\cdots$ & -- & 0.02 & 0.73 & 0.63 & 0.145 & 0.008 & 130 & 130 \\
\hline $13-17$ & 0950 & 0850 & 115 & 6.4 & -- & - & 0.01 & 0.72 & 1.20 & 0.090 & 0.008 & 130 & 140 \\
\hline $17-20$ & 0950 & 0850 & 110 & 24 & -- & -- & 0.02 & 0.78 & 1.20 & 0.160 & 0.006 & 130 & 150 \\
\hline $20-24$ & 1215 & 1115 & 80 & 33 & -- & -- & 0.02 & 1.3 & 1.20 & 0.195 & 0.008 & 130 & 160 \\
\hline $24-27$ & 0945 & 0845 & 72 & 26 & - & -- & 0.02 & 0.84 & 1.10 & 0.200 & 0.005 & 130 & 160 \\
\hline $27-29$ & 1000 & 1300 & 71 & 45 & 212 & 34 & 0.02 & 1.2 & 1.00 & 0.250 & 0.007 & 120 & 150 \\
\hline \multicolumn{14}{|l|}{ APR $29-$} \\
\hline MAY 01 & 1400 & 0900 & 83 & 75 & 391 & 63 & $<0.01$ & 1.5 & 1.20 & 0.360 & 0.005 & 120 & 150 \\
\hline $01-04$ & 1040 & 0940 & 274 & 95 & 902 & 135 & 0.04 & 4.4 & 0.82 & 0.955 & 0.007 & 100 & 9 \\
\hline $04-07$ & 1020 & 0120 & 148 & 80 & 497 & 58 & 0.04 & 2.4 & 0.90 & 0.550 & 0.009 & 110 & 110 \\
\hline $07-08$ & 0220 & 0920 & 486 & 290 & 1690 & 200 & 0.02 & 9.5 & 0.74 & 1.98 & 0.009 & 100 & 8 \\
\hline $08-10$ & 0955 & 1455 & 564 & 200 & 1180 & 156 & 0.03 & -- & 1.10 & 0.265 & 0.011 & 77 & 5 \\
\hline $10-11$ & 1555 & 0855 & 489 & 170 & 653 & 83 & 0.02 & -. & 1.10 & 0.165 & 0.010 & 90 & 66 \\
\hline $11-14$ & 1015 & 1315 & 560 & 200 & 883 & 113 & 0.05 & -- & 1.30 & 1.07 & 0.016 & 77 & 5 \\
\hline $14-15$ & 1415 & 0915 & 331 & 200 & 866 & 124 & 0.02 & -- & 1.20 & 1.19 & 0.013 & 87 & 7 \\
\hline $15-18$ & 1010 & 0910 & 277 & 110 & 525 & 77 & 0.05 & 3.5 & 1.00 & 0.655 & 0.013 & 80 & 66 \\
\hline $18-22$ & 1015 & 0915 & 152 & 65 & 344 & 50 & 0.04 & 2.8 & 1.20 & 0.505 & 0.015 & 100 & 92 \\
\hline $22-23$ & 1030 & 1830 & 123 & 65 & 344 & 46 & 0.06 & 2.2 & 1.20 & 0.470 & 0.018 & 98 & 120 \\
\hline $23-25$ & 1930 & 0930 & 131 & 90 & 482 & 72 & 0.03 & 2.3 & 1.20 & 0.625 & 0.016 & 96 & 110 \\
\hline $25-26$ & 0945 & 0345 & 125 & 80 & 408 & 47 & 0.09 & 2.2 & 1.30 & 0.540 & 0.013 & 90 & 130 \\
\hline $26-26$ & 0445 & 0845 & 131 & 200 & 887 & 129 & 0.09 & 3.2 & 1.30 & 0.190 & 0.014 & 96 & 120 \\
\hline $30-31$ & 1015 & 2120 & 135 & 380 & 1190 & 171 & 0.12 & 5.7 & 0.90 & 1.78 & 0.026 & 82 & $R$ \\
\hline $30 \ldots$ & 1030 & -- & 127 & 9.0 & -- & -- & 0.02 & 1.3 & 1.10 & 0.170 & 0.022 & 95 & 130 \\
\hline \multicolumn{14}{|l|}{ MAY $31-$} \\
\hline JUN 02 & 2220 & 0915 & 206 & 150 & 650 & 92 & 0.06 & 3.3 & 1.10 & 0.810 & 0.023 & 87 & 98 \\
\hline $02-05$ & 1030 & 0930 & 120 & 210 & 788 & 125 & 0.06 & 4.1 & 1.00 & 0.940 & 0.033 & 94 & 100 \\
\hline $05-08$ & 1000 & 0900 & 85 & 70 & 354 & 62 & 0.02 & 2.1 & 1.10 & 0.480 & 0.028 & 100 & 12 \\
\hline $07 \ldots$ & 0200 & -- & 79 & 5.4 & -- & -- & 0.02 & 0.88 & 1.20 & 0.080 & 0.022 & 110 & 13 \\
\hline $08-09$ & 1015 & 1715 & 87 & 170 & 637 & 78 & 0.05 & 3.3 & 1.10 & 0.920 & 0.034 & 90 & 11 \\
\hline $09-12$ & 1815 & 0915 & 190 & 220 & 921 & 113 & 0.11 & 4.0 & 0.78 & 1.21 & 0.037 & 71 & -1 \\
\hline $12-13$ & 1015 & 0315 & 106 & 160 & 563 & 78 & 0.04 & 3.2 & 1.10 & 0.950 & 0.036 & 95 & 10 \\
\hline $13-14$ & 0415 & 1215 & 137 & 160 & 692 & 88 & 0.05 & 3.7 & 0.92 & 0.200 & 0.008 & 87 & \\
\hline & & & 25 & 160 & 524 & 65 & 0.03 & 3.7 & 10 & 0175 & 0019 & 94 & \\
\hline
\end{tabular}




\section{Surface-Water Stations}

\section{A. Discharge and water quality}

0423205010 Irondequoit Creek Above Blossom Road, Rochester, N.Y.

\section{WATER-QUALITY RECORDS}

\begin{tabular}{|c|c|c|c|c|c|c|c|c|c|c|c|c|c|}
\hline & & & $\begin{array}{c}\text { DIS- } \\
\text { CHARGE, } \\
\text { IN } \\
\text { CUBIC }\end{array}$ & TUR- & $\begin{array}{l}\text { RES IDUE } \\
\text { TOTAL } \\
\text { AT } 105 \\
\text { DEG. C, }\end{array}$ & $\begin{array}{l}\text { RESIDUE } \\
\text { VOLA- } \\
\text { TILE, }\end{array}$ & $\begin{array}{l}\text { NITRO- } \\
\text { GEN, } \\
\text { AMMONIA } \\
\text { DIS- }\end{array}$ & $\begin{array}{l}\text { NITRO- } \\
\text { GEN, AM- } \\
\text { MONIA + } \\
\text { ORGANIC }\end{array}$ & $\begin{array}{c}\text { NITRO- } \\
\text { GEN, } \\
\mathrm{NO}_{2}+\mathrm{NO}_{3}\end{array}$ & $\begin{array}{l}\text { PHOS- } \\
\text { PHORUS }\end{array}$ & $\begin{array}{l}\text { PHOS- } \\
\text { PHORUS } \\
\text { ORTHO, } \\
\text { DIS- }\end{array}$ & $\begin{array}{l}\text { CHLO- } \\
\text { RIDE, } \\
\text { DIS- }\end{array}$ & $\begin{array}{l}\text { SULFATE } \\
\text { DIS- }\end{array}$ \\
\hline DATE & TIME & $\begin{array}{l}\text { ENDING } \\
\text { TIME }\end{array}$ & $\begin{array}{l}\text { FEET } \\
\text { PER } \\
\text { SECOND }\end{array}$ & $\begin{array}{l}\text { BID- } \\
\text { ITY } \\
\text { (NTU) }\end{array}$ & $\begin{array}{c}\text { SUS- } \\
\text { PENDED } \\
(\mathrm{mg} / \mathrm{L})\end{array}$ & $\begin{array}{l}\text { SUS- } \\
\text { PENDED } \\
(\mathrm{mg} / \mathrm{L})\end{array}$ & $\begin{array}{l}\text { SOLVED } \\
\text { ( } \mathrm{mg} / \mathrm{L} \\
\text { as } \mathrm{N})\end{array}$ & $\begin{array}{l}\text { TOTAL } \\
\text { (mg/L } \\
\text { as N) }\end{array}$ & $\begin{array}{l}\text { TOTAL } \\
\text { (mg/L } \\
\text { as N) }\end{array}$ & $\begin{array}{l}\text { TOTAL } \\
(\mathrm{mg} / \mathrm{L} \\
\text { as P) }\end{array}$ & $\begin{array}{l}\text { SOLVED } \\
(\mathrm{mg} / \mathrm{L} \\
\text { as P) }\end{array}$ & $\begin{array}{l}\text { SOLVE) } \\
(\mathrm{mg} / \mathrm{L} \\
\text { as } \mathrm{Cl})\end{array}$ & $\begin{array}{l}\text { SOLVED } \\
(\mathrm{mg} / \mathrm{L} \\
\left.\text { as } \mathrm{SO}_{4}\right)\end{array}$ \\
\hline
\end{tabular}

JUN-cont inued

\begin{tabular}{|c|c|c|c|}
\hline $15-16$ & & & \\
\hline $\begin{array}{l}15-16 \\
16-19\end{array}$ & 1010 & 1310 & 122 \\
\hline $\begin{array}{l}16-19 \\
19-20\end{array}$ & 1410 & 0910 & 350 \\
\hline $20-22$ & 0710 & 0910 & 498 \\
\hline $22-26$ & 0925 & 0825 & 203 \\
\hline $26-27$ & 1020 & 0020 & 115 \\
\hline $28-29$ & 0120 & 0920 & 117 \\
\hline JUN 29- & & & \\
\hline JUL 03 & 0955 & 0855 & 88 \\
\hline $03-06$ & 0920 & 0820 & 74 \\
\hline $06-10$ & 1045 & 0945 & 76 \\
\hline $10-13$ & 0955 & 0855 & 82 \\
\hline $13-17$ & 1020 & 0920 & 59 \\
\hline $17-20$ & 1010 & 0910 & 58 \\
\hline $20-23$ & 0950 & 0450 & 61 \\
\hline $23-24$ & 0550 & 0850 & 66 \\
\hline $24-27$ & 1040 & 0940 & 50 \\
\hline $27-31$ & 1000 & 0900 & 49 \\
\hline JUL 31- & & & \\
\hline AUG 03 & 0955 & 085 & 45 \\
\hline $03-04$ & 1150 & 1450 & 59 \\
\hline $04-05$ & 1550 & 1450 & 123 \\
\hline $05-07$ & 1550 & 0950 & 93 \\
\hline $07-10$ & 1000 & 0900 & 51 \\
\hline $10-11$ & 1100 & 1000 & 46 \\
\hline $10 \ldots$ & 1115 & -- & 44 \\
\hline $11 \ldots$ & 1110 & -- & 44 \\
\hline $14 \ldots$ & 1015 & -- & 41 \\
\hline $14-15$ & 1015 & 1815 & 43 \\
\hline $15-17$ & 1915 & 0915 & 47 \\
\hline $16 \ldots$ & 1400 & -- & 48 \\
\hline $17-19$ & 1000 & 1700 & 50 \\
\hline $19-21$ & 1800 & 0900 & 85 \\
\hline $21-24$ & 1015 & 0915 & 45 \\
\hline $24-28$ & 0933 & 0833 & 39 \\
\hline $28-31$ & 0955 & 0855 & 38 \\
\hline AUG 31- & & & \\
\hline SEP 01 & 0955 & 1955 & 46 \\
\hline $01-05$ & 2055 & 0855 & 47 \\
\hline $05-08$ & 1000 & 0900 & 36 \\
\hline $08-11$ & 0955 & 0855 & 36 \\
\hline $11-13$ & 0945 & 2045 & 38 \\
\hline $13 \ldots$ & 0200 & -- & 37 \\
\hline $13-14$ & 2145 & 0845 & 67 \\
\hline $14-16$ & 0950 & 1250 & 116 \\
\hline $16-18$ & 1350 & 0850 & 103 \\
\hline $18-21$ & 1000 & 0900 & 56 \\
\hline $21-22$ & 1000 & 1100 & 45 \\
\hline $22-22$ & 1130 & 1900 & 104 \\
\hline $22-24$ & 1930 & 1030 & 87 \\
\hline $24-26$ & 1200 & 1130 & 56 \\
\hline $26-28$ & 1230 & 0930 & 49 \\
\hline SEP 28- & & & \\
\hline OCT 02 & 1000 & 0100 & 47 \\
\hline
\end{tabular}

OCT

$\begin{array}{llll}02-02 & 0200 & 0900 & 8 \\ 02-04 & 1145 & 1045 & 6 \\ 04-06 & 1145 & 1045 & 5 \\ 06-10 & 1100 & 1000 & 4 \\ 10-13 & 1125 & 1025 & 5 \\ 13-14 & 1135 & 1335 & 4 \\ 14-16 & 1435 & 1035 & 9 \\ 16-18 & 1105 & 0205 & 10\end{array}$

WATER YEAR OCTOBER 1988 TO SEPTEMBER 1989-continued

\begin{tabular}{|c|c|c|c|c|c|c|c|}
\hline 451 & 59 & 0.05 & 1.8 & $<0.01$ & 0.700 & 0.039 & 91 \\
\hline 980 & 121 & 0.05 & 4.9 & 1.10 & 1.40 & 0.035 & 78 \\
\hline 440 & 55 & $<0.01$ & 2.5 & 1.30 & 0.630 & 0.046 & 88 \\
\hline 924 & 138 & $<0.01$ & 4.6 & 1.20 & 1.45 & 0.038 & 63 \\
\hline 396 & 64 & $<0.01$ & 2.6 & 1.40 & 0.675 & 0.056 & 79 \\
\hline 316 & 58 & 0.03 & 2.5 & 1.40 & 0.530 & 0.048 & 99 \\
\hline 231 & 34 & 0.02 & 2.6 & 1.40 & 0.340 & 0.039 & 93 \\
\hline - & -- & 0.02 & 1.3 & 1.20 & 0.200 & 0.039 & 93 \\
\hline 81 & 16 & 0.02 & 1.1 & 1.30 & 0.200 & 0.033 & 100 \\
\hline-- & - - & 0.02 & 0.92 & 1.20 & 0.180 & 0.030 & 96 \\
\hline - & -- & 0.04 & 1.7 & 1.20 & 0.270 & 0.029 & 92 \\
\hline-- & -- & 0.05 & 0.88 & 1.10 & 0.225 & 0.028 & 100 \\
\hline -- & -- & 0.02 & 0.77 & 0.95 & 0.160 & 0.012 & 110 \\
\hline-- & -- & 0.06 & 0.80 & 1.00 & 0.170 & 0.020 & 110 \\
\hline 163 & -- & 0.04 & 1.2 & 1.00 & 0.260 & 0.016 & 94 \\
\hline-- & -- & 0.02 & 0.94 & 0.94 & 0.190 & 0.017 & 100 \\
\hline -- & -- & 0.03 & 0.72 & 0.91 & 0.160 & 0.018 & 100 \\
\hline -- & -- & $<0.01$ & 0.52 & 0.85 & 0.120 & 0.014 & 100 \\
\hline -- & -- & $<0.01$ & 1.1 & 0.88 & 0.135 & 0.021 & 100 \\
\hline 468 & 63 & 0.04 & 3.1 & 0.88 & 0.765 & 0.029 & 83 \\
\hline 687 & 95 & 0.03 & 4.0 & 0.72 & 1.07 & 0.032 & 77 \\
\hline 266 & 44 & 0.01 & 1.9 & 0.83 & 0.370 & 0.031 & 110 \\
\hline 159 & 18 & 0.01 & 0.96 & 0.80 & 0.250 & 0.019 & 120 \\
\hline-- & -- & 0.02 & 0.76 & 0.78 & 0.120 & 0.019 & 110 \\
\hline -- & -- & 0.02 & 0.20 & 0.80 & 0.075 & 0.017 & 120 \\
\hline -- & -- & 0.02 & 0.30 & 0.81 & 0.070 & 0.016 & 100 \\
\hline-- & -- & 0.02 & 0.74 & 0.77 & 0.125 & 0.019 & 100 \\
\hline -- & -- & 0.02 & 0.68 & 0.76 & 0.115 & 0.017 & 99 \\
\hline-- & -- & $<0.01$ & 0.68 & 0.81 & 0.088 & 0.006 & 97 \\
\hline -- & - - & 0.01 & 0.56 & 0.68 & 0.085 & 0.014 & 100 \\
\hline-- & -- & 0.01 & 1.0 & 0.62 & 0.155 & 0.015 & 81 \\
\hline -- & -- & 0.04 & 0.77 & 0.78 & 0.115 & 0.016 & 100 \\
\hline-- & -- & 0.02 & 0.53 & 0.83 & 0.080 & 0.016 & 110 \\
\hline -- & -- & 0.02 & 0.68 & 0.86 & 0.090 & 0.015 & 110 \\
\hline-- & -- & 0.04 & 1.2 & 0.97 & 0.135 & 0.015 & 110 \\
\hline 88 & 18 & 0.02 & 1.2 & 0.91 & 0.185 & 0.017 & 100 \\
\hline-- & -- & 0.03 & 1.1 & 0.80 & 0.235 & 0.026 & 110 \\
\hline .. & - - & 0.03 & 0.89 & 0.78 & 0.155 & 0.014 & 110 \\
\hline -- & -- & 0.04 & 0.75 & 0.82 & 0.125 & 0.017 & 100 \\
\hline -- & -- & 0.02 & 0.49 & 0.86 & 0.060 & 0.018 & 110 \\
\hline 151 & 24 & 0.03 & 1.1 & 0.91 & 0.215 & 0.016 & 89 \\
\hline 158 & 30 & 0.04 & 1.3 & 0.91 & 0.275 & 0.023 & 71 \\
\hline 141 & 28 & 0.02 & 1.2 & 0.82 & 0.270 & 0.024 & 76 \\
\hline-- & -- & 0.02 & 0.77 & 0.87 & 0.170 & 0.023 & 96 \\
\hline- & - - & 0.01 & 0.53 & 0.91 & 0.140 & 0.020 & 110 \\
\hline$\cdots$ & -- & 0.02 & 0.39 & 0.92 & 0.110 & 0.020 & 120 \\
\hline 97 & 16 & 0.01 & 1.1 & 0.80 & 0.240 & 0.020 & 92 \\
\hline -- & -- & 0.02 & 0.48 & 0.86 & 0.095 & 0.022 & 100 \\
\hline-- & - - & $<0.01$ & 0.43 & 0.83 & 0.130 & 0.019 & 110 \\
\hline- & - & 0.02 & 0.50 & 0.81 & 0.090 & 0.018 & 96 \\
\hline
\end{tabular}

WATER YEAR OCTOBER 1989 TO SEPTEMBER 1990

$\begin{array}{llrll}-- & -- & 0.02 & 0.37 & 0.74 \\ -- & -- & 0.02 & 0.76 & 0.69 \\ -- & -- & <0.01 & 0.37 & 0.70 \\ -- & -- & <0.01 & 0.28 & 0.69 \\ -- & -- & 0.02 & 0.15 & 0.75 \\ -- & -- & 0.02 & 0.26 & 0.72 \\ -- & -- & 0.01 & 0.93 & 1.00 \\ -- & - & 0.02 & 0.76 & 0.64\end{array}$

$\begin{array}{rrr}0.014 & 97 & 200 \\ 0.016 & 87 & 160 \\ 0.013 & 110 & 190 \\ 0.011 & 98 & 170 \\ 0.012 & 100 & 190 \\ 0.012 & 110 & 190 \\ 0.014 & 80 & 150 \\ 0.015 & 80 & 150\end{array}$


0423205010 Irondequoit Creek Above Blossom Road, Rochester, N.Y.

\section{WATER-QUALITY RECORDS}

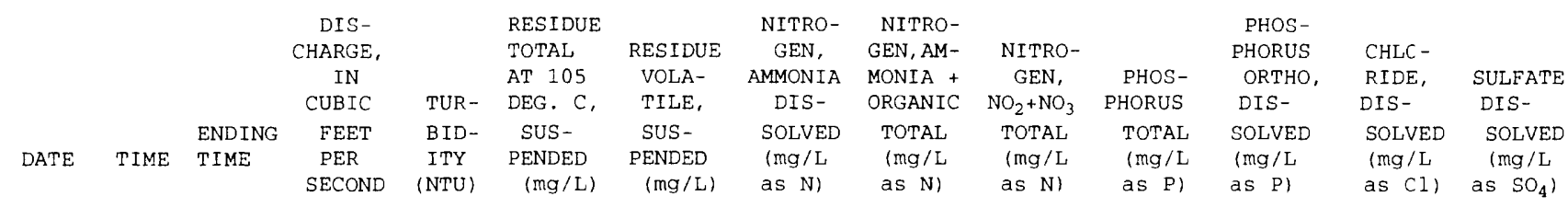

OCT-cont inued

\begin{tabular}{|c|c|c|c|}
\hline ( ) & & & \\
\hline $18-19$ & 0305 & 1005 & 124 \\
\hline $19-21$ & 1025 & 0925 & 173 \\
\hline $21-23$ & 1025 & 0925 & 120 \\
\hline $23-26$ & 1045 & 0945 & 61 \\
\hline $26-30$ & 1005 & 0905 & 58 \\
\hline \multicolumn{4}{|l|}{ OCT $30-$} \\
\hline NOV 02 & 1015 & 0915 & 54 \\
\hline $02-06$ & 1130 & 1030 & 67 \\
\hline $06-07$ & 0940 & 1440 & 65 \\
\hline $07-09$ & 1540 & 0840 & 94 \\
\hline $09-13$ & 1035 & 0935 & 81 \\
\hline $13-14$ & 1030 & 1830 & 64 \\
\hline $14-16$ & 1830 & 0330 & 71 \\
\hline $16-16$ & 0430 & 0930 & 93 \\
\hline $16-20$ & 1015 & 0915 & 70 \\
\hline $20-22$ & 1010 & 0910 & 105 \\
\hline $22-27$ & 1030 & 0930 & 76 \\
\hline $27-30$ & 1000 & 0900 & 71 \\
\hline \multicolumn{4}{|l|}{ Nov $30-$} \\
\hline DEC 02 & 1040 & 0540 & 67 \\
\hline $02-04$ & 0640 & 0940 & 58 \\
\hline $04-07$ & 1100 & 1000 & 56 \\
\hline $05 \ldots$ & 0200 & -- & - \\
\hline $07-11$ & 1030 & 0930 & 51 \\
\hline $11-14$ & 1045 & 0945 & 47 \\
\hline $14-15$ & 1045 & 1745 & 43 \\
\hline $15-18$ & 1845 & 0945 & 43 \\
\hline $18-22$ & 1055 & 0955 & 42 \\
\hline $26-29$ & 1130 & 1030 & 41 \\
\hline $29-31$ & 1055 & 0555 & 57 \\
\hline \multicolumn{4}{|l|}{ DEC $31-$} \\
\hline JAN 02 & 0655 & 0955 & 139 \\
\hline $02-03$ & 1015 & 1815 & 98 \\
\hline $03-05$ & 1915 & 0915 & 170 \\
\hline $05-08$ & 1045 & 0945 & 167 \\
\hline $08 \ldots$ & 1000 & -- & - \\
\hline $08-12$ & 1000 & 0900 & 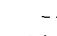 \\
\hline $12-16$ & 0940 & 0840 & 98 \\
\hline $16-18$ & 1015 & 0015 & 141 \\
\hline $18-19$ & 0115 & 0915 & 348 \\
\hline $19-22$ & 1015 & 0915 & 184 \\
\hline $22-25$ & 1030 & 0930 & 144 \\
\hline $25-29$ & 1000 & 0900 & 131 \\
\hline \multicolumn{4}{|l|}{ JAN 29- } \\
\hline FEB 01 & 1000 & 0800 & 93 \\
\hline $01-05$ & 0840 & 0740 & 222 \\
\hline $05-06$ & 1100 & 1300 & 177 \\
\hline $06-08$ & 1400 & 1000 & 222 \\
\hline $08 \ldots$ & 1000 & -- & 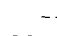 \\
\hline $08-09$ & 1015 & 0515 & 337 \\
\hline $09-12$ & 0615 & 0915 & 537 \\
\hline $15 \ldots$ & 1100 & - & 169 \\
\hline $16 \ldots$ & 0915 & -- & 405 \\
\hline $16 \ldots$ & 1235 & -- & 405 \\
\hline $20 \ldots$ & 1125 & -- & 233 \\
\hline $21 \ldots$ & 1330 & -- & 177 \\
\hline $22-23$ & 1535 & 0835 & 497 \\
\hline $23-24$ & 1050 & 0350 & 662 \\
\hline $26 \ldots$ & 1050 & -- & 232 \\
\hline $27 \ldots$ & 1345 & -- & 200 \\
\hline $28 \ldots$ & 1210 & -- & 183 \\
\hline \multicolumn{4}{|l|}{ MAR } \\
\hline 01. & 1000 & -- & 162 \\
\hline $02-05$ & 1530 & 0230 & 190 \\
\hline $05-08$ & 1105 & 0405 & 124 \\
\hline
\end{tabular}

\begin{tabular}{|c|c|c|c|c|c|c|}
\hline 22 & -- & -- & 0.01 & 0.70 & 0.60 & 0.140 \\
\hline 34 & 142 & 19 & 0.03 & 1.1 & 0.62 & 0.240 \\
\hline 21 & -- & -- & 0.02 & 0.69 & 0.71 & 0.145 \\
\hline 12 & -- & -- & 0.02 & 0.60 & 0.95 & 0.090 \\
\hline 3.1 & -- & -- & 0.02 & 0.59 & 0.78 & 0.080 \\
\hline 10 & -- & -- & 0.01 & 0.41 & 0.72 & 0.070 \\
\hline 2.4 & -- & -- & 0.03 & 0.32 & 0.68 & 0.065 \\
\hline 2.7 & -- & -- & 0.03 & 0.43 & 0.90 & 0.060 \\
\hline 8.5 & -- & -- & 0.02 & 0.79 & 0.92 & 0.125 \\
\hline 3.3 & -- & -- & 0.07 & 0.59 & 0.94 & 0.090 \\
\hline 2.3 & -- & -- & 0.04 & 0.48 & 0.93 & 0.075 \\
\hline 2.6 & -- & -- & 0.03 & 0.39 & 0.80 & 0.070 \\
\hline 3.8 & -- & -- & 0.02 & 0.53 & 0.79 & 0.080 \\
\hline 5.5 & -- & -- & 0.03 & 0.57 & 0.74 & 0.090 \\
\hline 19 & -- & -- & $<0.01$ & 1.0 & 0.80 & 0.170 \\
\hline 4.3 & -- & -- & $<0.01$ & 0.56 & 0.97 & 0.065 \\
\hline 2.4 & -- & $\cdots$ & $<0.01$ & 0.23 & 0.99 & 0.045 \\
\hline 2.6 & -- & -- & $<0.01$ & 0.56 & 1.00 & 0.070 \\
\hline 2.7 & -- & -- & $<0.01$ & 1.1 & 1.10 & 0.135 \\
\hline 3.7 & -- & -- & 0.01 & 0.85 & 1.20 & 0.080 \\
\hline 2.6 & -- & -- & 0.03 & 0.33 & 1.10 & 0.035 \\
\hline 2.1 & -- & -- & $<0.01$ & 0.52 & 1.00 & 0.055 \\
\hline 1.5 & -- & -- & $<0.01$ & 0.62 & 1.10 & 0.055 \\
\hline 1.4 & - & -- & 0.02 & 0.32 & 1.30 & 0.035 \\
\hline 1.9 & -- & -- & 0.01 & 0.41 & 1.30 & 0.045 \\
\hline$\ldots$ & -- & -- & 0.01 & 0.41 & 1.50 & 0.055 \\
\hline 2.2 & -- & -- & 0.03 & 0.60 & 1.60 & 0.035 \\
\hline 1.0 & -- & -- & 0.03 & 0.23 & 1.50 & 0.025 \\
\hline 6.3 & -- & -- & 0.06 & 1.0 & 1.50 & 0.100 \\
\hline 4.3 & -- & -- & 0.04 & 0.57 & 1.50 & 0.075 \\
\hline 38 & 135 & 25 & 0.06 & 1.6 & 1.40 & 0.240 \\
\hline 38 & 101 & 17 & 0.05 & 1.4 & 1.70 & 0.170 \\
\hline 18 & -- & -- & 0.12 & 1.5 & 1.70 & 0.150 \\
\hline 6.0 & -- & -- & 0.08 & 0.70 & 1.60 & 0.050 \\
\hline 4.4 & - & -- & 0.04 & 0.69 & 1.60 & 0.075 \\
\hline 26 & -- & -- & 0.03 & 1.1 & 1.70 & 0.140 \\
\hline 250 & 709 & 107 & 0.03 & 3.3 & 0.80 & 0.760 \\
\hline 37 & 93 & 17 & 0.02 & 1.2 & 1.80 & 0.160 \\
\hline 6.2 & -- & -- & 0.02 & 1.1 & 1.70 & 0.130 \\
\hline 13 & -- & -- & 0.02 & 0.89 & 1.60 & 0.070 \\
\hline 4.4 & -- & -- & 0.02 & 0.62 & 1.50 & 0.045 \\
\hline 42 & 101 & 16 & 0.02 & 1.1 & 1.60 & 0.170 \\
\hline 20 & -- & -- & 0.02 & 0.93 & 1.60 & 0.100 \\
\hline 23 & -- & -- & 0.01 & 0.78 & 1.60 & 0.110 \\
\hline 14 & 114 & 25 & 0.04 & 0.70 & 1.60 & 0.065 \\
\hline 40 & 119 & 19 & 0.02 & 1.4 & 1.60 & 0.175 \\
\hline 140 & 440 & 60 & 0.03 & 2.4 & 1.70 & 0.540 \\
\hline 5.4 & -- & -- & 0.04 & 0.70 & 0.80 & 0.045 \\
\hline 22 & -- & -- & 0.08 & 0.99 & 1.50 & 0.120 \\
\hline 17 & -- & -- & 0.02 & 1.3 & 1.50 & 0.120 \\
\hline 6.0 & - & -- & 0.05 & 0.73 & 1.60 & 0.040 \\
\hline 4.3 & -- & -- & 0.05 & 0.68 & 1.60 & 0.040 \\
\hline 130 & 554 & 81 & 0.07 & 2.2 & 1.40 & 0.610 \\
\hline 120 & 445 & 51 & 0.02 & 1.6 & 1.30 & 0.440 \\
\hline 5.9 & -- & -- & 0.04 & 0.66 & 1.60 & 0.040 \\
\hline 5.3 & -- & -- & 0.05 & 0.67 & 1.80 & 0.045 \\
\hline 110 & 194 & 15 & 0.05 & 0.51 & 1.80 & 0.040 \\
\hline 3.7 & -- & -- & 0.06 & 0.68 & 1.80 & 0.050 \\
\hline 20 & - & -- & 0.03 & 0.72 & 1.70 & 0.070 \\
\hline 7.5 & -- & -- & 0.02 & 0.72 & 1.60 & 0.055 \\
\hline
\end{tabular}

0.017

$79 \quad 140$

$\begin{array}{lll}0.023 & 81 & 120\end{array}$

$\begin{array}{lll}0.026 & 110 & 170\end{array}$

$\begin{array}{lll}0.019 & 110 & 180\end{array}$

$\begin{array}{lll}0.018 & 110 & 170\end{array}$

$\begin{array}{lll}0.016 & 100 & 170\end{array}$

$0.016 \quad 120 \quad 190$

$0.018 \quad 100 \quad 160$

$\begin{array}{lll}0.026 & 110 & 150\end{array}$

$0.030 \quad 120 \quad 170$

$\begin{array}{lll}0.023 & 110 & 180\end{array}$

$0.019 \quad 110 \quad 160$

$0.019 \quad 100 \quad 150$

$\begin{array}{lll}0.014 & 100 & 120 \\ 0.015 & 110 & 160\end{array}$

$0.011 \quad 110 \quad 170$

$\begin{array}{lll}0.011 & 140 \quad 180\end{array}$

$0.009 \quad 140 \quad 190$

$0.008 \quad 150 \quad 180$

$0.007 \quad 130 \quad 190$

$0.007 \quad 180 \quad 170$

$0.007 \quad 140 \quad 200$

$0.007 \quad 150 \quad 200$

$0.007 \quad 150 \quad 200$

$\begin{array}{lll}0.006 & 140 & 210\end{array}$

$\begin{array}{lll}0.006 & 140 & 200\end{array}$

$\begin{array}{lll}0.005 & 200 & 200\end{array}$

$\begin{array}{lll}0.005 & 470 \quad 150\end{array}$

$0.008 \quad 370 \quad 160$

$\begin{array}{lll}0.009 & 320 & 150\end{array}$

$0.012 \quad 240 \quad 140$

$0.011200 \quad 160$

$\begin{array}{lll}0.011 & 210 & 160\end{array}$

$0.011 \quad 220 \quad 170$

$0.014 \quad 210 \quad 160$

$0.015 \quad 170 \quad 100$

$0.014160 \quad 120$

$\begin{array}{lll}0.013 & 230 & 150\end{array}$

$0.011 \quad 170 \quad 150$

$\begin{array}{lll}0.008 & 250 & 170\end{array}$

$0.011250 \quad 120$

$0.009200 \quad 90$

$0.010 \quad 300 \quad 120$

$\begin{array}{lll}0.011 & 250 & 110\end{array}$

$\begin{array}{lll}0.016 & 220 & 92 \\ 0.016 & 150 & 74\end{array}$

$\begin{array}{lll}0.009 & 120 & 120\end{array}$

$0.014 \quad 290 \quad 87$

$0.014 \quad 260 \quad 84$

$\begin{array}{lll}0.009 & 140 & 110\end{array}$

$0.008 \quad 150 \quad 120$

$0.018 \quad 130 \quad 65$

$\begin{array}{lll}0.016 & 100 & 35 \\ 0.011 & 140 & 93\end{array}$

$\begin{array}{lll}0.011 & 150 & 120\end{array}$

$\begin{array}{lll}0.008 & 160 & 120\end{array}$

$0.008 \quad 150 \quad 120$

$0.007 \quad 160 \quad 110$

$\begin{array}{lll}0.004 & 150 & 130\end{array}$ 
0423205010 Irondequoit Creek Above Blossom Road, Rochester, N.Y.

\section{WATER-QUALITY RECORDS}

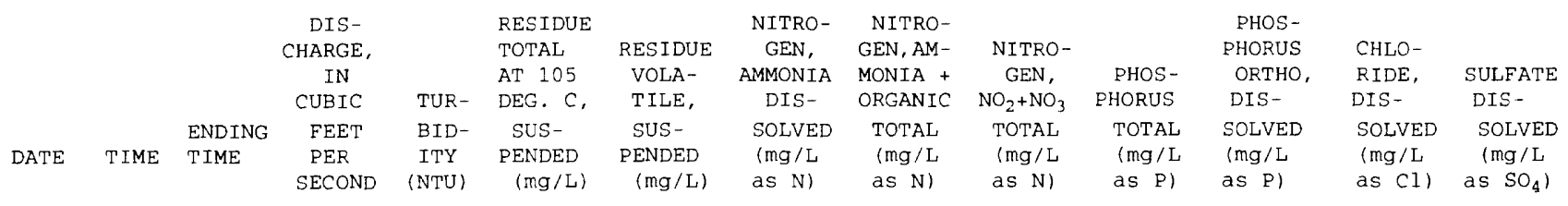

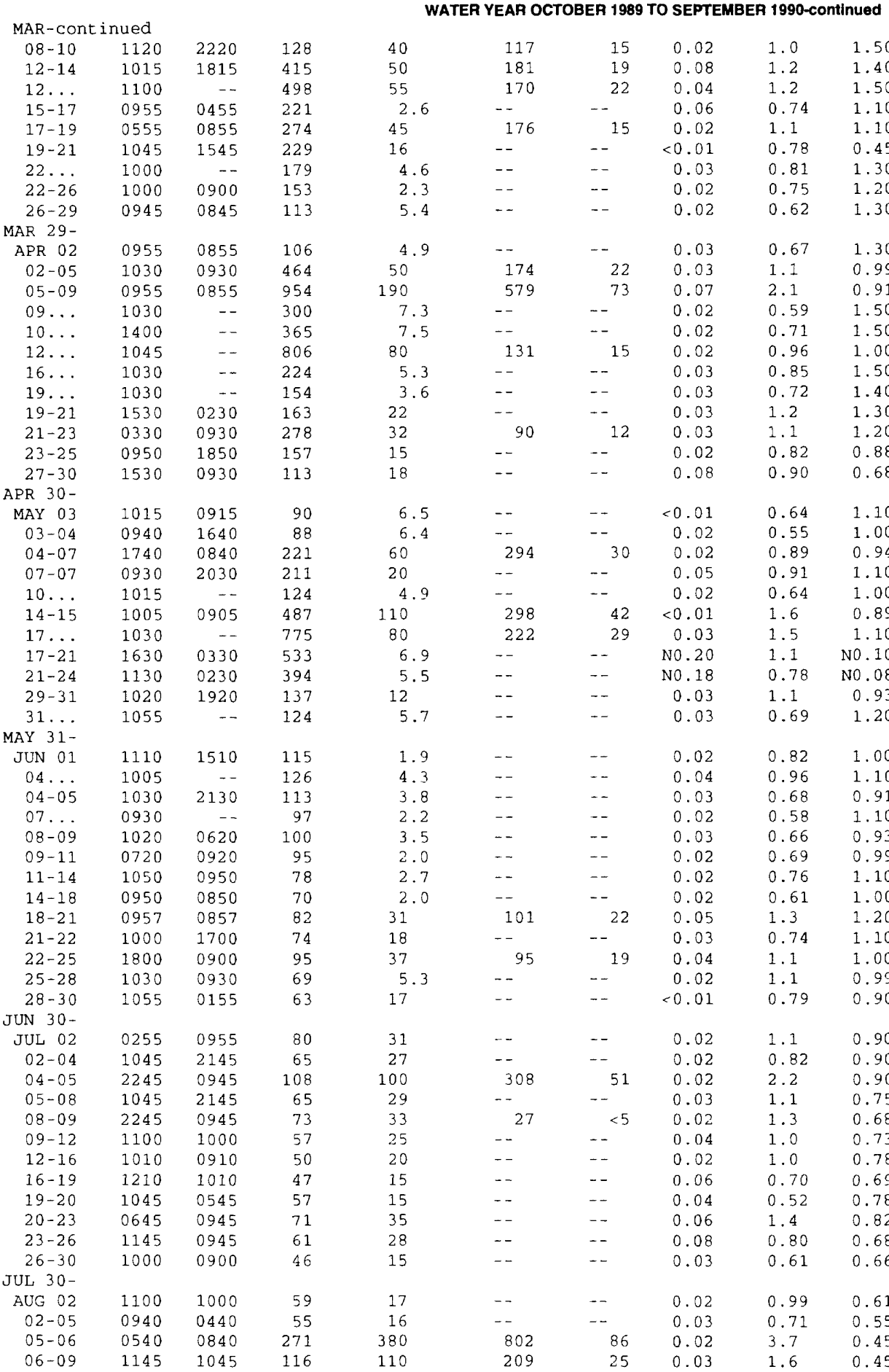

\begin{tabular}{|c|c|c|c|}
\hline 0.180 & 0.007 & 130 & 110 \\
\hline 0.200 & 0.011 & 100 & 81 \\
\hline 0.245 & 0.016 & 100 & 73 \\
\hline 0.065 & 0.004 & 120 & 110 \\
\hline 0.200 & 0.006 & 110 & 95 \\
\hline 0.085 & 0.002 & 120 & 110 \\
\hline 0.040 & 0.006 & 130 & 110 \\
\hline 0.050 & 0.010 & 130 & 130 \\
\hline 0.035 & 0.002 & 130 & 140 \\
\hline 0.055 & 0.004 & 130 & 160 \\
\hline 0.210 & 0.004 & 110 & 110 \\
\hline 0.590 & 0.009 & 120 & 59 \\
\hline 0.050 & 0.007 & 110 & 82 \\
\hline 0.055 & 0.005 & 120 & 88 \\
\hline 0.180 & 0.014 & 66 & 52 \\
\hline 0.110 & 0.007 & 110 & 84 \\
\hline 0.030 & 0.005 & 110 & 100 \\
\hline 0.115 & 0.002 & 110 & 110 \\
\hline 0.150 & 0.002 & 96 & 79 \\
\hline 0.065 & 0.003 & 100 & 90 \\
\hline 0.100 & 0.002 & 110 & 110 \\
\hline 0.050 & 0.003 & 130 & 130 \\
\hline 0.055 & 0.002 & 120 & 130 \\
\hline 0.300 & 0.003 & 96 & 91 \\
\hline 0.110 & 0.009 & 91 & 83 \\
\hline 0.045 & 0.005 & 110 & 110 \\
\hline 0.330 & 0.007 & 77 & 64 \\
\hline 0.310 & 0.026 & 64 & 54 \\
\hline 0.050 & 0.003 & 78 & 36 \\
\hline 0.060 & 0.003 & 110 & 36 \\
\hline 0.100 & 0.006 & 97 & 120 \\
\hline 0.035 & 0.012 & 99 & 120 \\
\hline 0.065 & 0.008 & 100 & 120 \\
\hline 0.060 & 0.015 & 98 & 100 \\
\hline 0.050 & 0.011 & 99 & 120 \\
\hline 0.030 & 0.011 & 100 & 140 \\
\hline 0.045 & 0.010 & 100 & 120 \\
\hline 0.030 & 0.006 & 100 & 140 \\
\hline 0.040 & 0.009 & 110 & 160 \\
\hline 0.035 & 0.013 & 110 & 160 \\
\hline 0.150 & 0.023 & 100 & 150 \\
\hline 0.095 & 0.022 & 110 & 170 \\
\hline 0.180 & 0.019 & 98 & 140 \\
\hline 0.122 & 0.016 & 110 & 150 \\
\hline 0.100 & 0.015 & 110 & 160 \\
\hline 0.150 & 0.015 & 100 & 150 \\
\hline 0.130 & 0.014 & 100 & 150 \\
\hline 0.460 & 0.017 & 79 & 120 \\
\hline 0.150 & 0.010 & 100 & 140 \\
\hline 0.170 & 0.006 & 110 & 160 \\
\hline 0.100 & 0.008 & 100 & 160 \\
\hline 0.120 & 0.004 & 110 & 180 \\
\hline 0.085 & 0.008 & 110 & 150 \\
\hline 0.075 & 0.015 & 100 & 170 \\
\hline 0.210 & 0.016 & 98 & 160 \\
\hline 0.130 & 0.011 & 100 & 150 \\
\hline 0.160 & 0.006 & 120 & 170 \\
\hline 0.130 & 0.007 & 100 & 160 \\
\hline 0.110 & 0.006 & 110 & 180 \\
\hline 0.950 & 0.008 & 550 & 84 \\
\hline 0.380 & 0.008 & 90 & 120 \\
\hline
\end{tabular}


Surface-Water Stations

A. Discharge and water quality

0423205010 Irondequoit Creek Above Blossom Road, Rochester, N.Y.

2. WATER-QUALITY RECORDS

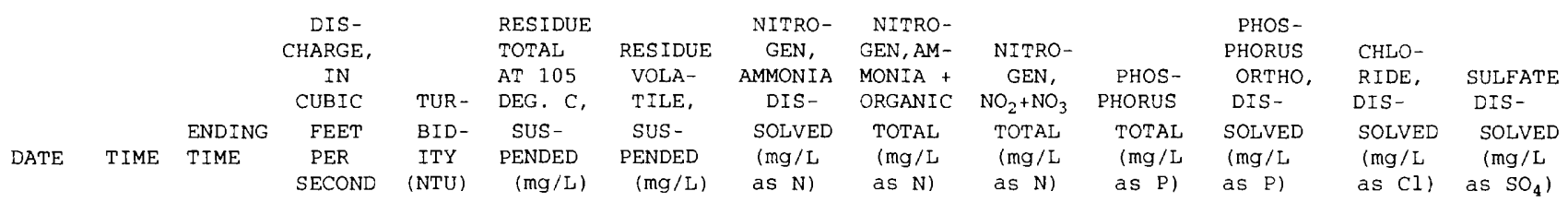

\begin{tabular}{ccc}
\multicolumn{2}{l}{ AUG-CONTINUED } & \\
$09-12$ & 1045 & 1345 \\
$12-13$ & 1445 & 0945 \\
$13-16$ & 1215 & 1115 \\
$16-20$ & 1100 & 1000 \\
$20-23$ & 1230 & 1030 \\
$23-27$ & 1115 & 1015 \\
$27-31$ & 1120 & 1020 \\
AUG 31- & & \\
SEP 04 & 1200 & 1100 \\
$04-05$ & 1205 & 0705 \\
$05-06$ & 0805 & 1105 \\
$06-07$ & 1215 & 0715 \\
$07-10$ & 0815 & 1115 \\
$10-13$ & 1215 & 1115 \\
$13-14$ & 1135 & 2235 \\
$14-17$ & 2335 & 1035 \\
$17-20$ & 1150 & 1050 \\
$20-24$ & 1115 & 1015 \\
$24-25$ & 1135 & 1935 \\
$25-27$ & 2035 & 1035 \\
$27-29$ & 1145 & 1845 \\
SEP $29-$ & & \\
$O C T-01$ & 1945 & 1045
\end{tabular}

WATER YEAR OCTOBER 1989 TO SEPTEMBER 1990-continued

OCT $01 \quad 1945 \quad 1045$

48
103
90
52
47
42
42

28
64
96
103
91
52
50
67
55
52
48
56
--
--

$\begin{array}{cc}27 & 78 \\ 75 & 212 \\ 50 & 147 \\ 30 & -77 \\ 21 & -- \\ 14 & -- \\ 18 & -- \\ & \\ 9.8 & -- \\ 14 & -- \\ 90 & 299 \\ 27 & -- \\ 65 & 229 \\ 26 & -- \\ 18 & -- \\ 29 & -- \\ 14 & -- \\ 11 & -- \\ 8.7 & -- \\ 18 & -- \\ 8.2 & --\end{array}$

$\begin{array}{cc}14 & 0.02 \\ 30 & 0.02 \\ 23 & -- \\ 15 & -- \\ -- & -- \\ -- & -- \\ -- & -- \\ & \\ -- & -- \\ -- & <0.04 \\ 50 & <0.04 \\ -- & <0.04 \\ 32 & <0.04 \\ -- & <0.01 \\ -- & <0.01 \\ -- & <0.01 \\ -- & 0.01 \\ -- & <0.01 \\ -- & <0.01 \\ -- & <0.01 \\ -- & 0.02\end{array}$

$\begin{array}{ll}1.2 & 0.52\end{array}$

$\begin{array}{ll}1.8 & 0.53 \\ 1.6 & 0.54\end{array}$

1.6

0.54

1.3

1.1
0.80

0.68

0.61

0.210

0.004

$\begin{array}{lll}0.96 & 0.61 & 0.105\end{array}$

0.76

2.4

0.55

1.3

0.81

0.095
0.510

0.180

0.93

0.87

0.53

0.290

0.140

0.56

0.110

1.3

0.69

0.51

1.0

0.59

0.150
0.095

0.070

0.68

0.66

0.55

0.65

0.070
0.110

0.070

$\begin{array}{lll}1.2 & 0.71 \quad 0.160\end{array}$

0.004
0.004
0.008

0.008

$0.016 \quad 100$

$0.006 \quad 100$

$0.006 \quad 110$

$0.007 \quad 110$

$\begin{array}{rr}0.0006 & 100 \\ 0.010 & 71\end{array}$

0.01199

$\begin{array}{rr}0.010 & 83 \\ 0.010 & 100\end{array}$

$0.014 \quad 120$

0.00998

$0.008 \quad 110$

$0.008 \quad 110$

$0.008 \quad 110$

$0.007 \quad 100$

0.02

0.008

89

97

OCT

$\begin{array}{rrrr}01-04 & 1200 & 1100 & 54 \\ 04-09 & 1130 & 1030 & 69 \\ 09-11 & 1210 & 1110 & 137 \\ 11-13 & 1125 & 0625 & 306 \\ 13-15 & 0725 & 1025 & 236 \\ 15-18 & 1150 & 1050 & 98 \\ 18-18 & 1100 & 1400 & 80 \\ 18-22 & 1500 & 1000 & 131 \\ 22-23 & 1130 & 0730 & 99 \\ 23-25 & 0830 & 1030 & 181 \\ 25-29 & 1100 & 1000 & 109 \\ \text { OCT } 29- & & & \\ \text { NOV } 01 & 1100 & 1000 & 87 \\ 01-05 & 1040 & 0940 & 76 \\ 05-09 & 1055 & 0855 & 115 \\ 09-10 & 0930 & 0030 & 86 \\ 10-13 & 0730 & 0830 & 207 \\ 13-15 & 1145 & 1045 & 142 \\ 15-19 & 1125 & 1025 & 138 \\ 21-22 & 0920 & 1420 & 71 \\ 22-26 & 1520 & 0820 & 121 \\ 26-27 & 1120 & 0420 & 103 \\ 27-29 & 0520 & 1020 & 158 \\ \text { NOV } 29- & & & \\ \text { DEC 03 } & 1045 & 0945 & 96 \\ 03-04 & 1205 & 0805 & 179 \\ 04-06 & 0905 & 0105 & 388 \\ 06-10 & 1100 & 0200 & 147 \\ 10-13 & 1100 & 0100 & 115 \\ 13-14 & 1035 & 1335 & 119 \\ 17-18 & 1130 & 1330 & 172 \\ 18-19 & 1415 & 0115 & 340 \\ 19-20 & 0215 & 0715 & 407 \\ 20-23 & 1000 & 0100 & 192 \\ 23-24 & 0200 & 0900 & 343 \\ 24-28 & 1025 & 0925 & 184 \\ 28-29 & 1125 & 0625 & 126 \\ 29-29 & 0725 & 2125 & 278\end{array}$

WATER YEAR OCTOBER 1990 TO SEPTEMBER 1991

\begin{tabular}{rrrllllrr}
-- & -- & $<0.01$ & 0.50 & 0.60 & 0.070 & 0.007 & 110 & 160 \\
101 & 18 & $<0.01$ & 0.99 & 0.49 & 0.180 & 0.009 & 100 & 150 \\
166 & 23 & 0.02 & 1.3 & 0.50 & 0.260 & 0.016 & 87 & 120 \\
275 & 37 & 0.02 & 1.8 & 0.48 & 0.410 & 0.012 & 70 & 72 \\
177 & 28 & 0.01 & 1.3 & 0.68 & 0.270 & 0.012 & 80 & 71 \\
77 & 12 & 0.02 & 1.1 & 0.71 & 0.150 & 0.013 & 100 & 120 \\
-- & -- & 0.03 & 0.92 & 0.77 & 0.140 & 0.015 & 110 & 140 \\
130 & \multicolumn{1}{l}{18} & 0.04 & 1.1 & 0.62 & 0.200 & 0.014 & 90 & 120 \\
-- & -- & 0.02 & 0.94 & 0.72 & 0.130 & 0.011 & 93 & 120 \\
132 & -15 & $<0.01$ & 0.98 & 0.69 & 0.200 & 0.012 & 82 & 120 \\
-- & -- & $<0.01$ & 0.73 & 0.88 & 0.110 & 0.016 & 97 & 120 \\
& & & & & & & & \\
-- & -- & 0.02 & 0.82 & 0.97 & 0.080 & 0.010 & 100 & 120 \\
-- & -- & 0.03 & 0.75 & 0.88 & 0.075 & 0.018 & 100 & 150 \\
-- & -- & 0.03 & 0.83 & 0.85 & 0.180 & 0.012 & 100 & 130 \\
-- & -- & 0.03 & 0.71 & 0.88 & 0.080 & 0.012 & 110 & 130 \\
-- & -- & 0.02 & 1.2 & 0.88 & 0.160 & 0.013 & 120 & 88 \\
-- & -- & $<0.01$ & 0.78 & 1.00 & 0.130 & 0.011 & 130 & 100 \\
-- & -- & 0.01 & 0.96 & 0.97 & 0.090 & 0.012 & 120 & 110 \\
-- & -- & 0.02 & 1.0 & 1.20 & 0.100 & 0.015 & 120 & 150 \\
-- & -- & 0.01 & 0.77 & 0.96 & 0.100 & 0.011 & 110 & 100 \\
-- & -- & $<0.01$ & 0.77 & 0.85 & 0.075 & 0.009 & 110 & 120 \\
-- & -- & $<0.01$ & 0.76 & 0.84 & 0.095 & 0.007 & 120 & 130 \\
& & & & & & & & \\
-- & -- & 0.03 & 0.84 & 1.00 & 0.095 & 0.011 & 120 & 120 \\
-- & -- & $<0.01$ & 1.2 & 1.10 & 0.200 & 0.008 & 170 & 78 \\
-- & -- & $<0.01$ & 2.0 & 0.98 & 0.360 & 0.011 & 120 & 69 \\
-- & -- & 0.01 & 1.0 & 1.20 & 0.105 & 0.012 & 120 & 110 \\
-- & -- & $<0.01$ & 0.80 & 1.50 & 0.070 & 0.010 & 110 & 120 \\
-- & -- & 0.01 & 0.74 & 1.40 & 0.090 & 0.008 & 130 & 130 \\
-- & -- & 0.02 & 0.90 & 1.20 & 0.110 & 0.008 & 100 & 91 \\
234 & 27 & 0.02 & 1.7 & 1.10 & 0.320 & 0.009 & 100 & 73 \\
304 & 41 & 0.02 & 2.2 & 1.20 & 0.420 & 0.019 & 77 & 58 \\
-- & -- & 0.02 & 0.81 & 1.30 & 0.140 & 0.013 & 92 & 73 \\
252 & 33 & 0.03 & 1.6 & 1.10 & 0.400 & 0.014 & 70 & 54 \\
89 & 11 & 0.01 & 1.0 & 1.40 & 0.150 & 0.018 & 110 & 91 \\
-- & -- & 0.02 & 0.50 & 1.60 & 0.125 & 0.016 & 120 & 130 \\
743 & 66 & 0.02 & 3.2 & 1.30 & 0.920 & 0.015 & 110 & 63
\end{tabular}

12
32
55
90
70
30
26
50
29
50
26

13
12
21
4.8
27
17
19
21
22
12
17
15
15
34
90
-9
12
16
28
80
130
33
100
40
24
220

0.50

0.007

0.016

0.012

0.013

0.014

0.011

$100 \quad 170$

140

130

180

180

190

190
130

130

N130

160

130
190

190

170

110 
0423205010 Irondequoit Creek Above Blossom Road, Rochester, N.Y.

2. WATER-QUALITY RECORDS

\begin{tabular}{|c|c|c|c|c|c|c|c|c|c|c|c|c|c|}
\hline & & & $\begin{array}{c}\text { DIS- } \\
\text { CHARGE, } \\
\text { IN } \\
\text { CUBIC }\end{array}$ & TUR- & $\begin{array}{l}\text { RESIDUE } \\
\text { TOTAL } \\
\text { AT } 105 \\
\text { DEG. C, }\end{array}$ & $\begin{array}{l}\text { RESIDUE } \\
\text { VOLA- } \\
\text { TILE, }\end{array}$ & $\begin{array}{l}\text { NITRO- } \\
\text { GEN, } \\
\text { AMMONIA } \\
\text { DIS- }\end{array}$ & $\begin{array}{l}\text { NITRO- } \\
\text { GEN, AM- } \\
\text { MONIA + } \\
\text { ORGANIC }\end{array}$ & $\begin{array}{c}\text { NITRO- } \\
\text { GEN, } \\
\mathrm{NO}_{2}+\mathrm{NO}_{3}\end{array}$ & $\begin{array}{l}\text { PHOS- } \\
\text { PHORUS }\end{array}$ & $\begin{array}{l}\text { PHOS- } \\
\text { PHORUS } \\
\text { ORTHO, } \\
\text { DIS- }\end{array}$ & $\begin{array}{l}\text { CHLO- } \\
\text { RIDE, } \\
\text { DIS- }\end{array}$ & $\begin{array}{l}\text { SULFATE } \\
\text { DIS- }\end{array}$ \\
\hline ATE & TIME & $\begin{array}{l}\text { ENDING } \\
\text { TIME }\end{array}$ & $\begin{array}{l}\text { FEET } \\
\text { PER } \\
\text { SECOND }\end{array}$ & $\begin{array}{l}\text { BID- } \\
\text { ITY } \\
\text { (NTU) }\end{array}$ & $\begin{array}{c}\text { SUS- } \\
\text { PENDED } \\
(\mathrm{mg} / \mathrm{L})\end{array}$ & $\begin{array}{l}\text { SUS- } \\
\text { PENDED } \\
(\mathrm{mg} / \mathrm{L})\end{array}$ & $\begin{array}{l}\text { SOLVED } \\
\text { (mg/L } \\
\text { as } \mathrm{N})\end{array}$ & $\begin{array}{l}\text { TOTAL } \\
\text { (mg/L } \\
\text { as } \mathrm{N})\end{array}$ & $\begin{array}{l}\text { TOTAL } \\
\text { (mg/L } \\
\text { as N) }\end{array}$ & $\begin{array}{l}\text { TOTAL } \\
(\mathrm{mg} / \mathrm{L} \\
\text { as } \mathrm{P})\end{array}$ & $\begin{array}{l}\text { SOLVED } \\
(\mathrm{mg} / \mathrm{L} \\
\text { as P) }\end{array}$ & $\begin{array}{l}\text { SOLVED } \\
\text { (mg/L } \\
\text { as C } 1 \text { ) }\end{array}$ & $\begin{array}{r}\text { SOLVED } \\
\left(\mathrm{mg}^{\prime} / \mathrm{L}\right. \\
\left.\text { as } \mathrm{SO}_{4}\right)\end{array}$ \\
\hline
\end{tabular}

JAN

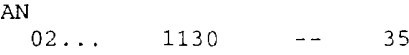

$\begin{array}{rrrc}02 \ldots & 1130 & -- & 357 \\ 02-04 & 1130 & 1030 & 279 \\ 04-07 & 1120 & 1020 & 183 \\ 07-10 & 1155 & 1055 & 144 \\ 10-11 & 1155 & 2255 & 126 \\ 11-14 & 2355 & 0355 & 117 \\ 14-15 & 1305 & 0105 & -- \\ 17-22 & 1210 & 1110 & 335 \\ 17 \ldots & 1215 & -- & 561 \\ 22-24 & 1215 & 1015 & 196 \\ 24-28 & 1110 & 1010 & 135 \\ 28-31 & 1120 & 1020 & 113 \\ \text { JAN 31- } & & & \\ \text { FEB 03 } & 1145 & 1445 & 112\end{array}$

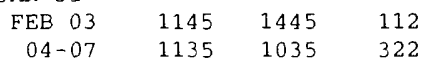

$\begin{array}{llll}07-11 & 1135 & 1035 & 272\end{array}$

$\begin{array}{llll}11-15 & 1150 & 0850 & 143 \\ 15-18 & 0930 & 2030 & 128\end{array}$

$\begin{array}{llll}15-18 & 0930 & 2030 & 128 \\ 18-19 & 2130 & 0830 & 131\end{array}$

$\begin{array}{llll}19-20 & 1155 & 1055 & 368\end{array}$

$\begin{array}{llll}19-21 & 1155 & 1055 & 351 \\ 21-25 & 1205 & 1105 & 174\end{array}$

$\begin{array}{llll}28 \ldots & 1125 \quad \ldots & 104\end{array}$

MAR

01-02 $1005 \quad 0905 \quad 139$

02-04 $\quad 1005 \quad 0305 \quad 440$

$06 \ldots \quad 1135 \quad \ldots \quad 697$

$07 \ldots \quad 1105 \quad--\quad 665$

$\begin{array}{rrrr}07-08 & 1105 & 1005 & 563 \\ 08 \ldots & 1320 & -- & 409\end{array}$

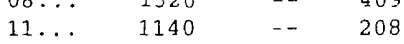

$14 \ldots \quad 1125 \quad \ldots \quad 169$

$\begin{array}{llll}14-15 & 1630 & 1430 & 165\end{array}$

$15-18 \quad 1520 \quad 1020 \quad 163$

$\begin{array}{llll}18-19 & 1130 & 0130 & 183 \\ 19-21 & 0230 & 1030 & 186\end{array}$

$\begin{array}{llll}121-23 & 1145 & 0745 & 139\end{array}$

$\begin{array}{llll}23-23 & 0845 & 1645 & 297\end{array}$

23-24 $1745 \quad 0445 \quad 385$

$24-24 \quad 0545 \quad 1045 \quad 444$

$25-27 \quad 1125 \quad 0425 \quad 223$

$25 \ldots \quad 1140 \quad--\quad 275$

$\begin{array}{llll}27-27 & 0525 & 2225 & 418\end{array}$

$27-28$

MAR $28-$

$\begin{array}{lllll}\text { APR } & 01 & 1130 & 1030 & 321\end{array}$

01-01 $1140 \quad 2240 \quad 248$

$01-04 \quad 2340 \quad 1040 \quad 354$

04-08 $1130 \quad 1030 \quad 149$

08-09 $1110 \quad 0110 \quad 193$

$09-10 \quad 0210 \quad 0110 \quad 239$

$\begin{array}{llll}10-11 & 0210 & 1010 & 318\end{array}$

$\begin{array}{llll}11-15 & 1120 & 1020 & 173\end{array}$

$\begin{array}{llll}15-16 & 1130 & 0130 & 266\end{array}$

$16-18 \quad 0230 \quad 1030 \quad 254$

$\begin{array}{llll}18-20 & 1125 & 0225 & 173\end{array}$

$\begin{array}{llll}20-22 & 0325 & 0925 & 449 \\ 22-25 & 1020 & 0920 & 694\end{array}$

$\begin{array}{llll}25-29 & 1020 & 0920 & 272\end{array}$

APR $29-$

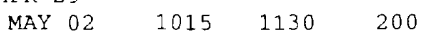

$01 \ldots \quad 1145 \quad--\quad 191$

02-05 $1205 \quad 2205 \quad 171$

06-09 $1015 \quad 0915 \quad 178$

$09-13 \quad 1115 \quad 1015 \quad 155$

13-16

WATER YEAR OCTOBER 1990 TO SEPTEMBER 1991-continued

\begin{tabular}{|c|c|c|c|c|c|c|c|c|c|}
\hline 19 & $\cdots$ & $\ldots$ & 0.06 & 0.76 & 1.50 & 0.095 & 0.028 & 87 & 60 \\
\hline 45 & 100 & 12 & 0.02 & 1.0 & 1.70 & 0.190 & 0.012 & 110 & 80 \\
\hline 20 & $\cdots$ & -- & 0.02 & 1.1 & 1.80 & 0.100 & 0.012 & 130 & 95 \\
\hline 13 & -- & - & 0.04 & 0.77 & 1.90 & 0.075 & 0.010 & 130 & 93 \\
\hline 13 & -- & -- & 0.02 & 0.76 & 1.60 & 0.085 & 0.011 & 130 & 120 \\
\hline 16 & - & $\ldots$ & 0.02 & 0.66 & 1.60 & 0.065 & 0.008 & 170 & 110 \\
\hline 16 & -- & -- & 0.03 & 0.77 & 1.80 & 0.090 & 0.010 & 170 & 120 \\
\hline 36 & 95 & 12 & 0.03 & 1.0 & 1.50 & 0.160 & 0.014 & 120 & 69 \\
\hline 26 & $\cdots$ & -- & 0.09 & 0.79 & 1.30 & 0.130 & 0.026 & 170 & 73 \\
\hline 15 & $-\cdots$ & -- & 0.02 & 0.77 & 1.80 & 0.070 & 0.010 & 120 & 93 \\
\hline 8.4 & $\cdots$ & -- & 0.01 & 0.65 & 1.90 & 0.050 & 0.009 & 130 & 130 \\
\hline 14 & - & -- & 0.02 & 0.73 & 1.80 & 0.085 & 0.008 & 180 & 110 \\
\hline 8.0 & -- & -- & -- & 0.64 & 1.90 & 0.065 & 0.006 & 210 & 140 \\
\hline 40 & 106 & 14 & 0.01 & 0.94 & 1.40 & 0.160 & 0.009 & 150 & 73 \\
\hline 24 & -- & -- & 0.01 & 0.86 & 1.40 & 0.095 & 0.009 & 120 & 78 \\
\hline 6.7 & - & -- & 0.01 & 0.62 & 1.50 & 0.040 & 0.004 & 180 & 110 \\
\hline 8.6 & - & -- & 0.01 & 0.70 & 1.50 & 0.065 & 0.005 & 200 & 120 \\
\hline 5.9 & - & -- & 0.01 & 0.50 & 1.50 & 0.045 & 0.003 & 200 & 130 \\
\hline 50 & 178 & 26 & 0.01 & 1.3 & 1.30 & 0.250 & 0.005 & 180 & 88 \\
\hline 90 & 208 & 23 & 0.01 & 1.3 & 1.20 & 0.260 & 0.006 & 110 & 66 \\
\hline 12 & -- & -- & 0.02 & 0.63 & 1.30 & 0.075 & 0.005 & 130 & 110 \\
\hline 1.9 & $\cdots$ & -- & 0.02 & 0.63 & 1.30 & 0.015 & 0.003 & 130 & 150 \\
\hline 8.2 & $\ldots$ & -- & 0.01 & 0.49 & 1.40 & 0.050 & 0.005 & 190 & 130 \\
\hline 270 & 926 & 91 & 0.02 & 2.6 & 1.10 & 1.05 & 0.010 & 130 & 77 \\
\hline 100 & 334 & 25 & 0.03 & 0.90 & 1.20 & 0.430 & 0.026 & 66 & 46 \\
\hline 30 & 234 & 140 & 0.04 & 0.97 & 1.20 & 0.140 & 0.022 & 77 & 45 \\
\hline 290 & 1090 & 92 & 0.17 & 3.8 & 0.47 & 1.25 & 0.006 & 69 & 43 \\
\hline 24 & - & - & 0.04 & 0.72 & 1.30 & 0.090 & 0.016 & 78 & 63 \\
\hline 6.2 & -- & -- & 0.03 & 0.56 & 1.60 & 0.040 & 0.009 & 95 & 94 \\
\hline 3.3 & - & - & 0.02 & 0.39 & 1.60 & 0.020 & 0.005 & 100 & 120 \\
\hline 3.9 & $-\infty$ & -- & 0.03 & 0.55 & 1.70 & 0.035 & -- & 100 & - \\
\hline 13 & -- & -- & 0.01 & 0.82 & 1.50 & 0.130 & 0.002 & 100 & 120 \\
\hline 16 & -- & -- & 0.01 & 0.88 & 1.30 & 0.085 & 0.004 & 110 & 120 \\
\hline 12 & - & -- & 0.01 & 0.88 & 1.30 & 0.075 & 0.004 & 110 & 120 \\
\hline 5.0 & -- & - & 0.02 & 0.67 & 1.30 & 0.045 & 0.003 & 110 & 120 \\
\hline 33 & 541 & 43 & 0.01 & 1.4 & 1.20 & 0.530 & 0.005 & 94 & 96 \\
\hline 190 & 480 & 50 & 0.01 & 1.8 & 1.00 & 0.650 & 0.007 & 79 & 67 \\
\hline 150 & 278 & 34 & 0.02 & 1.6 & 1.10 & 0.500 & 0.007 & 91 & 73 \\
\hline 35 & 81 & 11 & 0.03 & 0.91 & 1.20 & 0.160 & 0.005 & 92 & 83 \\
\hline 10 & -- & -- & 0.04 & 0.54 & 1.10 & 0.055 & 0.010 & 92 & 77 \\
\hline 90 & 248 & 29 & 0.02 & 1.2 & 1.10 & 0.280 & 0.005 & 84 & 74 \\
\hline 250 & 612 & 28 & 0.02 & 2.0 & 1.00 & 0.770 & 0.010 & 72 & 60 \\
\hline 55 & 206 & 24 & 0.02 & 1.2 & 1.10 & 0.270 & 0.006 & 82 & 56 \\
\hline 20 & -- & -- & 0.02 & 0.85 & 1.20 & 0.120 & 0.007 & 98 & 110 \\
\hline 10 & -- & -- & 0.01 & 0.72 & 1.30 & 0.090 & 0.005 & 100 & 110 \\
\hline 9.0 & - & -- & 0.02 & 0.82 & 1.20 & 0.085 & 0.006 & 110 & 97 \\
\hline 90 & 257 & 31 & 0.04 & 0.75 & 1.10 & 0.260 & 0.009 & 98 & 60 \\
\hline 40 & 139 & 21 & 0.04 & 0.85 & 1.10 & 0.190 & 0.011 & 89 & 98 \\
\hline 110 & 273 & 41 & 0.02 & 1.4 & 0.93 & 0.380 & 0.008 & 78 & 80 \\
\hline 24 & -- & -- & 0.03 & 0.90 & 0.97 & 0.095 & 0.005 & 95 & 110 \\
\hline 50 & 176 & 14 & 0.02 & 1.1 & 1.00 & 0.220 & 0.008 & 91 & 99 \\
\hline 75 & 190 & 24 & 0.02 & 1.2 & 0.83 & 0.240 & 0.007 & 81 & 85 \\
\hline 20 & -- & -- & 0.03 & 0.74 & 0.91 & 0.110 & 0.006 & 95 & 110 \\
\hline 150 & 395 & 33 & 0.01 & 2.0 & 0.85 & 0.535 & 0.007 & 73 & 73 \\
\hline 140 & 290 & 24 & 0.02 & 1.5 & 0.95 & 0.450 & 0.017 & 53 & 45 \\
\hline 40 & 105 & 12 & 0.02 & 0.98 & 0.95 & 0.170 & 0.011 & 76 & 84 \\
\hline 16 & -- & - & 0.02 & 0.99 & 1.00 & 0.120 & 0.008 & 90 & 110 \\
\hline 4.4 & -- & -- & $<0.01$ & 0.69 & 1.00 & 0.050 & 0.007 & 89 & 110 \\
\hline 14 & -- & -- & 0.02 & 0.58 & 1.10 & 0.095 & 0.008 & 91 & 120 \\
\hline 14 & -- & -- & $<0.01$ & 0.81 & 1.00 & 0.810 & 0.007 & 88 & 120 \\
\hline 11 & -- & -- & 0.01 & 1.0 & 1.00 & 0.080 & 0.010 & 92 & 120 \\
\hline 11 & -- & -- & -- & 0.92 & 0.94 & 0.095 & 0.010 & 94 & 140 \\
\hline
\end{tabular}


Surface-Water Stations

A. Discharge and water quality

0423205010 Irondequoit Creek Above Blossom Road, Rochester, N.Y. 2. WATER-QUALITY RECORDS

\begin{tabular}{|c|c|c|c|c|c|c|c|c|c|c|c|c|c|}
\hline & & & $\begin{array}{c}\text { DIS- } \\
\text { CHARGE, } \\
\text { IN } \\
\text { CUBIC }\end{array}$ & TUR- & $\begin{array}{l}\text { RESIDUE } \\
\text { TOTAL } \\
\text { AT } 105 \\
\text { DEG. C. }\end{array}$ & $\begin{array}{l}\text { RESIDUE } \\
\text { VOLA- } \\
\text { TILE, }\end{array}$ & $\begin{array}{l}\text { NITRO- } \\
\text { GEN, } \\
\text { AMMONIA } \\
\text { DIS- }\end{array}$ & $\begin{array}{l}\text { NITRO- } \\
\text { GEN, AM- } \\
\text { MONIA + } \\
\text { ORGANIC }\end{array}$ & $\begin{array}{c}\text { NITRO- } \\
\text { GEN, } \\
\mathrm{NO}_{2}+\mathrm{NO}_{3}\end{array}$ & $\begin{array}{l}\text { PHOS - } \\
\text { PHORUS }\end{array}$ & $\begin{array}{l}\text { PHOS- } \\
\text { PHORUS } \\
\text { ORTHO, } \\
\text { DIS- }\end{array}$ & $\begin{array}{l}\text { CHLO- } \\
\text { RIDI, } \\
\text { DIS- }\end{array}$ & $\begin{array}{l}\text { SULFATE } \\
\text { DIS- }\end{array}$ \\
\hline & LME & & $\begin{array}{c}\text { FEET } \\
\text { PER } \\
\text { SECOND }\end{array}$ & $\begin{array}{l}\text { BID- } \\
\text { ITY } \\
\text { (NTU) }\end{array}$ & $\begin{array}{l}\text { SUS- } \\
\text { PENDED } \\
(\mathrm{mg} / \mathrm{L})\end{array}$ & $\begin{array}{l}\text { SUS- } \\
\text { PENDED } \\
(\mathrm{mg} / \mathrm{L})\end{array}$ & $\begin{array}{l}\text { SOLVED } \\
\text { (mg/L } \\
\text { as N) }\end{array}$ & $\begin{array}{l}\text { TOTAL } \\
\text { (mg/L } \\
\text { as N) }\end{array}$ & $\begin{array}{l}\text { TOTAL } \\
\text { (mg/L } \\
\text { as N) }\end{array}$ & $\begin{array}{l}\text { TOTAL } \\
\text { (mg/L } \\
\text { as P) }\end{array}$ & $\begin{array}{l}\text { SOLVED } \\
\text { (mg/L } \\
\text { as P) }\end{array}$ & $\begin{array}{l}\text { SOLVED } \\
(\mathrm{mg} / \mathrm{L} \\
\text { as Cl) }\end{array}$ & $\begin{array}{l}\text { SOLVED } \\
\text { (mg/L } \\
\left.\text { as } \mathrm{SO}_{4}\right)\end{array}$ \\
\hline
\end{tabular}

\begin{tabular}{|c|c|c|c|}
\hline \multicolumn{4}{|c|}{ MAY-cont inued } \\
\hline $16-17$ & 0940 & 0840 & 12 \\
\hline $17-17$ & 0940 & 2040 & 19 \\
\hline $17-20$ & 2140 & 0840 & 16 \\
\hline $20-23$ & 1020 & 0920 & \\
\hline $23-26$ & 1150 & 1350 & \\
\hline $26-26$ & 1450 & 2350 & 13 \\
\hline $27-28$ & 0050 & 0950 & 14 \\
\hline $28-30$ & 1035 & 0935 & \\
\hline \multicolumn{4}{|l|}{ MAY $30-$} \\
\hline JUN 03 & 1020 & 0920 & \\
\hline $03-06$ & 1005 & 0905 & \\
\hline $06-10$ & 1230 & 0930 & \\
\hline $10-11$ & 1000 & 1200 & \\
\hline $11-12$ & 1300 & 0900 & \\
\hline $12-12$ & 1000 & 1800 & \\
\hline $12-13$ & 1900 & 0900 & \\
\hline $13-17$ & 1015 & 0915 & \\
\hline $17-20$ & 1015 & 0915 & \\
\hline $20-24$ & 1005 & 0905 & \\
\hline $24-27$ & 1015 & 0915 & \\
\hline \multicolumn{4}{|l|}{ JUN $27-$} \\
\hline JUL 01 & 1010 & 0910 & \\
\hline $01-04$ & 1015 & 1715 & \\
\hline $04-05$ & 1815 & 0915 & \\
\hline $05-06$ & 1240 & 2400 & \\
\hline $06-07$ & 0100 & 0600 & \\
\hline $07-07$ & 0700 & 1500 & \\
\hline $07-08$ & 1600 & 0900 & \\
\hline $08-09$ & 1015 & 1815 & \\
\hline $09-11$ & 1915 & 0915 & \\
\hline $11-15$ & 1010 & 0910 & \\
\hline $15-18$ & 1035 & 0935 & \\
\hline $18-21$ & 1010 & 1310 & \\
\hline $21-22$ & 1410 & 0910 & \\
\hline $22-23$ & 1015 & 1815 & \\
\hline $23-25$ & 1915 & 0915 & \\
\hline $25-29$ & 1020 & 0920 & \\
\hline $29-30$ & 1010 & 0910 & \\
\hline \multicolumn{4}{|l|}{ JUL $30-$} \\
\hline AUG 01 & 1025 & 0925 & \\
\hline $01-03$ & 1035 & 0135 & \\
\hline $03-03$ & 0235 & 1735 & \\
\hline $03-05$ & 1835 & 0935 & \\
\hline $05-08$ & 1010 & 0910 & \\
\hline $08-09$ & 1040 & 0140 & \\
\hline $09-09$ & 0240 & 1440 & \\
\hline $09-12$ & 1840 & 0940 & \\
\hline $12-14$ & 1045 & 1845 & \\
\hline $14-15$ & 1945 & 0045 & 10 \\
\hline $15-15$ & 0145 & 0945 & \\
\hline $15-19$ & 1025 & 0925 & \\
\hline $19-20$ & 1040 & 1840 & \\
\hline $20-21$ & 1940 & 0640 & \\
\hline $21-22$ & 0740 & 0940 & \\
\hline $22-26$ & 1015 & 0915 & \\
\hline $26-30$ & 0940 & 0840 & \\
\hline $30-31$ & 0900 & 0425 & \\
\hline \multicolumn{4}{|l|}{ AUG $31-$} \\
\hline SEP 03 & 0525 & 0825 & \\
\hline $03-04$ & 1000 & 0700 & \\
\hline $04-05$ & 0800 & 0900 & \\
\hline $05-09$ & 1120 & 1020 & \\
\hline $09-10$ & 1235 & 1735 & \\
\hline $10-12$ & 1835 & 1135 & \\
\hline
\end{tabular}

WATER YEAR OCTOBER 1990 TO SEPTEMBER 1991-continued

\begin{tabular}{|c|c|c|c|c|}
\hline-- & -- & -- & 0.84 & 0.98 \\
\hline-- & - & -- & 1.1 & 1.00 \\
\hline-- & $\ldots$ & - - & 1.3 & 0.91 \\
\hline-- & -- & 0.02 & 1.1 & 1.10 \\
\hline-- & -- & 0.02 & 0.88 & 0.82 \\
\hline 209 & 24 & 0.04 & 1.4 & 0.77 \\
\hline 230 & 28 & 0.05 & 1.9 & 0.70 \\
\hline-- & -- & 0.02 & 1.1 & 1.10 \\
\hline 115 & 16 & 0.02 & 1.8 & 1.30 \\
\hline 94 & 14 & $<0.01$ & 1.1 & 0.92 \\
\hline 104 & 28 & $<0.01$ & 0.20 & 1.20 \\
\hline-- & -- & $<0.01$ & 1.0 & 1.20 \\
\hline-- & -- & $<0.01$ & 1.5 & 1.50 \\
\hline-- & -- & 0.04 & 3.5 & 1.60 \\
\hline-- & -- & 0.02 & 3.9 & 1.50 \\
\hline 160 & 26 & $<0.01$ & 1.5 & 1.10 \\
\hline-- & -- & $<0.01$ & 1.0 & 0.97 \\
\hline-- & -- & 0.02 & 1.2 & 0.93 \\
\hline-- & -- & 0.03 & 0.98 & 0.85 \\
\hline-- & -. & 0.02 & 0.87 & 0.82 \\
\hline-- & -- & $<0.01$ & 0.92 & 0.82 \\
\hline-- & -- & $<0.01$ & 1.4 & 0.84 \\
\hline 108 & 18 & 0.04 & 0.72 & 1.02 \\
\hline 142 & 24 & 0.03 & 0.94 & 0.89 \\
\hline 129 & 24 & 0.03 & 0.86 & 0.75 \\
\hline 119 & 21 & 0.01 & 0.86 & 0.68 \\
\hline 103 & 16 & 0.03 & 1.4 & 0.84 \\
\hline-- & -- & 0.01 & 1.3 & 0.77 \\
\hline-- & -- & 0.20 & 0.92 & 0.70 \\
\hline -- & -- & 0.04 & 1.2 & 0.71 \\
\hline 60 & 11 & $<0.01$ & 1.0 & 0.72 \\
\hline 106 & 22 & $<0.01$ & 1.7 & 0.78 \\
\hline 82 & 14 & 0.03 & 0.96 & 0.83 \\
\hline-- & $\ldots$ & 0.03 & 0.98 & 0.71 \\
\hline$\ldots$ & -- & 0.01 & 0.78 & 0.59 \\
\hline-- & -- & $<0.01$ & 0.92 & 0.52 \\
\hline-- & -- & 0.03 & 1.0 & 0.56 \\
\hline-- & -- & 0.02 & 0.94 & 0.71 \\
\hline- & -- & 0.04 & 1.1 & 0.68 \\
\hline-- & -- & 0.02 & 1.2 & 0.83 \\
\hline-- & -- & 0.02 & 0.85 & 0.65 \\
\hline- & -- & $<0.01$ & 0.94 & 0.68 \\
\hline 130 & 32 & $<0.01$ & 1.7 & 0.69 \\
\hline 100 & 24 & $<0.01$ & 1.3 & 0.60 \\
\hline-- & -- & 0.04 & 0.99 & 0.66 \\
\hline 160 & 35 & 0.04 & 1.9 & 0.74 \\
\hline 110 & 26 & 0.05 & 1.2 & 0.86 \\
\hline- & -- & $<0.10$ & 1.3 & 0.54 \\
\hline-- & -- & $<0.01$ & 0.70 & 0.51 \\
\hline-- & -- & $<0.01$ & 0.99 & 0.56 \\
\hline-- & -- & 0.01 & 0.98 & 0.60 \\
\hline-- & -- & $<0.01$ & 1.0 & 0.63 \\
\hline-- & -- & $<0.01$ & 0.85 & 0.44 \\
\hline-- & -- & 0.03 & 0.85 & 0.51 \\
\hline-- & -- & 0.02 & 0.78 & 0.77 \\
\hline-- & -- & 0.01 & 0.90 & 0.67 \\
\hline-- & -- & $<0.01$ & 0.99 & 0.63 \\
\hline-- & -- & $<0.01$ & 0.88 & 0.56 \\
\hline-- & -- & 0.03 & 0.80 & 0.60 \\
\hline- & -- & 0.03 & 1.1 & 0.66 \\
\hline
\end{tabular}

\begin{tabular}{|c|c|c|c|}
\hline 0.110 & 0.015 & 93 & 150 \\
\hline 0.150 & 0.015 & 93 & 140 \\
\hline 0.170 & 0.011 & 82 & 120 \\
\hline 0.120 & 0.012 & 97 & 140 \\
\hline 0.120 & 0.054 & 110 & 160 \\
\hline 0.350 & 0.020 & 93 & 140 \\
\hline 0.360 & 0.017 & 76 & 12 \\
\hline 0.150 & 0.017 & 100 & 14 \\
\hline 0.210 & 0.026 & 110 & 150 \\
\hline 0.200 & 0.023 & 110 & 150 \\
\hline 0.200 & 0.022 & 110 & 180 \\
\hline 0.160 & 0.019 & 110 & 190 \\
\hline 0.290 & 0.021 & 110 & 150 \\
\hline 0.810 & 0.021 & 82 & 120 \\
\hline 0.760 & 0.023 & 64 & 100 \\
\hline 0.300 & 0.029 & 100 & 140 \\
\hline 0.185 & 0.023 & 98 & 180 \\
\hline 0.150 & 0.022 & 110 & 200 \\
\hline 0.130 & 0.017 & 110 & 220 \\
\hline 0.040 & 0.013 & 110 & 190 \\
\hline 0.140 & 0.015 & 110 & 190 \\
\hline 0.190 & 0.010 & 100 & 170 \\
\hline 0.250 & 0.024 & 87 & 160 \\
\hline 0.270 & 0.021 & 77 & 160 \\
\hline 0.270 & 0.016 & 82 & 160 \\
\hline 0.280 & 0.012 & 80 & 140 \\
\hline 0.220 & 0.024 & 89 & 180 \\
\hline 0.190 & 0.015 & 100 & 19 \\
\hline 0.150 & 0.015 & 110 & 17 \\
\hline 0.150 & 0.016 & 110 & 200 \\
\hline 0.130 & 0.016 & 110 & 210 \\
\hline 0.230 & 0.010 & 90 & 180 \\
\hline 0.190 & 0.024 & 78 & 140 \\
\hline 0.150 & 0.014 & 99 & 180 \\
\hline 0.130 & 0.015 & 110 & 200 \\
\hline 0.110 & 0.008 & 110 & 21 \\
\hline 0.170 & 0.011 & 100 & 200 \\
\hline 0.120 & 0.017 & 110 & 200 \\
\hline 0.130 & 0.017 & 100 & 180 \\
\hline 0.140 & 0.014 & 93 & 17 \\
\hline 0.130 & 0.014 & 99 & 19 \\
\hline 0.100 & 0.023 & 100 & 180 \\
\hline 0.270 & 0.026 & 91 & 15 \\
\hline 0.230 & 0.021 & 88 & 15 \\
\hline 0.150 & 0.019 & 110 & 17 \\
\hline 0.340 & 0.014 & $9 \epsilon$ & 11 \\
\hline 0.200 & 0.012 & $7 \varepsilon$ & 12 \\
\hline 0.120 & -- & 120 & 18 \\
\hline 0.110 & 0.015 & 120 & 19 \\
\hline 0.170 & 0.014 & 97 & 15 \\
\hline 0.160 & 0.013 & 83 & 16 \\
\hline 0.140 & 0.015 & 110 & 19 \\
\hline 0.130 & 0.007 & 120 & 22 \\
\hline 0.110 & 0.011 & 120 & 22 \\
\hline 0.130 & 0.010 & 100 & 19 \\
\hline 0.140 & 0.018 & 110 & 20 \\
\hline 0.120 & 0.012 & 100 & 20 \\
\hline 0.120 & 0.008 & $10 C$ & 22 \\
\hline 0.140 & 0.012 & $11 \mathrm{C}$ & \\
\hline 0.180 & 0.010 & 93 & 18 \\
\hline
\end{tabular}


Surface-Water Stations

A. Discharge and water quality

0423205010 Irondequoit Creek Above Blossom Road, Rochester, N.Y. 2. WATER-QUALITY RECORDS

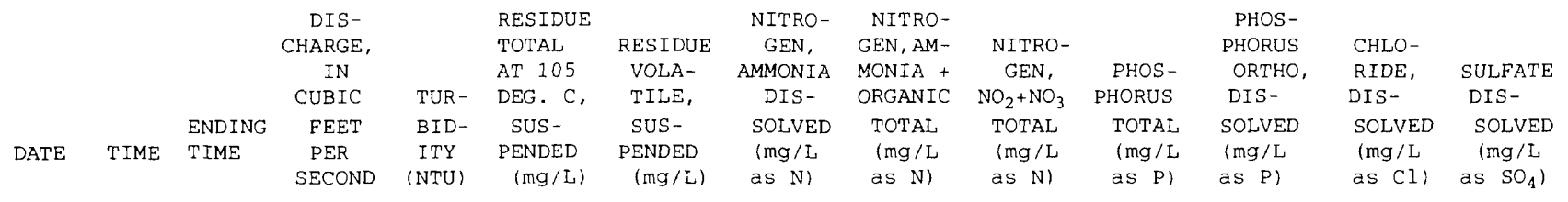

SEP-cont inued

$\begin{array}{lllr}12-15 & 1130 & 0630 & 39 \\ 15-16 & 0730 & 1030 & 63 \\ 16-19 & 1130 & 1135 & 39 \\ 19-23 & 1205 & 1105 & 4 \\ 23-25 & 1135 & 0135 & 41 \\ 25-25 & 0235 & 1335 & 65 \\ 25-26 & 1435 & 1035 & 100 \\ 26-30 & 1100 & 1000 & 4\end{array}$

WATER YEAR OCTOBER 1990 TO SEPTEMBER 1991-continued

$\mathrm{OCT}$

\begin{tabular}{|c|c|c|c|}
\hline $03-04$ & 1050 & 2150 & 39 \\
\hline $04-06$ & 2250 & 0550 & 41 \\
\hline $10-11$ & 1055 & 1955 & 67 \\
\hline $11-15$ & 2055 & 0955 & 52 \\
\hline $15-16$ & 1130 & 0230 & 81 \\
\hline $17-19$ & 1115 & 0615 & 54 \\
\hline \multicolumn{4}{|l|}{ NOV } \\
\hline 04-05 & 1050 & 0950 & 44 \\
\hline $05-06$ & 1050 & 1350 & 43 \\
\hline $08-10$ & 1125 & 1825 & 37 \\
\hline $12-14$ & 1150 & 1050 & 70 \\
\hline $21-24$ & 1115 & 0615 & 59 \\
\hline $24-25$ & 0715 & 1015 & 73 \\
\hline $25-25$ & 1100 & 1800 & 86 \\
\hline $26-27$ & 0100 & 1000 & 86 \\
\hline $27-28$ & 1030 & 2030 & 55 \\
\hline $28-29$ & 2130 & 1130 & 130 \\
\hline \multicolumn{4}{|l|}{ Nov $29-$} \\
\hline DEC 02 & 1230 & 0930 & 88 \\
\hline $02-03$ & 1040 & 0040 & 52 \\
\hline $03-03$ & 0140 & 1240 & 183 \\
\hline $03-05$ & 1340 & 0940 & 159 \\
\hline $05-07$ & 1155 & 1055 & 70 \\
\hline $07-07$ & 1155 & 2255 & 86 \\
\hline $07-09$ & 2355 & 1055 & 84 \\
\hline $09-12$ & 1040 & 0940 & 76 \\
\hline $12-16$ & 1040 & 0940 & 63 \\
\hline $16-19$ & 1100 & 1000 & 52 \\
\hline $19-23$ & 1045 & 0945 & 58 \\
\hline $23-26$ & 1050 & 0950 & 61 \\
\hline $26-27$ & 1205 & 1105 & 36 \\
\hline $30-31$ & 1200 & 1000 & 166 \\
\hline \multicolumn{4}{|l|}{ DEC $30-$} \\
\hline JAN 02 & 1200 & 1100 & 119 \\
\hline $02-06$ & 0950 & 0850 & 79 \\
\hline $06-09$ & 0955 & 0855 & 78 \\
\hline $09-13$ & 1115 & 1015 & 68 \\
\hline $13-14$ & 0935 & -- & -- \\
\hline $14-17$ & 0830 & -- & -- \\
\hline $23 \ldots$ & 1030 & -- & 123 \\
\hline $28-30$ & 1555 & 0855 & 62 \\
\hline \multicolumn{4}{|l|}{ FEB } \\
\hline $03-06$ & 0955 & 0855 & 59 \\
\hline $06-10$ & 1000 & 0500 & 58 \\
\hline $10-14$ & 1010 & 0910 & 63 \\
\hline $14-15$ & 0945 & 0345 & 56 \\
\hline $18-19$ & 1055 & 1355 & 144 \\
\hline $19-20$ & 1455 & 0855 & 186 \\
\hline $20-22$ & 0940 & 2040 & 160 \\
\hline $22-24$ & 2140 & 0840 & 188 \\
\hline $24-27$ & 1010 & 0910 & 158 \\
\hline $27-28$ & 0935 & 1635 & 128 \\
\hline $28-29$ & 1735 & 0435 & 134 \\
\hline
\end{tabular}

20
32
22
17
17
45
45
18
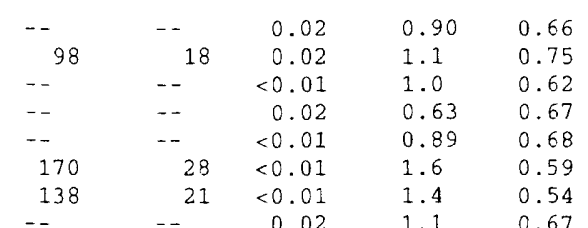

0.140
0.200
0.170
0.130
0.130
0.290
0.280
0.150

$\begin{array}{rrr}0.009 & 110 & 180 \\ 0.007 & 87 & 150 \\ 0.009 & 110 & 180 \\ 0.009 & 100 & 190 \\ 0.027 & 110 & 200 \\ 0.009 & 74 & 120 \\ 0.011 & 62 & 130 \\ 0.012 & 100 & 180\end{array}$

\section{WATER YEAR OCTOBER 1991 TO SEPTEMBER 1992}

\begin{tabular}{|c|c|c|c|c|c|c|c|c|c|}
\hline 16 & -. & -. & 0.01 & 0.80 & 0.79 & 0.120 & 0.012 & 120 & 200 \\
\hline 16 & -- & -- & 0.01 & 0.92 & 0.80 & 0.130 & 0.013 & 120 & 180 \\
\hline 21 & -. & - - & 0.02 & 0.85 & 0.73 & 0.150 & 0.015 & 97 & 180 \\
\hline 13 & -- & - - & 0.02 & 0.79 & 0.70 & 0.110 & 0.013 & 100 & 200 \\
\hline 16 & -- & -- & $<0.01$ & 0.53 & 0.60 & 0.120 & 0.012 & 96 & 200 \\
\hline 7.3 & -- & -- & 0.02 & 0.22 & 0.64 & 0.065 & 0.012 & 100 & \\
\hline 3.0 & -. & -. & 0.01 & 0.55 & 0.68 & 0.045 & 0.004 & 110 & 230 \\
\hline 44 & 706 & 184 & 0.05 & 3.1 & 0.66 & 1.75 & 0.005 & 110 & 240 \\
\hline 2.8 &.- & -- & 0.02 & 0.33 & 0.68 & 0.025 & 0.005 & 100 & 200 \\
\hline 5.5 & -- & -- & 0.04 & 0.65 & 0.71 & 0.055 & 0.008 & 130 & 180 \\
\hline 3.6 & -- & -- & -- & 0.68 & 1.00 & 0.035 & 0.009 & 170 & 270 \\
\hline 4.5 & -- & -- & -- & 0.43 & 0.94 & 0.045 & 0.008 & 130 & 210 \\
\hline 5.5 & -- & -- & 0.01 & 0.58 & 0.85 & 0.050 & 0.009 & 110 & 210 \\
\hline 8.4 & -- & -. & $<0.01$ & 0.55 & 0.70 & 0.050 & 0.008 & 130 & 180 \\
\hline 4.5 & -- & $\ldots$ & - - & 0.50 & 0.85 & 0.040 & 0.007 & 150 & 190 \\
\hline 23 & -- & -- & -- & 0.74 & 0.85 & 0.160 & 0.009 & 140 & 170 \\
\hline 12 & -. & -- & -- & 0.54 & 0.88 & 0.085 & 0.008 & 120 & 170 \\
\hline 2.9 & -- & $\ldots$ & 0.01 & 0.29 & 0.89 & 0.030 & 0.010 & 120 & 200 \\
\hline 35 & -- & -. & 0.01 & 1.2 & 0.74 & 0.220 & 0.012 & 210 & 150 \\
\hline 21 & -- & -- & 0.01 & 0.85 & 0.78 & 0.130 & 0.010 & 180 & 140 \\
\hline 9.1 & -- & - & 0.04 & 0.58 & 0.97 & 0.055 & 0.011 & 180 & \\
\hline 7.9 & -- & -. & 0.03 & 0.50 & 1.10 & 0.060 & 0.009 & 250 & \\
\hline 6.9 & -- & -- & 0.03 & 0.61 & 1.10 & 0.055 & 0.009 & 240 & \\
\hline 7.3 & -- & -. & $<0.01$ & 0.57 & 0.95 & 0.045 & 0.010 & 160 & 190 \\
\hline 8.5 & -- & - & 0.04 & 0.70 & 0.96 & 0.070 & 0.018 & 140 & 180 \\
\hline 7.2 & -. & -- & 0.03 & 0.64 & 1.10 & 0.040 & 0.010 & 170 & 220 \\
\hline 6.2 & -- & -- & 0.03 & 0.56 & 1.20 & 0.035 & 0.010 & 230 & 210 \\
\hline 4.5 & -- & -- & 0.02 & 0.56 & 1.10 & 0.030 & 0.008 & 220 & 200 \\
\hline 3.2 & -- & -- & 0.02 & 0.57 & 1.10 & 0.030 & 0.007 & 200 & 200 \\
\hline 21 & -- & -- & 0.04 & 1.0 & 0.97 & 0.090 & 0.011 & 210 & 140 \\
\hline 11 & -- & -- & 0.03 & 0.70 & 1.40 & 0.040 & 0.006 & 190 & 150 \\
\hline 3.3 & -- & -. & $<0.01$ & 0.59 & 1.20 & 0.030 & 0.002 & 160 & 19 \\
\hline 2.8 & -- & -- & $<0.01$ & 0.50 & 1.40 & 0.015 & 0.005 & 150 & 18 \\
\hline 6.4 & - & -- & 0.01 & 0.64 & 1.60 & 0.050 & 0.008 & 190 & 190 \\
\hline 2.4 & -- & -- & 0.01 & 0.47 & 1.40 & 0.025 & 0.006 & 230 & 190 \\
\hline 11 & -- & - & 0.02 & 0.73 & 1.40 & 0.045 & 0.006 & 220 & 170 \\
\hline 5.2 & 7 & $<5$ & 0.07 & 0.48 & 1.50 & 0.020 & 0.011 & 250 & 4 \\
\hline 4.3 & -- & -- & 0.02 & 0.46 & 1.50 & 0.020 & 0.004 & 180 & 200 \\
\hline 1.8 & .. & $\ldots$ & $<0.01$ & 0.53 & 1.40 & 0.015 & 0.004 & 180 & 21 \\
\hline 1.9 & - & -- & 0.02 & 0.50 & 1.40 & 0.020 & 0.003 & 230 & 22 \\
\hline 3.3 & -- & -- & 0.01 & 0.59 & 1.30 & 0.025 & 0.003 & 230 & 220 \\
\hline 1.6 & -- & -- & 0.05 & 0.44 & 1.20 & 0.015 & 0.006 & 290 & 22 \\
\hline 11 & -- & -- & 0.02 & 0.60 & 1.40 & 0.045 & 0.003 & 230 & 13 \\
\hline 12 & $\cdots$ & -- & 0.02 & 0.63 & 1.60 & 0.050 & 0.006 & 230 & 130 \\
\hline 7.0 & -- & - & 0.03 & 0.63 & 1.70 & 0.045 & 0.006 & 190 & 140 \\
\hline 8.0 & -- & -- & 0.02 & 0.77 & 1.70 & 0.050 & 0.004 & 190 & 13 \\
\hline 7.0 & $\cdots$ & -- & 0.02 & 0.64 & 1.90 & 0.035 & 0.003 & 170 & 130 \\
\hline 2.6 & -- & $\ldots$ & 0.02 & 0.68 & 1.90 & 0.030 & 0.004 & 140 & 15 \\
\hline 5.8 & -. & -. & 0.03 & 0.76 & 1.60 & 0.045 & 0.006 & 170 & 140 \\
\hline
\end{tabular}


0423205010 Irondequoit Creek Above Blossom Road, Rochester, N.Y. 2. WATER-QUALITY RECORDS

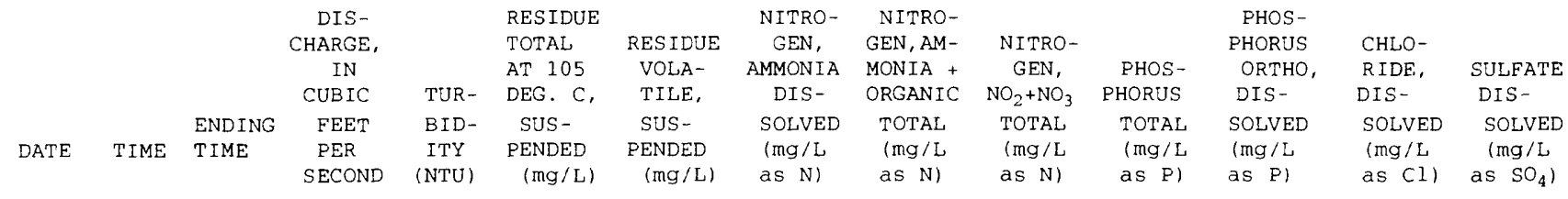

\begin{tabular}{|c|c|c|c|}
\hline \multicolumn{4}{|l|}{ FEB 29- } \\
\hline MAR 02 & 0535 & 0835 & 120 \\
\hline $02-05$ & 1000 & 0900 & 102 \\
\hline $07-08$ & 0130 & 0030 & 184 \\
\hline $08-09$ & 0130 & 0830 & 270 \\
\hline $12 \ldots$ & 1050 & -- & 110 \\
\hline $13-16$ & 1530 & 0830 & 110 \\
\hline $26 \ldots$ & 0930 & -- & 248 \\
\hline \multicolumn{4}{|l|}{ APR } \\
\hline $06-08$ & 1600 & 1500 & 143 \\
\hline $09-11$ & 0940 & 0440 & 106 \\
\hline $11-12$ & 0540 & 0440 & 256 \\
\hline $12-13$ & 0540 & 0840 & 437 \\
\hline $13-16$ & 0955 & 0855 & 194 \\
\hline $16-17$ & 0935 & 0835 & 585 \\
\hline $17-18$ & 0935 & 1635 & 610 \\
\hline $18-20$ & 1735 & 1735 & 356 \\
\hline $23-27$ & 0955 & 0855 & 315 \\
\hline $27-30$ & 0855 & 0855 & 167 \\
\hline \multicolumn{4}{|l|}{ APR $30-$} \\
\hline MAY 02 & 0950 & 1650 & 158 \\
\hline $02-03$ & 1750 & 0850 & 631 \\
\hline $03-07$ & 0930 & 0030 & 345 \\
\hline $07-11$ & 1020 & 0920 & 159 \\
\hline $11-14$ & 0955 & 0855 & 118 \\
\hline $14-17$ & 0940 & 2040 & 92 \\
\hline $18 \ldots$ & 0945 & -- & 200 \\
\hline $26 \ldots$ & 1010 & -- & 96 \\
\hline $28-30$ & 1000 & 1300 & 83 \\
\hline $30-31$ & 1400 & 0900 & 157 \\
\hline \multicolumn{4}{|l|}{ MAY 31- } \\
\hline JUN 01 & 1000 & 0100 & 180 \\
\hline $01-01$ & 0200 & 0900 & 217 \\
\hline $01-03$ & 1000 & 1240 & 150 \\
\hline $08-11$ & 1620 & 0920 & 92 \\
\hline $11-15$ & 0955 & 0855 & 68 \\
\hline $15-18$ & 0955 & 0855 & 59 \\
\hline $18-19$ & 0945 & 0445 & 68 \\
\hline $19-20$ & 0545 & 0045 & 111 \\
\hline $20-22$ & 0145 & 0845 & 91 \\
\hline $22-23$ & 1010 & 1210 & 65 \\
\hline $24-25$ & 1110 & 0310 & 106 \\
\hline $25-27$ & 1000 & 0100 & 76 \\
\hline $27-28$ & 0140 & 0040 & 96 \\
\hline $27-28$ & 0200 & 0100 & 96 \\
\hline $28-29$ & 0200 & 0900 & 81 \\
\hline \multicolumn{4}{|l|}{ ปUL, } \\
\hline $02-03$ & 0945 & 0845 & 71 \\
\hline $03-04$ & 0945 & 0445 & 91 \\
\hline $04-06$ & 0545 & 0845 & 86 \\
\hline $06-08$ & 0950 & 1750 & 68 \\
\hline $08-09$ & 1850 & 0850 & 131 \\
\hline $09-12$ & 0945 & 1645 & 96 \\
\hline $12-13$ & 1745 & 0845 & 102 \\
\hline $13-14$ & 1010 & 0610 & 137 \\
\hline $14-15$ & 0710 & 1510 & 330 \\
\hline $15-16$ & 1610 & 0910 & 437 \\
\hline $20 \ldots$ & 0945 & -- & 210 \\
\hline $20-23$ & 0955 & 0855 & 173 \\
\hline $23-26$ & 1035 & 1735 & 159 \\
\hline $31 \ldots$ & 0945 & -- & 240 \\
\hline \multicolumn{4}{|l|}{ AUG } \\
\hline $03 \ldots$ & 1020 & -- & 130 \\
\hline $04 \ldots$ & 1300 & -- & 924 \\
\hline $04 \ldots$ & 1700 & -- & 918 \\
\hline $05 \ldots$ & 0810 & -- & 559 \\
\hline
\end{tabular}

WATER YEAR OCTOBER 1991 TO SEPTEMBER 1992-continued

\begin{tabular}{|c|c|c|c|c|c|c|c|c|c|}
\hline 4.1 & -- & -- & 0.02 & 0.61 & 1.70 & 0.030 & 0.004 & 180 & 150 \\
\hline 2.4 & -- & -. & 0.01 & 0.55 & 1.50 & 0.020 & 0.003 & 170 & 170 \\
\hline 11 & -- & -- & 0.01 & 0.72 & 1.20 & 0.060 & $<0.002$ & 170 & 140 \\
\hline 33 & 124 & 22 & 0.01 & 1.2 & 1.30 & 0.160 & 0.002 & 150 & 200 \\
\hline 4.6 & -- & -- & 0.05 & 0.62 & 1.50 & 0.025 & 0.008 & 150 & 140 \\
\hline 2.4 & -. & -. & 0.03 & 0.81 & 1.30 & 0.035 & 0.003 & 210 & 150 \\
\hline 8.7 & -- & -- & 0.01 & 0.68 & 1.40 & 0.050 & 0.004 & 170 & 98 \\
\hline 8.7 & -- & -. & 0.01 & 0.98 & 0.98 & 0.050 & 0.002 & 150 & 130 \\
\hline 1.8 & -- & -. & 0.03 & 0.65 & 1.30 & 0.025 & 0.002 & 130 & 140 \\
\hline 35 & 117 & 15 & 0.01 & 1.2 & 1.30 & 0.140 & 0.002 & 130 & 110 \\
\hline 85 & 230 & 29 & 0.04 & 1.9 & 1.20 & 0.290 & 0.002 & 100 & 88 \\
\hline 4.6 & -- & -. & 0.01 & 0.63 & 1.20 & 0.030 & 0.002 & 120 & 110 \\
\hline 40 & 185 & 20 & -- & 0.93 & 1.20 & 0.210 & 0.006 & 97 & 79 \\
\hline 40 & 115 & 16 & -- & 0.91 & 1.30 & 0.190 & 0.004 & 83 & 65 \\
\hline 16 & - & -. & -- & 0.69 & 1.40 & 0.100 & 0.003 & 89 & 20 \\
\hline 5.9 & -- & -- & 0.03 & 0.80 & 0.96 & 0.070 & 0.003 & 98 & 92 \\
\hline 4.7 & -- & -- & 0.04 & 0.74 & 0.86 & 0.035 & 0.008 & 100 & 110 \\
\hline 0.20 & -- & -- & 0.03 & 0.52 & 1.10 & 0.020 & 0.002 & 100 & 150 \\
\hline 180 & 346 & 115 & 0.08 & 2.9 & 0.75 & 0.800 & 0.010 & 63 & 50 \\
\hline 4.7 & - & -- & 0.04 & 0.69 & 0.92 & 0.035 & 0.002 & 140 & 140 \\
\hline 43 & -- & -- & 0.06 & 1.0 & 0.69 & 0.130 & 0.009 & 95 & 120 \\
\hline 4.2 & - - & -- & 0.02 & 0.59 & 0.79 & 0.035 & 0.004 & 110 & 140 \\
\hline 5.0 & -- & -- & 0.03 & 0.61 & 1.00 & 0.030 & 0.003 & 110 & 150 \\
\hline 45 & 124 & 20 & 0.06 & 1.4 & 1.10 & 0.220 & 0.006 & 85 & 110 \\
\hline 5.1 & -- & .- & 0.04 & 0.57 & 1.10 & 0.040 & 0.010 & 110 & 290 \\
\hline 4.7 & -- & - & 0.02 & 0.62 & 1.00 & 0.040 & 0.005 & 110 & 150 \\
\hline 6.0 & -- & -- & 0.02 & 0.57 & 0.94 & 0.050 & 0.005 & 100 & 140 \\
\hline 10 & -. & -- & 0.03 & 0.71 & 0.87 & 0.060 & 0.005 & 86 & 120 \\
\hline 16 & - & -- & 0.03 & 0.91 & 0.92 & 0.110 & 0.006 & 87 & 120 \\
\hline 17 & - - & -- & -- & 0.88 & -- & 0.100 & 0.010 & 93 & 120 \\
\hline 1.4 & - & -- & 0.02 & 0.98 & 1.10 & 0.120 & 0.014 & 110 & 150 \\
\hline 18 & -- & -- & 0.01 & 0.98 & 0.84 & 0.090 & 0.012 & 120 & 160 \\
\hline 14 & $\cdots$ & -- & 0.01 & 0.88 & 1.00 & 0.080 & 0.012 & 130 & 170 \\
\hline 18 & -- & -. & 0.03 & 1.0 & 0.98 & 0.090 & 0.011 & 130 & 190 \\
\hline 45 & 156 & 21 & 0.05 & 1.5 & 1.00 & 0.240 & 0.016 & 110 & 170 \\
\hline 45 & 115 & 18 & 0.04 & 1.3 & 1.00 & 0.200 & 0.017 & 95 & 150 \\
\hline 22 & -- & -- & 0.02 & 0.92 & 1.00 & 0.115 & 0.018 & 120 & 170 \\
\hline 95 & -- & -- & 0.02 & 2.1 & 1.10 & 0.475 & 0.011 & 94 & 130 \\
\hline 40 & 121 & 17 & 0.02 & 1.3 & 1.10 & 0.300 & 0.021 & 110 & 160 \\
\hline 40 & 116 & 17 & 0.02 & 1.3 & 0.99 & 0.170 & 0.014 & 110 & 170 \\
\hline 36 & 101 & 15 & 0.01 & 1.3 & 0.98 & 0.140 & 0.016 & 110 & 160 \\
\hline 36 & 95 & 16 & 0.01 & 1.2 & 0.93 & 0.140 & 0.014 & 96 & 150 \\
\hline 15 & -- & -- & 0.02 & 0.83 & 0.87 & 0.075 & 0.011 & 120 & 210 \\
\hline 36 & 119 & 18 & 0.02 & 1.2 & 0.95 & 0.170 & 0.012 & 98 & 160 \\
\hline 25 & -- & -- & 0.02 & 0.99 & 0.83 & 0.120 & 0.012 & 94 & 160 \\
\hline 6.3 & -- & -- & -- & 0.68 & -- & 0.060 & 0.015 & 110 & 180 \\
\hline 30 & 123 & 16 & -- & 1.2 & -- & 0.140 & 0.010 & 95 & 160 \\
\hline 26 & $\cdots$ & -- & 0.01 & 1.1 & 0.80 & 0.110 & 0.014 & 97 & 160 \\
\hline 31 & 74 & 11 & 0.02 & 0.94 & 0.73 & 0.120 & 0.018 & 80 & 140 \\
\hline 21 & -- & $\ldots$ & 0.03 & 0.67 & -- & 0.110 & 0.015 & 87 & 120 \\
\hline 50 & 168 & 21 & 0.02 & 1.3 & -- & 0.210 & 0.016 & 68 & 90 \\
\hline 160 & 347 & 43 & 0.02 & 2.7 & -- & 0.470 & 0.012 & 51 & 60 \\
\hline 20 & - & -- & 0.03 & 0.95 & 1.20 & 0.110 & 0.037 & 78 & 85 \\
\hline 19 & $\cdots$ & -- & 0.02 & 1.0 & 0.90 & - & 0.017 & 88 & 100 \\
\hline 22 & 60 & 10 & 0.03 & 0.88 & 0.96 & 0.140 & 0.019 & 88 & 110 \\
\hline 55 & 119 & 13 & 0.02 & 1.0 & 0.81 & 0.240 & 0.027 & 71 & 92 \\
\hline 15 & -- & -- & 0.01 & 0.66 & 0.92 & 0.090 & 0.030 & 85 & 100 \\
\hline 160 & 270 & 27 & 0.08 & 1.2 & 0.87 & 0.480 & 0.070 & 35 & 35 \\
\hline 100 & -- & -- & 0.04 & 1.3 & 0.83 & 0.260 & 0.060 & 38 & 45 \\
\hline 40 & -- & -- & 0.03 & 0.92 & 0.83 & 0.180 & 0.040 & 57 & 54 \\
\hline
\end{tabular}




\section{$\therefore$ Discharge and water quality}

0423205010 Irondequoit Creek Above Blossom Road, Rochester, N.Y.

\section{WATER-QUALITY RECORDS}

\begin{tabular}{|c|c|c|c|c|c|c|c|c|c|c|c|c|c|}
\hline & & & $\begin{array}{c}\text { DIS- } \\
\text { CHARGE, } \\
\text { IN } \\
\text { CUBIC }\end{array}$ & TUR- & $\begin{array}{l}\text { RESIDUE } \\
\text { TOTAL } \\
\text { AT } 105 \\
\text { DEG. C, }\end{array}$ & $\begin{array}{l}\text { RESIDUE } \\
\text { VOLA- } \\
\text { TILE, }\end{array}$ & $\begin{array}{l}\text { NITRO- } \\
\text { GEN, } \\
\text { AMMONIA } \\
\text { DIS- }\end{array}$ & $\begin{array}{l}\text { NITRO- } \\
\text { GEN, AM - } \\
\text { MONIA + } \\
\text { ORGANIC }\end{array}$ & $\begin{array}{c}\mathrm{NITRO}- \\
\text { GEN, } \\
\mathrm{NO}_{2}+\mathrm{NO}_{3}\end{array}$ & $\begin{array}{l}\text { PHOS- } \\
\text { PHORUS }\end{array}$ & $\begin{array}{l}\text { PHOS- } \\
\text { PHORUS } \\
\text { ORTHO, } \\
\text { DIS- }\end{array}$ & $\begin{array}{l}\text { CHLO- } \\
\text { RIDE, } \\
\text { DIS- }\end{array}$ & $\begin{array}{l}\text { SULFATE } \\
\text { DIS- }\end{array}$ \\
\hline ATE & TIME & $\begin{array}{l}\text { ENDING } \\
\text { TIME }\end{array}$ & $\begin{array}{c}\text { FEET } \\
\text { PER } \\
\text { SECOND }\end{array}$ & $\begin{array}{l}\text { BID- } \\
\text { ITY } \\
\text { (NTU) }\end{array}$ & $\begin{array}{l}\text { SUS- } \\
\text { PENDED } \\
(\mathrm{mg} / \mathrm{L}\rangle\end{array}$ & $\begin{array}{l}\text { SUS- } \\
\text { PENDED } \\
(\mathrm{mg} / \mathrm{L})\end{array}$ & $\begin{array}{l}\text { SOLVED } \\
\text { (mg/L } \\
\text { as } N \text { ) }\end{array}$ & $\begin{array}{l}\text { TOTAL } \\
(\mathrm{mg} / \mathrm{L} \\
\operatorname{as~N)}\end{array}$ & $\begin{array}{l}\text { TOTAL } \\
(\mathrm{mg} / \mathrm{L} \\
\text { as N) }\end{array}$ & $\begin{array}{l}\text { TOTAL } \\
(\mathrm{mg} / \mathrm{L} \\
\text { as P) }\end{array}$ & $\begin{array}{l}\text { SOLVED } \\
(\mathrm{mg} / \mathrm{L} \\
\text { as P) }\end{array}$ & $\begin{array}{l}\text { SOLVED } \\
\text { (mg/L } \\
\text { as Cl) }\end{array}$ & $\begin{array}{l}\text { SOLVED } \\
(\mathrm{mg} / \mathrm{L} \\
\left.\text { as } \mathrm{SO}_{4}\right)\end{array}$ \\
\hline
\end{tabular}

AUG-cont inued

$\begin{array}{rrrr}05 \ldots & 1300 & -- & 498 \\ 05 \ldots & 1600 & -- & 473 \\ 06 \ldots & 0945 & -- & 294 \\ 07 \ldots & 0815 & -- & 156 \\ 07-08 & 1500 & 1100 & 193 \\ 08-08 & 1200 & 2300 & 205 \\ 10-13 & 0935 & 0835 & 147 \\ 13-17 & 0930 & 0830 & 122 \\ 17-20 & 0945 & 0845 & 101 \\ 20-24 & 1025 & 0915 & 79 \\ 24-24 & 0945 & 2045 & 72 \\ 24-25 & 2145 & 0845 & 137 \\ 25-27 & 0945 & 0845 & 147 \\ 31 \ldots & 1030 & -- & 298 \\ \text { SEP } & & & \\ 03 \ldots & 1530 & -- & 354 \\ 04 \ldots & 0855 & -- & 252 \\ 08 \ldots & 1030 & -- & 112 \\ 08-10 & 1110 & 1010 & 93 \\ 10-14 & 1140 & 0840 & 77 \\ 14-17 & 0935 & 0835 & 90 \\ 17-18 & 0945 & 1645 & 65 \\ 18-19 & 1745 & 0845 & 121 \\ 19-21 & 0945 & 0845 & 124 \\ 21-22 & 0950 & 2350 & 207 \\ 23-24 & 0050 & 0850 & 128 \\ 24-26 & 0925 & 0825 & 118 \\ 26-27 & 0925 & 2025 & 185 \\ 27-28 & 2125 & 0825 & 204 \\ \text { SEP 28- } & & & \\ \text { OCT 01 } & 0945 & 0845 & 134\end{array}$

OCT

$01-05 \quad 1000 \quad 0900$ $\begin{array}{rrrr}05-07 & 0910 & 0710 & 69 \\ 09 \ldots & 0920 & \ldots & 111\end{array}$

$09-10 \quad 1330 \quad 0430 \quad 299$

$\begin{array}{llll}10-13 & 0530 & 1030 & 160\end{array}$

$\begin{array}{rrrr}13-15 & 1040 & 0940 & 98 \\ 15-16 & 1045 & 0145 & 132\end{array}$

$\begin{array}{llll}16-19 & 0245 & 0545 & 122\end{array}$

$19-22$

$22-23$

$23-24$

26-29

OCT $29-$

NOV 02

$02-03$

$03-05$

$05-08$

$09-12$

$13-16$

$16-19$

$19-22$

$22-23$

$23-24$

$24-25$

$25-30$

NOV $30-$

DEC 03

03-07

07-10

$10-14$

14-16 $\begin{array}{lll}1055 & 0955 & 93 \\ 1025 & 2125 & 87\end{array}$

$2225 \quad 1325 \quad 112$

$1030 \quad 0830 \quad 130$

$0920 \quad 0820 \quad 94$

$\begin{array}{lll}1030 & 0930 & 312 \\ 1030 & 0430 & 342\end{array}$

$\begin{array}{lll}1115 & 1415 & 237\end{array}$

$1110 \quad 1010 \quad 138$

$\begin{array}{lll}1030 & 0930 & 136\end{array}$

11051005

$1050 \quad 1350$

$1450 \quad 0950$

$1105 \quad 2205$

$2305 \quad 1005$

$1120 \quad 1020$

$1110 \quad 1010 \quad 150$

$1055 \quad 0955 \quad 131$

$1120 \quad 1020$

$\begin{array}{ll}1100 & 1000 \\ 1205 & 0505\end{array}$
WATER YEAR OCTOBER 1991 TO SEPTEMBER 1992-continued

\begin{tabular}{|c|c|c|c|c|c|}
\hline 32 & -- & $\ldots$ & 0.03 & 0.90 & 0.76 \\
\hline 32 & 76 & 9 & 0.02 & 0.84 & 0.76 \\
\hline 17 & -- & -- & 0.02 & 0.79 & 0.84 \\
\hline 9.0 & -- & -- & 0.02 & 0.80 & 1.00 \\
\hline 13 & -- & -- & 0.03 & 0.81 & 0.66 \\
\hline 14 & -- & -- & 0.01 & 0.79 & 0.74 \\
\hline 6.9 & -- & -- & $<0.01$ & 0.64 & 0.80 \\
\hline 4.9 & $\cdots$ & -- & 0.02 & 0.66 & 0.87 \\
\hline 3.6 & -- & -- & $<0.01$ & 0.49 & 0.79 \\
\hline 2.5 & -- & - & 0.02 & 0.54 & 0.84 \\
\hline 2.1 & -- & -- & 0.01 & 0.56 & 0.77 \\
\hline 22 & $\ldots$ & -- & 0.05 & 0.87 & 0.82 \\
\hline 15 & -- & -- & 0.03 & 0.70 & 0.77 \\
\hline 12 & -- & - & 0.04 & 0.77 & 0.76 \\
\hline 31 & 135 & 13 & 0.03 & 0.89 & 0.89 \\
\hline 21 & -. & - & 0.03 & 0.72 & 0.79 \\
\hline 4.5 & -- & -- & 0.01 & 0.56 & 1.10 \\
\hline 3.8 & -- & -- & $<0.01$ & 0.66 & 1.00 \\
\hline 2.1 & $<6$ & $<6$ & $<0.01$ & 0.48 & 1.00 \\
\hline 1.8 & -- & - & $<0.01$ & 0.50 & 0.90 \\
\hline 18 & -- & -- & 0.01 & 0.50 & 0.83 \\
\hline 18 & -- & -- & 0.03 & 0.77 & 0.82 \\
\hline 5.0 & -- & -- & $<0.01$ & 0.59 & 0.73 \\
\hline 22 & -- & -- & 0.01 & 0.71 & 0.63 \\
\hline 40 & 96 & 15 & $<0.01$ & 0.92 & 0.53 \\
\hline 11 & -- & - & 0.02 & 0.64 & 0.69 \\
\hline 19 & -- & $\ldots$ & $<0.01$ & 0.77 & 0.60 \\
\hline 34 & 88 & 15 & $<0.01$ & 0.88 & 0.43 \\
\hline 17 & -- & -. & $<0.01$ & 0.70 & 0.61 \\
\hline
\end{tabular}

$0.180 \quad 0.444$

$0.180 \quad 0.040$

$0.100 \quad 0.037$

$0.085 \quad 0.012$

$0.090 \quad 0.014$

$0.060 \quad 0.013$

$0.050 \quad 0.008$

$0.040 \quad 0.006$

$0.035 \quad 0.004$

$0.030 \quad 0.006$

$0.015 \quad 0.011$

0.085

0.130

0.008

0.049

0.235

0.145

0.075

0.045

0.025

0.025

0.020

0.110

0.045

0.110

0.170

0.080

0.100

0.140

0.043

0.045

0.028
0.006

0.005

0.003

0.003

0.010

0.005

0.009

0.012

0.010

0.008
0.006

0.101

0.008

$\begin{array}{rr}61 & 59 \\ 57 & 59 \\ 66 & 70 \\ 76 & 84 \\ 80 & 96 \\ 82 & 88 \\ 91 & 110 \\ 95 & 130 \\ 98 & 140 \\ 100 & 160 \\ 100 & 170 \\ 86 & 140 \\ 87 & 140 \\ 60 & 61 \\ & \\ 65 & 63 \\ 67 & 85 \\ 110 & 140 \\ 97 & 140 \\ 110 & 160 \\ 110 & 180 \\ 110 & 190 \\ 93 & 150 \\ 86 & 140 \\ 75 & 100 \\ 73 & 97 \\ 90 & 140 \\ 79 & 110 \\ 68 & 88 \\ & \\ 84 & 110\end{array}$

WATER YEAR OCTOBER 1992 TO SEPTEMBER 1993

\begin{tabular}{|c|c|c|c|}
\hline 5.1 & -- & -- & $<0.01$ \\
\hline 3.2 & -- & -- & 0.02 \\
\hline 16 & -- & -- & 0.12 \\
\hline 70 & 227 & 38 & 0.07 \\
\hline 45 & 89 & 16 & 0.02 \\
\hline 6.0 & - & -- & $<0.01$ \\
\hline 13 & - & -- & 0.02 \\
\hline 21 & -- & -- & $<0.01$ \\
\hline 3.3 & -- & - & $<0.01$ \\
\hline 2.7 & -- & -- & $<0.01$ \\
\hline 5.0 & -- & -- & $<0.01$ \\
\hline 6.7 & 41 & $<5$ & $<0.01$ \\
\hline 3.4 & -- & -- & $<0.01$ \\
\hline 22 & -- & -- & $<0.01$ \\
\hline 40 & 76 & 11 & $<0.01$ \\
\hline 14 & -- & -- & $<0.01$ \\
\hline 5.0 & -- & -- & $<0.01$ \\
\hline 4.9 & -- & -- & $<0.01$ \\
\hline 4.4 & -- & -- & $<0.01$ \\
\hline 4.0 & -- & -- & $<0.01$ \\
\hline 30 & 70 & 12 & 0.01 \\
\hline 38 & 64 & 9 & $<0.01$ \\
\hline 45 & 99 & 14 & 0.01 \\
\hline 28 & $\cdots$ & -- & 0.01 \\
\hline 4.9 & -- & -- & $<0.01$ \\
\hline 4.8 & -- & -- & $<0.01$ \\
\hline 3.2 & -- & -- & $<0.01$ \\
\hline 4.5 & 10 & $<10$ & 0.02 \\
\hline 16 & 14 & $<6$ & $<0.01$ \\
\hline
\end{tabular}

$\begin{array}{ll}0.61 & 0.82 \\ 0.24 & 1.00 \\ 0.58 & 1.20 \\ 1.6 & 0.52 \\ 0.90 & 0.45 \\ 0.46 & 0.70 \\ 0.61 & 0.81 \\ 0.90 & 0.68 \\ 0.45 & 0.88 \\ 0.42 & 0.87 \\ 0.43 & 0.89 \\ 0.57 & 0.71 \\ 0.43 & 0.89 \\ 0.72 & 0.75 \\ 0.88 & 0.53 \\ 0.61 & 0.66 \\ 0.57 & 0.88 \\ 0.51 & 0.79 \\ 0.62 & 0.96 \\ 0.49 & 0.86 \\ 0.83 & 0.75 \\ 0.83 & 0.69 \\ 0.96 & 0.71 \\ 0.72 & 0.88 \\ 0.48 & 1.10 \\ 0.48 & 1.20 \\ 0.60 & 1.30 \\ 0.48 & 1.30 \\ 0.46 & 1.20\end{array}$

0.035

0.005

0.040

0.170

0.300

0.160

0.060

0.065

0.110

0.060

0.030

0.030

0.006

0.091

0.018

0.009

0.007

0.008

0.006

0.006

0.005

0.004

0.005

0.030

0.110

0.003

0.270

0.060

0.035

0.035

0.030

0.035

0.145
0.140

0.170

0.008
0.012

0.009

0.006

0.006

0.004

0.004

0.013

0.014

0.100

0.010

0.040

0.006

0.035

0.035

0.004
0.005

0.005

0.005

$\begin{array}{rr}100 & 160 \\ 110 & 180 \\ 110 & 160 \\ 67 & 100 \\ 74 & 110 \\ 97 & 140 \\ 98 & 140 \\ 92 & 140 \\ 100 & 160 \\ 100 & 100 \\ 100 & 160 \\ 88 & 120 \\ & \\ 99 & 160 \\ 78 & 110 \\ 60 & 75 \\ 74 & 90 \\ 92 & 130 \\ 89 & 120 \\ 140 & 140 \\ 100 & 110 \\ 88 & 93 \\ 77 & 79 \\ 68 & 68 \\ 76 & 93 \\ 92 & 120 \\ 120 & 120 \\ 160 & 140 \\ 250 & 130 \\ 180 & 120\end{array}$


Surface-Water Stations

A. Discharge and water quality

0423205010 Irondequoit Creek Above Blossom Road, Rochester, N.Y.

2. WATER-QUALITY RECORDS

\begin{tabular}{|c|c|c|c|c|c|c|c|c|c|c|c|c|c|}
\hline & & & $\begin{array}{c}\text { DIS- } \\
\text { CHARGE, } \\
\text { IN } \\
\text { CUBIC }\end{array}$ & TUR- & $\begin{array}{l}\text { RESIDUE } \\
\text { TOTAL } \\
\text { AT } 105 \\
\text { DEG. C, }\end{array}$ & $\begin{array}{l}\text { RESIDUE } \\
\text { VOLA- } \\
\text { TILE, }\end{array}$ & $\begin{array}{c}\text { NITRO- } \\
\text { GEN, } \\
\text { AMMONIA } \\
\text { DIS- }\end{array}$ & $\begin{array}{l}\text { NITRO- } \\
\text { GEN, AM- } \\
\text { MONIA + } \\
\text { ORGANIC }\end{array}$ & $\begin{array}{c}\text { NITRO- } \\
\text { GEN, } \\
\mathrm{NO}_{2}+\mathrm{NO}_{3}\end{array}$ & $\begin{array}{r}\text { PHOS- } \\
\text { PHORUS }\end{array}$ & $\begin{array}{l}\text { PHOS- } \\
\text { PHORUS } \\
\text { ORTHO, } \\
\text { DIS- }\end{array}$ & $\begin{array}{l}\mathrm{CH}^{-} \mathrm{OO}- \\
\mathrm{RIDE}, \\
\text { DIS- }\end{array}$ & $\begin{array}{l}\text { SULFATE } \\
\text { DIS- }\end{array}$ \\
\hline ATE & TIME & $\begin{array}{l}\text { ENDING } \\
\text { TIME }\end{array}$ & $\begin{array}{l}\text { FEET } \\
\text { PER } \\
\text { SECOND }\end{array}$ & $\begin{array}{l}\text { BID- } \\
\text { ITY } \\
\text { (NTU) }\end{array}$ & $\begin{array}{c}\text { SUS- } \\
\text { PENDED } \\
(\mathrm{mg} / \mathrm{L})\end{array}$ & $\begin{array}{l}\text { SUS- } \\
\text { PENDED } \\
(\mathrm{mg} / \mathrm{L})\end{array}$ & $\begin{array}{l}\text { SOLVED } \\
\text { (mg/L } \\
\text { as N) }\end{array}$ & $\begin{array}{l}\text { TOTAL } \\
(\mathrm{mg} / \mathrm{L} \\
\text { as } \mathrm{N})\end{array}$ & $\begin{array}{l}\text { TOTAL } \\
\text { (mg/L } \\
\text { as N) }\end{array}$ & $\begin{array}{l}\text { TOTAL } \\
\text { (mg/L } \\
\text { as P) }\end{array}$ & $\begin{array}{l}\text { SOLVED } \\
(\mathrm{mg} / \mathrm{L} \\
\text { as } \mathrm{P})\end{array}$ & $\begin{array}{l}\text { SOIJVED } \\
\text { (mg/L } \\
\text { as Cl) }\end{array}$ & $\begin{array}{r}\text { SOLVEL } \\
\left(\mathrm{mg}^{\prime} \mathrm{L}\right. \\
\left.\text { as } \mathrm{SO}_{4}\right)\end{array}$ \\
\hline
\end{tabular}

DEC-cont inued

$\begin{array}{llll}16-17 & 0605 & 1005 & 286 \\ 17-18 & 1100 & 0200 & 498 \\ 18-21 & 0300 & 1000 & 439 \\ 21-24 & 1130 & 0830 & 252 \\ 24-28 & 0910 & 0810 & 150 \\ 28-29 & 1015 & 1215 & 135 \\ 29-31 & 1315 & 0215 & 332\end{array}$

DEC $31-$

JAN 04

$\begin{array}{llll}05-07 & 1115 & 1015 & 417 \\ 07-111 & 1150 & 1015 & 425\end{array}$

$\begin{array}{llll}07-11 & 1050 & 0950 & 217\end{array}$

$\begin{array}{llll}11-12 & 1105 & 2205 & 168\end{array}$

$\begin{array}{llll}12-13 & 2305 & 2205 & 256\end{array}$

13-14 $2305 \quad 1205 \quad 415$

$\begin{array}{llll}15-19 & 1035 & 0935 & 226\end{array}$

$\begin{array}{llll}19-21 & 1110 & 1010 & 170\end{array}$

$\begin{array}{llll}21-23 & 1100 & 0200 & 289\end{array}$

$\begin{array}{llll}23-25 & 0300 & 1000 & 436\end{array}$

$25-28 \quad 1120 \quad 0950 \quad 290$

$28-29$

$\begin{array}{ll}1120 & 0950 \\ 1105 & 1805\end{array}$

206

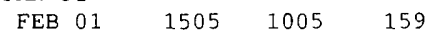

$\begin{array}{rrrr}04 \ldots & 1215 & -. & 147\end{array}$

$\begin{array}{llll}05-06 & 0930 & 2130 & 139\end{array}$

$12 \ldots \quad 1000 \quad--120$

$16 \ldots .1110 \quad--118$

$18 \ldots \quad 1100 \quad \ldots 114$

$22 \ldots \quad 1100 \quad \ldots \quad 110$

$23 \ldots \quad 1050 \quad-110$

$25 \ldots$

MAR

$01 \ldots \quad 1100 \quad--\quad 109$

$04 \ldots \quad 1045 \quad--123$

$\begin{array}{lrrr}08 \ldots & 1100 & -- & 181 \\ 09-10 & 0930 & 0230 & 198\end{array}$

$11 .-1105$

$\begin{array}{llll}11-15 & 1110 & 1010 & 159\end{array}$

$\begin{array}{llll}15-18 & 1035 & 0835 & 210\end{array}$

$\begin{array}{llll}18-22 & 0935 & 0835 & 191\end{array}$

$\begin{array}{llll}22-25 & 0920 & 0820 & 307 \\ 25-29 & 0915 & 0815 & 721\end{array}$

$\begin{array}{llll}29-30 & 0925 & 0025 & 1270\end{array}$

29-31 $0925 \quad 0025 \quad 1360$

APR

$\begin{array}{llll}01 \ldots & 0945 & -- & 1400\end{array}$

$\begin{array}{lllr}04-05 & 1750 & 0750 & 673 \\ 05-08 & 0935 & 0835 & 482\end{array}$

$\begin{array}{llll}10-12 & 1740 & 0040 & 398\end{array}$

$\begin{array}{llll}12-14 & 0925 & 2025 & 245\end{array}$

$\begin{array}{llll}15 \ldots & 1000 & -- & 278\end{array}$

$\begin{array}{llll}16-17 & 1005 & 1805 & 381\end{array}$

$\begin{array}{llll}17-19 & 1905 & 0905 & 388 \\ 19-22 & 0920 & 0820 & 246\end{array}$

$\begin{array}{llll}19-22 & 0920 & 0820 & 246 \\ 22-24 & 0940 & 0840 & 528\end{array}$

$\begin{array}{llll}24-26 & 2140 & 0840 & 314\end{array}$

$\begin{array}{llll}26-29 & 0950 & 0850 & 268\end{array}$

APR 29-

MAY $03 \quad 0930 \quad 0830 \quad 191$

$\begin{array}{llll}03-05 & 0920 & 0020 & 163\end{array}$

$\begin{array}{llll}05-05 & 0320 & 2320 & 187\end{array}$

$\begin{array}{llll}06-06 & 0020 & 0820 & 202 \\ 06-10 & 0915 & 0815 & 159\end{array}$
WATER YEAR OCTOBER 1992 TO SEPTEMBER 1993-continued

$\begin{array}{rlrrlllllr}55 & 132 & 27 & <0.01 & 1.7 & 1.10 & 0.240 & 0.005 & 190 & 94 \\ 45 & -- & -- & <0.01 & 1.1 & 0.96 & 0.250 & 0.007 & 140 & 58 \\ 35 & -- & -- & <0.01 & 1.2 & 1.00 & 0.200 & 0.007 & 97 & 59 \\ 12 & -- & -- & <0.01 & 0.72 & 0.59 & 0.055 & 0.009 & 98 & 90 \\ 7.3 & - & -- & 0.02 & 0.63 & 1.40 & 0.050 & 0.008 & 130 & 110 \\ 6.4 & -- & -- & 0.02 & 0.63 & 1.40 & 0.035 & 0.007 & 140 & 130 \\ 32 & -- & -- & 0.02 & 1.2 & 1.10 & 0.140 & 0.011 & 150 & 93\end{array}$

$\begin{array}{cccr}45 & 225 & 15 & 0.01 \\ 39 & 135 & 15 & 0.01 \\ 40 & 99 & 12 & 0.01 \\ 8.2 & -- & -- & <0.01 \\ 5.1 & -- & -- & <0.01 \\ 24 & - & -- & 0.02 \\ 40 & 122 & 18 & <0.01 \\ 7.7 & -- & -- & 0.01 \\ 4.6 & -- & -- & 0.01 \\ 26 & -- & -- & 0.02 \\ 36 & 91 & <8 & 0.01 \\ 22 & -- & -- & 0.01 \\ 5.8 & -- & -- & 0.01\end{array}$

$0.94 \quad 1.00$

0.160

$1.1 \quad 1.10 \quad 0.170$

$\begin{array}{lll}1.1 & 0.95 & 0.150\end{array}$

$\begin{array}{lll}0.69 & 1.20 & 0.045\end{array}$

$\begin{array}{lll}0.36 & 1.40 & 0.030\end{array}$

$\begin{array}{lll}0.72 & 1.30 & 0.100\end{array}$

$\begin{array}{lll}1.1 & 1.10 & 0.140\end{array}$

$\begin{array}{lll}0.52 & 1.20 & 0.050\end{array}$

$\begin{array}{lll}0.57 & 1.30 & 0.035\end{array}$

$0.69 \quad 1.20 \quad 0.110$

$\begin{array}{lll}0.73 & 1.10 & 0.140\end{array}$

$\begin{array}{lll}0.82 & 1.20 & 0.075\end{array}$

$0.62 \quad 1.20$

0.035

0.006

100

$0.011 \quad 120$

$0.014 \quad 87$

$0.005 \quad 120$

$0.004 \quad 160$

0.006300

0.005280

$0.004 \quad 160$

$\begin{array}{rr}0.004 & 150 \\ <0.005 & 180\end{array}$

$\begin{array}{rr}<0.005 & 180 \\ 0.006 & 130\end{array}$

$0.005 \quad 120$

$0.005 \quad 130$

0.045

$0.65 \quad 1.10$

$\begin{array}{ll}0.62 & 1.40 \\ 0.68 & 1.30\end{array}$

0.020

0.004

0.006

230

0.025

$\begin{array}{lll}0.58 & 1.30 & 0.025 \\ 0.51 & 1.40 & 0.025\end{array}$

$0.51 \quad 1.40 \quad 0.025$

$\begin{array}{lll}0.53 & 1.40 & 0.020\end{array}$

1.50

0.015

0.015

0.65

1.40

0.020

0.004

0.002

0.002

0.004

0.004

0.004

0.030

$0.45 \quad 1.50$

0.020

$0.49 \quad 1.30$

$0.67 \quad 1.20$

$0.57 \quad 1.30$

$0.63 \quad 1.30$

$0.47 \quad 1.30$

$0.64 \quad 1.30$

$0.84 \quad 1.60$

1.2

1.1

1.60
1.20

1.00

0.025

0.035

0.025

0.025

0.035

0.035

0.130

0.270

0.340

0.004

0.004

0.005

0.005

0.005

0.004

0.004

0.004

0.005

0.010

0.014

0.014

$\begin{array}{lll}0.75 & 1.00 & 0.160 \\ 0.58 & 1.20 & 0.095\end{array}$

0.027

0.011

0.004

0.004

$\begin{array}{ll}0.61 & 1.20 \\ 0.75 & 1.00\end{array}$

0.070

0.065

$0.51 \quad \mathrm{~N} 0.01 \quad 0.045$

0.54 N0.01

$\begin{array}{ll}0.75 & 1.00\end{array}$

$0.82 \quad 0.80$

0.99

1.2

0.99

0.60

0.99

0.045

0.100

0.110

0.085

0.190

0.095

0.065

0.005
0.007
0.004

0.004

0.006

0.004

0.009

0.006

0.045

0.59

1.10

0.5

1.00

0.045

0.080

0.170
0.070

0.004

0.005

0.003

$\begin{array}{ll}0.004 & 92 \\ 0.007 & 96\end{array}$

$\begin{array}{ll}1.2 & 1.10 \\ N 0.01 & 0.91\end{array}$

\section{0}

81

99

120

100

74
98

110

86
62

74

100

120

120
130

140

150

150

160

160

$\begin{array}{ll}170 & 160 \\ 270 & 140\end{array}$

$360 \quad 120$

$350 \quad 100$

$320 \quad 110$

$\begin{array}{ll}290 & 120 \\ 260 & 120\end{array}$

$220 \quad 100$

210

120

81

48
37

37

33

50
58

67

75

91

69
92

60

76
84

110

130

130

120 
0423205010 Irondequoit Creek Above Blossom Road, Rochester, N.Y.

\section{WATER-QUALITY RECORDS}

\begin{tabular}{|c|c|c|c|c|c|c|c|c|c|c|c|c|c|}
\hline & & & $\begin{array}{c}\text { DIS- } \\
\text { CHARGE, } \\
\text { IN } \\
\text { CUBIC }\end{array}$ & TUR- & $\begin{array}{l}\text { RESIDUE } \\
\text { TOTAL } \\
\text { AT } 105 \\
\text { DEG. C, }\end{array}$ & $\begin{array}{l}\text { RESIDUE } \\
\text { VOLA- } \\
\text { TILE, }\end{array}$ & $\begin{array}{l}\text { NITRO- } \\
\text { GEN, } \\
\text { AMMONIA } \\
\text { DIS- }\end{array}$ & $\begin{array}{l}\text { NITRO- } \\
\text { GEN, AM- } \\
\text { MONIA + } \\
\text { ORGANIC }\end{array}$ & $\begin{array}{c}\text { NITRO- } \\
\text { GEN, } \\
\mathrm{NO}_{2}+\mathrm{NO}_{3}\end{array}$ & $\begin{array}{l}\text { PHOS- } \\
\text { PHORUS }\end{array}$ & $\begin{array}{l}\text { PHOS- } \\
\text { PHORUS } \\
\text { ORTHO, } \\
\text { DIS- }\end{array}$ & $\begin{array}{l}\text { CHLO- } \\
\text { RIDE, } \\
\text { DIS- }\end{array}$ & $\begin{array}{l}\text { SULFATE } \\
\text { DIS- }\end{array}$ \\
\hline DATE & TIME & $\begin{array}{l}\text { ENDING } \\
\text { TIME }\end{array}$ & $\begin{array}{c}\text { FEET } \\
\text { PER } \\
\text { SECOND }\end{array}$ & $\begin{array}{l}\text { BID- } \\
\text { ITY } \\
\text { (NTU) }\end{array}$ & $\begin{array}{c}\text { SUS- } \\
\text { PENDED } \\
\text { mg/L) }\end{array}$ & $\begin{array}{l}\text { SUS- } \\
\text { PENDED } \\
(\mathrm{mg} / \mathrm{L})\end{array}$ & $\begin{array}{l}\text { SOLVED } \\
(\mathrm{mg} / \mathrm{L} \\
\text { as } \mathrm{N})\end{array}$ & $\begin{array}{l}\text { TOTAL } \\
(\mathrm{mg} / \mathrm{L} \\
\text { as } \mathrm{N})\end{array}$ & $\begin{array}{l}\text { TOTAL } \\
\text { (mg/L } \\
\text { as N) }\end{array}$ & $\begin{array}{c}\text { TOTAL } \\
\text { (mg/L } \\
\text { as P) }\end{array}$ & $\begin{array}{l}\text { SOLVED } \\
(\mathrm{mg} / \mathrm{L} \\
\text { as P) }\end{array}$ & $\begin{array}{l}\text { SOLVEI } \\
\text { (mg/L } \\
\text { as Cl) }\end{array}$ & $\begin{array}{l}\text { SOLVED } \\
(\mathrm{mg} / \mathrm{L} \\
\left.\text { as } \mathrm{SO}_{4}\right)\end{array}$ \\
\hline
\end{tabular}

MAY-cont inued

$10-13$

13-17 0930

$17-20 \quad 0930$

$20-24 \quad 0910$

28-3

$31-31$

MAY $31-$

JUN 01

$01-03$

05-06

$07 \ldots$

$07-08$

08-09

09-09

10-10

$10-11$

$14-17$

$\begin{array}{ll}14-17 & 0945 \\ 17-19 & 0925\end{array}$

$19-20$

$20-21$

21-24

24-27

$27-28$

JUN 28 -

JUL 02

$02-06$

$06-08$

$12-15$

15-19

19-19

$19-20$

$21-22$
$23-26$

$23-26$
$26-26$

26- 28

JUL 29 -

AUG 02

$02-03$

09 ...

10-11

11-11

$12-16$

16-17

$17-17$

19-20

20-21

$21-23$

23-26

$26-30$

$30-31$ SEP

$\begin{array}{lllr}01-02 & 0015 & 0815 & 70 \\ 03-04 & 0035 & 0035 & 159 \\ 04-06 & 0135 & 0735 & 71 \\ 07-07 & 0930 & 1430 & 113 \\ 09-09 & 0930 & 1815 & 64 \\ 09-10 & 1515 & 1815 & 129 \\ 10-13 & 1915 & 0815 & 86 \\ 13-16 & 0910 & 0810 & 60\end{array}$

$0825 \quad 134$

$0830 \quad 120$

$0520 \quad 109$

$\begin{array}{rr}0810 & 103 \\ 0800 & 87\end{array}$

$0350 \quad 81$

$1950 \quad 114$

$0750 \quad 135$

$\begin{array}{lr}0915 & 94 \\ 2025 & 318\end{array}$

$1225 \quad 238$

$\begin{array}{rr}-- & 137 \\ 1500 & 119\end{array}$

$0600 \quad 151$

$2400 \quad 166$

$0900 \quad 194$

$0205 \quad 145$

$\begin{array}{ll}0845 & 80 \\ 0025 & 74\end{array}$

$0825 \quad 102$

$0825 \quad 123$

0815

$0935 \quad 1635$

$1735 \quad 0835$

$0915 \quad 0715$

$0815 \quad 0715$

$0900 \quad 0800$

$\begin{array}{ll}0915 & 0815 \\ 0930 & 0830\end{array}$

$0930 \quad 1430$

$1530 \quad 1130$

$\begin{array}{ll}0900 & 0800 \\ 1200 & 0300\end{array}$

09252025

$2125 \quad 1125$

$0800 \quad 0900$

$0915 \quad 1215$

$\begin{array}{lr}1015 & = \\ 0920 & 1720\end{array}$

$1820 \quad 2320$

09350835

$1010 \quad 0010$

$0110 \quad 1510$

$1610 \quad 0910$

09350435

$\begin{array}{ll}0535 & 0835 \\ 0935 & 0835\end{array}$

$0930 \quad 0830$

$0905 \quad 0805$

$0915 \quad 1415$

1415
2315

93
71

92

83
69

69
55

53

48
216

111

56
48

48
74
61

59

65
51

47

67
49

52

78
55

49

98
58

47

42

42

94

70
159

159

13

64

86

60

\section{WATER YEAR OCTOBER 1992 TO SEPTEMBER 1993-continued}
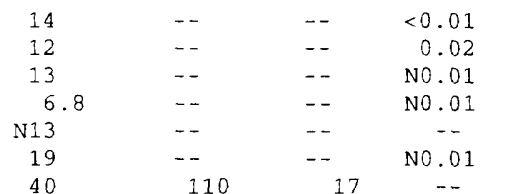

$\begin{array}{rr}0.67 & 0.99 \\ 0.66 & 1.00 \\ \text { N0.01 } & 1.10 \\ \text { N0.69 } & \text { N1.30 } \\ -- & 1.30 \\ \text { N0.01 } & 1.30\end{array}$

0.070

0.065

0.060

0.070

0.100

0.210

58
25
N3 10

N310

$\mathrm{N} 150$
9

32

32

35
50

36

14

24

36
65

65
37

23
29

31

31
16
11

4.8

14
55
50

50
10

4.9

23
25

23

18
4.

17

17
29

20

18
30

27

21

60

45

30
30

28

45

35

130

75

7.9

65
29

17

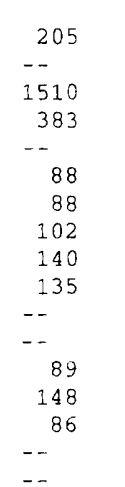

29
-
158

46

11
13
14

19

$$
{ }_{--}^{23}
$$$$
15
$$$$
25-0.01
$$$$
--\quad<0.01
$$$$
<0.01
$$

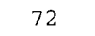

$\begin{array}{rr}12 & <0.01 \\ -- & <0.01 \\ -- & <0.01\end{array}$

$--\quad<0.01$

$\begin{array}{rr}-- & <0.01 \\ 41 \quad 0.06\end{array}$

340.32

0.02
0.02

0.04

0.03

$\begin{array}{rrr}-- & -- & 0.01 \\ -- & -- & <0.01 \\ -- & -- & 0.04 \\ -- & -- & 0.01\end{array}$

$\begin{array}{rrr}-- & -- & 0.01 \\ -- & -- & <0.01\end{array}$

$\begin{array}{lll}-- & < & <0.01\end{array}$

83

--

$--$

67

--

170

137
639
171

196

$\begin{array}{ccc}196 & - & 0.02 \\ - & -25 & 0.01 \\ 165 & 25 & 0.01 \\ -- & -- & 0.03\end{array}$
-- 1.30

0.320

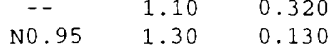

$3.5 \quad 1.00$

$2.4 \quad 1.00$

0.770

0.560

0.075

0.160

0.190

0.200

0.220

0.110

0.110

0.180

0.260

No. 170

$0.91 \quad 0.93$

0.87

1.00

0.130

$0.83 \quad 0.91$

0.64

0.59

0.61

0.54
2.0

2.0

NO. 7

$\begin{array}{rr}0.62 & \mathrm{~N} 0.90 \\ 0.45 & 0.63\end{array}$

$1.1 \quad \mathrm{NO} 01$

0.95

NO.01

N0.88 0.90

$\begin{array}{rr}\text { N0.01 } & 0.87 \\ 0.41 & \text { No.92 }\end{array}$

0.84 No.74

0.92 No.77

0.83

0.85

0.95

0.73

0.87

1. 5

1.1

No. 99

N1. 1

1. 1

1.6

1. 3

3.0

1. 4

1.4
1.3
0.70

0.70

1. 5

0.87

0.82

0.82

0.93
0.88

0.88

0.93

0.88

$-$

$+-$

0.140

0.100

0.070

0.060

0.180

0.240

0.260

0.070

0.045 


\section{Surface-Water Stations}

\section{A. Discharge and water quality}

\section{Irondequoit Creek Above Blossom Road, Rochester, N.Y.}

2. WATER-QUALITY RECORDS

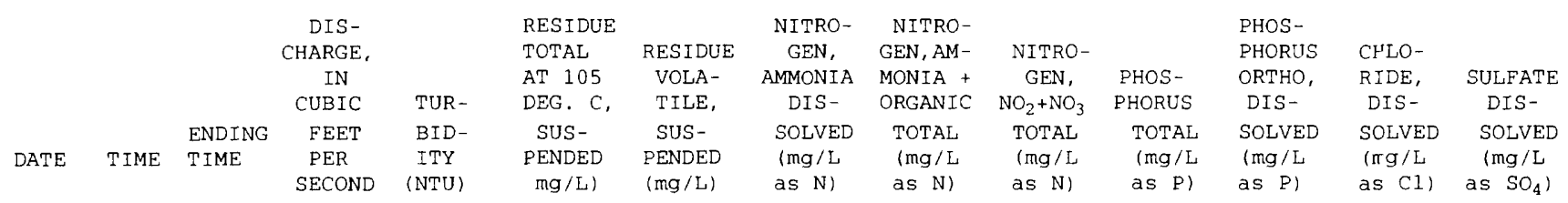

\begin{tabular}{lllrc}
\multicolumn{6}{l}{ SEP-cont inued } \\
$16-20$ & 0920 & 0820 & 55 & 12 \\
$20-23$ & 0920 & 0820 & 53 & 8.3 \\
$23-23$ & 0900 & 2400 & 109 & 22 \\
$24-25$ & 0100 & 2400 & 70 & 14 \\
$26-26$ & 0100 & 1600 & 198 & 80 \\
$26-27$ & 1700 & 0800 & 127 & 80 \\
$27-28$ & 0905 & 0805 & 107 & 48 \\
$28-30$ & 0905 & 0805 & 92 & 20 \\
$30-30$ & 0920 & 2300 & 75 & 14 \\
SEP 30- & & & & \\
OCT 04 & 0920 & 0820 & -- & 14
\end{tabular}

WATER YEAR OCTOBER 1992 TO SEPTEMBER 1993-continued

\begin{tabular}{|c|c|c|c|c|c|c|c|c|}
\hline-- & -- & $<0.01$ & 0.83 & 0.98 & 0.090 & 0.020 & 110 & 190 \\
\hline - & - & 0.01 & 0.60 & 0.95 & 0.065 & 0.012 & 110 & 200 \\
\hline-- & - & 0.02 & 0.93 & 0.92 & 0.150 & 0.024 & 90 & N170 \\
\hline-- & -- & 0.02 & 0.79 & 0.90 & 0.100 & 0.020 & 100 & N170 \\
\hline 201 & 37 & 0.01 & 1.2 & 0.64 & -- & 0.023 & 79 & 120 \\
\hline 160 & 31 & 0.01 & 0.99 & 0.70 & 0.280 & 0.020 & 70 & 130 \\
\hline 114 & 17 & 0.02 & 1.1 & 0.74 & 0.170 & 0.030 & 85 & 140 \\
\hline-- & -- & 0.01 & 0.82 & 0.76 & 0.110 & 0.018 & 87 & 150 \\
\hline- & -- & 0.02 & 0.75 & 0.90 & 0.085 & 0.014 & 110 & 170 \\
\hline-- & -- & 0.02 & 0.75 & 0.90 & 0.085 & 0.014 & 110 & 1 \\
\hline
\end{tabular}




\section{Surface-Water Stations}

\section{A. Discharge and water quality}

\section{Irondequoit Creek at Empire Boulevard, Rochester, N.Y.}

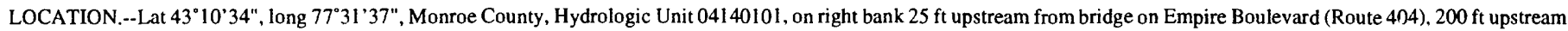
from mouth at south end of Irondequoit Bay, and 1.5 mi east of Rochester.

DRAINAGE AREA. - $151 \mathrm{mi}^{2}$, flow from $8.45 \mathrm{mi}^{2}$ noncontributing

\section{WATER-DISCHARGE RECORDS}

PERIOD OF RECORD.--June 1990 to current year.

GAGE.--Ultrasonic velocity meter, water-stage recorder, and crest-stage gage. Datum of gage is $242.66 \mathrm{ft}$ above sea level (levels by Corps of Engineers).

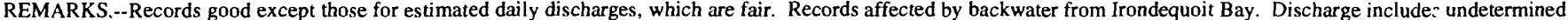

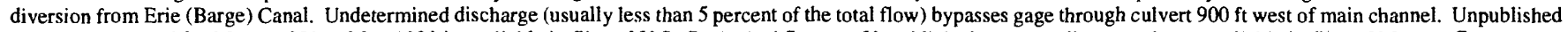
gage-height record for March 1989 to May 1990 is available in files of U.S. Geological Survey. Unpublished water-quality records are available in files of Monroe County Department of Health.

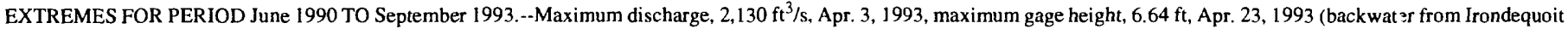
Bay), minimum daily discharge, $29 \mathrm{ft}^{3} / \mathrm{s}$, Aug. 2, 1991

STATISTICS OF MONTHLY MEAN DISCHARGE (in cubic feet per second) FOR WATER YEARS 1990-93, BY WATER YEAR

\begin{tabular}{|c|c|c|c|c|c|c|c|c|c|c|c|}
\hline & OCT & NOV & DEC & JAN & FEB & MAR & APR & MAY & JUN & JUL & AUG \\
\hline MEAN & 99.3 & 132 & 178 & 190 & 138 & 313 & 334 & 119 & 83.9 & 87.5 & 109 \\
\hline MAX & 124 & 208 & 226 & 286 & 170 & 351 & 481 & 150 & 99.2 & 181 & 262 \\
\hline$(W Y)$ & 1991 & 1993 & 1991 & 1993 & 1991 & 1993 & 1993 & 1992 & 1993 & 1992 & 1992 \\
\hline MIN & 54.9 & 63.3 & 88.6 & 93.6 & 109 & 267 & 259 & 93.9 & 60.4 & 50.3 & 50.4 \\
\hline (WY) & 1992 & 1992 & 1992 & 1992 & 1992 & 1992 & 1992 & 1993 & 1991 & 1991 & 1991 \\
\hline
\end{tabular}

\section{SUMMARY STATISTICS}

STATISTIC

ANNUAL TOTAL

ANNUAL MEAN

AVERAGE DISCHARGE

HIGHEST ANNUAL MEAN

LOWEST ANNUAL MEAN

HIGHEST DAILY MEAN

LOWEST DAILY MEAN

ANNUAL SEVEN-DAY MINIMUM

INSTANTANEOUS PEAK FLOW

INSTANTANEOUS PEAK STAGE

10 PERCENT EXCEEDS

50 PERCENT EXCEEDS

90 PERCENT EXCEEDS

$\begin{array}{lc}\text { FOR 1992 CALENDAR YEAR } & \text { FOR 1993 WATER YEAR } \\ 63990 & 66798 \\ 175 & 183\end{array}$

175

\begin{tabular}{|c|c|}
\hline 1350 & Mar 28 \\
\hline 49 & Jul \\
\hline 58 & Feb \\
\hline
\end{tabular}

a Backwater from Irondequopit Bay.

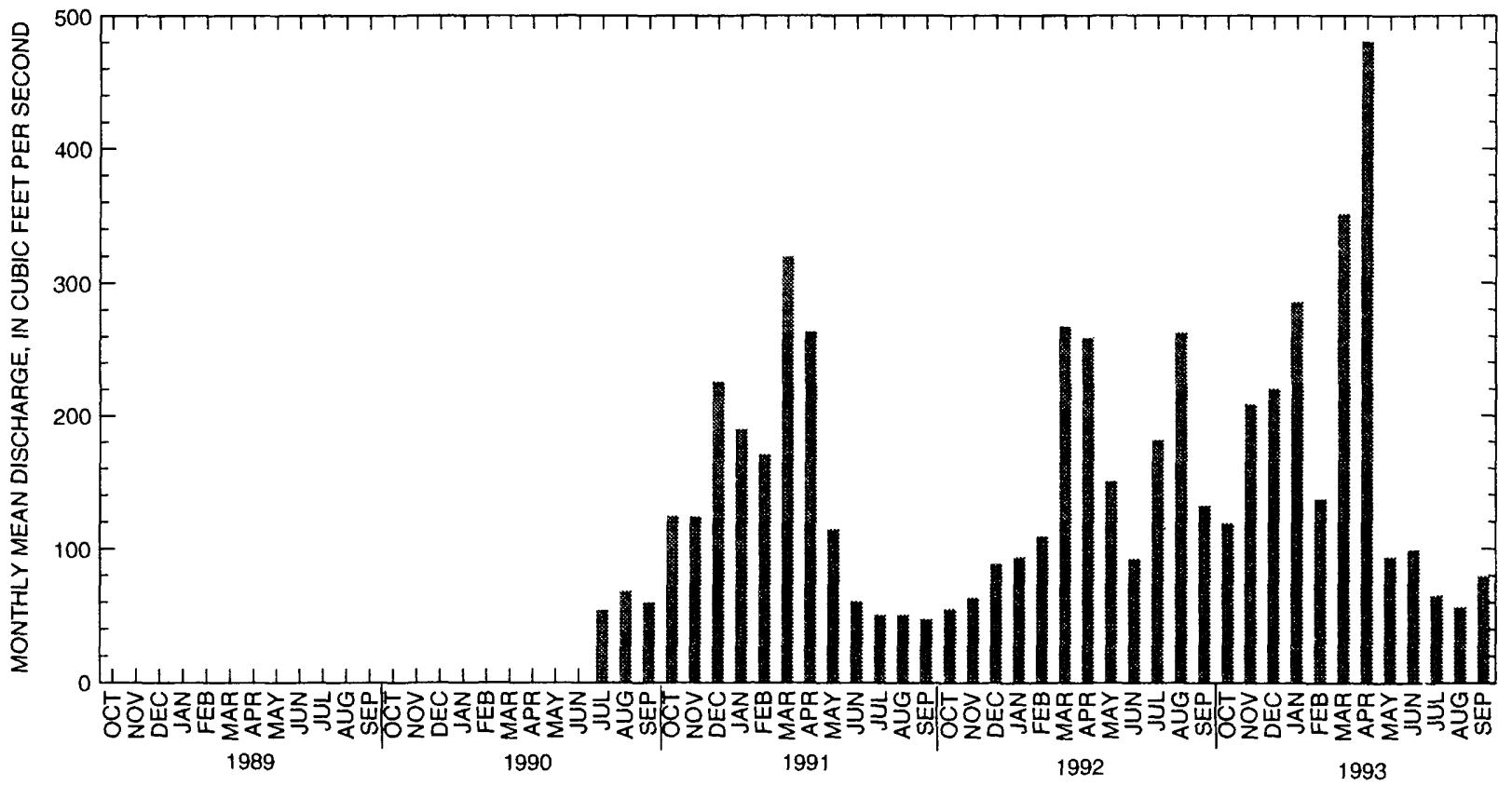




\section{Surface-Water Stations}

\section{A. Discharge and water quality}

0423205025 Irondequoit Creek at Empire Boulevard, Rochester, N.Y. 2. WATER-QUALITY RECORDS

PERIOD OF RECORD.--1989 to current year.

CHEMICAL DATA: $1989-93$ (e)

NUTRIENT DATA: $1989-93$ (e)

COOPERATION.--Water-quality samples were collected and analyzed by the Monroe County Environmental Health Laboratory at Rochester, N.Y.

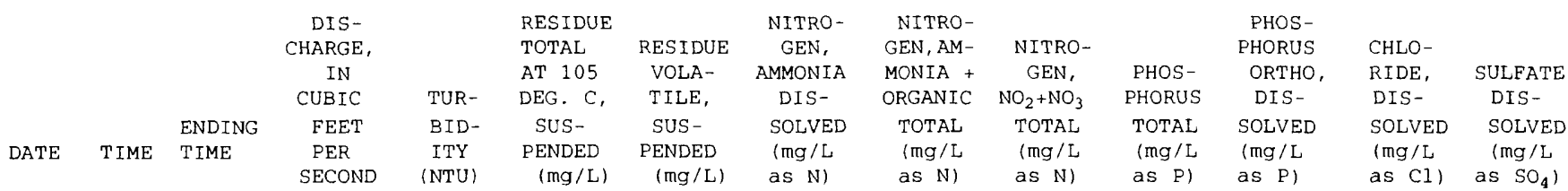

OCT

\begin{tabular}{|c|c|c|}
\hline $2-3$ & & \\
\hline $04-02$ & $\begin{array}{l}0125 \\
1120\end{array}$ & $\begin{array}{l}0825 \\
1020\end{array}$ \\
\hline $06-10$ & 1030 & 0930 \\
\hline $13 \ldots$ & 1035 & \\
\hline 16. & 1025 & \\
\hline $17-18$ & 1235 & 0535 \\
\hline $19 \ldots$ & 0950 & \\
\hline $23 \ldots$ & 1000 & - \\
\hline $23-26$ & 1000 & 0900 \\
\hline $26-30$ & 0925 & 0925 \\
\hline T $30-$ & & \\
\hline NOV 02 & 0940 & 0840 \\
\hline $02-06$ & 1115 & 1015 \\
\hline $06-07$ & 0910 & 1410 \\
\hline $07-09$ & 1510 & 0810 \\
\hline $09-13$ & 1000 & 0900 \\
\hline $13 \ldots$ & 0930 & \\
\hline $13-14$ & 1000 & 1800 \\
\hline $14-16$ & 1900 & 0900 \\
\hline $16-20$ & 0940 & 0840 \\
\hline $20-22$ & 0930 & 0830 \\
\hline $22-27$ & 1005 & 0905 \\
\hline $27-30$ & 0930 & 0830 \\
\hline Nov $30-$ & & \\
\hline DEC 02 & 1015 & 0915 \\
\hline $02-04$ & 1015 & 0915 \\
\hline $04-07$ & 1040 & 0940 \\
\hline $07-11$ & 1010 & 0910 \\
\hline $11-14$ & 1015 & 0915 \\
\hline $14-15$ & 1015 & 1515 \\
\hline $14-18$ & 1015 & 1115 \\
\hline $15-18$ & 1615 & 1115 \\
\hline $18-22$ & 1030 & 0930 \\
\hline $22-26$ & 1100 & 1000 \\
\hline $26-29$ & 1100 & 1000 \\
\hline $29-31$ & 1035 & 1735 \\
\hline DEC $31-$ & & \\
\hline JAN 02 & 1835 & 0935 \\
\hline $02-03$ & 0945 & 1745 \\
\hline $03-05$ & 1845 & 0845 \\
\hline $05-08$ & 1015 & 0915 \\
\hline $08-12$ & 0915 & 0815 \\
\hline $12-16$ & 0925 & 0825 \\
\hline $16-19$ & 0945 & 0845 \\
\hline $19-22$ & 0940 & 0840 \\
\hline $22-25$ & 1000 & 0900 \\
\hline $25-29$ & 0935 & 0835 \\
\hline JAN 29- & & \\
\hline $\begin{array}{llll}F E B & 01\end{array}$ & 0920 & 0820 \\
\hline $01-05$ & 0810 & 0710 \\
\hline $05-06$ & 1015 & 1215 \\
\hline $06-08$ & 1315 & 0915 \\
\hline $08-09$ & 0915 & 0415 \\
\hline $09-12$ & 0515 & 0815 \\
\hline $15-18$ & 1010 & 0710 \\
\hline $18-20$ & 0810 & 0910 \\
\hline $20-22$ & 1045 & 1545 \\
\hline $22-23$ & 1645 & 094 \\
\hline $3-26$ & 1015 & 0 \\
\hline
\end{tabular}

\section{WATER YEAR OCTOBER 1989 TO SEPTEMBER 1990}

$\begin{array}{cc}-- & 17 \\ -- & 12 \\ -- & 2.6 \\ -- & 2.0 \\ -- & 5.5 \\ -- & 45 \\ -- & 3.7 \\ -- & 8.0 \\ -- & 13\end{array}$

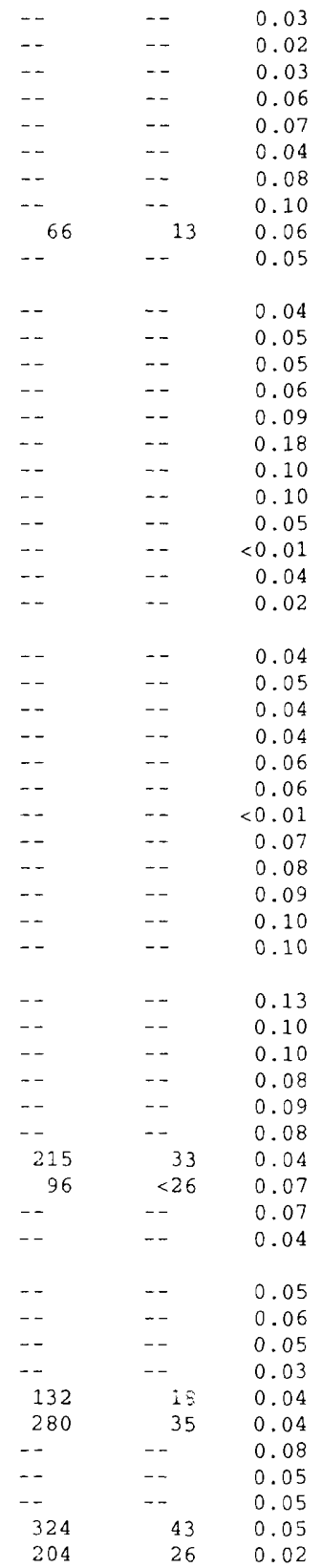

\begin{tabular}{|c|c|c|}
\hline 0.79 & 0.60 & 0.145 \\
\hline 0.66 & 0.56 & 0.115 \\
\hline 0.39 & 0.62 & 0.085 \\
\hline 0.23 & 0.57 & 0.065 \\
\hline 0.51 & 0.82 & 0.070 \\
\hline 1.5 & 0.49 & 0.280 \\
\hline 0.72 & 0.59 & 0.070 \\
\hline 0.51 & 0.67 & 0.065 \\
\hline 0.89 & 0.79 & 0.160 \\
\hline 0.39 & 0.65 & 0.095 \\
\hline 0.66 & 0.52 & 0.120 \\
\hline 0.54 & 0.56 & 0.080 \\
\hline 0.63 & 0.68 & 0.105 \\
\hline 0.83 & 0.73 & 0.155 \\
\hline 0.62 & 0.72 & 0.055 \\
\hline 0.80 & 0.69 & 0.065 \\
\hline 0.75 & 0.76 & 0.090 \\
\hline 0.60 & 0.60 & 0.105 \\
\hline 0.79 & 0.61 & $\ldots$ \\
\hline 0.75 & 0.69 & 0.110 \\
\hline 0.55 & 0.84 & 0.045 \\
\hline 0.53 & 0.88 & 0.055 \\
\hline 1.2 & 0.93 & 0.030 \\
\hline 0.47 & 0.96 & 0.040 \\
\hline 0.69 & 1.10 & 0.050 \\
\hline 0.57 & 0.96 & 0.040 \\
\hline 0.62 & 1.00 & 0.070 \\
\hline 0.48 & 1.10 & 0.025 \\
\hline 0.12 & $<0.01$ & 0.010 \\
\hline 0.66 & 1. 30 & 0.035 \\
\hline 0.42 & 1.30 & 0.050 \\
\hline 0.59 & 1.40 & 0.070 \\
\hline 0.60 & 1.50 & 0.035 \\
\hline 0.55 & 1.40 & 0.030 \\
\hline 0.82 & 1.40 & 0.045 \\
\hline 0.68 & 1.40 & 0.055 \\
\hline 1.1 & 1.40 & 0.130 \\
\hline 1.2 & 1.60 & 0.120 \\
\hline 0.73 & 1.60 & 0.070 \\
\hline 0.51 & 1.60 & 0.050 \\
\hline 1.8 & 1.60 & 0.260 \\
\hline 1.2 & 1.70 & 0.130 \\
\hline 0.92 & 1.60 & 0.090 \\
\hline 0.73 & 1.60 & 0.050 \\
\hline 0.73 & 1.40 & 0.045 \\
\hline 0.81 & 1.50 & 0.130 \\
\hline 0.73 & 0.84 & 0.075 \\
\hline 0.75 & 1.50 & 0.095 \\
\hline 1.1 & 1.70 & 0.175 \\
\hline 1.7 & 1.60 & 0.350 \\
\hline 1.0 & 1.60 & 0.130 \\
\hline 0.89 & 1.50 & 0.120 \\
\hline 0.84 & 1.50 & 0.060 \\
\hline 1.8 & 1.20 & 0.380 \\
\hline 1.4 & 1.50 & 0.260 \\
\hline
\end{tabular}

\begin{tabular}{|c|c|c|}
\hline 0.011 & 98 & 190 \\
\hline 0.010 & 110 & 180 \\
\hline 0.013 & 100 & \\
\hline 0.010 & 120 & 19 \\
\hline 0.013 & 84 & 1 \\
\hline 0.012 & 77 & 1. \\
\hline 0.019 & 92 & \\
\hline 0.019 & 99 & 13 \\
\hline 0.017 & 110 & 17 \\
\hline 0.025 & 110 & 18 \\
\hline 0.013 & 110 & \\
\hline 0.011 & 110 & 16 \\
\hline 0.012 & 120 & 18 \\
\hline 0.016 & 100 & 15 \\
\hline 0.027 & 110 & \\
\hline 0.024 & 120 & 16 \\
\hline 0.020 & 120 & 16 \\
\hline 0.014 & 120 & 1 \\
\hline 0.011 & 110 & 1 \\
\hline 0.010 & 110 & 12 \\
\hline 0.010 & 110 & 16 \\
\hline 0.008 & 120 & 17 \\
\hline 0.008 & 140 & 18 \\
\hline 0.007 & 140 & 19 \\
\hline 0.007 & 150 & 18 \\
\hline 0.006 & 190 & 18 \\
\hline 0.007 & 150 & 19 \\
\hline 0.006 & 160 & 18 \\
\hline 0.009 & - & \\
\hline 0.006 & 160 & 19 \\
\hline 0.005 & 150 & 20 \\
\hline 0.005 & 150 & 20 \\
\hline 0.005 & 150 & 21 \\
\hline 0.006 & 180 & 20 \\
\hline 0.006 & 520 & 1 \\
\hline 0.006 & 380 & \\
\hline 0.006 & 330 & 15 \\
\hline 0.011 & 250 & 14 \\
\hline 0.010 & 210 & 16 \\
\hline 0.008 & 230 & 1 \\
\hline 0.012 & 200 & 13 \\
\hline 0.011 & 160 & 12 \\
\hline 0.008 & 230 & 15 \\
\hline 0.008 & 170 & 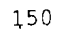 \\
\hline 0.005 & 250 & 1 \\
\hline 0.011 & 250 & 12 \\
\hline 0.007 & 210 & 12 \\
\hline 0.006 & 300 & 12 \\
\hline 0.014 & 250 & \\
\hline 0.019 & 140 & \\
\hline 0.010 & 190 & \\
\hline 0.010 & 140 & \\
\hline 0.008 & 150 & \\
\hline 0.016 & 140 & \\
\hline 0.010 & 120 & \\
\hline
\end{tabular}




\section{Surface-Water Stations}

A. Discharge and water quality

0423205025 Irondequoit Creek at Empire Boulevard, Rochester, N.Y.

\section{WATER-QUALITY RECORDS}

\begin{tabular}{|c|c|c|c|c|c|c|c|c|c|c|c|c|c|}
\hline & & & $\begin{array}{c}\text { DIS- } \\
\text { CHARGE, } \\
\text { IN } \\
\text { CUBIC }\end{array}$ & TUR- & $\begin{array}{l}\text { RESIDUE } \\
\text { TOTAL } \\
\text { AT } 105 \\
\text { DEG. C. }\end{array}$ & $\begin{array}{l}\text { RESIDUE } \\
\text { VOLA- } \\
\text { TILE, }\end{array}$ & $\begin{array}{l}\text { NITRO- } \\
\text { GEN, } \\
\text { AMMONIA } \\
\text { DIS- }\end{array}$ & $\begin{array}{l}\text { NITRO- } \\
\text { GEN, AM- } \\
\text { MONIA + } \\
\text { ORGANIC }\end{array}$ & $\begin{array}{c}\text { NITRO- } \\
\text { GEN, } \\
\mathrm{NO}_{2}+\mathrm{NO}_{3}\end{array}$ & $\begin{array}{l}\text { PHOS- } \\
\text { PHORUS }\end{array}$ & $\begin{array}{c}\text { PHOS- } \\
\text { PHORUS } \\
\text { ORTHO, } \\
\text { DIS- }\end{array}$ & $\begin{array}{l}\text { CHLO- } \\
\text { RIDE, } \\
\text { DIS- }\end{array}$ & $\begin{array}{l}\text { SULFATE } \\
\text { DIS- }\end{array}$ \\
\hline DATE & TIME & $\begin{array}{l}\text { ENDING } \\
\text { TIME }\end{array}$ & $\begin{array}{c}\text { FEET } \\
\text { PER } \\
\text { SECOND }\end{array}$ & $\begin{array}{l}\text { BID- } \\
\text { ITY } \\
\text { (NTU) }\end{array}$ & $\begin{array}{l}\text { SUS- } \\
\text { PENDED } \\
(\mathrm{mg} / \mathrm{L})\end{array}$ & $\begin{array}{c}\text { SUS- } \\
\text { PENDED } \\
(\mathrm{mg} / \mathrm{L})\end{array}$ & $\begin{array}{l}\text { SOLVED } \\
\text { (mg/L } \\
\text { as } N \text { ) }\end{array}$ & $\begin{array}{l}\text { TOTAL } \\
\text { (mg/L } \\
\text { as N) }\end{array}$ & $\begin{array}{l}\text { TOTAL } \\
\text { (mg/L } \\
\text { as N) }\end{array}$ & $\begin{array}{l}\text { TOTAL } \\
\text { (mg/L } \\
\text { as P) }\end{array}$ & $\begin{array}{l}\text { SOLVED } \\
(\mathrm{mg} / \mathrm{L} \\
\text { as } \mathrm{P})\end{array}$ & $\begin{array}{l}\text { SOLVED } \\
(\mathrm{mg} / \mathrm{L} \\
\text { as Cl) }\end{array}$ & $\begin{array}{c}\text { SOLVED } \\
(\mathrm{mg} / \mathrm{L} \\
\left.\text { as } \mathrm{SO}_{4}\right)\end{array}$ \\
\hline
\end{tabular}

FEB 26MAR 0

$\begin{array}{rrr}\text { MAR 01 } & 1030 & 0930 \\ 01-05 & 0920 & 0820 \\ 05-08 & 1040 & 0940 \\ 08 \ldots & 1040 & -- \\ 08-10 & 1045 & 0945 \\ 10-12 & 1045 & 0945 \\ 12-15 & 0945 & 0845 \\ 15-17 & 0925 & 0425 \\ 17-19 & 0525 & 0825 \\ 19-22 & 1015 & 0915 \\ 22-26 & 0920 & 0820 \\ 26-29 & 0925 & 0825\end{array}$

26-29 $0925 \quad 0825$

MAR $28-$

$A P R \quad 02$

$02-05$

$05-09$

$09-12$

$12-14$

$16 \ldots$

$19 \ldots$

$23 \ldots$

$26 \ldots$

$30 \ldots$
$01-03$

$03-04$

04-07

07-10

$10-13$

$13-14$

$14-17$

$17-21$

$21-25$
$25-29$

29-31

MAY 31-

JUN 02

$04 \ldots$

06-07

$07-08$

$08-11$

11-14

$14-18$

$18-21$

$21-22$

23-24

$24-25$

$28-30$

JUN $30-$

JUL 02

$02-04$
$04-05$

05-08

$09-12$

$09 \ldots$

$12-16$

$19 \ldots$

$19-20$

$20-23$

$23-26$

26-30

JUL $30-$

AUG 02
$0925 \quad 0825$

$0945 \quad 0845$

09450845

$2030 \quad 0430$

0940

0945
0915

1045

0940

$0840 \quad 0740$

$0915 \quad 1615$

$1715 \quad 0815$

$0910 \quad 0810$

$\begin{array}{ll}0945 & 1245 \\ 1345 & 0845\end{array}$

$0945 \quad 0845$

$0945 \quad 0845$

10550955

$1010 \quad 0910$

09250825

1040

0910

$1000 \quad 0900$

$0918 \quad 1218$

13180818

$\begin{array}{ll}1010 & 0910 \\ 0925 & 0825\end{array}$

$0930 \quad 0830$

$2115 \quad 2015$

$0115 \quad 1215$

17150815

$1018 \quad 0518$

$0618 \quad 0518$

$1015 \quad 2115$

22150915

10050105

$0920 \quad 0820$

1020

1015

1030

0630
1030

0920

1030
0100

0830
09150815

WATER YEAR OCTOBER 1989 TO SEPTEMBER 1990-continued

$\begin{array}{lll}-- & 15 & - \\ -- & 17 & - \\ -- & 12 & - \\ -- & 3.5 & - \\ -- & 9.0 & - \\ -- & 70 & \\ -- & 80 & \\ -- & 65 & \\ -- & 60 & \\ -- & 23 & - \\ -- & 5.8 & - \\ -- & 0.85 & - \\ & & \\ -- & 18 & - \\ -- & 55 & - \\ -- & 60 & \\ -- & 70 & \end{array}$

$\begin{array}{lll}-- & -- & 0.03 \\ -- & -- & 0.04 \\ -- & -- & 0.03 \\ -- & -- & 0.04 \\ -- & -- & 0.03 \\ 153 & 20 & 0.02 \\ 174 & 23 & 0.06 \\ 196 & 20 & 0.06 \\ 157 & 18 & 0.06 \\ -- & -- & 0.02 \\ -- & -- & 0.04 \\ -- & -- & 0.03\end{array}$

$0.72 \quad 1.70$

0.075

$0.65-1.70$

$0.77 \quad 1.60$

$\begin{array}{lll}0.74 & 1.70 & 0.040 \\ 0.85 & 1.70 & 0.070\end{array}$

0.080

$1.3 \quad 1.50$

$1.4 \quad 1.40$

$1.2 \quad 1.20$

$1.2 \quad 1.10$

$1.1 \quad 1.20$

$0.73 \quad 1.30$

0.040

0.165

0.230

0.240

0.200

0.125

$0.83 \quad 1.30 \quad 0.070$

$0.008 \quad 150$

$150-110$

0.008

$\begin{array}{lll}0.008 & 170 & 130 \\ 0.008 & 150 & 150\end{array}$

$0.008-150-160$

$0.007 \quad 140 \quad 100$

$0.042 \quad 100 \quad 72$

$0.023 \quad 120 \quad 100$

$0.014 \quad 120$

$0.008 \quad 120$

$0.008 \quad 130$

95
100

$\begin{array}{lll}- & - & 0.03\end{array}$

$\begin{array}{lll}154 & - & 0.02 \\ 156 & 20 & 0.06\end{array}$

$1.0 \quad 1.30$

$\begin{array}{ll}1.1 & 1.00\end{array}$

0.100

$0.005 \quad 130$

110

140

$\begin{array}{lrl}156 & 20 & 0.03 \\ - & - & 0.06\end{array}$

$1.2 \quad 1.20$

$0.75 \quad 1.10$

$0.93 \quad 1.50$

$0.75 \quad 1.40$

$0.83 \quad 2.80$

$1.0 \quad 1.10$

$\begin{array}{ll}0.97 & 0.81 \\ 1.5 & 0.90\end{array}$

0.290

$0.005 \quad 140$

0.250

0.060

0.060

0.040

0.085

0.075

0.090

$\begin{array}{ll}1.5 & 0.90 \\ 0.43 & 0.90\end{array}$

0.200

0.120

$\begin{array}{lll}0.73 & 0.95 & 0.150\end{array}$

$0.89 \quad \mathrm{~N} 1.10$

$0.64 \quad 0.98$

0.150
0.145

0.100

1.6

1.6

0.74

0.380

0.390

$1.6 \quad 0.95$

0.240

$\begin{array}{lll}1.2 & 1.10 & 0.200\end{array}$

$\begin{array}{ll}0.83 & 1.20\end{array}$

0.075

$0.008 \quad 120$

$0.010 \quad 120$

$0.012 \quad 97$

0.00988

$0.008 \quad 110$

$0.009 \quad 120$

$0.007 \quad 100$

$0.006 \quad 120$

$0.008 \quad 120$

0.005130

$0.005 \quad 130$

$0.004 \quad 100$

$0.007 \quad 110$

$0.009 \quad 110$

0.014

0.015

0.018

0.015

0.017

64
73

67

$\begin{array}{lll}0.81 & 1.20 \quad 0.070\end{array}$

0.018

110

$\begin{array}{ll}1.0 & 0.98\end{array}$

0.070

0.014

$0.016 \quad 100$

$0.015 \quad 110$

$0.72 \quad 0.95$

$0.63 \quad 0.96$

0.040

0.045

0.013

$0.92 \quad 3.20$

$0.59 \quad 0.88$

0.060

0.045

0.017

0.018

0.020

$\begin{array}{ll}0.89 & 0.97 \\ 0.82 & 0.99\end{array}$

$0.91 \quad 0.90$

0.110

0.028

0.020

0.130

0.016

$\begin{array}{ll}1.94 & 0.92 \\ 0.94 & 0.95\end{array}$

0.110

0.025

$\begin{array}{lll}0.70 & 0.90 & 0.110\end{array}$

$0.017 \quad 110$

0.90

1.3

0.90

0.93
0.73
0.78

0.170

0.025

0.014

0.150

0.029

$\begin{array}{lll}1.1 & 0.74 & 0.150\end{array}$

$\begin{array}{lll}0.71 & 0.69 & 0.080\end{array}$

0.019

0.018

0.97

0.74

1.6

0.79

0.150

0.018

0.010

0.037

$0.65 \quad 0.220$

0.77

$1.3 \quad 0.78$

0.220

0.021

0.025

$0.021 \quad 120$

140

90
61

71

51
84

100

84

100

110

130

130

91

96

49
54

48

68
100

110

107

$22-0.07$

$\begin{array}{llll}0930 & 49 & 50 & 113\end{array}$

$24 \quad 0.02$

1.2

0.75

0.230

$0.022 \quad 100$

110

120

120
130
130

130

140

150

170

160
120

150 


\section{Surface-Water Stations}

\section{A. Discharge and water quality}

0423205025 Irondequoit Creek at Empire Boulevard, Rochester, N.Y.

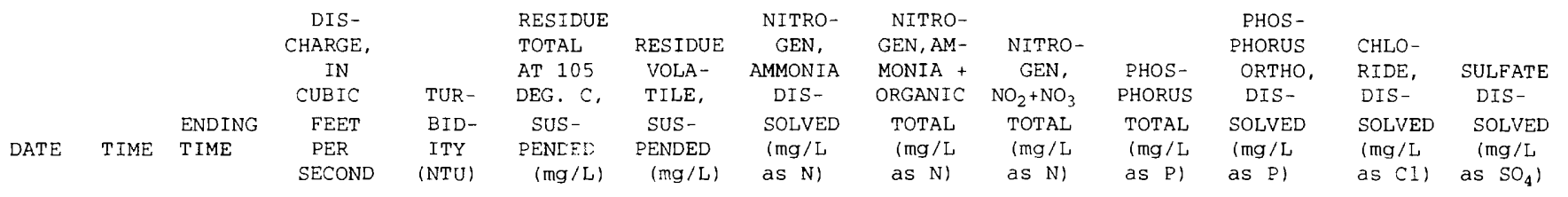

$\begin{array}{ccc}\text { AUG-cont inued } & \\ 06-09 & 1100 & 0900 \\ 09-12 & 0945 & 1245 \\ 12-13 & 1345 & 0845 \\ 13-16 & 1125 & 1025 \\ 16-20 & 1025 & 0925 \\ 20-23 & 1155 & 0955 \\ 23-27 & 1035 & 0935 \\ 27-31 & 1040 & 0940 \\ \text { AUG } 31- & & \\ \text { SEP 04 } & 1130 & 1030 \\ 04-05 & 1140 & 0640 \\ 05-06 & 0740 & 1040 \\ 06-07 & 1125 & 0625 \\ 07-10 & 0725 & 1025 \\ 10-13 & 1115 & 1015 \\ 13-14 & 1100 & 1800 \\ 14-17 & 1900 & 1000 \\ 17-20 & 1430 & 1030 \\ 20-24 & 1045 & 0945 \\ 24-27 & 1100 & 1000 \\ 27-29 & 1110 & 1810 \\ \text { SEP } 29- & & \\ \text { OCT } 01 & 1910 & 1010\end{array}$

WATER YEAR OCTOBER 1989 TO SEPTEMBER 1990-continued

OCT

$\begin{array}{lrrrr}\text { OCT } & & & & \\ 01-04 & 1115 & 1015 & 59 & 27 \\ 04-09 & 1050 & 0950 & -- & 50 \\ 09-11 & 1120 & 1020 & 153 & 70 \\ 11-13 & 1040 & 0540 & 280 & 110 \\ 13-15 & 0640 & 0940 & 264 & 85 \\ 15-18 & 1115 & 1015 & 96 & 40 \\ 18-18 & 1030 & 1330 & 72 & 60 \\ 18-22 & 1430 & 0930 & 115 & 55 \\ 22-23 & 1050 & 0650 & 98 & 55 \\ 23-25 & 0750 & 0920 & -- & 65 \\ 25-29 & 1030 & 0930 & 106 & 28 \\ \text { OCT 29- } & & & & \\ \text { NOV 01 } & 1030 & 0930 & 85 & 23 \\ 01-05 & 1010 & 0910 & 71 & 43 \\ 05-09 & 1540 & 0840 & 118 & 26 \\ 09-09 & 0850 & 2350 & 90 & 5.1 \\ 10-13 & 0050 & 0750 & 208 & 28 \\ 13-15 & 1110 & 1010 & 146 & 13 \\ 15-19 & 1040 & 0940 & 138 & 19 \\ 19-21 & 1100 & 0800 & 103 & 16 \\ 21-22 & 0850 & 1350 & 80 & 18 \\ 22-26 & 1450 & 0750 & 106 & 22 \\ 26-27 & 1040 & 0640 & 109 & 12 \\ 27-29 & 0740 & 0940 & 162 & 27 \\ \text { NOV 29- } & & & & \\ \text { DEC 03 } & 1015 & 0915 & 97 & 13 \\ 03-04 & 1125 & 0725 & 124 & 18 \\ 04-06 & 0825 & 1025 & 344 & 65 \\ 06-10 & 1030 & 0930 & 144 & 14 \\ 10-13 & 1035 & 0935 & 110 & 12 \\ 13-16 & 1010 & 0110 & 104 & 9.3 \\ 16-16 & 0210 & 0910 & 106 & 9.9 \\ 16-17 & 1010 & 0910 & 207 & 28 \\ 17-17 & 1100 & 1600 & 193 & 29 \\ 20 \ldots & 0920 & -- & 317 & 25 \\ 21 \ldots & 1015 & -- & 167 & 14 \\ 21-23 & 1015 & 0015 & 186 & 32 \\ 23-24 & 0115 & 0915 & 295 & 90 \\ 24-28 & 0955 & 0855 & 188 & 32\end{array}$

$\begin{array}{rrccc}220 & 26 & 0.03 & 1.5 & 0.64 \\ 144 & 23 & 0.02 & 1.5 & 0.75 \\ 170 & 25 & 0.02 & 1.7 & 0.62 \\ 153 & 28 & -- & 1.2 & 0.84 \\ 118 & 21 & -- & 1.1 & 0.80 \\ 95 & 18 & -- & 1.4 & 0.81 \\ 154 & 31 & -- & 1.4 & 0.73 \\ 111 & 22 & -- & 1.4 & 0.53 \\ & & & & \\ 109 & 23 & -- & 1.2 & 0.67 \\ 109 & 20 & <0.04 & 1.4 & 0.63 \\ 217 & 38 & <0.04 & 2.1 & 0.84 \\ 166 & 25 & <0.04 & 1.1 & 1.20 \\ 231 & 35 & <0.04 & 1.8 & 0.89 \\ 152 & 23 & <0.01 & 1.5 & 0.79 \\ 160 & 24 & <0.01 & 1.4 & 0.85 \\ 148 & 22 & <0.01 & 1.5 & 0.72 \\ 94 & 16 & 0.02 & 1.1 & 0.85 \\ 103 & 22 & <0.01 & 1.5 & 0.78 \\ 98 & 18 & <0.01 & 1.0 & 0.80 \\ 95 & 15 & 0.02 & 1.0 & 0.80 \\ & & & & \\ 143 & 22 & 0.02 & 1.2 & 0.80\end{array}$

$\begin{array}{rrrr}0.350 & 0.033 & 81 & 110 \\ 0.340 & 0.030 & 110 & 170 \\ 0.320 & 0.012 & 02 & 140 \\ 0.300 & 0.027 & 83 & 120 \\ 0.250 & 0.190 & 100 & 160 \\ 0.230 & 0.033 & 110 & 170 \\ 0.200 & 0.025 & 110 & 180 \\ 0.230 & 0.022 & 110 & 170 \\ 0.230 & 0.023 & 110 & 190 \\ 0.230 & 0.024 & 110 & 180 \\ 0.400 & 0.016 & 79 & 130 \\ 0.280 & 0.040 & \varepsilon 3 & 130 \\ 0.370 & 0.029 & 81 & 87 \\ 0.250 & 0.029 & 1 C 0 & 160 \\ 0.290 & 0.037 & 120 & 150 \\ 0.260 & 0.024 & 110 & 150 \\ 0.210 & 0.021 & 110 & 180 \\ 0.200 & 0.019 & 110 & 180 \\ 0.210 & 0.016 & 110 & 110 \\ 0.190 & 0.022 & 110 & 120\end{array}$

WATER YEAR OCTOBER 1990 TO SEPTEMBER 1991

\begin{tabular}{|c|c|c|c|c|c|c|c|c|}
\hline -. & -- & $<0.01$ & 1.1 & 0.74 & 0.180 & 0.029 & 100 & 140 \\
\hline 203 & 38 & $<0.01$ & 1.3 & 0.66 & 0.270 & 0.023 & 160 & 150 \\
\hline 229 & 40 & 0.03 & 1.8 & 0.68 & 0.340 & 0.020 & $\subseteq 4$ & 120 \\
\hline 385 & 56 & 0.02 & 1.6 & 0.60 & 0.530 & 0.025 & 69 & 77 \\
\hline 275 & 36 & 0.02 & 1.5 & 0.77 & 0.410 & 0.025 & 78 & 69 \\
\hline 125 & 20 & 0.02 & 1.3 & 0.92 & 0.230 & 0.026 & 100 & 110 \\
\hline -- & -- & 0.02 & 1.4 & 0.98 & 0.300 & 0.031 & $\varepsilon 3$ & 130 \\
\hline 131 & 25 & 0.03 & 1.3 & 0.78 & 0.240 & 0.025 & $\varsigma_{1}$ & 110 \\
\hline 188 & 27 & 0.02 & 1.1 & 0.95 & 0.260 & 0.023 & $\subseteq 7$ & 140 \\
\hline 209 & 28 & 0.01 & 1.4 & 0.87 & 0.290 & 0.021 & $\varepsilon 6$ & 120 \\
\hline-- & $\cdots$ & 0.01 & 1.0 & 1.10 & 0.190 & 0.021 & $\subsetneq 8$ & 110 \\
\hline - & -- & 0.02 & 1.1 & 1.10 & 0.160 & 0.017 & 100 & 120 \\
\hline 139 & 20 & 0.04 & 1.2 & 0.98 & 0.240 & 0.022 & 110 & 150 \\
\hline-- & -- & -. & 1.1 & 0.92 & 0.160 & 0.016 & 100 & 130 \\
\hline -. & -- & 0.03 & 0.76 & 0.93 & 0.085 & 0.013 & 110 & 140 \\
\hline-- & -- & 0.02 & 1.1 & 0.91 & 0.160 & 0.016 & 110 & 86 \\
\hline -- & -- & 0.06 & 0.82 & 1.10 & 0.120 & 0.014 & 140 & 100 \\
\hline.- & -- & 0.02 & 0.98 & 1.00 & 0.110 & 0.015 & $1<0$ & 100 \\
\hline -. & -- & 0.03 & 1.1 & 1.10 & 0.100 & 0.012 & 120 & 130 \\
\hline -- & -- & 0.02 & 1.1 & 1.20 & 0.120 & 0.017 & 120 & 140 \\
\hline-- & -. & 0.02 & 1.1 & 0.99 & 0.140 & 0.012 & 110 & 100 \\
\hline -- & -- & 0.02 & 1.0 & 0.91 & 0.110 & 0.014 & 120 & 110 \\
\hline -- & -- & 0.02 & 1.0 & 0.90 & 0.160 & 0.011 & 120 & 130 \\
\hline _. & -. & 0.01 & 0.73 & 0.04 & 0.075 & 0.011 & $1=0$ & 130 \\
\hline -. & -- & 0.02 & 1.0 & 1.20 & 0.110 & $<0.002$ & 140 & 77 \\
\hline- & -- & 0.01 & 1.8 & 0.97 & 0.300 & 0.015 & 140 & 68 \\
\hline - - & -. & 0.01 & 1.1 & 1.20 & 0.100 & 0.015 & 130 & 110 \\
\hline - - & -- & 0.02 & 0.87 & 1.40 & 0.080 & 0.013 & 120 & 120 \\
\hline- & -- & 0.02 & 0.90 & 1.30 & 0.065 & 0.008 & 130 & 140 \\
\hline - - & -. & $<0.01$ & 0.73 & 1.30 & 0.065 & 0.008 & 130 & 140 \\
\hline -- & -. & 0.03 & 0.94 & 1.20 & 0.130 & 0.008 & 120 & 100 \\
\hline -- & -. & 0.03 & 1.2 & 1.20 & 0.180 & 0.013 & 100 & 100 \\
\hline - - & -. & 0.06 & 0.86 & 1.10 & 0.110 & 0.019 & 100 & 58 \\
\hline-- & -- & 0.07 & 0.94 & 1.20 & 0.075 & 0.016 & 95 & 81 \\
\hline 85 & 14 & 0.03 & 1.1 & 1.30 & 0.190 & 0.018 & $\subseteq 8$ & 78 \\
\hline 255 & 28 & 0.02 & 1.4 & 1.10 & 0.330 & 0.015 & 83 & 59 \\
\hline 70 & 15 & 0.01 & 1.0 & 1.40 & 0.140 & 0.019 & 110 & 87 \\
\hline
\end{tabular}




\section{Irondequoit Creek at Empire Boulevard, Rochester, N.Y.}

\section{WATER-QUALITY RECORDS}

\begin{tabular}{|c|c|c|c|c|c|c|c|c|c|c|c|c|c|}
\hline & & & $\begin{array}{c}\text { DIS- } \\
\text { CHARGE, } \\
\text { IN } \\
\text { CUBIC }\end{array}$ & TUR- & $\begin{array}{l}\text { RESIDUE } \\
\text { TOTAL } \\
\text { AT } 105 \\
\text { DEG. C. }\end{array}$ & $\begin{array}{c}\text { RESIDUE } \\
\text { VOLA- } \\
\text { TILE, }\end{array}$ & $\begin{array}{c}\text { NITRO- } \\
\text { GEN, } \\
\text { AMMONIA } \\
\text { DIS- }\end{array}$ & $\begin{array}{l}\text { NITRO- } \\
\text { GEN, AM- } \\
\text { MONIA + } \\
\text { ORGANIC }\end{array}$ & $\begin{array}{c}\mathrm{NITRO}- \\
\mathrm{GEN} \\
\mathrm{NO}_{2}+\mathrm{NO}_{3}\end{array}$ & $\begin{array}{l}\text { PHOS- } \\
\text { PHORUS }\end{array}$ & $\begin{array}{c}\text { PHOS- } \\
\text { PHORUS } \\
\text { ORTHO, } \\
\text { DIS- }\end{array}$ & $\begin{array}{l}\text { CHLO- } \\
\text { RIDE, } \\
\text { DIS- }\end{array}$ & $\begin{array}{l}\text { SULFATE } \\
\text { DIS- }\end{array}$ \\
\hline DATE & TIME & $\begin{array}{l}\text { ENDING } \\
\text { TIME }\end{array}$ & $\begin{array}{l}\text { FEET } \\
\text { PER } \\
\text { SECOND }\end{array}$ & $\begin{array}{l}\text { BID- } \\
\text { ITY } \\
\text { (NTU) }\end{array}$ & $\begin{array}{l}\text { SUS- } \\
\text { PENDED } \\
(\mathrm{mg} / \mathrm{L})\end{array}$ & $\begin{array}{l}\text { SUS- } \\
\text { PENDED } \\
(\mathrm{mg} / \mathrm{L})\end{array}$ & $\begin{array}{l}\text { SOLVED } \\
(\mathrm{mg} / \mathrm{L} \\
\text { as } \mathrm{N} \text { ) }\end{array}$ & $\begin{array}{l}\text { TOTAL } \\
(\mathrm{mg} / \mathrm{L} \\
\text { as N) }\end{array}$ & $\begin{array}{l}\text { TOTAL } \\
(\mathrm{mg} / \mathrm{L} \\
\text { as N) }\end{array}$ & $\begin{array}{l}\text { TOTAL } \\
\text { (mg/L } \\
\text { as P) }\end{array}$ & $\begin{array}{l}\text { SOLVED } \\
\text { (mg/L } \\
\text { as P) }\end{array}$ & $\begin{array}{l}\text { SOLVED } \\
\text { (mg/L } \\
\text { as Cl) }\end{array}$ & $\begin{array}{c}\text { SOLVED } \\
(\mathrm{mg} / \mathrm{L} \\
\left.\text { as } \mathrm{SO}_{4}\right)\end{array}$ \\
\hline
\end{tabular}

DEC-cont inued \begin{tabular}{cc}
$28-30 \quad 1045$ \\
\hline
\end{tabular} DEC $30-$

$\begin{array}{rlll}\text { JAN } 02 & 1645 & 0945 & 747 \\ 02-04 & 1045 & 0945 & 262 \\ 04-07 & 1055 & 0955 & 157 \\ 07-10 & 1115 & 1015 & 119 \\ 10-14 & 1110 & 1010 & 127 \\ 14-16 & 1155 & 1355 & 112 \\ 16-17 & 1455 & 1055 & 288 \\ 17-22 & 1110 & 1010 & 281 \\ 22-24 & 1130 & 0930 & 171 \\ 24-28 & 1025 & 0925 & 115 \\ 28-31 & 1030 & 0930 & 100 \\ \text { JAN } 31- & & & \\ \text { FEB } 04 & 1055 & 0955 & 103 \\ 04-07 & 1045 & 0945 & 272 \\ 07-11 & 1045 & 0945 & 244 \\ 11-15 & 1055 & 0955 & 130 \\ 15-19 & 1100 & 1000 & 108 \\ 19-20 & 1105 & 1005 & 292 \\ 20-21 & 1105 & 1005 & 331 \\ 21-25 & 1110 & 1010 & 164 \\ 25-28 & 1050 & 0950 & 109\end{array}$

FEB $28-$

MAR 02

$02-04$

$06-08$

$06 .$.

$12 \ldots$

$12-14$

$14-18$

$18-19$

$19-21$

21-23

$23-23$

$23-25$

$25-27$
$27-27$

$27-28$

MAR 28 -

APR 01

01-01

04-08

08-09

09-10

10-11

11-15

15-16

18-20

20-22

22-25

25-29

APR $29-$

MAY 02

02-06

06-09

$09-13$

13-16

17-17

17-20

20-23

23-26 $\begin{array}{lll}1025 & 1725 & 112\end{array}$

$\begin{array}{lll}1825 & 0925 & 448\end{array}$

$\begin{array}{rrr}1110 & 1010 & 672 \\ 1115 & -- & 1010\end{array}$

$\begin{array}{llr}1115 & 0915 & 291\end{array}$

$\begin{array}{lrr}0955 & -- & 175 \\ 0955 & 0855 & 149\end{array}$

$1040 \quad 0940 \quad 134$

$1035 \quad 0035 \quad 130$

$0135 \quad 0935 \quad 162$

$1050 \quad 0550 \quad 122$

$0650 \quad 2150 \quad 222$

$2250 \quad 0950 \quad 309$

$1055 \quad 0555 \quad 218$

$0655 \quad 2155 \quad 277$

$2255 \quad 0955$

$1045 \quad 0945$

$\begin{array}{lll}1055 & 2155 & 179\end{array}$

$2255 \quad 0955 \quad 168$

$1045 \quad 0945 \quad 130$

$\begin{array}{lll}1020 & 0020 & 173\end{array}$

$0120 \quad 0020 \quad 206$

$\begin{array}{lll}0120 & 0920 & 260 \\ 1035 & 0935 & 142\end{array}$

$\begin{array}{llr}0145 & 0945 & - \\ 1045 & 0045 & 176\end{array}$

$\begin{array}{lll}1045 & 0045 & 176 \\ 1040 & 0140 & 127\end{array}$

$0240 \quad 0840 \quad 367$

$09350835 \quad 938$

$0940 \quad 0840 \quad 248$

$09350835 \quad 178$

$1100 \quad 0900 \quad 140$

$0940 \quad 0840 \quad 124$

$\begin{array}{lll}1040 & 0940 & 119 \\ 0945 & 0845 & 108\end{array}$

$1020 \quad 0920 \quad 96$

$1020 \quad 2120 \quad 98$

$2220 \quad 0920 \quad 201$

$\begin{array}{lll}0935 & 0835 & 85\end{array}$

WATER YEAR OCTOBER 1990 TO SEPTEMBER 1991 -contlnued

\begin{tabular}{|c|c|c|c|c|c|c|c|c|c|}
\hline 7.5 & -- & -- & 0.02 & 0.74 & 1.70 & 0.065 & 0.019 & 130 & 130 \\
\hline 110 & 326 & 24 & 0.02 & 1.4 & 1.20 & 0.390 & 0.023 & 100 & 41 \\
\hline 32 & 71 & 10 & 0.03 & 0.94 & 1.60 & 0.130 & 0.021 & 100 & 18 \\
\hline 20 & -- & -- & 0.02 & 0.82 & 1.80 & 0.120 & 0.019 & 120 & 94 \\
\hline 12 & -- & -- & 0.04 & 0.75 & 1.90 & 0.075 & 0.013 & 130 & 110 \\
\hline 12 & -- & -- & 0.02 & 0.75 & 1.80 & 0.070 & 0.014 & 150 & 100 \\
\hline 11 & -- & -- & 0.03 & 0.63 & 1.80 & 0.065 & 0.013 & 170 & 140 \\
\hline 40 & 105 & 15 & 0.04 & 0.85 & 1.40 & 0.170 & 0.012 & 220 & 84 \\
\hline 26 & -- & -- & 0.02 & 0.91 & 1.50 & 0.130 & 0.022 & 130 & 67 \\
\hline 14 & - & -- & 0.03 & 0.77 & 1.80 & 0.080 & 0.022 & 130 & 84 \\
\hline 7.8 & -- & -- & 0.01 & 0.78 & 1.90 & 0.065 & 0.015 & 130 & 120 \\
\hline 8.2 & -- & -- & 0.03 & 0.78 & 1.70 & 0.055 & 0.012 & 170 & 110 \\
\hline 6.7 & - & -- & -- & 0.66 & 1.70 & 0.050 & 0.008 & 210 & 140 \\
\hline 29 & -- & -- & 0.02 & 0.97 & 1.50 & 0.100 & 0.016 & 160 & 72 \\
\hline 16 & -- & -- & 0.01 & 0.85 & 1.50 & 0.120 & 0.014 & 120 & 74 \\
\hline 7.5 & - & -- & 0.02 & 0.68 & 1.50 & 0.055 & 0.007 & 170 & 100 \\
\hline 7.4 & -- & -- & 0.02 & 0.63 & 1.80 & 0.050 & 0.007 & 210 & 120 \\
\hline 27 & -- & -- & 0.02 & 0.83 & 1.40 & 0.120 & 0.008 & 200 & 100 \\
\hline 40 & 107 & 14 & 0.02 & 0.98 & 1.20 & 0.160 & 0.006 & 130 & 71 \\
\hline 13 & -- & -- & 0.02 & 0.72 & 1.30 & 0.065 & 0.007 & 130 & 99 \\
\hline 4.9 & -- & -- & 0.02 & 0.78 & 1.20 & 0.050 & 0.006 & 150 & 130 \\
\hline 9.9 & - & -- & $<0.01$ & 0.66 & 1.40 & 0.060 & 0.008 & 170 & 130 \\
\hline 190 & 488 & 46 & 0.02 & 1.7 & 1.00 & 0.490 & 0.014 & 130 & 66 \\
\hline 65 & 157 & 17 & 0.04 & 0.87 & 1.10 & 0.240 & 0.015 & 71 & 49 \\
\hline 45 & 66 & $<10$ & 0.05 & 0.75 & 1.10 & 0.120 & 0.024 & 62 & 34 \\
\hline 24 & -- & -- & 0.02 & 0.79 & 1.50 & 0.110 & 0.013 & 93 & 71 \\
\hline 6.2 & -- & -- & 0.04 & 0.61 & 1.40 & 0.030 & 0.009 & 100 & 100 \\
\hline 17 & -- & -- & 0.02 & 0.67 & 1.60 & 0.090 & 0.011 & 110 & 110 \\
\hline 18 & -- & - & 0.01 & 0.67 & 1.60 & 0.100 & 0.007 & 110 & 120 \\
\hline 25 & -- & -- & 0.02 & 0.70 & 1.40 & 0.110 & 0.006 & 110 & 120 \\
\hline 40 & 118 & 16 & 0.02 & 0.90 & 1.30 & 0.170 & 0.005 & 110 & 120 \\
\hline 32 & 114 & 15 & 0.03 & 0.94 & 1.20 & 0.150 & 0.006 & 110 & 120 \\
\hline 75 & 187 & 19 & 0.02 & 1.0 & 1.20 & 0.220 & 0.006 & 110 & 100 \\
\hline 90 & 206 & 25 & 0.02 & 1.2 & 1.00 & 0.270 & 0.007 & 93 & 71 \\
\hline 50 & 138 & 18 & 0.04 & 0.91 & 1.20 & 0.190 & 0.013 & 85 & 78 \\
\hline 75 & 209 & 26 & 0.03 & 1.1 & 1.10 & 0.240 & 0.008 & 93 & 82 \\
\hline 140 & 402 & 47 & 0.03 & 1.7 & 1.00 & 0.510 & 0.009 & 78 & 62 \\
\hline 95 & 213 & 25 & 0.02 & 1.2 & 1.10 & 0.230 & 0.016 & 80 & 49 \\
\hline 34 & 93 & 17 & 0.05 & 0.92 & 1.20 & 0.170 & 0.015 & 95 & 50 \\
\hline 34 & 114 & 20 & 0.02 & 0.95 & 1.30 & 0.200 & 0.011 & 100 & 100 \\
\hline 60 & 202 & 35 & 0.02 & 1.6 & 1.20 & 0.270 & 0.014 & 110 & 98 \\
\hline 65 & $\cdots$ & -- & 0.07 & 0.99 & 1.30 & 0.250 & 0.020 & 100 & 120 \\
\hline 95 & -- & -- & 0.04 & 1.4 & 1.20 & 0.340 & 0.022 & 95 & 100 \\
\hline 110 & 281 & 42 & 0.04 & 1.4 & 0.96 & 0.390 & 0.016 & 83 & 82 \\
\hline 45 & 119 & 19 & 0.03 & 1.3 & 0.98 & 0.190 & 0.015 & 93 & 100 \\
\hline 60 & 156 & 25 & 0.02 & 1.4 & 0.84 & 0.280 & 0.012 & 82 & 84 \\
\hline 50 & 149 & 19 & 0.03 & 1.3 & 1.10 & 0.230 & 0.017 & 100 & 110 \\
\hline 36 & 89 & 11 & $<0.01$ & 1.1 & 0.90 & 0.180 & 0.016 & 94 & 98 \\
\hline 60 & 178 & 17 & 0.01 & 1.4 & 0.80 & 0.260 & 0.011 & 82 & 83 \\
\hline 80 & 182 & 20 & 0.02 & 1.2 & 0.88 & 0.310 & 0.019 & 50 & 42 \\
\hline 40 & 114 & 16 & 0.01 & 1.2 & 0.92 & 0.200 & 0.017 & 71 & 74 \\
\hline 38 & 122 & 15 & 0.02 & 1.2 & 1.00 & 0.220 & 0.016 & 92 & 100 \\
\hline 26 & 80 & 12 & 0.02 & 0.97 & 1.10 & 0.170 & 0.015 & 93 & 120 \\
\hline 30 & 81 & 15 & $<0.01$ & 0.96 & 0.98 & 0.160 & 0.013 & 90 & 110 \\
\hline 28 & -- & -- & 0.02 & 1.2 & 0.87 & 0.150 & 0.015 & 96 & 130 \\
\hline 39 & -- & -- & -- & 1.2 & 0.69 & 0.180 & 0.008 & 99 & 130 \\
\hline 38 & -- & -- & -- & 1.1 & 0.68 & 0.180 & 0.009 & 100 & 140 \\
\hline 41 & 122 & 19 & -- & 1.5 & 0.73 & 0.190 & 0.010 & 96 & 140 \\
\hline 36 & -- & -- & -- & 1.2 & 0.80 & 0.220 & 0.013 & 88 & 120 \\
\hline 40 & 130 & 25 & 0.03 & 1.1 & 0.85 & 0.210 & 0.012 & 99 & 120 \\
\hline 50 & 156 & 30 & 0.04 & 1.2 & 0.60 & 0.240 & 0.012 & 110 & 140 \\
\hline
\end{tabular}


Surface-Water Stations

\section{A. Discharge and water quality}

0423205025 Irondequoit Creek at Empire Boulevard, Rochester, N.Y.

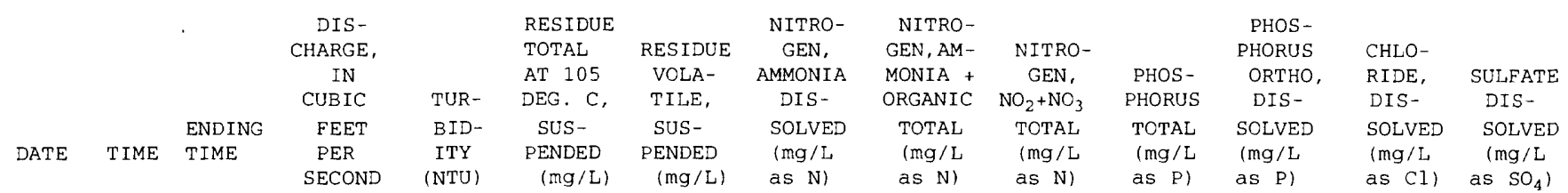

MAY-cont inued

\begin{tabular}{|c|c|c|c|c|}
\hline $26-26$ & 1405 & 2305 & 146 & 45 \\
\hline $27-28$ & 0005 & 0905 & 147 & 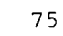 \\
\hline $28-30$ & 0955 & 0855 & 73 & \\
\hline \multicolumn{5}{|l|}{ MAY $30-$} \\
\hline JUN 03 & 0940 & 0840 & 60 & 4 \\
\hline $03-06$ & 0930 & 0930 & -- & \\
\hline $06 \ldots$ & 1140 & - & 43 & 2 \\
\hline $06-10$ & 1200 & 0900 & 56 & \\
\hline $06 \ldots$ & 1205 & -. & 43 & \\
\hline $10-11$ & 0925 & 1425 & 44 & 3 \\
\hline $11-12$ & 1525 & 1125 & 75 & \\
\hline $12-12$ & 1225 & 2025 & 129 & 3 \\
\hline $12-13$ & 2125 & 0825 & 152 & \\
\hline $13-17$ & 0930 & 0830 & 68 & 4 \\
\hline $17-20$ & 0940 & 0840 & 62 & 4 \\
\hline $20-24$ & 0930 & 0820 & 52 & 3 \\
\hline $24-27$ & 0935 & 0835 & 52 & 3 \\
\hline \multicolumn{5}{|l|}{ JUN $27-$} \\
\hline JUL 01 & 0935 & 0835 & 43 & 3 \\
\hline $01-04$ & 0935 & 1635 & 44 & 3 \\
\hline $04-05$ & 1735 & 0835 & 73 & 3 \\
\hline $05-06$ & 0925 & 0225 & 75 & 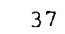 \\
\hline $06-07$ & 0325 & 0825 & 114 & \\
\hline $07-07$ & 0925 & 1725 & 86 & \\
\hline $07-08$ & 1825 & 0825 & 82 & 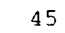 \\
\hline 08-09 & 0935 & 2035 & 45 & \\
\hline $09-10$ & 2135 & 2035 & 50 & \\
\hline $11-15$ & 0935 & 0835 & 51 & \\
\hline $15-18$ & 0950 & 0850 & 45 & \\
\hline $18-21$ & 0935 & 1635 & 40 & \\
\hline $21-22$ & 1735 & 0835 & 76 & \\
\hline $22-23$ & 0935 & 2035 & 55 & \\
\hline $23-25$ & 2135 & 0835 & 45 & \\
\hline $25-29$ & 0940 & 0840 & 39 & \\
\hline $29-30$ & 0930 & 0830 & 93 & \\
\hline \multicolumn{5}{|l|}{ IUL $30-$} \\
\hline AUG 01 & 0945 & 0845 & 39 & \\
\hline $01-03$ & 0945 & 0445 & 32 & \\
\hline $03-03$ & 0545 & 2045 & 93 & \\
\hline $03-05$ & 2145 & 0845 & 59 & \\
\hline $05-08$ & 0945 & 0845 & 49 & \\
\hline $08-09$ & 0945 & 0045 & 43 & \\
\hline $09-09$ & 0145 & 1645 & 101 & \\
\hline $09-11$ & 1745 & 0045 & 97 & \\
\hline $12 \ldots$ & 0940 & -- & 40 & \\
\hline $15 \ldots$ & 0920 & -- & 62 & \\
\hline $15-19$ & 0920 & 0820 & 42 & \\
\hline $22 \ldots$ & 0915 & - & 46 & \\
\hline $22-26$ & 2055 & 0855 & 38 & \\
\hline $26-30$ & 0910 & 0810 & 37 & \\
\hline $30-31$ & 0900 & 0400 & 29 & \\
\hline \multicolumn{5}{|l|}{ AUG $31-$} \\
\hline SEP 03 & 0500 & 0800 & 45 & \\
\hline $03-04$ & 0925 & 0825 & 40 & \\
\hline $04-05$ & 0925 & 0825 & 44 & \\
\hline $05-09$ & 1050 & 0950 & 39 & \\
\hline $09-11$ & 1450 & 0150 & 40 & \\
\hline $11-12$ & 0250 & 0950 & 49 & \\
\hline $12-15$ & 1045 & 0945 & 40 & \\
\hline $15-16$ & 1045 & 0945 & 81 & \\
\hline $16-19$ & 1100 & 1000 & 38 & $\mathrm{~N}_{2}$ \\
\hline $19-23$ & 1130 & 1030 & 40 & \\
\hline $23-25$ & 1055 & 0355 & 37 & \\
\hline $25-25$ & 0455 & 1555 & 141 & \\
\hline $25-26$ & 1655 & 0955 & 142 & \\
\hline $6-3$ & 1030 & 0930 & 47 & \\
\hline
\end{tabular}

WATER YEAR OCTOBER 1990 TO SEPTEMBER 1991-continued

\begin{tabular}{|c|c|c|c|c|c|c|c|c|c|}
\hline 45 & 132 & $<28$ & 0.03 & 1.3 & 0.60 & 0.240 & 0.012 & 98 & 150 \\
\hline 75 & -- & -- & 0.03 & 1.3 & 0.58 & 0.320 & 0.017 & 83 & 110 \\
\hline 50 & 150 & 19 & 0.03 & 1.4 & 0.88 & 0.250 & 0.017 & 96 & 120 \\
\hline 45 & 150 & 19 & 0.02 & 0.49 & 1.10 & 0.230 & 0.019 & 110 & 140 \\
\hline 50 & 130 & 18 & $<0.01$ & 1.4 & 0.94 & 0.300 & 0.037 & 120 & 160 \\
\hline 23 & -- & -- & 0.03 & 1.1 & 0.94 & 0.190 & 0.019 & 120 & 160 \\
\hline 39 & 110 & 18 & $<0.01$ & 0.24 & 0.79 & 0.240 & 0.019 & 96 & 160 \\
\hline 11 & -- & -- & 0.04 & 1.2 & 0.89 & 0.100 & 0.015 & 110 & 160 \\
\hline 39 & 109 & 16 & $<0.01$ & 1.3 & 0.82 & 0.200 & 0.030 & 120 & 160 \\
\hline 34 & 109 & 17 & $<0.01$ & 0.88 & 0.98 & 0.240 & 0.028 & 110 & 170 \\
\hline 34 & 93 & $<19$ & $<0.01$ & 1.3 & 1.20 & 0.220 & 0.023 & 100 & 140 \\
\hline 75 & -- & -- & $<0.01$ & 1.4 & 1.10 & 0.400 & 0.023 & 81 & 110 \\
\hline 45 & 100 & 16 & $<0.01$ & 1.0 & 0.87 & 0.240 & 0.025 & 95 & 130 \\
\hline 40 & 150 & 17 & $<0.01$ & 1.0 & 0.99 & 0.230 & 0.036 & 110 & 170 \\
\hline 37 & 123 & 18 & 0.05 & 1.2 & 0.67 & 0.230 & 0.005 & 110 & 180 \\
\hline 37 & 107 & 19 & 0.04 & 1.6 & 0.70 & 0.230 & 0.028 & 110 & 210 \\
\hline 32 & 131 & 29 & 0.02 & 1.3 & 0.52 & 0.050 & 0.019 & 120 & 180 \\
\hline 33 & 99 & 18 & $<0.01$ & 1.2 & 0.62 & 0.240 & 0.027 & 110 & 180 \\
\hline 37 & 102 & 21 & $<0.01$ & 1.3 & 0.60 & 0.240 & 0.019 & 110 & 190 \\
\hline 37 & 125 & 26 & 0.02 & 0.65 & 0.71 & 0.240 & 0.034 & 100 & 170 \\
\hline 55 & -- & -- & 0.03 & 0.74 & 0.88 & 0.280 & 0.035 & 82 & 140 \\
\hline 34 & 118 & 23 & 0.04 & 0.63 & 0.87 & 0.250 & 0.037 & 85 & 160 \\
\hline 45 & 102 & 20 & 0.05 & 0.59 & 0.73 & 0.240 & 0.031 & 82 & 140 \\
\hline 38 & 102 & 18 & $<0.01$ & 1.3 & 0.88 & 0.290 & 0.043 & 85 & 150 \\
\hline 34 & 92 & 16 & 0.02 & 1.2 & 0.75 & 0.270 & 0.031 & 96 & 170 \\
\hline 18 & -- & -- & 0.10 & 1.2 & 0.71 & 0.170 & 0.016 & 110 & 110 \\
\hline 33 & 98 & 20 & $<0.01$ & 1.2 & 0.62 & 0.200 & 0.034 & 110 & 200 \\
\hline 35 & 135 & 24 & $<0.01$ & 1.4 & 0.50 & 0.210 & 0.028 & 95 & 200 \\
\hline 38 & 142 & 25 & $<0.01$ & 1.3 & 0.52 & 0.230 & 0.018 & 110 & 190 \\
\hline 45 & 157 & 25 & 0.02 & 1.3 & 0.70 & 0.240 & 0.016 & 89 & 160 \\
\hline 40 & 146 & 25 & 0.02 & 1.2 & 0.66 & 0.270 & 0.020 & 90 & 150 \\
\hline 34 & 156 & 9 & 0.02 & 1.3 & 0.54 & 0.230 & 0.028 & 110 & 180 \\
\hline 31 & -- & -- & $<0.01$ & 1.3 & 0.43 & 0.240 & 0.020 & 110 & 200 \\
\hline 37 & 24 & $<24$ & 0.05 & 1.2 & 0.45 & 0.260 & 0.027 & 110 & 190 \\
\hline 38 & -- & -- & 0.02 & 0.93 & 0.58 & 0.180 & 0.031 & 110 & 200 \\
\hline 29 & -- & -- & 0.01 & 1.1 & 0.68 & 0.200 & 0.030 & 110 & 200 \\
\hline 35 & -- & -- & 0.03 & 1.3 & 0.83 & 0.210 & 0.021 & 94 & 110 \\
\hline 30 & 81 & 24 & 0.02 & 1.2 & 0.55 & 0.220 & 0.022 & 99 & 170 \\
\hline 30 & 71 & 19 & $<0.01$ & 1.2 & 0.70 & 0.230 & 0.037 & 110 & 170 \\
\hline 36 & 89 & 23 & $<0.01$ & 1.3 & 0.77 & 0.250 & 0.038 & 110 & 170 \\
\hline 40 & 110 & 26 & $<0.01$ & 1.2 & 0.80 & 0.350 & 0.038 & 110 & 120 \\
\hline 5.5 & -- & -- & 0.08 & 0.67 & 0.57 & 0.065 & 0.021 & 99 & 160 \\
\hline 30 & 45 & 9 & 0.15 & 0.91 & 0.70 & 0.160 & 0.013 & 84 & 97 \\
\hline 37 & -- & -- & 0.03 & 1.6 & 0.52 & 0.240 & - & 110 & 150 \\
\hline 8.9 & -- & -- & 0.02 & 0.59 & 0.66 & 0.065 & 0.021 & 89 & 160 \\
\hline 9.2 & -- & -- & $<0.01$ & 1.1 & 0.49 & 0.190 & 0.011 & 100 & 78 \\
\hline 29 & -- & -- & $<0.01$ & 1.0 & 0.51 & 0.180 & 0.022 & 120 & 200 \\
\hline 31 & $\cdots$ & -- & 0.03 & 1.1 & 0.42 & 0.180 & 0.012 & 120 & 210 \\
\hline 30 & 95 & 16 & 0.02 & 1.2 & 0.67 & 0.190 & 0.013 & 100 & 210 \\
\hline 32 & $\cdots$ & -- & 0.03 & 0.92 & 0.66 & 0.160 & 0.012 & 110 & 240 \\
\hline 32 & 86 & 14 & 0.02 & 0.88 & 0.74 & 0.180 & 0.016 & 110 & 210 \\
\hline 33 & 88 & 16 & 0.02 & 1.0 & 0.73 & 0.100 & 0.027 & 100 & 200 \\
\hline 32 & -- & -- & 0.03 & 1.1 & 0.67 & 0.230 & 0.032 & 110 & 170 \\
\hline 36 & -- & -- & 0.03 & 1.3 & 0.80 & 0.270 & 0.023 & 100 & 170 \\
\hline 36 & 90 & 16 & 0.03 & 1.2 & 0.79 & 0.210 & 0.032 & 110 & 170 \\
\hline 50 & 134 & 21 & 0.04 & 1.4 & 0.95 & 0.260 & 0.020 & 91 & 150 \\
\hline ง 25 & $\cdots$ & -- & 0.12 & 0.90 & 0.82 & 0.230 & 0.029 & 100 & 170 \\
\hline 30 & -- & -- & 0.01 & 0.75 & 0.81 & 0.200 & 0.027 & 110 & 180 \\
\hline 30 & 73 & 14 & $<0.01$ & 0.90 & 0.75 & 0.160 & 0.024 & 110 & 190 \\
\hline 40 & 96 & 16 & 0.03 & 1.1 & 0.79 & 0.220 & 0.020 & 98 & 160 \\
\hline 50 & 169 & 25 & $<0.01$ & 1.6 & 0.67 & 0.250 & 0.018 & 60 & 110 \\
\hline 22 & -- & -- & 0.01 & 0.97 & 0.80 & 0.160 & 0.026 & 98 & 170 \\
\hline
\end{tabular}


0423205025 Irondequoit Creek at Empire Boulevard, Rochester, N.Y.

\section{WATER-QUALITY RECORDS}

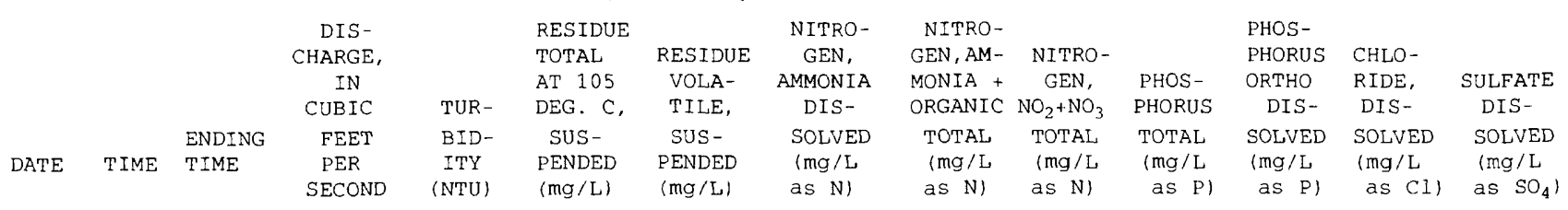

OCT

$\begin{array}{rrrr}03-05 & 1015 & 0015 & 44 \\ 05-07 & 0215 & 0915 & 57 \\ 10-15 & 1030 & 0030 & 67 \\ 15-16 & 1040 & 0540 & 79 \\ 17-19 & 1040 & 0940 & 53 \\ \text { NOV } & & & \\ 08-11 & 1045 & 2145 & 42 \\ 12-14 & 1115 & 1015 & 71 \\ 21-24 & 1045 & 0945 & 66 \\ 25 \ldots & 1115 & -- & 58 \\ 25-25 & 1115 & 1815 & 66 \\ 25-25 & 1915 & 2215 & 98 \\ 25-27 & 2315 & 1015 & 87 \\ 29-29 & 0300 & 1700 & 108 \\ \text { NOV } 29- & & & \\ \text { DEC } 02 & 1800 & 1000 & 95 \\ 02-03 & 1100 & 0400 & 61 \\ 03-03 & 0500 & 1600 & 218 \\ 03-05 & 1700 & 1000 & 155 \\ 05-07 & 1225 & 1525 & 71 \\ 07-08 & 1625 & 0325 & 90 \\ 08-09 & 0425 & 1125 & 86 \\ 09-12 & 1105 & 1005 & 77 \\ 12-16 & 1110 & 1010 & 66 \\ 16-19 & 1140 & 1040 & 46 \\ 19-22 & 1120 & 0620 & 53 \\ 23 \ldots & 1120 & -- & 62 \\ 24-26 & 1555 & 1155 & 56 \\ 26-29 & 1235 & 0335 & 55 \\ 29-30 & 0435 & 1135 & 256\end{array}$

$\begin{array}{rrrr}29-30 & 0435 & 1135 & 256\end{array}$

JAN $02 \quad 1230 \quad 1130 \quad 131$

$\begin{array}{lllr}02-06 & 1040 & 0940 & 82\end{array}$

$\begin{array}{llll}06-09 & 1035 & 0935 & 81 \\ 09-13 & 1210 & 1110 & 76\end{array}$

$\begin{array}{rrrr}09-13 & 1210 & 1110 & 76 \\ 13-14 & 1015 & -- & 80\end{array}$

$\begin{array}{rrrr}14-17 & 0830 & 0730 & 103\end{array}$

17-21 $1225 \quad 1025 \quad 69$

$\begin{array}{rrrr}21-23 & 1055 & 0955 & 67 \\ 23-24 & 1105 & 0205 & 147\end{array}$

$\begin{array}{llll}24-27 & 0305 & 1005 & 157\end{array}$

FEB

03-06 $1040 \quad 0940 \quad 62$

$\begin{array}{llll}06-10 & 1030 & 0930 & 59 \\ 10-14 & 1035 & 0935 & 58\end{array}$

$\begin{array}{llll}14-15 & 1015 & 1715 & 72\end{array}$

$\begin{array}{llll}15-16 & 1815 & 1715 & 151\end{array}$

16-17 $1815 \quad 1915 \quad 155$

$\begin{array}{llll}18-19 & 1130 & 1430 & 135\end{array}$

$\begin{array}{rrrr}19-20 & 1530 & 0930 & 160 \\ 20 \ldots & 1000 & -- & 170\end{array}$

$\begin{array}{rrrr}20-22 & 1015 & 2115 & 148\end{array}$

$\begin{array}{llll}22-24 & 2215 & 0915 & 176 \\ 24-27 & 1040 & 0940 & 168\end{array}$ MAR

$\begin{array}{llll}27-28 & 1000 & 1700 & 141\end{array}$

$\begin{array}{llll}02-05 & 1035 & 0935 & 110\end{array}$

$\begin{array}{llll}05-07 & 1005 & 0105 & 106\end{array}$

07-08 $0205 \quad 0105 \quad 191$

08-09 $0205 \quad 0905 \quad 284$

09-10 $1115 \quad 1915 \quad 225$

$10-12 \quad 2015 \quad 1015 \quad 133$

\section{WATER YEAR OCTOBER 1991 TO SEPTEMBER 1992}

\begin{tabular}{|c|c|c|c|c|c|c|c|c|c|}
\hline 26 & -- & -- & 0.01 & 1.0 & 0.90 & 0.210 & 0.025 & 110 & 200 \\
\hline 28 & -- & $\cdots$ & 0.01 & 1.1 & 0.86 & 0.210 & 0.020 & 120 & 200 \\
\hline 25 & - & $\ldots$ & 0.01 & 0.96 & 0.85 & 0.170 & 0.020 & 100 & 200 \\
\hline 18 & -- & -- & 0.01 & 1.1 & 0.72 & 0.110 & 0.014 & 110 & 210 \\
\hline 16 & -- & -- & 0.01 & 0.51 & 0.65 & 0.130 & 0.014 & 100 & \\
\hline 4.4 & -- & $\cdots$ & 0.04 & 0.43 & 0.72 & 0.045 & 0.007 & 110 & 220 \\
\hline 7.5 & -- & $\cdots$ & 0.10 & 0.61 & 0.74 & 0.065 & 0.009 & 150 & 160 \\
\hline 8.9 & - & -- & -- & 0.66 & 0.80 & 0.065 & 0.014 & 130 & 200 \\
\hline 7.9 & -- & -- & -- & 0.60 & 0.79 & 0.040 & 0.014 & 130 & 180 \\
\hline 13 & -- & -- & 0.05 & 0.74 & 0.84 & 0.050 & 0.008 & 120 & 190 \\
\hline 12 & -- & - & 0.02 & 0.67 & 0.84 & 0.065 & 0.010 & 120 & 200 \\
\hline 9.2 & -- & -- & 0.02 & 0.54 & 0.68 & 0.070 & 0.011 & 130 & 170 \\
\hline-- & -- & -- & -- & 0.63 & 0.42 & 0.095 & 0.012 & 150 & 150 \\
\hline 12 & -- & - &.- & 0.74 & 0.85 & 0.080 & 0.013 & 120 & 160 \\
\hline 12 & -- & -- & 0.05 & 0.50 & 0.91 & 0.055 & 0.010 & 130 & 190 \\
\hline 26 & -- & -- & 0.05 & 0.82 & 0.82 & 0.140 & 0.015 & 210 & 150 \\
\hline 22 & -- & -- & 0.04 & 0.69 & 0.78 & 0.120 & 0.018 & 200 & 140 \\
\hline 15 & -- & -- & 0.07 & 0.66 & 1.00 & 0.065 & 0.011 & 190 & \\
\hline 14 & -- & -- & 0.07 & 0.60 & 1.10 & 0.070 & 0.015 & 260 & \\
\hline 11 & -- & -- & 0.06 & 0.66 & 1.10 & 0.070 & 0.014 & 260 & \\
\hline 11 & -- & -- & 0.03 & 0.74 & 1.00 & 0.080 & 0.018 & 180 & 190 \\
\hline 5.1 & -- & -- & 0.02 & 0.46 & 0.92 & 0.025 & 0.009 & 130 & 190 \\
\hline 6.7 & -- & -- & 0.04 & 0.86 & 1.20 & 0.065 & 0.012 & 170 & 210 \\
\hline 6.7 & -- & -- & 0.06 & 0.60 & 1.20 & 0.045 & 0.011 & 210 & 220 \\
\hline 4.6 & -- & -- & 0.17 & 0.66 & 1.10 & 0.050 & 0.011 & 280 & 210 \\
\hline 5.5 & -- & -- & 0.06 & 0.55 & 1.10 & 0.040 & 0.012 & 230 & 190 \\
\hline 3.9 & -- & -- & 0.05 & 0.75 & 1.10 & 0.045 & 0.015 & 200 & \\
\hline 43 & 152 & 43 & 0.06 & 1.3 & 0.91 & 0.230 & 0.022 & 210 & 120 \\
\hline 14 & -- & -- & 0.05 & 0.90 & 1.30 & 0.085 & 0.016 & 210 & 130 \\
\hline 7.6 & - & $\cdots$ & 0.06 & 0.68 & 1.40 & 0.050 & 0.012 & 170 & 180 \\
\hline 6.5 & -- & -- & 0.05 & 0.60 & 1.40 & 0.035 & 0.011 & 160 & 190 \\
\hline 2.6 & -- & -- & $<0.01$ & 0.34 & 1.40 & 0.020 & 0.002 & 200 & 190 \\
\hline 5.7 & -- & -- & 0.05 & 0.68 & 1.40 & 0.040 & 0.010 & 240 & 180 \\
\hline 8.2 & -- & -- & 0.04 & 0.53 & 1.60 & 0.055 & 0.012 & 180 & 160 \\
\hline 3.2 & -- & -- & 0.17 & 0.57 & 1.70 & 0.035 & 0.010 & 200 & 200 \\
\hline 3.3 & -- & -- & 0.06 & 0.49 & 1.60 & 0.030 & 0.010 & 230 & 210 \\
\hline 12 & -- & -- & 0.07 & 0.74 & 1.40 & 0.080 & 0.010 & 350 & 170 \\
\hline 8.0 & -- & -- & 0.05 & 0.60 & 1.50 & 0.065 & 0.011 & 320 & 150 \\
\hline 3.7 & -- & -- & 0.05 & 0.52 & 1.60 & 0.030 & 0.010 & 200 & 190 \\
\hline 3.7 & -- & -- & 0.03 & 0.58 & 1.50 & 0.030 & 0.009 & 180 & 200 \\
\hline 2.5 & -- & -- & 0.04 & 0.45 & 1.50 & 0.025 & 0.008 & 240 & 210 \\
\hline 4.1 & - & $\cdots$ & 0.04 & 0.57 & 1.40 & 0.035 & 0.007 & 250 & 220 \\
\hline 3.7 & $<23$ & $<23$ & 0.05 & 0.43 & 1.20 & 0.030 & 0.009 & 270 & 200 \\
\hline 21 & 28 & $<21$ & 0.09 & 0.70 & 1.30 & 0.075 & 0.008 & 390 & 240 \\
\hline 18 & 33 & $<21$ & 0.08 & 0.77 & 1.30 & 0.085 & 0.009 & 2.80 & 130 \\
\hline 18 & -- & -- & 0.05 & 0.77 & 1.50 & 0.085 & 0.008 & 240 & 120 \\
\hline 23 & -- & -- & 0.05 & 0.86 & 1.50 & 0.095 & 0.009 & 230 & 120 \\
\hline- & -- & -- & - & -- & -- & -- & -- & -. & \\
\hline 9.0 & -- & -- & 0.04 & 0.64 & 1.70 & 0.060 & 0.009 & 190 & 140 \\
\hline 10 & -- & -- & 0.04 & 0.73 & 1.70 & 0.065 & 0.009 & 200 & 140 \\
\hline 11 & -- & -- & 0.03 & 0.59 & 1.90 & 0.050 & 0.009 & 180 & 130 \\
\hline 3.9 & -- & -- & 0.04 & 1.0 & 2.00 & 0.035 & 0.008 & 150 & 150 \\
\hline 3.0 & -- & -- & -- & 0.74 & 1.60 & 0.060 & 0.006 & 180 & 170 \\
\hline 4.2 & -- & -- & 0.03 & 0.55 & 1.30 & 0.030 & 0.006 & 140 & 160 \\
\hline 17 & -- & -- & 0.03 & 0.61 & 1.20 & 0.080 & 0.006 & 170 & 140 \\
\hline 31 & 84 & 12 & 0.03 & 0.90 & 1.40 & 0.130 & 0.010 & 150 & 110 \\
\hline 17 & -- & -- & 0.04 & 1.0 & 1.70 & 0.130 & 0.008 & 140 & 120 \\
\hline 17 & -- & -- & 0.04 & 0.84 & 1.50 & 0.130 & 0.007 & 140 & 130 \\
\hline
\end{tabular}


Surface-Water Stations

\section{A. Discharge and water quality}

0423205025 Irondequoit Creek at Empire Boulevard, Rochester, N.Y. 2. WATER-QUALITY RECORDS

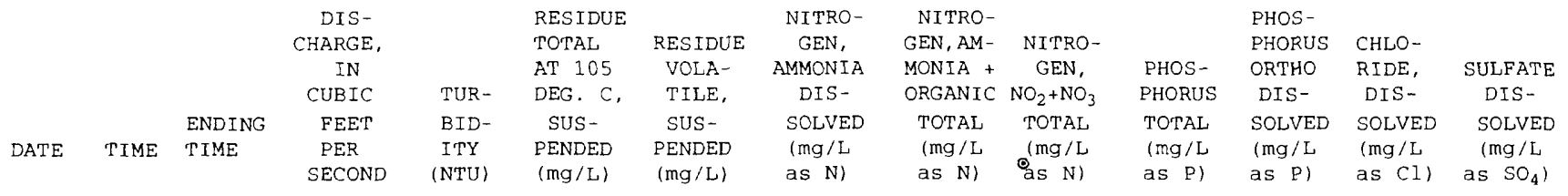

\begin{tabular}{|c|c|c|c|}
\hline \multicolumn{4}{|c|}{ MAR-cont inued } \\
\hline $12-16$ & 1135 & 0930 & 139 \\
\hline $16-19$ & 1020 & 0920 & \\
\hline $19-23$ & 1005 & 0905 & \\
\hline $23-25$ & 1010 & 1510 & \\
\hline $25-26$ & 1610 & 0910 & \\
\hline $26-28$ & 1000 & 0400 & \\
\hline $28-30$ & 0500 & 0900 & \\
\hline \multicolumn{4}{|l|}{ MAR $30-$} \\
\hline APR 02 & 1015 & 0915 & \\
\hline $02-06$ & 1010 & 0910 & \\
\hline $06-09$ & 1010 & 0910 & \\
\hline $09-11$ & 1005 & 0905 & \\
\hline $11-12$ & 1005 & 0905 & \\
\hline $12-13$ & 1005 & 0905 & \\
\hline $13-16$ & 1030 & 0930 & \\
\hline $16-17$ & 1005 & 1705 & 48 \\
\hline $17-19$ & 1805 & 0505 & \\
\hline $19-20$ & 0605 & 0905 & \\
\hline $20-21$ & 1020 & 1520 & \\
\hline $21-22$ & 1620 & 1220 & \\
\hline $22-23$ & 1320 & 0920 & \\
\hline $23-27$ & 1040 & 0940 & \\
\hline $27-30$ & 0925 & 0855 & \\
\hline \multicolumn{4}{|l|}{ APR $30-$} \\
\hline MAY 02 & 1020 & 1720 & \\
\hline $02-04$ & 1820 & 0920 & \\
\hline $04-07$ & 1025 & 0925 & 2 \\
\hline $07-11$ & 1045 & 0945 & \\
\hline $11-14$ & 1020 & 0920 & \\
\hline $14-18$ & 1005 & 0105 & \\
\hline $18-18$ & 0205 & 0905 & 1 \\
\hline $18-20$ & 1020 & 1045 & \\
\hline $26-26$ & 0830 & 1630 & \\
\hline $28-30$ & 1615 & 1115 & \\
\hline $31-31$ & 1215 & 2315 & \\
\hline \multicolumn{4}{|l|}{ JUN } \\
\hline $01-04$ & 1030 & 0630 & \\
\hline $04-07$ & 1025 & 0125 & \\
\hline $07-08$ & 0225 & 0125 & 14 \\
\hline $08-11$ & 1035 & 0935 & \\
\hline $11-15$ & 1020 & 0920 & \\
\hline $15-18$ & 1020 & 0920 & \\
\hline $18-19$ & 1005 & 0905 & \\
\hline $19-20$ & 1005 & 0505 & 1 \\
\hline $20-22$ & 0605 & 0905 & \\
\hline $22-24$ & 1030 & 0630 & \\
\hline $24-25$ & 0730 & 0930 & 11 \\
\hline $25-27$ & 1020 & 0520 & \\
\hline $27-28$ & 0620 & 0520 & \\
\hline $28-29$ & 0520 & 0920 & \\
\hline \multicolumn{4}{|l|}{ JUN $29-$} \\
\hline JUL 02 & 1010 & 0910 & \\
\hline $02-03$ & 1015 & 0915 & \\
\hline $03-04$ & 1015 & 0515 & 10 \\
\hline $04-06$ & 0615 & 0915 & \\
\hline $06-08$ & 1105 & 1905 & \\
\hline $08-09$ & 2005 & 1005 & 1 \\
\hline $09-12$ & 1005 & 1705 & \\
\hline $12-13$ & 1805 & 0905 & \\
\hline $13-14$ & 1035 & 0635 & 1 \\
\hline $14-15$ & 0735 & 1835 & 2 \\
\hline $15-16$ & 1935 & 0935 & \\
\hline $16-17$ & 1100 & 1000 & \\
\hline
\end{tabular}

WATER YEAR OCTOBER 1991 TO SEPTEMBER 1992-continued

\begin{tabular}{|c|c|c|c|c|c|c|c|c|c|}
\hline 4.4 & - & -- & 0.04 & 0.82 & 1.60 & 0.045 & 0.007 & 210 & 150 \\
\hline 5.9 & -- & -- & 0.04 & 0.69 & 1.70 & 0.035 & 0.006 & 240 & 150 \\
\hline 3.6 & -- & -- & 0.02 & 0.68 & 1.40 & 0.030 & 0.005 & 220 & \\
\hline 2.7 & -- & -- & 0.03 & 0.69 & 1.50 & 0.030 & 0.004 & 310 & \\
\hline 12 & -- & -- & 0.03 & 0.72 & 1.30 & 0.070 & 0.003 & 260 & \\
\hline 90 & 367 & 37 & 0.04 & 1.8 & 1.30 & 0.460 & 0.008 & 140 & \\
\hline 55 & 125 & 16 & 0.04 & 1.2 & 1.60 & 0.200 & 0.016 & 120 & \\
\hline 25 & - - & $\cdots$ & 0.03 & 0.82 & 1.40 & 0.100 & 0.006 & 140 & \\
\hline 15 & + & -- & 0.03 & 0.76 & 1.60 & 0.065 & 0.009 & 120 & \\
\hline 29 & -- & -- & 0.03 & 0.95 & 1.50 & 0.150 & 0.006 & 130 & 130 \\
\hline 16 & -- & -- & 0.04 & 0.93 & 1.40 & 0.100 & 0.006 & 140 & 14 \\
\hline 40 & 124 & 14 & 0.06 & 1.2 & 1.40 & 0.170 & 0.008 & 130 & \\
\hline 50 & 115 & 14 & 0.05 & 1.0 & 1.20 & 0.190 & 0.010 & 110 & \\
\hline 19 & -- & -- & 0.02 & 0.72 & 1.20 & 0.095 & 0.008 & 120 & \\
\hline 50 & 170 & 21 & -- & 0.40 & 1.30 & 0.210 & 0.009 & 98 & \\
\hline 36 & 92 & 12 & -- & 0.91 & 1.30 & 0.140 & 0.011 & 88 & \\
\hline 25 & -- & -- & -- & 0.70 & 1.30 & 0.130 & 0.010 & 91 & \\
\hline 25 & 81 & 11 & 0.03 & 0.84 & 1.20 & 0.120 & 0.007 & 100 & \\
\hline 34 & 103 & 15 & 0.02 & 1.1 & 1.20 & 0.190 & 0.007 & 110 & \\
\hline 34 & 97 & 13 & 0.02 & 0.90 & 1.10 & 0.180 & 0.011 & 100 & \\
\hline 25 & $\ldots$ & -- & 0.03 & 0.84 & 1.20 & 0.140 & 0.011 & 100 & \\
\hline 20 & -- & -- & 0.02 & 0.89 & 1.10 & 0.110 & 0.016 & 110 & \\
\hline 21 & $\ldots$ & -- & 0.04 & 0.85 & 1.20 & 0.130 & 0.010 & 110 & 11 \\
\hline 20 & 256 & 3 & 0.06 & 1.6 & 0.90 & 0.360 & 0.013 & 70 & \\
\hline 60 & 107 & 16 & 0.03 & 1.2 & 0.92 & 0.230 & 0.008 & 85 & \\
\hline 26 & -- & -- & 0.03 & 0.94 & 0.87 & 0.140 & 0.014 & 100 & 16 \\
\hline 21 & -- & -- & 0.03 & 0.94 & 0.80 & 0.140 & 0.010 & 110 & 13 \\
\hline 22 & -- & -- & 0.04 & 0.84 & 0.92 & 0.120 & 0.008 & 110 & 14 \\
\hline 32 & 82 & 16 & 0.04 & 1.1 & 0.98 & 0.150 & 0.008 & 120 & 15 \\
\hline 34 & -- & -- & 0.05 & 1.3 & 0.94 & 0.160 & 0.013 & 99 & 14 \\
\hline 20 & - & -- & 0.09 & 1.1 & 1.00 & 0.120 & 0.014 & 120 & 16 \\
\hline 6.0 & -- & -- & 0.04 & 0.70 & 0.99 & 0.056 & 0.009 & 110 & 15 \\
\hline 6.1 & -- & -- & 0.04 & 0.58 & 1.00 & 0.055 & 0.010 & 100 & \\
\hline 31 & 87 & $<13$ & -- & 1.1 & -- & 0.175 & 0.015 & 98 & \\
\hline 31 & 59 & 11 & 0.04 & 1.1 & 0.98 & 0.190 & 0.019 & 110 & 14 \\
\hline 34 & 84 & 13 & 0.03 & 1.3 & 1.00 & 0.210 & 0.019 & 110 & 14 \\
\hline 34 & -- & -- & 0.02 & 1.3 & 0.98 & 0.190 & 0.024 & 110 & 13 \\
\hline 30 & 76 & 16 & 0.00 & 1.3 & 0.76 & 0.170 & 0.020 & 120 & 15 \\
\hline 29 & -- & -- & $<0.01$ & 1.3 & 0.73 & 0.160 & 0.015 & 130 & 17 \\
\hline 31 & 78 & $<16$ & 0.03 & 1.3 & 0.78 & 0.180 & 0.020 & 130 & 19 \\
\hline 35 & 89 & 12 & 0.03 & 1.4 & 0.92 & 0.180 & 0.021 & 120 & 17 \\
\hline 40 & 94 & 13 & 0.04 & 1.2 & 1.00 & 0.210 & 0.023 & 89 & 14 \\
\hline 21 & -- & -- & 0.02 & 0.92 & 1.00 & 0.135 & 0.026 & 110 & 16 \\
\hline 38 & -- & -- & 0.03 & 1.1 & 1.10 & 0.170 & 0.022 & 110 & 16 \\
\hline 32 & 67 & 12 & 0.02 & 1.1 & 1.10 & 0.085 & 0.025 & 100 & 14 \\
\hline 29 & 74 & 12 & 0.01 & 1.2 & 0.97 & 0.140 & 0.025 & 110 & 17 \\
\hline 33 & 74 & 12 & 0.01 & 0.87 & 0.91 & 0.140 & 0.021 & 98 & \\
\hline 27 & -- & -- & $<0.01$ & 1.2 & 0.85 & 0.130 & 0.021 & 110 & \\
\hline 23 & -- & - & 0.02 & 1.3 & 0.75 & 0.150 & 0.017 & 100 & \\
\hline 32 & 90 & 14 & 0.02 & 1.2 & 0.78 & 0.160 & 0.018 & 120 & 17 \\
\hline 35 & -- & -- & 0.02 & 1.2 & 0.87 & 0.220 & 0.018 & 94 & \\
\hline 26 & -- & -- & - & 0.85 & -- & 0.140 & 0.022 & 110 & 16 \\
\hline 34 & 91 & 15 & -- & 1.0 & -- & 0.150 & 0.020 & 110 & \\
\hline 45 & 100 & 13 & 0.02 & 1.1 & 0.85 & 0.150 & 0.024 & 88 & 15 \\
\hline 40 & 101 & 14 & 0.02 & 1.2 & 0.93 & 0.200 & 0.026 & 110 & \\
\hline 45 & 116 & 15 & 0.03 & 1.1 & -- & 0.220 & 0.025 & 83 & \\
\hline 80 & 214 & 29 & 0.02 & 1.5 & -- & 0.340 & 0.027 & 75 & \\
\hline 50 & -- & -- & 0.03 & 2.4 & -- & 0.530 & 0.031 & 52 & \\
\hline 80 & 197 & 32 & 0.03 & 1.8 & 2.00 & 0.300 & 0.036 & 67 & \\
\hline
\end{tabular}


0423205025 Irondequoit Creek at Empire Boulevard, Rochester, N.Y.

\section{WATER-QUALITY RECORDS}

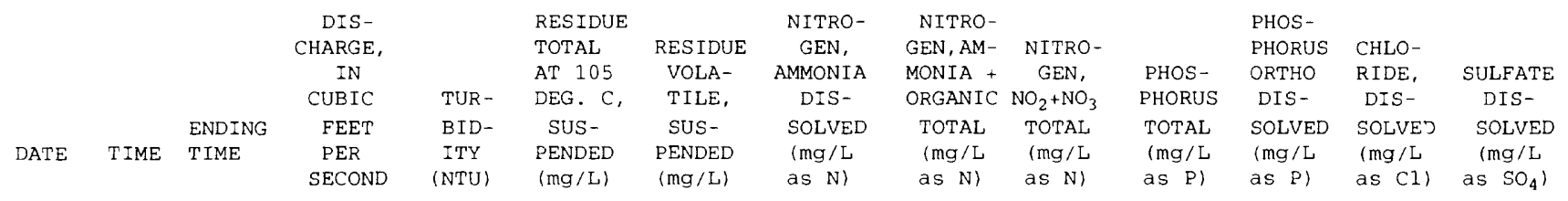

JUL-cont inued

$17-1801100$

$20-23$

$23-27$

$27-29$

$29 \ldots$

$29 \ldots$

$29 \ldots$

$29 .$.

$29 \ldots$

29.

29.

$29 \ldots$

29.

$29 .$.

$29 \ldots$

$29-31$

JUL $31-$

AUG 01

$01-03$

03-04

$04-06$

$06-08$

08-09

$09-10$

$10-13$

$13-17$

$17-20$

$20-24$

$24-24$

$24-25$

$25-27$

$27-27$

27-28

28-31

AUG 31 -

SEP 0

$03-03$
$03-04$

04-08

$08-10$

10-14

$14-17$

$17-18$

18-19

$19-21$

21-2

23-24

$24-26$

$25 \quad 27$

$27-28$

SEP 28-

OCT 01

1020

1000

1005
1010

1015

1020
1025

1030

1035
1040
1045

1045

1050

1055

1520

\section{1020}

0945

$1015 \quad 1515$

16150915

$1050 \quad 2150$

$2250 \quad 0950$

$\begin{array}{ll}1015 & 1315 \\ 1415 & 0415\end{array}$

$\begin{array}{ll}1415 & 0415 \\ 0515 & 0915\end{array}$

186

$\begin{array}{lll}1010 & 0910 & 86\end{array}$

$\begin{array}{lll}1055 & 0955 & 74\end{array}$

$1015 \quad 2115$

$2215 \quad 1215$

13150915

$1005 \quad 1305$

$1405 \quad 1305$

377

$1050 \quad 0550 \quad 194$

$0650 \quad 2150 \quad 229$

$2250 \quad 0850 \quad 295$

$0920 \quad 0820 \quad 146$

$\begin{array}{lll}1140 & 1040 & 88 \\ 1200 & 0900 & 73\end{array}$

$1000 \quad 0900 \quad 63$

$\begin{array}{lll}1005 & 1705 & 59\end{array}$

$1805 \quad 1305 \quad 133$

$1405 \quad 0905 \quad 110$

$\begin{array}{lll}1015 & 0315 & 199\end{array}$

$04150915 \quad 192$

$0955 \quad 0855 \quad 102$

$0955 \quad 2055 \quad 185$

$2155 \quad 0855 \quad 225$

$1005 \quad 0905$
$1000 \quad 0900 \quad 108$

WATER YEAR OCTOBER 1991 TO SEPTEMBER 1992-continued

\begin{tabular}{|c|c|c|c|c|c|c|c|c|}
\hline 329 & 40 & 0.02 & 1.9 & 1.50 & 0.460 & 0.034 & 59 & 62 \\
\hline 203 & 27 & 0.02 & 1.5 & 1.20 & 0.290 & 0.033 & 56 & 64 \\
\hline 91 & 13 & 0.03 & 1.2 & 1.00 & -- & 0.034 & 87 & 95 \\
\hline 60 & 10 & 0.03 & 0.92 & 1.10 & 0.220 & 0.032 & 92 & 110 \\
\hline - & $\cdots$ & 0.01 & 0.80 & 1.00 & 0.160 & 0.031 & 100 & 130 \\
\hline-- & -- & -- & 0.40 & 1.00 & 0.050 & -- & -- & - \\
\hline-- & -- & -- & 0.50 & 1.00 & 0.060 & -- & -- & \\
\hline-- & -- & -- & 0.40 & 1.00 & 0.050 & -- & -- & - \\
\hline -- & -- & -- & 0.40 & 1.00 & 0.050 & -- & - - & \\
\hline -. & -- & - & 0.50 & 1.10 & 0.040 & -- & -- & \\
\hline -- & -- & -- & 0.50 & 1.10 & 0.040 & -- & $\ldots$ & \\
\hline -. & -. & -- & 0.50 & 1.10 & 0.040 & -- & -- & \\
\hline -- & -- & -- & 0.50 & 1.10 & 0.040 & -- & -- & - \\
\hline - & -- & -- & 0.50 & 1.00 & 0.040 & - & -- & \\
\hline-- & -- & - & 0.50 & 1.00 & 0.050 & -- & - & - \\
\hline -- & -- & -- & 0.50 & 1.00 & 0.050 & -- & -- & \\
\hline-- & -- & -- & 0.40 & 1.00 & 0.030 & -- & - - & \\
\hline-- & -- & 0.01 & 0.96 & 0.95 & 0.140 & 0.023 & 99 & 130 \\
\hline 108 & 14 & 0.01 & 0.85 & 0.76 & 0.150 & 0.030 & 71 & 87 \\
\hline 97 & 14 & 0.02 & 1.1 & 0.68 & 0.240 & 0.030 & 63 & 71 \\
\hline 263 & 28 & 0.02 & 1.6 & 0.87 & 0.180 & 0.040 & 56 & 59 \\
\hline 146 & 19 & 0.02 & 1.3 & 0.73 & 0.220 & 0.042 & 54 & 69 \\
\hline 74 & 11 & 0.02 & 0.96 & 1.00 & 0.180 & 0.039 & 74 & 72 \\
\hline 76 & 12 & 0.03 & 1.4 & 1.10 & 0.180 & 0.035 & 84 & 88 \\
\hline 86 & 12 & 0.02 & 1.1 & 1.10 & 0.190 & 0.033 & 78 & 91 \\
\hline 64 & 11 & 0.01 & 0.91 & 0.99 & 0.180 & 0.038 & 89 & 110 \\
\hline -- & -- & 0.02 & 1.0 & 1.00 & 0.140 & 0.030 & 98 & 120 \\
\hline -- & -- & 0.01 & 0.84 & 0.95 & 0.140 & 0.026 & 100 & 140 \\
\hline -- & -- & 0.03 & 0.86 & 0.87 & 0.140 & 0.018 & 110 & 160 \\
\hline-- & -- & $<0.01$ & 0.79 & 0.78 & 0.130 & 0.013 & 110 & 160 \\
\hline - & -- & $<0.01$ & 0.79 & 0.77 & 0.130 & 0.013 & 100 & 150 \\
\hline-- & -- & 0.02 & 0.91 & 0.78 & 0.170 & 0.018 & 82 & 120 \\
\hline-- & -- & 0.04 & 1.1 & 0.83 & 0.200 & 0.025 & 99 & 130 \\
\hline 457 & 45 & 0.03 & 2.2 & 0.66 & 0.650 & 0.034 & 53 & 65 \\
\hline 153 & 17 & 0.03 & 1.3 & 0.49 & 0.300 & 0.047 & 44 & 41 \\
\hline -- & -- & 0.02 & 0.91 & 0.90 & 0.185 & 0.046 & 71 & 78 \\
\hline-- & -- & 0.02 & 0.81 & 0.49 & 0.190 & 0.040 & 79 & 98 \\
\hline 101 & 14 & 0.02 & 1.2 & 0.88 & 0.260 & 0.030 & 61 & 68 \\
\hline - - & -- & 0.01 & 0.86 & 0.90 & 0.160 & 0.037 & 93 & 100 \\
\hline-- & -- & 0.02 & 1.2 & 1.00 & 0.170 & 0.029 & 100 & 130 \\
\hline 46 & $<15$ & 0.02 & 0.94 & 1.00 & 0.135 & 0.027 & 110 & 160 \\
\hline-- & -- & 0.01 & 0.84 & 0.92 & 0.110 & 0.012 & 120 & 170 \\
\hline-- & -- & $<0.01$ & 0.88 & 0.83 & 0.130 & 0.010 & 110 & 180 \\
\hline-- & -- & $<0.01$ & 1.1 & 0.44 & 0.150 & 0.014 & 110 & 160 \\
\hline-- & -. & 0.01 & 0.92 & 0.84 & 0.140 & 0.021 & 84 & 140 \\
\hline 72 & 13 & 0.03 & 0.79 & 0.79 & 0.170 & 0.025 & 80 & 110 \\
\hline 69 & 12 & 0.02 & 0.93 & 0.75 & 0.160 & 0.032 & 73 & 96 \\
\hline-- & -- & 0.01 & 0.94 & 0.83 & 0.160 & 0.031 & 87 & 110 \\
\hline-- & -- & 0.02 & 0.91 & 0.46 & 0.180 & 0.028 & 84 & 120 \\
\hline 101 & 17 & 0.01 & 1.0 & 0.75 & 0.220 & 0.030 & 68 & 99 \\
\hline-- & -. & 0.01 & 0.98 & 0.84 & 0.170 & 0.029 & 84 & 110 \\
\hline
\end{tabular}

WATER YEAR OCTOBER 1992 TO SEPTEMBER 1993

$\begin{array}{rrrr}01-05 & 1025 & 0910 & 88 \\ 05-09 & 0940 & 0840 & 73 \\ 09-10 & 0945 & 0845 & 256 \\ 10-13 & 0945 & 0845 & 167 \\ 13-15 & 1100 & 1000 & 102 \\ 15-16 & 1505 & 0605 & 149 \\ 16-19 & 0705 & 1005 & 122\end{array}$
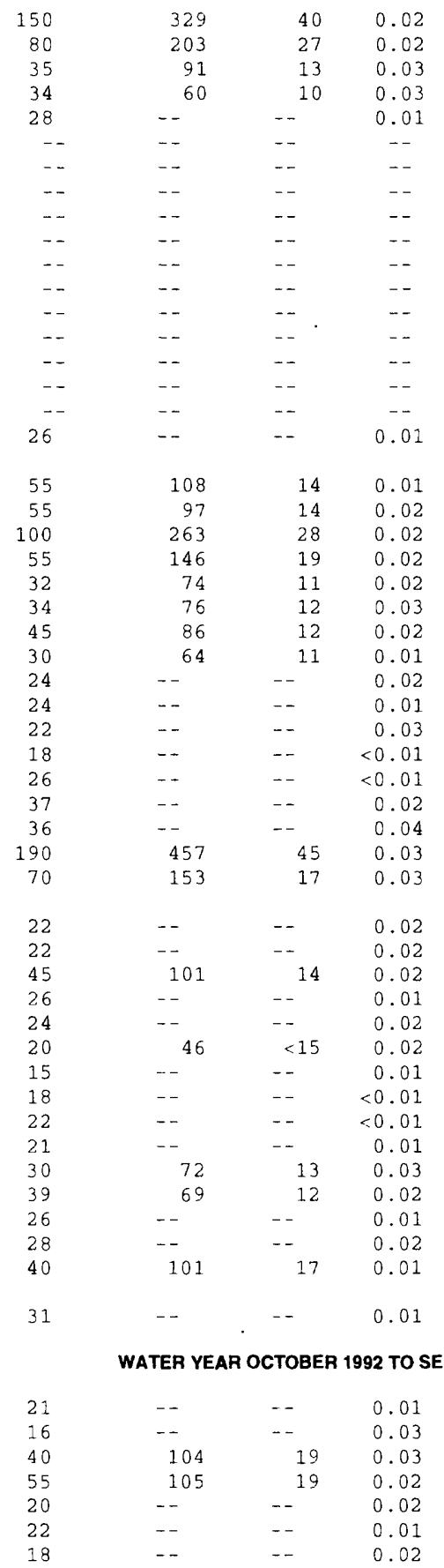

0.130
0.095
0.190
0.200
0.130
0.150
0.150

0.024
0.020
0.024
0.027
0.023
0.021
0.020
62 130 


\section{Surface-Water Stations}

\section{A. Discharge and water quality}

0423205025 Irondequoit Creek at Empire Boulevard, Rochester, N.Y.

2. WATER-QUALITY RECORDS

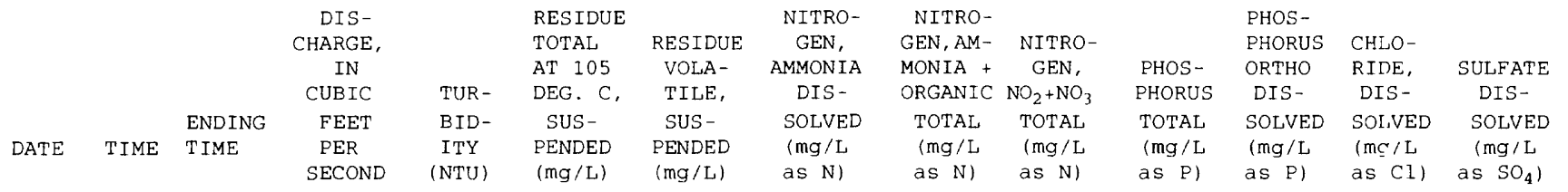

OCT-cont inued

\begin{tabular}{|c|c|c|c|}
\hline $19-22$ & 1115 & 1015 & 98 \\
\hline $22-24$ & 1050 & 0450 & 93 \\
\hline $24-26$ & 0550 & 0950 & 183 \\
\hline $26-29$ & 1115 & 0915 & 135 \\
\hline \multicolumn{4}{|l|}{ OCT $29-$} \\
\hline NOV 02 & 0945 & 0845 & 98 \\
\hline $02-03$ & 1010 & 2110 & 316 \\
\hline $03-05$ & -2210 & 0910 & 336 \\
\hline $05-09$ & 1030 & 0930 & 228 \\
\hline $09-12$ & 1045 & 0945 & 144 \\
\hline $12-13$ & 1005 & 1705 & 162 \\
\hline $13-16$ & 1805 & 0905 & 139 \\
\hline $16-19$ & 1035 & 0935 & 92 \\
\hline $19-22$ & 1025 & 1325 & 131 \\
\hline $22-25$ & 1425 & 0925 & 352 \\
\hline $23-25$ & 1030 & 0930 & 387 \\
\hline $25-30$ & 1050 & 0950 & 285 \\
\hline \multicolumn{4}{|l|}{ NOV $30-$} \\
\hline DEC 03 & 1040 & 0940 & 141 \\
\hline $03-06$ & 1025 & 0925 & 133 \\
\hline $07-10$ & 1025 & 0925 & 118 \\
\hline $10-14$ & 1025 & 0925 & 134 \\
\hline $14-16$ & 1125 & 0725 & 147 \\
\hline $16-17$ & 0825 & 0925 & 276 \\
\hline $17-18$ & 1025 & 0125 & 437 \\
\hline $18-21$ & 0225 & 0925 & 461 \\
\hline $21-24$ & 1050 & 0750 & 281 \\
\hline $24-28$ & 0825 & 0925 & 161 \\
\hline $28-29$ & 0915 & 2015 & 149 \\
\hline $29-31$ & 2115 & 0815 & 372 \\
\hline \multicolumn{4}{|l|}{ DEC 31- } \\
\hline JAN 04 & 0900 & 0800 & 449 \\
\hline $04-05$ & 1040 & 2140 & 407 \\
\hline $05-07$ & 2240 & 0940 & 437 \\
\hline $07-11$ & 1020 & 0920 & 224 \\
\hline $11-13$ & 1035 & 0135 & 170 \\
\hline $13-15$ & 0235 & 0935 & 334 \\
\hline $15-19$ & 1000 & 0900 & 229 \\
\hline $19-21$ & 1035 & 0935 & 171 \\
\hline $21-24$ & 1025 & 0525 & 326 \\
\hline $24-25$ & 0625 & 0925 & 439 \\
\hline $25-28$ & 1050 & 0920 & 314 \\
\hline \multicolumn{4}{|l|}{ JAN $28-$} \\
\hline FEB 01 & 1030 & 0930 & 165 \\
\hline $01-04$ & 1100 & 1000 & 162 \\
\hline $04-07$ & 1100 & 0200 & 156 \\
\hline $08-12$ & 1055 & 0855 & 142 \\
\hline $12-16$ & 0915 & 0815 & 130 \\
\hline $16-18$ & 1040 & 0940 & 139 \\
\hline $18-22$ & 1025 & 0925 & 123 \\
\hline $22-25$ & 1035 & 0935 & 130 \\
\hline \multicolumn{4}{|l|}{ FEB 25- } \\
\hline MAR 01 & 1020 & 0920 & 127 \\
\hline $01-04$ & 1020 & 0920 & 144 \\
\hline $04-08$ & 1020 & 0920 & 158 \\
\hline $08-11$ & 1030 & 0930 & 212 \\
\hline $11-15$ & 1035 & 0935 & 147 \\
\hline $15-18$ & 0945 & 0845 & 193 \\
\hline $18-22$ & 1015 & 0915 & 234 \\
\hline $22-25$ & 0950 & 0850 & 325 \\
\hline $25-29$ & 0950 & 0850 & 668 \\
\hline $29-31$ & 1005 & 0405 & 1260 \\
\hline $29-31$ & 1005 & 0605 & 1250 \\
\hline
\end{tabular}

WATER YEAR OCTOBER 1992 TO SEPTEMBER 1993-continued

\begin{tabular}{|c|c|c|c|c|c|c|c|c|c|}
\hline 13 & -- & - & 0.02 & 0.66 & 0.98 & 0.120 & 0.017 & 100 & 150 \\
\hline 17 & -- & -- & 0.01 & 0.65 & 1.10 & 0.095 & 0.017 & 110 & 160 \\
\hline 30 & 54 & 14 & 0.02 & 0.79 & 1.00 & 0.140 & 0.015 & 90 & 130 \\
\hline 18 & -- & -- & 0.02 & 0.75 & 0.89 & 0.095 & 0.016 & 89 & 120 \\
\hline 13 & -- & -. & 0.02 & 0.65 & 1.00 & 0.085 & 0.017 & 100 & 150 \\
\hline 45 & 135 & 15 & $<0.01$ & 1.1 & 0.87 & 0.310 & 0.026 & 79 & 110 \\
\hline 50 & 105 & 14 & $<0.01$ & 1.0 & 0.57 & 0.190 & 0.026 & 61 & 73 \\
\hline 21 & -- & -- & $<0.01$ & 0.69 & 0.82 & 0.120 & 0.022 & 77 & 90 \\
\hline 12 & -- & - & $<0.01$ & 0.66 & 1.20 & 0.060 & 0.017 & 93 & 120 \\
\hline 24 & -- & -- & $<0.01$ & 0.77 & 1.00 & 0.130 & 0.020 & 92 & 130 \\
\hline 12 & -- & -- & $<0.01$ & 0.60 & 0.91 & 0.070 & 0.018 & 94 & 120 \\
\hline 6.8 & -- & -- & 0.02 & 0.80 & 1.10 & 0.060 & 0.016 & 150 & 140 \\
\hline 8.6 & -- & -- & 0.02 & 0.64 & 1.00 & 0.070 & 0.014 & 120 & 120 \\
\hline 32 & 69 & 12 & 0.02 & 0.78 & 0.92 & 0.150 & 0.018 & 87 & 99 \\
\hline 45 & 86 & 12 & 0.01 & 0.85 & 0.76 & 0.180 & 0.020 & 77 & 78 \\
\hline 30 & 49 & 9 & 0.02 & 0.84 & 0.97 & 0.130 & 0.024 & $7 \varepsilon$ & 91 \\
\hline 7.3 & -- & -- & 0.02 & 0.63 & 1.40 & 0.065 & 0.020 & 94 & 120 \\
\hline 6.7 & -- & -- & 0.02 & 0.52 & 1.40 & 0.060 & 0.017 & 110 & 120 \\
\hline 5.2 & -. & -- & 0.02 & 0.63 & 1.40 & 0.060 & 0.016 & 170 & 140 \\
\hline 5.3 & $<10$ & $<10$ & 0.02 & 0.56 & 1.40 & 0.050 & 0.014 & 250 & \\
\hline 5.6 & 11 & $<8$ & 0.02 & 0.60 & 1.30 & 0.050 & 0.014 & 200 & 120 \\
\hline 16 & 34 & 8 & 0.02 & 0.69 & 1.20 & 0.085 & 0.014 & 200 & 96 \\
\hline 25 & -- & -- & 0.02 & 0.81 & 1.00 & 0.120 & 0.019 & 160 & 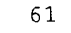 \\
\hline 22 & -- & -- & 0.02 & 0.69 & 1.00 & 0.120 & 0.021 & 100 & 5 \\
\hline 13 & -- & -- & 0.02 & 0.69 & 0.61 & 0.085 & 0.018 & 98 & 83 \\
\hline 6.1 & $\cdots$ & -- & 0.02 & 0.58 & 1.50 & 0.060 & 0.016 & $13 \mathrm{C}$ & 110 \\
\hline 6.7 & -- & -. & 0.03 & 0.71 & 1.50 & 0.055 & 0.016 & $14 \mathrm{C}$ & 120 \\
\hline 34 & -- & -- & 0.04 & 1.2 & 1.20 & 0.160 & 0.017 & $16 C$ & 88 \\
\hline 25 & -- & -- & 0.02 & 0.78 & 1.00 & 0.120 & 0.019 & $10 \mathrm{C}$ & 70 \\
\hline 30 & -. & -- & 0.02 & 0.79 & 1.10 & 0.130 & 0.019 & $13 c$ & 79 \\
\hline 34 & -- & -- & 0.02 & 0.76 & 0.98 & 0.130 & 0.019 & $8 \varepsilon$ & 68 \\
\hline 8.5 & -- & -- & 0.01 & 0.68 & 1.30 & 0.055 & 0.017 & $12 \mathrm{C}$ & 93 \\
\hline 6.5 & -. & -- & $<0.01$ & 0.51 & 1.50 & 0.045 & 0.013 & $16 \mathrm{C}$ & 120 \\
\hline 19 & -- & -- & 0.01 & 0.61 & 1.30 & 0.080 & 0.013 & $25 \mathrm{C}$ & 93 \\
\hline 8.5 & -- & -- & 0.01 & 0.55 & 1.30 & 0.050 & 0.013 & 170 & 95 \\
\hline 6.2 & -- & -- & 0.01 & 0.71 & 1.40 & 0.050 & 0.011 & 160 & 110 \\
\hline 18 & -- & -- & 0.02 & 0.60 & 1.30 & 0.075 & 0.012 & $17 \mathrm{C}$ & 81 \\
\hline 24 & -- & - - & - & 0.59 & 1.10 & 0.085 & 0.013 & $13 \mathrm{C}$ & 62 \\
\hline 18 & - & -- & 0.02 & 0.80 & 1.20 & 0.070 & 0.012 & $13 \mathrm{C}$ & 74 \\
\hline 5.8 & -- & -- & 0.02 & 0.64 & 1.30 & 0.045 & 0.011 & $16 \mathrm{C}$ & 110 \\
\hline 6.8 & 10 & $<7$ & 0.02 & 0.66 & 1.50 & 0.045 & 0.009 & $23 \mathrm{C}$ & 120 \\
\hline 5.6 & -- & -- & 0.02 & 0.60 & 1.40 & 0.045 & 0.009 & $22 \mathrm{C}$ & 120 \\
\hline 4.1 & - & -- & 0.01 & 0.61 & 1.40 & 0.045 & 0.008 & $18 \mathrm{C}$ & 130 \\
\hline 4.5 & -- & -- & $<0.01$ & 0.59 & 1.40 & 0.040 & 0.005 & $21 C$ & 140 \\
\hline 2.9 & -- & -- & $<0.01$ & 0.45 & 1.40 & 0.035 & 0.006 & $27 C$ & 140 \\
\hline 6.0 & -- & -- & 0.03 & 0.78 & 1.50 & 0.070 & 0.009 & $23 \mathrm{C}$ & 150 \\
\hline 4.2 & -- & -- & 0.04 & 0.55 & 1.40 & 0.045 & 0.008 & $21 C$ & 150 \\
\hline 4.0 & -- & -- & 0.05 & 0.61 & 1.40 & 0.040 & 0.011 & $20 \mathrm{C}$ & 15 \\
\hline 3.7 & -- & -- & 0.03 & 0.47 & 1.40 & 0.040 & 0.010 & $19 \mathrm{C}$ & 14 \\
\hline 4.4 & -- & -- & 0.04 & 0.64 & 1.40 & 0.035 & 0.009 & $38 \mathrm{C}$ & \\
\hline 4.6 & -. & -- & 0.03 & 0.66 & 1.30 & 0.035 & 0.010 & $36 c$ & 110 \\
\hline 4.2 & -- & -+ & 0.03 & 0.68 & 1.30 & 0.035 & 0.010 & $31 \mathrm{C}$ & 110 \\
\hline 3.8 & -- & -- & 0.04 & 0.58 & 1.30 & 0.035 & 0.009 & $27 \mathrm{C}$ & 13 \\
\hline 3.7 & -- & -- & 0.04 & 0.62 & 1.20 & 0.040 & 0.011 & $24 \mathrm{C}$ & 10 \\
\hline 16 & 22 & 4 & 0.04 & 0.60 & 1.20 & 0.055 & 0.011 & $23 C$ & \\
\hline 32 & 77 & 7 & 0.04 & 0.77 & 1.20 & 0.120 & 0.016 & $13 C$ & 50 \\
\hline 35 & -- & -- & 0.05 & 0.76 & 1.00 & 0.170 & 0.021 & 72 & 7 \\
\hline 34 & N90 & N8 & 0.05 & 0.76 & 1.00 & 0.160 & 0.021 & 73 & 37 \\
\hline
\end{tabular}




\section{Irondequoit Creek at Empire Boulevard, Rochester, N.Y.}

\section{WATER-QUALITY RECORDS}

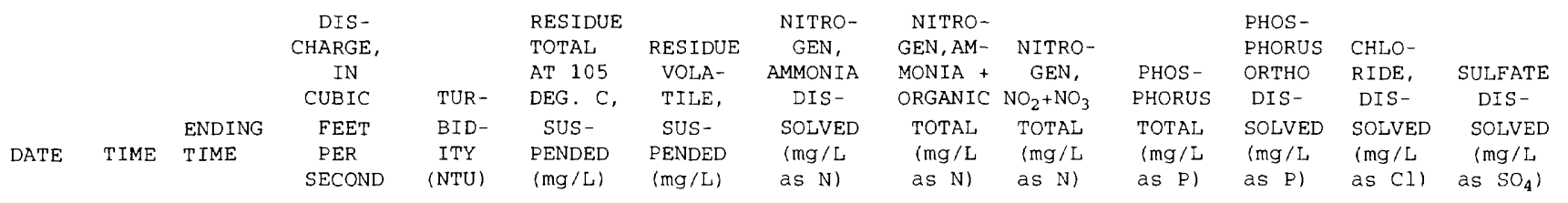

MAR $31-$

APR 0

$\begin{array}{llll}01-03 & 0705 & 0905 & 1360\end{array}$

$\begin{array}{llll}03-05 & 0230 & 0830 & 1150\end{array}$

06-06 $1015 \quad 1045 \quad 496$

06-08 $1045 \quad 0945 \quad 442$

$\begin{array}{llll}08-09 & 1010 & 2110 & 288\end{array}$

09-11 $2210 \quad 0110 \quad 291$

$\begin{array}{llll}11-12 & 0210 & 0910 & 384\end{array}$

$\begin{array}{llll}12-15 & 0950 & 0850 & 253\end{array}$

$\begin{array}{llll}15-16 & 1030 & 1330 & 284 \\ 16-18 & 1430 & 0130 & 389\end{array}$

$\begin{array}{llll}18-19 & 0230 & 0930 & 393\end{array}$

$19-22 \quad 1000 \quad 0900 \quad 250$

$\begin{array}{llll}22-23 & 1015 & 1315 & 464\end{array}$

$\begin{array}{llll}23-26 & 1415 & 0915 & 418\end{array}$

$26-29$

APR $29-$

MAY 03

$03-05$

$05-06$

$10-13$

$13-17$

$17-20$

$20-24$

$24-28$

28-31

MAY 31 -

JUN 01

$01-03$

$03-05$

$05-06$

06-07

$07-08$

$08-09$

$09-10$

$10-11$

$11-14$

$14-17$

$17-19$

19-20

$20-21$

$21-24$
$24-27$

$27-28$

JUN $28-$

JUL 02

$02-06$
$06-08$

$12-15$

$15-19$

$19-22$

$20 .$.

$20 \ldots$

20.

$20 .$.

$20 \ldots$

$20 \ldots$

$20 \ldots$

$20 \ldots$

$22-26$

$26-26$

$26-29$

29-31

\section{$09550855 \quad 149$}

$1000 \quad 0600 \quad 118$

$0700 \quad 0900 \quad 153$

$0955 \quad 0855 \quad 126$

$10150915 \quad 103$

$\begin{array}{lll}1000 & 0900 & 87\end{array}$

$\begin{array}{lll}0935 & 0835 & 90\end{array}$

$0925 \quad 0825 \quad 68$

$0515 \quad 0815 \quad 89$

$1050 \quad 0850 \quad 67$

$0945 \quad 0445 \quad 72$

$\begin{array}{lll}0545 & 0045 & 275\end{array}$

$0145 \quad 0845 \quad 174$

$\begin{array}{lll}1015 & 1815 & 103 \\ 1915 & 0915 & 137\end{array}$

$\begin{array}{lll}1015 & 0915 & 138\end{array}$

$10550555 \quad 113$

$\begin{array}{lll}0655 & 0955 & 83\end{array}$

$\begin{array}{lll}1015 & 0915 & 78 \\ 0950 & 0050 & 70\end{array}$

$0150 \quad 1250 \quad 105$

$1350 \quad 0850 \quad 141$

$0945 \quad 0845 \quad 100$

$\begin{array}{lll}0955 & 1655 & 70\end{array}$

$0935 \quad 0835 \quad 92$

$0840 \quad 0740$

$0945 \quad 0845$

$0950 \quad 0850$

$1000 \quad 0900$

$1310 \quad 0910$

1021

1022

1023
1024

1025

1026

1027

1029

09550855

09552055

$2155 \quad 0855$

$1000 \quad 0100$

WATER YEAR OCTOBER 1992 TO SEPTEMBER 1993-continued

\begin{tabular}{|c|c|c|c|c|c|c|c|c|c|}
\hline 45 & N71 & N7 & 0.03 & 0.66 & 1.00 & 0.120 & 0.020 & 57 & 34 \\
\hline 50 & -- & -- & 0.03 & 0.68 & 0.97 & 0.120 & 0.023 & 55 & 31 \\
\hline 31 & -- & -- & 0.02 & 0.55 & 1.10 & 0.085 & 0.022 & 56 & 37 \\
\hline 17 & -- & -- & 0.02 & 0.65 & 1.30 & 0.075 & 0.014 & 74 & 59 \\
\hline 18 & -- & -- & 0.02 & 0.56 & 1.20 & 0.080 & 0.014 & 73 & 59 \\
\hline 26 & -- & -- & 0.03 & 0.71 & 1.10 & 0.120 & 0.010 & 84 & 73 \\
\hline 38 & 74 & 13 & 0.04 & 0.91 & 1.00 & 0.170 & 0.011 & 87 & 75 \\
\hline 37 & 64 & $<31$ & 0.04 & 0.89 & 0.94 & 0.130 & 0.011 & 84 & 67 \\
\hline 24 & -- & -- & 0.03 & 0.72 & $<0.01$ & 0.110 & 0.011 & 90 & 76 \\
\hline 30 & 57 & $<11$ & 0.02 & 0.75 & 1.00 & 0.120 & 0.010 & 95 & 86 \\
\hline 34 & 63 & 11 & 0.03 & 0.86 & 0.99 & 0.130 & 0.010 & 96 & 83 \\
\hline 32 & 45 & $<11$ & 0.02 & 0.72 & 0.76 & 0.095 & 0.011 & 80 & 68 \\
\hline 24 & -- & -- & 0.03 & 0.78 & 0.94 & 0.100 & 0.010 & 98 & 82 \\
\hline 16 & -- & -- & 0.02 & $<0.10$ & 0.98 & 0.070 & 0.010 & 94 & 81 \\
\hline 24 & -- & -- & 0.02 & 0.71 & 0.85 & 0.095 & 0.010 & 80 & 65 \\
\hline 14 & -- & -- & 0.01 & 0.66 & 0.89 & 0.060 & 0.010 & 94 & 84 \\
\hline 11 & -- & -- & 0.02 & 0.73 & 0.85 & 0.065 & 0.010 & 100 & - \\
\hline 12 & -- & -- & 0.02 & 0.68 & 0.78 & 0.065 & 0.010 & 100 & 110 \\
\hline 14 & -- & -- & 0.03 & 0.76 & 0.76 & 0.070 & 0.009 & 100 & 110 \\
\hline 16 & -- &.- & 0.02 & $<0.10$ & 0.66 & 0.080 & 0.011 & 100 & 110 \\
\hline 13 & - & -- & 0.02 & 0.77 & 0.59 & 0.085 & 0.009 & 110 & - \\
\hline 14 & -- & -- & 0.02 & 0.74 & 0.60 & 0.090 & 0.010 & 110 & - \\
\hline 12 & -- & -- & $<0.01$ & $<0.10$ & 0.73 & 0.080 & 0.010 & 110 & 140 \\
\hline 8.6 & -- & -- & $<0.01$ & 0.65 & 0.82 & 0.075 & 0.012 & 110 & - \\
\hline $\mathrm{N} 12$ & -- & -- & -- & -- & 0.86 & 0.085 & 0.013 & 110 & 150 \\
\hline 12 & -- & -- & $<0.01$ & $<0.10$ & 0.86 & 0.110 & 0.013 & 120 & 160 \\
\hline 14 & -- & -- & $<0.01$ & $<0.10$ & 0.80 & 0.110 & 0.012 & 120 & 160 \\
\hline 13 & -- & -- & $<0.01$ & No. 77 & 0.87 & 0.100 & 0.016 & 110 & 150 \\
\hline 14 & $\ldots$ & -- & $<0.01$ & 0.85 & 0.90 & 0.100 & 0.016 & 120 & 160 \\
\hline N28 & 63 & $<10$ & -- & 1.1 & 0.87 & 0.160 & 0.017 & 99 & 140 \\
\hline N32 & 68 & $<12$ & -- & 0.91 & 0.82 & 0.145 & 0.020 & 89 & 100 \\
\hline 19 & -- & -- & $<0.01$ & 0.90 & 0.87 & 0.110 & 0.026 & 110 & 120 \\
\hline 22 & -- & -- & $<0.01$ & 0.90 & 0.81 & 0.110 & 0.026 & 110 & 120 \\
\hline 20 & $\cdots$ & -- & $<0.01$ & 0.78 & 0.81 & 0.120 & 0.027 & 110 & 120 \\
\hline 20 & -- & - & 0.07 & 0.80 & 0.80 & 0.140 & 0.030 & 100 & 110 \\
\hline 17 & -- & -- & 0.07 & 0.85 & 0.78 & 0.120 & 0.032 & 110 & 130 \\
\hline 26 & -- & -- & 0.05 & 0.88 & 0.74 & 0.130 & 0.029 & 120 & 160 \\
\hline 14 & -- & -- & 0.04 & 0.86 & 0.67 & 0.120 & 0.024 & 120 & 170 \\
\hline 14 & -- & -- & 0.06 & 0.83 & 0.71 & 0.130 & 0.031 & 120 & 170 \\
\hline 16 & -- & $\cdots$ & 0.07 & 0.86 & ง. 51 & 0.140 & 0.040 & 110 & 150 \\
\hline 14 & -- & -- & 0.07 & 0.73 & 0.76 & 0.110 & 0.042 & 100 & 140 \\
\hline 11 & -- & -- & 0.04 & 0.68 & 0.74 & 0.100 & 0.033 & 110 & 160 \\
\hline 13 & -- & -- & 0.02 & 0.69 & 0.68 & 0.120 & 0.026 & 120 & 170 \\
\hline 12 & -- & -- & 0.05 & 0.67 & $<0.05$ & 0.120 & 0.030 & 110 & 160 \\
\hline 10 & -- & - & 0.05 & 0.72 & 0.82 & 0.100 & 0.031 & 120 & 170 \\
\hline 9.7 & -- & -- & 0.04 & 0.77 & 0.64 & 0.110 & 0.028 & 120 & 190 \\
\hline 12 & -- & -- & 0.02 & 0.89 & 0.56 & 0.140 & 0.030 & 120 & 200 \\
\hline 8.8 & -- & -- & 0.03 & 0.82 & 0.63 & 0.120 & 0.029 & 120 & 200 \\
\hline 15 & -- & -- & 0.07 & 0.86 & No. 79 & 0.110 & 0.034 & 92 & $\mathrm{~N} 140$ \\
\hline-- & -- & -- & -- & 0.73 & -- & 0.120 & -- & -- & - \\
\hline-- & -- & -- & -- & 0.86 & -- & 0.120 & -- & -- & - \\
\hline-- & -- & -- & -- & 0.90 & -- & 0.130 & -- & -- & - \\
\hline- & -- & -- & -- & 0.85 & -- & 0.110 & -- & -- & - \\
\hline-- & -- & -- & -- & 0.88 & -- & 0.120 & -- & -- & - \\
\hline-- & -- & -- & -- & 0.92 & -- & 0.120 & -- & -- & - \\
\hline-- & & -- & -- & 0.93 & -- & 0.120 & -- & -- & - \\
\hline-- & -- & -- & -- & 0.80 & -- & 0.120 & -- & -- & - \\
\hline-- & -- & -- & -- & 0.78 & -- & 0.120 & -- & -- & - \\
\hline 9.3 & -- & -- & 0.02 & 0.57 & 0.68 & 0.100 & 0.023 & 120 & $>210$ \\
\hline 8.5 & -- & -- & 0.05 & 0.88 & $<0.01$ & 0.110 & 0.024 & 120 & 200 \\
\hline 8.0 & -- & -- & 0.05 & No. 75 & $<0.01$ & 0.095 & 0.037 & 110 & 190 \\
\hline 10 & -- & -- & 0.05 & No. 87 & 0.62 & 0.110 & 0.030 & 110 & 180 \\
\hline
\end{tabular}


Surface-Water Stations

\section{A. Discharge and water quality}

\section{Irondequoit Creek at Empire Boulevard, Rochester, N.Y.}

\section{WATER-QUALITY RECORDS}

\begin{tabular}{|c|c|c|c|c|c|c|c|c|c|c|c|c|c|}
\hline & & & $\begin{array}{c}\text { DIS- } \\
\text { CHARGE, } \\
\text { IN } \\
\text { CUBIC }\end{array}$ & TUR- & $\begin{array}{l}\text { RESIDUE } \\
\text { TOTAL } \\
\text { AT } 105 \\
\text { DEG. C. }\end{array}$ & $\begin{array}{l}\text { RESIDUE } \\
\text { VOLA- } \\
\text { TILE, }\end{array}$ & $\begin{array}{l}\text { NITRO- } \\
\text { GEN, } \\
\text { AMMONIA } \\
\text { DIS- }\end{array}$ & $\begin{array}{l}\text { NITRO- } \\
\text { GEN, AM- } \\
\text { MONIA + } \\
\text { ORGANIC }\end{array}$ & $\begin{array}{c}\text { NITRO- } \\
\text { GEN, } \\
\mathrm{NO}_{2}+\mathrm{NO}_{3}\end{array}$ & $\begin{array}{l}\text { PHOS- } \\
\text { PHORUS }\end{array}$ & $\begin{array}{l}\text { PHOS- } \\
\text { PHORUS } \\
\text { ORTHO } \\
\text { DIS- }\end{array}$ & $\begin{array}{l}\text { CHLO- } \\
\text { RIDE, } \\
\text { IIS- }\end{array}$ & $\begin{array}{l}\text { SULFATE } \\
\text { DIS- }\end{array}$ \\
\hline DATE & TIME & $\begin{array}{l}\text { ENDING } \\
\text { TIME }\end{array}$ & $\begin{array}{c}\text { FEET } \\
\text { PER } \\
\text { SECOND }\end{array}$ & $\begin{array}{l}\text { BID- } \\
\text { ITY } \\
\text { (NTU) }\end{array}$ & $\begin{array}{l}\text { SUS - } \\
\text { PENDED } \\
(\mathrm{mg} / \mathrm{L})\end{array}$ & $\begin{array}{l}\text { SUS- } \\
\text { PENDED } \\
(\mathrm{mg} / \mathrm{L})\end{array}$ & $\begin{array}{l}\text { SOLVED } \\
\text { (mg/L } \\
\text { as } N \text { ) }\end{array}$ & $\begin{array}{l}\text { TOTAL } \\
\text { (mg/L } \\
\text { as N) }\end{array}$ & $\begin{array}{l}\text { TOTAL } \\
\text { (mg/L } \\
\text { as N) }\end{array}$ & $\begin{array}{l}\text { TOTAL } \\
\text { (mg/L } \\
\text { as P) }\end{array}$ & $\begin{array}{l}\text { SOLVED } \\
\text { (mg/L } \\
\text { as P) }\end{array}$ & $\begin{array}{l}\text { SOLVED } \\
\text { (mg/L } \\
\text { as Cl) }\end{array}$ & $\begin{array}{c}\text { SOLVED } \\
(\mathrm{mg} / \mathrm{L} \\
\left.\text { as } \mathrm{SO}_{4}\right)\end{array}$ \\
\hline
\end{tabular}

$\begin{array}{rrrr}\text { JUL } 31- & & & \\ \text { AUG 02 } & 0200 & 0900 & 74 \\ 02-05 & 0945 & 0845 & 57 \\ 05-09 & 1250 & 0950 & 70 \\ 09-11 & 1045 & 1845 & 53 \\ 12-12 & 0020 & 0820 & 77 \\ 12-16 & 1005 & 0905 & 53 \\ 16-17 & 0940 & 0240 & 51 \\ 17-17 & 0340 & 2340 & 77 \\ 19-20 & 1015 & 0515 & 63 \\ 20-21 & 0615 & 0915 & 78 \\ 21-23 & 1015 & 0915 & 65 \\ 23-26 & 1005 & 0905 & 44 \\ 26-30 & 0940 & 0840 & 43 \\ 30-31 & 0945 & 1445 & 43 \\ 31-31 & 1545 & 2345 & 60 \\ \text { SEP } & & & \\ 01-02 & 0045 & 0845 & 71 \\ 02-04 & 2015 & 0615 & 150 \\ 04-07 & 0715 & 0915 & 106 \\ 07-09 & 1000 & 0900 & 81 \\ 09-10 & 1025 & 1725 & 100 \\ 10-13 & 1825 & 0925 & 84 \\ 13-16 & 0955 & 0855 & 41 \\ 16-20 & 1000 & 0900 & 50 \\ 20-23 & 0950 & 0850 & 45 \\ 23-24 & 0930 & 0030 & 85 \\ 24-26 & 0130 & 0430 & 64 \\ 26-26 & 0530 & 1630 & 224 \\ 26-27 & 1730 & 0830 & 140 \\ 27-28 & 0930 & 1130 & 99 \\ 28-30 & 1230 & 0830 & 82 \\ 30-30 & 0950 & 2300 & 74 \\ \text { SEP 30- } & & & \\ 0 C T 04 & 0950 & 0850 & 61\end{array}$

WATER YEAR OCTOBER 1992 TO SEPTEMBER 1993-continued 


\section{Surface-Water Stations}

\section{B. Partial-Record and Miscellaneous-Record Sites}

\section{Genesee River at Charlotte Pump Station Near Rochester, N.Y.}

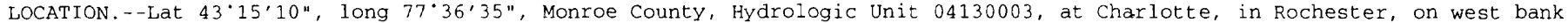
of the Genesee River, $1300 \mathrm{ft}$ downstream of stutson Street Bridge, $0.5 \mathrm{mi}$. upstream of mouth, and 5.0 mi. downstream from gaging station (04232000) at Rochester.

DRAINAGE AREA.--2,467 $\mathrm{mi}^{2}$ at station 04232000

PERIOD OF RECORD. - -Water years 1990 to current year.

CHEMICAL DATA: $1990-93$ (e)

NUTRIENT DATA: 1990-93 (e).

COOPERATION.--Water-quality samples were collected and analyzed by the Monroe County Environmental Health Laboratory at Rochester, NY.

\section{WATER-QUALITY DATA}

\begin{tabular}{|c|c|c|c|c|c|c|c|c|c|c|c|c|c|}
\hline & & & $\begin{array}{c}\text { DIS- } \\
\text { CHARGE, } \\
\text { IN } \\
\text { CUBIC }\end{array}$ & TUR - & $\begin{array}{l}\text { RESIDUE } \\
\text { TOTAL } \\
\text { AT } 105 \\
\text { DEG. C. }\end{array}$ & $\begin{array}{c}\text { RESIDUE } \\
\text { VOLA- } \\
\text { TILE, }\end{array}$ & $\begin{array}{l}\text { NITRO- } \\
\text { GEN, } \\
\text { AMMONIA } \\
\text { DIS- }\end{array}$ & $\begin{array}{l}\text { NITRO- } \\
\text { GEN, AM- } \\
\text { MONIA + } \\
\text { ORGANIC }\end{array}$ & $\begin{array}{c}\mathrm{NITRO}- \\
\text { GEN, } \\
\mathrm{NO}_{2}+\mathrm{NO}_{3}\end{array}$ & $\begin{array}{l}\text { PHOS- } \\
\text { PHORUS }\end{array}$ & $\begin{array}{l}\text { PHOS- } \\
\text { PHORUS } \\
\text { ORTHO, } \\
\text { DIS- }\end{array}$ & $\begin{array}{l}\text { CHLO- } \\
\text { RIDE, } \\
\text { DIS- }\end{array}$ & $\begin{array}{l}\text { SULFATE } \\
\text { DIS- }\end{array}$ \\
\hline DATE & TIME & $\begin{array}{l}\text { ENDING } \\
\text { TIME }\end{array}$ & $\begin{array}{l}\text { FEET } \\
\text { PER }\end{array}$ & $\begin{array}{l}\text { BID- } \\
\text { ITY }\end{array}$ & $\begin{array}{c}\text { SUS - } \\
\text { PENDED }\end{array}$ & $\begin{array}{l}\text { SUS- } \\
\text { PENDED }\end{array}$ & $\begin{array}{l}\text { SOLVED } \\
\text { (mg/L }\end{array}$ & $\begin{array}{l}\text { TOTAL } \\
\text { (mg/L }\end{array}$ & $\begin{array}{l}\text { TOTAL } \\
\text { (mg/L }\end{array}$ & $\begin{array}{l}\text { TOTAL } \\
\text { (mg/L }\end{array}$ & $\begin{array}{l}\text { SOLVED } \\
(\mathrm{mg} / \mathrm{L}\end{array}$ & $\begin{array}{l}\text { SOLVED } \\
(\mathrm{mg} / \mathrm{L}\end{array}$ & $\begin{array}{l}\text { SOLVED } \\
\text { (mg/L }\end{array}$ \\
\hline & & & SECOND & (NTU) & $(\mathrm{mg} / \mathrm{L})$ & $(\mathrm{mg} / \mathrm{L})$ & as $\mathrm{N}$ ) & as N) & as $\mathrm{N}$ ) & as P) & as $\mathrm{Pl}$ & as C11 & as $\mathrm{SO}_{4}$ ) \\
\hline
\end{tabular}

OCT

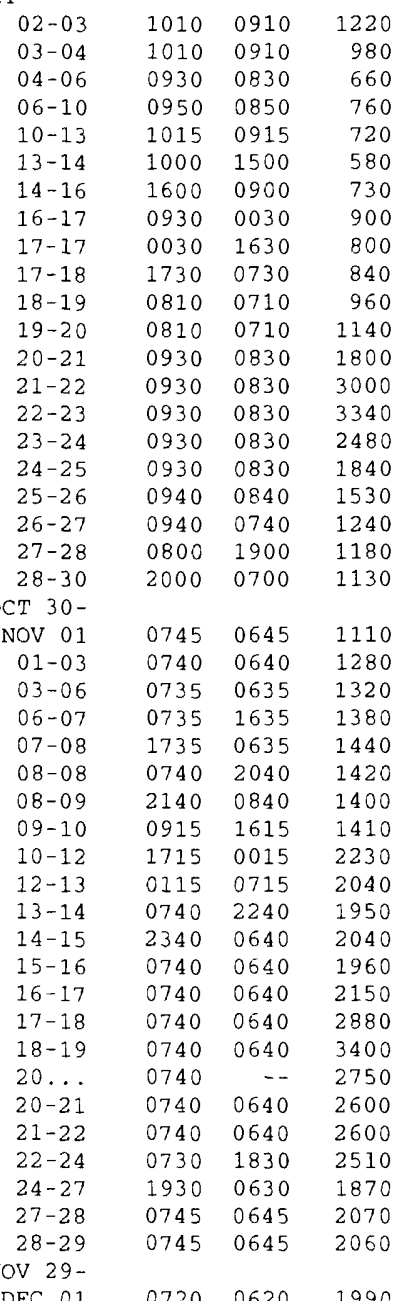

\begin{tabular}{|c|c|c|c|}
\hline 7.5 & -- & - & 0.13 \\
\hline 12 & -- & -- & 0.09 \\
\hline 15 & -- & -- & 0.14 \\
\hline 4.9 & -- & -- & 0.30 \\
\hline 1.9 & -- & -- & 0.18 \\
\hline 3.3 & -- & -- & 0.34 \\
\hline 5.4 & -- & -- & 0.37 \\
\hline 12 & -- & -- & 0.23 \\
\hline 13 & -- & -- & 0.21 \\
\hline 15 & -- & -- & 0.14 \\
\hline 11 & -- & -- & 0.15 \\
\hline 10 & -- & -- & 0.23 \\
\hline 7.8 & -- & -- & 0.34 \\
\hline 11 & -- & -- & 0.22 \\
\hline 32 & 40 & $<10$ & 0.12 \\
\hline 60 & 97 & 8 & 0.12 \\
\hline 55 & 71 & 6 & 0.14 \\
\hline 40 & 46 & 6 & 0.15 \\
\hline 24 & -- & -- & 0.21 \\
\hline 15 & -- & -- & 0.27 \\
\hline 13 & -- & -- & 0.28 \\
\hline 13 & -- & -- & -- \\
\hline 12 & -- & -- & 0.27 \\
\hline 5.4 & -- & -- & 0.24 \\
\hline 5.0 & -- & -- & 0.18 \\
\hline 7.3 & -- & -- & 0.14 \\
\hline 7.8 & -- & -- & 0.17 \\
\hline 7.9 & -- & -- & 0.17 \\
\hline 2.4 & -- & -- & 0.20 \\
\hline 5.7 & -- & -- & 0.22 \\
\hline 10 & -- & -- & 0.10 \\
\hline 13 & -- & -- & 0.11 \\
\hline 14 & -- & -- & 0.14 \\
\hline 13 & -- & -- & 0.05 \\
\hline 17 & -- & -- & 0.16 \\
\hline 16 & -- & -- & 0.09 \\
\hline 14 & -- & -- & 0.08 \\
\hline 64 & 82 & 7 & 0.09 \\
\hline 32 & 64 & 7 & 0.03 \\
\hline 24 & -- & -- & 0.08 \\
\hline 8.8 & -- & -- & 0.01 \\
\hline 12 & -- & -- & 0.09 \\
\hline 11 & $\cdots$ & -- & 0.08 \\
\hline 11 & -- & $\cdots$ & 0.13 \\
\hline 14 & -- & - & 0.13 \\
\hline
\end{tabular}

$\begin{array}{ll}0.54 & 0.82 \\ 0.34 & 0.78 \\ 0.52 & 0.73 \\ 0.64 & 0.73 \\ 0.54 & 0.78 \\ 0.78 & 0.77 \\ 0.95 & 0.80 \\ 0.79 & 0.77 \\ 0.71 & 0.76 \\ 0.68 & 0.72 \\ 0.66 & 0.74 \\ 0.68 & 0.72 \\ 0.77 & 0.71 \\ 0.59 & 0.66 \\ 0.65 & 0.96 \\ 0.74 & 0.80 \\ 0.77 & 0.73 \\ 0.66 & 0.73 \\ 0.71 & 0.71 \\ 0.68 & 0.70 \\ 0.64 & 0.74 \\ 0.64 & 0.72 \\ 0.77 & 0.76 \\ 0.85 & 0.68 \\ 0.43 & 0.62 \\ 0.49 & 0.66 \\ 0.72 & 0.66 \\ 0.56 & 0.65 \\ 0.86 & 0.64 \\ 0.74 & 0.62 \\ 0.65 & 0.63 \\ 0.55 & 0.67 \\ 0.69 & 0.67 \\ 0.50 & 0.79 \\ 0.57 & 0.74 \\ 0.57 & 0.73 \\ 0.77 & 0.67 \\ 0.71 & 0.56 \\ 0.57 & 0.67 \\ 0.66 & 0.70 \\ 0.41 & -. \\ 0.57 & 0.94 \\ 0.65 & 0.99 \\ 0.71 & 1.00 \\ 0.38 & 1.10 \\ 0.73\end{array}$

0.085

$0.006 \quad 120$

0.070

0.065

0.065

0.075

0.085

0.085

0.085

0.100

0.100

0.090

0.080

0.070

0.070

0.095

0.160

0.120

0.090

0.080

0.095

0.005

0.003

$0.013 \quad 77$

$0.011 \quad 150$

$0.008 \quad 100$

$0.011 \quad 72$

$0.017 \quad 67$

$0.018 \quad 96$

$0.019 \quad 98$

$0.017 \quad 110$

$0.017 \quad 120$

$0.021 \quad 100$

$0.022 \quad 100$

$0.021 \quad 96$

$0.019 \quad 57$

$0.016 \quad 60$

$0.016 \quad 62$

$0.015 \quad 60$

$0.016 \quad 71$

$0.016 \quad 100$

0.080

$0.018 \quad 120$

$\begin{array}{lll}0.075 & 0.022 & 120\end{array}$

$\begin{array}{lll}0.050 & 0.018 & 110\end{array}$

$\begin{array}{lll}0.055 & 0.019 & 110\end{array}$

$0.080 \quad 0.020 \quad 140$

$\begin{array}{lll}0.065 & 0.024 & 120\end{array}$

0.055

0.035

0.040

$0.017 \quad 110$

$0.016 \quad 96$

$0.016 \quad 110$

0.045

0.055

0.055

0.070

0.075

0.115

0.100

0.070

0.035

0.060

0.060

$0.015 \quad 130$

$0.015 \quad 120$

$0.002 \quad 85$

0.003

0.027

0.017

0.016

0.017

0.013

0.016

0.016

0.018

0.060

$0.020 \quad 77$ 
Surface-Water Stations

B. Partial-Record and Miscellaneous-Record Sites

431510077363501 Genesee River at Charlotte Pump Station Near Rochester, N." . WATER-QUALITY DATA

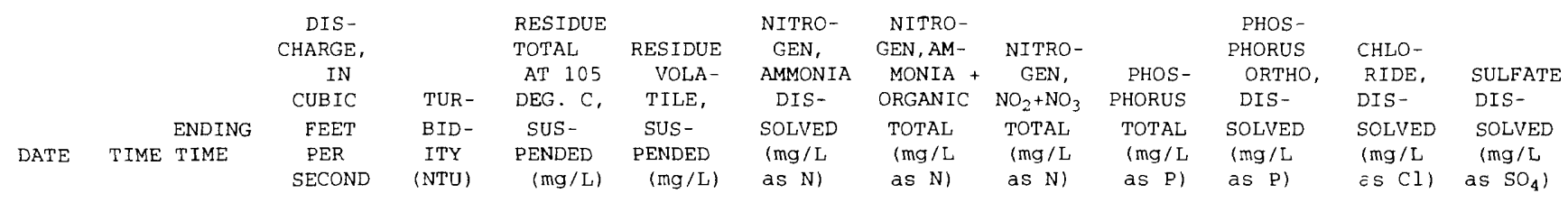

DEC

\begin{tabular}{|c|c|c|c|}
\hline $1-04$ & & & \\
\hline $04-06$ & $\begin{array}{l}0745 \\
0730\end{array}$ & $\begin{array}{l}0645 \\
0630\end{array}$ & $\begin{array}{l}1410 \\
1200\end{array}$ \\
\hline $06-08$ & 0735 & 0635 & 1420 \\
\hline $08-09$ & 0740 & 1840 & 1400 \\
\hline $09-11$ & 1940 & 0640 & 1130 \\
\hline $11-13$ & -- & -- & 1100 \\
\hline $11-13$ & 0930 & 0830 & 1100 \\
\hline $13-15$ & 1100 & 0900 & 1070 \\
\hline $15-16$ & 0940 & 2040 & 1040 \\
\hline $16-18$ & 2040 & 0840 & 1050 \\
\hline $18-20$ & 1000 & 0900 & 1030 \\
\hline $20-22$ & 1620 & 0920 & 990 \\
\hline $22-24$ & 0945 & 0845 & 95 \\
\hline $24-26$ & 0945 & 0845 & 940 \\
\hline $26-29$ & 1000 & 0900 & 970 \\
\hline $29-31$ & 0950 & 0850 & 1050 \\
\hline \multicolumn{4}{|l|}{ EC $31-$} \\
\hline JAN 02 & 0950 & 0850 & 1260 \\
\hline $02-03$ & 0735 & 0635 & 1480 \\
\hline $03-04$ & 0735 & 0635 & 1420 \\
\hline $04-05$ & 0745 & 0645 & 1760 \\
\hline $05-06$ & 0945 & 0845 & 2680 \\
\hline $06-07$ & 0945 & 0845 & 3270 \\
\hline $07-08$ & 0945 & 0645 & 3310 \\
\hline $08-08$ & 0735 & 2235 & 3200 \\
\hline $08-09$ & 2335 & 1435 & 920 \\
\hline $09-10$ & 1535 & 0635 & 2700 \\
\hline $10-10$ & 0740 & 2240 & 2770 \\
\hline $10-11$ & 2340 & 1440 & 2540 \\
\hline $11-12$ & 1540 & 0640 & 2390 \\
\hline $12-14$ & 0740 & 0640 & 2020 \\
\hline $14-16$ & 0740 & 0640 & 1760 \\
\hline $16-18$ & 0740 & 1340 & 2590 \\
\hline $22-23$ & 1940 & 0125 & 4880 \\
\hline $23-23$ & 0140 & 0725 & 4590 \\
\hline $23-24$ & 0730 & 0630 & 4910 \\
\hline $24-25$ & 0730 & 0630 & 5400 \\
\hline $25-26$ & 0730 & 0630 & 5710 \\
\hline $26-27$ & 0725 & 0625 & 5910 \\
\hline $27-28$ & 0725 & 0625 & 5820 \\
\hline $28-29$ & 0725 & 0625 & 5290 \\
\hline $29-29$ & 0735 & 2235 & 4960 \\
\hline $29-30$ & 2335 & 1435 & 4820 \\
\hline $30-31$ & 1535 & 0635 & 4560 \\
\hline \multicolumn{4}{|l|}{ AN 31- } \\
\hline $\mathrm{FEB} 01$ & 0900 & 0700 & 4370 \\
\hline $01-01$ & 0100 & 1600 & 4290 \\
\hline $01-02$ & 1700 & 0800 & 4120 \\
\hline $02-03$ & 0800 & 0700 & 5080 \\
\hline $03-04$ & 0800 & 0700 & 6590 \\
\hline $04-05$ & 0800 & 0700 & 6620 \\
\hline $05-06$ & 0920 & 0020 & 600 \\
\hline $06-06$ & 0120 & 1620 & 6690 \\
\hline $06-07$ & 1720 & 0820 & 6460 \\
\hline $07-07$ & 0745 & 2245 & 6220 \\
\hline $07-08$ & 2345 & 1445 & 6220 \\
\hline $08-09$ & 1545 & 0645 & 713 \\
\hline $09-11$ & 1000 & 0900 & 9400 \\
\hline & 1015 & - & 804 \\
\hline $21 \ldots$ & 1000 & - & 5300 \\
\hline $23 \ldots$ & 0900 & -- & 10900 \\
\hline$\cdots$ & 0930 & -- & \\
\hline & & & \\
\hline
\end{tabular}

WATER YEAR OCTOBER 1989 TO SEPTEMBER 1990-continued

\begin{tabular}{|c|c|c|c|c|c|c|c|c|c|}
\hline 7.0 & -- & -- & 0.14 & 0.71 & 1.10 & 0.060 & 0.024 & 68 & 78 \\
\hline 14 & -- & -- & 0.19 & 0.67 & 1.40 & 0.060 & 0.026 & 73 & 85 \\
\hline 9.1 & -- & -- & 0.31 & 0.79 & 1.30 & 0.060 & 0.021 & 93 & 48 \\
\hline 7.6 & -- & -- & 0.27 & 0.76 & 1.20 & 0.050 & 0.014 & 100 & 76 \\
\hline 12 & -- & -- & 0.26 & 0.68 & 1.10 & 0.045 & 0.013 & 78 & 70 \\
\hline 13 & 198 & -- & 0.31 & 0.75 & 1.10 & 0.045 & 0.010 & 79 & 71 \\
\hline 13 & -- & -- & 0.31 & 0.80 & 1.10 & 0.045 & 0.010 & 79 & 71 \\
\hline 8.9 & $\cdots$ & -- & 0.42 & -- & 1.10 & 0.055 & 0.016 & 93 & 91 \\
\hline 4.7 & -- & -- & 0.46 & 1.1 & 1.30 & 0.050 & 0.017 & 120 & 90 \\
\hline 4.4 & -- & -- & 0.42 & 0.94 & 1.30 & 0.055 & 0.014 & 97 & 91 \\
\hline -- & -- & -- & 0.43 & 0.81 & 1.60 & 0.060 & 0.015 & 100 & 95 \\
\hline- & - & -- & 0.40 & 0.96 & 1.40 & 0.065 & 0.013 & 120 & 110 \\
\hline 2.5 & -- & -- & 0.34 & 0.89 & 1.10 & 0.050 & 0.016 & 110 & 87 \\
\hline 2.7 & -- & -- & 0.34 & 0.89 & 1.00 & 0.045 & 0.014 & 85 & 79 \\
\hline 3.4 & -. & -- & 0.46 & 0.90 & 1.50 & 0.050 & 0.017 & 120 & 110 \\
\hline 2.9 & -- & -- & 0.40 & 0.94 & 1.40 & 0.040 & 0.019 & 140 & 100 \\
\hline 2.9 & -- & -- & 0.47 & 1.0 & 1.50 & 0.060 & 0.028 & 230 & 120 \\
\hline 2.8 & -- & -- & 0.49 & 1.0 & 1.50 & 0.065 & 0.025 & 260 & 120 \\
\hline 2.6 & -- & -- & 0.48 & 1.1 & 1.60 & 0.055 & 0.016 & 220 & 120 \\
\hline 5.4 & -- & -- & 0.36 & 0.93 & 1.50 & 0.070 & 0.022 & 200 & 110 \\
\hline 6.1 & -- & -- & 0.33 & 1.0 & 1.60 & 0.075 & 0.023 & 140 & 81 \\
\hline 16 & -- & -- & 0.24 & 1.0 & 1.60 & 0.100 & 0.026 & 100 & 66 \\
\hline 120 & 143 & 13 & 0.20 & 1.1 & 1.70 & 0.170 & 0.020 & 72 & 58 \\
\hline 120 & 179 & 18 & 0.22 & 0.97 & 1.50 & 0.170 & 0.013 & 170 & 56 \\
\hline 90 & 99 & 10 & 0.20 & 1.1 & 1.50 & 0.120 & 0.017 & 170 & 56 \\
\hline 70 & 76 & 9 & 0.22 & 1.1 & 1.60 & 0.120 & 0.018 & 180 & 55 \\
\hline 33 & 31 & $<5$ & 0.18 & 0.86 & 1.50 & 0.070 & 0.019 & 72 & 62 \\
\hline 30 & 35 & $<5$ & 0.17 & 0.83 & 1.40 & 0.065 & 0.016 & 70 & 63 \\
\hline 26 & -- & -- & 0.22 & 0.77 & 1.40 & 0.080 & 0.018 & 72 & 67 \\
\hline 14 & -- & -- & 0.28 & 0.68 & 1.60 & 0.060 & 0.020 & 77 & - \\
\hline 8.4 & -- & -- & 0.55 & 1.7 & 2.00 & 0.050 & 0.019 & 83 & 78 \\
\hline 16 & -. & -- & 0.28 & 0.98 & 1.60 & 0.080 & 0.017 & 90 & 77 \\
\hline 170 & 200 & 16 & 0.13 & 0.83 & 1.30 & 0.290 & 0.013 & 44 & 46 \\
\hline 180 & 203 & 16 & 0.14 & 0.83 & 1.20 & 0.330 & 0.012 & 240 & 48 \\
\hline 50 & 121 & 11 & 0.15 & 0.56 & 1.20 & 0.200 & 0.011 & 45 & 43 \\
\hline 40 & 101 & 10 & 0.13 & 0.52 & 1.20 & 0.110 & 0.013 & 47 & 44 \\
\hline 60 & 90 & 19 & 0.14 & 0.53 & 1.40 & 0.090 & 0.011 & 52 & 45 \\
\hline 60 & -- & -- & 0.16 & 0.85 & 1.50 & 0.140 & 0.016 & 50 & 76 \\
\hline 60 & 91 & 9 & 0.15 & 0.80 & 1.70 & 0.140 & 0.015 & 48 & 45 \\
\hline 70 & 85 & 8 & 0.10 & 0.67 & 1.50 & 0.110 & 0.014 & 44 & 44 \\
\hline 95 & 138 & 11 & 0.11 & 0.77 & 1.40 & 0.130 & 0.012 & 45 & 42 \\
\hline 70 & 136 & 11 & 0.12 & 0.60 & 1.30 & 0.120 & 0.012 & 47 & 41 \\
\hline 55 & 96 & 7 & 0.14 & 0.56 & 1.30 & 0.100 & 0.019 & 48 & 41 \\
\hline 35 & 50 & 5 & 0.13 & 0.47 & 1.30 & 0.070 & 0.005 & 47 & 44 \\
\hline 26 & -- & -- & 0.13 & 0.28 & 1.40 & 0.050 & 0.011 & 50 & 47 \\
\hline 24 & -- & -- & 0.12 & 0.39 & 1.40 & 0.060 & 0.012 & 54 & 47 \\
\hline 33 & 66 & $<10$ & 0.17 & 0.60 & 1.40 & 0.120 & 0.013 & 68 & 55 \\
\hline 90 & 161 & 13 & 0.15 & 1.0 & 1.70 & 0.180 & 0.016 & 74 & 47 \\
\hline 120 & 197 & 15 & 0.11 & 0.67 & 1.60 & 0.210 & 0.013 & 57 & 44 \\
\hline 160 & 156 & 11 & 0.07 & 0.93 & 1.40 & 0.240 & 0.013 & 55 & 41 \\
\hline 120 & 161 & 14 & 0.07 & 0.59 & 1.50 & 0.230 & 0.014 & 54 & 42 \\
\hline 88 & 141 & 12 & 0.05 & 0.57 & 1.50 & 0.170 & 0.012 & 57 & 43 \\
\hline 32 & 96 & 9 & 0.10 & 0.75 & 1.60 & 0.090 & 0.014 & 60 & 48 \\
\hline 38 & 116 & 10 & 0.10 & 0.55 & 1.50 & 0.110 & 0.013 & 61 & 46 \\
\hline 38 & 121 & 11 & 0.10 & 0.62 & 1.50 & 0.120 & 0.016 & 60 & 45 \\
\hline 100 & 366 & 31 & 0.11 & 1.6 & 1.90 & 0.360 & 0.024 & 53 & 48 \\
\hline 75 & 102 & 10 & 0.10 & 0.80 & 1.40 & 0.120 & 0.022 & 100 & 42 \\
\hline 140 & 198 & 16 & 0.14 & 0.83 & 1.60 & 0.200 & 0.018 & 44 & 42 \\
\hline 170 & 331 & 26 & 0.07 & 0.96 & 1.40 & 0.160 & 0.016 & 43 & 34 \\
\hline 120 & 160 & 13 & 0.07 & 0.62 & 1.50 & 0.150 & 0.013 & 36 & 31 \\
\hline 5.5 & -- & -- & 0.11 & 0.70 & 1.60 & 0.210 & 0.022 & 37 & 36 \\
\hline
\end{tabular}


431510077363501 Genesee River at Charlotte Pump Station Near Rochester, N.Y.

WATER-QUALITY DATA

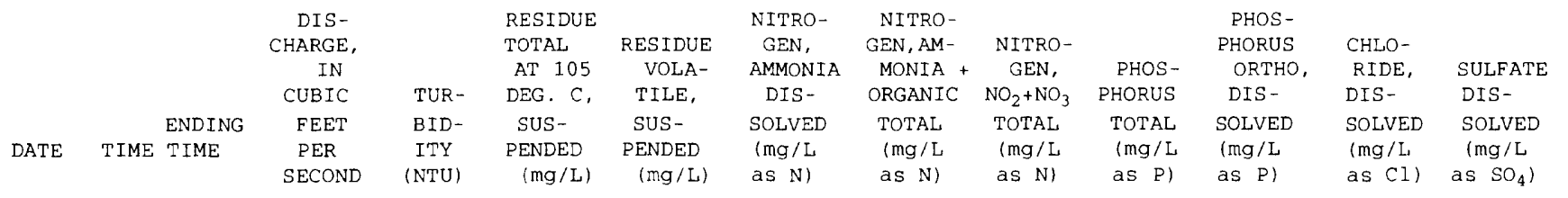

Feb-continued

\begin{tabular}{|c|c|c|c|c|c|c|c|c|c|c|c|c|c|}
\hline $28-28$ & 0740 & 1840 & 8350 & 110 & 186 & 12 & 0.04 & 0.71 & 1.70 & 0.200 & 0.006 & 39 & 40 \\
\hline \multicolumn{14}{|l|}{ MAR } \\
\hline $04-04$ & 1045 & 1315 & 5770 & 50 & -- & -- & 0.08 & 0.52 & 1.70 & 0.140 & 0.006 & 54 & 48 \\
\hline $07-09$ & 0950 & 0650 & 1930 & 27 & - & -- & 0.14 & 0.58 & 2.10 & 0.055 & 0.013 & 55 & 74 \\
\hline $09-10$ & 0740 & 1840 & 2440 & 8.0 & -- & -- & 0.20 & 0.71 & 2.20 & 0.045 & 0.014 & 93 & 86 \\
\hline $10-12$ & 1940 & 0640 & 3930 & 18 & -- & -- & 0.15 & 0.54 & 2.10 & 0.045 & 0.014 & 87 & 75 \\
\hline $12-13$ & 0725 & 0625 & 6280 & 39 & 83 & 7 & 0.11 & 0.66 & 2.00 & 0.090 & 0.019 & 65 & 64 \\
\hline $13-14$ & 0725 & 0625 & 6700 & 85 & 164 & 13 & 0.09 & 0.76 & 1.80 & 0.180 & 0.016 & 51 & 56 \\
\hline $14-15$ & 0725 & 0625 & 5810 & 95 & 158 & 10 & 0.07 & 0.71 & 1.60 & 0.150 & 0.017 & 46 & 50 \\
\hline $15-16$ & 0725 & 0625 & 4500 & 60 & 101 & 7 & 0.07 & 0.95 & 1.50 & 0.110 & 0.013 & 46 & 53 \\
\hline $16-17$ & 0705 & 1805 & 3650 & 29 & -- & -- & 0.09 & 0.51 & 1.40 & 0.080 & 0.023 & 51 & 58 \\
\hline $18-19$ & 1905 & 0605 & 6030 & 38 & 67 & 6 & 0.08 & 0.49 & 1.50 & 0.085 & 0.015 & 62 & 65 \\
\hline $19-20$ & 0945 & 0845 & 5740 & 280 & 441 & 26 & 0.05 & 1.2 & 1.10 & 0.280 & 0.012 & 51 & 50 \\
\hline $20-21$ & 0945 & 0645 & 5280 & 210 & 286 & 18 & 0.06 & 0.87 & 1.10 & 0.220 & 0.020 & 51 & 50 \\
\hline $21-22$ & 0725 & 0625 & 4900 & 58 & 97 & 7 & 0.07 & 0.73 & 1.20 & 0.110 & 0.011 & 52 & 52 \\
\hline $22-23$ & 0725 & 0625 & 4260 & 34 & 60 & $<5$ & 0.07 & 0.57 & 1.40 & 0.085 & 0.010 & 52 & 52 \\
\hline $23-24$ & 0740 & 1840 & 3740 & 17 & -- & -- & 0.07 & 0.52 & 1.40 & 0.040 & 0.012 & 52 & 54 \\
\hline $24-26$ & 1940 & 0640 & 3140 & 19 & -- & -- & 0.08 & 0.48 & 1.50 & 0.050 & 0.010 & 58 & 64 \\
\hline $26-28$ & 0735 & 0635 & 2530 & 16 & -- & -- & 0.08 & 0.47 & 1.50 & 0.050 & 0.011 & 63 & 68 \\
\hline $28-30$ & 0735 & 0635 & 2020 & 13 & -- & -- & 0.11 & 0.48 & 1.50 & 0.040 & 0.011 & 68 & 71 \\
\hline \multicolumn{14}{|l|}{ MAR $30-$} \\
\hline APR 02 & 0835 & 0735 & 2340 & 10 & -- & -- & 0.12 & 0.54 & 1.70 & 0.050 & 0.011 & 78 & 82 \\
\hline $02-03$ & 0750 & 1250 & 3120 & 13 & -- & -- & 0.06 & 0.57 & 1.70 & 0.050 & 0.010 & 80 & 82 \\
\hline $06 \ldots$ & 1115 & -- & 9290 & 130 & 260 & 19 & 0.06 & 0.80 & 1.30 & 0.250 & 0.016 & 56 & 49 \\
\hline $07 \ldots$ & 1035 & -- & 9090 & 100 & 205 & 14 & 0.05 & 0.88 & 1.30 & 0.150 & 0.014 & 49 & 47 \\
\hline $07-08$ & 1045 & 0645 & 8210 & 75 & 140 & 11 & 0.03 & 1.1 & 1.40 & 0.140 & 0.011 & 49 & 48 \\
\hline $08-09$ & 0745 & 0745 & 7000 & 60 & 102 & 8 & 0.03 & 0.61 & 1.30 & 0.095 & 0.010 & 50 & 49 \\
\hline $09-10$ & 0745 & 0645 & 6680 & 34 & 63 & 5 & -- & - & -- & 0.090 & 0.011 & 49 & - \\
\hline $10-11$ & 0745 & 0645 & 8970 & 38 & 88 & 8 & -- & -- & -- & 0.100 & 0.011 & 52 & - \\
\hline $11-13$ & 1645 & 0645 & 11400 & 390 & 617 & 50 & 0.04 & 1.1 & 1.30 & 0.600 & 0.022 & 37 & 37 \\
\hline $13-16$ & 0730 & 0630 & 9440 & 180 & 272 & 20 & 0.05 & 1.0 & 1.20 & 0.320 & 0.014 & 38 & 44 \\
\hline $16-18$ & 0710 & 0610 & 8750 & 190 & -- & -- & 0.05 & 0.86 & 1.10 & 0.260 & 0.012 & 31 & 40 \\
\hline $18-20$ & 0750 & 0650 & 7590 & 100 & 167 & 12 & 0.05 & 0.74 & 1.30 & 0.160 & 0.011 & 33 & 42 \\
\hline $20-23$ & 0740 & 0640 & 6220 & 50 & 83 & 7 & 0.05 & 0.92 & 1.40 & 0.090 & 0.011 & 38 & 48 \\
\hline $23-25$ & 0730 & 0630 & 3690 & 40 & 74 & 6 & 0.07 & 0.56 & 1.30 & 0.075 & 0.006 & 46 & 52 \\
\hline $25-27$ & 0730 & 0630 & 2660 & 30 & 49 & 5 & 0.06 & 0.59 & 1.00 & 0.050 & 0.010 & 57 & 53 \\
\hline $27-30$ & 0735 & 0635 & 1630 & 18 & -- & -- & 0.09 & 0.66 & 1.20 & 0.060 & 0.011 & 66 & 63 \\
\hline \multicolumn{14}{|l|}{ APR $30-$} \\
\hline MAY 01 & 0730 & 0230 & 2220 & 19 & -- & -- & 0.10 & 0.74 & 1.30 & 0.065 & 0.013 & 70 & 67 \\
\hline $02-02$ & 0800 & 1700 & 1220 & 18 & -- & -- & 0.11 & 0.83 & 1.20 & 0.070 & 0.009 & 75 & 71 \\
\hline $04 \ldots$ & 0735 & -- & 890 & 13 & -- & -- & 0.13 & 0.70 & 1.30 & 0.065 & 0.009 & 81 & 80 \\
\hline $04-05$ & 1520 & 0520 & 1420 & 20 & -- & -- & 0.12 & 0.95 & 1.10 & 0.070 & 0.006 & 73 & 67 \\
\hline $06-07$ & 0620 & 0320 & 6210 & 50 & -- & $\cdots$ & 0.11 & 0.80 & 1.30 & 0.105 & 0.007 & 82 & 69 \\
\hline $07-08$ & 0645 & 0545 & 5390 & 140 & 205 & 14 & 0.07 & 0.97 & 0.93 & 0.200 & 0.008 & 43 & 41 \\
\hline $07-08$ & 0710 & 0610 & 5390 & 27 & -- & $\ldots$ & 0.10 & 0.56 & 0.96 & 0.070 & 0.017 & 53 & 56 \\
\hline $08-09$ & 0645 & 0545 & 3990 & 50 & 79 & 6 & 0.07 & 0.67 & 0.88 & 0.140 & 0.007 & 45 & 46 \\
\hline $08-09$ & 0710 & 0610 & 3990 & 23 & -- & -- & 0.10 & 0.57 & 0.97 & 0.065 & 0.013 & 64 & 63 \\
\hline $12-12$ & 0735 & 2135 & 3300 & 19 & -- & -- & 0.11 & 0.57 & 0.87 & 0.055 & 0.011 & 47 & 65 \\
\hline $12-14$ & 2235 & 0635 & 4550 & 23 & -- & -- & 0.08 & 0.53 & 0.80 & 0.090 & 0.011 & 61 & 58 \\
\hline $14-15$ & 0735 & 0635 & 7120 & 50 & 97 & 9 & 0.08 & 0.69 & 1.00 & 0.120 & 0.016 & 57 & 52 \\
\hline $15-16$ & 0735 & 0635 & 7380 & 85 & -- & $\ldots$ & 0.06 & 0.90 & 1.10 & 0.240 & 0.014 & 43 & 45 \\
\hline $16-17$ & 0730 & 0630 & 8420 & 65 & 109 & 11 & 0.04 & 1.1 & 0.95 & 0.105 & 0.023 & 42 & 42 \\
\hline $17-18$ & 0730 & 0630 & 10300 & 80 & 218 & 40 & 0.04 & 1.7 & 1.00 & 0.190 & 0.026 & 42 & 38 \\
\hline $18-19$ & 0655 & 0555 & 9840 & 120 & 272 & 24 & 0.06 & 0.96 & 1.40 & 0.300 & 0.038 & 56 & 38 \\
\hline $19-20$ & 0655 & 0555 & 8440 & 80 & 150 & 14 & 0.06 & 0.71 & 1.10 & 0.150 & 0.028 & 37 & 38 \\
\hline $20-21$ & 0655 & 0555 & 8640 & 65 & 124 & 9 & 0.06 & 0.72 & 1.10 & 0.150 & 0.023 & 40 & 38 \\
\hline $21-22$ & 0725 & 0625 & 9340 & 85 & 144 & 11 & 0.06 & 0.80 & 0.97 & 0.210 & 0.026 & 37 & 43 \\
\hline $22-23$ & 0725 & 0625 & 9060 & 75 & 136 & 10 & 0.06 & 1.1 & 1.00 & 0.150 & 0.022 & 35 & 36 \\
\hline $23-24$ & 0730 & 0630 & 8060 & 55 & 116 & 9 & 0.06 & 0.72 & 1.10 & 0.115 & 0.022 & 34 & 40 \\
\hline $24-25$ & 0730 & 0630 & 7160 & 50 & 138 & 8 & 0.05 & 0.77 & 1.10 & -- & -- & 35 & 36 \\
\hline $25-27$ & 0940 & 0440 & 6120 & 15 & -- & -- & 0.02 & 0.46 & 1.00 & 0.045 & 0.020 & 35 & 38 \\
\hline $27-29$ & 0540 & 0040 & 4990 & 16 & -- & -- & 0.04 & 0.30 & 1.00 & 0.040 & 0.017 & 35 & 40 \\
\hline $29 \ldots$ & 0735 & -- & 4480 & 34 & 90 & 8 & 0.06 & 0.76 & 1.10 & 0.085 & 0.012 & 37 & 42 \\
\hline
\end{tabular}




\section{Surface-Water Stations}

B. Partial-Record and Miscellaneous-Record Sites

431510077363501 Genesee River at Charlotte Pump Station Near Rochester, N.Y.

WATER-QUALITY DATA

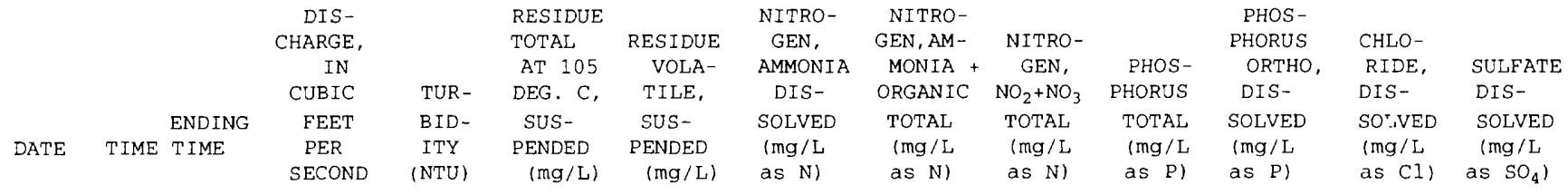

MAY-cont inued

\begin{tabular}{|c|c|c|c|}
\hline \multicolumn{4}{|c|}{ MAY-cont inued } \\
\hline $30 \ldots$ & 1635 & -- & 3510 \\
\hline \multicolumn{4}{|l|}{ MAY $30-$} \\
\hline JUN 01 & 1640 & 0740 & 4130 \\
\hline $01-03$ & 0800 & 1900 & 3110 \\
\hline $03-04$ & 2000 & 0700 & 2400 \\
\hline $04-05$ & 0735 & 0635 & 2270 \\
\hline $05-06$ & 0735 & 0635 & 2120 \\
\hline $06-07$ & 0700 & 0600 & 1980 \\
\hline $06-08$ & 0700 & 0600 & 1870 \\
\hline $08-09$ & 0715 & 1815 & 1600 \\
\hline $08-11$ & 1915 & 0615 & 1620 \\
\hline $11-13$ & 0735 & 0635 & 1440 \\
\hline $13-15$ & 0735 & 0835 & 1200 \\
\hline $15-18$ & 0745 & 0045 & 1060 \\
\hline $18 \ldots$ & 0800 & -- & 910 \\
\hline $18-19$ & 0800 & 1000 & 920 \\
\hline $20 \ldots$ & 0800 & -- & 1180 \\
\hline $20-22$ & 0800 & 0500 & 1180 \\
\hline $22-23$ & 0805 & 0105 & 1180 \\
\hline $23-25$ & 0205 & 0705 & 1170 \\
\hline $26-28$ & -- & -- & 1020 \\
\hline $28-30$ & 0945 & 0845 & 1000 \\
\hline \multicolumn{4}{|l|}{ JUN $30-$} \\
\hline JUL 02 & 0945 & 0745 & 960 \\
\hline $02-03$ & 0815 & 0845 & 920 \\
\hline $03-04$ & 0830 & 0730 & 830 \\
\hline $04-05$ & 0830 & 0330 & 760 \\
\hline $05-07$ & 0830 & 0730 & 720 \\
\hline $07-09$ & 0830 & 0730 & 680 \\
\hline $09-10$ & 0830 & 0730 & 740 \\
\hline $10-11$ & 0830 & 0730 & 850 \\
\hline $11-12$ & 0825 & 0725 & 870 \\
\hline $12-13$ & 0825 & 0725 & 780 \\
\hline $13-14$ & 0830 & 0730 & 1060 \\
\hline $14-15$ & 0830 & 0730 & 1350 \\
\hline $15-16$ & 0830 & 0730 & 1300 \\
\hline $16-17$ & 1045 & 0945 & 1220 \\
\hline $17-18$ & 1045 & 0845 & 1240 \\
\hline $18-19$ & 0915 & 0815 & 1120 \\
\hline $19-20$ & 0915 & 0815 & 880 \\
\hline $20-21$ & 0830 & 0730 & 860 \\
\hline $21-22$ & 0830 & 0730 & 910 \\
\hline $22-23$ & 0830 & 0730 & 920 \\
\hline $23-24$ & 0830 & 0730 & 1120 \\
\hline $25-26$ & 1015 & 0915 & 1400 \\
\hline $26-27$ & 1015 & 0915 & 1010 \\
\hline $27-28$ & 0940 & 0840 & 820 \\
\hline $28-29$ & 0940 & 0840 & 700 \\
\hline $29-30$ & 0940 & 0840 & 630 \\
\hline $30-31$ & 0830 & 0730 & 640 \\
\hline \multicolumn{4}{|l|}{ JUL 31 - } \\
\hline AUG 01 & 0830 & 0730 & 660 \\
\hline $01-02$ & 0830 & 0030 & 650 \\
\hline $03 \ldots$ & 0900 & -- & 590 \\
\hline $06 \ldots$ & 0830 & -- & 610 \\
\hline $08 \ldots$ & 0900 & -- & 710 \\
\hline $08-08$ & 1100 & 1600 & 710 \\
\hline $08-09$ & 1620 & 0720 & 680 \\
\hline $09-10$ & 0805 & 0705 & 600 \\
\hline $10-11$ & 1050 & 0950 & 530 \\
\hline $11-12$ & 1050 & 0950 & 520 \\
\hline
\end{tabular}

WATER YEAR OCTOBER 1989 TO SEPTEMBER 1990-continued

\begin{tabular}{|c|c|c|c|c|c|c|c|c|c|}
\hline 20 & -- & -- & 0.08 & 0.36 & 1.10 & 0.060 & 0.015 & 42 & 46 \\
\hline 1.5 & -. & -- & 0.08 & 0.64 & 1.10 & 0.045 & 0.019 & 49 & 54 \\
\hline 29 & -- & -- & 0.07 & 0.56 & 1.20 & 0.075 & 0.013 & 42 & 46 \\
\hline 15 & -- & -- & 0.08 & 0.72 & 1.10 & 0.060 & 0.013 & 52 & 42 \\
\hline 20 & -- & -- & 0.06 & 0.66 & 1.10 & 0.070 & 0.017 & 56 & 58 \\
\hline 19 & -- & -- & 0.08 & 0.70 & 1.10 & 0.070 & 0.014 & 64 & 58 \\
\hline 5.1 & -. & -- & 0.09 & 0.77 & 1.10 & 0.040 & 0.014 & 70 & 77 \\
\hline 16 & -- & - & 0.08 & 0.74 & 1.20 & 0.065 & 0.010 & 72 & 79 \\
\hline 2.7 & .. & -- & 0.08 & 0.57 & 1.20 & 0.030 & 0.010 & 95 & 82 \\
\hline 4.9 & -- & -- & 0.07 & 0.87 & 1.20 & 0.030 & 0.008 & 97 & 86 \\
\hline 7.0 & -- & -. & 0.04 & 0.63 & 1.10 & 0.050 & 0.005 & 87 & 93 \\
\hline 5.8 & -- & -- & 0.08 & 0.83 & 1.10 & 0.050 & 0.004 & 85 & 90 \\
\hline 2.1 & -- & -. & 0.08 & 0.67 & 1.20 & 0.040 & 0.007 & 90 & 89 \\
\hline 4.6 & -- & -- & 0.08 & 0.70 & 0.95 & 0.045 & 0.003 & 82 & 79 \\
\hline 5.5 & -. & -- & 0.14 & 0.85 & 0.91 & 0.060 & 0.017 & 85 & 88 \\
\hline 9.5 & -- & -- & 0.14 & 0.94 & 0.81 & 0.060 & 0.013 & 90 & 92 \\
\hline 15 & -- & -- & 0.17 & 1.0 & 1.00 & 0.080 & 0.014 & 99 & 97 \\
\hline 6.0 & -- & -- & 0.17 & 1.4 & 1.10 & 0.120 & 0.014 & 110 & 99 \\
\hline 10 & -- & $\cdots$ & 0.17 & 0.92 & 1.10 & 0.075 & 0.014 & 110 & 92 \\
\hline 4.4 & -- & $\ldots$ & 0.13 & 0.92 & 0.99 & 0.070 & 0.016 & 92 & 92 \\
\hline 4.4 & -- & -- & 0.27 & 1.1 & 1.20 & 0.095 & 0.015 & 130 & 100 \\
\hline 15 & -. & -- & 0.34 & 1.1 & 1.20 & 0.090 & 0.016 & 110 & 96 \\
\hline 9.3 & -. & -- & 0.25 & 1.3 & 1.40 & 0.080 & 0.014 & 97 & 91 \\
\hline 24 & -. & -- & 0.25 & 1.0 & 0.94 & 0.090 & 0.013 & 100 & 99 \\
\hline 22 & -- & -- & 0.19 & 0.93 & 0.92 & 0.090 & 0.008 & 97 & 100 \\
\hline 16 & -- & -- & 0.24 & 1.2 & 0.95 & 0.070 & 0.009 & 120 & 100 \\
\hline 22 & -- & -- & 0.35 & 1.3 & 0.80 & 0.080 & 0.006 & 110 & 90 \\
\hline 17 & -. & -- & 0.16 & 0.99 & 0.75 & 0.100 & 0.008 & 110 & 98 \\
\hline 24 & -. & -- & 0.22 & 1.6 & 0.77 & 0.080 & 0.009 & 120 & 93 \\
\hline 20 & -. & -- & 0.25 & 1.2 & 0.77 & 0.085 & 0.013 & 110 & 92 \\
\hline 18 & $\ldots$ & -- & 0.23 & 1.4 & 0.74 & 0.090 & 0.013 & 110 & 88 \\
\hline 16 & -. & -- & 0.30 & 1.1 & 0.80 & 0.095 & 0.016 & 120 & 110 \\
\hline 18 & -- & -- & 0.29 & 1.2 & 0.96 & 0.095 & 0.008 & 130 & 110 \\
\hline 28 & -- & -- & 0.20 & 1.5 & 1.00 & 0.130 & 0.011 & 120 & 91 \\
\hline 16 & -- & -- & 0.11 & 0.87 & 0.77 & 0.090 & 0.006 & 110 & 83 \\
\hline 20 & -- & -- & 0.14 & 0.89 & 0.76 & 0.080 & 0.009 & 95 & 66 \\
\hline 26 & -- & -. & 0.22 & 0.85 & 0.75 & 0.090 & 0.010 & 63 & 80 \\
\hline 26 & -- & -. & 0.22 & 0.94 & 0.86 & 0.110 & 0.009 & 70 & 92 \\
\hline 22 & -. & -- & 0.22 & 0.86 & 1.10 & 0.100 & 0.012 & 79 & 72 \\
\hline 19 & -. & -- & 0.22 & 0.93 & 0.96 & 0.110 & 0.012 & 77 & 64 \\
\hline 22 & -- & -- & 0.26 & 1.2 & 0.94 & 0.100 & 0.014 & 100 & 68 \\
\hline 0.25 & -- & -- & 0.15 & 0.70 & 0.84 & 0.095 & 0.007 & 150 & 88 \\
\hline 28 & .. & -- & 0.07 & 0.93 & 0.89 & 0.130 & 0.010 & 120 & 84 \\
\hline 25 & -. & -- & 0.09 & 0.70 & 0.94 & 0.100 & 0.010 & 130 & 69 \\
\hline 20 & -- & .. & 0.11 & 0.69 & 1.10 & 0.110 & 0.013 & 120 & 58 \\
\hline 16 & -- & -- & 0.18 & 0.61 & 1.00 & 0.100 & 0.013 & 77 & 62 \\
\hline 15 & .. & -. & 0.23 & 0.74 & 1.10 & 0.095 & 0.014 & 92 & 67 \\
\hline 9.2 & -. & -. & 0.11 & 0.70 & 0.92 & 0.075 & 0.009 & 140 & 70 \\
\hline 9.1 & -- & -- & 0.10 & 0.90 & 0.82 & 0.075 & 0.008 & 150 & 78 \\
\hline 8.2 & -. & -. & 0.05 & 0.78 & 0.73 & 0.060 & 0.003 & 120 & 77 \\
\hline 6.3 & -- & -- & 0.03 & 0.82 & 0.83 & 0.055 & 0.002 & 110 & 91 \\
\hline 7.0 & -. & -- & 0.15 & 0.55 & 0.51 & 0.060 & 0.013 & 120 & 96 \\
\hline 5.4 & -- & -. & $\ldots$ & 0.57 & 0.55 & 0.065 & 0.018 & 110 & 100 \\
\hline 5.0 & -- & -. & 0.04 & 0.81 & 0.56 & 0.065 & 0.007 & 110 & 83 \\
\hline 5.4 & -- & -- & 0.04 & 0.72 & 0.64 & 0.080 & 0.014 & 110 & 110 \\
\hline 5.1 & -. & -- & 0.05 & 0.75 & 0.60 & 0.060 & 0.005 & 100 & 110 \\
\hline 5.6 & -- & -- & 0.07 & 1.0 & 0.80 & 0.070 & 0.002 & 110 & 86 \\
\hline & -- & -. & & & & 0065 & ? 0 & & \\
\hline
\end{tabular}




\section{B. Partial-Record and Miscellaneous-Record Sites}

\section{Genesee River at Charlotte Pump Station Near Rochester, N.Y.} WATER-QUALITY DATA

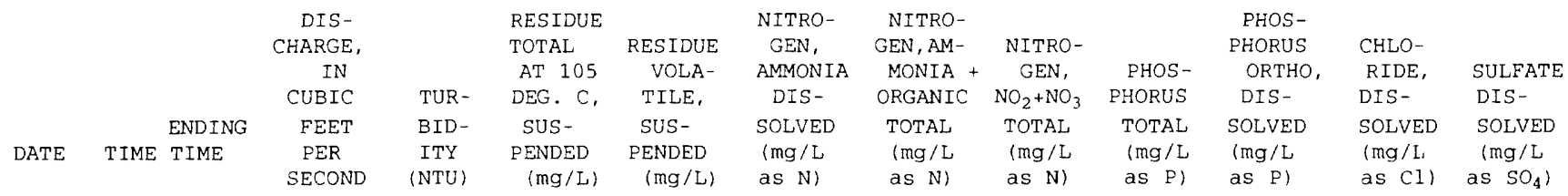

AUG-cont inued

$\begin{array}{rrrr}12-13 & 1050 & 0950 & 530 \\ 13-14 & 0930 & 0830 & 550 \\ 14-15 & 0930 & 0030 & 560 \\ 15 \ldots & 0830 & -- & 550 \\ 17-18 & 0930 & 0830 & 520 \\ 18-19 & 0930 & 0830 & 500 \\ 19-20 & 0930 & 0830 & 480 \\ 20-21 & 1110 & 1010 & 510 \\ 21-22 & 1110 & 1010 & 510 \\ 22-24 & 1050 & 0750 & 510 \\ 24-27 & 0950 & 0850 & 500 \\ 27-29 & 0805 & 0705 & 510 \\ 29-31 & 0825 & 0725 & 550 \\ \text { AUG } 31- & & & \\ \text { SEP 04 } & 0805 & 0535 & 510 \\ 04-05 & 1100 & 1000 & 460 \\ 05-06 & 1100 & 1000 & 510 \\ 06-07 & 1100 & 0900 & 680 \\ 07-08 & 0930 & 0830 & 760 \\ 08-09 & 0930 & 0830 & 700 \\ 09-10 & 0930 & 0830 & 860 \\ 10-11 & 1030 & 0930 & 1580 \\ 11-12 & 1030 & 0930 & 1680 \\ 12-13 & 0915 & 0815 & 1090 \\ 13-14 & 0915 & 0815 & 860 \\ 14-15 & 0915 & 2015 & 720 \\ 15-17 & 2115 & 0815 & 1010 \\ 17-19 & 1025 & 0725 & 1570 \\ 19-21 & 0750 & 0650 & 1130 \\ \text { EP 28- } & & & \\ 0 C T 01 & 0940 & 0840 & 920 \\ & & & \end{array}$

$\mathrm{OCT}$

$\begin{array}{rrrr}01-02 & 1030 & 0530 & 1540 \\ 02-03 & 1430 & 0930 & 1580 \\ 03-04 & 0940 & 0840 & 1380 \\ 04-05 & 0940 & 0840 & 1160 \\ 05-07 & 0940 & 0840 & 1350 \\ 07-09 & 0940 & 0840 & 1580 \\ 09-10 & 1040 & 0940 & 1600 \\ 10-11 & 1030 & 0930 & 2340 \\ 11-12 & 1030 & 0930 & 3140 \\ 12-13 & 0935 & 0835 & 4310 \\ 13-14 & 0935 & 0835 & 5990 \\ 14-15 & 0935 & 0835 & 5480 \\ 15-16 & 1035 & 0935 & 6220 \\ 16-17 & 1035 & 0935 & 6240 \\ 17-18 & 0950 & 0850 & 6520 \\ 18-19 & 0950 & 0850 & 6640 \\ 19-20 & 0950 & 0950 & 6780 \\ 22 \ldots & 0950 & -- & 4910 \\ 23 \ldots & 0745 & -- & 4730 \\ 23-24 & 1630 & 0830 & 4840 \\ 24-25 & 0940 & 0840 & 4640 \\ 25-26 & 0940 & 0840 & 3700 \\ 26-27 & 0945 & 0845 & 4000 \\ 27-28 & 0945 & 0845 & 5200 \\ 28-29 & 0945 & 0845 & 5320 \\ 29-30 & 0950 & 0850 & 4840 \\ 30-31 & 0950 & 0850 & 4280\end{array}$

\section{WATER YEAR OCTOBER 1989 TO SEPTEMBER 1990-continued}

\begin{tabular}{|c|c|c|c|c|c|c|c|c|c|}
\hline 3.8 & -- & -- & 0.26 & 1.0 & 0.84 & 0.055 & 0.002 & 110 & 110 \\
\hline 13 & - & -- & -- & 1.6 & 0.66 & 0.080 & 0.007 & 110 & 110 \\
\hline 8.8 & -- & - & -- & 1.3 & 0.62 & 0.070 & 0.019 & 99 & 100 \\
\hline 5.1 & -- & -- & - & 0.85 & 0.43 & 0.070 & 0.018 & 91 & 120 \\
\hline 4.6 & -- & - & -- & 0.70 & 0.98 & 0.065 & 0.008 & 88 & 120 \\
\hline 8.0 & - & -- & -- & 0.80 & 0.87 & 0.075 & 0.004 & 83 & 120 \\
\hline 8.5 & -- & - & -- & 0.77 & 0.84 & 0.070 & 0.014 & 88 & 120 \\
\hline 7.8 & -- & -- & -- & 0.87 & 0.87 & 0.085 & 0.024 & 90 & 120 \\
\hline 7.0 & - & - & - & 0.92 & 0.86 & 0.075 & 0.025 & 86 & 120 \\
\hline 7.7 & -- & -- & -- & 0.92 & 1.00 & 0.065 & 0.022 & 87 & 120 \\
\hline 4.6 & - & - - & - - & 0.71 & 1.00 & 0.045 & 0.003 & 90 & 110 \\
\hline 3.6 & - & -- & -- & 0.66 & 0.86 & 0.045 & 0.004 & 86 & 92 \\
\hline 4.5 & -- & -- & -- & 0.89 & 1.00 & 0.050 & 0.007 & 94 & 100 \\
\hline 4.2 & - & - & -- & 0.93 & 1.10 & 0.045 & 0.012 & 92 & 100 \\
\hline 5.9 & -- & -- & 0.09 & 0.78 & 0.83 & 0.055 & 0.003 & 95 & 100 \\
\hline 6.8 & -- & -- & 0.15 & 0.82 & 0.87 & 0.055 & 0.009 & 97 & 100 \\
\hline 6.7 & -- & -- & 0.17 & 0.82 & 0.87 & 0.055 & 0.012 & 99 & 94 \\
\hline 5.6 & -- & -- & 0.17 & 0.77 & 1.10 & 0.070 & 0.022 & 92 & 83 \\
\hline 5.3 & -- & - & 0.18 & 0.76 & 0.82 & 0.060 & 0.022 & 88 & 74 \\
\hline 5.1 & -- & -- & 0.30 & 0.73 & 0.96 & 0.060 & 0.024 & 95 & 80 \\
\hline 4.7 & -- & -- & 0.17 & 0.82 & 0.82 & 0.065 & 0.015 & 110 & 100 \\
\hline 9.4 & -- & -- & 0.13 & 0.75 & 0.81 & 0.085 & 0.029 & 130 & 88 \\
\hline 11 & $-\cdots$ & -- & 0.13 & 0.63 & 0.87 & 0.055 & 0.021 & 170 & 76 \\
\hline 12 & -- & -- & 0.20 & 0.65 & 0.90 & 0.065 & 0.021 & 170 & 56 \\
\hline 14 & -- & - & 0.19 & 0.76 & 0.79 & 0.065 & 0.021 & 100 & 46 \\
\hline 12 & $\cdots$ & - & 0.16 & 0.72 & 0.73 & 0.065 & 0.027 & 110 & 48 \\
\hline 13 & - & -- & 0.10 & 0.70 & 0.78 & 0.075 & 0.025 & 150 & 77 \\
\hline 12 & -- & -- & 0.10 & 0.83 & 0.61 & 0.060 & 0.020 & 110 & 56 \\
\hline 8.6 & $\ldots$ & - & 0.18 & 0.52 & 0.88 & 0.060 & 0.022 & 130 & 38 \\
\hline
\end{tabular}

WATER YEAR OCTOBER 1990 TO SEPTEMBER 1991

\begin{tabular}{|c|c|c|c|c|c|c|c|c|c|}
\hline 14 & $\ldots$ & - & 0.14 & 0.14 & 0.69 & 0.055 & 0.019 & 110 & 53 \\
\hline 14 & -- & -- & 0.15 & 0.15 & 0.70 & 0.060 & 0.018 & 130 & 60 \\
\hline 10 & -- & -- & 0.12 & 0.57 & 0.63 & 0.050 & 0.017 & 100 & 62 \\
\hline 15 & -- & -- & 0.11 & 0.58 & 0.71 & 0.065 & 0.019 & 96 & 49 \\
\hline 8.8 & - & - & 0.14 & 0.50 & 0.76 & 0.065 & 0.019 & 130 & 37 \\
\hline 10 & -- & $\cdots$ & 0.08 & 0.40 & 0.63 & 0.065 & 0.022 & 150 & 40 \\
\hline 17 & -- & -- & -- & 0.65 & 0.72 & 0.090 & 0.023 & 83 & 50 \\
\hline 12 & $\cdots$ & -- & 0.11 & 0.58 & 0.79 & 0.075 & 0.038 & 82 & 60 \\
\hline 16 & -- & -- & 0.10 & 0.60 & 0.56 & 0.075 & 0.031 & 110 & 50 \\
\hline 55 & 66 & $<7$ & 0.09 & 0.79 & 0.63 & 0.120 & 0.020 & 120 & 57 \\
\hline 220 & 322 & 26 & 0.06 & 1.3 & 0.94 & 0.310 & 0.019 & 120 & 42 \\
\hline 250 & 368 & 27 & 0.05 & 1.4 & 0.96 & 0.360 & 0.019 & 80 & 37 \\
\hline 320 & 391 & 27 & 0.04 & 1.3 & 0.85 & 0.450 & 0.018 & 68 & 35 \\
\hline 240 & 126 & 21 & 0.06 & 1.2 & 0.71 & 0.330 & 0.029 & 67 & 30 \\
\hline 230 & 244 & 12 & 0.05 & 1.1 & 0.65 & 0.300 & 0.025 & 60 & 24 \\
\hline 190 & 250 & 20 & 0.04 & 1.1 & 0.64 & 0.280 & 0.018 & 54 & 21 \\
\hline 120 & 200 & 15 & 0.04 & 0.87 & 0.74 & 0.210 & 0.018 & 40 & 24 \\
\hline 85 & 113 & 8 & 0.05 & 0.62 & 0.98 & 0.130 & 0.019 & 39 & 30 \\
\hline 120 & 146 & 11 & 0.05 & 0.79 & 0.80 & 0.210 & 0.019 & 41 & 28 \\
\hline 70 & 90 & $<10$ & 0.05 & 0.82 & - - & 0.150 & 0.022 & 41 & 32 \\
\hline 45 & 68 & 6 & 0.06 & 0.59 & 0.87 & 0.100 & 0.022 & 44 & 38 \\
\hline 55 & 80 & 6 & 0.06 & 0.69 & 1.10 & 0.110 & 0.024 & 48 & 50 \\
\hline 36 & 57 & $<5$ & 0.04 & 0.62 & 1.10 & 0.090 & 0.023 & 53 & 50 \\
\hline 65 & 94 & $<10$ & 0.03 & 0.54 & 0.95 & 0.110 & 0.018 & 58 & 40 \\
\hline 65 & 101 & 6 & 0.02 & 0.51 & 0.76 & 0.120 & 0.014 & 45 & 37 \\
\hline 36 & 61 & $<5$ & 0.04 & 0.39 & 0.80 & 0.070 & 0.016 & 44 & 35 \\
\hline 28 & -- & & 0.03 & 0.50 & 0.94 & 0.065 & 0.014 & 45 & 37 \\
\hline
\end{tabular}


Surface-Water Stations

B. Partial-Record and Miscellaneous-Record Sites

431510077363501 Genesee River at Charlotte Pump Station Near Rochester, N.Y.

WATER-QUALITY DATA

\begin{tabular}{|c|c|c|c|c|c|c|c|c|c|c|c|c|c|}
\hline DATE & TIME & $\begin{array}{l}\text { ENDING } \\
\text { TIME }\end{array}$ & $\begin{array}{l}\text { FEET } \\
\text { PER } \\
\text { SECOND }\end{array}$ & $\begin{array}{l}\text { BID- } \\
\text { ITY } \\
\text { (NTU) }\end{array}$ & $\begin{array}{l}\text { SUS- } \\
\text { PENDED } \\
(\mathrm{mg} / \mathrm{L})\end{array}$ & $\begin{array}{l}\text { SUS- } \\
\text { PENDED } \\
(\mathrm{mg} / \mathrm{L})\end{array}$ & $\begin{array}{l}\text { SOLVED } \\
\text { (mg/L } \\
\text { as N) }\end{array}$ & $\begin{array}{c}\text { TOTAL } \\
(\mathrm{mg} / \mathrm{L} \\
\text { as N) }\end{array}$ & $\begin{array}{l}\text { TOTAL } \\
\text { (mg/L } \\
\text { as N) }\end{array}$ & $\begin{array}{l}\text { TOTAL } \\
\text { (mg/L } \\
\text { as P) }\end{array}$ & $\begin{array}{l}\text { SOLVED } \\
(\mathrm{mg} / \mathrm{L} \\
\text { as } \mathrm{P})\end{array}$ & $\begin{array}{l}\text { SOSVED } \\
\text { (mI/L } \\
\text { as Cl) }\end{array}$ & $\begin{array}{l}\text { SOLVED } \\
(\mathrm{mg} / \mathrm{L} \\
\left.\text { as } \mathrm{SO}_{4}\right)\end{array}$ \\
\hline
\end{tabular}

OCT 31

NOV 02

$02-05$

$\begin{array}{llll}07-09 & 1000 & 0700 & 2040 \\ 0810 & 0710 & 2750\end{array}$

09-10 $1000 \quad 1700 \quad 3000$

$10-12 \quad 1800 \quad 0100 \quad 3830$

$\begin{array}{llll}12-13 & 0200 & 0900 & 4650\end{array}$

$\begin{array}{llll}13-16 & 1035 & 0835 & 3240 \\ 16-19 & 0915 & 0215 & 3040\end{array}$

$19 \ldots \quad 1020 \quad \ldots \quad 2790$

$\begin{array}{llll}20-21 & 0925 & 0825 & 2520\end{array}$

21-24 $1015 \quad 0715 \quad 2630$

$\begin{array}{llll}24-25 & 0815 & 0815 & 3080\end{array}$

$\begin{array}{llll}25-26 & 0915 & 0915 & 2860\end{array}$

$\begin{array}{llll}26-27 & 1000 & 0900 \quad 2680\end{array}$

27-28 $1000 \quad 0900 \quad 2500$

$28-29 \quad 0930 \quad 0830 \quad 2440$

$29-30$
IOV 30

NOV $30-$

$\begin{array}{llll}03-04 & 1045 & 0945 & 1620 \\ 04-06 & 0920 & 0935 & 1920\end{array}$

04-06 $0920 \quad 0820 \quad 4400$

$\begin{array}{llll}04-07 & 0920 & 0820 & 4450\end{array}$

$\begin{array}{llll}04-05 & 1035 & 0835 & 3880\end{array}$

07-10 $1030 \quad 0930 \quad 3270$

$\begin{array}{llll}10-12 & 1000 & 1100 & 2470\end{array}$

$\begin{array}{llll}12-13 & 1100 & 2000 & 2290\end{array}$

$17 \ldots \quad 1010 \quad \ldots \quad 3040$

$18 \ldots 0915 \quad \ldots \quad 3350$

$19 \ldots 0730 \quad-$ - 4370

$20 \ldots \quad 0800 \quad-\quad 5660$

$\begin{array}{llll}20-21 & 0815 & 0915 & 5510\end{array}$

$21 \ldots \quad 0930 \quad \ldots \quad 5360$

21-23 $\quad 1600 \quad 0900 \quad 5300$

23-24 $0100 \quad 0900 \quad 6100$

$\begin{array}{llll}24-26 & 0900 & 1200 & 6720\end{array}$

$\begin{array}{llll}24-25 & 0905 & 0805 & 6960\end{array}$

$\begin{array}{llll}24-26 & 0905 & 0805 & 6720 \\ 26-28 & 1300 & 0800 & 5350\end{array}$

$\begin{array}{llll}29-30 & 0935 & 0835 & 5890\end{array}$

30-31 $0935 \quad 0835 \quad 10300$

DEC 31 -

$\begin{array}{rrrr}\text { JAN } 01 & 1000 & 1400 & 12400 \\ 04-05 & 1020 & 0920 & 7350\end{array}$

05-06 $1020 \quad 0920 \quad 7330$

06-07 $1020 \quad 0920 \quad 7120$

07-08 $1030 \quad 0930 \quad 6740$

$\begin{array}{llll}08-09 & 1030 & 0930 & 6120\end{array}$

$\begin{array}{llll}10-11 & 1030 & 0930 & 4490\end{array}$

$11-12 \quad 0930 \quad 0830 \quad 3680$

$\begin{array}{llll}12-13 & 0930 & 0830 & 2990\end{array}$

$\begin{array}{llll}14-15 & 1100 & 1000 & 2550\end{array}$

15-16 $1100 \quad 0800 \quad 2600$

$\begin{array}{llll}16-17 & 0900 & 0900 & 3560\end{array}$

$17 \ldots \quad 0730 \quad \ldots \quad 4500$

17-18 $0900 \quad 0700 \quad 5140$

$\begin{array}{llll}18-20 & 0745 & 0645 & 5420\end{array}$

$\begin{array}{llll}20-22 & 0745 & 0645 & 4790\end{array}$

$\begin{array}{llll}22-23 & 1040 & 0740 & 3440\end{array}$

$\begin{array}{llll}23-25 & 0935 & 0835 & 2540\end{array}$

$\begin{array}{llll}25-26 & 0935 & 0835 & 2120\end{array}$

$\begin{array}{llll}26-27 & 0935 & 0835 & 2020\end{array}$

$27-28$

28-29
$0935 \quad 0835 \quad 2220$

\section{WATER YEAR OCTOBER 1990 TO SEPTEMBER 1991-continued}

\begin{tabular}{|c|c|c|c|c|c|c|c|c|c|}
\hline 22 & -- & -- & 0.04 & 0.48 & 0.98 & 0.055 & 0.043 & 46 & 42 \\
\hline 31 & 50 & $<5$ & 0.09 & 0.70 & 0.98 & 0.060 & 0.023 & 68 & 62 \\
\hline 21 & -- & -- & 0.05 & 0.62 & 1.00 & 0.070 & 0.021 & 81 & 64 \\
\hline 18 & -- & -- & 0.13 & 0.59 & 0.98 & 0.060 & 0.019 & 86 & 71 \\
\hline 26 & -- & -- & 0.08 & 0.70 & 0.88 & 0.085 & 0.020 & 80 & 48 \\
\hline 24 & -- & -- & 0.09 & 0.53 & 0.79 & 0.080 & 0.015 & 60 & 52 \\
\hline 65 & 105 & 11 & 0.06 & 0.68 & 0.92 & 0.130 & 0.013 & 42 & 46 \\
\hline 35 & -- & -- & 0.06 & 0.77 & 0.94 & 0.065 & 0.012 & 45 & 40 \\
\hline 17 & -- & -- & 0.08 & 0.59 & 1.10 & 0.055 & 0.012 & 63 & 44 \\
\hline 18 & -- & $\cdots$ & 0.09 & 0.55 & 1.00 & 0.045 & 0.015 & 56 & 42 \\
\hline 20 & -. & -- & 0.11 & 0.68 & 0.88 & 0.060 & 0.012 & 55 & 39 \\
\hline 7.6 & -- & -. & 0.08 & 0.45 & 0.89 & 0.030 & 0.014 & 68 & 43 \\
\hline 14 & -- & -- & 0.06 & 0.46 & 0.92 & 0.045 & 0.013 & 68 & 44 \\
\hline 23 & -- & -- & 0.05 & 0.61 & 1.00 & 0.055 & 0.012 & 55 & 36 \\
\hline 27 & -- & -- & 0.07 & 0.47 & 0.86 & 0.065 & 0.012 & 54 & 30 \\
\hline 15 & - & -- & 0.09 & 0.57 & 0.86 & 0.050 & 0.013 & 60 & 31 \\
\hline 13 & -- & -- & 0.11 & 0.50 & 0.89 & 0.045 & 0.014 & 66 & 36 \\
\hline 14 & -- & -- & 0.11 & 0.48 & 0.89 & 0.045 & 0.013 & 67 & 37 \\
\hline 12 & -- & -- & 0.13 & 0.60 & 1.10 & 0.045 & 0.012 & 77 & 50 \\
\hline 7.1 & -. & -- & 0.15 & 0.80 & 1.50 & 0.055 & 0.014 & 98 & 60 \\
\hline 65 & 102 & 8 & 0.07 & 1.4 & 1.30 & 0.130 & 0.021 & 80 & 48 \\
\hline 140 & 198 & 15 & 0.06 & 0.91 & 1.00 & 0.190 & 0.013 & 45 & 39 \\
\hline 8.7 & -- & - & 0.16 & 0.80 & 0.94 & 0.050 & 0.012 & 120 & 62 \\
\hline 33 & 49 & 5 & 0.07 & 0.71 & 1.00 & 0.190 & 0.013 & 52 & 44 \\
\hline 14 & -- & -- & 0.11 & 0.99 & 1.20 & 0.055 & 0.013 & 70 & 57 \\
\hline 9.2 & -- & -- & 0.14 & 0.70 & 1.60 & 0.040 & 0.014 & 69 & 57 \\
\hline 10 & - & -- & 0.12 & 0.55 & 1.20 & 0.030 & 0.009 & 72 & 57 \\
\hline 13 & -- & -- & 0.12 & 0.54 & 1.10 & 0.045 & 0.013 & 70 & 47 \\
\hline 13 & -- & -- & 0.12 & 0.54 & 1.10 & 0.045 & 0.013 & 55 & 45 \\
\hline 34 & 60 & $<5$ & 0.11 & 0.65 & 1.40 & 0.095 & 0.019 & 63 & 38 \\
\hline 95 & 171 & 10 & 0.09 & 0.68 & 1.10 & 0.150 & 0.016 & 44 & 33 \\
\hline 160 & 228 & 15 & 0.10 & 0.81 & 0.92 & 0.180 & 0.014 & 37 & 28 \\
\hline 80 & -- & -- & 0.08 & 0.82 & 0.93 & 0.120 & 0.020 & 44 & 31 \\
\hline 32 & $\cdots$ & - & 0.07 & 0.48 & 1.10 & 0.075 & 0.016 & 45 & 31 \\
\hline 110 & 166 & 12 & 0.03 & 0.73 & 1.10 & 0.140 & 0.017 & 29 & 35 \\
\hline 90 & 169 & 12 & 0.05 & 0.85 & 1.30 & 0.160 & 0.021 & 48 & 33 \\
\hline 170 & 290 & 19 & 0.03 & 1.1 & 1.10 & 0.420 & 0.017 & 97 & 28 \\
\hline 75 & 114 & 10 & 0.04 & 0.75 & 1.10 & 0.120 & 0.019 & 35 & 35 \\
\hline 24 & -- & -- & 0.08 & 0.65 & 1.30 & 0.070 & 0.018 & 58 & 42 \\
\hline 150 & 318 & 20 & 0.08 & 1.4 & 1.60 & 0.300 & 0.022 & 52 & 38 \\
\hline 300 & 713 & 44 & 0.09 & 1.3 & 1.50 & 0.550 & 0.026 & 37 & 24 \\
\hline 240 & 341 & 20 & 0.08 & 1.0 & 1.10 & 0.260 & 0.018 & 30 & 29 \\
\hline 210 & 303 & 20 & 0.06 & 0.90 & 1.10 & 0.260 & 0.016 & 31 & 31 \\
\hline 170 & 248 & 16 & 0.06 & 0.80 & 1.20 & 0.230 & 0.017 & 30 & 32 \\
\hline 140 & 199 & 14 & 0.06 & 0.83 & 1.30 & 0.180 & 0.014 & 44 & 29 \\
\hline 100 & 168 & 11 & 0.07 & 0.67 & 1.50 & 0.160 & 0.014 & 34 & 30 \\
\hline 40 & -- & -- & 0.06 & 0.53 & 1.70 & 0.070 & 0.016 & 33 & 35 \\
\hline 32 & 64 & $<5$ & 0.11 & 0.49 & 1.70 & 0.080 & 0.015 & 44 & 36 \\
\hline 26 & -. & -- & 0.11 & 0.54 & 1.70 & 0.080 & 0.012 & 92 & 35 \\
\hline 24 & -- & -- & 0.14 & 0.66 & 1.80 & 0.055 & 0.014 & 61 & 66 \\
\hline 40 & 79 & $<5$ & 0.18 & 0.59 & 1.90 & 0.070 & 0.013 & 73 & 71 \\
\hline 24 & -- & - & 0.17 & 0.63 & 1.70 & 0.055 & 0.013 & 84 & 68 \\
\hline 20 & -- & -- & 0.20 & 0.77 & 2.00 & 0.050 & 0.019 & 80 & 70 \\
\hline 40 & 106 & 7 & 0.17 & 0.69 & 1.70 & 0.110 & 0.016 & 85 & 64 \\
\hline 80 & 148 & 9 & 0.13 & 0.70 & 1.70 & 0.130 & 0.020 & 59 & 44 \\
\hline 34 & 69 & 5 & 0.09 & 0.50 & 1.70 & 0.070 & 0.020 & 55 & 46 \\
\hline 16 & -- & -- & 0.09 & 0.57 & 1.80 & 0.060 & 0.018 & 53 & 47 \\
\hline 15 & -- & - & 0.13 & 0.42 & 2.00 & 0.050 & 0.012 & 55 & 54 \\
\hline 11 & -- & -. & 0.14 & 0.51 & 2.00 & 0.050 & 0.014 & 70 & 66 \\
\hline 11 & -- & -- & 0.18 & 0.60 & 2.10 & 0.040 & 0.013 & 76 & 68 \\
\hline 10 & -- & -- & 0.17 & 0.61 & 2.10 & 0.040 & 0.015 & 72 & 71 \\
\hline 9.9 & -. & -- & 0.14 & 0.67 & 2.00 & 0.045 & 0.019 & 82 & 70 \\
\hline
\end{tabular}




\section{Genesee River at Charlotte Pump Station Near Rochester, N.Y.} WATER-QUALITY DATA

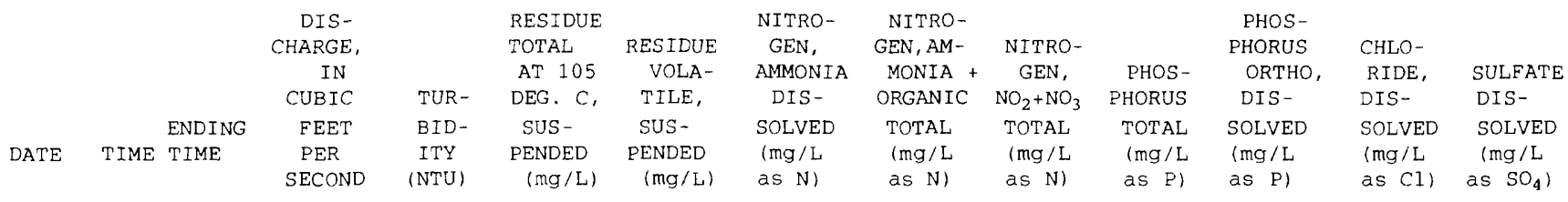

FEB

$\begin{array}{rrrrr}15-18 & 1015 & 1715 & 3170 & 1 \\ 21-22 & 1030 & 0830 & 6160 & 85 \\ 22-23 & 0905 & 2005 & 6130 & 120 \\ 23-25 & 2105 & 0805 & 4770 & 45 \\ \text { MAR } & & & & \end{array}$

WATER YEAR OCTOBER 1990 TO SEPTEMBER 1991-continued

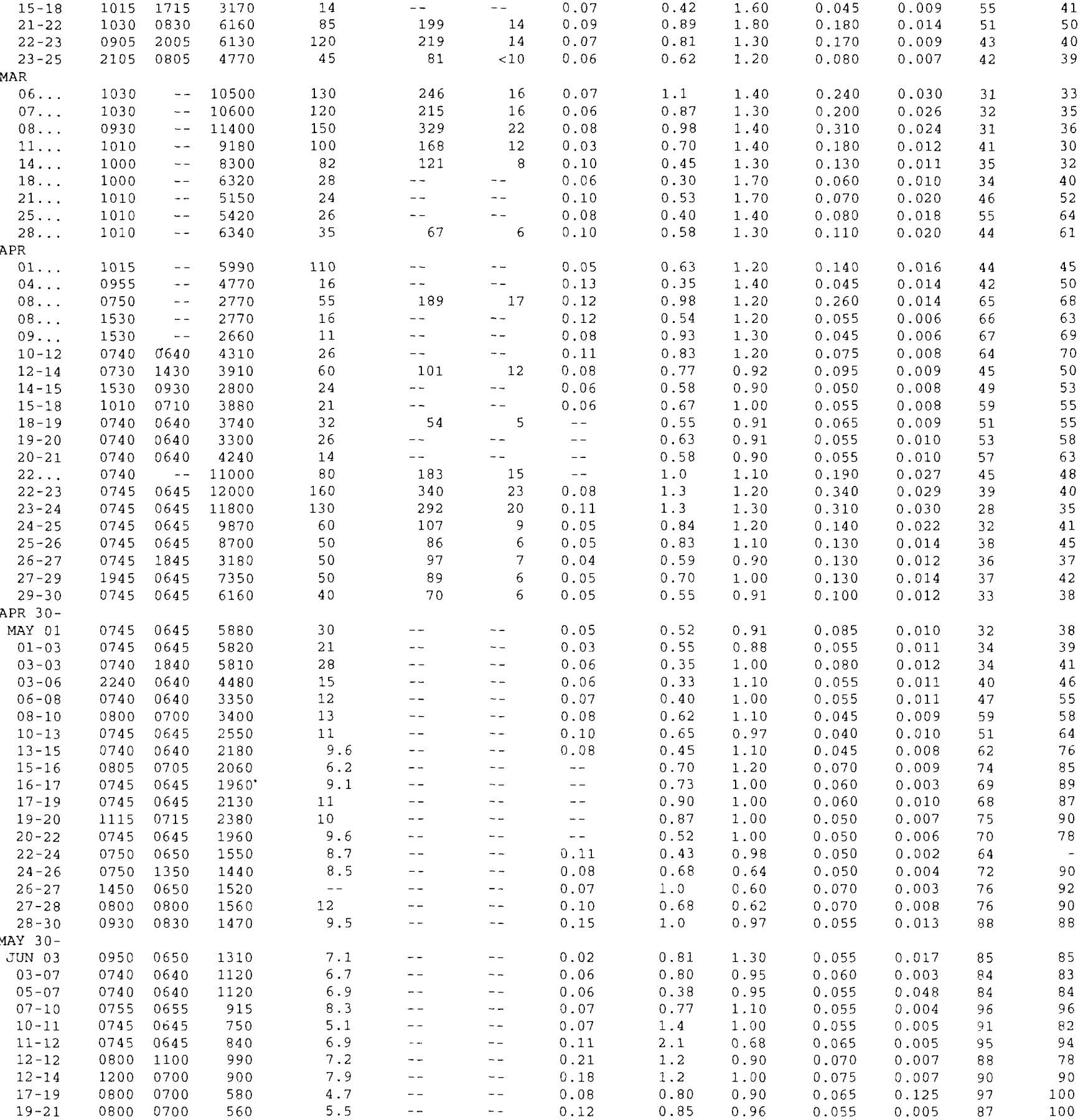




\section{Surface-Water Stations}

\section{B. Partial-Record and Miscellaneous-Record Sites}

\section{Genesee River at Charlotte Pump Station Near Rochester, N.Y.}

\section{WATER-QUALITY DATA}

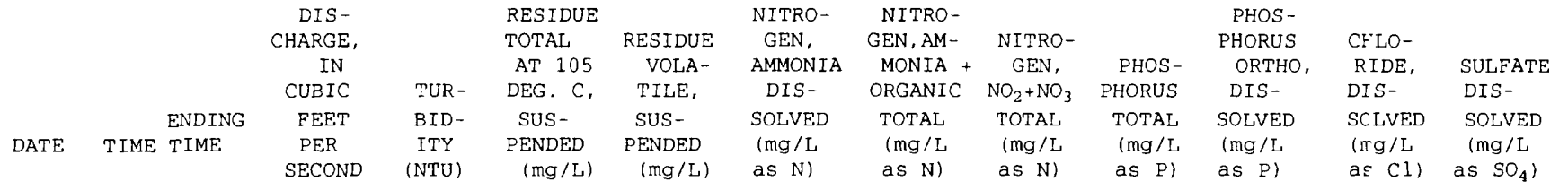

JUN-cont inued

\begin{tabular}{|c|c|c|c|}
\hline $21-24$ & 0755 & . 0655 & 550 \\
\hline $24-26$ & 0745 & 0745 & 490 \\
\hline $26-28$ & 0755 & 0050 & 460 \\
\hline $28 \ldots$ & 0750 & -- & 440 \\
\hline \multicolumn{4}{|l|}{ JUN 28 - } \\
\hline JUL 01 & 0750 & 0650 & 420 \\
\hline $01-03$ & 0805 & 0705 & 430 \\
\hline $03-0.5$ & 0840 & 0740 & 440 \\
\hline $05-06$ & 0905 & 0805 & 470 \\
\hline $06-07$ & 0905 & 0805 & 480 \\
\hline $07-08$ & 0905 & 0805 & 500 \\
\hline $10-12$ & 0900 & 0800 & 530 \\
\hline $12-13$ & 0820 & 1920 & 480 \\
\hline $13-15$ & 2020 & 0720 & 610 \\
\hline $15-17$ & 0745 & 0645 & 620 \\
\hline $17-19$ & 0750 & 0650 & 470 \\
\hline $19-20$ & 0750 & 0050 & 430 \\
\hline $22 \ldots$ & 0745 & -- & 410 \\
\hline $22-24$ & 0750 & 0650 & 390 \\
\hline $24-26$ & 0740 & 0640 & 400 \\
\hline $26-29$ & 0745 & 0645 & 380 \\
\hline $29-31$ & 0810 & 0710 & 360 \\
\hline \multicolumn{4}{|l|}{ JUL $31-$} \\
\hline AUG 02 & 0750 & 0650 & 380 \\
\hline $02-03$ & 0750 & 0650 & 400 \\
\hline $03-04$ & 0750 & 0650 & 390 \\
\hline $04-05$ & 0750 & 0650 & 380 \\
\hline $05-07$ & 0825 & 0725 & 400 \\
\hline $07-09$ & 0750 & 0650 & 370 \\
\hline $08-09$ & 0750 & 0650 & 370 \\
\hline $09-10$ & 0745 & 0645 & 410 \\
\hline $10-11$ & 0745 & 0645 & 510 \\
\hline $11-12$ & 0745 & 0645 & 580 \\
\hline $12-14$ & 0750 & 0650 & 530 \\
\hline $14-15$ & 0750 & 1650 & 440 \\
\hline $16 \ldots$ & 0740 & -- & 400 \\
\hline $16-19$ & 1050 & 0650 & 390 \\
\hline $19-21$ & 0825 & 0725 & 390 \\
\hline $21-22$ & 0750 & 0650 & 450 \\
\hline $22-23$ & 0750 & 0650 & 500 \\
\hline $23-26$ & 0740 & 0640 & 460 \\
\hline $26-26$ & 0845 & 2345 & 410 \\
\hline $27-28$ & 0845 & 2345 & 400 \\
\hline $28-30$ & 0800 & 0700 & 370 \\
\hline $30-31$ & 0750 & 2250 & 360 \\
\hline \multicolumn{4}{|l|}{ AUG $31-$} \\
\hline SEP 02 & 2350 & 1050 & 360 \\
\hline $03 \ldots$ & 0815 & -- & 350 \\
\hline $03-05$ & 0915 & 1015 & 360 \\
\hline $05-09$ & 1020 & 0920 & 370 \\
\hline $10-12$ & 1400 & 1000 & 350 \\
\hline $13-15$ & 0930 & 0230 & 34 \\
\hline $15-16$ & 0330 & 0830 & 340 \\
\hline $16-19$ & 1025 & 0925 & 340 \\
\hline $19-23$ & 1050 & 0950 & 340 \\
\hline $23-25$ & 1030 & 0130 & 36 \\
\hline $25-26$ & 0230 & 0930 & 39 \\
\hline $26-30$ & 1020 & 0920 & 42 \\
\hline
\end{tabular}

WATER YEAR OCTOBER 1990 TO SEPTEMBER 1991-continued

\begin{tabular}{|c|c|c|c|c|c|c|c|c|c|}
\hline 6.8 & -- & - & 0.02 & 0.92 & 0.69 & 0.050 & 0.008 & 88 & 100 \\
\hline 5.3 & -- & -- & 0.04 & 0.80 & 0.76 & 0.050 & 0.011 & 87 & 100 \\
\hline 5.6 & -- & -- & 0.07 & 0.63 & 0.65 & 0.050 & 0.008 & 86 & 100 \\
\hline 4.9 & -- & -. & 0.18 & 0.63 & 0.69 & 0.040 & 0.008 & 76 & 100 \\
\hline 5.5 & -. & -- & 0.17 & 0.75 & 0.71 & 0.040 & 0.011 & 71 & 90 \\
\hline 5.1 & -- & -- & 0.07 & 0.74 & 0.51 & 0.055 & 0.007 & 79 & 90 \\
\hline 7.7 & -- & - & 0.17 & 0.71 & 0.79 & 0.090 & 0.015 & 87 & 95 \\
\hline 6.9 & -- & -- & 0.14 & 0.29 & 0.62 & 0.065 & 0.019 & 85 & 88 \\
\hline 6.7 & -- & -- & 0.14 & 0.37 & 0.61 & 0.080 & 0.019 & 85 & 90 \\
\hline 6.2 & -- & -- & 0.14 & 0.33 & 0.60 & 0.080 & 0.021 & 69 & 82 \\
\hline 4.7 & $\cdots$ & -. & 0.10 & 0.71 & 0.71 & 0.050 & 0.008 & 82 & 110 \\
\hline 3.8 & - & -- & 0.20 & 0.71 & 0.70 & 0.045 & 0.012 & 91 & 170 \\
\hline 2.9 & -- & - & 0.36 & 0.88 & 0.81 & 0.055 & 0.018 & 81 & 150 \\
\hline 3.5 & -- & -- & 0.18 & 0.66 & 0.48 & 0.040 & 0.012 & 75 & 110 \\
\hline 4.3 & - & -- & 0.25 & 0.82 & 0.70 & 0.060 & 0.008 & 84 & 110 \\
\hline 2.8 & -- & -- & 0.22 & 1.0 & 0.73 & 0.055 & 0.004 & 93 & 110 \\
\hline 3.1 & -- & -- & 0.27 & 1.0 & 0.68 & 0.050 & 0.004 & 90 & 90 \\
\hline 2.8 & -- & -- & 0.15 & 0.86 & 0.52 & 0.055 & 0.008 & 74 & \\
\hline 3.7 & -- & -- & 0.26 & 0.91 & 0.50 & 0.065 & 0.018 & 57 & 74 \\
\hline 4.4 & -- & -. & 0.16 & 1.0 & 0.45 & 0.060 & 0.007 & 70 & 78 \\
\hline 6.8 & -. & -. & $<0.01$ & 0.91 & 0.50 & 0.065 & 0.008 & 77 & 82 \\
\hline 7.2 & -- & -- & 0.28 & 0.79 & 0.62 & 0.055 & 0.013 & 70 & 86 \\
\hline 2.8 & -- & -- & 0.25 & 0.89 & 0.54 & 0.050 & 0.014 & 80 & 94 \\
\hline 3.1 & -- & -- & 0.23 & 1.1 & 0.64 & 0.055 & 0.020 & 110 & 94 \\
\hline 2.7 & -- & - & 0.29 & 1.1 & 0.65 & 0.050 & 0.020 & 69 & 96 \\
\hline 3.5 & -- & -- & 0.18 & 0.75 & 0.53 & 0.055 & 0.025 & 110 & 96 \\
\hline 4.6 & -- & -- & 0.21 & 1.1 & 0.55 & 0.080 & 0.020 & 110 & 100 \\
\hline 4.1 & -- & -- & 0.30 & 1.0 & 0.74 & 0.090 & 0.017 & 91 & 100 \\
\hline 12 & -- & -- & 0.38 & 1.2 & 0.74 & 0.130 & 0.022 & 87 & 98 \\
\hline 5.1 & -- & -- & 0.28 & 0.93 & 0.66 & 0.090 & 0.022 & 120 & 92 \\
\hline 5.6 & -- & - & 0.32 & 1.2 & 0.59 & 0.070 & 0.023 & 150 & 94 \\
\hline 6.6 & -- & -- & 0.16 & 0.75 & 0.55 & 0.070 & 0.023 & 170 & 82 \\
\hline 7.3 & -- & -- & 0.18 & 0.85 & 0.54 & 0.060 & 0.016 & 210 & 100 \\
\hline 7.1 & -- & -- & 0.36 & 1.3 & 0.58 & 0.055 & 0.012 & 230 & 110 \\
\hline 8.6 & - & -- & 0.31 & 1.5 & 0.53 & 0.070 & -- & 200 & 92 \\
\hline 9.2 & -- & -- & 0.29 & 1.0 & 0.54 & 0.075 & 0.022 & 200 & 84 \\
\hline 12 & - & -- & 0.30 & 0.88 & 0.49 & 0.075 & 0.024 & 130 & 78 \\
\hline 10 & -. & -- & 0.32 & 0.76 & 0.55 & 0.070 & 0.023 & 100 & 76 \\
\hline 5.6 & -- & -- & 0.29 & 0.89 & 0.58 & 0.130 & 0.022 & 100 & 93 \\
\hline 3.4 & -. & -- & 0.18 & 0.76 & 0.45 & 0.055 & 0.011 & 90 & 75 \\
\hline 4.3 & -. & -- & 0.22 & 0.55 & 0.47 & 0.055 & 0.007 & 160 & 77 \\
\hline 7.9 & -- & -- & 0.24 & 0.74 & 0.49 & 0.050 & 0.007 & 170 & 96 \\
\hline 8.6 & $\cdots$ & -- & 0.21 & 0.84 & 1.00 & 0.065 & 0.012 & 170 & 77 \\
\hline 7.2 & -- & -- & 0.23 & 0.82 & 1.00 & 0.065 & 0.019 & 160 & 79 \\
\hline 9.0 & -- & -- & 0.27 & 1.2 & 0.90 & 0.120 & 0.026 & 120 & 91 \\
\hline 12 & -- & -- & 0.25 & 0.92 & 0.55 & 0.070 & 0.018 & 100 & 34 \\
\hline 2.0 & -- & -- & 0.15 & 0.83 & 0.47 & 0.045 & 0.015 & 100 & 92 \\
\hline 2.7 & -- & -- & 0.20 & 0.77 & 0.64 & 0.065 & 0.036 & 110 & 60 \\
\hline 3.8 & -- & - & 0.26 & 1.3 & -- & 0.055 & 0.023 & 130 & 75 \\
\hline 6.0 & -. & -- & 0.26 & 1.2 & -- & 0.065 & 0.025 & 140 & 84 \\
\hline 22 & -- & -- & 0.12 & 0.75 & 0.65 & 0.075 & 0.033 & 160 & 80 \\
\hline 4.3 & -- & -- & 0.25 & 0.76 & 0.72 & 0.065 & 0.036 & 130 & 76 \\
\hline 4.3 & - & -- & 0.26 & 0.85 & 0.78 & 0.060 & 0.034 & 140 & 30 \\
\hline 5.8 & -. & -- & 0.31 & 0.90 & 0.79 & 0.070 & 0.030 & 150 & \\
\hline 22 & -- & $\ldots$ & 0.31 & 0.78 & 0.84 & 0.055 & 0.026 & 150 & \\
\hline
\end{tabular}

OCT

$\begin{array}{llrr}03 \ldots & 1610 & -- & 540 \\ 04 \cdots & 0720 & -- & 436 \\ 07-10 & 1030 & 0930 & 650\end{array}$

WATER YEAR OCTOBER 1991 TO SEPTEMBER 1992

$\begin{array}{llllllllll}3.3 & -- & -- & 0.260 & 0.94 & 0.98 & 0.045 & 0.010 & 210 & 160 \\ 3.4 & -- & -- & 0.260 & 0.88 & 1.00 & 0.045 & 0.011 & 230 & 120 \\ 8.6 & -- & -- & 0.180 & 0.53 & 0.43 & 0.045 & 0.022 & 160 & 110\end{array}$




\section{Genesee River at Charlotte Pump Station Near Rochester, N.Y.}

WATER-QUALITY DATA

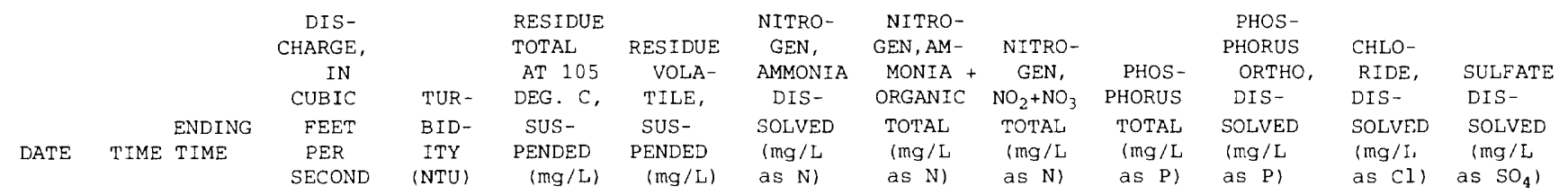

OCT-cont inued

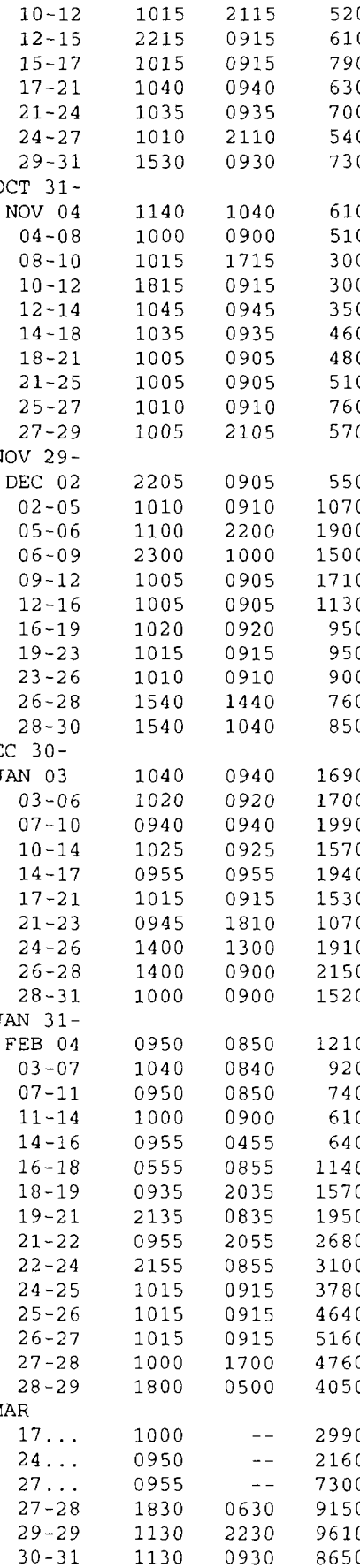

WATER YEAR OCTOBER 1991 TO SEPTEMBER 1992-continued

\begin{tabular}{|c|c|c|c|c|c|c|c|c|c|}
\hline 3.6 & $\cdots$ & -- & 0.29 & 0.90 & 0.78 & 0.055 & 0.025 & 150 & 100 \\
\hline 4.5 & -- & -- & 0.29 & 0.92 & 0.87 & 0.050 & 0.023 & 180 & 86 \\
\hline 18 & -- & -- & 0.25 & 1.5 & 0.79 & -- & 0.024 & 120 & 180 \\
\hline 4.6 & -- & -. & 0.26 & 0.49 & 0.83 & 0.045 & 0.016 & 140 & - \\
\hline 3.5 & -- & 4 & 0.21 & 0.46 & 0.76 & 0.045 & 0.021 & 110 & 84 \\
\hline 3.7 & -- & -- & 0.30 & 0.90 & 0.80 & 0.040 & 0.021 & 130 & 82 \\
\hline 4.1 & -- & -- & 0.29 & 0.82 & 0.64 & 0.040 & 0.017 & 100 & 82 \\
\hline 4.2 & -- & -- & - & 0.80 & -- & 0.055 & 0.016 & 150 & 86 \\
\hline 4.2 & -- & -- & 0.30 & 0.52 & 0.59 & 0.045 & 0.020 & 97 & 57 \\
\hline 3.2 & -- & -- & 0.25 & 0.75 & 0.72 & 0.035 & 0.016 & 140 & 89 \\
\hline 8.5 & -- & -- & 0.26 & 0.88 & 0.77 & 0.055 & 0.014 & 160 & 94 \\
\hline 2.8 & -- & -- & 0.52 & 1.0 & 0.87 & 0.040 & 0.015 & 200 & 110 \\
\hline 4.2 & -- & -- & 0.90 & 1.2 & 0.92 & 0.050 & 0.015 & 150 & 110 \\
\hline 1.5 & -- & -- & 0.93 & 1.4 & 0.46 & 0.045 & 0.012 & 150 & 140 \\
\hline 2.5 & -- & -- & -. & 0.83 & 0.77 & 0.035 & 0.012 & 170 & 100 \\
\hline 3.5 & -- & -- & 0.59 & -- & 0.99 & 0.055 & 0.019 & 250 & 130 \\
\hline 3.7 & -- & -- & -- & 0.56 & 0.67 & 0.040 & 0.020 & 200 & 100 \\
\hline 3.6 & -- & -- & -- & 0.50 & 0.73 & 0.035 & 0.014 & 200 & 91 \\
\hline 8.9 & -- & -- & 0.42 & - & 0.85 & 0.060 & 0.016 & 190 & 120 \\
\hline 5.8 & -- & -- & 0.42 & 0.86 & 0.83 & 0.055 & 0.026 & 200 & - \\
\hline 41 & -- & -- & 0.39 & 0.83 & 0.79 & 0.080 & 0.016 & 160 & . \\
\hline 17 & - - & -- & 0.22 & 1.0 & 0.90 & 0.050 & 0.024 & 210 & 90 \\
\hline 17 & -. & -- & 0.26 & 0.84 & 0.91 & 0.050 & 0.016 & 180 & 70 \\
\hline 7.5 & -- & -- & 0.32 & 0.95 & 1.10 & 0.040 & 0.017 & 180 & 93 \\
\hline 7.0 & -- & -. & 0.40 & 0.95 & 1.10 & 0.045 & 0.019 & 160 & 83 \\
\hline 6.3 & -- & -. & 0.24 & 0.79 & 0.96 & 0.035 & 0.016 & 130 & 87 \\
\hline 6.1 & -- & -- & 0.22 & 0.82 & 0.97 & 0.035 & 0.014 & 130 & 35 \\
\hline 9.0 & -- & -- & 0.24 & 0.88 & 1.10 & 0.050 & 0.014 & 120 & 50 \\
\hline 13 & -- &.- & 0.20 & 0.82 & 1.10 & 0.045 & 0.017 & 140 & 100 \\
\hline 18 & -- & -- & 0.20 & 0.66 & 1.20 & 0.040 & 0.016 & 79 & 74 \\
\hline 16 & -- & -- & 0.21 & 0.50 & 1.20 & 0.045 & 0.027 & 70 & 77 \\
\hline 8.1 & - - & -- & 0.23 & 0.61 & 1.50 & 0.035 & 0.016 & 75 & 80 \\
\hline 6.4 & -- & -- & 0.23 & 0.59 & 1.30 & 0.050 & 0.029 & 87 & 84 \\
\hline 25 & -- & -- & 0.37 & 0.58 & 1.20 & 0.050 & 0.014 & 74 & 72 \\
\hline 14 & -- & -- & 0.27 & 0.75 & 1.30 & 0.055 & 0.020 & 91 & 97 \\
\hline 7.0 & -- & - & 0.31 & 0.83 & 1.10 & 0.055 & 0.026 & 130 & 93 \\
\hline 1.2 & -- & - - & 0.17 & 0.47 & 1.20 & 0.040 & 0.013 & 93 & 77 \\
\hline 15 & -- & -- & 0.25 & 0.61 & 1.10 & 0.050 & 0.021 & 75 & 78 \\
\hline 5.9 & -- & -- & 0.26 & 0.54 & 1.20 & 0.050 & 0.025 & 77 & 80 \\
\hline 4.5 & -- & -- & 0.26 & 0.60 & 1.20 & -- & 0.022 & 100 & 89 \\
\hline 4.2 & $<5$ & $<5$ & 0.39 & 0.90 & 1.30 & 0.050 & 0.022 & 130 & 100 \\
\hline 2.6 & -- & -- & 0.29 & 0.79 & 1.00 & 0.035 & 0.015 & 130 & 97 \\
\hline 3.3 & -- & -. & 0.26 & 0.60 & 0.90 & 0.055 & 0.022 & 100 & 69 \\
\hline 4.8 & -- & - - & 0.52 & 1.1 & 1.20 & 0.050 & 0.022 & 170 & 110 \\
\hline 4.4 & -- & -- & 0.36 & 1.1 & 1.40 & 0.060 & 0.029 & 180 & 110 \\
\hline 6.4 & - & -- & 0.32 & 1.1 & 1.70 & 0.060 & 0.028 & 120 & 91 \\
\hline 9.0 & - - & -- & 0.24 & 0.66 & 1.70 & 0.055 & 0.024 & 96 & 90 \\
\hline 26 & -- & -- & 0.17 & 0.73 & 1.80 & 0.075 & 0.022 & 82 & 78 \\
\hline 24 & -- & -- & 0.13 & 0.57 & 1.80 & 0.060 & 0.021 & 71 & 73 \\
\hline 19 & -- & -- & 0.12 & 0.54 & 1.90 & 0.060 & 0.018 & 65 & 71 \\
\hline 38 & 42 & -- & 0.11 & 0.60 & 1.40 & 0.105 & 0.014 & 54 & 59 \\
\hline 26 & -- & -- & 0.11 & 0.65 & 1.30 & 0.110 & 0.016 & 49 & 60 \\
\hline 22 & -- & $\cdots$ & 0.13 & 0.55 & 1.40 & 0.085 & 0.014 & 51 & 60 \\
\hline 12 & -- & -- & 0.13 & 0.90 & 1.40 & 0.045 & 0.012 & 58 & 69 \\
\hline 6.7 & -- & -- & 0.13 & 0.45 & 1.70 & 0.030 & 0.012 & 83 & 75 \\
\hline 22 & -- & -- & 0.17 & 0.78 & 1.20 & 0.100 & 0.020 & 100 & 75 \\
\hline 85 & 198 & 20 & 0.04 & 1.7 & 2.30 & 0.210 & 0.005 & 78 & 57 \\
\hline 75 & 145 & 20 & 0.10 & 2.1 & 2.30 & 0.170 & 0.003 & 56 & 47 \\
\hline 55 & 97 & 10 & 0.06 & 1.0 & 2.50 & 0.110 & 0.013 & 55 & 53 \\
\hline
\end{tabular}


Surface-Water Stations

B. Partial-Record and Miscellaneous-Record Sites

431510077363501 Genesee River at Charlotte Pump Station Near Rochester, N."r. WATER-QUALITY DATA

\begin{tabular}{|c|c|c|c|c|c|c|c|c|c|c|c|c|c|}
\hline & & & $\begin{array}{c}\text { DIS- } \\
\text { CHARGE, } \\
\text { IN } \\
\text { CUBIC }\end{array}$ & TUR- & $\begin{array}{l}\text { RESIDUE } \\
\text { TOTAL } \\
\text { AT } 105 \\
\text { DEG. C, }\end{array}$ & $\begin{array}{c}\text { RESIDUE } \\
\text { VOLA- } \\
\text { TILE, }\end{array}$ & $\begin{array}{l}\text { NITRO- } \\
\text { GEN, } \\
\text { AMMONIA } \\
\text { DIS- }\end{array}$ & $\begin{array}{l}\text { NITRO- } \\
\text { GEN, AM- } \\
\text { MONIA + } \\
\text { ORGANIC }\end{array}$ & $\begin{array}{c}\text { NITRO- } \\
\text { GEN, } \\
\mathrm{NO}_{2}+\mathrm{NO}_{3}\end{array}$ & $\begin{array}{l}\text { PHOS- } \\
\text { PHORUS }\end{array}$ & $\begin{array}{l}\text { PHOS- } \\
\text { PHORUS } \\
\text { ORTHO, } \\
\text { DIS- }\end{array}$ & $\begin{array}{l}\text { CHLO- } \\
\text { RIDE, } \\
\text { EIS- }\end{array}$ & $\begin{array}{l}\text { SULFATE } \\
\text { DIS- }\end{array}$ \\
\hline ATE & TIME & $\begin{array}{l}\text { ENDING } \\
\text { TIME }\end{array}$ & $\begin{array}{l}\text { FEET } \\
\text { PER } \\
\text { SECOND }\end{array}$ & $\begin{array}{l}\text { BID- } \\
\text { ITY } \\
\text { (NTU) }\end{array}$ & $\begin{array}{l}\text { SUS- } \\
\text { PENDED } \\
(\mathrm{mg} / \mathrm{L})\end{array}$ & $\begin{array}{l}\text { SUS- } \\
\text { PENDED } \\
(\mathrm{mg} / \mathrm{L})\end{array}$ & $\begin{array}{l}\text { SOLVED } \\
\text { (mg/L } \\
\text { as N) }\end{array}$ & $\begin{array}{l}\text { TOTAL } \\
\text { (mg/L } \\
\text { as N) }\end{array}$ & $\begin{array}{l}\text { TOTAL } \\
\text { (mg/L } \\
\text { as N) }\end{array}$ & $\begin{array}{l}\text { TOTAL } \\
(\mathrm{mg} / \mathrm{L} \\
\text { as P) }\end{array}$ & $\begin{array}{l}\text { SOLVED } \\
\text { (mg/L } \\
\text { as P) }\end{array}$ & $\begin{array}{l}\text { SOLVED } \\
\text { (mg/L } \\
\text { as Cl) }\end{array}$ & $\begin{array}{l}\text { SOLVED } \\
(\mathrm{mg} / \mathrm{L} \\
\left.\text { as } \mathrm{SO}_{4}\right)\end{array}$ \\
\hline
\end{tabular}

MAR-cont inued

WATER YEAR OCTOBER 1991 TO SEPTEMBER 1992-continued

\begin{tabular}{|c|c|c|c|}
\hline $30 \ldots$ & 1600 & -. & 8810 \\
\hline \multicolumn{4}{|l|}{ MAR 31- } \\
\hline APR 02 & 1005 & 1305 & 7810 \\
\hline $02 \ldots$ & 0730 & -- & 7230 \\
\hline $03-07$ & 1015 & 0915 & 6380 \\
\hline $07-10$ & 1555 & 0855 & 5880 \\
\hline $10-11$ & 0950 & 1050 & 6620 \\
\hline $14-16$ & 0945 & 1330 & 4570 \\
\hline $16-17$ & 1400 & 2100 & 5320 \\
\hline $17-19$ & 2200 & 0500 & 6800 \\
\hline $19-20$ & 0600 & 1000 & 7220 \\
\hline $20-22$ & 0955 & 0855 & 7410 \\
\hline $22-24$ & 0955 & 0855 & 7640 \\
\hline $24-26$ & 0955 & 0855 & 7970 \\
\hline $26-28$ & 0955 & 0855 & 7990 \\
\hline $28-29$ & 0955 & 2055 & 7180 \\
\hline \multicolumn{4}{|l|}{ APR 29- } \\
\hline MAY 01 & 2155 & 0855 & 6770 \\
\hline $01-02$ & 0940 & 1640 & 6970 \\
\hline $05-06$ & 1005 & 0905 & 7800 \\
\hline $06-07$ & 1005 & 0905 & 7560 \\
\hline $07-08$ & 1005 & 0905 & 6920 \\
\hline $08-09$ & 1035 & 1735 & 6360 \\
\hline $09-11$ & 1835 & 0135 & 4430 \\
\hline $11-12$ & 0235 & 0935 & 2800 \\
\hline $12-13$ & 1015 & 0915 & 2560 \\
\hline $13-14$ & 1015 & 0915 & 2280 \\
\hline $14-15$ & 1015 & 0915 & 2120 \\
\hline $15-17$ & 0945 & 0845 & 1980 \\
\hline $17-19$ & 0945 & 0845 & 1820 \\
\hline $19-22$ & 0955 & 0855 & 1950 \\
\hline $22-24$ & 0755 & 0655 & 1470 \\
\hline $24-26$ & 0755 & 0655 & 1370 \\
\hline $26-27$ & 1020 & 2120 & 1540 \\
\hline $27-29$ & 2220 & 0920 & 1400 \\
\hline $29-31$ & 0940 & 0840 & 1410 \\
\hline \multicolumn{4}{|l|}{ MAY 31- } \\
\hline JUN 02 & 0940 & 0840 & 1660 \\
\hline $02-03$ & 1005 & 2105 & 1910 \\
\hline $03-05$ & 2205 & 0905 & 1740 \\
\hline $05-07$ & 0950 & 0850 & 1430 \\
\hline $07-09$ & 0950 & 0850 & 1550 \\
\hline $09-10$ & 0955 & 2055 & 1470 \\
\hline $10-12$ & 2155 & 0855 & 1170 \\
\hline $12-14$ & 1010 & 0110 & 940 \\
\hline $14-15$ & 0210 & 1510 & 890 \\
\hline $15-19$ & 1520 & 0920 & 790 \\
\hline $19-21$ & 1010 & 0910 & 820 \\
\hline $21-23$ & 1010 & 0910 & 900 \\
\hline $23-24$ & 1005 & 2105 & 740 \\
\hline $24-26$ & 2205 & 0905 & 750 \\
\hline $26-28$ & 0955 & 0855 & 660 \\
\hline $28-30$ & 0955 & 0855 & 710 \\
\hline \multicolumn{4}{|l|}{ JUN $30-$} \\
\hline JUL 02 & 1010 & 0810 & 640 \\
\hline $02-04$ & 0910 & 0810 & 570 \\
\hline $04-06$ & 0910 & 0810 & 620 \\
\hline $06-07$ & 0855 & 2250 & 530 \\
\hline $08-10$ & 0755 & 0855 & 640 \\
\hline $10-12$ & 0945 & 0945 & 810 \\
\hline $13 \ldots$ & 0920 & -- & 710 \\
\hline $13 \ldots$ & 1030 & -- & 710 \\
\hline $13-14$ & 1540 & 0640 & 930 \\
\hline $14-15$ & 0800 & 2300 & 1580 \\
\hline
\end{tabular}

\begin{tabular}{|c|c|c|c|c|c|}
\hline 65 & -- & -- & 0.07 & 0.82 & 2.60 \\
\hline 30 & 63 & 7 & 0.02 & 0.83 & 2.40 \\
\hline 40 & 61 & 6 & 0.10 & 0.73 & 2.50 \\
\hline 18 & -- & -- & 0.03 & 0.58 & 1.70 \\
\hline 22 & -. & -- & 0.10 & 0.68 & 1.70 \\
\hline 34 & 71 & 7 & 0.07 & 0.69 & 1.40 \\
\hline 3.5 & $\ldots$ & -- & 0.05 & 0.83 & 1.80 \\
\hline 34 & 64 & 7 & -- & 0.61 & 1.30 \\
\hline 36 & 72 & 7 & -- & 0.61 & 1.30 \\
\hline 45 & 72 & 6 & -- & 0.62 & 1.30 \\
\hline 37 & 61 & 6 & 0.05 & 0.55 & 1.30 \\
\hline 50 & 84 & 8 & 0.06 & 0.61 & 1.10 \\
\hline 38 & 72 & 7 & 0.06 & 0.49 & 0.97 \\
\hline 55 & 104 & 9 & 0.05 & 0.62 & 1.10 \\
\hline 28 & -- & -- & 0.06 & 0.44 & 1.10 \\
\hline 50 & 58 & 5 & 0.08 & 0.49 & 0.90 \\
\hline 55 & 87 & 8 & 0.06 & 0.59 & 0.92 \\
\hline 37 & 58 & 8 & 0.05 & 0.66 & 1.10 \\
\hline 65 & 87 & 8 & 0.07 & 0.66 & 1.00 \\
\hline 90 & 96 & 11 & 0.09 & 0.66 & 0.89 \\
\hline 48 & -- & -- & 0.10 & 0.53 & 0.87 \\
\hline 22 & -- & -- & 0.07 & 0.44 & 0.90 \\
\hline 18 & -- & -- & 0.10 & 0.50 & 1.10 \\
\hline 12 & -- & -- & 0.12 & 0.59 & 1.10 \\
\hline 16 & $\ldots$ & -- & 0.15 & 0.60 & 1.10 \\
\hline 14 & -- & -- & 0.17 & 0.59 & 1.10 \\
\hline 7.2 & -- & -- & 0.13 & 0.55 & 1.00 \\
\hline 7.2 & -- & -- & 0.11 & 0.54 & 1.10 \\
\hline 11 & -- & -- & 0.15 & 0.68 & 1.10 \\
\hline 8.2 & -- & -- & 0.19 & 0.69 & 1.00 \\
\hline 11 & -- & -- & 0.11 & 0.60 & 0.89 \\
\hline 7.0 & -- & -- & 0.10 & 0.66 & 0.89 \\
\hline 7.0 & -- & -- & 0.14 & 0.64 & 0.97 \\
\hline 5.7 & - & -- & 0.17 & 0.62 & 1.10 \\
\hline 7.4 & -- & -- & 0.18 & 0.62 & 1.10 \\
\hline 7.1 & -- & -- & 0.13 & 0.64 & 1.10 \\
\hline 6.7 & -- & $\ldots$ & 0.13 & 0.62 & 0.91 \\
\hline 5.1 & -- & -- & 0.15 & 0.72 & 1.00 \\
\hline 6.1 & -- & -- & 0.14 & 0.64 & 0.80 \\
\hline 4.9 & -- & -- & 0.12 & 0.69 & 0.89 \\
\hline 3.3 & -- & -- & 0.14 & 0.97 & 0.82 \\
\hline 4.2 & -- & -- & 0.14 & 0.81 & 0.76 \\
\hline 4.4 & -- & -- & 0.11 & 0.88 & 0.80 \\
\hline 7.3 & - & -- & 0.17 & 1.1 & 0.73 \\
\hline 5.0 & -- & -- & 0.25 & 0.95 & 0.70 \\
\hline 5.4 & -- & -- & 0.25 & 0.92 & 0.67 \\
\hline 7.0 & -- & -- & 0.28 & 1.1 & 0.74 \\
\hline 6.5 & -- & -- & 0.36 & 1.4 & 0.92 \\
\hline 5.4 & -- & -- & 0.30 & 1.5 & 0.88 \\
\hline 4.3 & -- & -- & 0.17 & 1.0 & 0.86 \\
\hline 3.6 & -- & -- & 0.14 & 0.85 & 0.90 \\
\hline 4.7 & -- & -- & 0.12 & 0.88 & 0.84 \\
\hline 6.7 & -- & -- & 0.13 & 0.77 & 0.74 \\
\hline 2.9 & -- & -- & 0.14 & 0.81 & 0.66 \\
\hline 4.0 & 9 & $<5$ & 0.14 & 0.87 & 0.67 \\
\hline 4.5 & -- & -- & 0.25 & 0.94 & 0.90 \\
\hline 5.2 & -- & -- & 0.23 & 0.87 & 0.72 \\
\hline 3.3 & $\cdots$ & -- & 0.23 & 0.93 & 0.80 \\
\hline 5.5 & -- & -- & 0.15 & 0.81 & 0.73 \\
\hline 6.8 & -- & -- & 0.20 & 0.93 & - \\
\hline
\end{tabular}

\begin{tabular}{|c|c|c|c|}
\hline 0.130 & 0.020 & 60 & 52 \\
\hline 0.090 & 0.004 & 55 & 54 \\
\hline 0.100 & 0.015 & 53 & 54 \\
\hline 0.040 & 0.004 & 43 & 49 \\
\hline 0.065 & 0.009 & 40 & 49 \\
\hline 0.130 & 0.010 & 31 & 46 \\
\hline 0.130 & 0.095 & 92 & 55 \\
\hline 0.095 & 0.012 & 58 & 56 \\
\hline 0.095 & 0.011 & 47 & 55 \\
\hline 0.110 & 0.011 & 43 & 53 \\
\hline 0.120 & 0.013 & 34 & 50 \\
\hline 0.100 & 0.012 & 35 & 48 \\
\hline 0.090 & 0.011 & 40 & 45 \\
\hline 0.150 & 0.012 & 28 & 45 \\
\hline 0.080 & 0.018 & 36 & 45 \\
\hline 0.085 & 0.018 & 36 & 42 \\
\hline 0.095 & 0.012 & 35 & 39 \\
\hline 0.100 & 0.017 & 39 & 46 \\
\hline 0.130 & 0.034 & 29 & 43 \\
\hline 0.150 & 0.036 & 31 & 45 \\
\hline 0.085 & 0.010 & 35 & 47 \\
\hline 0.050 & 0.011 & 33 & 53 \\
\hline 0.045 & 0.013 & 35 & 69 \\
\hline 0.050 & 0.019 & 34 & 75 \\
\hline 0.060 & 0.019 & 34 & 75 \\
\hline 0.055 & 0.014 & 34 & 70 \\
\hline 0.045 & 0.013 & 37 & 80 \\
\hline 0.045 & 0.013 & 79 & 80 \\
\hline 0.045 & 0.010 & 84 & 93 \\
\hline 0.040 & 0.005 & 73 & 98 \\
\hline 0.040 & 0.005 & 56 & 100 \\
\hline 0.030 & 0.005 & -- & 96 \\
\hline 0.030 & 0.006 & $\cdots$ & 110 \\
\hline 0.035 & 0.005 & 78 & 120 \\
\hline 0.040 & 0.009 & 89 & 120 \\
\hline 0.040 & 0.010 & 74 & 110 \\
\hline 0.045 & 0.014 & -- & 96 \\
\hline 0.040 & 0.022 & 82 & 92 \\
\hline 0.060 & 0.016 & 87 & 92 \\
\hline 0.045 & 0.016 & 46 & 100 \\
\hline 0.035 & 0.008 & 81 & 110 \\
\hline 0.040 & 0.004 & 78 & 100 \\
\hline 0.040 & 0.004 & 99 & 110 \\
\hline 0.040 & 0.003 & 98 & 100 \\
\hline 0.050 & 0.010 & 87 & 92 \\
\hline 0.045 & 0.011 & $\because 9$ & 98 \\
\hline 0.030 & 0.007 & 12.0 & 110 \\
\hline 0.045 & 0.010 & 12.0 & 120 \\
\hline-- & 0.008 & 12.0 & 110 \\
\hline-- & 0.004 & 93 & 100 \\
\hline 0.040 & 0.005 & 97 & 120 \\
\hline 0.040 & 0.002 & 100 & 130 \\
\hline 0.050 & 0.007 & 24 & 110 \\
\hline 0.045 & 0.016 & 83 & 110 \\
\hline 0.050 & 0.015 & 83 & 120 \\
\hline 0.050 & 0.015 & $s 0$ & 110 \\
\hline 0.040 & 0.016 & 86 & 130 \\
\hline 0.040 & 0.010 & $\subseteq 4$ & 130 \\
\hline 0.055 & 0.011 & 83 & 130 \\
\hline-- & 0.012 & 87 & 120 \\
\hline
\end{tabular}


Surface-Water Stations

B. Partial-Record and Miscellaneous-Record Sites

431510077363501 Genesee River at Charlotte Pump Station Near Rochester, N.Y. WATER-QUALITY DATA

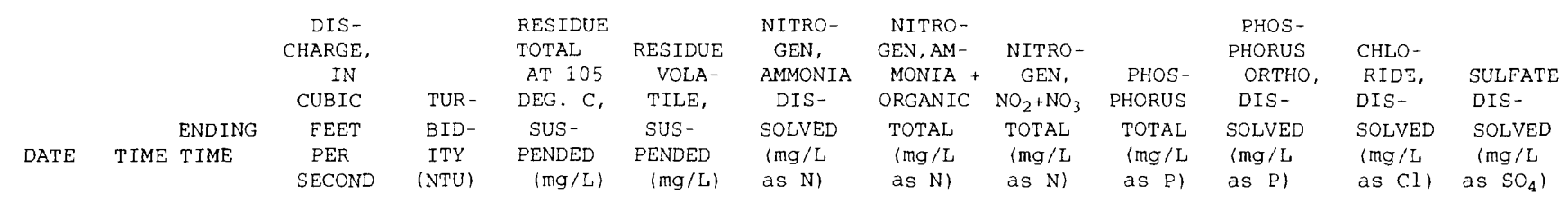

JUL-cont inued

$\begin{array}{rrrr}17-18 & 0800 & 1900 & 5850 \\ 18-21 & 2000 & 0700 & 2830 \\ 21-22 & 0750 & 1850 & 3560 \\ 22-24 & 1950 & 0650 & 4090 \\ 24-25 & 0800 & 1900 & 5100 \\ 25-27 & 2000 & 0700 & 5040 \\ 27-29 & 0800 & 0700 & 5470 \\ 29-31 & 0800 & 0700 & 5930 \\ \text { JUL } 31- & & & \\ \text { AUG 02 } & 0800 & 0700 & 7170 \\ 02-04 & 0800 & 0700 & 7110 \\ 04-05 & 0810 & 1910 & 7720 \\ 05-07 & 2010 & 0710 & 6960 \\ 07-09 & 1015 & 0915 & 5430 \\ 09-11 & 1015 & 0915 & 4900 \\ 11-12 & 0810 & 1910 & 5560 \\ 12-14 & 2010 & 0710 & 5370 \\ 14-15 & 1005 & 2105 & 3520 \\ 17-18 & 1230 & 0830 & 1770 \\ 18-21 & 0945 & 0845 & 1440 \\ 21-21 & 0935 & 1435 & 1230 \\ 24 . & 1000 & -- & 1010 \\ 24-25 & 1010 & 0910 & 1060 \\ 25-26 & 0950 & 2050 & 1140 \\ 26-28 & 2150 & 0850 & 1550 \\ 28-30 & 0945 & 0845 & 4050 \\ \text { AUG 30- } & & & \\ \text { SEP 01 } & 0945 & 945 & 4300 \\ 01-04 & 1045 & 145 & 2240 \\ 04-06 & 1005 & 9905 & 2390 \\ 06-08 & 1005 & 0905 & 1820 \\ 08-10 & 1110 & 1010 & 1400 \\ 11-15 & 1015 & 0915 & 1140 \\ 15-18 & 0955 & 0855 & 910 \\ 18-22 & 0940 & 0840 & 1680 \\ 22-23 & 1010 & 1810 & 3780 \\ 25-27 & 1005 & 0905 & 5670 \\ 27-29 & 1405 & 0905 & 5060 \\ 29-30 & 1025 & 2125 & 3810 \\ \text { SEP 30- } & & & \\ 0 \text { P } 02 & 2225 & 0925 & 1850\end{array}$

OCT $02 \quad 2225 \quad 0925 \quad 1850$

OCT

$\begin{array}{llll}02-03 & 0935 & 1635 & 1740 \\ 03-05 & 1735 & 0335 & 1270 \\ 06-09 & 1100 & 1000 & 1100 \\ 09-11 & 1420 & 1320 & 1810 \\ 11-13 & 1420 & 0920 & 2330 \\ 13-15 & 1000 & 0900 & 1780 \\ 15-17 & 0955 & 0855 & 1250 \\ 17-19 & 0955 & 0855 & 2420 \\ 19-22 & 0950 & 0850 & 1660 \\ 22-24 & 0950 & 0850 & 1860 \\ 24-26 & 0950 & 0850 & 4170 \\ 26-27 & 0955 & 2055 & 4510 \\ 27-29 & 2155 & 0855 & 3000 \\ 29-31 & 1215 & 1115 & 2120 \\ \text { CT } 31- & & & \\ \text { NOV } 02 & 1215 & 0915 & 2210 \\ 02-03 & 0935 & 1135 & 2580 \\ 05-07 & 1000 & 0900 & 6610 \\ 07-09 & 1000 & 0800 & 5760 \\ 09-12 & 0940 & 0840 & 3020 \\ 12-14 & 0930 & 0830 & 3610 \\ 14-16 & 0930 & 0830 & 6120\end{array}$

WATER YEAR OCTOBER 1991 TO SEPTEMBER 1992-continued

\begin{tabular}{|c|c|c|c|c|c|c|c|c|}
\hline 24 & $\cdots$ & -- & 0.10 & 0.81 & -- & 0.100 & 0.028 & 50 \\
\hline 85 & 134 & 12 & 0.07 & 1.1 & - - & 0.190 & 0.033 & 67 \\
\hline 39 & 50 & 5 & 0.10 & 0.73 & 1.50 & -- & 0.021 & 84 \\
\hline 37 & 49 & 5 & 0.11 & 0.79 & 1.30 & $\ldots$ & 0.024 & 100 \\
\hline 60 & 74 & 7 & 0.07 & 0.49 & 1.30 & 0.150 & 0.030 & 74 \\
\hline 38 & 47 & 5 & 0.08 & 0.71 & 1.30 & 0.110 & 0.019 & 71 \\
\hline 37 & 55 & 6 & 0.06 & 0.73 & 1.10 & 0.130 & 0.028 & 39 \\
\hline 38 & 58 & 6 & 0.07 & 0.74 & 0.94 & 0.130 & 0.028 & 31 \\
\hline 75 & 107 & 9 & 0.06 & 0.89 & 1.10 & 0.150 & 0.040 & 34 \\
\hline 80 & 114 & 10 & 0.06 & 0.85 & 0.93 & 0.130 & 0.040 & 36 \\
\hline 60 & 91 & 9 & 0.03 & 0.76 & 0.76 & 0.150 & 0.035 & 37 \\
\hline 50 & 84 & 9 & 0.05 & 0.87 & 0.85 & 0.140 & 0.033 & 35 \\
\hline 28 & -- & -- & 0.04 & 0.69 & 0.67 & 0.100 & 0.024 & 37 \\
\hline 26 & -- & -- & 0.04 & 0.67 & 0.66 & 0.075 & 0.024 & 40 \\
\hline 22 & -- & -. & 0.05 & 0.50 & 0.73 & 0.095 & 0.024 & 34 \\
\hline 26 & -- & -- & 0.04 & 0.56 & 0.51 & 0.100 & 0.022 & 36 \\
\hline 14 & - & - & 0.05 & 0.52 & 0.68 & 0.060 & 0.021 & 39 \\
\hline 18 & -- & -- & 0.09 & 0.71 & 0.91 & 0.080 & 0.020 & 62 \\
\hline 13 & -- & -- & 0.07 & 0.60 & 1.10 & 0.055 & 0.024 & 73 \\
\hline 8.6 & -- & -- & 0.07 & 0.66 & 1.10 & 0.060 & 0.022 & 75 \\
\hline 7.4 & -- & $\cdots$ & 0.13 & 0.85 & 1.00 & 0.050 & 0.016 & 95 \\
\hline 8.4 & -- & -- & 0.13 & 0.81 & 0.92 & 0.060 & 0.009 & 85 \\
\hline 6.7 & -- & -- & 0.10 & 0.53 & 0.83 & 0.050 & 0.008 & 86 \\
\hline 8.7 & 21 & $<5$ & 0.17 & 0.61 & 0.88 & 0.050 & 0.011 & 90 \\
\hline 13 & -- & -- & 0.11 & 0.67 & 0.78 & 0.085 & 0.030 & 80 \\
\hline 27 & 41 & $<5$ & 0.06 & 0.81 & 0.74 & 0.130 & 0.043 & 51 \\
\hline 15 & 23 & $<5$ & 0.09 & 0.73 & 0.70 & 0.110 & 0.043 & 49 \\
\hline 10 & -- & -- & 0.09 & 0.63 & 0.84 & 0.070 & 0.031 & 97 \\
\hline 14 & -- & -- & 0.08 & 0.58 & 0.80 & 0.080 & 0.033 & 74 \\
\hline 15 & -- & - & 0.08 & 0.70 & 0.72 & 0.085 & 0.032 & 53 \\
\hline 8.8 & -- & - & 0.12 & 0.73 & 0.68 & 0.070 & 0.022 & 66 \\
\hline 8.6 & 9 & $<5$ & 0.14 & 0.78 & 0.80 & 0.060 & 0.014 & 87 \\
\hline 6.9 & -- & -- & 0.16 & 0.86 & 0.90 & 0.070 & 0.022 & 100 \\
\hline 17 & -- & $\cdots$ & 0.12 & 0.64 & 0.83 & 0.100 & 0.035 & 65 \\
\hline 40 & 191 & 16 & 0.08 & 0.94 & 0.50 & 0.250 & 0.024 & 49 \\
\hline 50 & 75 & 6 & 0.07 & 0.62 & 0.59 & 0.120 & 0.026 & 44 \\
\hline 26 & -- & - & 0.08 & 0.63 & 0.85 & 0.085 & 0.031 & 44 \\
\hline 18 & -- & -- & 0.13 & 0.66 & 0.93 & 0.070 & 0.031 & 36 \\
\hline
\end{tabular}

\section{WATER YEAR OCTOBER 1992 TO SEPTEMBER 1993}

\begin{tabular}{|c|c|c|c|c|c|c|c|c|c|}
\hline 16 & -. & -. & 0.21 & 0.79 & 0.88 & 0.065 & 0.031 & 51 & 62 \\
\hline 18 & $\cdots$ & -- & 0.17 & 0.69 & 0.83 & 0.060 & 0.032 & 62 & 64 \\
\hline 8.0 & -- & -. & 0.30 & 0.72 & 1.10 & 0.060 & 0.029 & 82 & 82 \\
\hline 14 & -- & -- & 0.21 & 0.66 & 1.10 & 0.065 & 0.016 & 95 & 91 \\
\hline 15 & -- & - & 0.09 & 0.51 & 0.91 & 0.075 & 0.024 & 79 & 74 \\
\hline 19 & -- & - & 0.13 & 0.55 & 0.73 & 0.065 & 0.024 & 63 & 66 \\
\hline 18 & -- & -- & 0.22 & 0.77 & 0.79 & 0.075 & 0.021 & 61 & 71 \\
\hline 9.3 & -- & -- & 0.18 & 0.75 & 0.79 & 0.055 & 0.021 & 80 & 81 \\
\hline 16 & -- & -- & 0.14 & 0.48 & 0.68 & 0.080 & 0.019 & 43 & 65 \\
\hline 16 & -- & -- & 0.21 & 0.82 & 0.72 & 0.090 & 0.019 & 49 & 84 \\
\hline 22 & -- & -- & 0.07 & 0.45 & 0.61 & 0.100 & 0.019 & 64 & 56 \\
\hline 31 & 41 & $<5$ & 0.05 & 0.62 & 0.65 & 0.090 & 0.011 & 42 & 50 \\
\hline 28 & -. & -- & 0.09 & 0.66 & 0.56 & 0.060 & 0.015 & 40 & 49 \\
\hline 15 & 21 & $<5$ & 0.13 & 0.55 & 0.71 & 0.060 & 0.020 & 48 & $\epsilon 2$ \\
\hline 13 & 16 & $<6$ & 0.11 & 0.48 & 0.78 & 0.050 & 0.019 & 64 & 67 \\
\hline 12 & - & -- & 0.05 & 0.45 & 0.75 & 0.070 & 0.027 & 62 & 64 \\
\hline 50 & 71 & 6 & 0.05 & 0.64 & 0.61 & 0.140 & 0.016 & 37 & 47 \\
\hline 20 & -- & -- & 0.06 & 0.49 & 0.74 & 0.070 & 0.020 & 41 & 51 \\
\hline 12 & 14 & $<5$ & 0.08 & 0.48 & 0.77 & 0.045 & 0.017 & 47 & 61 \\
\hline 12 & -- & -- & 0.09 & 0.56 & 0.87 & 0.050 & 0.014 & 56 & 60 \\
\hline 55 & 75 & 6 & 0.08 & 0.60 & 0.70 & 0.110 & 0.015 & 41 & 44 \\
\hline
\end{tabular}


Surface-Water Stations

B. Partial-Record and Miscellaneous-Record Sites

431510077363501 Genesee River at Charlotte Pump Station Near Rochester, N.Y. WATER-QUALITY DATA

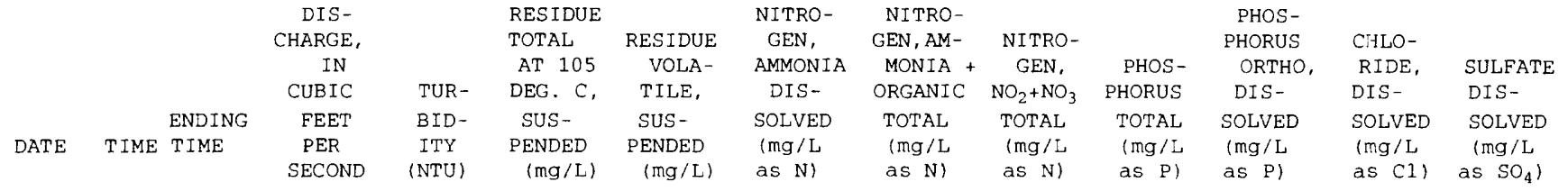

NOV-cont inued

\begin{tabular}{|c|c|c|c|c|c|c|c|c|c|c|c|c|c|}
\hline $16-19$ & 0955 & 0855 & 4080 & 29 & -- & -- & 0.08 & 0.45 & 0.75 & 0.065 & 0.014 & 43 & 41 \\
\hline $19-21$ & 0950 & 0850 & 3220 & 11 & -- & -- & 0.13 & 0.50 & 1.10 & 0.040 & 0.016 & 56 & 60 \\
\hline $23-24$ & 1000 & 1000 & 7380 & 30 & 48 & $<5$ & 0.05 & 0.55 & 0.97 & 0.090 & 0.022 & 47 & 47 \\
\hline $25-27$ & 1415 & 0515 & 8440 & 100 & 141 & 14 & 0.07 & 0.90 & 0.79 & 0.180 & 0.023 & 35 & 43 \\
\hline $27-28$ & 0615 & 2315 & 6090 & 45 & 62 & 6 & 0.05 & 0.58 & 0.89 & 0.093 & 0.021 & 37 & 48 \\
\hline \multicolumn{14}{|l|}{$\mathrm{DEC}$} \\
\hline $03-05$ & 0955 & 0855 & 4910 & 20 & -- & -- & 0.07 & 0.40 & 1.10 & 0.060 & 0.014 & 38 & 43 \\
\hline $05-07$ & 0955 & 0855 & 1820 & 18 & -- & -- & 0.07 & 0.40 & 1.10 & 0.045 & 0.014 & 37 & 45 \\
\hline $07-10$ & 0955 & 0855 & 3820 & 19 & -- & -- & 0.09 & 0.40 & 1.30 & 0.070 & 0.015 & 48 & 61 \\
\hline $10-12$ & 0950 & 0850 & 1600 & 16 & -- & -- & 0.14 & 0.55 & 1.40 & 0.050 & 0.019 & 52 & 57 \\
\hline $12-14$ & 0950 & 0850 & 2910 & 6.6 & 10 & $<5$ & 0.16 & 0.60 & 1.50 & 0.045 & 0.020 & 79 & 72 \\
\hline $14-15$ & 1055 & 2155 & 3630 & 8.3 & $-\div$ & -- & 0.10 & 0.37 & 1.40 & 0.035 & 0.013 & 55 & 110 \\
\hline $15-17$ & 2255 & 0855 & 3590 & 11 & -- & -- & 0.12 & 0.51 & 1.20 & 0.045 & 0.015 & 56 & 110 \\
\hline $17-19$ & 0950 & 0850 & 7550 & 50 & 101 & 8 & 0.10 & 0.73 & 1.20 & 0.120 & 0.024 & 54 & 44 \\
\hline $19-21$ & 0950 & 0850 & 7490 & 50 & 76 & 5 & 0.07 & 0.73 & 1.00 & 0.110 & 0.022 & 46 & 42 \\
\hline $21-22$ & 1010 & 2110 & 7660 & 45 & 79 & 6 & 0.08 & 0.61 & 0.49 & 0.085 & 0.018 & 37 & 43 \\
\hline $22-24$ & 2210 & 0710 & 7160 & 32 & 57 & 4 & 0.03 & 0.55 & 0.52 & 0.080 & 0.014 & 36 & 44 \\
\hline $24-28$ & 0755 & 0655 & 5070 & 28 & -- & -- & 0.07 & 0.47 & 1.10 & 0.070 & 0.015 & 38 & 43 \\
\hline $28-29$ & 0755 & 1555 & 2580 & 12 & - & -- & 0.09 & 0.43 & 1.40 & 0.040 & 0.017 & 45 & 58 \\
\hline $29-31$ & 1655 & 0655 & 3280 & 20 & -- & -- & 0.14 & 0.67 & 1.30 & 0.055 & 0.018 & 65 & 64 \\
\hline \multicolumn{14}{|l|}{ DEC 31} \\
\hline JAN 02 & 0815 & 0715 & 9640 & 120 & 271 & 23 & 0.09 & 1.0 & 1.20 & 0.260 & 0.019 & 47 & 46 \\
\hline $03-04$ & 1615 & 0715 & 7090 & 120 & 171 & 12 & 0.07 & 0.79 & 0.89 & 0.130 & 0.015 & 40 & 42 \\
\hline $04-05$ & 1000 & 0900 & 7010 & 95 & 120 & 10 & 0.08 & 0.80 & 0.96 & 0.120 & 0.015 & 39 & 46 \\
\hline $05-06$ & 1000 & 0900 & 8680 & 85 & -- & -- & 0.07 & 0.76 & 1.10 & 0.150 & 0.020 & 36 & 45 \\
\hline $06-07$ & 1000 & 0900 & 7370 & 80 & -- & -- & 0.08 & 0.81 & 1.10 & 0.160 & 0.027 & 36 & 47 \\
\hline $07-08$ & 0945 & 0845 & 7390 & 40 & 60 & 6 & 0.09 & 0.65 & 1.10 & 0.090 & -- & 59 & 79 \\
\hline $08-09$ & 0945 & 0845 & 7820 & 38 & 68 & 6 & 0.05 & 0.57 & 1.10 & 0.080 & 0.014 & 38 & 41 \\
\hline $09-10$ & 0945 & 0845 & 7830 & 50 & 82 & 7 & 0.05 & 0.51 & 1.10 & 0.100 & 0.012 & 30 & 39 \\
\hline $13-15$ & 1400 & 0900 & 7500 & 26 & -- & -- & 0.06 & 0.57 & 1.30 & 0.060 & 0.013 & 52 & 42 \\
\hline $15-17$ & 0920 & 0820 & 6960 & 24 & - & -- & 0.06 & 0.58 & 1.40 & 0.055 & 0.013 & 48 & 46 \\
\hline $17-19$ & 0920 & 0820 & 4580 & 20 & -- & -- & 0.06 & 0.39 & 1.30 & 0.050 & 0.012 & 47 & 52 \\
\hline $19-21$ & 1000 & 0900 & 2670 & 25 & -- & -- & 0.02 & 0.50 & 1.40 & 0.055 & 0.011 & 54 & 66 \\
\hline $21-23$ & 0945 & 0845 & 3310 & 14 & -- & -- & 0.29 & 0.72 & 1.50 & 0.050 & 0.014 & 74 & 74 \\
\hline $23-25$ & 0945 & 0845 & 6270 & 38 & 69 & $<12$ & 0.12 & 0.65 & 1.50 & 0.095 & 0.016 & 61 & 56 \\
\hline $25-26$ & 1000 & 2100 & 6720 & 38 & 63 & $<6$ & 0.12 & 0.73 & 1.50 & 0.075 & 0.017 & 48 & 49 \\
\hline $26-28$ & 2200 & 0900 & 5140 & 46 & 66 & 5 & 0.09 & 0.60 & 1.30 & 0.090 & 0.014 & 44 & 50 \\
\hline $28-30$ & 0955 & 0855 & 3370 & 18 & -- & -- & 0.12 & 0.66 & 1.20 & 0.045 & 0.012 & 49 & 59 \\
\hline \multicolumn{14}{|l|}{ FEB } \\
\hline $01-04$ & 1020 & 0920 & 2400 & 7.8 & -- & -- & 0.13 & 0.58 & 1.50 & 0.035 & 0.010 & 75 & 71 \\
\hline $04-06$ & 1020 & 0320 & 2630 & 8.7 & -- & -- & 0.14 & 0.55 & 1.50 & 0.035 & 0.010 & 68 & 72 \\
\hline $08-12$ & 1015 & 0715 & 1660 & 6.3 & -- & -- & 0.19 & 0.64 & 1.70 & 0.045 & 0.010 & 85 & 92 \\
\hline $14-16$ & 0800 & 0700 & 1510 & 4.8 & -- & - & 0.17 & 0.54 & 1.80 & 0.035 & 0.010 & 91 & 100 \\
\hline $16-18$ & 1000 & 0900 & 1420 & 3.8 & -- & -- & 0.16 & 0.57 & 1.80 & 0.030 & 0.008 & 100 & 100 \\
\hline $18-20$ & 0945 & 0845 & 1230 & 3.3 & -- & -- & 0.18 & 0.60 & 1.80 & 0.030 & 0.008 & 99 & 110 \\
\hline $20-22$ & 0945 & 0845 & 1170 & 4.4 & -- & -- & 0.22 & 0.74 & 1.80 & 0.035 & 0.007 & 100 & 110 \\
\hline $22-25$ & 1000 & 0900 & 1280 & 3.5 & -- & -- & 0.27 & 0.97 & 2.30 & 0.040 & 0.007 & 120 & 120 \\
\hline \multicolumn{14}{|l|}{ FEB 25- } \\
\hline MAR 01 & 0950 & 0850 & 1240 & 3.4 & -- & -- & 0.28 & 0.88 & 1.80 & 0.040 & 0.011 & 110 & 110 \\
\hline $03-04$ & 1245 & 0845 & 1400 & 4.4 & -- & -- & 0.28 & 0.89 & 1.90 & 0.050 & 0.008 & 130 & 110 \\
\hline $08-11$ & 0950 & 0850 & 1940 & 3.8 & $\cdots$ & -- & 0.22 & 0.90 & 1.50 & 0.035 & 0.009 & 140 & 93 \\
\hline $11-15$ & 1000 & 0800 & 1690 & 9.2 & -- & -- & 0.19 & 0.84 & 1.40 & 0.045 & 0.011 & 120 & 79 \\
\hline $15-18$ & 0845 & 0745 & 1640 & 5.4 & -- & -- & 0.23 & 0.80 & 1.40 & 0.040 & 0.011 & 120 & 92 \\
\hline $18-22$ & 0810 & 0710 & 2450 & 6.7 & -- & -- & 0.18 & 0.76 & 1.40 & 0.040 & 0.012 & 110 & 80 \\
\hline $22-23$ & 0800 & 1900 & 2530 & 5.8 & -- & -- & 0.16 & 0.52 & 1.40 & 0.035 & 0.012 & 93 & 75 \\
\hline $23-25$ & 2000 & 0700 & 3750 & 6.9 & 7 & -- & 0.17 & 0.76 & 1.40 & 0.050 & 0.013 & 89 & 74 \\
\hline $25-26$ & 0830 & 1530 & 6520 & 40 & $-\infty$ & -- & 0.15 & 0.86 & 1.30 & 0.100 & 0.018 & 79 & 56 \\
\hline $26-27$ & 1630 & 2330 & 8610 & 85 & -- & -- & 0.16 & 1.1 & 1.30 & 0.100 & 0.024 & 57 & 41 \\
\hline $28-29$ & 0030 & 0730 & 12400 & 130 & 242 & 15 & 0.15 & 1.4 & 1.30 & 0.200 & 0.027 & 45 & 34 \\
\hline $29-30$ & 0805 & 0705 & 13800 & 110 & -- & -- & 0.14 & 1.3 & 1.20 & 0.310 & 0.026 & 43 & 31 \\
\hline $30-31$ & 0805 & 0905 & 15800 & 140 & -- & - & 0.12 & 1.3 & 1.20 & 0.320 & 0.024 & 31 & 28 \\
\hline
\end{tabular}




\section{Surface-Water Stations}

\section{B. Partial-Record and Miscellaneous-Record Sites}

431510077363501 Genesee River at Charlotte Pump Station Near Rochester, N.Y. WATER-QUALITY DATA

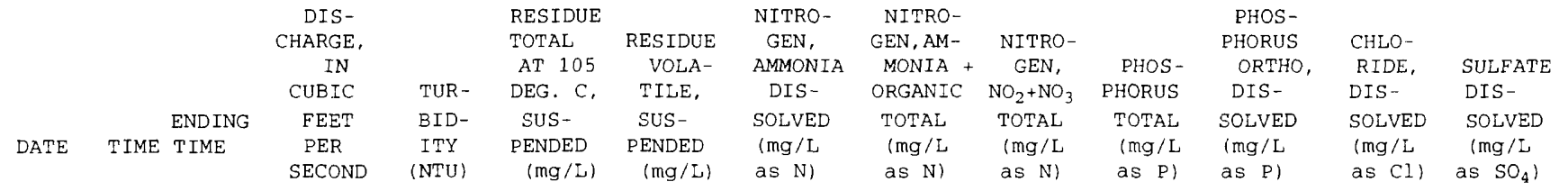

WATER YEAR OCTOBER 1992 TO SEPTEMBER 1993-continued

APR

\begin{tabular}{|c|c|c|c|c|c|c|c|}
\hline 01. & 0825 & -- & 18200 & 240 & -- & -- & 0.12 \\
\hline $01-02$ & 0830 & 1530 & 19000 & 130 & -- & -- & 0.08 \\
\hline $02-03$ & 1630 & 1930 & 19900 & 120 & -- & -- & 0.08 \\
\hline $04 \ldots$ & 1440 & -- & 14800 & 71 & -- & -- & 0.06 \\
\hline $04-05$ & 1450 & 0350 & 13500 & 56 & -- & -- & 0.05 \\
\hline $05-06$ & 0855 & 0755 & 7480 & 50 & -- & -- & 0.04 \\
\hline $06-07$ & 0855 & 0755 & 10200 & 70 & - & -- & 0.05 \\
\hline $07-08$ & 0855 & 0755 & 11500 & 160 & -- & -- & 0.08 \\
\hline $08-09$ & 0805 & 0705 & 11800 & 160 & 226 & 18 & 0.05 \\
\hline $09-10$ & 0805 & 0705 & 11800 & 190 & 202 & 16 & 0.05 \\
\hline $10-11$ & 0805 & 0705 & 11500 & 150 & 176 & 14 & 0.06 \\
\hline $11-12$ & 0805 & 0705 & 11500 & 130 & 173 & $<17$ & 0.06 \\
\hline $12-13$ & 0745 & 0645 & 11100 & 120 & 27 & 12 & 0.06 \\
\hline $13-14$ & 0745 & 0645 & 11000 & 130 & 106 & $<11$ & 0.06 \\
\hline $14-15$ & 0745 & 0645 & 10400 & 130 & 94 & $<12$ & 0.06 \\
\hline $15-16$ & 0740 & 0640 & 8790 & 140 & 56 & $<11$ & 0.05 \\
\hline $16-17$ & 0740 & 0640 & 10000 & 140 & 150 & 12 & 0.05 \\
\hline $17-18$ & 0740 & 0640 & 10600 & 130 & 158 & 13 & 0.05 \\
\hline $18-19$ & 0740 & 0640 & 10700 & 170 & 198 & 16 & 0.05 \\
\hline $19-20$ & 0830 & 0730 & 10400 & 100 & 145 & 10 & 0.06 \\
\hline $20-21$ & 0830 & 0730 & 9980 & 95 & 115 & $<10$ & 0.05 \\
\hline $21-22$ & 0830 & 0730 & 9530 & 95 & 77 & $<10$ & 0.05 \\
\hline $22-23$ & 0755 & 0655 & 10400 & 100 & -- & -- & 0.05 \\
\hline $23-24$ & 0755 & 0655 & 11100 & 100 & -- & -- & 0.05 \\
\hline $24-25$ & 0755 & 0655 & 10400 & 95 & -- & -- & 0.05 \\
\hline $25-26$ & 0755 & 0655 & 9420 & 90 & -- & -- & 0.05 \\
\hline $26-27$ & 0920 & 0820 & 8380 & 75 & -- & -- & 0.02 \\
\hline $27-28$ & 0920 & 0820 & 8990 & 140 & 167 & 14 & 0.03 \\
\hline $28-29$ & 0920 & 0820 & 9230 & 80 & $\cdots$ & -- & 0.04 \\
\hline $29-30$ & 0820 & 0720 & 9930 & 70 & 100 & 8 & $<0.01$ \\
\hline \multicolumn{8}{|l|}{ PR $30-$} \\
\hline MAY 01 & 0820 & 0720 & 10000 & 80 & 117 & 6 & 0.05 \\
\hline $01-02$ & 0820 & 0720 & 9550 & 75 & 97 & 7 & 0.04 \\
\hline $02-03$ & 0820 & 0720 & 9360 & 75 & 96 & 6 & 0.04 \\
\hline $03-04$ & 0815 & 0715 & 9150 & 75 & 95 & 7 & 0.01 \\
\hline $04-05$ & 0815 & 0715 & 9300 & 75 & 92 & 7 & 0.02 \\
\hline $05-06$ & 0815 & 0715 & 9310 & 75 & 91 & 7 & 0.04 \\
\hline $06-07$ & 0800 & 0700 & 9100 & 75 & +- & -- & 0.04 \\
\hline $07-08$ & 0800 & 0700 & 9110 & 80 & -- & -- & 0.03 \\
\hline $08-09$ & 0800 & 0700 & 9060 & 80 & -- & -- & 0.03 \\
\hline $09-10$ & 0800 & 0700 & 8950 & 70 & -- & -- & 0.02 \\
\hline $10-11$ & 0755 & 0655 & 8560 & 55 & 72 & $<5$ & 0.04 \\
\hline $11-12$ & 0755 & 0655 & 8720 & 55 & 78 & $<5$ & 0.04 \\
\hline $12-13$ & 0755 & 0655 & 8510 & 55 & 79 & $<5$ & 0.04 \\
\hline $13-14$ & 0805 & 0705 & 7580 & 34 & 51 & $<5$ & 0.05 \\
\hline $14-15$ & 0805 & 0705 & 6690 & 28 & -- & -- & 0.06 \\
\hline $15-16$ & 0805 & 0705 & 5100 & $3 I$ & 48 & $<5$ & 0.08 \\
\hline $16-17$ & 0805 & 0705 & 3300 & 24 & -- & -- & 0.07 \\
\hline $17-18$ & 0900 & 2000 & 2240 & 16 & -- & -- & $<0.01$ \\
\hline $18-20$ & 2100 & 0800 & 2020 & 15 & -- & -- & $<0.01$ \\
\hline $20-22$ & 0820 & 0720 & 1400 & 13 & -- & -- & $<0.01$ \\
\hline $22-24$ & 0820 & 0720 & 1300 & 8.4 & -- & -- & $<0.01$ \\
\hline $24-26$ & 0840 & 0740 & 1100 & N14 & -- & -- & $<0.01$ \\
\hline $26-27$ & 0840 & 2340 & 1050 & N1 1 & -- & -- & $<0.01$ \\
\hline $28-30$ & 0855 & 0755 & 930 & 8.5 & -- & -- & $<0.01$ \\
\hline \multicolumn{8}{|l|}{ IAY $30-$} \\
\hline JUN 01 & 0855 & 0755 & 840 & 9.1 & -- & -- & $<0.01$ \\
\hline $01-03$ & 1015 & 0915 & 1000 & 6.5 & -- & -- & $<0.01$ \\
\hline $03-03$ & 1030 & 2330 & 1100 & 7.4 & -- & -- & $<0.01$ \\
\hline $07 \ldots$ & 0800 & -- & 1100 & 8.2 & -- & -- & $<0.01$ \\
\hline $07-08$ & 0805 & 1905 & 1100 & 14 & -- & -- & $<0.01$ \\
\hline $08-10$ & 2005 & 0705 & 1200 & 14 & -- & -- & $<0.01$ \\
\hline $10-12$ & 0805 & 0705 & 1400 & 7.2 & $\cdots$ & -- & 0.18 \\
\hline $12-14$ & 0805 & 0705 & 1100 & 7.2 & -- & -- & 0.11 \\
\hline
\end{tabular}

\begin{tabular}{|c|c|}
\hline 1.6 & 1.30 \\
\hline 1.2 & 1.20 \\
\hline 1.2 & 1.30 \\
\hline 0.76 & 1.30 \\
\hline 0.67 & 1.30 \\
\hline 0.69 & 0.66 \\
\hline 0.78 & 0.69 \\
\hline 0.93 & 1.10 \\
\hline 0.77 & 0.47 \\
\hline 0.77 & 1.00 \\
\hline 0.76 & 1.00 \\
\hline 0.73 & 1.10 \\
\hline 0.65 & No. 94 \\
\hline 0.61 & N1. 10 \\
\hline 0.61 & $\mathrm{~N} 1.10$ \\
\hline 0.68 & 1.00 \\
\hline 0.69 & 0.99 \\
\hline 0.64 & 0.96 \\
\hline 0.83 & 0.78 \\
\hline 0.64 & 0.90 \\
\hline 0.48 & 0.96 \\
\hline 0.64 & 0.99 \\
\hline 0.60 & 1.00 \\
\hline 0.58 & 0.98 \\
\hline- & 0.94 \\
\hline 0.60 & 0.93 \\
\hline 0.51 & 0.90 \\
\hline 0.70 & 0.85 \\
\hline 0.55 & 0.88 \\
\hline-- & 0.85 \\
\hline-- & 0.87 \\
\hline -- & 0.84 \\
\hline -. & 0.82 \\
\hline 0.43 & 0.85 \\
\hline 0.48 & 0.82 \\
\hline 0.51 & 0.70 \\
\hline -- & 0.78 \\
\hline - & 0.76 \\
\hline -- & 0.75 \\
\hline -- & 0.78 \\
\hline 0.48 & 0.75 \\
\hline 0.58 & 0.77 \\
\hline 0.65 & 0.78 \\
\hline 0.39 & 0.76 \\
\hline 0.38 & 0.69 \\
\hline 0.49 & 0.71 \\
\hline 0.48 & 0.75 \\
\hline$<0.10$ & 0.78 \\
\hline$<0.10$ & 0.90 \\
\hline 0.66 & 1.00 \\
\hline 0.64 & 1.00 \\
\hline$<0.10$ & 1.10 \\
\hline$<0.10$ & 1.00 \\
\hline$<0.10$ & 1.10 \\
\hline$<0.1$ & 0.98 \\
\hline N0. 54 & 0.94 \\
\hline 0.57 & 0.90 \\
\hline N0.72 & 0.99 \\
\hline 0.60 & 1.00 \\
\hline 0.67 & 1.00 \\
\hline 0.69 & 1.10 \\
\hline 0.63 & 1.00 \\
\hline
\end{tabular}

0.026

0.020

0.021

0.023

0.210

0.140

0.110

0.130

0.180

0.290

0.240

0.210

0.230

0.120

0.120

0.180

0.180

0.090

0.200

0.260

0.140
0.130

0.130

0.160

0.160

0.160

0.120

0.095

0.180

0.100

0.020
0.015

0.015

0.012

0.012

0.011

0.012

0.014

0.014

0.016

0.010

0.012

0.013

N0.017

0.010

0.011

0.012

0.011

0.007

0.009

0.008

0.003

0.110

0.005

0.008

0.008

0.003

0.013

0.002

0.010

0.009

0.010

0.009

0.009

0.009

0.007

0.008

0.008

0.008

0.009

0.012

0.015

0.019

0.018

0.015

0.016

0.009

$\begin{array}{ll}29 & 28\end{array}$

30
31

30

34
38

43

29
29

29
26

27
30

30

30

27

27
26

27

23

28
27

28

26
26

26
28

28

27

13

26
29

27

26

13
24

26

25
26

28

28

27

27
25

26

30

30
33

36

41
46

65

83

83
75

75
83

0.006

0.006
0.005
0.006

0.006

0.014

0.014

0.016

0.018

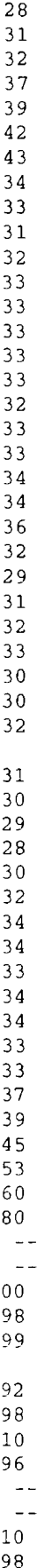




\section{Surface-Water Stations}

B. Partial-Record and Miscellaneous-Record Sites

\section{Genesee River at Charlotte Pump Station Near Rochester, N.Y.}

\section{WATER-QUALITY DATA}

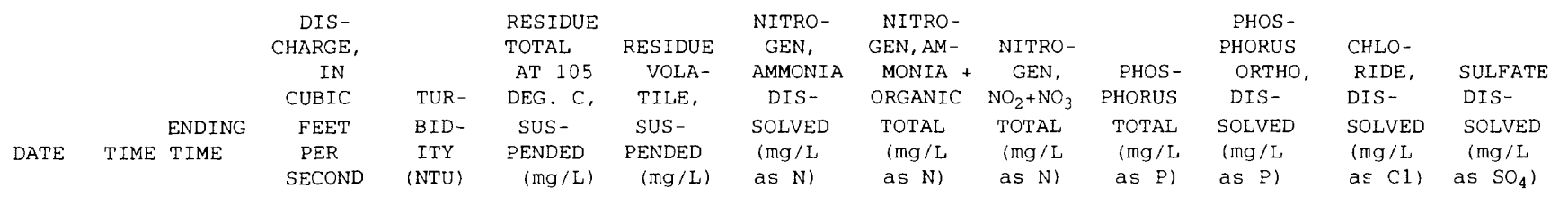

WATER YEAR OCTOBER 1992 TO SEPTEMBER 1993-continued

\begin{tabular}{|c|c|c|c|}
\hline \multicolumn{4}{|c|}{ JUN--cont Inued } \\
\hline $14-17$ & 0815 & 0715 & 650 \\
\hline $17-21$ & 0825 & 0725 & 550 \\
\hline $21-24$ & 0815 & 0715 & 550 \\
\hline $24-25$ & 0745 & 1845 & 510 \\
\hline $28 \ldots$ & 0840 & $-\infty$ & 44 \\
\hline $28-29$ & 1330 & 0830 & 50 \\
\hline JUN 30- & & & \\
\hline JUL 01 & 0800 & 2300 & 580 \\
\hline $02 \ldots$ & 0820 & -- & 0 \\
\hline $02-06$ & 0830 & 0730 & 40 \\
\hline $06-08$ & 0835 & 0835 & 3 \\
\hline $08-12$ & 0850 & 0750 & 3 \\
\hline $12-15$ & 0835 & 0735 & 32 \\
\hline $15-19$ & 0835 & 0735 & 29 \\
\hline $19-20$ & 0815 & 0715 & 3 \\
\hline $20-21$ & 0815 & 0715 & 3 \\
\hline $21-22$ & 0815 & 0715 & 31 \\
\hline $22-24$ & 0815 & 0715 & 3 \\
\hline $24-26$ & 0815 & 0715 & 27 \\
\hline $26-27$ & 0750 & 1850 & 4 \\
\hline $27-29$ & 1950 & 0650 & 6 \\
\hline $29-31$ & 0750 & 0650 & 37 \\
\hline JUL 31- & & & \\
\hline AUG 02 & 0750 & 0650 & 40 \\
\hline $05-07$ & 0825 & 0725 & 40 \\
\hline $07-09$ & 0825 & 0725 & 31 \\
\hline $09-10$ & 0815 & 1915 & 33 \\
\hline $10-12$ & 2015 & 0715 & 33 \\
\hline $12-14$ & 0835 & 0735 & 34 \\
\hline $14-16$ & 0835 & 0735 & חת \\
\hline $16-17$ & 0815 & 1915 & 34 \\
\hline $17-19$ & 2015 & 0715 & 600 \\
\hline $19-23$ & 0840 & 0740 & 60 \\
\hline $23-26$ & 1440 & 0940 & 38 \\
\hline $26-28$ & 0950 & 0850 & 290 \\
\hline $28-30$ & 0950 & 0850 & 25 \\
\hline AUG $31-$ & & & \\
\hline SEP 01 & 0910 & 0810 & 29 \\
\hline $01-03$ & 0910 & 0810 & 310 \\
\hline $03-04$ & 0950 & 1650 & 42 \\
\hline $04+06$ & 1750 & 0050 & 158 \\
\hline $06-07$ & 0150 & 0850 & 165 \\
\hline $07-08$ & 1110 & 1010 & 94 \\
\hline $08-09$ & 1110 & 0910 & 77 \\
\hline $09-10$ & 1005 & 1705 & 62 \\
\hline $10-12$ & 1805 & 0105 & 60 \\
\hline $12-13$ & 0205 & 0005 & 62 \\
\hline $13-14$ & 0950 & 2050 & 5 \\
\hline $14-16$ & 2150 & 0850 & . \\
\hline $16-18$ & 1010 & 0910 & 37 \\
\hline $18-20$ & 1010 & 0910 & 35 \\
\hline $20-21$ & 1010 & 2110 & 3 \\
\hline $22-23$ & 2210 & 0910 & 32 \\
\hline $23-25$ & 1115 & 1015 & 31 \\
\hline $27-28$ & 0955 & 2055 & 1 \\
\hline $28-30$ & 2155 & 0855 & 11 \\
\hline $30-30$ & 0950 & 2355 & 16 \\
\hline
\end{tabular}

\begin{tabular}{|c|c|c|c|}
\hline 7.8 & -- & -- & 0.07 \\
\hline 5.8 & -- & -- & 0.11 \\
\hline 6.8 & -- & -- & 0.17 \\
\hline 4.1 & -- & -- & 0.12 \\
\hline 5.8 & -- & -- & 0.14 \\
\hline 5.3 & -- & -- & 0.13 \\
\hline 6.5 & -- & -- & 0.16 \\
\hline 4.5 & -- & -- & 0.07 \\
\hline 4.0 & -- & -- & 0.15 \\
\hline 3.5 & -- & -- & 0.04 \\
\hline 4.4 & -- & -- & 0.06 \\
\hline 5.5 & -- & -- & 0.09 \\
\hline 8.8 & -- & - & 0.24 \\
\hline 8.0 & -- & -- & 0.20 \\
\hline 8.6 & -- & -- & 0.16 \\
\hline 9.4 & -- & -- & 0.20 \\
\hline 7.2 & -- & -- & 0.24 \\
\hline 7.4 & -- & - & 0.22 \\
\hline 5.8 & -- & - & 0.19 \\
\hline 6.4 & -- & - & 0.17 \\
\hline 6.5 & -- & -- & 0.26 \\
\hline 7.0 & -- & -- & 0.39 \\
\hline 9.5 & -- & $\rightarrow$ & 0.29 \\
\hline 8.5 & -- & -- & 0.29 \\
\hline 9.2 & -- & -- & 0.15 \\
\hline 4.9 & -- & - & 0.09 \\
\hline 3.3 & -- & -- & 0.06 \\
\hline 3.8 & -- & -- & 0.15 \\
\hline 3.6 & -- & -- & 0.14 \\
\hline 4.0 & -- & -- & 0.21 \\
\hline 12 & - & - - & 0.20 \\
\hline 3.4 & -- & -- & 0.15 \\
\hline 2.6 & - & - & 0.20 \\
\hline 0.25 & -- & - & 0.20 \\
\hline 3.2 & -- & -- & 0.00 \\
\hline 4.3 & -- & -- & 0.00 \\
\hline 4.2 & -- & -- & 0.24 \\
\hline 3.6 & -- & -- & 0.29 \\
\hline 6.5 & -- & -- & 0.12 \\
\hline 6.6 & -- & -- & 0.11 \\
\hline 9.9 & -- & -- & No. 10 \\
\hline 4.2 & -- & -- & NO. 11 \\
\hline 4.7 & -- & -- & 0.17 \\
\hline 3.6 & -- & -- & 0.21 \\
\hline 4.6 & -- & - & 0.08 \\
\hline 5.5 & -- & -- & 0.09 \\
\hline 3.2 & -- & -- & 0.12 \\
\hline 3.6 & -- & -- & 0.12 \\
\hline N3. 5 & -- & -- & 0.08 \\
\hline 3.6 & -- & - & 0.06 \\
\hline 3.5 & -- & -- & 0.14 \\
\hline 3.5 & -- & -- & 0.11 \\
\hline 4.0 & -- & -- & 0.21 \\
\hline 3.9 & -- & -- & 0.16 \\
\hline
\end{tabular}

\begin{tabular}{|c|c|c|}
\hline 0.63 & 0.91 & 0.060 \\
\hline 0.69 & 0.87 & 0.045 \\
\hline 0.79 & 0.91 & 0.060 \\
\hline 0.71 & 1.00 & 0.055 \\
\hline 0.63 & 0.84 & 0.045 \\
\hline 0.67 & 0.90 & 0.075 \\
\hline 0.79 & 0.97 & 0.070 \\
\hline 0.67 & 0.86 & 0.060 \\
\hline 0.80 & 0.91 & 0.055 \\
\hline 0.64 & 0.66 & 0.045 \\
\hline 0.71 & 0.62 & 0.050 \\
\hline 0.73 & 0.58 & 0.055 \\
\hline 1.0 & 0.74 & 0.075 \\
\hline 1.0 & No. 65 & 0.065 \\
\hline 1.1 & No. 64 & 0.085 \\
\hline 0.96 & No. 62 & 0.070 \\
\hline 1.0 & 0.71 & 0.070 \\
\hline 0.92 & 0.74 & 0.065 \\
\hline 0.93 & $<0.01$ & 0.065 \\
\hline 0.87 & $<0.01$ & 0.065 \\
\hline No. 71 & 0.75 & 0.060 \\
\hline NO. 71 & 0.74 & 0.060 \\
\hline 0.73 & No. 92 & 0.070 \\
\hline 1.0 & $\mathrm{~N} 1.10$ & 0.070 \\
\hline 0.94 & No. 87 & 0.070 \\
\hline 0.94 & No. 82 & 0.070 \\
\hline 0.69 & 0.89 & 0.055 \\
\hline 0.76 & 0.87 & 0.040 \\
\hline 0.89 & 0.74 & 0.060 \\
\hline 1.2 & 0.90 & 0.075 \\
\hline N1. 1 & 0.70 & 0.065 \\
\hline $\mathrm{N} 1.3$ & -- & 0.050 \\
\hline N1.1 & -- & 0.060 \\
\hline-- & $\cdots$ & 0.050 \\
\hline 0.86 & -- & 0.045 \\
\hline 0.96 & -- & 0.050 \\
\hline 1.0 & 0.74 & 0.055 \\
\hline 0.96 & 0.77 & 0.055 \\
\hline No. 93 & 0.64 & 0.065 \\
\hline 0.50 & 0.63 & 0.010 \\
\hline 0.44 & 0.64 & 0.055 \\
\hline 0.39 & 0.77 & 0.055 \\
\hline 0.50 & 0.93 & 0.065 \\
\hline 0.60 & 0.78 & 0.060 \\
\hline 0.57 & 0.74 & 0.060 \\
\hline 0.71 & 0.80 & 0.060 \\
\hline 0.92 & 0.88 & 0.045 \\
\hline 0.91 & 0.83 & 0.050 \\
\hline 0.70 & 0.65 & 0.045 \\
\hline 0.68 & 0.62 & 0.040 \\
\hline 0.71 & 0.79 & 0.040 \\
\hline 0.60 & 0.70 & 0.045 \\
\hline 0.82 & 0.85 & 0.045 \\
\hline 0.84 & 0.78 & 0 . \\
\hline
\end{tabular}

\begin{tabular}{|c|c|c|}
\hline 0.017 & 70 & 88 \\
\hline 0.011 & 78 & 100 \\
\hline 0.019 & 80 & 110 \\
\hline 0.024 & 99 & 110 \\
\hline 0.012 & 67 & 92 \\
\hline 0.015 & 69 & 91 \\
\hline 0.017 & 87 & 110 \\
\hline 0.011 & 82 & 110 \\
\hline 0.008 & 100 & 120 \\
\hline 0.007 & 87 & 82 \\
\hline 0.004 & 81 & 100 \\
\hline 0.011 & 80 & 98 \\
\hline 0.018 & 92 & 96 \\
\hline 0.020 & 100 & N92 \\
\hline 0.020 & 110 & N95 \\
\hline 0.022 & 110 & N93 \\
\hline 0.013 & 94 & N1 00 \\
\hline 0.013 & 87 & N99 \\
\hline 0.014 & 93 & 110 \\
\hline 0.013 & 89 & 110 \\
\hline 0.022 & 110 & 110 \\
\hline 0.022 & 110 & 110 \\
\hline 0.014 & 95 & 110 \\
\hline 0.011 & 120 & 120 \\
\hline 0.012 & 120 & 120 \\
\hline 0.015 & 120 & 110 \\
\hline 0.003 & 100 & 100 \\
\hline 0.006 & 86 & 110 \\
\hline 0.018 & 86 & 110 \\
\hline 0.028 & 96 & 120 \\
\hline 0.018 & 120 & 110 \\
\hline 0.020 & 140 & 100 \\
\hline 0.016 & 120 & 93 \\
\hline 0.029 & 120 & 86 \\
\hline 0.030 & 99 & 85 \\
\hline 0.028 & 89 & 94 \\
\hline 0.030 & 86 & 98 \\
\hline 0.033 & 110 & 97 \\
\hline 0.026 & 180 & 110 \\
\hline 0.028 & 75 & N7 3 \\
\hline 0.029 & 49 & N71 \\
\hline 0.030 & 47 & N5 1 \\
\hline 0.034 & 49 & N7 6 \\
\hline 0.034 & 51 & N77 \\
\hline 0.030 & 60 & 89 \\
\hline 0.028 & 95 & 96 \\
\hline 0.024 & 110 & $\mathrm{~N} 160$ \\
\hline 0.022 & 90 & 100 \\
\hline 0.018 & 110 & 100 \\
\hline 0.015 & 110 & 97 \\
\hline 0.016 & 100 & 110 \\
\hline 0.017 & 88 & 98 \\
\hline 0.021 & 110 & 100 \\
\hline 0.027 & 160 & 12 \\
\hline
\end{tabular}




\section{Surface-Water Stations}

\section{B. Partial-Record and Miscellaneous-Record Sites}

\section{Cartersville Waste Channel at Pittsford, N.Y.}

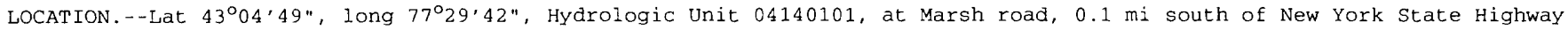
31 and $0.25 \mathrm{mi}$ north of Erie canal.

PERIOD OF RECORD. - - December 1984 to current year.

CHEMICAL DATA: $1984-86$ (a), 1988-91 (d), 1992 (c), 1993 (b).

NUTRIENT DATA: $1984-86$ (a), 1988-91 (d), 1992 (c), 1993 (b)

COOPERATION.--Water-quality samples were collected and analyzed by the Monroe County Environmental Health Laboratory at Rochester, N.Y.

\section{WATER-QUALITY DATA}

\begin{tabular}{|c|c|c|c|c|c|c|c|c|c|c|c|c|c|}
\hline & & $\begin{array}{c}\text { DIS- } \\
\text { CHARGE, } \\
\text { INST. } \\
\text { CUBIC }\end{array}$ & TUR- & OXYGEN, & $\begin{array}{l}\text { RESIDUE } \\
\text { TOTAL } \\
\text { AT } 105 \\
\text { DEG. C, }\end{array}$ & $\begin{array}{l}\text { RESIDUE } \\
\text { VOLA- } \\
\text { TILE, }\end{array}$ & $\begin{array}{l}\text { NITRO- } \\
\text { GEN, } \\
\text { AMMONIA } \\
\text { DIS- }\end{array}$ & $\begin{array}{l}\text { NITRO- } \\
\text { GEN, AM- } \\
\text { MONIA + } \\
\text { ORGANIC }\end{array}$ & $\begin{array}{c}\text { NITRO- } \\
\text { GEN, } \\
\mathrm{NO}_{2}+\mathrm{NO}_{3}\end{array}$ & $\begin{array}{l}\text { PHOS- } \\
\text { PHORUS }\end{array}$ & $\begin{array}{l}\text { PHOS- } \\
\text { PHORUS } \\
\text { ORTHO, } \\
\text { DIS- }\end{array}$ & $\begin{array}{l}\text { CHLO- } \\
\text { RIDE, } \\
\text { DIS- }\end{array}$ & $\begin{array}{l}\text { SULFATE } \\
\text { DIS- }\end{array}$ \\
\hline ATE & TIME & $\begin{array}{c}\text { FEET } \\
\text { PER } \\
\text { SECOND }\end{array}$ & $\begin{array}{l}\text { BID- } \\
\text { ITY } \\
\text { (NTU) }\end{array}$ & $\begin{array}{l}\text { DIS- } \\
\text { SOLVED } \\
(\mathrm{mg} / \mathrm{L})\end{array}$ & $\begin{array}{l}\text { SUS- } \\
\text { PENDED } \\
(\mathrm{mg} / \mathrm{L})\end{array}$ & $\begin{array}{l}\text { SUS- } \\
\text { PENDED } \\
(\mathrm{mg} / \mathrm{L})\end{array}$ & $\begin{array}{l}\text { SOLVED } \\
\text { (mg/L } \\
\text { as N) }\end{array}$ & $\begin{array}{c}\text { TOTAL } \\
\text { (mg/L } \\
\text { as N) }\end{array}$ & $\begin{array}{c}\text { TOTAL } \\
\text { (mg/L } \\
\text { as N) }\end{array}$ & $\begin{array}{c}\text { TOTAL } \\
\text { (mg/L } \\
\text { as P) }\end{array}$ & $\begin{array}{l}\text { SOLVED } \\
\text { (mg/L } \\
\text { as P) }\end{array}$ & $\begin{array}{l}\text { SOLVED } \\
\text { (mg/L } \\
\text { as Cl) }\end{array}$ & $\begin{array}{c}\text { SOLVED } \\
\text { (mg/L } \\
\text { as } \mathrm{SO}_{4} \text { ) }\end{array}$ \\
\hline
\end{tabular}

OCT

$06 \ldots 0900 \quad-. \quad 25$

$13 \ldots 1050 \quad--\quad 8.6$

$20 \ldots 1050 \quad-1 \quad 2.9$

$\begin{array}{llll}27 \ldots & 1030 & 1.4 & 7.8\end{array}$

JAN

31... $1050 \quad \ldots \quad 28$

$21 \ldots 0900 \quad \ldots \quad 80$

MAR

$20 \ldots \quad 1100 \quad \ldots \quad 26$

$30 \ldots 0950 \quad \ldots \quad 14$

APR

$03 \ldots 1030 \quad--\quad 16$

1130

UN

$28 \ldots \quad 1050 \quad 5.7 \quad 23$

JUL

$21 \ldots \quad 1130 \quad \ldots \quad 10$

$18 \ldots 0950 \quad \ldots \quad 16$

SEP

$01 \ldots 0950 \quad--\quad 22$

$13 \ldots 0950 \quad \ldots \quad 18$

OC$$
\begin{array}{r}
2 \\
\text { JUN } \\
1
\end{array}
$$$$
\text { JUL }
$$

$$
\begin{array}{r}
1 \\
2 \\
2 \\
\text { AUG } \\
0
\end{array}
$$$$
\text { SEP }
$$

$$
\begin{gathered}
\text { MAY } \\
08 \\
23 \\
\text { JUN } \\
15 \\
29 \\
\text { JUL } \\
13 \\
20 \\
25 \\
\text { AUG } \\
08 \\
24 \\
\text { SEP } \\
07 \\
21 \\
\\
\text { OCT } \\
09 \\
24
\end{gathered}
$$$$
\begin{aligned}
& 24 \\
& 3
\end{aligned}
$$

$\begin{array}{rr}36 & 6 \\ 19 & 3 \\ 6 & 1 \\ 8 & 1\end{array}$

0.05

$$
0.04
$$$$
0.01
$$$$
0.04
$$$$
13.5
$$$$
13.5
$$$$
13 .
$$$$
12.3
$$$$
--
$$$$
11
$$$$
\text { - } 0.09
$$$$
116
$$$$
\begin{array}{lll}
\ldots & \ldots & 0.13 \\
\ldots & \ldots & 0.02
\end{array}
$$

0.10
0.13$$
\begin{array}{ccc}
-- & - & 0.11 \\
25 & <5 & 0.06
\end{array}
$$$$
25
$$$$
48
$$$$
--
$$$$
7
$$$$
0.08
$$$$
\begin{array}{ll}
1.8 & 2.50
\end{array}
$$$$
0.64 \quad 1.10
$$$$
1.3 \quad 1.10
$$$$
0.03
$$$$
0.72
$$$$
0.81
$$$$
36
$$$$
48
$$$$
0.03
$$$$
0.64
$$$$
0.39
$$$$
0.78
$$$$
0.57
$$

\begin{tabular}{|c|c|c|c|c|c|c|c|c|}
\hline 25 & 4 & 0.02 & 0.86 & 0.30 & 0.065 & 0.009 & 92 & 88 \\
\hline 52 & 5 & 0.06 & 0.60 & 0.77 & 0.105 & 0.017 & 63 & 55 \\
\hline 29 & $<5$ & 0.02 & 0.74 & 1.10 & 0.070 & 0.006 & 91 & \\
\hline 21 & $<5$ & 0.03 & 0.87 & 0.86 & 0.080 & 0.026 & 64 & \\
\hline 12 & $<5$ & 0.05 & 0.57 & 0.99 & 0.040 & 0.005 & 91 & \\
\hline 23 & $<5$ & 0.04 & 0.58 & 0.84 & 0.095 & 0.026 & 99 & \\
\hline 27 & $<5$ & 0.15 & 0.80 & 0.80 & 0.070 & $<0.001$ & 96 & \\
\hline 137 & 43 & 0.90 & 3.9 & 2.20 & 0.570 & 0.210 & 25 & \\
\hline 25 & $<5$ & 0.06 & 0.54 & 0.61 & 0.075 & 0.025 & 96 & \\
\hline 27 & $<6$ & 0.83 & 0.83 & 0.45 & 0.080 & 0.315 & 80 & \\
\hline 24 & $<5$ & $<0.01$ & 0.31 & 0.66 & 0.070 & 0.030 & 64 & \\
\hline 26 & 5 & 0.04 & 0.51 & 0.50 & 0.080 & 0.036 & 65 & \\
\hline 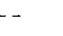 & - & 0.05 & 0.03 & 0.53 & 0.029 & 0.007 & 82 & \\
\hline 584 & & & & & & & & \\
\hline 12 & $<5$ & 0.05 & 0.62 & 0.63 & 0.070 & 0.030 & 190 & \\
\hline 22 & $<5$ & 0.04 & 0.43 & 0.95 & 0.065 & 0.021 & 40 & \\
\hline 17 & $<5$ & 0.04 & 0.43 & 0.83 & 0.045 & 0.016 & 51 & \\
\hline
\end{tabular}

0.06
0.10

0.61

0.57

WATER YEAR OCTOBER 1989 TO SEPTEMBER 1990 


\section{Surface-Water Stations}

\section{B. Partial-Record and Miscellaneous-Record Sites}

\section{Cartersville Waste Channel at Pittsford, N.Y. - continued}

\section{WATER-QUALITY DATA}

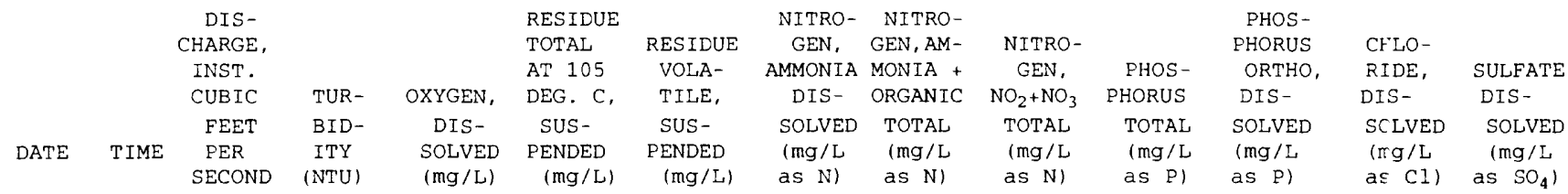

NOV

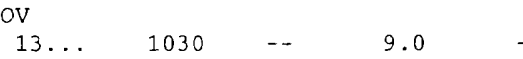

$\begin{array}{lllr}13 \ldots & 1030 & - & 9.0\end{array}$

$29 \ldots .0950 \quad--\quad 73$

MAY

$\begin{array}{llll}03 \ldots & 1030 & 3.1 & 22\end{array}$

JUN $10 \ldots 0900 \quad 09011$

JUL

$19 \ldots \quad 1030 \quad \ldots \quad 14$

$21 \ldots 0950 \quad \ldots \quad 7.6$

MAY

$\begin{array}{llll}21 \ldots & 1130 & 6.8 & 8.5\end{array}$

$04 \ldots \quad 1130 \quad \ldots \quad 14$

$24 \ldots \quad 1100 \quad--\quad 38$

JUL

08... $1030 \quad 6.6 \quad 16$

$22 \ldots \quad 1130 \quad \ldots \quad 18$

AUG

$12 \ldots 0950 \quad \ldots \quad 18$

$\begin{array}{llll}12 \ldots & 0900 & 8.3 & 11\end{array}$

SEP 1992

$09 . \cdots 1050$

$30 \ldots$

1050
$+-$

11.1

8.7

$--$

$$
-
$$

8.7

8.7

8.3

7.9

7.6

8.4

8.0

8.3

9.8

WATER YEAR OCTOBER 1990 TO SEPTEMBER 1991

\begin{tabular}{|c|c|c|c|c|c|c|c|c|}
\hline-- & -- & 0.18 & 0.70 & 1.10 & 0.080 & 0.027 & 110 & 65 \\
\hline-- & -- & 0.30 & 0.77 & 0.90 & 0.120 & 0.026 & 8 & 60 \\
\hline- & -- & 0.44 & 1.6 & 1.30 & 0.170 & 0.036 & 150 & 110 \\
\hline 32 & $<5$ & 0.03 & 0.61 & 0.85 & 0.080 & No. 001 & 41 & 47 \\
\hline 25 & 3 & 0.02 & 0.57 & 0.69 & 0.055 & 0.011 & $\varepsilon 4$ & 91 \\
\hline 28 & 3 & 0.02 & 0.62 & 0.58 & 0.065 & 0.018 & 79 & 92 \\
\hline 17 & 2 & 0.02 & 0.42 & 0.43 & 0.060 & 0.032 & $\varepsilon 7$ & 80 \\
\hline
\end{tabular}

WATER YEAR OCTOBER 1991 TO SEPTEMBER 1992

$\begin{array}{rrrrrrrrr}21 & 3 & 0.07 & 0.50 & 0.92 & 0.050 & 0.011 & 41 & 93 \\ 22 & 3 & 0.04 & 0.48 & 0.54 & 0.065 & 0.017 & 77 & 120 \\ 94 & 15 & 0.12 & 0.87 & 0.67 & 0.115 & 0.028 & 65 & 82 \\ 32 & 3 & 0.05 & 0.51 & 0.66 & 0.065 & 0.017 & \ldots & 120 \\ 34 & 4 & 0.10 & 0.85 & 0.31 & \text { N0.001 } & 0.066 & 59 & 52 \\ 29 & 4 & 0.02 & 0.43 & 0.31 & 0.090 & 0.025 & 45 & 44 \\ 18 & 2 & <0.01 & 0.42 & 0.68 & 0.050 & 0.019 & 72 & \text { N94 } \\ 19 & 2 & 0.030 & 0.6 & 1.00 & 0.070 & 0.041 & 64 & 79 \\ 18 & 2 & 0.04 & 0.44 & 0.59 & 0.065 & 0.031 & 51 & 48\end{array}$

WATER YEAR OCTOBER 1992 TO SEPTEMBER 1993

MAY

\begin{tabular}{|c|c|c|c|c|c|c|c|c|c|c|c|c|c|}
\hline 05 & 1030 & 15 & 25 & 101 & 30 & 4 & 001 & 058 & 068 & 0068 & בחم & $=0$ & 48 \\
\hline $19 \ldots$ & 0950 & 14 & 16 & 9.6 & 18 & 3 & 0.03 & 4.3 & 0.59 & 0.055 & 0.009 & 39 & 43 \\
\hline \multicolumn{14}{|l|}{ JUN } \\
\hline $02 \ldots$ & 1100 & 11 & N9. 0 & 9.1 & -- & - & No. 01 & No. 01 & No. 01 & 0.050 & 0.009 & $\varepsilon 6$ & 110 \\
\hline $23 \ldots$ & 1030 & 11 & N1 6 & 7.5 & 23 & 3 & 0.12 & 0.79 & 0.93 & 0.090 & 0.039 & 70 & 100 \\
\hline \multicolumn{14}{|l|}{ SEP } \\
\hline $15 \ldots$ & 1130 & 7.8 & 5.4 & 8.1 & 9 & $<1$ & 0.03 & 0.48 & No. 01 & No. 120 & 0.040 & NE 7 & 82 \\
\hline
\end{tabular}




\section{Surface-Water Stations}

\section{B. Partial-Record and Miscellaneous-Record Sites}

\section{East Branch Allen Creek Above Erie Canal Siphon near Pittsford, N.Y.}

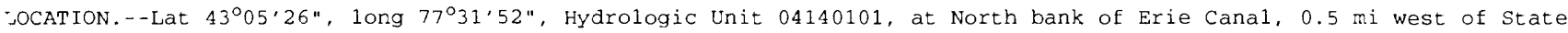
Highway 31.

PERIOD OF RECORD.--December 1984 to current year.

CHEMICAL DATA: $1984-86$ (a), $1988-89$ (c), 1990 (d), $1991-92$ (c), 1993 (a).

NUTRIENT DATA: $1984-86$ (a), 1988-89 (c), 1990 (d), 1991-92 (c), 1993 (a).

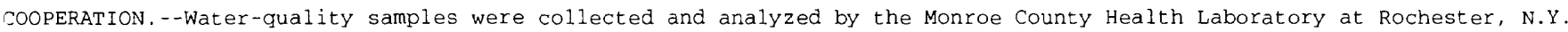

\section{WATER-QUALITY DATA}

\begin{tabular}{|c|c|c|c|c|c|c|c|c|c|c|c|c|c|}
\hline & & $\begin{array}{c}\text { DIS- } \\
\text { CHARGE, }\end{array}$ & & & $\begin{array}{l}\text { RES IDUE } \\
\text { TOTAL }\end{array}$ & RESIDUE & $\begin{array}{l}\text { NITRO- } \\
\text { GEN }\end{array}$ & $\begin{array}{l}\text { NITRO- } \\
\text { GEN, AM- }\end{array}$ & NIT & & $\begin{array}{l}\text { PHOS- } \\
\text { PHORUS }\end{array}$ & HLO- & \\
\hline & & $\begin{array}{l}\text { INST. } \\
\text { CUBIC }\end{array}$ & TUR- & OXYGEN, & $\begin{array}{l}\text { AT } 105 \\
\text { DEG. C, }\end{array}$ & $\begin{array}{l}\text { VOLA- } \\
\text { TILE, }\end{array}$ & $\begin{array}{c}\text { AMMONIA } \\
\text { DIS- }\end{array}$ & $\begin{array}{l}\text { MONIA + } \\
\text { ORGANIC }\end{array}$ & $\begin{array}{c}\mathrm{GEN} \\
\mathrm{NO}_{2}+\mathrm{NO}_{3}\end{array}$ & $\begin{array}{c}\text { PHOS- } \\
\text { PHORUS }\end{array}$ & $\begin{array}{l}\text { ORTHO, } \\
\text { DIS- }\end{array}$ & $\begin{array}{l}\text { RIDE, } \\
\text { DIS- }\end{array}$ & $\begin{array}{l}\text { SULFATE } \\
\text { DIS- }\end{array}$ \\
\hline ATE & TIME & $\begin{array}{c}\text { FEET } \\
\text { PER } \\
\text { SECOND }\end{array}$ & $\begin{array}{l}\text { BID- } \\
\text { ITY } \\
\text { (NTU) }\end{array}$ & $\begin{array}{c}\text { DIS- } \\
\text { SOLVED } \\
(\mathrm{mg} / \mathrm{L})\end{array}$ & $\begin{array}{l}\text { SUS - } \\
\text { PENDED } \\
(\mathrm{mg} / \mathrm{L})\end{array}$ & $\begin{array}{l}\text { SUS- } \\
\text { PENDED } \\
\text { (mg/L) }\end{array}$ & $\begin{array}{l}\text { SOLVED } \\
(\mathrm{mg} / \mathrm{L} \\
\text { as } \mathrm{N})\end{array}$ & $\begin{array}{l}\text { TOTAL } \\
\text { (mg/L } \\
\text { as N) }\end{array}$ & $\begin{array}{c}\text { TOTAL } \\
\text { (mg/L } \\
\text { as N) }\end{array}$ & $\begin{array}{l}\text { TOTAL } \\
\text { (mg/L } \\
\text { as P) }\end{array}$ & $\begin{array}{l}\text { SOLVED } \\
(\mathrm{mg} / \mathrm{L} \\
\text { as P) }\end{array}$ & $\begin{array}{l}\text { SOLVED } \\
(\mathrm{mg} / \mathrm{L} \\
\text { as Cl) }\end{array}$ & $\begin{array}{c}\text { SOLVED } \\
(\mathrm{mg} / \mathrm{L} \\
\left.\text { as } \mathrm{SO}_{4}\right)\end{array}$ \\
\hline
\end{tabular}

OCT

$06 \ldots 0950 \quad \ldots-11$

$13 \ldots .801130 \quad-\quad 8.3$

$20 . .1130 \quad--\quad 3.7$

$27 \ldots \quad 1130-0.80 \quad 4.2$

JUN

$\begin{array}{llll}28 \ldots & 0900 \quad 1.4 & 3.5\end{array}$

JUL

$21 \ldots \quad 1030 \quad--\quad 5.4$

AUC

$18 \ldots \quad 0950 \ldots$

$\begin{array}{llll}01 \ldots & 1130 & - & 9\end{array}$

$13 \ldots 20$

OCT

05... $1100 \quad 0.50$

$27 \ldots \quad 0900 \quad--\quad 2.2$

JUN

$\begin{array}{lllll}15 \ldots & 1130 & \ldots & 4.4 & 8.0\end{array}$

Ju

$06 \ldots 1030$

$13 \ldots .1050$

$20 \ldots$

$25 \ldots \quad 1030$

AUG

$01 \ldots \quad 1100$

$08 \ldots \quad 0950$

$17 \ldots \quad 0950$

$24 \ldots \quad 1130$

$30 \ldots \quad 0950$

$21 \ldots-1050$

OCT

$09+1050$

JUN

$10 \ldots \quad 1100$

$19 \ldots \quad 1130$

JUL

$10 \ldots \quad 0950$

$19 \ldots \quad 1130$

$25 \ldots \quad 1050$

21.

27.

1130

$\begin{array}{lll}-- & 5.2 & 9.3\end{array}$

$-\quad 4.7$

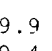

$(\mathrm{mg} / \mathrm{L}) \quad(\mathrm{mg} / \mathrm{L})$

\section{WATER YEAR OCTOBER 1988 TO SEPTEMBER 1989}

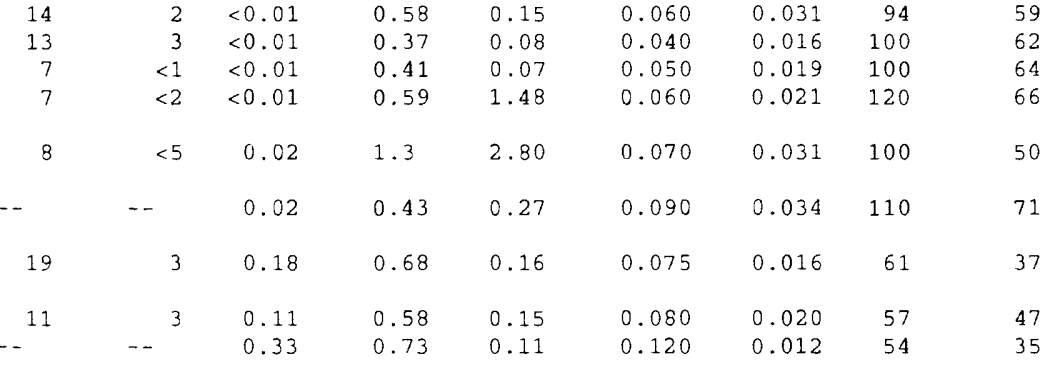

WATER YEAR OCTOBER 1989 TO SEPTEMBER 1990

\begin{tabular}{|c|c|c|c|c|c|c|c|c|}
\hline 4 & $<2$ & 0.01 & 0.37 & 0.01 & 0.055 & 0.022 & 120 & 98 \\
\hline 3 & $<2$ & 0.02 & 0.26 & 0.12 & 0.030 & 0.012 & 110 & 97 \\
\hline 8 & $<5$ & 0.04 & 0.84 & 0.74 & 0.085 & 0.052 & 110 & 110 \\
\hline$<5$ & $<5$ & 0.02 & 0.70 & 0.39 & 0.065 & 0.032 & 100 & \\
\hline$<5$ & $<5$ & 0.03 & N0. 01 & 0.59 & 0.050 & No. 001 & 80 & \\
\hline$<5$ & $<5$ & 0.08 & 0.68 & 0.28 & 0.055 & $\mathrm{~N} 0.001$ & 100 & 70 \\
\hline 590 & 90 & 0.15 & 3.0 & 0.85 & 0.770 & 0.057 & 41 & \\
\hline$<5$ & $<5$ & 0.05 & 0.51 & 0.18 & 0.080 & 0.032 & 96 & \\
\hline$<5$ & 9 & 0.06 & 0.04 & 0.12 & 0.040 & 0.003 & 100 & \\
\hline$<5$ & $<5$ & 0.77 & 0.77 & 0.32 & 0.070 & 0.045 & 100 & \\
\hline 5 & $<5$ & 0.45 & 0.45 & 0.21 & 0.050 & 0.024 & 95 & \\
\hline$<5$ & $<5$ & $<0.01$ & 0.59 & 0.04 & 0.040 & 0.024 & 110 & \\
\hline$<5$ & $<5$ & No.01 & 0.37 & 0.07 & 0.045 & 0.023 & 88 & \\
\hline & & 0.04 & 0.02 & 0.04 & 0.019 & 0.001 & 100 & \\
\hline
\end{tabular}

WATER YEAR OCTOBER 1990 TO SEPTEMBER 1991

$\begin{array}{rrrlllllr}12 & <5 & <0.01 & 0.46 & 0.16 & 0.085 & 0.037 & 94 & 60 \\ 10 & 2 & 0.04 & 0.69 & 0.57 & 0.060 & 0.025 & 100 & 64 \\ 9 & <5 & <0.01 & 0.66 & 0.72 & 0.075 & 0.037 & 92 & 68 \\ & & & & & & & & \\ 62 & -- & 0.02 & 0.67 & 0.18 & 0.090 & 0.045 & 110 & 120 \\ -7 & 2 & 0.03 & 1.2 & 0.09 & 0.060 & 0.030 & 110 & 110 \\ 18 & 4 & 0.01 & 0.69 & 0.38 & 0.085 & 0.021 & 36 & 36\end{array}$

WATER YEAR OCTOBER 1991 TO SEPTEMBER 1992

-. 62

18

0.38

0.085

$0.021 \quad 36$
36

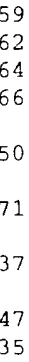

88

95

100
88

100
78

110

4

120

110

36
0.035

0.050
$0.008 \quad 110$

$0.010 \quad 83$

$\begin{array}{rrrrrrrrr}3 & <2 & 0.03 & 0.95 & 0.91 & 0.035 & 0.008 & 110 & 76 \\ 8 & <5 & 0.08 & 0.68 & 1.50 & 0.050 & 0.010 & 83 & 84\end{array}$




\section{Surface-Water Stations}

B. Partial-Record and Miscellaneous-Record Sites

\section{East Branch Allen Creek Above Erie Canal Siphon near Pittsford, N.Y.}

\begin{tabular}{|c|c|c|c|c|c|c|c|c|c|c|c|c|c|}
\hline & & & & & & ATER-C & JALITY & DATA & & & & & \\
\hline DATE & TIME & $\begin{array}{c}\text { DIS- } \\
\text { CHARGE, } \\
\text { INST. } \\
\text { CUBIC } \\
\text { FEET } \\
\text { PER } \\
\text { SECOND }\end{array}$ & $\begin{array}{l}\text { TUR- } \\
\text { BID- } \\
\text { ITY } \\
\text { (NTU) }\end{array}$ & $\begin{array}{c}\text { OXYGEN, } \\
\text { DIS- } \\
\text { SOLVED } \\
\text { (mg/L) }\end{array}$ & $\begin{array}{l}\text { RESIDUE } \\
\text { TOTAL } \\
\text { AT } 105 \\
\text { DEG. C, } \\
\text { SUS- } \\
\text { PENDED } \\
(\mathrm{mg} / \mathrm{L})\end{array}$ & $\begin{array}{l}\text { RESIDUE } \\
\text { VOLA- } \\
\text { TILE, } \\
\text { SUS - } \\
\text { PENDED } \\
\text { (mg/L) }\end{array}$ & $\begin{array}{l}\text { NITRO- } \\
\text { GEN, } \\
\text { AMMONIA } \\
\text { DIS- } \\
\text { SOLVED } \\
\text { (mg/L } \\
\text { as N) }\end{array}$ & $\begin{array}{c}\text { NITRO- } \\
\text { GEN, AM- } \\
\text { MONIA + } \\
\text { ORGANIC } \\
\text { TOTAL } \\
\text { (mg/L } \\
\text { as N) }\end{array}$ & $\begin{array}{c}\text { NITRO- } \\
\text { GEN, } \\
\mathrm{NO}_{2}+\mathrm{NO}_{3} \\
\text { TOTAL } \\
(\mathrm{mg} / \mathrm{L} \\
\text { as } \mathrm{N})\end{array}$ & $\begin{array}{c}\text { PHOS- } \\
\text { PHORUS } \\
\text { TOTAL } \\
\text { (mg/L } \\
\text { as P) }\end{array}$ & $\begin{array}{l}\text { PHOS- } \\
\text { PHORUS } \\
\text { ORTHO, } \\
\text { DIS- } \\
\text { SOLVED } \\
(\mathrm{mg} / \mathrm{L} \\
\text { as P) }\end{array}$ & $\begin{array}{l}\text { CHLO- } \\
\text { RIDE, } \\
\text { DIS- } \\
\text { SO_VED } \\
\text { (mJ/L } \\
\text { as CI) }\end{array}$ & $\begin{array}{l}\text { SULFATE } \\
\text { DIS- } \\
\text { SOLVED } \\
\text { (mg/L } \\
\left.\text { as } \mathrm{SO}_{4}\right)\end{array}$ \\
\hline UN & & & & & & & & & & & & & \\
\hline $04 \ldots$ & 1050 & -- & 4.4 & 8.9 & 6 & $<2$ & 0.05 & 0.83 & 1.10 & 0.060 & 0.028 & 99 & 82 \\
\hline $10 \ldots$ & 1050 & -- & 7.3 & - & 12 & 4 & 0.06 & 1.0 & 1.70 & 0.065 & 0.032 & 100 & 66 \\
\hline $24 \ldots$ & 0950 & -- & 26 & 8.1 & 133 & 14 & 0.05 & 1.3 & 1.20 & 0.145 & 0.030 & 350 & 34 \\
\hline JUL & & & & & & & & & & & & & \\
\hline $08 \ldots$ & 0950 & 0.10 & 6.6 & 7.2 & 5 & $<1$ & 0.08 & 0.53 & 0.39 & 0.055 & 0.024 & 55 & 55 \\
\hline SEP & & & & & & & & & & & & & \\
\hline $15 \ldots$ & 1130 & 0.50 & -- & 9.7 & 4 & $<1$ & 0.02 & 0.44 & 2.10 & 0.050 & 0.029 & 80 & 54 \\
\hline & & & & & IATE & $T$ & 199 & $\operatorname{Lim}$ & 93 & & & & \\
\hline$E P$ & & & & & & & & & & & & & \\
\hline $15 \ldots$ & 0950 & 0.50 & 7.8 & 7.5 & 9 & 2 & 0.04 & 1.1 & No. 01 & 0.060 & 0.028 & 120 & 81 \\
\hline
\end{tabular}




\section{Turface-Water Stations}

\section{Partial-Record and Miscellaneous-Record Sites}

\section{East Branch Allen Creek Below Erie Canal Siphon Near Pittsford, N.Y'.}

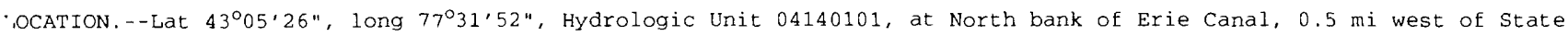
Highway 31.

PERIOD OF RECORD. --December 1984 to current year.

CHEMICAL DATA: $1984-86($ a), $1988-89$ (c), 1990 (d), $1991-92$ (2), 1993 (a)

NUTRIENT DATA: $1984-86$ (a), $1988-89$ (c), 1990 (d), $1991-92$ (2), 1993 (a).

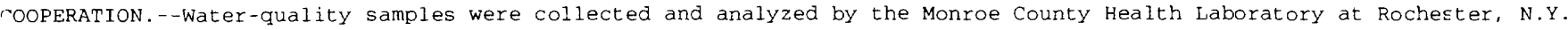

\section{WATER-QUALITY DATA}

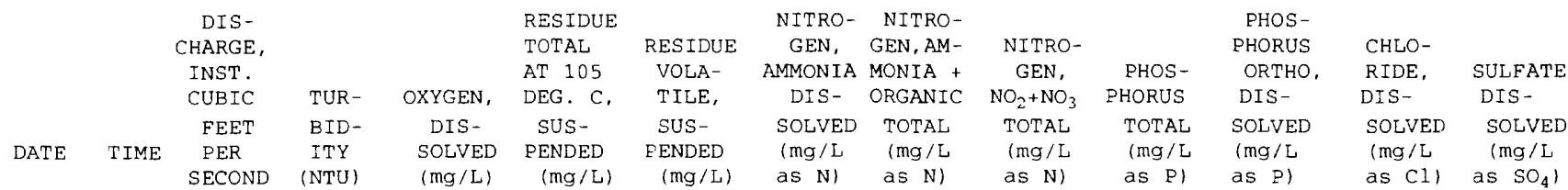

OCT

$\begin{array}{llll}\text { OCT } \\ 06 \ldots & 1130 & -- & 14 \\ 13 \ldots & 1130 & -- & 18 \\ 20 \ldots & 1130 & -- & 5.5 \\ 27 \ldots & 1130 & 3.2 & 8.8 \\ \text { JUN } & & & \\ 28 \ldots & 1100 & 2.3 & 17 \\ \text { AUG } & & & \\ 18 \ldots & 1130 & -- & 18 \\ \text { SEP } & & & \\ 01 \ldots & 1050 & -- & 25 \\ 13 \ldots & 1050 & -- & 20\end{array}$

OCT

JUN

$15 \ldots \quad 0950$

$29 \ldots \quad 0950$

JUL

$06 \ldots 0950$

$13 \ldots-1100$

$25 \ldots .0950 \quad-\cdots \quad 11$

AUG

$01 \ldots 1030$

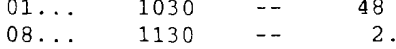

$17 \ldots 0950$

$24 \ldots \quad 1050$

$\mathrm{SEP}$

$21 \ldots 0900$

$\begin{array}{cc}2.3 & 6.4 \\ -- & 6.7 \\ -- & 19 \\ & \\ -- & 12 \\ -- & 10 \\ -- & 230 \\ -- & 11 \\ & \\ -- & 48 \\ -- & 2.6 \\ -- & 18 \\ -- & 12 \\ -- & 8.0\end{array}$

$(\mathrm{mg} / \mathrm{L})$

as N) as $\mathrm{N}$ )

as P)

\section{WATER YEAR OCTOBER 1988 TO SEPTEMBER 1989}

\begin{tabular}{|c|c|c|c|c|c|c|c|c|}
\hline 22 & 3 & 0.06 & 0.69 & 0.31 & 0.045 & 0.009 & 98 & 73 \\
\hline 33 & 4 & 0.11 & 0.61 & 0.42 & 0.060 & 0.018 & 140 & 72 \\
\hline 17 & 2 & 0.05 & 0.45 & 0.42 & 0.065 & 0.015 & 68 & 64 \\
\hline 12 & 2 & 0.06 & 0.81 & 0.83 & 0.085 & 0.023 & 120 & 79 \\
\hline 25 & $<5$ & 0.03 & 1.0 & 2.40 & 0.095 & 0.038 & 86 & 48 \\
\hline 33 & 5 & 0.02 & 0.61 & 0.52 & 0.080 & 0.005 & 93 & \\
\hline 5 & 6 & 0.09 & 0.86 & 0.54 & 0.130 & 0.011 & 80 & \\
\hline & -- & 0.15 & 0.69 & 0.47 & 0.115 & 022 & 61 & \\
\hline
\end{tabular}

WATER YEAR OCTOBER 1989 TO SEPTEMBER 1990

\begin{tabular}{|c|c|c|c|c|c|c|c|}
\hline 22 & 3 & 0.02 & 0.35 & 0.25 & 0.060 & 0.009 & 100 \\
\hline 13 & $<5$ & $<0.01$ & 0.79 & 1.00 & 0.060 & 0.007 & 98 \\
\hline 16 & $<5$ & 0.04 & 0.88 & 0.66 & 0.075 & 0.027 & 90 \\
\hline 92 & 14 & 0.02 & N0. 01 & 0.76 & 0.065 & N0.001 & 97 \\
\hline 15 & $<5$ & 0.14 & 0.86 & 0.54 & 0.055 & N0.001 & 87 \\
\hline 422 & 54 & 0.09 & 2.4 & 0.83 & 0.550 & 0.046 & 43 \\
\hline 48 & 7 & 0.04 & 0.69 & 0.49 & 0.100 & 0.015 & 98 \\
\hline 8 & 8 & 0.13 & 0.03 & 0.42 & 0.012 & 0.022 & 90 \\
\hline 12 & $<5$ & 0.44 & 0.44 & 0.31 & 0.080 & 0.046 & 100 \\
\hline 26 & $<5$ & No. 01 & 0.58 & 0.55 & 0.073 & 0.020 & 74 \\
\hline 20 & $<5$ & NO. 01 & 0.42 & 0.50 & 0.065 & 0.024 & 74 \\
\hline 15 & $<5$ & N0. 01 & No. 01 & 0.58 & 0.055 & 0.020 & 76 \\
\hline-- & - & 0.06 & 0.06 & 0.41 & 0.027 & 0.009 & 96 \\
\hline
\end{tabular}

OCT

JUN

$10 \ldots 0950$

$19 \ldots 0900$

$10 \ldots \quad 1130$

$19 \ldots .1130$

25... 0900

AUG

$21 \ldots \quad 1130$

$+-$

$-1$

$+-$

--
8.4
8.1
9.2
8.5
--
8.1
8.1
8.7
8.0
--
--
--

WATER YEAR OCTOBER 1990 TO SEPTEMBER 1991

MAY

$\begin{array}{lllll}21 \ldots & 0950 & 1.6 & 4.7 & 9.4\end{array}$

15

$<\quad 0.01$
$<2$

$0.43 \quad 0.27$

0.070

0.030

120

56

$-9.7$

$8.9 \quad 3$

0.56

0.80

0.070

0.005

0.075

0.017

0.100

\subsection{3}

0.50

0.40

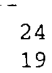

0.05
0.06

0.59
0.73

0.43
0.38

0.070

0.024

0.016

0.025

18

0.05

0.50

0.43

0.065

0.030

WATER YEAR OCTOBER 1991 TO SEPTEMBER 1992

JUN

$\begin{array}{ccccc}04 \ldots & 0900 & -- & 4.6 & 8.7 \\ 24 \ldots & 1130 & -- & 28 & 7.9\end{array}$

$\begin{array}{rrrlr}9 & 2 & 0.04 & 0.73 & 0.94 \\ 12 & <5 & 0.07 & 0.54 & 1.00 \\ 7 & 1 & 0.05 & 0.86 & 1.00 \\ \text { N58 } & 11 & 0.07 & 1.4 & 1.10\end{array}$

0.035

0.007

100

85
55

$\begin{array}{lr}8.7 & 7 \\ 7.9 & \text { N58 }\end{array}$

0.07

$1.4 \quad 1.10$

2.060

0.027

97
290

87 


\section{Surface-Water Stations}

\section{B. Partial-Record and Miscellaneous-Record Sites}

\section{East Branch Allen Creek Below Erie Canal Siphon Near Pittsford, N.Y.}

\begin{tabular}{|c|c|c|c|c|c|c|c|c|c|c|c|c|c|}
\hline & & & & & & ATER- & UALITY & DATA & & & & & \\
\hline DATE & TIME & $\begin{array}{c}\text { DIS- } \\
\text { CHARGE, } \\
\text { INST. } \\
\text { CUBIC } \\
\text { FEET } \\
\text { PER } \\
\text { SECOND }\end{array}$ & $\begin{array}{l}\text { TUR- } \\
\text { BID- } \\
\text { ITY } \\
\text { (NTU) }\end{array}$ & $\begin{array}{c}\text { OXYGEN, } \\
\text { DIS- } \\
\text { SOLVED } \\
\text { (mg/L) }\end{array}$ & $\begin{array}{l}\text { RESIDUE } \\
\text { TOTAL } \\
\text { AT } 105 \\
\text { DEG. C, } \\
\text { SUS- } \\
\text { PENDED } \\
(\mathrm{mg} / \mathrm{L})\end{array}$ & $\begin{array}{l}\text { RESIDUE } \\
\text { VOLA- } \\
\text { TILE, } \\
\text { SUS- } \\
\text { PENDED } \\
\text { (mg/L) }\end{array}$ & $\begin{array}{c}\text { NITRO- } \\
\text { GEN, } \\
\text { AMMONIA } \\
\text { DIS- } \\
\text { SOLVED } \\
\text { (mg/L } \\
\text { as N) }\end{array}$ & $\begin{array}{c}\text { NITRO- } \\
\text { GEN, AM- } \\
\text { MONIA + } \\
\text { ORGANIC } \\
\text { TOTAL } \\
(\mathrm{mg} / \mathrm{L} \\
\text { as N) }\end{array}$ & $\begin{array}{c}\mathrm{NITRO}- \\
\text { GEN, } \\
\mathrm{NO}_{2}+\mathrm{NO}_{3} \\
\mathrm{TOTAL} \\
(\mathrm{mg} / \mathrm{L} \\
\text { as } \mathrm{N})\end{array}$ & $\begin{array}{c}\text { PHOS- } \\
\text { PHORUS } \\
\text { TOTAL } \\
(\mathrm{mg} / \mathrm{L} \\
\text { as P) }\end{array}$ & $\begin{array}{l}\text { PHOS- } \\
\text { PHORUS } \\
\text { ORTHO, } \\
\text { DIS- } \\
\text { SOLVED } \\
\text { (mg/L } \\
\text { as P) }\end{array}$ & $\begin{array}{l}\text { CHLO- } \\
\text { RIDE, } \\
\text { DIS- } \\
\text { SO'.VED } \\
\text { (mg/L } \\
\text { as C1) }\end{array}$ & $\begin{array}{l}\text { SULFATE } \\
\text { DIS- } \\
\text { SOLVED } \\
(\mathrm{mg} / \mathrm{L} \\
\left.\text { as } \mathrm{SO}_{4}\right)\end{array}$ \\
\hline UL & & & & & & & & & & & & & \\
\hline $14 \ldots$ & 0950 & $\ldots$ & 60 & 7.6 & 77 & 9 & 0.04 & 0.93 & 1.10 & 0.180 & 0.040 & 55 & 39 \\
\hline $22 \ldots$ & 0950 & 2.0 & 4.6 & 9.0 & 9 & 1 & 0.03 & 0.94 & 3.40 & No.001 & 0.049 & 82 & 41 \\
\hline AUG & & & & & & & & & & & & & \\
\hline $12 \ldots$ & 1030 & -- & 3.3 & 8.9 & 5 & 2 & $<0.01$ & 0.66 & 2.50 & 0.070 & 0.047 & 83 & 45 \\
\hline SEP & & & & & & & & & & & & & \\
\hline $15 \ldots$ & 1030 & 2.3 & -- & 9.6 & 16 & 3 & $<0.01$ & 0.56 & 1.10 & 0.070 & 0.022 & 72 & 65 \\
\hline & & & & & WATER $Y$ & T & R 1992 TO S & SEPTEMBER & 1993 & & & & \\
\hline SEP & & & & & & & & & & & & & \\
\hline 15. & 1130 & 3.2 & 8.8 & 8.2 & 13 & 2 & 0.04 & 0.55 & No. 01 & 0.060 & 0.038 & 96 & 88 \\
\hline
\end{tabular}




\section{Surface-Water Stations}

B. Partial-Record and Miscellaneous-Record Sites

\section{East Branch Allen Creek Erie Canal Siphon Near Pittsford, N.Y.}

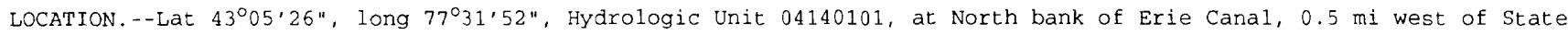
Highway 31

PERIOD OF RECORD. --December 1984 to current year.

CHEMICAL DATA: $1984-86$ (a), 1988-89 (c), 1990 (d), 1991-92 (c), 1993 (a).

NUTRIENT DATA: $1984-86$ (a), 1988-89 (c), 1990 (d), 1991-92 (c), 1993 (a).

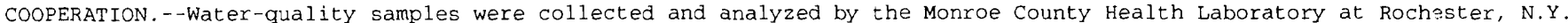

\section{WATER-QUALITY DATA}

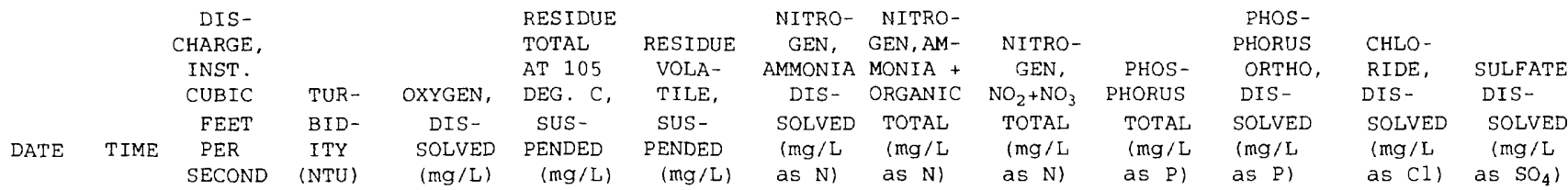

OCT

$13 .$.

$20 \ldots$

JUN

$28 .$.

JUL

21.

18.

SEP

$01 \ldots 0900 \quad--27$

$13 \ldots \quad 0900 \quad-. \quad 20$

OCT

JUN

JUN

15

29

$06 \ldots-1130$

$13 \ldots \quad 0900$

$13 \ldots \quad 1030$

$20 \ldots \quad 1050$

$25 \ldots \quad 1130$

AUG

$01 \ldots \quad 0950$

$17 \ldots \quad 1130$

$24 \ldots \quad 0900$

$30 \ldots \quad 1050$

SEP

$21 \ldots \quad 1100$

$1.8 \quad 14$

OC'

Jun

$10 \ldots \quad 1030$

JUL

$10 \ldots 1050$

$19 \ldots 0950$

$25 \ldots$

AUC

$21 \ldots \quad 0950 \quad \ldots \quad 9.6$

MAY $(\mathrm{mg} / \mathrm{L}) \quad(\mathrm{mg} / \mathrm{L})$

\section{WATER YEAR OCTOBER 1988 TO SEPTEMBER 1989}

\begin{tabular}{|c|c|c|c|c|c|c|c|}
\hline 21 & & 0.06 & 0.69 & 0.39 & 0.040 & 0.004 & 100 \\
\hline 36 & & 0.14 & 1.0 & 0.52 & 0.080 & 0.018 & 150 \\
\hline 18 & & 0.06 & 0.40 & 0.49 & 0.070 & 0.014 & 59 \\
\hline 14 & & 0.10 & 0.80 & 0.46 & 0.075 & 0.023 & 130 \\
\hline 76 & & 0.06 & 0.98 & 0.94 & 0.185 & 0.044 & 37 \\
\hline & -- & $<0.01$ & 0.69 & 0.75 & 0.085 & 0.003 & 83 \\
\hline 49 & & 0.04 & 0.67 & 0.55 & 0.090 & 0.003 & 94 \\
\hline 60 & - & 0.09 & $\begin{array}{l}0.94 \\
0.69\end{array}$ & $\begin{array}{l}0.55 \\
0.48\end{array}$ & 0.130 & 0.009 & 81 \\
\hline
\end{tabular}

\section{WATER YEAR OCTOBER 1989 TO SEPTEMBER 1990}

9.2
9.0
9.7
8.2
8.6
-
7.8
8.2
5.0
-
-

35
12
10
21
16
21
29
42
6
103
27
19

$\begin{array}{rl}4 & 0.02 \\ <5 & 0.01\end{array}$

$0.46 \quad 0.40$

0.070

0.010

$0.67 \quad 0.92$

0.03

$0.70 \quad 0.79$

0.040

0.075

0.003

No.01 0.80

\subsection{2}

0.65

0.17
0.16

0.75

0.58

0.60

0.74

0.51

0.060

0.045

0.060

0.200

0.05

0.59

0.090

\subsection{3 \\ 0.54}

0.09
0.84

0.84

0.47

0.69

No. 00

0.44

0.73

0.007

0.150

0.080

0.055

0.09

0.76

0.56

0.080

N0.00

No.00

N0. 00

0.005

0.009

0.020

0.018

0.024

0.020

0.029

0.040

0.024

160

45

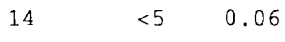

$0.60 \quad 0.49$

0.075

0.004

0.009

72
64

90

$\begin{array}{ll}-- & 11 \\ 2.4 & --\end{array}$

9.5
9.2

- $\quad 36$

$\begin{array}{ll}-- & 35 \\ -- & 27\end{array}$

0.02

0.58

0.88

0.52

0.085

0.065

0.009

0.08
0.07

0.60

0.45

0.66

0.41

0.060

0.008

0.019

66
74

$0.031 \quad 75$

0.035

0.006

0.005
0.96
0.78

0.035

\section{4 \\ 93 \\ 99 \\ 10}

82

88

79

100

140

130

84

80

120

94
92

50

\section{WATER YEAR OCTOBER 1991 TO SEPTEMBER 1992}

N9. 8
8.8

0.06

0.57

$\begin{array}{ll}89 & 100 \\ 46 & 110\end{array}$

(2) 


\section{Surface-Water Stations}

B. Partial-Record and Miscellaneous-Record Sites

\section{East Branch Allen Creek Erie Canal Siphon Near Pittsford, N.Y.} WATER-QUALITY DATA

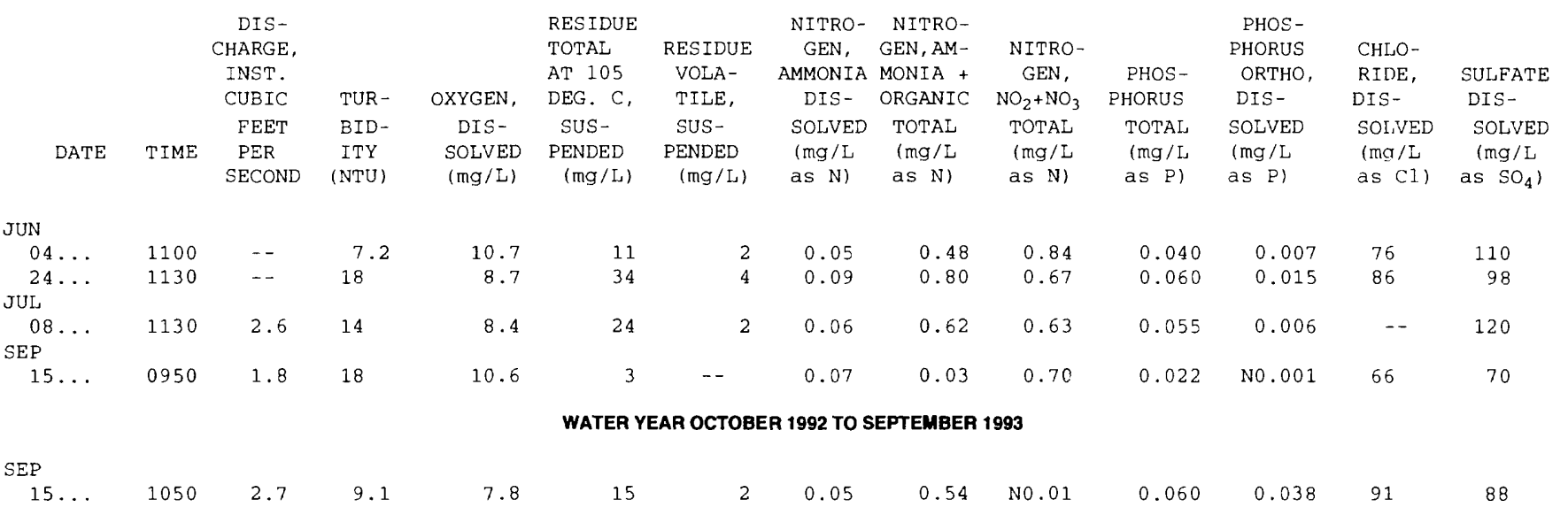




\section{Surface-Water Stations}

\section{B. Partial-Record and Miscellaneous-Record Sites}

\section{Allen Creek Below Erie Canal Siphon Near Rochester, N.Y.}

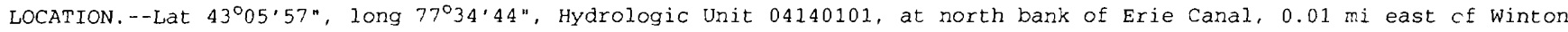
Road.

PERIOD OF RECORD.--December 1984 to current year.

CHEMICAL DATA: $1984-86$ (a), 1988-89 (c), 1990 (d), 1991-93 (c).

NUTRIENT DATA: $1984-86$ (a), $1988-89$ (c), 1990 (d), $1991-93$ (c)

COOPERATION.--Water-quality samples were collected and analyzed by the Monroe County Environmental Health Laboratory at Rochester, N.Y.

\section{WATER-QUALITY DATA}

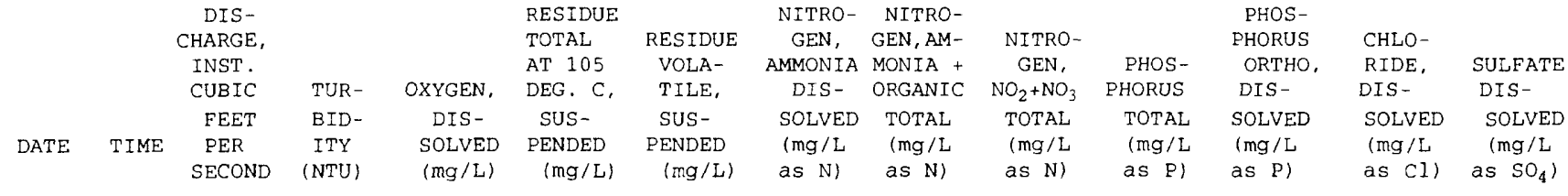

$\mathrm{OCT}$

$\begin{array}{rrrr}06 \ldots & 1100 & 6.1 & 13 \\ 13 \ldots & 1030 & -- & 16 \\ 20 \ldots & 0950 & -- & 6.8 \\ 27 \ldots & 1100 & \cdots & 8.5 \\ \text { JUN } & & & \\ \quad 28 \ldots & 1130 & 12 & 140 \\ \text { AUG } & & & \\ \quad 18 \ldots & 1030 & -- & 20 \\ \text { SEP } & & & \\ \quad 1 \ldots & 0950 & \text { N2.3 } & 22 \\ 13 \ldots & 1130 & -- & 15\end{array}$

OCT

$0950 \ldots 16$

JUN

$15 \ldots \quad 1030 \quad \ldots \quad 8.0$

$29 \ldots 0950 \quad \ldots \quad 34$

JUL

$13 \ldots 1130 \quad \ldots \quad 14$

$25 \ldots 0900 \quad \ldots \quad 13$

AUG

$08 \ldots 1100 \quad--\quad 15$

$24 \ldots 0950 \quad \ldots \quad 13$

$30 \ldots 0950 \ldots 140$

SEP

$21 \ldots \quad 1030 \quad \ldots \quad 11$

WATER YEAR OCTOBER 1988 TO SEPTEMBER 1989

\begin{tabular}{|c|c|c|c|c|c|c|c|c|}
\hline 23 & 3 & 0.15 & 1.0 & 0.38 & 0.045 & 0.013 & 100 & 71 \\
\hline 34 & 4 & 0.09 & 0.59 & 0.51 & 0.065 & 0.019 & 87 & 58 \\
\hline 13 & 2 & 0.10 & 0.65 & 0.42 & 0.060 & 0.015 & 75 & 69 \\
\hline 10 & 2 & 0.09 & 0.76 & 0.46 & 0.055 & 0.018 & 110 & 69 \\
\hline 143 & 12 & 0.06 & 1.0 & 0.72 & 0.235 & 0.030 & 50 & 45 \\
\hline-- & -- & 0.16 & 0.88 & 0.44 & 0.090 & 0.004 & 100 & 98 \\
\hline 58 & 12 & 0.16 & 2.0 & 0.52 & 0.155 & 0.047 & 95 & \\
\hline-- & -- & 0.08 & 0.99 & 0.33 & 0.085 & 0.014 & 100 & \\
\hline
\end{tabular}

WATER YEAR OCTOBER 1989 TO SEPTEMBER 1990

--
--
--
--
9.4
9.2
7.3
8.2
7.1
--
--
--

\begin{tabular}{|c|c|c|c|}
\hline 33 & 3 & 0.19 & 0.40 \\
\hline 30 & 3 & 0.13 & 0.44 \\
\hline - & -- & 0.10 & 0.83 \\
\hline 18 & $<5$ & 0.05 & 0.85 \\
\hline 18 & 6 & 0.02 & 0.78 \\
\hline 60 & 7 & 0.04 & 0.78 \\
\hline 22 & $<5$ & 0.15 & 0.73 \\
\hline 45 & 7 & 0.04 & 0.73 \\
\hline 26 & $<5$ & 0.47 & 0.47 \\
\hline 22 & $<5$ & No. 01 & 0.39 \\
\hline 23 & 33 & 0.05 & 1.1 \\
\hline- & -. & 0.08 & 0.52 \\
\hline
\end{tabular}

$\begin{array}{lrrr}0.080 & 0.009 & 77 & 72 \\ 0.070 & 0.016 & 75 & 75 \\ 0.055 & 0.002 & 110 & 73 \\ 0.080 & 0.026 & 87 & 44 \\ 0.060 & 0.003 & 95 & 82 \\ 0.105 & 0.024 & 73 & 76 \\ 0.015 & \text { N0.001 } & 85 & 130 \\ 0.110 & 0.006 & 130 & 41 \\ 0.080 & 0.024 & 71 & 83 \\ 0.070 & 0.021 & 60 & 92 \\ 0.320 & 0.050 & 19 & 15 \\ 0.065 & 0.027 & 65 & 53\end{array}$

WATER YEAR OCTOBER 1990 TO SEPTEMBER 1991

OC

$\begin{array}{lllll}24 \ldots & 1030 & -- & 31 & -- \\ 31 \ldots & 0900 & -- & 16 & -- \\ \text { MAY } & & & & \\ 03 \ldots & 0900 & 9.3 & 13 & 10.9 \\ 16 \ldots & 1050 & -- & 6.2 & -- \\ \text { JUN } & & & & \\ 14 \ldots & 1050 & -- & 7.1 & - \\ 14 \ldots & 1130 & -- & 5.5 & -- \\ \text { JUL } & & & & \\ \quad 19 \ldots & 0950 & -- & 12 & - \\ \text { AUG } & & & & \end{array}$

$\begin{array}{rrrllllll}31 & <5 & 0.07 & 0.60 & 0.60 & 0.080 & 0.020 & 59 & 32 \\ 16 & <5 & 0.04 & 0.44 & 0.84 & 0.045 & 0.014 & 58 & 35 \\ 17 & 2 & 0.05 & 0.73 & 0.64 & 0.065 & \text { N0.001 } & 73 & 47 \\ 16 & 3 & N 0.01 & 1.2 & 0.39 & 0.045 & 0.007 & 150 & 74 \\ 27 & 4 & 0.02 & 0.80 & 0.30 & 0.060 & 0.004 & 120 & 80 \\ 7 & 2 & 0.04 & 0.85 & 0.26 & 0.035 & 0.009 & 170 & 50 \\ 21 & 2 & 0.10 & 0.58 & 0.51 & 0.075 & 0.011 & 76 & 74 \\ 18 & 4 & 0.03 & 0.62 & 0.33 & 0.065 & 0.020 & 77 & 57\end{array}$




\section{Surface-Water Stations}

\section{B. Partial-Record and Miscellaneous-Record Sites}

\section{Allen Creek Below Erie Canal Siphon Near Rochester, N.Y.}

\section{WATER-QUALITY DATA}

\begin{tabular}{|c|c|c|c|c|c|c|c|c|c|c|c|c|c|}
\hline & & $\begin{array}{c}\text { DIS- } \\
\text { CHARGE, } \\
\text { INST. }\end{array}$ & & & $\begin{array}{l}\text { RESIDUE } \\
\text { TOTAL } \\
\text { AT } 105\end{array}$ & $\begin{array}{l}\text { RESIDUE } \\
\text { VOLA- }\end{array}$ & $\begin{array}{l}\text { NITRO- } \\
\text { GEN, } \\
\text { AMMONIA }\end{array}$ & $\begin{array}{l}\text { NITRO- } \\
\text { GEN, AM- } \\
\text { MONIA + }\end{array}$ & $\begin{array}{l}\text { NITRO- } \\
\text { GEN, }\end{array}$ & & $\begin{array}{r}\mathrm{PH} \\
\mathrm{PHO} \\
\mathrm{OP}\end{array}$ & $\begin{array}{l}\mathrm{CH} \\
\mathrm{RI}\end{array}$ & \\
\hline & WEE & $\begin{array}{c}\text { CUBIC } \\
\text { FEET } \\
\text { PER }\end{array}$ & $\begin{array}{l}\text { TUR- } \\
\text { BID- }\end{array}$ & $\begin{array}{c}\text { OXYGEN, } \\
\text { DIS- } \\
\text { SOLVED }\end{array}$ & $\begin{array}{l}\text { DEG. C, } \\
\text { SUS- } \\
\text { PENDED }\end{array}$ & $\begin{array}{l}\text { TILE, } \\
\text { SUS- } \\
\text { PENDED }\end{array}$ & $\begin{array}{l}\text { DIS- } \\
\text { SOLVED } \\
\text { /mg/L }\end{array}$ & $\begin{array}{c}\text { ORGANIC } \\
\text { TOTAL } \\
\text { (mg/L }\end{array}$ & $\begin{array}{c}\mathrm{NO}_{2}+\mathrm{NO}_{3} \\
\mathrm{TOTAL} \\
(\mathrm{mg} / \mathrm{L}\end{array}$ & $\begin{array}{r}\text { PHORUS } \\
\text { TOTAL } \\
\text { (mg/L }\end{array}$ & $\begin{array}{l}\text { DIS - } \\
\text { SOLVED } \\
\text { (mg/L }\end{array}$ & $\begin{array}{l}\text { DIS- } \\
\text { SJLVED } \\
(\mathrm{mg} / \mathrm{L}\end{array}$ & $\begin{array}{l}\text { DIS- } \\
\text { SOLVED } \\
\text { (mg/L }\end{array}$ \\
\hline & & SECOND & NTU) & $(\mathrm{mg} / \mathrm{L})$ & $\{\mathrm{mg} / \mathrm{L}\}$ & $(\mathrm{mg} / \mathrm{L})$ & as $N$ ) & as $\mathrm{N}$ ? & as $\mathrm{N}$ ) & as P) & as $\mathrm{Pl}$ & as Cll & $\left.\mathrm{S} \mathrm{SO}_{4}\right)$ \\
\hline
\end{tabular}

MAY

$\begin{array}{llll}\text { MAY } & & & \\ 21 \ldots & 1100 & -- & 8.5 \\ \text { JUN } & & & \\ 04 \ldots & 1030 & 6.4 & 6.7 \\ 25 \ldots & 0900 & -- & 14 \\ \text { JUL } & & & \\ 08 \ldots & 1050 & -- & 11 \\ 22 \ldots & 1130 & 2.9 & 14 \\ \text { AUG } & & & \\ 12 \ldots & 1130 & -- & 15 \\ 26 \ldots & 0950 & -- & 75 \\ \text { SEP } & & & \\ 09 \ldots & 0900 & 1.3 & 15 \\ 30 \ldots & 1130 & -. & 25\end{array}$

MAY

$\begin{array}{rrrr}05 \ldots & 1050 & 2.8 & 8.7 \\ 19 \cdots & 1130 & 0.90 & 4.6 \\ \text { JUN } & & & \\ 02 \ldots & 0950 & 2.9 & 15 \\ 23 \cdots & 1050 & 3.0 & \mathrm{~N} 16 \\ 23 \ldots & 1130 & 2.7 & 13 \\ \text { SEP } & & & \\ 15 \ldots & 1100 & 2.7 & 12\end{array}$

WATER YEAR OCTOBER 1991 TO SEPTEMBER 1992

9.1
9.0
8.0
7.6
8.7
8.2
7.4
9.6
10.8

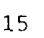

$$
10
$$

10
24

18

$$
18
$$$$
\begin{aligned}
& 18 \\
& 80
\end{aligned}
$$$$
\begin{aligned}
& 15 \\
& 27
\end{aligned}
$$

$\begin{array}{rl}3 & 0.03 \\ 2 & 0.02 \\ <5 & 0.10 \\ & \\ 2 & 0.14 \\ 3 & 0.05 \\ 3 & 0.04 \\ 8 & 0.07 \\ 2 & 0.03 \\ 4 & 0.03\end{array}$

$0.80 \quad 0.81$

$0.73 \quad 0.68$

$1.1 \quad 0.69$

$0.66 \quad 0.55$

1.20 .50

$0.81 \quad 0.22$

$0.91 \quad 0.30$

$0.80 \quad 0.44$

$\begin{array}{ll}0.80 & 0.44 \\ 0.71 & 0.47\end{array}$

WATER YEAR OCTOBER 1992 TO SEPTEMBER 1993

10.3
9.7
9.0
6.2
7.6
8.1

0.045

0.045

0.075

0.045

N0.001

0.060

0.130

0.060

0.060
$0.002 \quad 130$

$0.004 \quad 110$

$0.010 \quad 140$

$0.003 \quad--\quad 140$

$0.018 \quad 130 \quad 40$

$0.014 \quad 140$

$0.016 \quad 130$

$0.014 \quad 120$
89

98

\begin{tabular}{|c|c|c|c|c|c|c|c|c|}
\hline 8 & $<3$ & 0.01 & 0.85 & 0.25 & 0.035 & $<0.002$ & 200 & 58 \\
\hline 7 & 1 & 0.07 & No. 01 & 0.36 & 0.030 & 0.004 & 200 & 74 \\
\hline-- & - & No. 01 & N0.01 & 0.61 & 0.065 & 0.009 & 260 & 86 \\
\hline 19 & 3 & 0.16 & 0.94 & No. 00 & 0.080 & 0.034 & 110 & 95 \\
\hline 18 & 3 & 0.13 & 0.77 & 0.87 & 0.080 & 0.033 & 81 & 100 \\
\hline 20 & $<1$ & 0.02 & 0.66 & No. 01 & 0.075 & 0.019 & 90 & 78 \\
\hline
\end{tabular}




\section{Surface-Water Stations}

\section{B. Partial-Record and Miscellaneous-Record Sites}

\section{Allen Creek At Erie Canal Siphon Near Rochester, N.Y.}

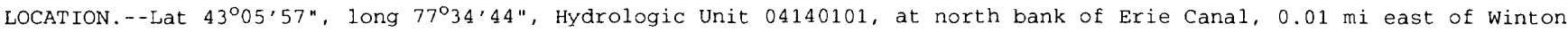
Road.

PERIOD OF RECORD. --December 1984 to current year.

CHEMICAL DATA: $1984-86$ (a), 1988-89 (c), 1990 (d), 1991-93 (c).

NUTRIENT DATA: $1984-86$ (a), 1988-89 (c), 1990 (d), 1991-93 (c).

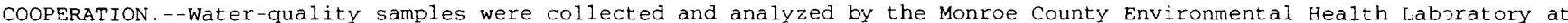
Rochester, N.Y.

\section{WATER-QUALITY DATA}

\begin{tabular}{|c|c|c|c|c|c|c|c|c|c|c|c|c|c|}
\hline & & $\begin{array}{c}\text { DIS- } \\
\text { CHARGE, } \\
\text { INST. } \\
\text { CUBIC }\end{array}$ & TUR- & OXYGEN, & $\begin{array}{l}\text { RESIDUE } \\
\text { TOTAL } \\
\text { AT } 105 \\
\text { DEG. C. }\end{array}$ & $\begin{array}{l}\text { RESIDUE } \\
\text { VOLA- } \\
\text { TILE, }\end{array}$ & $\begin{array}{l}\text { NITRO- } \\
\text { GEN, } \\
\text { AMMONIA } \\
\text { DIS- }\end{array}$ & $\begin{array}{l}\text { NITRO- } \\
\text { GEN, AM- } \\
\text { MONIA + } \\
\text { ORGANIC }\end{array}$ & $\begin{array}{c}\text { NITRO- } \\
\text { GEN, } \\
\mathrm{NO}_{2}+\mathrm{NO}_{3}\end{array}$ & $\begin{array}{l}\text { PHOS- } \\
\text { PHORUS }\end{array}$ & $\begin{array}{l}\text { PHOS- } \\
\text { PHORUS } \\
\text { ORTHO, } \\
\text { DIS - }\end{array}$ & $\begin{array}{l}\text { CHLO- } \\
\text { RIDI, } \\
\text { DIS- }\end{array}$ & $\begin{array}{l}\text { SULFATE } \\
\text { DIS- }\end{array}$ \\
\hline $\mathrm{E}$ & TIME & $\begin{array}{c}\text { FEET } \\
\text { PER } \\
\text { SECOND }\end{array}$ & $\begin{array}{c}\text { BID- } \\
\text { ITY } \\
\text { (NTU) }\end{array}$ & $\begin{array}{l}\text { DIS- } \\
\text { SOLVED } \\
(\mathrm{mg} / \mathrm{L})\end{array}$ & $\begin{array}{l}\text { SUS- } \\
\text { PENDED } \\
(\mathrm{mg} / \mathrm{L})\end{array}$ & $\begin{array}{l}\text { SUS- } \\
\text { PENDED } \\
(\mathrm{mg} / \mathrm{L})\end{array}$ & $\begin{array}{l}\text { SOLVED } \\
\text { (mg/L } \\
\text { as N) }\end{array}$ & $\begin{array}{l}\text { TOTAL } \\
(\mathrm{mg} / \mathrm{L} \\
\text { as N) }\end{array}$ & $\begin{array}{l}\text { TOTAL } \\
\text { (mg/L } \\
\text { as N) }\end{array}$ & $\begin{array}{l}\text { TOTAL } \\
\text { (mg/L } \\
\text { as P) }\end{array}$ & $\begin{array}{l}\text { SOLVED } \\
(\mathrm{mg} / \mathrm{L} \\
\text { as P) }\end{array}$ & $\begin{array}{l}\text { SOLVED } \\
\text { (mg/L } \\
\text { as Cl) }\end{array}$ & $\begin{array}{c}\text { SOLVED } \\
(\mathrm{mg} / \mathrm{L} \\
\left.\text { as } \mathrm{SO}_{4}\right)\end{array}$ \\
\hline
\end{tabular}

OCT

$\begin{array}{cccc}06 \ldots & 0900 & 4.6 & 15 \\ 13 \ldots & 0950 & -- & 14 \\ 20 \ldots & 0950 & -- & 6.8 \\ 27 \ldots & 0900 & -- & 8.3 \\ \text { JUN } & & & \\ 28 \ldots & 0950 & -- & 180 \\ \text { JUL } & & & \\ \quad 21 \ldots & 0900 & -- & 13 \\ \text { AUG } & & & \\ \quad 18 \ldots & 0900 & -- & 21 \\ \text { SEP } & & & \\ \quad 01 \ldots & 1030 & -- & 20 \\ 13 \ldots & 0950 & -- & 21\end{array}$

OCT

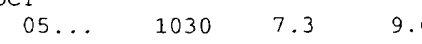

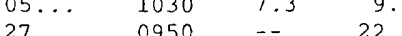

MAY

$04 \ldots \quad 1130 \quad-\cdots \quad 18$

$24 \ldots 1130$

JUN

$15 \ldots 0950 \quad--6.4$

$29 \ldots 1100$

JUL

$25 \ldots \quad 1050 \quad \ldots \quad 14$

AUG

$08 \ldots 0900 \quad \ldots \quad 18$

$24 \ldots 1030 \quad \ldots \quad 13$

$\mathrm{SEP}$

$07 \ldots 1030 \quad \ldots \quad 10$

$21 \ldots .1130 \quad \ldots \quad 11$

OCT

$24 \ldots 0950 \quad--\quad 40$

$31 \ldots 00950 \quad \ldots \quad 16$

MAY

$03 \ldots 1100 \quad 7.1 \quad 14$

$16 \ldots \quad 1130$

JUN

$14 \ldots 0950 \quad \ldots \quad 8$.

Jut

$19 \ldots \quad 1100 \quad--\quad 15$

AUG

$21 \ldots 0950 \quad \ldots \quad 12$

MAY

$\begin{array}{lllll}21 \ldots & 0950 & -- & 8.0 & \ldots \\ 04 \ldots & 1130 & 5.2 & 6.8 & 10.7\end{array}$

WATER YEAR OCTOBER 1988 TO SEPTEMBER 1989

\begin{tabular}{|c|c|c|c|c|c|c|c|}
\hline 24 & 3 & 0.18 & 1.2 & 0.43 & 0.055 & 0.019 & 100 \\
\hline 33 & 4 & 0.12 & 0.63 & 0.54 & 0.035 & 0.022 & 85 \\
\hline 11 & 2 & 0.11 & 0.56 & 0.44 & 0.070 & 0.018 & 68 \\
\hline 10 & 2 & 0.10 & 0.74 & 0.49 & 0.050 & 0.023 & 100 \\
\hline 147 & 12 & 0.07 & 0.99 & 0.79 & 0.260 & 0.031 & 30 \\
\hline-- & -- & 0.02 & 0.76 & 0.82 & 0.095 & 0.004 & 82 \\
\hline 51 & 6 & 0.17 & 0.87 & 0.49 & 0.095 & 0.003 & 91 \\
\hline 38 & 5 & 0.10 & 0.80 & 0.53 & 0.110 & 0.005 & 85 \\
\hline- & -- & 0.13 & 0.59 & 0.53 & 0.065 & 0.018 & 64 \\
\hline
\end{tabular}

WATER YEAR OCTOBER 1989 TO SEPTEMBER 1990

\begin{tabular}{|c|c|c|c|c|c|c|c|}
\hline 59 & 7 & 0.20 & 0.54 & 0.53 & 0.095 & 0.010 & 72 \\
\hline 30 & 3 & 0.13 & 0.44 & 0.76 & 0.075 & 0.016 & 72 \\
\hline 30 & $<5$ & 0.12 & 0.80 & 1.20 & 0.050 & 0.003 & 92 \\
\hline 23 & $<5$ & 0.06 & 0.91 & 0.56 & 0.095 & 0.038 & 56 \\
\hline 19 & 6 & 0.02 & 0.69 & 0.97 & 0.055 & 0.003 & 86 \\
\hline 34 & 6 & 0.06 & 0.68 & 0.73 & 0.080 & 0.007 & 69 \\
\hline 44 & $<8$ & 0.06 & 0.68 & 0.74 & 0.095 & 0.006 & 130 \\
\hline 25 & $<5$ & 0.52 & 0.52 & 0.43 & 0.080 & 0.025 & 70 \\
\hline 19 & $<5$ & N0.01 & 0.35 & 0.79 & 0.070 & 0.025 & 57 \\
\hline 21 & 4 & 0.26 & 0.66 & 0.51 & 0.070 & 0.033 & 71 \\
\hline - & -- & 0.09 & 1.0 & 0.50 & 0.060 & 0.030 & 60 \\
\hline
\end{tabular}

WATER YEAR OCTOBER 1990 TO SEPTEMBER 1991

$\begin{array}{rrrrrrrrr}42 & <5 & 0.07 & 0.56 & 0.70 & 0.090 & 0.020 & 43 & 32 \\ 18 & <5 & 0.04 & 0.45 & 0.94 & 0.040 & 0.016 & 48 & 30 \\ 22 & <4 & 0.06 & 0.50 & 0.77 & 0.055 & \text { N0.00 } & 42 & 45 \\ 21 & 3 & \text { N0.01 } & 0.65 & 0.95 & 0.055 & 0.004 & 78 & 90 \\ 24 & 3 & 0.07 & 0.67 & 0.90 & 0.055 & 0.004 & 110 & 94 \\ 32 & 3 & 0.11 & 0.57 & 0.49 & 0.075 & 0.010 & 73 & 78 \\ \text { N82 } & \text { N10 } & \text { N0.06 } & 0.56 & 0.42 & 0.070 & 0.033 & 63 & 160\end{array}$

WATER YEAR OCTOBER 1991 TO SEPTEMBER 1992

$\begin{array}{rrrrrrrrr}14 & 4 & 0.01 & 0.69 & 0.93 & 0.035 & 0.003 & 100 & 100 \\ 12 & <8 & 0.01 & 0.54 & 0.90 & 0.045 & 0.004 & 60 & 110 \\ 28 & <5 & 0.12 & 0.74 & 0.80 & 0.055 & 0.012 & 81 & 97\end{array}$




\section{Surface-Water Stations}

B. Partial-Record and Miscellaneous-Record Sites

\section{Allen Creek At Erie Canal Siphon Near Rochester, N.Y. -}

WATER-QUALITY DATA

\begin{tabular}{|c|c|c|c|c|c|c|c|c|c|c|c|c|c|}
\hline & & $\begin{array}{c}\text { DIS- } \\
\text { CHARGE, } \\
\text { INST. } \\
\text { CUBIC }\end{array}$ & TUR- & OXYGEN, & $\begin{array}{l}\text { RESIDUE } \\
\text { TOTAL } \\
\text { AT } 105 \\
\text { DEG. C, }\end{array}$ & $\begin{array}{l}\text { RESIDUE } \\
\text { VOLA- } \\
\text { TILE, }\end{array}$ & $\begin{array}{l}\text { NITRO- } \\
\text { GEN, } \\
\text { AMMONIA } \\
\text { DIS- }\end{array}$ & $\begin{array}{l}\text { NITRO- } \\
\text { GEN, AM- } \\
\text { MONIA + } \\
\text { ORGANIC }\end{array}$ & $\begin{array}{c}\text { NITRO- } \\
\text { GEN, } \\
\mathrm{NO}_{2}+\mathrm{NO}_{3}\end{array}$ & $\begin{array}{l}\text { PHOS- } \\
\text { PHORUS }\end{array}$ & $\begin{array}{c}\text { PHOS- } \\
\text { PHORUS } \\
\text { ORTHO, } \\
\text { DIS- }\end{array}$ & $\begin{array}{l}\text { CHLO- } \\
\text { RIDE, } \\
\text { DIS- }\end{array}$ & $\begin{array}{l}\text { SULFATE } \\
\text { DIS- }\end{array}$ \\
\hline DATE & TIME & $\begin{array}{c}\text { FEET } \\
\text { PER } \\
\text { SECOND }\end{array}$ & $\begin{array}{l}\text { BID- } \\
\text { ITY } \\
\text { (NTU) }\end{array}$ & $\begin{array}{c}\text { DIS- } \\
\text { SOLVED } \\
(\mathrm{mg} / \mathrm{L})\end{array}$ & $\begin{array}{l}\text { SUS- } \\
\text { PENDED } \\
(\mathrm{mg} / \mathrm{L})\end{array}$ & $\begin{array}{l}\text { SUS- } \\
\text { PENDED } \\
(\mathrm{mg} / \mathrm{L})\end{array}$ & $\begin{array}{l}\text { SOLVED } \\
\text { (mg/L } \\
\text { as } N \text { ) }\end{array}$ & $\begin{array}{c}\text { TOTAL } \\
\text { (mg/L } \\
\text { as N) }\end{array}$ & $\begin{array}{l}\text { TOTAL } \\
(\mathrm{mg} / \mathrm{L} \\
\text { as } \mathrm{N})\end{array}$ & $\begin{array}{l}\text { POTAL } \\
\text { (mg/L } \\
\text { as P) }\end{array}$ & $\begin{array}{l}\text { SOLVED } \\
\text { (mg/L } \\
\text { as P) }\end{array}$ & $\begin{array}{l}\text { SOLVED } \\
\text { (mg/L } \\
\text { as Cl) }\end{array}$ & $\begin{array}{c}\text { SOLVED } \\
(\mathrm{mg} / \mathrm{I} \\
\left.\text { as } \mathrm{SO}_{4}\right)\end{array}$ \\
\hline
\end{tabular}

\begin{tabular}{|c|c|c|}
\hline \multicolumn{3}{|l|}{ JUL } \\
\hline $08 \ldots$ & 1100 & -- \\
\hline $22 \ldots$ & 0900 & 0.50 \\
\hline \multicolumn{3}{|l|}{ AUG } \\
\hline $12 \ldots$ & 1130 & -- \\
\hline $26 \ldots$ & 1030 & -. \\
\hline \multicolumn{3}{|l|}{ SEP } \\
\hline $09 \ldots$ & 0950 & 0.10 \\
\hline $30 \ldots$ & 0950 & -. \\
\hline
\end{tabular}

\begin{tabular}{|c|c|c|c|c|c|c|c|c|c|}
\hline-- & 26 & 3 & 0.12 & 0.85 & 0.84 & 0.050 & 0.003 & -- & 140 \\
\hline 6.4 & 35 & 3 & 0.11 & 0.87 & 1.40 & 0.110 & 0.037 & 97 & 58 \\
\hline 7.9 & 38 & 4 & 0.03 & 0.45 & 0.66 & 0.090 & 0.023 & 44 & 37 \\
\hline 10.4 & 28 & 3 & 0.04 & 0.56 & 0.72 & 0.085 & 0.020 & 71 & N95 \\
\hline 9.2 & 25 & 3 & 0.03 & 0.56 & 0.82 & 0.090 & 0.035 & 62 & 80 \\
\hline 9.7 & 32 & 3 & 0.07 & 0.45 & 0.85 & 0.075 & 0.026 & 46 & 45 \\
\hline
\end{tabular}

MAY

$\begin{array}{ccccc}\text { MAY } & & & & \\ 05 \ldots & 1100 & 0.40 & 30 & 12.8 \\ 19 \ldots & 0900 & 0.40 & 6.6 & 11.5 \\ \text { JUN } & & & & \\ 02 \ldots & 1050 & 1.7 & 9.6 & 9.0 \\ 23 \ldots & 1100 & 2.5 & \mathrm{~N} 15 & -. \\ 23 \ldots & 1130 & 2.1 & \mathrm{~N} 14 & 7.4 \\ \text { SEP } & & & & \\ 15 \ldots & 0950 & 2.2 & 11 & --\end{array}$

WATER YEAR OCTOBER 1992 TO SEPTEMBER 1993

\begin{tabular}{|c|c|c|c|c|c|c|c|c|}
\hline 19 & $<3$ & $<0.01$ & 0.42 & 0.51 & 0.045 & 0.002 & 48 & 48 \\
\hline 8 & $<2$ & 0.01 & No. 01 & 0.53 & 0.035 & 0.002 & 47 & 57 \\
\hline-- & -- & No. 01 & NO. 01 & 0.93 & 0.050 & 0.006 & 170 & 110 \\
\hline 20 & 3 & 0.16 & 0.83 & 1.40 & 0.085 & 0.036 & 83 & 98 \\
\hline 18 & 3 & 0.16 & 0.91 & 0.84 & 0.080 & 0.029 & 75 & 120 \\
\hline 18 & 2 & $<0.01$ & 0.49 & No.01 & 0.060 & 0.023 & 78 & 82 \\
\hline
\end{tabular}




\section{Surface-Water Stations}

\section{B. Partial-Record and Miscellaneous-Record Sites}

\section{Fairport Waste Channel At Fairport, N.Y.}

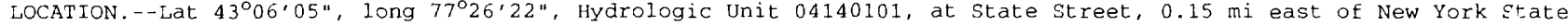
Highway 250 , and $0.05 \mathrm{mi}$ north of Erie canal. PERIOD OF RECORD. - - December 1984 to current year.

CHEMICAL DATA: $1984-86$ (a), $1988-89$ (d), 1990 (c), $1992-93$ (c)

NUTRIENT DATA: $1984-86$ (a), $1988-89$ (d), 1990 (c), $1992-93$ (c)

COOPERATION.--Water-quality samples were collected and analyzed by the Monroe County Environmental Health Laboratory at Rochester, N.Y.

WATER-QUALITY DATA

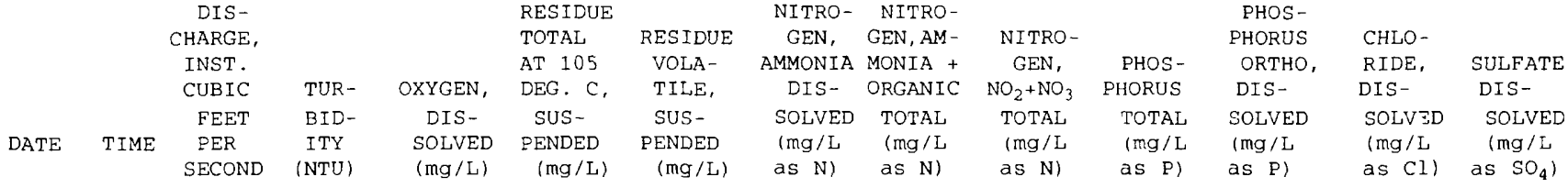

OCT

06... 1130 .-

13.

20.

27.

APR

26 .

JUN

JUL

21 ..

AUG

18 .

$\mathrm{SEP}$

$01 \ldots 1130 \quad--\quad 14$

$13 \ldots 1100 \quad--\quad 12$

OCT

$27 \ldots \quad 1130$

MAY

23... 0900

JUL

$25 \ldots \quad 1100$

AU

$30 \ldots 0900$

SEP

$07 . . \quad 1130 \quad \ldots \quad 7.3$

OCT

$24 \ldots \quad 1100 \quad \ldots-25$

MAY

JUN

08 .

$22 \ldots-0950$

AUG

$12 \ldots \quad 1100$

$26 \ldots \quad 1050$

SEP

09... 1130

$--$

6.9

4.1

20

5.4

MAY

$05 \ldots \quad 0950$

$19 \ldots \quad 1130$

JUN

$02 \ldots \quad 1030$

$23 \ldots$

1030

5.7

3.1

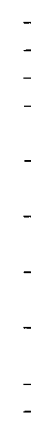

--
--
--
--
--
--
--
--
--
--
--
5.7
-

\section{WATER YEAR OCTOBER 1988 TO SEPTEMBER 1989}

\begin{tabular}{|c|c|c|c|c|}
\hline 16 & 3 & 0.03 & 0.56 & 0.39 \\
\hline 18 & 3 & 0.01 & 0.59 & 0.47 \\
\hline 21 & 3 & $<0.01$ & 0.54 & 0.50 \\
\hline 18 & 2 & 0.11 & 0.89 & 0.48 \\
\hline 62 & 8 & 0.07 & 0.88 & 0.96 \\
\hline 16 & 7 & 0.10 & 1.5 & 0.69 \\
\hline & $\cdots$ & 0.02 & 0.91 & 0.70 \\
\hline 21 & 3 & 0.01 & 0.70 & 0.44 \\
\hline 20 & 4 & 0.07 & 1.0 & 0.53 \\
\hline
\end{tabular}

0.040

0.050

0.075

0.060

0.006

0.009

0.004

0.015

0.130

0.018

0.120

0.017

0.085

0.004

0.065

0.011

0.080

0.085

0.019

0.020

WATER YEAR OCTOBER 1989 TO SEPTEMBER 1990

$\begin{array}{rrrrr}25 & 8 & 0.02 & 0.52 & 0.34 \\ 10 & 3 & 0.08 & 0.37 & 0.56 \\ 6 & <5 & 0.03 & 0.60 & 0.86 \\ 36 & 10 & 0.08 & 1.0 & 0.55 \\ 7 & <5 & \text { N0.01 } & 0.60 & 0.55 \\ 11 & 3 & 0.08 & 0.50 & 0.57\end{array}$

0.055

0.012

0.065

0.025

0.050

0.013

0.160

0.016

0.040

0.037

0.065

0.039

61

64

\section{WATER YEAR OCTOBER 1991 TO SEPTEMBER 1992}

6.8
4.5
3.7
3.7
4.3
5.7
11.2
6.5

17

$\begin{array}{rl}<5 & 0.06 \\ <2 & 0.10 \\ 3 & 0.18 \\ 4 & 0.10 \\ <2 & 0.12 \\ <1 & 0.07 \\ 2 & 0.03 \\ 2 & 0.04 \\ <1 & 0.03\end{array}$

$0.53 \quad 0.71$

0.070

0.021

$0.51 \quad 0.79$

0.045

0.016

0.80

0.72

0.070

0.040

$0.72 \quad 0.37$

0.060

0.027

0.043

$0.43 \quad 0.51$

0.065

0.028

0.034

$0.39 \quad 0.38$

0.070

0.025

0.045

$0.002 \quad 260$

55

7.3

7.4

5.8

3.7
0.040

0.100

$0.003 \quad 180$

0.018150

$\begin{array}{rrrr}<3 & 0.01 & 0.94 & 0.27 \\ 4 & <0.01 & 1.0 & 0.06 \\ 11 & 0.17 & 1.6 & 0.41 \\ & & & \\ - & \text { No.01 } & \text { N0.01 } & 0.46 \\ 2 & 0.18 & 0.76 & 0.34\end{array}$

0.011

$\begin{array}{lr}0.011 & 91 \\ 0.034 & 110\end{array}$

0.088
120 


\section{GROUND-WATER LEVELS}

Powder Mill Park

430252077283401. Local number Mo 10 (PM 83-1)

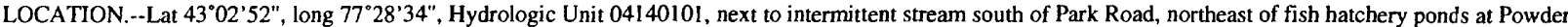
Mill Park near Bushnell basin. Owner: U.S. Geological Survey

AQUIFER.-- Water-table aquifer in sand of Pleistocene age.

WELL CHARACTERISTICS.--Drilled observation well, diameter 2 in, depth $48 \mathrm{ft}$, cased to $28 \mathrm{ft}$, screened $28 \mathrm{ft}$ to $48 \mathrm{ft}$. Filled in with silt to a depth of about $19 \mathrm{ft}$. INSTRUMENTATION.--Weekly measurement with chalked tape by Powder Mill Park personnel and occasional measurement by USGS and MCEHL.

DATUM.--Elevation of land-surface datum is $448.66 \mathrm{ft}$ above National Geodetic Vertical Datum of 1929. Measuring point: top of casing, $0.82 \mathrm{ft}$ above land-surface datum.

REMARKS.--This well is also a water-quality observation well. Water levels are monitored monthly and water samples taken quarterly by the Monroe County Environmental Health Laboratory. Water-temperature profiles are also taken by MCEHL on a quarterly basis.

PERIOD OF RECORD.--December 1983 to September 1993 (discontinued)

EXTREMES FOR PERIOD DECEMBER 1983 TO SEPTEMBER 1993.--Highest water level measured, 7.20 feet below land-surface datum, June 9, 198 4; lowest measured, 10.73 feet below land-surface datum, September 13, 1989.

WATER LEVEL, IN FEET BELOW LAND-SURFACE DATUM,

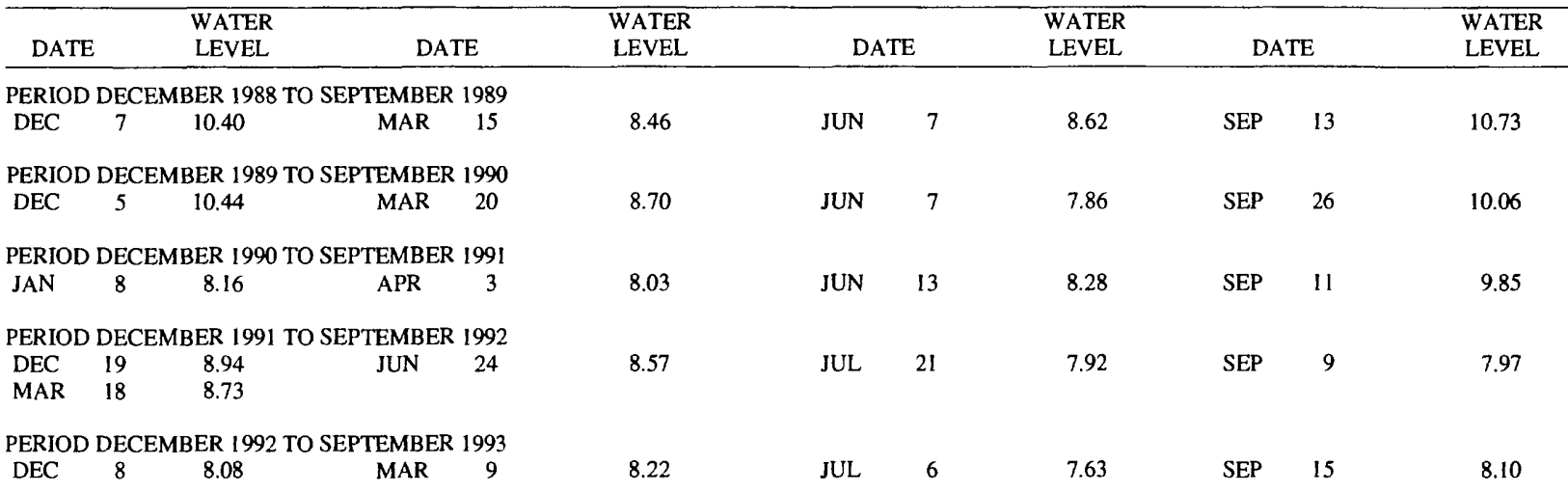

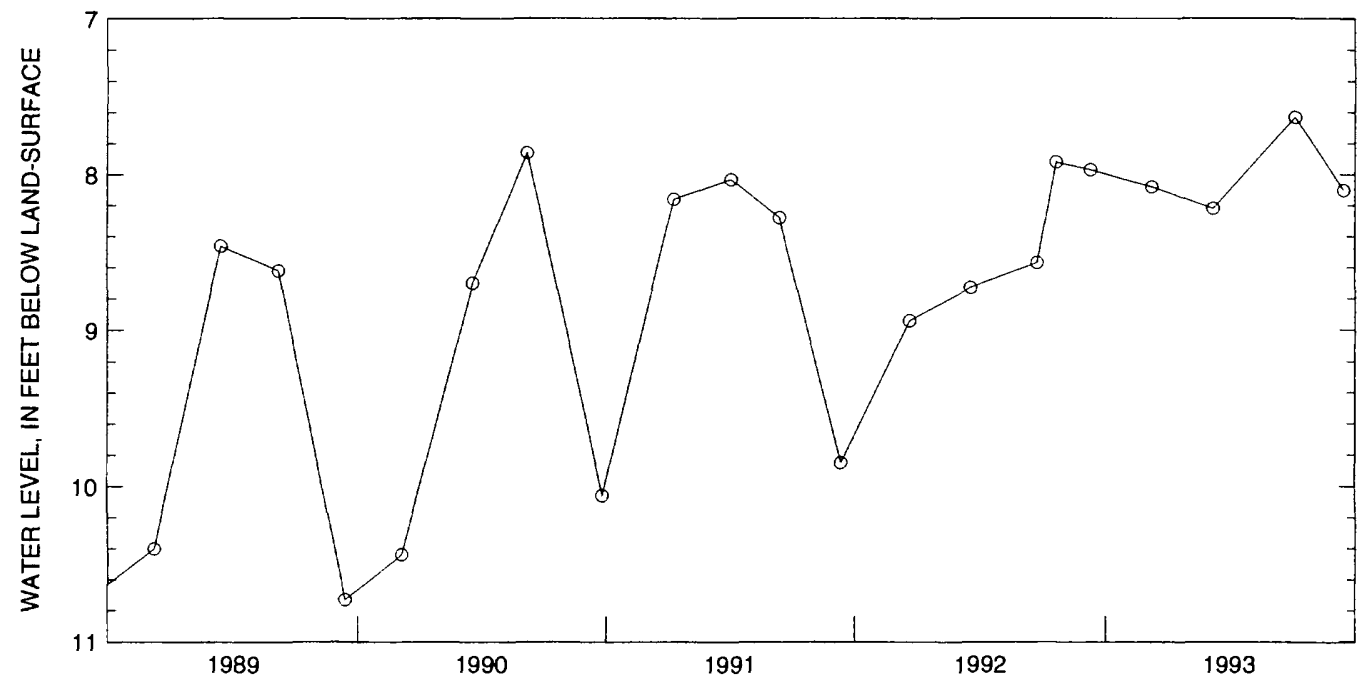


PERIOD OF RECORD.-- January 1986 to September 1993 (discontinued).

CHEMICAL DATA: 1986(a) 1987-93(b).

ORGANIC DATA: OC.--1986(a) 1987-93(b)

NUTRIENT DATA: 1986(a) 1987-93(b).

BIOLOGICAL DATA:

Bacteria.--1986(a) 1987-93(b).

COOPERATION-- Water-quality samples were collected and analyzed by the Monroe County Environmental Health Laboratory at Rochester, NY.

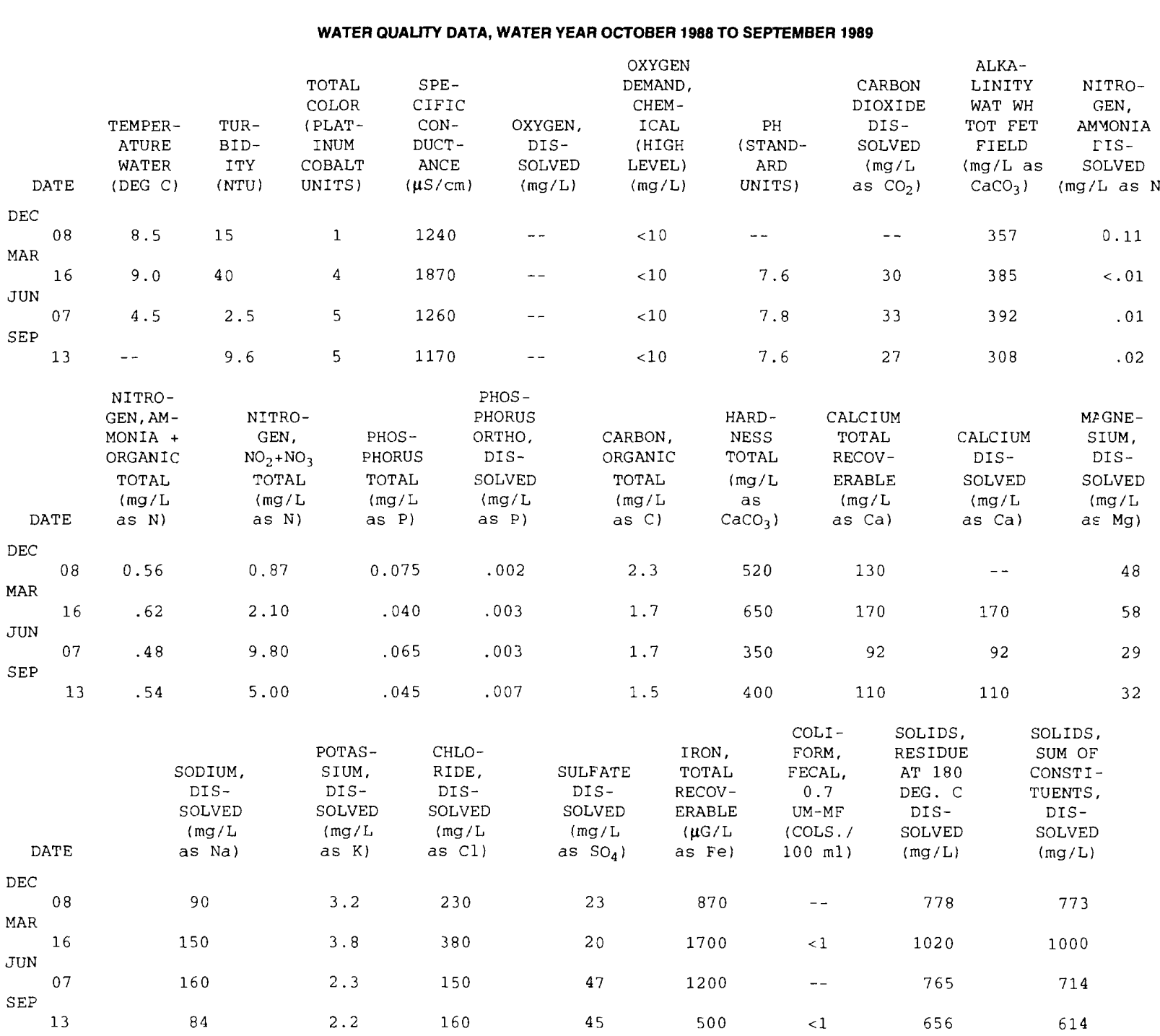


430252077283401. Local number Mo 10 (PM 83-1)--continued

WATER QUALITY DATA, WATER YEAR OCTOBER 1990 TO SEPTEMBER 1991

\begin{tabular}{|c|c|c|c|c|c|c|c|c|c|c|}
\hline & & & & & & OXYGEN & & & ALKA - & \\
\hline & & & TOTAL & SPE- & & DEMAND, & & CARBON & LINITY & NITRO- \\
\hline & & & COLOR & CIFIC & & CHEM- & & DIOXIDE & WAT WH & GEN， \\
\hline & TEMPER- & TUR - & (PLAT - & CON- & OXYGEN, & ICAL & PH & DIS - & TOT FET & AMMONIA \\
\hline & $\begin{array}{l}\text { ATURE } \\
\text { WATER }\end{array}$ & $\begin{array}{l}\text { BID- } \\
\text { ITYY }\end{array}$ & $\begin{array}{c}\text { INUM } \\
\text { COBALT }\end{array}$ & $\begin{array}{l}\text { DUCT - } \\
\text { ANCE }\end{array}$ & $\begin{array}{l}\text { DIS- } \\
\text { SOLVED }\end{array}$ & $\begin{array}{l}\text { (HIGH } \\
\text { LEVEL) }\end{array}$ & $\begin{array}{l}\text { (STAND- } \\
\text { ARD }\end{array}$ & $\begin{array}{c}\text { SOLVED } \\
(\mathrm{mg} / \mathrm{L}\end{array}$ & $\begin{array}{c}\text { FIELD } \\
\text { (mg/L as }\end{array}$ & $\begin{array}{c}\text { DIS- } \\
\text { SOLVED }\end{array}$ \\
\hline DATE & $(\mathrm{DEG} C)$ & (NTU) & $\begin{array}{l}\text { COBALT } \\
\text { UNITS) }\end{array}$ & $\begin{array}{c}\text { ANCE } \\
(\mu \mathrm{S} / \mathrm{Cm})\end{array}$ & $\begin{array}{l}\text { SOLVED } \\
(m g / L)\end{array}$ & $\begin{array}{l}\text { LEVEL) } \\
\text { (mg/L) }\end{array}$ & $\begin{array}{c}\text { ARD } \\
\text { UNITS) }\end{array}$ & $\begin{array}{c}(\mathrm{mg} / \mathrm{L} \\
\left.\text { as } \mathrm{CO}_{2}\right)\end{array}$ & & $\begin{array}{c}\text { COLVED } \\
\text { (mg/L as } N \text { ) }\end{array}$ \\
\hline
\end{tabular}

$$
\text { JAI }
$$

$\begin{array}{llllll}\text { JAN } & & & & & \\ \text { APR } & 08 & - & 4.7 & - & 1340 \\ \text { JUN } & 04 & -- & 7.9 & -- & 1380 \\ \text { SEP } & 13 & -- & 8.3 & -- & 1120 \\ & 12 & -- & 2.7 & -- & 937\end{array}$

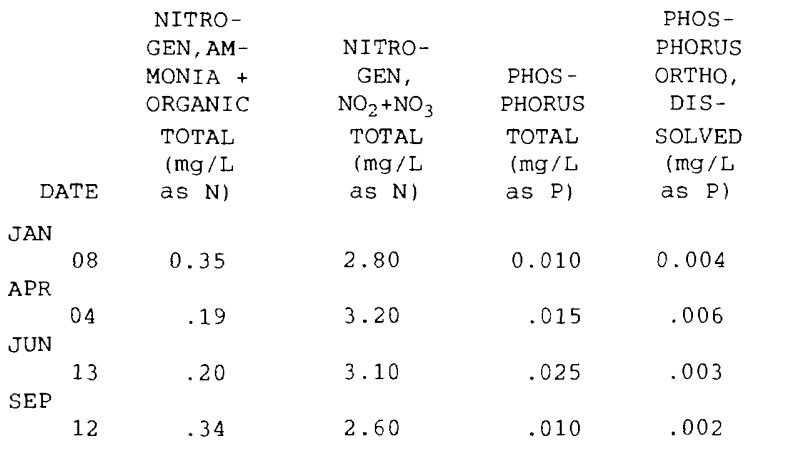

$\begin{array}{ccccc} & \text { HARD } & \text { CALCIUM } & & \text { MAGNE- } \\ \text { CARBON, } & \text { NESS } & \text { TOTAL } & \text { CALCIUM } & \text { SIUM, } \\ \text { ORGANIC } & \text { TOTAL } & \text { RECOV- } & \text { DIS- } & \text { DIS- } \\ \text { TOTAL } & (\mathrm{mg} / \mathrm{L} & \text { ERABLE } & \text { SOLVED } & \text { SOLVED } \\ (\mathrm{mg} / \mathrm{L} & \text { as } & (\mathrm{mg} / \mathrm{L} & (\mathrm{mg} / \mathrm{L} & (\mathrm{mg} / \mathrm{L} \\ \mathrm{as} \mathrm{C}) & \left.\mathrm{CaCO}_{3}\right) & \text { as Ca) } & \text { as Ca) } & \text { as Mg) }\end{array}$

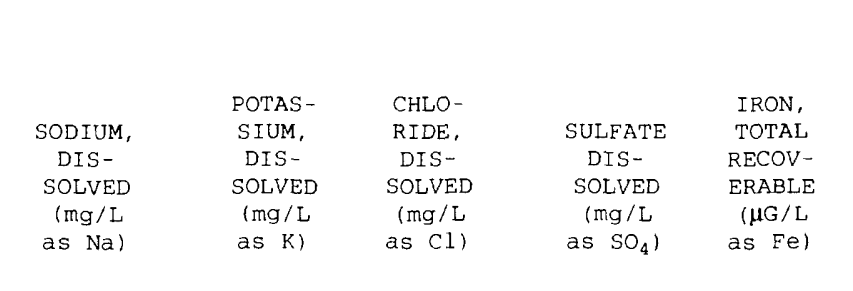

$\begin{array}{ccccc}-- & 500 & - & 130 & 32 \\ -- & 430 & - & 120 & 29 \\ -- & 400 & -- & 120 & 26 \\ - & 330 & \ldots & N 110 & 28\end{array}$

\begin{tabular}{|c|c|}
\hline DATE & as $N$ \\
\hline JAN & \\
\hline 08 & 120 \\
\hline APR & \\
\hline 04 & 140 \\
\hline JUN & \\
\hline 13 & 91 \\
\hline 12 & 76 \\
\hline
\end{tabular}

$\begin{array}{ll}2.9 & 240 \\ 2.8 & 210 \\ 2.4 & 150 \\ 2.1 & 120\end{array}$

31
42
36
33

$\begin{array}{llll}240 & - & - & 786 \\ 440 & -- & - & 799 \\ 500 & -- & -- & 671 \\ 130 & -- & -- & 542\end{array}$




\section{GROUND-WATER QUALITY}

\section{Powder Mill Park}

430252077283401. Local number Mo 10 (PM 83-1)--continued

\section{WATER QUALITY DATA, WATER YEAR OCTOBER 1991 TO SEPTEMBER 1992}

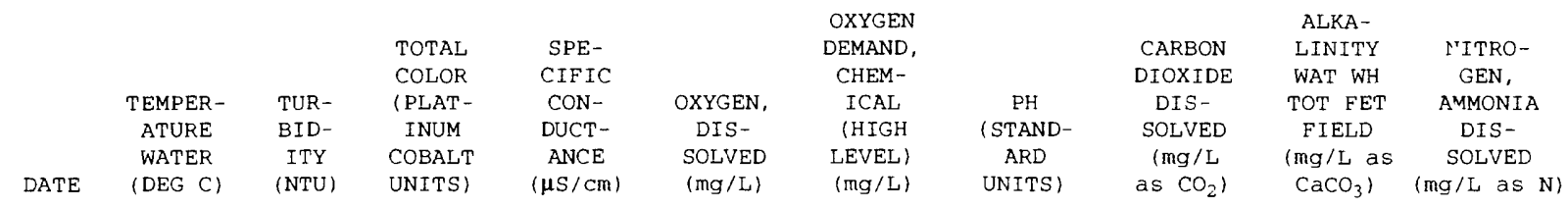
DEC

$\begin{array}{lccccc}\text { DEC } & 19 & \ldots & 6.0 & - & 1230 \\ \text { MAR } & 18 & \ldots & 12 & -- & 1640 \\ \text { JUN } & 14 & \ldots & 7.6 & -- & 1460 \\ \text { SEP } & 24 & & 1.0 & \ldots & 1100\end{array}$

7.0
-
-
-
-7.1
7.5

$<0.2$
--
--
24

$--$

0.01

$-$

7.5

NITROGEN, AM-

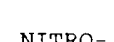

$\begin{array}{cc} & \text { PHOS- } \\ \text { PHORUS } \\ \text { PHOS - } & \text { ORTHO, } \\ \text { PHORUS } & \text { DIS- } \\ \text { TOTAL } & \text { SOLVED } \\ (\mathrm{mg} / \mathrm{L} & (\mathrm{mg} / \mathrm{L} \\ \text { aS P) } & \text { aS P) }\end{array}$

DEC

MONIA +

NITRO-

GEN, $\mathrm{NO}_{2}+\mathrm{NO}_{3}$

ORGANIC

TOTAL

(mg/L

DATE as N)

as N)

0.010

0.004

190.46

3.80

0.25

.004

.015

.004

$24 \quad .78$

3.40

.025

.003

$\begin{array}{ccc} & \text { HARD- } & \text { CALCIUM } \\ \text { CARBON, } & \text { NESS } & \text { TOTAL } \\ \text { ORGANIC } & \text { TOTAL } & \text { RECOV- } \\ \text { TOTAL } & (\mathrm{mg} / \mathrm{L} & \text { ERABLE } \\ (\mathrm{mg} / \mathrm{L} & \text { as } & (\mathrm{mg} / \mathrm{L} \\ \text { as C) } & \left.\mathrm{CaCO}_{3}\right) & \text { as Ca) }\end{array}$

$\begin{array}{cc} & \text { NAGNE- } \\ \text { CALCIUM } & \text { SIUM, } \\ \text { DIS- } & \text { DIS- } \\ \text { SOLVED } & \text { SOLVED } \\ \text { (mg/L } & \text { (mg/L } \\ \text { as Ca) } & \text { as Mg) }\end{array}$

1.80

$\begin{array}{cc} & \\ \text { POTAS- } & \text { CHLO- } \\ \text { SIUM, } & \text { RIDE, } \\ \text { DIS - } & \text { DIS- } \\ \text { SOLVED } & \text { SOLVED } \\ \text { (mg/L } & (\mathrm{mg} / \mathrm{L} \\ \text { MS } & \text { aS }\end{array}$

DATE

as K)

as Cl)

$\begin{array}{ll}2.8 & 170 \\ 4.4 & 280 \\ 2.6 & 230 \\ 2.6 & 160\end{array}$

SULFATE
DIS-
SOLVED
(mg/L

IRON,
TOTAL
RECOV-
ERABLE
( $\mu$ G / L
aS Fe)

$\begin{array}{cc}\text { COLI- } & \text { SOLIDS, } \\ \text { FORM, } & \text { RESIDUE } \\ \text { FECAL, } & \text { AT 180 } \\ 0.7 & \text { DEG. C } \\ \text { UM-MF } & \text { DIS- } \\ \text { (COLS.) } & \text { SOLVED } \\ 100 \mathrm{mi}) & (\mathrm{mg} / \mathrm{L})\end{array}$

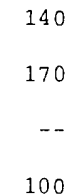

$<.01$

$<.01$

$<.01$

DEC

$\begin{array}{lll} & 19 & 100 \\ \text { MAR } & 18 & 130 \\ & 18 & 150 \\ \text { JUN } & & 24 \\ \text { SEP } & & 100\end{array}$

100

30

100

160

$\begin{array}{ll}50 & 250 \\ 64 & 570 \\ 16 & 460 \\ 46 & 640\end{array}$

$--$

$-$

731

$--$

$--\quad 986$

$--\quad--$

853

650 
430252077283401. Local number Mo 10 (PM 83-1)--continued

WATER QUALITY DATA, WATER YEAR OCTOBER 1992 TO SEPTEMBER 1993

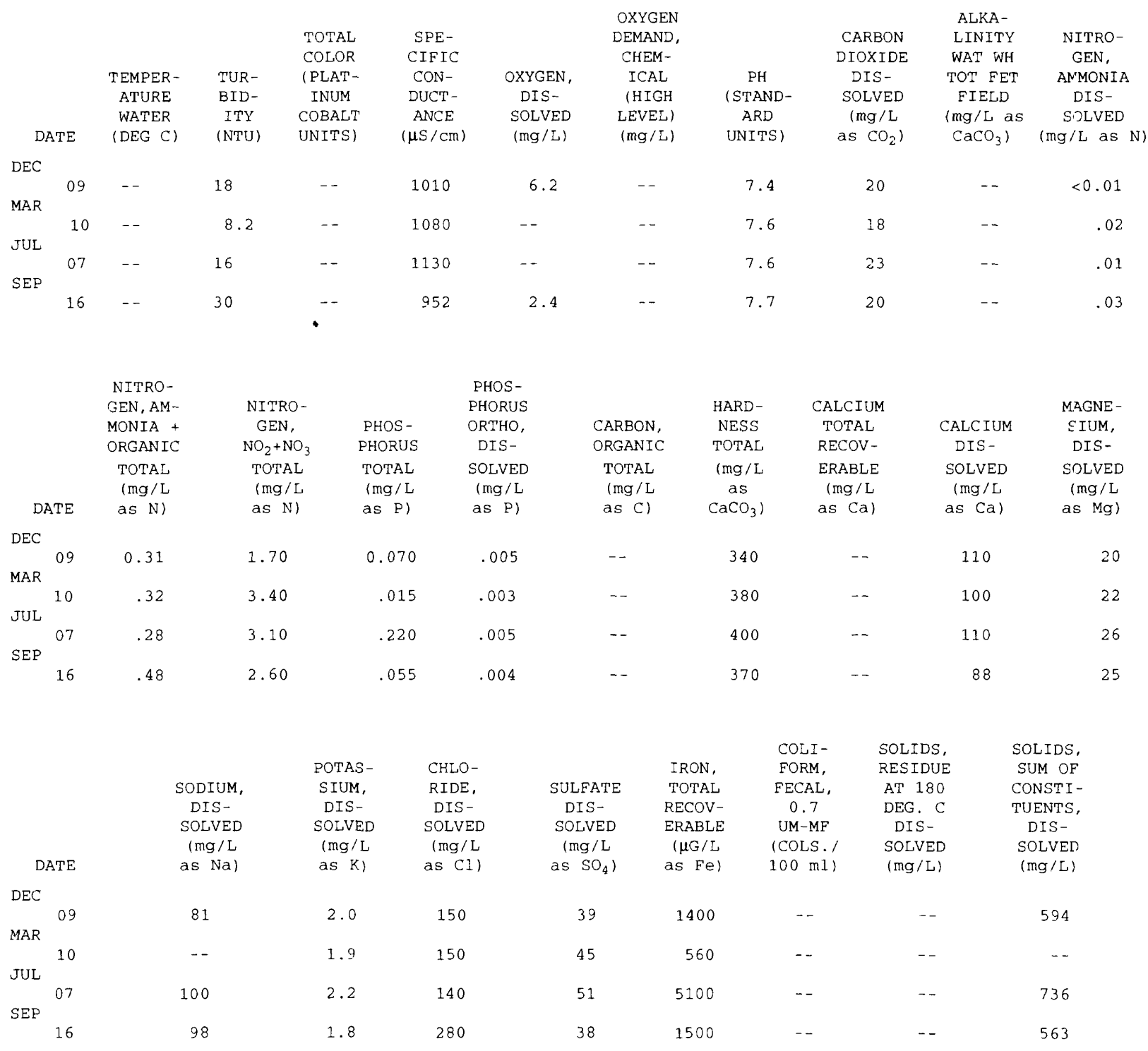


430252077283401. Local number Mo 10 (PM 83-1)--continued

WATER TEMPERATURE, IN DEGREES CELSIUS

\begin{tabular}{|c|c|c|c|c|c|c|c|c|c|c|c|c|c|c|c|c|c|c|c|c|}
\hline \multirow[b]{2}{*}{$\begin{array}{l}\text { depth } \\
\text { in feet }\end{array}$} & \multicolumn{4}{|c|}{1989 WY } & \multicolumn{4}{|c|}{$1990 \mathrm{WY}$} & \multicolumn{4}{|c|}{$1991 \mathrm{WY}$} & \multicolumn{4}{|c|}{1992 WY } & \multicolumn{4}{|c|}{$1993 \mathrm{WY}$} \\
\hline & $\begin{array}{c}\text { Dec } \\
7\end{array}$ & $\begin{array}{c}\text { Mar } \\
15\end{array}$ & $\begin{array}{c}\text { Jun } \\
7\end{array}$ & $\begin{array}{c}\text { Sep } \\
13\end{array}$ & $\begin{array}{c}\text { Dec } \\
5\end{array}$ & $\begin{array}{c}\text { Mar } \\
20\end{array}$ & $\begin{array}{c}\text { Jun } \\
7\end{array}$ & $\begin{array}{c}\text { Sep } \\
26\end{array}$ & $\begin{array}{c}\text { Jan } \\
8\end{array}$ & $\begin{array}{c}\text { Apr } \\
3\end{array}$ & $\begin{array}{c}\text { Jun } \\
13\end{array}$ & $\begin{array}{c}\text { Sep } \\
11\end{array}$ & $\begin{array}{c}\text { Dec } \\
19\end{array}$ & $\begin{array}{c}\text { Mar } \\
18\end{array}$ & $\begin{array}{c}\text { Jun } \\
24\end{array}$ & $\begin{array}{c}\text { Sep } \\
9\end{array}$ & $\begin{array}{c}\text { Dec } \\
8\end{array}$ & $\begin{array}{c}\text { Mar } \\
9\end{array}$ & $\begin{array}{c}\text { Jul } \\
6\end{array}$ & $\begin{array}{c}\text { Sep } \\
15\end{array}$ \\
\hline 7.68 & -. & -- & -- & -- & -- & -- & -- & -- & - & - & -- & -- & - & -- & -- & 2 & - & -- & 4.0 & - \\
\hline & -- & -- & -- & -- & - & -- & 2.0 & -. & - & 5.5 & - & -- & $\ldots$ & -- & -. & 16. & 5.5 & -- & & - \\
\hline & -- & -- & - - & -- & -- & -- & - & -. & 8.5 & -- & 13.0 & -- & -- & -- & -- & $\ldots$ & -- & 4.5 & 12.0 & 15.0 \\
\hline & - - & 3.5 & -- & -- & $\ldots$ & $\ldots$ & - & -- & -- & -- & -- & -- & - & -- & -- & - & -- & -- & $\ldots$ & $\ldots$ \\
\hline & -- & -- & -- & -- & - & 3.5 & - & -- & -- & -- & -- & -- & -- & -- & 11.5 & -- & -- & - & $\cdots$ & -- \\
\hline & -. & -. & - - & $\ldots$ & - & -- & - &.- & - - & -- & $\ldots$ & -. & -- & 4.5 & -- & - & $\ldots$ & -. & - - & - - \\
\hline & - - & -. & $\ldots$ & $\ldots$ & - - & -- & $\ldots$ & $\ldots$ & - - & -- & $\ldots$ & -- & 7.0 & -- & -- & - & $\ldots$ & -- & $\ldots$ & -- \\
\hline & -- & 3.5 & 6.0 & -- & -- & 4.0 & 8.5 & -- & 8.5 & 5.0 & 10.5 & -- & -- & -- & -- & 14. & 5.5 & 4.5 & 10.5 & 14.5 \\
\hline & - - & $\ldots$ & $\ldots$ & - - & - - & -- & -- & - & - - & -- & -- & - & -. & 5.0 & 9.5 & - & -- & -- & $\ldots$ & -- \\
\hline & -- & -- & -- & -- & -- & -- & -- & 15. & -- & - & -- & 16. & -- & $\cdots$ & -- & -- & -- & -- & -- & -- \\
\hline & -- & 3.5 & 3.5 & -- & - & 4.5 & 8.0 & $\ldots$ & 8.5 & 5.0 & 10.0 & 15. & 9.0 & - & -- & 14 & 5.5 & 4.5 & 9.5 & 14.0 \\
\hline & 9.5 & $\ldots$ & - & - . & 9.0 & -- & -- & -- & -- & -- & $\ldots$ & -- & $\ldots$ & -- & -- & - & -- & $\ldots$ & $\ldots$ & -- \\
\hline & -- & - & -- & $\ldots$ & - & -- & -- & -- & - & -- & -- & $\ldots$ & - & 5.0 & 8.5 & -- & -- & - - & - & - \\
\hline & 10.0 & 3.5 & 2.5 & 12.0 & 10,0 & 4.5 & 7.5 & 13. & 9.0 & 5.0 & 9.0 & 14.0 & 9.5 & $\ldots$ & $\cdots$ & 13.5 & 5.5 & 4.5 & 9.0 & 14.0 \\
\hline & - & -- & -- & $\ldots$ & $\ldots$ & -- & - - & $\ldots$ & -- & $\ldots$ & -- & -- & $\ldots$ & 5.0 & 8.0 & $\ldots$ & $\ldots$ & - & $\ldots$ & $\ldots$ \\
\hline & 10.0 & 3.5 & 2.0 & 11.5 & 10.5 & 5.0 & 7.0 & 13. & 9.0 & 5.5 & 8.5 & 13. & 9.5 & -- & -- & 12. & 5.5 & 4.5 & 8.5 & 13.0 \\
\hline & -- & -- & $\ldots$ & $\ldots$ & -- & $\ldots$ & -- & -- & -- & - & $\ldots$ & - & $\ldots$ & 5.5 & 7.5 & $\ldots$ & -- & $\ldots$ & $\ldots$ & -- \\
\hline & 10.5 & 5.0 & 1.5 & 11.5 & 10.5 & 5.0 & 6.5 & 12. & 9.0 & 5.5 & 8.0 & 13.0 & 9.5 & -- & -. & 12. & 5.5 & 4.5 & 8.0 & 12.5 \\
\hline & $\ldots$ & - & - & $\ldots$ & -- & -- & -- & -- & -- & -- & -- & -- & - & 5.5 & 7.0 & -- & -- & -- & -- & -- \\
\hline & 10.5 & 5.0 & 1.5 & 11.0 & 11.0 & 5.0 & 6.0 & 12. & 9.0 & 6.0 & 7.5 & 12.5 & 10.0 & $\ldots$ & -- & 12. & 5.5 & 4.5 & 8.0 & 12.0 \\
\hline & - & -- & -- & $\ldots$ & -- & -- & -- & -- & -- & -- & -- & - & - & 5.5 & 7.0 & -- & - - & -- & $\ldots$ & -- \\
\hline & 10.5 & 5.0 & 1.0 & 11.0 & 11.0 & 5.0 & 6.0 & 11.5 & 9.5 & 6.0 & 7.0 & - - & 10.0 & -- & -. & 11. & 5.5 & 4.5 & 7.5 & 11.5 \\
\hline & -- & $\ldots$ & -- & - & -- & -- & -- & -- & - & -- & -- & -- & -- & 6.0 & 7.0 & -- & - & $\ldots$ & $\ldots$ & -- \\
\hline & 10.5 & 5.5 & 1.0 & 10.5 & 11.0 & 5.0 & 6.0 & 11. & 9.5 & 6.0 & 7.0 & 12. & 10.5 & -- & -- & 11. & 5.5 & 4.5 & 7.5 & 11.0 \\
\hline & -- & -- & -- & - & -- & -- & -- & $\cdots$ & -- & -- & -- & -- & -- & 6.0 & 6.5 & - & -- & -- & $-\infty$ & - \\
\hline & 10.5 & 5.5 & 1.0 & 10.5 & 10.5 & 5.0 & 6.0 & 11.0 & 9.5 & 6.0 & 7.0 & 11.5 & 10.5 & -- & -- & 10.5 & 5.5 & 4.5 & 7.0 & 10.5 \\
\hline & - & - & -- & - & -- & -- & $\cdots$ & -- & -- & -- & - & -- & -- & 6.0 & 6.5 & -- & -- & -- & $\rightarrow$ & - \\
\hline & 10.5 & 5.5 & 1.0 & 10.0 & 10.5 & 5.5 & 6.0 & 10.5 & 9.5 & 6.0 & 6.5 & 11.0 & 10.5 & $\ldots$ & -- & 10.0 & 5.5 & 4.5 & 7.0 & 10.0 \\
\hline & 10.0 & -- & -- & $\ldots$ & $\ldots$ & -- & -- & -- & -- & - - & -- & -- & -- & 6.0 & 6.5 & -- & -- & -- & -- & -- \\
\hline & -- & 5.5 & 1.0 & 7.5 & 10.5 & 5.5 & 6.0 & 10.0 & 9.5 & 6.0 & 6.5 & 10.5 & 10.5 & - & -- & 9.5 & 5.5 & 4.5 & 7.0 & 9.5 \\
\hline & - & -- & -- & $\ldots$ & $\ldots$ & 5.5 & -- & -- & - & - - & -- & - & - - & -- & -- & - & - - & -- & $\cdots$ & -. \\
\hline & -- & -- & 1.0 & - & 10.5 & -- & 6.0 & -- & -- & 6.5 & -- & -- & -- & 6.5 & 6.5 & -- & -- & -- & -- & -- \\
\hline & -- & - - & $-\ldots$ & .. & -- & -- & -- & 9. & 9.5 & $\ldots$ & 6.5 & 10.5 & .. & - - & $\ldots$ & 9.5 & -- & 4.5 & 7.0 & $\ldots$ \\
\hline & -- & - & $\ldots$ & 7.5 & -- & - - & -- & -- & -- & -- & -- & -- & 10.5 & - & -- & - & 5.5 & -- & -- & 9.0 \\
\hline
\end{tabular}

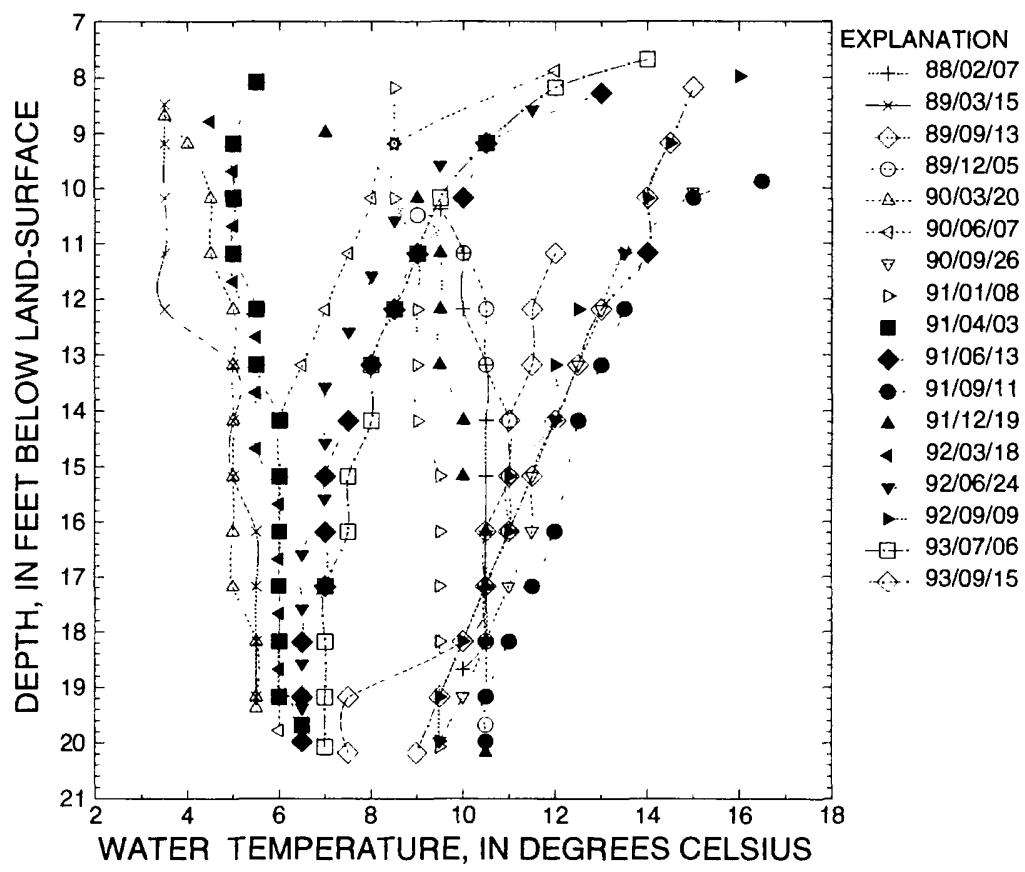


LOCATION.--Lat $43^{\circ} 02^{\prime} 52^{\prime \prime}$, long $77^{\circ} 28^{\prime} 34^{\prime \prime}$, Hydrologic Unit 04140101 , next to intermittent stream south of Park Road, northeast of fish hatchery ponds at Powder Mill Park near Bushnell basin. Owner: U.S. Geological Survey.

AQUIFER.--Water-table aquifer in sand of Pleistocene age.

WELL CHARACTERISTICS.--Drilled observation well, diameter $2 \mathrm{in}$, depth $16 \mathrm{ft}$, cased to $6 \mathrm{ft}$, screened $6 \mathrm{ft}$ to $16 \mathrm{ft}$.

INSTRUMENTATION.--Weekly measurement with chalked tape by Powder Mill Park personnel and occasional measurement by USGS and MCEHL.

DATUM.--Elevation of land-surface datum is $448.66 \mathrm{ft}$ above National Geodetic Vertical Datum of 1929. Measuring point: top of casing, $0.88 \mathrm{ft}$ above land-surface datum.

REMARKS.--This well is also a water-quality observation well. Water levels are monitored monthly and water samples taken quarterly by the Monroe County Environmental Health Laboratory. Water-temperature profiles are also taken by MCEHL on a quarterly basis.

PERIOD OF RECORD.--December 1983 to September 1993 (discontinued).

EXTREMES FOR PERIOD DECEMBER 1983 TO SEPTEMBER 1993.--Highest water level measured, 7.25 feet below land-surface datum, June 9, 1984; lowest measured, 10.84 feet below land-surface datum, September 13, 1989.

WATER LEVEL, IN FEET BELOW LAND-SURFACE DATUM,

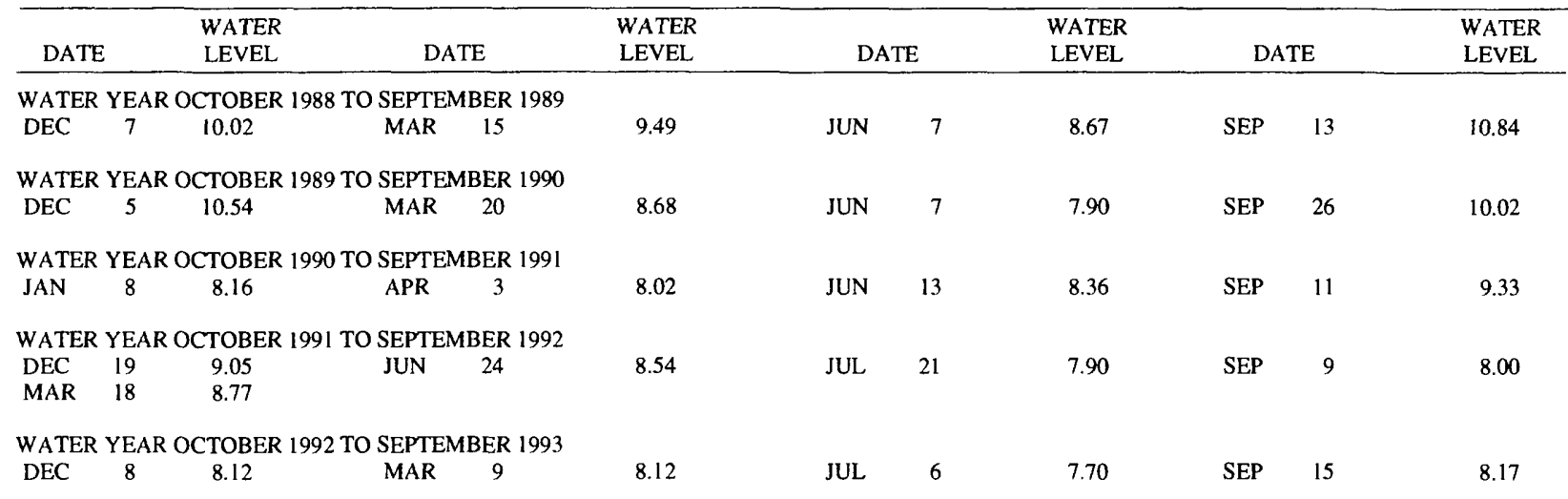

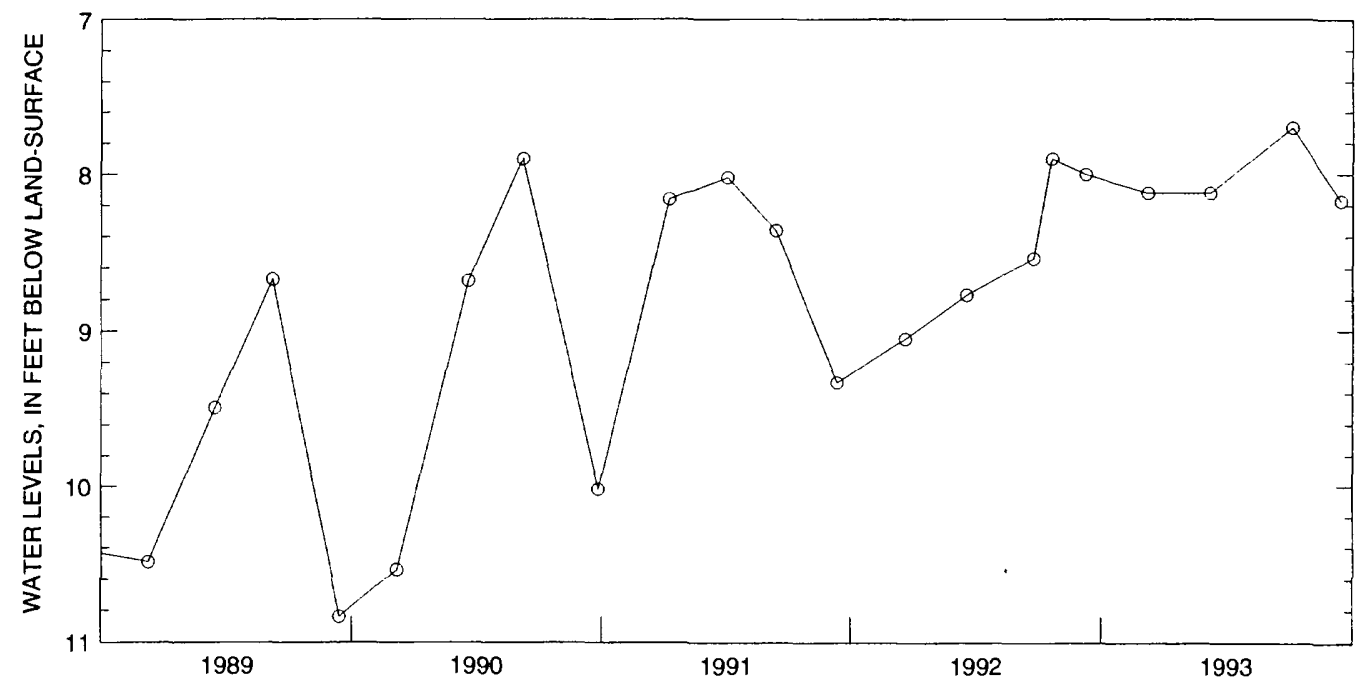


PERIOD OF RECORD.-- January 1986 to September 1993 (discontinued).

CHEMICAL DATA: 1986(a) 1987-93(b).

ORGANIC DATA: OC.--1986(a) 1987-93(b)

NUTRIENT DATA: 1986(a) 1987-93(b).

BIOLOGICAL DATA:

Bacteria.--1986(a) 1987-93(b).

COOPERATION.- Water-quality samples were collected and analyzed by the Monroe County Environmental Health Laboratory at Rochester, NY

WATER QUALTY DATA, PERIOD JANUARY 1988 TO SEPTEMBER 1989

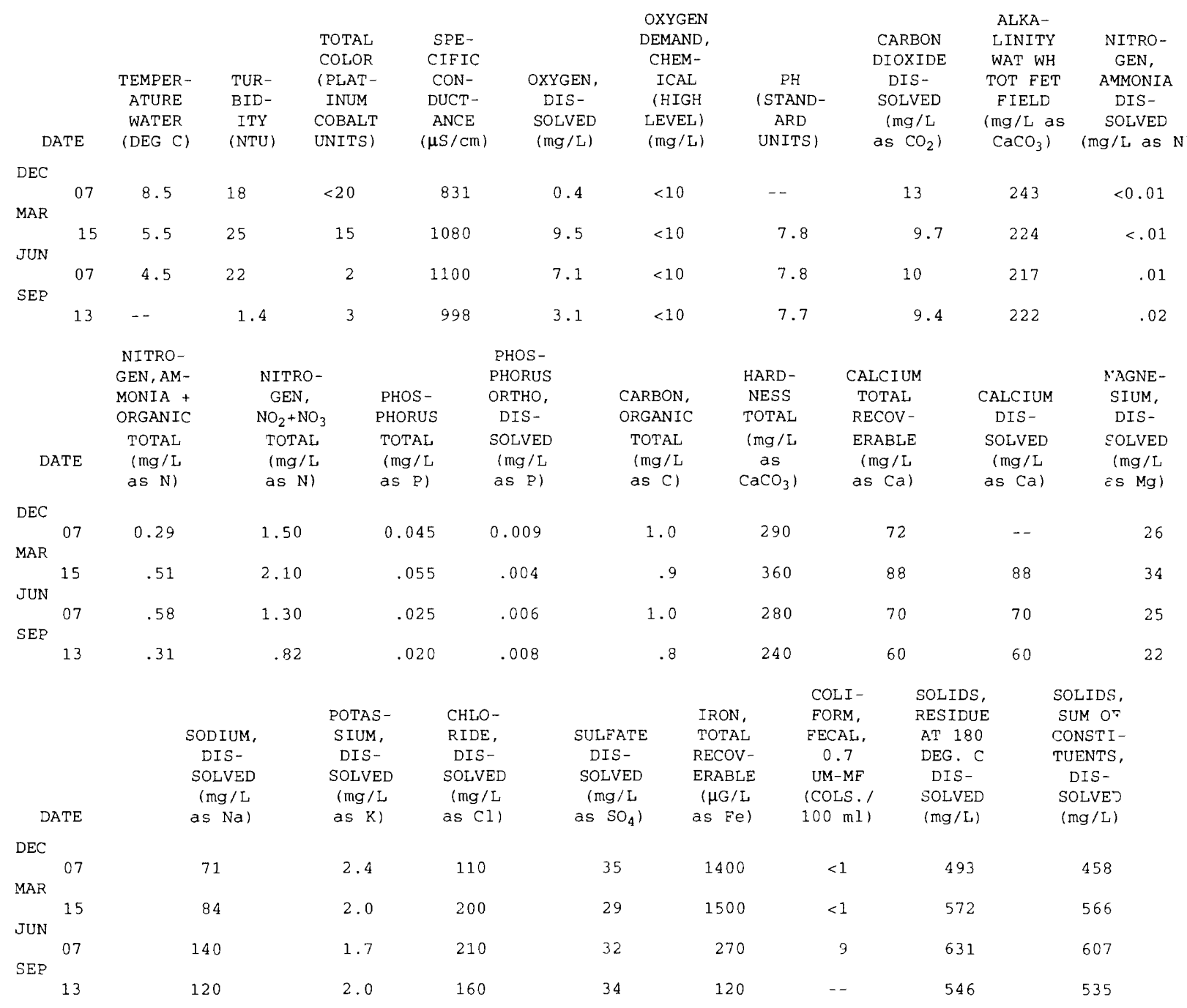


WATER QUALITY DATA, WATER YEAR OCTOBER 1989 TO SEPTEMBER 1990

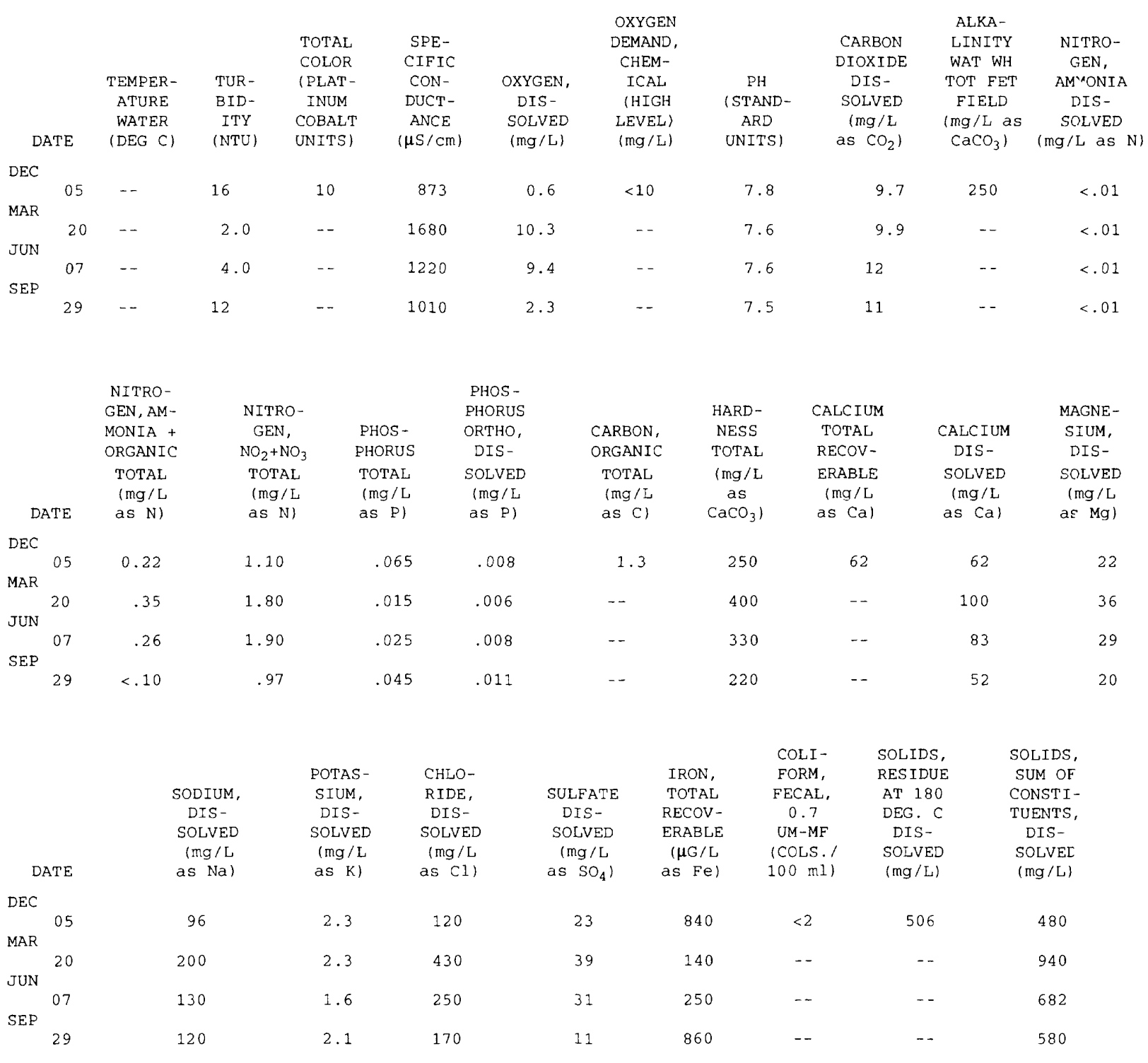


WATER QUALTY DATA, WATER YEAR OCTOBER 1990 TO SEPTEMBER 1991

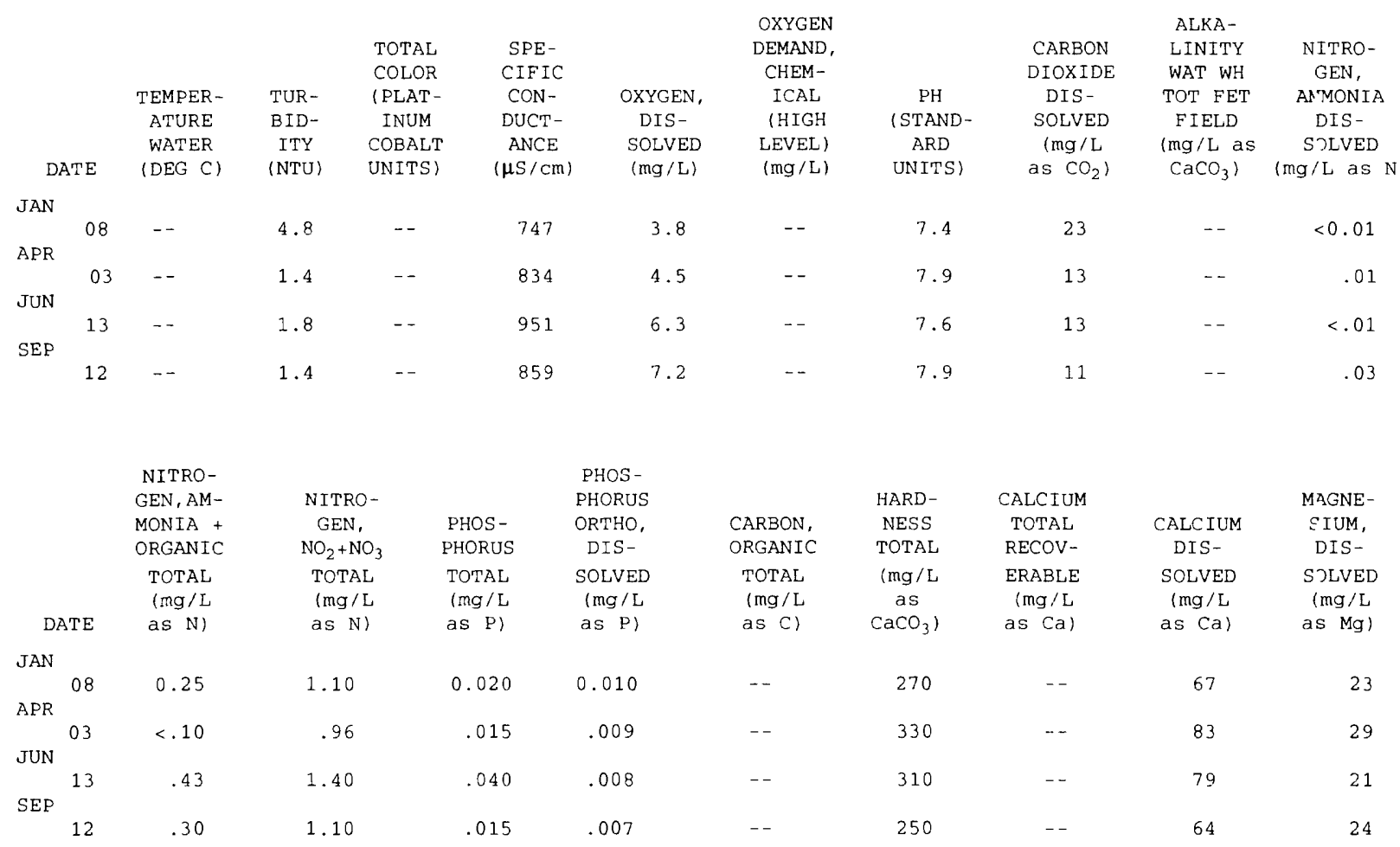

\begin{tabular}{|c|c|}
\hline DATE & \\
\hline \multicolumn{2}{|l|}{ JAN } \\
\hline 08 & 57 \\
\hline APR & \\
\hline 03 & 70 \\
\hline JUN & \\
\hline 13 & 81 \\
\hline SEP & \\
\hline
\end{tabular}

POTAS-
SIUM,
DIS-
SOLVED
$(\mathrm{mg} / \mathrm{L}$
$\mathrm{aS} \mathrm{K)}$

$\begin{array}{ccc} & & \\ \text { CHLO- } & & \text { IRON, } \\ \text { RIDE, } & \text { SULFATE } & \text { TOTAL } \\ \text { DIS- } & \text { DIS- } & \text { RECOV- } \\ \text { SOLVED } & \text { SOLVED } & \text { ERABLE } \\ (\mathrm{mg} / \mathrm{L} & \left(\mathrm{mg} / L^{2}\right. & (\mu \mathrm{G} / \mathrm{L} \\ \text { as Cl) } & \left.\text { as } \mathrm{SO}_{4}\right) & \text { as Fe })\end{array}$

$\begin{array}{ccc}\text { COLI- } & \text { SOLIDS, } & \text { SOLIDS, } \\ \text { FORM, } & \text { RESIDUE } & \text { SUM OF } \\ \text { FECAL, } & \text { AT } 180 & \text { CONSTI- } \\ 0.7 & \text { DEG. C } & \text { TUENTS, } \\ \text { UM-MF } & \text { DIS- } & \text { DIS- } \\ \text { (COLS. } & \text { SOLVED } & \text { SOLVED } \\ 100 \mathrm{ml}) & (\mathrm{mg} / \mathrm{L}) & \text { (mg } / \mathrm{L})\end{array}$

$\begin{array}{rrrrrrr}1.5 & 87 & 16 & 410 & \ldots & \ldots 18 \\ 1.7 & 130 & 30 & 90 & \ldots & -\ldots & 457 \\ 2.0 & 160 & 32 & 220 & - & 534 \\ 2.4 & 110 & 28 & 100 & -- & -- & 478\end{array}$


430252077283402. Local number Mo 11 (PM 83-2)--continued

\section{WATER QUALTTY DATA, WATER YEAR OCTOBER 1991 TO SEPTEMBER 1992}

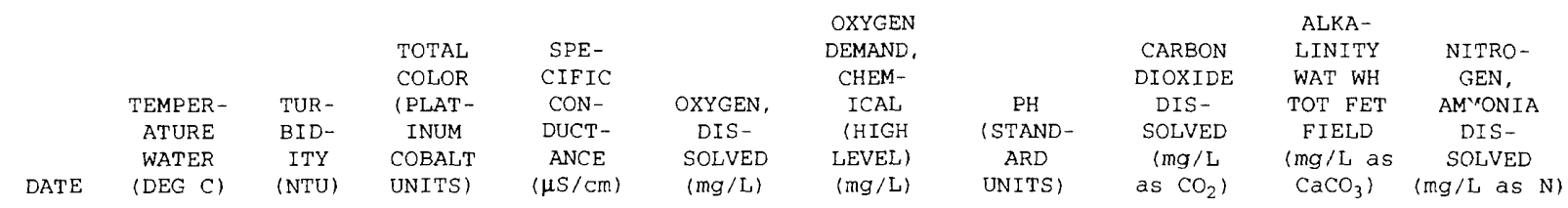
DEC

$\begin{array}{rrrrrr}\text { MAR } & 19 & -- & 1.6 & -- & 739 \\ \text { JUN } & 18 & -- & 1.5 & -- & 979 \\ & 24 & -- & 1.4 & -- & 1160 \\ \text { SEP } & 09 & -- & 1.8 & -- & 859\end{array}$

$2.5-10$

$\begin{array}{llll}7.7 & 11 & - & 0.01 \\ 7.6 & 16 & -- & <.01 \\ 7.5 & 15 & -- & <.01 \\ 7.5 & 16 & -- & <.01\end{array}$

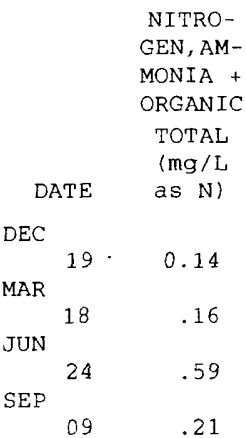

$\begin{array}{ccc} & & \text { PHOS - } \\ \text { PITRO- } & & \text { PHORUS } \\ \text { GEN, } & \text { PHOS- } & \text { ORTHO, } \\ \mathrm{NO}_{2}+\mathrm{NO}_{3} & \text { PHORUS } & \text { DIS- } \\ \mathrm{TOTAL} & \text { TOTAL } & \text { SOLVED } \\ \langle\mathrm{mg} / \mathrm{L} & (\mathrm{mg} / \mathrm{L} & (\mathrm{mg} / \mathrm{L} \\ \mathrm{aS} \text { ) } & \text { aS P) } & \text { aS P) }\end{array}$

$\begin{array}{ccc} & \text { HARD- } & \text { CALCIUM } \\ \text { CARBON, } & \text { NESS } & \text { TOTAL } \\ \text { ORGANIC } & \text { TOTAL } & \text { RECOV- } \\ \text { TOTAL } & (\mathrm{mg} / \mathrm{L} & \text { ERABLE } \\ \text { (mg } / \mathrm{L} & \text { as } & \text { (mg/L } \\ \text { as C) } & \left.\mathrm{CaCO}_{3}\right) & \text { as Ca) }\end{array}$

$\begin{array}{cc} & \text { MFGNE- } \\ \text { CALCIUM } & \text { SIUM, } \\ \text { DIS- } & \text { IIS- } \\ \text { SOLVED } & \text { SCLVED } \\ (\mathrm{mg} / \mathrm{L} & (\mathrm{mg} / \mathrm{L} \\ \text { aS Ca) } & \text { as } \mathrm{Mg})\end{array}$

1.30
.13
1.20
.80

0.010

0.008

240

$--$

61

22

$\begin{array}{llll}.010 & .007 & -- & 330\end{array}$

$--$

73

350

92

240

64

DATE

$\begin{array}{ccc} & & \\ & \text { POTAS- } & \text { CHLO- } \\ \text { SODIUM, } & \text { SIUM, } & \text { RIDE, } \\ \text { DIS- } & \text { DIS- } & \text { DIS- } \\ \text { SOLVED } & \text { SOLVED } & \text { SOLVED } \\ \text { (mg/L } & \text { (mg/L } & \text { (mg/L } \\ \text { as Na) } & \text { as K) } & \text { as Cl) }\end{array}$

$D E C$

$\begin{array}{lrr}\text { MER } & 19 & 62 \\ & 18 & 68 \\ \text { JUN } & & 130 \\ & 24 & 92\end{array}$

$\begin{array}{rr}2.2 & 81 \\ 2.8 & 170 \\ 2.4 & 280 \\ 1.7 & 110\end{array}$

SULFATE
DIS-
SOLVED
$(\mathrm{Mg} / \mathrm{L}$
aS $\left.\mathrm{SO}_{4}\right)$

IRON,
TOTAL
RECOV-
ERABLE
$(\mu G / L$
as $\mathrm{Fe})$

$\begin{array}{cc}\text { COLI- } & \text { SOLIDS, } \\ \text { FORM, } & \text { RESIDUE } \\ \text { FECAL, } & \text { AT } 180 \\ 0.7 & \text { DEG. C } \\ \text { UM-MF } & \text { DIS- } \\ \text { (COLS.) } & \text { SOLVED } \\ 100 \mathrm{ml}) & \text { (mg } / \mathrm{L})\end{array}$

SOLIDS, SUM OF CONSTITUENTS, DISSOLVED $(\mathrm{mg} / \mathrm{L})$

$\begin{array}{rrrrr}<10 & 70 & -- & -- & 411 \\ 35 & 110 & -- & -- & 525 \\ 39 & 160 & -- & -- & 691 \\ 27 & 170 & -- & -- & 484\end{array}$




\section{GROUND-WATER QUALITY}

Powder Mill Park

430252077283402. Local number Mo 11 (PM 83-2)--continued

\section{WATER QUALTT DATA, WATER YEAR OCTOBER 1992 TO SEPTEMBER 1993}

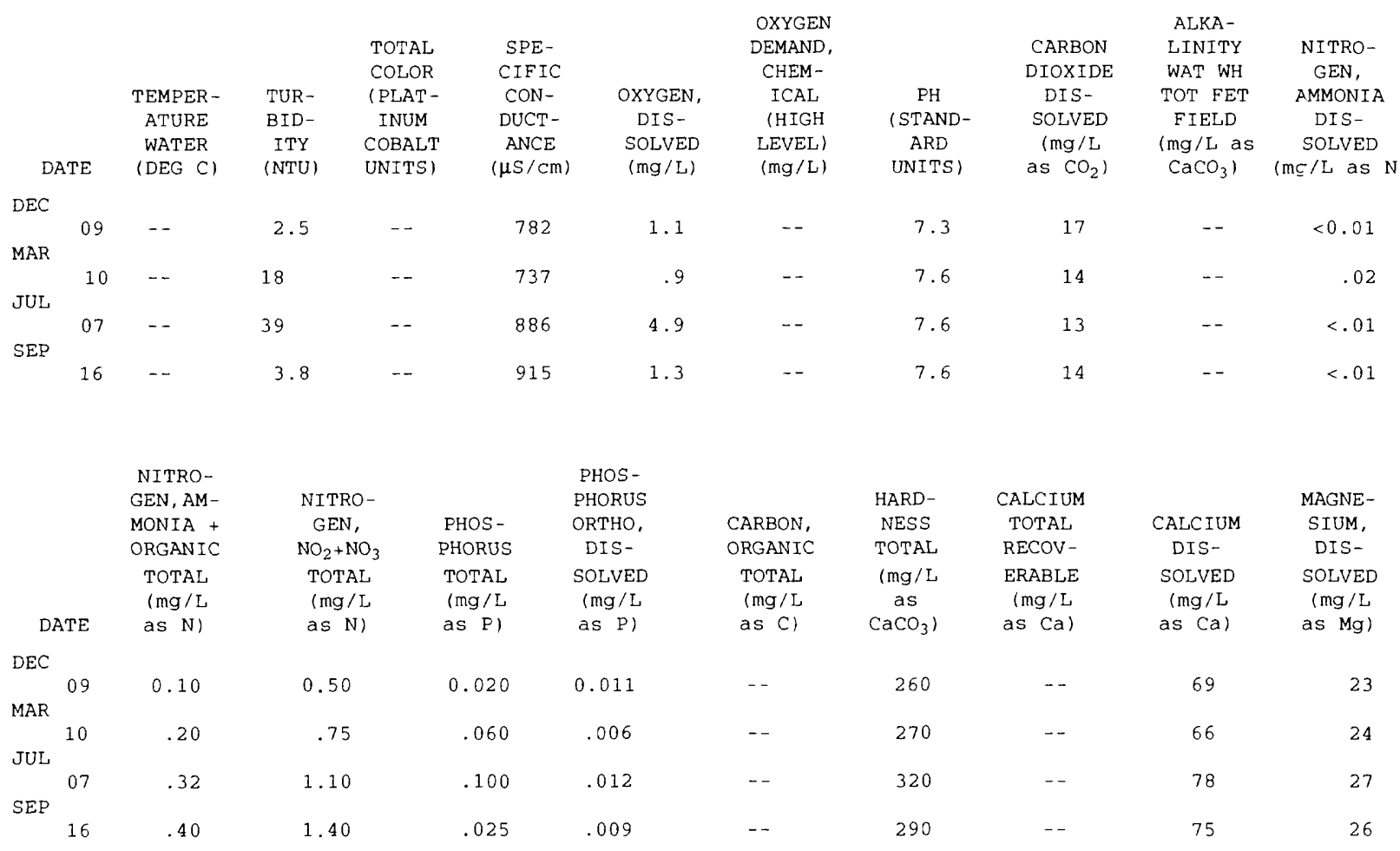

DATE

$\mathrm{DEC}$

MAR 09

10

07

EP
.40

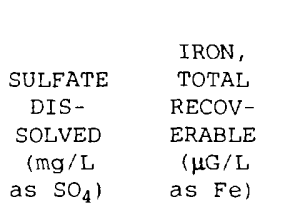

COLI -
FORM,
FECAL,
0.7
UM-MF
(COLS. '
$100 \mathrm{~m} 1$ )

SOLIDS,

\section{RESIDUE}

AT 180

DEG. C

DIS -

SOLVED

$(\mathrm{mg} / \mathrm{L})$

$$
\begin{aligned}
& \text { SOLIDS, } \\
& \text { SUM OF } \\
& \text { CONSTI- } \\
& \text { TUENTS, } \\
& \text { DIS- } \\
& \text { SOLVED } \\
& \text { (mg/L) }
\end{aligned}
$$

$\begin{array}{rrrrr}24 & 230 & -- & -- & 46 \varepsilon \\ 21 & 1400 & -- & -- & -- \\ 25 & 2200 & -- & -- & 516 \\ 31 & 230 & -- & -- & 528\end{array}$


430252077283402. Local number Mo 11 (PM 83-2)--continued

WATER TEMPERATURE, IN DEGREES CELSIUS

\begin{tabular}{|c|c|c|c|c|c|c|c|c|c|c|c|c|c|c|c|c|c|c|c|}
\hline \multirow[b]{2}{*}{$\begin{array}{l}\text { Depth, } \\
\text { in feet }\end{array}$} & \multicolumn{3}{|c|}{$1989 \mathrm{Wr}$} & \multicolumn{4}{|c|}{$1990 \mathrm{WY}$} & \multicolumn{4}{|c|}{$1991 \mathrm{WY}$} & \multicolumn{4}{|c|}{$1992 \mathrm{WY}$} & \multicolumn{4}{|c|}{$1993 \mathrm{Wr}$} \\
\hline & $\begin{array}{c}\text { Dec } \\
7\end{array}$ & $\begin{array}{c}\text { Mar } \\
15\end{array}$ & $\begin{array}{c}\text { Sep } \\
13\end{array}$ & $\begin{array}{c}\text { Dec } \\
5\end{array}$ & $\begin{array}{c}\text { Mar } \\
20\end{array}$ & $\begin{array}{c}\text { Jun } \\
7\end{array}$ & $\begin{array}{c}\text { Sep } \\
26\end{array}$ & $\begin{array}{c}\operatorname{Jan} \\
8\end{array}$ & $\begin{array}{c}\text { Apr } \\
3\end{array}$ & $\underset{13}{\text { Jun }}$ & $\begin{array}{c}\text { Sep } \\
11\end{array}$ & $\begin{array}{c}\text { Dec } \\
19\end{array}$ & $\begin{array}{c}\text { Mar } \\
18\end{array}$ & $\begin{array}{c}\text { Jun } \\
24\end{array}$ & $\begin{array}{c}\text { Sep } \\
9\end{array}$ & $\begin{array}{c}\text { Dec } \\
8\end{array}$ & $\begin{array}{c}\text { Mar } \\
9\end{array}$ & $\begin{array}{c}\text { Jul } \\
6\end{array}$ & $\begin{array}{c}\text { Sap } \\
15\end{array}$ \\
\hline 7.72 & $=$ & -- & $\overline{--}$ & -- & -- & -- & -- & -- & -- & -- & -- & -- & -- & -- & -- & -- & -- & 19.0 & -- \\
\hline 8.12 & -- & -- & -- & -- & -- & 9.0 & -- & 7.5 & 5.5 & 9.5 & -- & -- & -- & -- & 15.0 & 7.5 & 4.5 & 15.0 & 15.0 \\
\hline 8.52 & -- & -- & -- & -- & 4.0 & -- & -- & -- & -- & -- & -- & -- & -- & 10.0 & -- & -- & -- & -- & -- \\
\hline 9.12 & $\ldots$ & -- & -- & -. & 4.0 & 8.0 & -- & 8.5 & 5.0 & 9.5 & -- & 7.5 & 2.5 & $-\rightarrow$ & 14.0 & 9.0 & 5.0 & 13.5 & 15.0 \\
\hline 9.52 & -- & 3.5 & -- & -- & -- & -- & -- & -- & -- & -- & -- & -- & -- & 9.0 & $\cdots$ & -- & -- & -- & -- \\
\hline 9.82 & -- & -- & -- & -- & -- & -- & -- & -- & -- & $-\sim$ & 13.5 & -- & 3.0 & -- & -- & -- & -- & -- & -- \\
\hline 10.12 & - & 3.5 & -- & -- & 4.0 & 7.5 & 13.5 & 8.5 & 5.0 & 9.0 & -- & 9.0 & -- & -- & 13.5 & 9.5 & 5.0 & 12.0 & 14.5 \\
\hline 10.52 & 9.5 & -- & 12.0 & 10.5 & -- & -- & -- & -- & - & -- & -- & -- & 3.5 & 8.5 & -- & -- & -- & -- & -- \\
\hline 11.12 & 9.5 & 3.5 & 12.0 & 10.5 & 4.5 & 7.0 & 13.0 & 9.0 & 5.0 & 8.5 & 13.5 & 9.5 & -- & -- & 13.0 & 10.0 & 4.5 & 10.5 & 14.0 \\
\hline 11.52 & -- & -- & -- & -- & -- & -- & -- & -- & -- & -- & -- & -- & -- & 8.0 & -- & -- & -- & -- & + \\
\hline 12.12 & 10.0 & 3.5 & 11.5 & 11.0 & 4.5 & 7.0 & 12.5 & 9.0 & 5.5 & 8.0 & 13.0 & 10.0 & 3.5 & $\cdots$ & 12.5 & 10.5 & 4.5 & 9.5 & 14.0 \\
\hline 12.52 & -- & -- & -- & -- & -- & -- & -- & -- & - & -- & -- & -- & -- & 7.5 & -- & -- & -- & -- & -- \\
\hline 13.12 & 10.0 & 5.0 & 11.0 & 11.0 & 5.0 & 6.5 & 12.5 & 9.0 & 5.5 & 7.5 & 12.5 & 10.0 & 4.0 & -- & 12.0 & 10.5 & 4.5 & 9.0 & $1: .5$ \\
\hline 13.52 & -- & -- & -- & -- & $\cdots$ & -- & -- & -- & -- & -- & -- & -- & -- & 7.0 & -- & -- & -- & -- & -- \\
\hline 14.12 & 10.5 & 5.0 & 11.0 & 11.0 & 5.0 & 6.5 & 12.0 & 9.0 & 6.0 & 7.0 & 12.5 & 10.0 & 4.5 & -- & 11.5 & 10.5 & 4.5 & 8.5 & 12.5 \\
\hline 14.52 & -- & -- & -- & +- & -- & -- & -- & -- & -- & -- & -- & -- & -- & 7.0 & -- & -- & -- & -- & -- \\
\hline 15.12 & 10.5 & 5.0 & 10.5 & 11.0 & 5.5 & 6.0 & 11.5 & 9.0 & 6.0 & 7.0 & 12.0 & 10.5 & 4.5 & -- & 11.0 & 10.5 & 4.5 & 8.0 & 12.5 \\
\hline 15.52 & -- & -- & -- & -- & -- & -- & $\rightarrow$ & -- & -- & -- & -- & -- & -- & 6.5 & -- & -- & -- & -- & -- \\
\hline 15.72 & -- & -- & - & -- & -- & -- & -- & -- & -- & -- & -- & -- & -- & -- & -- & 10.5 & -- & -- & -- \\
\hline 16.12 & 10.5 & 5.0 & 10.5 & 11.0 & 5.5 & 6.0 & 11.0 & 9.0 & 6.0 & 7.0 & 11.5 & 10.5 & 4.5 & -- & 10.5 & -- & 4.5 & 7.5 & 11.5 \\
\hline 16.62 & -- & -- & -- & 11.0 & 5.5 & 6.0 & 11.0 & 9.5 & 6.0 & 7.0 & -- & 10.5 & -- & 6.5 & 10.5 & -- & -- & 7.5 & -- \\
\hline 16.92 & - & -- & -- & -- & -- & -- & -- & -- & -- & -- & 11.5 & -- & -- & $\ldots$ & -- & -- & -- & -- & -- \\
\hline
\end{tabular}

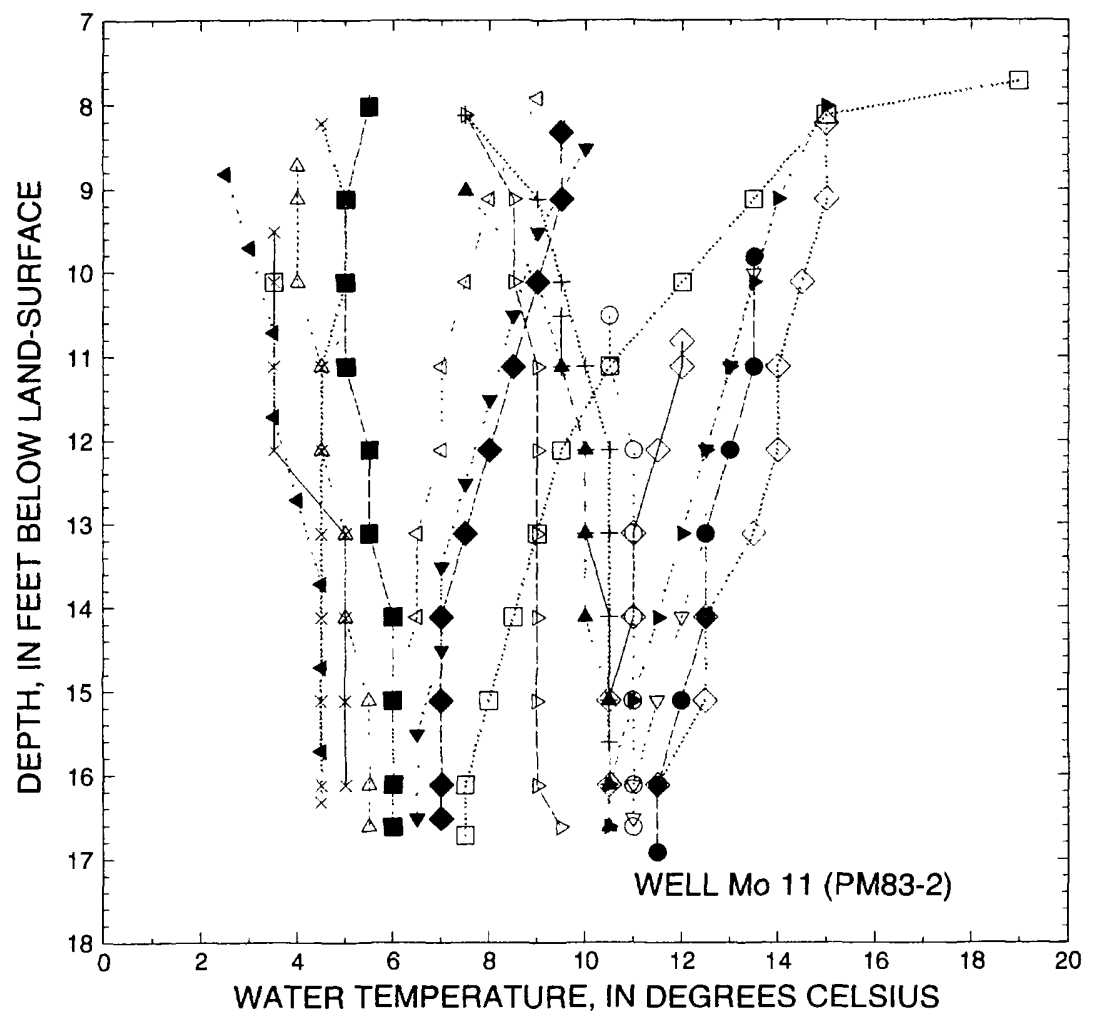

EXPLANATIO"

$+\quad 88 / 12 / 07$

$\rightarrow \quad 89 / 03 / 15$

$\curvearrowright 89 / 09 / 13$

.... 89/12/05

$\triangle 90 / 03 / 20$

..ङ

$\nabla \quad 90 / 09 / 26$

$-\theta-91 / 01 / 08$

- $91 / 04 / 03$

- $91 / 06 / 13$

- $91 / 09 / 11$

A . $91 / 12 / 19$

4. $92 / 03 / 18$

ᄀ. $92 / 06 / 24$

-. 92/09/09

$\ldots+\cdots 92 / 12 / 98$

........ 93/03/09

........ 93/07/06

....... 93/09/15 


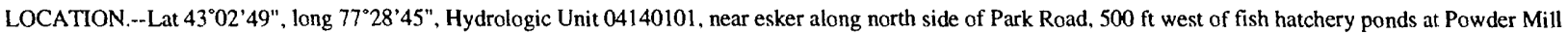
Park near Bushnell basin. Owner: U.S. Geological Survey.

AQUIFER.--Confined aquifer in sand of Pleistocene age.

WELL CHARACTERISTICS.--Drilled observation well, diameter 2 in, depth $43 \mathrm{ft}$, cased to $43 \mathrm{ft}$, open end.

INSTRUMENTATION.--Weekly measurement with chalked tape by Powder Mill Park personnel and occasional measurement by USGS and MCEHL.

DATUM.--Elevation of land-surface datum is $431.82 \mathrm{ft}$ above National Geodetic Vertical Datum of 1929 . Measuring point: top of casing, $0.25 \mathrm{ft}$ below land-surface datum.

REMARKS.--This well is also a water-quality observation well. Water levels are monitored monthly and water samples taken quarterly by the Monroe County Environmental Health Laboratory. Water-temperature profiles are also taken by MCEHL on a quarterly basis.

PERIOD OF RECORD.--December 1983 to September 1993 (discontinued).

EXTREMES FOR PERIOD DECEMBER 1983 TO SEPTEMBER 1993..-Highest water level measured, 0.47 feet below land-surface datum, July 6, 19 ` $_{\text {; }}$ lowest measured, 1.88 feet below land-surface datum, December 5, 1989.

WATER LEVEL, IN FEET BELOW LAND-SURFACE DATUM,

\begin{tabular}{|c|c|c|c|c|c|c|c|c|c|c|c|}
\hline DATE & & $\begin{array}{l}\text { WATER } \\
\text { LEVEL }\end{array}$ & & & $\begin{array}{l}\text { WATER } \\
\text { LEVEL }\end{array}$ & & & $\begin{array}{l}\text { WATER } \\
\text { LEVEL }\end{array}$ & & & $\begin{array}{l}\text { WATER } \\
\text { LEVEL }\end{array}$ \\
\hline WATER & YE & CTOBER & SEPTE & 3ER & & & & & & & \\
\hline $\mathrm{DEC}$ & 7 & 1.71 & MAR & 15 & 1.78 & JUN & 7 & 1.77 & SEP & 13 & 2.05 \\
\hline WATER & YE & CTOBER & SEPTE & BER & & & & & & & \\
\hline DEC & 5 & 1.88 & MAR & 20 & 1.59 & JUN & 7 & 1.36 & SEP & 26 & 1.64 \\
\hline WATER & YE & CTOBER & SEPTE & 3ER & & & & & & & \\
\hline JAN & 8 & 1.28 & APR & 4 & 1.10 & JUN & 13 & 1.17 & SEP & 12 & 1.54 \\
\hline WATER & & TOBER & SEPTE & 36 & & & & & & & \\
\hline $\begin{array}{l}\text { DEC } \\
\text { MAR }\end{array}$ & & $\begin{array}{l}1.45 \\
1.35\end{array}$ & $\begin{array}{l}\text { MAR } \\
\text { JUN }\end{array}$ & $\begin{array}{l}19 \\
25\end{array}$ & $\begin{array}{l}1.35 \\
1.27\end{array}$ & JUL & 21 & 1.20 & SEP & 9 & 1.14 \\
\hline WATER & & TOBER & EPT & 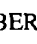 & & & & & & & \\
\hline $\mathrm{DEC}$ & 8 & 0.99 & MAR & 9 & 0.75 & JUL & 6 & 0.47 & SEP & 15 & 0.62 \\
\hline
\end{tabular}

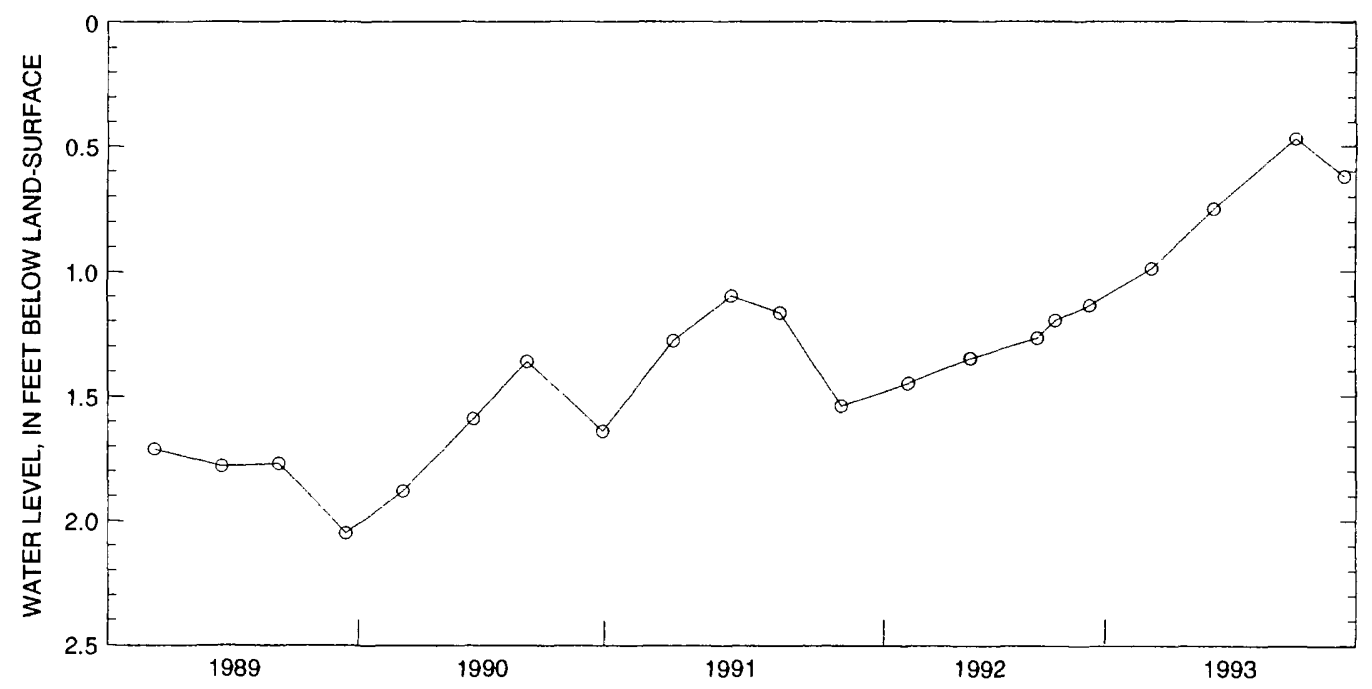


PERIOD OF RECORD.-- January 1986 to September 1993 (discontinued)..

CHEMICAL DATA: 1986(a) 1987-93(b).

ORGANIC DATA: OC.--1986(a) 1987-93(b).

NUTRIENT DATA: 1986(a) 1987-93(b).

BIOLOGICAL DATA:

Bacteria.--1986(a) 1987-93(b).

COOPERATION-- Water-quality samples were collected and analyzed by the Monroe County Environmental Health Laboratory at Rochester, NY.

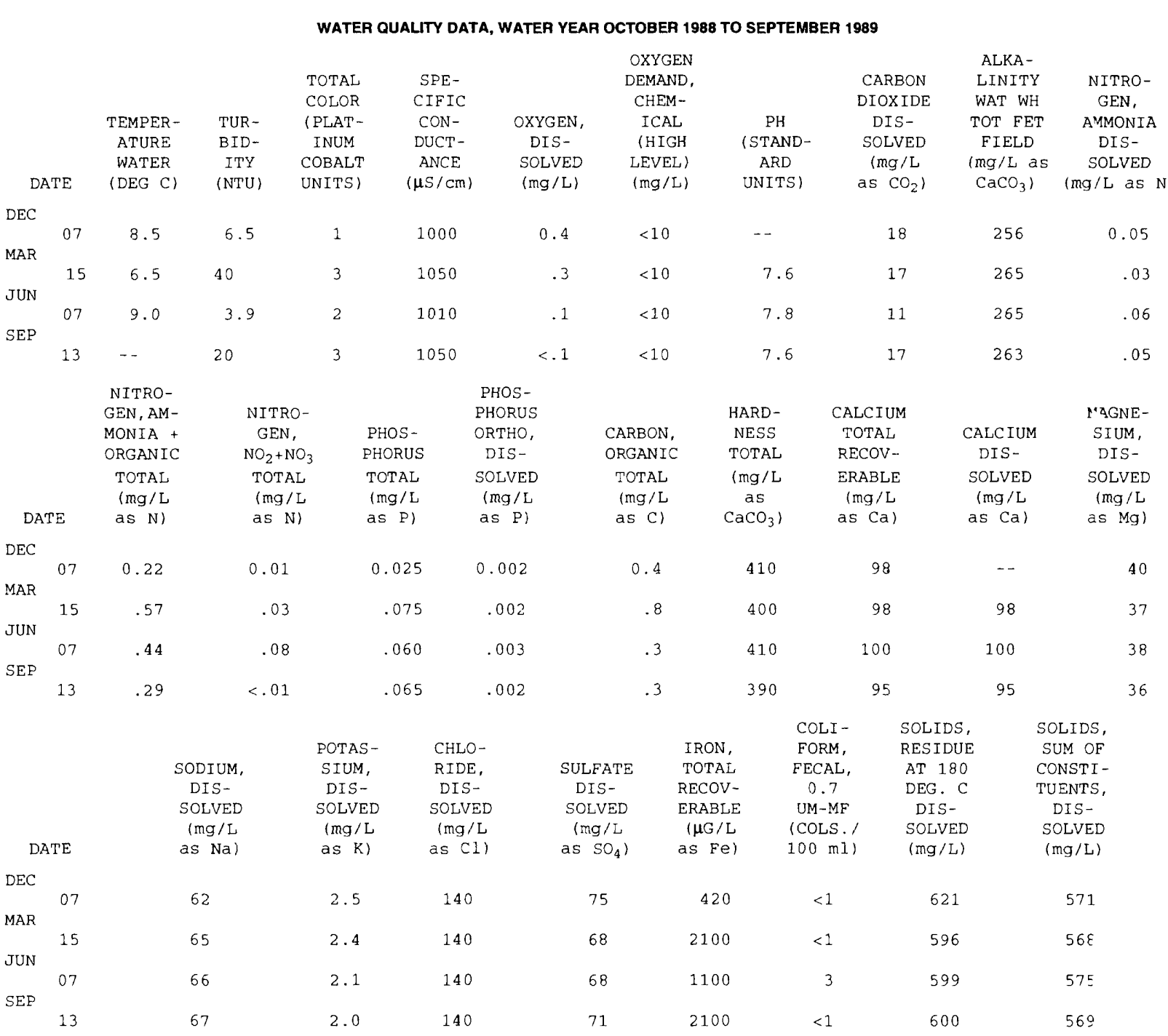


430249077284501. Local number Mo 12 (PM 83-4)--continued

\section{WATER QUALITY DATA, WATER YEAR OCTOBER 1989 TO SEPTEMBER 1990}

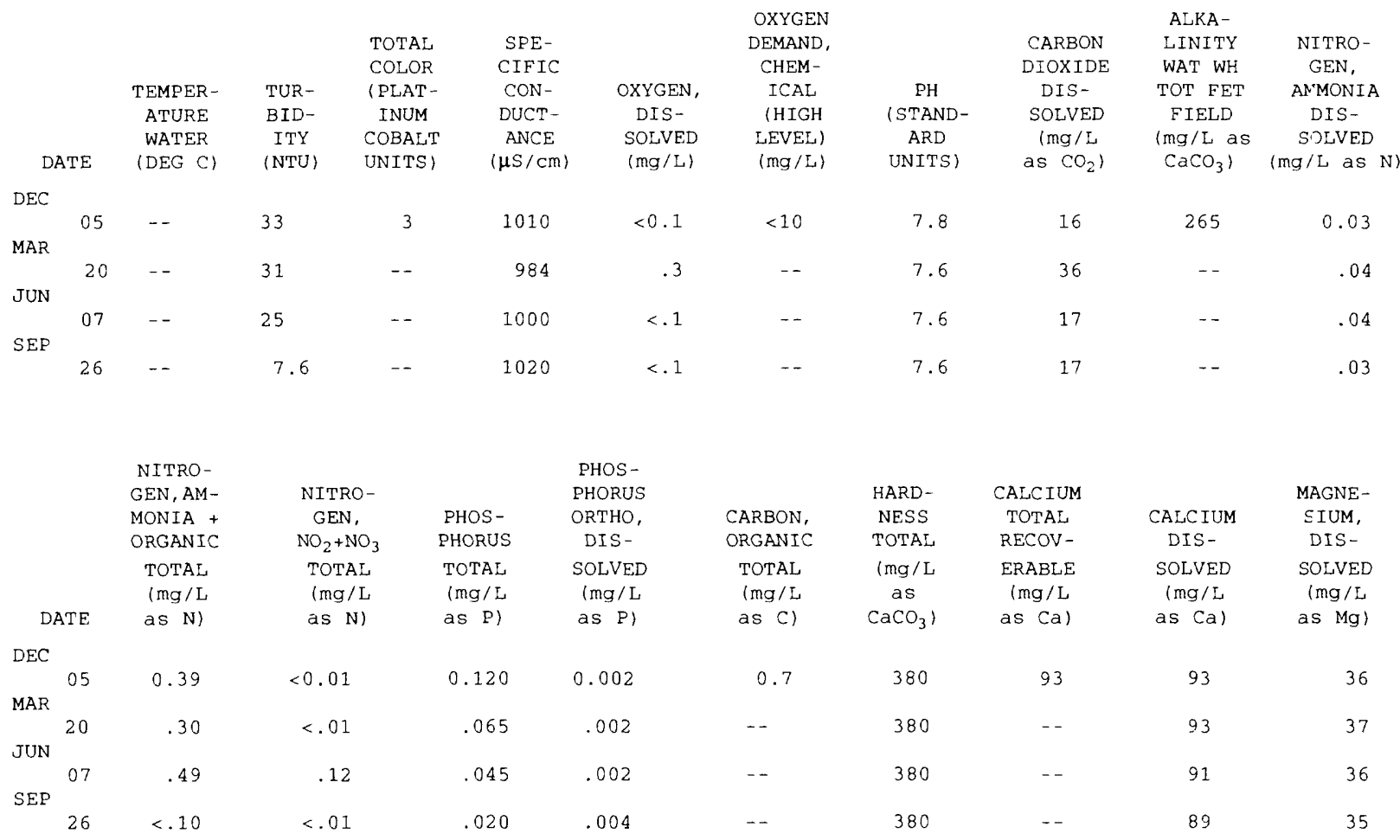

DATE

DEC

$05 \quad 67$

20

JUN

SEP

$\begin{array}{cc} & \\ \text { SODIUM, } & \text { POTAS- } \\ \text { DIS- } & \text { DIUM, } \\ \text { SOLVED } & \text { SOLVED } \\ (\mathrm{mg} / \mathrm{L} & (\mathrm{mg} / \mathrm{L} \\ \text { as } \mathrm{Na}) & \text { as K) }\end{array}$

$\begin{array}{ll}2.3 & 140 \\ 2.0 & 140 \\ 2.1 & 140 \\ 1.9 & 140\end{array}$

SULFATE
DIS-
SOLVED
$(\mathrm{mg} / \mathrm{L}$
as $\left.\mathrm{SO}_{4}\right)$

IRON,
TOTAL
RECOV-
ERABLE
$(\mu G / L$
aS Fe $)$

$\begin{array}{cc}\text { COLI- } & \text { SOLIDS, } \\ \text { FORM, } & \text { RESIDUE } \\ \text { FECAL, } & \text { AT } 180 \\ 0.7 & \text { DEG. C } \\ \text { UM-MF } & \text { DIS- } \\ \text { (COLS./ } & \text { SOLVED } \\ 100 \mathrm{ml}) & (\mathrm{mg} / \mathrm{L})\end{array}$

SOLIDS, SUM OF CONST ITUENTS, DISSOLVED $(\mathrm{mg} / \mathrm{L})$

$\begin{array}{rrrrr}74 & 2100 & <4 & 582 & 568 \\ 70 & 1800 & -- & -- & 578 \\ 70 & 1200 & -- & -- & 605 \\ 38 & 470 & -- & -- & 612\end{array}$


430249077284501. Local number Mo 12 (PM 83-4)--continued

\section{WATER QUALTY DATA, WATER YEAR OCTOBER 1990 TO SEPTEMBER 1991}

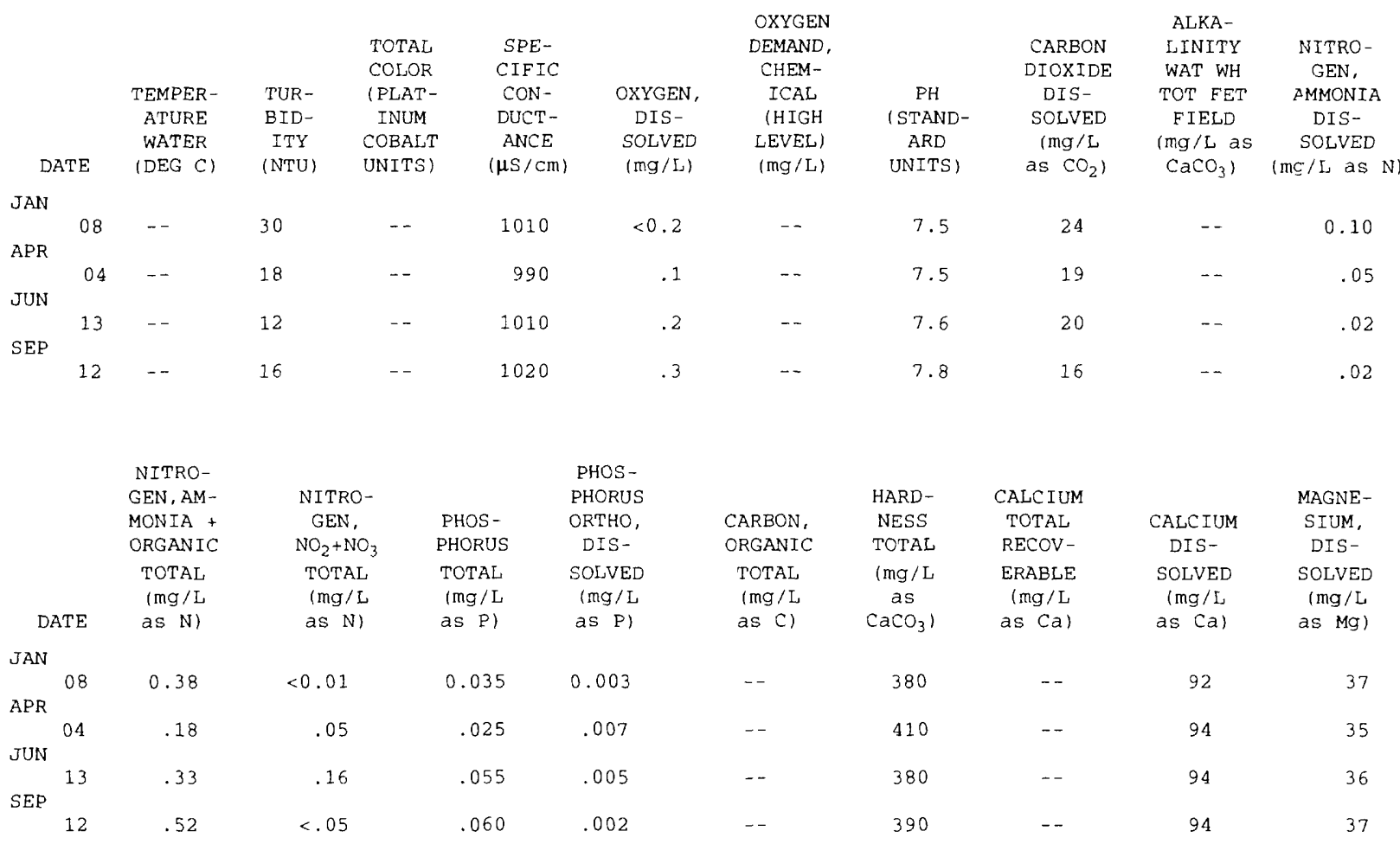

DATE

JAN 08

APR 04

JUN

SEP 13

$\begin{array}{cc} & \\ & \text { POTAS- } \\ \text { SODIUM, } & \text { SIUM, } \\ \text { DIS- } & \text { DIS- } \\ \text { SOIJED } & \text { SOLVED } \\ (\mathrm{mg} / \mathrm{L} & (\mathrm{mg} / \mathrm{L} \\ \text { aS Na) } & \text { aS K) }\end{array}$

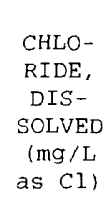

$\begin{array}{ll}2.4 & 130 \\ 2.3 & 140 \\ 2.4 & 130 \\ 2.2 & 140\end{array}$
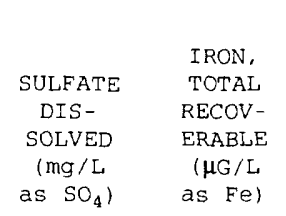
COLI - SOLIDS, FORM, RESIDUE FECAL, AT 180 0.7 DEG. UM-MF DIS- (COLS.) SOLVED $100 \mathrm{ml}) \quad(\mathrm{mg} / \mathrm{L})$

$\begin{array}{rrrrr}77 & 1500 & -- & - & 6 \subset 2 \\ 79 & 1100 & -- & -- & 575 \\ 62 & 1300 & - & -- & 616 \\ 74 & 960 & -- & -- & 589\end{array}$

$\begin{array}{rrrrr}77 & 1500 & -- & -- & 6 c 2 \\ 79 & 1100 & -- & -- & 575 \\ 62 & 1300 & -- & -- & 616 \\ 74 & 960 & -- & -- & 589\end{array}$

$\begin{array}{rrrrr}77 & 1500 & -- & -- & 6 \subset 2 \\ 79 & 1100 & -- & -- & 575 \\ 62 & 1300 & -- & -- & 616 \\ 74 & 960 & -- & -- & 589\end{array}$

SOLIDS, SUM OF CONSTITUEN?S, DISSOLVED (mg/L) 
430249077284501. Local number Mo 12 (PM 83-4)--continued

WATER QUALITY DATA, WATER YEAR OCTOBER 1991 TO SEPTEMBER 1992

\begin{tabular}{|c|c|c|c|c|c|c|c|c|c|c|}
\hline DATE & $\begin{array}{c}\text { TEMPER- } \\
\text { ATURE } \\
\text { WATER } \\
\text { (DEG C) }\end{array}$ & $\begin{array}{c}\text { TUR- } \\
\text { BID- } \\
\text { ITY } \\
\text { (NTU) }\end{array}$ & $\begin{array}{l}\text { TOTAL } \\
\text { COLOR } \\
\text { (PLAT- } \\
\text { INUM } \\
\text { COBALT } \\
\text { UNITS) }\end{array}$ & $\begin{array}{c}\text { SPE- } \\
\text { CIFIC } \\
\text { CON- } \\
\text { DUCT- } \\
\text { ANCE } \\
(\mu \mathrm{S} / \mathrm{Cm})\end{array}$ & $\begin{array}{l}\text { OXYGEN, } \\
\text { DIS- } \\
\text { SOLVED } \\
(\mathrm{mg} / \mathrm{L})\end{array}$ & $\begin{array}{c}\text { OXYGEN } \\
\text { DEMAND, } \\
\text { CHEM- } \\
\text { ICAL } \\
\text { (HIGH } \\
\text { LEVEL) } \\
(\mathrm{mg} / \mathrm{L})\end{array}$ & $\begin{array}{c}\text { PH } \\
\text { (STAND- } \\
\text { ARD } \\
\text { UNITS) }\end{array}$ & $\begin{array}{c}\text { CARBON } \\
\text { DIOXIDE } \\
\text { DIS- } \\
\text { SOLVED } \\
(\mathrm{mg} / \mathrm{L} \\
\left.\text { as } \mathrm{CO}_{2}\right)\end{array}$ & $\begin{array}{l}\text { ALKA- } \\
\text { LINITY } \\
\text { WAT WH } \\
\text { TOT FET } \\
\text { FIELD } \\
(\mathrm{mg} / \mathrm{L} \text { as } \\
\left.\mathrm{CaCO}_{3}\right)\end{array}$ & $\begin{array}{c}\text { NITRO- } \\
\text { GEN, } \\
\text { AMYONIA } \\
\text { DIS- } \\
\text { SOLVED } \\
\text { (mg/L as N }\end{array}$ \\
\hline \multicolumn{11}{|l|}{$\mathrm{DEC}$} \\
\hline $\begin{array}{ll} & 19 \\
\text { MAR } & \end{array}$ & - & 30 & -- & 1020 & 0.4 & -- & 7.6 & 18 & -- & 0.02 \\
\hline $\begin{array}{ll} & 18 \\
\text { JUN } & \end{array}$ & -- & 3.9 & - & 1010 & - & -- & 7.6 & 19 & -- & .02 \\
\hline \multirow[t]{4}{*}{ SEP } & -. & 3.7 & -. & 1030 & N. 2 & -. & 7.3 & $\mathrm{~N} 21$ & - & .01 \\
\hline & $\cdots$ & 3.5 & -- & 1030 & .4 & -- & 7.4 & 20 & -- & .02 \\
\hline & $\begin{array}{l}\text { NITRO- } \\
\text { GEN, AM- } \\
\text { MONIA + } \\
\text { ORGANIC }\end{array}$ & $\begin{array}{l}\mathrm{NITRO}- \\
\text { GEN, } \\
\mathrm{NO}_{2}+\mathrm{NO}\end{array}$ & & $\begin{array}{l}\text { PHOS- } \\
\text { PHORUS }\end{array}$ & $\begin{array}{l}\text { PHOS- } \\
\text { PHORUS } \\
\text { ORTHO, } \\
\text { DIS- }\end{array}$ & $\begin{array}{l}\text { CARBON, } \\
\text { ORGANIC }\end{array}$ & $\begin{array}{l}\text { HARD- } \\
\text { NESS } \\
\text { TOTAL }\end{array}$ & $\begin{array}{c}\text { CALCIUM } \\
\text { TOTAL } \\
\text { RECOV- }\end{array}$ & $\begin{array}{l}\text { CALCIUM } \\
\text { DIS- }\end{array}$ & $\begin{array}{c}\text { MASNE- } \\
\text { SIUM, } \\
\text { IIS- }\end{array}$ \\
\hline & $\begin{array}{l}\text { TOTAL } \\
(\mathrm{mg} / \mathrm{L} \\
\text { as N) }\end{array}$ & $\begin{array}{l}\text { TOTAL } \\
(\mathrm{mg} / \mathrm{L} \\
\mathrm{as} \mathrm{N})\end{array}$ & & $\begin{array}{l}\text { TOTAL } \\
\text { (mg/L } \\
\text { as P) }\end{array}$ & $\begin{array}{l}\text { SOLVED } \\
(\mathrm{mg} / \mathrm{L} \\
\mathrm{as} \mathrm{P})\end{array}$ & $\begin{array}{l}\text { TOTAL } \\
\text { (mg/L } \\
\text { as C) }\end{array}$ & $\begin{array}{c}(\mathrm{mg} / \mathrm{L} \\
\mathrm{as} \\
\left.\mathrm{CaCO}_{3}\right)\end{array}$ & $\begin{array}{c}\text { ERABLE } \\
\text { (mg/L } \\
\text { as Ca) }\end{array}$ & $\begin{array}{l}\text { SOLVED } \\
\text { (mg/L } \\
\text { as Ca) }\end{array}$ & $\begin{array}{c}\text { SOLVED } \\
\text { (mg/L } \\
\text { as Mg) }\end{array}$ \\
\hline DEC & & & & & & & & & & \\
\hline 19 & 0.27 & $<0.05$ & & 0.035 & 0.003 & -- & 390 & -- & 90 & 35 \\
\hline JUN 18 & .17 & $<.05$ & & .015 & .003 & - & 390 & -- & 95 & 38 \\
\hline SEP 24 & .48 & $<.05$ & & .008 & .002 & -- & 390 & -- & 94 & 38 \\
\hline 09 & .32 & $<.05$ & & .020 & .003 & -- & 390 & -- & 100 & 43 \\
\hline
\end{tabular}

DATE DEC

$\begin{array}{lrr} & 19 & 69 \\ \text { MAR } & & 18 \\ \text { JUN } & & 63 \\ & 24 & \text { N76 } \\ \text { SEP } & & 70\end{array}$

$\begin{array}{cc}\text { POTAS- } & \text { CHLO- } \\ \text { SIUM, } & \text { RIDE, } \\ \text { DIS- } & \text { DIS- } \\ \text { SOLVED } & \text { SOLVED } \\ \text { (mg/L } & (\mathrm{mg} / \mathrm{L} \\ \text { as K) } & \text { as Cl) }\end{array}$

2.3

140

2.9

140

2.2

140

2.5

140

$\begin{array}{cc} & \\ & \text { IRON, } \\ \text { SULFATE } & \text { TOTAL } \\ \text { DIS- } & \text { RECOV- } \\ \text { SOLVED } & \text { ERABLE } \\ (\mathrm{mg} / \mathrm{L} & (\mu \mathrm{G} / \mathrm{L} \\ \left.\text { as } \mathrm{SO}_{4}\right) & \text { as } \mathrm{Fe})\end{array}$

COLI -

RESIDUE

AT 180

0.7

DEG. C

$\mathrm{UM}-\mathrm{MF}$

(COLS.)

DIS-

SOLVED

$(\mathrm{mg} / \mathrm{L})$

$\begin{array}{rrrrr}34 & 1600 & - & - & 605 \\ 76 & 410 & -- & -- & 612 \\ 21 & 450 & - & -- & 588 \\ 85 & 610 & -- & -- & 625\end{array}$

SOLIDS,

SUM OF

CONSTI-

TUENTS

DIS-

SOLVED

( $\mathrm{mg} / \mathrm{L}$ ) 
430249077284501. Local number Mo 12 (PM 83-4)--continued

WATER QUALITY DATA, WATER YEAR OCTOBER 1992 TO SEPTEMBER 1993

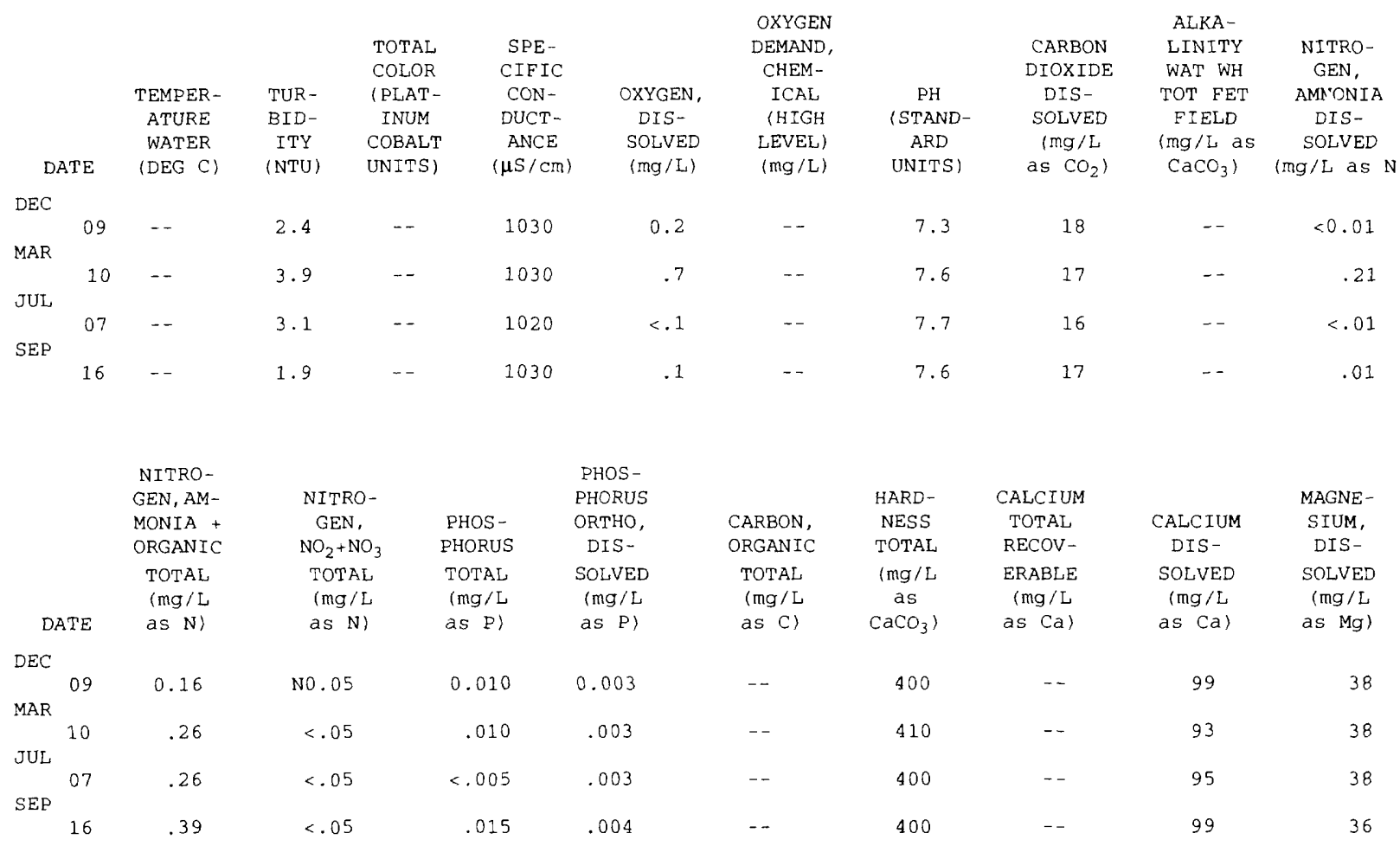

\begin{tabular}{|c|c|c|c|c|c|c|c|c|}
\hline DATE & $\begin{array}{c}\text { SODIUM, } \\
\text { DIS- } \\
\text { SOLVED } \\
\text { (mg/L } \\
\text { as Na) }\end{array}$ & $\begin{array}{l}\text { POTAS- } \\
\text { SIUM, } \\
\text { DIS- } \\
\text { SOLVED } \\
\text { (mg/L } \\
\text { as K) }\end{array}$ & $\begin{array}{l}\text { CHLO- } \\
\text { RIDE, } \\
\text { DIS- } \\
\text { SOLVED } \\
\text { (mg/L } \\
\text { as Cl) }\end{array}$ & $\begin{array}{l}\text { SULFATE } \\
\text { DIS- } \\
\text { SOLVED } \\
\text { (mg/L } \\
\text { as } \mathrm{SO}_{4} \text { ) }\end{array}$ & $\begin{array}{c}\text { IRON, } \\
\text { TOTAL } \\
\text { RECOV- } \\
\text { ERABLE } \\
(\mu \mathrm{G} / \mathrm{L} \\
\text { as } \mathrm{Fe})\end{array}$ & $\begin{array}{c}\text { COLI- } \\
\text { FORM, } \\
\text { FECAL, } \\
0.7 \\
\text { UM-MF } \\
\text { (COLS., } \\
100 \mathrm{ml} \text { ) }\end{array}$ & $\begin{array}{l}\text { SOLIDS, } \\
\text { RESIDUE } \\
\text { AT } 180 \\
\text { DEG. C } \\
\text { DIS- } \\
\text { SOLVED } \\
\text { (mg/L) }\end{array}$ & $\begin{array}{c}\text { SOLIDS, } \\
\text { SUM O } \\
\text { CONSTI- } \\
\text { TUENTE, } \\
\text { DIS- } \\
\text { SOLVE) } \\
\text { (mg/L) }\end{array}$ \\
\hline & & & & & & & & \\
\hline 09 & 68 & 2.1 & 140 & 88 & 460 & 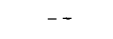 & -- & 604 \\
\hline 10 & 67 & 2.0 & 140 & 83 & 560 & -- & - & - \\
\hline 07 & 68 & 2.2 & 140 & 82 & 300 & -- & -- & 644 \\
\hline 16 & 71 & 1.8 & 140 & 82 & 440 & -- & - & 608 \\
\hline
\end{tabular}


430249077284501. Local number Mo 12 (PM 83-4)--continued

WATER TEMPERATURE, IN DEGREES CELSIUS

\begin{tabular}{|c|c|c|c|c|c|c|c|c|c|c|c|c|c|c|c|c|c|c|c|c|}
\hline \multirow[b]{2}{*}{$\begin{array}{l}\text { Depth, } \\
\text { in feet }\end{array}$} & \multicolumn{4}{|c|}{$1989 \mathrm{Wr}$} & \multicolumn{4}{|c|}{$1990 \mathrm{Wr}$} & \multicolumn{4}{|c|}{$1991 \mathrm{Wr}$} & \multicolumn{4}{|c|}{$1992 \mathrm{WY}$} & \multicolumn{4}{|c|}{$1993 \mathrm{WY}$} \\
\hline & $\begin{array}{c}\mathrm{Dec} \\
7\end{array}$ & $\begin{array}{c}\text { Mar } \\
15\end{array}$ & Jun & $\begin{array}{l}\text { Sep } \\
13\end{array}$ & $\begin{array}{c}\text { Dec } \\
5\end{array}$ & $\begin{array}{c}\text { Mar } \\
20 \\
\end{array}$ & Jun & $\begin{array}{l}\text { Sep } \\
26\end{array}$ & $\underset{8}{\operatorname{Jan}}$ & Apr & $\underset{13}{\operatorname{Jun}}$ & Sep & $\begin{array}{c}\text { Dec } \\
19\end{array}$ & $\begin{array}{c}\mathrm{Mar} \\
19\end{array}$ & $\begin{array}{l}\text { Jun } \\
25 \\
\end{array}$ & $\begin{array}{c}\text { Sep } \\
9\end{array}$ & $\begin{array}{c}\text { Dec } \\
8\end{array}$ & $\begin{array}{c}\text { Mar } \\
9\end{array}$ & $\begin{array}{c}\text { Jul } \\
6 \\
\end{array}$ & Sep \\
\hline 0.65 & -- & -- & -- & -- & - & -- & -- & $-\cdots$ & $\tau$ & -- & -- & -- & 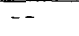 & 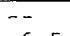 & 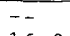 & 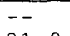 & 6.5 & & 3.0 & 17.0 \\
\hline & -- & -- & & -- & - & -- & 6.0 & - & 5.0 & 10.5 & 7.5 & - & -- & 6.5 & 16.0 & 21.0 & -- & & & 17. \\
\hline & -- & -- & - & -- & -- & 5.0 & -- & $\cdots$ & -- & -- & -- & 1.0 & 6.0 & -- & -- & -- & -- & & - & -- \\
\hline & 8.0 & 3.0 & 1.0 & -- & 7.0 & -- & -- & 7. & - & +- & - & - & -- & -- & -- & -- & & & - & -- \\
\hline & 8.0 & - & - & 17.0 & 7.0 & 5.5 & - & -- & -- & 8.5 & 16.5 & 20.0 & 7.0 & 5.5 & 15.0 & 19.5 & 7.0 & & 8.0 & 17.0 \\
\hline & -- & -- & -- & -- & -- & -- & -- & $\cdots$ & -- & -- & -- & -- & - & -- & - & $\ldots$ & 8.0 & & - & 16.0 \\
\hline & 9.0 & 3.0 & 8.5 & 16.0 & 8.5 & 6.0 & 13.0 & 16.5 & 6.5 & 8.0 & 14.0 & 18.0 & 8.0 & 6.0 & 13.5 & 17.0 & 8 & & 5.0 & 15.5 \\
\hline & -- & -- & -- & -- & -- & -- & -- & -- & +- & -- & -- & -- & -- & -- & -- & -- & & & - & 15. \\
\hline 5 & 9.5 & 3.0 & 6.5 & 15.5 & 9.0 & 6.5 & 11.5 & 15.5 & 7.5 & 7.5 & -- & 16.5 & 8.5 & 6.5 & 12.0 & 16.0 & 9.0 & & 13.5 & 14. \\
\hline & $\ldots$ & -- & - & -- & -- & -- & -- & -- & -- & -- & -- & -- & -- & -- & -- & -- & 9.5 & & . & 14. \\
\hline & 9.5 & 6.0 & 5.5 & 12.5 & 10.0 & 7.0 & 11.0 & 14.5 & 8.0 & 7.5 & 12.0 & 15.5 & 9.0 & 7.0 & 11.5 & 14.5 & b & & 2.0 & 13.5 \\
\hline & - & -- & -- & -- & -- & -- & -- & -- & -- & -- & -- & -- & -- & -- & -- & -- & & & -- & 13.0 \\
\hline & 10.0 & 6.5 & 4.0 & 12.0 & 10.5 & 7.0 & 10.0 & 13.5 & 8.5 & 8.0 & 10.5 & 14.0 & 9.5 & 7.5 & 10.5 & 13. & & & 11.0 & 12.5 \\
\hline & -- & - & -- & - & -- & -- & -- & -- & -- & $\cdots$ & -- & -- & -- & -- & -- & -- & & & -- & 12.0 \\
\hline & 10.5 & 7.0 & 4.0 & 11.5 & 10.5 & 7.5 & 10.0 & 12.5 & 9.5 & 8.0 & 10.0 & 13.0 & 9.5 & 8.0 & -- & 12 & & & 0.5 & 11.5 \\
\hline & -- & - & -- & -- & -- & -- & -- & $\cdots$ & -- & -- & -- & -- & -- & -- & -- & -- & & & & 11.0 \\
\hline & 10.5 & 7.5 & 4.0 & 11.0 & 11.0 & 8.0 & 9.5 & 12.0 & 9.5 & 8.0 & 9.5 & 12.5 & 10.0 & 8.5 & 9.5 & 11. & & & 0.0 & 10.5 \\
\hline & - & -- & -- & -- & -- & -- & -- & -- & -- & -- & -- & 11.5 & -- & -- & -- & -- & & & -- & 10.5 \\
\hline & 10.5 & 8.0 & 4.0 & 10.5 & 10.5 & 8.5 & 9.5 & 11.0 & 10.0 & 8.5 & 9.5 & 11.0 & 10.0 & 9.0 & 9.5 & 11. & & & 10.0 & 10.0 \\
\hline & $\cdots$ & -- & -- & -- & -- & -- & -- & -- & - & -- & -- & -- & -- & $\cdots$ & - & -- & & & 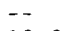 & 9.5 \\
\hline & 10.5 & 8.0 & 4.0 & 10.5 & 10.5 & 9.0 & 9.5 & 10.5 & 10.0 & 9.0 & 9.5 & 10.5 & 10.0 & 9.0 & 9.5 & 10 & & & 0.0 & 9.5 \\
\hline & $\ldots$ & -- & -- & -- & -- & -- & -- & -- & -- & $\ldots$ & -- & -- & -- & -- & -- & -- & & & -- & 9.5 \\
\hline & 10.0 & 8.0 & 4.0 & 10.0 & 10.0 & 9.0 & 9.5 & 10.0 & 10.0 & 9.0 & 9.5 & 10.5 & 9.5 & 9.5 & 9.5 & 10 & & & 10.0 & 9.0 \\
\hline & 10.0 & 8.0 & 4.0 & 10.0 & 10.0 & 9.5 & 9.5 & 10.0 & 0.0 & 9.0 & 9.5 & 10 & 9. & 9.5 & 9.5 & & & & 9.5 & 9.0 \\
\hline & 9.5 & 8.0 & 4.0 & 10. & 10.0 & 9.5 & 9.5 & 10.0 & .0 & 9.0 & 9.5 & 10.0 & 9. & 9.5 & 9.5 & 10. & & & 9.5 & 9.0 \\
\hline & $-\infty$ & -- & -- & -- & -- & -- & -- & -- & -- & -- & -- & -- & - & -- & - & -- & & & -- & - \\
\hline & 9.5 & 8.0 & 4.0 & 10.0 & 9.5 & 9.5 & 10.0 & 10.0 & 10.0 & 9.0 & 9.5 & 10.0 & 9.5 & 9.5 & 9.5 & 10. & & & 9.5 & 9.0 \\
\hline & - & -- & $\ldots$ & -- & -- & $\ldots$ & -- & $\ldots$ & $\ldots$ & -- & -- & -- & -- & - & - & -- & & & -- & -- \\
\hline & 9.5 & 8.0 & 4.0 & 10.0 & 9.5 & 9.5 & 10.0 & 10.0 & 10.0 & 9.5 & 9.5 & 10.0 & 9.5 & 10.0 & 9.5 & 10. & -- & & 9.5 & 9.0 \\
\hline & $\cdots$ & -- & -- & -- & -- & -- & -- & -- & -- & -- & -- & -- & -- & -- & -- & -- & 10 & & -- & -- \\
\hline & 9.5 & 8.0 & 4.0 & 7.5 & 9.5 & 9.5 & 10.0 & 10.0 & 9.5 & 9.5 & 9.5 & 10.0 & 9.5 & 10.0 & 10.0 & 10. & & & 0.0 & 9.0 \\
\hline & $\sim$ & -- & -- & -- & -- & - & -- & -- & -- & -- & -- & -- & -- & -- & -- & -- & 1 & & - & - \\
\hline & 9.5 & 8.0 & 4.0 & 7.5 & 9.5 & 9.5 & 10.0 & 9.5 & 9.5 & 9.5 & 9.5 & 10.0 & 9.5 & 10.0 & 10.0 & 10. & & & 10.0 & 9.0 \\
\hline & $\therefore$ & -- & -- & -- & -- & -- & -- & $\ldots$ & - & -- & -- & -- & -- & & & -- & 10 & & & -- \\
\hline & 9.5 & 8.0 & 4.0 & 7.5 & 9.5 & 9.5 & 10.0 & 9.5 & 9.5 & 9.5 & 9.5 & 10.0 & 9.5 & 9.5 & 10.0 & 10. & -- & & 10.0 & 9.0 \\
\hline & $\cdots$ & -- & -- & -- & -- & -- & -- & -- & -- & $\cdots$ & -- & -- & -- & -- & -- & - & 9 & & 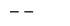 & -- \\
\hline & 9.5 & 8.0 & 4.0 & 7.5 & 9.5 & 9.5 & 10.0 & 9. & 9. & 9.5 & 9.5 & 10.0 & 9.5 & 9.5 & 9.5 & 10. & $\cdots$ & & -- & 9. \\
\hline & $\cdots$ & -- & -- & -- & -- & -- & - & -. & -- & -- & -- & -- & $\cdots$ & -- & -- & -- & 9.5 & & -- & -- \\
\hline & 9.5 & 8.0 & 4.0 & 7.5 & 9.5 & 9.5 & 10.0 & 3.5 & 9.5 & 9.5 & 9.5 & 10.0 & 9.5 & 9.5 & 9.5 & 10. & - & & -- & 9.0 \\
\hline & $\cdots$ & -- & -- & -- & -- & - & -- & $\cdots$ & -- & -- & -- & -- & -- & -- & -- & -- & 9.5 & & -- & -- \\
\hline & 9.5 & 8.0 & 4.0 & 7.5 & 9.5 & 9.5 & 10.0 & 9.5 & 9.5 & 9.0 & 9.5 & 10.0 & 9.5 & 9.5 & 9.5 & 9.5 & $\cdots$ & & - & 9.0 \\
\hline & $\cdots$ & -- & -- & -- & -- & -- & -- & -- & -- & -- & -- & - & -- & - & -- & - & 9 & & -- & -- \\
\hline & $-\sim$ & -- & -- & -- & -- & -- & -- & -- & -- & -- & -- & -- & -- & -- & -- & -- & 9.5 & & -- & -- \\
\hline & 9.5 & 8.0 & 4.0 & 7.5 & 9.5 & 9.5 & 10.0 & 9.5 & 9.5 & 9.0 & 9.5 & 9.5 & 9.0 & 9.5 & 9.5 & 9. & -- & & -- & 9.0 \\
\hline & $\cdots$ & -- & 4.0 & -- & 9.5 & -- & 10.0 & -- & -- & $\cdots$ & -- & -- & -- & -- & -- & 9. & -- & & -- & -- \\
\hline & -- & -- & -- & 7.5 & -- & 9.5 & -- & -- & -- & -- & -- & -- & -- & -- & -- & -- & $\cdots$ & & -- & -- \\
\hline & -- & -- & -- & -- & -- & -- & -- & 9.5 & -- & $\cdots$ & -- & -- & -- & -- & -- & -- & -- & & -- & $\ldots$ \\
\hline
\end{tabular}




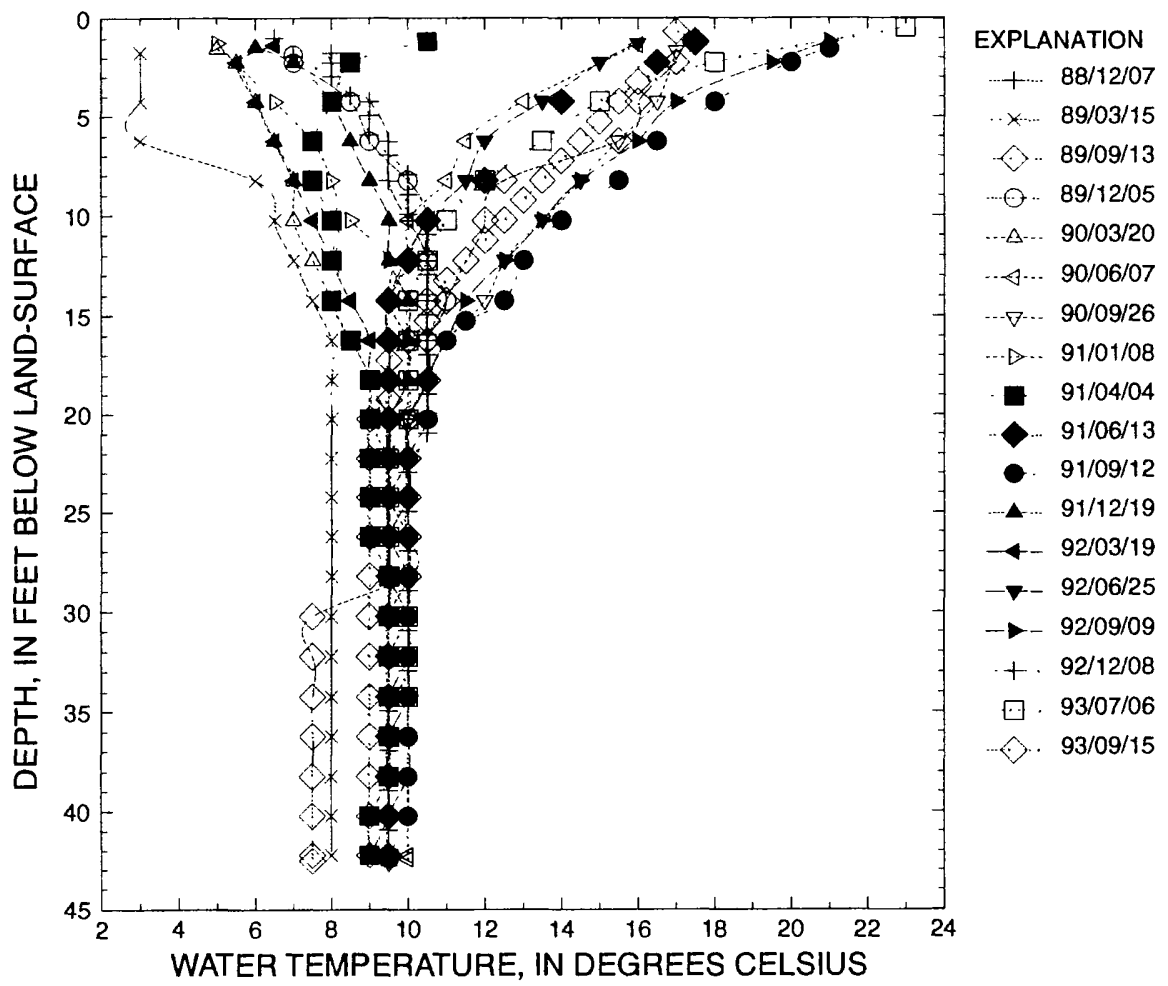




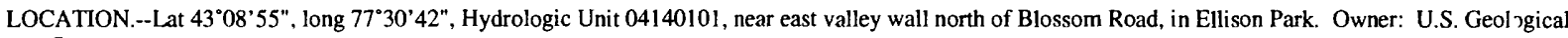
Survey.

AQUIFER.--Water-table aquifer in alluvium of Holocene age.

WELL CHARACTERISTICS.--Drilled observation well, diameter 2 in, depth $26.5 \mathrm{ft}$, cased to $23.5 \mathrm{ft}$, screened $23.5 \mathrm{ft}$ to $26.5 \mathrm{ft}$.

INSTRUMENTATION.--Monthly measurement with chalked tape by Monroe County Environmental Health Laboratory personnel and occasional measurement by USGS personnel.

DATUM.--Elevation of land-surface datum is $252.60 \mathrm{ft}$ above National Geodetic Vertical Datum of 1929. Measuring point: arrow at top of casing, $3.26 \mathrm{ft}$ above landsurface datum.

REMARKS.--Water level may be affected by stage of Irondequoit Creek. This well is also a water-quality observation well. Water levels are monitored monthly and water samples taken quarterly by the Monroe County Environmental Health Laboratory. Water-temperature profiles are also taken by MCEHL on a quarterly basis. PERIOD OF RECORD.--September 1984 to November 1990, (discontinued).

EXTREMES FOR PERIOD SEPTEMBER 1984 TO NOVEMBER 1990.--Highest water level measured, 0.80 feet above land-surface datum, April 9, 1987 ; lowest measured, 1.59 feet below land-surface datum, September 13,1989.

WATER LEVEL, IN FEET ABOVE (+) OR BELOW LAND-SURFACE DATUM,

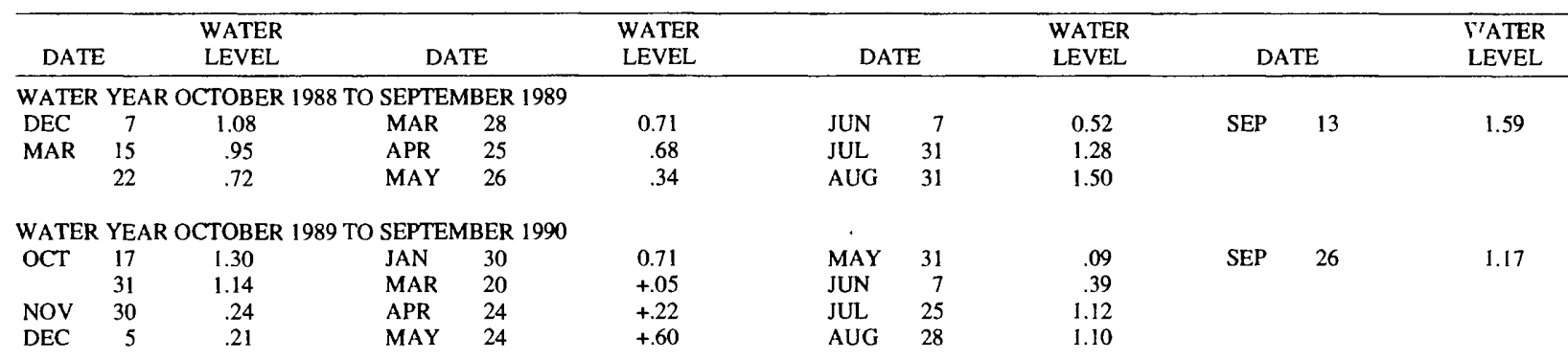

WATER YEAR OCTOBER 1990 TO SEPTEMBER 1991

$\begin{array}{llllll}\text { OCT } & 30 & 0.76 & \text { NOV } & 28 & 0.08\end{array}$

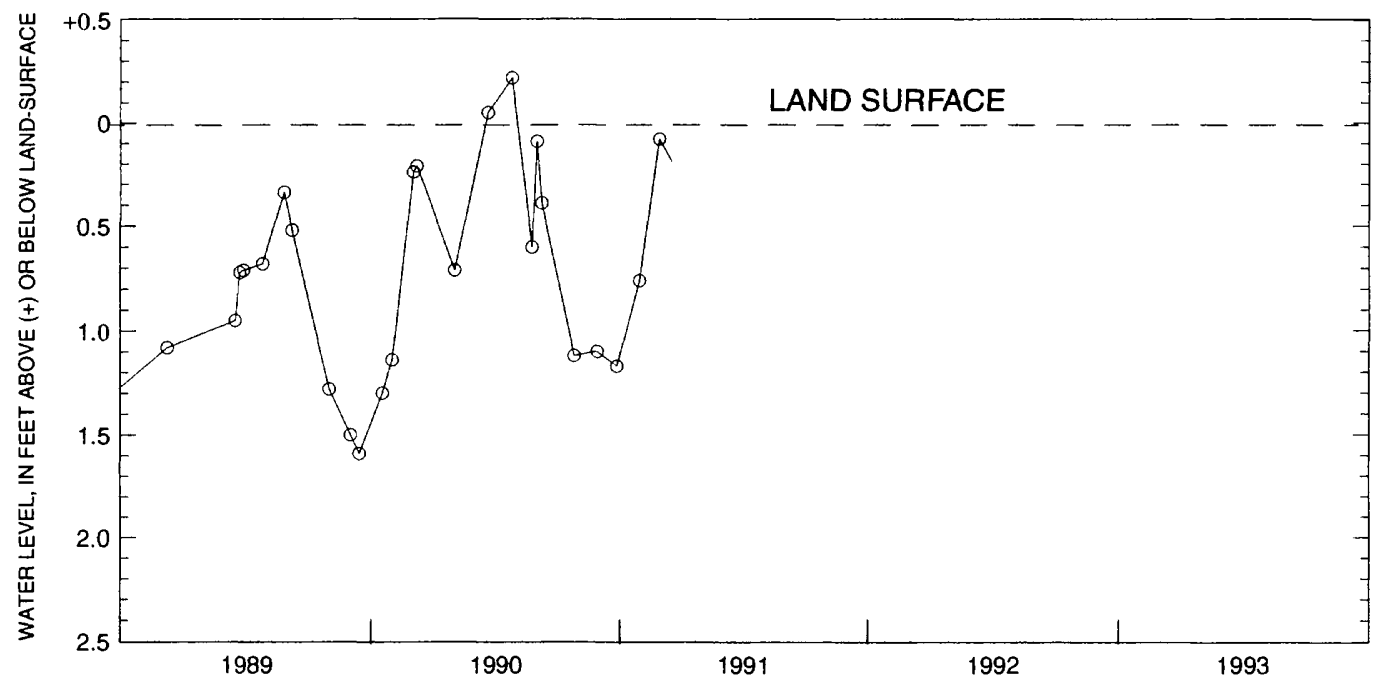


PERIOD OF RECORD.-- January 1986 to November 1990, (discontinued).

CHEMICAL DATA: 1986(a) 1987-90(b).

ORGANIC DATA: OC.--1986(a) 1987-90(b).

NUTRIENT DATA: 1986(a) 1987-90(b).

BIOLOGICAL DATA

Bacteria.--1986(a) 1987-90(b)

COOPERATION-- Water-quality samples were collected and analyzed by the Monroe County Environmental Health Laboratory at Rochester, NY

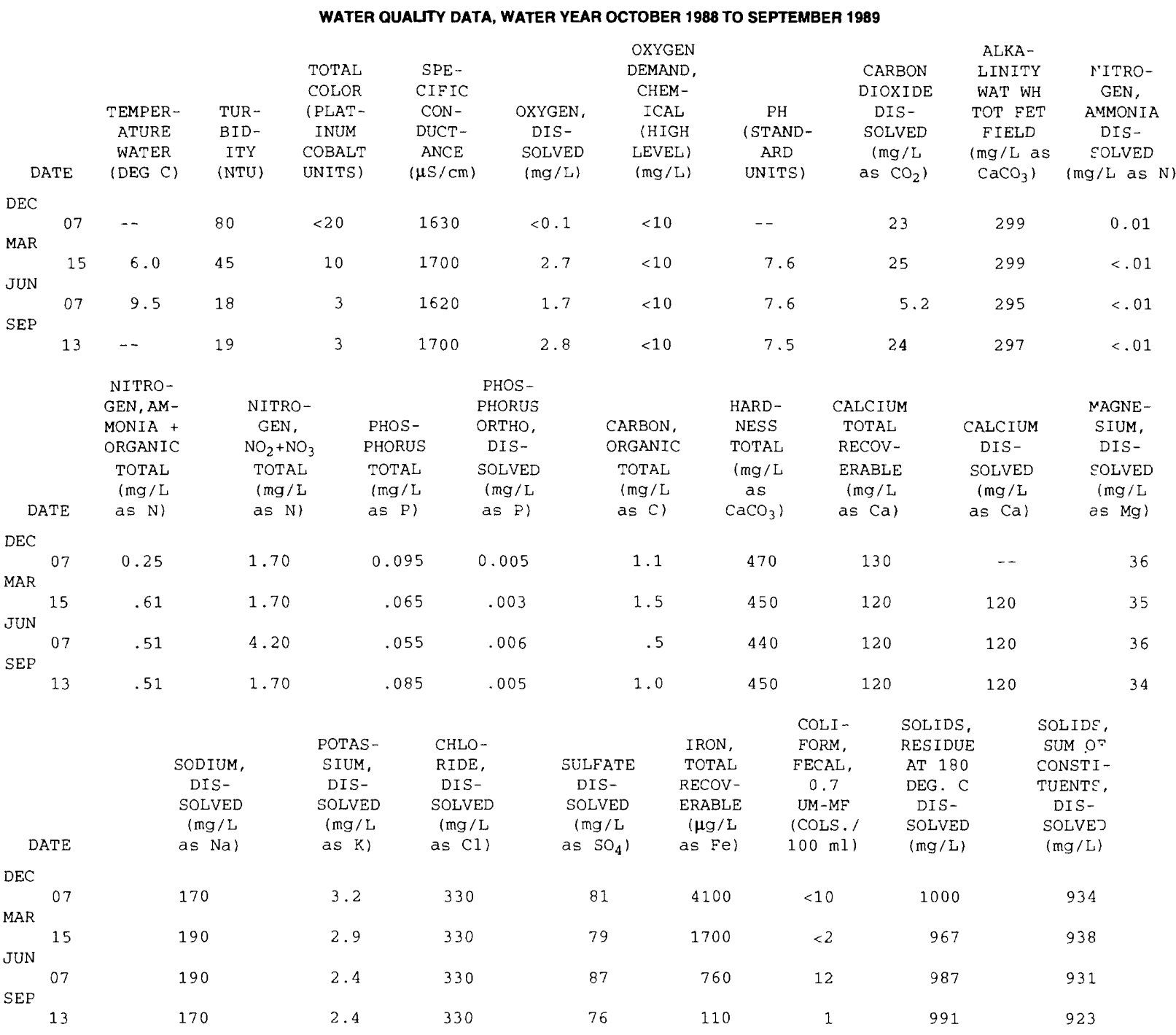




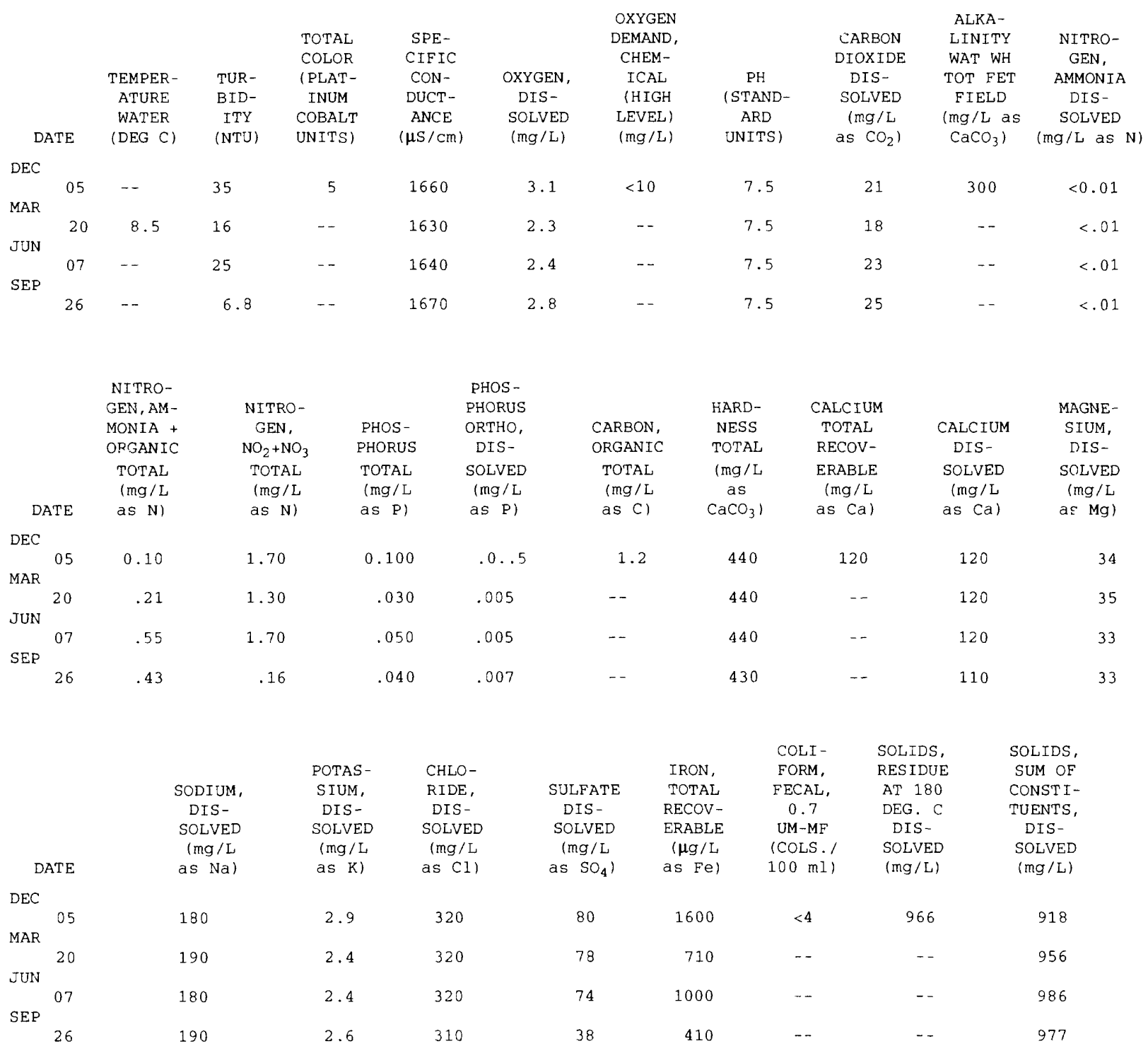


430855077304201. Local number Mo 1 (E1 84-1)--continued

WATER TEMPERATURE, IN DEGREES CELSIUS

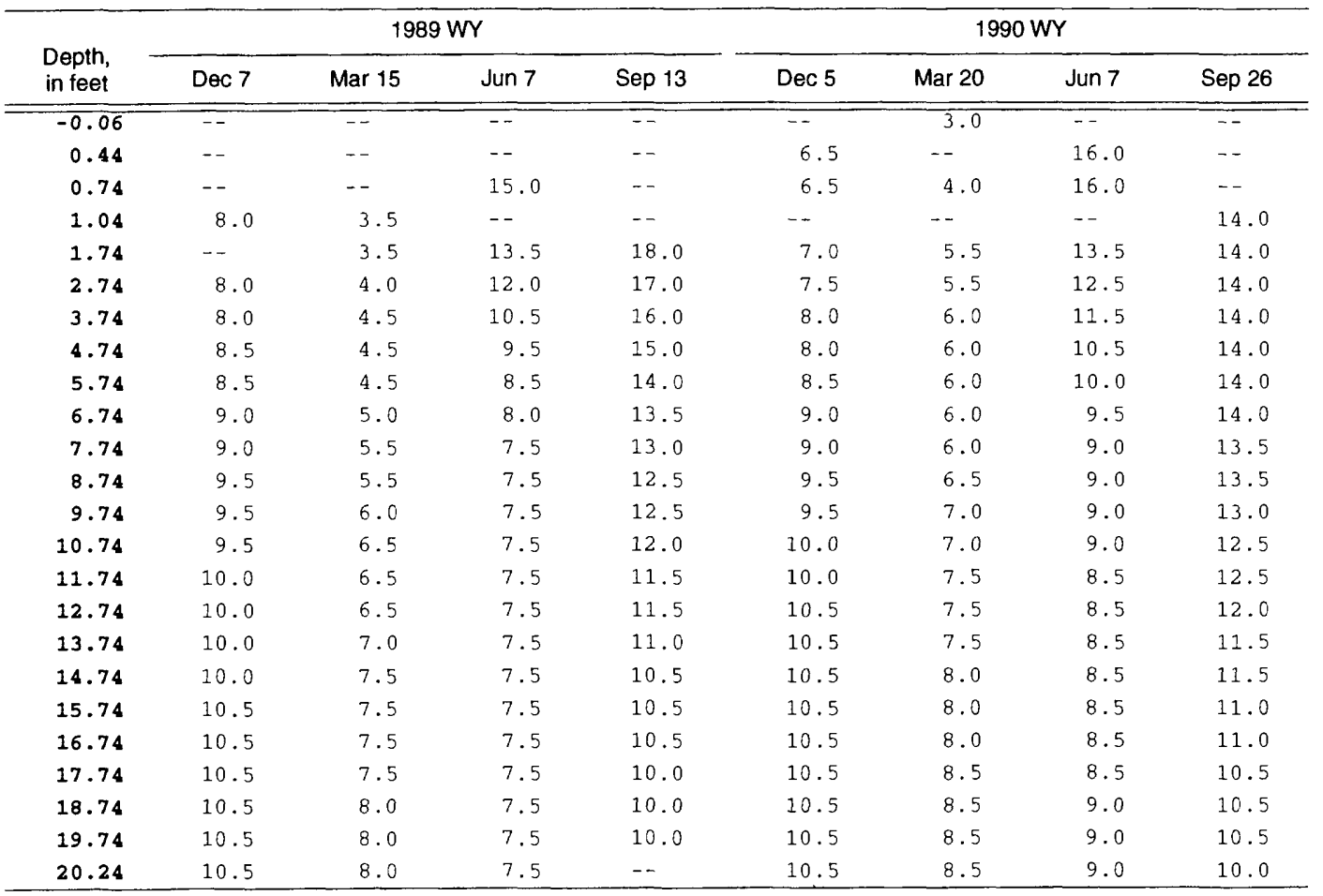

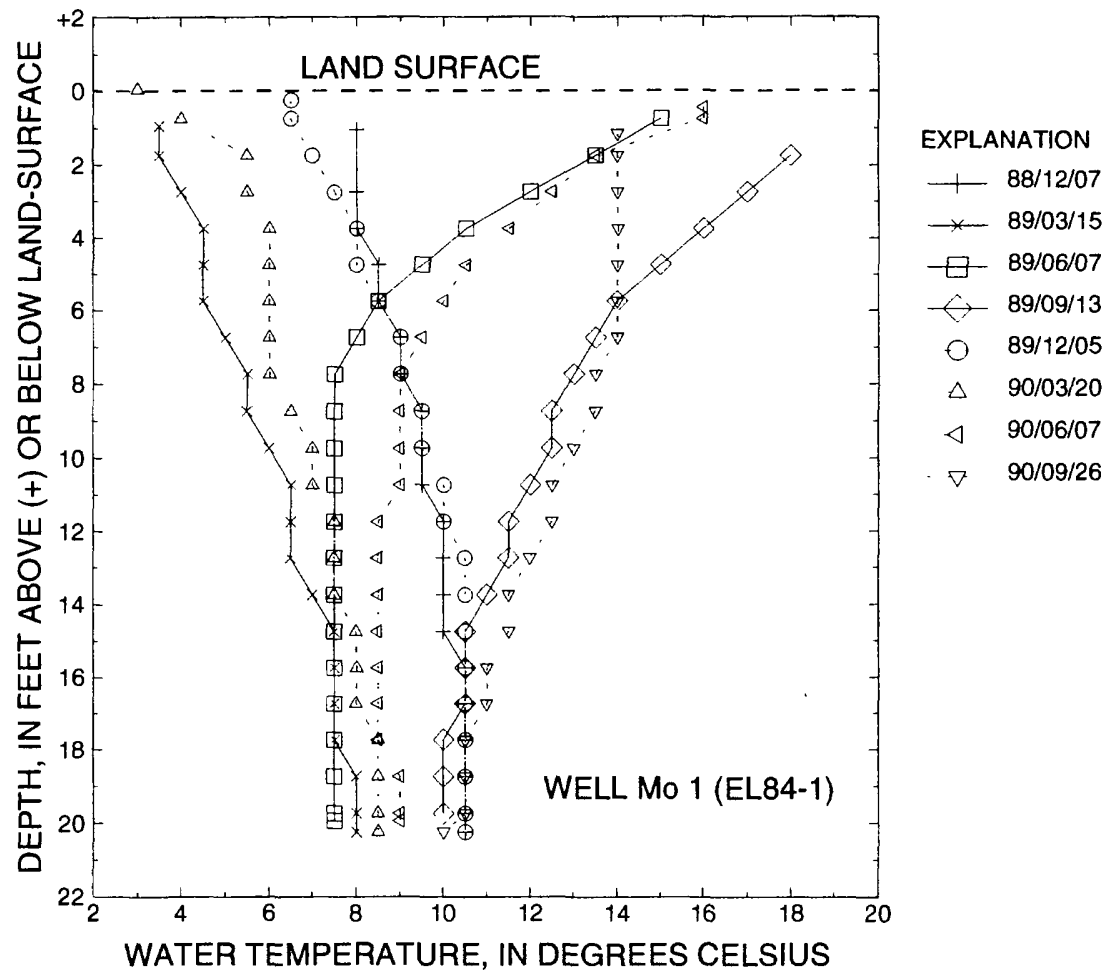




\section{GROUND-WATER LEVELS}

\section{Ellison park}

430855077304202. Local number Mo 2 (El 84-2)

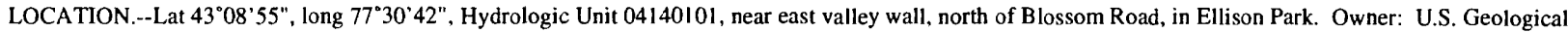
Survey.

AQUIFER.--Water-table aquifer in coarse sand and gravel of Pleistocene age.

WELL CHARACTERISTICS.--Drilled observation well, diameter 2 in, depth $45 \mathrm{ft}$, cased to $41 \mathrm{ft}$, screened 41 to $45 \mathrm{ft}$.

INSTRUMENTATION.--Monthly measurement with chalked tape by Monroe County Environmental Health Laboratory personnel and occasional measurements by USGS personnel.

DATUM.--Elevation of land-surface datum is $252.60 \mathrm{ft}$ above National Geodetic Vertical Datum of 1929. Measuring point: arrow at top of casing, $4.08 \mathrm{ft}$ above land-surface datum.

REMARKS.--This well is also a water-quality observation well. Water levels are monitored monthly and water samples taken quarterly by the Monree County Environmental Health Laboratory. Water-temperature profiles are also taken by MCEHL on a quarterly basis.

PERIOD OF RECORD.--September 1984 to current year.

EXTREMES FOR PERIOD SEPTEMBER 1984 TO SEPTEMBER 1993.--Highest water level measured, 1.24 feet above land-surface datum, April 26, 1991; lowest measured, 3.32 feet below land-surface datum, October 29, 1991.

WATER LEVEL, IN FEET ABOVE (+) OR BELOW LAND-SURFACE DATUM,

\begin{tabular}{|c|c|c|c|c|c|c|c|c|c|c|c|}
\hline \multicolumn{2}{|c|}{ DATE } & $\begin{array}{l}\text { WATER } \\
\text { LEVEL }\end{array}$ & \multicolumn{2}{|c|}{ DATE } & \multirow{2}{*}{$\begin{array}{l}\text { WATER } \\
\text { LEVEL }\end{array}$} & \multicolumn{2}{|c|}{ DATE } & \multirow{2}{*}{$\begin{array}{l}\text { WATER } \\
\text { LEVEL }\end{array}$} & \multicolumn{2}{|c|}{ DATE } & $\begin{array}{l}\text { WATER } \\
\text { LEVEL }\end{array}$ \\
\hline \multicolumn{10}{|c|}{ WATER YEAR OCTOBER 1988 TO SEPTEMBER 1989} & & \\
\hline DEC & 7 & 1.08 & MAR & 28 & 0.72 & JUN & 7 & 0.60 & SEP & 13 & 1.55 \\
\hline \multirow[t]{2}{*}{ MAR } & 15 & .95 & APR & 25 & .66 & JUL & 31 & 1.25 & & & \\
\hline & 22 & .79 & MAY & 26 & .30 & AUG & 31 & 1.47 & & & \\
\hline \multicolumn{12}{|c|}{ WATER YEAR OCTOBER 1989 TO SEPTEMBER 1990} \\
\hline \multirow[t]{2}{*}{ OCT } & 17 & 1.33 & JAN & 30 & 0.71 & MAY & 31 & .07 & SEP & 26 & 1.16 \\
\hline & 31 & 1.13 & MAR & 20 & +.06 & JUN & 7 & .37 & & & \\
\hline NOV & 30 & .20 & APR & 24 & +.23 & JUL & 25 & 1.09 & & & \\
\hline DEC & 5 & .20 & MAY & 24 & +0.61 & AUG & 28 & 1.5 & & & \\
\hline \multicolumn{12}{|c|}{ WATER YEAR OCTOBER 1990 TO SEPTEMBER 1991} \\
\hline OCT & 30 & 0.75 & JAN & 23 & +0.16 & APR & 26 & +1.24 & JUL & 26 & 1.20 \\
\hline NOV & 28 & .03 & FEB & 26 & .27 & MAY & 25 & .34 & AUG & 22 & 1.25 \\
\hline JAN & 9 & +.04 & APR & 3 & +.51 & JUN & 12 & .66 & SEP & 11 & 1.38 \\
\hline \multicolumn{12}{|c|}{ WATER YEAR OCTOBER 1991 TO SEPTEMBER 1992} \\
\hline OCT & 29 & 3.32 & JAN & 31 & 0.83 & JUN & 24 & 0.83 & SEP & 10 & 0.13 \\
\hline NOV & 27 & .34 & MAR & 18 & .69 & JUL & 21 & +.11 & & & \\
\hline DEC & 18 & .90 & MAY & 28 & .56 & AUG & 21 & .52 & & & \\
\hline \multicolumn{12}{|c|}{ WATER YEAR OCTOBER 1992 TO SEPTEMBER 1993} \\
\hline OCT & 28 & 0.48 & JAN & 27 & +0.28 & APR & 30 & +0.75 & JUL & 30 & 0.74 \\
\hline NOV & 24 & +60 & FEB & 26 & .43 & MAY & 27 & .17 & AUG & 27 & .96 \\
\hline DEC & 8 & .26 & MAR & 9 & .42 & JUN & 22 & .14 & SEP & 15 & .87 \\
\hline
\end{tabular}

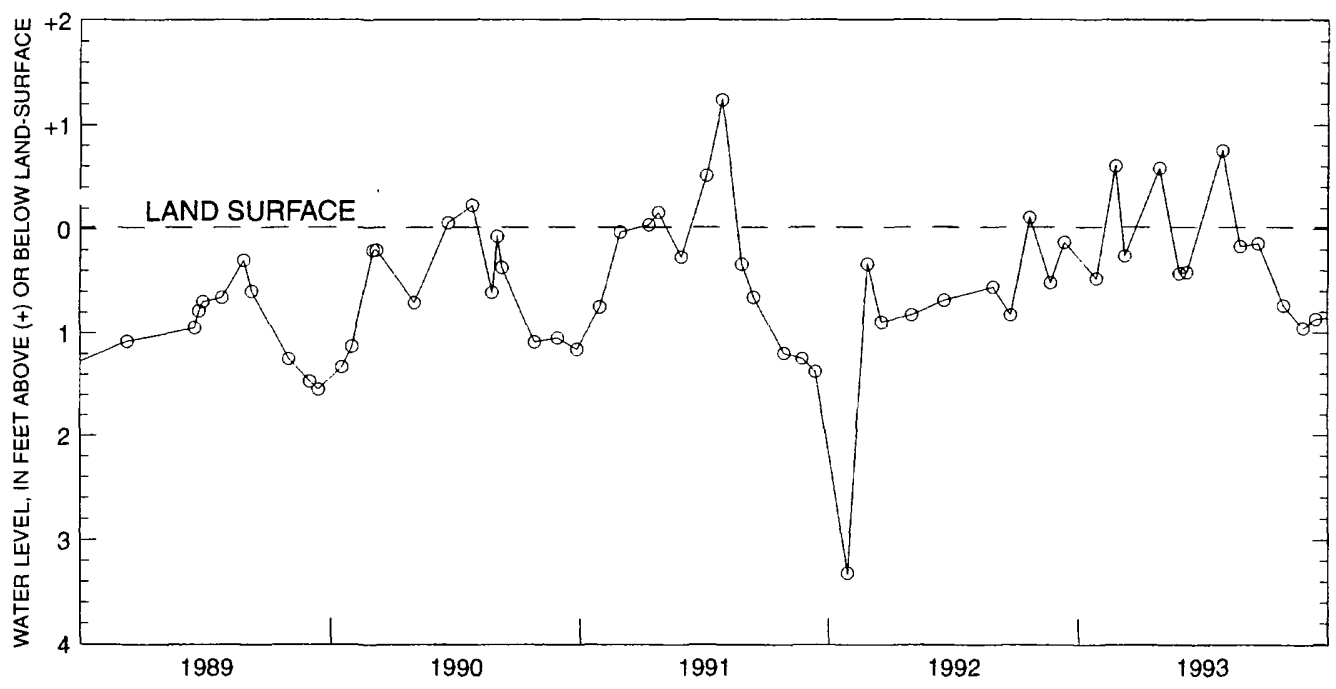


PERIOD OF RECORD.-- January 1986 to current year.

CHEMICAL DATA: 1986(a) 1987-93(b).

ORGANIC DATA: OC.--1986(a) 1987-93(b).

NUTRIENT DATA: 1986(a) 1987-93(b).

BIOLOGICAL DATA

Bacteria.-- 1986(a) 1987-93(b).

COOPERATION -- Water-quality samples were collected and analyzed by the Monroe County Environmental Health Laboratory at Rochester, NY.

\section{WATER QUALTYY DATA, WATER YEAR OCTOBER 1988 TO SEPTEMBER 1989}

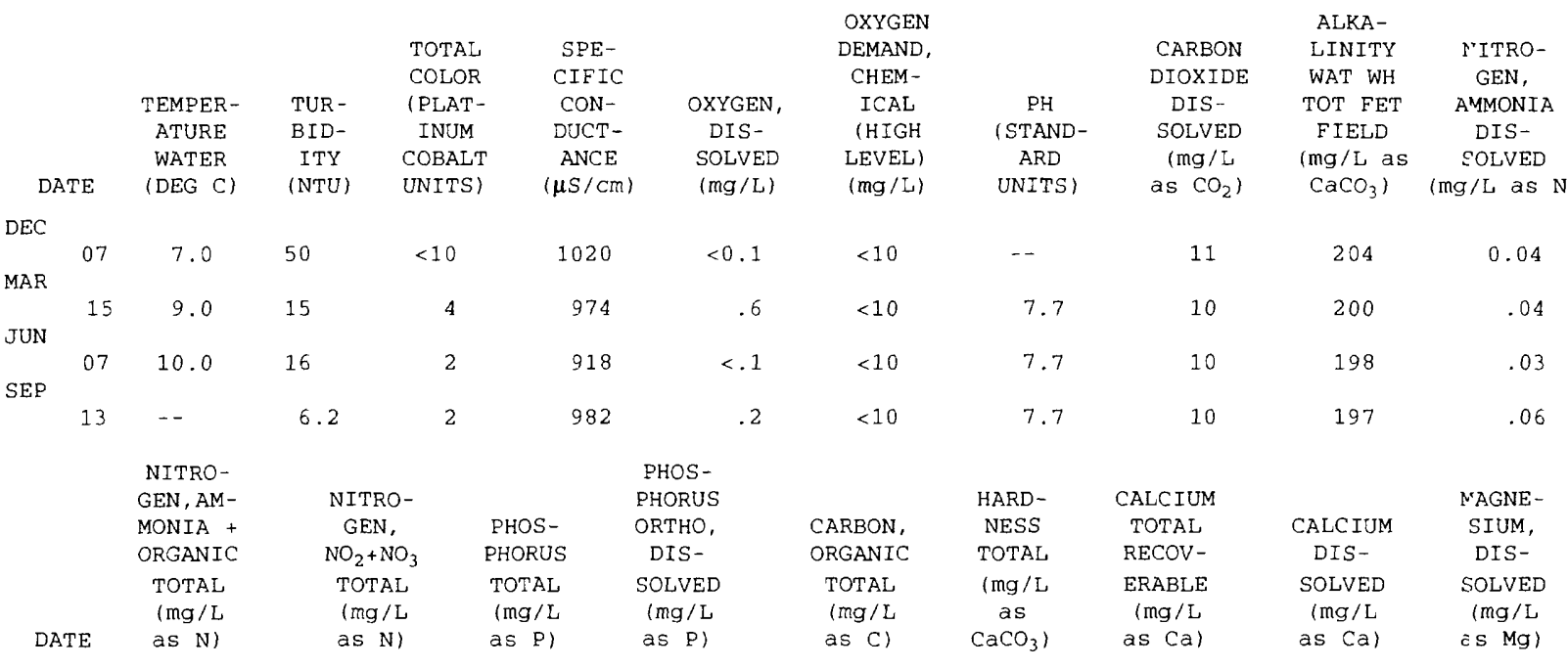

$$
\text { DEC }
$$

$\begin{array}{lrrrrr}\text { MAR } & 07 & 0.26 & 0.20 & 0.080 & 0.002 \\ & 15 & .48 & <.01 & .060 & <.002 \\ \text { JUN } & 07 & 44 & <.01 & .050 & .002 \\ & & & & & \\ \text { SEP } & 13 & .23 & <.01 & .040 & .003\end{array}$

$\begin{array}{rr}0.5 & 330 \\ 1.0 & 320 \\ .9 & 320 \\ 1.2 & 300\end{array}$

94

$$
-
$$

24

92

92

22

91

91

23

84

84

21

POTAS - CHLO-

$\begin{array}{ccc} & \text { POTAS- } & \text { CHLO- } \\ \text { SODIUM, } & \text { SIUM, } & \text { RIDE, } \\ \text { DIS- } & \text { DIS- } & \text { DIS- } \\ \text { SOLVED } & \text { SOLVED } & \text { SOLVED } \\ (\mathrm{mg} / \mathrm{L} & (\mathrm{mg} / \mathrm{L} & (\mathrm{mg} / \mathrm{L} \\ \text { as Na) } & \text { as } \mathrm{K}) & \text { as } \mathrm{Cl})\end{array}$

$\begin{array}{cc} & \\ \text { SULFATE } & \text { IRON, } \\ \text { DIS- } & \text { RETAL } \\ \text { SOLVED } & \text { ERABLE } \\ (\mathrm{mg} / \mathrm{L} & (\mu \mathrm{g} / \mathrm{L} \\ \left.\text { aS } \mathrm{SO}_{4}\right) & \text { aS } \mathrm{Fe})\end{array}$

COLI -

SOLIDS, SOLIDS,

FORM, RESIDUE SUM OF

FECAL, AT 180 CONSTI-

0.7 DEG. C TUENTS

UM-MF DIS- DIS -

(COLS.) SOLVED SOLVE?

$100 \mathrm{ml}) \quad(\mathrm{mg} / \mathrm{L}) \quad(\mathrm{mg} / \mathrm{L})$

$\begin{array}{lll}\text { DEC } & & 84 \\ & 07 & 79 \\ \text { MAR } & & \\ & 15 & 74 \\ \text { JUN } & & \\ & 07 & 88\end{array}$

$\begin{array}{ll}1.9 & 170 \\ 1.6 & 150 \\ 1.5 & 140 \\ 1.5 & 150\end{array}$

74
73
74
72

1800
890
780
440

$<1$
$<1$
$<2$
$<1$

619

573

533

524

536 


\section{GROUND-WATER LEVELS}

\section{Ellison park}

430855077304202. Local number Mo 2 (El 84-2)--continued

WATER QUALITY DATA, WATER YEAR OCTOBER 1989 TO SEPTEMBER 1990

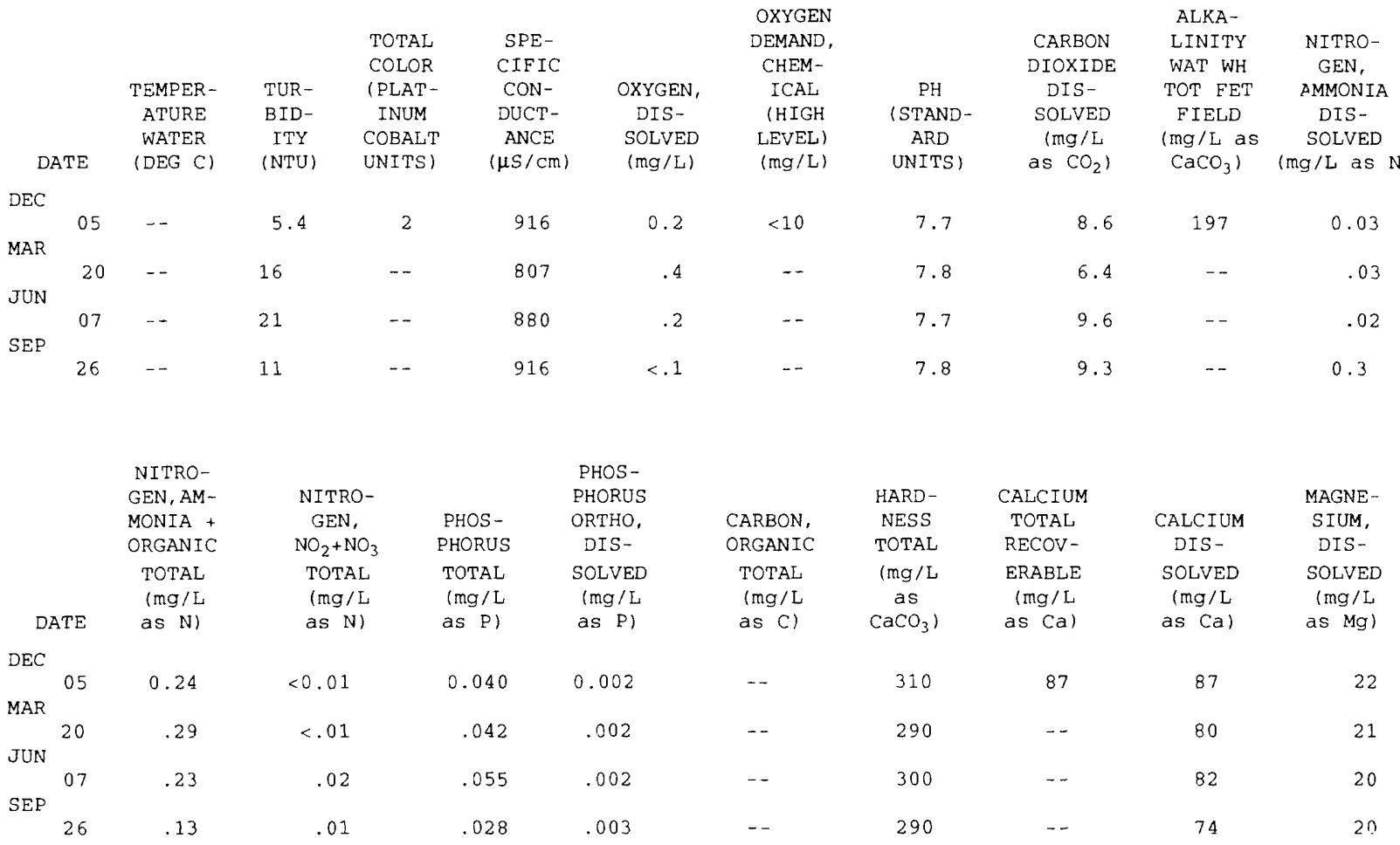

DATE DEC

MAR 05

JUN 20

07

26

$\begin{array}{cc}\text { POTAS- } & \text { CHLO- } \\ \text { SIUM, } & \text { RIDE, } \\ \text { DIS- } & \text { DIS- } \\ \text { SOLVED } & \text { SOLVED } \\ (\mathrm{mg} / \mathrm{L} & (\mathrm{mg} / \mathrm{L} \\ \text { as K) } & \text { as Cl) }\end{array}$

1.6

140

1.3

120

1.5

130

1.3

140

SULFATE
DIS-
SOLVED
$(\mathrm{mg} / \mathrm{L}$
as $\left.\mathrm{SO}_{4}\right)$

$$
\begin{aligned}
& \text { IRON, } \\
& \text { TOTAL } \\
& \text { RECOV- } \\
& \text { ERABLE } \\
& (\mu \mathrm{g} / \mathrm{L} \\
& \text { as Fe) }
\end{aligned}
$$

\section{COLI -}

FECAL RESIDUE

0.7

DEG.

DIS -

SOLVED

$100 \mathrm{ml}) \quad(\mathrm{mg} / \mathrm{L})$

340
630
820
470

$<2$

542

488

570

536 


\section{GROUND-WATER LEVELS}

\section{Ellison park}

\section{Local number Mo 2 (El 84-2)--continued}

\section{WATER QUALITY DATA, WATER YEAR OCTOBER 1990 TO SEPTEMBER 1991}

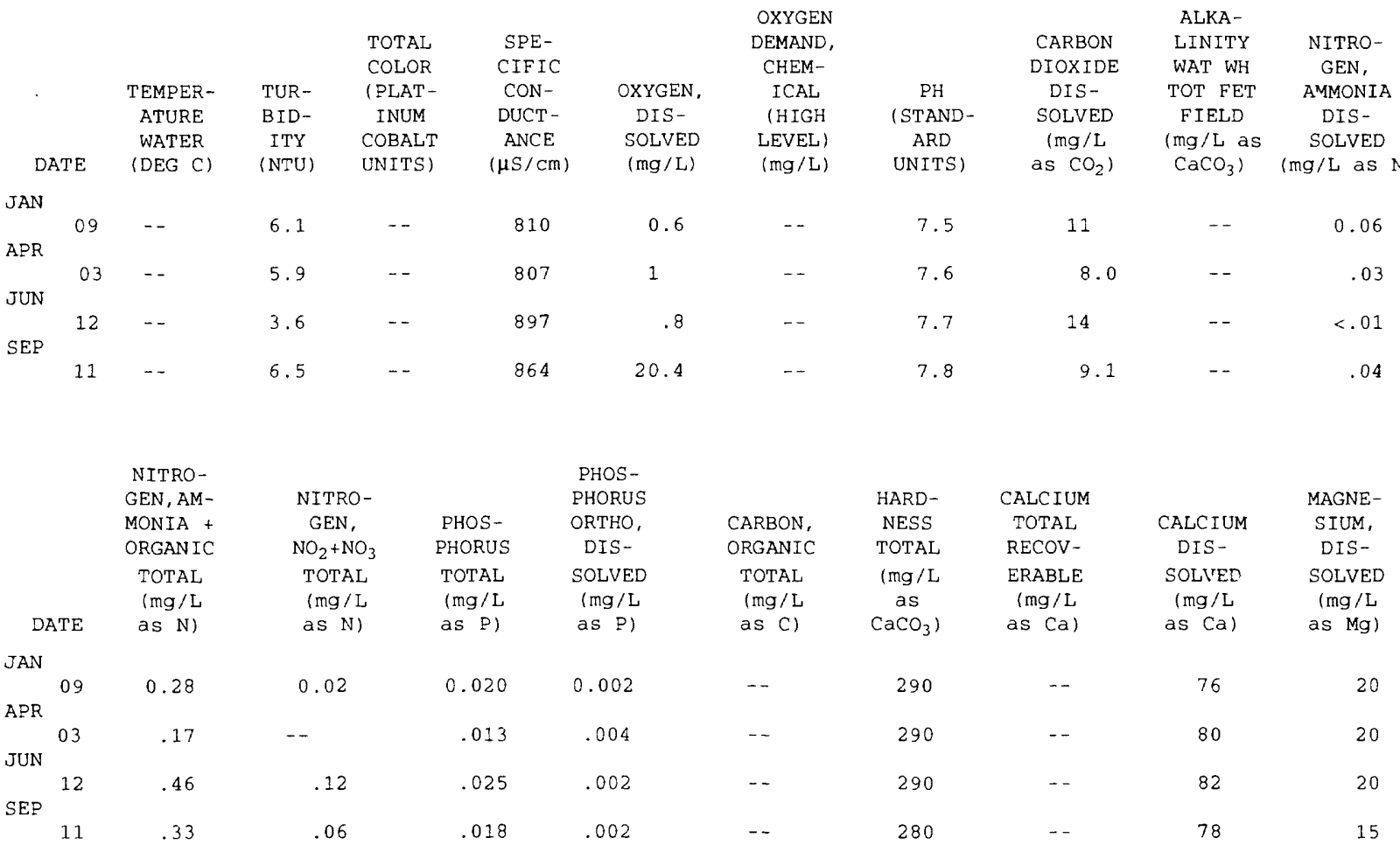

\begin{tabular}{|c|c|c|c|c|c|c|c|c|}
\hline DATE & $\begin{array}{c}\text { SODIUM, } \\
\text { DIS- } \\
\text { SOLVED } \\
\text { (mg/L } \\
\text { as Na) }\end{array}$ & $\begin{array}{l}\text { POTAS- } \\
\text { SIUM, } \\
\text { DIS- } \\
\text { SOLVED } \\
\text { (mg/L } \\
\text { as K) }\end{array}$ & $\begin{array}{l}\text { CHLO- } \\
\text { RIDE, } \\
\text { DIS- } \\
\text { SOLVED } \\
\text { (mg/L } \\
\text { as C1) }\end{array}$ & $\begin{array}{l}\text { SULFATE } \\
\text { DIS- } \\
\text { SOLVED } \\
\text { (mg/L } \\
\left.\text { as } \mathrm{SO}_{4}\right)\end{array}$ & $\begin{array}{c}\text { IRON, } \\
\text { TOTAL } \\
\text { RECOV- } \\
\text { ERABLE } \\
(\mu \mathrm{g} / \mathrm{L} \\
\text { as } \mathrm{Fe})\end{array}$ & $\begin{array}{c}\text { COLI- } \\
\text { FORM, } \\
\text { FECAL, } \\
0.7 \\
\text { UM-MF } \\
\text { (COLS.' } \\
100 \mathrm{~m} 1 \text { ) }\end{array}$ & $\begin{array}{l}\text { SOLIDS, } \\
\text { RESIDUE } \\
\text { AT } 180 \\
\text { DEG. C } \\
\text { DIS- } \\
\text { SOLVED } \\
(\mathrm{mg} / \mathrm{L})\end{array}$ & $\begin{array}{c}\text { SOLID3, } \\
\text { SUM CF } \\
\text { CONSTI- } \\
\text { TUENTS, } \\
\text { DIS- } \\
\text { SOLVED } \\
\left(\mathrm{mg} / \mathrm{L}_{1}\right)\end{array}$ \\
\hline JAN & & & & & & & & \\
\hline 09 & 62 & 1.6 & 110 & 68 & 340 & -- & - & 500 \\
\hline 03 & 58 & 1.5 & 110 & 77 & 330 & -- & -- & 468 \\
\hline $\begin{array}{ll}\text { JUN } & \\
& 12\end{array}$ & 78 & 1.6 & 150 & 68 & 400 & -- & - - & 538 \\
\hline 11 & 72 & 1.5 & 130 & 74 & 320 & - & -- & 516 \\
\hline
\end{tabular}


430855077304202. Local number Mo 2 (El 84-2)--continued

WATER QUALTYY DATA, WATER YEAR OCTOBER 1991 TO SEPTEMBER 1992

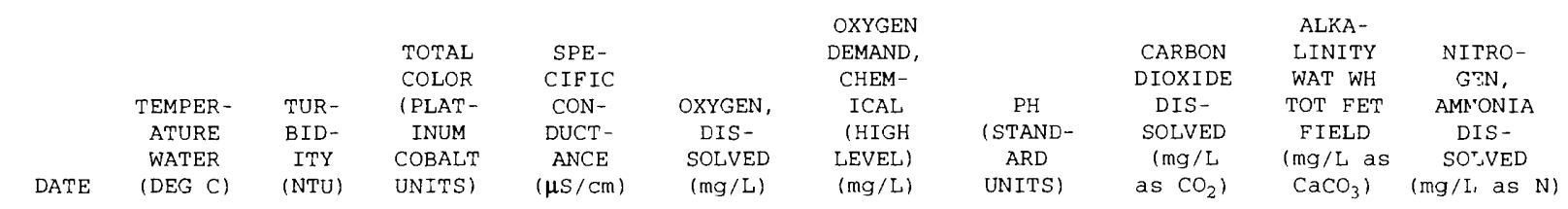

$$
\text { DEC }
$$

$\begin{array}{cccccc}\text { DEC } & 18 & -- & 4.6 & -- & 106 \\ \text { MAR } & 18 & -- & 4.9 & -- & 85 \\ \text { JUN } & 18 & -- & 7.3 & -- & 93 \\ \text { SEP } & 24 & - & 14 & -- & 89\end{array}$

1060

7.5
7.8
7.6
7.6

11

$$
9.2
$$$$
-
$$$$
0.06
$$$$
\begin{array}{lll}
2.8 & -- & 7.8
\end{array}
$$$$
10
$$$$
.03
$$$$
.2 \quad--
$$

9.6

$\begin{array}{lll}<.1 & - & 7.6\end{array}$

$--$

.04

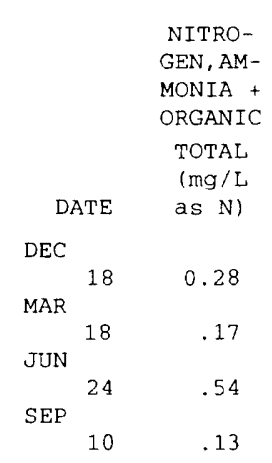

$\begin{array}{ccc} & & \text { PHOS- } \\ \text { NITRO- } & & \text { PHORUS } \\ \text { GEN, } & \text { PHOS- } & \text { ORTHO, } \\ \mathrm{NO}_{2}+\mathrm{NO}_{3} & \text { PHORUS } & \text { DIS- } \\ \mathrm{TOTAL} & \text { TOTAL } & \text { SOLVED } \\ (\mathrm{mg} / \mathrm{L} & \text { (mg/L } & \text { (mg/L } \\ \text { aS } \mathrm{N}) & \text { as P) } & \text { as P) }\end{array}$

$\begin{array}{ccc} & \text { HARD- } & \text { CALCIUM } \\ \text { CARBON, } & \text { NESS } & \text { TOTAL } \\ \text { ORGANIC } & \text { TOTAL } & \text { RECOV- } \\ \text { TOTAL } & (\mathrm{mg} / \mathrm{L} & \text { ERABLE } \\ (\mathrm{mg} / \mathrm{L} & \text { as } & (\mathrm{mg} / \mathrm{L} \\ \text { as C) } & \left.\mathrm{CaCO}_{3}\right) & \text { as } \mathrm{Ca})\end{array}$

$\begin{array}{cc} & \text { MAGNE- } \\ \text { CALCIUM } & \text { SIUM, } \\ \text { DIS- } & \text { DIS- } \\ \text { SOLVED } & \text { SO'NED } \\ \text { (mg/L } & \text { (mg/L } \\ \text { as Ca) } & \text { as Mg) }\end{array}$

$<0.05$
$<.05$
$<.05$
$<.05$

$\begin{array}{ll}0.018 & 0.004 \\ .025 & .003 \\ .030 & .002 \\ .045 & .006\end{array}$

$\begin{array}{ll}-- & 290 \\ -- & 300 \\ -- & 310 \\ -- & 300\end{array}$

--

\begin{tabular}{|c|c|c|c|c|c|c|c|c|}
\hline DATE & $\begin{array}{c}\text { SODIUM, } \\
\text { DIS- } \\
\text { SOLVED } \\
\text { (mg/L } \\
\text { as } \mathrm{Na} \text { ) }\end{array}$ & $\begin{array}{l}\text { POTAS- } \\
\text { SIUM, } \\
\text { DIS- } \\
\text { SOLVED } \\
\text { (mg/L } \\
\text { as } \mathrm{K} \text { ) }\end{array}$ & $\begin{array}{l}\text { CHLO- } \\
\text { RIDE, } \\
\text { DIS- } \\
\text { SOLVED } \\
\text { (mg/L } \\
\text { as Cl) }\end{array}$ & $\begin{array}{c}\text { SULFATE } \\
\text { DIS- } \\
\text { SOLVED } \\
(\mathrm{mg} / \mathrm{L} \\
\left.\text { as } \mathrm{SO}_{4}\right)\end{array}$ & $\begin{array}{c}\text { IRON, } \\
\text { TOTAL } \\
\text { RECOV- } \\
\text { ERABLE } \\
(\mu \mathrm{g} / \mathrm{L} \\
\text { as Fe) }\end{array}$ & $\begin{array}{c}\text { COLI - } \\
\text { FORM, } \\
\text { FECAL, } \\
0.7 \\
\text { UM-MF } \\
\text { (COLS., } \\
100 \mathrm{ml} \text { ) }\end{array}$ & $\begin{array}{l}\text { SOLIDS, } \\
\text { RES IDUE } \\
\text { AT } 180 \\
\text { DEG. C } \\
\text { DIS- } \\
\text { SOLVED } \\
(\mathrm{mg} / \mathrm{L})\end{array}$ & $\begin{array}{c}\text { SOLIDS, } \\
\text { SUM OF } \\
\text { CONSTI- } \\
\text { TUENTS, } \\
\text { DIS- } \\
\text { SOLVED } \\
(\mathrm{mg} / \mathrm{L})\end{array}$ \\
\hline DEC & & & & & & & & \\
\hline 18 & 77 & 1.7 & 140 & 80 & 290 & -- & -- & 555 \\
\hline JUN 18 & 66 & 2.1 & 130 & 74 & 200 & -- & -- & 491 \\
\hline SEP 24 & 75 & 1.6 & 140 & 72 & 460 & -. & -- & 544 \\
\hline 10 & 70 & 1.6 & 140 & 84 & 1600 & -. & -- & 544 \\
\hline
\end{tabular}

$\begin{array}{ll}81 & 20 \\ 82 & 22 \\ 88 & 22 \\ 84 & 21\end{array}$


430855077304202. Local number Mo 2 (El 84-2)--continued

WATER QUALITY DATA, WATER YEAR OCTOBER 1992 TO SEPTEMBER 1993

\begin{tabular}{|c|c|c|c|c|c|c|c|c|c|c|}
\hline DATE & $\begin{array}{c}\text { TEMPER- } \\
\text { ATURE } \\
\text { WATER } \\
(\text { DEG C) }\end{array}$ & $\begin{array}{l}\text { TUR- } \\
\text { BID- } \\
\text { ITY } \\
\text { (NTU) }\end{array}$ & $\begin{array}{l}\text { TOTAL } \\
\text { COLOR } \\
\text { (PLAT- } \\
\text { INUM } \\
\text { COBALT } \\
\text { UNITS) }\end{array}$ & $\begin{array}{c}\text { SPE- } \\
\text { CIFIC } \\
\text { CON- } \\
\text { DUCT- } \\
\text { ANCE } \\
(\mu \mathrm{S} / \mathrm{Cm})\end{array}$ & $\begin{array}{l}\text { OXYGEN, } \\
\text { DIS- } \\
\text { SOLVED } \\
\text { (mg/L) }\end{array}$ & $\begin{array}{c}\text { OXYGEN } \\
\text { DEMAND, } \\
\text { CHEM- } \\
\text { ICAL } \\
\text { (HIGH } \\
\text { LEVEL) } \\
\text { (mg/L) }\end{array}$ & $\begin{array}{c}\mathrm{PH} \\
\text { (STAND- } \\
\text { ARD } \\
\text { UNITS) }\end{array}$ & $\begin{array}{c}\text { CARBON } \\
\text { DIOXIDE } \\
\text { DIS- } \\
\text { SOLVED } \\
(\mathrm{mg} / \mathrm{L} \\
\left.\text { as } \mathrm{CO}_{2}\right)\end{array}$ & $\begin{array}{l}\text { ALKA- } \\
\text { LINITY } \\
\text { WAT WH } \\
\text { TOT FET } \\
\text { FIELD } \\
(\mathrm{mg} / \mathrm{L} \text { as } \\
\left.\mathrm{CaCO}_{3}\right)\end{array}$ & $\begin{array}{c}\text { NITRO- } \\
\text { GEN, } \\
\text { AMMONIA } \\
\text { DIS- } \\
\text { SOLVED } \\
(\mathrm{mg} / \mathrm{L} \text { as } \mathrm{N})\end{array}$ \\
\hline $\mathrm{DEC}$ & & & & & & & & & & \\
\hline MAR 08 & -- & 5.8 & -- & 875 & 0.7 & -- & 7.4 & 9.2 & -- & 0.04 \\
\hline JUN & -- & 14 & -- & 897 & 1.4 & -- & 7.5 & 11 & -- & .02 \\
\hline SEP $\quad 22$ & -- & 9.4 & -- & 907 & -- & -- & 7.8 & 8.3 & -- & .03 \\
\hline 16 & -- & 7.6 & -- & 887 & $<.1$ & - & 7.8 & 8.0 & -- & .04 \\
\hline
\end{tabular}

\begin{tabular}{|c|c|c|c|c|c|c|c|c|c|}
\hline & $\begin{array}{l}\text { NITRO- } \\
\text { GEN, AM- } \\
\text { MONIA + } \\
\text { ORGANIC }\end{array}$ & $\begin{array}{l}\text { NITRO- } \\
\text { GEN, } \\
\mathrm{NO}_{2}+\mathrm{NO}_{3}\end{array}$ & $\begin{array}{l}\text { PHOS- } \\
\text { PHORUS }\end{array}$ & $\begin{array}{l}\text { PHOS- } \\
\text { PHORUS } \\
\text { ORTHO, } \\
\text { DIS- }\end{array}$ & $\begin{array}{l}\text { CARBON, } \\
\text { ORGANIC }\end{array}$ & $\begin{array}{l}\text { HARD- } \\
\text { NESS } \\
\text { TOTAL }\end{array}$ & $\begin{array}{l}\text { CALCIUM } \\
\text { TOTAL } \\
\text { RECOV- }\end{array}$ & $\begin{array}{l}\text { CALC IUM } \\
\text { DIS- }\end{array}$ & $\begin{array}{l}\text { MAGNE- } \\
\text { SIUM, } \\
\text { DIS- }\end{array}$ \\
\hline & $\begin{array}{l}\text { TOTAL } \\
(\mathrm{mg} / \mathrm{L}\end{array}$ & $\begin{array}{l}\text { TOTAL } \\
\text { /mg/L }\end{array}$ & $\begin{array}{l}\text { TOTAL } \\
\text { (mg/L }\end{array}$ & $\begin{array}{c}\text { SOLVED } \\
\text { (mg/L }\end{array}$ & $\begin{array}{l}\text { TOTAL } \\
\text { (mg/L }\end{array}$ & $\begin{array}{c}(\mathrm{mg} / \mathrm{L} \\
\mathrm{as}\end{array}$ & $\begin{array}{c}\text { ERABLE } \\
\text { (mg/L }\end{array}$ & $\begin{array}{l}\text { SOLVED } \\
(\mathrm{mg} / \mathrm{L}\end{array}$ & $\begin{array}{c}\text { SOLVED } \\
\text { (mg / L }\end{array}$ \\
\hline DATE & as N) & as $\mathrm{N}$ ) & as P) & as P) & as C) & $\left(\mathrm{CaCO}_{3}\right)$ & as (a) & as $\mathrm{Ca}$ & as $\mathrm{Mg}$ ) \\
\hline & & & & & & & & & \\
\hline 08 & 0.17 & $<0.05$ & 0.015 & 0.003 & -- & 300 & - & 85 & 22 \\
\hline 09 & .20 & .05 & .025 & .002 & -- & 310 & -- & 84 & 22 \\
\hline 22 & .32 & $<.05$ & .063 & .003 & - & 300 & - & 90 & 23 \\
\hline 16 & .43 & $<.05$ & .025 & .007 & -- & 300 & -- & 85 & 20 \\
\hline
\end{tabular}

DATE

$\mathrm{DEC}$

MAR 08

JUN 09

SEP 22

$\begin{array}{ccc} & & \\ & \text { POTAS- } & \text { CHLO- } \\ \text { SODIUM, } & \text { SIUM, } & \text { RIDE, } \\ \text { DIS- } & \text { DIS- } & \text { DIS- } \\ \text { SOLVED } & \text { SOLVED } & \text { SOLVED } \\ (\mathrm{mg} / \mathrm{L} & (\mathrm{mg} / \mathrm{L} & (\mathrm{mg} / \mathrm{L} \\ \text { aS } \mathrm{Na}) & \mathrm{aS} \mathrm{K}) & \text { as } \mathrm{C} 1)\end{array}$

$\begin{array}{cc} & \\ & \text { IRON, } \\ \text { SULFATE } & \text { TOTAL } \\ \text { DIS- } & \text { RECOV- } \\ \text { SOLVED } & \text { ERABLE } \\ (\mathrm{mg} / \mathrm{L} & (\mu \mathrm{g} / \mathrm{L} \\ \left.\text { as } \mathrm{SO}_{4}\right) & \text { as } \mathrm{Fe})\end{array}$

100

$\begin{array}{lll}85 & 400 & - \\ 81 & 810 & -- \\ 81 & 760 & -- \\ -- & 480 & --\end{array}$

SOLIDS, RESIDUE AT 180 DEG. C DISSOLVED ( $\mathrm{mg} / \mathrm{L}$ )
SOLIDE SUM OT CONSTITUENTE, DISSOLVE? (mg/L)

61
61
80
75

$\begin{array}{ll}1.4 & 130 \\ 1.3 & 140 \\ 1.4 & 140 \\ 1.2 & 140\end{array}$

140

140

480

$\begin{array}{lr}- & 553 \\ -- & -- \\ - & 580 \\ - & 520\end{array}$


430855077304202. Local number Mo 2 (EI 84-2)--continued

WATER TEMPERATURE, IN DEGREES CELSIUS

\begin{tabular}{|c|c|c|c|c|c|c|c|c|c|c|c|c|c|c|c|c|c|c|c|c|}
\hline \multirow[b]{2}{*}{$\begin{array}{l}\text { Depth, } \\
\text { in feet }\end{array}$} & \multicolumn{4}{|c|}{$1989 \mathrm{WY}$} & \multicolumn{4}{|c|}{$1990 \mathrm{WY}$} & \multicolumn{4}{|c|}{$1991 \mathrm{WY}$} & \multicolumn{4}{|c|}{$1992 \mathrm{WY}$} & \multicolumn{4}{|c|}{$1993 \mathrm{WY}$} \\
\hline & $\begin{array}{c}\text { Dec } \\
7 \\
\end{array}$ & $\begin{array}{c}\text { Mar } \\
15\end{array}$ & $\begin{array}{c}\text { Jun } \\
7 \\
\end{array}$ & $\begin{array}{c}\text { Sep } \\
13 \\
\end{array}$ & $\begin{array}{c}\text { Dec } \\
5 \\
\end{array}$ & $\begin{array}{c}\text { Mar } \\
20 \\
\end{array}$ & $\begin{array}{c}\text { Jun } \\
7 \\
\end{array}$ & $\begin{array}{c}\text { Sep } \\
26\end{array}$ & $\begin{array}{c}\text { Jan } \\
9 \\
\end{array}$ & $\begin{array}{c}\text { Apr } \\
3 \\
\end{array}$ & $\begin{array}{c}\text { Jun } \\
12 \\
\end{array}$ & $\begin{array}{c}\text { Sep } \\
11\end{array}$ & $\begin{array}{c}\text { Dec } \\
18 \\
\end{array}$ & $\begin{array}{c}\text { Mar } \\
18 \\
\end{array}$ & $\begin{array}{c}\text { Jun } \\
24 \\
\end{array}$ & $\begin{array}{c}\text { Sep } \\
10 \\
\end{array}$ & $\begin{array}{c}\text { Dec } \\
8 \\
\end{array}$ & $\begin{array}{c}\text { Mar } \\
9 \\
\end{array}$ & $\begin{array}{c}\text { Jun } \\
22 \\
\end{array}$ & $\begin{array}{c}\text { Sep } \\
15 \\
\end{array}$ \\
\hline-0.48 & -- & 10.0 & 9.5 & 9.5 & 9.0 & 10.5 & 10.0 & 10.0 & 10.0 & 10.5 & 10.0 & 10.0 & 10.0 & 10.5 & - & 10.0 & 7.0 & -- & 10.5 & -- \\
\hline .42 & -- & 10.0 & 9.5 & 9.5 & 9.0 & 10.5 & 10.5 & 10.0 & 10.0 & 10.5 & 10.0 & 10.0 & 10.5 & 10.5 & 9.0 & 10.0 & 7.0 & 10.5 & 10.5 & 9.5 \\
\hline 1.12 & 8.0 & -- & -- & 9.5 & -- & -- & -- & 14.5 & 10.0 & -- & -- & -- & -- & -- & -- & -- & -- & -- & 10.5 & 9.5 \\
\hline 1.22 & -- & - & -- & -- & $-\infty$ & -- & 10.5 & - & - & -- & -- & -- & -- & 10.5 & - & -- & -- & -- & 17.5 & -- \\
\hline 1.32 & -- & 10.0 & 9.5 & -- & -- & 10.5 & -- & 10.5 & -- & 10.5 & 10.0 & 10.5 & 10.5 & -- & 9.0 & -- & & -- & -- & - \\
\hline 1.42 & -- & -- & -- & -- & -- & -- & -- & -- & -- & -- & -- & 19.0 & -- & -- & -- & 10.0 & 7.0 & -- & - & -- \\
\hline 1.92 & 8.0 & 4.0 & 14.0 & 16.5 & 7.0 & 5.5 & 12.5 & 14.0 & 4.5 & 6.0 & 16.5 & 18.0 & 7.0 & 5.5 & 13.5 & 10.5 & -- & 4.5 & 15.0 & 17.0 \\
\hline 2.92 & -- & -- & -- & -- & - & -- & 11.5 & 14.0 & -- & - & 15.0 & -- & 8.0 & 5.5 & 13.0 & -- & 8.0 & 5.0 & 13.0 & 16.5 \\
\hline 3.92 & 8.5 & 4.5 & 11.0 & 15.5 & 8.0 & 5.5 & 11.0 & 14.0 & 6.0 & 6.0 & 13.5 & 16. & 8.0 & 5.5 & 12.5 & 15.0 & 8.0 & 5.0 & 2.0 & 6.0 \\
\hline 4.92 & -- & -- & - & -- & -- & -- & -- & 14.0 & -- & -- & 13.0 & -- & 8.5 & 5.5 & 12.0 & -- & 8.0 & 5.0 & 11.0 & \\
\hline 5.92 & 8.5 & 4.5 & 9.0 & 13.5 & 8.5 & 6.0 & 9.5 & 14.0 & 7.0 & 6.5 & 12.0 & 15.5 & 8.5 & 5.5 & 11.0 & 14.0 & 8.0 & 5.5 & 10.0 & \\
\hline 6.92 & -- & - & -- & -- & -- & -- & -- & & -- & -- & 11.5 & -- & & 5.5 & 10.5 & -- & 8.5 & & 9.5 & \\
\hline 7.92 & 9.0 & 5.5 & 8.0 & 13.0 & 9.0 & 6.0 & 9.0 & 13.5 & 7.5 & 6.5 & 10.5 & 14.5 & & 6.5 & 10.5 & & 8.5 & & 9.0 & \\
\hline 8.92 & $\ldots$ & -- & -- & -- & -- & -- & -- & 13.5 & -- & -- & 10.5 & -- & 9.5 & 6.5 & 10.5 & -- & 9.0 & 6.0 & 9.0 & \\
\hline 9.92 & 9.5 & 6.0 & 8.0 & 12.0 & 9.5 & 6.5 & 9.0 & 13.0 & 8.0 & 7.0 & 9.5 & & 9.5 & 7.0 & 10.0 & & 9.0 & 6.5 & 8.5 & 13.0 \\
\hline 10.92 & -- & -- & -- & -- & -- & -- & -- & 12.5 & - & -- & 9.5 & -- & .0 & 7.0 & 9.5 & -- & 5 & 7.0 & 8.5 & 12.5 \\
\hline & 10.0 & 6.5 & 8.0 & 11.5 & 10.0 & 7.0 & 9.0 & & 8.5 & 7.5 & 9.0 & & & 7.5 & 9.5 & & 5 & 7.0 & 8.5 & 12.0 \\
\hline & -- & -- & -- & -- & -- & -- & -- & 12.0 & $-\cdots$ & -- & 9.0 & -- & & 8.0 & 9.0 & -- & 0 & 7.5 & 8.0 & 11.5 \\
\hline 13.92 & 10.0 & 7.0 & 8.0 & 11.0 & 10.0 & 8.0 & 9.0 & 11.5 & 9.0 & 7.5 & 9.0 & 12.0 & .5 & 8.0 & 9.0 & & & 7.5 & 8.0 & 11.5 \\
\hline 14.92 & -- & -- & -- & -- & -- & -- & -- & 11.5 & -- & -- & 9.0 & -- & 10.5 & 8.5 & 9.0 & -- & 10.0 & 8.0 & 8.0 & 11.0 \\
\hline 15.92 & 10.0 & 7. & 8.0 & 10.5 & 10.5 & 8.5 & 9.0 & 11.0 & 9.5 & 8.0 & 9.0 & 11.5 & 10.5 & 8.5 & 9.0 & 10.5 & 10.5 & 8.0 & 8.0 & 11.0 \\
\hline 16.92 & -- & -- & $\cdots$ & -- & -- & -- & -- & 11.0 & -- & - & 9.0 & -- & 10.5 & 8.5 & 9.0 & $\ldots$ & 10.5 & 8.5 & $\ldots$ & -- \\
\hline 17.92 & 10.5 & 8.0 & 8.0 & 10.0 & 10.5 & 8.5 & 9.0 & 10.5 & 10.0 & 8.5 & 9.0 & 11.0 & 10.5 & 9.0 & 9.0 & 10.5 & 10.5 & 8.5 & 8.5 & 10. \\
\hline 18.92 & -- & -- & -- & -- & -- & -- & -- & 10.5 & - & -- & -- & -- & -- & 9.0 & 9.0 & -- & 10.5 & 9.0 & -- & -- \\
\hline 19.92 & 10.5 & 8.5 & 8.0 & 10.0 & 10.5 & 9.0 & 9.0 & 10.5 & 10.0 & 9.0 & 9.0 & 10.5 & 10.5 & 9.5 & 9.0 & 10.0 & 10.5 & 9.0 & 8.5 & 10. \\
\hline 20.92 & -- & -- & -- & -- & - & -- & -- & 10.0 & -- & -- & -- & -- & -- & 9.5 & 9.0 & -- & 10.5 & 9.0 & -- & -- \\
\hline 21.92 & 10.5 & 9.0 & 8.5 & 8.5 & 10.5 & 9.5 & 9.0 & 10.0 & 10.0 & 9.0 & 9.5 & 10.5 & 11.0 & 9.5 & 9.5 & 10. & -- & 9.5 & 8.5 & 10. \\
\hline 22.92 & -- & -- & -- & -- & -- & -- & -- & 10.0 & -- & -- & -- & -- & -- & 9.5 & -- & -- & 10.5 & 9.5 & -- & -- \\
\hline 23.92 & 10.5 & 9.0 & 8.5 & 8.5 & 10.5 & 9.5 & 9.5 & 10.0 & 10.0 & 9.5 & 9.5 & 10.0 & 10.5 & 9.5 & 9.5 & 10. & -- & 10.0 & 8.5 & 10. \\
\hline .92 & -- & -- & -- & $\cdots$ & -- & -- & - & 10.0 & $\ldots$ & -- & - & -- & -- & 9.5 & -- & -- & 10.5 & 10.0 & - & -- \\
\hline 25.92 & 10.0 & 9.5 & 9.0 & 8.5 & 10.5 & 10.0 & 9.5 & 10.0 & 10.5 & 9.5 & 9.5 & 10.0 & 10.5 & 10.0 & 9.5 & 9. & -- & 10.0 & 9.0 & 10. \\
\hline 26.92 & -- & -- & -- & -- & -- & $-\cdots$ & -- & 10.0 & -- & -- & -- & -- & -- & 10.0 & -- & -- & 10.5 & -- & -- & -- \\
\hline 27.92 & 10.0 & 9.5 & 9.0 & 8.5 & 10.5 & 10.0 & 9.5 & 10.0 & 10.5 & 9.5 & 10.0 & 10.0 & 10.5 & 9.5 & 9.5 & 8.0 & -- & 10.0 & 9.0 & 10.0 \\
\hline 29.92 & 10.0 & 9.5 & 9.0 & 8.5 & 10.5 & 10.0 & 10.0 & 10.0 & 10.5 & 10.0 & 10.0 & 10.0 & 10.5 & -- & 9.5 & 7.5 & 10.5 & 10.0 & 9.0 & 10.0 \\
\hline 30.92 & -- & -- & -- & -- & - & -- & - & - & -- & -- & -- & -- & -- & 9.5 & -- & -- & 10.5 & -- & -- & -- \\
\hline 31.92 & 10.0 & 9.5 & 9.0 & 9.0 & 10.5 & 10.0 & 10.0 & 10.0 & 10.5 & 10.0 & 10.0 & 10.0 & 10.5 & -- & 10.0 & 7.5 & -- & 10.0 & 9.0 & 10.0 \\
\hline 33.92 & 10.0 & 9.5 & 9.5 & 9.0 & 10.0 & 10.0 & 10.0 & 10.0 & 10.5 & 10.0 & 10.0 & 10.0 & 10.5 & -- & 10.0 & 7.0 & 10.5 & 10.5 & 9.5 & 10.0 \\
\hline 34.92 & -- & -- & - & - & -- & - & -- & -- & -- & -- & - & -- & - & 9.5 & - & -- & 10.5 & $\ldots$ & $\ldots$ & -- \\
\hline 35.92 & 10.0 & 9.5 & 9.5 & 9.0 & 10.0 & 10.0 & 10.0 & 10.0 & 10.5 & 10.0 & 10.0 & 10.0 & 10.5 & -- & 10.0 & 7.0 & -- & 10.5 & 9.5 & 10.0 \\
\hline 36.92 & -- & -- & -- & -- & - & -- & $\cdots$ & -- & -- & - & - & - & -- & 9.5 & -- & -- & 10.5 & -- & -- & -- \\
\hline 37.92 & 10.0 & 9.5 & 9.5 & 9.0 & 10.5 & 10.0 & 10.0 & 10.0 & 10.5 & 10.0 & 10.0 & 10.0 & 10.5 & -- & 10.0 & 7.0 & -- & 10.5 & 9.5 & 10.0 \\
\hline 38.92 & -- & -- & $\cdots$ & $\ldots$ & -- & -- & -- & -- & -- & -- & -- & -- & -- & 9.0 & - & -- & 10.5 & 10.5 & -- & -- \\
\hline 39.92 & 10.0 & 9.5 & 9.5 & 9.0 & 10.5 & 10.5 & 10.0 & 10.0 & 10.5 & 10.0 & 10.0 & 10.5 & 10.5 & -- & 10.0 & 7.0 & -- & 10.5 & 9.5 & 10.0 \\
\hline 41.72 & 10.0 & 9.5 & 9.5 & -- & 10.5 & 10.5 & 10.5 & 10.0 & 10.5 & 10.0 & 10.5 & 10.5 & 10.5 & 9.0 & 10.0 & 7.0 & $\ldots$ & 10.5 & 9.5 & -- \\
\hline
\end{tabular}




\section{GROUND-WATER LEVELS}

\section{Ellison park}

430855077304202. Local number Mo 2 (El 84-2)--continued

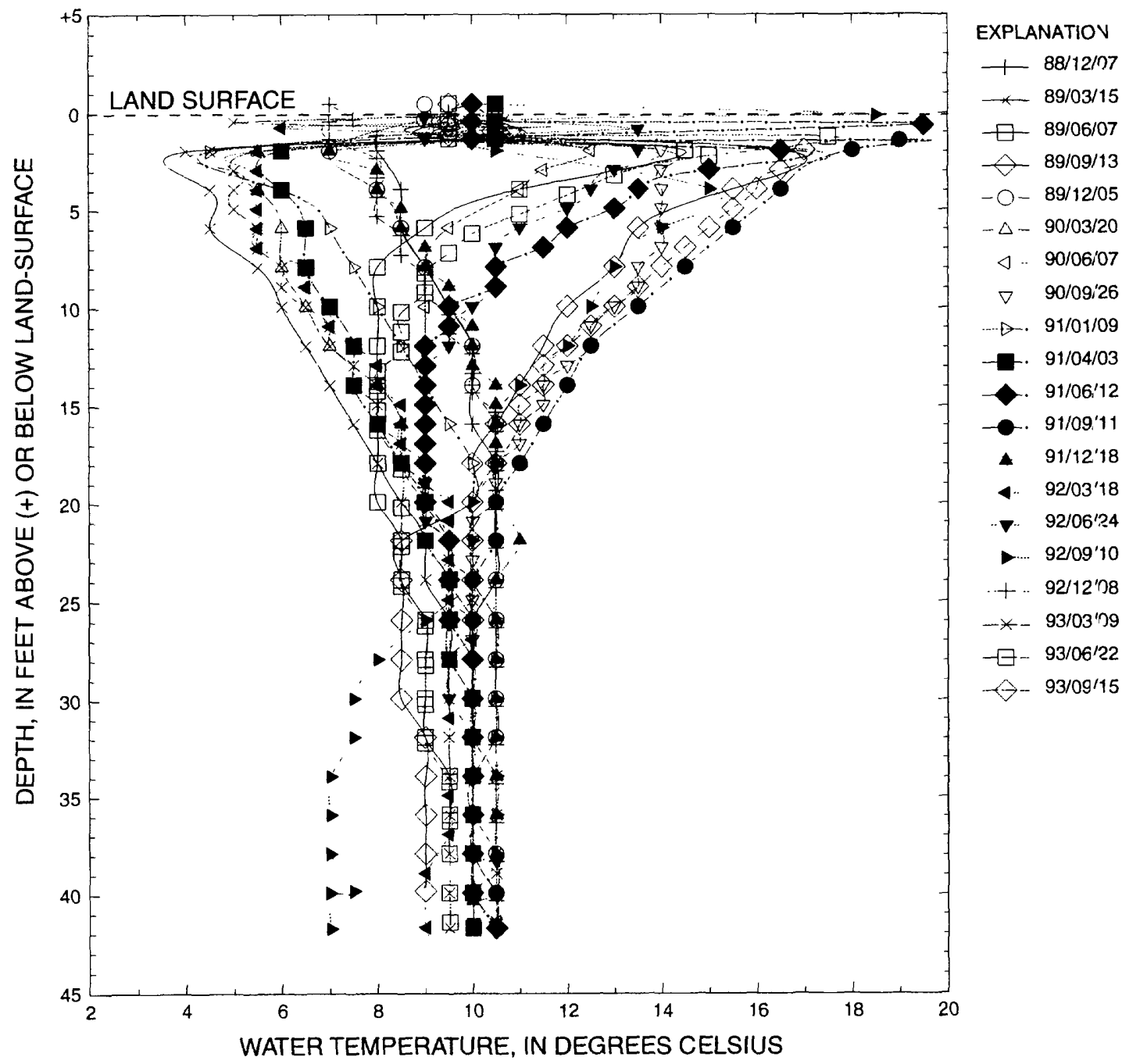




\section{GROUND-WATER LEVELS}

Ellison park

430854077304601. Local number Mo 3 (El 84-3)

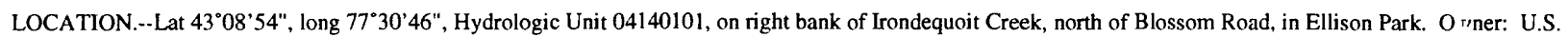
Geological Survey.

AQUIFER.--Water-table aquifer in alluvium of Holocene age.

WELL CHARACTERISTICS.--Drilled observation well, diameter 2 in, depth $16 \mathrm{ft}$, cased to $13.5 \mathrm{ft}$, screened $13.5 \mathrm{ft}$ to $16 \mathrm{ft}$.

INSTRUMENTATION.--Monthly measurement with chalked tape by Monroe County Environmental Health Laboratory personnel and occasional measurement by USGS personnel.

DATUM.--Elevation of land-surface datum is $253.2 \mathrm{ft}$ above National Geodetic Vertical Datum of 1929. Measuring point: arrow at top of casing, $3.74 \mathrm{ft}$ above landsurface datum.

REMARKS.--Water level affected by stage of Irondequoit Creek. This well is also a water-quality observation well. Water levels are monitored monthly and water samples taken quarterly by the Monroe County Environmental Health Laboratory. Water-temperature profiles are also taken by MCEHL on a quarterly basis.

PERIOD OF RECORD.--September 1984 to current year.

EXTREMES FOR PERIOD SEPTEMBER 1984 TO SEPTEMBER 1993.--Highest water level measured, 2.03 feet above land-surface datum, February 27, 1985; lowest measured, 4.11 feet below land-surface datum, September 13, 1989.

WATER LEVEL, IN FEET BELOW LAND-SURFACE DATUM,

\begin{tabular}{|c|c|c|c|c|c|c|c|c|c|c|c|}
\hline \multicolumn{3}{|r|}{$\begin{array}{l}\text { WATER } \\
\text { LEVEL }\end{array}$} & \multicolumn{2}{|c|}{ DATE } & $\begin{array}{l}\text { WATER } \\
\text { LEVEL } \\
\end{array}$ & \multicolumn{2}{|c|}{ DATE } & $\begin{array}{l}\text { WATER } \\
\text { LEVEL }\end{array}$ & \multicolumn{2}{|c|}{ DATE } & $\begin{array}{l}\text { WATER } \\
\text { LEVEL }\end{array}$ \\
\hline \multicolumn{12}{|c|}{ WATER YEAR OCTOBER 1988 TO SEPTEMBER 1989} \\
\hline DEC & 7 & 3.63 & MAR & 28 & 2.81 & JUN & 7 & 3.12 & SEP & 13 & 4.11 \\
\hline \multirow[t]{2}{*}{ MAR } & 15 & 3.00 & APR & 25 & 3.24 & JUL & 31 & 3.87 & & & \\
\hline & 22 & 2.69 & MAY & 26 & 2.64 & AUG & 31 & 4.07 & & & \\
\hline \multicolumn{12}{|c|}{ WATER YEAR OCTOBER 1989 TO SEPTEMBER 1990} \\
\hline \multirow[t]{2}{*}{ OCT } & 17 & 3.52 & JAN & 30 & 3.09 & MAY & 24 & 1.82 & AUG & 28 & 3.66 \\
\hline & 31 & 3.62 & FEB & 28 & 2.09 & & 31 & 2.65 & SEP & 26 & 3.57 \\
\hline NOV & 30 & 3.16 & MAR & 20 & 2.00 & JUN & 7 & 3.01 & & & \\
\hline DEC & 5 & 3.17 & APR & 24 & 2.31 & JUL & 25 & 3.65 & & & \\
\hline \multicolumn{12}{|c|}{ WATER YEAR OCTOBER 1990 TO SEPTEMBER 1991} \\
\hline OCT & 30 & 3.11 & JAN & 23 & 1.93 & APR & 26 & 1.01 & SEP & 11 & 3.85 \\
\hline NOV & 28 & 2.59 & FEB & 26 & 2.62 & MAY & 25 & 2.73 & & & \\
\hline JAN & 9 & 2.38 & APR & 3 & 1.87 & JUN & 12 & 3.12 & & & \\
\hline \multicolumn{12}{|c|}{ WATER YEAR OCTOBER 1991 TO SEPTEMBER 1992} \\
\hline NOV & 27 & 3.15 & MAR & 18 & 2.84 & JUN & 24 & 3.38 & AUG & 21 & 3.03 \\
\hline DEC & 18 & 3.40 & MAY & 28 & 3.00 & JUL & 21 & 2.09 & SEP & 10 & 2.74 \\
\hline JAN & 31 & 3.18 & & & & & & & & & \\
\hline \multicolumn{12}{|c|}{ WATER YEAR OCTOBER 1992 TO SEPTEMBER 1993} \\
\hline OCT & 28 & 2.74 & JAN & 27 & 1.48 & APR & 30 & 1.73 & JUL & 30 & 3.41 \\
\hline NOV & 24 & 1.49 & FEB & 26 & 2.72 & MAY & 27 & 2.81 & AUG & 27 & 3.63 \\
\hline DEC & 8 & 2.64 & MAR & 9 & 2.21 & JUL & 7 & 2.61 & SEP & 15 & 3.42 \\
\hline
\end{tabular}

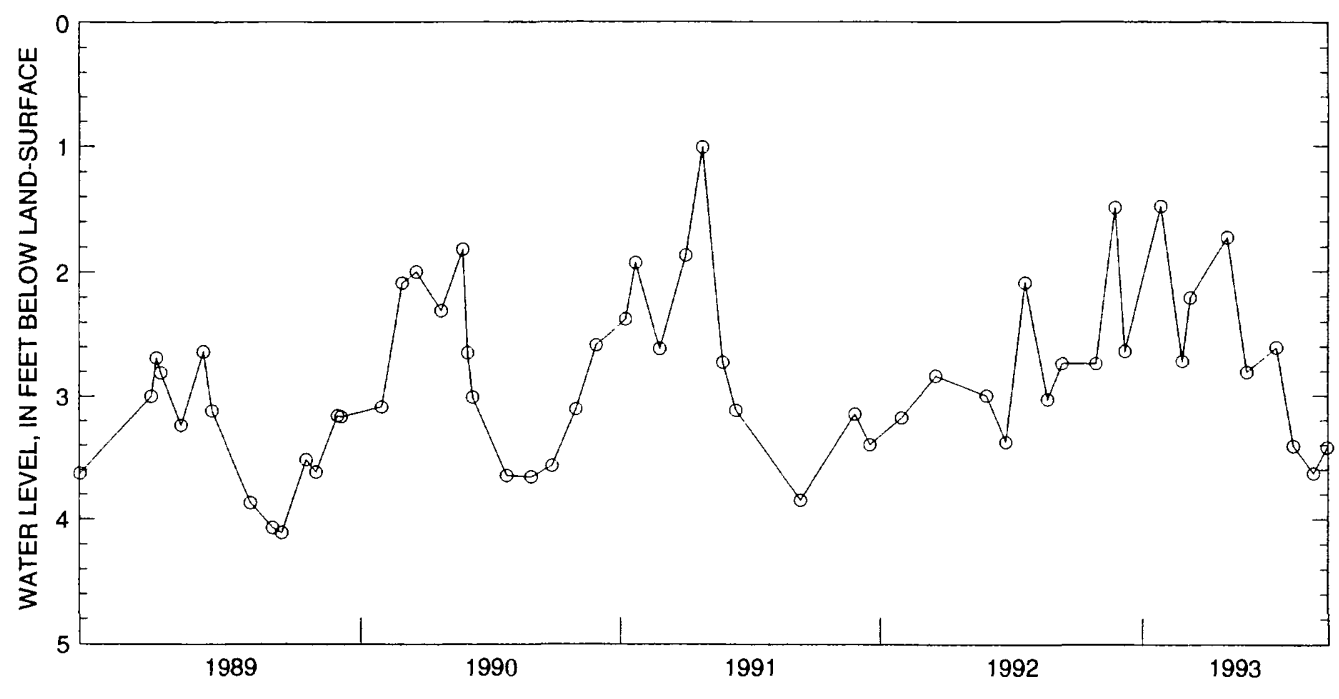


PERIOD OF RECORD.-- January 1986 to current year.

CHEMICAL DATA: 1986(a) 1987-93(b).

ORGANIC DATA: OC.--1986(a) 1987-93(b).

NUTRIENT DATA: 1986(a) 1987-93(b).

BIOLOGICAL DATA

Bacteria.--1986(a) 1987-93(b).

COOPERATION-- Water-quality samples were collected and analyzed by the Monroe County Environmental Health Laboratory at Rochester, NY.

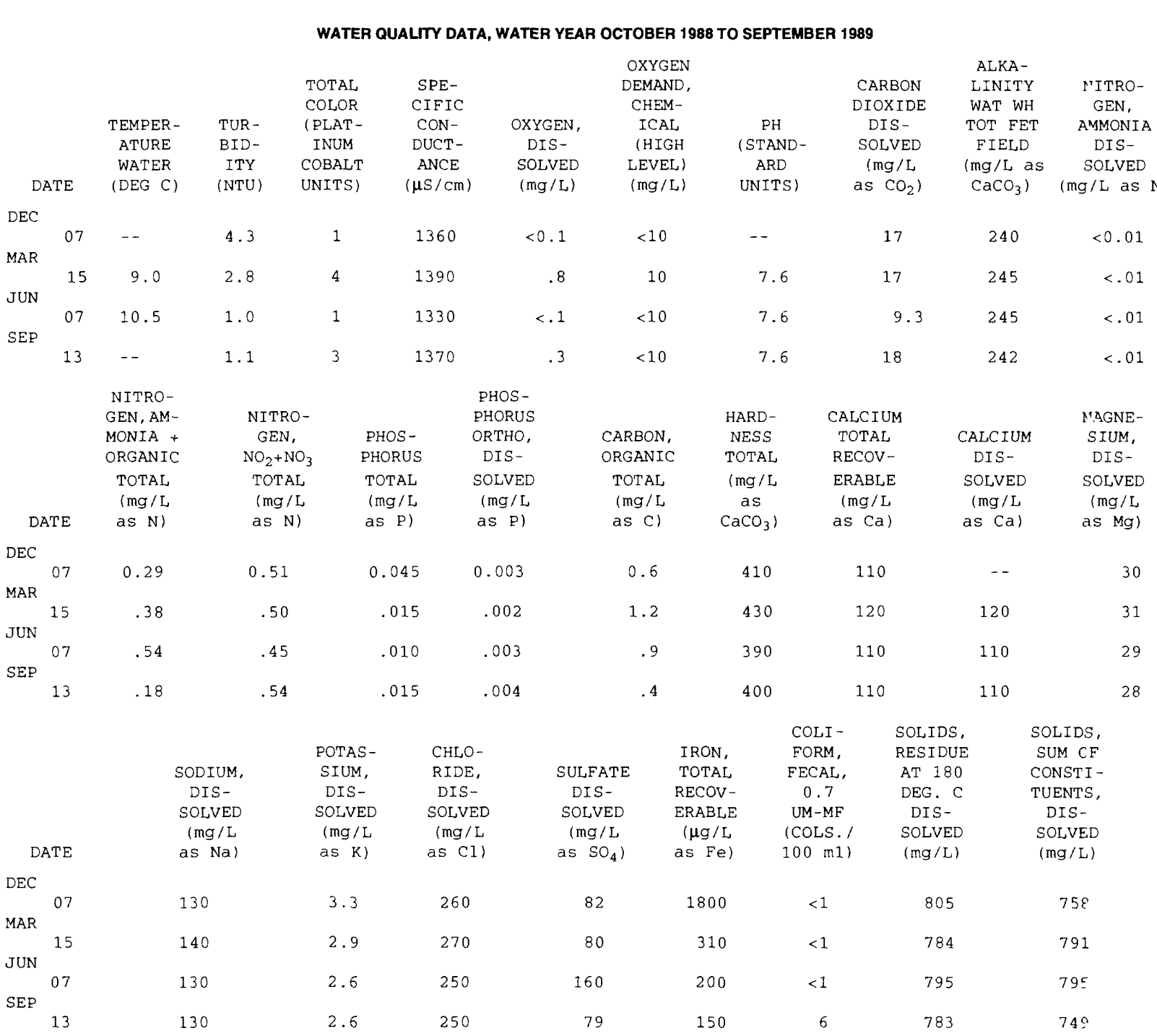


WATER QUALTY DATA, WATER YEAR OCTOBER 1989 TO SEPTEMBER 1990

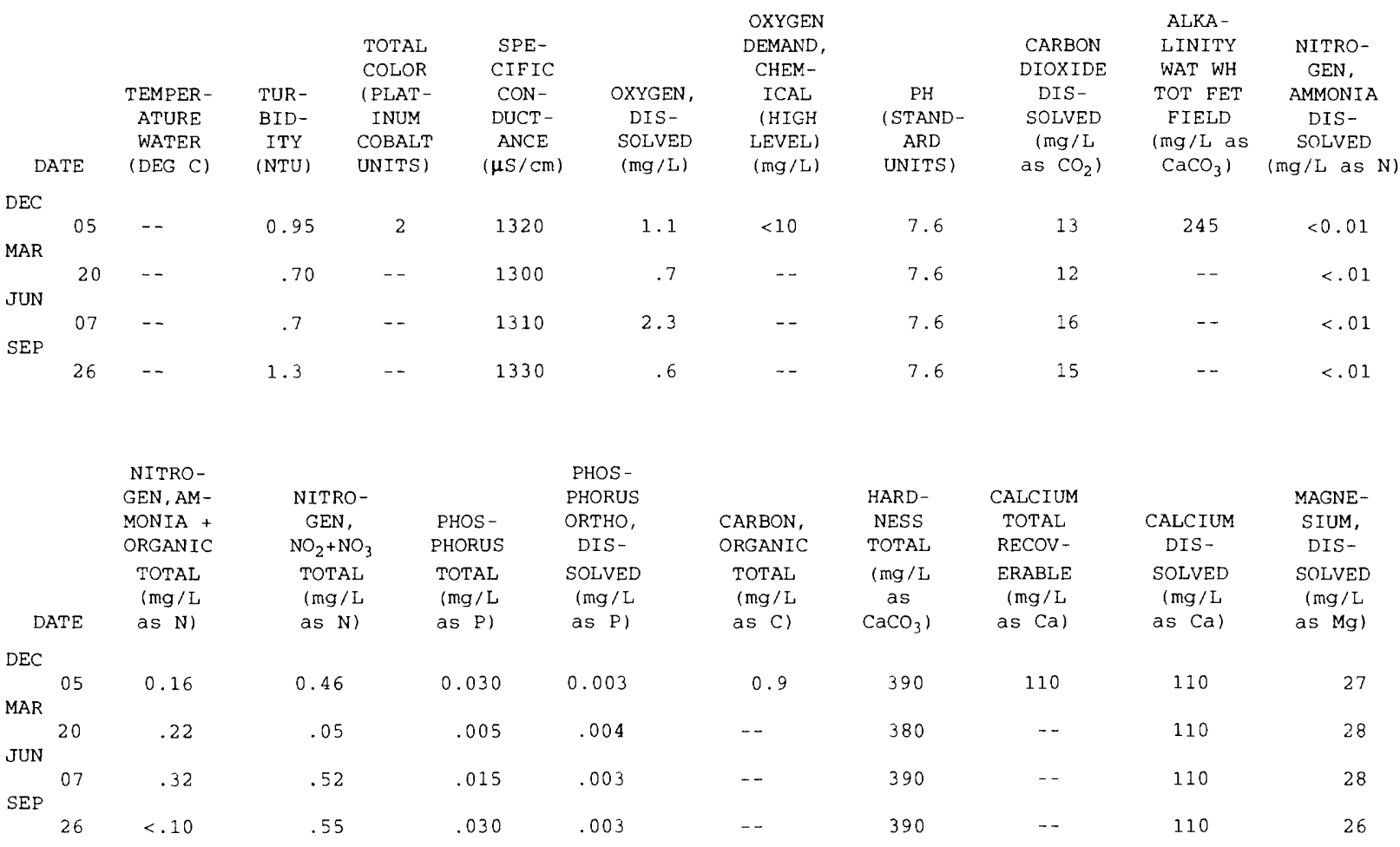

\begin{tabular}{ccc}
\multicolumn{1}{c}{ DATE } & $\begin{array}{c}(\mathrm{mg} / \mathrm{L} \\
\text { DEC }\end{array}$ \\
MAR & 05 & 130 \\
& 20 & 130 \\
JUN & & \\
& 07 & 130 \\
SEP & 26 & 120
\end{tabular}

$\begin{array}{ccc} & & \\ & \text { POTAS- } & \text { CHLO- } \\ \text { SODIUM, } & \text { SIUM, } & \text { RIDE, } \\ \text { DIS- } & \text { DIS- } & \text { DIS- } \\ \text { SOLVED } & \text { SOLVED } & \text { SOLVED } \\ \text { (mg/L } & \text { (mg/L } & (\mathrm{mg} / \mathrm{L} \\ \text { as } \mathrm{Na}) & \text { as K) } & \text { as Cl) }\end{array}$

\begin{tabular}{|c|c|c|c|c|}
\hline & IRON, & $\begin{array}{l}\text { COLI - } \\
\text { FORM, }\end{array}$ & $\begin{array}{l}\text { SOLIDS, } \\
\text { RESIDUE }\end{array}$ & $\begin{array}{l}\text { SOLIDS } \\
\text { SUM OF }\end{array}$ \\
\hline SULFATE & TOTAL & FECAL, & AT 180 & CONSTI \\
\hline DIS- & RECOV- & 0.7 & DEG. C & TUENTS \\
\hline $\begin{array}{c}\text { SOLVED } \\
(\mathrm{mg} / \mathrm{L}\end{array}$ & $\begin{array}{c}\text { ERABLE } \\
(\mu \mathrm{g} / \mathrm{L}\end{array}$ & $\begin{array}{l}\text { UM-MF } \\
\text { COLS. }\end{array}$ & $\begin{array}{l}\text { DIS- } \\
\text { SOLVED }\end{array}$ & $\begin{array}{l}\text { DIS- } \\
\text { SOLVEI }\end{array}$ \\
\hline as $\mathrm{SO}_{4}$ ) & as $\mathrm{Fe}$ ) & $100 \mathrm{ml})$ & $(\mathrm{mg} / \mathrm{L})$ & (mg/L) \\
\hline
\end{tabular}

$\begin{array}{lllllll}3.0 & 240 & 80 & 150 & <2 & 765 & 740 \\ 2.4 & 240 & 79 & 130 & -- & -- & 777 \\ 2.5 & 240 & 75 & 120 & -- & -- & 790 \\ 2.5 & 240 & 38 & 360 & -- & -- & 766\end{array}$


430854077304601. Local number Mo 3 (El 84-3)--continued

WATER QUALTYY DATA, WATER YEAR OCTOBER 1991 TO SEPTEMBER 1992

\begin{tabular}{|c|c|c|c|c|c|c|c|c|c|c|}
\hline DATE & $\begin{array}{c}\text { TEMPER- } \\
\text { ATURE } \\
\text { WATER } \\
\text { (DEG C) }\end{array}$ & $\begin{array}{l}\text { TUR- } \\
\text { BID- } \\
\text { ITY } \\
\text { (NTU) }\end{array}$ & $\begin{array}{l}\text { TOTAL } \\
\text { COLOR } \\
\text { (PLAT- } \\
\text { INUM } \\
\text { COBALT } \\
\text { UNITS) }\end{array}$ & $\begin{array}{c}\text { SPE- } \\
\text { CIFIC } \\
\text { CON- } \\
\text { DUCT - } \\
\text { ANCE } \\
(\mu S / C m)\end{array}$ & $\begin{array}{c}\text { OXYGEN, } \\
\text { DIS- } \\
\text { SOLVED } \\
(\mathrm{mg} / \mathrm{L})\end{array}$ & $\begin{array}{c}\text { OXYGEN } \\
\text { DEMAND, } \\
\text { CHEM- } \\
\text { ICAL } \\
\text { (HIGH } \\
\text { LEVEL) } \\
\text { (mg/L) }\end{array}$ & $\begin{array}{c}\text { PH } \\
\text { (STAND- } \\
\text { ARD } \\
\text { UNITS) }\end{array}$ & $\begin{array}{c}\text { CARBON } \\
\text { DIOXIDE } \\
\text { DIS- } \\
\text { SOLVED } \\
(\mathrm{mg} / \mathrm{L} \\
\left.\text { as } \mathrm{CO}_{2}\right)\end{array}$ & $\begin{array}{l}\text { ALKA- } \\
\text { LINITY } \\
\text { WAT WH } \\
\text { TOT FET } \\
\text { FIELD } \\
(\mathrm{mg} / \mathrm{L} \text { as } \\
\left.\mathrm{CaCO}_{3}\right)\end{array}$ & $\begin{array}{c}\text { NITRO- } \\
\text { GEN, } \\
\text { AMMONIA } \\
\text { DIS- } \\
\text { SOLVED } \\
\text { (mg/L as N) }\end{array}$ \\
\hline$E C$ & & & & & & & & & & \\
\hline JUN & -- & 1.7 & -- & 1290 & 1.2 & -- & 7.6 & 15 & - & $<.01$ \\
\hline$E P$ & -- & .75 & -- & 1320 & 2.2 & -- & 7.5 & 15 & -. & $<.01$ \\
\hline 10 & -- & 1.0 & -- & 1320 & 1.4 & -- & 7.6 & 13 & -- & $<.01$ \\
\hline
\end{tabular}

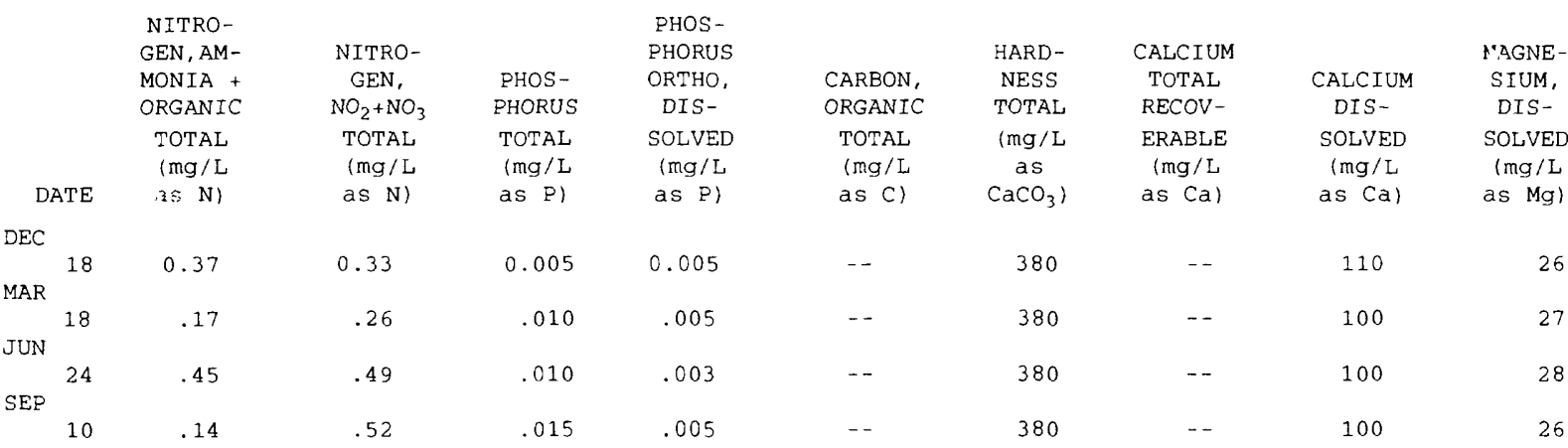

DATE

DEC

$\begin{array}{lll} & 18 & 130 \\ \text { MAR } & & 130 \\ & 18 & 130 \\ \text { JUN } & & \\ \text { SEP } & 24 & 130\end{array}$

$\begin{array}{cc}\text { POTAS- } & \text { CHLO- } \\ \text { SIUM, } & \text { RIDE, } \\ \text { DIS- } & \text { DIS- } \\ \text { SOLVED } & \text { SOLVED } \\ \text { (mg/L } & (\mathrm{mg} / L \\ \text { aS K) } & \text { aS Cl) }\end{array}$

3.2

230

3.8

2.8

3.0

SULFATE
DIS-
SOLVED
$\langle\mu g / L$
aS $\left.\mathrm{SO}_{4}\right\rangle$

$$
\begin{gathered}
\text { IRON, } \\
\text { TOTAL } \\
\text { RECOV- } \\
\text { ERABLE } \\
\text { (mg/L }
\end{gathered}
$$

as $\mathrm{Fe})$

86
77
80
91

70
180
340
290

$\begin{array}{ccc}\text { COLI- } & \text { SOLIDS, } & \text { SOLIDS, } \\ \text { FORM, } & \text { RESIDUE } & \text { SUM OF } \\ \text { FECAL, } & \text { AT } 180 & \text { CONSTI- } \\ 0.7 & \text { DEG. C } & \text { TUENTS, } \\ \text { UM-MF } & \text { DIS- } & \text { DIS- } \\ \text { (COLS.) } & \text { SOLVED } & \text { SOLVED } \\ 100 \mathrm{ml}) & (\mathrm{mg} / \mathrm{L}) & \left(\mathrm{mg} / \mathrm{I}_{1}\right)\end{array}$


430854077304601. Local number Mo 3 (EI 84-3)--continued

WATER QUALITY DATA, WATER YEAR OCTOBER 1992 TO SEPTEMBER 1993

\begin{tabular}{|c|c|c|c|c|c|c|c|c|c|c|}
\hline & & & & & & OXYGEN & & & ALKA- & \\
\hline & & & TOTAL & SPE- & & DEMAND， & & CARBON & LINITY & NITRO- \\
\hline & & & COLOR & CIFIC & & CHEM - & & DIOXIDE & WAT WH & GEN, \\
\hline & TEMPER - & TUR- & (PLAT - & $\mathrm{CON}-$ & OXYGEN, & ICAL & $\mathrm{PH}$ & DIS - & TOT FET & $A M$ ONIA \\
\hline & ATURE & BID- & INUM & DUCT - & DIS- & ( $\mathrm{HIGH}$ & (STAND- & SOLVED & FIELD & DIS - \\
\hline & WATER & ITY & COBALT & ANCE & SOLVED & LEVEL) & ARD & (mg/L & $(\mathrm{mg} / \mathrm{L}$ as & SOLVED \\
\hline DATE & $(\mathrm{DEG} C)$ & (NTU) & UNITS) & $(\mu \mathrm{S} / \mathrm{cm})$ & $(\mathrm{mg} / \mathrm{L})$ & $(\mathrm{mg} / \mathrm{L})$ & UNITS) & as $\left.\mathrm{CO}_{2}\right)$ & $\left.\mathrm{CaCO}_{3}\right)$ & $(\mathrm{mg} / \mathrm{L}$ as $\mathrm{N})$ \\
\hline
\end{tabular}

$\begin{array}{lccccc}\text { DEC } & & & 1.1 & -- & 1300 \\ \text { MAR } & 08 & -- & 1.2 & -- & 1280 \\ \text { JUN } & 09 & -- & .60 & -- & 1270 \\ \text { SEP } & 22 & -- & .90 & -- & 1280\end{array}$

$\begin{array}{lll}0.5 & -- & 7.4 \\ 1.2 & -- & 7.6 \\ .6 & - & 7.8 \\ .5 & -- & 7.7\end{array}$

14
16
13

$\begin{array}{ll}-- & <0.01 \\ -- & <.01 \\ -- & <.01 \\ -- & <.01\end{array}$

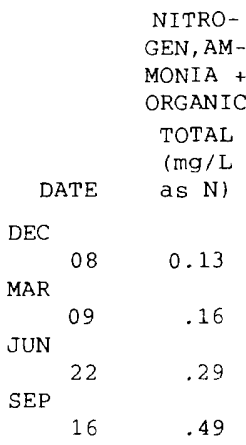

$\begin{array}{ccc} & & \text { PHOS - } \\ \text { NITRO- } & & \text { PHORUS } \\ \text { GEN, } & \text { PHOS - } & \text { ORTHO, } \\ \mathrm{NO}_{2}+\mathrm{NO}_{3} & \text { PHORUS } & \text { DIS- } \\ \mathrm{TOTAL} & \text { TOTAL } & \text { SOLVED } \\ (\mathrm{mg} / \mathrm{L} & (\mathrm{mg} / \mathrm{L} & (\mathrm{mg} / \mathrm{L} \\ \mathrm{as} \mathrm{N}) & \text { as P) } & \text { as P) }\end{array}$

$\begin{array}{ccc} & \text { HARD- } & \text { CALCIUM } \\ \text { CARBON, } & \text { NESS } & \text { TOTAL } \\ \text { ORGANIC } & \text { TOTAL } & \text { RECOV- } \\ \text { TOTAL } & (\mathrm{mg} / \mathrm{L} & \text { ERABLE } \\ (\mathrm{mg} / \mathrm{L} & \text { as } & \text { (mg/L } \\ \text { as C) } & \left.\mathrm{CaCO}_{3}\right) & \text { as Ca) }\end{array}$

$\begin{array}{cc} & \text { MAGNE- } \\ \text { CALCIUM } & \text { SIUM, } \\ \text { DIS- } & \text { IIS- } \\ \text { SOLVED } & \text { SCLVED } \\ (\mathrm{mg} / \mathrm{L} & (\mathrm{mg} / \mathrm{L} \\ \text { as Ca) } & \text { as Mg) }\end{array}$

$\begin{array}{lll}0.45 & 0.010 & .004 \\ .39 & .010 & .004 \\ .45 & .005 & .003 \\ .51 & .010 & .006\end{array}$

370
370
370
370

--
--
--

110
100
120
100

$\begin{array}{ccc} & & \\ \text { SODIUM, } & \text { POTAS- } & \text { CHLO- } \\ \text { DIS- } & \text { SIUM, } & \text { RIDE, } \\ \text { SOLVED } & \text { DIS- } & \text { DIS- } \\ (\mathrm{mg} / \mathrm{L} & \text { (mg } / \mathrm{L} & \text { SOLVED } \\ \text { as Na) } & \text { as K) } & \text { as Cl) }\end{array}$

DEC

$\begin{array}{lll}\text { DEC } & & 130 \\ \text { MAR } & 08 & 120 \\ & 09 & 120 \\ \text { JUN } & 22 & 130 \\ \text { SEP } & 16 & \end{array}$

$\begin{array}{ll}2.5 & 240 \\ 2.3 & 230 \\ 5.2 & 220 \\ 2.1 & 230\end{array}$

SULFATE
DIS-
SOLVED
$\left(\mathrm{mg}^{-1}\right.$
aS $\left.\mathrm{SO}_{4}\right)$

IRON,
TOTAL
RECOV-
ERABLE
$(\mu g / L$
as Fe)

$\begin{array}{cc}\text { COLI- } & \text { SOLIDS, } \\ \text { FORM, } & \text { RESIDUE } \\ \text { FECAL, } & \text { AT } 180 \\ 0.7 & \text { DEG. C } \\ \text { UM-ME } & \text { DIS- } \\ \text { (COLS.) } & \text { SOLVED } \\ 100 \mathrm{ml}) & (\mathrm{mg} / \mathrm{L})\end{array}$

$\begin{array}{rrrrr}100 & 360 & - & - & 760 \\ 87 & 180 & -- & -- & -- \\ 86 & 140 & -- & -- & 753 \\ 88 & 310 & -- & -- & 760\end{array}$


430854077304601. Local number Mo 3 (El 84-3)--continued

WATER TEMPERATURE, IN DEGREES CELSIUS

\begin{tabular}{|c|c|c|c|c|c|c|c|c|c|c|c|c|c|c|c|c|c|c|c|c|}
\hline \multirow[b]{2}{*}{$\begin{array}{l}\text { Depth, } \\
\text { in feet }\end{array}$} & \multicolumn{4}{|c|}{$1989 \mathrm{Wr}$} & \multicolumn{4}{|c|}{$1990 \mathrm{Wr}$} & \multicolumn{4}{|c|}{$1991 \mathrm{Wr}$} & \multicolumn{4}{|c|}{$1992 \mathrm{WY}$} & \multicolumn{4}{|c|}{1993 IVY } \\
\hline & $\begin{array}{c}\text { Dec } \\
7\end{array}$ & $\begin{array}{c}\text { Mar } \\
15\end{array}$ & $\begin{array}{c}\text { Jun } \\
7\end{array}$ & $\begin{array}{c}\text { Sep } \\
13\end{array}$ & $\begin{array}{c}\text { Dec } \\
5\end{array}$ & $\begin{array}{c}\text { Mar } \\
20\end{array}$ & $\underset{7}{\text { Jun }}$ & $\begin{array}{l}\text { Sep } \\
26\end{array}$ & $\begin{array}{c}\text { Jan } \\
9\end{array}$ & $\begin{array}{c}\text { Apr } \\
3\end{array}$ & $\begin{array}{c}\text { Jun } \\
12\end{array}$ & $\begin{array}{c}\text { Sep } \\
11\end{array}$ & $\begin{array}{c}\text { Dec } \\
18\end{array}$ & $\begin{array}{c}\text { Mar } \\
18\end{array}$ & $\begin{array}{c}\text { Jun } \\
24\end{array}$ & $\begin{array}{c}\text { Sep } \\
10\end{array}$ & $\begin{array}{c}\text { Dec } \\
8\end{array}$ & $\begin{array}{c}\text { Mar } \\
9\end{array}$ & $\begin{array}{c}\text { Jun } \\
22\end{array}$ & $\begin{array}{c}\text { Sep } \\
15\end{array}$ \\
\hline 1.96 & -- & -- & -- & $\cdots$ & - & 5.5 & -- & $\overline{--}$ & -- & 8.5 & -- & $\overline{-1}$ & 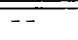 & - & -- & $=-$ & -- & - & -- & -- \\
\hline 2.26 & - & -- & -- & -- & -- & 5.5 & - & - & 6.5 & 7.0 & -- & -- & -- & -- & -- & -- & 8.5 & 4.0 & 15.0 & -- \\
\hline 2.76 & -- & -- & -- & -- & -- & -- & -- & - & -- & -- & -- & -- & -- & & -- & 19.5 & -- & -- & -- & -- \\
\hline 3.26 & -- & 4.5 & 14.5 & -- & 8.5 & 6.0 & 14.5 & -- & 6.5 & 6.5 & 17.0 & -- & 7.5 & 5.5 & 14.0 & 18.5 & 9.5 & 4.5 & 14.0 & -- \\
\hline 3.76 & 6.5 & - & -- & -- & $\ldots$ & -- & $\ldots$ & 17.0 & -- & -- & -- & 19.0 & -- & -- & -- & -. & $\ldots$ & -- & -- & 18.0 \\
\hline 4.26 & 7.5 & 4.5 & 14.0 & 17.0 & 9.5 & 6.0 & 13.5 & 17.0 & 7.0 & 6.5 & 15.0 & 18.0 & 8.5 & 5.5 & 13.5 & 17.5 & 10.0 & 5.0 & 13.0 & 18.0 \\
\hline 5.26 & 8.0 & 4.5 & 13.0 & 16.5 & 10.0 & 6.0 & 12.5 & 17.0 & 7.5 & 6.5 & 14.0 & 17.5 & 9.5 & 6.0 & 12.5 & 17.0 & 10.5 & 5.5 & 11.5 & 17.0 \\
\hline 6.26 & 8.0 & 5.5 & 11.5 & 16.0 & 10.5 & 6.5 & 11.5 & 16.5 & 8.0 & 6.5 & 13.0 & 16.5 & 9.5 & 6.5 & 12.5 & 16.5 & 10.5 & 6.0 & 10.5 & 16.0 \\
\hline 7.26 & 8.5 & 6.0 & 10.5 & 15.5 & 11.0 & 6.5 & 11.0 & 16.0 & 8.5 & 7.0 & 12.5 & 15.5 & 10.0 & 6.5 & 11.5 & 15.5 & 11.0 & 6.0 & 10.0 & 15.5 \\
\hline 8.26 & 8.5 & 6.5 & 9.5 & 13.5 & 11.0 & 7.0 & 10.5 & 15.5 & 9.0 & 7.0 & 11.5 & 15.0 & 10.0 & 7.0 & 11.0 & 15.0 & 11.0 & 6.5 & 9.5 & 15.0 \\
\hline 9.26 & 8.5 & 6.5 & 9.0 & 13.0 & 11.5 & 7.0 & 10.0 & 15.0 & 9.5 & 7.0 & 11.0 & 14.0 & 10.5 & 7.0 & 10.5 & 14.5 & 11.5 & 7.0 & 9.0 & 14.0 \\
\hline 10.26 & 9.0 & 6.5 & 9.0 & 12.5 & 11.5 & 7.0 & 9.5 & 14.0 & 9.5 & 7.5 & 10.5 & 13.5 & 11.0 & 7.5 & 10.0 & 13.5 & 11.5 & 7.5 & 9.0 & 13.5 \\
\hline 11.26 & 9.0 & 7.0 & 9.0 & 12.0 & 11.5 & 7.5 & 9.5 & 13.5 & 10.0 & 7.5 & 10.0 & 13.0 & 11.0 & 7.5 & 9.5 & 13.0 & 11.5 & 7.5 & 8.5 & 12.5 \\
\hline 12.26 & 9.0 & 7.0 & 9.0 & 11.5 & 11.5 & 8.0 & 9.5 & 13.0 & 10.5 & 7.5 & 10.0 & 12.5 & 11.5 & 8.0 & 9.5 & 12.5 & 11.5 & 8.0 & 8.5 & 12.0 \\
\hline 13.26 & 9.0 & 7.5 & 9.0 & 11.5 & 12.0 & 8.0 & 9.5 & 12.5 & 10.5 & 8.0 & 10.0 & 12.0 & 11.5 & 8.0 & 9.5 & 12.0 & 12.0 & 8.0 & 8.5 & 12.0 \\
\hline 14.26 & 9.0 & 8.0 & 9.0 & 11.0 & 12.0 & 8.5 & 9.5 & 12.0 & 11.0 & 8.5 & 10.0 & 11.5 & 11.5 & 8.5 & 9.5 & 11.5 & 12.0 & 8.5 & 8.5 & 11.5 \\
\hline 15.26 & 9.5 & 8.0 & 9.0 & 11.0 & 12.0 & 8.5 & 9.5 & 11.5 & 11.0 & 8.5 & 10.0 & 11.5 & 11.5 & 8.5 & 9.5 & 11.5 & 12.0 & 8.5 & 8.5 & 11.0 \\
\hline 16.16 & 9.5 & 8.0 & 9.0 & 11.0 & 12.0 & 8.5 & 9.5 & 11.5 & 11.0 & 8.5 & 10.0 & 11.5 & 11.5 & 9.0 & 9.5 & 11.0 & 9.5 & 8.5 & 8.5 & 11.0 \\
\hline
\end{tabular}

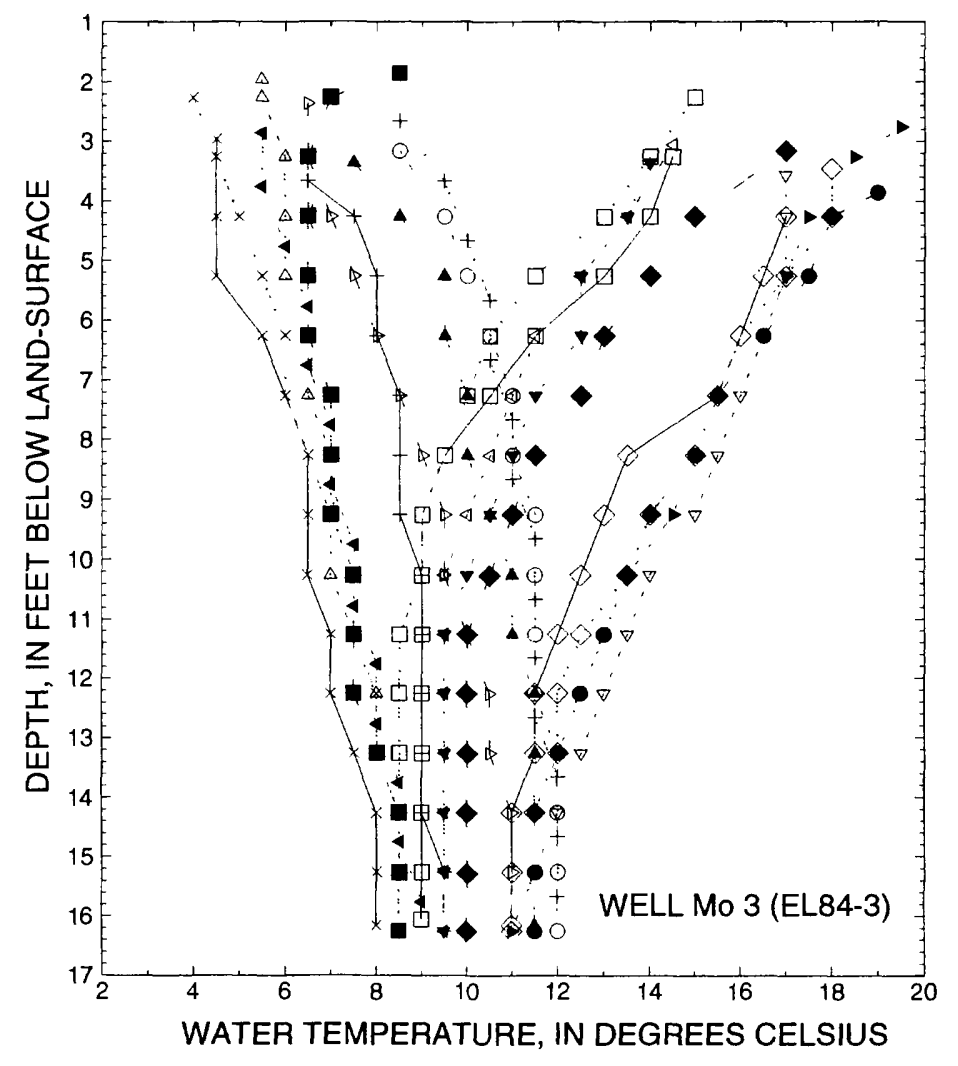

EXPLANATION

$+88 / 12 / 07$

$\times \quad 89 / 03 / 15$

—- 89/06/07

$\curvearrowright$ 89/09/13

○. $89 / 12 / 05$

$\triangle 90 / 03 / 20$

4 90/06/07

$\nabla \quad 90 / 09 / 26$

.- $D-91 / 01 / 09$

- 91/04/03

- $91 / 06 / 12$

- $91 / 09 / 11$

- $91 / 12 / 18$

4 92/03/18

จ. $92 / 06 / 24$

-.. $92 / 09 / 10$

+.., $92 / 12 / 08$

※. 93/03/09

‥ 93/06/22

C. $93 / 09 / 15$ 


\section{Ellison park}

\section{Local number Mo 4 (EI 84-4)}

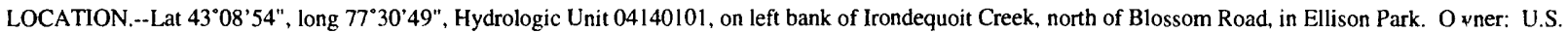
Geological Survey.

AQUIFER.-.-Water-table aquifer in alluvium of Holocene age.

WELL CHARACTERISTICS.--Drilled observation well, diameter 2 in, depth $16 \mathrm{ft}$, cased to $13.5 \mathrm{ft}$, screened $13.5 \mathrm{ft}$ to $16 \mathrm{ft}$.

INSTRUMENTATION.--Monthly measurement with chalked tape by Monroe County Environmental Health Laboratory personnel and occasional measirement by USGS personnel.

DATUM.--Elevation of land-surface datum is $252.70 \mathrm{ft}$ above National Geodetic Vertical Datum of 1929. Measuring point: arrow at top of casing, $3.31 \mathrm{ft}$ above landsurface datum.

REMARKS.--Water level affected by stage of Irondequoit Creek. This well is also a water-quality observation well. Water levels are monitored monthly and water samples taken quarterly by the Monroe County Environmental Health Laboratory. Water-temperature profiles are also taken by MCEHL on a quarterly basis.

PERIOD OF RECORD.--September 1984 to November 1990, (discontinued).

EXTREMES FOR PERIOD SEPTEMBER 1984 TO NOVEMBER 1990.--Highest water level measured, 2.03 feet above land-surface datum, February 27, 1985 ; lowest measured, 3.63 feet below land-surface datum, September 13, 1989.

WATER LEVEL, IN FEET BELOW LAND-SURFACE DATUM

\begin{tabular}{|c|c|c|c|c|c|c|c|c|c|c|c|}
\hline \multicolumn{2}{|c|}{ DATE } & \multirow{2}{*}{$\begin{array}{l}\text { WATER } \\
\text { LEVEL }\end{array}$} & \multicolumn{2}{|c|}{ DATE } & \multirow[t]{2}{*}{$\begin{array}{l}\text { WATER } \\
\text { LEVEL }\end{array}$} & \multicolumn{2}{|c|}{ DATE } & \multirow[t]{2}{*}{$\begin{array}{l}\text { WATER } \\
\text { LEVEL }\end{array}$} & \multicolumn{2}{|c|}{ DATE } & \multirow[t]{2}{*}{$\begin{array}{l}\text { WATER } \\
\text { LEVEL }\end{array}$} \\
\hline \multicolumn{8}{|c|}{ WATER YEAR OCTOBER 1988 TO SEPTEMBER 1989} & & & & \\
\hline DEC & 7 & 3.13 & MAR & 28 & 2.33 & JUN & 7 & 2.60 & SEP & 13 & 3.63 \\
\hline \multirow[t]{2}{*}{ MAR } & 15 & 2.45 & APR & 25 & 2.72 & JUL & 31 & 3.53 & & & \\
\hline & 22 & 2.22 & MAY & 26 & 2.11 & AUG & 31 & 3.56 & & & \\
\hline \multicolumn{12}{|c|}{ WATER YEAR OCTOBER 1989 TO SEPTEMBER 1990} \\
\hline \multirow[t]{2}{*}{ OCT } & 17 & 3.04 & JAN & 30 & 2.59 & MAY & 24 & 1.32 & AUG & 28 & 3.19 \\
\hline & 31 & 3.13 & FEB & 28 & 1.59 & & 31 & 2.14 & SEP & 26 & 3.13 \\
\hline NOV & 30 & 2.67 & MAR & 20 & 1.49 & JUN & 7 & 2.51 & & & \\
\hline DEC & 5 & 2.69 & APR & 24 & 1.80 & JUL & 25 & 3.16 & & & \\
\hline \multicolumn{12}{|c|}{ WATER YEAR OCTOBER 1990 TO SEPTEMBER 1991} \\
\hline OCT & 30 & 2.61 & NOV & 28 & 2.13 & & & & & & \\
\hline
\end{tabular}

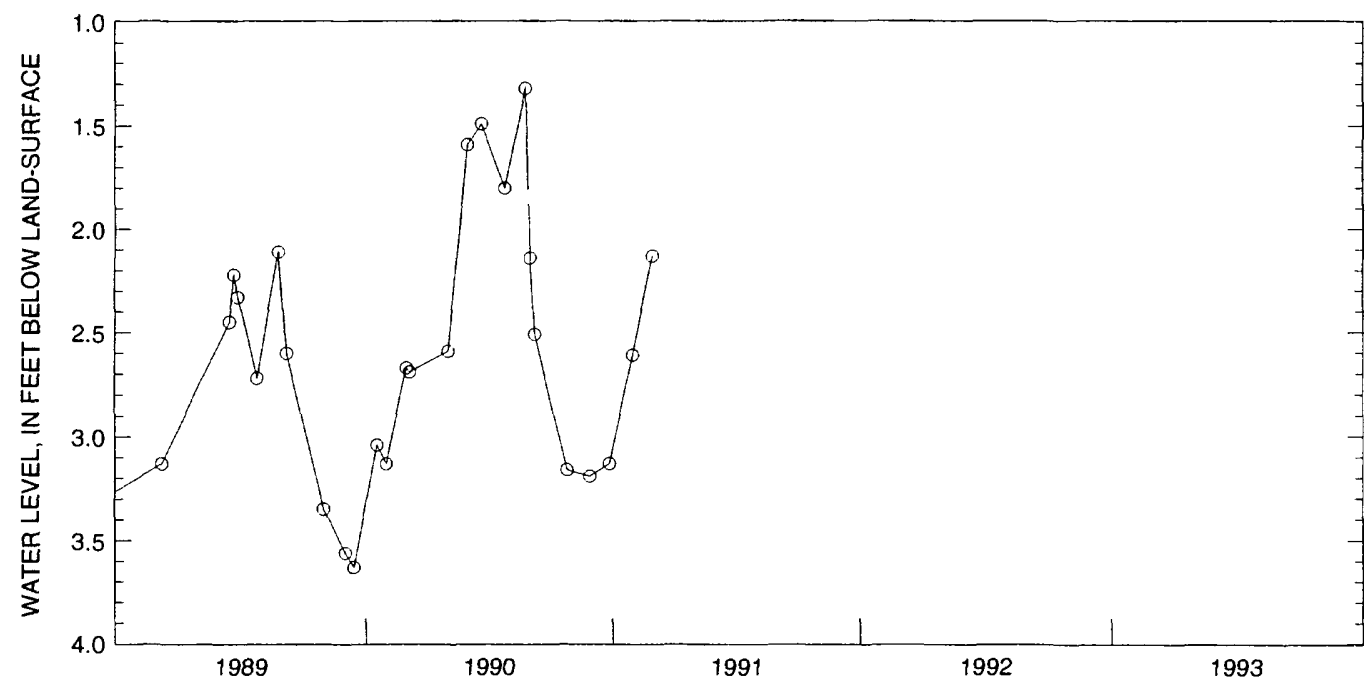


PERIOD OF RECORD.-- January 1986 to November 1990 (discontinued)..

CHEMICAL DATA: 1986(a) 1987-90(b).

ORGANIC DATA: OC.--1986(a) 1987-90(b).

NUTRIENT DATA: 1986(a) 1987-90(b).

BIOLOGICAL DATA:

Bacteria.--1986(a) 1987-90(b).

COOPERATION-- Water-quality samples were collected and analyzed by the Monroe County Environmental Health Laboratory at Rochester, NY

\section{WATER QUALITY DATA, WATER YEAR OCTOBER 1988 TO SEPTEMBER 1989}

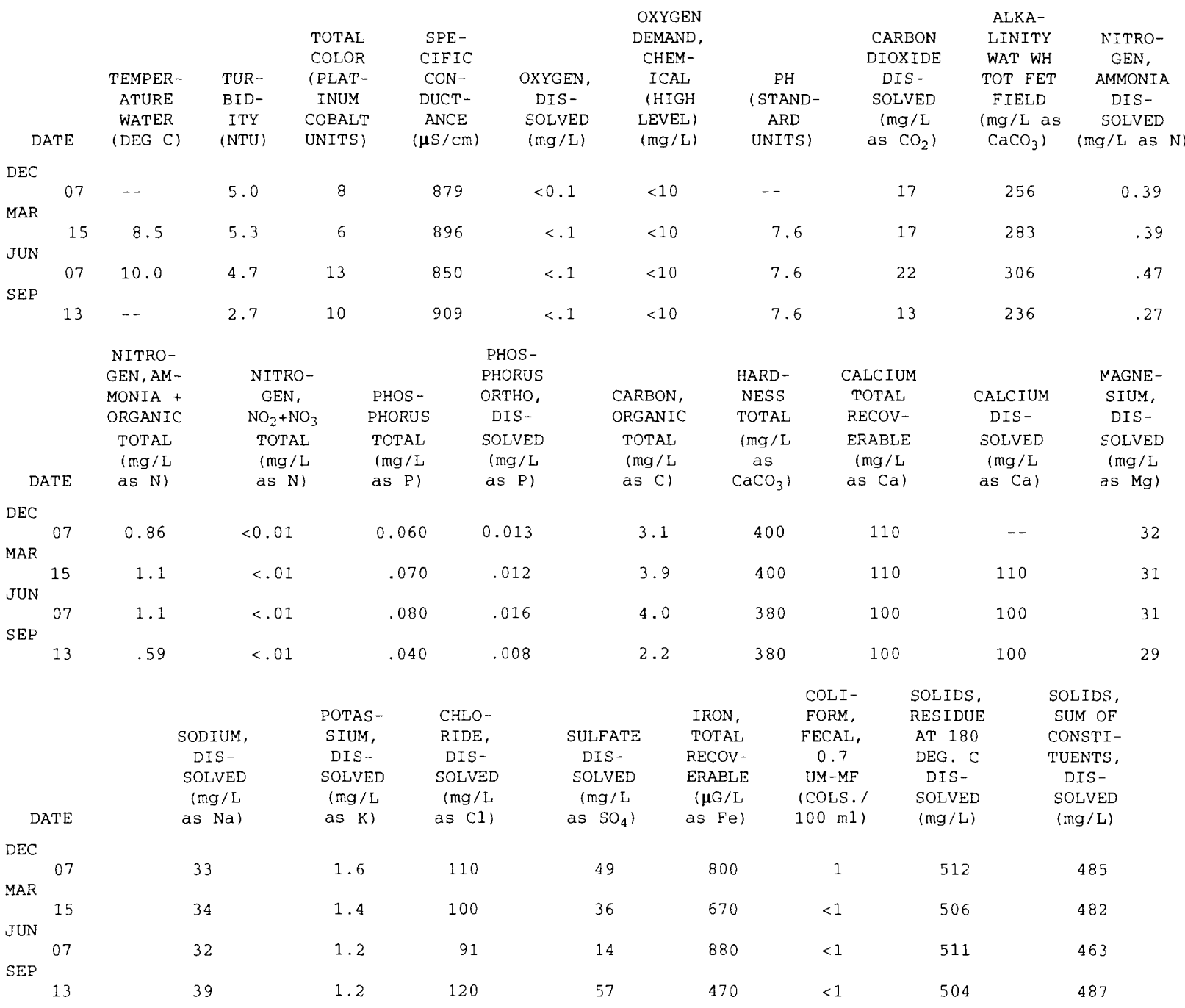


430854077304901. Local number Mo 4 (El 84-4)--continued

WATER QUALTYY DATA, WATER YEAR OCTOBER 1989 TO SEPTEMBER 1990

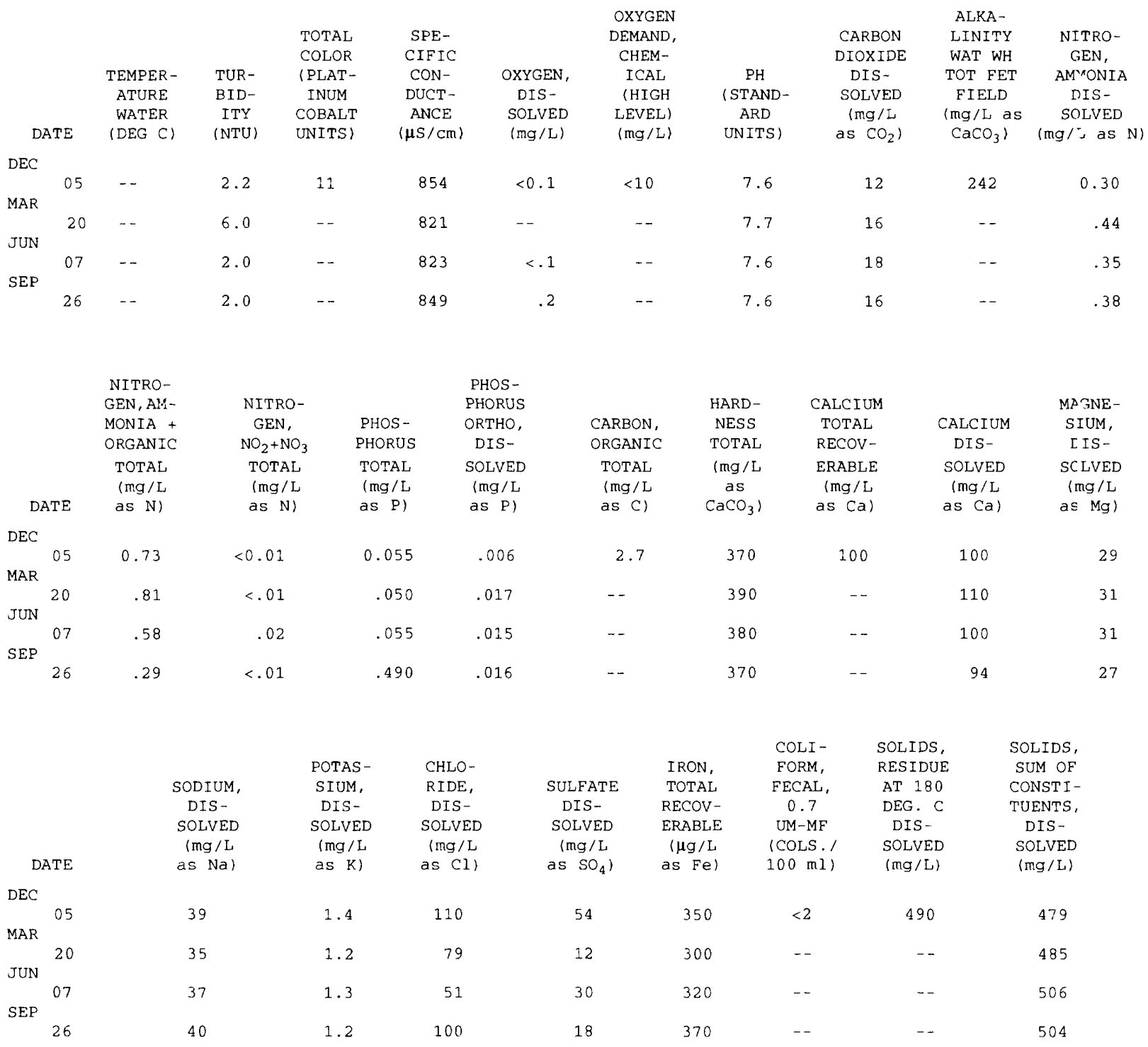




\section{GROUND-WATER TEMPERATURE PROFILES}

\section{Ellison park}

430854077304901. Local number Mo 4 (El 84-4)--continued

WATER TEMPERATURE, IN DEGREES CELSIUS

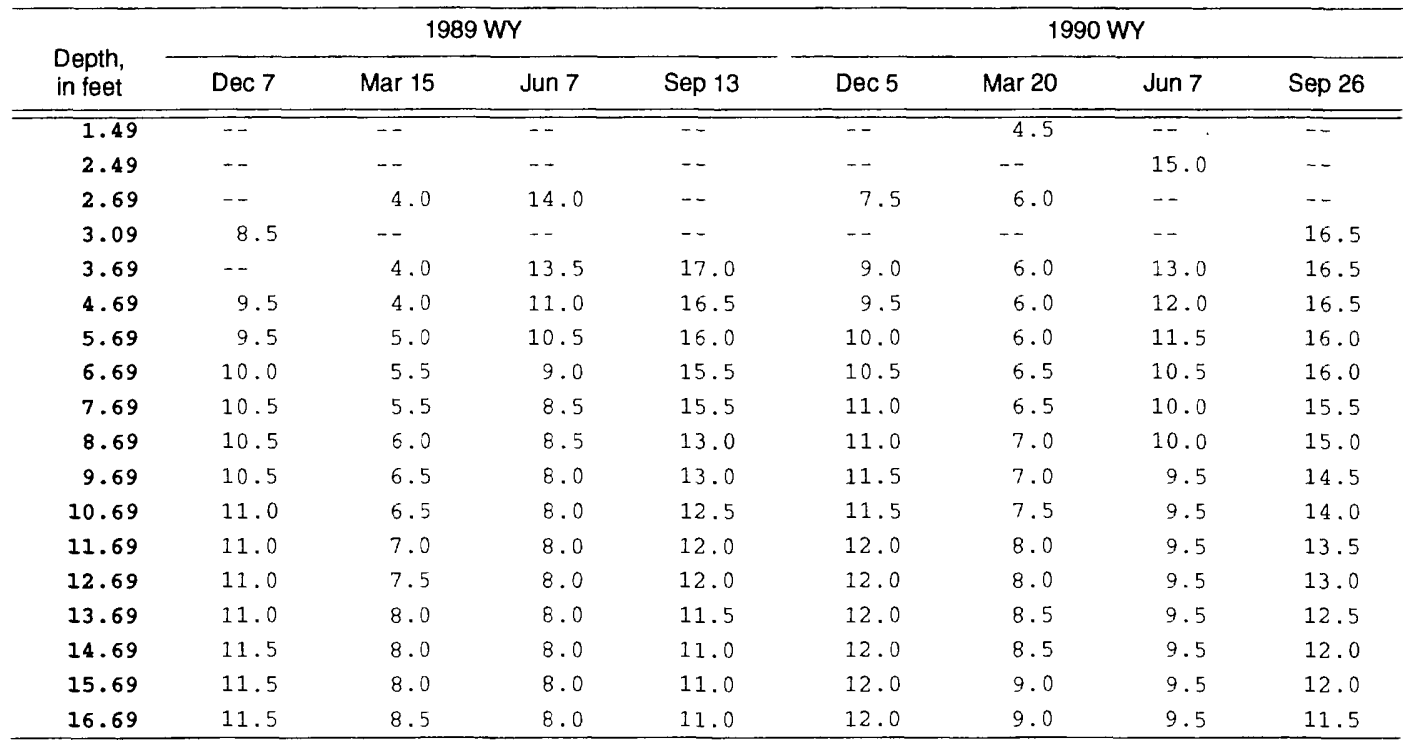

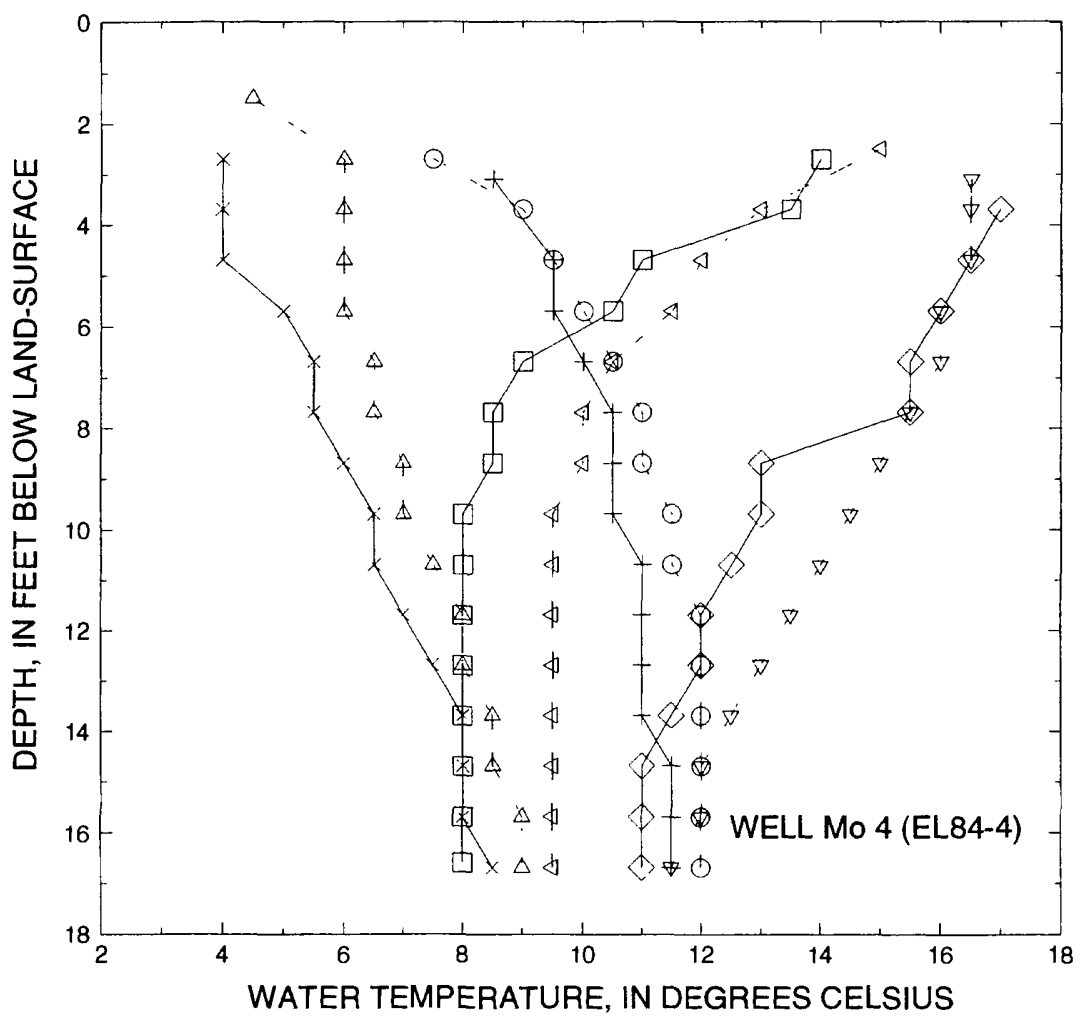

EXPLANATION

$+88 / 12 / 07$

$\rightarrow \quad 89 / 03 / 15$

$\square-89 / 06 / 07$

๑ 89/09/13

$-\mathrm{O}-89 / 12 / 05$

.. $\triangle . .90 / 03 / 20$

$\checkmark$.. $90 / 06 / 07$

$\nabla \quad 90 / 09 / 26$ 


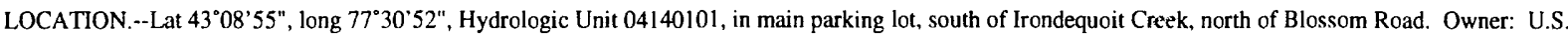
Geological Survey.

AQUIFER.--Water-table aquifer in sand of Pleistocene age.

WELL CHARACTERISTICS.--Drilled observation well, diameter 2 in, depth $43 \mathrm{ft}$, cased to $39 \mathrm{ft}$, screened $39 \mathrm{ft}$ to $43 \mathrm{ft}$.

INSTRUMENTATION.--Monthly measurement with chalked tape by Monroe County Environmental Health Laboratory personnel and occasional measurement by USGS personnel.

DATUM.--Elevation of land-surface datum is $251.1 \mathrm{ft}$ above National Geodetic Vertical Datum of 1929. Measuring point: arrow at top of casing, $3.64 \mathrm{ft}$ above landsurface datum.

REMARKS.--This well is also a water-quality observation well. Water levels are monitored monthly and water samples taken quarterly by the Monroe County Environmental Health Laboratory. Water-temperature profiles are also taken by MCEHL on a quarterly basis.

PERIOD OF RECORD.--September 1984 to August 1989, (discontinued).

EXTREMES FOR PERIOD SEPTEMBER 1984 TO AUGUST 1989.--Highest water level measured, 1.63 feet above land-surface datum, April 17, 1986; lowest measured, 2.09 feet below land-surface datum, August 21, 1985.

WATER LEVEL, IN FEET BELOW LAND-SURFACE DATUM,

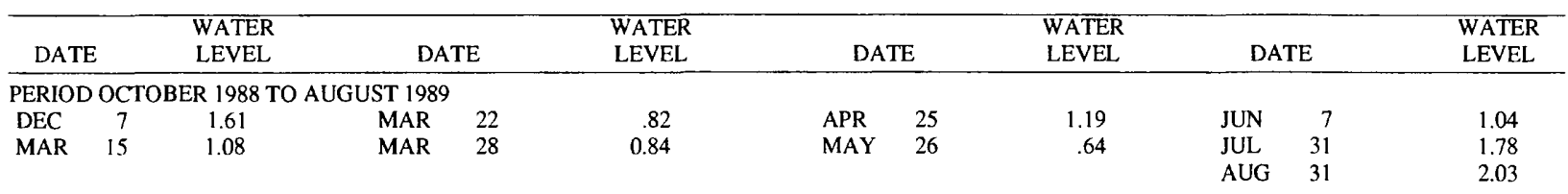

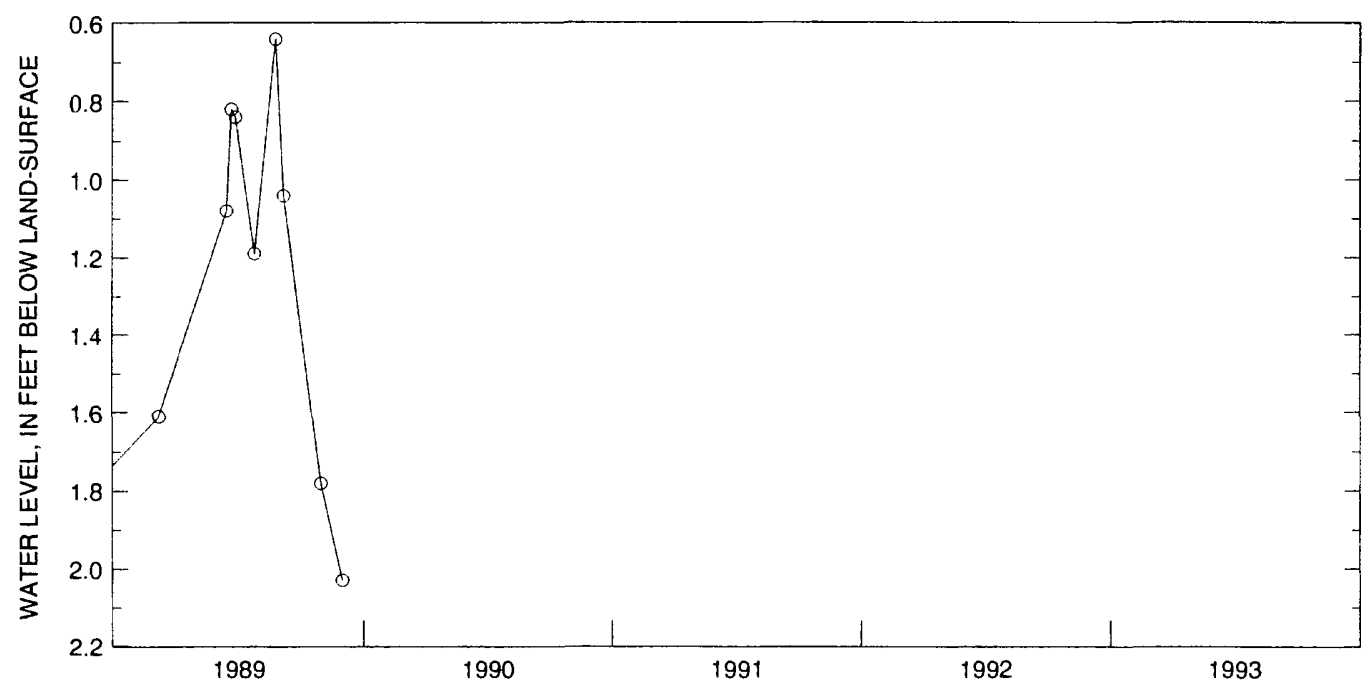


PERIOD OF RECORD.-- January 1986 to August 1989 (discontinued).

CHEMICAL DATA: 1986(a) 1987-89(b).

ORGANIC DATA: OC.--1986(a) 1987-89(b).

NUTRIENT DATA: 1986(a) 1987-89(b).

BIOLOGICAL DATA:

Bacteria.--1986(a) 1987-89(b).

COOPERATION-- Water-quality samples were collected and analyzed by the Monroe County Environmental Health Laboratory at Rochester, NY.

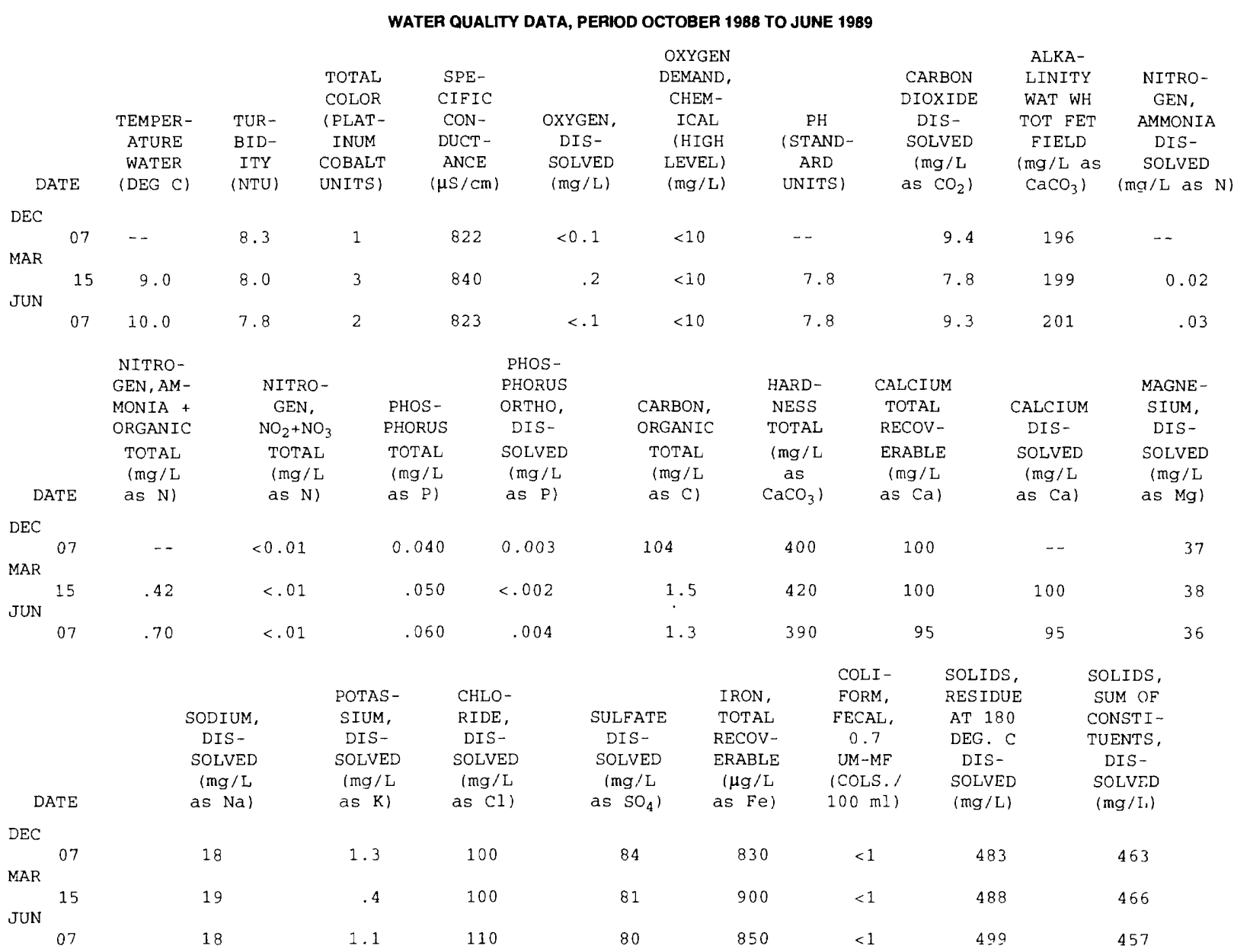


430855077305201. Local number Mo 5 (EI 84-5)--continued

WATER TEMPERATURE, IN DEGREES CELSIUS

\begin{tabular}{|c|c|c|c|}
\hline \multirow{2}{*}{$\begin{array}{l}\text { Depth, } \\
\text { in feet }\end{array}$} & \multirow{2}{*}{$\frac{1988 W Y}{\operatorname{Dec} 7}$} & \multicolumn{2}{|c|}{$1989 \mathrm{WY}$} \\
\hline & & Jun 7 & Sep 15 \\
\hline 1.06 & -- & 17.5 & 1.5 \\
\hline 1.66 & 8.0 & -- & -- \\
\hline 2.36 & 8.0 & 14.0 & 3.0 \\
\hline 4.36 & 9.0 & 11.0 & 4.0 \\
\hline 6.36 & 9.5 & 9.0 & 4.0 \\
\hline 8.36 & 10.0 & 8.0 & 5.5 \\
\hline 10.36 & 10.5 & 8.0 & 6.0 \\
\hline 12.36 & 11.0 & 8.0 & 7.0 \\
\hline 14.36 & 11.0 & 8.0 & 7.5 \\
\hline 16.36 & 11.0 & 8.5 & 8.0 \\
\hline 18.36 & 11.0 & 8.5 & 8.5 \\
\hline 20.36 & 11.0 & 8.5 & 9.0 \\
\hline 22.36 & 11.0 & 9.0 & 9.0 \\
\hline 24.36 & 10.5 & 9.0 & 9.0 \\
\hline 26.36 & 10.5 & 9.0 & 9.0 \\
\hline 28.36 & 10.5 & 9.0 & 9.0 \\
\hline 30.36 & 10.5 & 9.0 & 9.0 \\
\hline 32.36 & 10.5 & 9.0 & 9.0 \\
\hline 34.36 & 10.5 & 9.0 & 9.0 \\
\hline 36.36 & 10.5 & 9.0 & 9.0 \\
\hline 38.36 & 10.5 & 9.0 & 9.0 \\
\hline 40.36 & 10.5 & 9.0 & 9.0 \\
\hline 40.56 & -- & 9.0 & -- \\
\hline
\end{tabular}

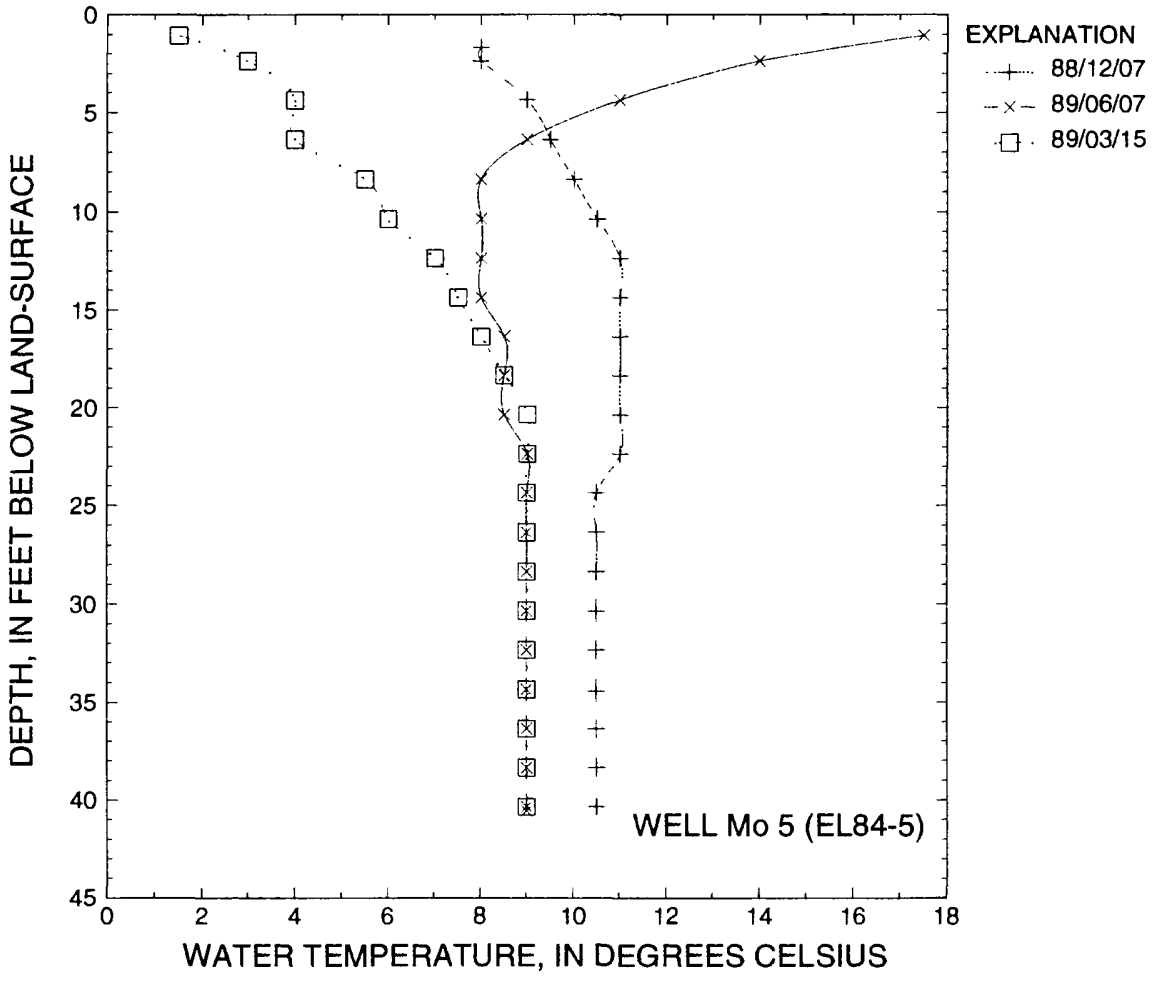




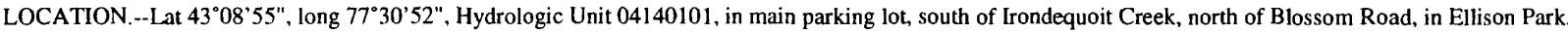
Owner: U.S. Geological Survey. AQUIFER.-Water-table aquifer in alluvium of Holocene age.

WELL CHARACTERISTICS.--Drilled observation well, diameter 2 in, depth $16 \mathrm{ft}$, cased to $13 \mathrm{ft}$, screened $13 \mathrm{ft}$ to $16 \mathrm{ft}$.

INSTRUMENTATION.--Monthly measurement with chalked tape by Monroe County Environmental Health Laboratory personnel and occasional measurement by USGS personnel.

DATUM.--Elevation of land-surface datum is $251.1 \mathrm{ft}$ above National Geodetic Vertical Datum of 1929. Measuring point: arrow at top of casing, $4.53 \mathrm{ft}$ above landsurface datum; prior to September 30,1985, $4.26 \mathrm{ft}$ above land-surface datum.

REMARKS.--This well is also a water-quality observation well. Water levels are monitored monthly and water samples taken quarterly by the Monroe County Environmental Health Laboratory. Water-temperature profiles are also taken by MCEHL on a quarterly basis.

PERIOD OF RECORD.--September 1984 to August 1989 (discontinued).

EXTREMES FOR PERIOD SEPTEMBER 1984 TO AUGUST 1989.--Highest water level measured, 1.64 feet above land-surface datum, February 24, 19?5; lowest measured, 2.22 feet below land-surface datum, August 31, 1989.

WATER LEVEL, IN FEET BELOW LAND-SURFACE DATUM,

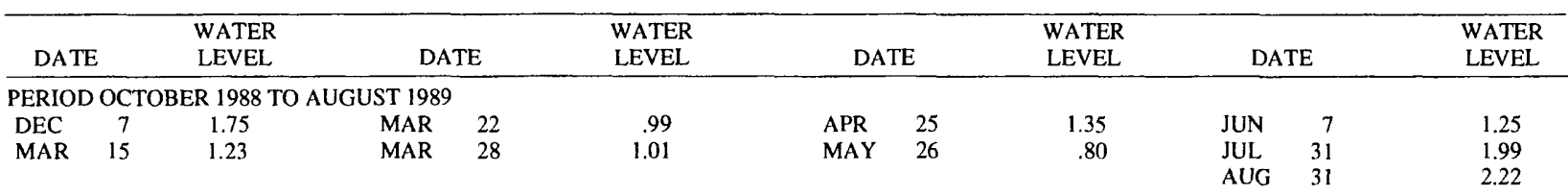

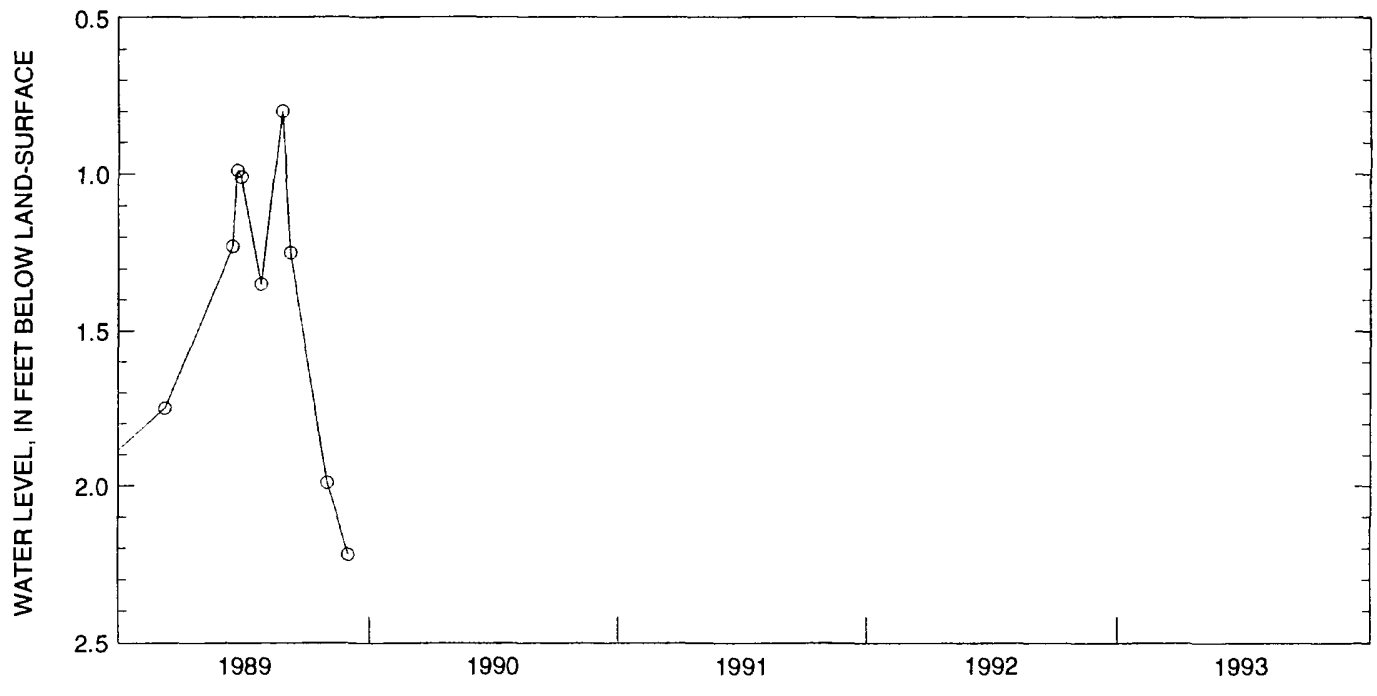


PERIOD OF RECORD.-- January 1986 to August 1989 (discontinued).

CHEMICAL DATA: 1986(a) 1987-89(b).

ORGANIC DATA: OC.--1986(a) 1987-89(b).

NUTRIENT DATA: 1986(a) 1987-89(b).

BIOLOGICAL DATA:

Bacteria.--1986(a) 1987-89(b).

COOPERATION-- Water-quality samples were collected and analyzed by the Monroe County Environmental Health Laboratory at Rochester, NY.

WATER QUALTY DATA, PERIOD OCTOBER 1988 TO JUNE 1989

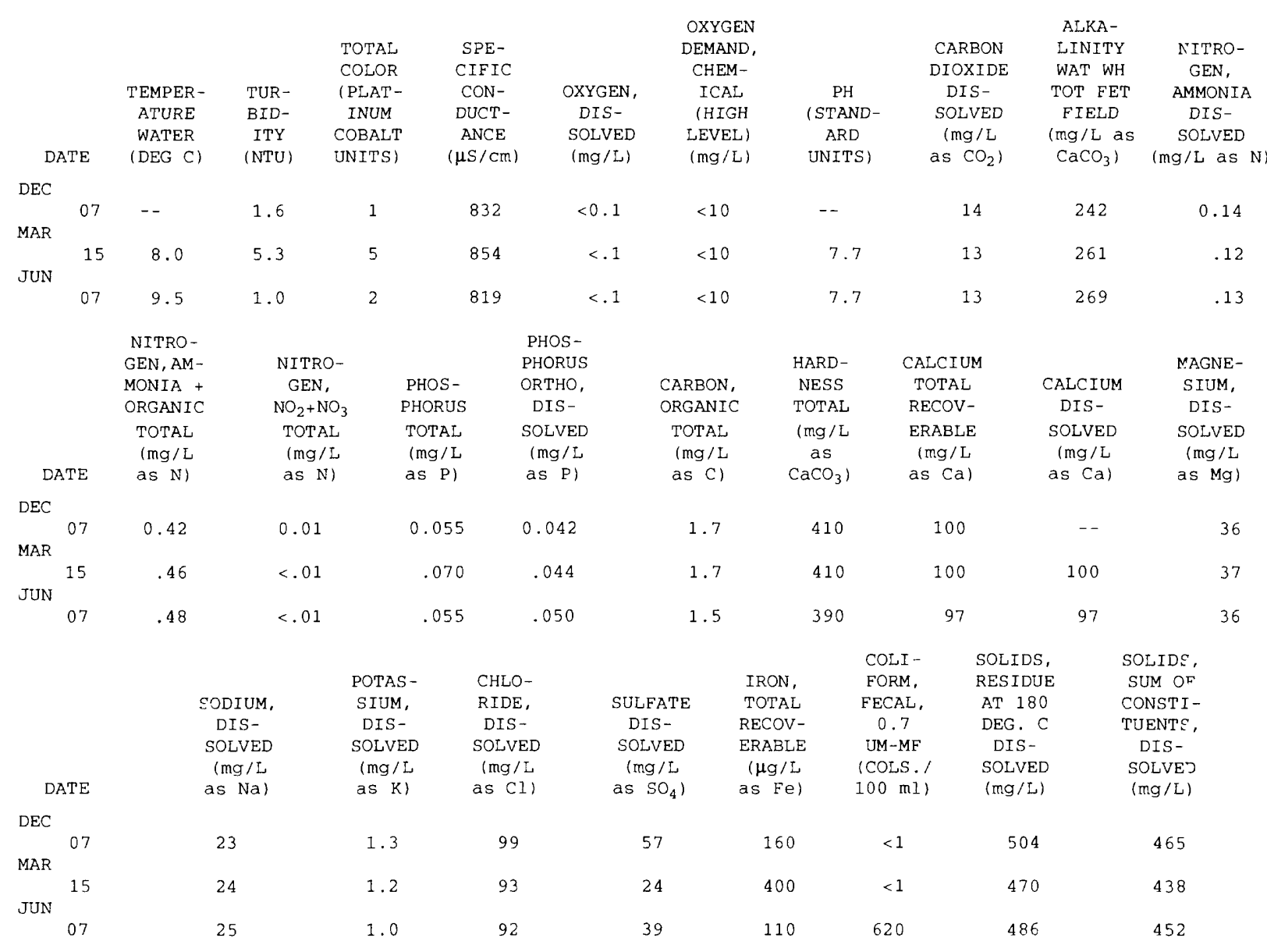


GROUND-WATER TEMPERATURE PROFILES

Ellison park

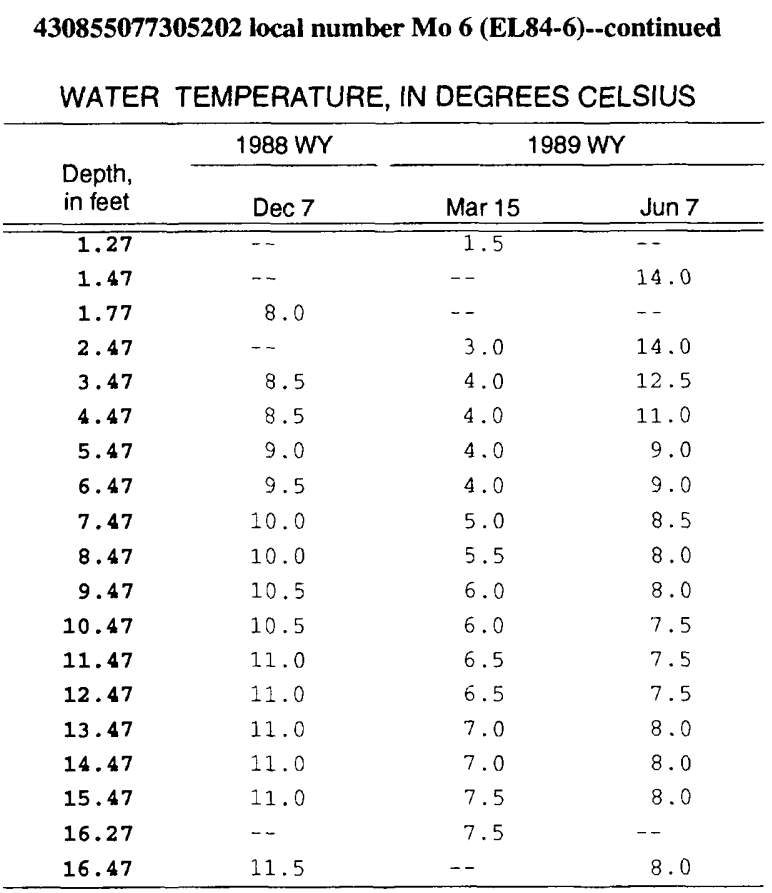

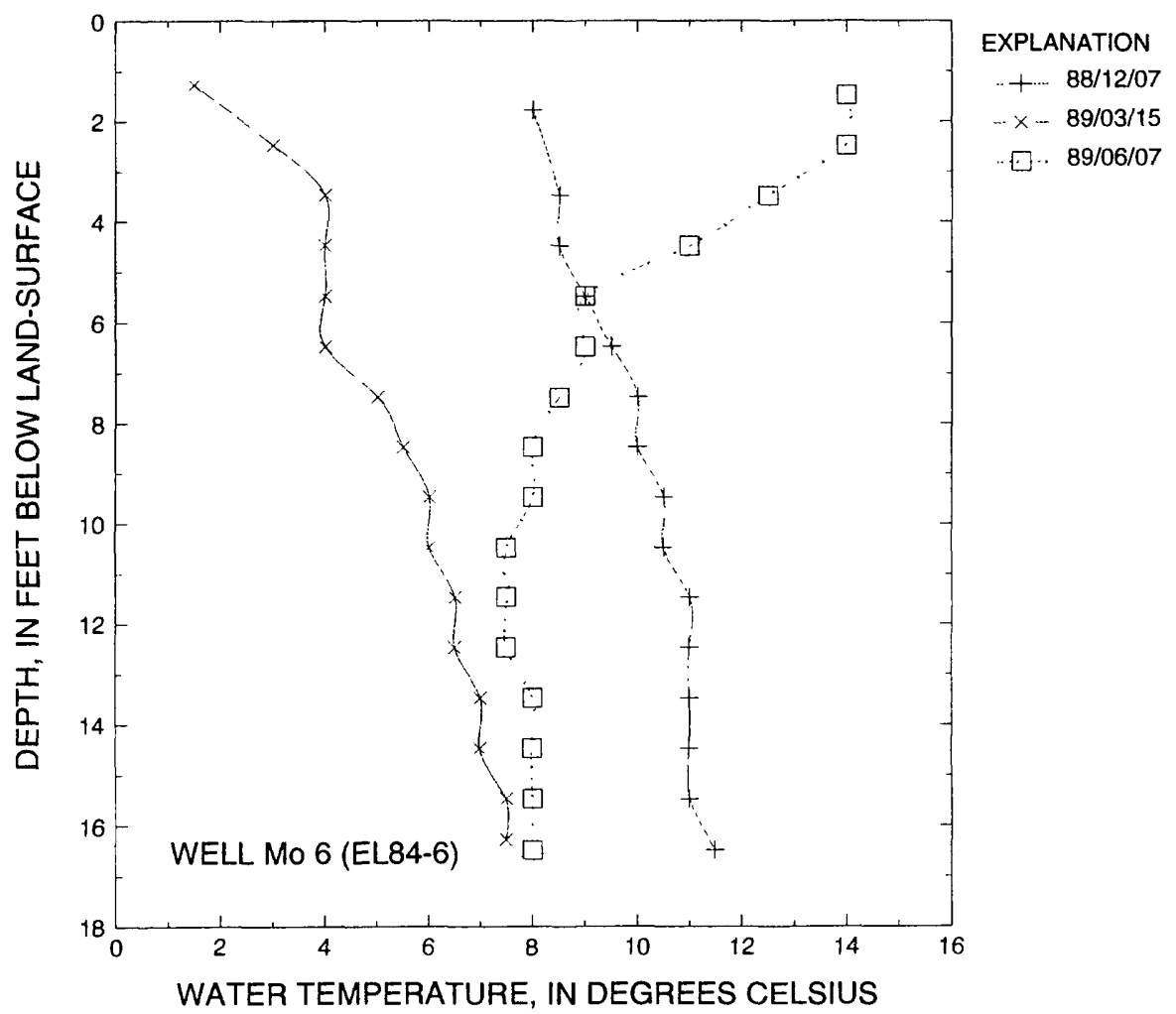


LOCATION.--Lat $43^{\circ} 09^{\prime} 32^{\prime \prime}$, long $77^{\circ} 31^{\prime} 15^{\prime \prime}$, Hydrologic Unit 04140101 , at top of right bank about $400 \mathrm{ft}$ north east of bridge over Irondequoit Creek overflow channel at Old Browncroft Boulevard. Owner: U.S. Geological Survey.

AQUIFER.--Confined aquifer in sand and gravel of Pleistocene age.

WELL CHARACTERISTICS.--Drilled observation well, diameter 6 in, depth $215 \mathrm{ft}$, cased to $215 \mathrm{ft}$, perforated 80 to $90 \mathrm{ft}$ and 160 to $170 \mathrm{ft}$, openended at $215 \mathrm{ft}$. INSTRUMENTATION.--Monthly measurement with chalked tape by Monroe County Environmental Health Laboratory personnel; periodic measurement by USGS personnel.

DATUM.--Elevation of land-surface datum is $266.58 \mathrm{ft}$ above sea level. Measuring point: arrow at top of casing, $1.80 \mathrm{ft}$ above land-surface datum.

REMARKS.--Well also sampled for water-quality.

PERIOD OF RECORD.--December 1986 to current year.

EXTREMES FOR PERIOD DECEMBER 1986 TO SEPTEMBER 1993.--Highest water level measured, $15.89 \mathrm{ft}$ below land-surface datum, Apr. 30, 1993 ; lowest measured, $17.82 \mathrm{ft}$ below land-surface datum, Sept. 12, 1988, Aug. 31, 1989.

WATER LEVEL, IN FEET BELOW LAND-SURFACE DATUM,

\begin{tabular}{|c|c|c|c|c|c|c|c|c|c|c|c|}
\hline DATE & & $\begin{array}{l}\text { WATER } \\
\text { LEVEL }\end{array}$ & \multicolumn{2}{|c|}{ DATE } & \multirow[t]{2}{*}{$\begin{array}{l}\text { WATER } \\
\text { LEVEL }\end{array}$} & \multicolumn{2}{|c|}{ DATE } & \multirow[t]{2}{*}{$\begin{array}{l}\text { WATER } \\
\text { LEVEL }\end{array}$} & \multicolumn{2}{|c|}{ DATE } & $\begin{array}{l}\text { WATER } \\
\text { LEVEL }\end{array}$ \\
\hline \multicolumn{10}{|c|}{ PERIOD SEPTEMBER 1988 TO SEPTEMBER 1989} & & \\
\hline SEP & 12 & 17.82 & APR & 25 & 17.37 & JUL & 31 & 17.50 & SEP & 26 & 17.80 \\
\hline FEB & 28 & 17.69 & MAY & 26 & 16.95 & AUG & 31 & 17.82 & & 29 & 17.79 \\
\hline MAR & 28 & 17.45 & JUN & 30 & 16.90 & & & & & & \\
\hline
\end{tabular}

WATER YEAR OCTOBER 1989 TO SEPTEMBER 1990

$\begin{array}{llllll}\text { OCT } & 31 & 17.71 & \text { FEB } & 28 & 16.90 \\ \text { NOV } & 30 & 17.58 & \text { MAR } & 30 & 17.10 \\ \text { DEC } & 29 & 17.78 & \text { APR } & 24 & 16.72 \\ \text { JAN } & 30 & 17.47 & & 26 & 16.76\end{array}$

WATER YEAR OCTOBER 1990 TO SEPTEMBER 1991

$\begin{array}{llllll}\text { OCT } & 30 & 17.41 & \text { JAN } & 23 & 16.65 \\ \text { NOV } & 28 & 17.26 & \text { FEB } & 26 & 16.88\end{array}$

$\begin{array}{lr}\text { JAN } \quad 9 & 16.90\end{array}$

APR 4

WATER YEAR OCTOBER 1991 TO SEPTEMBER 1992

\begin{tabular}{|c|c|c|c|c|c|}
\hline OCT & 29 & 17.74 & JAN & 31 & 17.47 \\
\hline NOV & 27 & 17.51 & MAR & 18 & 17.45 \\
\hline DEC & 18 & 17.58 & MAY & 1 & 16.87 \\
\hline \multicolumn{6}{|c|}{ WATER YEAR OCTOBER 1992 TO SEPTEMBER 1993} \\
\hline OCT & 28 & 17.17 & FEB & 18 & 16.78 \\
\hline NOV & 24 & 16.91 & & 26 & 16.89 \\
\hline DEC & 8 & 17.12 & MAR & 9 & 16.83 \\
\hline \multirow[t]{2}{*}{ JAN } & 6 & 16.44 & APR & 14 & 16.04 \\
\hline & 27 & 16.45 & & 30 & 15.89 \\
\hline
\end{tabular}

\subsection{5}

16.57

16.78

16.89

16.04

15.89

$\begin{array}{lll}\text { MAY } & 31 & 16.73 \\ \text { JUN } & 22 & 17.07 \\ \text { JUL } & 25 & 17.52\end{array}$

AUG $28 \quad 17.51$

$\begin{array}{lll}M A Y & 28 & 16.71\end{array}$

$\begin{array}{lll}\text { JUN } & 12 & 16.85\end{array}$

$\begin{array}{lll}\text { JUL } & 26 & 17.58 \\ \text { AUG } & 22 & 17.75 \\ \text { SEP } & 11 & 17.80\end{array}$

$\begin{array}{lll}\text { MAY } & 28 & 17.33\end{array}$

17.28

$\begin{array}{lll}\text { JUN } & 24 & 17.28 \\ & 21 & 17.15\end{array}$

16.91

$\begin{array}{lll}\text { MAY } & 25 \quad 16.21\end{array}$

16.30

SEP 15

17.46

$\begin{array}{lll}\text { JUL } & 20 & 16.87\end{array}$

$\begin{array}{lll}\text { AUG } & 27 & 17.05 \\ & & 17.40\end{array}$

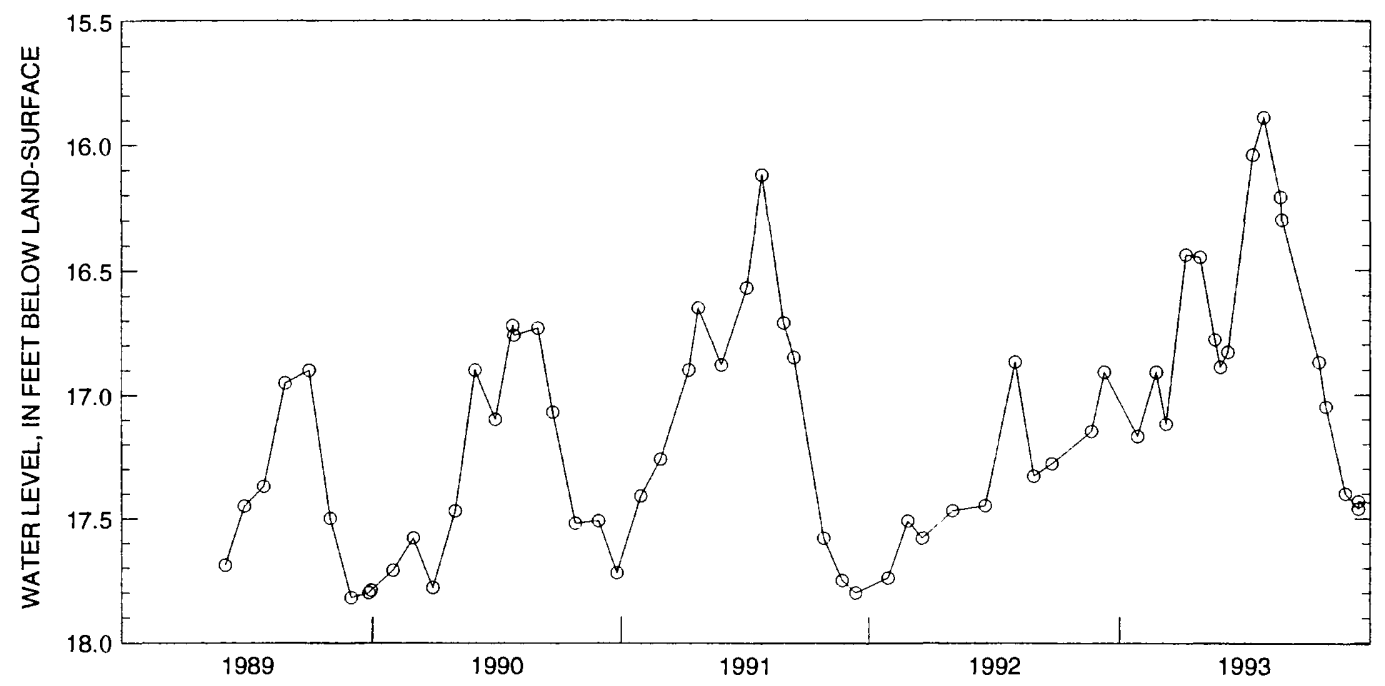


PERIOD OF RECORD.-- January 1991 to current year.

CHEMICAL DATA: 1991-93(b).

ORGANIC DATA: OC.--1991-93(b).

NUTRIENT DATA: 1991-93(b).

COOPERATION-- Water-quality samples were collected and analyzed by the Monroe County Environmental Health Laboratory at Rochester, NY.

\section{WATER QUAUTY DATA, PERIOD JANUARY 1991 TO SEPTEMBER 1992}

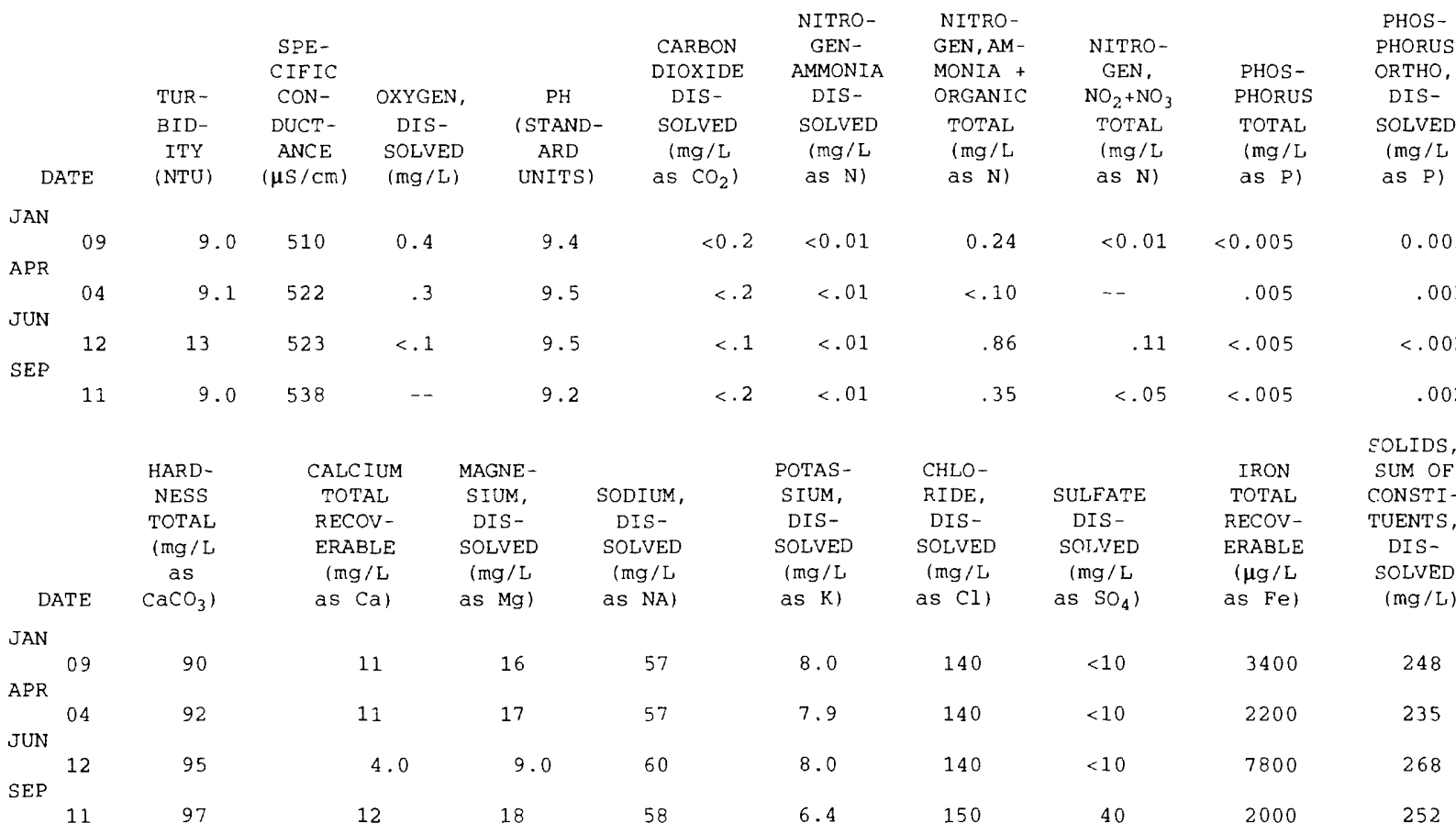

WATER QUALITY DATA, WATER YEAR OCTOBER 1991 TO SEPTEMBER 1992

PHOS-


430932077311501. Local number Mo 659 (B86-2)--continued

WATER QUALTY DATA, WATER YEAR OCTOBER 1992 TO SEPTEMBER 1993




GROUND-WATER TEMPERATURE PROFILES

Ellison park

430932077311501. Local number Mo 659 (B86-2)--continued

WATER TEMPERATURE, IN DEGREES CELSIUS

\begin{tabular}{|c|c|c|c|c|c|c|c|c|c|c|c|c|}
\hline \multirow{2}{*}{$\begin{array}{l}\text { Depth, } \\
\text { in feet }\end{array}$} & \multicolumn{4}{|c|}{$1991 \mathrm{WY}$} & \multicolumn{4}{|c|}{$1992 \mathrm{WY}$} & \multicolumn{4}{|c|}{$1993 \mathrm{WY}$} \\
\hline & $\operatorname{Jan} 9$ & Apr 4 & Jun 12 & Sep 11 & Dec 18 & Mar 18 & Jun 24 & Sep 9 & Dec 8 & Mar 9 & Jul 7 & Sep 15 \\
\hline 15.70 & -- & -- & -- & 10.0 & -- & -- & -- & -- & $\ldots$ & -- & 16.5 & -- \\
\hline 16.60 & - & 9.5 & -- & - & -- & -- & -- & -- & -- & -- & - & -- \\
\hline 16.90 & 9.0 & $\ldots$ & 11.5 & - & - & -- & $\ldots$ & 11.0 & 10.0 & 8.5 & 11.0 &.- \\
\hline 17.50 & -- & -- & -- & 12.0 & 4.5 & 9.0 & 9.5 & -- & -- & -- & - & 10.5 \\
\hline 18.20 & 9.5 & 9.0 & 10.0 & -- & 9.0 & 9.0 & 9.0 & 10.5 & 10.0 & 9.0 & 9.5 & 10.0 \\
\hline 19.20 & -- & - & $\ldots$ & -- & $-\cdots$ & 9.0 & 9.0 & - & 9.5 & 9.0 & 9.0 & 9.5 \\
\hline 20.20 & 9.5 & 9.0 & 10.0 & 10.5 & 9.5 & 9.0 & 9.0 & 10.0 & 9.5 & 9.0 & 9.0 & 9.0 \\
\hline 22.20 & 9.5 & 9.0 & 9.5 & 10.0 & 9.5 & 9.0 & 8.5 & 10.0 & 9.5 & 9.0 & 9.0 & 9.0 \\
\hline 24.20 & 9.5 & 9.0 & 9.5 & 10.3 & 9.5 & 9.5 & 8.5 & 9.5 & 9.5 & 9.5 & 9.0 & 8.5 \\
\hline 26.20 & 9.5 & 9.0 & 9.5 & 9.5 & 9.5 & 9.5 & 8.5 & 9.5 & 9.5 & 9.5 & 9.0 & 8.5 \\
\hline 28.20 & 9.5 & 9.0 & 9.5 & 9.5 & 9.5 & 9.5 & 8.5 & 9.5 & 9.5 & 9.5 & 9.0 & 8.5 \\
\hline 30.20 & 9.5 & 9.0 & 9.5 & 9.5 & 9.5 & 9.5 & 9.0 & 9.5 & 9.5 & 9.5 & 9.0 & 8.5 \\
\hline 32.20 & 9.5 & 9.0 & -- & 9.5 & 9.0 & 9.5 & 9.0 & 9.5 & 9.5 & 9.5 & 9.0 & 8.5 \\
\hline 34.20 & 9.0 & 9.0 & 9.5 & 9.5 & 9.0 & 9.5 & 9.0 & 9.5 & 9.5 & -- & 9.0 & 8.5 \\
\hline 36.20 & 9.0 & 9.0 & -- & 9.5 & 9.0 & 9.5 & 9.0 & 9.5 & 9.5 & -- & 9.0 & 8.5 \\
\hline 38.20 & 9.0 & 9.0 & 9.5 & 9.5 & 9.0 & 9.5 & 9.0 & 9.5 & 9.0 & 9.5 & 9.0 & 8.5 \\
\hline 40.20 & 9.0 & 9.0 & $-\infty$ & 9.5 & 9.0 & 9.5 & 9.0 & $\ldots$ & 9.0 & -- & 9.0 & $\ldots$ \\
\hline 42.20 & 9.0 & 9.0 & 9.5 & 9.5 & 9.0 & 9.5 & 9.0 & 9.5 & 9.0 & 9.5 & 9.0 & 8.5 \\
\hline 44.20 & 9.0 & 9.0 & - & 9.5 & 9.0 & 9.0 & 9.0 & -- & 9.0 & -- & 9.0 & $\ldots$ \\
\hline 46.20 & 9.0 & 9.0 & 9.5 & 9.5 & 9.0 & 9.0 & 9.0 & 9.5 & 9.0 & - & 9.0 & -- \\
\hline 48.20 & 9.0 & 9.0 & - & 9.5 & 9.0 & 9.0 & 9.0 & $\ldots$ & 9.0 & 9.5 & 9.5 & 8.5 \\
\hline 50.20 & 9.0 & $\ldots$ & 9.5 & -- & -- & 9.0 & 9.0 & 9.5 & 9.0 & - & -- & -- \\
\hline 52.20 & 9.0 & 9.0 & -- & 9.5 & 9.0 & 9.0 & 9.0 & -- & 9.0 & 9.5 & 9.5 & 8.5 \\
\hline 54.20 & 9.0 & $\ldots$ & 9.5 & -- & $\ldots$ & 9.0 & 9.0 & 9.5 & 9.0 & -- & - & $\ldots$ \\
\hline 56.20 & 9.0 & -- & -- & 9.5 & -- & 9.0 & 9.0 & -- & 9.0 & -- & -- & -- \\
\hline 58.20 & 9.0 & 9.0 & 9.5 & -- & 9.0 & -- & -- & 9.5 & -- & 9.5 & 9.5 & 9.0 \\
\hline 60.20 & 9.0 & -- & -- & 9.5 & - & 9.5 & 9.0 & -- & 9.0 & $\ldots$ & $\ldots$ & -- \\
\hline 62.20 & $\ldots$ & 9.0 & 9.5 & -- & 9.0 & & & -- & -- & 9.5 & 9.5 & - \\
\hline 64.20 & 9.0 & -- & $\ldots$ & 9.5 & $\ldots$ & $-\infty$ & -- & -. & -- & -- & -- & $\ldots$ \\
\hline 66.20 & $-\cdots$ & - & 9.5 & -- & - & 9.5 & 9.0 & -- & 8.5 & -- & -- & $\ldots$ \\
\hline 68.20 & 9.0 & 9.0 & -- & 9.5 & 9.0 & - & -- & 9.5 & -- & 9.5 & 9.5 & 9.0 \\
\hline 70.20 & $\ldots$ & $-\ldots$ & 9.5 & -- & -- & -- & -- & -- & 8.0 & -- & -- & -- \\
\hline 72.20 & 9.5 & - & -- & -- & -- & 9.5 & 9.0 & -- & -- & -- & -- & -- \\
\hline 78.20 & 9.5 & 9.0 & 9.5 & 9.5 & 9.5 & -- & -- & 9.5 & -- & 9.5 & 9.5 & 9.0 \\
\hline 86.20 & -- & $-\infty$ & -- & -- & $\ldots$ & -- & -- & -- & 7.5 & $\ldots$ & -- & -- \\
\hline 96.20 & -- & -- & -- & -- & -- & -- & -- & -- & 7.0 & -. & -- & $\ldots$ \\
\hline 97.20 & -- & -- & -- & -- & -- & 9.5 & 9.5 & -- & -- & -- & $\ldots$ & -- \\
\hline 98.20 & -- & 9.0 & 9.5 & 9.5 & 9.5 & -- & -- & 9.5 & $\cdots$ & 9.5 & 9.5 & 9.0 \\
\hline 108.20 & - & 9.0 & 9.5 & 9.5 & 9.5 & 9.5 & 9.5 & 9.5 & -- & 9.5 & 9.5 & 9.0 \\
\hline 118.20 & -- & 9.0 & 9.5 & 9.5 & 9.5 & 9.5 & 9.5 & 9.5 & -- & 9.5 & 9.5 & 9.0 \\
\hline 128.20 & -- & 9.0 & 9.5 & 9.5 & 9.5 & 9.5 & 9.5 & 9.5 & 7.0 & 9.5 & -- & 9.0 \\
\hline 138.20 & - & 9.0 & 9.5 & 10.0 & 9.5 & 9.5 & 9.5 & 9.5 & 6.5 & 9.5 & - & 9.0 \\
\hline 142.20 & -- & -- & -- & -- & -- & -- & 9.5 & -- & -- & -- & -- & -- \\
\hline 146.20 & -- & -- & -- & - & -- & -- & -- & -- & 6.5 & -- & -- & -- \\
\hline 147.20 & $\ldots$ & -- & -- & -- & -- & 9.5 & -- & -- & $\ldots$ & -- & - & -- \\
\hline 148.20 & -- & 9.5 & 10.0 & 10.0 & 9.5 & -- & -- & 9.5 & -- & 9.5 & -- & 9.0 \\
\hline 158.20 & - & 9.5 & 10.0 & 10.0 & 9.5 & 9.5 & -- & 10.0 & 6.5 & 10.0 & $\cdots$ & 9.0 \\
\hline 161.20 & -- & 9.5 & 10.0 & -- & -- & 9.5 & -- & - & $-\infty$ & $\ldots$ & -- & -- \\
\hline
\end{tabular}




\section{GROUND-WATER TEMPERATURE PROFILES}

Ellison park

430932077311501. Local number Mo 659 (B86-2)--continued

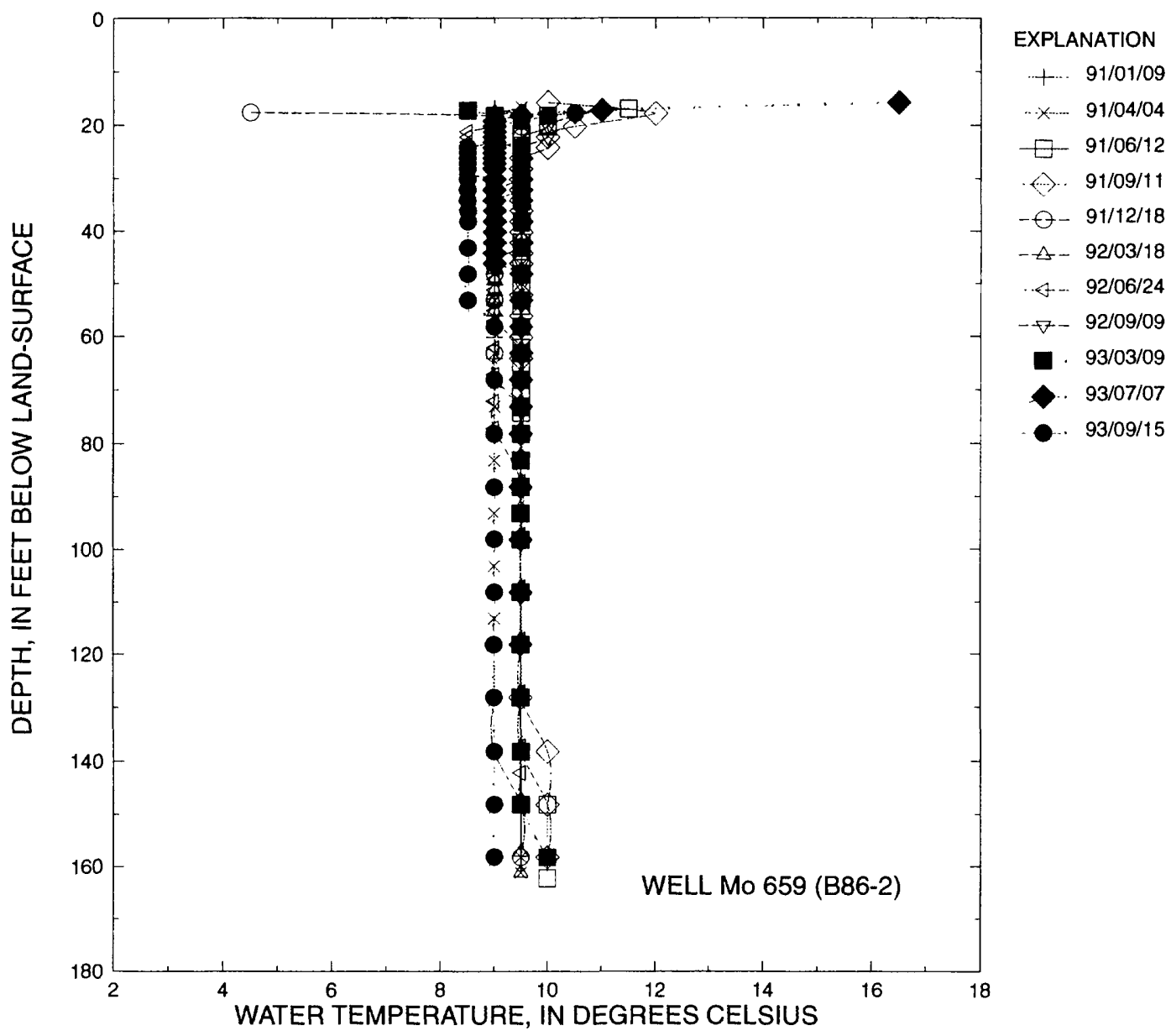




\section{Local number Mo 663}

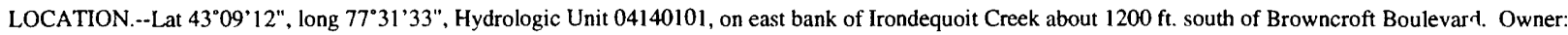

U.S. Geological Survey.

AQUIFER.--Unconfined aquifer in fluvial sediments of Holocene age.

WELL CHARACTERISTICS.--Drilled observation well, diameter 2 in, depth $10 \mathrm{ft}$, cased to $10 \mathrm{ft}$, screened $7.5 \mathrm{ft}$ to $10 \mathrm{ft}$.

INSTRUMENTATION.--Monthly measurement with chalked tape by Monroe County Environmental Health Laboratory personnel.

DATUM.--Elevation of land-surface datum is $251.16 \mathrm{ft}$ above sea level. Measuring point: arrow at top of casing, $3.60 \mathrm{ft}$ above land-surface datum.

REMARKS.--Well also sampled for water quality.

PERIOD OF RECORD.--September 1988 to current year.

EXTREMES FOR PERIOD SEPTEMBER 1988 TO SEPTEMBER 1993.--Highest water level measured, $1.02 \mathrm{ft}$ below land-surface datum, April 26, 1991; lowest measured, $4.32 \mathrm{ft}$ below land-surface datum, Aug. 31, 1989, Sept. 11, 1991.

WATER LEVEL, IN FEET BELOW LAND-SURFACE DATUM,

\begin{tabular}{|c|c|c|c|c|c|c|c|c|c|c|c|}
\hline \multicolumn{2}{|c|}{ DATE } & $\begin{array}{l}\text { WATER } \\
\text { LEVEL }\end{array}$ & \multicolumn{2}{|c|}{ DATE } & \multirow[t]{2}{*}{$\begin{array}{l}\text { WATER } \\
\text { LEVEL }\end{array}$} & \multicolumn{2}{|c|}{ DATE } & \multirow[t]{2}{*}{$\begin{array}{l}\text { WATER } \\
\text { LEVEL }\end{array}$} & \multicolumn{2}{|c|}{ DATE } & $\begin{array}{l}\text { WATER } \\
\text { LEVEL } \\
\end{array}$ \\
\hline \multicolumn{10}{|c|}{ PERIOD SEPTEMBER 1988 TO SEPTEMBER 1989} & & \\
\hline SEP & 07 & 3.46 & MAR & 28 & 2.12 & JUN & 30 & 2.96 & SEP & 29 & 4.00 \\
\hline OCT & 26 & 2.80 & APR & 25 & 3.27 & JUL & 31 & 3.98 & & & \\
\hline MAR & 22 & 1.80 & MAY & 26 & 2.60 & AUG & 31 & 4.32 & & & \\
\hline \multicolumn{12}{|c|}{ WATER YEAR OCTOBER 1989 TO SEPTEMBER 1990} \\
\hline OCT & 17 & 3.64 & MAR & 30 & 2.72 & MAY & 31 & 2.71 & AUG & 28 & 3.92 \\
\hline JAN & 30 & 2.80 & APR & 24 & 2.28 & JUN & 22 & 3.55 & SEP & 25 & 3.97 \\
\hline FEB & 26 & 1.90 & MAY & 24 & 1.92 & JUL & 25 & 3.85 & & & \\
\hline \multicolumn{12}{|c|}{ WATER YEAR OCTOBER 1990 TO SEPTEMBER 1991} \\
\hline OCT & 30 & 2.89 & APR & 3 & 1.89 & JUN & 12 & 3.35 & AUG & 22 & 4.03 \\
\hline \multirow[t]{2}{*}{ FEB } & 13 & 2.19 & & 26 & 1.02 & JUL & 9 & 3.58 & SEP & 11 & 4.32 \\
\hline & 26 & 2.19 & MAY & 7 & 1.84 & & 26 & 4.12 & & & \\
\hline MAR & 22 & 1.61 & & 28 & 2.70 & AUG & 21 & 3.82 & & & \\
\hline \multicolumn{12}{|c|}{ WATER YEAR OCTOBER 1991 TO SEPTEMBER 1992} \\
\hline \multirow[t]{2}{*}{ OCT } & 29 & 4.05 & JAN & 31 & 2.84 & MAY & 28 & 3.08 & AUG & 21 & 3.12 \\
\hline & 31 & 3.81 & MAR & 18 & 2.36 & JUN & 24 & 3.54 & SEP & 9 & 2.85 \\
\hline DEC & 18 & 3.24 & MAY & 1 & 2.32 & JUL & 21 & 1.94 & & & \\
\hline \multicolumn{12}{|c|}{ WATER YEAR OCTOBER 1992 TO SEPTEMBER 1993} \\
\hline OCT & 28 & 2.44 & JAN & 27 & 1.25 & APR & 30 & 1.50 & JUL & 30 & 3.52 \\
\hline NOV & 24 & 1.07 & FEB & 26 & 2.43 & MAY & 27 & 2.55 & AUG & 27 & 4.02 \\
\hline DEC & 8 & 2.31 & MAR & 9 & 1.48 & JUL & 6 & 2.14 & SEP & 15 & 3.67 \\
\hline
\end{tabular}

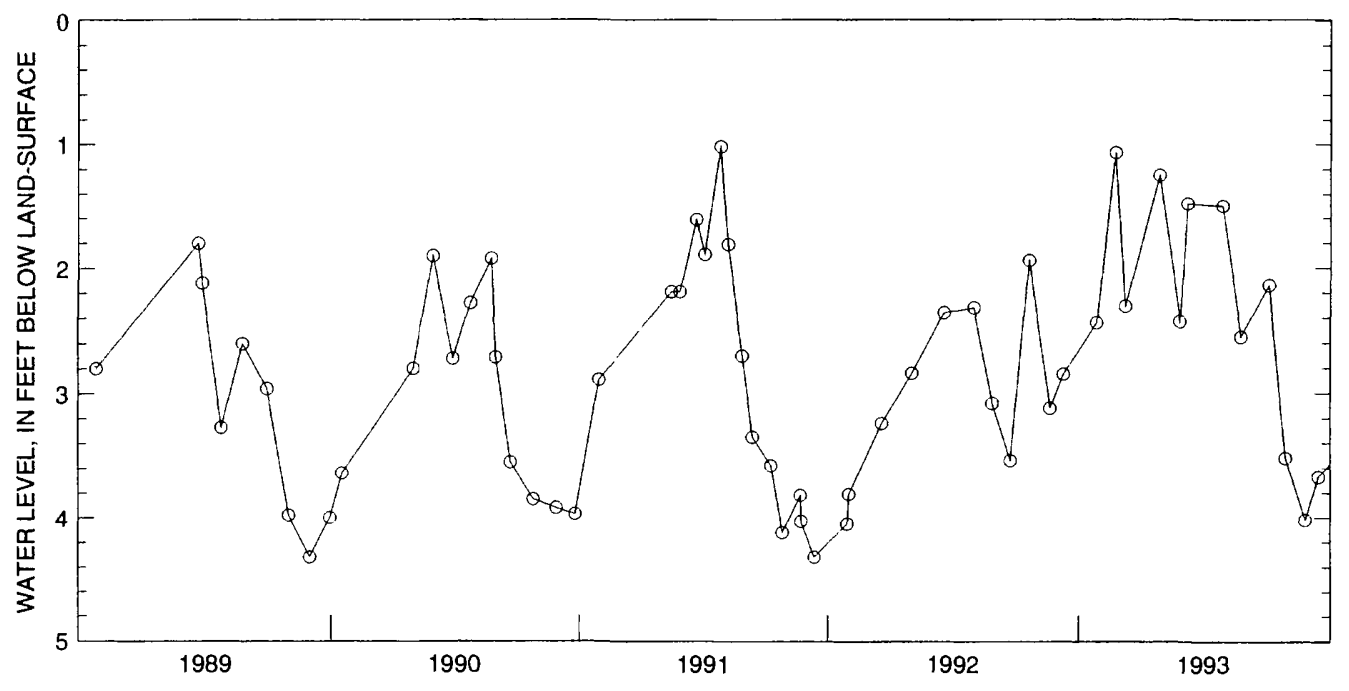


PERIOD OF RECORD.-- April 1991 to current year.

CHEMICAL DATA: 1991-93(b).

ORGANIC DATA: OC.--1991-93(b).

NUTRIENT DATA: 1991-93(b).

COOPERATION-- Water-quality samples were collected and analyzed by the Monroe County Environmental Health Laboratory at Rochester, NY.

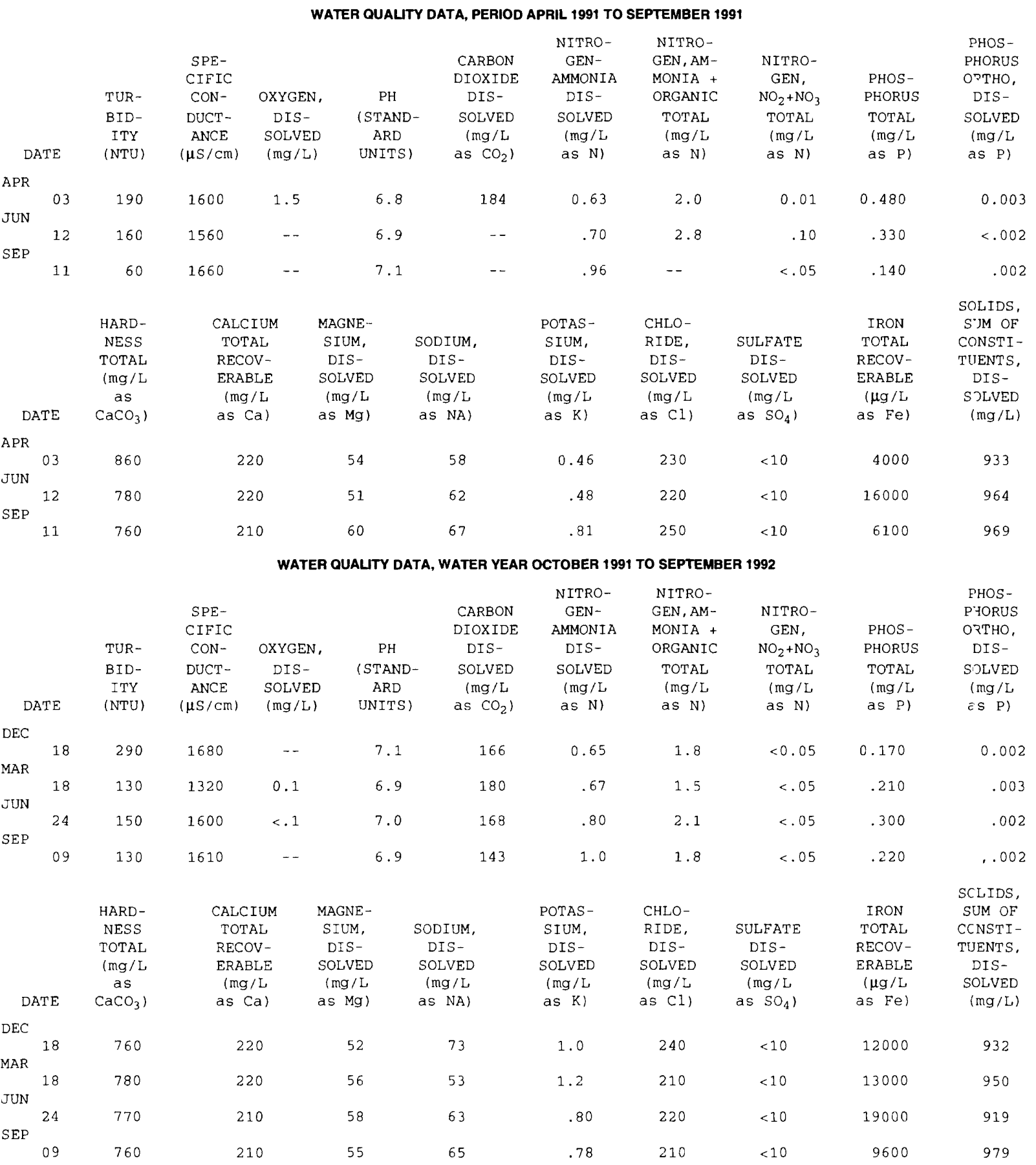


430912077313301. Local number Mo 663 (B88-3s)--continued

\section{WATER QUALITY DATA, WATER YEAR OCTOBER 1992 TO SEPTEMBER 1993}

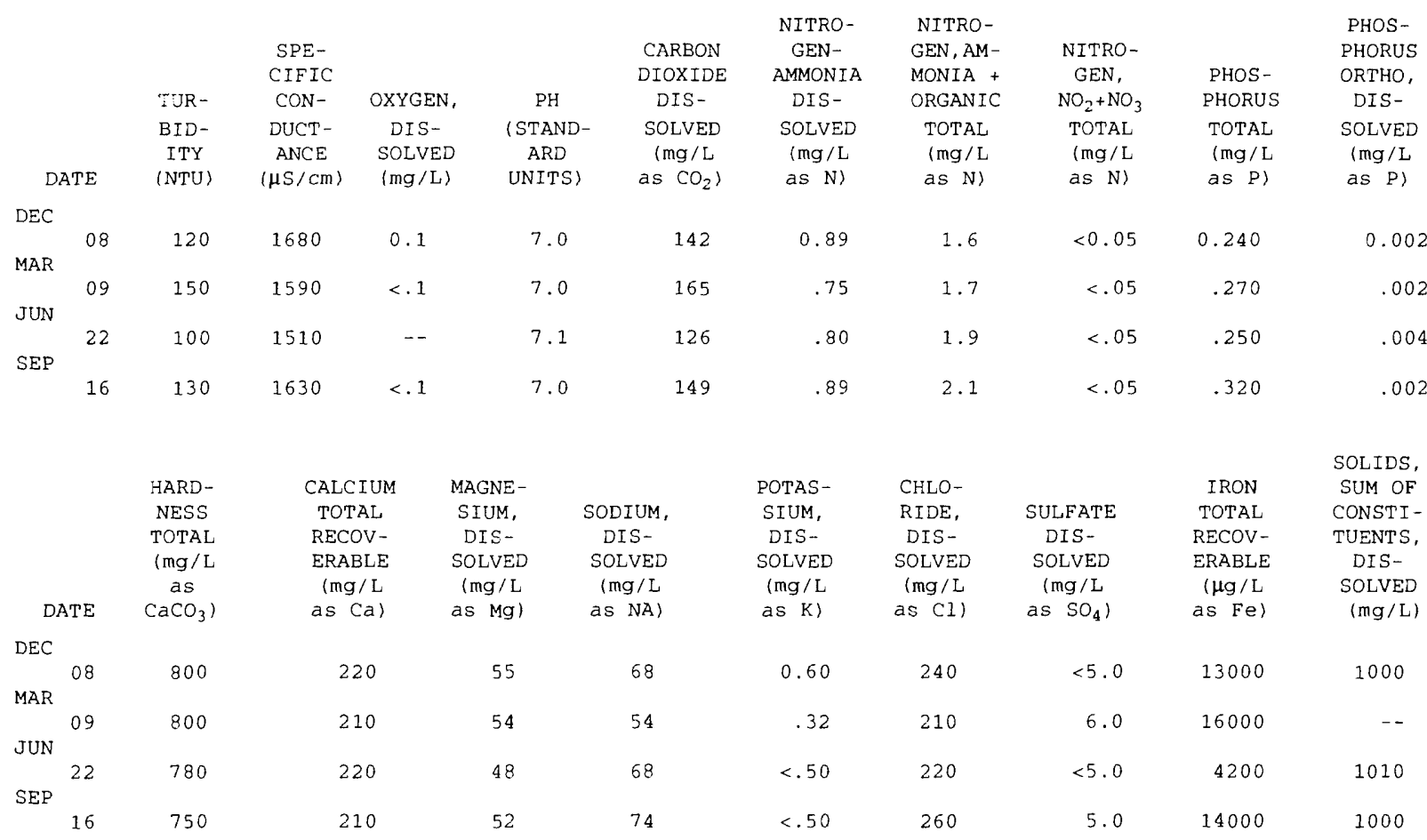


430912077313301 local number Mo 663 (B88-3s)--continued

WATER TEMPERATURE, IN DEGREES CELSIUS

\begin{tabular}{|c|c|c|c|c|c|c|c|c|c|c|c|}
\hline \multirow{2}{*}{$\begin{array}{l}\text { Depth, } \\
\text { in feet }\end{array}$} & \multicolumn{3}{|c|}{$1991 \mathrm{Wr}$} & \multicolumn{4}{|c|}{$1992 \mathrm{Wr}$} & \multicolumn{4}{|c|}{$1993 \mathrm{WY}$} \\
\hline & Apr 3 & Jun 12 & Sep 11 & Dec 18 & Mar 18 & Jun 24 & Sep 9 & Dec 8 & Mar 9 & Jun 22 & Sep 15 \\
\hline 1.90 & 7.0 & $=$ & -- & -- & - & -- & -- & - & 2.0 & -- & -- \\
\hline 2.40 & 6.5 & -- & -- & -- & 3.5 & -- & -- & 7.0 & 2.0 & 17.5 & -- \\
\hline 2.90 & -- & -- & $\ldots$ & -- & - & -- & 20.5 & -- & -- & -- & -- \\
\hline 3.40 & 6.0 & 18.0 & -- & 6.0 & 4.0 & 15.0 & 20.0 & 7.5 & 3.5 & 16.5 & -- \\
\hline 3.90 & -- & -- & -- & -- & -- & -- & -- & -- & -- & -- & 18.0 \\
\hline 4.40 & 6.0 & 15.5 & 21.5 & 7.5 & 4.0 & 14.0 & 18.0 & 8.0 & 4.0 & 15.0 & 17.5 \\
\hline 5.40 & 6.0 & 14.5 & 19.5 & 9.0 & 4.5 & 13.0 & 17.5 & 8.5 & 4.5 & 13.5 & 17.0 \\
\hline 6.40 & 6.0 & 13.5 & 18.5 & 9.0 & 4.5 & 12.0 & 16.5 & 9.0 & 4.5 & 12.5 & 16.5 \\
\hline 7.40 & 6.0 & 12.5 & 17.5 & 9.0 & 4.5 & 11.5 & 16.0 & 9.5 & 5.0 & 11.5 & 16.0 \\
\hline 8.00 & -- & -- & -- & -- & -- & 11.0 & -- & -- & -- & -- & -- \\
\hline 8.40 & 6.5 & 12.0 & 16.5 & 9.5 & 4.5 & -- & 15.0 & 9.5 & 5.5 & 11.0 & 15.0 \\
\hline 9.20 & -- & -- & -- & -- & -- & -- & -- & 10.0 & -- & 10.5 & -- \\
\hline 9.40 & 6.5 & 11.5 & 16.0 & 9.5 & 4.5 & -- & 14.5 & -- & 5.5 & 10.5 & 14.5 \\
\hline 9.60 & -- & 11.0 & -- & 10.0 & -- & -- & 14.5 & -- & -- & - & -- \\
\hline 9.90 & -- & -- & 15.5 & -- & -- & -- & -- & - & -- & -. & -- \\
\hline
\end{tabular}

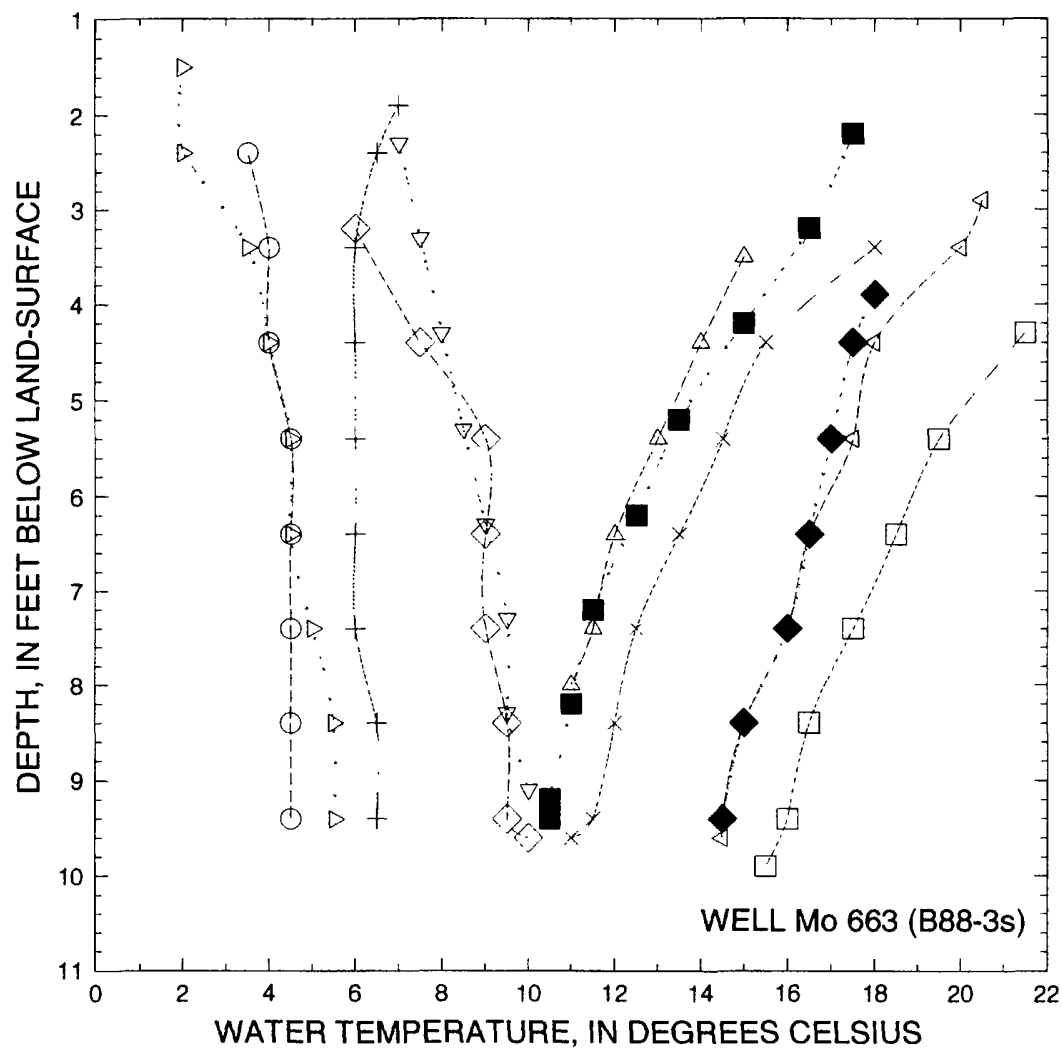

EXPLANATION

.. $+. . .91 / 04 / 03$

$\times \quad 91 / 06 / 12$

— $91 / 09 / 11$

$\rightarrow-92 / 12 / 18$

- (-). $92 / 03 / 18$

$-A-92 / 06 / 24$

$-4-92 / 09 / 09$

$\nabla \cdot 93 / 12 / 08$

.... $93 / 03 / 09$

[. . $93 / 06 / 22$

. $93 / 09 / 15$ 


\section{GROUND-WATER LEVELS}

\section{Ellison park}

\section{Local number Mo 664}

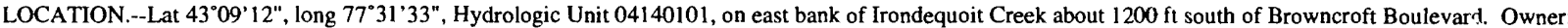
U.S. Geological Survey.

AQUIFER.--Unconfined aquifer in fluvial sediments of Holocene age.

WELL CHARACTERISTICS.--Drilled observation well, diameter 2 in, depth $27 \mathrm{ft}$, cased to $27 \mathrm{ft}$, screened $22 \mathrm{ft}$ to $27 \mathrm{ft}$

INSTRUMENTATION.--Monthly measurement with chalked tape by Monroe County Environmental Health Laboratory personnel.

DATUM.--Elevation of land-surface datum is $251.18 \mathrm{ft}$ above sea level. Measuring point: arrow at top of casing, $3.20 \mathrm{ft}$ above land-surface datum.

REMARKS.--Well also sampled for water quality.

PERIOD OF RECORD.--September 1988 to current year.

EXTREMES FOR PERIOD SEPTEMBER 1988 TO SEPTEMBER 1993.--Highest water level measured, $1.10 \mathrm{ft}$ below land-surface datum, April 26, 1991; lowest measured, $4.01 \mathrm{ft}$ below land-surface datum, Aug. 31, 1989.

WATER LEVEL, IN FEET BELOW LAND-SURFACE DATUM.

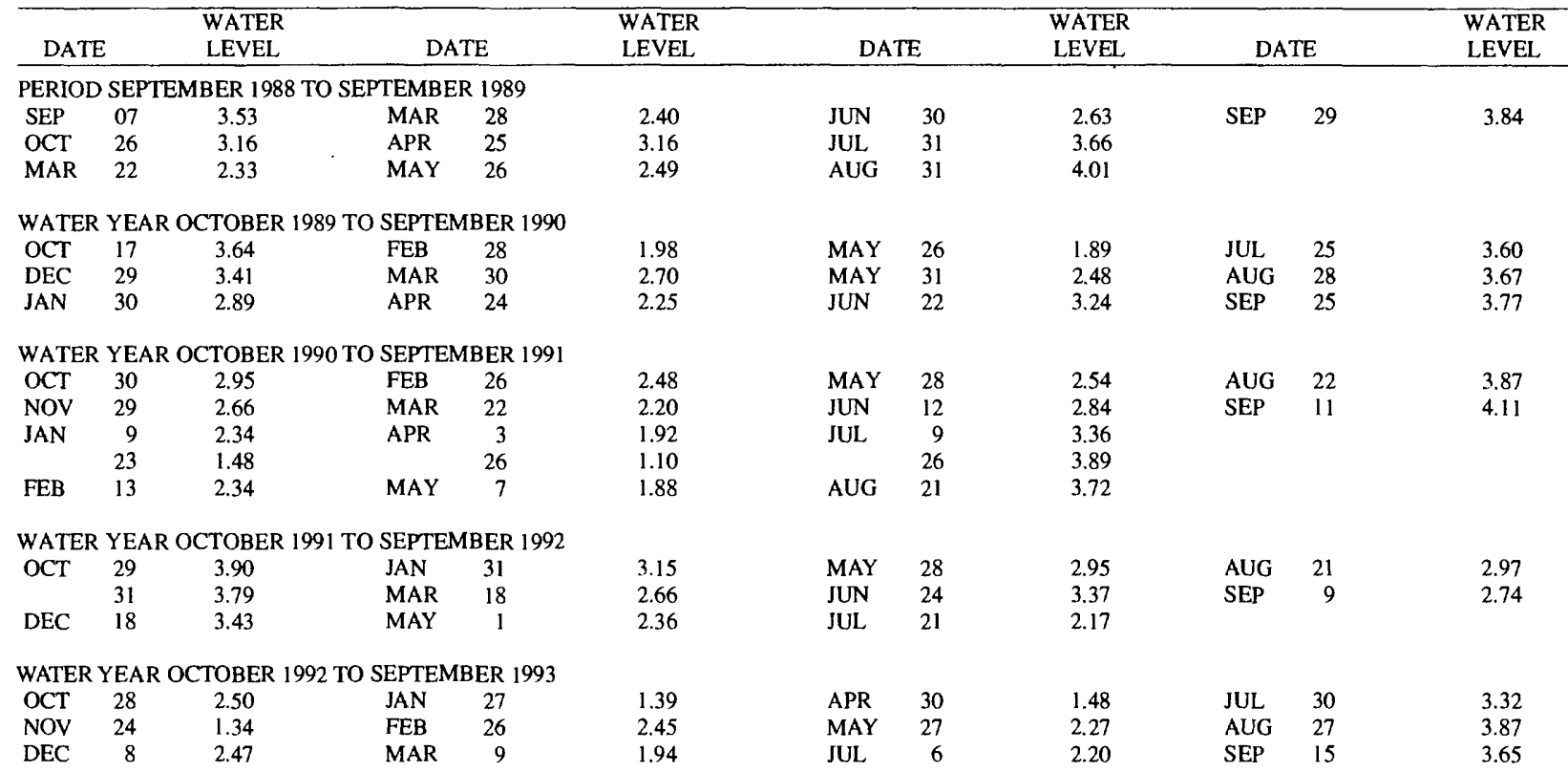

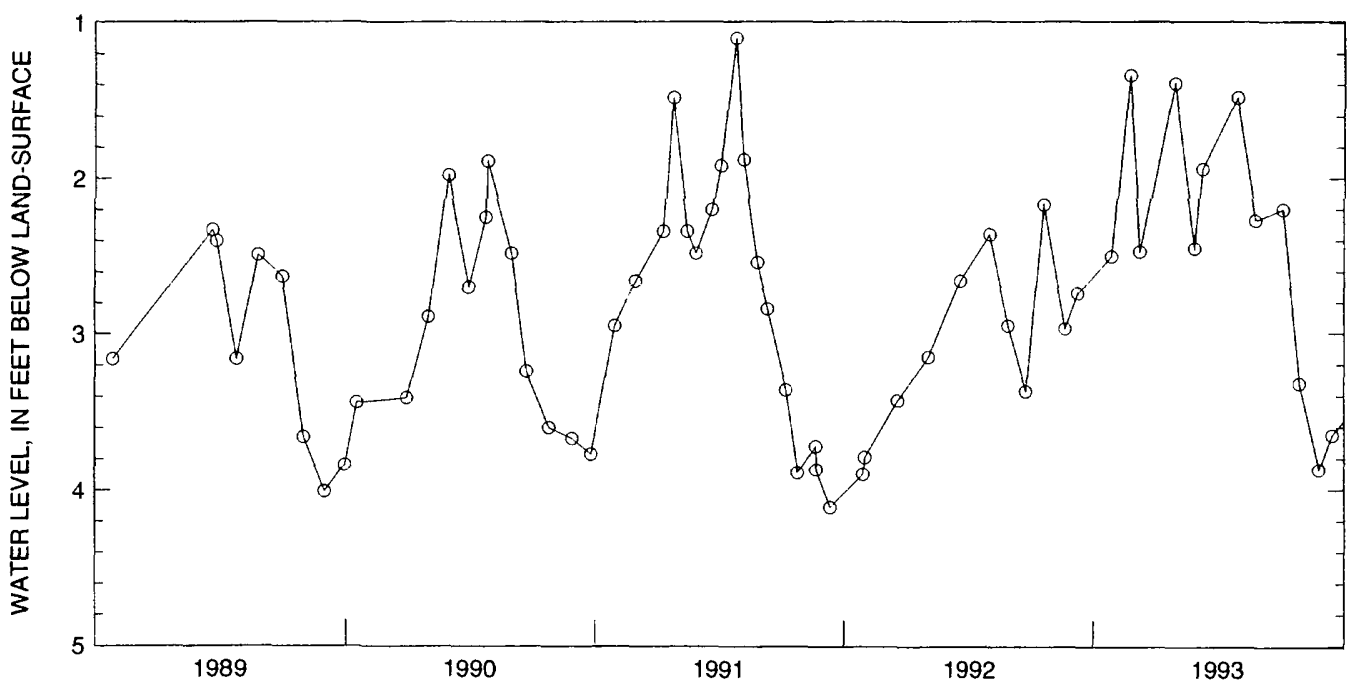


PERIOD OF RECORD.-- January 1991 to current year.

CHEMICAL DATA: 1991-93(b).

ORGANIC DATA: OC.--1991-93(b)

NUTRIENT DATA: 1991-93(b).

COOPERATION-- Water-quality samples were collected and analyzed by the Monroe County Environmental Health Laboratory at Rochester. NY WATER QUALTY DATA, PERIOD JANUARY 1991 TO SEPTEMBER 1991

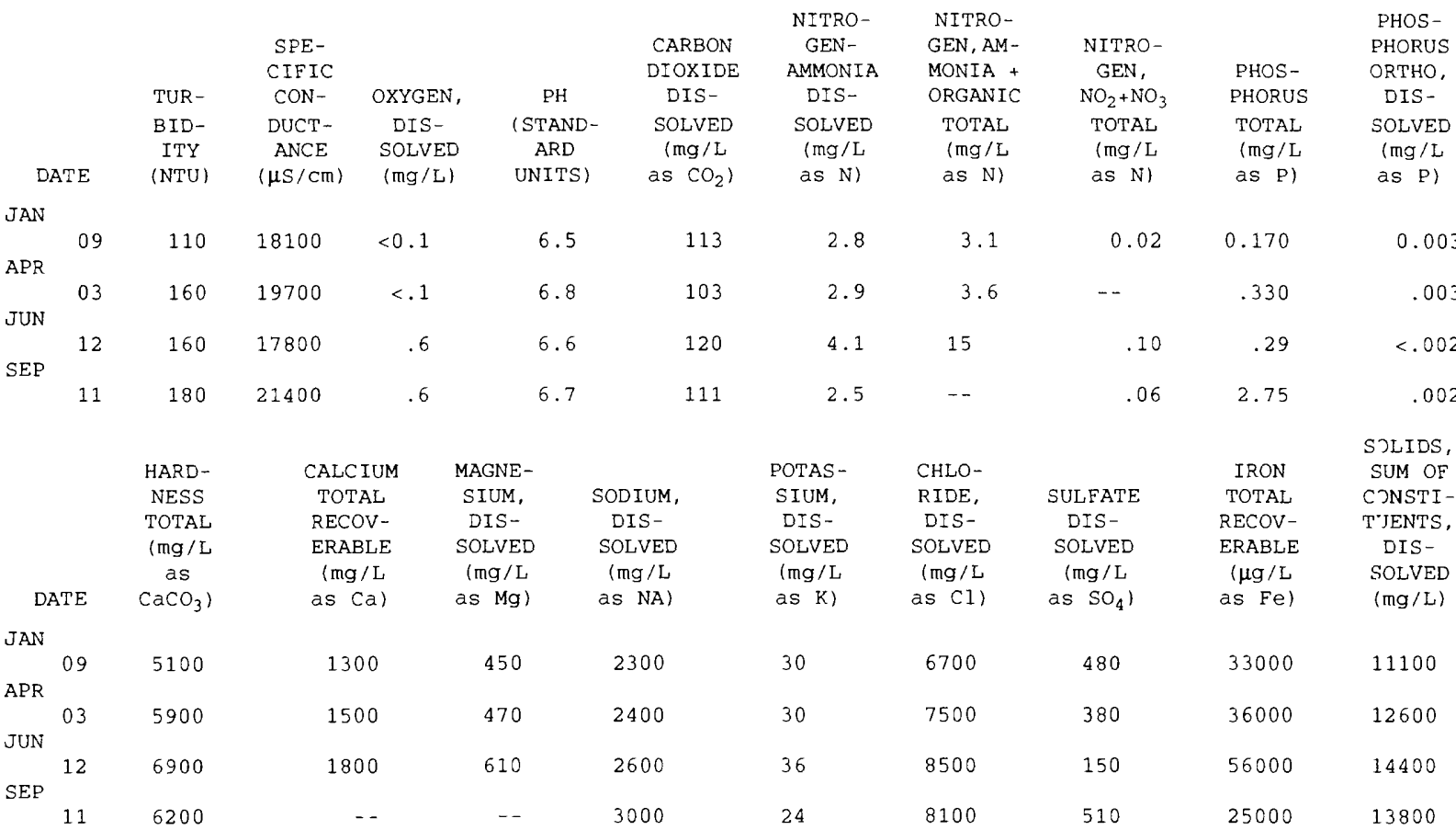

\section{WATER QUALITY DATA, WATER YEAR OCTOBER 1991 TO SEPTEMBER 1992}

PHOS-


430912077313302. Local number Mo 664 (B88-3d)--continued

WATER QUALTY DATA, WATER YEAR OCTOBER 1992 TO SEPTEMBER 1993

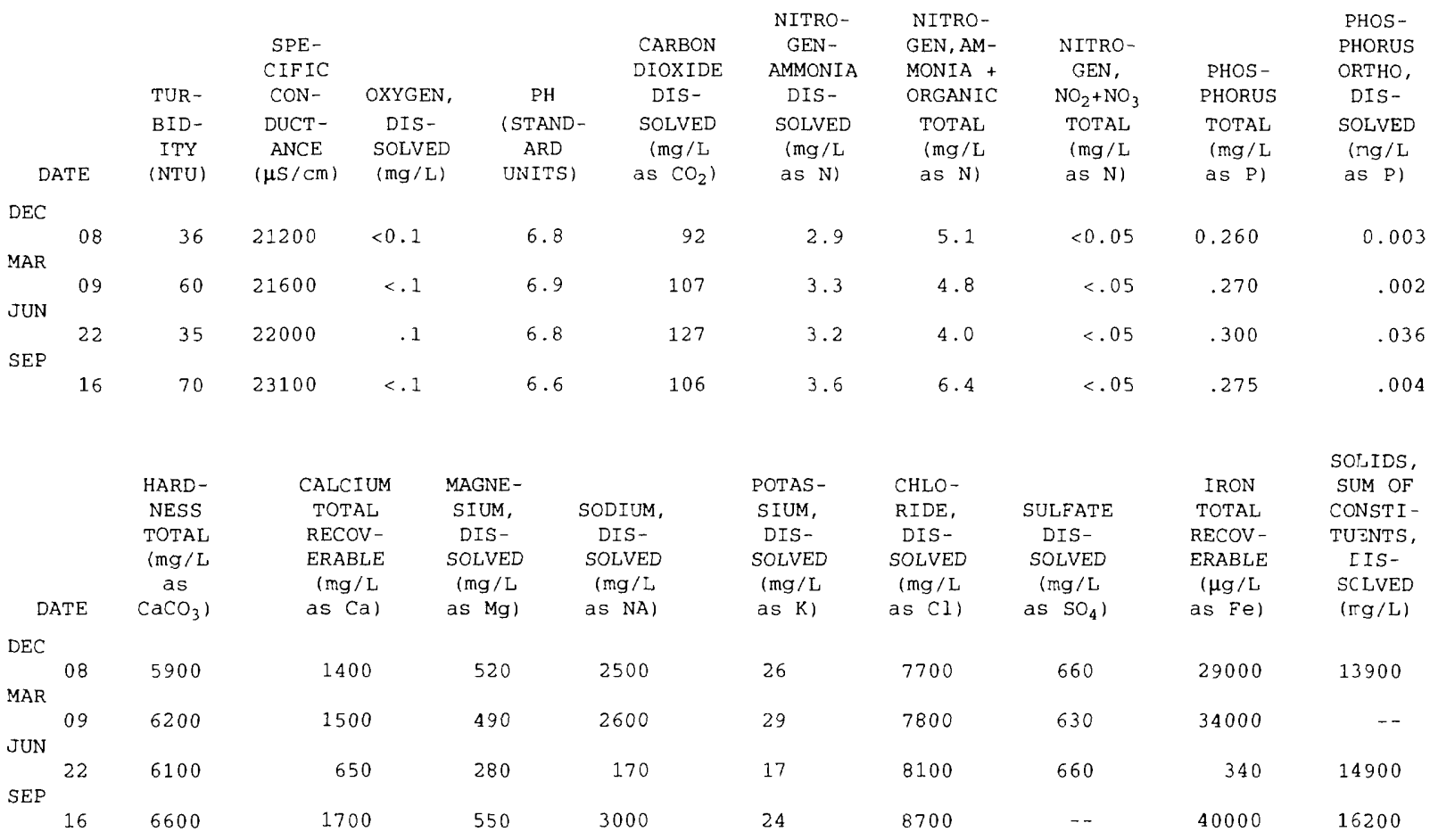


430912077313302 local number Mo 664 (B88-3d)--continued

WATER TEMPERATURE, IN DEGREES CELSIUS

\begin{tabular}{|c|c|c|c|c|c|c|c|c|c|c|c|}
\hline \multirow{2}{*}{$\begin{array}{l}\text { Depth, } \\
\text { in feet }\end{array}$} & \multicolumn{4}{|c|}{$1991 \mathrm{WY}$} & \multicolumn{4}{|c|}{$1992 W Y$} & \multicolumn{3}{|c|}{$1993 \mathrm{WY}$} \\
\hline & $\operatorname{Jan} 9$ & Apr 3 & Jun 12 & Sep 11 & Dec 18 & Mar 18 & Jun 24 & Sep 9 & $\operatorname{Dec} 8$ & $\operatorname{Mar} 9$ & Sep 15 \\
\hline 2.00 & $=$ & 6.5 & -- & - & -- & -- & $\overline{-1}$ & -- & -- & 2.0 & -- \\
\hline 2.80 & 6.0 & 6.0 & 17.0 & -- & -- & 4.0 & -- & 20.5 & 6.5 & 2.0 & -- \\
\hline 3.40 & -- & -- & -- & -- & 6.0 & -- & 15.5 & -- & -- & -- & - - \\
\hline 3.80 & $\ldots$ & - & 16.0 & 20.5 & 7.5 & 4.0 & -- & 18.5 & 7.0 & 2.5 & 17.5 \\
\hline 4.80 & 7.0 & 6.5 & 14.5 & 19.0 & 8.5 & 4.5 & 14.5 & 17.5 & 7.5 & 3.5 & 17.0 \\
\hline 5.80 & -- & - & 13.5 & 18.5 & 9.0 & 4.5 & 13.5 & 17.0 & 8.5 & 4.5 & 16.5 \\
\hline 6.80 & 7.5 & 6.5 & 13.0 & 17.5 & 9.0 & 5.0 & 13.0 & 16.0 & 9.5 & 6.5 & 16.0 \\
\hline 7.80 & -- & -- & 12.5 & 17.0 & 9.5 & 6.5 & 12.0 & 15.5 & 10.5 & 7.0 & 15.0 \\
\hline 8.80 & 8.5 & 6.5 & 11.5 & 16.0 & 9.5 & 7.0 & 11.5 & 15.0 & 11.0 & 7.0 & 15.0 \\
\hline 9.80 & -- & -- & 11.0 & 15.5 & 10.5 & 7.5 & 11.0 & 14.0 & 11.5 & 7.5 & 14.5 \\
\hline 10.80 & 9.5 & 8.0 & 10.5 & 15.0 & 11.5 & 7.5 & 10.5 & 14.0 & 11.5 & 7.5 & 14.0 \\
\hline 11.80 & $\ldots$ & -- & 10.0 & 14.5 & 11.5 & 8.0 & 10.0 & 13.5 & 11.5 & 8.0 & 13.0 \\
\hline 12.80 & 10.0 & 8.5 & 10.0 & 13.5 & 12.0 & 8.0 & 10.0 & 13.0 & 12.0 & 8.5 & 12.5 \\
\hline 13.80 & -- & -- & 9.5 & 13.0 & 12.0 & 8.5 & 9.5 & 12.5 & 12.0 & 8.5 & 12.5 \\
\hline 14.80 & 10.5 & 8.5 & 9.5 & 13.0 & 12.0 & 8.5 & 9.5 & 12.5 & 12.0 & 9.0 & 12.0 \\
\hline 15.80 & -- & -- & 9.5 & 12.5 & 12.0 & 9.0 & 9.5 & 12.0 & 12.0 & 9.5 & 11.5 \\
\hline 16.80 & 11.0 & 9.0 & 9.5 & 12.0 & 12.0 & 10.0 & 9.5 & 11.5 & 12.0 & 9.5 & 11.5 \\
\hline 17.80 & $\ldots$ & $\ldots$ & 9.5 & 12.0 & 12.0 & 9.5 & 9.5 & 11.5 & 12.0 & 9.5 & -- \\
\hline 18.80 & 11.0 & 9.0 & 9.5 & 11.5 & 12.0 & 9.5 & 9.5 & 11.0 & 12.0 & 10.0 & 11.0 \\
\hline 19.80 & -- & -- & 9.5 & -- & 12.0 & 10.0 & 9.5 & 11.0 & 12.0 & 10.0 & -- \\
\hline 20.80 & 11.5 & 9.5 & 9.5 & 11.0 & 12.0 & 10.0 & 9.5 & 11.0 & 12.0 & 10.5 & 10.5 \\
\hline 21.80 & -- & -- & 10.0 & -- & $\cdots$ & 10.0 & 9.5 & 11.0 & 11.5 & 10.5 & -- \\
\hline 22.80 & 11.5 & 10.0 & 10.0 & 11.0 & 12.0 & 10.0 & 9.5 & 10.5 & -- & 10.5 & 10.5 \\
\hline 23.80 & -- & -- & -- & -- & -- & 10.0 & 10.0 & 10.5 & 11.5 & 10.5 & -- \\
\hline 24.80 & 11.5 & 10.5 & 10.0 & 11.0 & 11.5 & 10.0 & 10.0 & 10.5 & $\ldots$ & 10.5 & 10.5 \\
\hline 25.30 & -- & -- & -- & -- & -- & -- & $\ldots$ & 10.5 & - & -- & 10.0 \\
\hline 25.80 & 11.5 & 10.5 & 10.5 & 10.5 & 11.5 & 11.0 & -- & -- & 11.5 & 11.0 & -- \\
\hline
\end{tabular}

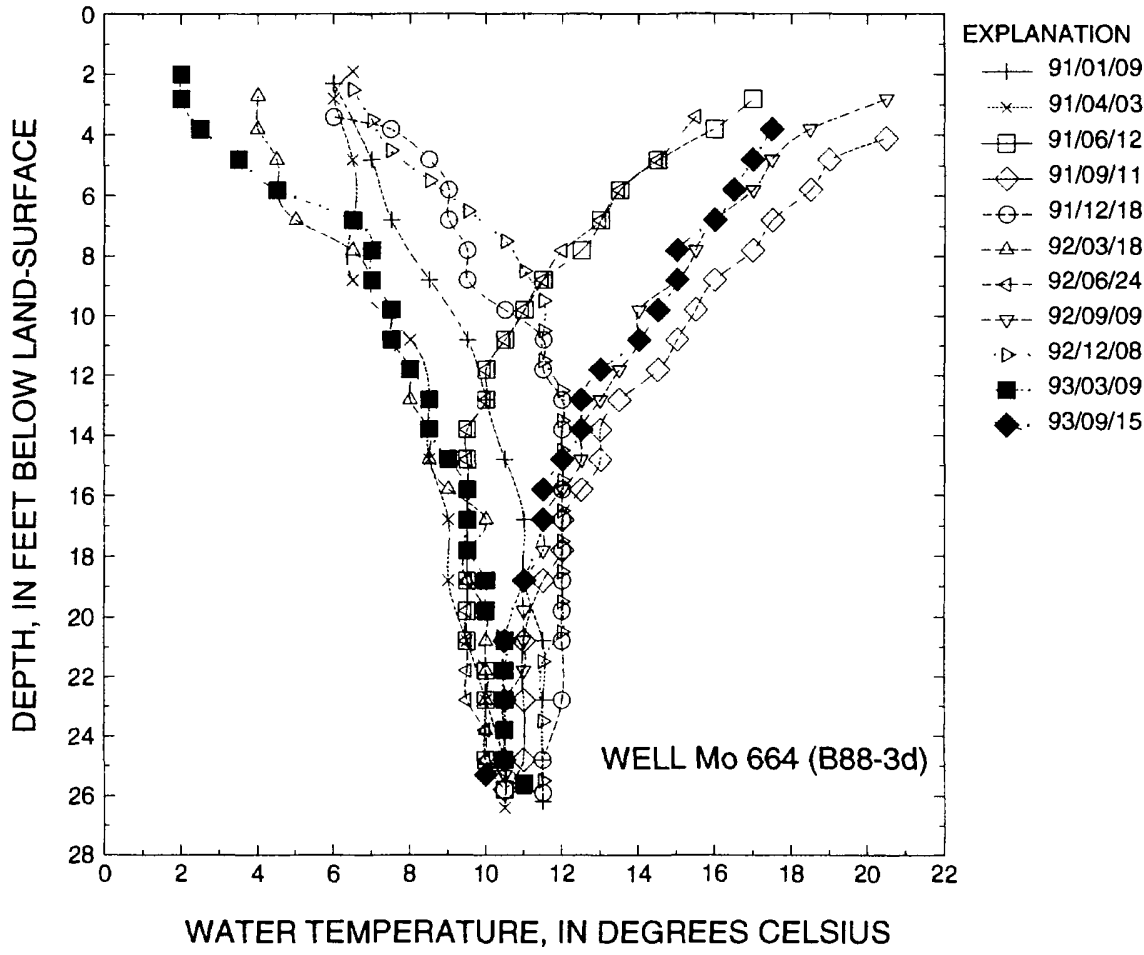




\section{GROUND-WATER LEVELS}

\section{Ellison park}

\section{Local number Mo 665}

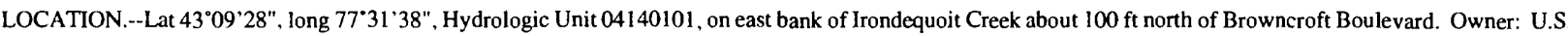
Geological Survey.

AQUIFER.--Unconfined aquifer in fluvial sediments of Holocene age.

WELL CHARACTERISTICS.--Drilled observation well, diameter 2 in, depth $17 \mathrm{ft}$, cased to $17 \mathrm{ft}$, screEned $12 \mathrm{ft}$ to $17 \mathrm{ft}$.

INSTRUMENTATION.--Monthly measurement with chalked tape by Monroe County Environmental Health Laboratory personnel.

DATUM.--Elevation of land-surface datum is $254.14 \mathrm{ft}$ sea level. Measuring point: arrow at top of casing, $2.45 \mathrm{ft}$ above land-surface datum.

REMARKS.--Well also sampled for water-quality.

PERIOD OF RECORD.--September 1988 to current year.

EXTREMES FOR PERIOD SEPTEMBER 1988 TO SEPTEMBER 1993.--Highest water level measured, $4.00 \mathrm{ft}$ below land-surface datum, May 7, 1991 ; lowest measured, $7.48 \mathrm{ft}$ below land-surface datum, Oct. 31, 1989.

WATER LEVEL, IN FEET BELOW LAND-SURFACE DATUM,

\begin{tabular}{|c|c|c|c|c|c|c|c|c|c|c|c|}
\hline \multicolumn{2}{|c|}{ DATE } & $\begin{array}{l}\text { WATER } \\
\text { LEVEL } \\
\end{array}$ & \multicolumn{2}{|c|}{ DATE } & \multirow[t]{2}{*}{$\begin{array}{l}\text { WATER } \\
\text { LEVEL }\end{array}$} & \multicolumn{2}{|c|}{ DATE } & \multirow[t]{2}{*}{$\begin{array}{l}\text { WATER } \\
\text { LEVEL } \\
\end{array}$} & \multicolumn{2}{|c|}{ DATE } & $\begin{array}{l}\text { WATER } \\
\text { LEVEL } \\
\end{array}$ \\
\hline \multicolumn{10}{|c|}{ PERIOD SEPTEMBER 1988 TO SEPTEMBER 1989} & & \\
\hline SEP & 07 & 6.40 & MAR & 22 & 6.86 & JUN & 30 & 5.50 & SEP & 26 & 6.67 \\
\hline & 12 & 6.47 & & 28 & 5.80 & JUL & 31 & 6.38 & & 29 & 6.73 \\
\hline OCT & 26 & 6.18 & APR & 25 & 6.03 & AUG & 8 & 6.40 & & & \\
\hline FEB & 28 & 6.20 & MAY & 26 & 5.55 & & 31 & 6.83 & & & \\
\hline \multicolumn{12}{|c|}{ WATER YEAR OCTOBER 1989 TO SEPTEMBER 1990} \\
\hline OCT & 31 & 7.48 & FEB & 13 & 5.07 & APR & 26 & 5.35 & JUL & 25 & 6.47 \\
\hline NOV & 30 & 6.27 & & 28 & 5.17 & MAY & 31 & 6.39 & AUG & 1 & 6.52 \\
\hline DEC & 29 & 6.60 & MAR & 30 & 5.72 & JUN & 12 & 5.75 & & 28 & 6.45 \\
\hline JUN & 30 & 6.12 & APR & 24 & 5.25 & & 22 & 5.93 & SEP & 25 & 6.70 \\
\hline \multicolumn{12}{|c|}{ WATER YEAR OCTOBER 1990 TO SEPTEMBER 1991} \\
\hline OCT & 30 & 5.93 & FEB & 13 & 5.24 & MAY & 7 & 4.00 & AUG & 21 & 6.61 \\
\hline \multirow[t]{2}{*}{ NOV } & 7 & 5.98 & & 26 & 5.52 & & 28 & 5.52 & & 22 & 6.79 \\
\hline & 28 & 5.71 & MAR & 22 & 5.27 & JUN & 12 & 5.65 & SEP & 11 & 7.07 \\
\hline \multirow[t]{2}{*}{ JAN } & 9 & 5.36 & APR & 3 & 5.13 & JUL & 9 & 5.61 & & & \\
\hline & 23 & 4.80 & & 26 & 4.31 & & 26 & 6.62 & & & \\
\hline \multicolumn{12}{|c|}{ WATER YEAR OCTOBER 1991 TO SEPTEMBER 1992} \\
\hline \multirow[t]{2}{*}{ OCT } & 29 & 6.91 & DEC & 18 & 6.48 & MAY & 1 & 5.33 & JUL & 21 & 5.25 \\
\hline & 31 & 6.67 & JAN & 31 & 6.07 & & 28 & 5.81 & AUG & 21 & 5.76 \\
\hline NOV & 27 & $6.5 \mathrm{I}$ & MAR & 18 & 5.92 & JUN & 24 & 6.02 & SEP & 9 & 5.46 \\
\hline \multicolumn{12}{|c|}{ WATER YEAR OCTOBER 1992 TO SEPTEMBER 1993} \\
\hline OCT & 28 & 5.74 & JAN & 27 & 4.61 & APR & 30 & 4.39 & JUL & 30 & 5.93 \\
\hline NOV & 24 & 5.08 & FEB & 26 & 5.53 & MAY & 27 & 5.03 & AUG & 27 & 6.32 \\
\hline DEC & 8 & 5.68 & MAR & 9 & 5.31 & JUL & 6 & 5.50 & SEP & 15 & 6.30 \\
\hline
\end{tabular}

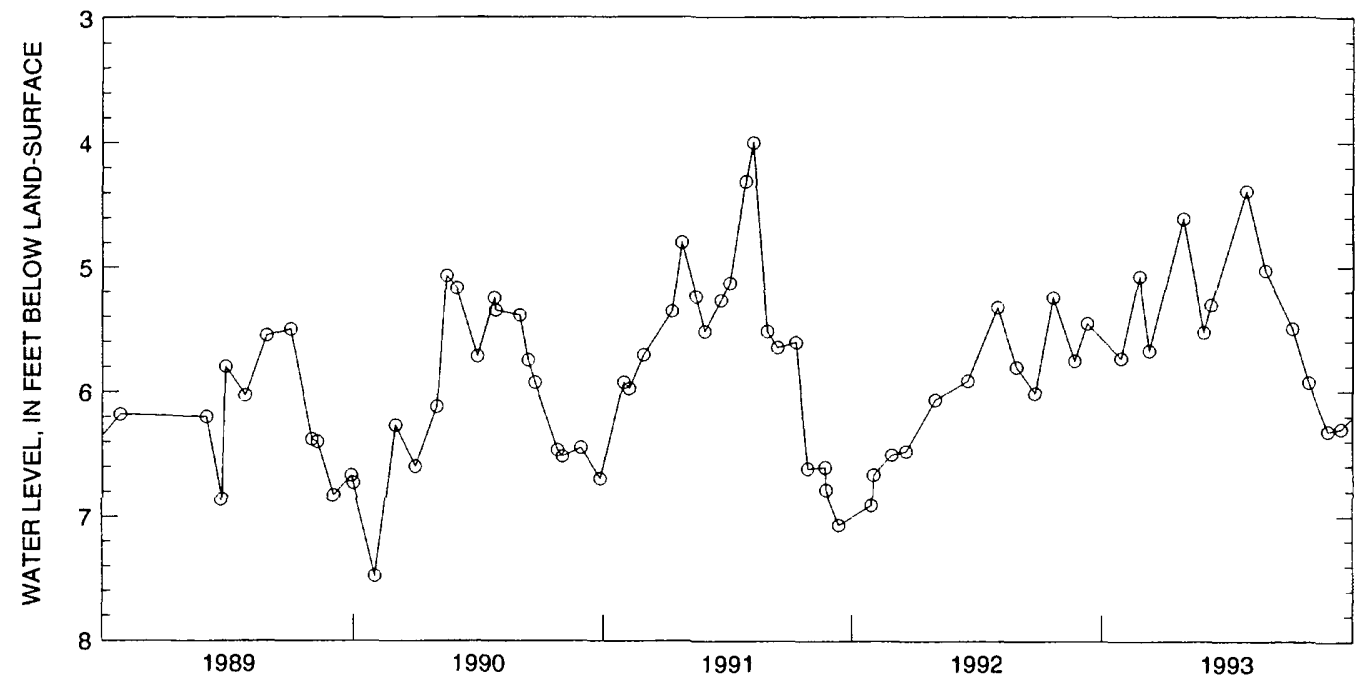


PERIOD OF RECORD.-- January 1991 to current year.

CHEMICAL DATA: 1991-93(b).

ORGANIC DATA: OC.--1991-93(b).

NUTRIENT DATA: 1991-93(b).

COOPERATION-- Water-quality samples were collected and analyzed by the Monroe County Environmental Health Laboratory at Rochester, NY.

\section{WATER QUALTY DATA, PERIOD JANUARY 1991 TO SEPTEMBER 1991}

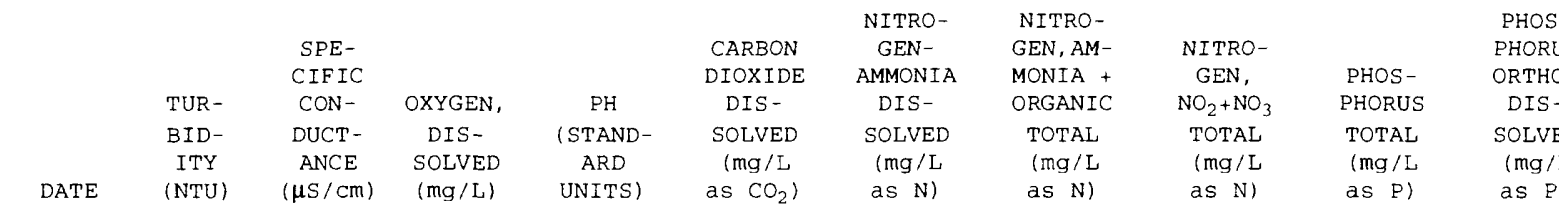

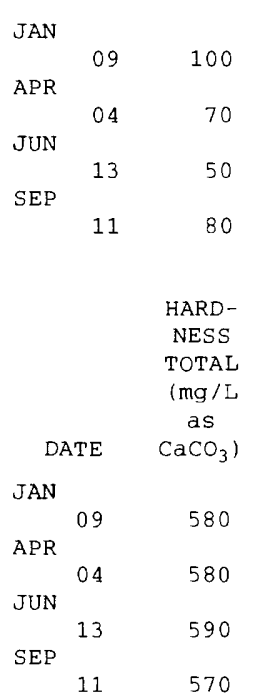

$\begin{array}{rrrr}1900 & - & 7.0 & -- \\ 1940 & <.1 & 7.1 & 117 \\ 900 & <.1 & 7.1 & 142 \\ 1970 & .1 & 7.3 & 111\end{array}$

\begin{tabular}{rrrr}
1.9 & 3.5 & 0.03 & 0.330 \\
1.8 & 1.7 & \multicolumn{1}{c}{--} & .240 \\
1.7 & 3.6 & .14 & .160 \\
2.0 & - & $<.05$ & .260
\end{tabular}

0.004

$\begin{array}{ccc}\text { CALCIUM } & \text { MAGNE- } & \\ \text { TOTAL } & \text { SIUM, } & \text { SODIUM, } \\ \text { RECOV- } & \text { DIS- } & \text { DIS- } \\ \text { ERABLE } & \text { SOLVED } & \text { SOLVED } \\ \text { (mg/L } & (\mathrm{mg} / \mathrm{L} & (\mathrm{mg} / \mathrm{L} \\ \text { as } \mathrm{Ca}) & \text { as } \mathrm{Mg}) & \text { as NA) }\end{array}$

\section{POTAS- CHLO- $\quad$ IRON} SIUM, RIDE, SULFATE TOTAL DIS- DIS- DIS- RECOV -

SOLVED SOLVED SOLVED ERABLE

$(\mathrm{mg} / \mathrm{L} \quad(\mathrm{mg} / \mathrm{L}$

as $\mathrm{K}$ )

as $\mathrm{Cl}$ )

( $\mathrm{mg} / \mathrm{L}$

ERABLE

$(\mu \mathrm{g} / \mathrm{L}$

CONSTI-

TUENTS,

DIS-

SOLVED

(mg/L)

$\begin{array}{rrrrrrrr}180 & 35 & 230 & 0.80 & 240 & <10 & 12000 & 1210 \\ 170 & 34 & 240 & .76 & 230 & <10 & 27000 & 1220 \\ 170 & 33 & 260 & .70 & 240 & <10 & 6900 & 1240 \\ 160 & 7.0 & 260 & .88 & 240 & <10 & 13000 & 1240\end{array}$

WATER QUALITY DATA, WATER YEAR OCTOBER 1991 TO SEPTEMBER 1992

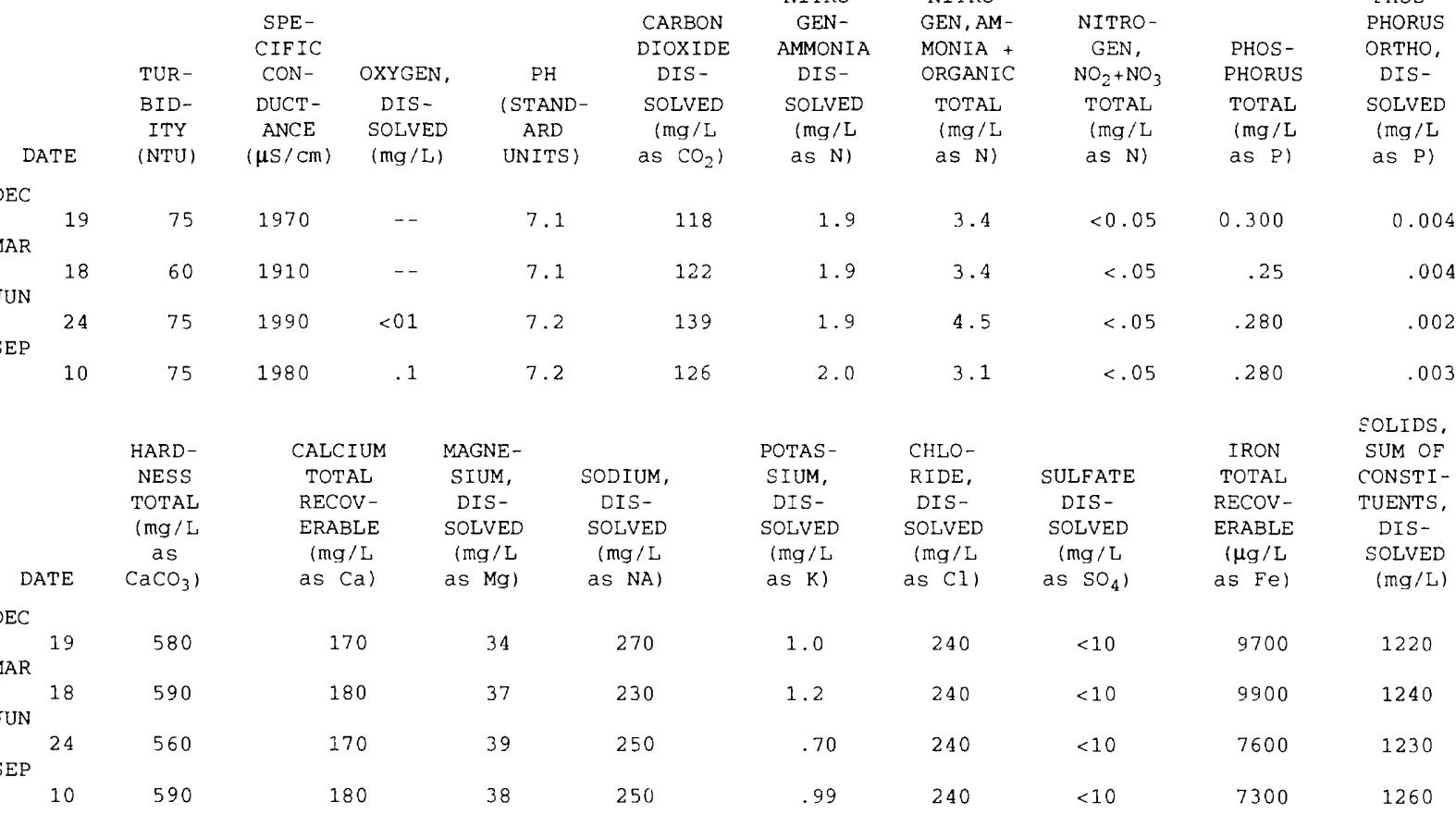




\section{GROUND-WATER QUALITY}

\section{Ellison park}

430928077313802. Local number Mo 665 (B88-1s)--continued

WATER QUALTY DATA, WATER YEAR OCTOBER 1992 TO SEPTEMBER 1993

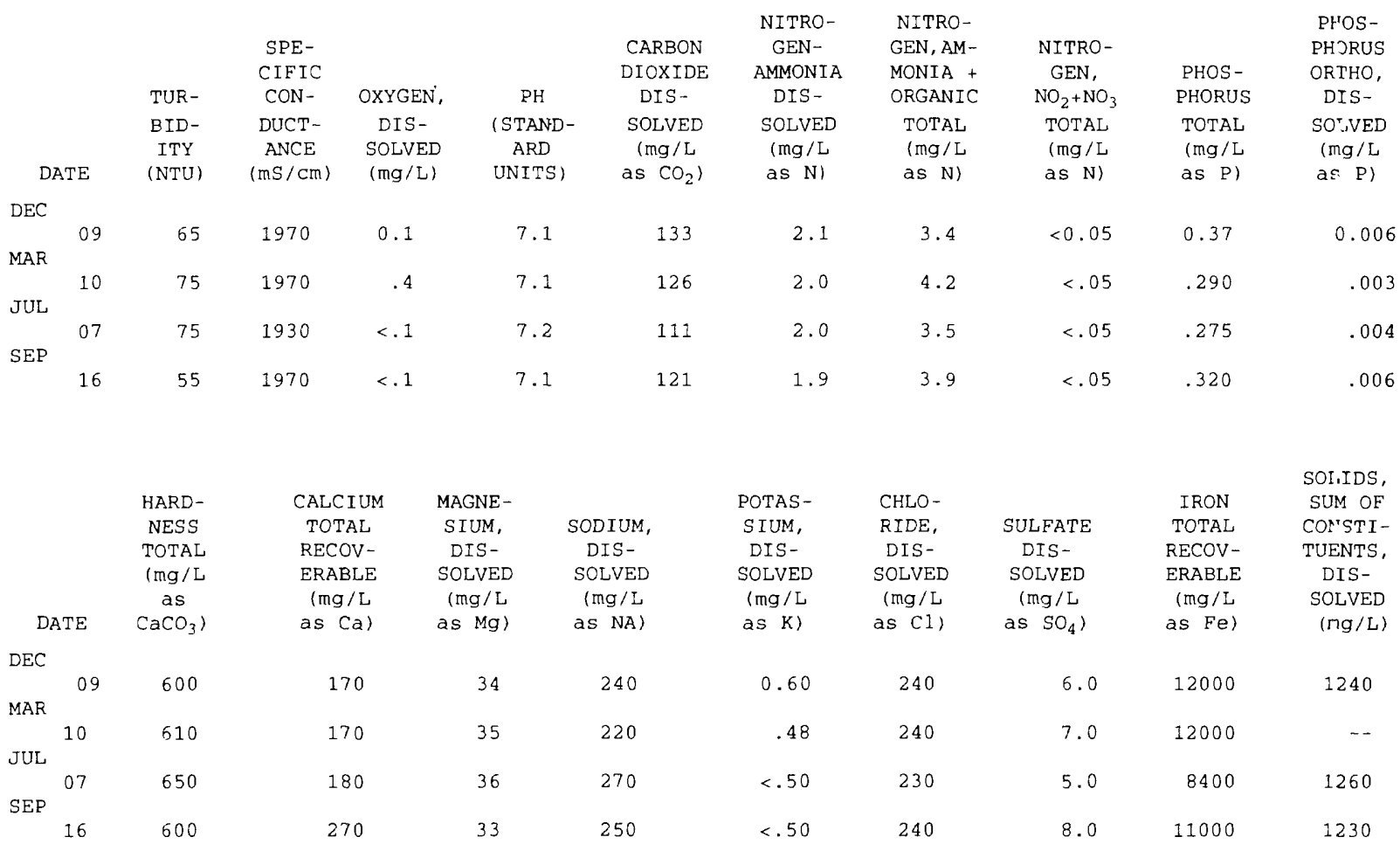




\section{GROUND-WATER TEMPERATURE PROFILES}

\section{Ellison park}

430928077313802. Local number Mo 665 (B88-1s)--continued

WATER TEMPERATURE, IN DEGREES CELSIUS

\begin{tabular}{|c|c|c|c|c|c|c|c|c|c|c|c|}
\hline \multirow{2}{*}{$\begin{array}{l}\text { Depth, } \\
\text { in feet }\end{array}$} & \multicolumn{4}{|c|}{$1991 \mathrm{WY}$} & \multicolumn{4}{|c|}{$1992 \mathrm{WY}$} & \multicolumn{3}{|c|}{$1993 \mathrm{WY}$} \\
\hline & $\operatorname{Jan} 9$ & Apr 3 & Jun 12 & Sep 11 & Dec 18 & Mar 18 & Jun 24 & Sep 9 & Mar 9 & Jul 6 & Sep 15 \\
\hline 5.15 & 8.0 & 8.5 & -- & -- & -- & -- & -- & -- & -- & -- & $\overline{--}$ \\
\hline 5.55 & -- & 8.5 & 14.0 & -- & -- & -- & -- & 17.0 & 6.5 & 18.5 & -- \\
\hline 5.95 & -- & -- & -- & -- & -- & 8.5 & -- & -- & -- & -- & -- \\
\hline 6.05 & $\rightarrow$ & -- & -- & -- & -- & -- & 12.0 & -- & -- & -- & -- \\
\hline 6.45 & -- & -- & -- & -- & 7.5 & -- & -- & -- & -- & -- & -- \\
\hline 6.55 & -- & 8.0 & 12.5 & -- & -- & 8.5 & -- & 16.0 & 7.5 & 14.0 & 15.5 \\
\hline 7.05 & -- & -- & -- & 15.5 & -- & -- & 11.0 & -- & -- & -- & -- \\
\hline 7.55 & 9.5 & 7.5 & 11.5 & 15.5 & 10.0 & 8.5 & -- & 14.5 & 7.5 & 12.5 & 14.5 \\
\hline 8.05 & -- & -- & -- & -- & -- & -- & 10.5 & -- & -- & -- & -- \\
\hline 8.55 & -- & 8.0 & 11.0 & 15.0 & 11.0 & 8.5 & -- & 14.0 & 8.0 & 12.0 & 14.0 \\
\hline 9.05 & -- & -- & -- & -- & -- & $\cdots$ & 10.0 & -- & -- & -- & -- \\
\hline 9.55 & 10.0 & 8.0 & 10.5 & 14.5 & 11.5 & 9.0 & -- & 13.5 & 8.0 & 11.5 & 13.5 \\
\hline 10.05 & -- & -- & -- & -- & -- & -- & 10.0 & -- & -- & -- & -- \\
\hline 10.55 & -- & 8.0 & 10.5 & 14.0 & 11.5 & 9.0 & -- & 13.0 & 8.5 & 11.0 & 13.0 \\
\hline 11.05 & -- & -- & -- & -- & -- & -- & 10.0 & -- & -- & -- & -- \\
\hline 11.55 & 10.5 & 8.0 & 10.0 & 13.5 & 12.0 & 9.0 & -- & 13.0 & 8.5 & 10.5 & 13.0 \\
\hline 12.05 & -- & -- & -- & -- & -- & $\ldots$ & 9.5 & -- & -- & -- & -- \\
\hline 12.55 & -- & 8.5 & 10.0 & 13.5 & 12.0 & 9.5 & -- & 12.5 & 9.0 & 10.0 & 12.5 \\
\hline 13.05 & -- & -- & -- & -- & -- & -- & 9.5 & -- & -- & -- & -- \\
\hline 13.55 & 11.0 & 8.5 & 10.0 & 13.0 & 12.0 & 9.5 & -- & 12.0 & 9.0 & 10.0 & 12.0 \\
\hline 14.05 & -- & -- & -- & -- & -- & -- & 9.5 & -- & -- & -- & -- \\
\hline 14.55 & -- & 8.5 & 10.0 & 12.5 & 12.0 & 10.0 & -- & 12.0 & 9.5 & 10.0 & 11.5 \\
\hline 15.05 & -- & -- & -- & -- & -- & -- & 9.5 & -- & -- & -- & -- \\
\hline 15.55 & 11.0 & 9.0 & 10.0 & 12.0 & 12.0 & -- & -- & 11.5 & 9.5 & 10.0 & 11.5 \\
\hline 16.05 & -- & 9.0 & -- & -- & 12.5 & -- & -- & -- & 9.5 & 10.0 & 11.5 \\
\hline 16.35 & 11.5 & -- & 10.0 & 12.0 & -- & -- & -- & 11.5 & -- & -- & -- \\
\hline
\end{tabular}

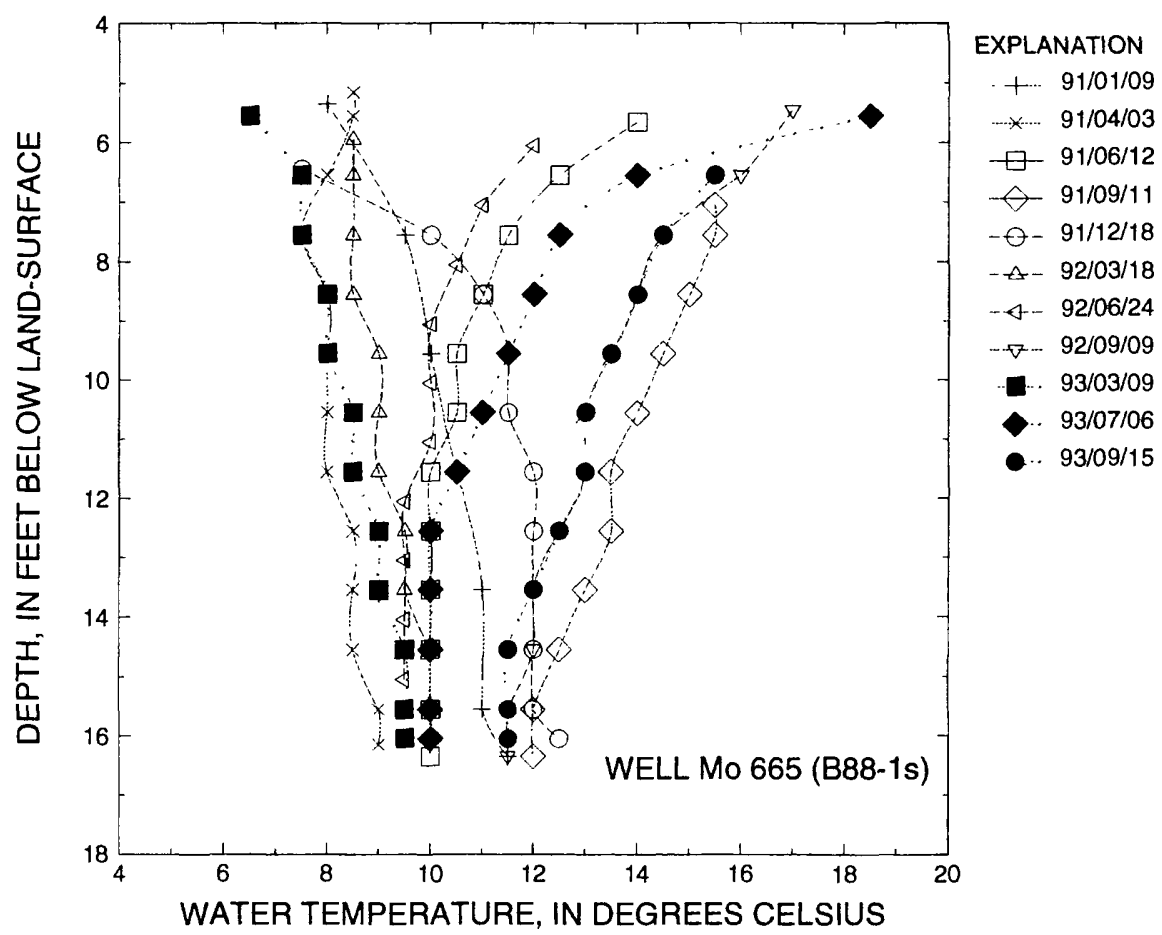




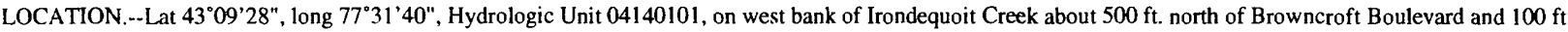
west of Irondequoit Creek. Owner: U.S. Geological Survey.

AQUIFER.--Unconfined aquifer in fluvial sediments of Holocene age.

WELL CHARACTERISTICS.--Drilled observation well, diameter 2 in, depth $15 \mathrm{ft}$, cased to $15 \mathrm{ft}$, screened $10 \mathrm{ft}$ to $15 \mathrm{ft}$.

INSTRUMENTATION.--Monthly measurement with chalked tape by Monroe County Environmental Health Laboratory personnel.

DATUM.--Elevation of land-surface datum is $255.38 \mathrm{ft}$ above sea level. Measuring point: arrow at top of casing, $2.05 \mathrm{ft}$ above land-surface datum.

REMARKS.--Well also sampled for water-quality.

PERIOD OF RECORD.--September 1988 to current year.

EXTREMES FOR PERIOD SEPTEMBER 1988 TO SEPTEMBER 1993.--Highest water level measured, $0.65 \mathrm{ft}$ below land-surface datum, Apr 30, 1993; lowest measured, $6.06 \mathrm{ft}$ below land-surface datum, Oct. 29, 1991.

WATER LEVEL, IN FEET BELOW LAND-SURFACE DATUM,

\begin{tabular}{|c|c|c|c|c|c|c|c|c|c|c|c|}
\hline \multicolumn{2}{|c|}{ DATE } & $\begin{array}{l}\text { WATER } \\
\text { LEVEL }\end{array}$ & \multicolumn{2}{|c|}{ DATE } & \multirow[t]{2}{*}{$\begin{array}{l}\text { WATER } \\
\text { LEVEL } \\
\end{array}$} & \multicolumn{2}{|c|}{ DATE } & \multirow[t]{2}{*}{$\begin{array}{l}\text { WATER } \\
\text { LEVEL }\end{array}$} & \multicolumn{2}{|c|}{ DATE } & $\begin{array}{l}\text { "NATER } \\
\text { LEVEL }\end{array}$ \\
\hline \multicolumn{10}{|c|}{ PERIOD SEPTEMBER 1988 TO SEPTEMBER 1989} & & \\
\hline SEP & 7 & 4.05 & MAR & 28 & 2.88 & MAY & 26 & 2.27 & SEP & 26 & 5.06 \\
\hline OCT & 26 & 4.00 & APR & 25 & 2.64 & AUG & 31 & 4.96 & & 29 & 5.01 \\
\hline FEB & 28 & 3.38 & & & & & & & & & \\
\hline \multicolumn{12}{|c|}{ WATER YEAR OCTOBER 1989 TO SEPTEMBER 1990} \\
\hline OCT & 31 & 4.47 & FEB & 28 & 1.90 & JUN & 12 & 2.64 & SEP & 25 & 5.26 \\
\hline NOV & 30 & 3.72 & MAR & 30 & 2.18 & & 22 & 2.95 & & & \\
\hline DEC & 29 & 4.33 & APR & 24 & 1.88 & JUL & 25 & 4.46 & & & \\
\hline JAN & 30 & 2.57 & MAY & 31 & 1.91 & AUG & 28 & 4.51 & & & \\
\hline \multicolumn{12}{|c|}{ WATER YEAR OCTOBER 1990 TO SEPTEMBER 1991} \\
\hline OCT & 30 & 3.45 & FEB & 26 & 1.65 & MAY & 28 & 2.67 & AUG & 22 & 5.02 \\
\hline NOV & 28 & 2.49 & MAR & 22 & 1.61 & JUN & 13 & 3.33 & SEP & 11 & 5.47 \\
\hline JAN & 9 & 1.49 & APR & 4 & 1.51 & JUL & 9 & 4.08 & & & \\
\hline & 23 & 1.23 & & 26 & 1.03 & & 26 & 4.70 & & & \\
\hline FEB & 13 & 1.28 & MAY & 7 & 1.61 & AUG & 21 & 4.92 & & & \\
\hline \multicolumn{12}{|c|}{ WATER YEAR OCTOBER 1991 TO SEPTEMBER 1992} \\
\hline OCT & 29 & 6.06 & DEC & 18 & 4.38 & MAY & l & 1.04 & JUL & 21 & 2.71 \\
\hline & 31 & 5.56 & JAN & 31 & 1.90 & & 28 & 2.30 & AUG & 21 & 2.18 \\
\hline NOV & 27 & 5.33 & MAR & 18 & 1.60 & JUN & 24 & 3.07 & SEP & 9 & 1.84 \\
\hline \multicolumn{12}{|c|}{ WATER YEAR OCTOBER 1992 TO SEPTEMBER 1993} \\
\hline OCT & 28 & 2.98 & JAN & 27 & 0.73 & APR & 30 & 0.65 & JUL & 30 & 3.69 \\
\hline NOV & 24 & 1.80 & FEB & 26 & 2.28 & MAY & 27 & 2.25 & AUG & 27 & 4.16 \\
\hline DEC & 8 & 2.03 & MAR & 9 & 2.84 & JUL & 6 & 3.12 & SEP & 15 & 4.15 \\
\hline
\end{tabular}

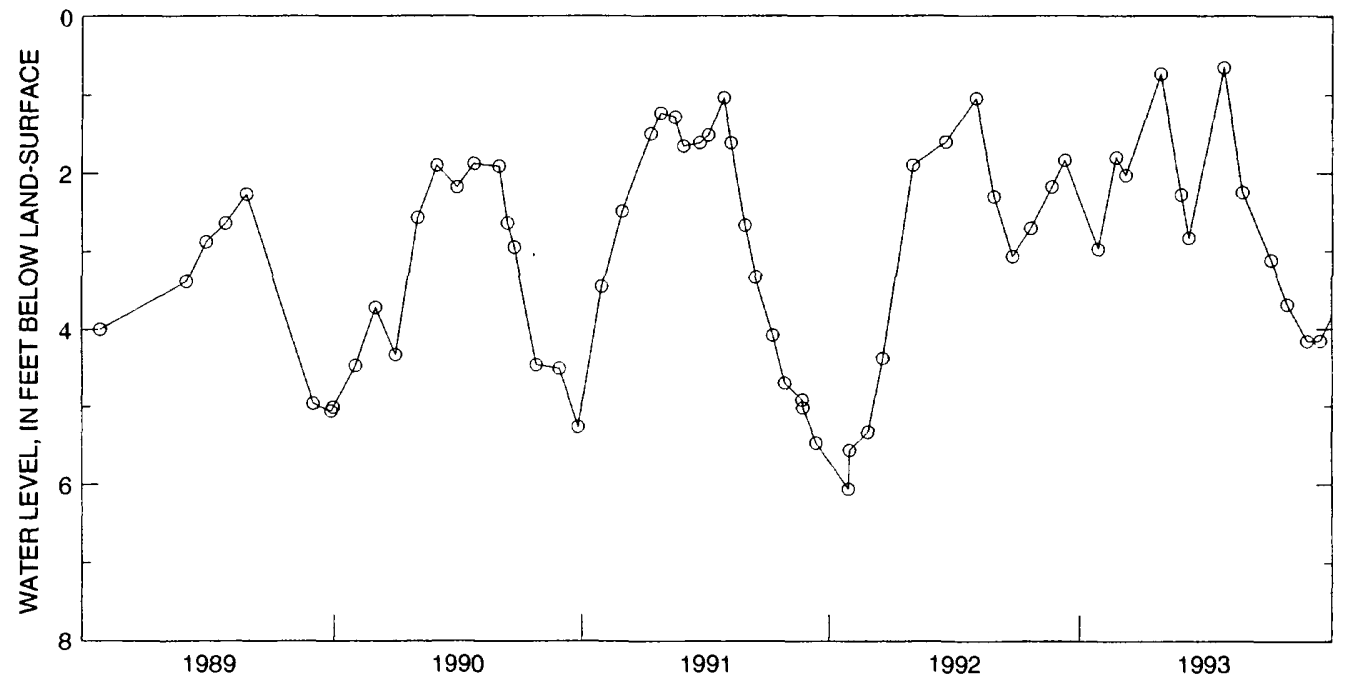


PERIOD OF RECORD.-- January 1991 to current year.

CHEMICAL DATA: $1991-93(\mathrm{~b})$

ORGANIC DATA: OC.--1991-93(b)

NUTRIENT DATA: 1991-93(b)

COOPERATION-- Water-quality samples were collected and analyzed by the Monroe County Environmental Health Laboratory at Rochester, NY.

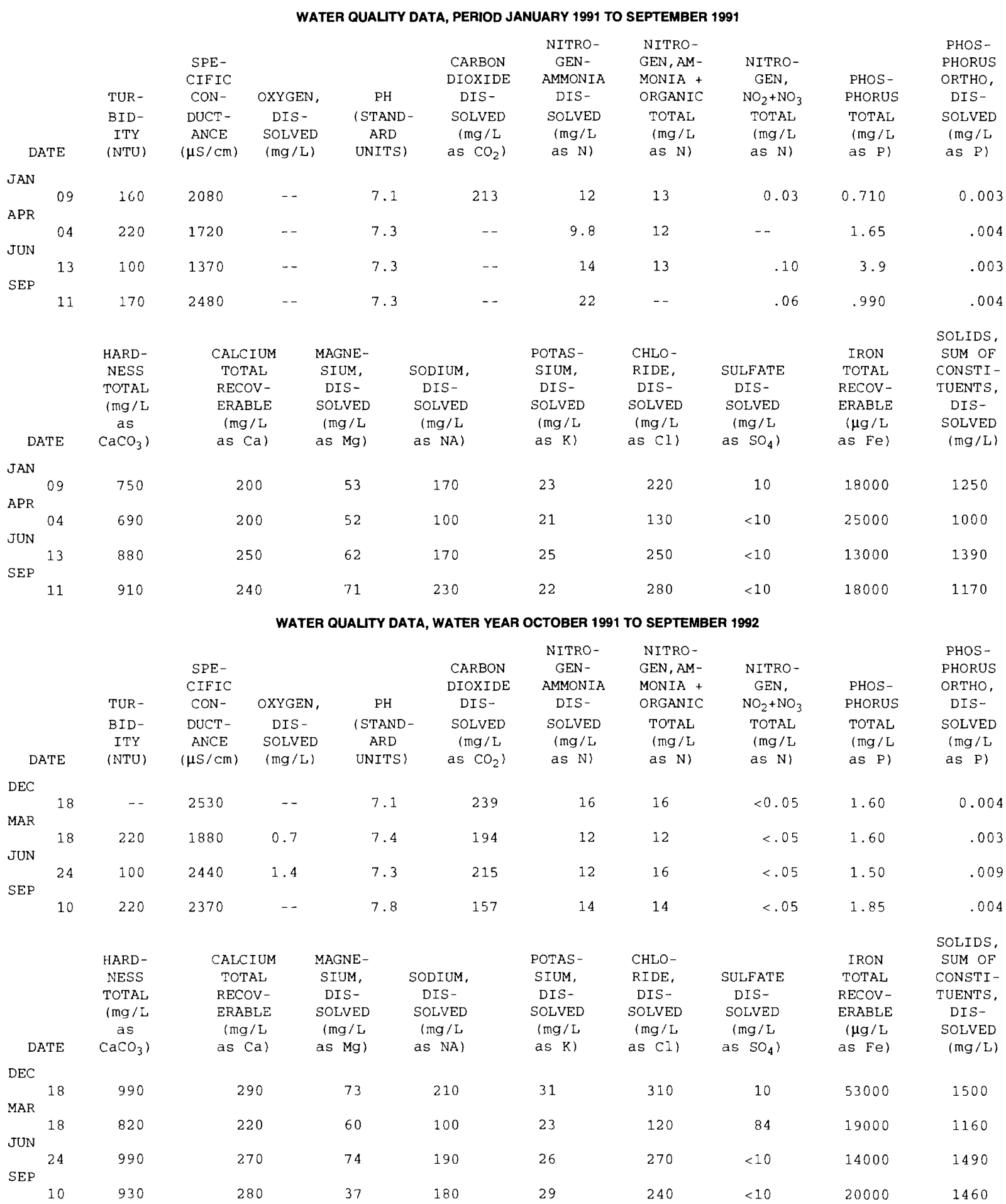


430928077314001. Local number Mo 667 (B88-2s)--continued

WATER QUALTY DATA, WATER YEAR OCTOBER 1992 TO SEPTEMBER 1993

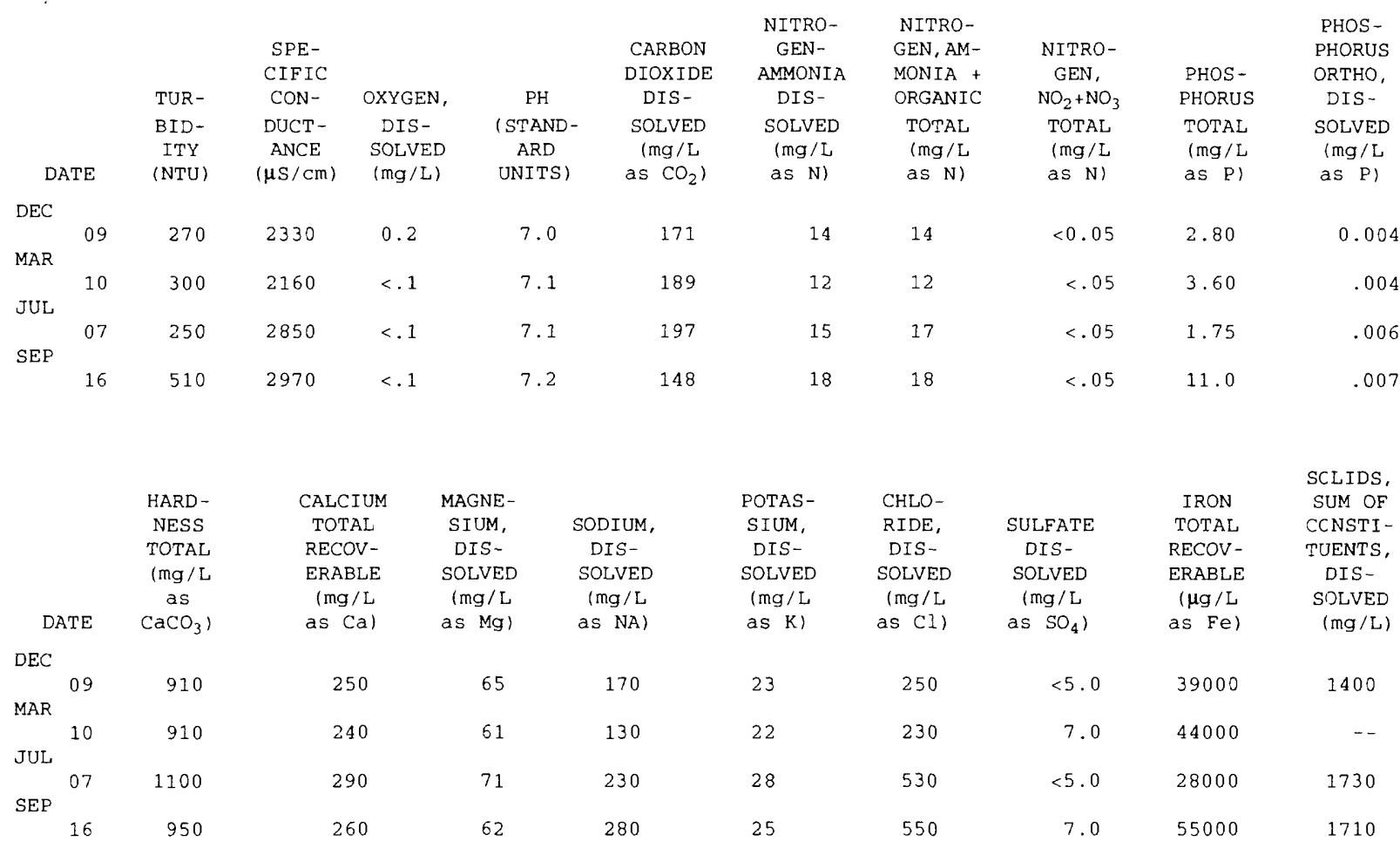


430928077314001. Local number Mo 667 (B88-2s)--continued

WATER TEMPERATURE, IN DEGREES CELSIUS

\begin{tabular}{|c|c|c|c|c|c|c|c|c|c|c|c|c|}
\hline \multirow{2}{*}{$\begin{array}{l}\text { depth, } \\
\text { in feet }\end{array}$} & \multicolumn{4}{|c|}{$1991 \mathrm{WY}$} & \multicolumn{4}{|c|}{$1992 \mathrm{WY}$} & \multicolumn{4}{|c|}{$1993 \mathrm{WY}$} \\
\hline & $\operatorname{Jan} 9$ & Apr 4 & Jun 13 & Sep 11 & Dec 18 & Mar 18 & Jun 24 & Sep 9 & Dec 8 & Mar 9 & Jul 6 & Sep 15 \\
\hline 1.55 & 4.0 & 9.0 & -- & -- & -- & 2.0 & -- & $\cdots$ & -- & -- & -- & -- \\
\hline 1.95 & -- & 7.5 & -- & -- & -- & -- & -- & 20.5 & 6.5 & -- & -- & -- \\
\hline 2.55 & -. & -- & -- & -- & -- & 2.0 & -- & -- & -- & - & -- & -- \\
\hline 2.95 & 5.0 & 6.5 & -- & -- & -- & -. & 14.5 & 19.0 & 7.0 & 4.5 & -- & -- \\
\hline 3.15 & -- & -- & -- & -- & -- & -- & -- & -- & -- & -+ & 20.0 & -- \\
\hline 3.35 & -. & -- & 18.5 & -- & -- & -- & -- & -- & -- & -- & -- & $\ldots$ \\
\hline 3.55 & -- & -- & - & -- & -- & 3.0 & 14.0 & -- & -- & -- & -- & -- \\
\hline 3.95 & 5.0 & 5.5 & 17.5 & -- & -- & +- & $\ldots$ & 17.5 & 7.5 & 4.5 & 16.5 & -- \\
\hline 4.55 & $\ldots$ & -- & -+ & - & 6.5 & 3.5 & 13.5 & -- & -- & -- & -- & 16.5 \\
\hline 4.95 & 5.5 & 6.0 & 16.0 & -- & -- & -- & -- & 17.0 & 8.0 & 4.5 & 15.0 & 16.5 \\
\hline 5.45 & -- & -- & -- & 17.5 & -- & $\ldots$ & +- & - & -+ & -- & $\ldots$ & -- \\
\hline 5.55 & -- & -- & -- & - & -- & 3.5 & 12.5 & -- & -- & -- & -- & -- \\
\hline 5.95 & 6.5 & 5.5 & 15.0 & 17.0 & 7.0 & -- & -- & 16.5 & 8.0 & 4.5 & 14.0 & 16.0 \\
\hline 6.55 & - & - & -- & -- & -- & 4.0 & 11.5 & -- & -- & -- & -- & -- \\
\hline 6.95 & 6.5 & 5.5 & 12.5 & 16.5 & 8.5 & -. & -- & 16.0 & 8.5 & 4.5 & 13.0 & 15.5 \\
\hline 7.55 & -- & -- & -- & $\ldots$ & -- & 4.0 & 11.0 & -- & -- & -- & -- & -- \\
\hline 7.95 & 7.0 & 5.5 & 12.0 & 16.0 & 8.5 & -- & -- & 15.0 & 9.0 & 4.5 & 12.5 & 15.0 \\
\hline 8.55 & -- & -- & -- & -- & -- & 4.5 & 10.5 & -- & -- & -- & $\ldots$ & - \\
\hline 8.95 & 7.5 & 5.5 & 11.0 & 15.5 & 9.0 & -- & -- & 14.5 & 9.5 & 4.5 & 11.5 & 14.5 \\
\hline 9.55 & -- & -- & -- & -- & +- & 4.5 & 10.0 & -- & -- & -- & $\ldots$ & $\ldots$ \\
\hline 9.95 & 7.5 & 6.0 & 10.5 & 15.0 & 9.5 & -- & -- & 14.0 & 9.5 & 5.0 & 11.0 & 14.0 \\
\hline 10.55 & $\ldots$ & -- & - & - & -- & 5.0 & 9.5 & - & -- & -- & $+\infty$ & -- \\
\hline 10.95 & 8.0 & 6.0 & 10.0 & 14.5 & 9.5 & + & -- & 13.5 & 10.0 & 5.0 & 10.5 & 13.5 \\
\hline 11.55 & -- & -- & -- & -- & -- & 5.0 & 9.0 & -- & -. & -- & -- & -- \\
\hline 11.95 & 8.5 & 6.0 & 9.5 & 13.5 & 9.5 & -- & -- & 13.0 & 10.0 & 5.0 & 10.0 & 13.0 \\
\hline 12.55 & -- & -- & -- & -- & -- & 3.5 & 9.0 & + & -- & -- & -- & -- \\
\hline 12.95 & 8.5 & 6.0 & 9.0 & 13.5 & 10.0 & -- & -- & 12.5 & 10.0 & 5.5 & 9.5 & 12.5 \\
\hline 13.55 & $\ldots$ & - & -- & -- & -- & 4.0 & 9.0 & -- & -- & -- & -- & -- \\
\hline 13.95 & 8.5 & 6.0 & 9.0 & 12.5 & 10.5 & -- & -- & 12.0 & 10.5 & 6.0 & 9.0 & 12.0 \\
\hline 14.55 & -. & -- & $\ldots$ & -- & -- & 4.0 & -- & $\ldots$ & & -- & -- & -- \\
\hline 14.85 & 9.0 & 6.5 & 9.0 & 12.0 & -- & -- & -- & 11.5 & -- & 6.0 & 9.0 & 11.5 \\
\hline 15.55 & $\ldots$ & -- & -- & -- & -- & 4.5 & -- & -- & -- & -- & $\cdots$ & -- \\
\hline
\end{tabular}

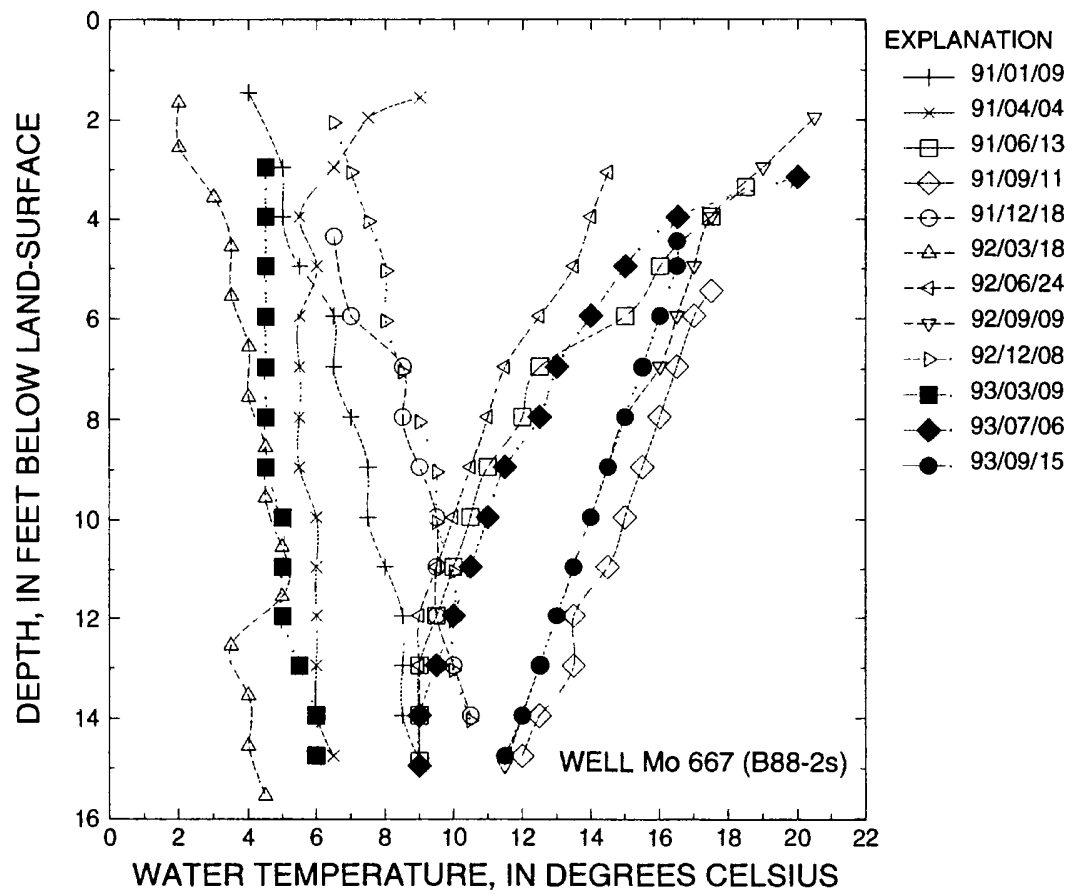

186 Water Resources of Monroe County, N.Y., 1989-93, with Emphasis on Water Quality in the Irondequoit Creek Basin 
LOCATION.--Lat 4309'28', long $77^{\circ} 31^{\prime} 40^{\prime \prime}$, Hydrologic Unit 04140101, on west bank of Irondequoit Creek about $500 \mathrm{ft}$ north of Browncroft Boulevard and $100 \mathrm{ft}$ west of Irondequoit Creek. Owner: U.S. Geological Survey.

AQUIFER.--Unconfined aquifer in fluvial sediments of Holocene age.

WELL CHARACTERISTICS.--Drilled observation well, diameter 2 in, depth $36 \mathrm{ft}$, cased to $36 \mathrm{ft}$, screened $31 \mathrm{ft}$ to $36 \mathrm{ft}$.

INSTRUMENTATION.--Monthly measurement with chalked tape by Monroe County Environmental Health Laboratory personnel.

DATUM.--Elevation of land-surface datum is $255.32 \mathrm{ft}$ above sea level. Measuring point: arrow at top of casing, $1.40 \mathrm{ft}$ above land-surface datum.

REMARKS.--Well also sampled for water quality.

PERIOD OF RECORD.--September 1988 to current year.

EXTREMES FOR PERIOD SEPTEMBER 1988 TO SEPTEMBER I993.--Highest water level measured. $5.83 \mathrm{ft}$ below land-surface datum, Apr. 30, 1973: lowest measured, $8.54 \mathrm{ft}$ below land-surface datum, Sept. 11, 1991.

WATER LEVEL, IN FEET BELOW LAND-SURFACE DATUM,

\begin{tabular}{|c|c|c|c|c|c|c|c|c|c|c|c|}
\hline $\mathrm{DA}$ & & $\begin{array}{l}\text { WATER } \\
\text { LEVEL }\end{array}$ & & & $\begin{array}{l}\text { WATER } \\
\text { LEVEL }\end{array}$ & DA & & $\begin{array}{l}\text { WATER } \\
\text { LEVEL }\end{array}$ & DA & & $\begin{array}{l}\text { WATER } \\
\text { LEVEL }\end{array}$ \\
\hline PERIO & SEP & BER 198 & TEMB & 198 & & & & & & & \\
\hline SEP & 7 & 7.90 & MAR & 28 & 7.32 & JUN & 30 & 6.94 & SEP & 26 & $8.1 \mathrm{I}$ \\
\hline $\mathrm{OCT}$ & 26 & 7.64 & APR & 25 & 7.61 & JUL & 31 & 7.74 & & 29 & 8.18 \\
\hline FEB & 28 & 7.71 & MAY & 26 & 7.09 & AUG & 31 & 8.18 & & & \\
\hline WATE & YEA & CTOBER & SEPTE & BER & & & & & & & \\
\hline $\mathrm{OCT}$ & 31 & 8.03 & FEB & 28 & 6.76 & JUN & 12 & 7.25 & SEP & 25 & 8.27 \\
\hline NOV & 30 & 7.84 & MAR & 30 & 7.34 & & 22 & 7.43 & & & \\
\hline $\mathrm{DEC}$ & 29 & 8.05 & APR & 24 & 6.87 & JUL & 25 & 7.90 & & & \\
\hline JAN & 30 & 7.70 & MAY & 31 & 6.97 & AUG & 28 & 8.03 & & & \\
\hline WATE & YEA & CTOBER & SEPTE & BER & & & & & & & \\
\hline OCT & 30 & 7.59 & FEB & 26 & 7.16 & MAY & 28 & 7.00 & AUG & 22 & 8.22 \\
\hline NOV & 28 & 7.28 & MAR & 22 & 6.92 & JUN & 13 & 7.18 & SEP & 11 & 8.54 \\
\hline JAN & 9 & 7.08 & APR & 4 & 6.81 & JUL & 9 & 7.55 & & & \\
\hline & 23 & 6.42 & & 26 & 5.95 & & 26 & 8.06 & & & \\
\hline FEB & I3 & 6.98 & MAY & 7 & 6.56 & AUG & 21 & 8.06 & & & \\
\hline WATE & YEA & CTOBER & SEPTE & BER & & & & & & & \\
\hline OCT & 29 & 8.45 & $\mathrm{DEC}$ & 18 & 8.06 & MAY & 1 & 7.00 & JUL & 21 & 6.91 \\
\hline & 31 & 8.26 & JAN & 31 & 7.77 & & 28 & $7.3 I$ & AUG & $2 \mathrm{I}$ & 7.35 \\
\hline NOV & 27 & 8.14 & MAR & 18 & 7.54 & JUN & 24 & 7.55 & SEP & 9 & 7.14 \\
\hline WATES & YEA & TOBER & SEPTEN & ER & & & & & & & \\
\hline $\mathrm{OCT}$ & 28 & 7.33 & JAN & 27 & 6.28 & APR & 30 & 5.83 & JUL & 30 & 7.36 \\
\hline NOV & 24 & 6.54 & FEB & 26 & 7.10 & MAY & 27 & 6.37 & AUG & 27 & 7.86 \\
\hline $\mathrm{DEC}$ & 8 & 7.35 & MAR & 9 & 6.78 & JUL & 6 & 7.01 & SEP & 15 & 7.88 \\
\hline
\end{tabular}

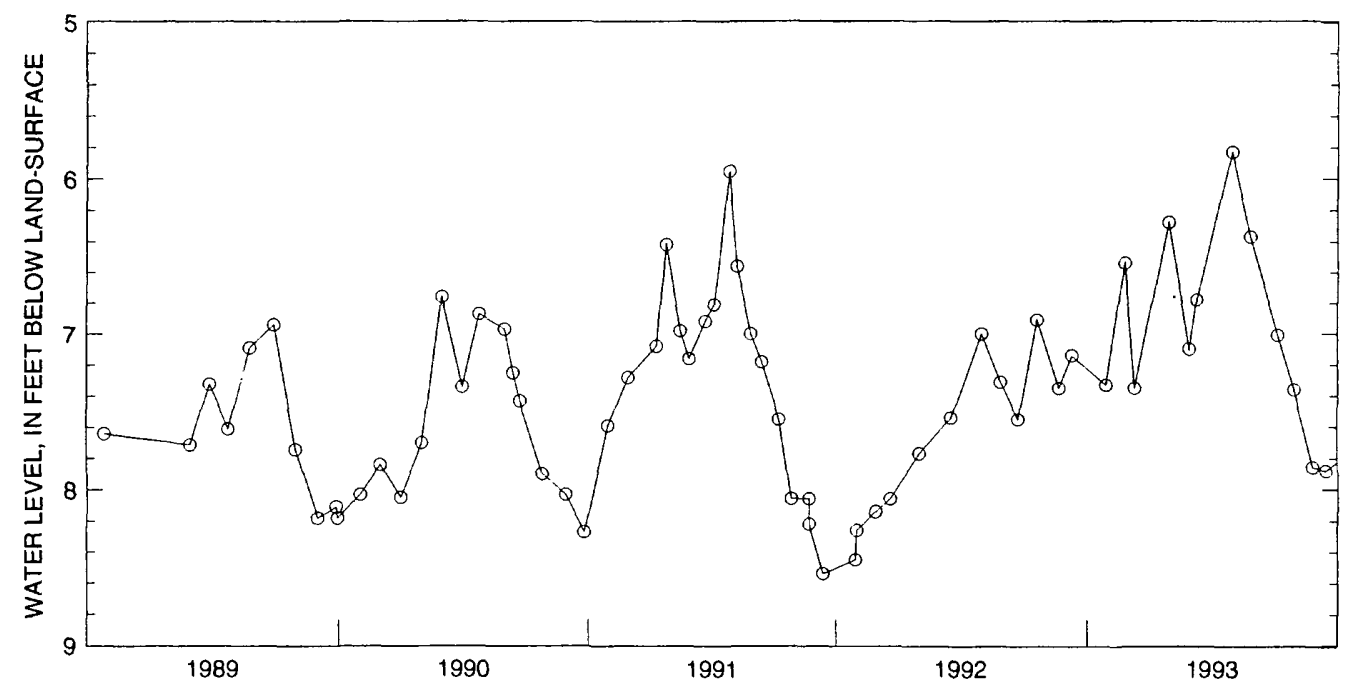


PERIOD OF RECORD.-- January 1991 to current year.

CHEMICAL DATA: 1991-93(b)

ORGANIC DATA: OC.--1991-93(b)

NUTRIENT DATA: 1991-93(b)

COOPERATION-- Water-quality samples were collected and analyzed by the Monroe County Environmental Health Laboratory at Rochester, NY.

\section{WATER QUALTY DATA, PERIOD JANUARY 1991 TO SEPTEMBER 1991}

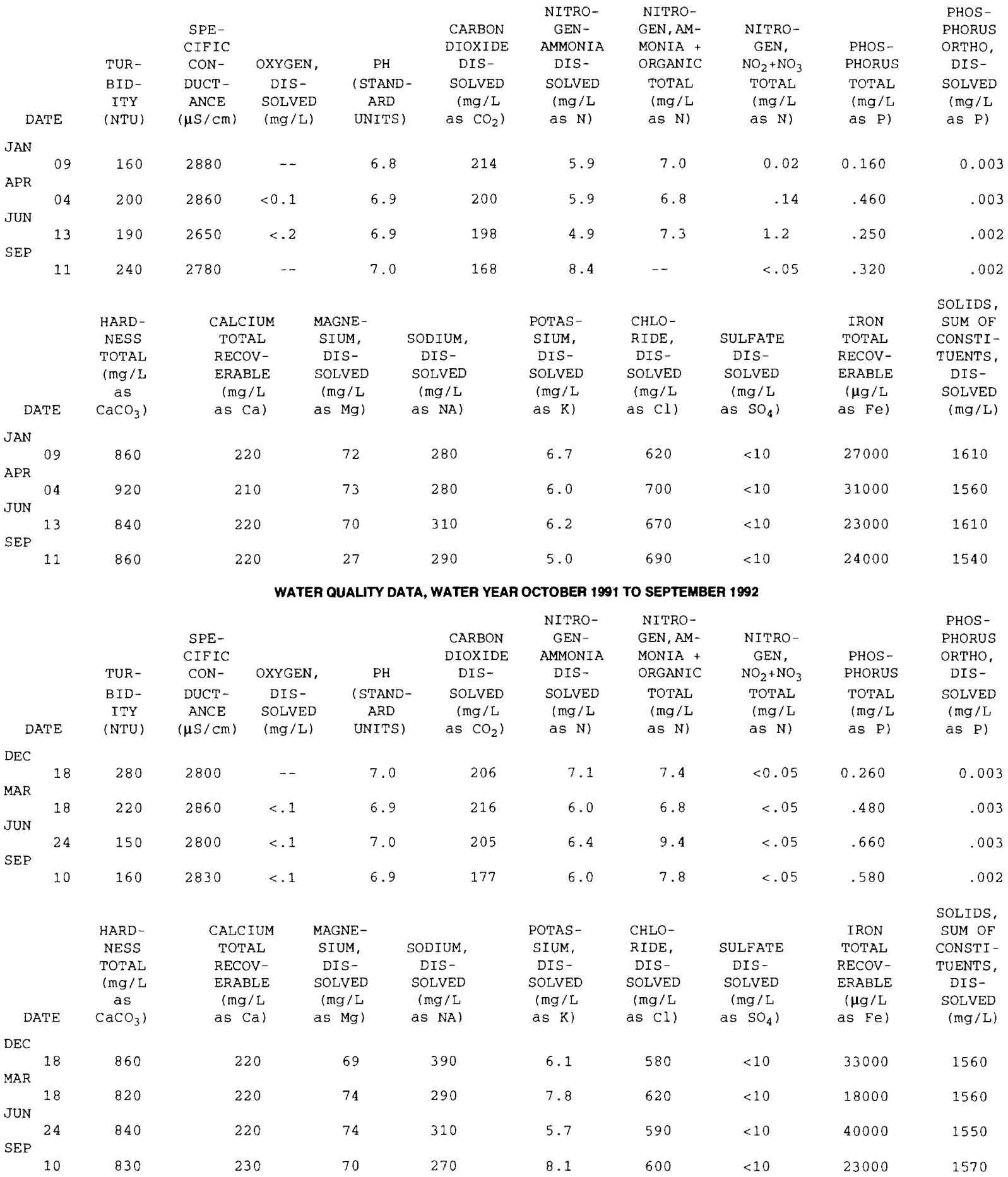




\section{GROUND-WATER QUALITY}

\section{Ellison park}

430928077314002. Local number Mo 668 (B88-2d)--continued

WATER QUALTY DATA, WATER YEAR OCTOBER 1992 TO SEPTEMBER 1993

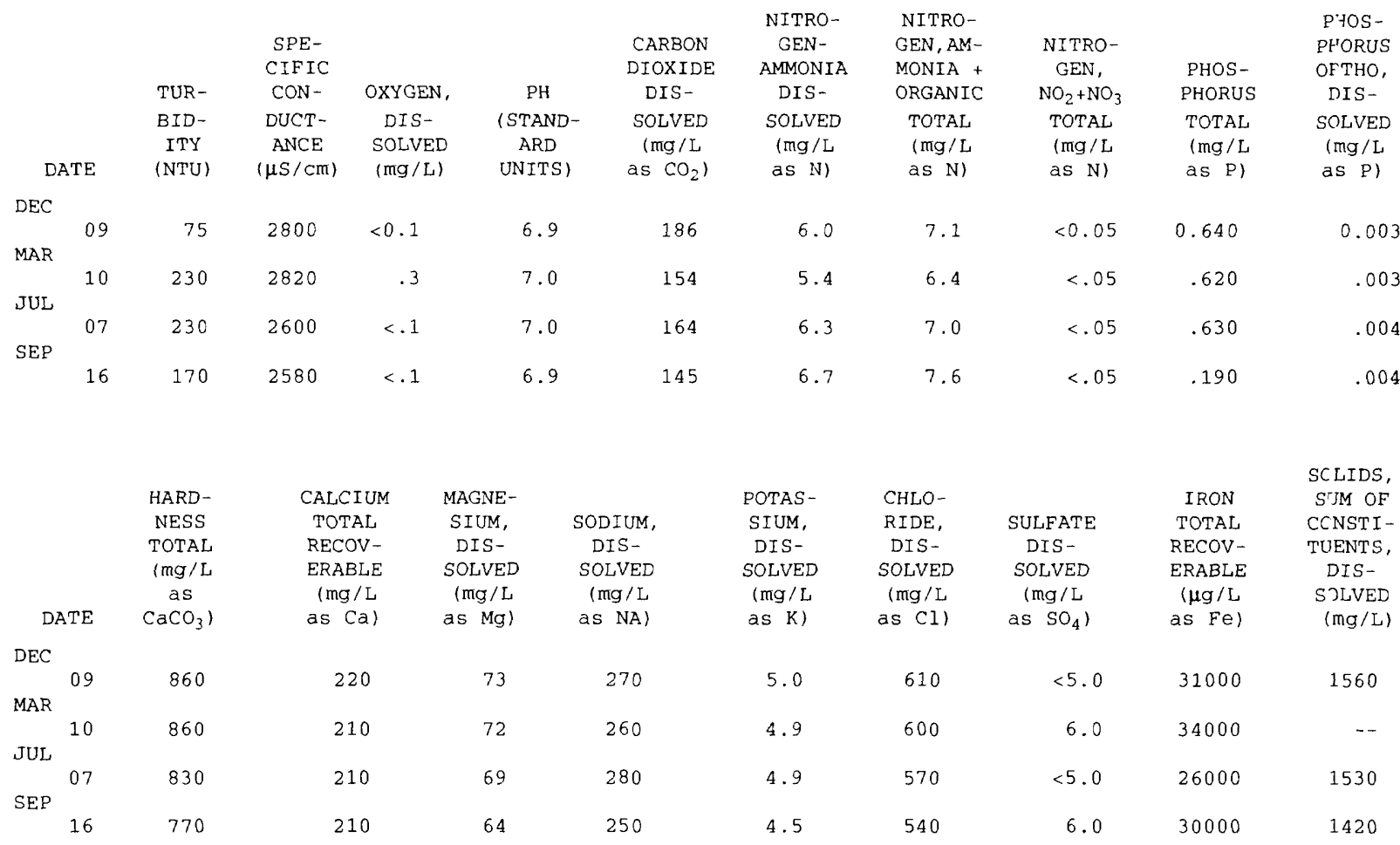


430928077314002. Local number Mo 668 (B88-2d)--continued

WATER TEMPERATURE, IN DEGREES CELSIUS

\begin{tabular}{|c|c|c|c|c|c|c|c|c|c|c|c|c|}
\hline \multirow{2}{*}{$\begin{array}{l}\text { Depth, } \\
\text { in feet }\end{array}$} & \multicolumn{4}{|c|}{$1991 \mathrm{WY}$} & \multicolumn{4}{|c|}{$1992 \mathrm{WY}$} & \multicolumn{4}{|c|}{$1993 \mathrm{WY}$} \\
\hline & $\operatorname{Jan} 9$ & Apr 4 & Jun 13 & Sep 11 & Dec 18 & Mar 18 & Jun 24 & Sep 9 & $\operatorname{Dec} 8$ & $\operatorname{Mar} 9$ & Jul 6 & Sep 15 \\
\hline 7.00 & 7.0 & 7.5 & 11.5 & -- & -- & -- & -- & 16.5 & -- & 5.5 & 17.5 & -- \\
\hline 7.60 & -- & 6.5 & -- & -- & -- & 3.5 & 11.0 & 15.5 & 9.0 & 5.5 & 13.5 & -- \\
\hline 8.10 & -- & -- & -- & -- & 8.5 & -- & -- & -- & -- & $\ldots$ & $\begin{array}{ll}10 \\
--2\end{array}$ & 15.0 \\
\hline 8.60 & 8.5 & 6.5 & 11.0 & 15.0 & 9.0 & 3.5 & 10.5 & 14.5 & 9.5 & 6.0 & 12.5 & 14.5 \\
\hline 9.60 & $\ldots$ & 6.5 & 10.0 & 15.0 & 10.0 & 4.0 & 10.0 & 14.0 & 10.0 & 6.0 & $\ldots$ & 14.0 \\
\hline 10.60 & 9.0 & 6.5 & 9.5 & 14.5 & 10.5 & 4.0 & 9.5 & 13.5 & 10.5 & 6.5 & 11.0 & 13.5 \\
\hline 11.60 & -- & 6.5 & 9.5 & 14.0 & 10.5 & 4.5 & 9.0 & 13.5 & 10.5 & 7.0 & -- & 13.0 \\
\hline 12.60 & 9.0 & 7.0 & 9.0 & 13.5 & 11.0 & 5.0 & 9.0 & 12.5 & 10.5 & 7.0 & 10.0 & 12.5 \\
\hline 13.60 & -- & 7.0 & 9.0 & 12.5 & 11.0 & 5.0 & 9.0 & 12.0 & 10.5 & 7.5 & -- & 12.0 \\
\hline 14.60 & 9.5 & 7.5 & 9.0 & 12.0 & 11.0 & 5.5 & 9.0 & 11.5 & 11.0 & 7.5 & 9.5 & 11.5 \\
\hline 15.60 & -- & 8.0 & 9.0 & 11.5 & 11.0 & 5.5 & 9.0 & 11.0 & 11.0 & 8.0 & -- & 11.0 \\
\hline 16.60 & 10.0 & 8.0 & 9.0 & 11.5 & 11.5 & 8.0 & 9.0 & 11.0 & 11.0 & 8.0 & 9.0 & 10.5 \\
\hline 17.60 & - & 8.0 & 9.0 & 11.0 & 11.5 & 8.5 & 9.0 & 10.5 & 11.0 & 8.5 & -- & 10.5 \\
\hline 18.60 & 10.5 & 8.5 & 9.0 & 11.0 & 11.5 & 8.5 & 9.0 & 10.5 & 11.5 & 9.0 & 9.0 & 10.5 \\
\hline 19.60 & - & 9.0 & 9.0 & 10.5 & 11.5 & 9.0 & 9.0 & 10.0 & 11.0 & 9.0 & -- & -- \\
\hline 20.60 & 11.0 & 9.0 & 9.5 & 10.5 & 11.5 & 9.0 & 9.5 & 10.0 & 11.0 & 9.5 & 9.5 & 10.0 \\
\hline 21.60 & -- & 9.0 & 9.5 & 10.5 & 11.0 & 9.5 & 9.5 & 10.0 & 11.0 & 9.5 & -- & -- \\
\hline 22.60 & 11.0 & 9.0 & 9.5 & 10.5 & 11.0 & 9.5 & 9.5 & 10.0 & 11.0 & 9.5 & 9.5 & 10.0 \\
\hline 23.60 & - & 9.5 & 9.5 & 10.0 & 11.0 & 9.5 & 9.5 & 10.0 & 11.0 & 9.5 & -- & -- \\
\hline 24.60 & 10.5 & 9.5 & 9.5 & 10.0 & 11.0 & 10.0 & 9.5 & 10.0 & 11.0 & 9.5 & 9.5 & 10.0 \\
\hline 25.60 & -- & 9.5 & 9.5 & 10.0 & - & 10.0 & 9.5 & 10.0 & 10.5 & 9.5 & - & -- \\
\hline 26.60 & 10.5 & 9.5 & 10.0 & 10.0 & 11.0 & 10.5 & 10.0 & 10.0 & 10.5 & 10.0 & 9.5 & 9.5 \\
\hline 27.60 & -- & 10.0 & 10.0 & -- & -- & 10.5 & 10.0 & 10.0 & 10.5 & 10.0 & -- & -- \\
\hline 28.60 & 10.5 & 10.0 & 10.0 & 10.0 & 10.5 & 10.5 & -- & 10.0 & -- & 10.0 & 10.0 & 9.5 \\
\hline 29.60 & -- & 10.0 & 10.0 & - & -- & 10.5 & 10.0 & 10.0 & 10.5 & -. & $\ldots$ & $\ldots$ \\
\hline 30.60 & 10.5 & 10.0 & 10.0 & 10.0 & 10.5 & 10.5 & $\ldots$ & 10.0 & $\ldots$ & 10.0 & 10.0 & 10.0 \\
\hline 31.60 & -- & 10.0 & 10.0 & -- & - & 10.5 & 10.0 & 10.0 & 10.5 & -- & -- & -- \\
\hline 32.60 & 10.5 & 10.0 & 10.0 & 10.0 & 10.5 & 10.5 & -- & 10.0 & -- & 10.0 & 10.0 & 10.0 \\
\hline 33.60 & $=$ & -- & 10.5 & $\ldots$ & -- & 10.5 & 10.0 & 10.0 & 10.5 & -- & -- & -- \\
\hline 34.60 & 10.5 & 10.0 & 10.5 & 10.5 & 10.5 & 10.5 & -- & 10.0 & $\ldots$ & 10.0 & 10.5 & 10.0 \\
\hline 35.60 & -- & 10.0 & 10.5 & 10.5 & -- & 10.5 & 10.5 & 10.0 & 10.5 & 10.0 & 10.5 & 10.0 \\
\hline 36.00 & 10.5 & $\ldots$ & - & - & 10.5 & -- & -- & 10.0 & -- & $\ldots$ & $\ldots$ & -- \\
\hline
\end{tabular}

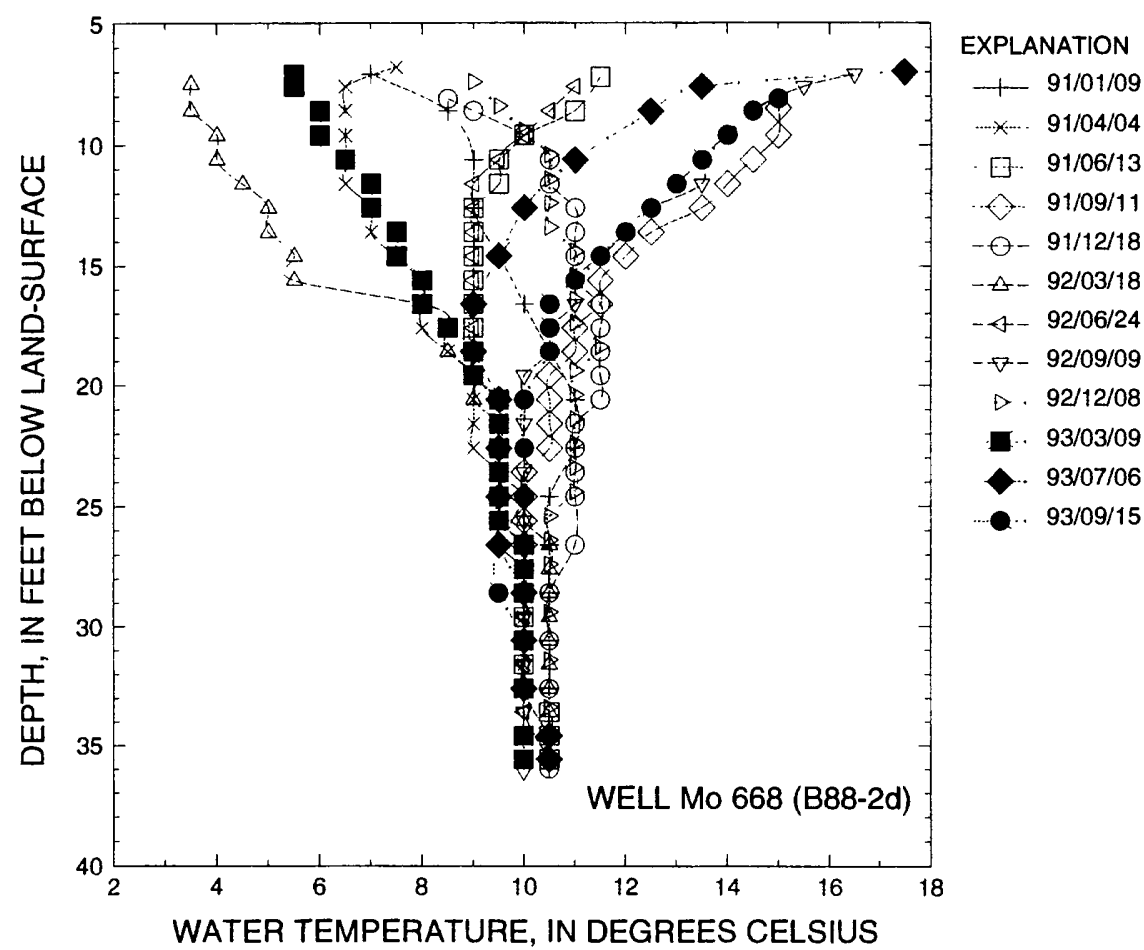




\section{Precipitation Stations}

\section{At Mendon Ponds, Rochester, N.Y.}

LOCATION.--Lat $43^{\circ} 01^{\prime} 17^{\prime \prime}$, long $77^{\circ} 35^{\circ} 01^{\prime \prime}$, Monroe County, Hydrologic Unit 04130003 , in Mendon Ponds County Park, $200 \mathrm{ft}$ east of rangers' quarters, $300 \mathrm{ft}$ east of State Highway 65 , and $1.7 \mathrm{mi}$ south of Interstate Highway 90.

PERIOD OF RECORD.--May 1985 to current year.

EQUIPMENT...Weighing bucket rain gage with graphic recorder.

REMARKS.--Records poor. Because of large amounts of missing or questionable data, no attempt has been made to estimate the missing record.

1. RAINFALL ACCUMULATED - DAILY SUM VALUES (INCHES)

\begin{tabular}{|c|c|c|c|c|c|c|c|c|c|c|c|c|}
\hline DAY & OCT & NoV & $D E C$ & JAN & FEB & MAR & APR & MAY & JUN & JUL & AUG & SEP \\
\hline 1 & .00 & .00 & .00 & -- & .00 & $\cdots$ & .80 & .40 & .50 & .10 & .00 & .30 \\
\hline 2 & .20 & .35 & .00 & --- & --- & .00 & .00 & .80 & .20 & .00 & .20 & .00 \\
\hline 3 & .20 & .00 & .00 & --- & --- & .00 & .20 & .00 & .10 & .00 & 1.10 & .00 \\
\hline 4 & $\cdots$ & .00 & $\cdots$ & $\cdots$ & $\cdots$ & .20 & .35 & .00 & .00 & .00 & .00 & .00 \\
\hline 5 & .10 & .40 & $\cdots$ & .00 & $\cdots$ & .20 & .05 & .00 & .00 & .00 & .00 & .00 \\
\hline 6 & -- & .00 & $\cdots$ & .15 & $\cdots$ & $\cdots$ & .00 & .00 & .00 & .00 & .00 & .00 \\
\hline 7 & -. & .05 & .00 & .00 & $\cdots$ & .10 & .00 & -- & .00 & .00 & .00 & .00 \\
\hline 8 & .00 & .10 & .00 & .05 & $\cdots$ & -- & .00 & $\cdots$ & .00 & .00 & .00 & .00 \\
\hline 9 & -.- & .00 & $\cdots$ & $\cdots$ & $\cdots$ & .00 & .00 & $\cdots$ & 1.10 & .00 & .00 & .00 \\
\hline 10 & $\cdots$ & .00 & $\cdots$ & --- & $\cdots$ & .00 & .00 & -- & .00 & .00 & .00 & .10 \\
\hline 11 & $\ldots$ & .00 & $\cdots$ & .00 & .00 & .00 & .00 & $\cdots$ & .00 & .00 & .00 & .00 \\
\hline 12 & .00 & .00 & $\cdots$ & .00 & .00 & .10 & .00 & $\cdots$ & .10 & .10 & .00 & .00 \\
\hline 13 & .00 & .00 & $\cdots$ & .00 & .00 & .00 & .10 & $\cdots$ & .30 & .00 & .00 & .10 \\
\hline 14 & .00 & $\cdots$ & .10 & .05 & .00 & .00 & .00 & $\cdots$ & .30 & .10 & .00 & .90 \\
\hline 15 & .20 & -- & .00 & .00 & .10 & .00 & .00 & $\cdots$ & .00 & .00 & .00 & .20 \\
\hline 16 & .05 & .10 & $\cdots$ & .00 & .00 & .00 & .00 & $\ldots$ & .40 & .00 & .20 & .30 \\
\hline 17 & .00 & .00 & --- & -.- & .00 & .10 & .00 & $\ldots$ & .40 & .00 & .00 & .50 \\
\hline 18 & .40 & .00 & --- & .00 & .00 & .40 & .00 & $\cdots$ & .00 & .00 & .00 & .00 \\
\hline 19 & .00 & .00 & -- & .00 & --- & .05 & .00 & $\cdots$ & .00 & .10 & .60 & .00 \\
\hline 20 & .00 & .85 & -- & .10 & $\cdots$ & .15 & .00 & $\cdots$ & 1.40 & .00 & .00 & .00 \\
\hline 21 & .15 & $\cdots$ & .00 & .00 & .20 & .05 & .00 & --- & .00 & .20 & .00 & .00 \\
\hline 22 & 1.05 & -- & .00 & $-\cdots$ & .00 & $\cdots$ & .00 & $\ldots$ & .00 & .00 & .00 & .40 \\
\hline 23 & .00 & .00 & .10 & -- & -- & .00 & .00 & $\cdots$ & .10 & .10 & .00 & .50 \\
\hline 24 & .00 & .00 & .20 & $-\cdots$ & $\cdots$ & .10 & .00 & $\cdots$ & .00 & .10 & .00 & .00 \\
\hline 25 & .00 & $\cdots$ & $\cdots$ & .00 & .00 & .05 & .00 & --- & .00 & .00 & .10 & .00 \\
\hline 26 & .00 & --- & $\cdots$ & .30 & .10 & .00 & .00 & .00 & .00 & .10 & .00 & .00 \\
\hline 27 & .00 & - - & $-\ldots$ & .00 & $\cdots$ & .00 & .00 & .00 & .20 & .00 & .00 & .00 \\
\hline 28 & .05 & --. & .05 & .00 & .00 & .05 & .00 & .00 & .10 & .00 & .00 & .00 \\
\hline 29 & .00 & -- & .00 & .00 & --- & .00 & .10 & .00 & .00 & .10 & .10 & .00 \\
\hline 30 & .00 & .00 & -- & .05 & -- & .45 & .00 & .30 & .00 & .10 & .00 & .00 \\
\hline 31 & .00 & --- & $\cdots$ & .00 & $\cdots$ & .40 & $\cdots$ & .20 & --- & .00 & .00 & $\cdots$ \\
\hline TOTAL & -- & --- & $-\cdots$ & $\ldots$ & $-\cdots$ & $\ldots$ & 1.60 & -. & 5.20 & 1.10 & 2.30 & 3.30 \\
\hline
\end{tabular}




\section{At Mendon Ponds, Rochester, N.Y. -- continued}

RAINFALL ACCUMULATED - DAILY SUM VALUES (INCHES)

October 1989 To September 1990

\begin{tabular}{|c|c|c|c|c|c|c|c|c|c|c|c|c|}
\hline DAY & OCT & NOV & $D E C$ & JAN & FEB & MAR & APR & MAY & JUN & JUL & AUG & SEP \\
\hline 1 & .00 & .20 & .00 & $\ldots$ & -- & - - & $\cdots$ & .00 & --- & .10 & .00 & .00 \\
\hline 2 & .40 & .00 & .00 & $\ldots$ & -- & .00 & --- & .00 & $\cdots$ & .00 & .00 & .00 \\
\hline 3 & .00 & .20 & .00 & -- & --- & .00 & --- & .10 & -- & .00 & .00 & .00 \\
\hline 4 & .00 & .00 & .00 & -- & -- & .00 & $\cdots$ & --- & .00 & .30 & .00 & .00 \\
\hline 5 & .00 & .00 & .10 & --- & $-\cdots$ & .00 & $\cdots$ & $\ldots$ & .00 & .20 & 1.30 & .80 \\
\hline 6 & .00 & .00 & .20 & $\cdots$ & --- & .00 & --- & $\ldots$ & .00 & .10 & .50 & .00 \\
\hline 7 & .10 & .40 & .00 & --- & $\ldots$ & .45 & $\cdots$ & --- & .00 & .00 & .00 & .30 \\
\hline 8 & .00 & .00 & .00 & $\cdots$ & $\ldots$ & .00 & -- & --- & .40 & .20 & .00 & .00 \\
\hline 9 & .00 & .20 & .00 & -- & --- & .00 & $-\cdots$ & $\cdots$ & .00 & .50 & .00 & .20 \\
\hline 10 & .00 & .10 & .00 & $\ldots$ & --- & .00 & $\cdots$ & -- & .00 & .00 & .10 & .00 \\
\hline 11 & .00 & .00 & .00 & $\ldots$ & --- & -- & $\ldots$ & - - & .00 & .00 & .00 & .00 \\
\hline 12 & .00 & .00 & .00 & $\cdots$ & $\cdots$ & --- & --- & --- & .00 & .10 & 1.00 & .00 \\
\hline 13 & .00 & .00 & .00 & --- & --- & $\cdots$ & --- & -- & .00 & .00 & .60 & .00 \\
\hline 14 & .00 & .00 & .00 & $\cdots$ & --- & $\cdots$ & $\cdots$ & $\cdots$ & .00 & .00 & .00 & .20 \\
\hline 15 & .30 & .00 & .00 & --- & $\cdots-$ & --- & --- & $\cdots$ & .00 & .10 & .00 & .10 \\
\hline 16 & .00 & .40 & .00 & --- & --- & $\cdots$ & --- & $\cdots$ & .00 & .20 & .00 & .00 \\
\hline 17 & .10 & .10 & .00 & $\cdots$ & $\cdots$ & $\cdots$ & --- & $\ldots$ & .00 & .10 & .00 & .10 \\
\hline 18 & .60 & .00 & .00 & $\cdots$ & -- & $-\cdots$ & -- & --- & .20 & .00 & .00 & .10 \\
\hline 19 & .10 & .00 & .00 & $\cdots$ & $-\cdots$ & $--\infty$ & -- & $-\cdots$ & .10 & .00 & .00 & .10 \\
\hline 20 & .20 & .40 & --- & $\cdots$ & $\cdots$ & $\cdots$ & $-\cdots$ & $-\cdots$ & .00 & .40 & .00 & .00 \\
\hline 21 & .70 & .00 & $\cdots$ & $\cdots$ & --- & --- & --- & $\cdots$ & .00 & .00 & .00 & .00 \\
\hline 22 & .00 & .00 & --- & $-\cdots-$ & .15 & $\cdots$ & --- & $\cdots$ & .70 & .00 & .00 & .10 \\
\hline 23 & .10 & .00 & $\cdots$ & $\cdots$ & .15 & $\cdots$ & $\cdots$ & $\cdots$ & .00 & .30 & .00 & .00 \\
\hline 24 & .00 & .00 & --- & $\cdots-$ & .30 & $\cdots$ & --- & $-\cdots$ & .00 & .10 & .00 & .00 \\
\hline 25 & .00 & .00 & --- & $\cdots$ & --- & $\cdots-$ & --- & $\cdots$ & .00 & .00 & .00 & .10 \\
\hline 26 & .00 & .10 & --- & $-\cdots$ & $\cdots$ & $\cdots$ & --- & $\cdots$ & .00 & .00 & .00 & .20 \\
\hline 27 & .00 & .10 & --- & $\cdots$ & +-- & $\cdots$ & $\cdots$ & $-\cdots$ & .00 & .10 & .00 & .00 \\
\hline 28 & .00 & .00 & --- & $-\cdots$ & --- & -- & --- & $\cdots$ & .00 & .10 & .10 & .00 \\
\hline 29 & .00 & .00 & $\ldots$ & $-\cdots$ & $\ldots$ & $\cdots$ & $\cdots$ & --- & .00 & .10 & .00 & .30 \\
\hline 30 & .00 & .00 & --- & $\cdots$ & $\cdots$ & --- & --- & $\cdots$ & .40 & .00 & .00 & .50 \\
\hline 31 & .00 & $\cdots$ & --- & --- & --- & --- & $\cdots$ & --- & $\cdots$ & .40 & .00 & - - \\
\hline TOTAL & 2.60 & 2.20 & $-\cdots$ & -- & -- & $-\cdots$ & $-\cdots-$ & --- & $-\cdots$ & 3.40 & 3.60 & 3.10 \\
\hline
\end{tabular}


Precipitation Stations

430117077350101. At Mendon Ponds, Rochester, N.Y. -- continued

RAINFALL ACCUMULATED (INCHES), DAILY SUM VALUES

October 1990 To September 1991

\begin{tabular}{|c|c|c|c|c|c|c|c|c|c|c|c|c|}
\hline DAY & OCT & NOV & $\mathrm{DEC}$ & JAN & FEB & MAR & APR & MAY & JUN & JUL & AUG & SEP \\
\hline 1 & .00 & .00 & $\ldots$ & --- & $\cdots$ & $\ldots$ & $-\cdots$ & .10 & .00 & -- & .00 & .00 \\
\hline 2 & .00 & .00 & -- & 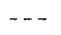 & $\ldots$ & -.- & $\cdots$ & .10 & .00 & .00 & .00 & .00 \\
\hline 3 & .00 & -- & -- & -- & --- & $\ldots$ & $\cdots$ & .00 & .00 & .00 & .60 & .00 \\
\hline 4 & .70 & $\ldots$ & $\ldots$ & $\cdots$ & $\ldots$ & $\ldots$ & -- & .00 & .00 & .10 & .00 & .50 \\
\hline 5 & .10 & $\cdots$ & $\ldots$ & --- & --- & --- & --- & .10 & .00 & 1.00 & .00 & .00 \\
\hline 6 & .00 & $\cdots$ & $\ldots$ & --- & $\ldots$ & $\ldots$ & $\ldots$ & .20 & .00 & .00 & .00 & .10 \\
\hline 7 & .00 & --- & -- & $\cdots$ & -- & -. & $\cdots$ & .00 & .00 & .40 & .00 & .00 \\
\hline 8 & .10 & $\ldots$ & --- & --- & $\cdots$ & --- & -- & .00 & .00 & .00 & .00 & .00 \\
\hline 9 & .70 & $\ldots$ & $\ldots$ & $\ldots$ & -- & -- & $-\cdots$ & .00 & .00 & .00 & 1.00 & .00 \\
\hline 10 & .30 & $-\cdots$ & -- & $\ldots$ & -- & -. & --- & .10 & .00 & .00 & .00 & .30 \\
\hline 11 & 1.10 & $\ldots$ & - & $\ldots$ & .20 & -- & --- & .00 & .00 & .00 & .10 & .00 \\
\hline 12 & .30 & $\ldots$ & --- & $\cdots$ & .00 & -- & -- & .00 & .20 & .00 & .00 & .00 \\
\hline 13 & .70 & --- & $\cdots$ & --- & .10 & $\cdots$ & --- & .00 & .00 & .20 & .00 & .10 \\
\hline 14 & .00 & --- & --- & $\cdots$ & .20 & $\cdots$ & --- & .30 & $\cdots$ & .00 & .20 & .00 \\
\hline 15 & .00 & $-\cdots$ & --- & $\ldots$ & .00 & $\cdots$ & $\cdots$ & .00 & -- & .00 & .00 & .10 \\
\hline 16 & .00 & $\ldots$ & $\ldots$ & --- & .00 & --- & $\ldots$ & .00 & $-\cdots$ & .10 & .00 & .00 \\
\hline 17 & .00 & --- & --- & --- & .00 & -- & -- & .40 & --- & .00 & .00 & .00 \\
\hline 18 & .50 & -. & -- & -- & .30 & $\cdots$ & .00 & .00 & -. & .00 & .00 & .10 \\
\hline 19 & .00 & --- & --- & -- & .00 & -- & .20 & .00 & --- & .00 & .00 & .20 \\
\hline 20 & .00 & --- & --- & --- & .00 & $\cdots$ & .50 & .00 & --- & .00 & .70 & .00 \\
\hline 21 & .00 & $\ldots$ & $\ldots$ & --- & .00 & $\cdots$ & 1.20 & .00 & --- & .30 & .00 & .00 \\
\hline 22 & .30 & --- & $\ldots$ & --- & .00 & --- & .60 & .00 & --- & .10 & .00 & .00 \\
\hline 23 & .60 & $\cdots$ & --- & --- & .10 & --- & .00 & .00 & -- & .00 & .00 & .20 \\
\hline 24 & .00 & --- & --- & --- & .00 & $\cdots$ & .10 & .00 & --- & .00 & .00 & .20 \\
\hline 25 & .10 & --- & -- & -- & .10 & $-\cdots$ & .00 & .00 & --- & .00 & .00 & .70 \\
\hline 26 & .00 & $\ldots$ & $\cdots$ & --- & .00 & $\ldots$ & .00 & .60 & $\ldots$ & .00 & .00 & .00 \\
\hline 27 & .00 & $\ldots$ & $\ldots$ & $\cdots$ & .20 & $\cdots$ & .00 & .00 & -- & .00 & .00 & .00 \\
\hline 28 & .20 & --- & $\cdots$ & --- & .00 & --- & .00 & .00 & -- & .00 & .00 & .00 \\
\hline 29 & .00 & $\cdots$ & -- & $\cdots$ & -- & $-\cdots$ & .00 & .00 & $\cdots$ & .00 & .00 & .00 \\
\hline 30 & .00 & -- & --- & $\cdots$ & --- & --- & .00 & .00 & --- & .10 & .00 & .00 \\
\hline 31 & .00 & --- & $-\cdots$ & $\cdots$ & $\cdots$ & -- & $-\cdots$ & .00 & --- & .00 & .10 & $-\cdots$ \\
\hline TOTAL & 5.70 & $\ldots$ & $\ldots$ & $\ldots$ & -- & $\ldots$ & --- & 1.90 & $\ldots$ & $\ldots$ & 2.70 & 2.50 \\
\hline
\end{tabular}




\section{Precipitation Stations}

430117077350101. At Mendon Ponds, Rochester, N.Y. -- continued

RAINFALL ACCUMULATED (INCHES), DAILY SUM VALUES

October 1991 To September 1992

\begin{tabular}{|c|c|c|c|c|c|c|c|c|c|c|c|c|}
\hline DAY & OCT & NOV & DEC & JAN & FEB & MAR & APR & MAY & JUN & JUL & AUG & SEP \\
\hline 1 & .40 & .00 & .00 & .00 & --- & --- & .20 & .00 & .20 & --- & $\ldots-$ & -- \\
\hline 2 & .00 & .00 & .10 & .00 & --- & -- & .00 & 1.30 & .00 & --- & $\ldots$ & --- \\
\hline 3 & .00 & .00 & .70 & .00 & --- & --- & .00 & .00 & .00 & $\cdots$ & $\ldots$ & $\cdots$ \\
\hline 4 & .10 & .00 & .30 & .10 & $\cdots$ & .10 & .00 & .00 & .10 & -- & --- & -- \\
\hline 5 & .30 & .00 & .30 & .10 & $\cdots$ & .10 & .00 & .00 & .20 & --- & --- & $\ldots$ \\
\hline 6 & .00 & .00 & .10 & .00 & --- & .00 & .00 & .00 & .20 & --- & - - & -- \\
\hline 7 & .00 & .30 & .10 & .00 & $\cdots$ & .60 & .10 & .00 & .00 & --- & -.- & --- \\
\hline 8 & .00 & .00 & .00 & --- & $\cdots$ & .00 & .00 & .00 & .00 & --- & --- & --- \\
\hline 9 & .00 & .00 & .10 & --- & $\cdots$ & .10 & .10 & .20 & .00 & --- & $\cdots$ & $\cdots$ \\
\hline 10 & .60 & .30 & .00 & -- & --- & .00 & .00 & .00 & .20 & --- & --- & -- \\
\hline 11 & .00 & .70 & .00 & $\ldots$ & --- & 1.10 & .80 & .00 & .00 & --- & --- & $\ldots$ \\
\hline 12 & .00 & .00 & .10 & --- & --- & .00 & .00 & .00 & .10 & --- & --- & - - \\
\hline 13 & .10 & .00 & .00 & -- & --- & .00 & .00 & .00 & .00 & $\cdots$ & --- & -- \\
\hline 14 & .00 & .00 & .10 & $\cdots$ & --- & .00 & .00 & .00 & .00 & --- & $\cdots$ & --- \\
\hline 15 & .40 & .20 & .10 & $\cdots$ & -- & .00 & .00 & .00 & $\cdots$ & --- & --- & $\ldots$ \\
\hline 16 & .00 & .00 & .00 & $\cdots-$ & --- & .00 & 1.00 & .00 & --- & --- & --- & --- \\
\hline 17 & .00 & .00 & .10 & --- & --- & .00 & .00 & .70 & --- & --- & --- & --- \\
\hline 18 & .00 & .00 & --- & $\cdots$ & --- & .00 & .30 & .20 & $\cdots$ & --- & --- & .90 \\
\hline 19 & .20 & .00 & .00 & --- & -- & .00 & .00 & .00 & --- & --- & --- & .00 \\
\hline 20 & .00 & .20 & .00 & $\cdots$ & -- & .00 & .00 & .00 & --- & $-\cdots$ & $\cdots$ & .00 \\
\hline 21 & .00 & .10 & .10 & --- & --- & .00 & .40 & .00 & --- & $\cdots$ & --- & 1.00 \\
\hline 22 & .00 & .00 & .00 & -- & --- & .10 & .00 & .00 & --- & --- & --- & .30 \\
\hline 23 & .00 & .00 & .00 & $-\cdots$ & --- & .00 & .10 & .00 & -- & --- & $\cdots$ & .00 \\
\hline 24 & .00 & .20 & .10 & --- & --- & .00 & .30 & .30 & --- & -- & -- & .00 \\
\hline 25 & .00 & .00 & .00 & -- & $-\cdots$ & .00 & .10 & .00 & --- & --- & $\cdots$ & .00 \\
\hline 26 & .00 & .10 & .00 & --- & $\cdots$ & .40 & .00 & .50 & --- & -- & --- & .40 \\
\hline 27 & .00 & .00 & .00 & --- & --- & 1.40 & .00 & .10 & $\cdots$ & $\cdots$ & $\cdots$ & .40 \\
\hline 28 & .00 & .30 & .00 & -- & -- & .10 & .00 & .00 & --- & --- & -- & .00 \\
\hline 29 & .00 & .40 & 1.30 & --- & --- & .00 & .00 & .10 & --- & $-\cdots$ & $\cdots$ & .00 \\
\hline 30 & .00 & .00 & .00 & --- & -- & .00 & .10 & .70 & -- & --- & --- & .00 \\
\hline 31 & .00 & $-\cdots$ & .00 & --- & --- & .00 & $\cdots$ & .40 & $\cdots$ & --- & --- & --- \\
\hline TOTAL & 2.10 & 2.80 & $\cdots$ & --- & $\cdots$ & --- & 3.50 & 4.50 & -- & --- & --- & -- \\
\hline
\end{tabular}


Precipitation Stations

430117077350101. At Mendon Ponds, Rochester, N.Y. -- continued

RAINFALL ACCUMULATED (INCHES), DAILY SUM VALUES

October 1992 To September 1993

\begin{tabular}{|c|c|c|c|c|c|c|c|c|c|c|c|c|}
\hline DAY & OCT & NOV & DEC & JAN & FEB & MAR & APR & MAY & JUN & JUL & AUG & SEP \\
\hline 1 & .00 & .00 & .00 & .00 & .00 & --- & .80 & .00 & .10 & .00 & .00 & .10 \\
\hline 2 & .00 & 1.00 & .00 & .00 & .10 & .00 & .70 & .00 & .00 & .40 & .10 & .50 \\
\hline 3 & .00 & .10 & .00 & .30 & .00 & .00 & .10 & .00 & .00 & .00 & .00 & .00 \\
\hline 4 & .00 & .10 & .10 & .40 & .00 & .30 & .00 & .00 & .00 & .00 & .00 & .00 \\
\hline 5 & .00 & .40 & .10 & .00 & .10 & .20 & .00 & .20 & 1.30 & .00 & .00 & .00 \\
\hline 6 & .00 & .00 & .10 & .00 & .00 & .00 & .00 & .00 & .00 & .00 & .00 & 1.00 \\
\hline 7 & .00 & .00 & .10 & .00 & .00 & .00 & .00 & .00 & .00 & .00 & .30 & .00 \\
\hline 8 & .00 & .00 & .20 & .10 & .00 & .10 & .00 & .00 & .30 & .00 & .00 & .00 \\
\hline 9 & .90 & .00 & .00 & .00 & .00 & .00 & .00 & .00 & .30 & .30 & .00 & .30 \\
\hline 10 & .00 & .10 & .20 & .10 & .00 & .20 & .40 & .00 & .20 & .00 & .00 & .40 \\
\hline 11 & .40 & .10 & .80 & .10 & .00 & .00 & .00 & .10 & .00 & .00 & .00 & .00 \\
\hline 12 & .00 & .00 & .40 & .00 & .40 & .00 & .00 & .00 & .10 & .50 & .00 & .10 \\
\hline 13 & .00 & .00 & .30 & .80 & .10 & .90 & .00 & .00 & .00 & .00 & .10 & .00 \\
\hline 14 & .00 & .00 & .10 & .10 & .00 & .50 & .00 & .00 & .00 & .00 & .20 & .00 \\
\hline 15 & .30 & .30 & .00 & .10 & .00 & .00 & .00 & .00 & .00 & .00 & .00 & .00 \\
\hline 16 & .20 & .10 & .00 & .00 & .30 & .20 & .60 & .00 & .00 & .00 & .40 & .00 \\
\hline 17 & .00 & .00 & .20 & .00 & .00 & .10 & .20 & .00 & .00 & .00 & .10 & .00 \\
\hline 18 & .00 & -- & .00 & .00 & .00 & .00 & .00 & .00 & .00 & .00 & .00 & .00 \\
\hline 19 & .00 & -- & .00 & .00 & .00 & .00 & .00 & .00 & .30 & .60 & .00 & .00 \\
\hline 20 & .00 & --- & .10 & .00 & --- & .10 & .20 & .00 & .10 & .00 & .60 & .00 \\
\hline 21 & .00 & --- & .00 & .10 & $-\cdots$ & .00 & .10 & .00 & .10 & .00 & .00 & .00 \\
\hline 22 & .00 & $\cdots$ & .00 & .10 & --- & .00 & .70 & .10 & .00 & .00 & .10 & .00 \\
\hline 23 & .00 & .00 & .00 & .10 & $\cdots$ & .30 & .00 & .00 & .00 & .00 & .00 & .40 \\
\hline 24 & .80 & .30 & .10 & .20 & --- & .00 & .00 & .10 & .00 & .00 & .00 & .00 \\
\hline 25 & .10 & .10 & .00 & .00 & $\cdots$ & .00 & .30 & .10 & .00 & .00 & .00 & .00 \\
\hline 26 & .00 & .00 & .00 & .00 & --- & .00 & .00 & .00 & .00 & .20 & .10 & .80 \\
\hline 27 & .00 & .00 & .00 & .00 & --- & .00 & .00 & .00 & .10 & .00 & .00 & .40 \\
\hline 28 & .00 & .00 & .00 & .10 & --- & .10 & .00 & .10 & .20 & .00 & .00 & .10 \\
\hline 29 & .00 & .00 & .30 & .00 & --- & .10 & .00 & .00 & .10 & .00 & .10 & .10 \\
\hline 30 & .00 & .00 & .70 & .20 & --- & .00 & .00 & .00 & .00 & .20 & .00 & .10 \\
\hline 31 & .00 & --- & .10 & .00 & --- & .10 & --- & .50 & $\cdots$ & .00 & .70 & $\ldots$ \\
\hline TOTAL & 2.70 & $\ldots$ & 3.90 & 2.80 & $-\cdots$ & $\ldots$ & 4.10 & 1.20 & 3.20 & 2.20 & 2.80 & 4.30 \\
\hline
\end{tabular}




\section{Precipitation Stations}

\section{At Mendon Ponds, Rochester, N.Y. -- continued}

PERIOD OF RECORD.--June 1980 to current year (monthly composite)

June 1980 to current year (monthly wetfall).

June 1980 to current year (monthly dustfall).

INSTRUMENTATION.-- The composite sample collector is a straight-sided polyethlyene funnel approximately 6.5 in. in diameter that drains into a Teflon receiving bottle. A looped plastic tubing connects the funnel with the receiving bottle to retard evaporation. The polyethlyene funnel is heated during the cold-weather season to aid in complete collection of snow. The receiving bottle is enclosed in an insulated box. The opening for the collector is approximately $5 \mathrm{ft}$ above ground level.

Wetfall and dustfall sample collector. An automatic sensor detects precipitation and activates a motor that removes the cover from the wetfall-collection vessel and covers the dustfall-collection vessel. When precipitation ceases, the cycle is reversed. The sampling vessels are polyethlyene and have a collection diameter of 11.26 in. and a capacity of about 3.4 gallons. The openings of the collectors are approximately $8 \mathrm{ft}$ above ground level.

REMARKS.--Inches of precipitation are obtained from an onsite recording weighing-bucket rain gage.

COOPERATION.--Water-quality samples were collected and analyzed by the Monroe County Health Laboratory at Rochester, NY.

\section{CHEMICAL QUALITY OF PRECIPITATION - MONTHLY DUSTFALL}

\begin{tabular}{|c|c|c|c|c|c|c|c|c|c|c|}
\hline & DATE & $\begin{array}{l}\text { RAIN } \\
\text { FALL } \\
\text { ACCUM } \\
(I N)\end{array}$ & $\begin{array}{l}\text { CALCIUM } \\
\text { DIS- } \\
\text { SOLVED } \\
\text { (mg/L } \\
\text { as Ca) }\end{array}$ & $\begin{array}{l}\text { MAGNE- } \\
\text { SIUM, } \\
\text { DIS- } \\
\text { SOLVED } \\
\text { (mg/L } \\
\text { as Mg) }\end{array}$ & $\begin{array}{l}\text { SODIUM, } \\
\text { DIS- } \\
\text { SOLVED } \\
\text { (mg/L } \\
\text { as Na) }\end{array}$ & $\begin{array}{l}\text { POTAS- } \\
\text { SIUM, } \\
\text { DIS- } \\
\text { SOLVED } \\
\text { (mg/L } \\
\text { as K) }\end{array}$ & $\begin{array}{l}\text { CHLO- } \\
\text { RIDE, } \\
\text { DIS- } \\
\text { SOLVED } \\
\text { (mg/L } \\
\text { as Cl) }\end{array}$ & $\begin{array}{l}\text { SULFATE } \\
\text { DIS- } \\
\text { SOLVED } \\
\text { (mg/L } \\
\text { as } \mathrm{SO}_{4} \text { ) }\end{array}$ & $\begin{array}{c}\text { NITRO- } \\
\text { GEN, } \\
\text { AMMONIA } \\
\text { DIS- } \\
\text { SOLVED } \\
\text { (mg/L } \\
\text { as N) }\end{array}$ & $\begin{array}{l}\text { NITRO } \\
\text { GEN, AM- } \\
\text { MONIA } \\
\text { ORGANIC } \\
\text { TOTAL } \\
\text { (mg/L } \\
\text { as N) }\end{array}$ \\
\hline \multicolumn{11}{|c|}{ WATER YEAR OCTOBER 1988 TO SEPTEMBER 1989} \\
\hline $\mathrm{OCT}$ & $05-\mathrm{NOV} 03$ & e2.35 & 1.9 & 0.38 & 0.17 & 0.20 & 0.80 & $<10$ & 0.63 & 1.1 \\
\hline NOV & $03-D E C \quad 01$ & e1.50 & 0.63 & 0.30 & 0.13 & 0.20 & 0.30 & $<5.0$ & 0.57 & 0.86 \\
\hline DEC & 01 -JAN 05 & $\mathrm{e} 0.45$ & 7.2 & 0.59 & 2.1 & 0.09 & 3.4 & 5.0 & 0.42 & 0.76 \\
\hline JAN & $05-F E B \quad 02$ & $\mathrm{e} 0.70$ & 2.0 & 0.59 & 1.6 & 0.13 & 4.2 & 7.0 & 0.90 & 1.1 \\
\hline FEB & 02 -MAR 02 & $\mathrm{e} 0.40$ & 2.0 & 0.49 & 3.3 & $<0.05$ & 4.3 & 10 & 0.58 & 0.95 \\
\hline MAR & $02-A P R \quad 05$ & e3.75 & 2.3 & 0.68 & 1.4 & 0.25 & 2.5 & 10 & 0.85 & 1.8 \\
\hline APR & $05-M A Y \quad 04$ & 1.40 & 3.4 & 0.88 & 0.33 & 0.31 & 0.70 & 10 & 0.80 & 1.2 \\
\hline MAY & 04 -JUN 01 & 4.50 & 2.2 & 0.46 & 0.21 & 1.0 & 0.80 & 11 & 1.70 & 6.6 \\
\hline JUN & 01 -JUL 05 & 5.30 & 3.4 & 1.1 & 0.42 & 6.1 & 2.5 & 14 & 2.50 & 16 \\
\hline JUL, & 05-AUG 01 & 1.00 & 1.3 & 0.25 & 0.04 & 0.21 & 0.30 & $<5.0$ & 0.12 & 0.66 \\
\hline AUG & 01 -SEP 01 & 2.30 & 1.8 & 0.51 & 0.04 & 1.2 & 0.70 & 9.0 & 0.45 & 3.8 \\
\hline SEP & $01-$ OCT $\quad 02$ & 3.70 & 1.7 & 0.35 & 0.10 & 0.40 & 0.50 & 7.0 & 0.65 & 1.4 \\
\hline
\end{tabular}

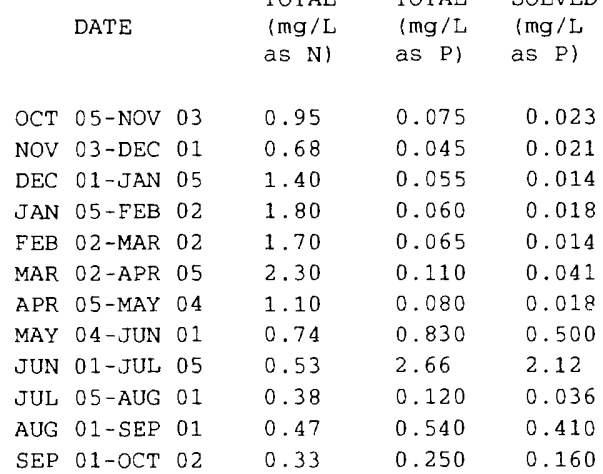

SPE-

CIFIC

CON-

DUCT-

$\mathrm{ANCE}$

$(\mu \mathrm{s} / \mathrm{cm})$

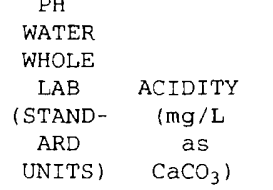

LEAD,

TOTAL TOTAL

RECOV- RECOV-

ERABLE ERABLE

$(\mu \mathrm{g} / \mathrm{L} \quad(\mu \mathrm{g} / \mathrm{L}$

UNITS ) $\mathrm{CaCO}_{3}$ ) as $\left.\mathrm{Pb}\right)$ as $\mathrm{Zn}$

$\begin{array}{lll}29 & 5.4 & 2.6 \\ 24 & 5.1 & 2.9 \\ 41 & 5.3 & 2.4 \\ 48 & 4.7 & 4.0 \\ 50 & 5.0 & 4.4 \\ 63 & 4.4 & 6.8 \\ 42 & 5.7 & 3.2 \\ 49 & 4.9 & 7.5 \\ 80 & 6.6 & 9.7 \\ 21 & 6.0 & 3.8 \\ 33 & 5.4 & 6.4 \\ 27 & 5.6 & 3.5\end{array}$

$\begin{aligned} 5 & -- \\ 7 & -- \\ 14 & -- \\ 10 & -- \\ 12 & -- \\ <5 & -- \\ 14 & - \\ 15 & - \\ 11 & - \\ <5 & - \\ 7 & - \\ 5 & -\end{aligned}$




\section{At Mendon Ponds, Rochester, N.Y. -- continued}

CHEMICAL QUALITY OF PRECIPITATION - MONTHLY DUSTFALL

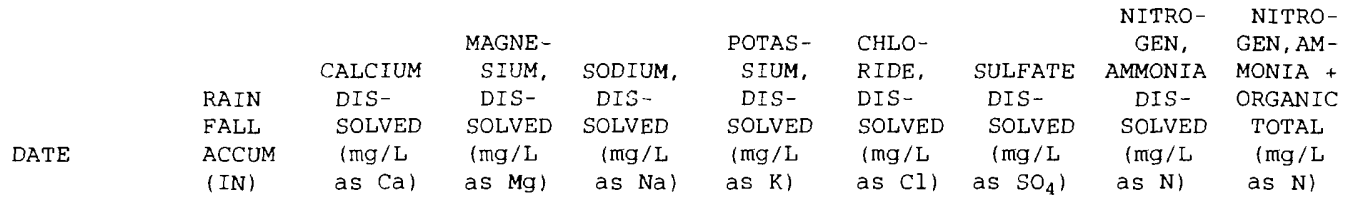

$\begin{array}{lll}\text { OCT } & 02-\text { NOV } & 01 \\ \text { NOV } & 01 \text {-DEC } & 01 \\ \text { DEC } & 01 \text {-JAN } & 02 \\ \text { JAN } & 02-\text { FEB } & 02 \\ \text { FEB } & 02-M A R & 01 \\ \text { MAR } & 01-A P R & 02 \\ \text { APR } & 02-\text { MAY } & 01 \\ \text { MAY } & 01 \text {-JUN } & 04 \\ \text { JUN } & 04-J U L & 02 \\ \text { JUL } & 02-A U G & 01 \\ \text { AUG } & 01-S E P & 04 \\ \text { SEP } & 04-O C T & 02\end{array}$

WATER YEAR OCTOBER 1989 TO SEPTEMBER 1990

$\begin{array}{lllllllll}2.40 & 1.8 & 0.45 & 0.08 & 0.67 & 0.20 & 5.0 & 0.32 & 0.87 \\ 2.00 & 1.9 & 0.39 & 0.89 & 0.10 & 1.4 & 5.0 & 0.78 & 1.2 \\ -- & 1.2 & 0.31 & 3.2 & <0.05 & 4.7 & 6.0 & 0.48 & 0.58 \\ -- & 1.4 & 0.52 & 1.3 & <0.05 & 1.4 & 7.0 & 0.93 & 1.3 \\ -- & 0.97 & 0.28 & 0.54 & 0.01 & 0.80 & <5.0 & 0.34 & 0.40 \\ -- & 2.7 & 0.74 & 1.1 & 0.11 & 1.1 & 10 & 0.55 & 0.94 \\ -- & 2.8 & 0.66 & 0.32 & 0.81 & 0.60 & 9.0 & 0.62 & 5.1 \\ 3.70 & 3.8 & 0.96 & 0.13 & 0.42 & 0.60 & 9.0 & 0.26 & 2.2 \\ 1.90 & 2.3 & 0.58 & 0.12 & 1.1 & 2.3 & 8.0 & 0.43 & 3.2 \\ 3.30 & 1.9 & 0.47 & 0.10 & 0.25 & 0.40 & 7.0 & 0.26 & 1.1 \\ 3.60 & 1.5 & 0.32 & 0.07 & 0.12 & 0.40 & 9.0 & <0.01 & 0.60 \\ 3.10 & 1.9 & 0.37 & 0.10 & 0.16 & 0.20 & 14 & 1.00 & 1.7\end{array}$

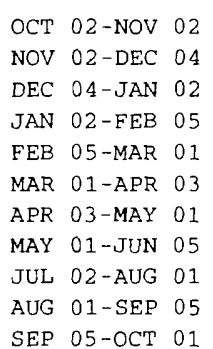

$\begin{array}{lll}\text { OCT } & 02-\text { NOV } & 02 \\ \text { NOV } & 02-D E C & 04 \\ \text { DEC } & 04-J A N & 02 \\ \text { JAN } & 02-\text { FEB } & 05 \\ \text { FEB } & 05-M A R & 01 \\ \text { MAR } & 01-A P R & 03 \\ \text { APR } & 03-M A Y & 01 \\ \text { MAY } & 01-\text { JUN } & 05 \\ \text { JUL } & 02-A U G & 01 \\ \text { AUG } & 01-\text { SEP } & 05 \\ \text { SEP } & 05-O C T & 01\end{array}$

$\begin{array}{lll}5.70 & 2.1 & 0.76 \\ -- & 0.89 & 0.16 \\ -- & 0.18 & 0.04 \\ -- & 1.0 & 0.32 \\ -- & 1.8 & 0.45 \\ -- & 0.64 & 0.23 \\ -- & 2.7 & 0.54 \\ 1.90 & 2.4 & 0.67 \\ 2.30 & 0.67 & 0.32 \\ 3.20 & 1.8 & 0.47 \\ 2.90 & 2.3 & 0.44\end{array}$

$\begin{array}{ll}0.54 & 2.0 \\ 0.56 & 0.72 \\ 0.15 & 0.16 \\ 0.52 & 0.62 \\ 0.69 & 0.75 \\ 0.43 & 0.68 \\ 0.72 & 2.6 \\ 0.37 & 3.9 \\ 0.08 & 5.2 \\ 0.08 & 0.80 \\ 0.28 & 1.1\end{array}$

DATE

$\begin{array}{lll}\text { OCT } & 02-N O V & 01 \\ \text { NOV } & 01-D E C & 01 \\ \text { DEC } & 01 \text {-JAN } & 02 \\ \text { JAN } & 02-\text {-FEB } & 02 \\ \text { FEB } & 02-\text { MAR } & 01 \\ \text { MAR } & 01 \text {-APR } & 02 \\ \text { APR } & 02 \text {-MAY } & 01 \\ \text { MAY } & 01 \text {-JUN } & 04 \\ \text { JUN } & 04-J U L & 02 \\ \text { JUL } & 02-A U G & 01 \\ \text { AUG } & 01 \text {-SEP } & 04 \\ \text { SEP } & 04-O C T & 02\end{array}$

OCT $02-\mathrm{NOV} 02$ NOV $02-D E C \quad 04$ DEC 04-JAN 02 JAN $02-F E B \quad 05$ FEB 05-MAR 01 MAR 01-APR 03 APR 03-MAY 01 MAY 01-JUN 05 JUL 02-AUG 01 AUG 01-SEP 05 SEP 05-OCT 01

\begin{tabular}{|c|c|c|}
\hline & & PHOS- \\
\hline NITRO- & & PHORUS \\
\hline GEN, & PHOS- & ORTHO, \\
\hline $\mathrm{NO} 2+\mathrm{NO} 3$ & PHORUS & DIS- \\
\hline $\begin{array}{l}\text { TOTAL } \\
1 \mathrm{mg} / \mathrm{L}\end{array}$ & $\begin{array}{l}\text { TOTAL } \\
\text { (mg/L }\end{array}$ & $\begin{array}{l}\text { SOLVED } \\
\text { (mg/L }\end{array}$ \\
\hline as $N$ ) & as P) & as P) \\
\hline
\end{tabular}

$\begin{array}{lcc} & \text { PH } & \\ \text { SPE- } & \text { WATER } & \\ \text { CIFIC } & \text { WHOLE } & \\ \text { CON- } & \text { LAB } & \text { ACIDITY } \\ \text { DUCT- } & \text { (STAND- } & (\mathrm{mg} / \mathrm{L} \\ \text { ANCE } & \text { ARD } & \text { as } \\ (\mu \mathrm{S} / \mathrm{cm}) & \text { UNITS) } & \left.\mathrm{CaCO}_{3}\right)\end{array}$

$\begin{array}{ll}\text { LEAD, } & \text { ZINC, } \\ \text { TOTAL } & \text { TOTAL } \\ \text { RECOV- } & \text { RECOV- } \\ \text { ERABLE } & \text { ERABLE } \\ (\mu g / L & (\mu g / L \\ \text { as Pb) } & \text { as Zn) }\end{array}$

\section{WATER YEAR OCTOBER 1989 TO SEPTEMBER 1990}

$$
\begin{aligned}
& 0.80 \\
& 1.50 \\
& 1.80 \\
& 1.70 \\
& 0.85 \\
& 1.60 \\
& 0.88 \\
& 1.00 \\
& 0.71 \\
& 0.54 \\
& 0.11 \\
& 0.85
\end{aligned}
$$

1.30

1.00

0.22

1.70

1. 50

0.87

0.93

0.55

0.60

0.36

0.76
0.120

0.065

0.025

0.025

0.015

0.055

0.310

0.260

0.410

0.130

0.035

0.085
5.5

5.7

4.4

4. 4

4. 5

4.7

5.8

5.8

5. 6

5.0

5.6

4. 5
2.2

6.2

6.1

5.0

3.1

2.7

4.7

2. 2

3.1

3.6

2. 2

4.3
0.87

0.58

. 3

0.94

5.1

2
3. 2

.1
.60

1.7

\section{WATER YEAR OCTOBER 1990 TO SEPTEMBER 1991}

$\begin{array}{lllllrr}0.590 & 0.450 & 51 & 4.3 & 9.5 & 8 & <40 \\ 0.020 & 0.006 & 52 & 4.0 & 6.8 & <5 & <40 \\ 0.010 & 0.009 & 14 & 4.6 & 3.4 & <5 & <40 \\ 0.020 & 0.013 & 73 & 4.0 & 8.2 & 12 & <40 \\ 0.010 & 0.008 & 36 & 5.0 & 2.2 & 9 & 40 \\ 0.015 & 0.009 & 36 & 4.5 & 7.0 & 22 & 40 \\ 0.040 & 0.069 & 36 & 5.6 & 2.6 & 7 & 50 \\ 0.490 & 0.305 & 36 & 6.0 & 4.5 & 9 & 130 \\ 0.770 & 0.520 & 26 & 5.8 & 3.6 & 6 & 40 \\ 0.090 & 0.039 & 21 & 6.3 & 1.8 & 7 & 50 \\ 0.085 & 0.015 & 25 & 6.1 & 1.7 & 10 & <40\end{array}$


430117077350101. At Mendon Ponds, Rochester, N.Y. -- continued CHEMICAL QUALITY OF PRECIPITATION - MONTHLY DUSTFALL

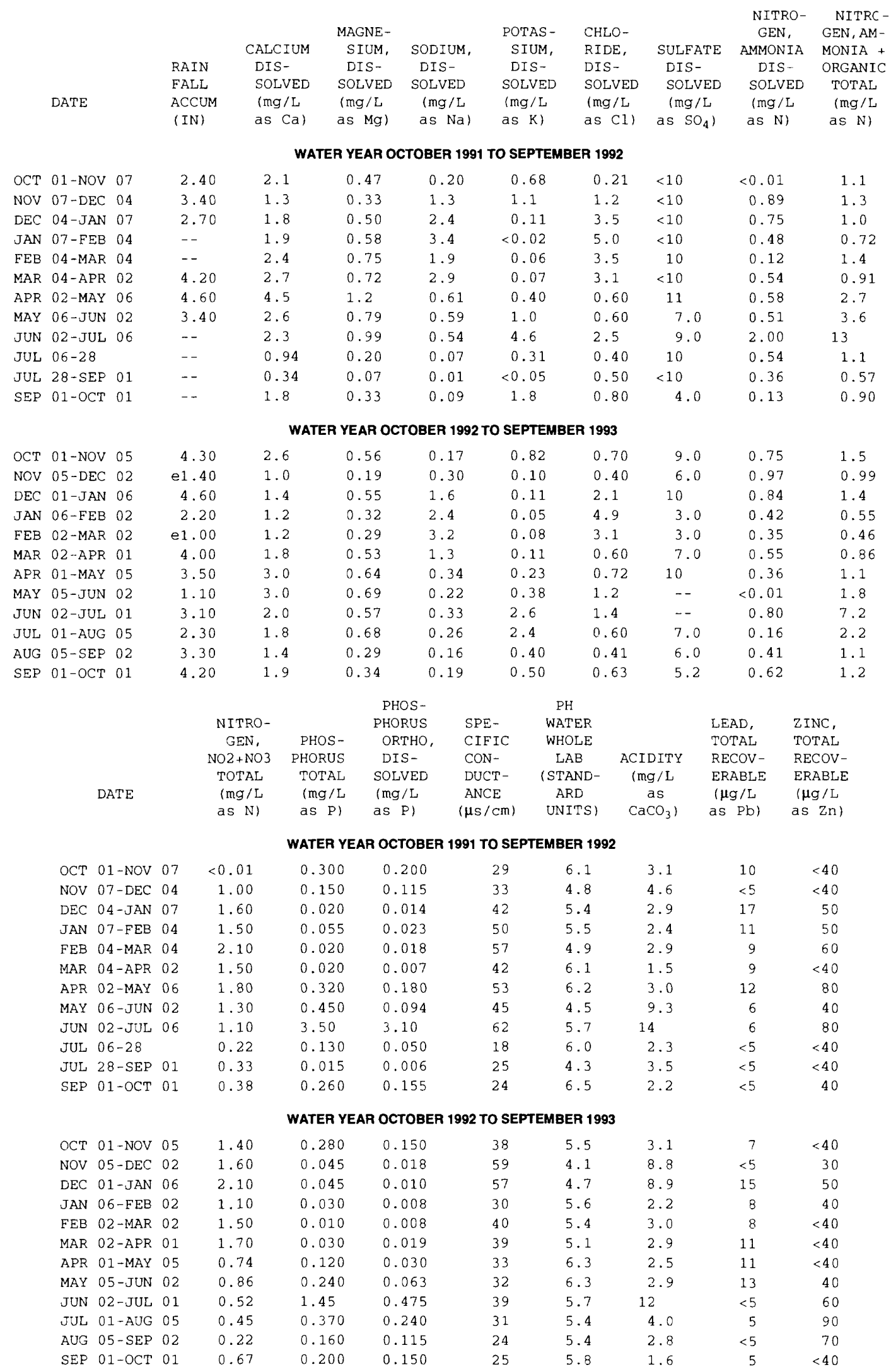


430117077350101. At Mendon Ponds, Rochester, N.Y. -- continued CHEMICAL QUALITY OF PRECIPITATION - MONTHLY WETFALL

$\begin{array}{lll}\text { OCT } & 05-\text { NOV } & 03 \\ \text { NOV } & 03-D E C & 01 \\ \text { DEC } & 01-J A N & 05 \\ \text { JAN } & 05-F E B & 02 \\ \text { FEB } & 02-M A R & 02 \\ \text { MAR } & 02-A P R & 05 \\ \text { APR } & 05-M A Y & 04 \\ \text { MAY } & 04-J U N & 01 \\ \text { JUN } & 01-J U L & 05 \\ \text { JUL } & 05-A U G & 01 \\ \text { AUG } & 01-S E P & 01 \\ \text { SEP } & 01-O C T & 02 \\ & & \\ \text { OCT } & 02-N O V & 01 \\ \text { NOV } & 01-D E C & 01 \\ \text { DEC } & 01-J A N & 02 \\ \text { JAN } & 02-F E B & 02 \\ \text { FEB } & 02-M A R & 01 \\ \text { MAR } & 01-A P R & 02 \\ \text { APR } & 02-M A Y & 01 \\ \text { MAY } & 01-J U N & 04 \\ \text { JUN } & 04-J U L & 02 \\ \text { JUL } & 02-A U G & 01 \\ \text { AUG } & 01-S E P & 04 \\ \text { SEP } & 04-O C T & 02\end{array}$

DATE

\begin{tabular}{|c|c|c|c|c|c|c|c|c|}
\hline & $\begin{array}{l}\text { CALCIUM } \\
\text { DIS- }\end{array}$ & $\begin{array}{l}\text { MAGNE- } \\
\text { SIUM, } \\
\text { DIS- }\end{array}$ & $\begin{array}{c}\text { SODIUM, } \\
\text { DIS- }\end{array}$ & $\begin{array}{c}\text { POTAS- } \\
\text { SIUM, } \\
\text { DIS- }\end{array}$ & $\begin{array}{l}\text { CHLO- } \\
\text { RIDE, } \\
\text { DIS- }\end{array}$ & $\begin{array}{l}\text { SULFATE } \\
\text { DIS- }\end{array}$ & $\begin{array}{l}\text { NITRO- } \\
\text { GEN, } \\
\text { AMMONIA } \\
\text { DIS- }\end{array}$ & $\begin{array}{l}\text { NITRO- } \\
\text { GEN, AM- } \\
\text { MONIA + } \\
\text { ORGANIC }\end{array}$ \\
\hline $\begin{array}{l}\text { FALL } \\
\text { ACCUM }\end{array}$ & $\begin{array}{l}\text { SOLVED } \\
\text { (mg/L }\end{array}$ & $\begin{array}{l}\text { SOLVED } \\
\text { (mg/L } \\
\text { as } \mathrm{Mg} \text { ) }\end{array}$ & $\begin{array}{c}\text { SOLVED } \\
\text { (mg/L } \\
\text { as } \mathrm{Na} \text { ) }\end{array}$ & $\begin{array}{l}\text { SOLVED } \\
\text { (mg/L } \\
\text { as K) }\end{array}$ & $\begin{array}{l}\text { SOLVED } \\
\text { (mg/L } \\
\text { as Cl) }\end{array}$ & $\begin{array}{c}\text { SOLVED } \\
(\mathrm{mg} / \mathrm{L} \\
\left.\text { as } \mathrm{SO}_{4}\right)\end{array}$ & $\begin{array}{l}\text { SOLVED } \\
\text { (mg/L } \\
\text { as N) }\end{array}$ & $\begin{array}{l}\text { TOTAL } \\
\text { (mg/L } \\
\text { as N) }\end{array}$ \\
\hline
\end{tabular}

\section{WATER YEAR OCTOBER 1988 TO SEPTEMBER 1989}

$\begin{array}{ccccccccc}\text { e2.35 } & 0.39 & 0.11 & 0.08 & 0.06 & 0.80 & <10 & 0.34 & 0.46 \\ \text { e1.50 } & 0.13 & 0.16 & 0.08 & 0.08 & <0.20 & <5.0 & 0.18 & 0.24 \\ \text { e0.45 } & 0.14 & 0.05 & 0.20 & 0.05 & <0.20 & <5.0 & 0.16 & 0.21 \\ \text { e0.70 } & 0.15 & 0.03 & 0.50 & 0.34 & 2.7 & <5.0 & 0.16 & 0.48 \\ \text { e0.40 } & 0.90 & 0.26 & 1.2 & <0.05 & 6.0 & 10 & 0.21 & 0.68 \\ \text { e3.75 } & 0.42 & 0.08 & 0.24 & 0.10 & 1.4 & 6.0 & 0.23 & 0.33 \\ 1.40 & 0.14 & 0.04 & 0.10 & 0.04 & 0.40 & 5.0 & 0.47 & 0.54 \\ 4.50 & 0.12 & <0.01 & 0.08 & 0.04 & 0.40 & <5.0 & 0.30 & 0.54 \\ 5.30 & 0.06 & <0.01 & <0.01 & 0.01 & <0.20 & 6.0 & 0.21 & 0.34 \\ 1.00 & 0.08 & 0.02 & 0.04 & 0.05 & 0.50 & <5.0 & 0.25 & 0.40 \\ 2.30 & 0.19 & <0.04 & 0.04 & 0.02 & 0.30 & 8.0 & 0.38 & 0.42 \\ 3.70 & 0.31 & 0.03 & 0.08 & 0.04 & 0.50 & 6.0 & 0.24 & 0.30\end{array}$

\section{WATER YEAR OCTOBER 1989 TO SEPTEMBER 1990}

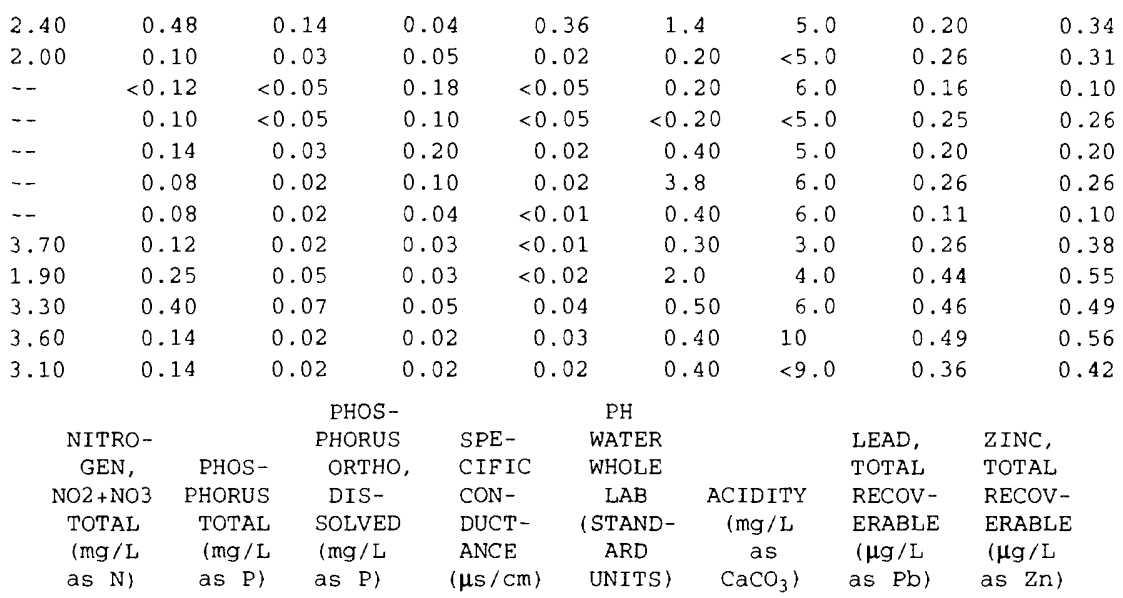

OCT 05 -NOV 03 NOV $03-D E C \quad 01$ DEC 01-JAN 05 JAN $05-$ FEB 02 FEB 02 -MAR 02 MAR 02 -APR 05 APR 05 -MAY 04 MAY 04-JUN 01 JUN 01-JUL 05 JUL 05-AUG 01 AUG $01-S E P 01$ SEP 01 -OCT 02

OCT 02 -NOV 01 NOV $01-D E C \quad 01$ DEC 01-JAN 02 JAN $02-F E B \quad 02$ FEB 02 -MAR 01 MAR 01 -APR 02 APR 02 -MAY 01 MAY 01 -JUN 04 JUN 04 -JUL 02 JUL 02 -AUG 01 AUG 01-SEP 04 SEP $04-O C T \quad 02$
WATER YEAR OCTOBER 1988 TO SEPTEMBER 1989

$\begin{array}{llllll}0.85 & 0.015 & 0.008 & 40 & 4.2 & 6.0 \\ 0.42 & 0.005 & 0.003 & 21 & 4.4 & 4.0 \\ 0.34 & 0.010 & 0.004 & 20 & 4.5 & 3.3 \\ 0.35 & 0.010 & 0.006 & 27 & 4.3 & 5.3 \\ 0.98 & 0.045 & 0.015 & 37 & 4.5 & 4.8 \\ 0.51 & 0.025 & 0.006 & 29 & 4.3 & 5.8 \\ 0.51 & 0.010 & 0.002 & 31 & 4.3 & 5.4 \\ 0.44 & 0.010 & 0.002 & 32 & 4.2 & 5.2 \\ 0.38 & 0.010 & <0.002 & 29 & 4.3 & 4.7 \\ 0.28 & 0.010 & 0.006 & 20 & 4.5 & 5.3 \\ 0.40 & 0.015 & 0.004 & 40 & 4.2 & 6.0 \\ 0.57 & 0.015 & 0.002 & 44 & 4.1 & 6.4\end{array}$

WATER YEAR OCTOBER 1989 TO SEPTEMBER 1990

\begin{tabular}{lrrrrr}
0.46 & 0.072 & 0.036 & 23 & 4.5 & 4.0 \\
0.50 & 0.010 & 0.005 & $\mathrm{~N} 34$ & 4.5 & 4.8 \\
0.60 & 0.005 & 0.005 & \multicolumn{1}{c}{-} & 4.4 & 4.8 \\
0.60 & 0.007 & 0.004 & $\mathrm{~N} 23$ & 4.4 & 4.7 \\
0.50 & 0.005 & 0.003 & 4 & 4.3 & 6.2 \\
0.48 & 0.005 & $<0.002$ & 33 & 4.2 & 5.7 \\
0.36 & 0.005 & 0.002 & $\mathrm{~N} 24$ & $\mathrm{~N} 6.0$ & $\mathrm{~N} 2.7$ \\
0.44 & 0.012 & $<0.002$ & $\mathrm{~N} 28$ & 4.2 & 6.0 \\
0.48 & 0.008 & 0.003 & 34 & 4.2 & 6.5 \\
0.67 & 0.015 & 0.010 & 47 & 4.1 & 22 \\
0.50 & $<0.005$ & $<0.002$ & 60 & 4.0 & 8.7 \\
0.53 & $<0.005$ & 0.002 & 39 & 4.0 & 6.2
\end{tabular}

0.46

0.21

0.48

0.33

0.34

0.42

0.30 
430117077350101. At Mendon Ponds, Rochester, N.Y. -- continued

CHEMICAL QUALITY OF PRECIPITATION - MONTHLY WETFALL

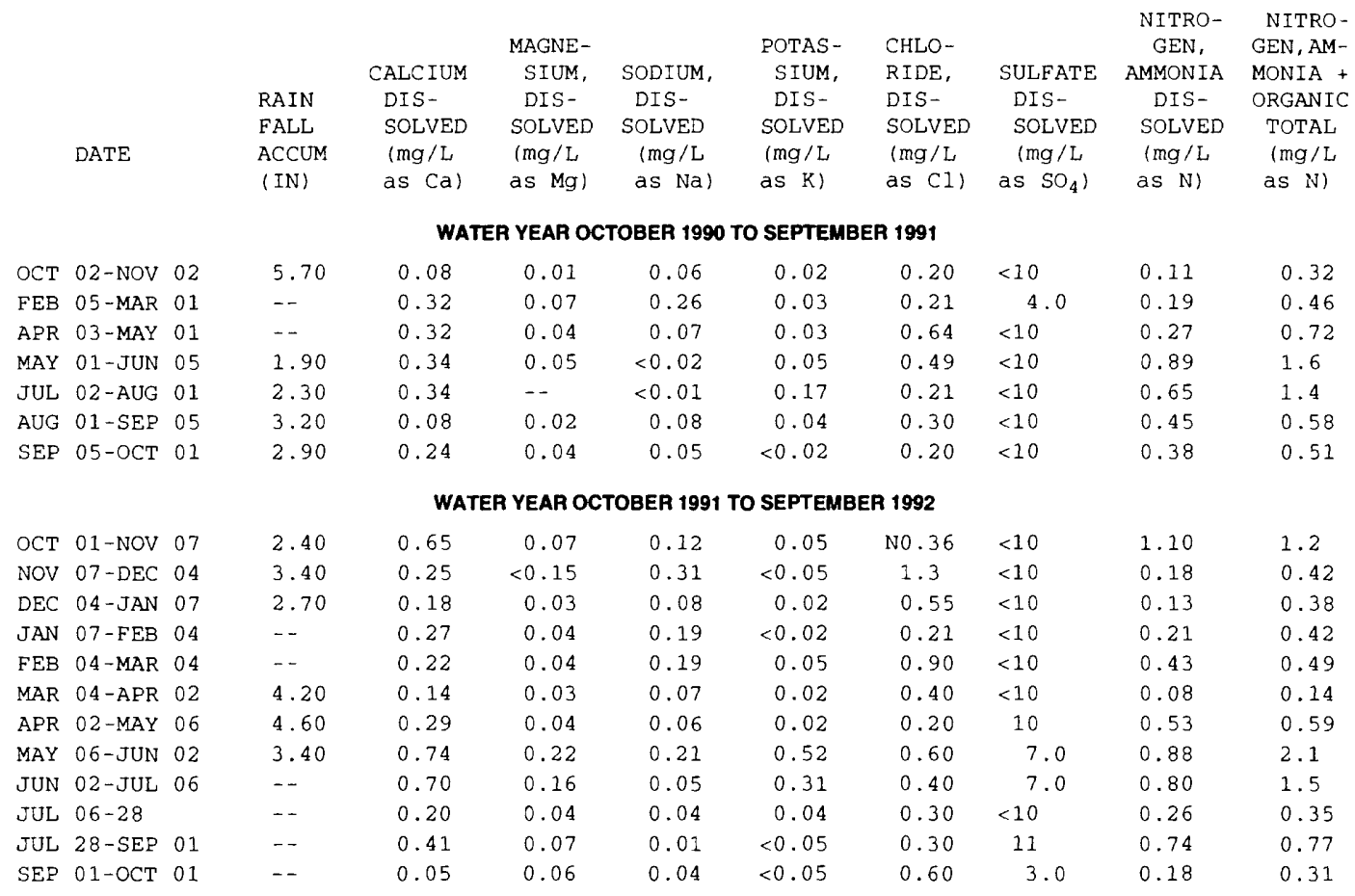

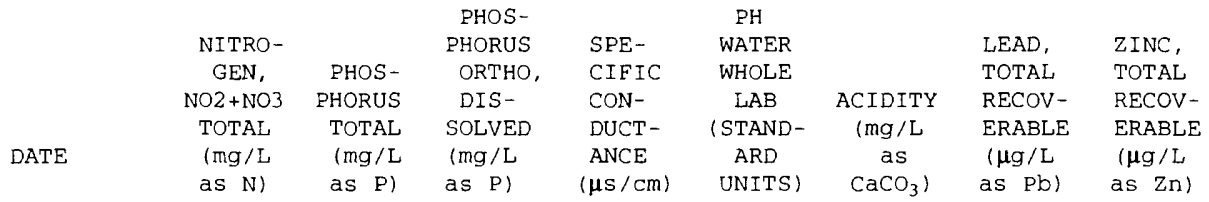

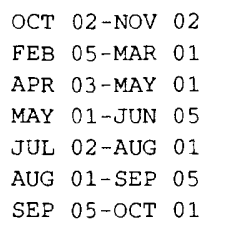

OCT 01-NOV 07 NOV $07-D E C \quad 04$ DEC $04-J A N \quad 07$ JAN $07-$ FEB 04 FEB $04-$ MAR 04 MAR $04-A P R \quad 02$ APR 02-MAY 06 MAY 06-JUN 02 JUN 02-JUL 06 JUL $06-28$ JUL 28-SEP 01 SEP 01-OCT 01

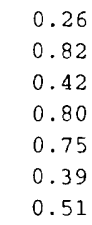

0.26

0.82

0.80

0.75

0.51

$<0.01$

0.35

0.27

0.43

0.38

0.25

0.65

0.80

1.10

0.34

1.00

0.22

WATER YEAR OCTOBER 1990 TO SEPTEMBER 1991

$\begin{array}{rr}<0.005 & <0.002 \\ 0.010 & 0.006 \\ 0.005 & <0.002 \\ 0.070 & <0.002 \\ 0.055 & 0.021 \\ <0.005 & <0.002 \\ 0.012 & <0.002\end{array}$

$\begin{array}{ll}20 & 4.4 \\ 30 & 4.3 \\ 19 & 4.5 \\ 54 & 4.2 \\ 58 & 3.9 \\ 33 & 4.2 \\ 30 & 4.3\end{array}$

4.0
5.5
3.4
7.4
9.2
6.6
5.0

WATER YEAR OCTOBER 1991 TO SEPTEMBER 1992

$\begin{array}{rrrrrrr}0.018 & 0.002 & 50 & 4.1 & 7.9 & 8 & <40 \\ <0.005 & <0.002 & 19 & 4.7 & 3.3 & <5 & <40 \\ <0.005 & <0.002 & 14 & 5.2 & 2.7 & 5 & <40 \\ 0.005 & 0.006 & 19 & 5.0 & 4.3 & 7 & <40 \\ 0.005 & 0.003 & 34 & 4.4 & 5.0 & 8 & 40 \\ 0.005 & 0.002 & 14 & 5.9 & 2.8 & <5 & <40 \\ 0.005 & <0.002 & 36 & 4.4 & 4.6 & <5 & 20 \\ 0.225 & 0.043 & 40 & 4.4 & 7.4 & 7 & <20 \\ 0.120 & 0.036 & 72 & 4.0 & 9.4 & 5 & <40 \\ 0.010 & <0.002 & 31 & 4.4 & 4.8 & <5 & <40 \\ 0.010 & <0.002 & 76 & 3.7 & 12 & <5 & <40 \\ 0.010 & 0.002 & 20 & 4.6 & 3.1 & 5 & 40\end{array}$




\section{At Mendon Ponds, Rochester, N.Y. -- continued}

CHEMICAL QUALITY OF PRECIPITATION - MONTHLY WETFALL

\begin{tabular}{|c|c|c|c|c|c|c|c|c|c|}
\hline & & $\begin{array}{l}\text { CALCIUM } \\
\text { DIS- }\end{array}$ & $\begin{array}{l}\text { MAGNE- } \\
\text { SIUM, } \\
\text { DIS- }\end{array}$ & $\begin{array}{l}\text { SODIUM, } \\
\text { DIS- }\end{array}$ & $\begin{array}{l}\text { POTAS- } \\
\text { SIUM, } \\
\text { DIS- }\end{array}$ & $\begin{array}{l}\text { CHLO- } \\
\text { RIDE, } \\
\text { DIS- }\end{array}$ & $\begin{array}{l}\text { SULFATE } \\
\text { DIS- }\end{array}$ & $\begin{array}{l}\text { NITRO- } \\
\text { GEN, } \\
\text { AMMONIA } \\
\text { DIS- }\end{array}$ & $\begin{array}{l}\text { NITRO- } \\
\text { GEN, AM- } \\
\text { MONIA + } \\
\text { ORGANIC }\end{array}$ \\
\hline & $\begin{array}{l}\text { RAIN } \\
\text { FALL }\end{array}$ & $\begin{array}{l}\text { DIS- } \\
\text { SOLVED }\end{array}$ & $\begin{array}{l}\text { DIS- } \\
\text { SOLVED }\end{array}$ & $\begin{array}{l}\text { DIS- } \\
\text { SOLVED }\end{array}$ & $\begin{array}{l}\text { DIS- } \\
\text { SOLVED }\end{array}$ & $\begin{array}{l}\text { DIS- } \\
\text { SOLVED }\end{array}$ & $\begin{array}{l}\text { DIS- } \\
\text { SOLVED }\end{array}$ & $\begin{array}{l}\text { DIS- } \\
\text { SOLVED }\end{array}$ & $\begin{array}{l}\text { ORGANIC } \\
\text { TOTAL }\end{array}$ \\
\hline & $\begin{array}{l}\text { ACCUM } \\
\text { (IN) }\end{array}$ & $\begin{array}{l}(\mathrm{mg} / \mathrm{L} \\
\text { as Ca) }\end{array}$ & $\begin{array}{l}\text { (mg/L } \\
\text { as } \mathrm{Mg} \text { ) }\end{array}$ & $\begin{array}{l}(\mathrm{mg} / \mathrm{L} \\
\text { as } \mathrm{Na})\end{array}$ & $\begin{array}{l}(\mathrm{mg} / \mathrm{L} \\
\text { as K) }\end{array}$ & $\begin{array}{l}(\mathrm{mg} / \mathrm{L} \\
\text { as C1) }\end{array}$ & $\begin{array}{c}(\mathrm{mg} / \mathrm{L} \\
\left.\operatorname{as} \mathrm{SO}_{4}\right)\end{array}$ & $\begin{array}{l}(\mathrm{mg} / \mathrm{L} \\
\operatorname{as~N})\end{array}$ & $\begin{array}{l}(\mathrm{mg} / \mathrm{L} \\
\mathrm{as} \mathrm{N})\end{array}$ \\
\hline
\end{tabular}

\section{WATER YEAR OCTOBER 1992 TO SEPTEMBER 1993}

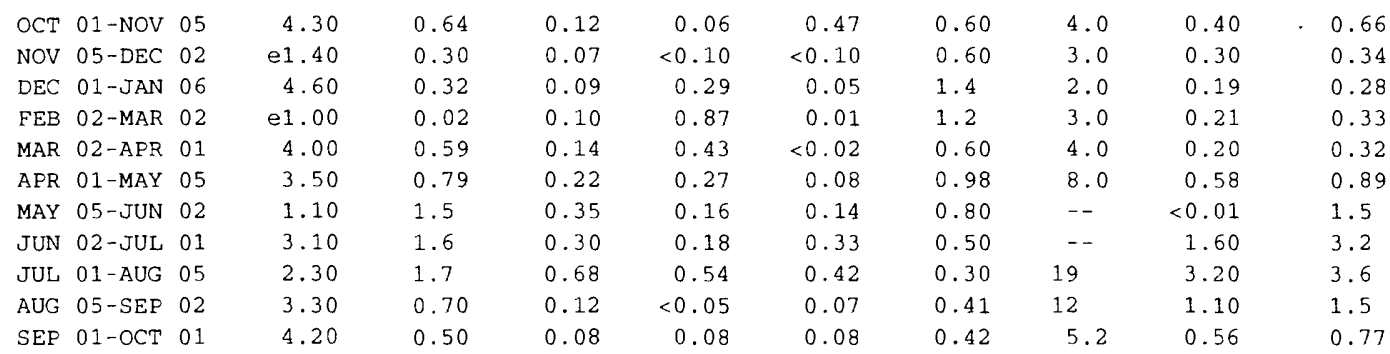

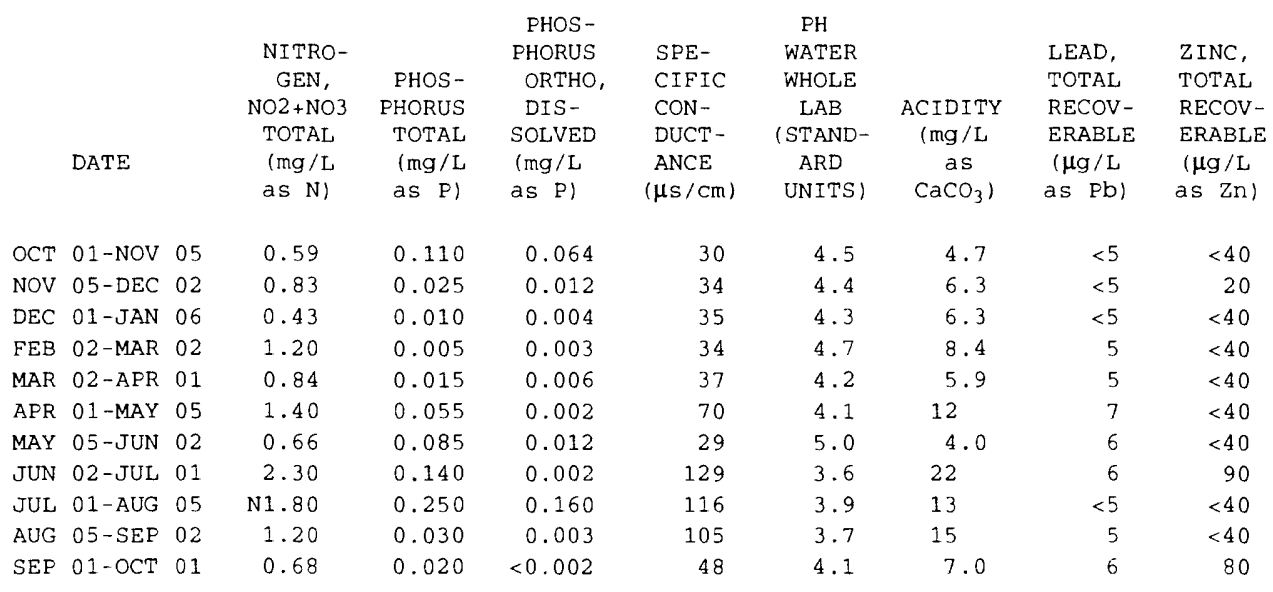


430117077350101 At Mendon Ponds, Rochester, N.Y.--continued

\section{CHEMICAL QUALITY OF PRECIPITATION - MONTHLY COMPOSITE}

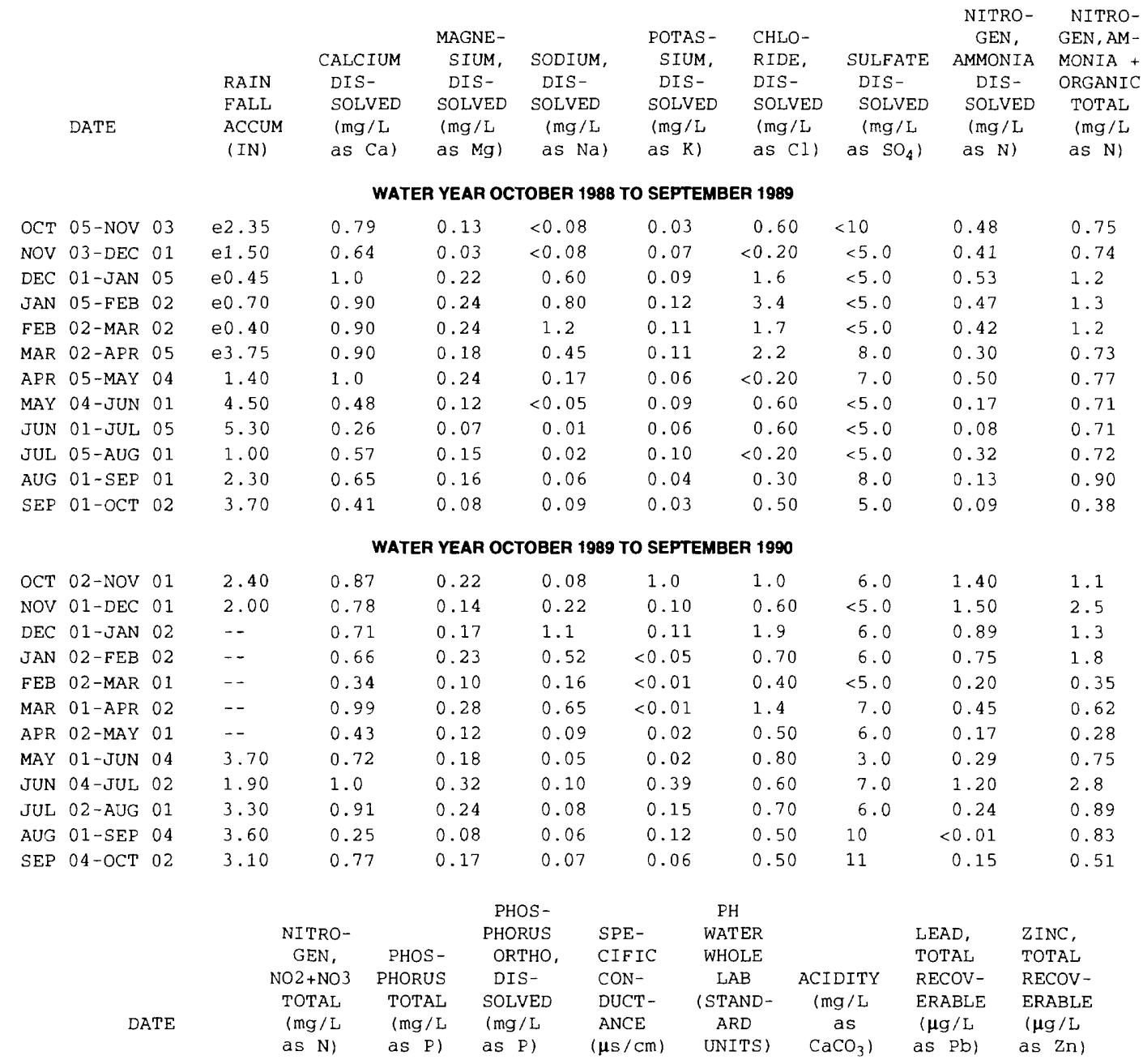

$\begin{array}{llll}\text { OCT } & 05-\text { NOV } & 03 & 0.77 \\ \text { NOV } & 03-\text { DEC } & 01 & 0.69 \\ \text { DEC } & 01-J A N & 05 & 0.62 \\ \text { JAN } & 05-\text {-FEB } & 02 & 0.81 \\ \text { EEB } & 02-\text {-MAR } & 02 & 1.00 \\ \text { MAR } & 02-A P R & 05 & 0.88 \\ \text { APR } & 05-\text { MAY } & 04 & 0.87 \\ \text { MAY } & 04-\text { JUN } & 01 & 0.64 \\ \text { JUN } 01-\text { JUL } & 05 & 0.45 \\ \text { JUL } & 05-A U G & 01 & 0.18 \\ \text { AUG } & 01-S E P & 01 & 0.50 \\ \text { SEP } & 01-\text { OCT } & 02 & 0.55\end{array}$

OCT $02-$ NOV 01 NOV $01-D E C \quad 01$ DEC 01 -JAN 02 JAN $02-F E B \quad 02$ FEB $02-M A R \quad 01$ MAR $01-A P R \quad 02$ APR $02-$ MAY 01 MAY 01-JUN 04 JUN 04 -JUL 02 JUL, 02 -AUG 01 AUG 01 -SEP 04 SEP 04 -OCT 02

WATER YEAR OCTOBER 1988 TO SEPTEMBER 1989

$\begin{array}{lllllll}0.055 & 0.004 & 30 & 4.4 & 4.4 & <5 & \ldots \\ 0.020 & 0.011 & 25 & 4.5 & 4.6 & 6 & - \\ 0.090 & 0.045 & 19 & 5.5 & 2.6 & 5 & \ldots \\ 0.060 & 0.034 & 24 & 5.0 & 2.3 & 6 & -- \\ 0.085 & 0.035 & 35 & 4.5 & 4.0 & 5 & -- \\ 0.045 & 0.014 & 45 & 4.2 & 7.0 & 5 & -- \\ 0.025 & <0.002 & 43 & 4.2 & 7.2 & <5 & -- \\ 0.020 & 0.002 & 46 & 4.1 & 8.7 & <5 & -- \\ 0.030 & 0.006 & 49 & 4.0 & 13 & 6 & - \\ 0.050 & 0.017 & 18 & 4.7 & 11 & <5 & - \\ 0.045 & 0.003 & 56 & 4.0 & 13 & <5 & - \\ 0.075 & 0.009 & 50 & 4.0 & 10 & <5 & --\end{array}$

\section{WATER YEAR OCTOBER 1989 TO SEPTEMBER 1990}

\begin{tabular}{lrrrrrrr}
0.77 & 0.580 & 0.450 & 29 & 5.7 & 3.9 & $<5$ & -- \\
1.10 & 0.120 & 0.052 & 29 & 5.1 & 4.6 & $<5$ & -- \\
0.98 & 0.120 & 0.085 & \multicolumn{1}{c}{-1} & 5.0 & 5.0 & $<5$ & -- \\
1.20 & 0.135 & 0.038 & 46 & 4.3 & 6.5 & 8 & $<40$ \\
0.49 & 0.015 & 0.003 & 3 & 4.3 & 4.9 & 12 & $<40$ \\
0.94 & 0.025 & 0.008 & 48 & 4.1 & 7.0 & $<5$ & $<40$ \\
0.58 & 0.005 & $N 0.006$ & 21 & 6.3 & 2.7 & 8 & $<40$ \\
0.65 & 0.020 & $<0.002$ & 38 & 4.1 & 8.8 & 5 & $<40$ \\
0.64 & 0.130 & 0.088 & 38 & 4.5 & 7.6 & 6 & $<40$ \\
0.85 & 0.030 & 0.010 & 70 & 3.9 & 16 & 7 & $<40$ \\
0.42 & 0.075 & 0.046 & 67 & 3.9 & 13 & $<5$ & 60 \\
0.87 & 0.015 & 0.002 & 70 & 3.8 & 13 & 7 & $<40$
\end{tabular}


430117077350101 At Mendon Ponds, Rochester, N.Y.--continued

CHEMICAL QUALITY OF PRECIPITATION - MONTHLY COMPOSITE

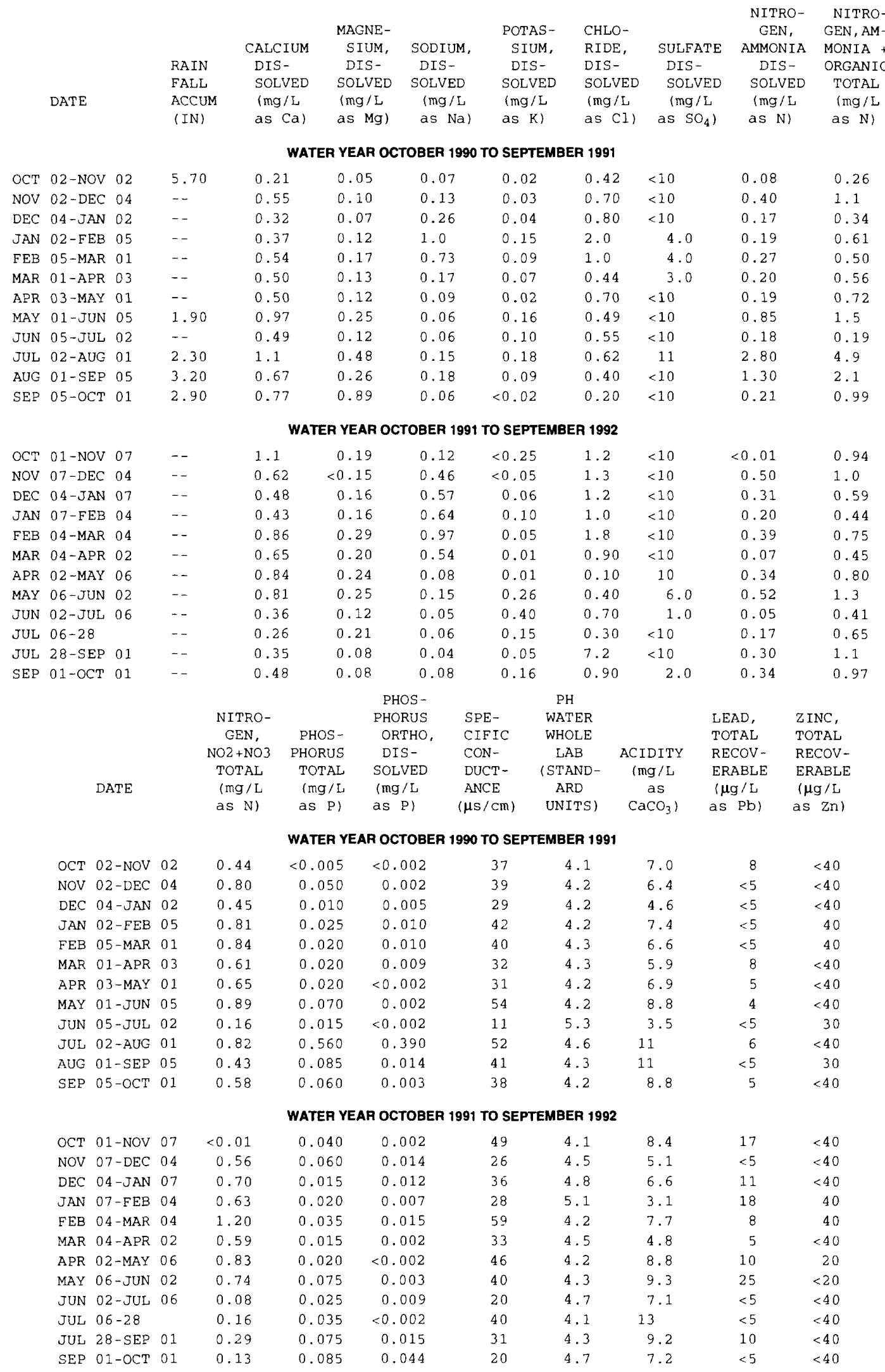




\section{At Mendon Ponds, Rochester, N.Y.--continued}

\section{CHEMICAL QUALITY OF PRECIPITATION - MONTHLY COMPOSITE}

\begin{tabular}{|c|c|c|c|c|c|c|c|c|c|c|}
\hline & DATE & $\begin{array}{l}\text { RAIN } \\
\text { FALL } \\
\text { ACCUM } \\
\text { (IN) }\end{array}$ & $\begin{array}{l}\text { CALCIUM } \\
\text { DIS- } \\
\text { SOLVED } \\
\text { (mg/L } \\
\text { as Ca) }\end{array}$ & $\begin{array}{l}\text { MAGNE- } \\
\text { SIUM, } \\
\text { DIS- } \\
\text { SOLVED } \\
\text { (mg/L } \\
\text { as Mg) }\end{array}$ & $\begin{array}{l}\text { SODIUM, } \\
\text { DIS- } \\
\text { SOLVED } \\
\text { (mg/L } \\
\text { as } \mathrm{Na})\end{array}$ & $\begin{array}{l}\text { POTAS- } \\
\text { SIUM, } \\
\text { DIS- } \\
\text { SOLVED } \\
\text { (mg/L } \\
\text { as K) }\end{array}$ & $\begin{array}{l}\text { CHLO- } \\
\text { RIDE, } \\
\text { DIS- } \\
\text { SOLVED } \\
\text { (mg/L } \\
\text { as Cl) }\end{array}$ & $\begin{array}{l}\text { SULFATE } \\
\text { DIS- } \\
\text { SOLVED } \\
\left(\mathrm{mg}^{2} \mathrm{~L}\right. \\
\left.\text { as } \mathrm{SO}_{4}\right)\end{array}$ & $\begin{array}{l}\text { NITRO- } \\
\text { GEN, } \\
\text { AMMONIA } \\
\text { DIS- } \\
\text { SOLVED } \\
\text { (mg/L } \\
\text { as N) }\end{array}$ & $\begin{array}{l}\text { NITRO } \\
\text { GEN, AM } \\
\text { MONIA } \\
\text { ORGANIC } \\
\text { TOTAL } \\
\text { (mg/L } \\
\text { as N) }\end{array}$ \\
\hline \multicolumn{11}{|c|}{ WATER YEAR OCTOBER 1992 TO SEPTEMBER 1993} \\
\hline NOV & 05 -DEC 02 & e1. 40 & 0.40 & 0.07 & 0.10 & 0.20 & 0.20 & 4.0 & 0.16 & 0.40 \\
\hline JAN & $06-F E B \quad 02$ & 2.20 & 0.37 & 0.12 & 1.0 & 0.17 & 3.5 & 4.0 & 0.29 & 0.29 \\
\hline MAR & $02-A P R \quad 01$ & 4.00 & 0.59 & 0.15 & 0.65 & 0.12 & 1.0 & 4.0 & 0.22 & 0.95 \\
\hline APR & $01-M A Y \quad 05$ & 3.50 & 0.64 & 0.15 & 0.06 & 0.04 & 0.82 & 5.0 & 0.17 & 0.48 \\
\hline MAY & 05-JUN 02 & 1.10 & 1.3 & 0.31 & 0.16 & 0.66 & 1.9 & -- & $<0.01$ & 1.4 \\
\hline
\end{tabular}

\begin{tabular}{|c|c|c|c|c|c|c|c|c|c|}
\hline & DATE & $\begin{array}{c}\text { NITRO- } \\
\text { GEN, } \\
\text { NO2 + NO3 } \\
\text { TOTAL } \\
(\mathrm{mg} / \mathrm{L} \\
\text { as N) }\end{array}$ & $\begin{array}{c}\text { PHOS- } \\
\text { PHORUS } \\
\text { TOTAL } \\
\text { (mg/L } \\
\text { as P) }\end{array}$ & $\begin{array}{l}\text { PHOS- } \\
\text { PHORUS } \\
\text { ORTHO, } \\
\text { DIS- } \\
\text { SOLVED } \\
\text { (mg/L } \\
\text { as P) }\end{array}$ & $\begin{array}{l}\text { SPE- } \\
\text { CIFIC } \\
\text { CON- } \\
\text { DUCT- } \\
\text { ANCE } \\
(\mu \mathrm{S} / \mathrm{cm})\end{array}$ & $\begin{array}{c}\text { PH } \\
\text { WATER } \\
\text { WHOLE } \\
\text { LAB } \\
\text { (STAND- } \\
\text { ARD } \\
\text { UNITS) }\end{array}$ & $\begin{array}{c}\text { ACIDITY } \\
(\mathrm{mg} / \mathrm{L} \\
\mathrm{as} \\
\left.\mathrm{CaCO}_{3}\right)\end{array}$ & $\begin{array}{l}\text { LEAD, } \\
\text { TOTAL } \\
\text { RECOV- } \\
\text { ERABLE } \\
(\mu \mathrm{g} / \mathrm{L} \\
\text { as } \mathrm{Pb})\end{array}$ & $\begin{array}{l}\text { ZINC, } \\
\text { TOTAL } \\
\text { RECOV- } \\
\text { ERABLE } \\
(\mu \mathrm{g} / \mathrm{L} \\
\text { as } \mathrm{Zn})\end{array}$ \\
\hline OCT & 01-NOV 05 & 0.39 & 0.020 & $<0.002$ & 31 & 4.5 & 5.5 & $<5$ & $<40$ \\
\hline Nov & $05-D E C \quad 02$ & 0.28 & 0.020 & 0.002 & 38 & 4.3 & 6.6 & $<5$ & 30 \\
\hline JAN & 06 -FEB 02 & 0.92 & 0.040 & $<0.002$ & 48 & 4.1 & 7.3 & 8 & $<40$ \\
\hline MAR & $02-A P R \quad 01$ & 1.00 & 0.060 & 0.004 & 45 & 4.1 & 7.4 & 12 & 120 \\
\hline$A P R$ & $01-M A Y \quad 05$ & 0.82 & 0.015 & $<0.002$ & 47 & 4.1 & 8.4 & $<5$ & 70 \\
\hline MAY & 05 -JUN 02 & 0.65 & 0.040 & 0.002 & 27 & 4.8 & 5.4 & 7 & $<40$ \\
\hline JUN & 02 -JUL 01 & 0.68 & 0.170 & 0.115 & 38 & 4.3 & 9.8 & 5 & 100 \\
\hline AUG & $05-S E P \quad 02$ & 0.45 & 0.100 & $<0.002$ & 65 & 4.0 & 15 & $<5$ & 60 \\
\hline SEP & $01-O C T \quad 01$ & 0.68 & 0.020 & $<0.002$ & 48 & 4.1 & 7.0 & 6 & 80 \\
\hline
\end{tabular}




\section{Precipitation Stations}

\section{At Empire Boulevard, Rochester, N.Y.}

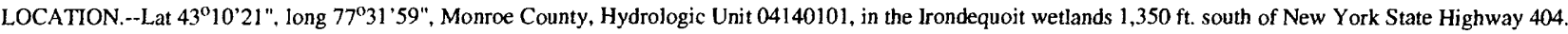
PERIOD OF RECORD.--October 1992 to current year (monthly dustfall).

October 1992 to current year (monthly wetfall).

INSTRUMENTATION.--Wetfall and dustfall sample collector. An automatic sensor detects precipitation and activates a motor that removes the cover from the wetfail-collection vessel and covers the dustfall-collection vessel. When precipitation ceases, the cycle is reversed. The sampling vessels are poiyethlyene and have a collection diameter of

11.26 in. and a capacity of about 3.4 gallons. The openings of the collectors are approximately $8 \mathrm{ft}$ above ground level.

COOPERATION.--Water-quality samples were collected and analyzed by the Monroe County Health Laboratory at Rochester, NY

CHEMICAL QUALITY OF PRECIPITATION, MONTHLY DUSTFALL

WATER YEAR OCTOBER 1992 TO SEPTEMBER 1993

\begin{tabular}{|c|c|c|c|c|c|c|c|c|c|c|}
\hline & DATE & & $\begin{array}{l}\text { CALCIUM } \\
\text { DIS- } \\
\text { SOLVED } \\
\text { (mg/L } \\
\text { as Ca) }\end{array}$ & $\begin{array}{l}\text { MAGNE- } \\
\text { SIUM, } \\
\text { DIS- } \\
\text { SOLVED } \\
\text { (mg/L } \\
\text { as Mg) }\end{array}$ & $\begin{array}{l}\text { SODIUM, } \\
\text { DIS- } \\
\text { SOLVED } \\
\text { (mg/L } \\
\text { as Na) }\end{array}$ & $\begin{array}{l}\text { POTAS- } \\
\text { SIUM, } \\
\text { DIS- } \\
\text { SOLVED } \\
\text { (mg/L } \\
\text { as K) }\end{array}$ & $\begin{array}{l}\text { CHLO- } \\
\text { RIDE, } \\
\text { DIS- } \\
\text { SOLVED } \\
\text { (mg/L } \\
\text { as Cl) }\end{array}$ & $\begin{array}{l}\text { SULFATE } \\
\text { DIS- } \\
\text { SOLVED } \\
(\mathrm{mg} / \mathrm{L} \\
\left.\text { as } \mathrm{SO}_{4}\right)\end{array}$ & $\begin{array}{c}\text { NITRO- } \\
\text { GEN, } \\
\text { AMMONIA } \\
\text { DIS- } \\
\text { SOLVED } \\
\text { (mg/L } \\
\text { as N) }\end{array}$ & $\begin{array}{l}\text { NITRO- } \\
\text { GEN, AM- } \\
\text { MONIA + } \\
\text { ORGANIC } \\
\text { TOTAL } \\
\text { (mg/L } \\
\text { as N) }\end{array}$ \\
\hline NOV & $05-D E C \quad 0$ & 02 & 0.20 & 0.04 & $<0.10$ & 0.10 & 0.20 & 2.0 & 0.07 & 0.10 \\
\hline $\mathrm{DEC}$ & 02 -JAN 0 & 05 & 0.46 & 0.14 & 0.85 & 0.07 & 1.3 & 5.0 & 0.30 & 0.28 \\
\hline APR & 14-MAY 0 & 07 & 2.3 & 0.56 & 0.21 & 0.18 & 0.52 & 8.0 & 0.70 & 1.6 \\
\hline MAY & $07-J U N \quad 0$ & 02 & 3.0 & 0.81 & 1.9 & 20 & 9.2 & -- & $<0.01$ & 24 \\
\hline JUN & 02-JUL 0 & 01 & 1.8 & 0.48 & 0.26 & 0.30 & 0.30 & -- & 0.14 & 0.76 \\
\hline JUL & $01-A U G \quad 0$ & 05 & 3.3 & 0.76 & 0.76 & 0.22 & 2.8 & 10 & 0.18 & 11 \\
\hline AUG & $05-$ SEP 0 & 02 & 1.4 & 0.44 & 0.12 & 0.44 & 0.92 & 6.0 & 0.24 & 1.4 \\
\hline SEP & $01-\mathrm{OCT} 0$ & 01 & 2.2 & 0.68 & 0.86 & 2.4 & 2.1 & 11 & 3.49 & 17 \\
\hline & DATE & & $\begin{array}{c}\text { NITRO- } \\
\text { GEN, } \\
\mathrm{NO}_{2}+\mathrm{NO}_{3} \\
\text { TOTAL } \\
\text { (mg/L } \\
\text { as N) }\end{array}$ & $\begin{array}{c}\text { PHOS- } \\
\text { PHORUS } \\
\text { TOTAL } \\
\text { (mg/L } \\
\text { as P) }\end{array}$ & $\begin{array}{l}\text { PHOS- } \\
\text { PHORUS } \\
\text { ORTHO, } \\
\text { DIS- } \\
\text { SOLVED } \\
\text { (mg/L } \\
\text { as P) }\end{array}$ & $\begin{array}{l}\text { SPE- } \\
\text { CIFIC } \\
\text { CON- } \\
\text { DUCT- } \\
\text { ANCE } \\
(\mu \mathrm{S} / \mathrm{cm})\end{array}$ & $\begin{array}{c}\text { PH } \\
\text { WATER } \\
\text { WHOLE } \\
\text { LAB } \\
\text { (STAND- } \\
\text { ARD } \\
\text { UNITS) }\end{array}$ & $\begin{array}{c}\text { ACIDITY } \\
(\mathrm{mg} / \mathrm{L} \\
\text { as } \\
\left.\mathrm{CaCO}_{3}\right)\end{array}$ & $\begin{array}{l}\text { LEAD, } \\
\text { TOTAL } \\
\text { RECOV- } \\
\text { ERABLE } \\
(\mu \mathrm{g} / \mathrm{L} \\
\text { as Pb) }\end{array}$ & $\begin{array}{l}\text { ZINC, } \\
\text { TOTAL } \\
\text { RECOV- } \\
\text { ERABLE } \\
\text { ( } \mu \mathrm{g} / \mathrm{L} \\
\text { as } \mathrm{Zn})\end{array}$ \\
\hline NOV & 05-DEC 02 & 02 & 0.16 & 0.015 & 0.005 & 4 & 6.5 & 0.9 & $<5$ & 20 \\
\hline DEC & 02-JAN 0 & 05 & 0.46 & 0.015 & 0.008 & 38 & 4.3 & 4.6 & 10 & $<40$ \\
\hline APR & 14-MAY & 07 & 1.00 & 0.300 & 0.215 & 42 & 4.6 & 5.6 & 8 & 50 \\
\hline MAY & 07 -JUN 02 & 02 & 1.00 & 0.490 & 3.40 & 79 & 4.9 & 23 & 10 & $<40$ \\
\hline JUN & 02 -JUL 0 & 01 & 0.45 & 0.120 & 0.039 & 22 & 6.1 & 2.2 & 6 & 100 \\
\hline JUL & 01 -AUG 0 & & N0. 51 & 1.45 & 0.258 & 45 & 5.1 & 9.8 & 6 & 90 \\
\hline AUG & $05-S E P \quad 02$ & 22 & 0.35 & 0.250 & 0.077 & 23 & 6.0 & 2.0 & $<5$ & $<40$ \\
\hline SEP & $01-\mathrm{OCT} \quad 0$ & 01 & 0.68 & 2.05 & 1.85 & 99 & 7.0 & 5.3 & 11 & 50 \\
\hline
\end{tabular}




\section{At Empire Boulevard, Rochester, N.Y.--Continued}

\section{CHEMICAL QUALITY OF PRECIPITATION, MONTHLY WETFALL}

WATER YEAR OCTOBER 1992 TO SEPTEMBER 1993

CALCIUM
DIS-
SOLVED
( $\mathrm{mg} / \mathrm{L}$
as Ca)

$\begin{array}{lc}\text { MAGNE- } & \\ \text { SIUM, } & \text { SODIUM, } \\ \text { DIS- } & \text { DIS- } \\ \text { SOLVED } & \text { SOLVED } \\ \text { (mg/L } & \text { (mg/L } \\ \text { as Mg) } & \text { as Na) }\end{array}$

POTAS-
SIUM,
DIS-
SOLVED
(mg /L
as K)

$\begin{array}{lc}\text { CHLO- } & \\ \text { RIDE, } & \text { SULFATE } \\ \text { DIS- } & \text { DIS- } \\ \text { SOLVED } & \text { SOLVED } \\ (\mathrm{mg} / \mathrm{L} & (\mathrm{mg} / \mathrm{L} \\ \text { as Cl) } & \left.\text { as } \mathrm{SO}_{4}\right)\end{array}$

NITRO- NITROGEN, GEN, AMAMMONIA MONIA +

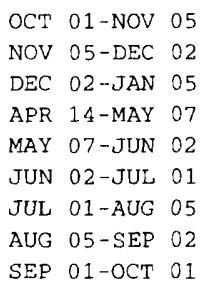

DATE

$\begin{array}{lll}0.74 & 0.09 & 0.07 \\ 0.60 & 0.15 & 0.20 \\ 0.63 & 0.17 & 1.0 \\ 1.8 & 0.47 & 0.38 \\ 0.62 & 0.13 & 0.13 \\ 0.61 & 0.15 & 0.10 \\ 0.88 & 0.34 & 0.22 \\ 0.75 & 0.18 & 0.12 \\ 0.30 & 0.07 & 0.19\end{array}$

$$
0.32
$$

0.80

$<0.10$

0.09

0.16

0.04

0.08

0.22

0.13

-.

0.19

PHOSPHORUS

$\begin{array}{cr}\text { NITRO- } & \\ \text { GEN, } & \text { PHOS- } \\ \mathrm{NO}_{2}+\mathrm{NO}_{3} & \text { PHORUS } \\ \text { TOTAL } & \text { TOTAL } \\ (\mathrm{mg} / \mathrm{L} & (\mathrm{mg} / \mathrm{L} \\ \text { as N) } & \text { as P) }\end{array}$
ORTHO

DIS -

SOLVED

( $\mathrm{mg} / \mathrm{L}$

as P)

OCT 01-NOV 05 NOV 05-DEC 02 DEC 02-JAN 05 APR 14-MAY 07 MAY 07-JUN 02 JUN 02 -JUL 01 JUL 01 -AUG 05 AUG $05-S E P \quad 02$ SEP 01-OCT 01

$\begin{array}{rrr}0.60 & 0.075 & 0.047 \\ 0.97 & 0.020 & 0.011 \\ 0.34 & 0.025 & 0.006 \\ 1.70 & 0.050 & 0.015 \\ 0.68 & 0.030 & 0.003 \\ 0.94 & 0.025 & <0.002 \\ N 0.91 & 0.015 & <0.002 \\ 1.40 & 0.020 & 0.006 \\ 0.48 & 0.010 & 0.003\end{array}$

730
53
17
78
36
78
104
113
38

$\begin{array}{lc}\text { PH } & \\ \text { WATER } & \\ \text { WHOLE } & \\ \text { LAB } & \text { ACIDITY } \\ \text { (STAND- } & \text { (mg/L } \\ \text { ARD } & \text { as } \\ \text { UNITS) } & \mathrm{CaCO}_{3} \text { ) }\end{array}$

LEAD, TOTAL RECOVERABLE $(\mu g / L$ as $\mathrm{Pb}$ )

$\begin{array}{cc}4.7 & 3.3 \\ 4.2 & 8.1 \\ 6.1 & 0.9 \\ 3.9 & 11 \\ 4.0 & 5.8 \\ 3.8 & 11 \\ 3.8 & 14 \\ 3.7 & 15 \\ 4.2 & 5.9\end{array}$

OLVED TOTAL

as $\mathrm{N}$ )

$\begin{array}{rr}<5 & <40 \\ <5 & 30 \\ 8 & <40 \\ 7 & 60 \\ 25 & <40 \\ 5 & 90 \\ <5 & <40 \\ <5 & 50 \\ 6 & 40\end{array}$




\section{Precipitation Stations}

\section{At State University of New York at Brockport, N.Y.}

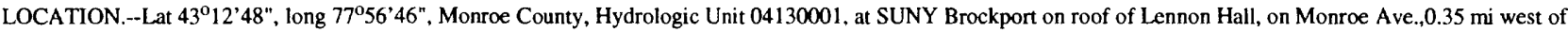
New York State Highway 19 and 31

PERIOD OF RECORD.-- June 1990 to current year (monthly dustfall).

June 1990 to current year (monthly wetfall).

June 1990 to current year (monthly composite).

INSTRUMENTATION.--The composite sample collector is a straight-sided polyethlyene funnel approximately 6.5 inch in diameter that drains into a Teflon receiving bottle. A looped plastic tubing connects the funnel with the receiving bottle to retard evaporation. The polyethlyene funnel is heated during the cold-weather season to aid in complete collection of snow. The receiving bottle is enclosed in an insulated box.

Wet/dry precipitation collector used for wetfall and dustfall samples. An automatic sensor detects precipitation and activates a motor that removes the cover fiom the wetfallcollection vessel and covers the dustfall-collection vessel. When precipitation ceases, the cycle is reversed. The sampling vessels are polyethlyene and have a collection diameter of 11.26 inch and a capacity of about 3.4 gallons.

COOPERATION.--Water-quality samples were collected and analyzed by the Monroe County Health Laboratory at Rochester, NY.

REMARKS.--Analytical results of samples from two sample collectors at this site (SUNY Brockport East and SUNY Brockport West) were combined to produse a complete record of chemical quality of precipitation.

CHEMICAL QUALITY OF PRECIPITATION - MONTHLY DUSTFALL

PERIOD JUNE 1990 TO SEPTEMBER 1990

\begin{tabular}{|c|c|c|c|c|c|c|c|c|}
\hline DATE & $\begin{array}{l}\text { CALCIUM } \\
\text { DIS- } \\
\text { SOLVED } \\
\text { (MG/L } \\
\text { AS Ca) }\end{array}$ & $\begin{array}{l}\text { MAGNE- } \\
\text { SIUM, } \\
\text { DIS- } \\
\text { SOLVED } \\
\text { (MG/L } \\
\text { as Mg) }\end{array}$ & $\begin{array}{l}\text { SODIUM, } \\
\text { DIS- } \\
\text { SOLVED } \\
\text { (mg/L } \\
\text { as Na) }\end{array}$ & $\begin{array}{l}\text { POTAS- } \\
\text { SIUM, } \\
\text { DIS- } \\
\text { SOLVED } \\
(\mathrm{mg} / \mathrm{L} \\
\text { as K) }\end{array}$ & $\begin{array}{l}\text { CHLO- } \\
\text { RIDE, } \\
\text { DIS- } \\
\text { SOLVED } \\
\text { (mg/L } \\
\text { as Cl) }\end{array}$ & $\begin{array}{l}\text { SULFATE } \\
\text { DIS- } \\
\text { SOLVED } \\
(\mathrm{mg} / \mathrm{L} \\
\left.\text { as } \mathrm{SO}_{4}\right)\end{array}$ & $\begin{array}{l}\text { NITRO- } \\
\text { GEN, } \\
\text { AMMONIA } \\
\text { DIS- } \\
\text { SOLVED } \\
\text { (mg/L } \\
\text { as N) }\end{array}$ & $\begin{array}{l}\text { NITRO- } \\
\text { GEN, AM- } \\
\text { MONIA + } \\
\text { ORGANIC } \\
\text { TOTAL } \\
\text { (mg/L } \\
\text { as N) }\end{array}$ \\
\hline JUN $01-30$ & 2.9 & 0.76 & 0.13 & 0.36 & 0.40 & 9.0 & 0.21 & 1.4 \\
\hline JUL $01-31$ & 2.8 & 0.41 & 0.17 & 0.38 & 0.80 & 6.0 & 0.22 & 1.6 \\
\hline AUG $01-31$ & 2.0 & 0.51 & 0.16 & 0.10 & 0.50 & 10 & $<0.01$ & 1.1 \\
\hline AUG 31-OCT 01 & 2.5 & 0.54 & 0.11 & 0.13 & 0.60 & 10 & 0.35 & 1.2 \\
\hline DATE & $\begin{array}{c}\text { NITRO- } \\
\text { GEN, } \\
\mathrm{NO}_{2}+\mathrm{NO}_{3} \\
\text { TOTAL } \\
\text { (mg/L } \\
\text { as } \mathrm{N})\end{array}$ & $\begin{array}{c}\text { PHOS- } \\
\text { PHORUS } \\
\text { TOTAL } \\
\text { (mg/L } \\
\text { as P) }\end{array}$ & $\begin{array}{l}\text { PHOS- } \\
\text { PHORUS } \\
\text { ORTHO, } \\
\text { DIS- } \\
\text { SOLVED } \\
\text { (mg/L } \\
\text { as P) }\end{array}$ & $\begin{array}{l}\text { SPE- } \\
\text { CIFIC } \\
\text { CON- } \\
\text { DUCT- } \\
\text { ANCE } \\
(\mu \mathrm{S} / \mathrm{Cm})\end{array}$ & $\begin{array}{c}\text { PH } \\
\text { WATER } \\
\text { WHOLE } \\
\text { LAB } \\
\text { (STAND- } \\
\text { ARD } \\
\text { UNITS) }\end{array}$ & $\begin{array}{c}\text { ACIDITY } \\
(\mathrm{mg} / \mathrm{L} \\
\text { as } \\
\left.\mathrm{CaCO}_{3}\right)\end{array}$ & $\begin{array}{c}\text { LEAD, } \\
\text { TOTAL } \\
\text { RECOV- } \\
\text { ERABLE } \\
(\mu g / L \\
\text { as Pb) }\end{array}$ & $\begin{array}{l}\text { ZINC, } \\
\text { TOTAL } \\
\text { RECOV- } \\
\text { ERABLE } \\
(\mu \mathrm{g} / \mathrm{L} \\
\text { as } \mathrm{Zn})\end{array}$ \\
\hline JUN $01-30$ & 0.76 & 0.220 & 0.058 & 34 & 6.0 & 4.4 & 8 & - \\
\hline JUL 01 -JUL 31 & 0.89 & 0.230 & 0.077 & 28 & 6.3 & 4.5 & 13 & 40 \\
\hline AUG $01-31$ & 0.74 & 0.160 & 0.110 & 27 & 5.9 & 2.4 & $<5$ & 40 \\
\hline AUG 31-OCT 01 & 0.98 & 0.110 & 0.012 & 32 & 6.0 & 2.8 & 6 & 50 \\
\hline
\end{tabular}


431248077564601 At State University of New York at Brockport, N.Y. CHEMICAL QUALITY OF PRECIPITATION - MONTHLY DUSTFALL

OCT 01-NOV 01 NOV $02-D E C \quad 04$ DEC 04-JAN 02 JAN 01 -FEB 01 FEB 01-MAR 01 MAR 01 -APR 03 APR 01-MAY 02 MAY $02-J U N \quad 05$ JUL 01-31 JUL 31 -SEP 03

NOV $01-D E C \quad 02$ DEC $01-J A N \quad 01$ JAN 01-31 JAN 31-FEB 29 FEB 29-MAR 31 MAR 31-MAY 05 MAY 05-JUN 01 JUN 01-JUL 01 JUL 01-27

JUL 28-SEP 01 SEP 01 -OCT 01

DATE

OCT 01-NOV 01 NOV $02-D E C \quad 04$ DEC 04-JAN 02 JAN OI-FEB 01 FEB 01 -MAR 01 MAR 01 -APR 03 APR 01-MAY 02 MAY 02-JUN 05 JUL $01-31$

JUL 31-SEP 03

NOV $01-\mathrm{DEC} 02$ DEC 01-JAN 01 JAN 01-31

JAN 31-FEB 29 FEB 29-MAR 31 MAR 31-MAY 05 MAY 05-JUN 01 JUN 01-JUL 01 JUL $01-27$

JUL 28-SEP 01 SEP 01 -OCT 01

\begin{tabular}{|c|c|c|c|c|c|c|c|}
\hline$A I$ & $\begin{array}{l}\text { MAGNE- } \\
\text { SIUM, }\end{array}$ & SODI & $\begin{array}{l}\text { POTAS- } \\
\text { SIUM }\end{array}$ & $\begin{array}{l}\text { CHLO- } \\
\text { RIDE, }\end{array}$ & ULFATE & $\begin{array}{l}\text { NITRO- } \\
\text { GEN, } \\
\text { AMMONIA }\end{array}$ & $\begin{array}{l}\text { NITRO- } \\
\text { GEN, AM- } \\
\text { MONIA + }\end{array}$ \\
\hline DIS - & DIS- & DIS - & DIS- & DIS- & DIS - & DIS- & ORGANIC \\
\hline $\begin{array}{l}\text { SOLVED } \\
\text { (MG/L }\end{array}$ & $\begin{array}{l}\text { SOLVED } \\
\text { (MG/L }\end{array}$ & $\begin{array}{c}\text { SOLVED } \\
(\mathrm{mg} / \mathrm{L}\end{array}$ & $\begin{array}{l}\text { SOLVED } \\
\text { (mg/L }\end{array}$ & $\begin{array}{l}\text { SOLVED } \\
(\mathrm{mg} / \mathrm{L}\end{array}$ & $\begin{array}{l}\text { SOLVED } \\
\text { (mg/L }\end{array}$ & $\begin{array}{l}\text { SOLVED } \\
\text { (mg/L }\end{array}$ & $\begin{array}{l}\text { TOTAL } \\
\text { (mg/L }\end{array}$ \\
\hline AS Ca) & as $\mathrm{Mg}$ ) & as $\mathrm{Na}$ ) & as $\mathrm{K}$ ) & as Cll & as $\left.\mathrm{SO}_{4}\right)$ & as $N$ ) & as $N$ ) \\
\hline
\end{tabular}

\section{WATER YEAR OCTOBER 1990 TO SEPTEMBER 1991}

$\begin{array}{llllllll}1.8 & 0.38 & 0.20 & 0.11 & 0.62 & <10 & 0.18 & 0.75 \\ 2.7 & 0.41 & 0.52 & 0.09 & 1.1 & <10 & 0.38 & 0.92 \\ 2.8 & 0.46 & 1.4 & 0.06 & 1.9 & <10 & 0.20 & 0.62 \\ 2.0 & 0.45 & 2.8 & 0.12 & 3.9 & 1.0 & 0.49 & 0.88 \\ 3.3 & 0.53 & 2.7 & 0.09 & 3.1 & 7.0 & 0.50 & 1.0 \\ 3.5 & 0.49 & 0.81 & 0.10 & 1.6 & 5.0 & 0.29 & 0.92 \\ 3.2 & 0.64 & 0.52 & 0.37 & 1.1 & <10 & 0.15 & 1.5 \\ 3.0 & 0.51 & 0.50 & 0.62 & 0.85 & <10 & 0.12 & 0.05 \\ 0.90 & 0.11 & <0.01 & 0.02 & 0.21 & <10 & 0.04 & 0.33 \\ 3.1 & 0.51 & 0.12 & 0.12 & 0.50 & 10 & 0.08 & 1.1\end{array}$

\section{WATER YEAR OCTOBER 1991 TO SEPTEMBER 1992}

$\begin{array}{llllllll}2.8 & 0.61 & 0.57 & 0.07 & 1.3 & <10 & 0.53 & 1.5 \\ 3.0 & 0.88 & 1.8 & 0.09 & 2.9 & <10 & 0.60 & 1.3 \\ 1.9 & 0.48 & 1.9 & 0.04 & 1.3 & <10 & 0.52 & 0.84 \\ 3.6 & 0.88 & 3.2 & 0.04 & 4.4 & <10 & 0.98 & 1.2 \\ 4.8 & 0.90 & 4.1 & 0.08 & 5.4 & <10 & 0.55 & 1.4 \\ 4.5 & 0.75 & 1.2 & 0.20 & 1.0 & 8.0 & 0.20 & 1.6 \\ 3.4 & 1.3 & 0.69 & 0.88 & 0.60 & 5.0 & 0.38 & 2.6 \\ 2.9 & 0.42 & 0.28 & 0.43 & 1.1 & 3.0 & 0.12 & 1.8 \\ 1.9 & 0.44 & 0.08 & 0.11 & 0.50 & 10 & 0.22 & 1.4 \\ 2.7 & 0.50 & 0.11 & 0.16 & 0.30 & <10 & 0.12 & 0.76 \\ 2.9 & 0.35 & 0.15 & 0.12 & 0.60 & 3.0 & 0.12 & 0.80\end{array}$

\begin{tabular}{|c|c|c|c|c|c|c|c|}
\hline & & PHOS- & & PH & & & \\
\hline $\begin{array}{c}\text { NITRO- } \\
\text { GEN }\end{array}$ & PHOS - & $\begin{array}{c}\text { PHORUS } \\
\text { ORTHO }\end{array}$ & $\begin{array}{l}\text { SPE- } \\
\text { CIFIC }\end{array}$ & $\begin{array}{l}\text { WATER } \\
\text { WHOLE }\end{array}$ & & $\begin{array}{l}\text { LEAD, } \\
\text { TOTAL }\end{array}$ & $\begin{array}{l}\text { ZINC, } \\
\text { TOTAI }\end{array}$ \\
\hline $\mathrm{NO}_{2}+\mathrm{NO}_{3}$ & PHORUS & DIS- & CON- & LAB & ACIDITY & RECOV- & RECOV- \\
\hline $\begin{array}{l}\text { TOTAL } \\
\text { /mg/L }\end{array}$ & $\begin{array}{l}\text { TOTAL } \\
(\mathrm{mg} / \mathrm{L}\end{array}$ & $\begin{array}{l}\text { SOLVED } \\
\text { (mg/L }\end{array}$ & $\begin{array}{l}\text { DUCT- } \\
\text { ANCE }\end{array}$ & $\begin{array}{c}\text { (STAND- } \\
\text { ARD }\end{array}$ & $\begin{array}{c}(\mathrm{mg} / \mathrm{L} \\
\mathrm{as}\end{array}$ & $\begin{array}{l}\text { ERABLE } \\
(\mu g / L\end{array}$ & $\begin{array}{l}\text { ERABIE } \\
(\mu \mathrm{g} / \mathrm{L}\end{array}$ \\
\hline as $N$ ) & as $\mathrm{Pl}$ & as P) & $(\mu \mathrm{S} / \mathrm{cm})$ & UNITS) & $\left.\mathrm{CaCO}_{3}\right)$ & as $\mathrm{Pb}$ & as $\mathrm{Zn}$ ) \\
\hline
\end{tabular}

\section{WATER YEAR OCTOBER 1990 TO SEPTEMBER 1991}

$\begin{array}{rrrrrrrr}0.76 & 0.060 & 0.008 & 26 & 6.4 & 1.1 & 8 & 120 \\ 0.90 & 0.045 & 0.007 & 30 & 6.5 & 3.2 & 13 & 40 \\ 0.59 & 0.025 & 0.005 & 32 & 6.3 & 1.1 & 12 & 40 \\ 1.40 & 0.020 & 0.002 & 44 & 5.9 & 1.6 & 20 & 40 \\ 1.40 & 0.025 & 0.003 & 50 & 6.7 & 1.1 & 5 & 50 \\ 1.20 & 0.055 & 0.005 & 37 & 6.5 & 2.2 & 24 & 40 \\ 1.10 & 0.130 & 0.023 & 40 & 6.4 & 1.9 & 6 & 50 \\ 1.20 & 0.260 & 0.080 & 33 & 6.1 & 3.7 & 8 & 50 \\ 0.25 & 0.030 & 0.011 & 11 & 6.5 & 0.6 & <5 & <40 \\ 0.72 & 0.070 & 0.002 & 24 & 6.3 & 0.6 & 12 & 40\end{array}$

\section{WATER YEAR OCTOBER 1991 TO SEPTEMBER 1992}

1.10
1.60
1.20
1.80
1.80
1.70
1.10
0.89
0.46
0.70
0.67

$\begin{array}{lr}0.060 & 0.004 \\ 0.035 & 0.004 \\ 0.035 & 0.007 \\ 0.030 & <0.002 \\ 0.040 & 0.002 \\ 0.180 & 0.029 \\ 0.430 & 0.088 \\ 0.200 & 0.050 \\ 0.095 & 0.006 \\ 0.065 & 0.016 \\ 0.055 & 0.009\end{array}$

$\begin{array}{lll}27 & 5.8 & 2.7 \\ 48 & 7.2 & 0.9 \\ 36 & 6.0 & 2.4 \\ 58 & 6.1 & 2.0 \\ 65 & 6.6 & 1.2 \\ 46 & 6.6 & 0.6 \\ 31 & 6.3 & 5.8 \\ 28 & 6.5 & 1.2 \\ 21 & 5.9 & 3.4 \\ 23 & 6.1 & 1.2 \\ 23 & 6.4 & 1.8\end{array}$

$\begin{array}{rr}11 & <40 \\ 21 & 70 \\ 9 & 50 \\ 29 & 80 \\ 31 & 70 \\ 11 & 70 \\ 23 & 40 \\ 13 & 60 \\ 12 & <40 \\ 6 & <40 \\ 5 & 40\end{array}$




\section{At State University of New York at Brockport, N.Y. \\ CHEMICAL QUALITY OF PRECIPITATION - MONTHLY DUSTFALL}

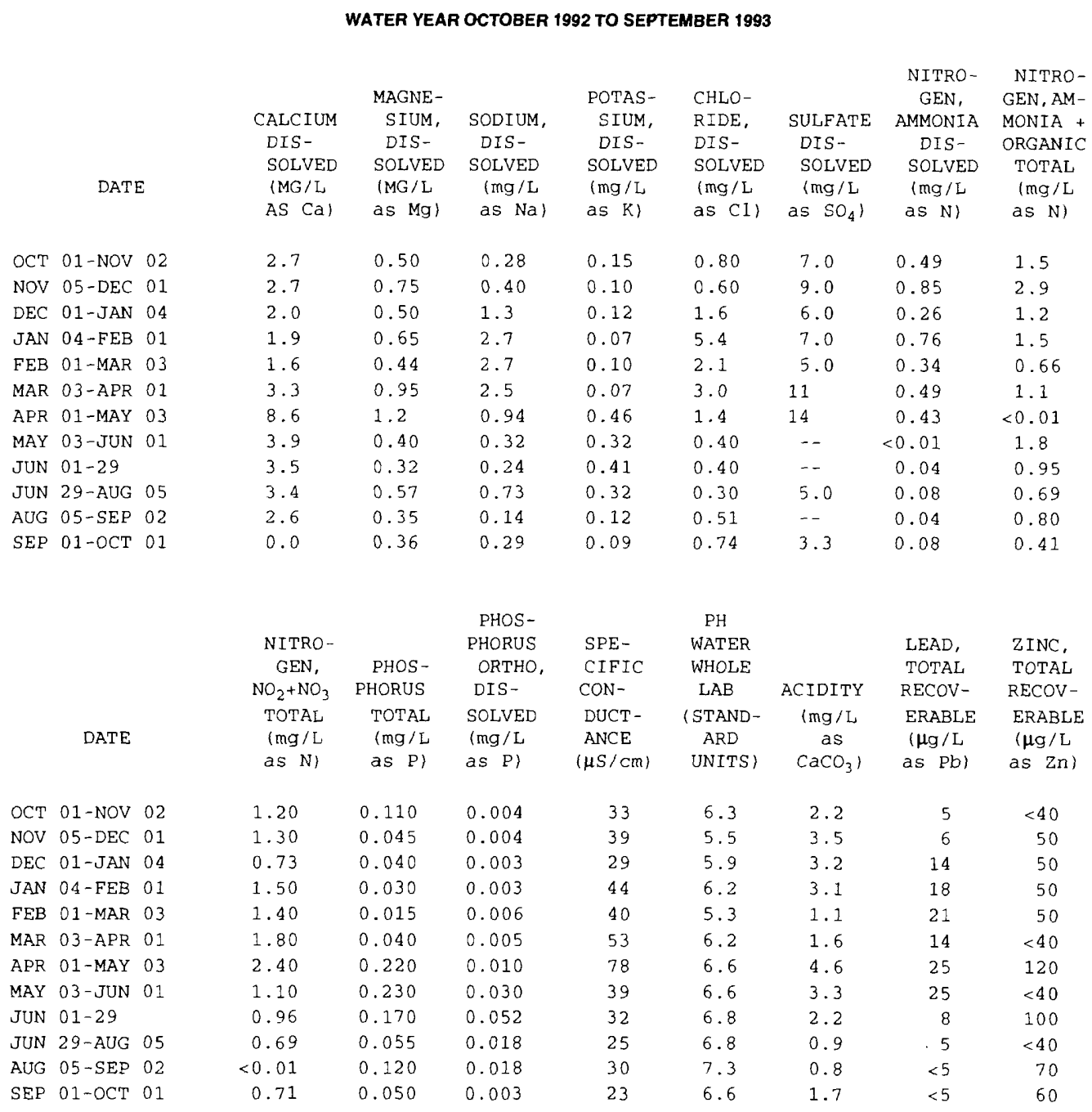


431248077564601 At State University of New York at Brockport, N.Y.

CHEMICAL QUALITY OF PRECIPITATION - MONTHLY WETFALL

JUN $01-30$

JUL 01 -JUL 31

AUG 01-31

AUG 31 -OCT 01

OCT 01-NOV 01 NOV $01-30$ DEC 04-JAN 02 JAN 01 -FEB 01 FEB 01-MAR 01 MAR 01-APR 03 APR 01 -MAY 02 MAY 02 -JUN 05 JUL 01-31

JUL 31 -SEP 03

DATE

JUN 01-30

JUL 01 -JUL 31 AUG 01-31

AUG 31-OCT 01

OCT 01-NOV 01 NOV $01-30$ DEC 04-JAN 02 JAN 01 -FEB 01 FEB 01-MAR 01 MAR 01 -APR 03 APR 01-MAY 02 MAY 02-JUN 05 JUL 01-31

JUL 31 -SEP 03

\begin{tabular}{|c|c|c|c|c|c|c|c|}
\hline & MAGNE- & & POTAS- & CHLO- & & $\begin{array}{l}\text { NITRO- } \\
\text { GEN, }\end{array}$ & $\begin{array}{l}\text { NITRO- } \\
\text { GEN, AM- }\end{array}$ \\
\hline $\begin{array}{l}\text { CALCIUM } \\
\text { DIS- }\end{array}$ & $\begin{array}{l}\text { SIUM, } \\
\text { DIS- }\end{array}$ & $\begin{array}{l}\text { SODIUM, } \\
\text { DIS - }\end{array}$ & $\begin{array}{l}\text { SIUM, } \\
\text { DIS- }\end{array}$ & $\begin{array}{l}\text { RIDE, } \\
\text { DIS - }\end{array}$ & $\begin{array}{c}\text { SULFATE } \\
\text { DIS- }\end{array}$ & $\begin{array}{c}\text { AMMONIA } \\
\text { DIS- }\end{array}$ & $\begin{array}{l}\text { MONIA + } \\
\text { ORGANIC }\end{array}$ \\
\hline SOLVED & SOLVED & SOLVED & SOLVED & SOLVED & SOLVED & SOLVED & TOTAL \\
\hline (MG/L & (MG / L & $(\mathrm{mg} / \mathrm{L}$ & ( $\mathrm{mg} / \mathrm{L}$ & $(\mathrm{mg} / \mathrm{L}$ & ( $\mathrm{mg} / \mathrm{L}$ & $(\mathrm{mg} / \mathrm{L}$ & $(\mathrm{mg} / \mathrm{L}$ \\
\hline AS Ca) & as $\mathrm{Mg}$ ) & as $\mathrm{Na}$ ) & as $\mathrm{K}$ ) & as Cl) & as $\left.\mathrm{SO}_{4}\right)$ & as $\mathrm{N}$ ) & \\
\hline
\end{tabular}

PERIOD JUNE 1990 TO SEPTEMBER 1990

$\begin{array}{rrrrrrrr}0.12 & <0.01 & 0.01 & 0.01 & <0.20 & <5.0 & 0.30 & 0.41 \\ 0.32 & 0.05 & 0.06 & 0.03 & 0.30 & 6.0 & 0.31 & 0.53 \\ 0.14 & 0.02 & 0.02 & 0.02 & 0.50 & 10 & <0.01 & 0.70 \\ 0.21 & 0.04 & 0.03 & 0.02 & 0.40 & 9.0 & 0.50 & 0.52\end{array}$

WATER YEAR OCTOBER 1990 TO SEPTEMBER 1991

$\begin{array}{lccccccc}0.21 & 0.04 & 0.07 & 0.03 & <0.20 & <10 & 0.22 & 0.41 \\ 0.26 & 0.04 & 0.08 & 0.03 & 0.40 & <10 & 0.37 & 0.96 \\ 0.11 & 0.04 & 0.10 & 0.60 & 0.60 & <10 & 0.14 & 0.30 \\ 0.56 & 0.08 & 0.28 & 0.02 & 1.2 & <1.0 & 0.16 & 0.19 \\ 0.52 & 0.12 & 0.44 & 0.03 & 0.50 & 4.0 & 0.28 & 0.45 \\ 0.25 & 0.03 & 0.08 & 0.04 & 0.29 & 2.0 & 0.21 & 0.32 \\ 0.28 & 0.06 & 0.07 & 0.03 & 0.43 & <10 & 0.24 & 1.1 \\ 0.31 & 0.06 & 0.03 & 0.04 & 0.41 & <10 & 0.49 & <0.04 \\ 0.31 & 0.04 & <0.01 & <0.01 & <0.20 & <10 & 0.48 & 0.78 \\ 0.30 & 0.04 & 0.16 & 0.04 & 0.40 & <10 & 0.41 & 0.48\end{array}$

\begin{tabular}{|c|c|c|c|c|c|c|c|}
\hline & & PHOS - & & $\mathrm{PH}$ & & & \\
\hline $\begin{array}{l}\text { NITRO- } \\
\text { GEN, }\end{array}$ & PHOS - & $\begin{array}{c}\text { PHORUS } \\
\text { ORTHO, }\end{array}$ & $\begin{array}{l}\text { SPE- } \\
\text { CIFIC }\end{array}$ & $\begin{array}{l}\text { WATER } \\
\text { WHOLE }\end{array}$ & & $\begin{array}{l}\text { LEAD, } \\
\text { TOTAL }\end{array}$ & $\begin{array}{l}\text { ZINC, } \\
\text { TOTAL }\end{array}$ \\
\hline $\mathrm{NO}_{2}+\mathrm{NO}_{3}$ & PHORUS & DIS - & $\mathrm{CON}-$ & LAB & ACIDITY & RECOV- & RECOV- \\
\hline $\begin{array}{l}\text { TOTAL } \\
(\mathrm{mg} / \mathrm{L}\end{array}$ & $\begin{array}{l}\text { TOTAL } \\
\text { (mg/L }\end{array}$ & $\begin{array}{l}\text { SOLVED } \\
(\mathrm{mg} / \mathrm{L}\end{array}$ & $\begin{array}{l}\text { DUCT - } \\
\text { ANCE }\end{array}$ & $\begin{array}{l}\text { (STAND- } \\
\text { ARD }\end{array}$ & $\begin{array}{c}(\mathrm{mg} / \mathrm{L} \\
\text { as }\end{array}$ & $\begin{array}{l}\text { ERABLE } \\
(\mu \mathrm{g} / \mathrm{L}\end{array}$ & $\begin{array}{l}\text { ERABLE } \\
(\mu g / L\end{array}$ \\
\hline as N) & as $\mathrm{Pl}$ & as P) & $(\mu \mathrm{S} / \mathrm{cm})$ & UNITS) & $\left.\mathrm{CaCO}_{3}\right\}$ & as $\mathrm{Pb}$ ) & as $\mathrm{Zn \}}$ \\
\hline
\end{tabular}

PERIOD JUNE 1990 TO SEPTEMBER 1990

$\begin{array}{lrrrrrrr}0.40 & <0.005 & <0.002 & 34 & 4.2 & 6.0 & 5 & -- \\ 0.62 & 0.010 & 0.005 & 42 & 4.1 & 6.7 & 9 & <40 \\ 0.76 & <0.005 & <0.002 & 76 & 3.8 & 11 & <5 & <40 \\ 0.66 & 0.005 & 0.003 & 38 & 4.1 & 5.8 & <5 & <40\end{array}$

WATER YEAR OCTOBER 1990 TO SEPTEMBER 1991

$\begin{array}{lrrrrrrr}0.60 & <0.005 & <0.002 & 37 & 4.1 & 5.9 & <5 & <40 \\ 0.60 & 0.005 & 0.002 & 33 & 4.2 & 5.8 & <5 & <40 \\ 0.32 & 0.020 & 0.018 & 19 & 4.4 & 3.4 & <5 & <40 \\ 0.56 & 0.005 & 0.003 & 19 & 4.5 & 3.3 & 8 & <40 \\ 0.68 & 0.005 & 0.006 & 33 & 4.2 & 5.0 & <5 & <40 \\ 0.36 & 0.005 & 0.002 & 19 & 4.6 & 3.8 & <5 & <40 \\ 0.46 & 0.010 & <0.002 & 27 & 4.4 & 4.5 & 6 & <40 \\ 0.77 & 0.020 & <0.002 & 56 & 4.0 & 8.4 & 6 & <40 \\ 0.82 & 0.013 & <0.002 & 68 & 3.8 & 10 & <5 & <40 \\ 0.37 & 0.005 & <0.002 & 30 & 4.3 & 4.6 & <5 & 20\end{array}$


431248077564601 At State University of New York at Brockport, N.Y.

CHEMICAL QUALITY OF PRECIPITATION - MONTHLY WETFALL

NOV 01-DEC 02 DEC $01-J A N \quad 01$ JAN 01-31

JAN $31-F E B \quad 29$ FEB 29-MAR 31 MAR 31-MAY 05 MAY 05-JUN 01 JUN 01-JUL 01 JUL $01-27$

JUL 28 -SEP 01 SEP 01-OCT 01

OCT $01-$ NOV 02 NOV 05-DEC 01 DEC 01-JAN 04 JAN $04-F E B \quad 01$ FEB 01 -MAR 03 MAR 03-APR 01 APR 01 -MAY 03 MAY 03-JUN 01 JUN 01-29

JUL 01-AUG 05 AUG 05-SEP 02 SEP 01-OCT 01

DATE

NOV 01-DEC 02 DEC $01-J A N \quad 01$ JAN 01-31

JAN 31-FEB 29 FEB 29-MAR 31 MAR 31-MAY 05 MAY 05-JUN 01 JUN 01-JUL 01 JUL $01-27$

JUL 28-SEP 01 SEP 01-OCT 01

OCT 01-NOV 02 NOV 05-DEC 01 DEC 01-JAN 04 JAN $04-F E B \quad 01$ FEB 01-MAR 03 MAR 03-APR 01 APR 01-MAY 03 MAY 03-JUN 01 JUN $01-29$

JUL 01-AUG 05 AUG 05-SEP 02 SEP 01-OCT 01

\begin{tabular}{|c|c|c|c|c|c|c|c|}
\hline CALCIUM & $\begin{array}{l}\text { MAGNE- } \\
\text { SIUM, }\end{array}$ & SODIUM, & $\begin{array}{l}\text { POTAS- } \\
\text { SIUM, }\end{array}$ & $\begin{array}{l}\text { CHLO- } \\
\text { RIDE, }\end{array}$ & SULFATE & $\begin{array}{l}\text { NITRO- } \\
\text { GEN, } \\
\text { AMMONIA }\end{array}$ & $\begin{array}{l}\text { NITRO- } \\
\text { GEN, AM- } \\
\text { MONIA + }\end{array}$ \\
\hline DIS- & DIS- & DIS- & DIS- & DIS- & DIS- & DIS- & ORGANIC \\
\hline SOLVED & SOLVED & SOLVED & SOLVED & SOLVED & SOLVED & SOLVED & TOTAL \\
\hline $\begin{array}{c}(\mathrm{MG} / \mathrm{L} \\
\mathrm{AS} \mathrm{Ca})\end{array}$ & $\begin{array}{l}\text { (MG/L } \\
\text { as Mg) }\end{array}$ & $\begin{array}{l}(\mathrm{mg} / \mathrm{L} \\
\text { as } \mathrm{Na})\end{array}$ & $\begin{array}{l}(\mathrm{mg} / \mathrm{L} \\
\operatorname{as~K)}\end{array}$ & $\begin{array}{l}(\mathrm{mg} / \mathrm{L} \\
\text { as Cl) }\end{array}$ & $\begin{array}{c}(\mathrm{mg} / \mathrm{L} \\
\left.\text { as } \mathrm{SO}_{4}\right)\end{array}$ & $\begin{array}{l}(\mathrm{mg} / \mathrm{L} \\
\operatorname{as~N})\end{array}$ & $\begin{array}{l}(\mathrm{mg} / \mathrm{L} \\
\text { as } \mathrm{N})\end{array}$ \\
\hline
\end{tabular}

\section{WATER YEAR OCTOBER 1991 TO SEPTEMBER 1992}

$\begin{array}{lrrrcccl}0.21 & <0.15 & 0.23 & 0.07 & 0.90 & <10 & 0.27 & 0.37 \\ 0.13 & 0.03 & 0.06 & 0.04 & 0.70 & <10 & 0.11 & 0.15 \\ 0.38 & 0.09 & 0.35 & 0.05 & <0.20 & <10 & 0.21 & 0.35 \\ 0.34 & 0.68 & 0.29 & -- & 1.0 & <10 & 0.43 & 0.62 \\ 0.31 & 0.06 & 0.08 & 0.01 & 0.30 & <10 & 0.14 & 0.27 \\ 0.33 & 0.04 & 0.04 & 0.04 & 0.0 & 5.0 & 0.55 & 0.67 \\ 0.50 & 0.20 & 0.49 & 0.11 & 0.60 & 2.0 & 0.20 & 0.50 \\ 0.42 & 0.08 & 0.18 & 0.16 & 1.1 & 4.0 & 0.50 & 0.69 \\ 0.11 & 0.02 & <0.02 & 0.01 & <0.20 & 10 & 0.22 & 0.34 \\ 0.17 & 0.03 & 0.01 & 0.06 & 0.20 & <10 & 0.31 & 0.32 \\ 0.14 & <0.03 & 0.01 & <0.05 & 0.60 & 2.0 & 0.14 & 0.23\end{array}$

\section{WATER YEAR OCTOBER 1992 TO SEPTEMBER 1993}

\begin{tabular}{|c|c|c|c|c|c|c|c|}
\hline 0.38 & 0.05 & 0.08 & 0.03 & 0.50 & 3.0 & 0.32 & 0.49 \\
\hline 0.10 & 0.03 & $<0.10$ & $<0.10$ & 0.20 & 2.0 & 0.16 & 0.40 \\
\hline 0.45 & 0.12 & 0.64 & 0.06 & 0.60 & 2.0 & 0.18 & 0.41 \\
\hline 0.22 & 0.03 & 0.30 & 0.04 & 1.6 & 2.0 & 0.18 & 0.27 \\
\hline 0.07 & 0.01 & 1.4 & 0.01 & 0.20 & $<2.0$ & 0.08 & 0.20 \\
\hline 0.41 & 0.10 & 0.31 & $<0.02$ & 0.40 & 3.0 & 0.15 & 0.37 \\
\hline 0.22 & 0.05 & 0.06 & 0.04 & 0.36 & 4.0 & 0.24 & $<0.01$ \\
\hline 0.95 & 0.15 & 0.18 & 0.14 & 1.2 & -- & $<0.01$ & 0.78 \\
\hline 0.39 & 0.09 & 0.09 & 0.13 & 0.20 & - & 0.51 & 0.60 \\
\hline 0.50 & 0.10 & 0.44 & 0.10 & 0.45 & 5.0 & 0.31 & 0.44 \\
\hline 0.22 & 0.04 & $<0.05$ & 0.02 & 0.41 & 4.0 & 0.32 & 0.40 \\
\hline$<0.01$ & 0.04 & 0.12 & 0.04 & 0.21 & 1.8 & 0.26 & 0.32 \\
\hline & & PHOS- & & PH & & & \\
\hline NITRO- & & PHORUS & SPE- & WATER & & LEAD, & ZINC, \\
\hline $\begin{array}{r}\mathrm{GEN}, \\
\mathrm{NO}_{2}+\mathrm{NO}\end{array}$ & $\begin{array}{l}\text { PHOS- } \\
\text { PHORUS }\end{array}$ & $\begin{array}{l}\text { ORTHO, } \\
\text { DIS- }\end{array}$ & $\begin{array}{l}\text { CIFIC } \\
\text { CON- }\end{array}$ & $\begin{array}{l}\text { WHOLE } \\
\text { LAB }\end{array}$ & ACIDITY & $\begin{array}{l}\text { TOTAL } \\
\text { RECOV- }\end{array}$ & $\begin{array}{l}\text { TOTAL } \\
\text { RECOV- }\end{array}$ \\
\hline TOTAL & TOTAL & SOLVED & DUCT- & (STAND- & (mg/L & ERABLE & ERABLE \\
\hline$(\mathrm{mg} / \mathrm{L}$ & $(\mathrm{mg} / \mathrm{L}$ & $(\mathrm{mg} / \mathrm{L}$ & ANCE & ARD & as & $(\mu \mathrm{g} / \mathrm{L}$ & $(\mu \mathrm{g} / \mathrm{L}$ \\
\hline N) & as P) & as P) & $(\mu \mathrm{s} / \mathrm{cm})$ & UNITS) & $\left.\mathrm{CaCO}_{3}\right)$ & as $\mathrm{Pb}$ ) & as $\mathrm{Zn}$ ) \\
\hline
\end{tabular}

\section{WATER YEAR OCTOBER 1991 TO SEPTEMBER 1992}

$\begin{array}{lrrrrrrr}0.50 & 0.005 & 0.002 & 26 & 4.4 & 5.3 & 6 & <40 \\ 0.28 & <0.005 & 0.003 & 14 & 5.4 & 1.3 & <5 & <40 \\ 0.41 & <0.005 & 0.004 & 20 & 4.8 & 3.3 & 6 & <40 \\ 1.00 & 0.005 & <0.002 & 49 & 4.1 & 8.5 & 5 & <40 \\ 0.36 & 0.005 & 0.003 & 20 & 4.6 & 3.4 & 11 & <40 \\ 0.64 & 0.010 & <0.002 & 36 & 4.4 & 5.2 & <5 & 40 \\ 0.31 & 0.005 & 0.002 & 17 & 4.5 & 4.6 & 11 & <20 \\ 0.88 & 0.010 & 0.002 & 45 & 4.0 & 7.7 & 5 & 50 \\ 0.53 & <0.005 & <0.002 & 48 & 4.0 & 6.9 & <5 & <40 \\ 0.44 & 0.005 & <0.002 & 41 & 4.1 & 6.9 & <5 & <40 \\ 0.26 & <0.005 & <0.002 & 22 & 4.4 & 3.9 & <5 & <40\end{array}$

\section{WATER YEAR OCTOBER 1990 TO SEPTEMBER 1991}

$\begin{array}{rrrrrrrr}0.49 & 0.010 & <0.002 & 27 & 4.5 & 4.4 & 10 & <40 \\ 0.46 & 0.005 & 0.002 & 22 & 4.9 & 3.9 & <5 & 20 \\ 0.52 & 0.015 & 0.005 & 19 & 4.8 & 4.3 & 8 & <40 \\ 0.44 & 0.005 & 0.004 & 21 & 4.5 & 4.4 & 8 & <40 \\ 0.23 & 0.005 & <0.002 & 10 & 5.0 & 2.2 & 7 & <40 \\ 0.57 & 0.010 & 0.003 & 27 & 4.4 & 4.7 & 16 & <40 \\ 0.56 & 0.005 & 0.003 & 32 & 4.2 & 6.1 & <5 & <40 \\ 0.63 & 0.026 & <0.002 & 26 & 4.6 & 4.7 & 9 & <40 \\ 0.54 & 0.010 & <0.002 & 37 & 4.9 & 5.7 & <5 & 110 \\ 0.57 & 0.005 & <0.002 & 45 & 4.1 & 7.1 & <5 & <40 \\ 0.55 & <0.005 & <0.002 & 41 & 4.2 & 6.4 & <5 & <40 \\ 0.42 & 0.010 & <0.002 & 24 & 4.4 & 4.2 & <5 & <40\end{array}$




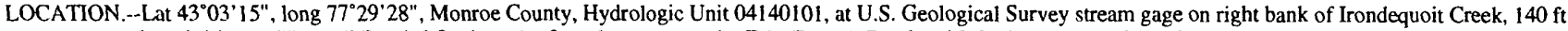
upstream from bridge on Thornell Road, $0.9 \mathrm{mi}$ south of creek passage under Erie (Barge) Canal and 2.7 mi southeast of Pittsford.

PERIOD OF RECORD.--October 1983 to current year.

EQUIPMENT.--Iowa-type precipitation gage with 8-in diameter receiver funnel mounted on roof of stream gage shelter, and 4-in diameter PVC collector pife mounted inside of gage shelter. The bottom portion of the receiver funnel is wrapped with heat tape to prevent freezing and to facilitate the rapid melting of snow. A float-driven punched tape recorder stores 15 -min values of water level in the collector pipe. Values recorded are to the nearest 0.01 in.

REMARKS.--Records poor. Because of large amounts of missing or questionable data, no attempt has been made to estimate the missing record

RAINFALL ACCUMULATED - DAILY SUM VALUES (INCHES)

WATER YEAR OCTOBER 1988 TO SEPTEMBER 1989

\begin{tabular}{|c|c|c|c|c|c|c|c|c|c|c|c|c|}
\hline DAY & OCT & NOV & $\mathrm{DEC}$ & JAN & FEB & MAR & APR & MAY & JUN & JUL & AUG & SEP \\
\hline 1 & --- & .00 & .00 & .00 & .00 & .00 & .01 & .24 & .27 & .00 & .00 & .31 \\
\hline 2 & --- & .14 & .00 & .00 & .01 & .00 & .00 & .46 & .09 & .00 & .10 & .05 \\
\hline 3 & --- & .00 & .00 & .00 & .07 & .00 & .16 & .00 & .08 & .00 & .01 & .01 \\
\hline 4 & $\cdots$ & .00 & .00 & .00 & .00 & .21 & .15 & .00 & .00 & .00 & 2.00 & .00 \\
\hline 5 & $-\cdots$ & .47 & .00 & .00 & .00 & .03 & .01 & .00 & .00 & .00 & .08 & .00 \\
\hline 6 & -- & .01 & .00 & .00 & .01 & .00 & .01 & .02 & .00 & .00 & .00 & .00 \\
\hline 7 & --- & .01 & .00 & .00 & .00 & .00 & .01 & 1.96 & .00 & .00 & .00 & .00 \\
\hline 8 & --- & .05 & .00 & .00 & .01 & .00 & .00 & .03 & .00 & .00 & .00 & .00 \\
\hline 9 & --- & .00 & .00 & .00 & .02 & .00 & .03 & .00 & .91 & .00 & .00 & .00 \\
\hline 10 & --- & .00 & .00 & .00 & .00 & .00 & .01 & .11 & .01 & .58 & .00 & .09 \\
\hline 11 & --- & .00 & .07 & .00 & .00 & .00 & .00 & 00 & .00 & .00 & .00 & .01 \\
\hline 12 & -- & .00 & .10 & .00 & .00 & .00 & .00 & 1.02 & .01 & .00 & .00 & .00 \\
\hline 13 & -- & .00 & .00 & .00 & .01 & .00 & .03 & .00 & .18 & .00 & .00 & .10 \\
\hline 14 & --- & .01 & .04 & .01 & .00 & .00 & .00 & .38 & .13 & .03 & .00 & 2.11 \\
\hline 15 & --- & .00 & .00 & .00 & .06 & .06 & .00 & .00 & .00 & .00 & .04 & .12 \\
\hline 16 & $\cdots$ & .01 & .00 & .00 & .00 & .00 & .00 & --- & .25 & .00 & .22 & .26 \\
\hline 17 & --- & .02 & .00 & .00 & .00 & .00 & 1.08 & $\cdots$ & .27 & .01 & .00 & .52 \\
\hline 18 & --- & .00 & .00 & .00 & .00 & .48 & .01 & --- & .08 & .00 & .00 & .00 \\
\hline 19 & --- & .00 & .00 & .00 & .00 & .00 & .00 & .00 & .00 & .00 & .77 & .00 \\
\hline 20 & $\cdots$ & .54 & .00 & .02 & .00 & .05 & .00 & .00 & .78 & .06 & .01 & .00 \\
\hline 21 & $\ldots$ & .01 & .00 & .00 & .19 & .00 & .00 & .00 & .00 & .00 & .00 & .00 \\
\hline 22 & --- & .00 & .00 & .00 & .00 & .00 & .00 & .00 & .00 & .00 & .00 & .24 \\
\hline 23 & $\cdots$ & .00 & .06 & .00 & .00 & .00 & .00 & .07 & .00 & .00 & .03 & .23 \\
\hline 24 & $\ldots$ & .00 & .19 & .00 & .00 & .02 & .00 & .00 & .00 & .00 & .00 & .04 \\
\hline 25 & $-\cdots$ & .00 & .00 & .00 & .00 & .00 & .01 & .02 & .00 & .00 & .00 & .01 \\
\hline 26 & $\cdots$ & .00 & .00 & .20 & .00 & .00 & .00 & .13 & .02 & .00 & .00 & .00 \\
\hline 27 & 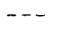 & .00 & .00 & .00 & .00 & .00 & .00 & .00 & .00 & .00 & .00 & .00 \\
\hline 28 & -- & .00 & .16 & .00 & .01 & .11 & .00 & .00 & .21 & .04 & .00 & .00 \\
\hline 29 & .00 & .02 & .00 & .05 & $\cdots$ & .00 & .07 & .00 & .00 & .00 & .08 & .00 \\
\hline 30 & .00 & .00 & .00 & .00 & --- & .43 & .00 & .19 & .00 & .00 & .02 & .00 \\
\hline 31 & .01 & --- & .00 & .00 & $\cdots$ & .74 & $\cdots$ & .41 & $\cdots$ & .00 & .00 &.- \\
\hline TOTAL & --- & 1.29 & 0.62 & 0.28 & 0.39 & 2.13 & 1.59 & $-\cdots$ & 3.29 & 0.72 & $3.3 \epsilon$ & 4.10 \\
\hline
\end{tabular}


430315077292801. Near Pittsford, N.Y.-continued

\section{RAINFALL ACCUMULATED - DAILY SUM VALUES (INCHES)}

WATER YEAR OCTOBER 1989 TO SEPTEMBER 1990

\begin{tabular}{|c|c|c|c|c|c|c|c|c|c|c|c|c|}
\hline DAY & ОСт & NOV & DEC & JAN & FEB & MAR & APR & MAY & JUN & JUL & AUG & SEP \\
\hline 1 & .00 & .22 & .00 & .02 & 1.06 & .00 & .29 & --- & .00 & .09 & .00 & .00 \\
\hline 2 & .50 & .02 & .01 & .00 & .26 & .00 & .44 & $\cdots$ & .00 & .00 & .00 & .00 \\
\hline 3 & .03 & .22 & .00 & .00 & .00 & .00 & .24 & -- & .28 & .00 & .00 & .00 \\
\hline 4 & .00 & .00 & .03 & .02 & .00 & .00 & 2.22 & $\cdots$ & .25 & .21 & .00 & .00 \\
\hline 5 & .00 & .00 & .00 & .00 & .27 & .01 & .16 & --- & .00 & .12 & 1.97 & .65 \\
\hline 6 & .08 & 1.04 & .18 & .00 & .30 & .00 & 1.19 & -- & .02 & .05 & .16 & .00 \\
\hline 7 & .00 & .40 & .01 & .00 & .00 & .00 & .00 & - - & .00 & .01 & .00 & .90 \\
\hline 8 & .00 & .01 & .00 & .00 & .00 & .00 & 2.00 & $\cdots$ & .34 & .00 & .01 & .00 \\
\hline 9 & .00 & .16 & .00 & .00 & 1.19 & .05 & .00 & -- & .00 & .37 & .00 & .21 \\
\hline 10 & .35 & .09 & .00 & .12 & .10 & .00 & .20 & -.. & .00 & .01 & .00 & .00 \\
\hline 11 & .01 & .01 & .02 & .07 & .00 & .20 & .00 & $\ldots$ & .00 & .00 & .00 & .00 \\
\hline 12 & .01 & .01 & .00 & .01 & .00 & .13 & .00 & --- & .00 & .07 & 30 & .00 \\
\hline 13 & .02 & .00 & .01 & .00 & .03 & .00 & .00 & $\cdots$ & .00 & .00 & .44 & .00 \\
\hline 14 & .03 & .01 & .00 & .00 & .04 & .00 & .03 & -- & .00 & .01 & .00 & .00 \\
\hline 15 & .35 & .06 & .00 & .00 & .33 & .00 & .00 & --- & .00 & .05 & .00 & .00 \\
\hline 16 & .00 & .47 & .03 & .05 & 1.72 & .00 & .00 & -- & .00 & .00 & .01 & .00 \\
\hline 17 & .00 & .01 & .16 & .00 & .01 & .40 & .08 & -.- & .00 & .00 & .00 & .00 \\
\hline 18 & 1.26 & .01 & .05 & .35 & .01 & .07 & .00 & -- & .27 & .00 & .00 & .00 \\
\hline 19 & .13 & .00 & .00 & .02 & .00 & .21 & .00 & $\cdots$ & .09 & .00 & .06 & .00 \\
\hline 20 & .73 & .45 & .00 & .00 & .00 & .08 & .18 & --- & .00 & .36 & .00 & .00 \\
\hline 21 & .08 & .02 & .00 & .09 & .01 & .00 & .01 & $\ldots$ & .13 & .00 & .00 & .00 \\
\hline 22 & .01 & .00 & .01 & 1.03 & .48 & .00 & .00 & -. & .40 & .05 & .02 & .00 \\
\hline 23 & .01 & .00 & .00 & .00 & 1.17 & .09 & .71 & $\ldots$ & .06 & .28 & .00 & .00 \\
\hline 24 & .00 & .00 & .02 & .04 & .14 & .01 & .00 & $\ldots$ & .00 & .02 & .00 & .00 \\
\hline 25 & .00 & .00 & .00 & .05 & .00 & .00 & -- & -. & .00 & .00 & .00 & .18 \\
\hline 26 & .06 & .10 & .00 & .00 & .22 & .01 & $\ldots$ & -- & .00 & .00 & .00 & .24 \\
\hline 27 & .00 & .00 & .00 & .00 & .01 & .00 & $\ldots$ & $\ldots$ & .01 & .00 & .00 & .01 \\
\hline 28 & .00 & .04 & .04 & .00 & .01 & .00 & $\ldots$ & ... & .00 & .00 & .14 & .01 \\
\hline 29 & .00 & .00 & .00 & .27 & -- & .00 & -- & -.- & .04 & .00 & .00 & .31 \\
\hline 30 & .00 & .07 & .05 & .49 & $\ldots$ & .23 & -.. & $\ldots$ & .25 & .00 & .00 & .42 \\
\hline 31 & .00 & --- & 1.52 & .00 & --- & .00 & $\cdots$ & .00 & -- & .31 & .00 & -- \\
\hline TOTAL & 3.66 & 3.42 & 2.14 & 2.63 & 7.36 & 1.49 & -- & -- & 2.14 & 2.01 & 3.11 & 2.93 \\
\hline
\end{tabular}


430315077292801. Near Pittsford, N.Y.-continued

RAINFALL ACCUMULATED - DAILY SUM VALUES (INCHES) WATER YEAR OCTOBER 1990 TO SEPTEMBER 1991

\begin{tabular}{|c|c|c|c|c|c|c|c|c|c|c|c|c|}
\hline DAY & OCT & Nov & DEC & JAN & FEB & MAR & APR & MAY & JUN & JUL & AUG & SEP \\
\hline 1 & .04 & .00 & .00 & .03 & .03 & .03 & .17 & --- & - - & $\ldots$ & -- & $\ldots$ \\
\hline 2 & .00 & .00 & .00 & .00 & .00 & .49 & .02 & --- & -- & --- & $\ldots$ & $\cdots$ \\
\hline 3 & .00 & .00 & .52 & .09 & .00 & .28 & .00 & $-\cdots$ & -. & --- & -- & --- \\
\hline 4 & .69 & .00 & .29 & .00 & .00 & .90 & .00 & --- & --- & -- & -- & $-\cdots$ \\
\hline 5 & .04 & 1.52 & .05 & .00 & .00 & .01 & .00 & --- & --- & --- & --- & $\cdots$ \\
\hline 6 & .00 & .03 & .01 & .07 & .11 & .16 & .07 & --- & --- & -- & $\cdots$ & $-\cdots$ \\
\hline 7 & .00 & .05 & .00 & 2.01 & .16 & .10 & .00 & --- & --- & --- & -- & $-\cdots$ \\
\hline 8 & 1.05 & .02 & .00 & .00 & .00 & .00 & .26 & --- & -- & --- & -- & -- \\
\hline 9 & .67 & .18 & .00 & .01 & .00 & .00 & .38 & --- & --- & $\cdots$ & -- & -- \\
\hline 10 & .18 & 1.62 & .00 & .00 & .00 & .00 & .01 & --- & $\cdots$ & --- & --- & -- \\
\hline 11 & 1.05 & .22 & .00 & .01 & .03 & .00 & .00 & $\cdots$ & --- & --- & -- & -- \\
\hline 12 & 1.11 & .32 & .00 & .20 & .02 & .00 & .00 & $-\cdots$ & --- & --- & --- & $\cdots$ \\
\hline 13 & .70 & .06 & .18 & .08 & .00 & .00 & .00 & --- & --- & --- & -- & -- \\
\hline 14 & .00 & .00 & .00 & .33 & .20 & .00 & .21 & $\cdots$ & --- & $\cdots$ & --- & -- \\
\hline 15 & .00 & .00 & .04 & .02 & .00 & .06 & .48 & --- & $\cdots$ & -- & -- & --- \\
\hline 16 & .01 & .07 & .40 & .30 & .01 & .00 & .00 & $\cdots$ & $\cdots$ & -- & -- & -- \\
\hline 17 & .00 & .07 & .03 & .07 & .00 & .00 & .00 & $-\cdots$ & $\cdots$ & $\cdots$ & -- & -- \\
\hline 18 & .51 & .01 & .66 & .00 & .10 & .16 & .00 & $-\cdots$ & $\ldots$ & $\cdots$ & --- & -- \\
\hline 19 & .04 & .00 & .01 & .01 & .08 & .03 & .07 & --- & --- & --- & --- & --- \\
\hline 20 & .00 & .00 & .00 & .00 & .01 & .00 & .46 & -- & --- & --- & -- & $-\cdots$ \\
\hline 21 & .01 & .00 & .09 & .00 & .00 & .00 & .97 & -- & --- & $\cdots$ & --- & -- \\
\hline 22 & .24 & .29 & .07 & .04 & .00 & .08 & .51 & $-\cdots$ & $\ldots$ & -- & -- & -- \\
\hline 23 & 1.59 & .01 & .35 & .00 & .00 & .41 & .04 & $\cdots$ & $\ldots$ & $\cdots$ & --- & -- \\
\hline 24 & .02 & .01 & .17 & .00 & .05 & .06 & .05 & --- & $-\cdots$ & --- & -- & -- \\
\hline 25 & .07 & .00 & .06 & .01 & .01 & .00 & --- & $-\cdots$ & $-\cdots$ & - - & --- & -- \\
\hline 26 & .00 & .00 & .00 & .00 & .00 & .00 & $\cdots$ & $\cdots$ & --- & --- & --- & $-\cdots$ \\
\hline 27 & .02 & .19 & .02 & .01 & -- & .83 & - & --- & --- & --- & $\cdots$ & -- \\
\hline 28 & .18 & .00 & 1.22 & .06 & .12 & .20 & $\cdots$ & --- & --- & $\cdots$ & --- & $\ldots$ \\
\hline 29 & .04 & .00 & .14 & .00 & --- & .00 & -- & --- & --- & --- & -- & $-\ldots$ \\
\hline 30 & .02 & .01 & 2.24 & .10 & -- & .00 & -- & --- & --- & --- & --- & --- \\
\hline 31 & .00 & --- & .19 & .09 & $-\cdots$ & .00 & --- & $\cdots$ & --- & --- & --- & --- \\
\hline TOTAL & 8.28 & 4.68 & 6.74 & 3.54 & $-\cdots$ & 3.80 & --- & --- & -- & --- & --- & $\ldots$ \\
\hline
\end{tabular}




\section{$430622077274401 \quad$ At Fairport, N.Y.}

LOCATION.--Lat $43^{\circ} 06^{\prime} 22^{\prime \prime}$, long $77^{\circ} 27^{\prime} 44^{\prime \prime}$, Monroe County, Hydrologic Unit 04140101, at U.S. Geological Survey stream gage on right bank of Thomas Creek , 48 ft upstream from culvert on Foreman Center Road, 0.5 mi northwest of Fairport, and 0.8 mi upstream from the mouth of Thomas Creek.

PERIOD OF RECORD.--October 1983 to current year.

EQUIPMENT.--Iowa-type precipitation gage with 8-in diameter receiver funnel mounted on roof of stream gage shelter, and 4-in diameter PVC collector pipe mounted inside of gage shelter. The bottom portion of the receiver funnel is wrapped with heat tape to prevent freezing and to facilitate the rapid melting of snow. A float-driven punchedtape recorder stores 15 -min values of water level in the collector pipe. Values recorded are to the nearest $0.01 \mathrm{in}$.

REMARKS.--Records poor. Because of large amounts of missing or questionable data, no attempt has been made to estimate the missing record

RAINFALL ACCUMULATED - DAILY SUM VALUES (INCHES) WATER YEAR OCTOBER 1988 TO SEPTEMBER 1989

\begin{tabular}{|c|c|c|c|c|c|c|c|c|c|c|c|c|}
\hline DAY & OCT & NOV & $\mathrm{DEC}$ & JAN & FEB & MAR & $\begin{array}{c}\text { APR } \\
.\end{array}$ & MAY & JUN & JUL & AUG & SEP \\
\hline 1 & $\ldots$ & .00 & .00 & .00 & .00 & .00 & .77 & .26 & .33 & .00 & .00 & .37 \\
\hline 2 & -- & .27 & .00 & .00 & .00 & .00 & .03 & .47 & .10 & .00 & .11 & .04 \\
\hline 3 & --- & .00 & .00 & .01 & .01 & .00 & .25 & .00 & .09 & .00 & .01 & .00 \\
\hline 4 & --- & .00 & .00 & .00 & .00 & .34 & .21 & .00 & .01 & .03 & .63 & .00 \\
\hline 5 & -- & .34 & .00 & .07 & .14 & .26 & .01 & .01 & .02 & .00 & .25 & .00 \\
\hline 6 & --- & .07 & .00 & .00 & .05 & .00 & .00 & .02 & .01 & .00 & .00 & .00 \\
\hline 7 & --- & .03 & .00 & .06 & .12 & .00 & .03 & 1.04 & .00 & .00 & .00 & .00 \\
\hline 8 & --- & .10 & .00 & .03 & .03 & .02 & .00 & .12 & .01 & .00 & .00 & .00 \\
\hline 9 & --- & .00 & .00 & .00 & .01 & .00 & .00 & .00 & 1.03 & .00 & .00 & .00 \\
\hline 10 & --- & .00 & .00 & .00 & .00 & .00 & .00 & .37 & .01 & .37 & .00 & .07 \\
\hline 11 & --- & .00 & .00 & .00 & .02 & .00 & .01 & .53 & .00 & .00 & .00 & .00 \\
\hline 12 & -- & .00 & .07 & .00 & .01 & .00 & .01 & .11 & .09 & .00 & .00 & .00 \\
\hline 13 & --- & .03 & .00 & .00 & .00 & .00 & .08 & .00 & .45 & .00 & .00 & .00 \\
\hline 14 & $-\cdots$ & .00 & .28 & .03 & .05 & .00 & .00 & .47 & .26 & .00 & .00 & .34 \\
\hline 15 & --- & .00 & .07 & .00 & .13 & .09 & .06 & .02 & .01 & .00 & .01 & .83 \\
\hline 16 & $\ldots$ & .03 & .02 & .00 & .00 & .01 & .00 & .11 & .28 & .00 & .20 & .19 \\
\hline 17 & $\cdots$ & .06 & .00 & .00 & .00 & .04 & .22 & .00 & .62 & .00 & .01 & .34 \\
\hline 18 & --- & .00 & .01 & .00 & .03 & .39 & .00 & .00 & 1.08 & .00 & .00 & .01 \\
\hline 19 & $\ldots$ & .00 & .00 & .00 & .00 & .18 & .00 & .00 & .00 & .00 & .47 & .00 \\
\hline 20 & --- & .59 & .00 & .09 & .00 & .00 & .00 & 1.03 & 1.06 & .21 & .01 & .00 \\
\hline 21 & --- & .04 & .00 & .00 & .39 & .17 & .00 & .00 & .01 & .00 & .00 & .00 \\
\hline 22 & --- & .00 & .00 & .01 & .00 & .00 & .00 & .00 & .00 & .00 & .06 & .00 \\
\hline 23 & --- & .00 & .10 & .00 & .00 & .00 & .00 & .19 & .00 & .00 & .00 & .32 \\
\hline 24 & $\cdots$ & .00 & .23 & .00 & .00 & .13 & .01 & .03 & .00 & .00 & .01 & .01 \\
\hline 25 & --- & .00 & .00 & .00 & .00 & .01 & .06 & .03 & .00 & .00 & .00 & .07 \\
\hline 26 & -- & .00 & .00 & .27 & .00 & .00 & .00 & .11 & .00 & .00 & .00 & $\ldots$ \\
\hline 27 & .00 & .02 & .01 & .01 & .06 & .00 & .00 & .00 & .00 & .00 & .00 & -- \\
\hline 28 & .04 & .04 & .31 & .00 & .00 & .21 & .00 & .00 & .28 & .02 & .00 & --- \\
\hline 29 & .00 & .03 & .03 & .06 & -- & .00 & .09 & .00 & .00 & .00 & .06 & $\cdots$ \\
\hline 30 & .01 & .00 & .00 & .00 & $\cdots$ & .70 & .00 & .55 & .00 & .00 & .00 & .00 \\
\hline 31 & .00 & --- & .00 & .00 & --- & .31 & --- & .38 & --- & .00 & .00 & -- \\
\hline TOTAL & $\ldots$ & 1.65 & 1.13 & 0.64 & 1.05 & 2.86 & 1.84 & 5.85 & 5.75 & 0.63 & 1.83 & -- \\
\hline
\end{tabular}




\section{At Fairport, N.Y. -- continued \\ RAINFALL ACCUMULATED - DAILY SUM VALUES (INCHES) WATER YEAR OCTOBER 1989 TO SEPTEMBER 1990}

\begin{tabular}{|c|c|c|c|c|c|c|c|c|c|c|c|c|}
\hline DAY & OCT & NOV & DEC & JAN & FEB & MAR & APR & MAY & JUN & JUL & AUG & SEP \\
\hline 1 & .00 & .00 & .00 & .00 & .04 & $\ldots$ & $\ldots$ & $\ldots$ & - - & ... & $\ldots$ & ... \\
\hline 2 & .42 & .00 & .00 & .00 & .19 & -. & -. & $\ldots$ & $\ldots$ & -- & $\ldots$ & $-\ldots$ \\
\hline 3 & .00 & .51 & .02 & .00 & .00 & -. & --- & -- & -.. & 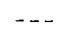 & $\ldots$ & --. \\
\hline 4 & .00 & .00 & .03 & .00 & .00 & -- & -- & -- & -- & -- & $\ldots$ & -.. \\
\hline 5 & .00 & .01 & .05 & .00 & .00 & -- & -- & -- & $\cdots$ & $-\cdots$ & $\ldots$ & -.. \\
\hline 6 & .13 & .05 & .10 & .00 & .22 & -.. & - - - & --- & $\ldots$ & $-\ldots$ & $\ldots$ & --. \\
\hline 7 & .02 & .35 & .00 & .01 & .00 & -- & - - &.- & -. & --- & -. & --- \\
\hline 8 & .00 & .01 & .00 & .00 & .00 & --- & --- & --- & -- & --- & $\cdots$ & - \\
\hline 9 & .02 & .15 & .00 & .01 & .13 & --- & -. & -- & $\cdots$ & -- & $\cdots$ & --- \\
\hline 10 & .15 & .10 & .00 & .16 & .06 & -- & -- & $\cdots$ & -- & -- & $\ldots$ & --- \\
\hline 11 & .01 & .04 & .00 & .06 & .00 & -.. & -.- & --- & -.. & $\ldots$ & $\ldots$ & ... \\
\hline 12 & .00 & .02 & .00 & .00 & .00 & -.- & --- &.- & $\ldots$ & --- & $\ldots$ & \\
\hline 13 & .00 & .00 & .00 & .00 & .02 & -- & -- &.- & -.. & $\ldots$ & $\ldots$ & \\
\hline 14 & .23 & .04 & .00 & .00 & .06 & --- & -- & $\ldots$ & -.. & -- &.-- & -.. \\
\hline 15 & .01 & .07 & .00 & .01 & .01 & -.. & $\ldots$ & $\ldots$ & $\ldots$ & $\ldots$ & $\ldots$ & $-\ldots$ \\
\hline 16 & .16 & .36 & .00 & .06 & .33 & -.. & $\ldots$ & -. & $\ldots$ & $\ldots$ & $\ldots$ & -.. \\
\hline 17 & .62 & .00 & .00 & .03 & .00 & $\cdots$ & --- & --- & $\cdots$ & --- & -- & \\
\hline 18 & .02 & .00 & .00 & .23 & .00 & -- & $\cdots$ & -- & -- & -- & $\cdots$ & -.- \\
\hline 19 & .08 & .00 & .00 & .00 & .00 & -. & --- & -- & -.. & 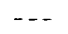 & $\ldots$ & \\
\hline 20 & .50 & .54 & .00 & .00 & .00 & --. & -- & -- & $\cdots$ & $\cdots$ & $\cdots$ & -- \\
\hline 21 & .08 & .01 & .00 & .00 & - - & -- & $\ldots$ & $\ldots$ & -.- & $\ldots$ & $\ldots$ & - \\
\hline 22 & .00 & .00 & .00 & .02 & - - & -- & -- & $-\ldots$ & -.- & 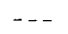 & -.. & $\ldots$ \\
\hline 23 & .00 & .00 & .00 & .01 & -.- & -- & $\cdots$ & $\ldots$ & $\ldots$ & $\cdots$ & $\ldots$ & -.. \\
\hline 24 & .00 & .01 & .00 & .06 &.-- &.- & -- & -.. & -- & $\ldots$ & -.. & \\
\hline 25 & .00 & .00 & .00 & .03 & $\ldots$ & --- &.- & -.- & -.. & -- & $\ldots$ &.- \\
\hline 26 & .00 & .12 & .00 & .01 & --- & -.- & --- & -.. & -.. & - . & $\ldots$ & - \\
\hline 27 & .00 & .01 & .00 & .00 & $\cdots$ & --- & -- & -. & -.. & $\ldots$ & $\ldots$ & \\
\hline 28 & .00 & .02 & .00 & .00 & -- & -- & -- & -. & $\ldots$ & -- & $\ldots$ & \\
\hline 29 & .00 & .00 & .00 & .01 & --- & --- &.- & -. & -.. & -- & $\cdots$ & -- \\
\hline 30 & .00 & .03 & .00 & .25 & -.- & $\ldots$ & $\ldots$ & --. & -.. & $\ldots$ & -- & - \\
\hline 31 & .22 & . & .00 & .27 & -.. & -- & -- & -- & $\cdots$ & --- & $\cdots$ & - \\
\hline TOTAL & 2.67 & 2.45 & 0.20 & 1.23 & -.. & -.- & $\ldots$ & -.. & $\ldots$ & -.. & $\ldots$ & - \\
\hline
\end{tabular}


Precipitation Stations

\section{At Blossom Road, Rochester, N.Y.}

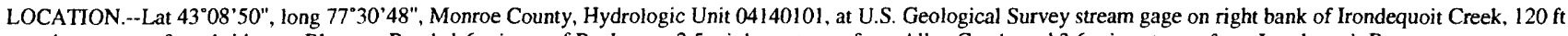
downstream from bridge on Blossom Road, 1.6 mi east of Rochester, 2.5 mi downstream from Allen Creek, and 3.6 mi upstream from Irondequoit Bay.

PERIOD OF RECORD.--October 1983 to current year.

EQUIPMENT.--lowa-type precipitation gage with 8-in diameter receiver funnel mounted on roof of stream gage shelter, and 4-in diameter PVC collector pipe mounted inside of gage shelter. The bottom portion of the receiver funnel is wrapped with heat tape to prevent freezing and to facilitate the rapid melting of snow. A float-driven punchedtape recorder stores 15 -min values of water level in the collector pipe. Values recorded are to the nearest 0.01 in.

REMARKS.--Records poor. Because of large amounts of missing or questionable data, no attempt has been made to estimate the missing record

RAINFALL ACCUMULATED - DAILY SUM VALUES (INCHES)

WATER YEAR OCTOBER 1989 TO SEPTEMBER 1990

\begin{tabular}{|c|c|c|c|c|c|c|c|c|c|c|c|c|}
\hline DAY & OCT & NOV & $\mathrm{DEC}$ & JAN & FEB & MAR & APR & MAY & JUN & JUL & AUG & SEP \\
\hline 1 & --- & --- & --- & -- & --- & --- & $\cdots$ & .00 & .00 & .08 & .01 & -- \\
\hline 2 & -- & --- & $\cdots$ & $\cdots$ & $\cdots$ & --- & $\cdots$ & .00 & .00 & .00 & .00 & $\cdots$ \\
\hline 3 & --- & --- & --- & --- & 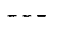 & --- & --- & .00 & .22 & .00 & .00 & $-\cdots$ \\
\hline 4 & --- & -- & $\cdots$ & $\cdots$ & $\cdots$ & --- & -- & .31 & .25 & .30 & .00 & -- \\
\hline 5 & --- & --- & --- & -- & -- & -- & --- & .50 & .00 & .01 & 1.43 & --- \\
\hline 6 & --- & --- & --- & --- & $\cdots$ & --- & - - & .17 & .00 & .00 & .07 & $\ldots$ \\
\hline 7 & --- & --- & $\cdots$ & --- & --- & --- & $\cdots$ & .08 & .00 & .00 & .00 & -- \\
\hline 8 & --- & 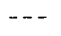 & --- & -- & --- & --- & -- & .01 & .27 & .00 & .00 & --- \\
\hline 9 & $\cdots$ & --- & $\cdots$ & --- & --- & $-\cdots$ & $\cdots$ & .00 & .00 & .32 & .00 & --- \\
\hline 10 & --- & --- & --- & $\cdots$ & $-\cdots$ & -- & --- & .14 & .00 & .00 & .00 & --- \\
\hline 11 & $-\cdots$ & --- & --- & $\cdots$ & --- & --- & --- & .01 & .00 & .00 & .00 & $\ldots$ \\
\hline 12 & --- & --- & --- & $\ldots$ & $\cdots$ & $\cdots$ & $\cdots$ & .08 & .00 & .02 & .64 & -- \\
\hline 13 & --- & $\cdots$ & --- & --- & $-\cdots$ & $-\cdots$ & --- & 1.18 & .00 & .00 & .59 & $\ldots$ \\
\hline 14 & $-\cdots$ & $\cdots$ & --- & --- & --- & $\cdots$ & --- & .01 & .00 & .00 & .03 & -- \\
\hline 15 & -- & --- & $\cdots$ & --- & $-\cdots$ & -- & $\cdots-$ & .00 & .00 & .18 & .00 & --- \\
\hline 16 & $-\cdots$ & $\cdots$ & --- & $-\cdots$ & $\cdots$ & $\cdots$ & --- & .84 & .00 & .00 & .00 & -- \\
\hline 17 & --- & --- & $\cdots$ & $\cdots$ & --- & $\cdots$ & --- & .31 & .00 & .00 & .00 & --- \\
\hline 18 & --- & --- & --- & --- & --- & --- & $\cdots$ & .21 & .45 & .00 & .00 & --- \\
\hline 19 & $-\cdots$ & --- & -- & $\cdots$ & --- & --- & -- & .02 & .01 & .00 & .10 & --- \\
\hline 20 & --- & --- & $\cdots$ & --- & -- & -- & --- & .58 & .00 & .34 & .00 & .00 \\
\hline 21 & --- & $\cdots$ & --- & --- & --- & $-\cdots$ & $\cdots$ & .03 & .02 & .00 & .00 & .03 \\
\hline 22 & $\ldots$ & $\cdots$ & --- & --- & --- & --- & --- & .01 & .50 & .07 & .00 & .11 \\
\hline 23 & --- & --- & --- & $\cdots$ & --- & --- & --- & .00 & .12 & .46 & .00 & .00 \\
\hline 24 & --- & --- & --- & $-\cdots$ & $\cdots$ & --- & --- & .00 & .00 & .00 & .00 & .00 \\
\hline 25 & --- & --- & -- & -- & $\cdots$ & $\cdots$ & $\cdots$ & .00 & .00 & .00 & .00 & .14 \\
\hline 26 & --- & --- & --- & --- & --- & --- & --- & .00 & .00 & .00 & .00 & .17 \\
\hline 27 & --- & --- & --- & --- & -- & --- & --- & .03 & .06 & .00 & .00 & .00 \\
\hline 28 & --- & --- & -- & $\cdots$ & --- & --- & --- & .00 & .00 & .00 & .07 & .00 \\
\hline 29 & --- & --- & --- & --- & --- & $\cdots$ & --- & .71 & .02 & .05 & --- & .26 \\
\hline 30 & --- & $\cdots$ & --- & $\cdots$ & --- & $\cdots$ & --- & .00 & .17 & .00 & $-\cdots$ & .37 \\
\hline 31 & --- & --- & --- & --- & $\cdots$ & --- & $\cdots$ & .00 & $-\infty-$ & .42 & --- & $\cdots$ \\
\hline TOTAL & --- & --- & --- & $-\cdots$ & --- & --- & $\cdots$ & 5.23 & 2.09 & 2.25 & $\cdots$ & --- \\
\hline
\end{tabular}


Precipitation Stations

430850077304801 At Blossom Road, Rochester, N.Y. -- continued

RAINFALL ACCUMULATED - DAILY SUM VALUES (INCHES)

WATER YEAR OCTOBER 1990 TO SEPTEMBER 1991

\begin{tabular}{|c|c|c|c|c|c|c|c|c|c|c|c|c|}
\hline DAY & $\mathrm{OCT}$ & NOV & $\mathrm{DEC}$ & JAN & FEB & MAR & APR & MAY & JUN & JUL & AUG & SEP \\
\hline 1 & .00 & .00 & .00 & .00 & .00 & .03 & .18 & .00 & .00 & .00 & .00 & .00 \\
\hline 2 & .00 & .00 & .00 & .00 & .00 & .76 & .00 & .15 & .00 & .00 & .00 & .00 \\
\hline 3 & .00 & .00 & .63 & .01 & .00 & .60 & .00 & .00 & .00 & .00 & .08 & .00 \\
\hline 4 & .58 & .00 & .28 & .01 & .00 & 1.49 & .00 & .00 & .00 & .01 & .00 & .09 \\
\hline 5 & .12 & .48 & .00 & .03 & .01 & .01 & .00 & .00 & .00 & .10 & .00 & .00 \\
\hline 6 & .00 & .06 & .01 & .00 & .09 & .27 & .09 & .26 & .00 & .10 & .00 & .00 \\
\hline 7 & .00 & .10 & .00 & .00 & .10 & .02 & .00 & .02 & .00 & .00 & .00 & .00 \\
\hline 8 & .05 & .03 & .00 & .01 & .00 & .01 & .40 & .02 & .00 & .00 & .00 & .00 \\
\hline 9 & .51 & .13 & .00 & .00 & .00 & .00 & .19 & .03 & .00 & .00 & .01 & .00 \\
\hline 10 & .26 & .48 & .00 & .00 & .00 & .00 & .00 & .05 & .00 & .00 & .01 & .16 \\
\hline 11 & .85 & .30 & .00 & .09 & .00 & .00 & .00 & .00 & .04 & .00 & .01 & .02 \\
\hline 12 & .13 & .01 & .00 & .39 & .00 & .00 & .00 & .00 & .02 & .01 & .02 & .00 \\
\hline 13 & .76 & .00 & .08 & .00 & .01 & .00 & .00 & .00 & .00 & .00 & .00 & .00 \\
\hline 14 & .00 & .00 & .00 & .00 & .15 & .00 & .13 & .00 & .00 & .00 & .56 & .00 \\
\hline 15 & .00 & .00 & .02 & .00 & .00 & .11 & .31 & .00 & .00 & .00 & .00 & .55 \\
\hline 16 & .00 & .02 & .23 & .33 & .02 & .00 & .00 & .00 & .00 & .00 & .05 & .00 \\
\hline 17 & .00 & .05 & .01 & .01 & .00 & .00 & .00 & .00 & .00 & .00 & .11 & .00 \\
\hline 18 & .51 & .00 & .59 & .02 & .07 & .22 & .00 & .00 & .00 & .00 & .01 & .05 \\
\hline 19 & .03 & .01 & .01 & .01 & .11 & .00 & .09 & .00 & .00 & .00 & .00 & .16 \\
\hline 20 & .00 & .00 & .00 & .00 & .01 & .00 & .31 & .02 & .00 & .00 & .24 & .19 \\
\hline 21 & .00 & .00 & .05 & .00 & .00 & .01 & 1.83 & .00 & .00 & .00 & .01 & .01 \\
\hline 22 & .25 & .37 & .10 & .01 & .00 & .15 & .55 & .00 & .00 & .00 & .00 & .00 \\
\hline 23 & .47 & .00 & .52 & .00 & .00 & .71 & .00 & .38 & .00 & .00 & .00 & .07 \\
\hline 24 & .00 & .00 & .23 & .00 & .02 & .09 & .01 & .00 & .00 & .00 & .00 & .15 \\
\hline 25 & .09 & .00 & .01 & .00 & .00 & .01 & .00 & .00 & .00 & .00 & .00 & .93 \\
\hline 26 & .01 & .01 & .00 & .00 & .01 & .00 & .00 & .00 & .00 & .00 & .00 & .06 \\
\hline 27 & .00 & .12 & .00 & .01 & .00 & .59 & .00 & .00 & .00 & .00 & .00 & .00 \\
\hline 28 & .11 & .00 & .06 & .06 & .01 & .14 & .00 & .00 & .00 & .00 & .00 & .00 \\
\hline 29 & .01 & .00 & .12 & .00 & $\ldots$ & .00 & .00 & .00 & .03 & .00 & .00 & .00 \\
\hline 30 & .00 & .00 & .89 & .12 & $\cdots-$ & .00 & .06 & .01 & .01 & .00 & .00 & .00 \\
\hline 31 & .00 & --- & .03 & .01 & --- & .00 & --- & .00 & $\cdots$ & .00 & .30 & -- \\
\hline TOTAL & 4.74 & 2.17 & 3.87 & 1.12 & 0.61 & 5.22 & 4.15 & 0.94 & 0.10 & 0.22 & 1.41 & 2.44 \\
\hline
\end{tabular}


Precipitation Stations

430850077304801 At Blossom Road, Rochester, N.Y. -- continued

RAINFALL ACCUMULATED - DAILY SUM VALUES (INCHES)

WATER YEAR OCTOBER 1991 TO SEPTEMBER 1992

\begin{tabular}{|c|c|c|c|c|c|c|c|c|c|c|c|c|}
\hline DAY & OCT & NOV & $\mathrm{DEC}$ & JAN & FEB & MAR & APR & MAY & JUN & JUL & AUG & SEP \\
\hline 1 & .24 & .00 & .00 & .00 & .00 & .02 & .08 & .07 & .38 & .00 & .12 & .00 \\
\hline 2 & .02 & .00 & .02 & .00 & .00 & .00 & .00 & .62 & .00 & .00 & .00 & .00 \\
\hline 3 & .03 & .00 & .00 & .00 & .00 & .00 & .00 & .00 & .00 & .30 & 1.01 & .52 \\
\hline 4 & .07 & .00 & .01 & .09 & .00 & .00 & .00 & .01 & .00 & .03 & .14 & .00 \\
\hline 5 & .16 & .00 & .00 & .05 & .00 & .00 & .00 & .00 & .18 & .11 & .00 & .00 \\
\hline 6 & .02 & .00 & .09 & .00 & .00 & .00 & .00 & .00 & .15 & .01 & .00 & .00 \\
\hline 7 & .00 & .09 & .00 & .00 & .05 & .34 & .01 & .00 & .17 & .00 & .00 & .02 \\
\hline 8 & .00 & .02 & .00 & .00 & .03 & .00 & .00 & .00 & .00 & .36 & .43 & .00 \\
\hline 9 & .00 & .00 & .08 & .04 & .04 & .07 & .02 & .07 & .00 & e. 17 & .23 & .00 \\
\hline 10 & .47 & .17 & .00 & .00 & .06 & .03 & .01 & .02 & .00 & e.06 & .11 & .00 \\
\hline 11 & .00 & .54 & .00 & .01 & .00 & .08 & .03 & .00 & .00 & e.00 & .02 & .00 \\
\hline 12 & .15 & .04 & .05 & .01 & .05 & .09 & .00 & .00 & .00 & e. 55 & .00 & .00 \\
\hline 13 & .07 & .00 & .01 & .02 & .08 & .00 & .00 & .00 & .00 & e. 15 & .23 & .00 \\
\hline 14 & .01 & .01 & .03 & .16 & .01 & .00 & .00 & .00 & .00 & e. 80 & .00 & .00 \\
\hline 15 & .33 & .21 & .03 & .00 & .15 & .00 & .00 & .00 & .00 & e1. 20 & .02 & .00 \\
\hline 16 & .01 & .01 & .06 & .01 & .00 & .00 & .08 & .00 & .00 & .00 & .19 & .00 \\
\hline 17 & .00 & .00 & .01 & .02 & .00 & .00 & .64 & .19 & .00 & .06 & .01 & .00 \\
\hline 18 & .00 & .00 & .01 & .00 & .07 & .00 & .12 & .14 & .00 & .00 & .00 & .04 \\
\hline 19 & .00 & .01 & .00 & .01 & .01 & .00 & .00 & .00 & .27 & .00 & .10 & .00 \\
\hline 20 & .00 & .13 & .00 & .35 & .01 & .00 & .00 & .00 & .00 & .04 & .00 & .00 \\
\hline 21 & .00 & .03 & .03 & .00 & .06 & .00 & .35 & .00 & .00 & .14 & .00 & .50 \\
\hline 22 & .00 & .00 & .00 & .00 & .07 & .03 & .08 & .00 & .02 & .00 & .00 & .78 \\
\hline 23 & .00 & .00 & .00 & .28 & .08 & .04 & .01 & .00 & .00 & .53 & .00 & .00 \\
\hline 24 & .00 & .17 & .01 & .00 & .06 & .00 & .48 & .18 & .42 & .00 & .00 & .00 \\
\hline 25 & .00 & .01 & .01 & .01 & .00 & .00 & .04 & .00 & .00 & .00 & .34 & .00 \\
\hline 26 & .00 & .01 & .00 & .00 & .00 & .15 & .05 & .33 & .00 & .16 & .00 & .41 \\
\hline 27 & .04 & .00 & .00 & .00 & .01 & .62 & .00 & .12 & .21 & .00 & 1.02 & .28 \\
\hline 28 & .01 & .29 & .00 & .00 & .13 & .00 & .00 & .01 & .00 & .00 & .72 & .00 \\
\hline 29 & .00 & .26 & .73 & .00 & .01 & .00 & .00 & .00 & .01 & .20 & .01 & .00 \\
\hline 30 & .00 & .00 & .01 & .00 & --- & .01 & .08 & .33 & .00 & .00 & .02 & .00 \\
\hline 31 & .00 & $-\cdots$ & .00 & .00 & --- & .00 & -- & .32 & $-\cdots$ & .67 & .01 & $-\cdots$ \\
\hline TOTAL & 1.63 & 2.00 & 1.19 & 1.06 & 0.98 & 1.48 & 2.08 & 2.41 & 1.81 & 5.54 & 4.73 & 2.55 \\
\hline
\end{tabular}

